\title{
NEUROIMAGING - CLINICAL APPLICATIONS
}

Edited by Peter Bright 


\section{Neuroimaging - Clinical Applications}

Edited by Peter Bright

\section{Published by InTech}

Janeza Trdine 9, 51000 Rijeka, Croatia

\section{Copyright $\odot 2012$ InTech}

All chapters are Open Access distributed under the Creative Commons Attribution 3.0 license, which allows users to download, copy and build upon published articles even for commercial purposes, as long as the author and publisher are properly credited, which ensures maximum dissemination and a wider impact of our publications. After this work has been published by InTech, authors have the right to republish it, in whole or part, in any publication of which they are the author, and to make other personal use of the work. Any republication, referencing or personal use of the work must explicitly identify the original source.

As for readers, this license allows users to download, copy and build upon published chapters even for commercial purposes, as long as the author and publisher are properly credited, which ensures maximum dissemination and a wider impact of our publications.

\section{Notice}

Statements and opinions expressed in the chapters are these of the individual contributors and not necessarily those of the editors or publisher. No responsibility is accepted for the accuracy of information contained in the published chapters. The publisher assumes no responsibility for any damage or injury to persons or property arising out of the use of any materials, instructions, methods or ideas contained in the book.

Publishing Process Manager Sandra Bakic

Technical Editor Teodora Smiljanic

Cover Designer InTech Design Team

First published March, 2012

Printed in Croatia

A free online edition of this book is available at www.intechopen.com

Additional hard copies can be obtained from orders@intechweb.org

Neuroimaging - Clinical Applications, Edited by Peter Bright

p. $\mathrm{cm}$.

ISBN 978-953-51-0200-7 


\section{INTECH open science | open minds}

free online editions of InTech Books and Journals can be found at www.intechopen.com 



\section{Contents}

Preface IX

Chapter 1 Congenital Malformation of the Brain 1

Shahina Bano, Vikas Chaudhary and Sachchidanand Yadav

Chapter 2 Neuroimaging in Inborn Errors of Metabolism 37

Carlos Casimiro, Paula Garcia, Miguel Cordeiro,

Isabel Fineza, Teresa Garcia and Luísa Diogo

Chapter 3 Acquired Demyelinating

Disorders of the CNS in Children 61

R. Govender, Jo M. Wilmshurst and Nicky Wieselthaler

Chapter 4 Landau Kleffner Syndrome: Neuroradiology Aspect 97

José Guevara Campos and Lucía González Guevara

Chapter 5 Neurocristopathies: Role of Glial Cells, Genetic Basis

and Relevance of Brain Imaging for Diagnosis 109

$\mathrm{M}^{\mathrm{a}}$ Carmen Carrascosa Romero and Carlos de Cabo de la Vega

Chapter 6 Role of Neuroimaging in Brain Radiosurgery 129

Tomoyuki Koga and Nobuhito Saito

Chapter 7 The Role of Magnetic Resonance Spectroscopy in the Diagnosis of Ring Enhancing Lesions 145

Eftychia Kapsalaki, Efstathios D. Gotsis,

loannis Tsougos and Konstantinos N. Fountas

Chapter 8 The Role of Functional MRI in

Intracranial Glioma Resection 159

Eftychia Z. Kapsalaki, Dimitrios Verganelakis, Ioannis Z. Kapsalakis,

Efstathios D. Gotsis and Kostas N. Fountas

Chapter 9 Neuroimaging in Epileptic Disorders 173

José Augusto Bragatti

Chapter 10 MRI Abnormalities Induced by Seizures 191

Nuno Canas and Pedro Soares 
Chapter 11 Central Nervous System Findings on

Magnetic Resonance Imaging in Children with Epilepsy 211

Teodoro Durá-Travé, Maria Eugenia Yoldi-Petri,

Joaquin Esparza-Estaún, Fidel Gallinas-Victoriano,

Sergio Aguilera-Albesa and Amaia Sagastibelza-Zabaleta

Chapter 12 Robotic Arm and Imaging in Neurosurgical

Stereotactic Interventions: Oblique Insular

Electrodes Implanted in Patients with Epilepsy 251

Afif Afif

Chapter 13 Multimodal MRI of Cerebral Small Vessel Disease 277

Bence Gunda, György Várallyay and Dániel Bereczki

Chapter 14 Neuroimaging of Intracranial Atherosclerotic Disease 301

Maria Khan, Imama Naqvi and Ayeesha Kamran Kamal

Chapter 15 Neuroimaging in Multiple Sclerosis 317

Elisabeth Andreadou

Chapter 16 Impact of Gray Matter Pathology on

Cognitive Function in Multiple Sclerosis 355

Mike Andrea, Guttmann Charles R.G. and Illes Zsolt

Chapter 17 Pseudotumor Cerebri

(Idiopathic Intracranial Hypertension) an Update 373

Eldar Rosenfeld and Anat Kesler

Chapter 18 Dopamine Transporter Imaging for

Distinguishing Between Idiopathic

Parkinson's Disease and Secondary Parkinsonism 401

Chin-Chang Huang, Tzu-Chen Yen and Chin-Song Lu

Chapter 19 Neuroimaging in Fragile

X-Associated Tremor/Ataxia Syndrome (FXTAS) 423

Laia Rodriguez-Revenga, Beatriz Gómez-Ansón,

Esther Granell Moreno, Javier Pagonabarraga and Montserrat Mila

Chapter 20 Non-Conventional MRI Techniques in Neurophychiatric Systemic Lupus Erythematosus (NPSLE):

Emerging Tools to Elucidate the Pathophysiology and

Aid the Diagnosis and Management 441

Efrosini Z. Papadaki and Dimitrios T. Boumpas

Chapter 21 Central Nervous System Tuberculosis 467

Shahina Bano, Vikas Chaudhary and Sachchidanand Yadav

Chapter 22 Imaging of

Metabotropic Glutamate Receptors (mGluRs) 499

Zhaoda Zhang and Anna-Liisa Brownell 
Chapter 23 Molecular Imaging of $\alpha 7$ Nicotinic Acetylcholine Receptors In Vivo: Current Status and Perspectives

Peter Brust and Winnie Deuther-Conrad

Chapter 24 Advances in MR Imaging of Leukodystrophies

559

Eva-Maria Ratai, Paul Caruso and Florian Eichler 



\section{Preface}

Modern neuroimaging tools allow unprecedented opportunities for understanding brain neuroanatomy and function in health and disease. Each available technique carries with it a particular balance of strengths and limitations, such that converging evidence based on multiple methods provides the most powerful approach for advancing our knowledge in the fields of clinical and cognitive neuroscience. In addition to offering fine-grained in-vivo neuroanatomical specification, neuroimaging methods also provide the opportunity to non-invasively explore brain function. The latter - widely employed in the study of cognition, and instrumental in the emergence of the field of cognitive neuroscience - is being increasingly used for clinical purposes (for example, to enhance pre-surgical lesion mapping, to avoid critical networks serving sensory and cognitive functions, and to improve prognostic evaluation for patients in minimally conscious states). Molecular imaging is providing a window on the biological mechanisms associated with disease progression, but rapid advancement in this area is contingent upon the specificity, sensitivity and delivery of the probes and the speed and resolution of the imaging method. Clinical near-infrared spectroscopy (NIRS) is a form of optical imaging increasingly employed to estimate location and extent of cerebral activation via the monitoring of blood haemoglobin levels. Its portability provides the possibility of bedside measurement, and its relatively low cost and straightforward application lends itself well to clinical use. Magnetic resonance spectroscopy (MRS), unlike conventional magnetic resonance imaging (MRI), provides information on the brain's chemical environment (rather than neuroanatomical structure) and the data are most commonly presented as line spectra. This capacity for determining brain metabolite concentrations provides the basis for clinical investigation of, and differentiation between, neurological and neurosurgical conditions. For example, MRS is particularly important for differentiating between high and low grade gliomas, between Alzheimer's disease and non-progressive neurological conditions, and in the detection of changes associated with mild cognitive impairment (often a precursor for dementia). Neurological conditions carry different prognoses and require different forms of immediate treatment and longer term clinical management, and MRS (particularly when combined with clinical information and MRI) can provide critically important information for diagnostic and evaluation purposes.

The scope of this book is not to provide a comprehensive overview of methods and their clinical applications but to provide a "snapshot" of current approaches using 
well established and newly emerging techniques. Richly illustrated overviews of MRI/MRS features associated with congenital brain malformations (Chapter 1, Bano) and inborn errors of metabolism (Chapter 2, Casimiro et al.) provide important reference documents for diagnostic purposes. Chapters 3 (Govender, Wilmshurst \& Wieselthaler) and 4 (Guevara Campos \& González Guevara) focus on acquired disorders in children. In children, disseminated encephalomyelitis is more common than (and a major risk factor for) multiple sclerosis (MS). Following recovery, the potential risk of a relapse occurring is a critical issue for long-term prognosis. Govender et al. present an important overview of criteria employed to predict recurrence and progression to MS, with particular attention given to radiological findings. Guevara Campos and González Guevara focus specifically on Landau Kleffner syndrome (LKS; sometimes called infantile acquired aphasia), in which language comprehension and expression impairments emerge after an initial period of normal development. Diagnosis (and differentiation from autism and other forms of learning disability) can be difficult and contingent upon encephalography (EEG) recordings acquired during sleep. The authors reflect on the advantages of magnetoencephalography (MEG) employed on its own, or in combination with EEG, for differential diagnosis, evaluation and management.

In Chapter 5, Carrascosa Romero and de Cabo de la Vega consider the relevance of genetics and brain imaging findings in the diagnosis of diseases which result from abnormal development or neoplasia of cells derived from the neural crest (neurocristopathies). Koga and Saito (Chapter 6) provide a beautifully illustrated discussion of the use of neuroimaging tools to guide gamma knife radiosurgery. Advances in neuroimaging methods and software have greatly improved precision of radiosurgery for the treatment of structural abnormalities, thereby preserving the function of surrounding tissue. Combined methods have led to further refinements with, for example, high visuospatial resolution afforded by MRI combined with metabolic information from the lesion site (measured with 18F-fluorodeoxyglucose positron emission tomography; FDG-PET) to indicate location and malignancy of gliomas, which in turn informs radiosurgical targeting. Kapsalaki et al. (Chapter 7) consider MRS spectroscopic profiles for differentiation among ring enhancing lesions including high grade gliomas, abscesses and post-radiation necrosis. The potential for misdiagnosis of ring enhancing lesions is an important clinical concern and this overview of spectroscopic signatures associated with various candidate pathologies is a welcome contribution to the literature. In Chapter 8 Kapsalaki et al. turn to the viability of functional MRI (fMRI) for maximising glioma extirpation and limiting the likelihood or level of neurocognitive deficit. The chapter includes a careful consideration of issues associated with fMRI design and analysis, its inherent inferential limitations and a comprehensive review of its application during presurgical planning.

The role of neuroimaging methods in managing epilepsy and identifying suspected areas of abnormality are described by Bragatti (Chapter 9). He includes an overview of the utility of structural, diffusion and functional MRI, MRS, EEG, MEG, PET, single 
photon emission computed tomography (SPECT) and combined approaches. Canas and Soares (Chapter 10) discuss transient abnormal MRI findings associated with seizure activity. This reversibility of neuroimaging features indicates that they are specifically caused by the seizures but their localization significance is unclear. The authors consider the role of MRI techniques for investigating and characterising these abnormalities. Durá-Travé et al. (Chapter 11) present an epidemiological study of epilepsy and epileptic syndromes in 457 children for whom MRI was performed at diagnosis. A number of common MRI abnormalities were observed, particularly when diagnosed in the first years of life, raising the possibility that imaging findings previously considered incidental might be clinically significant with respect to emergence and likely form of epileptic disorder. A role for the insula as part of a distributed network involved in medial temporal lobe epilepsy has been implicated as far back as the 1940s, but its deep position behind the operculum presents difficulties for investigation with depth electrodes. Afif (Chapter 12) describes the advantages of using an oblique (rather than orthogonal) robotic arm guided approach to explore the insular cortex during invasive pre-surgical evaluation of patients with epilepsy.

Cerebral small vessel disease (cSVD) is widely prevalent in elderly people and a risk factor for motor and cognitive impairment (particularly of executive function) and possibly for parkinsonian disorders and dementia. Gunda, Várallyay and Bereczki (Chapter 13) explore the utility of non-conventional MRI techniques (including diffusion and perfusion weighted imaging (DWI/PWI) and diffusion tensor imaging (DTI)) for enhancing our understanding of cSVD. Intracranial atherosclerotic disease (ICAD) is a major cause of ischemic stroke worldwide, but particularly associated with Asian populations. Khan, Naqvi and Kamal (Chapter 14) describe neuroimaging tools available for diagnosis and management of the disease, including diffusion weighted images of infarct patterns and vessel imaging using conventional angiography as well as other complementary approaches, such as transcranial Doppler ultrasound for measuring blood velocity.

The advantages and limitations of conventional structural MRI for the diagnosis and monitoring of MS are discussed by Andreadou (Chapter 15). There is often a disparity between the level of structural abnormality seen on MRI and level of cognitive difficulties expressed by the patient with MS. Clinical interview and neuropsychological assessment are vitally impairment, not only from an initial diagnostic perspective but also in terms of longer term planning and care. Mike, Guttman and Illes (Chapter 16) consider cognitive impairment in MS and its relationship to grey matter findings with MRI. Although significant correlations between quantified measures of grey matter integrity and level of cognitive impairment are observed, this relationship requires further exploration and refinement if more targeted and appropriate long term management is to be realised.

Idiopathic intracranial hypertension (also known as pseudotumor cerebri) is a neurological condition (disproportionately occurring in obese women of childbearing age) associated with raised intracranial pressure which is not caused by intracranial 
mass or other disease. Rosenfeld and Kesler (Chapter 17) review presenting signs and the role of neuroradiological evaluation in excluding tumors or vascular lesions that might otherwise explain raised intracranial pressure.

Huang, Yen and Lu (Chapter 18) review the role of SPECT in the diagnosis of idiopathic Parkinson's disease and its differentiation from other conditions characterised by parkinsonisms such as dementia with Lewy Bodies (DLB) and vascular parkinsonism. Accurate diagnosis is clearly critical for treatment and prognosis, and the authors provide a very useful overview of recent developments in dopamine transporter imaging which have led to important advances in this area. Fragile X-associated tremor/Ataxia syndrome (FXTAS), formally identified in 2001, is a late-onset neurodegenerative condition occurring in carriers of a fragile $\mathrm{X}$ gene (FMR1) premutation. Rodriguez-Revenga et al. (Chapter 19) consider the possible role of MRI in the early diagnosis of patients for whom the underlying pathogenic process may begin early in life. Papadaki and Boumpas (Chapter 20) present a comprehensive review of the application of non-conventional neuroimaging techniques in neuropsychiatric systemic lupus erythematosus (NPSLE). Wide variability in the clustering of symptoms presents considerable challenges for diagnosis, and the authors present strong arguments for more widespread use of techniques sensitive to microstructural, haemodynamic and biochemical tissue characteristics. Bano (Chapter 21) reviews important clinical and imaging features associated with tuberculosis of the central nervous system. Conventional MRI has an important role in ensuring accurate clinical diagnosis but the diagnostic sensitivity of infra-tentorial lesions and initial cerebral changes identified with this approach is questionable. MRS can be particularly helpful in differentiating causes of ring enhancing lesions and magnetization transfer imaging (MTI), diffusion and perfusion imaging can also aid in diagnosis and management of the condition.

Molecular cloning has identified eight metabotropic glutamate receptor (mGluR) subtypes, each with distinct patterns of expression. However, the characteristic roles of these different mGluRs in physiological processes are not well understood. Identification of subtype-specific compounds may potentially act as therapeutic agents for the treatment of a wide range of conditions (including progressive brain disease, epilepsy and cerebral ischemia) all of which are associated with abnormal mGLuR activation. Zhang and Brownell (Chapter 22) present a superb overview of mGluR functions, radiotracer development and imaging findings to date, and raise important clinical implications and recommendations for further research. Brust and DeutherConrad (Chapter 23) provide an overview of the current status of molecular imaging of the $\alpha 7$ nicotinic acetylcholine receptor, considered an important regulator of cellular function with probable involvement in a range of neurodegenerative diseases, schizophrenia, and other disorders. Ratai, Caruso and Eichler (Chapter 24) consider the role of advanced magnetic resonance technologies for the imaging of leukodystrophies which are (mostly inherited) genetic mutations that produce progressive cognitive and physical impairment in children previously considered well. 
These disorders are caused by maturational white matter abnormalities and therefore white matter tractography (using DTI) can offer advantages over conventional MRI in the detectability of clinically relevant microstructural features. High field strength structural MR scanning (up to 7 tesla for clinical and research use in humans) is now available in some countries, and this greatly enhances the spatial resolution over that offered by standard clinical facilities (typically 1.5 or 3 tesla). Similarly, better MRS spectral resolution is achieved at higher field strengths due to improved signal-tonoise and chemical-shift dispersion. The authors describe how developments in MR technology have provided clear clinical and practical benefits but also acknowledge their methodological drawbacks and limitations. Their overriding conclusions and recommendations are common to all fields of research in clinical neuroscience covered in this book: that it is only though the combined application of different neuroimaging methods, in which both strengths and limitations of each mode of investigation are taken into account, that rapid advances in knowledge of health and disease are likely to be realised.

Peter Bright Anglia Ruskin University in Cambridge United Kingdom 



\title{
Congenital Malformation of the Brain
}

\author{
Shahina Bano, Vikas Chaudhary ${ }^{2}$ and Sachchidanand Yadav ${ }^{3}$ \\ ${ }^{1}$ Department of Radiodiagnosis, G.B. Pant Hospital \& Maulana \\ Azad Medical College, New Delhi \\ ${ }^{2}$ Department of Radiodiagnosis, Employees' State Insurance \\ Corporation (ESIC) Model Hospital, Gurgaon, Haryana, New Delhi \\ ${ }^{3}$ Department of Radiodiagnosis, Dr. Ram Manohar \\ Lohia Hospital \& PGIMER, New Delhi \\ India
}

\section{Introduction}

More than 2000 different congenital malformations of the brain have been described in the literature, and their incidence is reported to be about 1 percent of all live births. ${ }^{1}$ Since the congenital anomalies of the brain are commonly encountered in day to day practice, it is very important for every radiologist to be familiar with the basic imaging findings of common congenital anomalies to make a correct diagnosis necessary for optimum management of these conditions. Magnetic resonance imaging (MRI) is very useful in studying these malformations. The aim of this chapter is to provide an overview of all important and routinely encountered congenital malformations of the brain.

\section{Normal brain development}

Congenital anomalies of the brain are extremely complex and are best studied by correlating with embryological development. Basic events in normal brain development includes the following four stages: ${ }^{1}$

Stage 1: Dorsal Induction: Formation and closure of the neural tube

- Occurs at 3-5 weeks

- Three phases: Neurulation, canalization, retrogressive differentiation

- Failure: Neural tube defects (Anencephaly, Cephalocele, Chiari malformations) and Spinal dysraphic disorders.

Stage 2: Ventral Induction: Formation of the brain segments and face

- Occurs at 5-10 weeks of gestation

- Three vesicles (prosencephalon, mesencephalon, and rhombencephalon) form the cerebral hemispheres/thalamus, midbrain, and cerebellum/brain stem respectively.

- Development of face

- Failure: Holoprosencephalies, Dandy Walker malformation, Cerebellar hypoplasia/dysplasia (Chiari-IV), Joubert syndrome, Rhombencephalosynapsis, Septooptic dysplasia, and facial anomalies. 


\section{Stage 3: Migration and Histogenesis}

- Occurs at 2-5 months of gestation

- Neuronal migration from germinal matrix to the cortex

- Cortical organization

- Failure of Migration: Heterotopias, Agyria-pachygyria, Polymicrogyria, Lissencephaly, Schzencephaly, Corpus callosal agenesis, Lhermitte-Duclos disease.

- Failure of Histogenesis: Aqueductal stenosis, Arachnoid cyst, Megalencephaly, Micoencephaly, Neurocutaneous syndrome (phakomatoses), Congenital vascular malformation, and Congenital neoplasms.

\section{Stage 4: Myelination}

- $\quad$ Begins at 6 months of gestation; matures by 3 years.

- Progresses from caudal to cephalad, from dorsal to ventral, and from central to peripheral

- $\quad$ Failure: Dysmyelinating diseases, Metabolic disorders.

\section{Classification of congenital malformation of brain}

A number of classification systems have been proposed, but with regards to our basic purpose, simplified classification of brain malformations has been taken into account, which is as follows: 1,2

\section{a. Disorders of Organogenesis}

- Neural tube closure

- Diverticulation and cleavage

- Sulcation/cellular migration

- Cerebellar hypoplasia/dysplasia

b. Disorders of Histogenesis

- Neurocutaneous syndromes (Phakomatoses)

\section{c. Disorders of Cytogenesis}

- Congenital vascular malformations

- Congenital neoplasms of brain

d. Disorders of Myelination

- Leukodystrophies

\section{Imaging features of congenital malformations of the brain}

\subsection{Disorders of organogenesis}

\subsubsection{The chiari malformations}

Chiari I Malformation ${ }^{1,2}$ (Figure 1)

- $\quad$ Termed as tonsillar ectopia

- Herniation of elongated peg like cerebellar tonsils into the upper cervical canal through the foramen magna.

- Abnormal tonsillar descent below the opisthion-basion line

- At least $6 \mathrm{~mm}$ in first decade

- $5 \mathrm{~mm}$ in the $2^{\text {nd }} / 3^{\text {rd }}$ decade 
- $4 \mathrm{~mm}$ between $4^{\text {th }}-8^{\text {th }}$ decade, and

- $3 \mathrm{~mm}$ by $9^{\text {th }}$ decade

- Causes of abnormal tonsillar descent

- Congenital asymptomatic tonsillar ectopia

- Intracranial hypotension due to chronic CSF leak (sagging brain)

- Long-standing compensated hydrocephalus

- Craniovertebral anomalies (Basilar invagination, Platybasia, Atlantooccipital assimilation)

- Associated anomalies

- Syringohydromyelia(30-60\%)

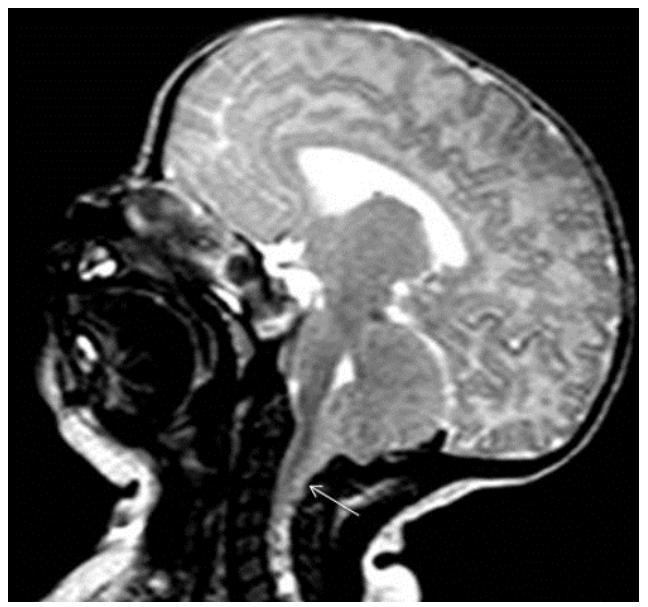

Fig. 1. Chiari I Malformation. Sagittal T1W MR scan of brain shows peg like low lying tonsil in upper posterior cervical canal (thin white arrow). The cerebellar tonsils lie more than $10 \mathrm{~mm}$ below the foramen magnum. Note mild enlargement of supratentorial ventricular system.

\section{Chiari II Malformation1,2 (Figure 2)}

- Complex anomaly involving skull, dura, brain, spine and the cord

- Skull and dural involvement

- Luckenschadel (lacunar skull), concave clivus and petrous ridges

- Small and shallow posterior fossa with low lying transverse sinuses and torcular herophilli

- Hypoplastic tentorium cerebelli with gaping (heart shaped) incisura

- Hypoplastic, fenestrated falx cerebri with interdigitating gyri

- Gaping foramen magnum

- Brain involvement

- Cascading protrusion of vermian nodulus, fourth ventricle choroid plexus and the medulla into the spinal canal with formation of cervicomedullary spur and kink

- Upward herniation of cerebellar hemispheres and vermis through gaping incisura (towering cerebellum) producing tectal deformity (beaked tectum)

- Cerebellar hemispheres creep around to engulf the brain stem

- Large massa intermedia

- Hydrocephalus (90\%) with serrated appearance of lateral ventricles 

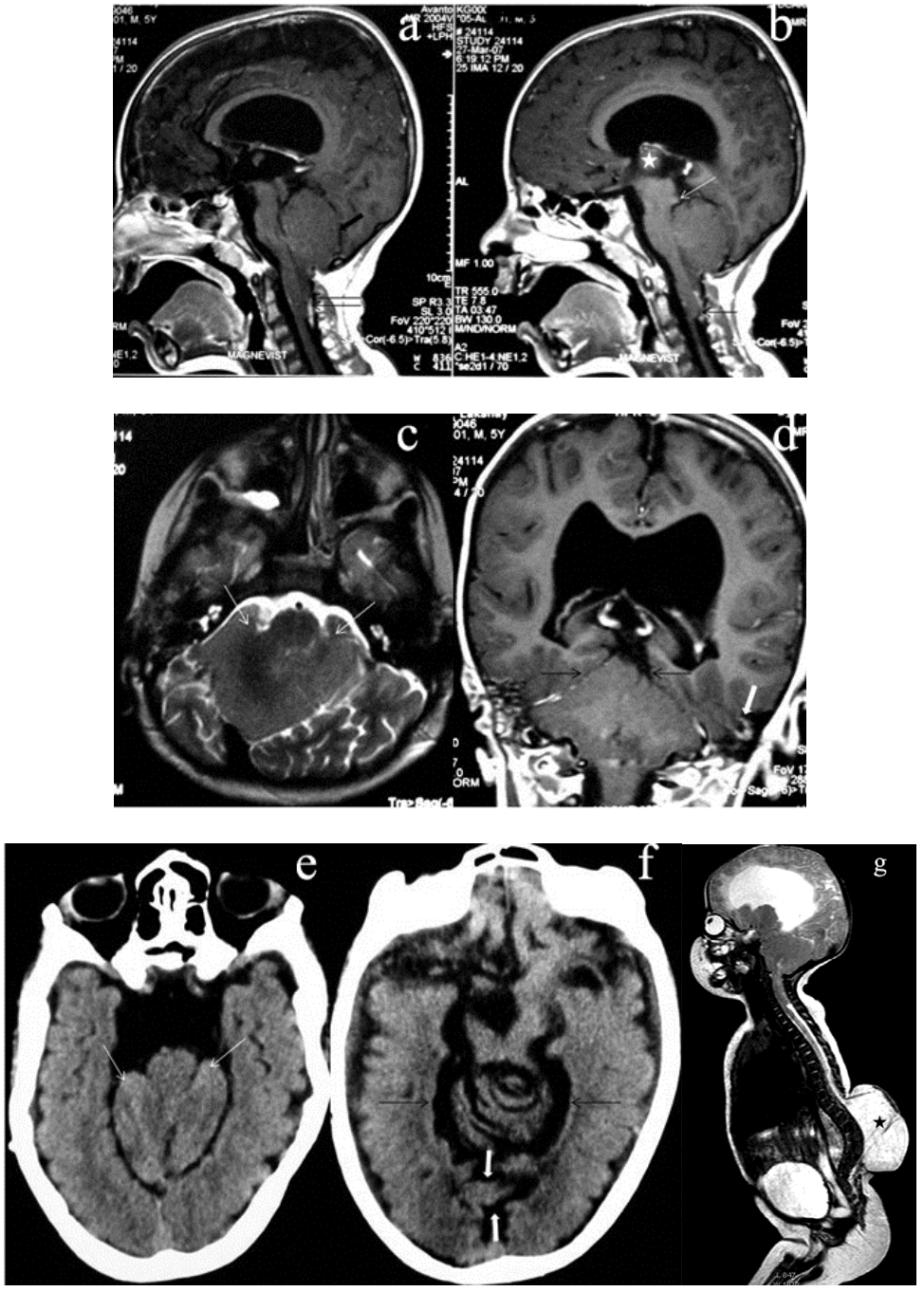

Fig. 2. Chiari II Malformation. Sagittal T1W MR images(a,b) of brain show a small posterior fossa, low lying tetorium cerebelli (thick black arrow), cascade of inferiorly displaced vermis and choroid plexus into upper posterior cervical canal (double thin black arrow), medullary spur (thin white arrow), large massa intermedia(asterix). The cerebellar hemispheres extend antero-medially(thin white arrow) to engulf the brain stem on axial T2W(c) image. Coronal T1W image(d) reveals low lying torcular herophili(thick white arrow), gaping incisura and towering cerebellum(thin black arrow). Also note bilateral hydrocephalus. Plain axial CT scan $(e, f)$ another patient shows cerebellar hemispheres engulfing the brain stem, wide gaping incisura plugged with upward herniating dysmorphic cerebellum(thin black arrow), gross gyral interdigitation and stenogyria (thick white arrow). Screening of whole spine in another patient with ACM-II demonstrates associated lumber myelomeningocele (asterix) on sagittal T2W image (g). 
- $\quad$ Spine and cord involvement

- Myelomeningocele (nearly $100 \%$ )

- Syringohydromyelia $(70-90 \%)$

- Diastematomyelia

- Associated anomalies

- Corpus callosal dysgenesis, heterotopias, polymicrogyria

Chiari III Malformation 1,2,3 (Figure 3)

- $\quad$ Features of Chiari II malformation with a low occipital or high cervical encephalocele

- The encephalocele may contain meninges, cerebellum, occipital lobe or brain stem. Cisterns and dural sinuses may also be present

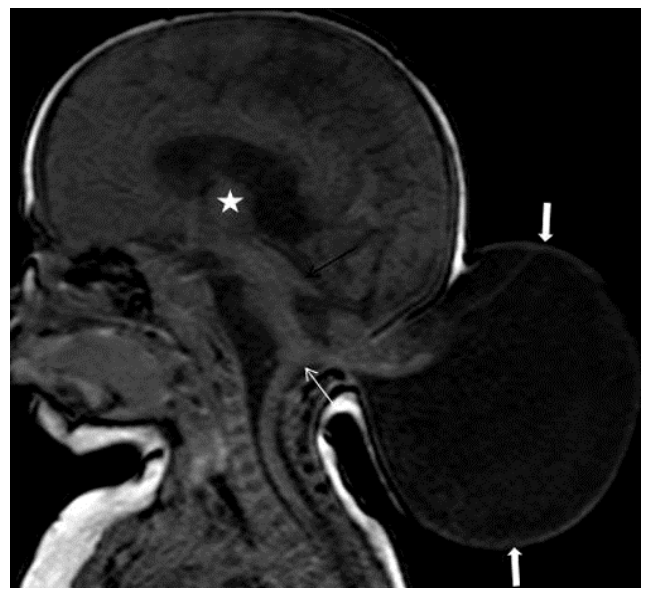

Fig. 3. Chiari III Malformation. Sagittal T1w MR scan shows features of ACM II, evidenced as a large massa intermedia (asterix), tectal beaking (thin black arrow), inferior herniation of the cerebellar tissue through the foramen magnum into the upper posterior cervical canal (thin white arrow), and a low occipiltal meningoencephalocele (thick white arrow).

\section{Chiari IV Malformation ${ }^{1,2}$}

- Severe cerebellar hypoplasia or dysplasia, small brain stem and large posterior fossa CSF space

- No hydrocephalus and other CNS anomalies

\section{Cephaloceles ${ }^{1,2,4}$ (Figure 4-10)}

- Characterized by protrusion of intracranial contents through a congenital defect in the dura and skull

- Usually located at or near the midline.

- Pathological classification of cephaloceles (based on the contents of the herniated sac)

- $\quad$ Meningocele (leptomeninges and CSF)

- Meningoencephalocele (leptomeninges, CSF and brain)

- Meningoencephalocystocele (leptomeninges, CSF, brain and ventricles)

- Atretic cephalocele (small nodule of fibrous fatty tissue)

- $\quad$ Gliocele (CSF lined by glial tissue) 
- $\quad$ Anatomic classification of cephalocele (based on location)

- Occipital - most common in America, Europe

- Parietal

- Temporal

- Frontal or Frontoethmoidal (Sincipital) - most common in Asia

- Transsphenoidal - uncommon

- Nasal

- The herniated brain dysgenetic and non-functional

- Absence or erosion of the crista galli with enlargement of foramen cecum is a constant feature of a nasal cephalocele.

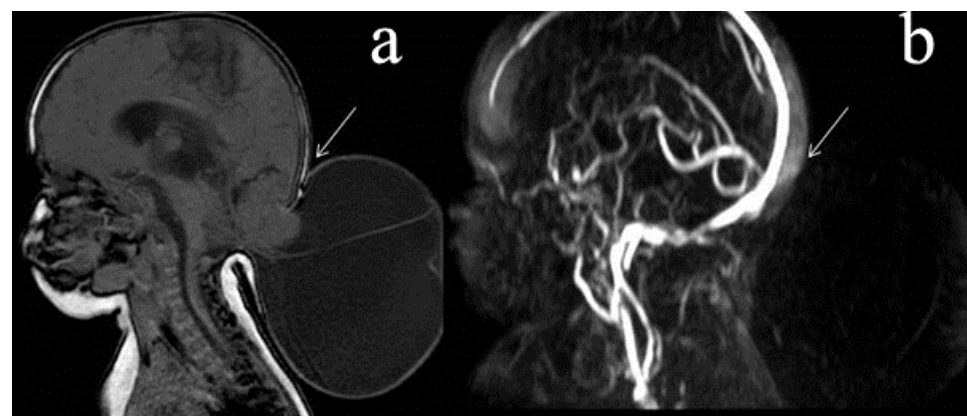

Fig. 4. Occipital cephalocele. Sagittal T1W (a) image shows herniation of severely dysplastic cerebellar tissue and the occipital lobe into a large CSF containing sac through an osseous defect in the occipital bone(thin white arrow). Thin strand of dysplastic brain tissue or septa can be seen traversing the CSF within the sac. Also note small posterior fossa, and deformed brain stem. 2D Time-of- flight venogram (b) demonstrates no herniation of dural venous sinuses in the cephalocele sac. This is important information for the surgeons.

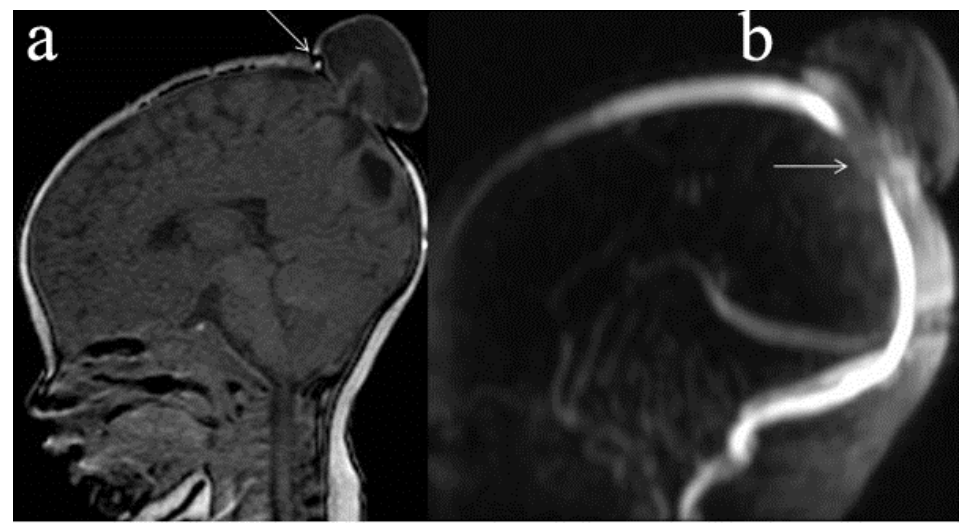

Fig. 5. Parietal cephalocele. Sagittal T1W(a) image of brain shows a small parietal cephalocele containing CSF and dysplastic brain tissue(thin white arrow). 2D TOF venogram $(b)$ shows non visualization of small segment of superior sagittal sinus in the region of osseous defect consistent with sinus thrombosis(thin white arrow). 
- Differential diagnosis of a nasal cephalocele includes congenital nasal masses (e.g. dermoid) where crista galli is present but split.

- Antenatal ultrasound and MRI are useful in evaluation of content of the sac. MR venography is useful to assess major dural sinuses within the herniated sac, which may be responsible for major bleeding during surgery. CT is useful in demonstrating bony defect.

- Associated anomalies

- $\quad$ Chiari II and III malformation (seen with occipital cephalocele)

- Corpus callosum agenesis, Dandy-Walker malformation (seen with parietal cephalocele)

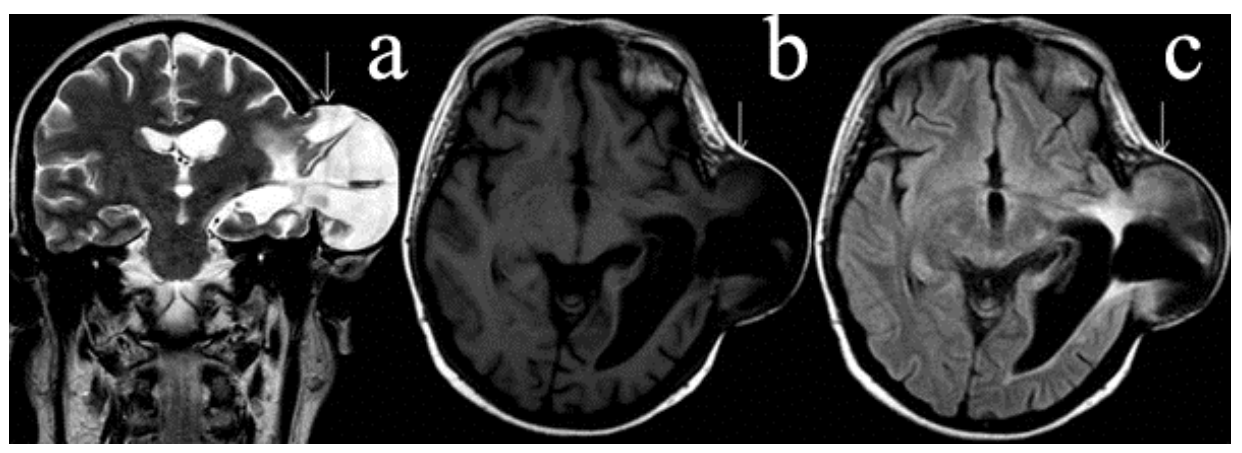

Fig. 6. Temporal cephalocele. Coronal T2W (a), axial T1W (b) and FLAIR (c) image of brain shows a left temporal meningoencephalocele (thin white arrow) containing dysplastic and disorganized left temporal lobe and CSF. Ipsilateral lateral ventricle is dilated and the temporal horn appears stretched towards the bony defect.

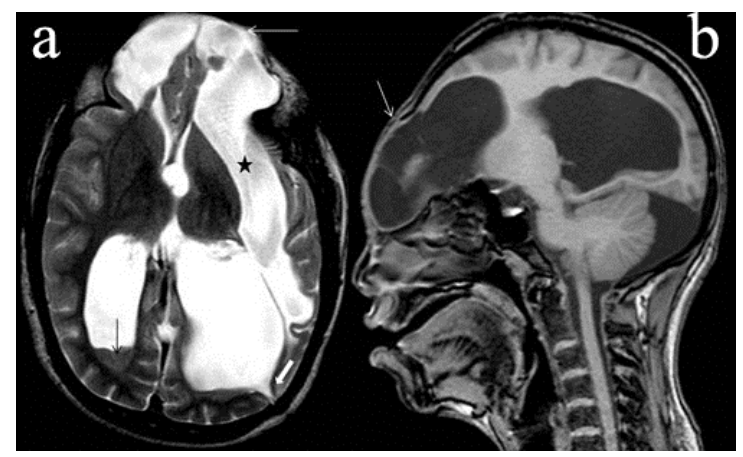

Fig. 7. Frontal cephalocele. Axial T2W(a) and sagittal T1W(b) image of brain demonstrates a large frontal midline meinigoencephalocele (thin white arrow) containing dysplastic frontal lobes, stretched frontal horns and the CSF. Left cerebral hemisphere drsraphism is present, seen as a widely open CSF cleft(asterix) extending across the left external capsule region, and communicating posteriorly with the dilated occipital horn and anteriorly with the cephalocele. Ipsilateral basal ganglion show abnormal morphology. Subependymal heterotopia of right occipital horn (thin black arrow) and a small, narrow left sided posterior scizencephalic cleft (thick white arrow) is also noted. 


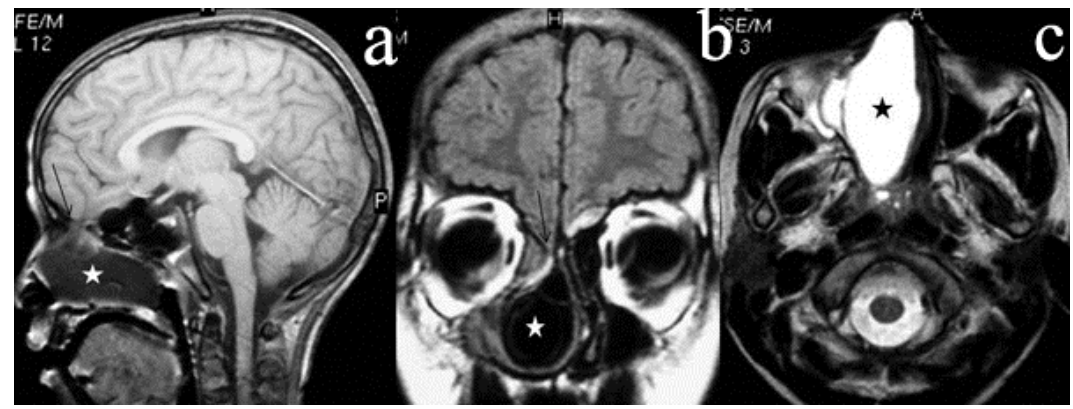

Fig. 8. Nasal cephalocele. Sagittal T1W (a), Coronal FLAIR (b), and axial T2W(c) images reveal a large right sided nasal meningocele containing the CSF (asterix). There is a defect in the skull base in the region of the cribriform plate and crista galli (thin arrow).

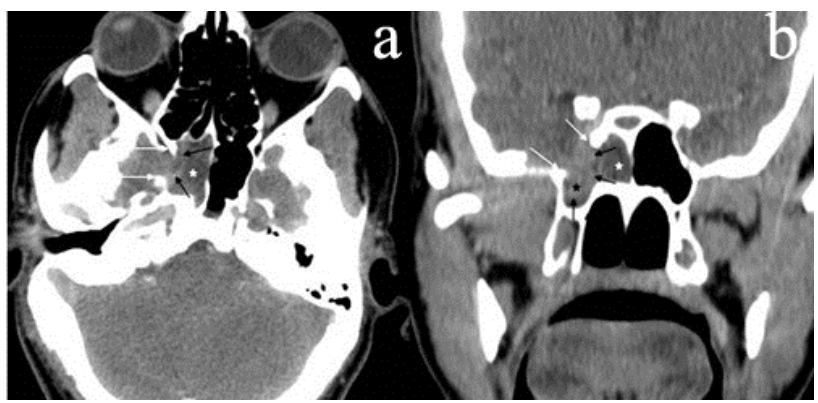

Fig. 9. Sphenoid encephalocele. Axial (a) and coronal (b) NCCT head shows a defect in right lateral wall of sphenoid sinus(thin white arrow) with herniation of small portion of inferomedial part of ipsilateral temporal lobe (black asterix and thin black arrow). Associated sinusitis is seen involving the ipsilateral sphenoid sinus (white asterix).

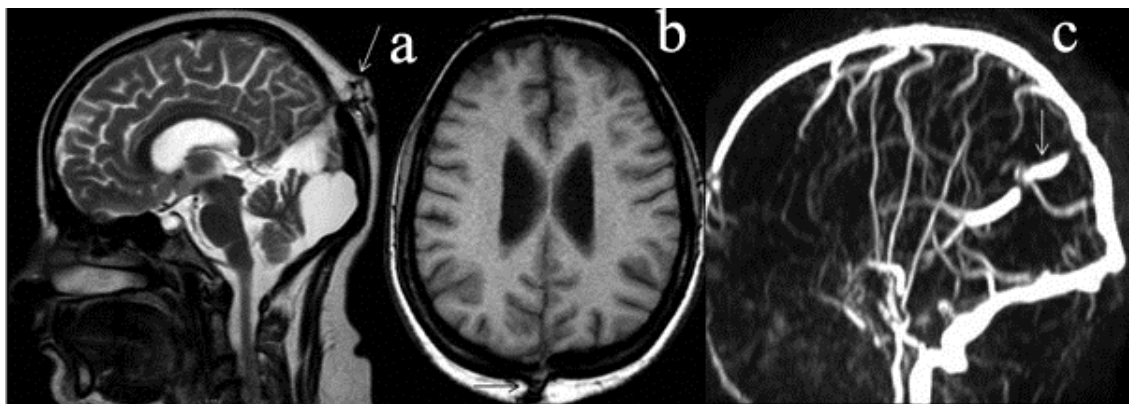

Fig. 10. Atretic occipital cephalocele. Sagittal T2W(a), axial T1W(b) image shows a small subcutaneous mass (thin white arrow) in high occipital region just external to a small defect in the calvarium. Note that the brain is not entering the cephalocele; instead, a thin strand of fibrous tissue is seen extending across the osseous defect, from the surface of the brain to the subcutaneous mass. Small posterior fossa arachnoid cyst is also seen. 2D TOF venogram (c) shows presence of median procencephalic vein within embryonic falcine sinus(thin white arrow) and absence of sagittal sinus. 
Corpus Callosum Agenesis 1,2 (Figure 11,12,13)

- Corpus callosum, a midline commissure connects two cerebral hemispheres

- Develops in cephalocaudal direction, beginning with genu then followed by the body and splenium. The rostrum is last to develop

- Two types: complete or partial

- $\quad$ Complete callosal agenesis

- Absence of entire corpus callosum, cingulate gyrus and sulcus

- High riding $3^{\text {rd }}$ ventricle with spoke like orientation of gyri around it

- Widely separated, parallel and non-converging lateral ventricles.

- Probst bundles (longitudinal white matter tracts) indent superomedial lateral ventricles

- Colpocephaly (dilated occipital horns) common, frontal horns small and pointed

- High incidence of dorsal interhemispheric cyst

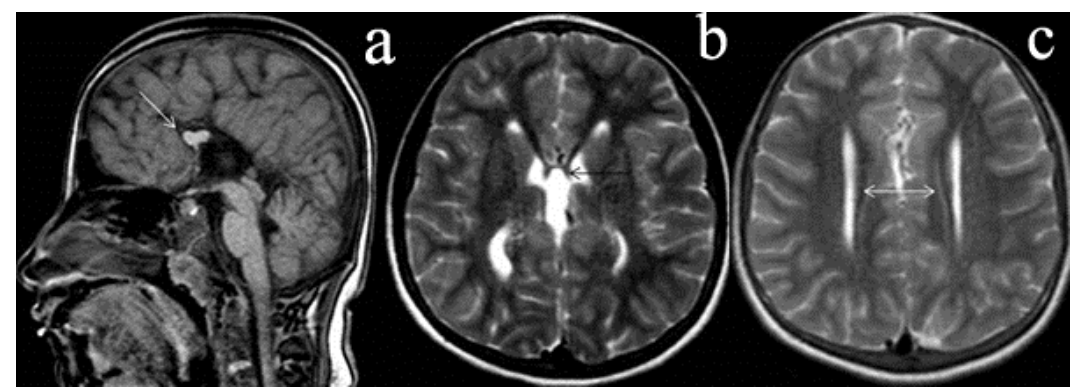

Fig. 11. Corpus callosal agenesis (partial). Sagittal T1W(a) and axial T2W (b,c) image shows presence of only the genu of the corpus callosum (thin white arrow), high riding third ventricle(thin black arrow), widely separated and parallel lateral ventricles(double thin white arrow).

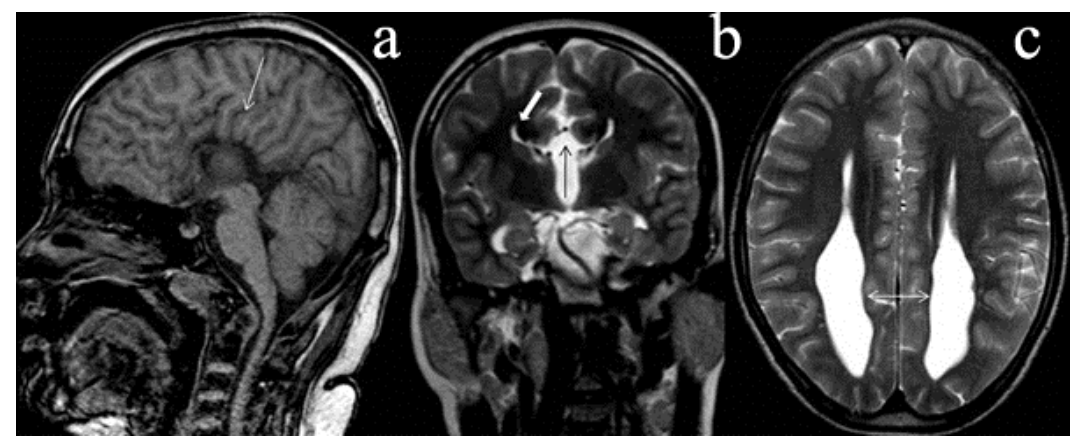

Fig. 12. Corpus callosal agenesis(complete). Sagittal T1W(a) and coronal T2W (b) image shows complete absence of the corpus callosum and cingulate sulcus (thin white arrow), high riding third ventricle communicating with the interhemispheric fissure(thin black arrow), and crescent shaped frontal horns indented medially by white matter tracts of Probst's bundles(thick white arrow). Widely separated and parallel lateral ventricles with colpocephaly are also seen (double thin white arrow) on axial T2W image(c). 
- Partial callosal agenesis

- Splenium and rostrum absent or hypoplastic

- Genu and body present to various degrees

- Associated anomalies

- Migration disorders (heterotopias, lissencephaly, schizencephaly)

- Chiari II malformation

- Dandy-Walker malformation

- Holoprosencephaly

- Corpus callosal lipoma

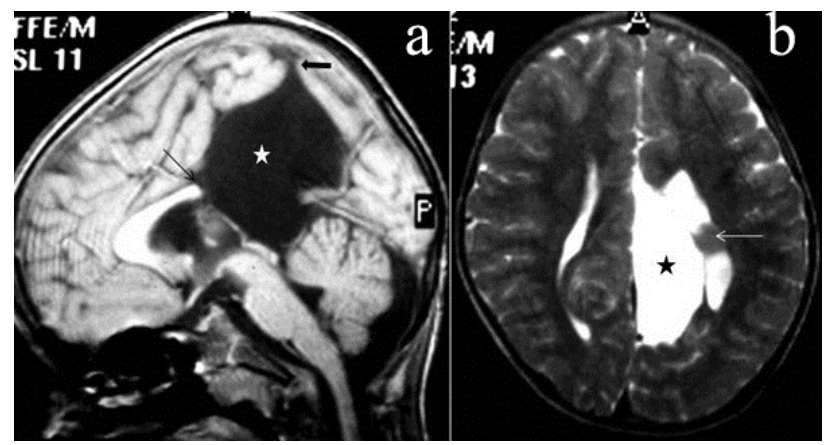

Fig. 13. Corpus callosal agenesis(partial) with dorsal interhemispheric cyst. Sagittal T1W(a) and axial $\mathrm{T} 2 \mathrm{~W}(\mathrm{~b})$ image shows presence of the genu and anterior part of body of the corpus callosum while, the posterior body, splenium and rostrum is absent (thin white arrow). The lateral ventricles are widely separated and a moderately large dorsal interhemispheric cyst (asterix) is present which is seen communicating with the overlying subarachnoid space via a narrow schizencephalic cleft (thin black arrow). Solitary nodular heterotopia can be seen within the body of left lateral ventricle which is mildly dilated (thin white arrow).

\subsection{Disorders of diverticulation and cleavage}

Holoprosencephaly1,2,5 (Figure 14,15,16)

- Characterized by complete or partial failure of cleavage and differentiation of developing cerebrum (prosencephalon) into hemispheres and lobes.

- Cerebellum and brain stem relatively normal

- Classified into three types on the basis of degree of severity

- Alobar (most severe)

- Semilobar ( moderately severe)

- Lobar (mildest form)

- Precise boundaries among these three groups does not exist, intermediate cases may be identified

- Alobar Holoprosencephaly

- Near complete lack of hemispheric cleavage

- Cresent-shaped monoventricle

- Absence of septum pellucidum, corpus callosum, falx cerebri, and interhemispheric fissure

- $\quad$ Fused thalami and basal ganglia 
- Associated anomalies: Dorsal interhemispheric cyst, severe craniofacial anomaly

- Semilobar Holoprosencephaly

- Partial brain diverticulation

- H-shaped holoventricle with rudimentary occipital and temporal horns. Rudimentary third ventricle may be present.

- Septum pellucidum absent, callosal splenium may be formed, falx cerebri and interhemispheric fissure partially developed posteriorly.

- Thalami and basal ganglia partially separated

- Associated anomalies: Dorsal interhemispheric cyst, variable craniofacial anomalies

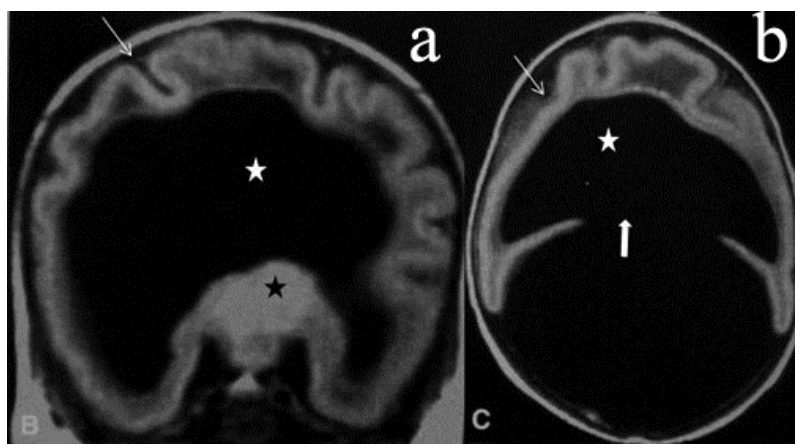

Fig. 14. Alobar holoprosencephaly. T1W coronal(a) and axial(b) MRI brain shows a large central monoventricule(white asterix), thin cortical mantle(thin white arrow) and fused deep gray nuclei seen as a small central mass of gray matter(black asterix). The crescentric monoventricle communicates with a large dorsal cyst (thick white arrow). (From Barcovich AJ. Pediatric Neuroimaging ((4th edn). Philadelphia: Lippincott Williams \& Wilkins).

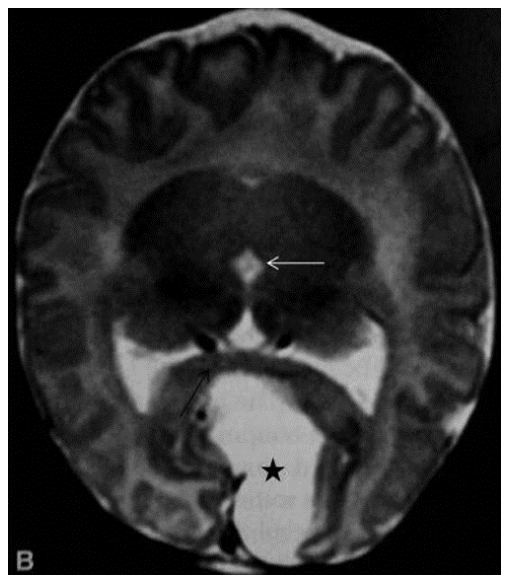

Fig. 15. Semilobar holoprosencephaly. Axial T2W MRI brain shows H-shaped monoventricle with rudimentary third ventricle (arrow). Basal ganglia and thalami are partially fused. Note the presence of callosal splenium(thin black arrow) and a small dorsal interhemispheric cyst(asterix). (From Barcovich AJ. Pediatric Neuroimaging (( $4^{\text {th }}$ edn). Philadelphia: Lippincott Williams \& Wilkins). 


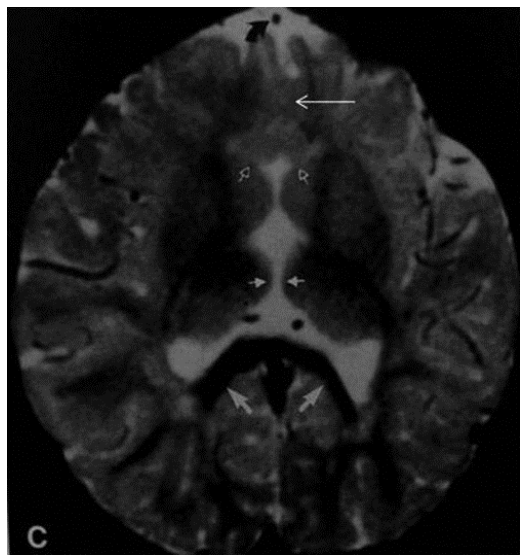

Fig. 16. Lobar holoprosencephaly. Axial T2W MRI brain shows separated basal ganglia and thalami, well formed third ventricle(small closed arrows) and splenium(large closed arrows), rudimentary frontal horns(small open arrows) and partial midline fusion of the frontal lobe cortex(thin large white arrow). The azygous anterior cerebral is also present(curved black arrow). (From Barcovich AJ. Pediatric Neuroimaging ((4th edn). Philadelphia: Lippincott Williams \& Wilkins).

- Lobar Holoprosenceohaly

- Nearly complete brain cleavage

- Squared-off or boxlike configuration of frontal horns due to absence of septum pellucidum

- Septum pellucidum absent, variable degree of anterior extension of corpus callosum, well formed falx cerebri and interhemispheric fissure with some anteroinferior fusion of hemispheres

- Thalami and basal ganglia well separated

- Associated anomalies: Azygous anterior cerebral artery, mild or absent craniofacial anomalies

\section{Septooptic Dysplasia (de Morsier syndrome) 1,2,6}

- $\quad$ Milder form of lobar holoprosencephaly

- Characterized by hypoplastic optic nerves and optic chiasma, and absent septum pellucidum

- Associated hypoplasia of hypothalamic-pituitary axis seen in $2 / 3^{\text {rd }}$ cases

- MR picture of small optic nerves and chiasma, widened anterior recess of $3^{\text {rd }}$ ventricle and suprasellar cistern, and squared frontal horns

- Associated anomalies: Scizencephaly (most common)

\subsection{Disorders of sulcation/cellular migration}

\section{Lissencephaly (Agyria-Pachygyria) 1,2 (Figure 17,18)}

- Refers to "smooth brain" with absent or poor sulcation.

- Can be complete (agyria) or incomplete (pachygyria). Intermediate features of agyria and pachygyria may coexist 
- $\quad$ Three types: Type I, II, and III

- $\quad$ Type I (classical) lissencephaly

- Typical figure eight configuration of brain with oblique and shallow sylvian fissures.

- $\quad$ Thickened cortex with flat broad gyri and smooth gray-white matter interface.

- Colpocephaly

- $\quad$ Associated with Miller-Dieker syndrome.

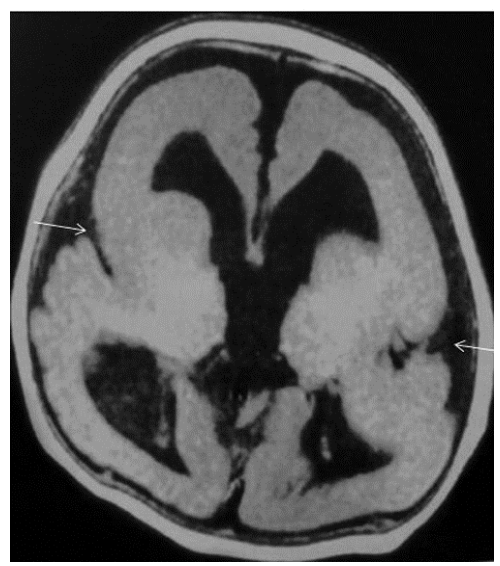

Fig. 17. Lissencephaly. Axial T1W image shows a complete smooth brain with thickened cortex and shallow sylvian fissures(arrows) giving the brain characteristic figure of eight appearance.

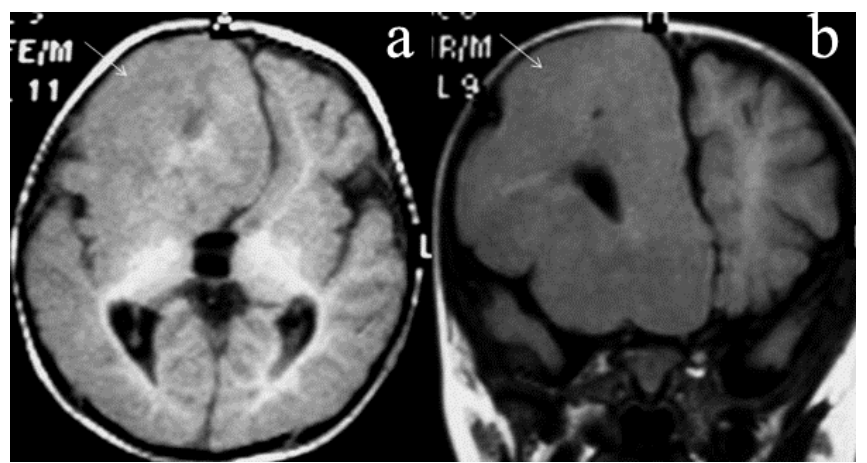

Fig. 18. Lissencephaly with hemimegalencephaly. Axial (a) and coronal (b) T1W image of brain shows right sided hemimegalencephaly and lissencephaly. Right frontal lobe is particularly enlarged, has a disorganized, thickened, nearly agyric cortex with complete loss of cortico-medullary differentiation (arrow). The anterior interhemispheric fissure is displaced to the opposite side by the hypertrophied frontal lobe; ipsilateral frontal horn is also enlarged.

- $\quad$ Type II(Cobblestone) lissencephaly 
- Thickened cortex with polymicrogyric appearance. Concurrent hypomyelination of underlying white matter present.

- Associated with Fukuyama congenital muscular dystrophy, Walker-Warburg syndrome and muscle-eye-brain syndrome.

- $\quad$ Type III(cerebrocerebellar) lissencephaly

- Microcephaly with moderately thickened cortex and hypoplastic cerebellum and brain stem

Nonlissencephalic Cortical Dysplasia 1,2 (Figure 19,20,21)

- $\quad$ Two types: Polymicrogyria and pachygyria

- Polymicrogyria

- characterized by diffusely thickened cortex with irregular, bumpy gyral pattern

- MRI: thick cortex with flat surface, irregular gray-white matter junction

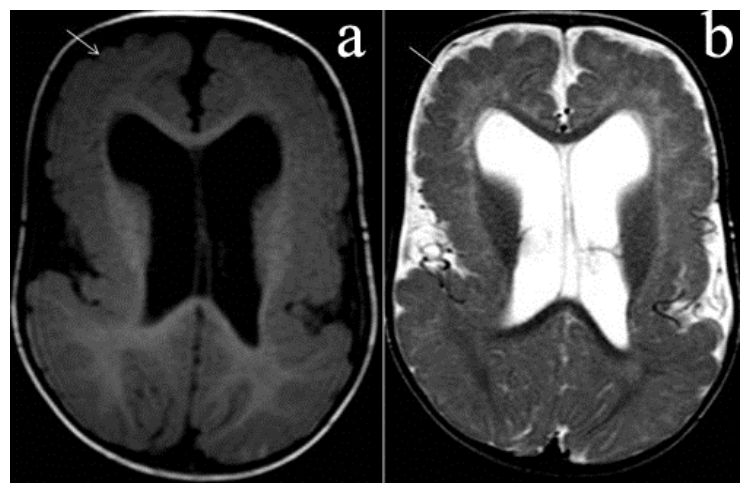

Fig. 19. Polymycrogyria. Axial T1W(a) and T2W(b) image shows shallow sylvian fissures giving figure of eight appearance to the brain. The cortex is diffusely thickened and has irregular bumpy gyral pattern (arrow) with relative paucity of underlying white matter. The cortico-medullary junction is also irregular.

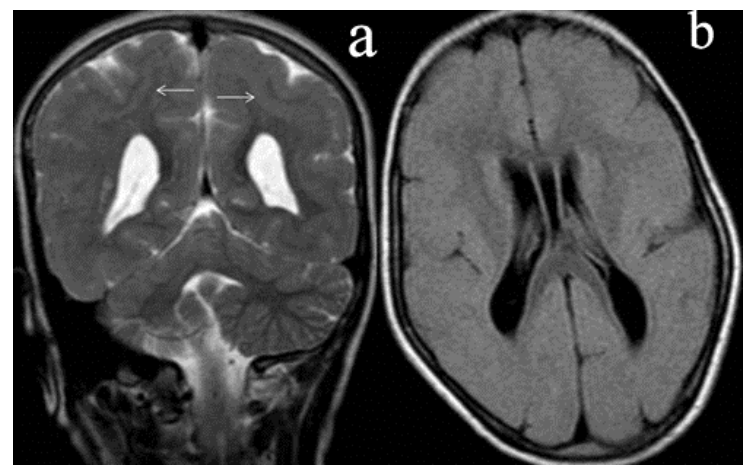

Fig. 20. Patchygyria with band heterotopia. Coronal T2W (a) and axial FLAIR (b) image shows very few, shallow sulci and thickened gyri. Also note band heterotopia, seen as bands of gray matter alternating with the white matter (arrow). 
- Pachygyria

- Focal areas of thickened and flattened cortex with blurred gray-white matter junction

- $\quad$ Both types of cortical dysplasia show relative paucity and marked T2 prolongation of underlying white matter

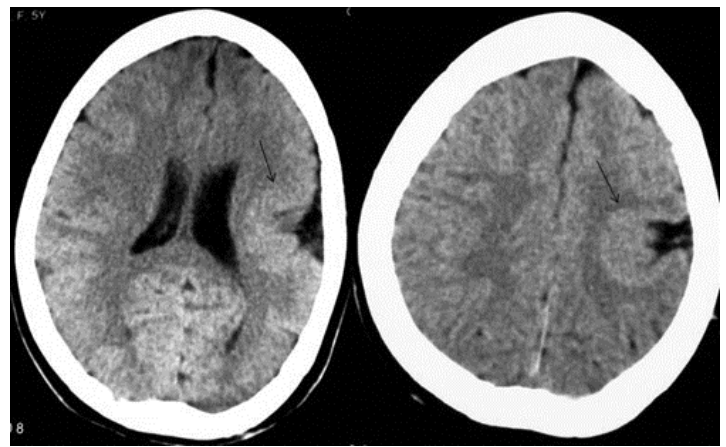

Fig. 21. Focal cortical dysplasia. Axial NCCT brain shows focal cortical dysplasia seen as localized thickening and infolding of the cortex (arrow) in left fronto-parietal junction region with paucity of underlying white matter and prominence of overlying sulcus.

Heterotopias 1,2,7 (Figure 22,23)

- Characterized by the presence of normal neurons at abnormal sites

- Result of arrested neuronal migration from periventricular germinal zone to the cortex along the radial glial fibers

- Two types: Nodular type(common), band (laminar) type (uncommon)

- Nodular type:

- Multiple masses of gray matter which are of variable size

- Common location: subependymal or subcortical

- Focal or diffuse

- Subependymal focal nodules indent the ventricular wall, whereas diffuse heterotopias border the walls of the lateral ventricle and are X-linked.

- Heterotopias are isointense to normal gray matter in all pulse sequences and do not enhance on administration of intravenous contrast. They are best appreciated on medium tau inversion recovery sequences.

- The differential diagnosis is subependymal nodules (SENs) of tuberous sclerosis. On MRI, SENs are not precisely isointense to gray matter, however, occasionally show enhancement after contrast administration. They are often calcified.

- Large dysplastic and disorganized masses of ectopic gray matter may mimic intracranial mass, and produce severe deformity of ipsilateral ventricle.

- Subcortical heterotopias are less frequent.

- Band or laminar type

- A layer of neurons interposed between the ventricle and cortex, seen as alternating layer of gray and white matter band

- The cortex overlying the heterotopia is nearly always abnormal with pachygyria or polymicrogyria. 


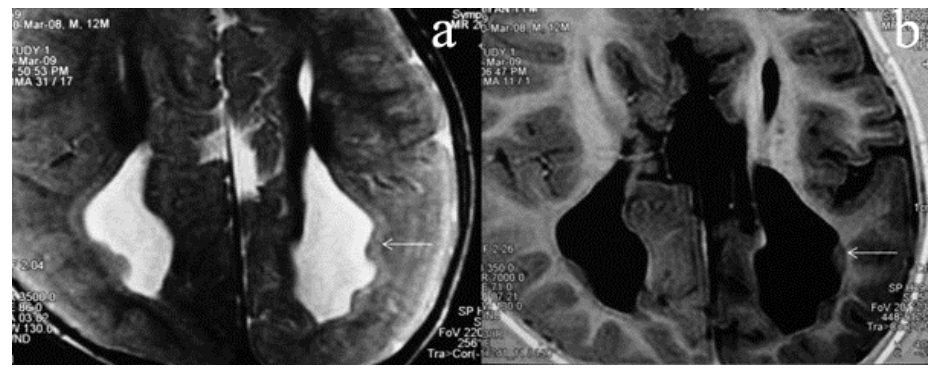

Fig. 22. Nodular heterotopia. Axial T2W(a) and T1W medium tau inversion recovery(b) image shows subependymal nodular heterotopias(arrow). Also note colpocephaly, widely separated and parallel lateral ventricles along with a small dorsal interhemispheric cyst in a patient of partial callosal agenesis.

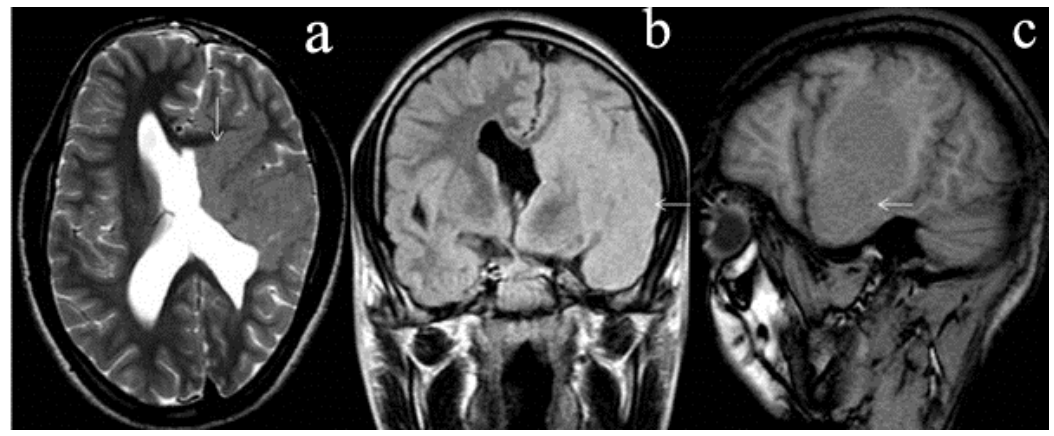

Fig. 23. Focal cortical dysplasia with mass heterotopia. Axial T2W (a) image demonstrates a heterotopic mass of gray matter indenting the left lateral ventricle(arrow). On FLAIR coronal(b) and T1W sagittal(c) image, the ipsilateral temporal lobe has a thickened, agyric cortex and shows complete loss of gray-white matter differentiation.

\section{Schizencephaly (split brain) $1,2,8,9$ (Figure 24,25)}

- Characterized by presence of heterotopic gray matter lined cleft that extends from the ventricular (ependyma) to the periphery (pial surface) of the brain, traversing through the white matter.

- Can be unilateral or bilateral

- $\quad$ Two types: Closed lip (type I) or open lip (type II)

- $\quad$ Closed lip (type I) schizencephaly

- Walls of the cleft oppose each other and there is no intervening CSF

- Imaging shows an outpouching or nippling at the ependymal surface of the cleft

- $\quad$ Open lip (type II) schizencephaly

- Walls of the cleft are widely separated and the cleft is occupied by CSF

- Severe form of open lip schizencephaly has an appearance which is called "basket brain".

- Closest differential is porencephalic cyst in which CSF space is lined by gliotic white matter, in contrast to gray matter lined schizencephaly. 
- Associated anomalies: heterotopias, septo-optic dysplasia, absence of septum pellucidum and callosal dysgenesis

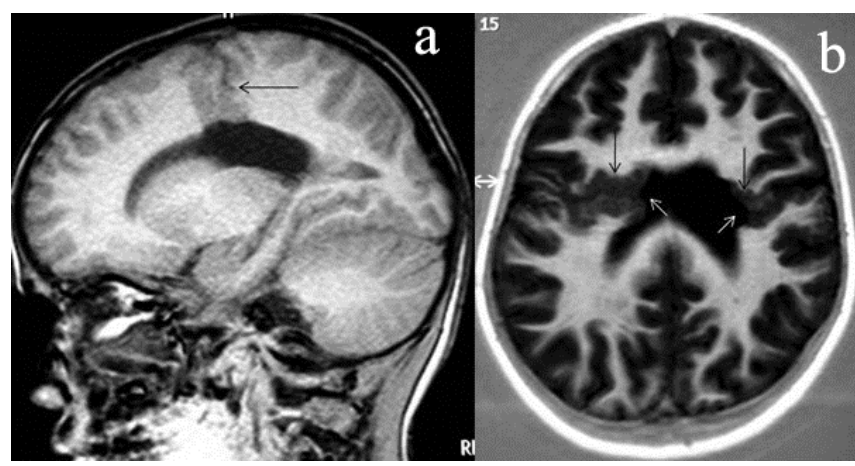

Fig. 24. Bilateral closed lip scizencephaly. Sagittal T1W(a) and axial T1W medium tau inversion recovery $(b)$ image shows bilateral gray matter lined tract with closely opposed walls(thin black arrow), extending from the lateral ventricle to the cerebral convexity. Note nippling in the lateral wall of bilateral lateral ventricles (thin white arrow).

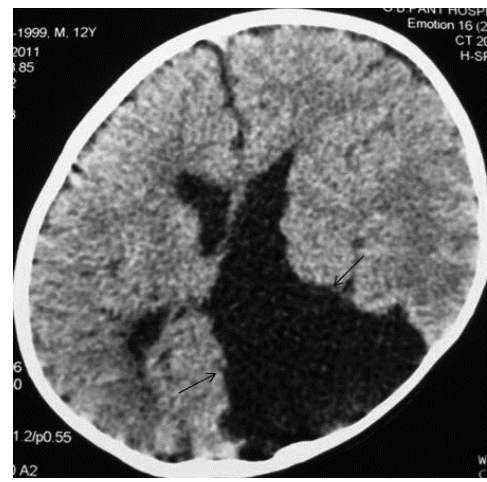

Fig. 25. Unilateral open lip scizencephaly. Axial NCCT brain shows a wide open, gray matter lined CSF cleft extending from left lateral ventricle to the cerebral cortex (arrow).

\section{Hemimegalencephaly $1,2,9,10$ (Figure 18)}

- Hamartomatous overgrowth of a part or all of one cerebral hemisphere

- MR shows enlargement of a part or whole of one cerebral hemisphere, ipsilateral ventricle is frequently dilated and the frontal horn is stretched. The cortex is affected by diffuse migration anomaly (polymicrogyria, pachygyria) and the underlying white matter is gliotic and dysmyelinated

- Rarely, associated enlargement and dysplasia of ipsilateral cerebellar hemisphere and brain stem may be present, a condition referred as total hemimegalencephaly

- Heterotopias may be present

- Associated anomalies: Epidermal nevus syndrome, Klippel-Trenaunay-Weber syndrome, Neurofibromatosis type 1 


\subsection{Disorders of cerebellar hypoplasia/dysplasia}

\section{Dandy-Walker Complex 1,2,11 (Figure 26,27)}

- Includes Dandy-Walker Malformation and Dandy-Walker Variant

- Dandy-Walker Malformation Characterized by

- Large posterior fossa with cystic dilatation of the fourth ventricle; that elevates the tentorium, torcular Herophili and the transverse sinuses above the lamdoid suture (lamdoid torcula inversion)

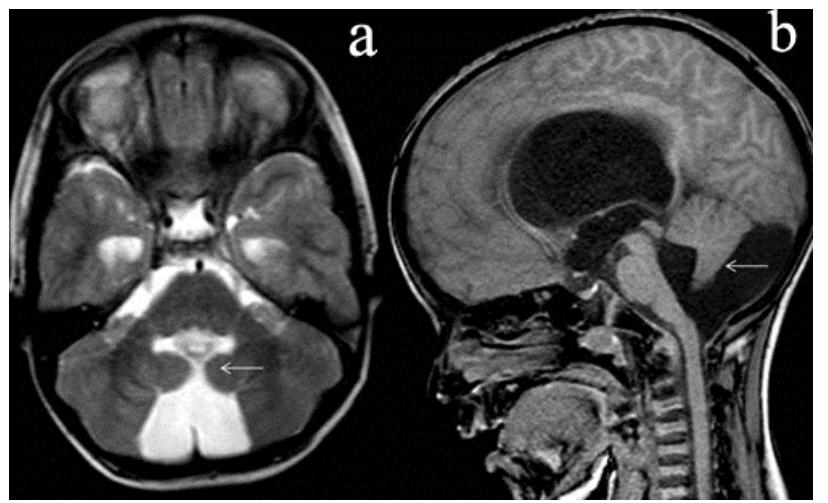

Fig. 26. Dandy-Walker Variant. Axial T2W (a) and sagittal T1W(b) image shows that inferior half of the vermis is hypoplastic (arrow) and the fourth ventricle communicates with enlarged cistern magna. Associated hydrocephalus is also present.

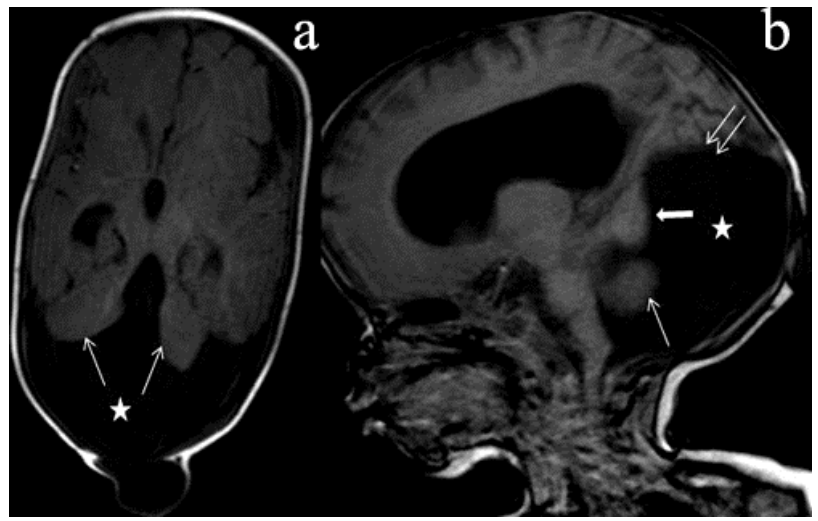

Fig. 27. Dandy-Walker Malformation. T1W axial(a) and sagittal (b) image of brain snows the classic findings of a Dandy-Walker malformation. The posterior fossa is enlarged and filled with a large cyst that is actually a huge fourth ventricle (asterix). The vermis is small, hypogenetic and is everted over the posterior fossa cyst (thick white arrow). The cerebellar hemispheres are hypoplastic (thin white arrow), and appear to be pushed laterally by the cyst on axial image and located beneath the everted vermis on sagittal image. The tentorium is elevated (double thin arrow). Note moderate hydrocephalus and small occipital encephalocele communicating with the posterior fossa cyst. 
- Partial or complete vermian agenesis associated with hypoplastic cerebellar hemispheres. The hypoplastic superior vermis is everted above the cyst and the cerebellar hemispheres which are displaced anterolaterally against the petrous ridge appear "winged outward".

- Brain stem compressed against the clivus

- The cistern magna, albeit present, is effaced

- Hydrocephalus present in $80 \%$ of untreated cases

- Associated anomalies: Corpus callosum agenesis (20-25\%), heterotopias, schizencephaly, cephaloceles

- Closest differentials: Mega cisterna magna and posterior fossa arachnoid cyst where vermis is well developed.

- Dandy-Walker Variant characterized by

- Inferior vermian hypoplasia with communication of fourth ventricle and cistern magna through an enlarged vallecula giving characteristic "Key-hole deformity".

- The tentorial position and posterior fossa size are normal

- Brain stem is usually normal and hydrocephalus is uncommon.

Mega Cistern Magna 1,2 (Figure 28)

- Characterized by variable size dilatation of the cistern magna which, however, freely communicates with both the fourth ventricle and the adjacent subarachnoid spaces (can extend up to the straight sinus superiorly and C1-C2 level inferiorly). In severe cases enlargement of posterior fossa and scalloping of the occipital squamae may be present.

- The fourth ventricle, vermis and cerebellar hemispheres are normal. No hydrocephalus.

- Vermian hypoplasia may mimic mega cistern magna, hence, it is important to look at the size of the vermis for vemian hypoplasia which can be symptomatic.

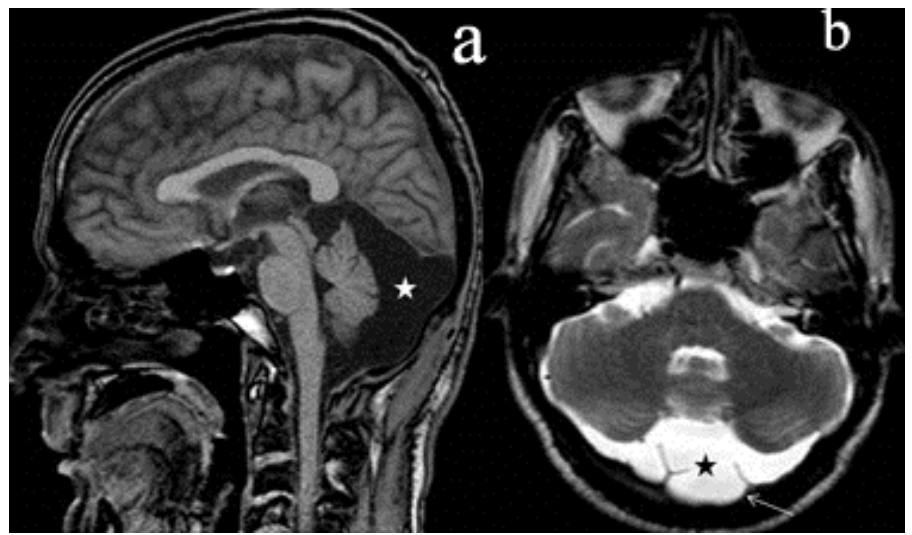

Fig. 28. Mega Cisterna Magna. Sagittal T1W(a) and axial T2W(b) image demonstrates an intact vermis with enlarged posterior fossa CSF space(asterix) that extends superiorly above the vermis and communicates with adjacent CSF space. Prominent scalloping of the occipital squamae is also seen (arrow). No hydeocephalus present. 


\section{Posterior Fossa Arachnoid Cyst 1,2,12 (Figure 29)}

- The posterior fossa arachnoid cyst is a CSF collection within the layers of arachnoid membrane which does not communicates fully with the fourth ventricle or adjacent subarachnoid spaces.

- The fourth ventricle, vermis and cerebellar hemispheres are normal but may be displaced by the cyst.

- On imaging, cyst appears as a non-enhancing round to oval lesion attenuation and signal intensity that closely parallels CSF.

- Differential diagnosis includes Dandy-Walker Malformation, Mega cisterna Magna.

- Note: In general the arachnoid cysts are more frequently supratentorial (75\%), particularly at the temporal poles. Other relative common location includes the posterior fossa, the suprasellar and quadrigeminal cisterns.

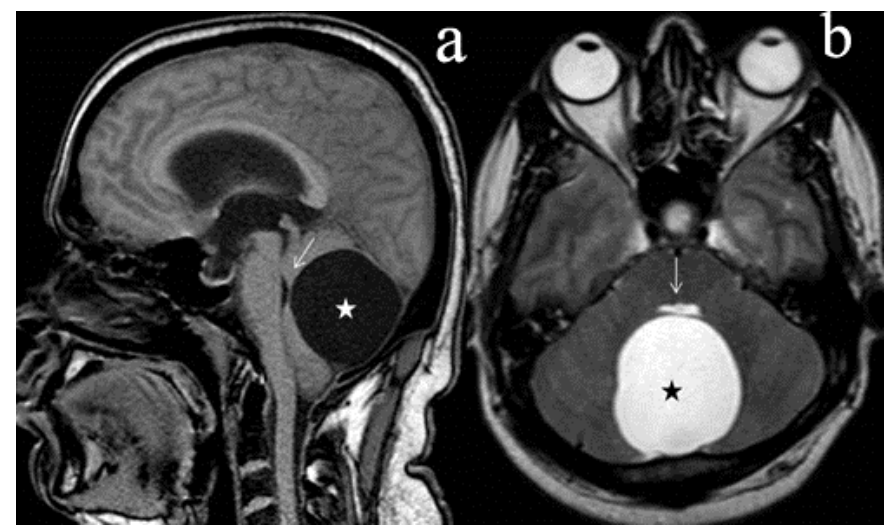

Fig. 29. Posterior fossa arachnoid cyst. Sagittal T1W(a) and axial T2W(b) image shows a classical posterior fossa arachnoid cyst(asterix). Note normally formed but displaced fourth ventricle (arrow) and vermis.

\section{Chiari IV Malformation ${ }^{1,2}$}

- Severe cerebellar hypoplasia or dysplasia, small brain stem and large posterior fossa CSF space

- No hydrocephalus and other CNS anomalies

Joubert's Syndrome (Congenital Vermian Hypoplasia) 1,2,13,14 (Figure 30)

- Characterized by inherited vermian dysgenesis, enlarged superior cerebellar peduncles and a high riding fourth ventricle.

- On MR imaging, the vermis is completely or partially absent. Superior fourth ventricle is bat-wing or umbrella shaped (on axial image) and has a convex roof (on sagittal image). The superior cerebellar peduncles are elongated, thin, running parallel to each other. Isthmus (area of pontomesencephalic junction) is narrow. Midbrain has the typical "molar tooth" appearance.

- Hydrocephalus is absent

- All patients of Joubert's syndrome should be screened for occipital encephalocele (30\%), callosal dysgenesis, cortical dysplasia, hypothalamic hamartoma, and ocular, hepatic \& renal diseases. 


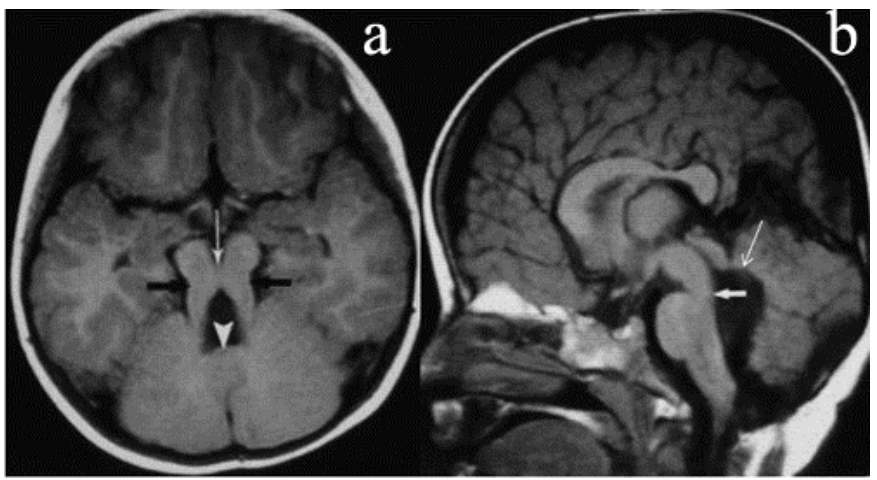

Fig. 30. Joubert's syndrome (Congenital vermian hypoplasia). Axial T1W image(a) at level of mid brain shows characteristic "molar tooth" appearance of the midbrain secondary to the narrow isthmus (thin white arrow) and elongated superior cerebellar peduncles (thick black arrow). Note severe vermian hypoplasia(arrow head). On sagittal T1W(b) image the fourth ventricle has a peculiar upward convex appearance(thin white arrow). Note narrow isthmus(thick white arrow).

\section{Rhombencephalosynapsis 1,2,15 (Figure 31)}

- Rare abnormality characterized by vermian agenesis or hypogenesis combined with midline fusion of cerebellar hemispheres, peduncles and dentate nuclei.

- On MR imaging, the folia and sulci are continuous throughout the midline on axial section and monolobated cerebellum lies in mid-sagittal plane on sagittal image. The Posterior fossa is small.

- Associated anomalies: ventriculomegaly (common), callosal dysgenesis, absent septum pellucidum, cephalocele and schizencephaly.

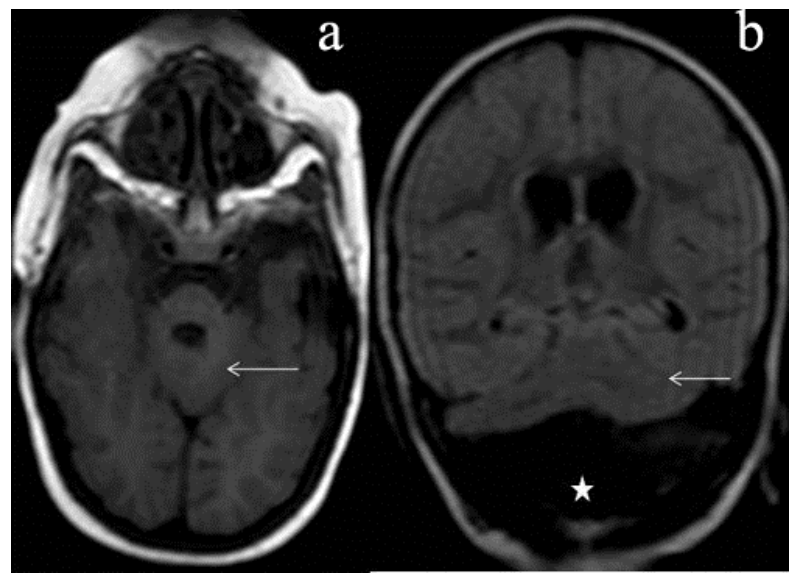

Fig. 31. Rhombenencephalosynapsis. T1W axial(a) and FLAIR coronal(b) MR image demonstrates lack of normal vermis and midline fusion of the cerebellar hemispheres(thin white arrow). Also note markedly enlarged cisterna magna(asterix) indenting the posterior aspect of fused cerebellar hemispheres. 


\section{Tectocerebellar Dysraphism ${ }^{1}$}

- Rare abnormality characterized by vermian hypo-aplasia, occipital cephalocele, and marked deformation of quadrigeminal plate and the brain stem. Fusion of colliculi forms a tectal beak which points towards the site of the cephalocele. The cerebellar hemispheres usually engulf the brain stem.

- Associated anomalies: hydrocephalus and other supratentorial abnormalities.

Lhermitte-Duclos Disease 1,2 (Figure 32)

- Also known as dysplastic gangliocytoma of the cerebellum

- Uncommon cerebellar dysplasia characterized by gross thickening of the cerebellar folias with or without mass effect.

- MR imaging demonstrates a pseudomass having laminated or folial pattern of increased signal on T2-weighted scans. The lesion may or may not enhance after administration of contrast. Mass effect and displacement of fourth ventricle may occur. Calcification or hydrocephalus may be present.

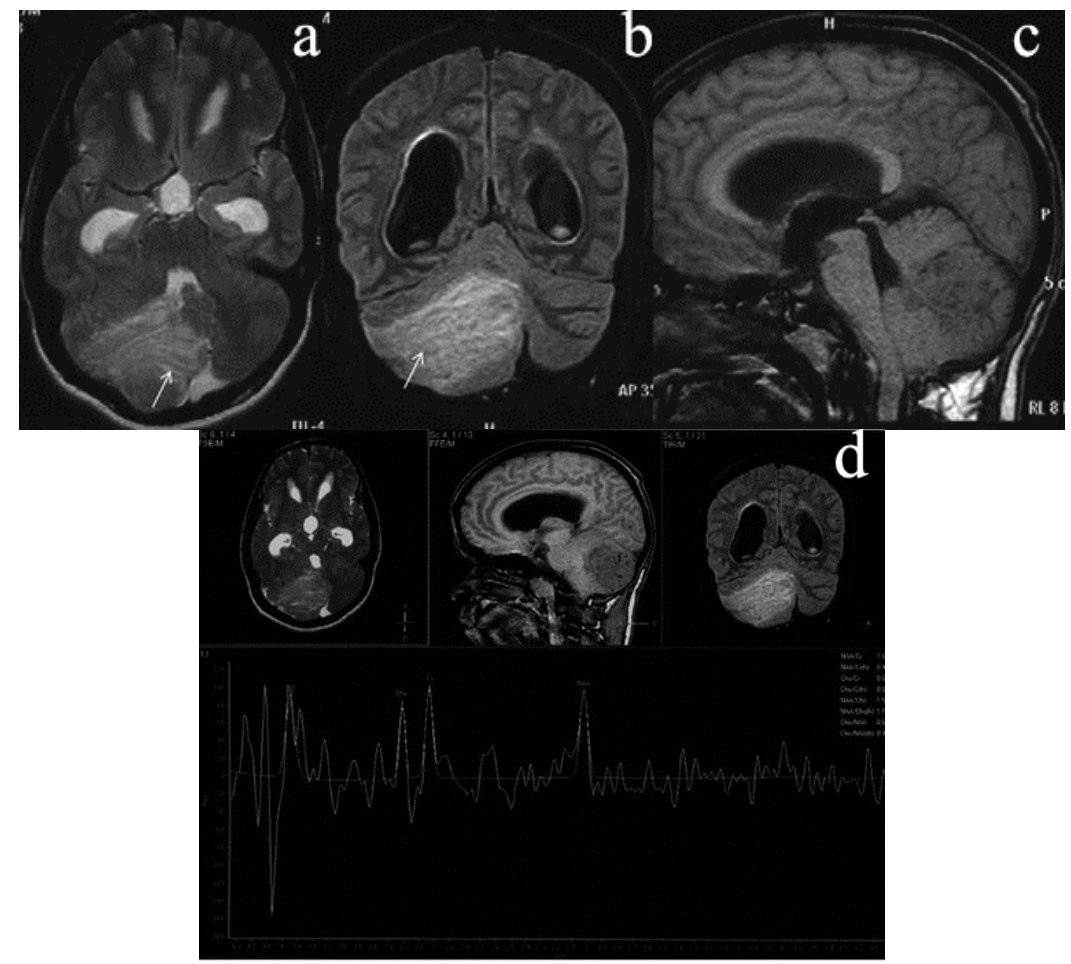

Fig. 32. Lhermitte-Duclos syndrome. Axail T2W (a), coronal FLAIR(b), and sagittal T1W(c) MR image demonstrates a large laminated-appearing T2W/FLAIR hyperintense and T1weighted hypointense mass involving the right cerebellar hemisphere. Note gross thickening of the cerebellar folias (arrow). No perilesional edema present. However, mass effect on the fourth ventricle with moderate hydrocephalus can be seen. Proton MR spectroscopy $(\mathrm{d})$ reveals normal metabolites peak. 
- Associated anomalies: Cowden syndrome (common), megalencephaly, heterotopias, cortical dysplasia, multiple visceral hamartomas and neoplasms.

\subsection{Disorders of histogenesis}

"Neurocutaneous syndromes" or "Phakomatoses" constitute a group of congenital malformations which are characterized by cutaneous lesions associated with CNS anomalies. Some of the common neurocutaneous syndromes are described below.

Neurofibromatosis 1,16,17 (Figure 33-42)

Neurofibromatosis type 1 (NF 1)

- Also known as Von Recklinghausen disease or peripheral neurofibromatosis

- Accounts for $>90 \%$ of all NF cases

- $\quad$ Incidence $=1: 2000$ to 3000 live births

- Diagnostic criteria: two or more of the following findings are present

- Six or more café-au-lait spots $(\geq 5 \mathrm{~mm}$ in pre-pubertal children and $\geq 15 \mathrm{~mm}$ in postpubertal period)

- One plexiform neurofibroma or two or more neurofibromas of any type

- Two or more pigmented iris hamartomas(Lisch nodules)

- Optic nerve glioma

- Axillary or inguinal freckling

- Osseous lesions such as dysplasia of greater wing of sphenoid, pseudoarthrosis

- $\quad$ First degree relative with NF-1

- CNS lesions present in $15-20 \%$ cases. These include

- Optic nerve glioma (most common CNS lesion), may extend to involve the optic chiasma, optic tract, optic radiation and the lateral geniculate bodies.

- Nonoptic gliomas may involve the brain stem, tectum, and periaqueductal region.

- Plexiform neurofibroma is a hallmark of NF-1. It is an unencapsulated neurofibroma along the path of major cutaneous nerve of the scalp and neck, which commonly involves the first (ophthalmic) division of trigeminal nerve. It is often associated with dysplasia of sphenoid bone and bony orbit.

- Non-neoplastic hamartomatous lesions $(80 \%)$ of basal ganglia and white matter. Majority of lesions show no mass effect or contrast enhancement. These lesions may increase in size or number in early childhood, diminishes with age and rarely observed into adulthood.

- Other intracranial lesions include astrocytic proliferation of the retina, intracranial aneurysms, vascular ectasia and a progressive cerebral arterial occlusion disease akin to moya-moya pattern.

- Spinal lesions may include cord astrocytoma / hamartoma, dural ectasia and lateral/anterior intrathoracic meningoceles.

- Skeletal dysplasias may include hypoplasia of sphenoid bone and bony orbit, kyphoscoliosis, scalloping of posterior aspect of the vertebral bodies

Neurofibromatosis type 2 (NF 2)

- Also known as central neurofibromatosis

- $\quad$ Incidence $=1: 50,000$ live births

- Cutaneous manifestations rare 
- CNS lesions present in $100 \%$ cases. These include

- Bilateral acoustic schwannomas, hallmark of NF-2

- Schwannomas of other cranial nerves. Trigeminal nerve is next most frequently involved nerve, albeit, any cranial nerve may be affected (with the exception of the olfactory and optic nerves).

- Meningiomas, often multiple

- Choroid plexus calcification

- Spinal lesions include cord ependymomas, meningiomas, or multilevel bulky schwannomas of exiting roots
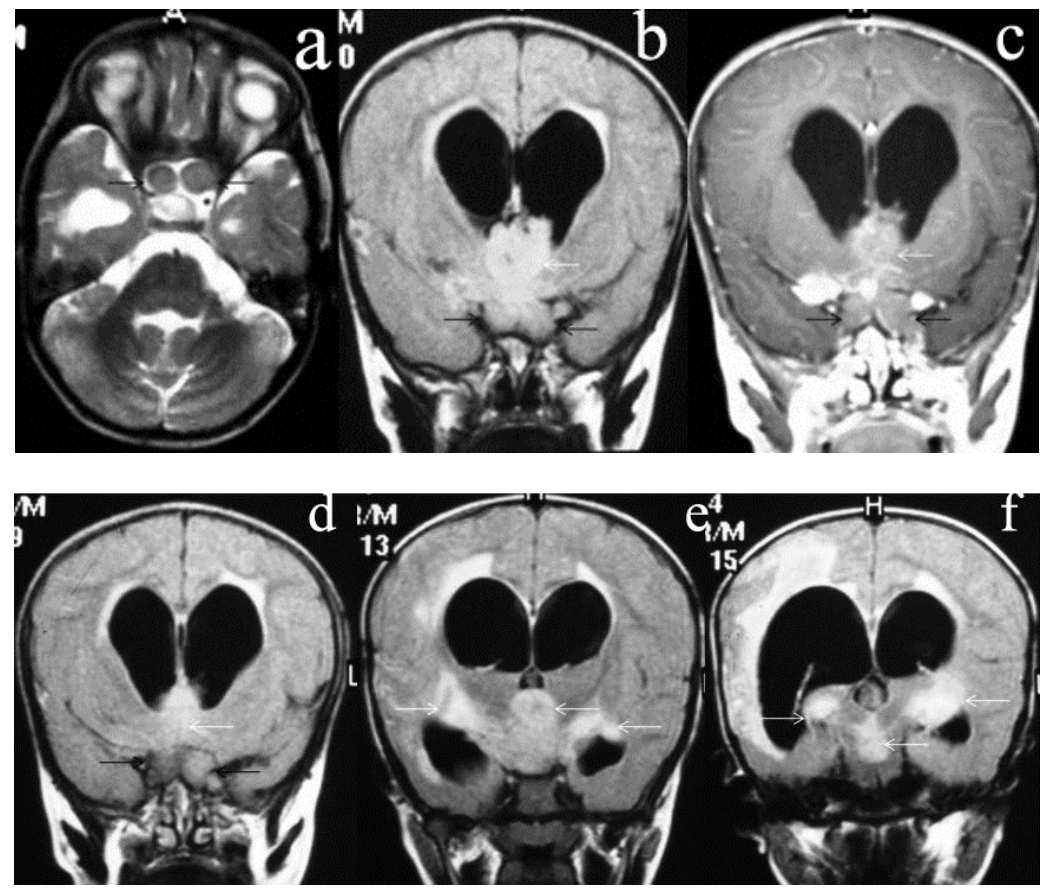

Fig. 33. Neurofibromatosis type 1: Opticochiasmatic-hypothalamic pilocytic astrocytomas. Axial T2-weighted (a) and coronal FLAIR (b) MR image shows enlargement of bilateral optic chiasma (thin black arrows) and ill-defined hyperintensity involving the hypothalamus(thin white arrow) and adjacent brain. Coronal T1-weighted post contrast image (c) demonstrates mild to moderate enhancement of the optic chiasma/hypothalamus but marked enhancement of the lesions involving the adjacent brain parenchyma. Moderate obstructive hydrocephalus is also present. FLAIR coronal images (d-f) of the same patient shows further extension of the optic pathway glioma to involve bilateral medial temporal lobes, basal ganglia region, mid brain and pons (thin white arrow). These lesions appear as ill-defined areas of high signal intensity on Flair images. The enlarged optic chiasma (thin black arrow) and obstructive hydrocephalus are also seen in these images. 


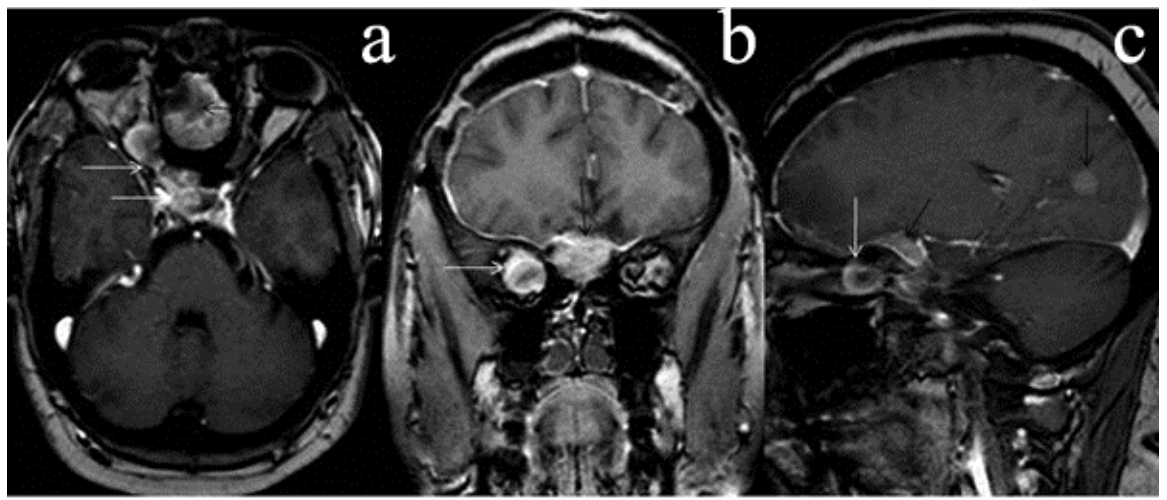

Fig. 34. Neurofibromatosis type 1: Optic nerve glioma with multiple meningiomas. Post contrast T1-weighted axial(a), coronal(b) and sagittal(c) MR images of brain reveal markedly enhancing right optic nerve glioma(thin white arrow) which extends posteriorly to involve the optic chiasma. Associated multiple enhancing meninigiomas are also present (thin black arrow).

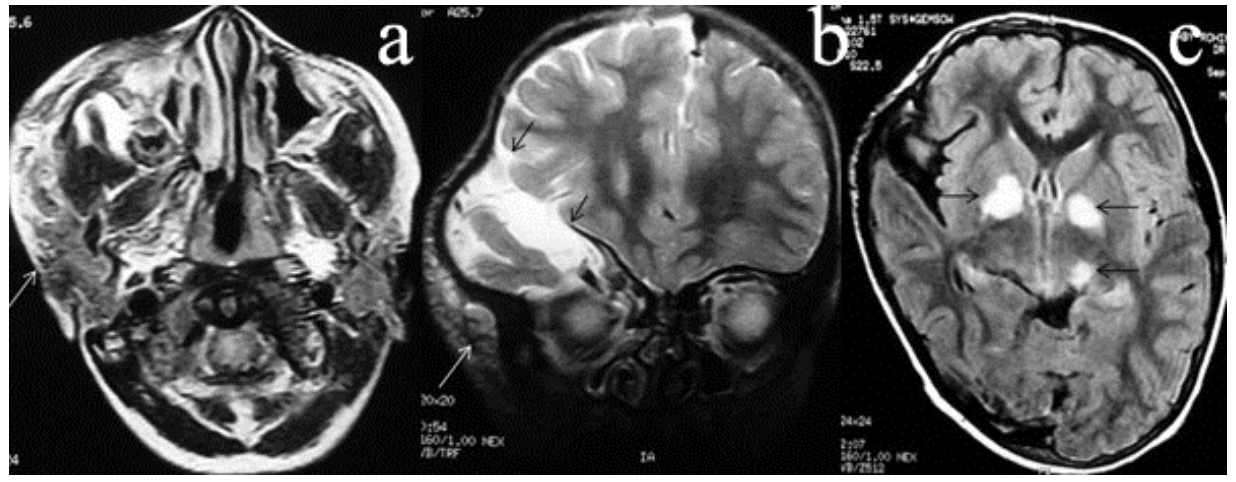

Fig. 35. Neurofibromatosis type 1: Plexiform neurofibroma with sphenoid wing dysplasia. T2W axial(a) and coronal(b) MRI brain shows right temporal and infratemporal plexiform neurofibroma(thin white arrows). Lateral part of the right sphenoid wing is absent and the dysplastic temporal lobe is seen protruding through the dehiscent sphenoid bone into the orbit(thin black arrow). FLAIR axial image(c) of the same patient shows multiple hyperintense nonneoplastic hamartomatous lesions involving bilateral basal ganglia and left midbrain region(arrows). 


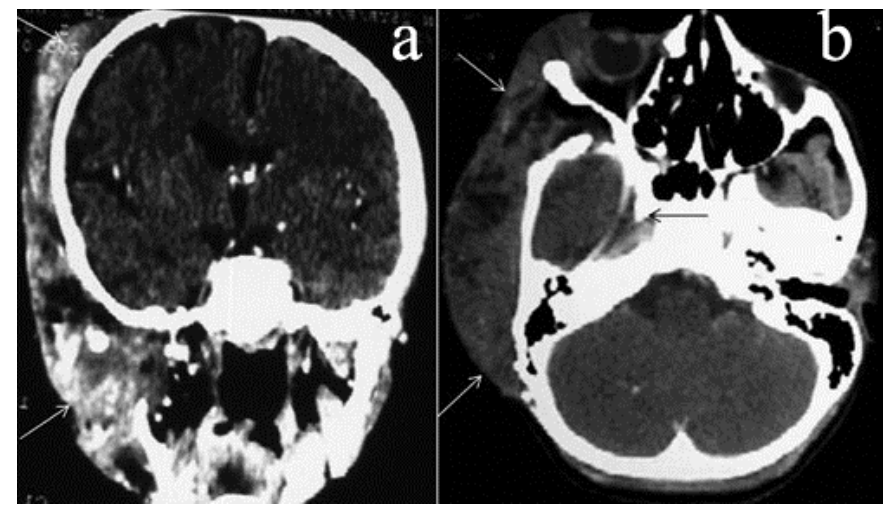

Fig. 36. Neurofibromatosis type 1: Plexiform neurofibroma with sphenoid wing dysplasia. Contrast enhanced CT brain, coronal (parenchymal window)(a) and axial (bone window)(b) view shows left sided plexiform neurofibroma of subcutaneous soft tissue(thin white arrow) and sphenoid wing dysplasia(thin black arrow).

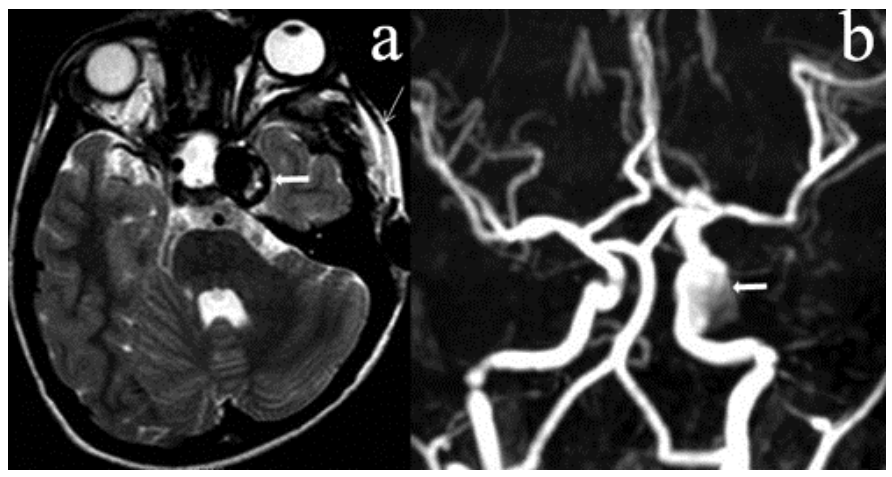

Fig. 37. Neurofibromatosis type 1: Vascular abnormalities. Axial T2W MR image of brain(a) shows severe left sphenoid wing dysplasia and subcutaneous soft tissue plexiform neurofibroma(thin white arrow). Associated left internal carotid artery aneurysm (thick white arrow) is seen on T2-weighted axial image(a) and TOF angiogram(b). 


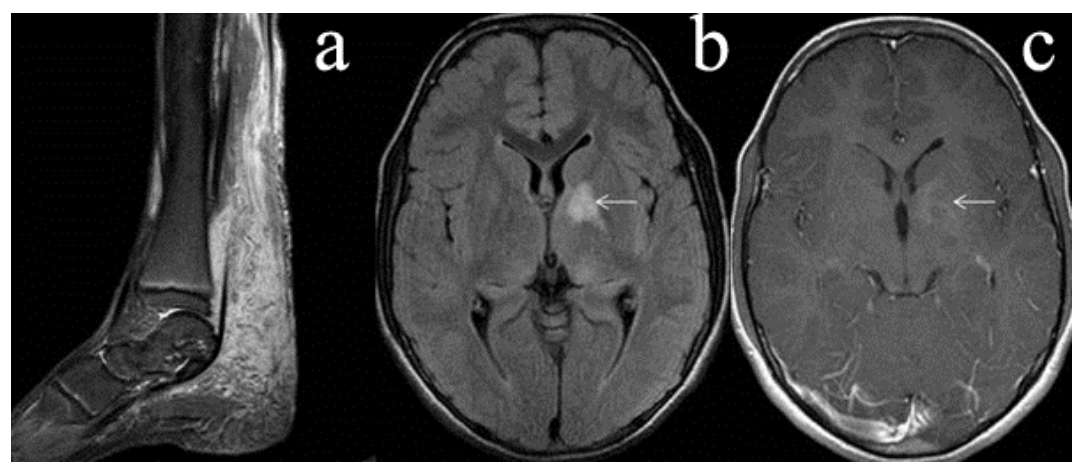

Fig. 38. Neurofibromatosis type 1: Peripheral Plexiform neurofibroma. Sagittal T1W post contrast image of lower limb (a) of another patient shows diffuse plexiform neurofibroma of left leg. Screening of brain revealed T2-hyperintense (b) focus in left basal ganglia region showing minimal contrast enhancement(c) but no mass effect, consistent with nonneoplastic hamartomatous lesion of brain. The patient had no neurological complains.

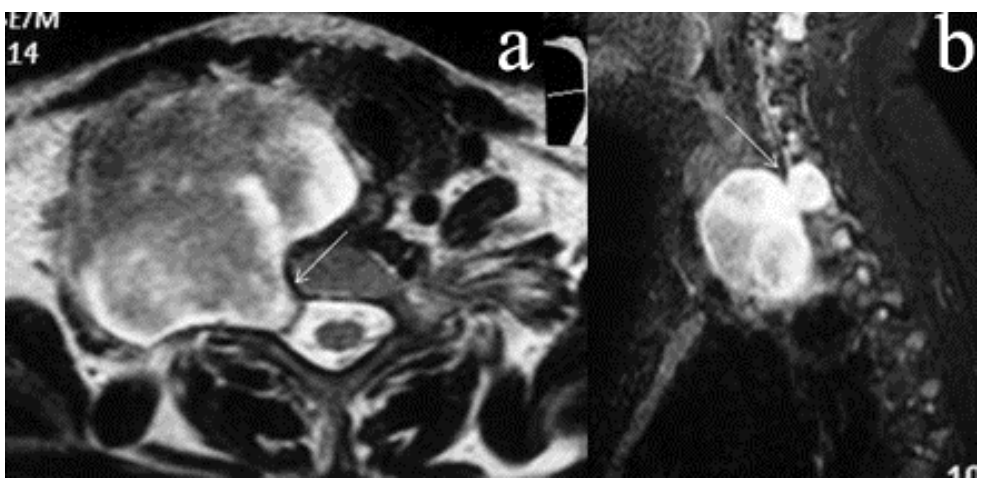

Fig. 39. Neurofibromatosis type 1: various spinal lesions. MRI of three different patients showing various spinal lesions found in NF-1. Axial T2W (a) and sagittal T1W post contrast (b) image of cervical spine demonstrates a large right paravertebral neurofibroma with intraspinal extension causing widening of ipsilateral neural foramen(thin white arrow). Sagittal T2W MRI of cervico-thoracic spine(c) shows multiple intradural extamedullary neurofibromas (thin white arrows). Solitary intramedullary hyperintense lesion (thin black arrow), which did not enhance after contrast administration (not shown) probably represents the benign white matter lesion similar to that observed in the brain. T2W-sagittal MRI spine (d) of another patient with NF-1 shows lumbar canal dural ectasia with posterior vertebral scalloping (arrows). 


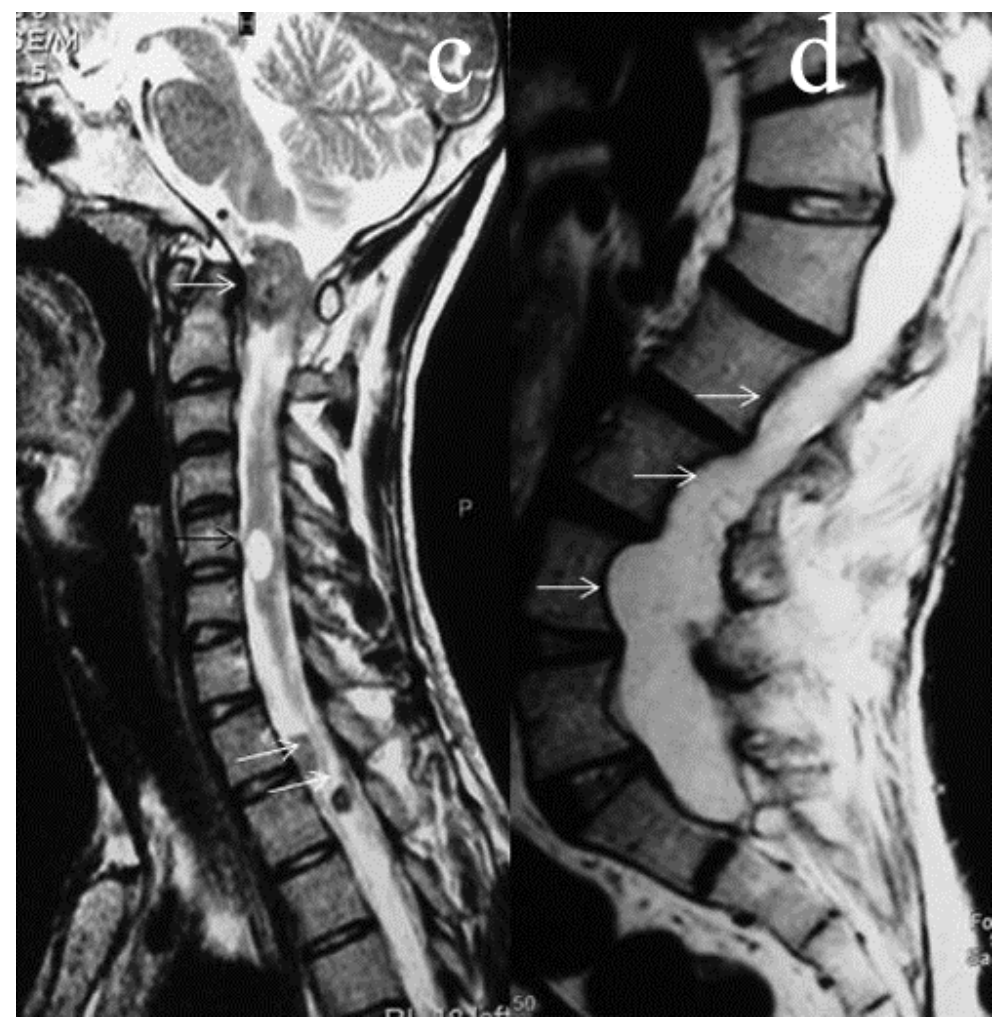

Fig. 40. Neurofibromatosis type 1: various spinal lesions. MRI of three different patients showing various spinal lesions found in NF-1. Axial T2W (a) and sagittal T1W post contrast (b) image of cervical spine demonstrates a large right paravertebral neurofibroma with intraspinal extension causing widening of ipsilateral neural foramen(thin white arrow). Sagittal T2W MRI of cervico-thoracic spine(c) shows multiple intradural extamedullary neurofibromas (thin white arrows). Solitary intramedullary hyperintense lesion (thin black arrow), which did not enhance after contrast administration (not shown) probably represents the benign white matter lesion similar to that observed in the brain. T2W-sagittal MRI spine (d) of another patient with NF-1 shows lumbar canal dural ectasia with posterior vertebral scalloping (arrows). 

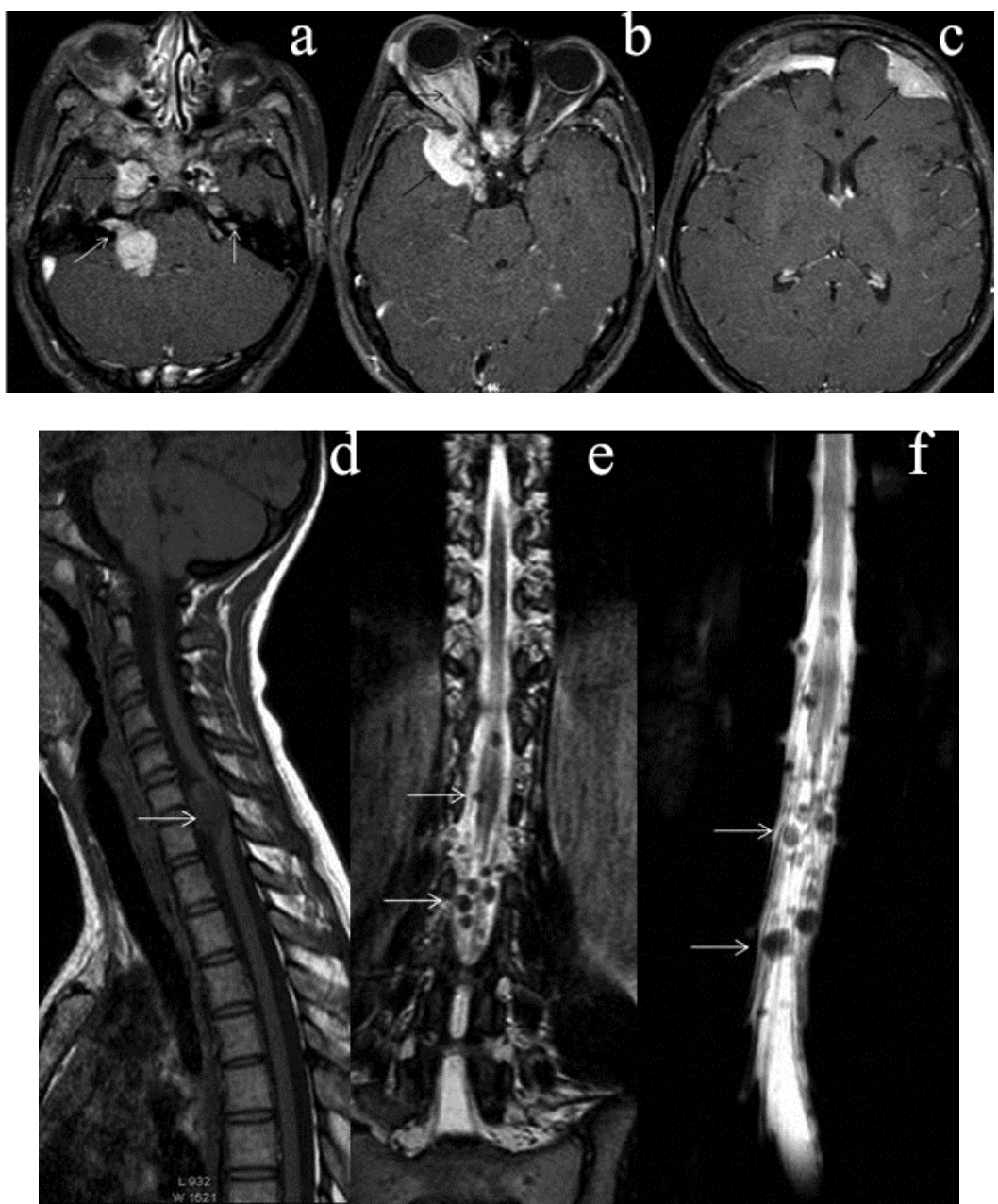

Fig. 41. Neurofibromatosis type 2: Axial T1W post contrast MR images(a-c) of brain demonstrate bilateral acoustic schwannomas(thin white arrows). Right schwannoma appears as a large homogenous enhancing right $\mathrm{CP}$ angle mass with intracnalicular extension and the left one is seen as a small intracanalicular enhancing mass. Multiple meningiomas(thin black arrows) are also present seen as enhancing extra-axial masses in right medial temporal and bilateral frontal regions. Right optic nerve meningioma is also seen completely filling the intraconal space. Non-contrast sagittal T1W(d) and coronal T2W image(e) of whole spine of the same patient demonstrates low cervical region meningioma(d) and multiple rounded lumbar region nerve root schwannomas(e), best appreciated on MR myelogram(f). 


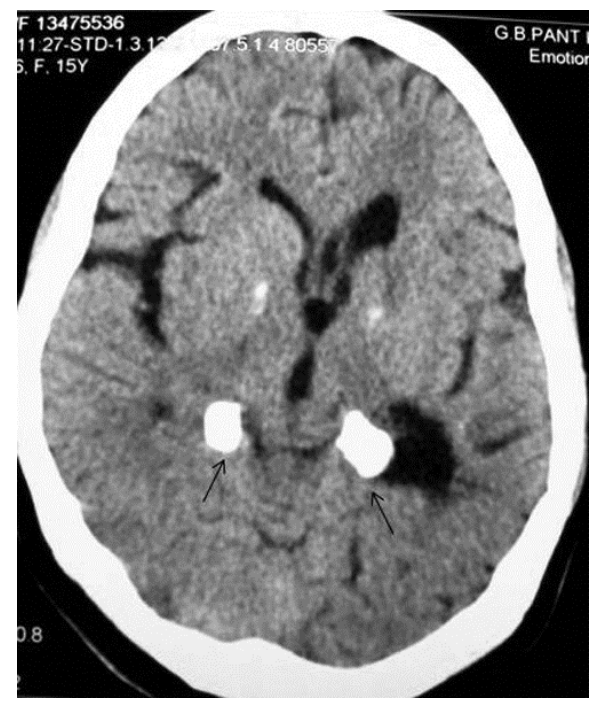

Fig. 42. Neurofibromatosis type 2: Nonneoplastic choroid plexus lesions. Axial NCCT brain of another patient with NF-2 shows extensive nonneoplastic bilateral choroid plexus calcification(arrows).

\section{Tuberous sclerosis1,16,18 (Figure 43,44)}

- Also known as Bourneville disease or multiple hamartomatous syndrome

- Incidence $=1: 10,000$ to 50,000 live births

- Classical triad of popular facial lesions (adenoma sebaceum), seizure disorder and mental retardation

- CNS lesions include

- Cortical tubers or hamartomas present in 95\% cases of TS, are characteristic lesions at pathologic examination. On MR imaging, these lesions may expand and distort the affected gyri and show age-related signal changes. Enhancement following contrast administration occurs in less than 5\% cases. Calcification of theses lesions increases with age.

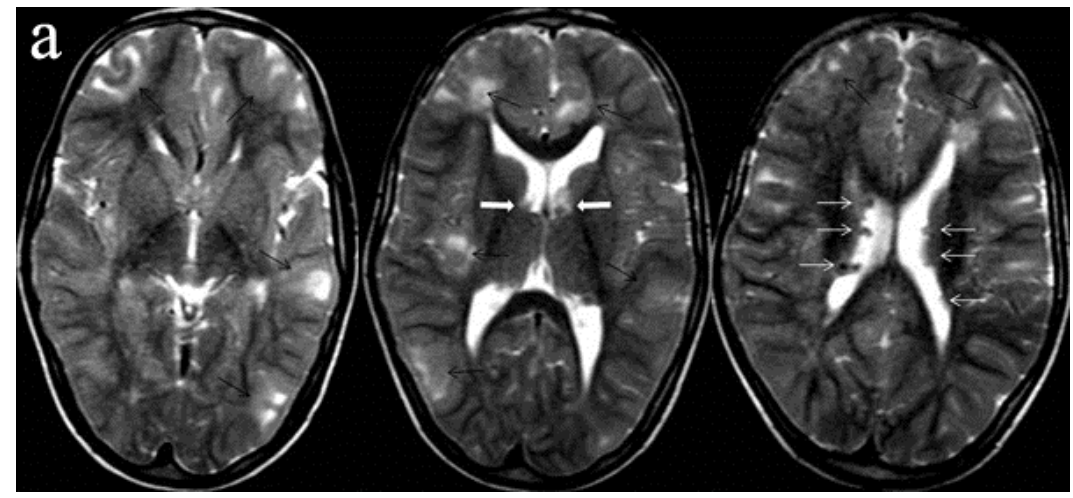




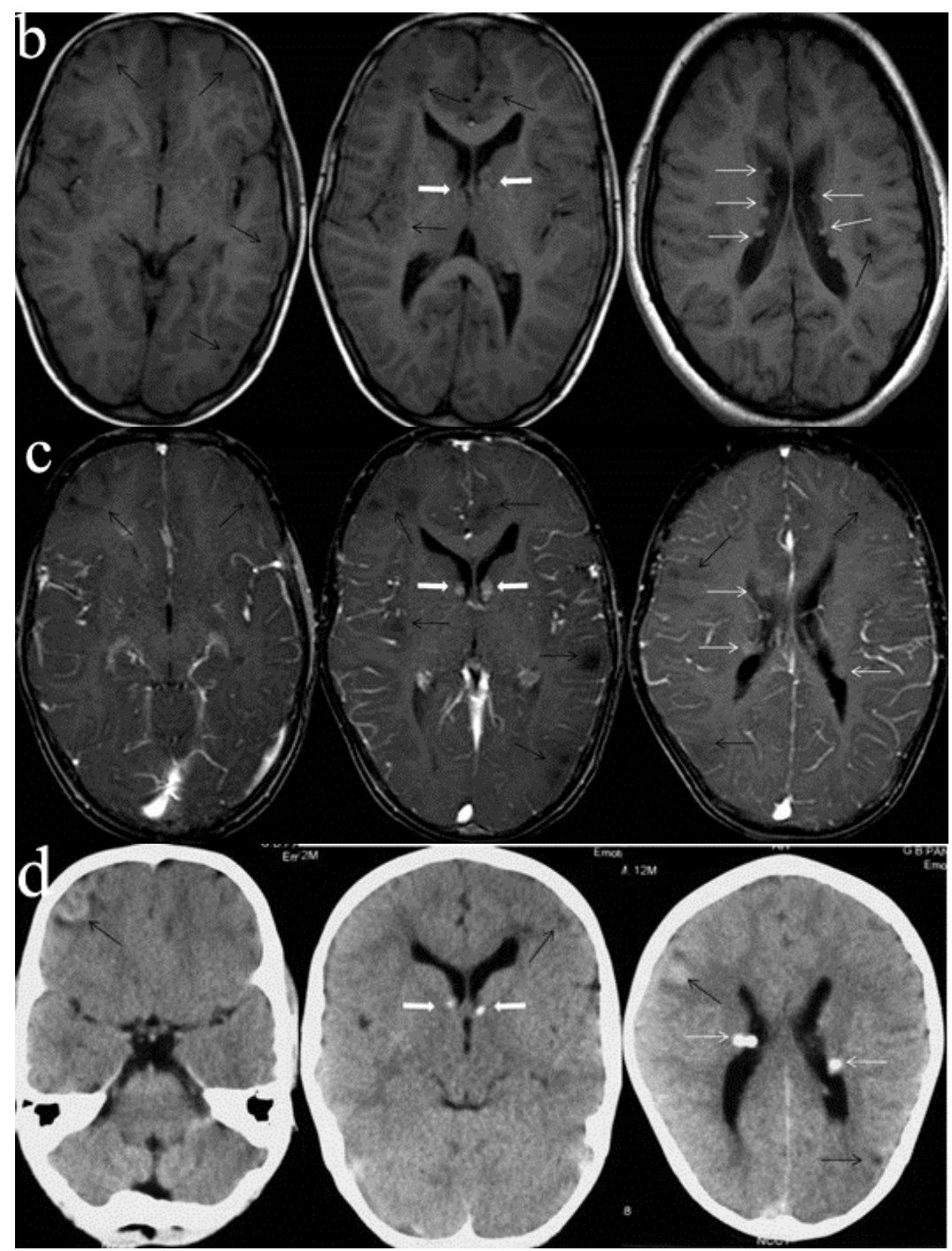

Fig. 43. Tuberous sclerosis. Axial T2W MRI brain(a) demonstrates multiple hyperintense cortical tubers(thin black arrows), bilateral hyperintense giant cell tumors at the foramen of monro (thick white arrows) and multiple hypointense subependymal nodules(thin white arrows). On corresponding axial T1W images (b) the cortical tubers appear hypointense to gray matter; while the giant cell tumors and subependymal nodules appear isointense to the gray matter. Corresponding axial T1W post contrast images(c) demonstrate nonenhancement of the cortical tubers, mild enhancement of the subependymal nodules and marked enhancement of giant cell tumors. On corresponding axial NCCT(d) the cortical tubers are hypo to hyperdense in attenuation, while the giant cell tumors and subependymal nodules appear calcified. 
- Subependymal nodules or hamartomas (SENs) present in 95\% cases of TS, are the hallmark of tuberous sclerosis. They are most commonly located on the ventricular surface of caudate nucleus, just behind the foramen of Monro followed by atria and temporal horns of lateral ventricles, third and fourth ventricle. On MR imaging, these nodules are usually hypointense to white matter on T2-weighted sequences (because of calcification) and may show minimal contrast enhancement. Closest differential includes nodular heterotopias which parallel cortex in signal on all MR pulse sequences and do not enhance after administration of intravenous contrast.

- Subependymal giant cell astrocytoma (SGCAs) present in 15\% cases of TS, are located at or near the foramen Monro. On MR imaging, SGCAs show intense, uniform contrast enhancement. These lesions are frequently calcified. Obstructive hydrocephalus is common with SGCAs.

- Dysplastic/disorganized benign white matter lesions. MR imaging shows four distinct patterns of these lesions: (i) straight or curvilinear bands that extends from the ventricle through the white matter to the cortex, (ii) wedge-shaped lesions, (iii) tumefactive or conglomerate foci, and (iv) cerebellar radial bands. Like cortical tubers, these lesions also show age-related signal changes, and contrast enhancement in about $15 \%$ cases.

- Other CNS lesions include retinal hamartomas (50\%), intracranial aneurysms, vascular ectasia and a progressive cerebral arterial occlusion disease akin to moyamoya pattern.

- $\quad$ Non-CNS lesions include hamartomatous growths in multiple organ system.

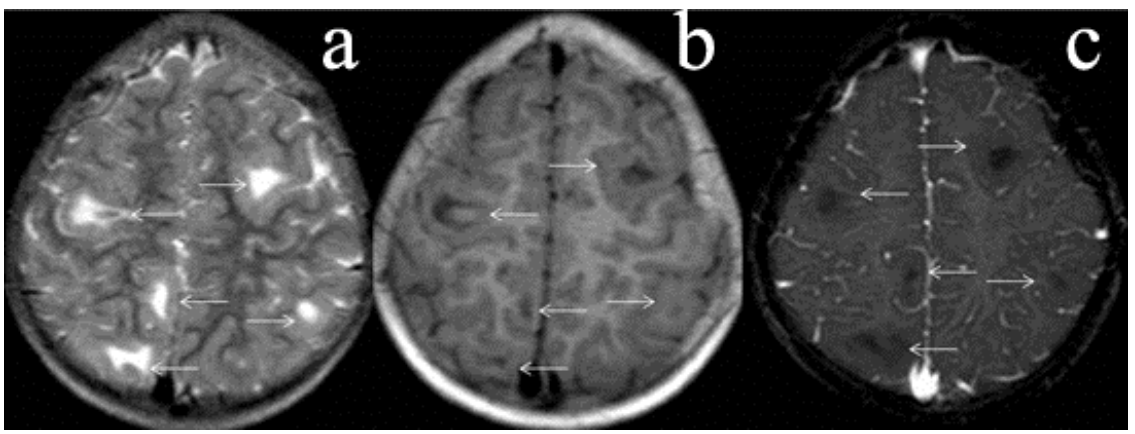

Fig. 44. Tuberous sclerosis. Axial T2W(a), T1W(b) and T1W post contrast image(c) of another patient demonstrates multiple cortical tubers appearing hyperintense on T2WI, hypointense on T1WI and showing no post contrast enhancement.

Sturge-Weber Syndrome 1,16 (Figure 45,46)

- Also known as encephalotrigeminal angiomatosis

- Characterized by facial port wine vascular nevus flammeus in the trigeminal nerve distribution (1 $1^{\text {st }}$ division most commonly involved), leptomeningeal venous angiomatosis of ipsilateral brain, hemiparesis, homonymous hemianopia and seizure.

- Pathologically, there is leptomeningeal venous angiomatosis with congenital absence of cortical veins; therefore the blood is shunted towards the hypertrophied deep medullary veins and thence to the choroid plexus. This results in venous stasis and vascular congestion with hypoxia of the affected cortex. Slowly progressive atrophy 
and dystrophic calcification of brain underlying the angioma occurs. The angioma itself does not calcify.

- Usually unilateral, rarely bilateral

- Occipital and posterior parietal lobe on the side of facial angioma is most commonly involved.

- Tram-track or gyriform pattern of cortical calcification underlying the leptomeningeal angioma is diagnostic of the syndrome. The calcification is unusual before two years of age. Calcifications are best seen on plain CT, T2W and GRE image.

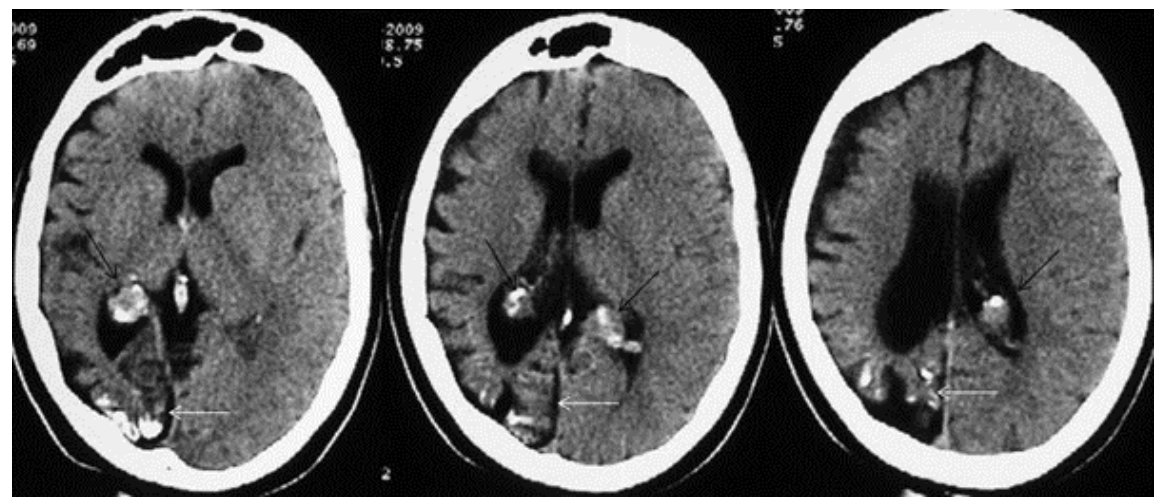

Fig. 45. Sturge-Weber syndrome. Unenhanced axial CT brain shows diffuse right cerebral hemispheric atrophy with dystrophic clacification (gyriform) of occipito-parietal cortex(thin white arrows). Associated enlargement of ipsilateral subarachnoid space, lateral ventricle and the frontal sinus is also present. Bilateral choroid plexus are enlarged (thin black arrows).

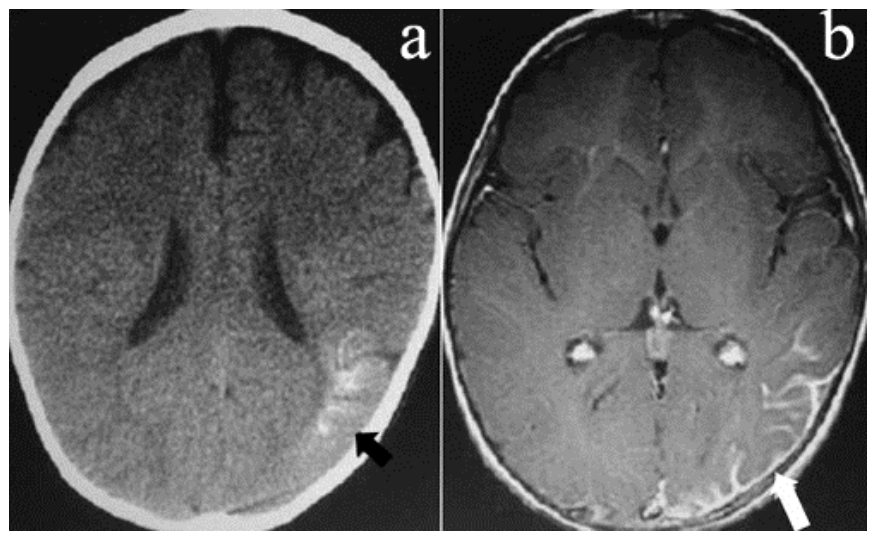

Fig. 46. Sturge-Weber syndrome. Unenhanced axial CT of brain(a) demonstrates gyriform calcification (thick black arrow) of left parieto-occipital cortex. After administration of contrast (b) there is marked enhancement of overlying leptomeningeal venous angiomas (thick white arrow). 
- Severe cortical atrophy results in marked dilatation of ipsilateral ventricle, thickening of calvarium and prominence of ipsilateral sinuses.

- The pial angiomas, cortical infarcts and enlarged ipsilateral choroid plexus show intense post contrast enhancement. Abnormal medullary and subependymal veins are demonstrated on CEMR and MR angiogram.

- Ocular lesions in the form of sclera and choroidal angiomas may be seen on MRI in onethird cases.

- Associated anomalies: Klippel-Trenaunay syndrome

Von Hippel-Lindau syndrome1,16 (Figure 47)

- Is a multisystem disease characterized by presence of cysts, angiomas, and neoplasms of the CNS and abdominal viscera.

- CNS lesions include

- Cerebellar (75\%), retinal (50\%) and spinal cord (25\%) hemangioblatomas. Supratentorial hemangioblatomas are extremely rare.

- On MR imaging, majority of hemangioblastomas have cystic appearance with intensely enhancing mural nodule. Between $20-40 \%$ tumors are solid. Contrast enhanced MRI has increased sensitivity for detection of small lesions. Flow voids in the afferent and efferent vessels supplying the tumor can often be detected. The angiographic appearance of the hemangioblastoma is highly characteristic, showing tangles of tightly packed vessels that become opacified in the early arterial phase.

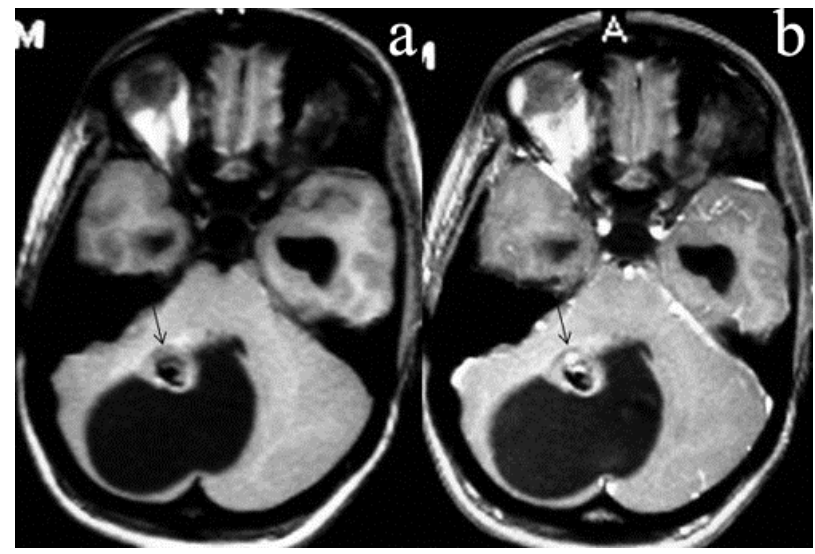

Fig. 47. Von Hipple-Lindau syndrome: Cerebellar hemangioblastoma. Axial T1W (a) MRI brain shows a right cerebellar hemispheric cyst with a mural nodule containing a curvelinear flow voids (arrow). Corresponding post contrast image (b) shows enhancing vessels within the mural nodule (arrow).

\section{Summary}

Congenital malformations of the brain are both complex and multiple. The neuroradiologic diagnosis of such anomalies requires a basic understanding of normal brain development and pathogenesis. The aetiologies associated with development anomalies may result from a 
variety of insults from genetic to environmental. Abnormalities associated with the neural tube and the neural plate generally occur within the first 28 days of gestation. On the other hand, abnormalities associated with cellular proliferation and migration in the CNS generally occur after the 28th day of gestation. This chapter will cover malformations associated with both of these periods.

Congenital anomalies of the brain are commonly encountered in day to day practice. Nevertheless, diagnosing it correctly is of paramount importance. Imaging plays an important role in reaching the correct diagnosis necessary for optimum management of these unfortunate conditions. It is as important for every radiologist to be familiar with basic imaging findings of common congenital anomalies, as it is for the paediatrician.

\section{References}

[1] Osborn AG. Brain development and congenital malformation. In: Diagnostic Neuroradiology (edn). Mosby Year Book; 1994: 3-116.

[2] Barkovich AJ. Congenital Malformations of the brain and skull. In: Pediatric Neuroimaging (4th edn). Philadelphia: Lippincott Williams \& Wilkins; 2005: 291439.

[3] Castillo M, Quencer RM, Dominguez R. Chiari III malformation: imaging features. Am J Neuroradiol. 1992;13:107-113.

[4] Naidich TP, Altman NR, Braffman BH et al. Cephaloceles and related malformations. Am J Neuroradiol 1992;13:655-690.

[5] Hahn JS, Barnes PD. Neuroimaging advances in holoprosencephaly: Refining the spectrum of the midline malformation. Am J Med Genet C Semin Med Genet 2010;15:120-132.

[6] Morishima A, Aranoff G, Syndrome of septo-optic pituitary dysplasia: The clinical spectrum brain dev 1986;8:233-235.

[7] Barkovich AJ, Kuzniecky RI. Gray matter heterotopias. Neurology 2005;55:1603-1608.

[8] Barkovich AJ, Kjos BO. Schizencephaly: correlation of clinical findings with MR characteristics. AM J Neuroradiol 1992;13:85-94.

[9] Abdel Razek AA, Kandell AY, Elsorogy LG, Elmongy A, Basett AA. Disorders of cortical formaton: MR imaging features. AJNR Am J Neuroradiol 2009;30:4-11.

[10] Flores- Sarnat L. Hemimegalencephaly: Part 1. Genetic, clinical and imaging aspects. J Child Neurol. 2002;17:373-384.

[11] Altman N, Naidich T, Braffman B. Posterior fossa malformation AJNR Am J Neuroradiol 1992;13:691-724.

[12] Naidich TP, McLone DG and Radhkowski MA. Intracranial arachnoid cysts. Pediatric Neurosci 1986;12:112-122.

[13] Joubert M, Eisenring JJ, Robb JP, Andermann F. Familial agenesis of the cerebellar vermis: a syndrome of episodic hyperpnea, abnormal eye movements, ataxia, and retardation. Neurology 1969;19:813-825.

[14] Maria BL, Quisling RG, Rosainz LC, Yachnis AT, Gitten J, Dede D, Fennell E. Molar tooth sign in Joubert syndrome: clinical, radiologic, and pathologic significance. J Child Neurol 1999;14:368-376.

[15] Mendonca JL, Natal MR, Viana SL, Coimbra PP, Viana MA, Matsumine M. Rhombencephalosynapsis: CT and MRI findings. Neurol India 2004;52:118-20. 
[16] Barkovich AJ. The Phakomatoses. In Barkovich AJ. Pediatric Neuroimaging (4th edn.) Philadelphia: Lippincott Williams and Wilkins 2005;476-481.

[17] Mulvihill JJ. Neurofibromatosis 1 (Von Recklinghausen's disease) and Neurofibromatosis 2 (bilateral acoustic neurofibromatosis) an update. Ann Inter Med 1990;113:39-52.

[18] Smirniotopoulous JG, Murphy FM. Central nervous system manifestations of the phakomatoses and other inherited syndromes. In Atlas SW(Ed): Magnetic Resonance Imaging of Brain and Spine( $3^{\text {rd }}$ edn). Philadelphia: Lippincott Williams and Wilkins 2002;371-413. 


\title{
Neuroimaging in Inborn Errors of Metabolism
}

\author{
Carlos Casimiro, Paula Garcia, Miguel Cordeiro, \\ Isabel Fineza, Teresa Garcia and Luísa Diogo \\ Centro Hospitalar Universitário de Coimbra \\ Portugal
}

\section{Introduction}

Inborn errors of metabolism (IEM) are a heterogeneous group of genetic disorders, classically caused by enzyme deficiency. They are associated with different pathogenic mechanisms from enzyme substract accumulation or product deficiency to formation of an abnormal, toxic molecule. Occasionally, the deficient protein has non-enzymatic functions such as membrane transport or others, making the boundaries of IEM difficult to establish. IEM are individually rare (orphan diseases) but relatively numerous as a group, since more than 500 different entities have been identified (Scriver et al., 2001). Overall, their incidence is estimated to be 1:1,500 (Raghuveer et al., 2006).

The age of onset varies. Signs and symptoms of IEM present a considerable overlap among the diverse IEM and many other diseases, not allowing the differential diagnosis on a clinical basis. There is no correlation between genotype and phenotype, in general. Most symptoms are apparent at or soon after birth, but clinical onset may occur prenatally or at any age, including adulthood. Multisystem involvement is frequent, with the presence of nervous system manifestations in most patients, either at disease onset or during the evolution.

IEM are most probably underdiagnosed. In spite of all contributions from varied fields of medical science, etiological diagnosis is not achieved in a significant percentage of suspected patients. Despite being frequently difficult, diagnosis may be done selectively based on clinical features or pre-symptomatically by neonatal screening and achieved by biochemical, enzymatic and/or genetic studies. The diagnosis of IEM is challenging due to their rarity and clinical heterogeneity. To address these diagnostic problems, several schemes based on clinical, biochemical, neuroradiological, morphologic, enzymatic and genetic criteria have been proposed.

Neuroimaging techniques are essential for assessing brain structures, namely white matter and/or gray matter involvement (Barkovich, 2007). They are undoubtedly useful in neurologically affected patients' diagnosis and follow-up. Neuroradiological features of many IEM overlap and are stage-dependent. Patients occasionally show distinctive patterns of central nervous system involvement in magnetic resonance imaging (MRI). These patterns may characterise some disorders, especially during the early stages, or they can show guiding characteristics, or reveal non-specific changes. In later phases, the MRI findings are similar for most IEM with neurological involvement, often presenting diffuse loss of brain tissue and increased water in the remaining tissue. For this reason, it is 
important to submit the patient to a brain MRI early in the course of the disease, when some key features are more evident (Barkovich, 2007).

Although the majority of patients present non-specific changes on MRI, a systematic pattern recognition approach to involved brain structures is useful, as it narrows differential diagnosis. It occasionally points to a diagnosis and allows the reduction of the biochemical and genetic work-ups. According to brain MRI in early stages, IEM can be classified into disorders primarily involving gray matter, diseases primarily involving white matter and disorders involving both gray matter and white matter (Barkovich, 2006, 2007). The proton magnetic resonance spectroscopy (MRS) may be more sensitive to detect early abnormalities in the brain. However, only a few metabolic diseases present with specific MRS findings.

Other neuroimaging techniques, such as positron emission tomography (PET) and single photon emission computed tomography (SPECT), could be useful in the diagnosis and follow-up of IEM. Nonetheless, they are not widely available on a routine clinical basis.

This section will review the most relevant IEM, based on a practical pattern-recognition approach to brain MRI and its correlation to clinical, biochemical and genetic features.

\section{Disorders primarily involving gray matter}

IEM primarily involving gray matter, known as poliodystrophies, can affect cortical or deep gray matter in the early phases. This differentiation should be made in order to accurately discriminate a range of diseases.

\subsection{Disorders primarily involving cortical gray matter}

During an acute phase, neuroimaging reveals sulcal effacement, cortical swelling and restricted diffusion when the damage affects primarily the cortical gray matter (Barkovich, 2005). The imaging findings during a chronic phase are: sulcal volume increasing, cortical thinning and cortical low attenuation on computed tomography (CT) scan, and T1 and T2 prolongation on MRI (Campistol, 1999). Nevertheless, in later phases, lesions can progress and spread to other brain structures (including deep gray matter, white matter and the cerebellum) (van der Knaap \& Valk, 2005), showing non-specific patterns.

The primary involvement of cortical gray matter is commonly found in neuronal ceroid lipofuscinoses, mucolipidosis type I and GM1 gangliosidosis. Generally, findings on imaging studies are non-specific in these disorders.

\subsubsection{Neuronal ceroid lipofuscinoses}

The neuronal ceroid lipofuscinoses (NCL) are inherited lysosomal storage diseases characterized by the accumulation of autofluorescent ceroid lipopigments in the lysosomes of neurons and other cell types. Together, they constitute the commonest group of progressive neurodegenerative diseases in children, and are inherited in an autosomal recessive mode. The adult form of NCL is rare and demonstrates either an autosomal recessive or a dominant mode of inheritance (Haltia, 2003). The main features of these diseases include visual failure, seizures, progressive physical and mental decline, and premature death. Diagnosis, based on the age of clinical presentation, is made by enzymatic or genetic studies. Ultra-structural analysis of white blood cells or skin can be used to orientate biochemical and genetic tests in atypical cases (Williams et al., 2006). 
The major imagiological features in NCL are cerebral and cerebellar atrophy, cortical thinning associated with mild hyperintensity of the cerebral white matter and hipointensity of the thalami on T2-weighted images (T2-WI) (Incerti, 2000). Infantile and late infantile variants show rapid progression of enlargement of ventricles and sulci of the brain. In the juvenile and adult forms, however, cerebral atrophy is frequently slight in the course of the disease, or may remain totally absent (Autti et al., 1996). Cerebellar atrophy is common and may be the earliest finding in late-infantile and juvenile forms of NCL. In late infantile form, the first finding is usually a rapid progression of cerebellar atrophy (Fig. 1.), whilst in infantile and juvenile forms this progression is slower (Järvelä et al., 1997). T2-WI hyperintensity in white matter, which is usually less intense than in leukodystrophies, tends to spare the subcortical areas and initially involves the posterior periventricular region. Later in the disease, corpus callosum may also become thinner (Incerti, 2000).

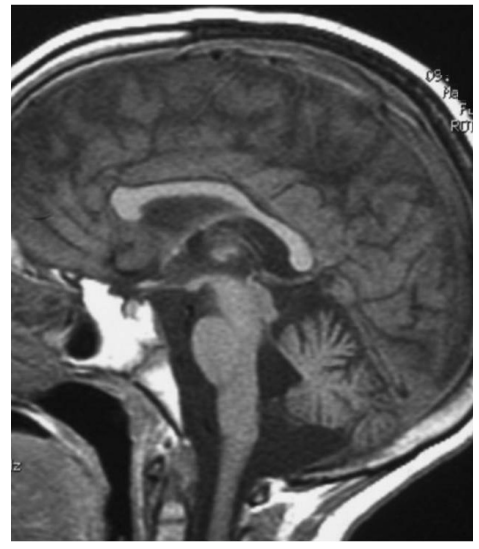

(a)

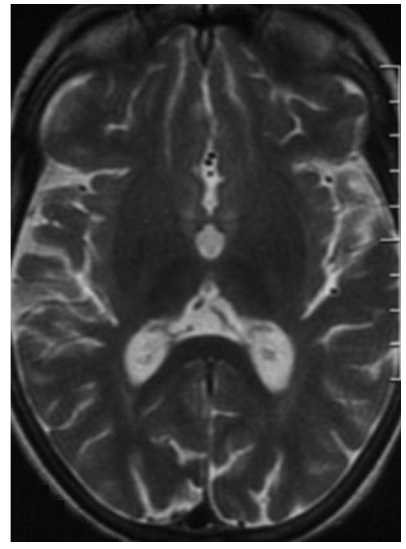

(b)

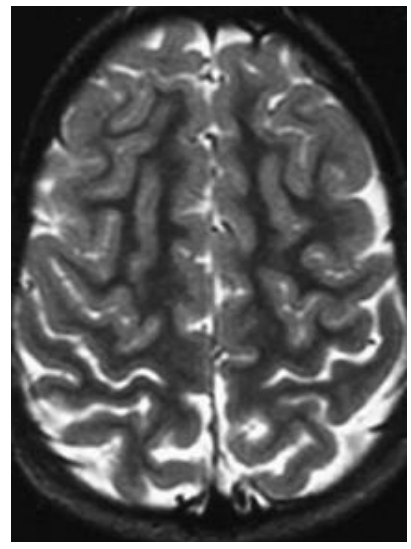

(c)

Fig. 1. Late infantile NCL. (a) Midline sagittal T1-WI demonstrates cerebellar atrophy. Axial T2-WI show mild thalamic hypointensity (b) and cortical thinning (c).

Diffusion-weighted imaging (DWI) can be very useful in detecting variances in abnormalities of cerebral water diffusion in the late infantile form, showing increased whole-brain apparent diffusion coefficient (ADC) values. It correlates with patient's age, disease severity and duration (Dyke et al., 2007). MRS can give additional hints to the diagnosis of infantile and late infantile forms, and be helpful in the differential diagnosis. In infantile NCL, MRS spectrum revealed progressive changes, with a complete loss of Nacetylaspartate (NAA), as well as marked reduction of creatine and choline, and increase in myo-inositol and lactate in gray matter and white matter. In late-infantile NCL, MRS spectrum revealed reduction of NAA in gray matter and white matter and an increase of myo-inositol, creatine and choline in white matter (Brockmann et al., 1996).

\subsection{Disorders primarily involving deep gray matter}

When the damage primarily affects the deep gray matter, neuroimaging reveals many different patterns, with involvement of particular structures. Some of them are more specific in a group of diseases, allowing for the narrowing in differential diagnosis. CT scan can be 
normal, or disclose calcifications or hypodensity areas in deep gray matter. MRI sensibility is higher, although CT is best suited for calcification detection.

The list of IEM affecting deep gray matter is extensive. Globus pallidus hyperintensity in T2WI can be seen in succinic semialdehyde dehydrogenase deficiency, methylmalonic acidemia, urea cycle disorders, guanidinoacetate methyltransferase deficiency, pyruvate dehydrogenase deficiency and isovaleric acidemia (Barkovich, 2005, 2007). Additionally, globus pallidus hypointensity, with or without central hyperintensity, is highly characteristic of Hallervorden-Spatz disease. Striatum hyperintensity in T2-WI can be seen in some mitochondrial respiratory chain disorders (MRCD) such as Leigh's syndrome and mitochondrial encephalopathy with lactic acidosis and stroke-like episodes - MELAS, the glutaric acidurias, propionic acidemia and molybdenum co-factor deficiency (Barkovich, 2005 , 2007). Many of these IEM can concomitantly involve the white matter so some will be discussed later in this chapter.

\subsubsection{Pantothenate kinase-associated neurodegeneration}

Pantothenate kinase-associated neurodegeneration (PKAN), formerly known as Hallervorden-Spatz disease is an autosomal recessive disorder characterized by neurodegeneration with brain iron accumulation. Many patients have mutations in the pantothenate kinase 2 gene (PANK2). Two distinct groups were identified. Classical PKAN presents in early childhood, usually before age 6 years, has uniform presentation and is characterized by rapid progression of extrapyramidal and pyramidal signs, intellectual impairment, pigmentary retinal degeneration and abnormal eye movements. Atypical PKAN, which is less common, has a wider clinical spectrum and slower progression. PANK2 mutations are associated with all classic PKAN and one third of atypical disease cases (Hayflick et al., 2003). PKAN is suggested by typical MRI features, and the diagnosis is made by molecular genetic testing (Zhang et al., 2006).

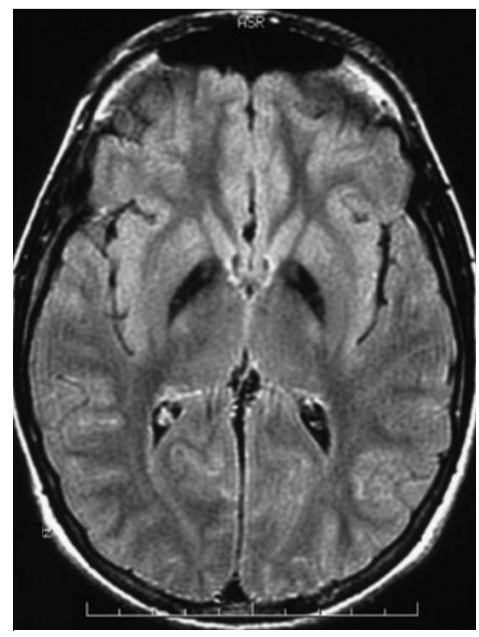

(a)

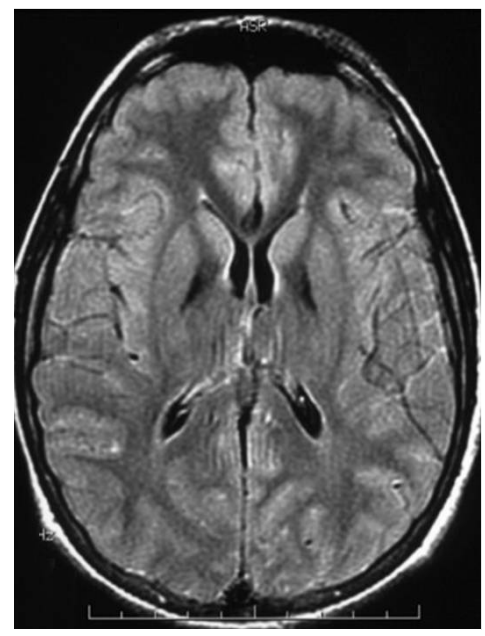

(b)

Fig. 2. Pantothenate kinase-associated neurodegeneration (PKAN). (a) and (b) Axial FLAIR show hypointensity with a central region of hyperintensity in the globus pallidi. 
Some patients' CT scans show low or high density areas in the globus pallidus. Lowdensity foci are a sign of tissue destruction, while the high-density foci reflect dystrophic calcification (Barkovich, 2005). All patients with PANK2 mutations had the specific pattern of T2-WI globus pallidus central hyperintensity (destruction and gliose) with surrounding hypointensity (iron deposition), known as the eye-of-the-tiger sign (Fig. 2.). This sign was not present in patients without mutations. Patients with PANK2 mutations could be distinguished by the occurrence of isolated globus pallidus hyperintensity on T2-WI before the hypointensity developed (Hayflick et al., 2006). Patients without PANK2 mutations revealed globus pallidus T2-WI hypointensity, without central hyperintensity. In the later stage of the disease, it can be seen evidence of iron deposition in the substantia nigra pars reticulata, cerebral and cerebellar atrophy. These signs were frequently and more severely seen in PANK2 mutation-negative patients (Hayflick et al., 2006).

MRS can show markedly decreased NAA/creatine ratio in the globus pallidus and substancia nigra, with increased myo-inositol/creatine ratio (Parashari et al., 2010).

\subsubsection{Creatine deficiency syndromes}

Creatine deficiency syndromes (CDS) are a newly described group of IEM affecting creatine metabolism. Three disorders have been described: guanidinoacetato methyltransferase (GAMT) deficiency, arginine:glycine amidinotransferase (AGAT) deficiency and creatine transporter defect (SLC6A8). GAMT e AGAT deficiencies (creatine synthesis pathway) have an autosomal recessive inheritance, whereas SLC6A8 defect is an X-linked disorder. All CDS can cause developmental delay, intellectual disability, behavioural problems, movement disorders, seizures, and severe disturbance of expressive language (Schulze, 2003). These clinical manifestations can be partially reversed by oral creatine supplementation and dietary manipulation, even in same patients with the creatine transporter defect (Chilosi et al., 2008; Mercimek-Mahmutoglu et al., 2010). CDS are suggested by marked reduction or complete absent of the creatine peak on MRS. Diagnosis relies on measurement of guanidinoacetate, creatine, and creatinine in urine and plasma and molecular genetic testing of the gene involved. If molecular test results are inconclusive, AGAT enzyme activity, GAMT enzyme activity, or creatine uptake in fibroblasts can be evaluated (MercimekMahmutoglu \& Stöckler-Ipsiroglu, 2009).

MRI and mainly MRS are very important tools to suggest CDS diagnosis and follow therapy response (Chilosi et al., 2008). In GAMT deficiency MRI can be normal or reveal T2-WI hyperintensity on globus pallidus, mild myelination delay or white matter hyperintensity. MRI is normal in AGAT deficiency (Barkovich, 2005); SLC6A8 defect MRI can show brain atrophy (Póo-Argüelles et al., 2006).

MRS shows spectrum changes even without MRI signal abnormally. Markedly reduction or absence of creatine peak, which is easily seen on long echo time (TE) spectrum, is the classical sign shared by all three disorders, in gray matter and white matter. GAMT deficiency reveals a broad guanidinoacetate peak at $3.78 \mathrm{ppm}$, on short TE sequences, which can be reduced (but not normalized) with dietary restriction of arginine associated to the supplementation of creatine and ornithine. In GAMT and AGAT deficient patients, but not in most of those with SLC6A8 deficiency, the creatine peak slowly increases under treatment (Fig. 3.). 


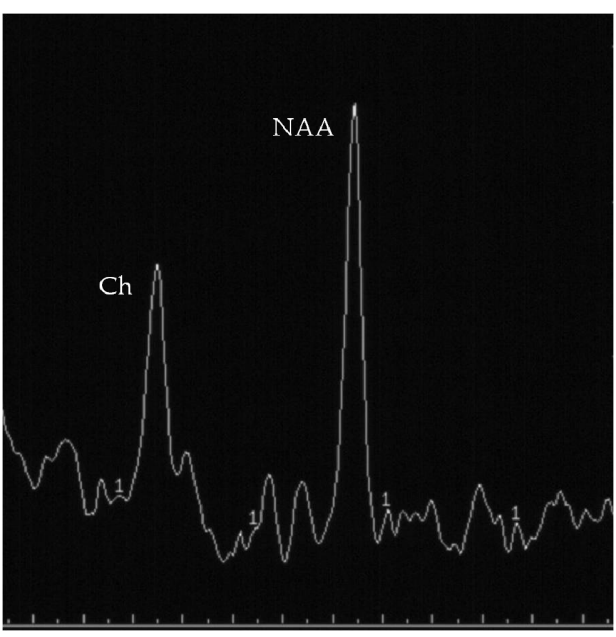

(a)

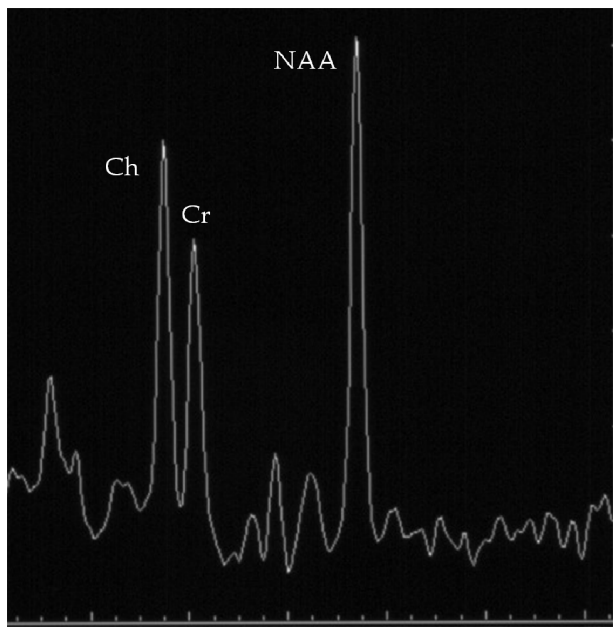

(b)

Fig. 3. Creatine deficiency due to GAMT deficiency. (a) Multivoxel MRS spectrum (TE =144 ms) of the basal ganglia, before therapy, shows a markedly reduced creatine peak, and normal choline (Ch) and NAA peaks. (b) Multivoxel MRS spectrum (TE $=144 \mathrm{~ms}$ ) of the basal ganglia, after 6 months therapy, shows a creatine $(\mathrm{Cr})$ peak increasing.

\section{Disorders primarily involving white matter}

When the damage affects primarily the white matter, there is hypodensity on CT scan, and $\mathrm{T} 1$ and T2 prolongation on MRI. In later phases, the atrophy is the main feature. Knowledge of normal brain myelination, its appearance on different MRI sequences and normal variations, is crucial to accurately approach the range of disorders primarily involving white matter. When the damage affects primarily the white matter, it is important to find out if the white matter has never myelinated completely (hypomyelination), or if the myelin has been developed and destroyed afterwards (demyelination) (Barkovich, 2007). Since many of these IEM can also involve the gray matter, some will be discussed later in this chapter.

\subsection{Hypomyelination diseases}

The hypomyelination is observed in a small number of IEM, like Pelizaeus-Merzbacher disease and Salla disease. MRS findings may be useful in their differentiation (Barkovich, 2005).

\subsubsection{Pelizaeus-Merzbacher disease}

Pelizaeus-Merzbacher disease (PMD) is a recessive X-linked neurological disorder caused by a mutation in the proteolipidic protein 1 (PLP1) gene, which results in defective central nervous system myelination. The connatal PMD phenotype presents during the neonatal period or in the first weeks of life, with nystagmus, stridor, hypotonia, severe spasticity and motor deficits, cognitive impairment, seizures and, later on, absence of speech. Death usually occurs in the period from infancy to third decade. The classic PMD phenotype presents in the first 5 years of life, with nystagmus, hypotonia, titubation, ataxia, slow motor 
development and extrapyramidal movements, like dystonia and athetosis. Death usually occurs between the third and the seventh decades. PMD is suggested by typical neurologic findings, X-linked inheritance pattern, and general changes on MRI. Molecular genetic testing of PLP1 is also available.

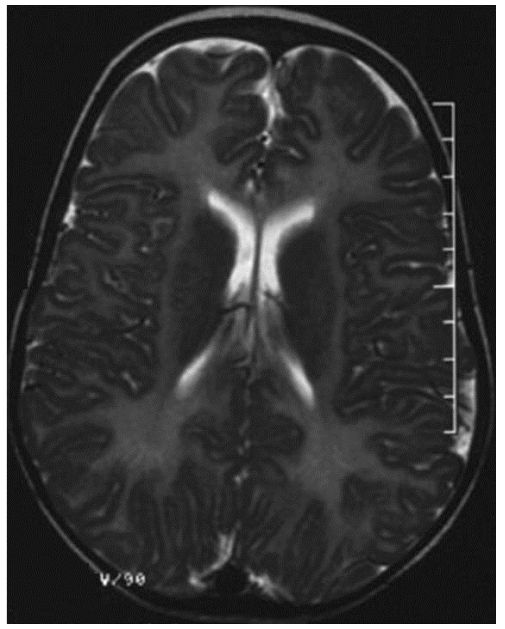

(a)

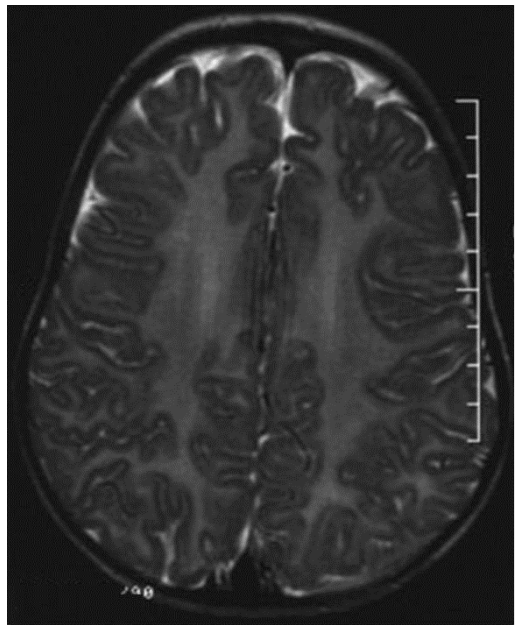

(b)

Fig. 4. Pelizaeus-Merzbacher disease. (a) and (b) Axial T2-WI show abnormal high signal intensity in cerebral white matter, representing hypomyelination.

As in most white matter diseases, CT scan changes are non-specific, revealing white matter hypodensity and atrophy in later stages. MRI shows discordance of myelin maturation caused by a lack of myelination. In the connatal form, a complete absence of myelin in the brain is demonstrated (Fig. 4.) (van der Knaap \& Valk, 1989). In classical forms in the early stages, MRI shows a brain with normal appearance, but more immature than expected. During infancy, myelin development of the brain progresses in an orderly and predictable fashion. Absence of these predictable patterns should raise the consideration for PMD. In late and severe cases, MRI reveals diffusely T2 hyperintensity and generalized volume reduction in the white matter with thinned corpus callosum and cortical sulci enlargement (Plecko et al., 2003).

MRS is very useful for evaluating both axonal integrity and myelination. However, reports may vary, which might be related to different clinical phenotypes, genotypes, or stages of disease progression (Pizzini et al, 2003). The pattern of metabolite abnormalities in individuals with PLP1 duplication appears to be distinctive, showing increased levels of NAA, creatine, glutamine and myo-inositol, which helps the differentiation of PMD from other leukodystrophies (Hanefeld et al., 2005).

\subsection{Demyelination diseases}

MR imaging has become the primary imaging modality in demyelinating diseases, playing an essential role in the identification, localization, and characterization of these pathologies. Demyelination processes can primarily involve subcortical white matter or periventricular 
white matter. This differentiation should be made to ensure an effective imagiological approach and facilitate differential diagnosis.

\subsubsection{Disorders primarily involving subcortical cerebral white matter}

Early subcortical white matter demyelination can be seen in megalencephalic leukoencephalopathy with subcortical cysts, Alexander disease, some organic acidurias, Kearns-Sayre syndrome, galactosemia and diverse MRCD.

\subsubsection{Megalencephalic leukoencephalopathy with subcortical cysts}

Megalencephalic leukoencephalopathy with subcortical cysts (MLC), also known as van der Knaap disease, is an autosomal recessive disorder caused by mutations in the MLC1 gene. This gene encodes a protein mainly expressed in astrocytic endfeet at the blood-brain and cerebrospinal fluid-brain barriers (Boor et al., 2007). MLC is characterized by development of macrocephaly through the first year of life, generally up to the 98th percentile. Head circumference stabilizes afterwards. Initially, the psychomotor development is relatively normal or only mildly delayed. Later on, motor development delay, cerebellar ataxia, dysarthria, spasticity, and sometimes extrapyramidal signs ensue. Seizures, usually responsive to medication, are also observed in some children. MLC diagnosis can be made by recognition of typical neurologic signs and symptoms, and its distinguished brain MRI features. Molecular genetic testing of MLC1 is available (van der Knaap \& Scheper, 2008).

The MRI is characterized by diffusely abnormal and mildly swollen cerebral white matter, showing enlargement of the gyri, and subcortical cysts in posterior frontal and temporal lobes (Barkovich, 2005). Central white matter is better preserved, especially the corpus callosum, internal capsule and brainstem. The presence of cerebellar white matter T2 hyperintensity is mild. Follow-up MRI reveals cerebral atrophy, and subcortical cyst growth can be present in some cases (van der Knaap et al, 1995).

Abnormal white matter shows increased diffusivity on DWI (Gelal, 2002). MRS reveals reduced NAA in T2 hyperintensity areas (Morita et al., 2006).

Tc-99m-ethyl cysteinate dimer SPECT reveals hypoperfusion in the abnormal cerebral white matter seen on MRI (Kiriyama et al., 2007).

\subsubsection{Alexander disease}

Alexander disease, or fibrinoid leukodystrophy, is caused by a gene mutation encoding glial fibrillary acidic protein (GFAP), leading to profound cellular dysfunction (Cecil \& Kos, 2006). The pathological hallmark is the accumulation of ubiquitinated intracytoplasmic inclusions in astrocytes, called Rosenthal fibers. The infantile form, the most frequent, is present in the first 2 years of life, typically with macrocephaly and frontal bossing, psychomotor retardation, seizures, pyramidal signs and ataxia. Patients survive weeks to several years. Diagnosis is based on MRI features. Molecular genetic testing, for GFAP gene, is available (Gorospe, 2010).

CT scan shows low attenuation in frontal white matter with posterior progression to involve parietal region, internal capsules, and sometimes caudate heads. Frequently the tips of frontal horns show contrast-enhancement. There are 5 criteria on MRI to diagnose Alexander disease, according to van der Knaap: (1) extensive cerebral white matter changes with frontal predominance; (2) a periventricular rim with high signal on T1-WI and low signal on T2-WI; (3) signal abnormalities with swelling or volume loss in the basal ganglia and thalami; (4) brainstem signal abnormalities; and (5) contrast enhancement of one or 
more of the following structures: ventricular lining, periventricular rim tissue, white matter of the frontal lobes, optic chiasm, fornix, basal ganglia, thalamus, dentate nucleus, or brainstem structures. The association of 4 or more criteria is relatively specific for Alexander disease. The imagiological pattern of enhancement provides specific information that can lead to correct diagnosis. Subcortical white matter is affected early in the course of the disease. The frontal changes correspond to prolonged T1 and T2 relaxation times, and tend to involve the parietal white matter and the internal and external capsules (van der Knaap et al., 2001). The progression of disease can lead to cavitations in the white matter (Vargas, 2009).

DWI reveals increased diffusion in the affected regions (Barkovich, 2005). MRS shows elevated myo-inositol and decreased NAA in the lesions (Cecil \& Kos, 2006).

\subsubsection{Disorders primarily involving periventricular cerebral white matter}

Early periventricular white matter lesions can be seen in Krabbe disease, GM2 gangliosidoses, metachromatic leukodystrophy, X-linked adrenoleukodystrophy, vanishing white matter disease, phenylketonuria, maple syrup urine disease, Lowe syndrome, Sjögren-Larsson syndrome and mucolipidosis type IV.

\subsubsection{Metachromatic leukodystrophy}

Metachromatic leukodystrophy (MLD) is a lysosomal storage disease caused by decreased activity of arylsulfatase A, resulting in failure of myelin breakdown and reutilization in the central and peripheral nervous systems. There are three clinical forms: late infantile MLD (50$60 \%$ of the cases), juvenile MLD (20-30\%), and adult MLD (15-20\%) (Fluharty, 2008). The late infantile MLD presents before age 2 years with weakness, hypotonia, delayed psychomotor development, impairment of speech, spasticity, seizures and compromised vision and hearing. Death usually occurs before age 5. MLD is suspected by neurodegeneration and evidence

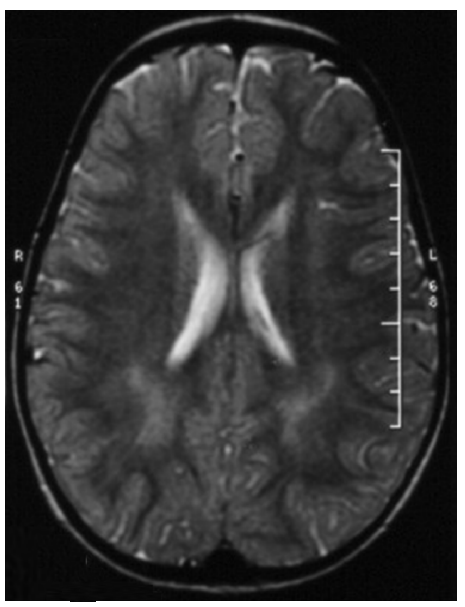

(a)

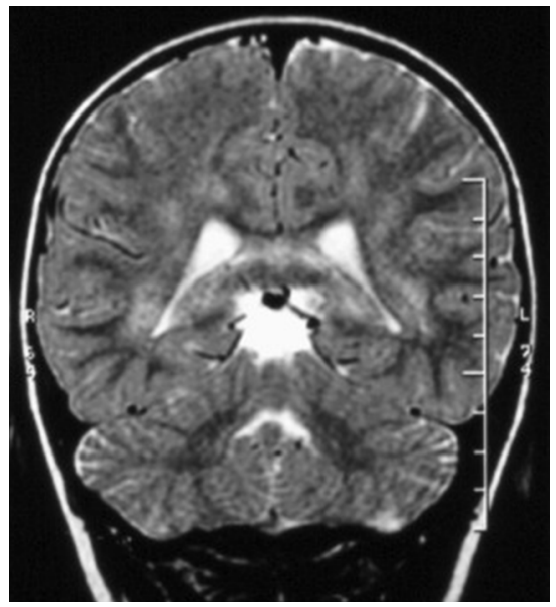

(b)

Fig. 5. Metachromatic leukodystrophy. Axial (a) and coronal (b) T2-WI show abnormal hyperintensity in the periventricular white matter, predominantly around the posterior body and trigones of the lateral ventricles, sparing subcortical U fibers. 
of leukodystrophy on MRI. Diagnosis is suggested by increased urinary excretion of sulfatides and/or decreased arylsulfatase A activity, and is confirmed by genetic testing. Metachromatic lipids deposits can be seen in the nervous system tissue (Fluharty, 2008).

CT scan shows confluent attenuation of periventricular white matter density. MRI reveals progressive symmetrical prolongation of $\mathrm{T} 1$ and $\mathrm{T} 2$ relaxation times in periventricular white matter. The cerebral white matter located around the posterior body and trigones of lateral ventricles is involved earlier in late infantile MLD (Fig. 5.), whereas frontal white matter is involved in cases of late onset. Subcortical U fibers are spared early but not in the later stages of the disease course. No enhancement has been reported. Later signs include cerebral atrophy. Higher resolution images reveal leopard skin pattern or tigroid pattern in the centrum semiovale that correspond to areas combining demyelination and normal regions. The progression of disease leads to involvement of the internal capsule, corpus callosum and corticospinal tracts.

DWI reveals restricted diffusion in affected areas (Sener, 2002). MRS shows decreased levels of NAA and increased levels of myo-inositol and choline (Cecil \& Kos, 2006).

\subsubsection{Krabbe disease}

Krabbe disease, or globoid cell leukodystrophy, is an autosomal recessive neurodegenerative disorder, caused by a deficiency of the lysosomal enzyme galactocerebrosidase, a key enzyme in metabolic pathways of myelin turnover and breakdown. Its deficiency results in galactosylsphingosine accumulation, a central and peripheral nervous system neurotoxin. The most frequent (85-90\%) form of Krabbe disease has an infantile onset (Wenger, 2008). It presents with irritability between 3 and 6 months after birth and progresses with motor deterioration, feeding problems and atypical seizures. Eventually, the child develops decerebrate posture (Cecil \& Kos, 2006) and dies before age 2 years. Diagnosis is made by measuring the activity of galactocerebrosidade in leukocytes or in cultured skin fibroblasts and can be confirmed by genetic studies. A molecular genetic test is available for carrier detection.

CT features during the initial stage of the disease may show symmetric high-attenuation in the thalami, caudate nuclei, corona radiate, posterior limbs of the internal capsule, brainstem and cerebellar dentate nuclei. Early in the course of the disease, MRI shows T1 and T2 prolongation in the cerebellar nuclei, posterior limbs of the internal capsules and cerebellar white matter. The subcortical U fibers are spared until late in Krabbe disease (Fig. 6.). Symmetric enlargement of the optic nerves may be seen (Cecil \& Kos, 2006).

Enhancement in junction between deep white matter and subcortical U fibers is rare (Vargas et al., 2009), but may be a common feature for cranial nerves and the cauda equina (Given et al., 2001). Thalami can be normal in the early stages, or reveal decreased T1 and T2 relaxation times due to calcium deposition (Cecil \& Kos, 2006). Later in the disease course, hyperintensity predominantly involves the parietal lobes, with extension to the callosal splenium, and severe, progressive atrophy.

DWI reveals restriction diffusion in the early phases of the disease, mainly in the subcortical white matter, caudate head, and anterior limb of internal capsule; in later stages it shows increased diffusion in the white matter (Engelbrecht et al., 2002). Elevated choline and myoinositol, and reduced NAA have been found in the white matter MRS of infantile onset. Lactate elevation has also been reported (Zarifi et al., 2001). On the other hand, in an adult onset case, white matter spectrum changes were much less marked, and revealed only a mild elevation of creatine (Farina et al, 2000). 


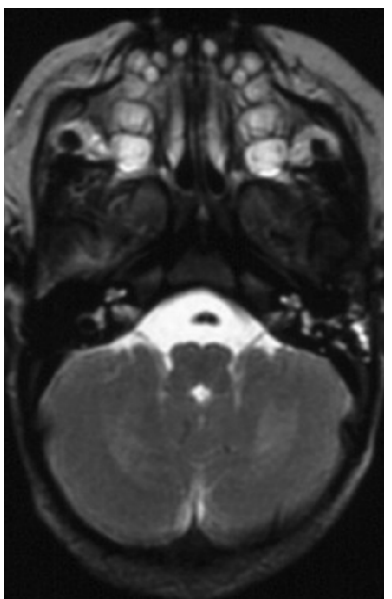

(a)

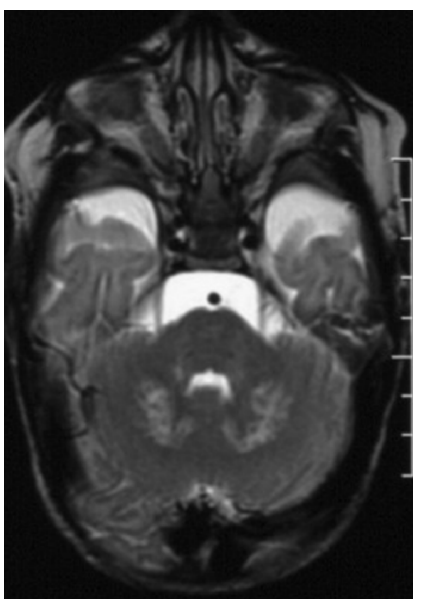

(b)

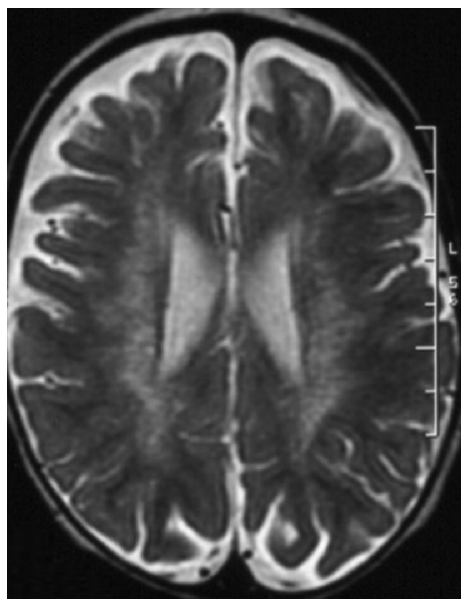

(c)

Fig. 6. Krabbe disease. Axial T2-WI show high signal intensity in the cerebellar white matter (a), cerebellar dentate nuclei (b), and periventricular white matter with sparing of the subcortical white matter (c).

\subsubsection{X-linked adrenoleukodystrophy}

X-linked adrenoleukodystrophy (ALD) is a peroxisomal disorder affecting the central nervous system, adrenal cortex, and testicles. It is caused by mutations in the ALD gene that encodes for a peroxisomal membrane protein. It is associated to accumulation of very long chain of fatty acids in different tissues and in plasma. Several different forms of ALD have been reported. The childhood cerebral form, the most common, with a clinical onset between ages 4 and 8 years, manifests with behavioural and school performance problems, progressive impairment of cognition, vision, hearing and motor function. Within 2 years, it leads to total disability, and to death within 5 to 8 years (Cecil \& Kos, 2006). The adrenomyeloneuropathy (AMN) generally manifests in the late twenties and progresses over decades with paraparesis and sphincter and sexual dysfunction. Clinical manifestations associated to MRI features may lead to diagnosis. High concentration of very long chain fatty acids in plasma and/or cultured skin fibroblasts reinforces it. Molecular genetic testing is clinically available.

Three distinct zones have been described in white matter lesions, with direct influence in imagiologic features. An inner zone (Zone A) of astrogliosis and scarring; an intermediate zone (Zone B) of active inflammation and demyelination, with axons spared; and an outer zone (Zone $\mathrm{C}$ ) of ongoing demyelination in absence of inflammation. A Zone $\mathrm{D}$ has been referred, which is peripheral to Zone $\mathrm{C}$ and characterized by impending demyelination (Eichler et al., 2002). In early stages, ALD CT scan and MRI have a typical appearance. Symmetric white matter changes occur predominantly in the peritrigonal regions and across the corpus callosum splenium with relative preservation of the subcortical U fibers. Spread progression occurs outwardly and cephalad as a confluent lesion, until most of the white matter is involved. CT shows symmetric low attenuation in a butterfly distribution, across the corpus callosum splenium, surrounded by an enhancing zone peripherally, due to inflammation. In the earliest stage, the lesion may be restricted to the splenium (Barkovich, 
2005). On MRI the Zone A reveals T1 and T2 prolongation; the Zone B appears isointense on T1-WI and isointense or slightly hypointense on T2-WI, showing enhancement if paramagnetic contrast is administered; and the Zone $\mathrm{C}$ reveals minimally hypointense on T1-WI and hyperintense on T2-WI, without enhancement (Melhem et al., 2000). Symmetric abnormal T2 hyperintensity along the corticopontine and corticospinal tracts, and auditory pathways are common (Fig. 7.). Cases of predominantly frontal lobe involvement may occur with lesion of corpus callosum genu, anterior limbs and genu of the internal capsules, sporadically with lesion of cerebellar white matter. Atypical cases with unilateral involvement have also been reported, as well as calcifications in parieto-occipital region (Barkovich, 2005). MRI in AMN, compared to ALD, shows more frequently an involvement of cerebellar white matter and brainstem corticospinal tract and less commonly, cerebral lesion (Barkovich, 2005).

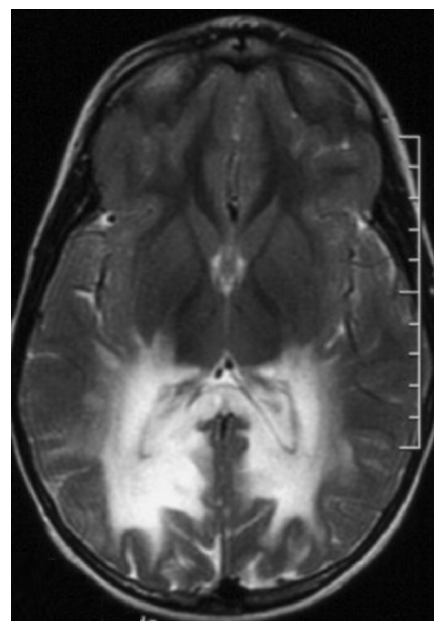

(a)

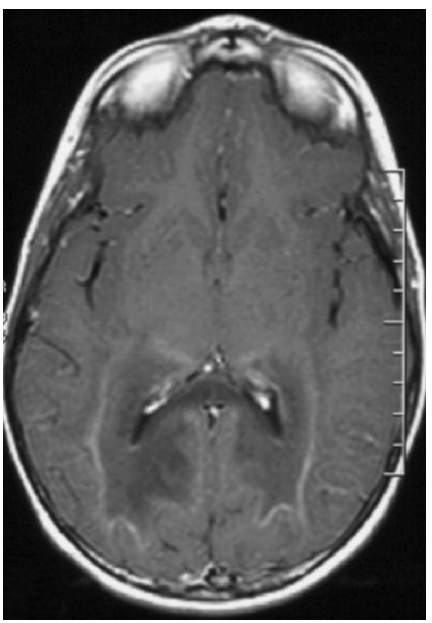

(b)

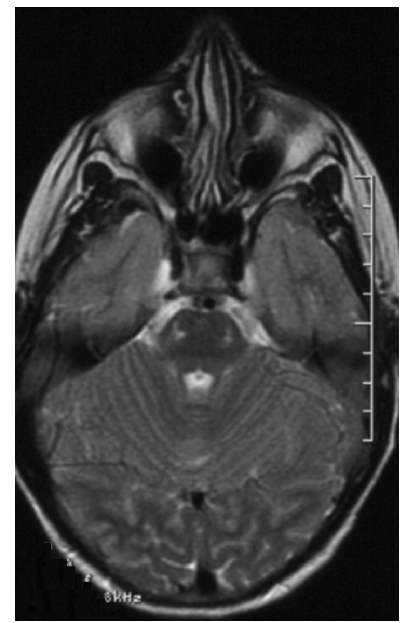

(c)

Fig. 7. X-linked adrenoleukodistrophy. (a) Axial T2-WI shows hyperintensity in the occipital white matter and corpus callosum splenium. (b) Axial T1-WI postcontrast shows enhancement leading edge of inflammation. (c) Axial T2-WI shows hyperintensity in the corticospinal tracts within the pons.

DWI shows an increased apparent diffusion coefficient (ADC) in Zone A (Schneider et al., 2003). MRS reveals abnormal spectrum within regions of abnormal imaging, as well as normal appearing white matter. Spectrum profile in normal appearing white matter discloses elevated choline levels. Raised choline and myo-inositol levels reflect the onset of demyelination. An increase in choline, myo-inositol and glutamine levels suggests active demyelination and glial proliferation. Decreased NAA and glutamine levels reflect neuronal loss and injury. High lactate peak is consistent with inflammation (Cecil \& Kos, 2006). Detection of MRS abnormalities in asymptomatic patients or in those with stable MRI can predict disease progression.

\subsubsection{Vanishing white matter disease}

Vanishing White Matter (VWM), or childhood ataxia with central nervous system hypomyelination, is an autosomal recessive disease caused by mutation in one of the five 
genes involved in eukaryotic translation initiation 2B. It may result in impairment of the ability of cells to regulate protein synthesis in response to stress (Pronk et al., 2006). Its phenotype varies widely from antenatal onset with early death, to adult onset with slowly progressive disease. In late onset cases, motor and mental development is normally or mildly delayed at first. Chronic progressive or subacute neurological deterioration, with cerebellar ataxia, spasticity, and variable optic atrophy frequently occurs between the ages of 2 and 6 years. Epilepsy is not a major sign of the disease, and, unlike motor abilities, mental capacities are relatively preserved. Episodes of rapid deterioration can occur after minor trauma or infection, ending in unexpected coma. Death happens a few years after onset (van der Knaap et al., 1998). Diagnosis is based on clinical manifestations, MRI features, and mutation identification in one of the five mentioned genes (Schiffmann et al., 2010).

CT scan reveals symmetric and diffuse white matter low density in the cerebral hemispheres. MRI is typical, showing symmetric and diffuse white matter anomalies. Subcortical involvement occurs during early stages of disease, with swelling and enlargement of gyri. On the later stages, white matter reveals signal intensity which is close to, or similar to cerebrospinal fluid in every sequence (Fig. 8.). On FLAIR and T1-WI there is a radiating, stripe-like pattern, on sagittal and coronal images; and a dot-like pattern in the centrum semiovale, on the axial images, which corresponds to remain tissue strands. Overtime, cystic lesions develop. Cerebellar atrophy may be seen, mainly involving the vermis. Dorsal pons hyperintensity is seen at the beginning; in later stages, involvement of the ventral pons also occurs (van der Knaap et al., 2006).

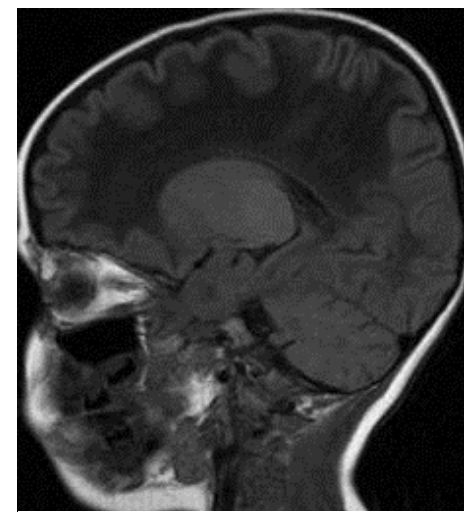

(a)

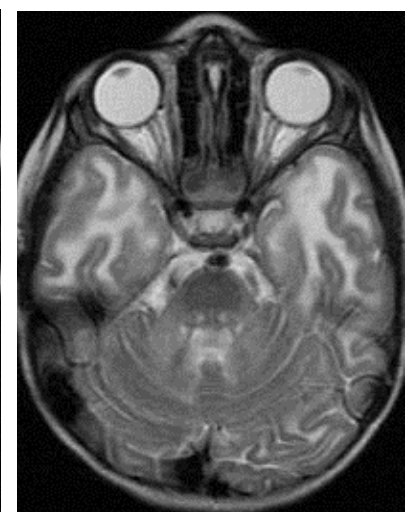

(b)

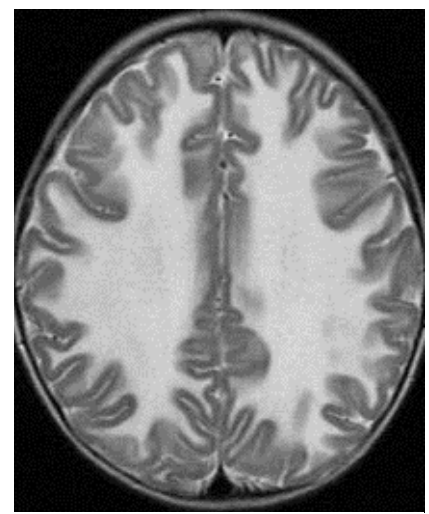

(c)

Fig. 8. Vanishing white matter disease. (a) Sagittal T1-WI shows abnormal cerebral white matter hypointensity. T2-WI shows hyperintensity involving central tegmental tract in the dorsal pons (b) and cerebral white matter (c).

MRS in the white matter shows marked decrease in NAA, creatine and choline peaks, or even virtual absence of all parenchymal metabolites, with presence of lactate and glucose. Gray matter spectrum is nearly normal, with a small increase in lactate and glucose, and a decrease in NAA peaks (van der Knaap et al., 1998). 


\section{Disorders involving both gray and white matter}

When the damage affects the gray matter and white matter, it is important to recognize if the lesions involve deep gray matter, or if, in addition to the white matter, the cortical gray matter is the only one to be affected (Barkovich, 2005, 2007).

\subsection{Disorders involving white matter and cortical gray matter}

This group of IEM includes peroxisomal disorders such as Zellweger syndrome, Alpers disease, Menkes disease, mucopolysaccharidoses and mucolipidoses (Barkovich, 2005).

\subsection{Disorders involving white matter and deep gray matter}

The list of IEM with white matter and deep gray matter involvement is wide, but the identification of the nuclei that is early affected can help narrowing the differential diagnosis. The involvement of thalami is present in GM1 and GM2 gangliosidoses, Krabbe disease, and Wilson's disease. The lesion of globi pallidi is present in Canavan disease, methylmalonic acidemia, MSUD, Kearns-Sayre syndrome, L-2-hydroxyglutaric aciduria, and urea cycle disorders (Barkovich, 2005). When the involvement is seen in striata, the differential diagnosis should include glutaric aciduria type I, propionic acidemia, ethylmalonic acidemia, Wilson's disease, MELAS and Leigh syndrome.

\subsubsection{Canavan disease}

Canavan disease, or spongiform leukodystrophy, is an autosomal recessive disorder, characterized by deficiency of $\mathrm{N}$-acetylaspartylase resulting in abnormal accumulation of $\mathrm{N}$ acetylaspartic acid. Three clinical forms - neonatal, infantile, and late onset - are recognized (Traeger \& Rapin, 1998). Most commonly, Canavan disease presents within the first 6 months of life with hypotonia, lack of head control, macrocephaly, irritability and development delay. Later on, spasticity, optic atrophy and seizures ensue (Cecil \& Kos, 2006). Death occurs within the teens. Suspicion of diagnosis is based on clinical manifestation and MRI features. Diagnosis is confirmed by demonstration of high concentration of $\mathrm{N}$-acetylaspartic acid in the urine. Molecular genetic testing is clinically available (Matalon \& Bhatia, 2009).

CT scan shows diffuse hypodensity in the cerebral and cerebellar white matter (Barkovich, 2005). MRI shows symmetric areas of diffuse confluent white matter areas of T1 and T2 prolongation. The subcortical $U$ fibers are preferentially affected in the beginning of the disease. Globi pallidi are frequently involved, as well as thalami. In some cases internal and external capsules (Cheon et al, 2002), cerebellar white matter and brain stem can also be affected (Matalon \& Bhatia, 2009). In the later stages, there is a diffuse atrophy of white matter.

MRS in white matter shows marked elevation of the NAA peak, which is classically assumed to be exclusive for Canavan disease, although it can also be seen some times in Salla disease and PMD (Varho et al, 1999).

\subsubsection{Maple syrup urine disease}

Maple syrup urine disease (MSUD), an autosomal recessive disorder, is caused by deficiency of the branched-chain alpha-ketoacid dehydrogenase (BCKAD) complex, leading to accumulation of the branched-chain amino acids (BCAAs), allo-isoleucine and branched- 
chain ketoacids (BCKAs) in tissues and plasma (Strauss et al., 2009). MSUD manifests as heterogeneous clinical and molecular phenotypes. Several clinical forms have been described with manifestations from early acute neonatal to chronic intermittent forms diagnosed in adolescents. Classic MSUD causes maple syrup odor in urine and cerumen, soon after birth. At age 2-3 days newborns present with irritability, poor feeding and ketonuria. At age 4-5 days lethargy, apneia, opisthotonus and stereotyped movements occur; followed by coma and central respiratory failure at age 7-10 days. High protein ingestion or any cause of enhanced catabolism like infection, injury or surgery, can lead to acute leucine intoxication with cerebral oedema and neurological impairment (Morton et al., 2002). In less severe cases, patients reveal normal or moderately retarded neurodevelopment, later presenting with metabolic crises similar to classic MSUD. Suspicion of diagnosis, based on clinical manifestations (and eventually MRI features in late onset cases), can be confirmed by elevation of BCAAs, allo-isoleucine and BCKAs in tissues and plasma. Molecular genetic testing is also clinically available (Strauss et al., 2009). In Portugal, extended newborn screening detects MSUD, frequently in a pre-symptomatic stage.

Cranial ultrasonography can be useful in symptomatic neonates, showing symmetric hyperechogenicity of periventricular white matter, basal ganglia (mainly globi pallidi) and thalami (Fariello et al., 1996).

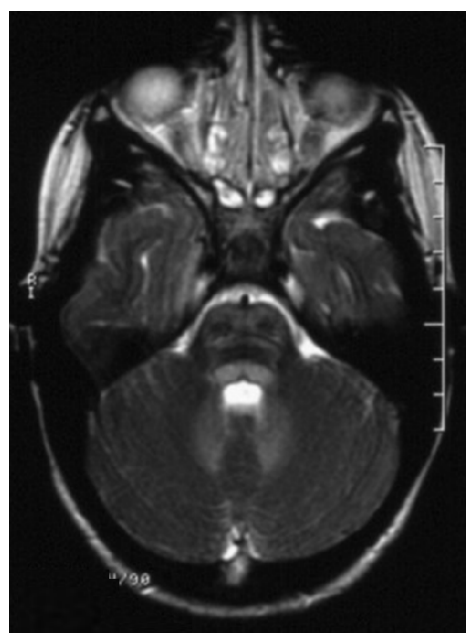

(a)

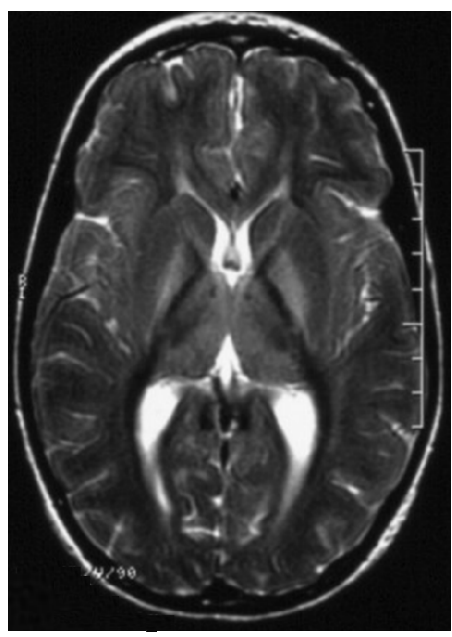

(b)

Fig. 9. Maple syrup urine disease. Axial T2-WI show hyperintensity in the brainstem and cerebellar white matter (a), globus pallidi and thalami (b).

Acute classic MSUD form shows signs of diffuse oedema, characterized by hypodensity on CT scan, and T1 and T2 prolongation on MRI. Severe localized oedema (MSUD oedema) is seen in myelinated areas at birth, namely deep cerebellar white matter, posterior brain stem, cerebral peduncles, thalami, posterior limb of internal capsule, posterior centrum semiovale, and globi pallidi (Fig. 9.) (Brismar et al., 1990). Forms with later onset reveal lack of myelination superimposed upon lesions located at the same regions affected by MSUD 
oedema in acute classic form. Brain sequel depends on the time it takes to reverse metabolic decompensation (Barkovich, 2005).

DWI is very important, as the oedema may be hard to identify in T2-WI due to nonmyelinated brain hyperintensity. Regions with acute MSUD oedema show restricted diffusion with decreased ADC value. Diffusion-tensor imaging (DTI) reveals decreased anisotropy in the same areas (Parmar et al., 2004). In acute phase, MRS with long TE reveals elevated levels of lactate and presence of an abnormal BCAAs and BCKAs peak at $0.9 \mathrm{ppm}$. These changes can reverse completely after metabolic correction (Jan et al., 2003).

\subsubsection{L-2-hydroxyglutaric aciduria}

L-2-hydroxyglutaric aciduria is an autosomal recessive disease caused by mutations in L-2hydroxyglutarate dehydrogenase gene, with accumulation of L-2-hydroxyglutac acid in urine, cerebrospinal fluid and plasma. It is characterized by slowly progressive neurological dysfunction with cerebellar ataxia, psychomotor retardation, seizures, macrocephaly, and extrapyramidal and pyramidal signs (Steenweg et al., 2010). It presents in childhood, frequently after a period of normal psychomotor development. Diagnosis is suggested by typical MRI features and by measurement of L-2-hydroxyglutaric acid in urine, cerebrospinal fluid or serum. Molecular genetic testing is clinically available (Seashore, 2009).

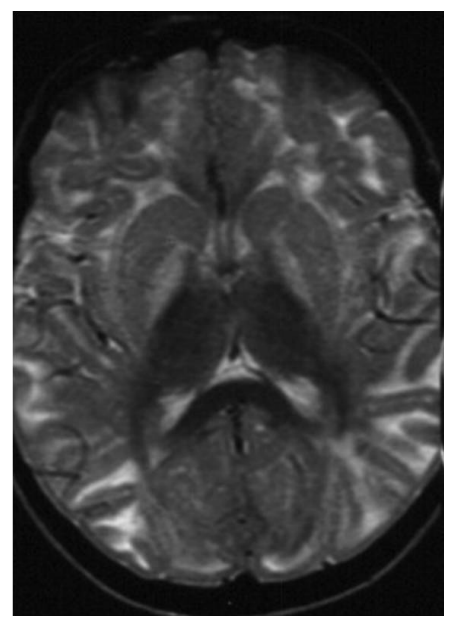

(a)

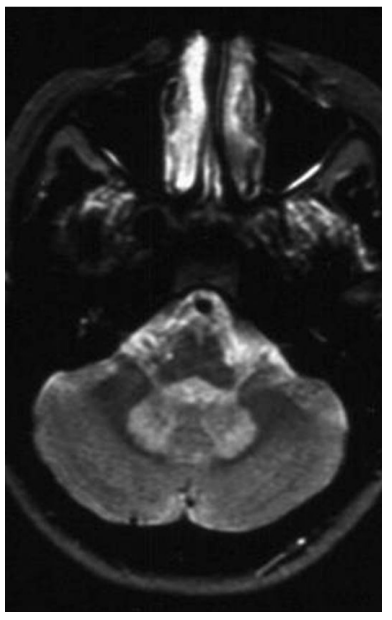

(b)

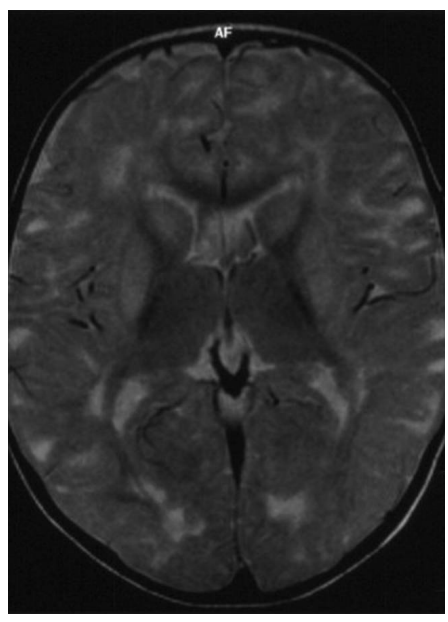

(c)

Fig. 10. L-2-hydroxyglutaric aciduria. Axial T2-WI show hyperintensity of the subcortical WM and globus pallidi (a), dentate nuclei (b), putamina and caudate nuclei (c).

CT scan shows symmetrical hypodensity in subcortical white matter, globi pallidi, and frequently dentate nuclei (Topçu et al., 1996). MRI reveals hyperintensity in the same locations listed above, with a preferential involvement of the frontal over the occipital white matter. The periventricular white matter is spared, as well as internal capsule, corpus callosum, cerebellar white matter, and brainstem. Sometimes involvement of putamina and caudate nuclei are seen (Fig. 10.). Globi pallidi, and sometimes cerebellar vermis and 
hemisphere, reveal atrophy. Swelling of the cerebral white matter with broadening of gyri, rarefaction of the subcortical white matter and atrophy of cerebral white matter were also reported (Steenweg et al., 2009).

DWI shows increased diffusion in the white matter lesions. MRS is usually normal, but it can reveal a slight decrease of NAA peak, in short TE (Aydin et al, 2003).

\subsubsection{Glutaric aciduria type I}

Glutaric aciduria type $\mathrm{I}$ is an autosomal recessive disorder resulting from a deficiency in glutaryl-CoA dehydrogenase with accumulation of glutaric, 3-hydroxyglutaric and glutaconic acids and secondary carnitine deficiency. Typically the disorder presents with an acute encephalopathy between 6 and 18 months of age, in a previously healthy or mildly motor retarded, macrocephalic child. In some cases, a slowly progressive course with mental retardation, hypotonia, dystonia, choreoathetosis, spastic quadriplegia and macrocephaly is seen (Hoffmann \& Zschocke, 1999). Diagnosis is suggested by classical MRI features that are highly typical, and made by measurement of glutaric, 3-hydroxyglutaric and glutaconic acids in urine. Molecular genetic testing is clinically available (Seashore, 2009). In Portugal, broad neonatal screening detects glutaric aciduria type I in a presymptomatic phase.

CT scan shows diffuse white matter hypodensity and/or cerebral atrophy, most prominent in the frontal and temporal regions (Yager et al., 1988). MRI reveals symmetric widening of the Sylvian fissure, frontotemporal volume loss, and delayed myelination. Putamen T2 hyperintensity is predominantly seen, either alone or in combination with the caudate nucleus. Globus pallidus is less affected. Later in the course of disease, periventricular white matter T2 hyperintensity, basal ganglia and cerebral atrophy are seen. Sometimes imagiological studies reveal acute or chronic subdural haematomas (Fig. 11.), implying differential diagnosis with nonaccidental trauma (Neumaier-Probst et al., 2004).

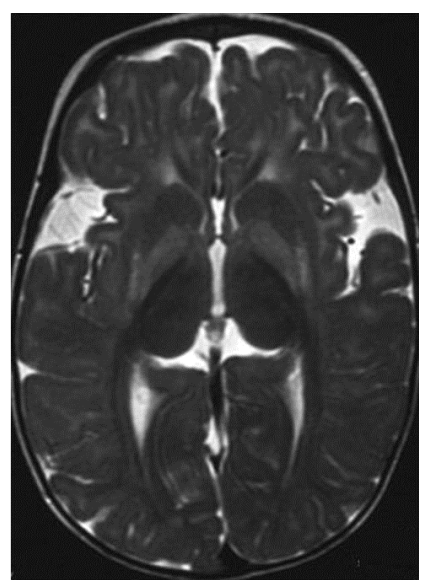

(a)

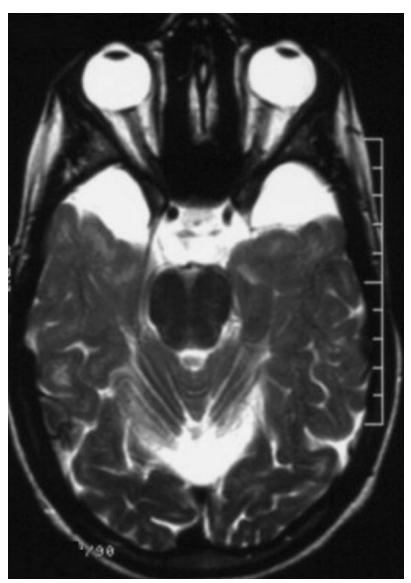

(b)

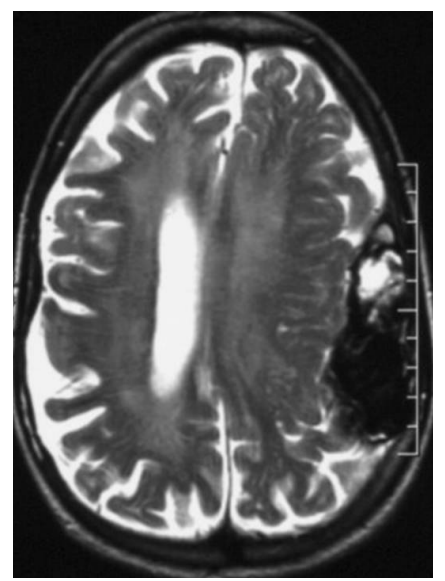

(c)

Fig. 11. Glutaric aciduria type I. Axial T2-WI show putamina and globi pallidi hyperintensity (a), expansion of pericerebral fluid spaces anterior to the temporal lobes (b), periventricular white matter hyperintensity, cerebral atrophy and left frontoparietal subdural hematoma (c). 
DWI shows restricted diffusion in acute basal ganglia lesions. MRS shows decreased NAA/creatine ratio at the basal ganglia in encephalopathic patients (Pérez-Dueñas at al., 2009).

Fluoro-2-deoxyglucose PET reveals decreased glucose uptake in the cerebral cortex, basal ganglia and thalami (Al-Essa et al., 1998).

\subsubsection{Mitochondrial encephalomyopathy with lactic acidosis and stroke-like episodes (MELAS)}

MELAS is a multisystem mitochondrial disorder, with early normal psychomotor development and onset typically between the ages 2 and 10 years. It is characterised by neurological manifestations including seizures, encephalopathy, recurrent headaches and stroke-like episodes (hemiparesis and hemianopsia). Diagnosis is based on an association of clinical findings, MRI features and molecular genetic testing. Mitochondrial DNA mutation A3243G is found in $80 \%$ of MELAS patients. Sometimes, pathogenic mutations may be undetectable in mtDNA from leukocytes, and it is necessary to resort to other tissues, such as skeletal muscle, which is the most reliable for diagnosis (DiMauro \& Hirano, 2010).

Stroke-like lesions are often transient, affecting mainly the gray matter and are not restricted to specific vascular territories, unlike embolic and thrombotic infarction (Barkovick, 2005). They are usually fluctuating. Acute ischemic episodes show swelling and hypodensity on CT scan, and T1 and T2 prolongation on MRI, commonly involving the temporo-parietooccipital lobes and basal ganglia (Fig. 12.).

With regard to DWI findings, reports are discordant. Some early reports demonstrate an increased diffusion in stroke-like lesions. However the number of reports revealing a decreased diffusion in these areas has increased. Thus, the absence of vasogenic oedema should not weaken the possibility of MELAS in favour of ischemic stroke. MRS shows a decrease in NAA and an increase in lactate in stroke-like lesions (Fig. 12.) (Tzoulis \& Bindoff, 2009). An increase in lactate is also seen in embolic and thrombotic infarction, so this finding is not specific of MELAS.

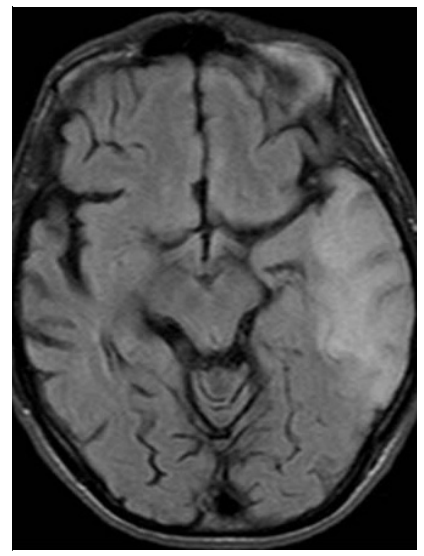

(a)

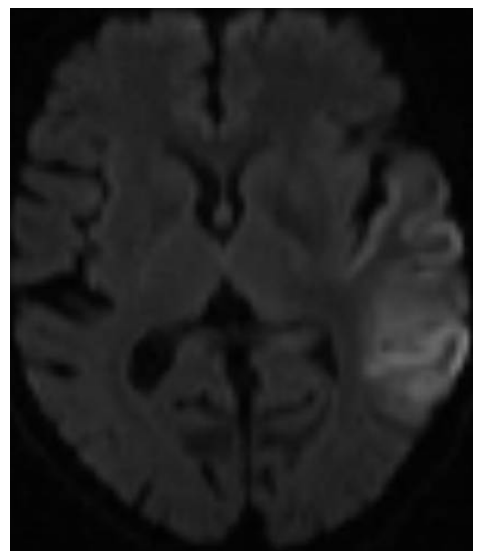

(b)

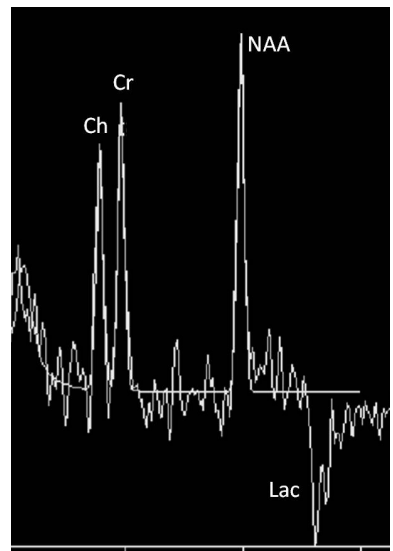

(c)

Fig. 12. MELAS. (a) Axial FLAIR shows hyperintensity in left temporal lobe. (b) Axial DWI $(b=1000)$ shows restriction diffusion of the lesion. (c) Single voxel proton MRS (TE = $135 \mathrm{~ms})$ of the lesion shows a lactate doublet (Lac). (This case is a courtesy of Dr. Fernando Matias) 
SPECT shows hyperperfusion in the acute stage of stroke-like episodes, and hypoperfusion in the chronic stage (Tzoulis \& Bindoff, 2009). PET and xenon reveal increased blood flow and decreased glucose uptake and oxygen extraction fraction in the lesion areas (Barkovich, 2005).

\subsubsection{Leigh syndrome}

Leigh syndrome, or subacute necrotizing encephalomyelopathy, is caused, in the majority of the cases, by a dysfunction of the mitochondrial respiratory chain (particularly complexes I, II, IV or V), the coenzyme Q, or the pyruvate dehydrogenase complex (Finsterer, 2008). Most children are normal at birth and manifested usually by the end of the first year of life. The onset is commonly insidious, and the course may be intermittently progressive for some years. Clinical presentation can be highly variable, and includes psychomotor retardation, feeding difficulties, recurrent episodes of vomiting, failure thrive, signs of brainstem, cerebellar and basal ganglia dysfunction, and lactic acidosis (Medina et al., 1990). Diagnosis is suggested by clinical criteria and MRI features. Molecular genetic testing allows for a specific etiological diagnosis.

Neuroradiological findings in Leigh syndrome are symmetrical hypodensities on CT, and T1 and T2 prolongation on MRI, in the basal ganglia and thalami. Lesions can involve the substantia nigra, periaqueductal gray matter within the midbrain, inferior colliculus, inferior olivary nuclei, inferior cerebellar peduncles, medulla, solitary tract in the medulla, central tegmental tract and reticular formation in the dorsal pons (Barkovich 2005). Less commonly, the red nuclei and cerebellar dentate nuclei are involved. Basal ganglia are often affected before the brainstem, but in some patients, brainstem lesions appear without basal ganglia alterations (Fig. 13.). Sometimes, MRI reveals delayed myelination. In most patients,

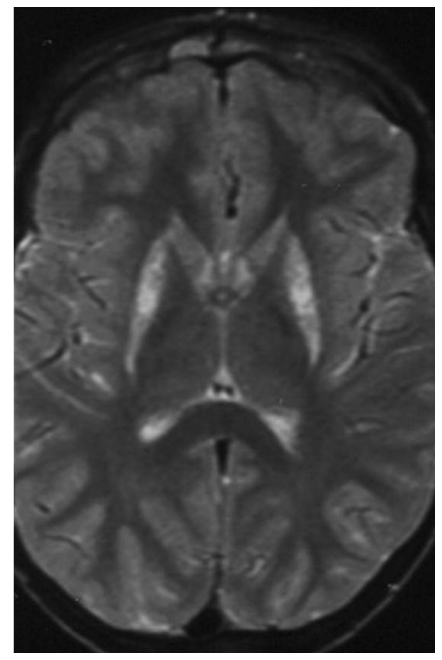

(a)

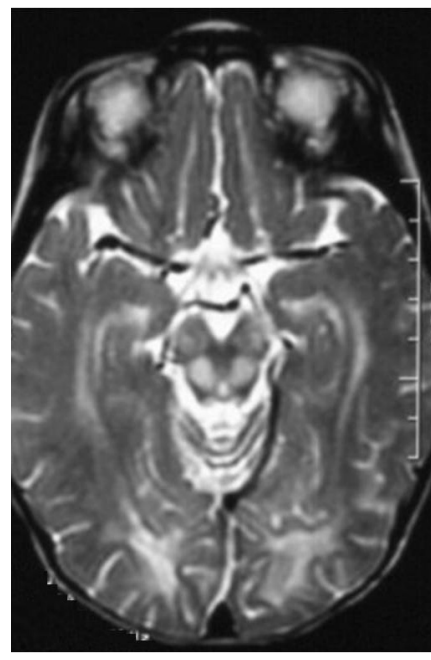

(b)

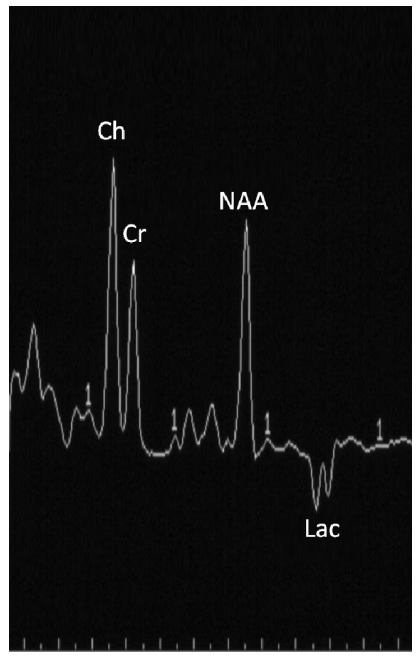

(c)

Fig. 13. Leigh syndrome. Axial T2-WI show hyperintensity in the striata (a), dorsal midbrain and cerebral peduncles (b). (c) Multivoxel proton MRS (TE $=144 \mathrm{~ms}$ ) of the basal ganglia shows a decreased of NAA peak and a lactate doublet (Lac). 
cerebral white matter is generally involved in the later stages of disease (Arii \& Tanabe, 2000). In some cases, a marked global atrophy can be seen, over time. Some MRI patterns may suggest an implicated mutation. In SURF-1 mutation, associated to complex IV deficiency, basal ganglia are less involved, and the brainstem, subthalamic nuclei, cerebellar nuclei and cerebellar peduncles are commonly involved. ATPase 6 mutation, which is associated to maternally inherited Leigh syndrome (MILS), shows anterior putamina, globi pallidi and dorsal mesencephalon and pons lesions. In the absence of hypoxia, ischemia or infection, symmetric involvement of deep gray matter is very suspicious of a mitochondrial defect (Saneto et al., 2008).

DWI shows reduced diffusion in acute lesions and increased diffusion in chronic ones. MRS reveals increase in lactate and a small decrease of NAA in lesions, and alongside the imagiological features of conventional MR it supports the diagnosis of Leigh syndrome (Barkovich, 2005).

\section{Conclusion}

MRI plays an essential role in the diagnosis of IEM. It shows high sensitivity in the detection of some of these disorders and evaluation of their severity. Despite of the nonspecificity of many features, a systematic pattern recognition approach to brain structures involved is useful, as it narrows differential diagnosis. MRS can help in this process and may disclose anomalies, even if there are no lesions detectable with conventional MRI. Further investigation and accurate characterization of neuroradiological features are needed in order to gather a wider range of specific patterns. These would allow more patients to be adequately classified.

\section{References}

Al-Essa, M. e tal.. (1998). Fluro-2-deoxyglucose (18FDG) PET scan of the brain in glutaric aciduria type I: clinical and MRI correlations. Brain Dev, Vol.20, No.5, (August 1998), pp. 295-301, ISSN 0387-7604

Arii, J. \& Tanabe, Y. (2000). Leigh syndrome: serial MR imaging and clinical follow-up. Am J Neuroradiol, Vol.21, No.8, (September 2000), pp. 1502-1509, ISSN 0195-6108

Autti, T. et al.. (1996). MRI of neuronal ceroid lipofuscinosis. I. Cranial MRI of 30 patients with juvenile neuronal ceroid lipofuscinosis. Neuroradiology, Vol.38, No.5, (July 1996), pp. 476-482, ISSN 0028-3940

Aydin, K. et al.. (2003). Single-voxel MR spectroscopy and diffusion-weighted MRI in two patients with L-2-hydroxyglutaric aciduria. Pediatr Radiol, Vol.33, No.12, (December 2003), pp. 872-876, ISSN 0301-0449

Barkovich, A. (2005). Pediatric Neuroimaging (4th edition) Lippincott Williams \& Wilkins, ISBN 0-7817-5766-5, Philadelphia

Barkovich, A. (2006). A magnetic resonance approach to metabolic disorders in childhood. Rev Neurol, Vol.10, No.43, (October 2006), pp. 5-16, ISSN 0210-0010

Barkovich, A. (2007). An approach to MRI of metabolic disorders in children. Journal of neuroradiology, Vol.34, (2007), pp. 75-88, ISSN 0195-6108

Boor, I. et al.. (2007). MLC1 is associated with the dystrophin-glycoprotein complex at astrocytic endfeet. Acta Neuropathol. Vol.114, No.4, (October 2007), pp. 403-410, ISSN 0001-6322 
Brismar, J. et al.. Maple syrup urine disease: findings on CT and MR scans of the brain in 1o infants. Am J Neuroradiol, Vol.11, No.6, (November-December 1990), pp. 1219-1228, ISSN 0195-6108

Brokmann, K. et al.. (1996). Localized proton magnetic resonance spectroscopy of cerebral metabolic disturbances in children with neuronal ceroid lipofuscinosis. Neuropediatrics, Vol.27, No.5, (October 1996), pp. 242-248, ISSN 1439-1899

Campistol, J. (1999). Aproximación al diagnostic de los errors congénitos del metabolismo por la neuroimagen. Rev Neurol, Vol.28, No.161, (1999), pp. 16-23, ISSN 1576-6578

Cecil, K. \& Kos, R. (2006). Magnetic resonance spectroscopy and metabolic imaging in white matter diseases and pediatric disorders. Top Magn Reson Imaging, Vol.17, No.4, (August 2006), pp. 275-293, ISSN 1536-1004

Cheon, J. et al.. (2002). Leukodystrophy in children: a pictorial review of MR imaging features. Radiographics, Vol.22, No.3, (May-June 2002), pp. 461-476, ISSN 0271-5333

Chilosi, A. et al.. (2008). Treatment with L-arginine improves neuropsychological disorders in a child with creatine transporter defect. Neurocase, Vol.14, No.2, (2008),pp. 151161, ISSN 1465-3656

DiMauro, S. \& Hirano, M. (October 2010). MELAS, In: GeneReviews, 03.02.2011, Available from http://www.ncbi.nlm.nih.gov/books/NBK1233/\#melas.Summary

Dyke, J. et al.. (2007). Assessing disease severity in late infantile neuronal ceroid lipofuscinosis using quantitative MR diffusion-weighted imaging. Am J Neuroradiol, Vol.28, (August 2007), pp. 1232-1236, ISSN 0195-6108

Eichler, F. et al.. (2002). Proton MR spectroscopy and diffusion tensor brain MR imaging in X-linked adrenoleukodystrophy: initial experience. Radiology, Vol.225, No.1, (October 2002), pp. 245-252, ISSN 0033-8419

Engelbrecht, V. et al.. (2002). Diffusion-weight MR imaging in the brain in children: findings in the normal brain and in the brain with white matter diseases. Radiology, Vol.222, No.2, (February 2002), pp. 410-418, ISSN 0033-8419

Fariello, G. et al.. (1996). Cranial ultrasonography in maple syrup urine disease. Am J Neuroradiol, Vol.17, No.2, (February 1996), pp. 311-315, ISSN 0195-6108

Farina, L. et al.. (2000). MR imaging and proton MR spectroscopy in adult Krabbe disease. Am J Neuroradiol, Vol.21, No.8, (September 2000), pp. 1478-1482, ISSN 0195-6108

Finsterer, J. (2008). Leigh and leigh-like syndrome in children and adults. Pediatr Neurol, Vol.39, No.4, (October 2008), pp. 223-235, ISSN 0887-8994

Fluharty, A. (September 2008). Arysulfatase A deficiency. In: GeneReviews, 24.02.2011, Available from http://www.ncbi.nlm.nih.gov/books/NBK1130/\#mld.Summary

Gelal, F. et al.. (2002). van der Knaap's leukoencephalopathy: report of five new cases with emphasis on diffusion-weighted MRI findings. Neuroradiology, Vol.44, No.7, (July 2002), pp. 625-630, ISSN 0028-3940

Given, C. et al.. (2001). Intracranial and spinal MR imaging findings associated with Krabbe's disease: case report. Am J Neuroradiol, Vol.22, No.9, (October 2001), pp. 1782-1785, ISSN 0195-6108

Gorospe, J. (April 2010). Alexander disease, In: GeneReviews, 28.02.2011, Available from http://www.ncbi.nlm.nih.gov/books/NBK1172/

Haltia, M. (2003). The neuronal ceroid-lipofuscinoses. J Neuropathol Exp Neurol, Vol.62, No.1, (January 2003), pp. 1-13, ISSN 0022-3069 
Hanefeld, F. et al.. (2005). Quantitative proton MRS of Pelizaeus-Merzbacher disease: evidence of dys- and hypomyelination. Neurology, Vol.65, No.5, (September 2005), pp.701-706, ISSN 0028-3878

Hayflick, S. et al.. (2003). Genetic, clinical and radiographic delineation of HallervordenSpatz syndrome. N Engl J Med, Vol.348, No.1, (January 2003), pp. 33-40, ISSN 00284793

Hayflick, S. et al.. (2006). Brain MRI in neurodegeneration with brain iron accumulation with and without PANK2 mutations. Am J Neuroradiol. Vol.27, No.6. (June-July 2006), pp.1230-1233, ISSN 0195-6108

Hoffmann, G. \& Zschocke, J. (1999). Glutaric aciduria type I: from clinical, biochemical and molecular diversity to successful therapy. J Inherit Metab Dis, Vol.22, No.4, (June 1999), pp. 381-391, ISSN 0141-8955

Incerti, L. (2000). MRI in neuronal ceroid lipofuscinosis. Neuro Sci, Vol.21, No.1, (2000), pp. 71-73, ISSN 1590-1874

Jan, W. et al.. (2003) MR diffusion imaging and MR spectroscopy of maple syrup urine disease during acute metabolic decompensation. Neuroradiology, Vol.45, No.6, (June 2003), pp. 393-399, ISSN 0028-3940

Järvelä, I. et. al.. (1997). Clinical and magnetic resonance imaging findings in Batter disease: analysis of the major mutation (1.02-kb deletion). Ann Neurol, Vol.42, No.5, (1997), pp. 799-802, ISSN 0364-5134

Kiriyama, T. et al.. (2007). SPECT revealed cortical dysfunction in a patient ho had genetically definite megalencephalic leukoencephalopathy with subcortical cysts. Clin Neurol Neurosurg, Vol.109, No.6, (July 2007), pp. 526-530, ISSN 0303-8467

Matalon, R. \& Bhatia, G. (October 2009). Canvan disease, In: GeneReviews, 02.03.2011, Available from http://www.ncbi.nlm.nih.gov/books/NBK1234/

Medina, L. et al.. (1990). MR findings in patients with subacute necrotizing encephalomyelopathy (Leigh syndrome): correlation with biochemical defect. Am J Neuroradiol, Vol.11, No.2, (March-April 1990), pp. 379-384, ISSN 0195-6108

Melhem, E. et al.. X-linked adrenoleukodystrophy: the role of contrast-enhanced MR imaging in predicting disease progression. Am J Neuroradiol, Vol.21, No.5, (May 2000), pp. 839-844, ISSN 0195-6108

Mercimek-Mahmutoglu, S. \& Stöckler-Ipsiroglu, S. (January 2009). Creatine deficiency syndromes, In: GeneReviews, 21.02.2011, Available from http://www.ncbi.nlm.nih.gov/books/NBK3794/\#creatine.Clinical_Description

Mercimek-Mahmutoglu, S. et al.. (2010). Treatment of intractable epilepsy in a femalewith SLC6A8 deficiency. Mol Genet Metab, Vol.101, No.4, (December 2010), pp. 409-412, ISSN 1096-7192

Morita, H. et al.. (2006). MR imaging and 1H-MR spectroscopy of a case of van der Knaap disease. Brain Dev, Vol.28, No.7, (August 2006), pp. 466-469, ISSN 0387-7604

Morton, D. et al.. (2002). Diagnosis and treatment of maple syrup disease: a study of 36 patients. Pediatrics, Vol.109, No.6, (June 2002), pp. 999-1008, ISSN 0031-4005

Neumaier-Probst, E. et al.. (2004). Neuroradiological findings in glutaric aciduria type I (glutaryl-CoA dehydrogenase deficiency). Vol.27, No.6, (2004), pp. 869-876, ISSN 0141-8955 
Parashari, U. et al.. (2010). Case report: MR spectroscopy in pantothenate kinase-2 associated neurodegeneration. Indian J Radiol Imaging, Vol.20, No.3, (August 2010), pp. 188191, ISSN 0971-3026

Parmar, H. et al.. (2004). Maple syrup urine disease: diffusion-weighted and diffusion-tensor magnetic resonance imaging findings. J Comput Assist Tomogr, Vol.28, No.1, (January-February 2004), pp. 93-97, ISSN 0363-8715

Pérez-Dueñas, B. et al.. (2009). Brain injury in glutaric aciduria type I: the value of functional techniques in magnetic resonance imaging. Eur J Paediatric Neurol, Vol.13, No.6, (November 2009), pp. 534-540, ISSN 1090-3798

Pizzini, F. et al.. (2003). Proton MR spectroscopy imaging in Pelizaeus-Merzbacher disease. Am J Neuroradiol, Vol.24, No.8, pp.1683-1689, ISSN 0195-6108

Plecko, B. et al.. (2003). Degree of hypomyelination and magnetic resonance spectroscopy findings in patients with Pelizaeus Merzbacher phenotype. Neuropediatrics, Vol.34, No.3, (June 2003), pp.127-136, ISSN 1439-1899

Póo-Arqüelles, P. et al.. (2006). X-linked creatine transporter deficiency in two patients with severe mental retardation and autism. J Inherit Metab Dis, Vol.29, No.1, (February 2006), pp. 220-223, ISSN 1573-2665

Pronk, J. et al.. (2006). Vanishing white matter disease: a review with focus on its genetics. Ment Retard Dev Disabil Res Rev, Vol.12, No.2, (2006), pp.123-128, ISSN 1080-4013

Raghuveer, T. et al.. (2006). Inborn errors of metabolism in infancy and early childhood: an update. Am Fam Physician, Vol.73, No.11, (June 2006), pp. 1981-90, ISSN 0002-838X

Saneto, R. et al.. (2008). Neuroimaging of mitochondrial disease. Mitochondrion, Vol.8, No.56, (December 2008), pp. 396-413, ISSN 1567-7249

Schiffmann, R. et al.. (February 2010). Childhood ataxia with central nervous system hypomyelination/vanishing white matter, In: GeneReviews, 27.02.2011, Available from http:/ / www.ncbi.nlm.nih.gov/books/NBK1258/

Schneider, J. et al.. (2003). Diffusion tensor imaging in cases of adrenoleukodystrophy: preliminary experience as a marker for early demyelination? Am J Neuroradiol, Vol.24, No.5, (May 2003), pp. 819-824, ISSN 0195-6108

Schulze, A. (2003). Creatine deficiency syndromes. Mol Cell Biochem, Vol.244, No.1-2, (February 2003), pp. 143-150, ISSN 0330-8177

Scriver, C. et al.. (2001). The metabolic and molecular basis of inherited diseases, ISBN 9780079130358, New York

Seashore, M. (December 2009). The organic acidemias: an overview, In: GeneReviews, 03.03.2011, Available from http://www.ncbi.nlm.nih.gov/books/NBK1134/

Sener, R. (2002). Metachromatic leukodystrophy: diffusion MR imaging findings. Am J Neuroradiol, Vol.23, No.8, (September 2002), pp. 1424-1426, ISSN 0195-6108

Steenweg, M. et al.. (2009) L-2-hydroxyglutaric aciduria: pattern of MR imaging abnormalities in 56 patients. Radiology, Vol.251, No.3, (June 2009), pp. 856-865, ISSN 0033-8419

Steenweg, M. et al.. (2010). An overview of L-2-hydroxyglutarate dehydrogenase gene (L"HGDH) variants: a genotype-phenotype study. Hum Mutat, Vol.31, No.4, (April 2010), pp. 380-390, ISSN 1059-7794

Strauss, K. et al.. (December 2009). Maple syrup urine disease, In: GeneReviews, 27.02.2011, Available from http://www.ncbi.nlm.nih.gov/pubmed/20301495 
Traeger, E. \& Rapin, I. (1998). The clinical course of Canavan disease. Pediatric Neurol, Vol.18, No.3, (March 1998), pp. 207-212, ISSN 0887-8994

Topçu, M. et al.. (2009). Clinical and magnetic resonance imaging features of L-2hydroxyglutaric acidemia: report of three cases in comparison with Canavan disease. J Child Neurol, Vol.11, No.5, (September 1996), pp. 373-377, ISSN 0883-0738

Tzoulis, C. \& Bindoff, L. (2009). Serial diffusion imaging in a case of mitochondrial encephalomyopathy, lactic acidosis, and stroke-like episodes. Stroke, Vol.40, No.2, (February 2009), pp. 15-17, ISSN 0039-2499

van der Knaap, M. \& Valk, J. (1989). The reflection of histology in MR imaging of PelizaeusMerzbacher disease. Am J Neuroradiol, Vol.10, No.1, (January-February 1989), pp. 99-103, ISSN 0195-6108

van der Knaap, M. et al. (1995). Leukoencephalopathy with swelling and a discrepantly mild clinical course in eight children. Ann Neurol, Vol.37, No.3, (March 1995), pp. 324334, ISSN 0364-5134

van der Knaap, M. et al.. (1998). Phenotypic variation in leukoencephalopathy with vanishing white matter. Neurology, Vol.51, No.2, (August 1998), pp. 540-547, ISSN 0028-3878

van der Knaap, M. et al.. (2001). Alexander disease: diagnosis with MR imaging. Am J Neuroradiol, Vol.6, No.4, (March 2001), pp.541-552, ISSN 0195-6108

van der Knaap, M. \& Valk, J. (2005). Magnetic resonance of myelin, myelination and myelin disorders (3rd edition), Springer, ISBN 978-3-540-22286-6, Germany

van der Knaap, M. et al.. (2006). Vanishing white matter disease. Lancet Neurol, Vol.5, No.5, (May 2006), pp. 413-423, ISSN 1474-4422

van der Knaap, M. \& Scheper, G. (July 2008). Megalencephalic leukoencephalopathy with subcortical cysts, In: GeneReviews, 24.02.2011, Available from http://www.ncbi.nlm.nih.gov/books/NBK1535/\#mlc.Summary

Vargas, M. et al.. (December 2009). Magnetic resonance imaging of metabolic disease of the cerebral white matter. Top Magn Reson Imaging, Vol.20, No.6, (December 2009), pp. 333-341, ISSN 0899-3459

Varho, T. et al.. (1999). A new metabolite contributing to N-acetyl signal in 1H MRS of the brain in Salla disease. Neurology, Vol.52, No.8, (May 1999), pp. 1668-72, ISSN 00283878

Yager, J. et al.. (1988). CT-scan findings in an infant with glutaric aciduria type I. Dev Med Child Neurol, Vol.30, No.6, (December 1988), pp. 808-811, ISSN 0012-1622

Wenger, D. (August 2008). Krabbe disease, In: GeneReviews, 26.02.2011, Available from http://www.ncbi.nlm.nih.gov/books/NBK1238/\#krabbe.Summary

Williams, R. et al.. (2006). Diagnosis of the neuronal ceroid lipofuscinoses: an update. Biochim Biophys Acta, Vol.1762, No.10, (October 2006), pp. 865-872, ISSN 0006-3002

Zarifi, M. et al.. (2001). Magnetic resonance spectroscopy and magnetic resonance imaging findings in Krabbe's disease. J Child Neurol, Vol.16, No.7, (July 2001), pp. 522-526, ISSN 0883-0738

Zhang, Y. et al.. (2006). Biochemical properties of human pantothenate kinase 2 isoforms and mutations linked to pantothenato kinase-associated neurodegeneration. J Biol Chem, Vol.281, No.1, (January 2006), pp. 107-114, ISSN 


\title{
Acquired Demyelinating Disorders of the CNS in Children
}

\author{
R. Govender ${ }^{1}$, Jo M. Wilmshurst ${ }^{2}$ and Nicky Wieselthaler ${ }^{2}$ \\ ${ }^{1}$ University of Kwa-Zulu Natal, Durban \\ ${ }^{2}$ University of Cape Town, Cape Town \\ South Africa
}

\section{Introduction}

Acquired Demyelinating disorders of the central nervous system in children span a wide spectrum. These conditions may be mono-phasic and self limiting or multi-phasic. Children may present with mono-focal (optic neuritis) or multi-focal (Acquired demyelinating encephalomyelitis) clinical findings.

These demyelinating disorders also share many common clinical, radiological and laboratory features. Early classification of whether the disease is either mono- or multiphasic has diagnostic and therapeutic implications. Identification of patients who present with a first demyelinating event and are at risk for evolution to multiple sclerosis, allows disease modifying therapeutic agents to be initiated early and thus preserve brain function. The aetiology of acquired demyelinating conditions is multi-factorial namely - genetic, postinfectious, post-immunization and possibly due to a T-cell mediated auto-immune response to myelin basic protein triggered by an infection or immunization.

This chapter will cover the aetiologies, consensus definitions, clinical presentation, neuroimaging, evolution and therapeutic advances in acquired demyelinating disorders in children. The pivotal role of neuro-imaging in unraveling the pathology, aetiology and diagnosis of these disorders is also highlighted.

Clinical and neuro-imaging features of other acquired white matter lesions (via infections, toxins, nutritional deficiencies, and osmotic myelinolysis) disease are also discussed.

\section{Definitions}

The International Paediatric Multiple Sclerosis Study group in 2007 proposed consensus definitions for the demyelinating disorders in children (Krupp et al., 2007). This group was convened to define an operational classification system for the demyelinating disorders. Consensus definitions aid in standardization of diagnosis, investigation, management and further research of these conditions.

\section{Imaging techniques for white matter disorders}

Magnetic Resonance Imaging (MRI) is the diagnostic modality of choice for evaluating white matter disorders. Sophisticated applications of magnetic resonance technology, such 


\begin{tabular}{|c|c|}
\hline & Definition \\
\hline $\begin{array}{c}\text { Acquired Demyelinating } \\
\text { Encephalomyelitis (ADEM) }\end{array}$ & $\begin{array}{l}\text { A first clinical event with a presumed inflammatory or } \\
\text { demyelinating cause, with acute or sub-acute onset that } \\
\text { affects multifocal areas of the CNS and must include } \\
\text { encephalopathy }\end{array}$ \\
\hline Recurrent ADEM & $\begin{array}{l}\text { New event of ADEM with a recurrence of the initial } \\
\text { symptoms and signs, } 3 \text { or more months after the first } \\
\text { ADEM event, without involvement of new clinical } \\
\text { areas by history, examination, or neuro-imaging }\end{array}$ \\
\hline $\begin{array}{l}\text { Multi-phasic Demyelinating } \\
\text { Encephalomyelitis (MDEM) }\end{array}$ & $\begin{array}{l}\text { ADEM followed by a new clinical event also meeting } \\
\text { criteria for ADEM, but involving new anatomic areas } \\
\text { of the CNS as confirmed by history, neurologic } \\
\text { examination, and neuro-imaging. The event must } \\
\text { develop within } 3 \text { months of the initial event. }\end{array}$ \\
\hline Neuromyelitis Optica (NMO) & $\begin{array}{l}\text { Must have optic neuritis and acute myelitis as major } \\
\text { criteria and a spinal MRI lesion extending over three or } \\
\text { more segments or be NMO positive on antibody testing }\end{array}$ \\
\hline $\begin{array}{l}\text { Acute Transverse Myelitis } \\
\text { (ATM) }\end{array}$ & $\begin{array}{l}\text { A focal inflammatory disorder of the spinal cord } \\
\text { resulting in motor, sensory and autonomic dysfunction }\end{array}$ \\
\hline Schilder's Disease & $\begin{array}{l}\text { A sub-acute demyelinating disorder characterized by } \\
\text { bilateral large and vaguely symmetrical lesions }\end{array}$ \\
\hline $\begin{array}{l}\text { Paediatric Multiple Sclerosis } \\
\text { (MS) }\end{array}$ & $\begin{array}{l}\text { A clinical syndrome of multiple clinical demyelinating } \\
\text { events involving } \\
\text { more than one area of the central nervous system with } \\
\text { dissemination in time and space on imaging* }\end{array}$ \\
\hline $\begin{array}{l}\text { Clinically Isolated Syndromes } \\
\text { (CIS) }\end{array}$ & $\begin{array}{c}\text { A first acute clinical episode of } \\
\text { CNS symptoms (without encephalopathy) with a } \\
\text { presumed inflammatory demyelinating cause; for } \\
\text { which there is no prior history of a demyelinating } \\
\text { event }\end{array}$ \\
\hline
\end{tabular}

*Important caveats in the definition of Paediatric MS put forward by the study group include:

1. The combination of an abnormal CSF (presence of Oligoclonal bands or an elevated IgG index) and two lesions on the MRI, of which one must be in the brain, can also meet dissemination in space criteria.

2. The MRI can meet the dissemination in space criteria if it shows 3 of the following 4 criteria (1) nine or more white matter lesions or one gadolinium enhancing lesion, 2) three or more periventricular lesions, 3) one juxta-cortical lesion, 4) an infra-tentorial lesion.

3. MRI can be used to satisfy criteria for dissemination in time following the initial clinical event, even in the absence of a new clinical event if new T2 or gadolinium enhancing lesions develop within 3 months of the initial clinical event.

4. A second non-ADEM event in a patient is insufficient to make the diagnosis of paediatric MS if the first event meets the criteria for ADEM. MS can only be diagnosed if there is further evidence of dissemination in time on the MRI (new T2 lesions > 3months since the second event) or a new clinical event (> 3months since the second event).

Table 1. Definitions of Acquired Demyelinating Disorders (Adapted from Krupp et al 2007) 
as magnetization transfer imaging, magnetic resonance spectroscopy, and diffusion tensor imaging, provide quantitative information about the extent of damage that occurs in the white matter.

1H magnetic resonance spectroscopy (MRS) is a valuable technique to non-invasively acquire in-vivo information about biochemical processes in patients with neurologic disorders. MRS measures N-acetyl-aspartate (NAA), total creatine, choline-containing compounds, and lactate. NAA has been considered a marker of neuronal integrity, whereas the levels of choline and lactate are indicative of cell membrane turnover and anaerobic glycolysis (Bizzi et al., 2001). NAA is located almost exclusively in neurons and neuronal processes and thus provides information about neuronal integrity (De Stefano et al., 1995).

MRS studies on patients with white matter disorders have shown reduction in NAA in areas that appear normal on conventional MRI studies suggesting that a significant amount of axonal damage is present in these patients (Arnold et al., 1990; van Der Knaap et al., 1992). Abnormalities in NAA on MRS have also shown a correlation with long term functional outcomes. The author suggests that the extent of axonal damage rather than demyelination may be more reliable in monitoring disease evolution in primary white matter disorders (De Stefano et al., 2000).

Diffusion-weighted MR imaging (DWI) provides further information that may not be apparent on conventional MR images. Engelbrecht et al showed that diffusion restriction precedes brain myelination and is further increased during myelination (Engelbrecht $e t$ al., 2002).

\section{Acquired Demyelinating Encephalomyelitis (ADEM)}

ADEM initially described by Lucas (1790) is characterized by acute onset of diffuse neurological signs with multi-focal white matter involvement. In 1931 a series of case studies was reported in The Lancet. McAlpine described 3 sets of patients with ADEM: -post-vaccination, post-infectious, and those with spontaneous occurring disease. The International Paediatric MS study group further defined ADEM (see Table 1).

\subsection{Pathogenesis}

The aetiology of ADEM is not completely understood. The pathogenesis is thought to be auto-immune mediated. The seasonal distribution and high rate of antecedent infections (Dale et al., 2000; Murthy et al., 2002; Govender et al., 2010) reported in ADEM suggest a link to an infectious aetiology. Infectious diseases are common in childhood however the rate of preceding infection reported in these series exceed the rate of childhood infectious diseases described (30-50\%). The auto-immune reaction is thought to be on the basis of molecular mimicry. The offending infection serves as an antigenic trigger and shares epitopes with various autoantigens of myelin such as myelin basic protein, proteolipid protein, and myelin oligodendrocyte protein (Alvord et al., 1987). This theory shares many similarities with experimental allergic encephalitis. A second theory is that the antigenic trigger activates $T$ cells which cross the blood-brain barrier and react against similar myelin epitopes. ADEM was associated with the class II alleles HLA-DRB1*01 and HLA-DRB*03 in a Russian study (Idrissova et al., 2003). Pathological studies of children 
with ADEM showed that peripheral and cerebrospinal lymphocytes have increased reactivity to myelin basic protein (Lisak and Zweiman, 1977). Viral infections described in the context of ADEM include Herpes Simplex Virus-1, Cytomegalovirus (CMV), HIV (Human Immunodeficiency virus), Measles, Mumps, Rubella, Ebsteinne Barre Virus (EBV) and Varicella Zoster Virus (VZV). ADEM occurs after one of every 1000 cases of measles, with a fatality rate of $20 \%$ (Tselis and Lisak, 1998). Bacterial antigens implicated include Mycoplasma, Campylobacter, Streptococci and Borrelia Burgdofferi. Dale et al. described a subgroup of ADEM associated with Group A $\beta$ hemolytic streptococcus, abnormal basal ganglia imaging and elevated antibasal ganglia antibodies (Dale et al., 2001). Some studies fail to identify the agent responsible for the pre-demyelinating infection (Murthy et al., 2002). The authors postulate that the inciting agents are uncommon or unusual organisms that can not be identified by routine laboratory testing. Vaccines, specifically the influenza, rabies and smallpox vaccines have also been reported to precipitate ADEM (Saito et al., 1998). Post-vaccination ADEM is thought to be the result of immune mediated mechanisms rather than the cyto-pathic effects of the virus. ADEM is reported after the administration of drugs such as sulfonamides and streptomycin, further supporting an immunological basis of the pathogenesis.

\subsection{Epidemiology}

There are few epidemiological studies of ADEM in children. Prevalence studies are also complicated by the use of inconsistent case-definitions of ADEM. The estimated incidence in California is $0.4 / 100,000$ population per year (Leake et al., 2004) and in Canada is 0.2/100,000 per year (Banwell et al., 2009). The mean age of presentation of ADEM in children ranges from 5-8 years (Hynson et al., 2001; Tenembaum et al., 2007). There is no specific ethnic distribution (Leake et al., 2004). Some studies indicate a slight male predominance (Murthy et al., 2002; Tenembaum et al., 2007). Prevalence in resource poor countries would be expected to be higher because of the significant frequency of childhood infections. However it is probably under-estimated because of limited access to health care facilities and MRI facilities.

\subsection{Clinical presentation}

ADEM has a wide clinical spectrum of presentation. The hallmark of the disease is an acute presentation of multifocal neurological signs with encephalopathy consistent with diffuse brain involvement usually following a viral infection or immunization. However, events may range from sub-clinical episodes diagnosed by MRI showing mult-ifocal white matter lesions, to a more fulminant presentation with seizures and encephalopathy. Seizures are reported to be more common in children compared to adults with ADEM (Tenembaum et al., 2007). Fever and meningism are also common in ADEM prompting treatment for meningo-encephalitis in the initial management (Dale et al., 2000). Multi-focal neurological signs are pathognomic for ADEM and include hemiparesis, paraparesis, cranial nerve involvement and ataxia. Atypical presentations include concomitant peripheral nervous system involvement (Kinoshita et al., 1996), presentation as an isolated acute psychotic episode (Moscovich et al., 1995) or with an extra-pyramidal syndrome (dystonia and behaviour disturbances) (Dale et al., 2001). 


\subsection{Laboratory investigations}

In the absence of specific biological markers diagnosis is based on a combination of historical features, clinical and MRI characteristics. Other investigations are usually done to exclude other differential diagnosis (e.g. meningitis, metabolic encephalopathies). In resource poor settings where the burden of disease is predominantly infectious illnesses and because of the overlap of symptoms, infectious aetiologies must be excluded first. Peripheral blood leucocytosis is documented in ADEM (Jacobs et al., 1994). CSF studies in ADEM are usually abnormal (in $>67 \%$ of cases), typically showing a moderate pleocytosis with an elevated protein content (Miller et al., 1956; Govender et al., 2010). CSF Oligoclonal bands synthesis may occur in ADEM; however this tends to disappear when the patient recovers.

Electrophysiological studies have limited value in ADEM. Slow-wave abnormalities on electro-encephalogram are compatible with an encephalopathic state (Dale et al., 2000). The spindle coma pattern has been described in a child with post measles ADEM (Bortone et al., 1996). Visual evoked potentials though are useful in detecting asymptomatic optic tract lesions (Dale et al., 2000).

\subsection{Neuro-imaging}

MRI is the investigation of choice. Since CT is often non-diagnostic for white matter lesions in patients with ADEM, this study is often normal or shows non-specific hypo-densities in the white matter.

Lesions are most easily recognized on T2 weighted (T2WI) and FLAIR MRI sequences. T2WI are more sensitive than T1 weighted images (T1WI) in detecting lesions (Sheldon et al., 1985). T1WI shows hypo-intense lesions. The lesions of ADEM are multi-focal and often do not correlate with clinical signs. Lesions tend to involve the cerebellum, the cerebral cortex and brainstem (Figure $\mathbf{1} \mathbf{~ a - g ) . ~ T h e y ~ u s u a l l y ~ i n v o l v e ~ t h e ~ s u b - c o r t i c a l , ~ c e n t r a l ~ a n d ~}$ periventricular white matter. Lesions are typically hyper-intense, patchy, asymmetric and ill-defined. Diffusion-weighted imaging (DWI) and apparent diffusion co-efficient (ADC) maps may be helpful to prognosticate outcome. Low ADC values and restricted diffusion on DWI may suggest a worse outcome as this may indicate permanent tissue damage (Barkovich., 2007). A case study of ADEM with MRS reported reduced NAA and an elevation of choline and lactate (Gabis et al., 2004). These authors suggest a place for H MRS studies in longitudinal follow-up studies of ADEM to assess the response to immunomodulating therapies. Deep grey matter lesions in the thalami and basal ganglia have also been described (Baum et al., 1994; Govender et al., 2010) (Figure 2a-e). Lesions in the corpus callosum are uncommon and considered atypical for ADEM (Figure 3). Contrast enhancement of lesions post gadolinium administration indicates activity of the lesions (Figure 4). This correlates with the pathological finding of inflammation and demyelination in experimental allergic encephalitis. Non-enhancing and partially enhancing lesions in the presence of enhancing lesions have been described in ADEM and are thought to be because of lesions of differing ages and the evolution of the disease over several weeks (Schwaz et al., 2001; Govender et al., 2010).

Concomitant spinal cord lesions have been described in ADEM (Hynson et al., 2001; Murthy et al., 2002; , Govender et al., 2010) (Figure 5a,b). Spinal cord lesions in ADEM typically have ill-defined margins, extend over multiple vertebral segments, are thoracic in location and result in mild cord expansion (Singh et al., 2002). 


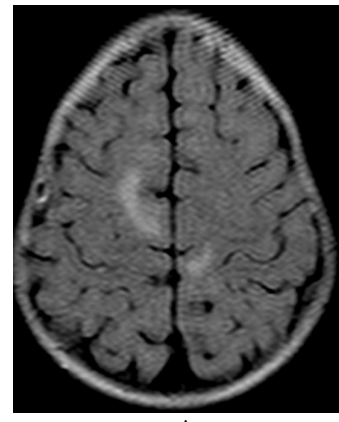

A

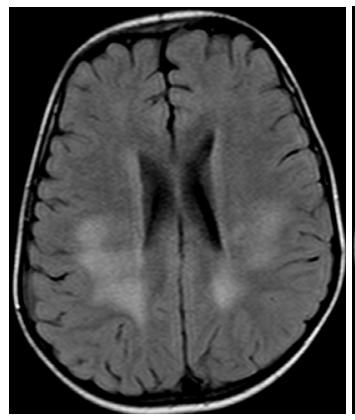

C

$\mathrm{E}$

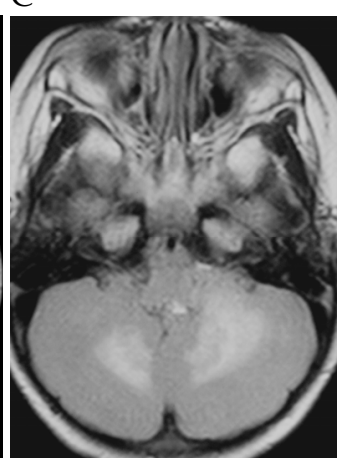

$\mathrm{F}$

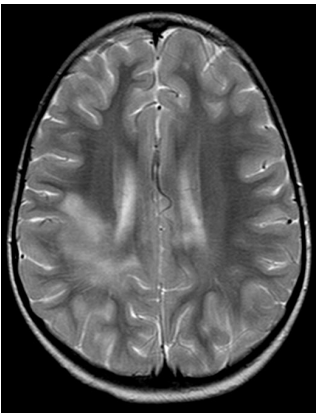

B

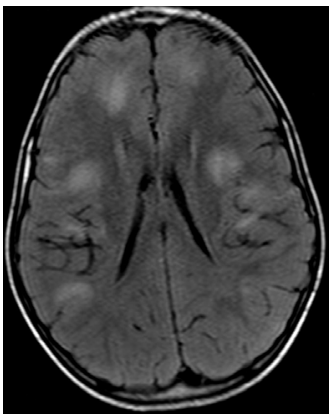

$\mathrm{D}$

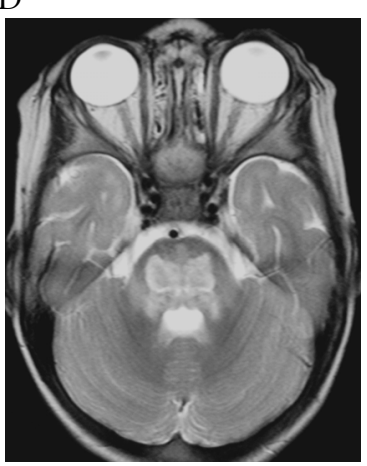

G

Fig. 1. A - Flair axial MRI demonstrating bilateral asymmetrical cortex and sub-cortical white matter high signal intensities consistent with ADEM. B- T2 axial MRI demonstrating right ill-defined peritrigonal white matter high signal intensity lesion consistent with ADEM. C- Flair axial MRI demonstrating bilateral, fairly symmetrical high signal intensities in the posterior white matter consistent with ADEM. D - Flair axial MRI demonstrating bilateral asymmetrical high signal intensity lesions in the sub-cortical and deep white matter consistent with ADEM. E- Flair axial MRI demonstrating bilateral asymmetrical high signal intensity lesions in the cortex and sub-cortical and deep white matter consistent with ADEM. F - Flair axial MRI demonstrating hyper-intense lesions in the brachium ponti consistent with ADEM. G - T2 axial MRI of the brain demonstrating large hyper-intense pontine lesion with surrounding oedema 


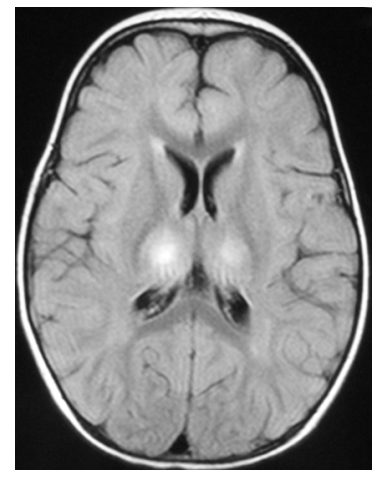

A

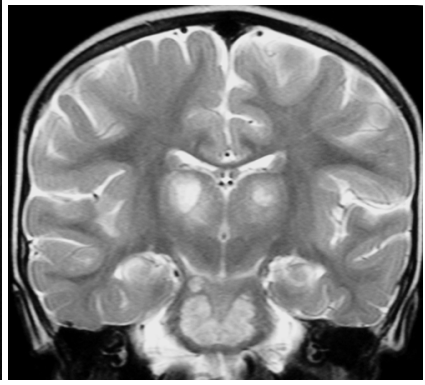

B

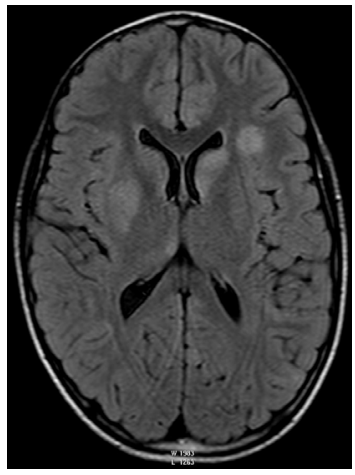

C

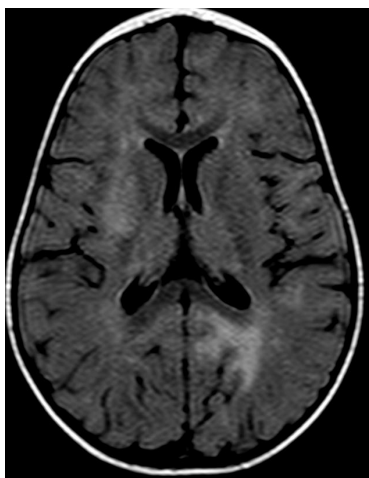

$\mathrm{D}$

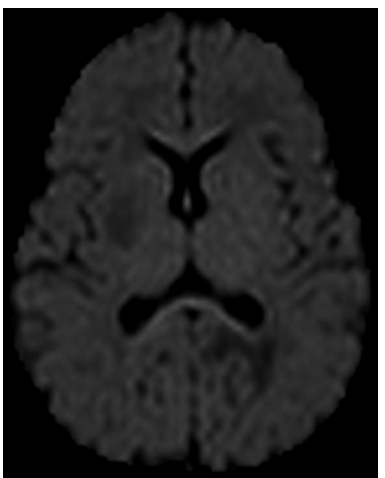

E

Fig. 2. A- Flair axial MRI of the brain demonstrating bilateral symmetrical hyper-intense thalamic lesions. B- T2 coronal MRI of the brain demonstrating bilateral symmetrical rounded hyper-intense thalamic lesions as well as a large hyper-intense pontine lesion. CFlair axial MRI demonstrating bilateral high signal intensity lesions in the basal ganglia and deep white matter on the left consistent with ADEM. D- Flair axial MRI demonstrating asymmetrical basal ganglia and white matter high signal intensities consistent with ADEM. E- DWI of 2D shows no evidence of restricted diffusion.

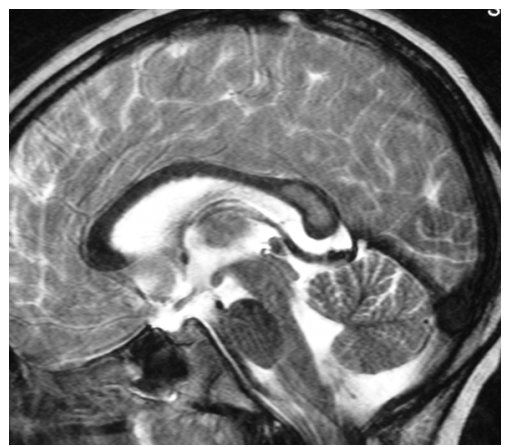

Fig. 3. T2 sagittal midline brain MRI demonstrating a well-defined rounded lesion in the splenium of the corpus callosum, which is unusual for ADEM. 


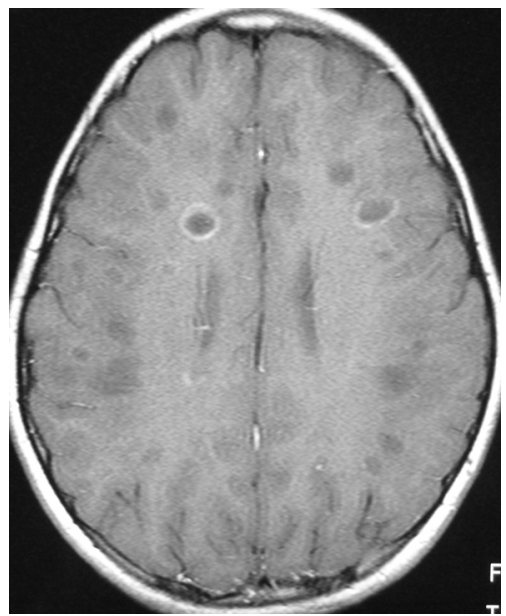

Fig. 4. T1 with contrast axial MRI demonstrating multiple hypo-intense white matter lesions of varying sizes, some with ring enhancement. This patient was diagnosed with ADEM.

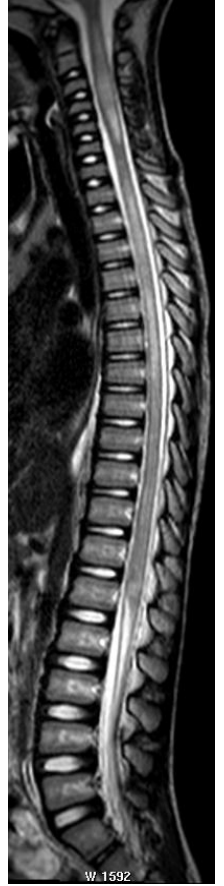

A

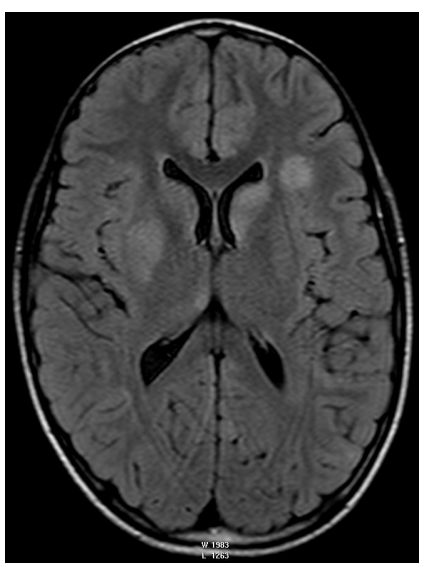

B

Fig. 5. A- T2 sagittal MRI of the cord showing diffuse abnormal high signal intensity throughout the cord with cord expansion in the cervical region. There was no associated enhancement. This together with the brain lesions seen in 5B is consistent with ADEM. BFlair axial MRI demonstrating bilateral high signal intensity lesions in the basal ganglia and deep white matter on the left consistent with ADEM. 


\subsection{Treatment}

There are no standard treatment protocols as there are insufficient large scale studies to form consensus for optimal management. Supportive care (e.g. respiratory support for patients with brainstem involvement, anti-epileptics for seizure control) in the acute phase is vital.

Therapies recommended are mainly immunomodulating agents targeting the immunebased mechanism of the disease. Corticosteroids are considered the mainstay of treatment based on the rapid improvement in symptoms following therapy (Straub et al., 1997; Tenembaum et al., 2007). However widely varying doses, formulations, duration of therapy and tapering have been reported with corticosteroid use (Hynson et al., 2001; Murthy et al., 2002; Tenembaum et al., 2007; Govender et al., 2010). A single study reported worse outcomes in patients who received corticosteroids (Boe et al., 1965). Methylprednisone, dexamethasone and ACTH are used. Most reports in paediatric patients have used IV methylprednisolone (10 to $30 \mathrm{mg} / \mathrm{kg} /$ day) or dexamethasone $(1 \mathrm{mg} / \mathrm{kg})$ for 3 to 5 days (Dale et al., 2000; Hyson et al 2001; Tenembaum et al 2002; Govender et al., 2010) followed by a taper for 4 to 6 weeks with full recovery reported in 50 to $80 \%$ of patients. In resource poor countries high dose corticosteroids must be used with caution and only commenced once commonly occurring infections like tuberculosis and cytomegalovirus (CMV) are excluded. Outcomes on efficacy of corticosteroid treatment are mainly compared to historical controls. Worse outcomes are linked to shorter duration of treatment (Tenembaum et al., 2007). Other treatment modalities suggested include intravenous immunoglobulin, plasmapheresis and glatiramer acetate (Abramsky et al., 1977; Stricker et al., 1992; Finsterer et al., 1998). There is some evidence to suggest that patients may respond to a combination of methylprednisolone and immunoglobulin if they fail to respond to either separately (Straussberg et al., 2001).

\subsection{Prognosis}

ADEM is by definition a monophasic illness (variants are discussed in Section 4.8). Mortality during the post-measles ADEM period in the 1950's was reported as 10-30\% (Johnson et al., 1985). At follow-up, approximately $60-80 \%$ of children have no neurologic deficits (Menge et al., 2007). This study also reports a mortality rate of $5 \%$. The extent and site of lesions on the initial MRI do not predict the clinical outcome. Motor deficits persist in 8-30\% (Dale et al., 2000; Tenembaum et al., 2007) of patients and include paraparesis, hemiparesis and ataxia. Neuro-cognitive deficits are also documented post ADEM (Hahn et al., 2003; Jacobs et al., 2004). These include deficits in short term memory, verbal processing skills and complex attention. Patient with early onset ADEM (<5year of age) were also more likely to have cognitive deficits and behaviour problems (Kumar et al., 1998). Follow-up MRI's showed complete or partial resolution of abnormalities in the majority of cases (Kesslering et al., 1990; Dale et al., 2000; Tenembaum et al 2007; Govender et al., 2010). However, residual gliosis and demyelination persist in some patients (Kesselring et al., 1990). Clinical as well as imaging follow-up (at least 3 months later) (Figure 6) is important to monitor for evolution to MS. Risk factors for relapse are discussed in greater detail in Section 10.

\subsection{Variants of ADEM}

Definitions for Recurrent and Multi-phasic ADEM are described in Table 1.

Acute Hemorrhagic Leukoencephalitis is a rare, hyper-acute form of ADEM with a mortality rate of about 70\% (Davies et al., 2006). Pathological studies show a necrotizing vasculitis 
with haemorrhage, oedema and a neutrophilic infiltration (Stone and Hawkins, 2007). Seventy percent of survivors have neurological deficits (Stone and Hawkins, 2007).

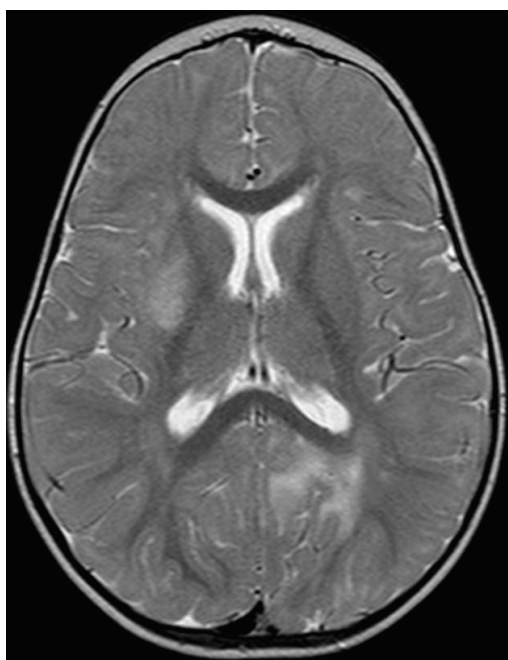

A

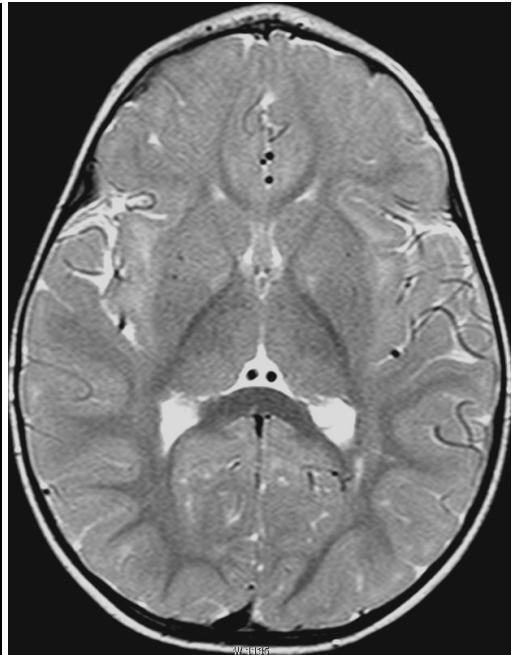

B

Fig. 6. A- T2 axial MRI demonstrating asymmetrical basal ganglia and white matter high signal intensities consistent with ADEM. B- T2 axial MRI in same patient at 3 months followup showing resolution of lesions.

\section{Transverse myelitis}

\subsection{Epidemiology}

Acute Transverse myelitis (ATM) is a focal inflammatory disorder of the spinal cord resulting in motor, sensory and autonomic dysfunction with evidence of inflammation on CSF or MRI studies. The initial definition was proposed by the Transverse Myelitis Consortium Working Group in 2002 and refined by the more recent consensus definitions for paediatric demyelinating disease (Krupp et al., 2007). The incidence is reported as 1- 8 per million people per year (Berman et al., 1981). There are no gender or ethnic differences in the prevalence of ATM (Berman et al., 1981). ATM is often difficult to distinguish clinically from ischaemic cord lesions, fibro-cartilagenous emboli or traumatic spinal cord lesions. ATM is also an important differential diagnosis for acute flaccid paralysis in childhood.

\subsection{Pathogenesis}

The aetiology of ATM is thought to be immune-mediated. In $30-60 \%$ of patients ATM is para-infectious (Jeffrey et al., 1993; Kalra et al., 2009). Molecular mimicry and super-antigen mediated mechanisms have been postulated (Kaplin et al., 2005). Positive anti-GM1 antibodies following Campylobacter and CMV infections have been implicated in the aetiopathogenesis. Neuromyelitis optica-immunoglobulin $G$ is an aquaporin-4-specific water channel antibody, which has been associated with neuromyelitis optica and longitudinally extensive transverse myelitis in adults (Lennon et al., 2004). This is discussed further in Section 6. 


\subsection{Clinical presentation}

Clinical presentation is that of acute or sub-acute onset of bilateral spinal cord dysfunction (that may be asymmetrical) with associated sphincter dysfunction and pain. A sensory level may not be easily elicitable in the paediatric population. The clinical features depend on the location of the lesion. High cervical cord lesions can present with respiratory failure. The thoracic segment is the commonest site of cord involvement in ATM (Kneubusch et al., 1998).

\subsection{Investigations}

Initial evaluation of a patient with an evolving myelopathy must include a gadolinium enhanced MRI of the spine to exclude a compressive myelopathy. If there is no evidence of a compressive lesion a lumbar puncture should be performed. CSF pleocytosis and an elevated protein (IgG index) on the CSF support a diagnosis of ATM. Brain MRI and eye examination with visual evoked potentials are recommended to exclude demyelination in other parts of the neuro-axis.

Other investigations recommended include para-infectious markers- EBV, VZV, CMV, Herpes Simplex virus serology and stool for campylobacter cultures. If there are signs of a systemic inflammatory disorder, auto-immune screens and serum angiotensin converting enzyme levels should be performed to exclude other causes of an acute myelopathy (e.g. vasculitides).

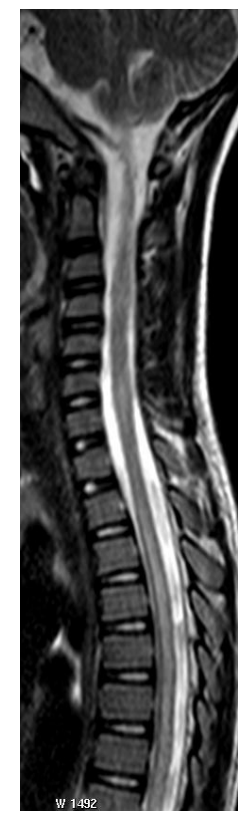

A

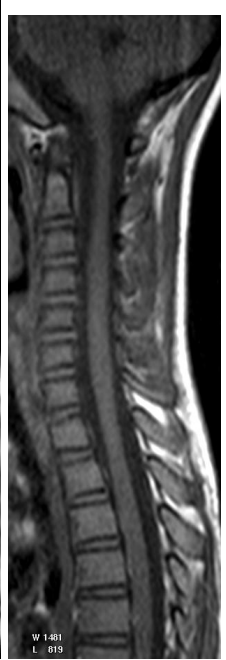

B

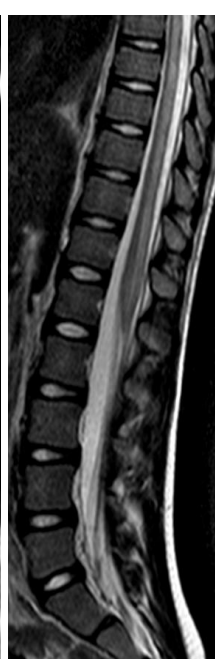

C

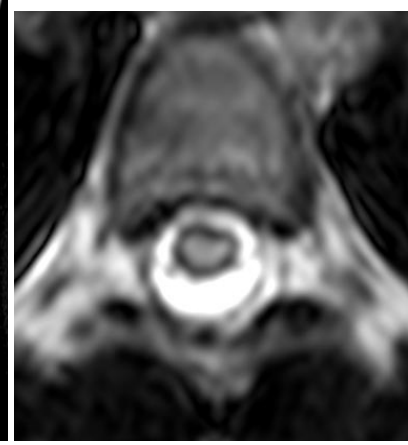

$\mathrm{D}$

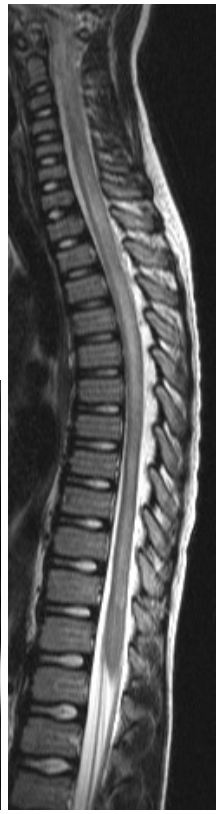

E

Fig. 7. A- T2 sagittal MRI of the cord showing diffuse abnormal increased signal intensity consistent with transverse myelitis. B- T1 sagittal MRI of the cord showing no abnormal signal intensity. There was no contrast enhancement. C- T2 sagittal MRI of the distal cord showing diffuse abnormal increased signal intensity consistent with transverse myelitis. DT2 axial MRI of the cord showing abnormal increased signal intensity consistent with transverse myelitis. E- T2 sagittal MRI of the cord showing diffuse abnormal increased signal intensity and cord swelling consistent with transverse myelitis. 


\subsection{Neuro-imaging}

MRI of the spinal cord usually shows nonspecific localized hyper-intense signal on T2WI sequences with, in some cases, segmental cord enlargement and/or focal enhancement (Figure 7 a-e). Acute partial transverse myelitis described in adults is characterized by MRI lesions that are asymmetrically placed and spanning fewer than 2 vertebral segments in length; these patients have been found to have a greater risk of progression to multiple sclerosis (Ford et al., 1992). Longitudinally extensive myelitis (spanning $>3$ vertebral segments) is shown to have a lower risk of progression to MS in adults (Pittock et al, 2006). Lesions of ATM in children are typically longitudinal, demonstrate rim enhancement and are centrally located.

\subsection{Treatment}

Previous case series did not demonstrate any benefit from the use of low dose corticosteroids (Dunn et al., 1986; Adams et al., 1990). Recent reports demonstrate the benefit of high dose corticosteroids (10-30mg/ $\mathrm{kg}$ per day for 3-5 days) on recovery (Defrense et al., 2001; Sebire et al., 1997). Compared to historical controls patients treated with steroids walked independently sooner. If there is no clinical response to steroids within 5-7 days plasma exchange was used as adjunctive therapy in isolated case reports. Supportive measures include respiratory support and early management of a neuropathic bladder.

\subsection{Prognosis}

Various studies have looked at prognostic indicators for ATM. Jain et al. (1983) described backache at onset, acute course (within hours), spinal shock and a cervical sensory level as poor prognostic features. Other studies did not demonstrate this (Govender et al., 2010). Early recovery (within one week of presentation), age less than 10 years at presentation and lumbosacral spinal level on clinical assessment were significant predictors of a good outcome (De Goede et al., 2010). The extent of lesions on MRI has not shown consistent correlation with outcome (Pradhan et al., 1997; Adronikou et al., 2003). Berman et al's series (1987) described more than one-third of the patients with ATM making a good recovery; in one-third of patients recovery was only fair; 14 patients failed to improve and 3 demised.

In the series by Dunne et al (1986) that assessed the risk of progression to multiple sclerosis in children with ATM, definite evidence of multiple sclerosis did not develop in any of the patients. In the series by Pidcock et al of the 47 children with acute transverse myelitis, 2 experienced recurrent transverse myelitis, 1 was diagnosed with neuromyelitis optica, and 1 developed multiple sclerosis on follow-up (Pidcock et al., 2007).

\section{Neuromyelitis Optica (NMO)}

The association between myelitis and optic problems was first described in 1870 by Thomas Clifford Allbutt (Murray, 2005). In 1894 Eugene Devic described 16 patients with visual impairment who developed paraparesis, sensory deficits and sphincter dysfunction within weeks. They recognized that these symptoms were the result of inflammation of the optic nerve and spinal cord. NMO is a recurrent demyelinating disorder affecting the optic nerves and the spinal cord. Modifications to the definition of NMO in 2005 incorporated the inclusion of patients with brain lesions, and included the NMO-IgG antibody as a confirmatory test. 
The Mayo Clinic proposed a revised set of criteria in 2006. The new guidelines for diagnosis requires both absolute criteria and two of the three supportive criteria to be present to make a diagnosis of NMO (Wingerchuck et al., 2006).

Absolute criteria:

1. Optic neuritis

2. Acute myelitis

Supportive criteria:

1. Brain MRI not meeting criteria for MS at disease onset

2. Spinal cord MRI showing contiguous T2-weighted signal abnormality extending over 3 or more vertebral segments, indicating a relatively large lesion in the spinal cord

3. NMO-IgG seropositive status.

The association of NMO with the serum autoantibody marker NMO-IgG was reported in 2004 (Lennon et al., 2004). NMO-IgG is 73\% sensitive and 91\% specific for distinguishing NMO from classical MS. The new diagnostic criteria allows for the diagnosis of NMO in patients who are NMO-IgG antibody negative. NMO antibodies play a key role in the pathogenesis. These antibodies are directed against the aquaporin-4- receptors located in the cell membrane of astrocytes (Pearce, 2005). Aquaporin-4 is the most abundant channel facilitating water transport across membranes in the brain. NMO-IgG is also detected more commonly in patients with NMO symptoms who have clinical or serological evidence for SLE than in those who do not (McAdam et al., 2002).

\subsection{Clinical characteristics}

Clinical characteristics include painful visual loss, weakness, sphincter dysfunction and sensory deficits. Loss of red color vision, a relative afferent pupillary defect and visual field defects are other features of optic neuritis in children. Other complications such as ataxia and respiratory failure result from extension of cervical cord lesions into the brainstem.

\subsection{Investigations}

Diagnostic evaluation includes an MRI of the brain. During acute optic neuritis attacks, an orbital MRI may identify optic nerve gadolinium-enhancement. MRI of the brain is usually normal. However, brain lesions located in the hypothalamus, brainstem, and periventricular areas have been described in children who have typical features of NMO (Pittock et al., 2005). These are considered to be the aquaporin- 4 rich areas of the brain.

Patients with signs of myelitis should have a spinal MRI with contrast. The lesions are typically longitudinally extensive, centrally based in the cord and extend over three or more vertebral segments. All patients should have a serological test for the NMO-IgG antibody. A negative test however does not exclude the diagnosis. CSF pleocytosis also supports the diagnosis. In patients with longitudinal myelitis and no visual symptoms, visual evoked potentials can sometimes detect asymptomatic visual pathway dysfunction.

\subsection{Treatment}

The recommended treatment for acute attacks of myelitis or optic neuritis is high dose methylprednisone. Prophylactic long-term immunosuppression is recommended for established NMO and patients who have a single attack of myelitis and are NMO-IgG positive (Wingerchuck et al., 2005). There are no efficacious preventative therapies demonstrated by controlled trials in NMO. Intravenous immunoglobulin is an alternative for patients who do not respond to corticosteroids. 
Characteristics of NMO that help to distinguish it from classical MS include:

- Prominent CSF pleocytosis (more than $50 \mathrm{WBC}$ ) with a polymorphonuclear cell predominance (Mandler et al., 1993; O Riordan et al., 1996)

- Lower frequency of CSF oligoclonal banding (15-30\% in NMO compared to 85\% in MS)

- Bilateral symmetrical optic neuritis

- At disease onset, the brain MRI scan is normal or reveals nonspecific white matter lesions

- $\quad$ MRI of the spinal cord showing longitudinally extensive, central lesions (MS lesions are more peripherally located in the cord and extend over one to two segments in length)

\section{Schilder's disease/myelinoclastic diffuse sclerosis}

This disorder was initially described by Schilder in 1912 and later clarified by Poser (1992). There are further reported cases of solitary, large plaque like lesions, which were histologically confirmed to be foci of demyelination (Kumar et al., 1998; Gutling and Landis, 1998). The aetiology is unclear; however an association with tuberculosis was described in 3 South African children (Pretorius et al., 1998). Schilder's Disease occurs predominantly in children (peak age 5-14 years) (Afifi et al., 1994).

The Poser criteria (1992) for diagnosis are:

- $\quad$ one or two roughly symmetrical large plaques (greater than $2 \mathrm{~cm}$ diameter)

- pathological analysis is consistent with sub-acute or chronic myelinoclastic diffuse sclerosis

- $\quad$ adrenoleukodystrophy and peripheral nervous system involvement must be excluded.

\subsection{Clinical presentation}

The clinical presentation is non-specific and includes neuroregression, seizures, ataxia or signs of raised intra-cranial pressure.

\subsection{Neuro-imaging}

The lesions of Schilder's Disease are typically large and plaque-like and have also been termed tumefactive demyelination. MRI is the most accurate modality of delineating the lesions that are often confused with brain neoplasms or abscesses. Making the distinction between demyelination and infection/malignancies early is important to prevent unnecessary surgical procedures and toxic therapies like radiation and chemotherapy (McAdam et al., 2002). MRI studies demonstrate 1 or 2 large confluent lesions in the deep white matter, usually the centrum semiovale (Figure 8 a-f). Lesions are at least $2 \mathrm{~cm}$ in size in 2 of 3 dimensions. No additional lesions should be observed on imaging of the brain or spinal cord- this would suggest MS or ADEM. On T1WI, tumefactive demyelination lesions reveal a hypo-intense central area with a thick surrounding band of moderately increased intensity. Lesions are centrally hyper-intense on T2WI. Enhancement, when present, is incomplete. The lesions are characterized by enhancement limited to one side of the lesion; usually the rim facing the lateral ventricles (McAdam et al., 2007). Demyelination can be distinguished from other ring enhancing lesions (brain abscesses, tumors, parasitic infections) by the presence of other demyelinating plaques elsewhere in the central nervous system. 

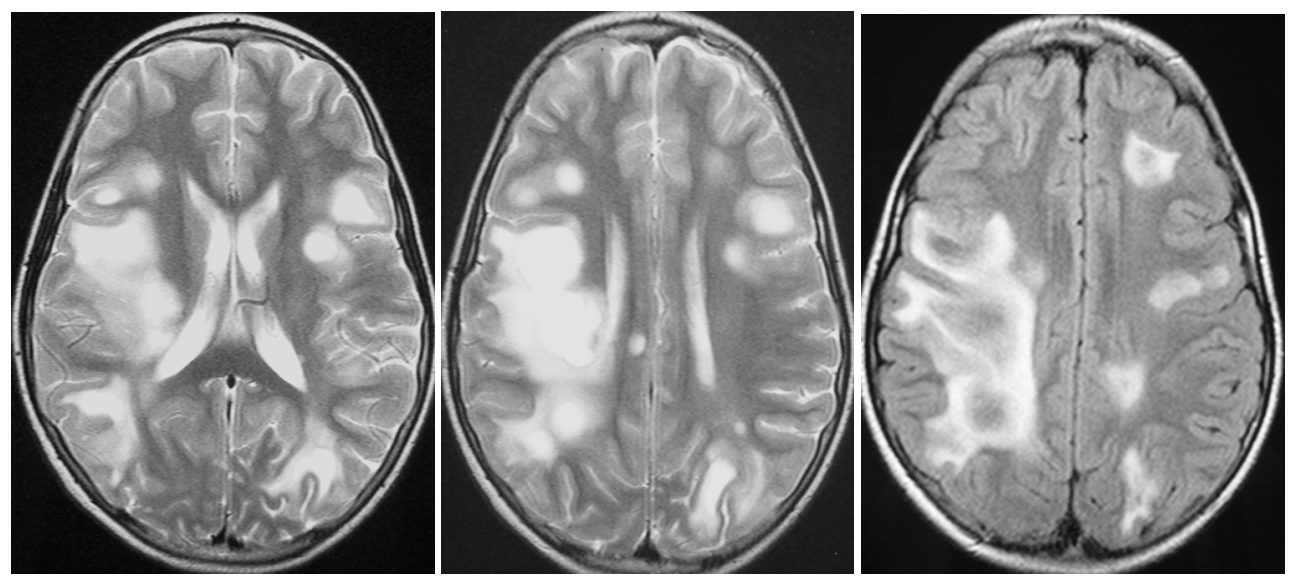

A

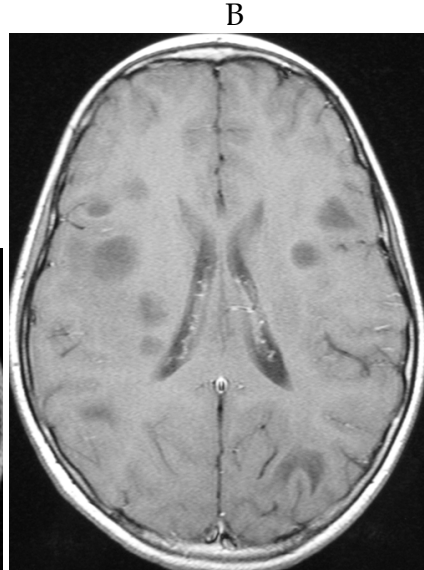

$\mathrm{E}$

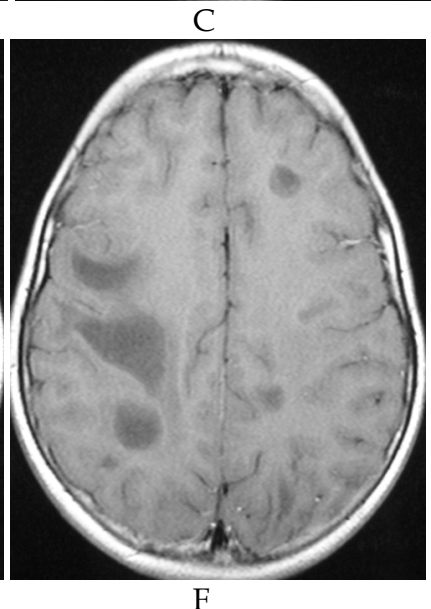

$\mathrm{F}$

Fig. 8. A \& B - T2 axial MRI demonstrating multiple well-defined hyper-intense white matter foci surrounded by more ill-defined areas of increased white matter signal intensity consistent with Schilder's Disease. C- Flair axial MRI demonstrating multiple hyper-intense lesions of varying sizes within the white matter with some areas of suppression within the plaques consistent with Schilder's Disease. D- Flair parasagittal MRI demonstrating large flame- shaped white matter plaque with some areas of suppression within the lesions consistent with Schilder's Disease. E \& F- T1 with contrast axial MRI demonstrating multiple non- enhancing hypo-intense lesions of varying shapes and sizes within the white matter.

Other supportive diagnostic tests include an elevated CSF protein and an elevation of CSF IgG in 50-60\% of patients with Schilder's Disease. Many patients with a large ring enhancing lesion will have a brain biopsy mainly to exclude other disorders.

\subsection{Treatment}

The treatment of choice is high dose intravenous corticosteroids. A rapid clinical and radiological response to high dose corticosteroids favors the diagnosis of demyelination. 


\section{Multiple Sclerosis (MS)}

MS is defined in Table 1 (Krupp et al., 2007). MS in children is likely an under-recognized phenomenon that poses a unique set of challenges in terms of diagnosis and management. Early accurate diagnosis of MS is vital to facilitate early institution of disease modifying agents.

\subsection{Epidemiology}

Childhood onset MS is an uncommon entity however, an estimated 2- 5\% of patients with MS have onset of symptoms of MS before 16 years of age (Duquette et al., 1987; Boiko et al., 2002). The youngest reported patient with MS presented at 10 months of age. This was an indigenous African child who died at 6 years of age after 11 episodes of relapsing neurological symptoms (Shaw and Alvord, 1987). Similar to adult studies, a female preponderance is reported for MS in adolescence (Duquette et al., 1987; Govender et al., 2010). However there is no gender predilection in children presenting with MS under 6 years of age (Banwell et al., 2007).

A crude period prevalence for patients of European ancestry was 25.63 per 100000 and for patients of indigenous African descent was 0.22 per 100000 (Bhigjee et al., 2007). Adult studies have described a more severe clinical and radiological phenotype in patients of African indigenous ancestry compared to patients of European ancestry (Kaufmann et al., 2003; Bhigjee et al., 2007). A retrospective single centre analysis showed a significantly higher relapse-rate in African-American children, compared with whites, suggesting a more aggressive disease course in the former group (Boster et al., 2009).

\subsection{Pathogenesis}

Genetic and environmental factors are implicated in the aetiology of MS. Twin studies show a $20-30 \%$ higher risk of disease in monozygotic twins compared to dizygotic twins. Allelic variation in the MHC class II region exerts the single strongest effect on genetic risk (Ramgopalan SV et al., 2009). The HLA DR1B is the gene marker associated with higher risk of MS (Ness et al., 2007). Alleles of IL2RA, IL7RA (Hafler et al., 2007), the ecotropic viral integration site 5 (EVI5) (Hoppenbrouwers et al., 2008) and kinesin family member 1B (KIF1B) genes (Aulchenko et al., 2008) have recently been shown to increase susceptibility to MS.

Epidemiological studies implicate environmental factors such as geographical variations (Kurtzke and Hyllested, 1979), season of birth (Sadovnick et al., 2007) and migration patterns (Pugliatti et al., 2006) in the aetiology of MS. Emerging evidence supports sunlight or vitamin D as an important environmental factor in aetiology (Ramgopalan SV et al., 2009). Children exposed to parental smoking also have a higher risk of MS (Mikaeloff et al., 2007).

\subsection{Sub-types of MS}

The National MS Society in the US in 1996 categorized MS into four internationally recognized forms (Lublin and Reingold, 1996).

Relapsing-remitting: refers to MS that has exacerbations/relapses followed by symptom-free periods of remission. This is the commonest form of MS in children (Ruggierri et al., 2004).

Primary Progressive: It is characterized by gradual clinical decline from the time of disease onset with no distinct periods of remission or relapses. There maybe plateau periods during the disease but no periods of being symptom free. This entity, though rare in children, is reported (Duquette et al., 1987; Govender et al., 2010).

Secondary Progressive: This type begins with a relapsing remitting course which may last several years before the onset of the secondary progressive stage. Secondary progressive 
multiple sclerosis is a second-stage, chronic, progressive form of the disease where there are no periods of remission, only breaks in attack duration with no recovery from symptoms.

Relapsing progressive: have a steady neurologic decline but also suffer clear superimposed attacks. This is the least common of all subtypes.

An acute/ Malignant MS (Marburg variant) form presenting with a fulminant, rapidly fatal disease has also been described.

\subsection{Clinical presentation}

Children present with a wide variety of clinical symptomatology including motor, sensory, visual, cerebellar and brainstem dysfunction (Shaw and Alvord., 1987; Sindern et al., 1992; Ghezzi et al., 1997; Dale et al., 2000; Boiko et al., 2002; Pohl et al., 2006; Govender et al., 2010). Motor manifestations are described as the most common clinical presentation (Duquette et al., 1987; Sindern et al., 1992; Pohl et al., 2006). Polysymptomatic presentation is reported to be more frequent in childhood onset MS compared to adults (Ghezzi et al., 1997; Dale et al., 2000; Boiko et al., 2002). However monosymptomatic presentation is also reported in children (Duquette et al., 1987). Encephalopathy and seizures also occur in MS (Gusev et al., 2002). Eye involvement is described in up to $50 \%$ of children with MS (Pohl et al., 2006). Optic neuritis in MS is more likely to be unilateral (Dale et al., 2000). Optic tract involvement may be asymptomatic and diagnosed only by abnormal visual evoked potentials (Pohl et al., 2006). Fatigue in children is more frequent compared to adults with MS (Gusev et al., 2002). Cognitive decline is reported in 30$66 \%$ of children with MS (Banwell and Anderson 2005; Banwell et al., 2007a).

\subsection{Laboratory evaluation}

Diagnostic evaluation is to exclude other conditions affecting predominantly the white matter and to look for supportive evidence for MS. The workup should also include CSF studies (including cell count, total protein, IgG index, evidence of oligoclonal bands, and cytology) (Hahn et al., 2007). CSF Oligoclonal bands are reported in $72-84 \%$ of children with MS (Sindern et al., 1992; Dale et al., 2000). Oligoclonal bands may be absent initially and only develop during the course of the illness. Leucocytosis in the peripheral blood, though described in MS (Dale et al., 2000; Govender et al., 2010), is uncommon and non-specific. Neuro-physiological testing such as visual and auditory evoked potentials are also of diagnostic importance in detecting sub-clinical evidence of demyelination.

\subsection{Imaging}

Lesions of MS are typically multiple, discrete, plaque-like and involve predominantly the white matter (Mikaeloff et al., 2004). Commonly involved areas in MS include the corpus callosum, periventricular and sub-cortical white matter (Fig 9a-g). Lesions of MS are typically iso- or hypo-intense on T1WI, and hyper-intense on T2W1 and FLAIR sequences. Enhancement of active lesions post-gadolinium may be solid, ring-like or arc-like (Fig 10a-c). Children tend to have fewer lesions and less enhancement (Banwell et al., 2007b). However, some children lack typical MRI findings of MS and have either large tumefactive lesions with peri-lesional oedema (Hahn et al., 2004) or deep grey matter involvement. Basal ganglia affectation in MS, though described, is uncommon (Figure $11 \mathbf{a}, \mathbf{b})$. Younger children with MS may also have more diffuse, bilateral ill defined lesions (Mikaeloff et al., 2004). The International Pediatric MS Study Group strongly recommended additional imaging of the entire spinal cord to identify other sites of demyelination (Figure $12 \mathbf{a}, \mathbf{b}$ ). The cervical spinal cord is the commonest region involved in MS. 


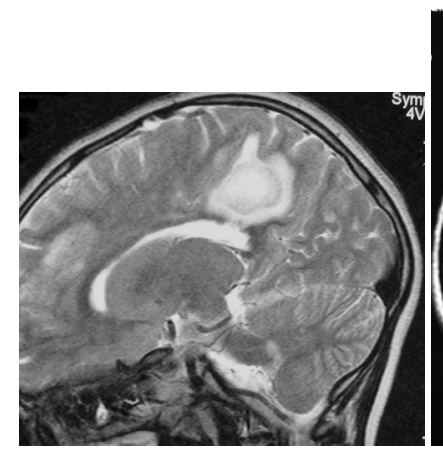

A
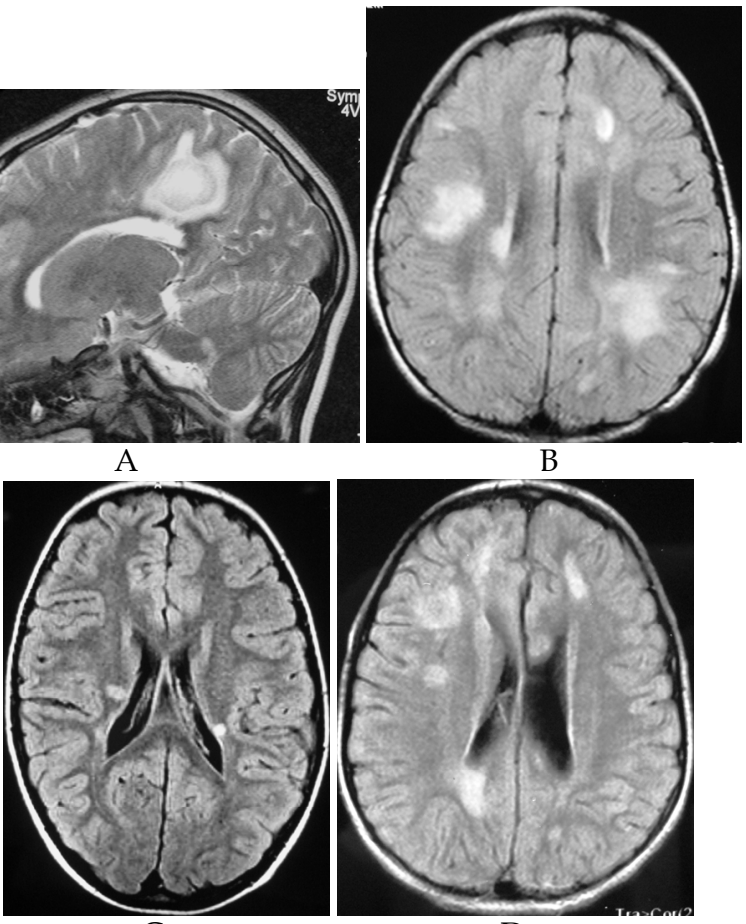

B

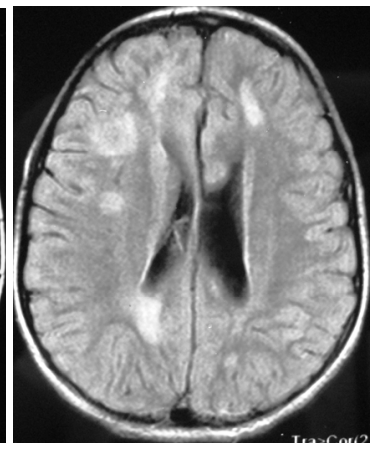

$\mathrm{D}$

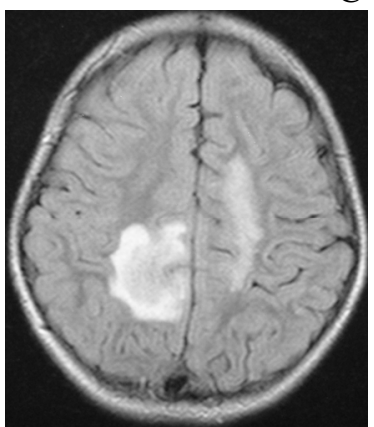

$\mathrm{E}$

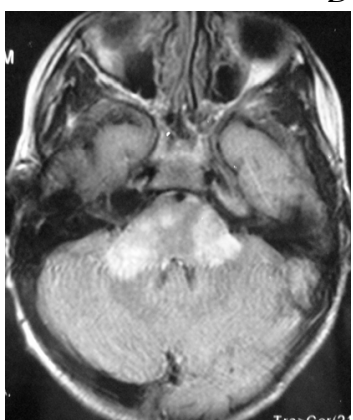

F

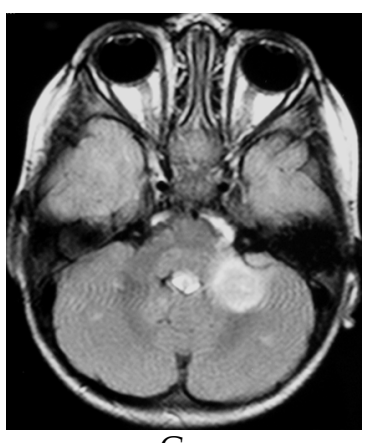

G

Fig. 9. A- T2 parasagittal MRI of the brain demonstrating flame-shaped hyper-intense lesion perpendicular to lateral ventricle consistent with MS. B- Flair axial MRI demonstrating multiple asymmetrical hyper-intense plaque-like lesions in the centrum-semiovale. Features are consistent with MS. C-Flair axial MRI of brain demonstrating 2 periventricular hyperintense white matter lesions consistent with MS. D- Flair axial at level of lateral ventricles demonstrating asymmetrical hyper-intense white matter lesions consistent with demyelination and MS. E- Flair axial of brain demonstrating hyper-intense right parietal white matter plaque-like lesion and left subtle white matter hyperintensity consistent with MS. F- Flair axial of brainstem demonstrating pontine and brachium pontis high signal intensity lesions consistent with demyelination. G-Flair axial MRI demonstrating rounded hyper-intense lesion in left brachium pontis consistent with demyelination. 


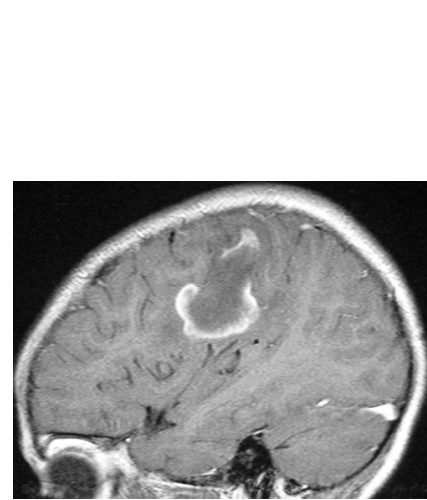

A

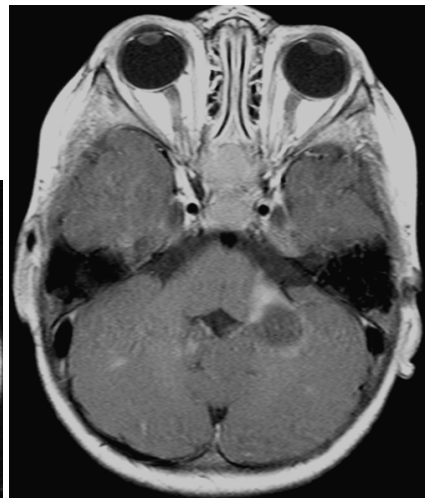

B

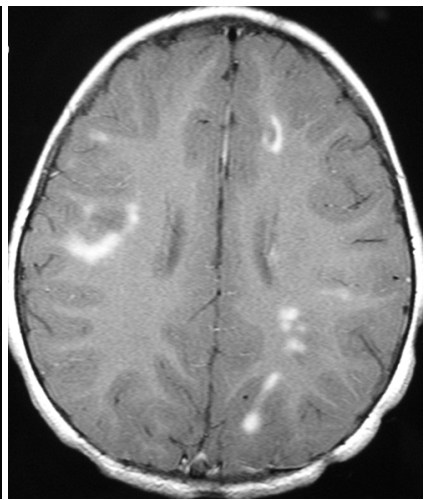

C

Fig. 10. A- T1 with contrast parasagittal MRI of brain demonstrating rim-enhancing plaquelike lesion typical of active demyelination in a patient with MS. B- T1 with contrast axial MRI demonstrating ring enhancement of left brachium pontis lesion consistent with active demyelination in a patient with MS. C- T1 with contrast axial MRI demonstrating ill-defined irregular marginal enhancement of the plaque-like lesions consistent with active demyelination in a patient with MS.

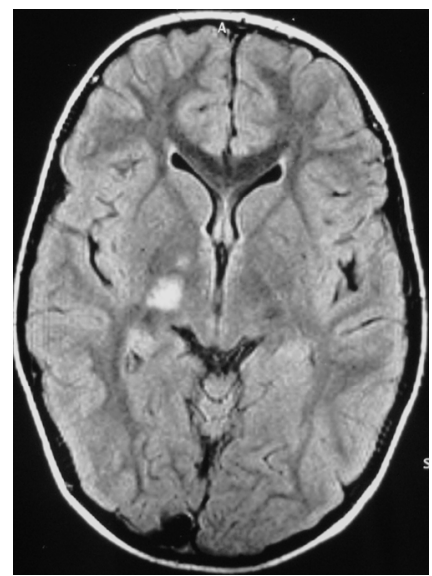

A

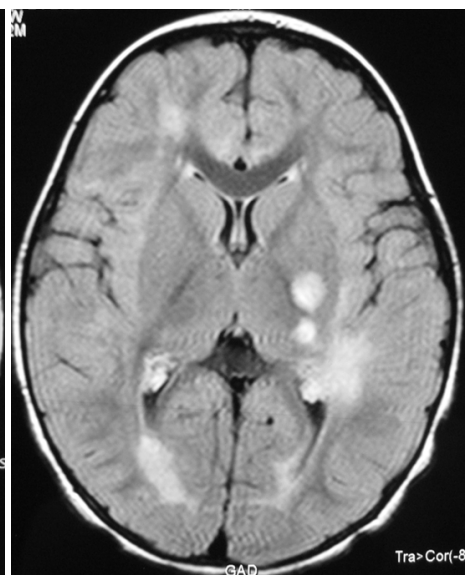

B

Fig. 11. A- Flair axial MRI of brain demonstrating 2 lesions in the right basal ganglia. This is unusual for MS. B- Flair axial MRI demonstrating multiple hyper-intense lesions in the periventricular white matter as well as left basal ganglia(atypical) consistent with MS.

MRS reveals a reduction in NAA and an elevation in choline, lipids and lactate in active lesions (Smith AB, 2009). Volumetric MRI studies reveal progressive loss of tissue in white matter tracts early in the course of the disease (Miller et al., 2002). A single study of Magnetization transfer imaging and Diffusion tensor imaging in children with MS suggested that there was no evidence of white matter degeneration in normal appearing white matter areas (Tintore et al., 2000). 


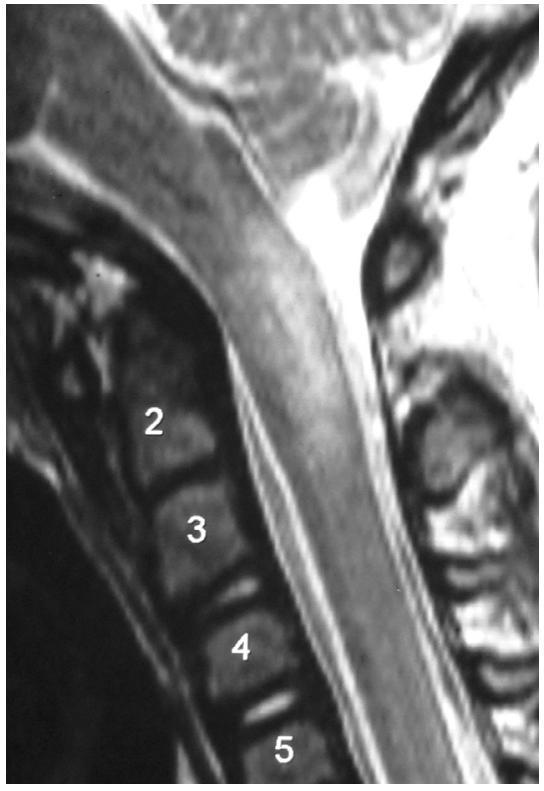

A

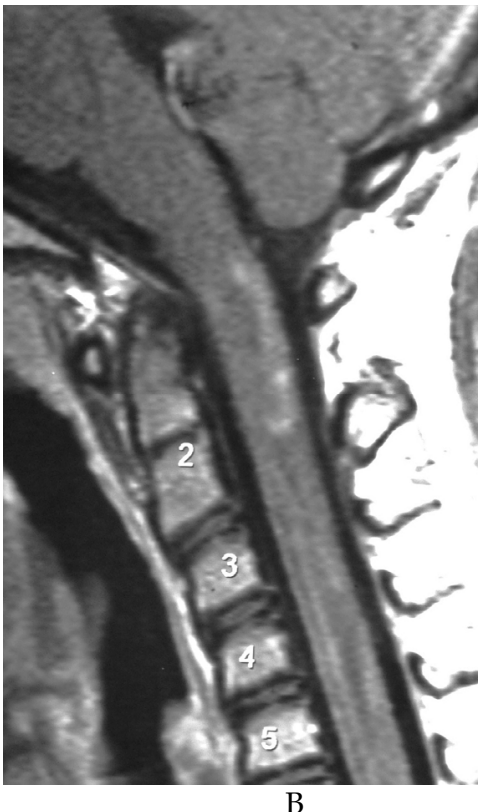

B

Fig. 12. A- T2 sagittal MRI of cervical spine in a patient with MS demonstrating an ill-defined expansile hyper-intense lesion in the proximal cord consistent with demyelination. B- T1 with contrast sagittal MRI of cervical spine in a patient with MS demonstrating ill-defined contrast enhancement of the lesion in 12A consistent with active demyelination.

\subsection{Treatment}

MS is a chronic condition with significant impact on all aspects of the family's life. Management should be trans-disciplinary involving psychologists, physiotherapists, occupational therapists and school teachers.

\subsubsection{Management of relapses}

The mainstay of managing relapses is high dose corticosteroids. High dose IV corticosteroids $(10-30 \mathrm{mg} / \mathrm{kg} /$ day) for 3-5 days, is usually used with an optional oral tapering dose. High dose oral steroids were found to be efficacious in adults (Morrow et al., 2004). Plasmapharesis and IVIG are alternatives to be considered if steroids are not effective (Hahn et al., 1996; Duzova and Bakkaloglu, 2008).

\subsubsection{Disease modifying therapy}

These therapies are known to alter the disease course and outcomes. They reduce the frequency and severity of relapses (Mikaeloff et al., 2001; Kornek et al., 2003; Tenembaum and Segura, 2006;). Patients on therapy are shown to have better outcomes compared to untreated patients (Mikaeloff et al., 2008). First line agents include Interferon beta 1a, 1b and Glatimer acetate. Case reports of second line therapies used include Natalizumab, Cyclophosphamide and Mitoxantrone. 


\begin{tabular}{|l|l|l|l|}
\hline Study & No. of patients & Treatment & Outcomes \\
\hline Ghezzi et al, 2007 & 52 & Interferon beta 1a & $\begin{array}{l}\text { Reduction in } \\
\text { relapse rate } \\
\text { Reduction in *EDS } \\
\text { score }\end{array}$ \\
\hline $\begin{array}{l}\text { Tenembaum and } \\
\text { Segura, 2006 }\end{array}$ & 24 & Interferon beta 1a & $\begin{array}{l}\text { Reduction in } \\
\text { relapse rate }\end{array}$ \\
\hline Kornek et al 2003 & 7 & Glatimer Acetate & $\begin{array}{l}\text { Reduction in } \\
\text { relapse-2/7 } \\
\text { Stable EDSS- 3/7 }\end{array}$ \\
\hline Huppke et al, 2008 & 3 & Natalizumab & $\begin{array}{l}\text { Induction of } \\
\text { remission in all }\end{array}$ \\
\hline Makhani et al, 2009 & 17 & Cyclophosphamide & $\begin{array}{l}\text { Reduction in } \\
\text { relapse rate } \\
\text { Stabilization of } \\
\text { EDSS }\end{array}$ \\
\hline
\end{tabular}

${ }^{*}$ EDSS: Extended Disability Status Scale

Table 2. Studies of specific treatment interventions in MS

\subsection{Prognosis}

Most children with MS follow a relapsing, remitting course with increasing neurodisability (Boiko et al., 2002). A slower rate of progression of disease compared to adults suggests more plasticity and potential for recovery in the developing CNS (Simone et al., 2002). Children tend to have more relapses in the first 2 years of the disease (Simone et al., 2002; Mikaeloff et al., 2006). Patients with childhood-onset MS also take longer to reach the stage of severe disability but reach irreversible neurological disability at a younger age compared to patients with adult onset disease (Renoux et al., 2007). More severe disease was noted in girls; when the time between the first and second attacks was <1 year; for childhood-onset multiple sclerosis fulfilling MRI diagnostic criteria at onset; for an absence of severe mental state changes at onset; and for a progressive course (Mikaeloff $e t$ al., 2006).

\section{Clinically isolated syndromes}

These episodes may be mono-focal (the clinical features can be attributed to a single CNS site) or multi-focal if the clinical features can not be explained by a single lesion. These include isolated optic neuritis, transverse myelitis, brainstem (Fig 13 a-d) or cortical lesions. Typically in contrast to ADEM there is no associated fever or encephalopathy. A CIS often poses a diagnostic and therapeutic challenge. Multiple lesions (> 4 lesions) (Morissey et al., 1993) on the MRI increase the risk of evolution to MS. In adult studies up to $80 \%$ of patients with a CIS evolve MS (Brex et al., 2002). Brainstem lesions in CIS are associated with a worse prognosis (Tintore et al., 2010). Children with CIS tend to have more infra-tentorial lesions 


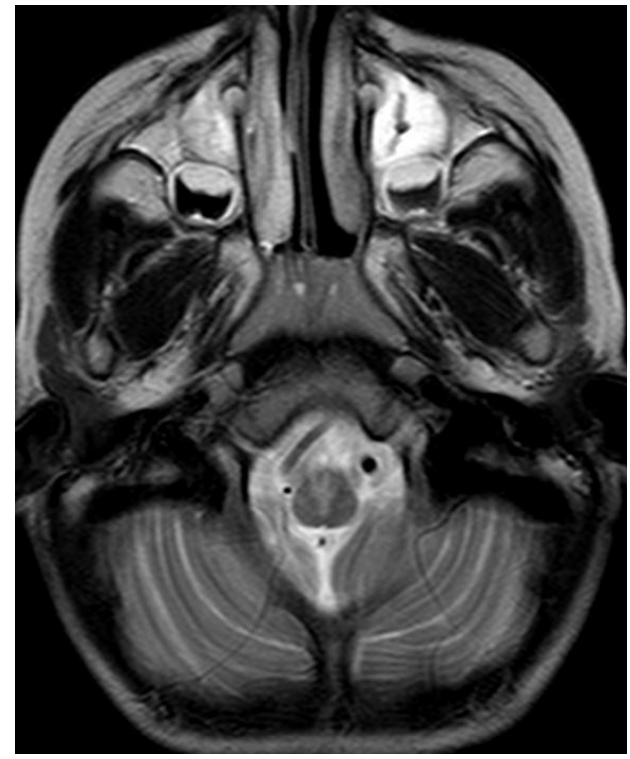

A

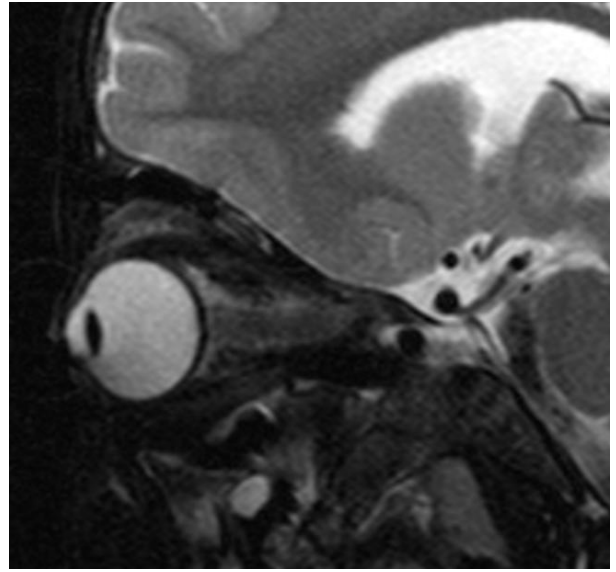

C

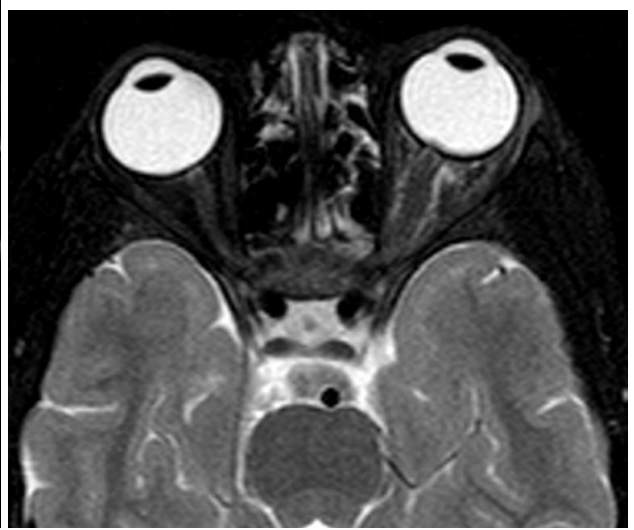

B

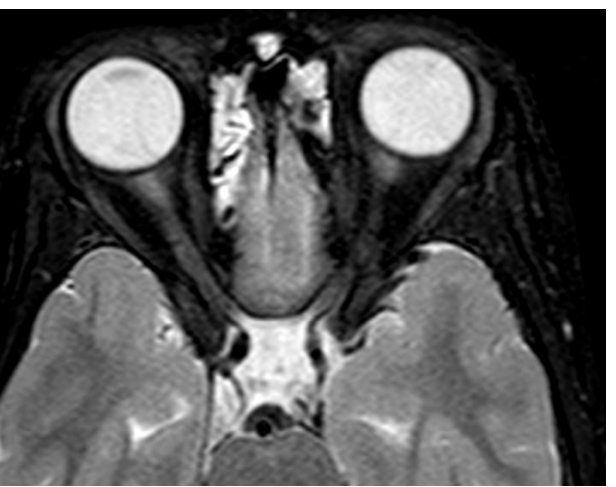

$\mathrm{D}$

Fig. 13. (B\&C same patient) Clinically Isolated Syndrome. A- T2 axial MRI demonstrating abnormal increased signal in the brainstem which was the only abnormal lesion. Clinically this patient had a CIS. B- T2 fat saturation axial MRI demonstrating a left swollen hyperintense optic nerve with resultant proptosis. Features consistent with a unilateral Optic Neuritis. Remainder of brain and spine were normal. C- T2 fat saturation sagittal oblique MRI demonstrating a left swollen hyper-intense optic nerve. Features consistent with a unilateral Optic Neuritis. Remainder of brain and spine were normal. D- T2 fat saturation axial MRI of the optic nerves demonstrating abnormal high signal intensity within the proximal portions of the nerves and swelling of the nerves. Features consistent with Optic Neuritis (worse on the right). Remainder of brain and spine were normal. 


\begin{tabular}{|c|c|c|c|}
\hline Characteristic & & ADEM & $1^{\text {st }}$ MS event \\
\hline \multirow[t]{5}{*}{ Demographics } & Age of presentation & Younger & Onset $>10 y r s$ \\
\hline & Sex & $\begin{array}{l}\text { Slight male } \\
\text { predominance }\end{array}$ & $\begin{array}{l}\text { Female } \\
\text { predominance }\end{array}$ \\
\hline & $\begin{array}{l}\text { History of Pre- } \\
\text { demyelinating event }\end{array}$ & More frequent & Less frequent \\
\hline & Seasonal distribution & More frequent & Less frequent \\
\hline & $\begin{array}{l}\text { Family history of } \\
\text { demyelinating disease }\end{array}$ & Not present & More frequent \\
\hline \multirow{5}{*}{$\begin{array}{l}\text { Clinical } \\
\text { Presentation }\end{array}$} & Seizures & More frequent & Less frequent \\
\hline & Encephalopathy & More frequent & Less frequent \\
\hline & Headache, fever & More frequent & Less frequent \\
\hline & Optic Neuritis & Bilateral & $\begin{array}{l}\text { More frequent } \\
\text { Unilateral }\end{array}$ \\
\hline & $\begin{array}{l}\text { Mono-focal vs. Poly- } \\
\text { focal signs }\end{array}$ & Polysymptomatic & Mono-focal \\
\hline \multirow{4}{*}{$\begin{array}{l}\text { Laboratory } \\
\text { features }\end{array}$} & Elevated CSF protein & Less frequent & More frequent \\
\hline & Leucocytosis in CSF & More frequent & Less frequent \\
\hline & $\begin{array}{l}\text { Oligoclonal bands in } \\
\text { CSF }\end{array}$ & $\begin{array}{l}\text { Less/ Usually } \\
\text { transient }\end{array}$ & $\begin{array}{l}\text { More frequent } \\
\text { Persistent }\end{array}$ \\
\hline & Serum Leucocytosis & More frequent & Less frequent \\
\hline \multirow{10}{*}{$\begin{array}{l}\text { MRI } \\
\text { Characteristics }\end{array}$} & Lesion definition & Ill Defined & Well-defined \\
\hline & Lesion load & Greater & Lower \\
\hline & Periventricular Lesions & Less frequent & More frequent \\
\hline & Juxta-cortical Lesions & More frequent & Less frequent \\
\hline & Cortical Lesions & More frequent & Less frequent \\
\hline & $\begin{array}{l}\text { Corpus Callosum } \\
\text { Involvement }\end{array}$ & Less frequent & More frequent \\
\hline & Brainstem/ Cerebellum & More frequent & Less frequent \\
\hline & $\begin{array}{l}\text { Spinal Cord } \\
\text { Involvement }\end{array}$ & More frequent & Less frequent \\
\hline & $\begin{array}{l}\text { Deep Grey matter } \\
\text { involvement }\end{array}$ & More frequent & Less frequent \\
\hline & Contrast enhancement & Less frequent & More frequent \\
\hline \multirow[t]{2}{*}{ Outcome } & Cognitive deficits & Less frequent & More frequent \\
\hline & $\begin{array}{l}\text { No neuro deficit after } 1^{\text {st }} \\
\text { event }\end{array}$ & Less likely & More likely \\
\hline
\end{tabular}

Table 3. Markers comparing ADEM to a first episode of MS 


\begin{tabular}{|c|c|c|c|}
\hline Disorder & Example & Clinical/ Laboratory & Radiological \\
\hline \multirow[t]{2}{*}{ Infections } & $\begin{array}{l}\text { HIV } \\
\text { Encephalopathy }\end{array}$ & $\begin{array}{l}\text { Developmental delay, } \\
\text { pyramidal tract signs, } \\
\text { microcephaly }\end{array}$ & $\begin{array}{l}\text {-Confluent bilateral } \\
\text { symmetrical white } \\
\text { matter changes } \\
\text {-cerebral atrophy } \\
\text {-basal ganglia } \\
\text { calcification }\end{array}$ \\
\hline & $\begin{array}{l}\text { Progressive Multi- } \\
\text { focal } \\
\text { Leucoencephalopa } \\
\text { thy (JC Virus) }\end{array}$ & Immunocompromised & $\begin{array}{l}\text {-Multi-focal } \uparrow \mathrm{T} 2 \mathrm{WI} \\
\text { lesions } \\
\text {-Propensity for } \\
\text { frontal/ parieto- } \\
\text { occipital areas } \\
\text {-Subcortical U } \\
\text { fibres involved }\end{array}$ \\
\hline Other infections & \multicolumn{3}{|c|}{$\begin{array}{l}\text { Sub-acute Sclerosing Panencephalitis, Lymes Disease, } \\
\text { Neurosyphillus, HTLV1, Borreliosis }\end{array}$} \\
\hline \multirow[t]{2}{*}{$\begin{array}{l}\text { Auto- } \\
\text { immune/Vasculitides }\end{array}$} & $\begin{array}{l}\text { Systemic Lupus } \\
\text { Erythematosus }\end{array}$ & $\begin{array}{l}\text { Multi-system auto- } \\
\text { immune disorder } \\
\text { Anti-nuclear factor } \\
\text { positive }\end{array}$ & $\begin{array}{l}\text {-Multi-focal } \\
\uparrow T 2 W I / F L A I R \\
\text {-Infarcts } \\
\text {-Contrast } \\
\text { enhancement } \\
\text { of active } \\
\text { lesions }\end{array}$ \\
\hline & \multicolumn{3}{|c|}{ Isolated CNS angiitis, CADASIL (adult disorder-rare in children) } \\
\hline \multirow[t]{2}{*}{ Tumor } & CNS Lymphoma & $\begin{array}{l}\text { Insiduous onset, CSF } \\
\text { cytospin -malignant cells }\end{array}$ & $\begin{array}{l}-\downarrow \text { T1WI } \\
-\uparrow \text { T2WI } \\
- \text { GM involved more } \\
\text { frequently } \\
- \text { MRS may help }\end{array}$ \\
\hline & \multicolumn{3}{|c|}{ Medulloblastoma, Astrocytoma } \\
\hline Leukodystrophies & $\begin{array}{l}\text { Adrenoleukody- } \\
\text { strophy }\end{array}$ & $\begin{array}{l}\text { Boys with } \\
\text { hyperpigmentation of } \\
\text { skin, behaviour and } \\
\text { learning problems, } \\
\text { Abnormal very long chain } \\
\text { fatty acids }\end{array}$ & $\begin{array}{l}\text {-Symmetrical, } \\
\text { confluent } \\
\text {-predominantly } \\
\text { posterior } \\
\text { involvement } \\
\text {-Splenium and } \\
\text { cortico-spinal tracts } \\
\text { involved } \\
\text {-Leading edge } \\
\text { enhancement in } \\
\text { peri-trigonal } \\
\text { area }\end{array}$ \\
\hline
\end{tabular}




\begin{tabular}{|c|c|c|c|}
\hline \multirow[b]{2}{*}{ Mitochondrial } & \multicolumn{3}{|c|}{$\begin{array}{l}\text { Metachromic Leukodystrophy, Krabbe's Disease, Alexander's } \\
\text { disease }\end{array}$} \\
\hline & Leigh's Disease & $\begin{array}{l}\text { Neuro-regression with } \\
\text { dystonia/opthalmoplegia } \\
\text { Hyperlactataemia }\end{array}$ & $\begin{array}{l}\text {-Bilateral } \\
\text { symmetrical } \\
\uparrow T 2 W I / F L A I R \text { of } \\
\text { Putamen and } \\
\text { caudate nuclei } \\
\text {-Can have diffuse } \\
\text { cortical WM } \\
\text { hyperintensities }\end{array}$ \\
\hline \multirow[t]{2}{*}{$\begin{array}{l}\text { Nutritional } \\
\text { Deficiencies }\end{array}$} & $\begin{array}{l}\text { Vitamin B12 } \\
\text { deficiency }\end{array}$ & $\begin{array}{l}\text { Anaemia, peripheral } \\
\text { neuropathy, Myelopathy }\end{array}$ & $\begin{array}{l}\text {-Periventricular } \\
\uparrow \mathrm{T} 2 \mathrm{WI}\end{array}$ \\
\hline & \multicolumn{3}{|c|}{ Vitamin E, Folate deficiency } \\
\hline \multirow[t]{2}{*}{ Toxins/ Drugs } & Radiation & History of exposure & $\begin{array}{l}\text {-Diffuse, bilateral } \\
\text { periventricular and } \\
\text { central WM } \\
\text { involved } \\
\text {-T1WI } \downarrow, \mathrm{T} 2 \mathrm{WI} \uparrow \\
\text {-Sparing of sub- } \\
\text { cortical U Fibres }\end{array}$ \\
\hline & \multicolumn{3}{|l|}{ Lead, Isoniazid } \\
\hline \multirow[t]{2}{*}{ Infiltrative } & Sarcoidosis & $\begin{array}{l}\text { Cranial neuropathies, } \\
\text { aseptic meningitis, visual } \\
\text { disturbances }\end{array}$ & $\begin{array}{l}\text {-Discrete } \\
\text { periventricular } \\
\text { lesions } \\
\text {-May have } \\
\text { hypothalamic and } \\
\text { meningeal } \\
\text { enhancement }\end{array}$ \\
\hline & Histiocytosis & $\begin{array}{l}\text { Visual disturbances, } \\
\text { hypothalamic dysfunction }\end{array}$ & $\begin{array}{l}\text {-Hypothalamic/ } \\
\text { cerebellar T1WI } \downarrow \text {, } \\
\text { T2WI } \uparrow \\
\text {-Skull/ mastoid } \\
\text { lesions }\end{array}$ \\
\hline $\begin{array}{l}\text { Osmotic } \\
\text { Demyelination } \\
\text { Syndrome }\end{array}$ & & $\begin{array}{l}\text { Rapid correction of Hypo- } \\
\text { or hypernatraemia }\end{array}$ & $\begin{array}{l}\text {-Symmetric } \\
\text { changes in BG/ } \\
\text { Cerebral cortical } \\
\text { WM } \\
\text {-T1WI } \downarrow \\
-\uparrow \text { FLAIR/T2WI } \uparrow \\
\text {-Pontine : Usual } \\
\text { central with } \\
\text { sparing of cortico- } \\
\text { spinal tracts }\end{array}$ \\
\hline
\end{tabular}

Table 4. Main differential diagnosis of acquired white matter diseases on MRI 
(Ghassemi et al., 2008). This may be related to the differences in myelination patterns and maturation in children compared to adults. A radiologically isolated syndrome (RIS) is defined by incidental MRI findings suggestive of MS in an asymptomatic patient lacking any history, symptoms, or signs of MS (Okuda et al., 2009).

\section{Risk of recurrence after a first demyelinating event}

Predicting the risk of a first episode of demyelination evolving on to MS is important as new immunomodulating therapies become available. Early initiation of disease modifying therapy reduces the risk of relapse and long-term disability (Jacobs et al., 2000). Patients with "ADEM" progressing to MS vary from 0-29\% (Belman et al., 2007). Multiple historical, clinical, laboratory and radiological criteria are used to predict the risk of recurrence/ progression to MS (Table 3).

A seasonal pattern, a history of a precipitant, seizures, bilateral optic neuritis and encephalopathy are considered more likely in ADEM compared to MS (Dale et al., 2005). Inflammatory markers, a high cerebrospinal fluid protein and leucocytosis are also more common in ADEM (Kesselring et al., 1990). MRI characteristics that are predictive of evolution to MS include well defined lesions that are peri-aqueductal or perpendicular to the corpus callosum (Dale et al., 2000), deep grey matter involvement and lesions that enhance post contrast (Govender et al., 2010).

\section{Differential diagnosis of white matter disease in children}

The differential diagnosis for a child who presents with a neurological symptom and white matter lesions on neuro-imaging is vast and includes infectious diseases, leukodystrophies, tumors, vasculitides, toxins and vitamin deficiencies. In resource poor countries, CNS infections must be excluded first as they are common and have acute therapeutic implications.

CNS infections must be excluded in children presenting acutely especially with fever and encephalopathy. CNS infections that may present with multifocal white matter lesions include HTLV-1, Borreliosis and Subacute Sclerosing Panencephalitis. In resource poor settings HIV Encephalopathy (Figure $\mathbf{1 4} \mathbf{a}, \mathbf{b}$ ) is common and is also characterized by confluent white matter lesions. Progressive Multi-focal Leukoencephalopathy (Figure 14 c), is also common in immuno-compromised patients.

Neurometabolic disorders, such as Adrenoleukodystrophy (Figure $14 \mathbf{d}$,e), presents with primary white matter disease.

Osmotic Myelinolysis (Figure $14 \mathbf{f}, \mathbf{g}$ ) is thought to be related to osmotic shifts associated with rapid correction of fluid and electrolyte abnormalities (especially sodium abnormalities). Malnourished children are at greater risk for developing myelinolysis. Lesions typically occur in the pons but have also been reported in extra-pontine sites such as the basal ganglia, cerebral cortex and cerebellar peduncles.

Nutritional deficiencies such as vitamins B12, E and folate deficiencies may also cause white matter lesions.

Drugs and toxins implicated in demyelination include tin, lead, isoniazid and radiation.

Collagen Vascular Diseases refer to a group of auto-immune mediated disorders. The neurological manifestations are diverse. Neuro-imaging may show multi-focal white matter lesions- involving the cortex, cerebellum or spinal cord. 


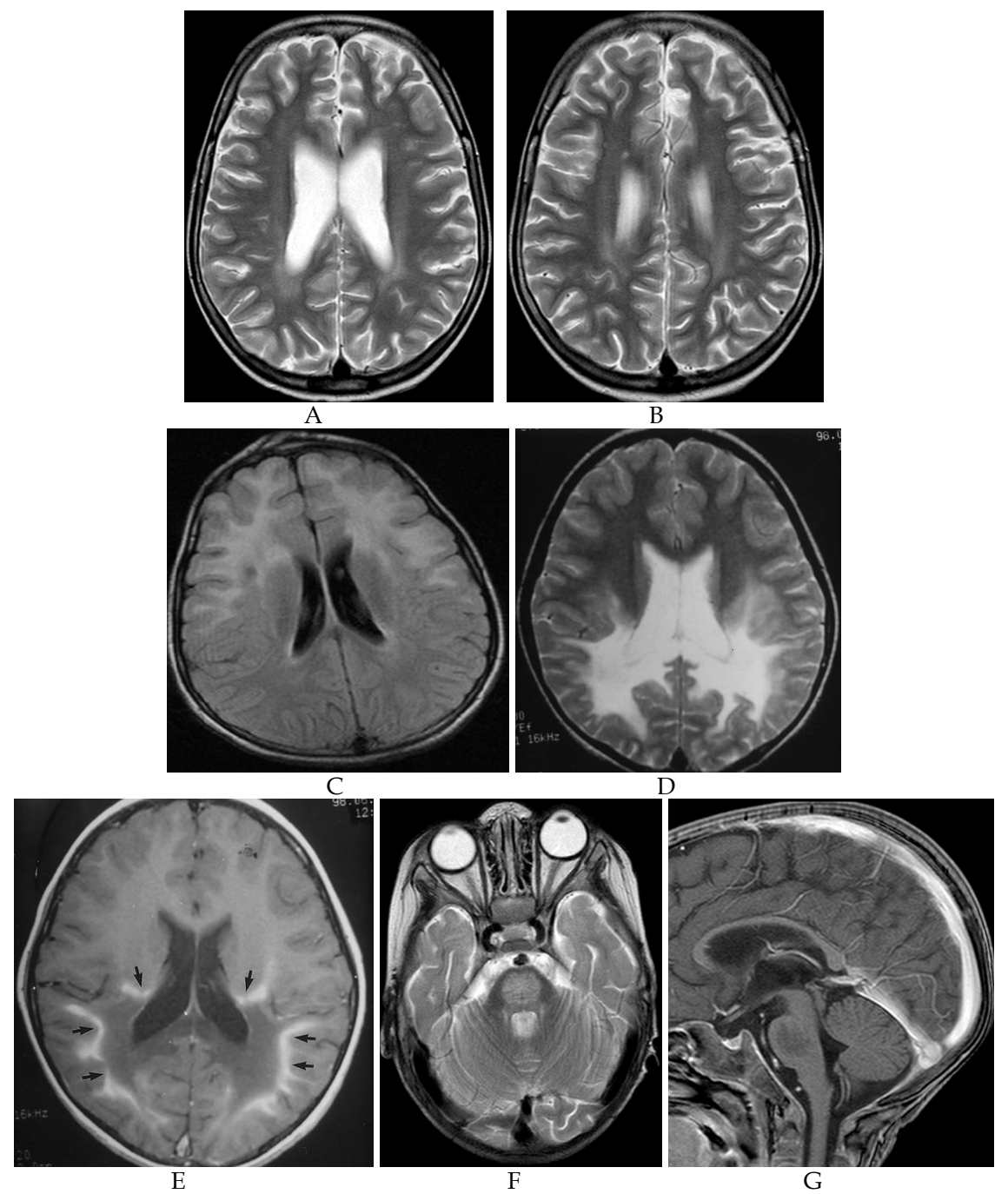

Fig. 14. A \& B- T2 axial MRI demonstrating diffuse brain shrinkage/atrophy with ex-vacuo dilatation of the ventricles and abnormal increased signal intensity in the deep white matter bilaterally. This patient has features of HIV encephalopathy. C- Flair axial MRI demonstrating bilateral symmetrical increased signal intensity in the frontal white matter. This patient was diagnosed with PML. D- T2 axial MRI demonstrating bilateral symmetrical confluent abnormal increased white matter signal intensity in the posterior white matter consistent with Adrenoleukodystrophy. E- T1 with contrast axial MRI of same patient as 14D demonstrating peripheral enhancement of the white matter lesions. F- T2 axial MRI demonstrating well-defined rounded hyper-intense lesion in the pons. Note peripheral sparing of the pons. (Compared to figure 14G). Features consistent with Osmotic/ Pontine Myelinolysis. G- T1 with contrast sagittal MRI of the same pontine lesion as 14F showing no contrast enhancement. Features consistent with Pontine Myelinolysis. 


\section{Conclusion}

Acquired demyelinating disorders in children are a diverse, challenging group of conditions that are probably under-diagnosed. Early recognition is essential for optimal patient management as some of these disorders cause significant long-term sequelae. Advances in the last decade include establishing consensus definitions and improvement in neuroimaging techniques. These advances set the stage for international collaborative studies to better define other areas such as understanding the aetio-pathogenesis, identifying biomarkers and standardizing treatment protocols of this diverse group of conditions.

\section{References}

Abramsky O, Teitelbaum D, Arnon R (1977). Effect of a synthetic polypeptide (cop1) on patients with multiple sclerosis and with acute disseminated encephalomyelitis. $J$ Neurol Sci 31: 433-8.

Adams C, Armstrong D (1990). Acute transverse myelopathy in children. Can J Neurol Sci 17: 40-5.

Afifi AK, Bell WE, Menezes AH, Moore SA (1994). Myelinoclastic diffuse sclerosis (Schilder's disease): report of a case and review of the literature. J. Child Neurol. 9 (4): 398-403

Alvord EC Jr, Jahnke U, Fischer EH, Kies MW, Driscoll BF, Compston DA (1987). The multiple Causes of Multiple sclerosis: the importance of age of infections in childhood. J Child Neurol. 2: 313-21

Andronikou S, Albuquerque-Jonathan G, Wilmshurst J, Hewlett R (2003). MRI findings in acute idiopathic transverse myelopathy in children. Pediatr Radiol 33:624-629

Arnold DL, Matthews PM, Francis G, Antel J (1990). Proton magnetic resonance spectroscopy of human brain in vivo in the evaluation of multiple sclerosis: assessment of the load of disease. Magnet Reson Med 14: 154 -159.

Aulchenko YS, Hoppenbrouwers IA, Ramagopalan SV, Broer L, Jafari N, Hillert J, Link J, Lundström W, Greiner E, Dessa Sadovnick A, Goossens D, Van Broeckhoven C, Del-Favero J, Ebers GC, Oostra BA, van Duijn CM, Hintzen RQ (2008). Genetic variation in the KIF1B locus influences susceptibility to multiple sclerosis. Nat Genetics 40(12): 1402-3

Banwell B, Kennedy J, Sadovnick D, Magalhaes S et al (2009). Incidence of acquired demyelination of the CNS in Canadian children. Neurology 72(3): 232-239

Banwell B, Ghezzi A, Bar-Or A, Mikaeloff Y, Tardieu M (2007a). Multiple sclerosis in children: Clinical diagnosis, therapeutic strategies, and future directions. Lancet Neurol. 6: 887-902.

Banwell BL, Anderson PE (2005). The cognitive burden of multiple sclerosis in children. Neurology 64: 891-4.

Banwell B, Shroff M, Ness JM, Jeffery D, Schwid S, Weinstock-Guttman, B; for the International Pediatric MS Study Group (2007b). MRI features of pediatric multiple sclerosis. Neurology 68(16) Suppl 2, pp S46-S53

Barkovich JA, Moore KR, Grant E, Jones BV, Vezina G, Koch BL, Raybaud C, Blaser S, Hedlund GB, Illner A (2007). Diagnostic Imaging: Pediatric Neuroradiology (1 ${ }^{\text {st }}$ edition), Amirsys-Elsevier, ISBN 1-4160-4918-5 Salt Lake City, Utah 
Baum PA, James Barkovich A, Koch TK (1994). Deep gray matter involvement in children with acute disseminated encephalomyelitis. AJNR 15: 1275-1283

Belman A, Tanuja C, Renoux C, Waubant E for the International Pediatric MS Study Group (2007). Challenges in the classification of Paediatric MS and future directions. Neurology 68(16): pp S70-74

Berman M, Feldman S, Alter M, Zilber N, Kahana E (1981). Acute transverse myelitis: incidence and etiological considerations. Neurology 31: 966-971

Bhigjee AI, Moodley K, Ramkissoon K (2007). Multiple sclerosis in KwaZulu Natal, South Africa: an Epidemiological and clinical study. Multiple Sclerosis 13(9): 1095-1099

Boe J, Solberg CO, Saeter T. Corticosteroid treatment of acute meningo encephalitis: Retrospective study of 346 cases (1965). BMJ 1: 1094-5

Boiko A, Vorobeychik G, Paty D, Devonshire V, Sadovnick V (2002). Early onset multiple sclerosis: A Longitudinal study. Neurology 59: 1006-1010

Bortone E, Bettoni L, Buzio S, Delsoldato S, Giorgi C, Mancia D (1996). Spindle coma and alternating pattern in the course of measles encephalitis. Clin Electroencephalogr 27: 210-4.

Boster AL, Endress CF, Hreha SA, Caon C, Perumal JS, Khan OA (2009). Pediatric-onset multiple sclerosis in African- American black and European-origin white patients. Pediatr Neurol 40: 31-33.

Bizzi A, Ulug AM, Crawford TO, Passe T, Bugiani M, Bryan RN, Barker PB (2001). Quantitative proton MR spectroscopic imaging in acute disseminated encephalomyelitis. Am J Neuroradiol 22: 1125-30

Brex PA, Ciccarelli O, O'Riordan JI, Sailer M, Thomson AJ, Miller DH (2002). A longitudinal study of abnormalities on MRI and disability from multiple sclerosis. $N$ Engl J Med 346: 158-164

Dale RC, Branson JA (2005). ADEM vs. MS Can initial presentation help in establishing a correct diagnosis? Archive of Disease in Childhood 90: 636-639

Dale RC, de Sousa C, Chong WK, Cox TC, Harding B, Neville BG (2000). Acute Disseminated Encephalomyelitis, Multiphasic disseminated encephalomyelitis and Multiple Sclerosis in Children. Brain 123: 2407-2422

Dale RC, Church AJ, Cardoso F, Goddard E, Cox TC, Kling Chong WK, Williams A, Klein NJ, Neville BG, Thomson EJ, Giovannoni G (2001). Post streptococcal acute disseminated encephalomyelitis with basal ganglia involvement and auto-reactive antibasal ganglia antibodies. Ann Neurol 50(5): 588-595.

Davies NW, Sharief MK, Howard RS ((2006). Infection-associated encephalopathies: their investigation, diagnosis, and treatment. J. Neurol. 253 (7): 833-45

Defresne P, Meyer L, Tardieu M, Scalais E, Nuttin C, De Bont B, Loftus G, Landrieu P, Kadhim H, Sébire G (2001). Efficacy of high dose steroid therapy in children with severe acute transverse myelitis. J Neurol Neurosurg Psychiatr 71: 272-4

De Goede CG, Holmes EM, Pike MG (2010). Acquired transverse myelopathy in children in the United Kingdom - A 2 year prospective study. European Journal of Paediatric Neurology 14: 479-487

De Stefano N, Matthews PM, Arnold DL (1995). Reversible decreases in N-acetylaspartate after acute brain injury. Magnet Reson Med 34: $721 \pm 727$ 
De Stefano N, Narayanan S, Matthews PM, Mortilla M, Dotti MD, Federico A, Arnold DL (2000). Proton MR spectroscopy to assess axonal damage in multiple sclerosis and other white matter disorders. Journal of NeuroVirology 6 (2): S121- S129

De Stefano N, Narayanan S, Matthews PM, Mortilla M, Dotti MD, Federico A, Arnold DL (2000). Proton MR spectroscopy to assess axonal damage in multiple sclerosis and other white matter disorders. Journal of NeuroVirology 6 (2): S121- S129

Dunne K, Hopkins IJ, Shield LK (1986). Acute transverse myelopathy in childhood. Dev Med Child Neurol 28: 198-204.

Duquette P, Murray TJ, Pleines J, Ebers GC, Sadovnick D, Weldon P, Warren S, Paty DW, Upton A, Hader W (1987). Multiple sclerosis in childhood: clinical profile in 125 patients. J Pediatr 111(3): 359-63.

Duzova A, Bakkaloglu A (2008). Central nervous system involvement in pediatric rheumatic diseases: current concepts in treatment. Curr Pharm Des 14: 1295-301

Engelbrecht V, Scherer A, Rassek M, Witsack HJ, Mödder U (2002). Diffusion-weighted MR Imaging in the Brain in Children: Findings in the Normal Brain and in the Brain with White Matter Diseases. Radiology 222: 410-418

Finsterer J, Grass R, Stollberger C, Mamoli B (1998). Immunoglobulins in acute, parainfectious, disseminated encephalo-myelitis. Clin Neuropharmacol 21: 258-61.

Ford B, Tampori D, Francis G (1992). Long term follow up of acute partial transverse myelopathy. Neurology 42: 250-252

Gabis LV, Panasci DJ, Andriola MR, Huang W (2004). Acute disseminated encephalomyelitis: An MRI/MRS longitudinal study. Pediatr Neurol 30: 324-329.

Ghassemi R, Antel SB, Narayanan S, Francis SJ, Bar-Or A, Sadovnick AD, Banwell B, Arnold DL; Canadian Pediatric Demyelinating Disease Study Group (2008). Lesion distribution in children with clinically isolated syndromes. Ann Neurol. 63(3): 401-5

Ghezzi A, Deplano V, Faroni J, Grasso MG, Liguori M, Marrosu G, Pozzilli C, Simone IL, Zaffaroni M (1997). Multiple sclerosis in childhood: clinical features of 149 cases. Mult Scler 3: 43-46.

Ghezzi A, Amato MP, Capobianco M, Gallo P, Marrosu MG, Martinelli V, Milanese C, Moiola L, Milani N, La Mantia L, Patti F, Pozzilli C, Trojano M, Comi G, Zaffaroni M; Immunomodulatory Treatment of Early-onset MS (ITEMS) Group (2007). Treatment of early-onset multiple sclerosis with intramuscular interferon beta-1a: long-term results. Neurol Sci. 28(3): 127-32

Govender R, Wieselthaler N, Ndondo AP, Wilmshurst JM (2010). Acquired Demyelinating Disorders of Childhood in the Western Cape, South Africa. J Child Neurol 25: 48-56

Gusev E, Boiko A, Bikova O, Maslova O, Guseva M, Boiko S, Vorobeichik G, Paty D (2002). The natural history of early onset multiple sclerosis: Comparison of data from Moscow and Vancouver. Clin Neurol Neurosurg 104: 203-7.

Gutling E, Landis T (1989). CT ring sign imitating tumour, disclosed as multiple sclerosis by MRI: A case report. J Neurol Neurosurg Psychiatry 52: 903-6.

Hafler DA, Compston A, Sawcer S, Lander ES, Daly MJ, De Jager PL, de Bakker PI, Gabriel SB, Mirel DB, Ivinson AJ, Pericak-Vance MA, Gregory SG, Rioux JD, McCauley JL, Haines JL, Barcellos LF, Cree B, Oksenberg JR, Hauser SL International Multiple Sclerosis Genetics Consortium (2007) Risk Alleles for Multiple Sclerosis Identified by a Genomewide Study. N Engl J Med 357(9): 851-62. 
Hahn CD, Miles BS, MacGregor DL, Blaser SI, Banwell BL, Hetherington CR (2003). Neurocognitive outcome after acute disseminated encephalomyelitis. Pediatr. Neurol. 29 (2): 117-23.

Hahn J S, Pohl, D, Rensel, M, Sanjai DO for the International Pediatric MS Study Group (2007). Differential diagnosis and evaluation in pediatric multiple sclerosis. Neurology 68(16) Suppl 2: pp S13-S22

Hahn CD, Shroff MM, Blaser SI, Banwell BL (2004). MRI criteria for multiple sclerosis: Evaluation in a pediatric cohort. Neurology 62: 806-808

Hahn JS, Siegler DJ, Enzmann D (1996). Intravenous gammaglobulin therapy in recurrent acute disseminated encephalomyelitis. Neurology 46: 1173-4.

Hung KL, Liao HT, Tsai ML (2000). Postinfectious encephalomyelitis: etiologic and diagnostic trends. J Child Neurol. 15(10): $666-670$

Hoppenbrouwers IA, Aulchenko YS, Ebers GC, Ramagopalan SV, Oostra BA, van Duijn CM, Hintzen RQ (2008). EVI5 is a risk gene for multiple sclerosis. Genes Immun 9(4): 334-7

Huppke P., Stark W., Zurcher C., Huppke B., Bruck W. and Gartner J. (2008). Natalizumab use in pediatric multiple sclerosis. Arch Neurol 65: 1655-1658.

Hynson JL, Kornberg AJ, Coleman LT, Shield L, Harvey AS, Kean MJ (2001). Clinical and neuroradiologic features of acute disseminated encephalomyelitis in children. Neurology 56: 1308-1312

Idrissova ZR., Boldyreva MN, Dekonenko EP, Malishev NA, Leontyeva IY, Martinenko IN (2003). Acute disseminated encephalomyelitis in children: clinical features and HLA-DR linkage. Eur J Neurol 10: 537-546.

Jacobs RK, Anderson VA, Neale JL, Shield LK, Kornberg AJ (2004). Neuropsychological outcome after acute disseminated encephalomyelitis: impact of age at illness onset. Pediatr. Neurol. 31 (3): 191-7.

Jacobs LD, Beck RW, Simon JH, Kinkel RP, Brownscheidle CM, Murray TJ, Simonian NA, Slasor PJ, Sandrock AW, and the CHAMPS Study Group (2000). Intramuscular interferon beta-1-a therapy initiated during a first demyelinating event in multiple sclerosis. N Engl J Med 343: 898-904

Jain AP, Gupta OP, Jajoo UN (1983). A study of some prognostic factors in acute transverse Myelitis. Journal Assoc Physicians India. 31(8): 497-9.

Jeffery DR, Mandler RN, Davis LE (1993). Transverse myelitis: retrospective analysis of 33 cases with differentiation of cases associated with multiple sclerosis and parainfectious agents. Arch Neurol. 50: 532-535

Johnson, R. T. (1994), The virology of demyelinating diseases. Annals of Neurology 36: S54S60

Johnson RT, Griffin DE, Gendelman HE (1985). Postinfectious encephalomyelitis. Semin Neurol 5: 180-90.

Kalra V, Sharma S, Sahu J, Sankhyan N, Chaudry R, Dhawan B, Mridula B (2009). Childhood acute transverse myelitis: clinical profile, outcome, and association with antiganglioside antibodies. J Child Neurol. 24: 466-471.

Kaplin AI, Krishnan C, Deshpande DM, Pardo CA, Kerr DA(2005). Diagnosis and management of acute myelopathies. Neurologist 11: 2-18 
Kaufman MD, Johnson SK, Moyer D, Bivens J, Norton HJ (2003). Multiple sclerosis: severity and progression rate in African Americans compared with whites. Am J Phys Med Rehabil 82: 582-90.

Kesselring J, Miller DH, Robb SA, Kendall BE, Moseley IF, Kingsley D, du Boulay EP, McDonald WI (1990). Acute disseminated encephalomyelitis. MRI findings and the distinction from multiple sclerosis. Brain 113: 291-302.

Kinoshita A, Hayashi M, Miyamoto K, Oda M, Tanabe H (1996). Inflammatory demyelinating polyradiculitis in a patient with acute disseminated encephalomyelitis (ADEM). J Neurol Neurosurg Psychiatry 60: 87-90.

Knebusch M, Strassburg HM, Reiners K (1998). ATM in Childhood: 9 cases and a review of the literature. Dev Med Child. Neurol 40: 631-639

Kornek B, Bernert G, Balassy C, Geldner J, Prayer D, Feucht M (2003). Glatiramer acetate treatment in patients with childhood and juvenile onset multiple sclerosis. Neuropediatrics 34(3): 120-6.

Krupp Lauren B, Banwell B, and Tenembaum $S$ for the International Pediatric MS Study Group (2007). Neurology. Vol 68(16): pp S7-S12

Kumar K, Toth C, Jay V (1998). Focal plaque of demyelination mimicking cerebral tumor in a pediatric patient. Pediatr Neurosurg 29: 60-3.

Kurtzke, J.F. and Hyllested, K (1979). Multiple sclerosis in the Faroe Islands: I. Clinical and epidemiological features. Ann Neurol 5(1): 6-21.

Leake JA, Albani S, Kao AS, Senac MO, Billman GF, Nespeca MP (2004). Acute disseminated encephalomyelitis in childhood: Epidemiologic clinical and laboratory features. Pediatr Infect Dis J 23: 756-64.

Lennon VA, Wingerchuk DM, Kryzer TJ, Pittock SJ, Lucchinetti CF, Fujihara K, Nakashima I, Weinshenker BGL (2004). A serum autoantibody marker of neuromyelitis optica: distinction from multiple sclerosis. Lancet 364: 2106-2112.

Lisak RP, Zweiman B (1977). In vitro cell-mediated immunity of cerebrospinal-fluid lymphocytes to myelin basic protein in primary demyelinating diseases. $N$ Engl J Med 297(16): 850-3.

Lublin FD, Reingold SC (1996). Defining the clinical course of multiple sclerosis: results of an international survey. National Multiple Sclerosis Society (USA) Advisory Committee on Clinical Trials of New Agents in Multiple Sclerosis. Neurology 46 (4): 907-11

Lucas J (1790). An account of uncommon symptoms succeeding the measles with additional remarks on the infection of measles and smallpox. London Med J. 11: 325 -331

Makhani N, Gorman MP, Branson HM, Stazzone L, Banwell BL, Chitnis T (2009). Cyclophosphamide use in pediatric multiple sclerosis. Neurology 72(24): 2064-5.

Mandler RN, Davis LE, Jeffery DR, Kornfeld M.(1993). Devic's neuromyelitis optica: a clinicopathological study of 8 patients. Ann Neurol 34(2): 162-8.

McAdam LC, Blaser SI, Banwell BL (2002). Pediatric tumefactive demyelination: Case series and review of the literature. Pediatr Neurol 26: 18-25

McAlpine D (1931). Acute disseminated encephalomyelitis: its sequelay and its relationship to disseminated sclerosis. Lancet $846-852$ 
Menge T, Kieseier BC, Nessler S, Hemmer B, Hartung HP, Stüve O (2007). Acute disseminated encephalomyelitis: an acute hit against the brain. Curr. Opin. Neurol. 20 (3): 247-54.

Mikaeloff Y, Adamsbaum C, Husson B, Vallée L, Ponsot G, Confavreux C, Tardieu M, Suissa S; KIDMUS Study Group on Radiology (2004). MRI prognostic factors for relapse after acute CNS inflammatory demyelination in childhood. Brain 127(9): 1942-7

Mikaeloff Y, Caridade G, Rossier M, Suissa S, Tardieu M (2007). Parental smoking at home and the risk of childhood onset multiple sclerosis in children. Brain 130: 2589-95

Mikaeloff Y, Moreau T, Debouverie M, Pelletier J, Lebrun C, Gout O, Pedespan JM, Van Hulle C, Vermersch P, Ponsot G (2001) Interferon-beta treatment in patients with childhood-onset multiple sclerosis. J Pediatr 139: 443-6.

Mikaeloff Y, Caridade G, Tardieu M, Suissa S; KIDSEP study group of the French Neuropediatric Society (2008). Effectiveness of early beta interferon on the first attack after confirmed multiple sclerosis: A comparative cohort study. Eur J Paediatr Neurol 12: 205-9.

Mikaeloff Y, Caridade G, Assi S, Suissa S, Tardieu M (2006). Prognostic factors for early severity in a childhood multiple sclerosis cohort. Pediatrics 118: 1133-9.

Miller HG, Stanton JB, Gibbons JL (1956). Parainfectious encephalomyelitis and related syndromes. QJM 25: 427-505

Miller DH, Barkhof F, Frank JA, Parker GJ, Thompson AJ (2002). Measurement of atrophy in multiple sclerosis: pathological basis, methodological aspects and clinical relevance. Brain 125: 1676-1695

Morrow SA, Stoian CA, Dmitrovic J, Chan SC, Metz LM (2004). The bioavailability of IV methylprednisolone and oral prednisone in multiple sclerosis. Neurology 63: 107980.

Moscovich DG, Singh MB, Eva FJ, Puri BK (1995). Acute disseminated encephalomyelitis presenting as an acute psychotic state. J Nerv Ment Dis 183: 116-117

Morissey SP, Miller DH, Kendall BE, Kingsley DPE,Kelly MA, Francis DA, Macmanus DG, Mcdonald WI. The significance of brain magnetic resonance imaging abnormalities at presentation with clinically isolated syndromes suggestive of multiple sclerosis. Brain (1993) 116 (1): 135-146

Murray TJ (2005). Multiple Sclerosis: The History of a Disease, Demos Medical Publishing, ISBN 1-888799-80-3, New York

Murthy SN, Faden HS, Cohen ME, Bakshi R (2002). Acute disseminated Encephalomyelitis in children. Paediatrics 110: e21

Ness, J.M., Chabas, D., Sadovnick, A. Pohl D, Banwell B, Weinstock-Guttman B; International Pediatric MS Study Group (2007). Clinical features of children and adolescents with mulptiple sclerosis. Neurology 68: S37-45.

O'Riordan JI, Gallagher HL, Thompson AJ, Howard RS, Kingsley DP, Thompson EJ, McDonald WI, Miller DH (1996). Clinical, CSF, and MRI findings in Devic's neuromyelitis optica. J Neurol Neurosurg Psychiatry 60(4): 382-7.

Okuda DT, Mowry EM, Beheshtian A, Waubant E, Baranzini SE, Goodin DS, Hauser SL, Pelletier D (2009). Incidental MRI anomalies suggestive of multiple sclerosis: the radiologically isolated syndrome. Neurology 72(9): 800-805 
Pearce JM (2005). "Neuromyelitis optica" (abstract). Spinal Cord 43 (11): 631-634.

Pidcock FS, Krishnan C, Crawford TO, Salorio CF, Trovato M, Kerr DA (2007). Acute transverse myelitis in childhood: center-based analysis of 47 cases. Neurology 68: 1474-1480.

Pittock SJ, Lucchinetti CF (2006). Inflammatory transverse myelitis: evolving concepts. Curr Opin Neurol. 19: 362-368

Pittock SJ, Wingerchuk DM, Krecke K, Lennon VA, Lucchinetti CF, Weinshenker BG (2005). Brain abnormalities in patients with neuromyelitis optica (NMO). Neurology 2005;64: A39.

Pohl D, Rostasy K, Treiber-Held S Brockmann K, Gärtner J, Hanefeld F (2006). Pediatric multiple sclerosis: detection of clinically silent lesions by multimodal evoked potentials. J Pediatr 149: 125-127.

Poser S, Luer W, Bruhn H, Frahm J, Briick Y, Felgenhauer K (1992). Classification and Noninvasive diagnosis. Acta Neurologica Scand. 86: 579-585

Pradhan S, Gupta RK, Ghosh D (1997). Parainfectious myelitis: three distinct clinicoimagiological patterns with prognostic implications. Acta Neurol Scand 5: 241-7.

Pretorius ML, Loock DB, Ravenscroft A, Schoeman JF (1998). Demyelinating Disease of the Schilder Type in 3 young SA children: dramatic response to Steroids. J Child. Neurol 13: 197- 201

Pugliatti M, Riise T, Sotgiu MA, Satta WM, Sotgiu S, Pirastru MI, Rosati G (2006). Evidence of early childhood in the susceptibility period in multiple sclerosis: space-time cluster analysis in a Sardinian population. Am J Epidemiol 164. 326-33

Ramagopalan SV, Maugeri NJ, Handunnetthi L, Lincoln MR, Orton S, Dyment DA, DeLuca G, Herrera BM, Chao MJ, Sadovnick DA, Ebers GC, Knight JC (2009). Expression of the Multiple Sclerosis-Associated MHC Class II Allele HLA-DRB1*1501 Is Regulated by Vitamin D. PLoS Genet 5(2): e1000369

Renoux C, Vukusic S, Mikaeloff Y, Edan G, Clanet M, for the Adult Neurology Departments KIDMUS Study Group (2007). Natural History of Multiple Sclerosis with Childhood Onset. NEJM 356 (5): 2603-2613

Ruggieri M, Iannetti P, Polizzi A, Pavone L, Grimaldi LM (2004). Multiple sclerosis in children under 10 years of age. Neurol Sci 25 (4): S326-S335.

Sadovnick AD, Duquette, P, Herrera B, Yee IM, Ebers GC (2007). A timing-of-birth effect on multiple sclerosis clinical phenotype. Neurology 69. 60-2.

Saito H, Endo M, Takase S, Itahara K (1998). Acute disseminated encephalomyelitis after influenza vaccination. Arch Neurol 37: 564-6

Schwaz S, Mohar A, Knauth M (2001). Acute disseminated encephalomyelitis: a follow-up study of 40 patients. Neurology 56: 1383-1318

Sebire G, Hollenberg H, Meyer L, Huault G, Landrieu P, Tardieu M (1997). High dose methylprednisolone in severe acute transverse myelopathy. Arch Dis Child 76: 167-8.

Shaw CM, Alvord EC (1987). Multiple sclerosis beginning in infancy. Journal of Child Neurology 2: 252-256

Sheldon JJ, Siddharthan R, Tobias J, Sherenata WA, Soila K, Viamonte M (1985). MR imaging of multiple sclerosis: Comparison with clinical and CT examinations in 74 patients. Am J Roentgenol 145 (5): 957-64. 
Simone IL, Carrara D, Tortorella C, Liguori M, Lepore V, Pellegrini F, Bellacosa A, Ceccarelli A, Pavone I, Livrea (2002). Course and prognosis in early-onset MS: comparison with adult-onset forms. Neurology 24; 59(12): 1922-8.

Sindern E, Haas J, Stark E, Wurster U (1992). Early onset MS under the age of 16: clinical and paraclinical features. Acta Neurol Scand 86: 280-4.

Singh S, Prabhakar S, Korah IP, Warade SS, Alexander M (2002). Acute disseminated encephalomyelitis and multiple sclerosis: Magnetic resonance imaging differentiation. Australas Radiol 44: 404-411

Smith AB (2009). Imaging of Demyelinating diseases, In: Radiological Pathology, $8^{\text {th }}$ edition, Craig DW, Chung EM, Galvin JR, Glassman LM, Lewis RB, Murphey MD, Smith AB (eds), pp1103-9, American Registry of Pathology,ISBN 1-933477-28-8, Washington DC

Stone MJ, Hawkins CP (2007). A medical overview of encephalitis. Neuropsychol Rehabil 17 (4-5): 429-49

Straub J, Chofflon M, Delavelle J (1997). Early high dose intravenous methylprednisolone in acute disseminated encephalomyelitis: A successful recovery. Neurology 49: 1145-7.

Straussberg R, Schonfeld T, Weitz R, Karmazyn B, Harel L (2001). Improvement of atypical acute disseminated encephalomyelitis with steroids and intravenous immunoglobulins. Pediatr. Neurol. 24 (2): 139-43

Stricker RB, Miller RG, Kiprov DD (1992). Role of plasmapharesis in acute disseminated (postinfectious) encephalomyelitis. J Clin Apheresis 7: 173-9.

Tenembaum S, Chitnis Tanuja MD, Ness Jayne, Hahn Jin S for the International Pediatric MS Study Group (2007). Acute Disseminated Encephalomyelitis. Neurology 68(16): s23-s26

Tenembaum S, Chamoles N, Fejerman N (2002). Acute disseminated encephalomyelitis: a long-term follow-up study of 84 pediatric patients. Neurology 59: 1224-1231.

Tenembaum SN, Segura MJ (2006). Interferon beta-1a treatment in childhood and juvenileonset multiple sclerosis. Neurology 67: 511-13

Tintore M, Rovira A, Arrambide G, Mitjana R, Río J, Auger C, Nos C, Edo MC, Castillo J, Horga A, Perez-Miralles F, Huerga E, Comabella M, Sastre-Garriga J, Montalban X (2010). Brainstem lesions in clinically isolated syndromes. Neurology 75 (21): 19331938

Tintoré M, Rovira A, Martínez MJ, Rio J, Díaz-Villoslada P, Brieva L, Borrás C, Grivé E, Capellades J, Montalban X (2000). Isolated demyelinating syndromes: comparison of different MR imaging criteria to predict conversion to clinically definite multiple sclerosis. AJNR Am J Neuroradiol 21: 702-706.

Transverse Myelitis Consortium Working Group (2002). Proposed diagnostic criteria and nosology of acute transverse myelitis. Neurology 59: 499-505

Tselis AC, Lisak RP (1998). Acute disseminated encephalomyelitis. In: Clinical neuroimmunology. Antel J, Birnbaum G, Hartung HP, eds. pp116-47, Blackwell Science,, Boston

Van der Knaap MS, van der Grond J, Luyten PR, den Hollander JA, Nauta JJP, Valk J (1992). $1 \mathrm{H}$ and $31 \mathrm{P}$ magnetic resonance spectroscopy of the brain in degenerative cerebral disorders. Ann Neurol 31: $202 \pm 211$ 
Wingerchuk DM, Lennon VA, Pittock SJ, Lucchinetti CF, Weinshenker BG (2006).Revised diagnostic criteria for neuromyelitis optica. Neurology 66 (10): 1485-1489.

Wingerchuk DM, Weinshenker BG (2005). Neuromyelitis Optica. Curr Treat Options Neurol 7(3): 173-82 


\title{
Landau Kleffner Syndrome: Neuroradiology Aspect
}

\author{
José Guevara Campos ${ }^{1}$ and Lucía González Guevara ${ }^{2}$ \\ ${ }^{1 P e d i a t r i c ~ S e r v i c e ~ o f ~ t h e ~ H o s p i t a l ~ " F e l i p e ~ G u e v a r a ~ R o j a s ", ~}$ \\ Universidad de Oriente, El Tigre, \\ ${ }^{2}$ Epilepsy and Electroencephalography Unit, El Tigre \\ Venezuela
}

\section{Introduction}

\subsection{Magnetoencephalography}

The first recording of electromagnetic fields in the brain, or magnetoencephalography (MEG), were performed in 1968 at the Massachussets Institute of Techonology in Boston, by the David Cohen team, exploring alpha rhythm with the aid of a single sensor machine (Cohen,1968). In 1972 Cohen again studied alpha rhythm in normal subjects, and response to hyperventilation in a patient with partial complex seizures (Cohen, 1972). Until the mideighties, MEG equipment had a single sensor that in practice meant, in addition to spatial limitation of the study, that is was necessary to prolong the studies excessively until it was possible to gather the required data.

Magnetoencefalography, with up to 148 channels, has been more useful in dipole localization and evaluating transcallosal spread than traditional EEG, given the limited channels in the 10-20 system. At present, over hundred MEG installations worldwide contribute to our knowledge about the function and development of the human brain (Pateau, 2002). Most MEG studies have been conducted with adult subjects, but some MEG data already exist on children. Pediatric MEG studies have mainly focused on epilepsy surgery, on the Landau-Kleffner syndrome and related disorders, on sensory cortex properties in progressive myoclonus epilepsies, and on dyslexia.

MEG is a noninvasive technique that measure the magnetic fields generated by intracellular current flow in the dendrites of the pyramidal neurons of the brain cortex. The fluctuations of the magnetic field produced by this flow of current can be recorded from the outside of scalp and can subsequently be used to locate the cortical generators of that activity with a temporal resolution measured in millisecond. These characteristic made MEG an invalidly useful tool to study the brain centers of cognitive functions. The way, MEG had served to show that there is a neuronal dynamic neuronal involved in the language process (Pulvermuller,et al.,2003;Wilson,et al.,2005;Pammer,et al.,2004) and that, measuring the magnetic fields derived from that activity helps to locate the sources of electrical activity electric in the human brain in a noninvasive manner(Ray \& Bowyer,2010).

Despite being a relatively new technique, MEG is fast becoming an essential tool for clinical physicians, including neurophysiologist, to give an accurate diagnosis Although the main applications of the technique are found in the area of presurgical evaluation, epilepsy and 
brain tumors (Frye,et al.,2009), especially in the functional localization of epileptic focus, another condition in for which it may be useful include autism (Roberts,et al.,2008), stroke vascular, (Tecchio,et al.,2007) schizophrenia,(Rutter,et al.,2009) Parkinson's disease (Stoffers,et al.,2008) and language disorders.

The integration of MEG with other functional techniques such as PET (positron emission tomography, SPECT (single photon emission computed tomography) and fMRI (functional magnetic resonance) and MRS (magnetic resonance spectroscopy) has been an important contribution to increasing our knowledge of the biologic substrates of the various neurocognitive process and shows the importance of certain areas determine in behaviors. However, these techniques fail to provide information on the functional organization paths of the neuronal networks involved in cognition (Del Rio,et al., 2005).

The capacity of MEG to define the epileptogenic area and its propagation paths has been confirmed by validating the data obtained by using that technique with those obtained by means of electroccorticography or recording made with intracranial electrodes (Del Rio,et al.,2005).

Once of the aspects in which MEG can more quickly define its contributions are without and doubt its application to presurgical study of patients with epilepsy resisted to medical treatment (Shibasaki,et al., 2007).

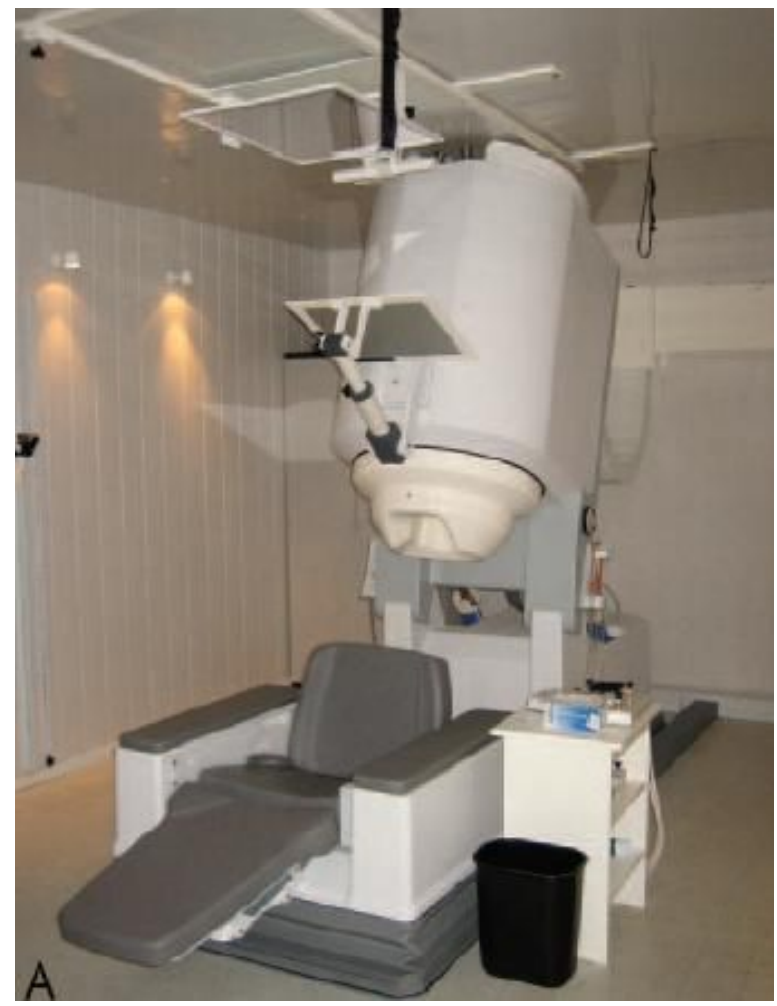

Fig. 1. A, Biomagnetometer in a electrically shielded room. Patients can be comfortably studied in a seated or supine position. 
MEG's special sensitivity towards the currents discharges in the cerebral sulci and fissures have fostered the study of patients with Landau-Kleffner syndrome, for whom an epileptogenic area is proposed in the persylvian region. In these patients, MEG has had an impotant role for the selection of patients who are candidates to multiple subpial transection treatment (Pateau,et al.,1999).

The contribution of MEG to epilepsy surgery does not simple lie in more accurate or exact definition of the epileptogenic area alone, but, in addition, MEG contributes non invasive alternatives to locate eloquent cortex area that must be preserved during a surgical procedure (Sibasaki,et al., 2007).

\subsection{Basic principles of MEG}

A moving electric charge is always associated with an electric field and concomitant magnetic field surrounding the axis movement fig 2a. Electroencephalogram (EEG) and MEG signals are belied to reflect synchronous postsynatic currents in thousands of parallel apical dendrites. Despite being ultimately due to the same primary currents, EEG and MEG signals differ at some important points (Paetau, 2002). First, only tangential currents, parallel to the head surface, give rise to an extracranial magnetic field. Because the apical dendrites typically run perpendicular to the cortex surface, MEG signal mainly arise in fissure walls fig2b. The EEG signals, on the other hand, are dominated by radial currents, while the tangential ones may require signal averaging to be detected. The complementary sensitivity to current warrants combined use of EEG and MEG whenever possible. Second, in homogeneous tissue conductivity of the human head tends to spread out the signal, but does not alter the magnetic fields. Therefore, tumors, cysts, calcified lesions and skull defects cause less distortion on MEG than EEG signals (Vander,et al., 1998). Third, signal attenuation in EEG is caused by poorly conducting tissue, while the magnetic field fades off proportionally to the second power of the distance from the source. Infant and persons with small heads should preferably be studies with systems composed of two part-head devices adjustable according to the head size or with specially designed baby devices. Fourth, different practical problems hamper data acquisition. MEG sensors are in a rigid helmet and the head as to be kept immobile with respect to the helmet. Long Term recordings or recordings of major motor seizures area far not possible with MEG, but continuous monitoring of the head position may offer relief to some of the movement problems. Finally, MEG and EEG have partly differing artifact profiles: MEG is less sensitive to muscle artifacts than EEG. On the other hand, magnetic materials moving with respiration (Traces from craniotomy drills, some shunt materials, tooth braces, cochlear implants, etc) may cause serious artifacts or even destroy the MEG data.

\subsection{MEG in acquired epileptiform regression syndromes}

Acquired epileptic regression syndromes are disabling childhood disorder, were a previously healthy child deteriorates over a week to months. The children may or may not show overt seizures, but their EEG usually displays almost continuous epileptiform spike and wave activity, especially during sleep. In acquired epileptic aphasia or the LandauKleffner syndrome (Landau, 1957), the regression affects receptive language and /or auditory perception. Epileptiform spike-and wave discharges appear during the first few weeks, and have been proposed to causally related to cognitive disability. Some LKS children develop giant auditory N100m response with similar auditory cortex sources and 


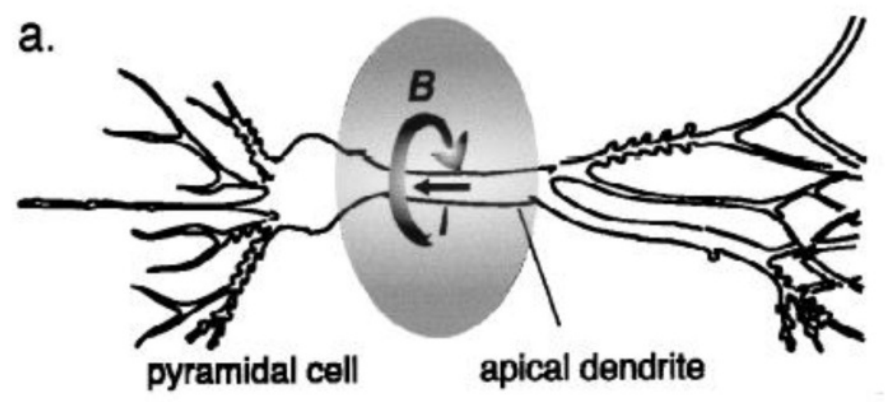

b. C.

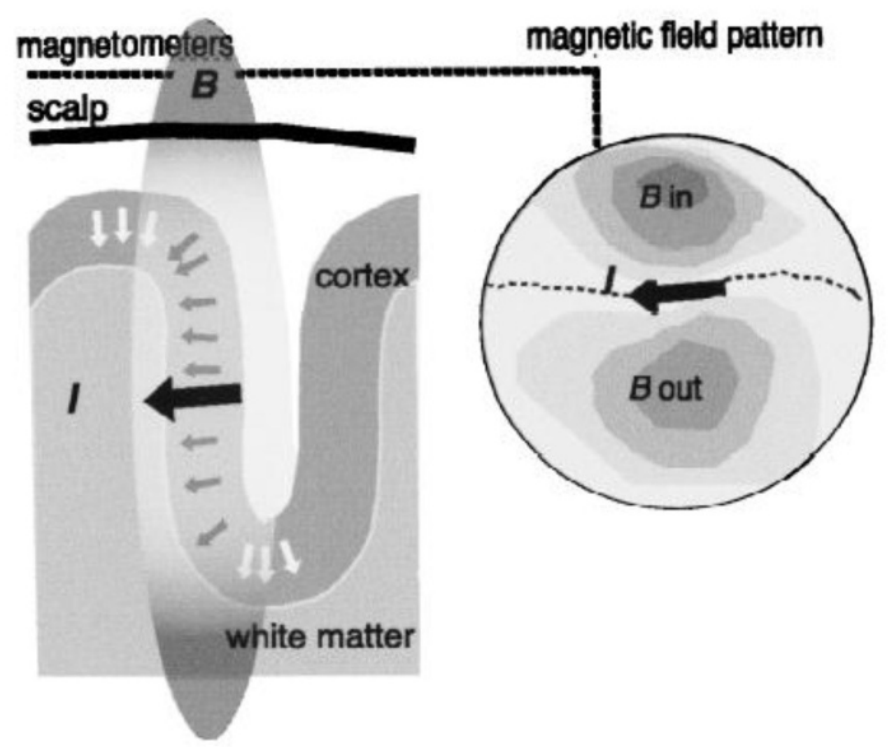

Fig. 2. Physical basis of MEG signals. (a) The intracellular current I in the apical dendrite of a pyramidal cell is associated with a surrounding magnetic field B. (b) In the brain, pyramidal cells typically are perpendicular to cortex surface, and may be radial (white arrows), oblique or tangential (dark arrows) to the scalp. Only the tangential currents and the tangential component of oblique currents contribute to the extracranial magnetic field, which can be detected by sensitive SQUID magnetometers. (c) Dipolar magnetic field pattern viewed above. B in indicates the magnetic flux into the head and B out the flux out from the head. An equivalent current dipole (ECD; the thick black arrow) represents the concerted action lines of all fissural dipoles. Its orientation is parallel to the isofield lines and it is located underneath the steepest gradient halfway between the in- and out-flowing flux. Its depth determines the distance between the two extremes, and its strength is proportional to the number of active pyramidal cells. 
reactivity as the normal $\mathrm{N} 100 \mathrm{~m}$, but with morphology and amplitude identical lo the patient's epileptic spikes (Pateau, 2002). LKS Patient's verbal auditory agnosia can, therefore, be understood as local epilepsy of the auditory cortex, activated by sounds. MEG alone or combined with EEG has proved useful in identifying the sources of epileptic activity in LK, as well as in childhood epileptic autistic regression disorders (Sobel et al.,2000;Muñoz Yunta et al.,2008).

\section{Landau-Kleffner syndrome}

In 1957 Landau and Kleffner described six children with acquired aphasia syndrome and epileptic seizures with encephalographic alterations (Landau, 1957). The syndrome had two basic symptoms: on the one hand an acute or sub-acute acquired aphasia in previously normal children, and on the other, a paroxysmal encephalographic plot with spikes and waves more frequently located in region temporal region that may reach an epileptic stage during the slow stage. Convulsive seizures, if shown come before or follow the beginning of the slow speech impairment but these epileptic discharges in the electroencephalogram are invariably present (Guevara Campos \& González Guevara, 2007). It may be accompanied by behavior or demeanor disorders, such as hyperactivity, expression of aggressive, rebelliousness and autistc features.

The etiology of the LKS is unknown. Although encephalitis has been proposed as an etiology, some of empirical evidence has raised doubts. For example, the course of LKS is very different from that of Rasmussen chronic encephalitis and temporal lobotomy histology in two cases of LKS was not consistent with encephalitis (Rasmussen \& Mc Cann 1968; Coke et al., 1988). Based on the results of conventional angiography in some patients with LKS, Pascual-Castroviejo (Castroviejo et al., 1992) proposed vasculitis as an etiology. These investigators treated patients with calcium blockers with a favorable response. Others have considered whether an underlying autoimmune mechanism may contribute to the pathogenesis of LKS give the fact autoimmune processes may be involved in the pathogenesis of child development disorders and epilepsy (Nesimalova et al., 1992). This idea is supported by Connolly (Connolly et al., 2006). Who reported an increase in IgM autoantibodies to endothelial cells and IgG autoantibodies to myelin basic protein as compared to healthy controls. A small number of brain lesions have been reported in association with the LKS, including neurocysticercosis (Bhatia et al., 1994), toxoplasmosis (Michalowiez et al., 1989), temporal lobe astrocytoma (Solomon et al., 1993), and temporal lobe glioma (Solomon et al., 1993). The increased risk of LKS in monozygoyic twins and siblings suggests a genetic etiology (Feekery et al., 1993). Thus, it is very likely that the etiology of the LKS is variable with several etiologies. It would be useful to classify LKS as cryptogenic or symptomatic. The cause of epilepsy and continuous aphasia still remains a mystery (Deonna, 1991).

\section{Clinical manifestations}

\subsection{Aphasia}

Single photon emission computed tomography imaging demonstrated hypometabolism in 22 of his 25 patients (O’Regan et al., 1998), suggested that aphasia occurs as a manifestation of a hypometabolic encephalopathy secondary to a local postictal state (O'Regan et al., 1998). Positron emission tomography has shown dynamic changes in the metabolism of glucose in 
the temporal lobe during episopdes of aphasia (Luat, et al., 2006). Temporary changes in the positron emission tomography images suggest that the metabolism of glucose in LKS cannot be attributed only to the epileptiform interictal activity (Luat, et al., 2006).

MEG offers the possibility to study the organization of the neuronal networks involved in language processes and shows with high precision, and in a noinvasive fashion, the spatial and temporal patterns of brain activity related to this cognitive function in order to determine the neurocognitive reorganization of language in several diseases such as aphasia or epilepsy, and the relationship of this functional reorganization to behavioral language problems (Ortíz et al., 2009).

Although aphasia is present in all patients, it constitutes the first manifestations in only half. Its onset may be abrupt or progressive over several days or weeks (Guevara Campos, et al., 1989). Typically, the aphasia is receptive; the child begins to have difficulty-understanding language to the point that deafness may suspected. The capacity for oral expression gets impaired quickly; mutism is frequent. Non-verbal skills are usually preserved, thereby allowing sign language to be used for some degree o communication. Writing is preserved in older children (Deonna, 1991). The integrity of the thalamic auditoy pathways is confirmed by normal brainstem potentials. Alterations the evoked cortical latencies support posterior temporal region dysfunction (Zouari \& Choyakh, 1997).

Expressive aphasia was present in two of the original Landau -Kleffner patients (Landau \& Kleffner, 1957), and 6 of the 77 cases studies by Dugas, (Dugas, et al., 1992). A detailed study of aphasia shows that it is possible to find virtually every type, including semantic pragmatic disorders (Paquier, et al., 1992).

The course of the aphasia found in the LKS is one of its most disconcerting characteristic. A relationship between one age of onset and the long term results has been reported the younger the child the worse the prognosis for the recuperation of speech. The effect are devastating in young child that have no yet developed linguistic abilities since the cortical mechanisms to process speech cannot develop. In older children, the effects are less serious because they have already learned language (Bishop, 1985). In general total recovery is very difficult, and variable difficulties persist in oral communication in most case. Non -verbal communication can be unaffected, but there is the possibility that it may not be even when it is present (Baynes,et al.,1998).

\subsection{Epilepsy}

Most researchers support the hypothesis than paroxysmal activity produces a functional disconnection of the cortical areas related to speech. The exact origin of epileptic activity has been shown through brain mapping techniques. Paroxysms have been shown to be located in the depths of Sylvian fissure through dipole modeling (Nakano,et al.,1989). This is supported by results of positron emission tomography, single photron emission computed tomography and magnetoencephalography studies (Baumgartner \& Pataraira, 2006).

MEG and EEG record synchronous postsynaptic currents in thousands of cortical pyramidal cell apical dendrites, with a temporal resolution at the level of the millisecond. Although EEG reflects cortical currents of all orientations, MEG is mainly sensitive to fissure currents and, therefore, provides an optimal method to identify the fissure generators of LKS spikes. During a single spike-wave complex, spike propagation from a fissure wall to the adjoining convexity can take between 10 and $30 \mathrm{~ms}$, and propagation time between left and right perisylvian areas are similar. Both MEG and EEG can differentiate between primary 
(independent) spike foci and secondary spikes (Paetau, 2009) MEG and EEG are necessary for the spatial and temporal understanding of the perisylvian region and epileptic networks in Landau -Kleffner syndrome.

The 4 potential roles for MEG in the localization of the ictal onset zones (IOZ) and functional cortex: 1) MEG confirmations of localization based on traditional noninvasive methods, allowing epilepsy surgery to proceed without long term intracaneal EEG monitoring; 2) MEG demonstration of bilateral, multifocal, or diffuse ictal onset, indicating an unfavorable candidate for epilepsy surgery; 3) MEG locations adjacent to or distant from that of traditional methods or focally, when traditional methods suggest multifocal activity, potentially altering clinical decision making regarding epilepsy surgery ; and 4)MEG localization of eloquent cortex to guide surgical trajectory. These data will serve to refine prospective studies on the optimal use of MEG in the localization of the IOZ and functional cortex in children with treatment-resistant epilepsy (Schawartz,et al.,2008).

Epileptic seizures constitute the first manifestation in approximately half the cases of LKS. Beaumanior (Beaumanior, 1992) found that nightly partial seizures were most common, followed by generalized tonic-clonic, atypical absences and, rarely, myoclonic absences. Extremely rare cases have been reported of partial complex seizure patients with automatism (Deonna, 1991; Guevara Campos et al., 1989). Clinical seizures only appear in around $70 \%$ of the patients. A third of clinical seizure patients have isolated single seizures. Status epileptic occurs in some (Hirsch, et al.,1990).Seizures are not very frequent in patients between the ages of 5 to 10 years of age (Pablo,et al.,2002) After the age of 10 years old, only one fifth of the patients experiences sporadic seizures. Seizures rarely persist in older adolescence. (Guevara Campos, et al., 1989)

In most cases, monotherapy with anti-epileptic is effective to control the seizure but not the aphasia (Deonna, 1991). In most the patients, no close relationship is established between the frequency of the seizures and the degree aphasia, which can be serious even in absence of seizures (Guevara Campos \&González Guevara, 2007). Seizures almost always disappear in adulthood, although rare cases have been reported in the literature or refractory epilepsy that persists until adulthood (Beaumanoir, 1992).

\subsection{Paroxysmal alterations of the EEG}

The behavioral and cognitive alterations characteristic of patients with LKS are believed to be secondary to epilepsy rather than structural damage to the central nervous system. The affirmation is based on the fact that frequent and intense epileptic discharges are focally located in language areas important for speech and the fact recovery, either spontaneous or induced by treatment, is always associated with the disappearance of EEG paroxysmal activity (Guevara Campos \& González Guevara,2007).

In the theory of the pathogenic mechanisms that lead to the LKS syndrome there is general consensus on the epileptiform discharges normally active in the non REM sleep stage and the regression of speech. The spikes on the etectroencephalograms are usually temporal, central-temporal or bilateral parietal-occipital and synchronous. Experiences with brain injuries have shown that a healthy hemisphere is sufficient to acquire normal speech. Therefore, the regression of speech in LKS suggests a bilateral dysfunction of the auditory cortex and related speech cortex, both hemisphere having independent epileptic injuries or a unilateral injury that induces the functions paralysis of the healthy hemisphere due to bombarding of epileptic discharges through commissural injuries (Paetau, 2009). 
Magnetoencephalography detect extra-cranial magnetic fields. Spike generated in the perisylvian convexities are detected only with the EEG, while the spikes generated by the intrasylvian region are selectively seen by MEG. The spike generated by EEG and the MEG are detected by using equivalent dipoles that represent the cortical activity of the pyramidal neurons in the cortex. MEG localizes 1-2-cm generated cortical spikes with spatial precision and a one millisecond resolution.

MEG studies that, in approximately $80 \%$ of LKS patients, epileptic discharges are bilateral and are generated in the auditory and speech cortex, and are related. Approximately $20 \%$ of children with LKS have a unilateral pacemaker in the perisylvian region that triggers the secondary bilateral synchrony of the spikes. This $20 \%$ could recover the speck ability after performing a supial intracortical transaction in the pacemaker (Paetau, 2009).

EEG abnormalities are present in all patients. The background rhythms are usually normal, but theta slowing is occasionally present in the same regions as the spike. Spike paroxysms or spike and wave complexes can be unilateral or bilateral and may be focal, multifocal or diffuse (Beaumanoir, 1992). Discharges are bitemporal in 50\% of the patients and in the parietal-occipital regions in $30 \%$ of the patients. Discharges are usually active by sleep. Paroxysms are frequently similar to those found in Benign Rolandic Epilepsy. There is a clear activation of the epileptiform activity in Rolandic epilepsy during sleep that can transform into continuous spike and wave. In such patients the sleep index never reaches 85\% (Deonna, 1991). Most of the foci are localized to the temporal or temporal central region and are more commonly found in the left hemisphere.

Bilateral foci are frequent (Beaumanoir, 1992); they tend increase by more than $85 \%$ during sleep and can transform into continuous spike and wave sleep (CSWS) or electrical status epilepticus during slow sleep (ESES). The term CSWS is used synonymously with that ESS. The discharges in ESES are located in the frontal or frontocentral region, while they tend to located in the temporal or parietal areas in LKS. The seizures seen in patients with ESES are similar to the ones of LKS, but drop, myoclonic and unilateral clonic seizures may be more common. The nature of the cognitive deterioration is more diffuse in ESES than in LKS. The correlation between the EEG paroxysmal activity and the neuropsychological deficits supports the opinion that these are a direct result of the epileptic process, thus permitting the consideration that both LKS and ESES syndrome belong to the group epileptic encephalopathies (Aicardi, 1999).

Hyperventilation and photostimulation activation mancuvers are rare positive. The disappearance of the spike and wave complexes in sleep fortells the improvement of aphasia and it may be a good index of the efficacy of treatment (Li et al., 1996). However, neither full nor partial recovery of aphasia is never noted before CSWS disappears (Aicardi, 1999).

The EEG constitutes the basic tool to follow up on anti-epileptic therapy aimed at suppressing the marked paroxysmal alterations shown in the EEG, most specially the activity of the CSWS (Li et al., 1996).

\subsection{Behavioral changes}

Behavioral changes are extremely common in patients with LKS (Dugas,et al.,1992). These include hyperkinesias, outburst of wrath, rebelliousness and aggressive expressions. Some of these behaviors may be a related the frustration associated to speech difficulties. Autistic features and autism with all its components are very frequent and in some cases, they can even control the clinical manifestations (Nesimalova, et al., 1992). Hyperkinesia is usually 
described as psychomotor disorder; hyperkinesia and slight verbal auditory agnosia occurs in the early stages of LKS; it can be mistaken for cases of attention deficit with hyperkinesia. Personality disorders, aggressiveness and depression are also evident (Guevara Campos \& González Guevara, 2007). Neville (Neville,et al.,1998) have pointed out the fact that many children with LKS show various degrees of motor disorder. Thirteen out a total of 30 children with LKS showed moderate motor disorders expressed as clumsiness, slowness and imprecise movements with six showing serious dysfunctions (Neville,et al.,1998).

\section{Diagnostic studies}

In general diagnostic research has not clear defined the etiology of LKS. In a child with language regression or apparent deafness, it is mandatory to perform an EEG that includes a recording during extended sleep (Aicardi, 1999). Neither the evoked potentials nor auditory test have contributed useful data for diagnosis (Zouari \& Choyakh, 1997; Pablo et al., 2002). The analysis of cerebral fluid is almost always normal, although case in which a slight elevation of total protein has been reported (Perniola,et al.,1993). Axial computer tomography and magnetic imaging are always normal, although non-specific finding, such as white matter changes or structural damage, have been reported (Perniola,et al.,1993). In a small number of patients single photon emission computed tomography and positron emission tomography have revealed perfusion and glucose metabolism abnormalities in the temporal lobes (Luat,et al.,2006;Guerreiro,et al.,1996;Da Silva et al.,1997).There have been case report about the use of MEG in patients with LKS (Sobel,et al.,2000). Magnetoencephalography can provide excellent pre-surgery localization of epileptiform activity as well as functional language mapping if surgical treatment, such as multiple subpial transection, the role MEG in evaluating patients with epilepsy continues to evoive. MEG is sensitive to epileptic activity originating from depths of sulci, such as the fissure, because it reveals magnetic fields generated by transmembrane synaptic currents oriented in a plane tangential to the skull surface (Seppo,et al.,2010). The precise locations afforded by MEG compared with EEG can sometimes obviate the need for invasive video EEG recording as well as direct the placement of intraoperative electrodes when surgery is being considered for patients with medically intractable epilepsy, Specially, for patients with LKS, MEG is useful in confirming the diagnosis and may prove useful in preoperative localitation if subpial intracortical trasction becomes widely accepted for the treatment of intractable cases. Magnetoencephalography has added valuable data in presurgical planning in determining the location of the dominant focus along and within the sylvian fissure during secondary discharges see in CSWS (Smith, 2004).

\section{Conclusions}

MEG and EEG are fundamentally different functional magnetic resonance imaging (fMRI), positron emission tomography (PET) or other anatomical imaging methods, where the spatial resolution is based on voxel size. The most important advantage of MEG and EEG over any present functional imaging modality is their submillisecond temporal resolution. At present, MEG provides the most efficient single tool for real time tracking of distributed brain activities during a number of cognitive tasks or epileptic discharges. The MEG finding have impacted patient management in nearly every case and have been universally accepted by pediatric neurologist and pediatric neurosurgeon as beneficial for their patients. 
Specially, these benefits include substantiated decisions not to offer surgical resection, decisions to move ahead with intracranial grid placements; and lesion detection in the setting of nonlesional structural imaging.

\section{References}

Aicardi J, (1999) Landau-Kleffner syndrome. Rev Neurol 29:380-385.

Baumgartner C, Pataraira E. (2006) Revisiting the role of magnetoencephalography in epilepsy. Curr Opin Neurol 19:181-186.

Baynes K, Kegel J.A, Brentari D, Kussmaul C, Poizner H (1998) chronic auditory agnosia following Landau-Kleffner syndrome: a 23 year outcome study. Brain Lang 63:381425.

Beaumanoir A (1992) The Landau-Kleffner syndrome, in: Epileptic Syndromes in Infancy, Childhood and Adolescence, Roger J, Bureau M, Dravet C, Dreifuss FE, Perret A, Wolf P, 213-243. John Libbey, London.

Bhatia M.S, Shome S, Chadda P.K and Saurabh II (1994) Landau-Kleffner syndrome in cerebral cysticercosis. Indian Pediatr 31: 584-587.

Bishop D.V, (1985) Age of onset and outcome in "acquired aphasic with convulsive disorders" (Landau-Kleffner syndrome). Dev Med Child Neurol 27:705-712.

Castroviejo-Pascal I, López Martin V, Martinez Bermejo A and Pérez-Higueras A (1992) Is cerebral arteritis the cause of Landau-Kleffner syndrome? Four cases in childhood with angiographic study. Camn J Neurol Sci 19: 46-52.

Cohen D (1968) Magnetoencephalography: evidence of magnetic fields produced by alpha rhthm current. Science 161: 784-786.

Cohen D (1972) Magnetoencephalography: detection of the brain's electrical activity with a superconducting magntometer. Science 175: 664-666

Coke A.J, Andermann F, Taylor L (1988) The Landau-Kleffner syndrome of acquired aphasia: unusual clinical outcome, surgical experience, and absence of encephalitis, Neurolgy 38:31-38.

Connolly A.M, Chez M, Streif E.M (2006) Brain derived neurotrophic factor and autoantibodies to neural antigens in serum of children with autistic spectrum disorders, Landau-Kleffner syndrome, and epilepsy. Biol Psychiatry 59:354-363.

Da Silva E, Chugani D, Muzik O, Chugani H (1997) Landau-Kleffner syndrome: metabolic abnormalities in temporal lobe are a common feature. J Child Neurol 12:489-495.

Del Rio, Santiuste M, Capilla A, Maestú F, Campo P, Fernández Lucas A, Ortiz T (2005) Bases neurológicas del lenguaje. Aportaciones desde la magnetoencefalografía. Rev Neurol 41: 109-114.

Deonna T.W (1991) Acquired epileptiform aphasia in children (Landau-Kleffner Syndrome), J Clin Neurophysiology 8: 288-298.

Dugas M, Manson M, Le Heuzey M F, Regnier N (1992) Childhood acquired aphasia with epilepsy (Landau-Kleffner syndrome), 12 personal cases. Rev Neurol (Paris) 138:817-823.

Feekery C.J, Parry-Fielder B and Hopkins I.J (1993) Landau-kleffner syndrome six patients including discordant monozygote twins. Pediatr Neurol 9: 49-53.

Frye RE, Rezaie R, Papanicolaou AC (2009) Functional neuroimaging of Language using Magnetoencephalography. Phys Life Rev 6(1):1-13.

Guerreiro M.M, Camargo E, Kato M (1996) Brain single photon emission computed tomography imaging in Landau-Kleffner syndrome. Epilepsia 37:60-67. 
Guevara Campos J, Guevara González L, and Dravet Ch (1989) Sindrome de LandauKleffner.Rev Fund José Ma Vargas 13:14-16.

Guevara Campos J, González de Guevara L (2007) Sindrome de Landau-Kleffner: análisis de 10 Casos en Venezuela, Rev Neurol 44(11):652-656.

Guevara Campos J, González Guevara L (2007) Landau-Kleffner syndrome. J Pediatr Neurol 5:93-99.

Hirsch E, Marescaux C, Maquet P (1990) Landau-Kleffner syndrome: a clinical and EEG study of five cases. Epilepsia 31:756-767.

Landau W and KLeffner F (1957) Syndrome of acquired aphasia with convulsive disorder in children. Neurology 7: 523-530.

Li M, Hao XY, Qing J, Wu R (1996) Correlation between CSWS and aphasia in LandauKleffner syndrome:a study of three cases. Brain Dev 18:197-200.

Luat A.F, Chuagani H.T, Asano E (2006) Episode receptive aphasia in a child with LandauKleffner syndrome: PET correlates. Brain Dev 28: 592-596.

Michalowiez R, Jozwiak S, Szwabwska-Orzeszo E, IgnatowiezL and Ignatowiez R (1989) Landau-Kleffner Wiad Lek 42: 256-259.

Muñoz-Yunta JA, Ortiz T, Palau- Badell M, Martín -Muñoz L, Salvadó-Salvadó B, VallsSantasusana A (2008) Magnetoencephalographic patterm of epileptiform activity in children with early -onset autism spectrum disorders. Clin Neurophysiol 119: 626-34.

Nakano S, Okuno T, Mikawa H. (1989) Landau Kleffner syndrome: EEG topographic studies. Brain Dev 11:43-50.

Nesimalova S, Tauberova A, Doutilik S, Kucera V and Diouha O (1992) A role of autoimmunity in the etiophathogenesis of Landau- Kleffner syndrome? Brain Dev 14: 342-345.

Neville B, Burch V, Cass H (1998) Motor disorder in Landau-Kleffner syndrome.Epilepsia 39:123 A.

O’Regan M.E , Brown J.K, Geodiwin G.M and Clark M (1998) Epileptic aphasia: a consequence of regional hypometabolic encephalopathy? Dev Med Child Neurol 40: 508-516.

Ortiz T, Palau- Baduel M, Salvadó-Salvadó B, Valls-Santasusana A (2009) Estudio de los trastornos del espectro autista $\mathrm{y}$ tratstornos del lenguaje mediante magnetoencefalografía. Aportación científica del Dr. Muñoz Yunta. Rev Neurol 48(2):3-12.

Pablo MJ, Valdizán JR, Carvajal P (2002) Landau-Kleffner syndrome. Rev Neurol 34:262-264.

Paetau R, Granstom ML, Blomstedt G, Jousmaki V, Korkman M, Liukkone E(1999) Magnetoencephalography in presurgical of children with the Landau-kleffner syndrome Epilepsia 40: 326-335.

Paetau Ritva (2002) Magnetoencephalography: in pediatric neuroimaging. Developmental Science 5:3,361-370.

Paetau Ritva, (2009) Magnetoencephalography in Landau-Kleffner syndrome. Epilepsia 50:51-54.

Pammer K, Hansen PC, Kringelbach ML, Holliday I, Barnes G, Hillebrand A (2004) Visual word recognition: the first half second. Neuroimage 22: 1819-25.

Paquier. P.F Van Dongen H.R and Loonen C.B (1992) The Landau Kleffner syndrome acquired aphasia with convulsive disorder Long-term follow-up of six children and review after the recent literature. Arch Neurol 49:354-359. 
Perniola T, Margari L, Buttiglione M, Andreula C, Simone I, Santostasi R (1993) A case of Landau-Kleffner syndrome secondary to inflammatory demyelinating disease. Epilepsia 34:551-556.

Pulvermuller F, Shtyrov Y, IImoniemi R (2003) Spatiotemporal dynamics of neural language processing: an MEG study using minimum -norm current estimates. Neuroimage 20: 1020-1025.

Rasmussen T and Mc Cann W (1968) Clinical studies of patients with focal epilepsy due to chronic encephalitis, Trans Am Neurol 93: 89-94.

Ray A, Bowyer SM (2010) Clinical applications of magnetoencephalography in epilepsy. An Indian Acad Neurol 13(1): 14-22.

Roberts T.P, Schmidt G.L, Egeth M, Blaskey L, Rey M.M, Edgar J.C, Levy S.L (2008) Electrophysiological signatures: magnetoencephalography studies of the neural correlates of language impairment in autism spectrum disorders. Int $J$ Psytchophysiol 68:149-80.

Rutter L, Carver F.W, Hoiroyd T, Nadare S.R, Mitchell-Francis J, Apud J,(2009) Magnetoencephalography gamma power reduction in patients with schizophrenia during resting condition. Hum Brain Mapp 30: 3254-64.

Schawartz E.S, Dlugos D.J, Storm P.B, Dell J, Magee R, Flynn T.P, Zarnow D.M, Zimmerman R.A, Roberts TPL (2008) Magnetoencephalography for Pediatric Epilepsy: How We Do It. Am J Neuroradiol 29:832-837.

Seppo P, Ahlfors Jooman Han, John W. Belliveau, Matti S. Hamalainen (2010) Sensitivity of MEG and EEG to Source Orientation. Brain Topogr 23:227-232.

Shibasaki H, Ikeda A, Nagamine T (2007) Use of magnetoencephalograpy in presurgical evaluation of epilepsy patients. Clin Neurophysiol 118: 1438-48.

Smith M (2004) The utility of Magnetoencephalography in the evaluation of secondary bilateral synchrony: A case report. Epilepsia 45:57-60.

Sobel DF, Aung M, Otsubo H, Smith MC(2000) Magnetoencephalography in children with Landau- Kleffner syndrome and acquired epileptic aphasia. Am J Neuroradiol 21: 301-307.

Solomon G.L, CarsonD, Pavlaskis S, Fraser R and Labar D (1993) Intracranial EEG monitoring in Landau- Kleffner syndrome associated with a left temporal lobe astrocytoma. Epilepsia 34: 557-560.

Stoffers D, Bosboom JL, Deijen JB, Wolters ECh, Stam CJ, Berendse HW (2008) Increased cortico cortical functional connectivity in early-sttage Parkinso's disease: an MEG study. Neuroimage 41:212-22.

Tecchio F, Zappasodi F, Tombini M, Caulo M, Vermieri F, Rossini PM (2007) Interhemispheric asymmetry of primary hand representation and recovery afther stoke: a MEG study. Neuroimage 36: 1057-64.

Vander Broek S, Reinders F, Donderwinkel M ,Peters M (1998) Volume conduction effects in EEG and MEG. Electroeencephalography and Clinical Neurophyology 106: 522-534.

Wilson T.W, Leuthold A.C, Lewis S.M, Georgopoulos A.P, Pardo P.J (2005) The time and space of lexicality : a neuromagnetic view. Exp Brain Res 162: 1-13.

Zouari N, Choyakh F (1997) Early middle latency and late auditory evoked potentials a case of acquired epileptic aphasia (Landau-Kleffner syndrome).Rev Laryngol Otol Rhinol 118:267-270. 


\title{
Neurocristopathies: Role of Glial Cells, Genetic Basis and Relevance of Brain Imaging for Diagnosis
}

\author{
$\mathrm{M}^{\mathrm{a}}$ Carmen Carrascosa Romero ${ }^{1}$ and Carlos de Cabo de la Vega ${ }^{2}$ \\ ${ }^{1}$ Neuropediatrics and ${ }^{2}$ Neuropsychopharmacology Units, Albacete General Hospital \\ Spain
}

\section{Introduction}

The concept of neurocristopathy was introduced by Bolande in 1974 to describe a group of diseases arising from aberrations in the development, migration and differentiation of the embryonic neural crest (NC). This cell lineage differentiates into pigmentary and neural cells and forms part of the autonomous nervous system, nervous enteric plexus, as well as endocrine glands (adrenals, parathyroid gland) and chemoreceptors (carotid and aortic bodies). Neurocristopathies derived from a failure in NC development and range from alterations in intestinal ganglion cells, as seen in Hirschsprung's Disease or in intestinal neuronal dysplasia, to alterations in skin pigmentation (such as neurofibromatosis, Waardenburg-Shah syndrome and piebaldism) (Spritz, 1997). In recent years, fostered by the increasing research on the NC ontogeny (Trainor, 2005), the notion of cristopathies has widened (Bolande, 1997), particularly with the inclusion of craniofacial syndromes of cranial crest mesoectodermal origin, often accompanied by morphological brain abnormalities (Couly \& Aicardi, 1988), as well as the association with other diseases (Martucciello et al., 2005), including chromosomopathies (Down syndrome), embryopathies (fetal alcohol and fetal cocaine syndromes) and tumors of the endocrine system (multiple endocrine neoplasia type IIB).

Based on the accumulating knowledge of the role of NC in development, Jones (1990) proposed a new classification of cristopathies according to the pathological mechanism involved. The first group includes the defects and disorders originally defined as neurocristopathies including pheochromocytoma, neurofibromatosis, and the multiple endocrine adenomatoses. These diseases can be explained as dysplasia of neural crest derivatives. Affected individuals rarely exhibit actual morphological malformations but do carry a risk for impaired growth of crest-derived tissue. The second group corresponds to defects and disorders which derive from migrational abnormalities primarily of cranial NC cells such as frontonasal dysplasia, the DiGeorge sequence, velo-cardio-facial syndrome and Waardenberg syndrome represent true malformations. The genetic origins of some of these syndromes have been identified: microdeletions at the locus 22q11.2 (DiGeorge sequence and velo-cardio-facial syndromes) and mutations in the PAX3, MITF, EDNRB, EDN3 and SOX10 genes (Waardenberg syndrome I-IV types) 
Hirschsprung's disease (HSCR) or aganglionic megacolon is perhaps the best-studied neurocristopathy. HSCR is a congenital defect characterized by complete absence of intramural neuronal ganglion cells in the myenteric (Auerbach's) plexus and the submucosal (Meissner's) plexus from distal portions of the intestinal tract caused by failure in the migration of these cells from the NC. HSCR is a disorder with multifactorial etiology including genetic factors (Kusafuka \& Puri, 1998). Mutations in at least 8 genes have been associated with HSCR, most of the mutations occurring in the RET gene. According to epidemiology studies (Amiel \& Lyonnet, 2001; Luis et al., 2006; Polly \& Coran, 1993), HSCR appears as an isolated trait in $70 \%$ of cases. HSCR is associated with a chromosomal abnormality in $12 \%$ of cases, of which trisomy 21 (Down syndrome) represents $>90 \%$. A recent report raises the incidence of Down syndrome up to a $17.6 \%$ of HSCR patients (Carrascosa-Romero et al., 2007). Association with other congenital multimalformative syndromes and isolated dysmorphic conditions anomalies are found in up to $18 \%$ of HSCR patients. The ones occurring at a frequency above that expected by chance include gastrointestinal malformation, cleft palate, polydactyly, cardiac septal defects, and craniofacial anomalies. HSCR has also been connected with nervous system malformations related to alterations in the development of the anterior segment of the NC: defects in the closure of the neural tube such as anencephalia and myelomeningocele, as well as impairment of neuronal migration and brain dysgenesis (Carrascosa-Romero et al., 2007; Juliá et al., 2003; Shahar \& Shinawi, 2003).

\section{Evidence that glial cell are critical participants in every major aspect of brain development, function, and disease}

Astrocytes are the most abundant cell type in the mammalian brain. Interest in astrocyte function has increased dramatically in recent years because of their newly discovered roles in synapse formation, maturation, efficacy, and plasticity. However, our understanding of astrocyte development has lagged behind that of other brain cell types. We do not know the molecular mechanism by which astrocytes are specified, how they grow to assume their complex morphologies, and how they interact with and sculpt developing neuronal circuits. Recent work has provided a basic understanding of how intrinsic and extrinsic mechanisms govern the production of astrocytes from precursor cells and the generation of astrocyte diversity. Moreover, new studies of astrocyte morphology have revealed that mature astrocytes are extraordinarily complex, interact with many thousands of synapses, and tile with other astrocytes to occupy unique spatial domains in the brain. A major challenge for the field is to understand how astrocytes talk to each other, and to neurons, during development to establish appropriate astrocytic and neuronal network architectures (Freeman, 2010). Astrocytes influence synaptic transmission in many ways. They secrete distinct factors that promote synaptogenesis, neurotransmitter release, and postsynaptic receptors (Barres, 2008); in addition, they release so-called gliotransmitters in response to stimulation and contribute to the calcium waves that correlate with blood flow (Haydon \& Carmignoto, 2006; Volterra A \& Meldolesi, 2005). However, they also participate directly in synaptic transmission through the expression of high-affinity transporters for neurotransmitters.

Emerging evidence indicates that signalling between perisynaptic astrocytes and neurons at the tripartite synapse plays an important role during the critical period when neural circuits 
are formed and refined. Cross-talk between astrocytes and neurons during development mediates synaptogenesis, synapse elimination and structural plasticity through a variety of secreted and contact-dependent signals. Recent live imaging studies reveal a dynamic and cooperative interplay between astrocytes and neurons at synapses that is guided by a variety of molecular cues. A unifying theme from these recent findings is that astrocytes can promote the development and plasticity of synaptic circuits. Insight into the molecular mechanisms by which astrocytes regulate the wiring of the brain during development could lead to new therapeutic strategies to promote repair and rewiring of neural circuits in the mature brain following central nervous system (CNS) injury and neurodegenerative disease (Stevens, 2008).

\section{Neuron-to-glia signalling in the central and enteric nervous system - Implications for neural disease}

Dysfunction of non-neuronal cells such as astrocytes and microglia have been involved in the process of neurodegeneration in the CNS. In fact, they have been proposed as therapeutic targets since their selective survival is capable of slowing down the process of neuronal death in animal models of neurodegenerative disease (Boillee et al., 2006; Yamanaka et al. 2008). In a healthy individual, astrocytes seem to respond to synaptic activity in the CNS in a synapse-specific way and, in turn, they appear to precisely regulate synaptic activity. These processes have been shown to involve the activity and expression of plasma membrane transporters (Bergles et al., 1999). The excitatory amino acid transporters (EAATs) control spillover of glutamate from one synapse to another. Besides this, they also prevent accumulation of glutamate at the synapse and subsequent toxicity and they serve to recycle the released transmitter for packaging and subsequent release. Most transporters are expressed at the nerve terminal, where they are well positioned to serve both functions. However, the major EAAT isoforms GLAST (human EAAT1) and GLT1 (human EAAT2) are expressed by glia and localize to astrocytic processes that reside at varying distances from the synapse. Recent experimental in vivo work has shown that presynaptic terminals regulate astroglial GLT1/EAAT2 expression (Yang et al., 2009). The regulation of GLT1 expression by presynaptic input also raises important questions about cause and effect in neural degeneration. It is very clear that loss of GLT1/EAAT2 causes severe toxicity, and downregulation may occur in ALS. However, several works (Bergles et al., 1999; Yang et al., 2009) suggest that the downregulation observed may reflect rather than cause neuronal loss. Indeed, downregulation in the absence of neural input might have less deleterious consequences than in the intact state, where the release of more glutamate has greater potential to produce toxicity. Neuronal regulation presumably serves to coordinate glutamate clearance with glutamate release. However, a defect in the signalling mechanism might trigger the degenerative process without a primary disorder of the neuron, and even secondary changes in EAATs expression may propagate the neuronal injury.

Glia in the peripheral nervous system also respond to neuronal activity. Enteric glia are intimately associated with the neurons from the enteric nervous system (ENS) This association is similar in morphology and molecular nature to that shown by neurons and glia in the CNS. Astrocyte-like enteroglial cells are actively involved in enteric neuronal activity via neurotransmitter receptors. In the ENS, the purine adenosine triphosphate (ATP) is released together with noradrenaline and acetylcholine by enteric neurons (Al-Humayyd 
\& White, 1985; Nurgali et al., 2003). ATP plays a pivotal role in regulating synaptic transmission in CNS astrocytes (Abbracchio \& Ceruti, 2006) and it is involved in controlling gastrointestinal motility, secretomotor function, blood flow, and synaptic transmission (Bornstein, 2008; Christofi, 2008; Ren \& Bertrand, 2008). Enteric glia express purinergic receptors and has been reported to respond to ATP in vitro (Gomes et al., 2007; Zhang et al., 2003) which suggested enteric glia participation in functional gastrointestinal responses to nerve signalling. More recently, activation of enteric neurons using electrical field stimulation of interganglionic fiber tracts in a longitudinal muscle myenteric plexus preparation from Guinea pig colon was able to elicit enteric glial cell activity as assesses by intracellular Ca2+ imaging in situ through P2Y4 ATP receptors (Gulbransen \& Sharkey, 2009), providing the first evidence of neuron-to-glia signalling in the ENS. Further to this, the same team using a combination of techniques to selectively stimulate or eliminate intrinsic and extrinsic populations of neurons found that enteric glia are specifically activated by sympathetic postganglionic neurons innervating the colonic myenteric plexus (Gulbransen et al., 2010). This result supports the notion that glia are not indiscriminate detectors of neuronal activity and can discern activity from specific neural pathways.

Interestingly, the case of a HSCR patient with a SOX10 gene mutation has recently been reported showing neurological impairment entirely due to glial maldevelopment including peripheral dysmyelinating neuropathy and enteric neuroglia deficiency (Shimotake et al., 2007). This discovery has driven renewed interest for neuron-to-glia interactions and neuroimaging in the field of neurocristopathies.

\section{Hirschsprung disease (HSCR) or aganglionic megacolon and its association with other malformations}

HSCR is a cause of functional intestinal obstruction with an incidence of 1/5000 alive newborns (NB) (Lister \& Irving, 1990; Amiel \& Lyonnet, 2001). However, the incidence has been shown to vary significantly among ethnic groups: 1.5, 2.1, and 2.8 per 10000 live births in Caucasians, African-Americans, and Asians, respectively, in the state of California (Amiel \& Lyonnet, 2001). For comparison, in our Albacete Health Service Area the incidence is 1.6 per 5000 alive NB (Carrascosa-Romero et al., 2007). Although there are many sporadic cases, a heterogeneous familial incidence has also been described involving several genetic factors (Amiel \& Lyonnet, 2001).

There is abundant literature connecting HSCR with other malformations (Table 1), frequently including cleft palate, iris coloboma and congenital cardiac defects, among others (Amiel \& Lyonnet, 2001; Scriver et al., 2002). Often, HSCR may appear as part of a wellknown neurocristopathy syndrome such as piebaldism (MIM 172800), Shah-Waardenburg (MIM 277580), congenital central hypoventilation (Haddad, MIM 209880) or Riley-Day (MIM 223900) syndromes, or associated to other types of dysmorphic syndromes, such as Aarskog (MIM 100050), Rubinstein-Taybi (MIM 180849), or Smith-Lemli-Opitz syndromes (MIM 270400), where HSCR is not always present but shows an incidence higher than expected by chance. Of interest, Carrascosa-Romero et al., 2007 also reported an unusual case of HSCR associated with FG syndrome (MIM 305450), a X-linked heterogeneous genetic disorder characterized by hair whorls, broad thumbs and severe constipation (Romano et al., 1994), presenting with severe mental retardation. 


\begin{tabular}{|c|c|c|}
\hline SYNDROMES & MIM & KEY CLINICAL FEATURES \\
\hline - NEUROCRESTOPATHIES & & \\
\hline WS4 (Shah-Waardenburg) & 277580 & $\begin{array}{l}\text { Pigmentary anomalies (white forelock, iris } \\
\text { hypoplasia, patchy hypopigmentation), deafness. }\end{array}$ \\
\hline $\begin{array}{l}\text { Hipopigmentación- Sordera- } \\
\text { Ceguera Yemenite }\end{array}$ & 601706 & $\begin{array}{l}\text { Hearing loss, eye anomalies (microcornea, } \\
\text { coloboma, nystagmus), pigmentary anomalies }\end{array}$ \\
\hline $\begin{array}{l}\text { BADS (black locks, } \\
\text { oculocutaneous albinism, } \\
\text { sensorineural deafness) }\end{array}$ & 227010 & $\begin{array}{l}\text { Hearing loss, hypopigmentation of the skin and } \\
\text { retina }\end{array}$ \\
\hline Piebaldism & 172800 & Patchy hypopigmentation of the skin and hair \\
\hline Haddad & 209880 & Congenital central hypoventilation \\
\hline MEN2A & 171400 & $\begin{array}{l}\text { Medullary thyroid carcinoma, } \\
\text { phaeochromocytoma, hyperplasia of the } \\
\text { parathyroid }\end{array}$ \\
\hline Riley-Day & 223900 & Autonomic nervous system anomalies \\
\hline \multicolumn{3}{|l|}{ HSCR MANDATORY } \\
\hline Goldberg-Shprintzen & $\begin{array}{l}609460 \\
235740\end{array}$ & $\begin{array}{l}\text { Cleft palate, hypotonia, microcephaly, mental } \\
\text { retardation, dysmorphic facial features } \\
\text { Hypertelorism, deafness, polydactyly, unilateral } \\
\text { renal agenesis }\end{array}$ \\
\hline HSCR with limbs anomalies & $\begin{array}{l}235750 \\
235760 \\
604211 \\
306980 \\
\end{array}$ & $\begin{array}{l}\text { Postaxial polydactyly, ventricular septal defect } \\
\text { Hypoplasia of distal phalanges and nails, } \\
\text { dysmorphic features } \\
\text { Preaxial polydactyly, heart defect, laryngeal } \\
\text { anomalies. } \\
\text { Brachydactyly type D }\end{array}$ \\
\hline BRESHECK & 300404 & $\begin{array}{l}\text { Brain abnormalities, Retardation, Ectodermal } \\
\text { dysplasia, Skeletal malformation, HSCR, Ear/eye } \\
\text { anomalies, Cleft palate/Cryptorchidism and } \\
\text { Kidney dysplasia/hypoplasia }\end{array}$ \\
\hline $\begin{array}{l}\text { Mesomelic dysplasia, Werner } \\
\text { type }\end{array}$ & & Mesomelia, polydactyly \\
\hline \multicolumn{3}{|l|}{$\begin{array}{l}\text { HSCR FREQUENTLY } \\
\text { ASSOCIATED }\end{array}$} \\
\hline Mowat-Wilson & 255730 & $\begin{array}{l}\text { Dysmorphic facial features, microcephaly, mental } \\
\text { retardation, agenesis of corpus callosum, heart } \\
\text { disease, urogenital/renal anomalies }\end{array}$ \\
\hline \multicolumn{3}{|l|}{$\begin{array}{l}\text { HSCR OCCASIONALLY } \\
\text { ASSOCIATED }\end{array}$} \\
\hline Bardet-Biedl & 209900 & $\begin{array}{l}\text { Pigmentary retinopathy, obesity, hypogenitalism, } \\
\text { mild mental retardation, postaxial polydactyly }\end{array}$ \\
\hline Kauffman-McKusick & 236700 & $\begin{array}{l}\text { Hydrometrocolpos, postaxial polydactyly, } \\
\text { congenital heart defect. }\end{array}$ \\
\hline
\end{tabular}




\begin{tabular}{|l|l|l|}
\hline Smith-Lemli-Opitz & 270400 & $\begin{array}{l}\text { Growth retardation, microcephaly, mental } \\
\text { retardation, hypospadias, 2-3 toes syndactyly, } \\
\text { dysmorphic features }\end{array}$ \\
\hline Cartilage-hair hypoplasia & 250250 & $\begin{array}{l}\text { Short limb dwarfism, metaphyseal dysplasia, } \\
\text { immunodeficiency }\end{array}$ \\
\hline HSCR RARELY ASSOCIATED & 253800 & $\begin{array}{l}\text { Muscular dystrophy, polymicrogyria, } \\
\text { hydrocephalus, mental retardation, seizures. }\end{array}$ \\
\hline $\begin{array}{l}\text { Fukuyama congenital } \\
\text { muscular dystrophy }\end{array}$ & 258840 & $\begin{array}{l}\text { Dysmorphic features, ichthyosis, hypoplastic toes } \\
\text { and nails }\end{array}$ \\
\hline Clayton-Smith & 304100 & $\begin{array}{l}\text { Agenesis of corpus callosum, adducted thumbs, } \\
\text { ptosis, muscle weakness }\end{array}$ \\
\hline Kaplan & 308840 & $\begin{array}{l}\text { Agenesis of corpus callosum, hydrocephalus, cleft } \\
\text { palate. }\end{array}$ \\
\hline Okamoto &
\end{tabular}

Table 1. Syndromes associated with HSCR (reprinted and adapted from Scriver CM et al, eds. "The metabolic and molecular bases of inherited diseases" 8th ed. Chap 251. New York: McGraw-Hill: 6231-55.)

For other syndromes, HSCR is a mandatory feature for diagnosis such as BRESHECK (Brain Abnormalities, Retardation, Ectodermal dysplasia, Skeletal malformation, Hirschsprung disease, Ear/eye anomalies, Cleft palate/Cryptorchidism and Kidney dysplasia) (MIM 300404) and the subtypes of HSCR with limb anomalies (MIM 235750, 235760, 604211 and 306980). HSCR is also a mandatory feature for Goldberg-Shprintzen megacolon syndrome (GOSHS MIM 609460)(Goldberg \& Shprintzen, 1981). This syndrome is characterized by microcephaly, hypertelorism, short stature, cleft palate, learning problems, and seems to be caused by homozygous nonsense mutations in KIAA 1279 located at 10q22.1. GOSHS presents various common characteristics with the Mowat-Wilson syndrome (MIM 235730), which will be discussed below in section 5 .

HSCR has also been connected with malformations of the nervous system: defects in the neural tube closure such as anencephaly (Mathew, 1998) and meningomyelocele (Merkler et al., 1985), as well as anomalies in neuronal migration and or cerebral dysgenesis (Cass, 1990), predominantly agenesis of corpus callosum (Sayed \& Al-Alaigan, 1996). These phenomena have been regarded as alterations in the embryonic development of the anterior portion of the NC (Currie et al., 1986; Hurst et al., 1986). The actual incidence of associated brain anomalies has been studied for other neurocristopathies. For example, Couly \& Aicardi (1983) reported that of a group of 3000 children presenting with facial dysembryoplasias, $13 \%$ also showed brain malformations. Another study showed up to 82 $\%$ (18 out of 25) of children with uni- or bilateral maxillomandibular neurocristopathies (Goldenhar's, Franceschetti's first arch syndromes or transient forms) also presented morphological and motor anomalies of the brain stem and its corresponding cranial nerves, as assessed by neurological and cerebral computed tomography examinations (Couly \& Le Lievre-Ayer, 1983). However, we have failed to find previous literature studying the incidence of brain malformations associated with HSCR. Available reports dealt with isolated cases (Turkdogan-Sozuer et al., 1998), sometimes under other denominations such as GOSHS. All these cases nonetheless share a common denominator: psychomotor delay, 
associated to alterations in the CNS, generally: microcephay, agenesis of corpus callosum, white matter atrophy, ventricular dilatation. Few reports describe pachygyria, polymicrogyria as well as cerebellar hypoplasia in HSCR patients (Carrascosa-Romero et al., 2007), perhaps due to the fact that neuroimaging studies are rarely performed on this type of patients. Our team did study the incidence of brain malformations associated with HSCR in our Albacete Health Service Area. In our study we found 10 cases of isolated HSCR versus 7 cases of HSCR associated with other structural anomalies or psychomotor retardation, which indicated a high incidence of anomalies associated with HSCR $(41,1 \%)$ in our territory.

\subsection{Molecular genetics in HSCR: The RET proto-oncogene}

Segregation studies in nonsyndromic HSCR have shown sibling recurrence risk ranging from 1 to $33 \%$, and it is considered a multifactorial disorder, the effect of genes predominating over environmental factors (relative risk of 200). The higher susceptibility for HSCR in some families allowed establishing the locus 10q11. 2q21.2 as a genetic origin of the disease (Fewtrell et al., 1994.) The RET proto-oncogen is located in this region and has been shown to be highly involved in neurocristopathies (Amiel \& Lyonnet, 2001). The RET protooncogen is a tyrosine-protein kinase receptor essential for the glial cell-derived neurotrophic factor (GDNF) actions preventing neuroectodermal cell apoptosis (Mograbi et al., 2001). De Pontual et al., 2006, genotyped RET in 143 patients with two syndromic HSCR entities: congenital central hypoventilation (CCHS) and Mowat-Wilson syndrome (MWS), caused by PHOX2B and ZFHX1B gene mutations, respectively, finding that there were both RET dependent and RET independent HSCR cases. Besides this, RET mutations have been found only in $50 \%$ of familial and $15 \%$ to $20 \%$ sporadic HSCR cases (Attie et al., 1995). Therefore, notwithstanding the importance of the mutations in the RET gene, there are other genes involved in human HSRC: neurturin (NTN), endothelin B receptor (EDNRB), endothelin-3 (EDN3), endothelin-converting enzyme (ECE1), as well as the SOX10 and SIP1 genes (Amiel \& Lyonnet, 2001; Mollaaghababa \& Pavan, 2003).

Substance $\mathrm{P}$-a neurotransmitter specific marker of neuronal differentiation- has been found downregulated in HSCR suggesting a role for peptidergic innervation in the pathogeny of this disease (Tam, 1986). The possible connection between the above mentioned genes, substance $\mathrm{P}$ and GDNF and the causal mechanism the alteration of neuronal migration in the NC morphogenesis is not yet clear, since a high proportion of genetic anomalies cannot be identified using a standard screening of genomic DNA. A multiple origin is likely including other types of molecular parameters. For example, elevated levels of maternal homocysteine have been established as a risk toxicity factor for congenital defects during embryonic development, particularly anomalies in the neural tube closure and neurocristopathies (Brauer \& Tierney, 2004), whereas a prophylactic role has been observed for folic acid in both types of pathologies (Antony, 2007).

\section{Mowat-Wilson syndrome}

Mowat-Wilson syndrome (MWS, MIM 235730) is a condition that involves multiple congenital defects described by Mowat et al., 1998. They reported a series of six children presenting mental retardation, microcephaly, and short stature with a distinctive facial 
phenotype accompanied by other variable types of congenital anomalies. Five of these patients also had HSCR and one presented an interstitial deletion of chromosome 2, del (2)(q21q23). These children strongly resembled the patient earlier reported by Lurie et al. 1994, with HSCR and dysmorphic features also associated with del (2) (q22q23). They concluded that these children had a distinct syndrome for which HSCR was not a mandatory feature and that could be caused by a contiguous gene syndrome, or a single gene disorder, or disruption of a critical region within 2q22-23. In 2001, two independent teams (Cacheux et al., 2001; Wakamatsu et al., 2001) identified the cause of MWS as either a heterozygous deletion or truncation mutations in the Zinc finger E-box-binding homeobox 2 gene, ZEB2, (MIM 605802, previously called ZFHX1B) which encodes a smad interacting protein 1 (SIP1) located in the above mention chromosome region 2q22-23.

Since the first description by Mowat et al (1998), approximately 200 patients have been reported and over 100 mutations have been described (Dastot-Le Moal et al., 2007). The phenotype/genotype correlation for these mutations is very variable, not only for a given mutation (Cerruti-Mainardi et al., 2005; Zweier et al., 2003) but also within the same family (McGaughran et al., 2005). The syndrome has been identified in several ethnic groups (Dastot-Le Moal et al., 2007), with similar clinical features in all populations. The male/female ratio is approximately 1,42:1 (Adam et al., 2006; Horn et al., 2004).

The prevalence of MWS is currently unknown, but it seems probable that the syndrome is under-diagnosed, particularly in patients without HSCR (Cerruti-Mainardi et al., 2005). For this reason the identification of the facial phenotype is of special relevance for the initial clinical diagnosis (Garavelli L \& Cerruti-Mainardi, 2007). The clinical features of the face are: high forehead, frontal bossing, eyebrows are large, medially flaring and sparse in the middle part, hypertelorism with hollow but large eyes, big and uplifted ear lobes with a central depression, saddle nose, open mouth, with M-shaped upper lip, frequent smiling and occasional drooling (Fig. 1, a and b), and a prominent but narrow and triangular pointed chin that further elongates with age ( Fig. 2, a and b).

At birth, patients show growth parameters with values within normal percentiles but they develop microcephaly and short stature progressively with age. The facial phenotype also evolves in older children. For example, the eyebrows become thicker, broad and horizontal, with an increased wide middle separation and medial sparseness. The nasal tip lengthens and becomes more depressed, the columella is more prominent, the nasal profile becomes convex, the face tends to elongate and the jaw is more pronounced. The uplifted ear lobes do not change significantly with time and are an excellent diagnostic clue (Mowat et al., 2003) (Fig. 3).

The clinical manifestations of MWS in the about 200 cases described presenting with ZEB2 mutations have been recently reviewed Garavelli and Cerruti-Mainardi, 2007. In brief, the distinct facial phenotype is present in $97 \%$ of the patients, at least moderate but usually severe mental retardation in all the cases, microcephaly is present in $81 \%$, epilepsy in $73 \%$, HSCR in $57 \%$, constipation in $26 \%$, and congenital heart disease (principally, patent ductus arteriosus, pulmonary stenosis, ventricular and atrial septal defects, pulmonary artery sling, Tetralogy of Fallot and aortic coarctation), in 52\% of the cases; urogenital/renal anomalies were demonstrated in $51 \%$ of the patients (of which $51 \%$ presented hypospadias, 36\% cryptorchidism and $12.8 \%$ several renal defects). Oropharyngeal and gastrointestinal malformations include pyloric stenosis, arched palate, among others. Musculoskeletal 
anomalies occur in many patients and eye defects have also been demonstrated $(4.1 \%$ of the published cases). Microcephaly is a common feature and is present in $81 \%$ of the published cases. Brain anomalies reported so far include hypoplasia or agenesis of corpus callosum, present in $43 \%$ of the published cases, cortical atrophy (Garavelli et al., 2003), pachygyria and cerebellar hypoplasia (Silengo et al., 2004), hippocampal formation hypoplasia (Kääriäinen et al., 2001) and frontotemporal hypoplasia with temporal dysplasia (Cacheux et al., 2001, Mowat et al., 2003). These findings may be under-represented because not all published cases employed brain imaging

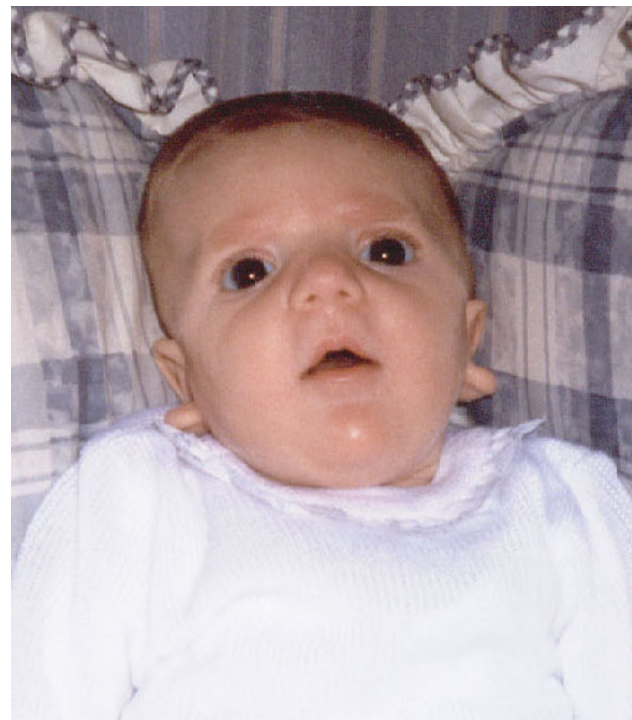

(a)

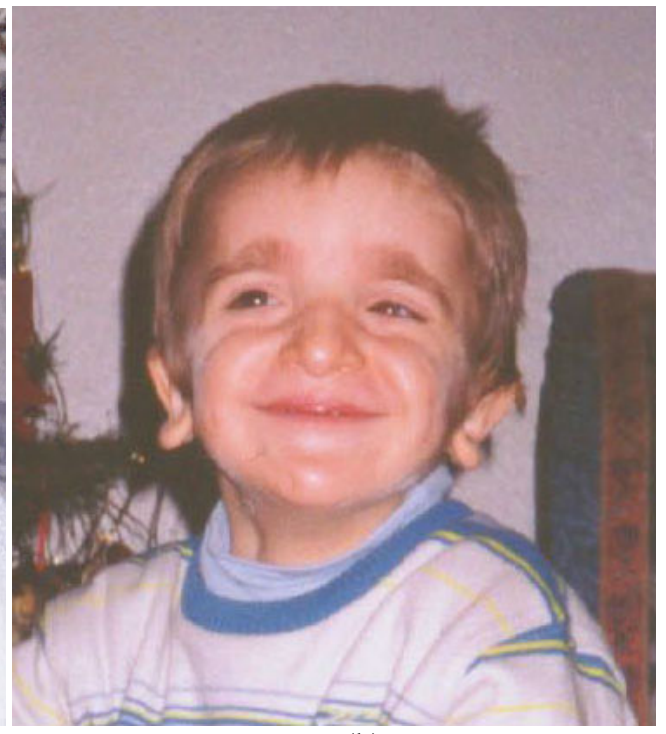

(b)

Fig. 1. Clinical facial features in MWS: a) In the neonate, excess nuchal skin and scarce fine hair can be observed together with high forehead, prominent frontal bone, large wide eyebrows, but thinning in the middle part, hypertelorism, strabismus, epicanthus, large hollow eyes that originate prominent cheek bones, wide nasal bridge and open mouth, with M-shaped upper lip b) Some years later: saddle nose, prominent rounded nasal tip. The large and uplifted ear lobes with a central depression do not change significantly with age.

Regarding differential diagnosis, the facial phenotype of patients with MWS is very characteristic. However, due to the frequent presence of HSCR, epilepsy and mental retardation it may initially be mistaken as GOSHS, as we previously mentioned in section 4. The patients with GOSHS share clinical features such as HSCR, epilepsy and mental retardation, but have different facial features (high nasal bridge, synophrys, long curled eyelashes, palpebral ptosis, and cleft palate). The differential diagnosis can be carried out on the basis of facial phenotype and confirmed by mutational analysis of the ZEB2 gene. This is important for genetic counseling, since GOSHS is autosomal recessive, whereas MWS is a sporadic condition. Since individuals with MWS often show an ataxic-like gait and a smiling, sociable personality, combined with absent speech, microcephaly and seizures, they can be given a presumptive diagnosis of Angelman syndrome (Williams et al., 2001). 


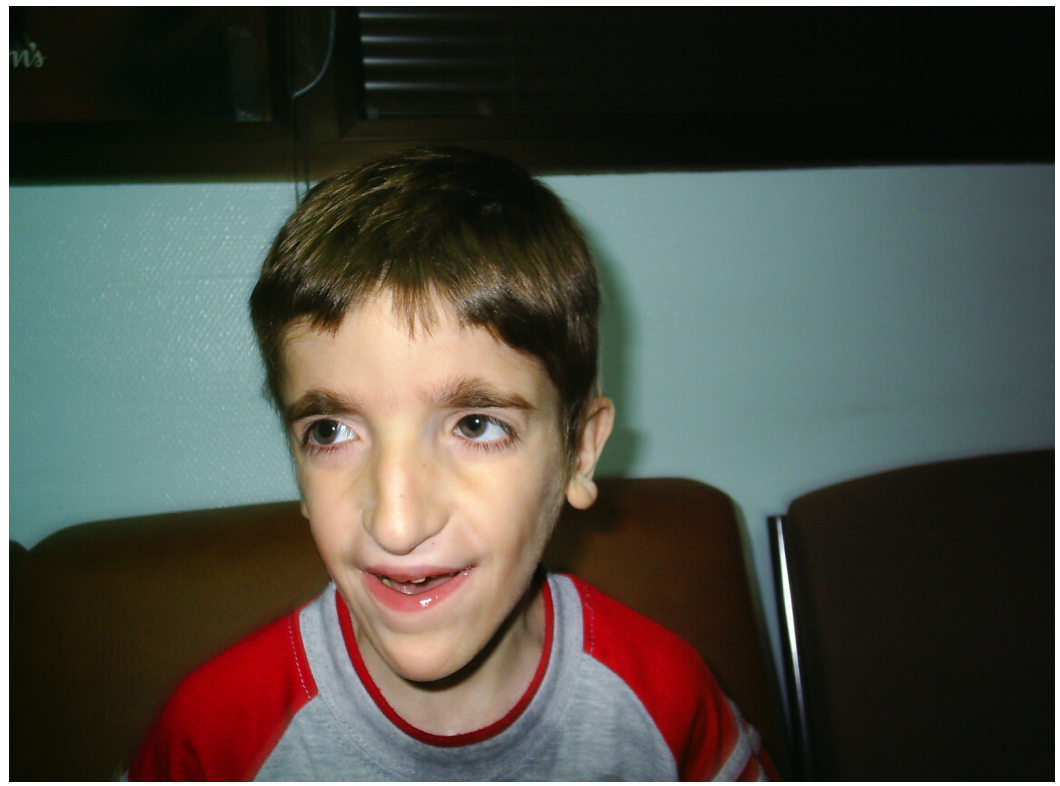

(a)

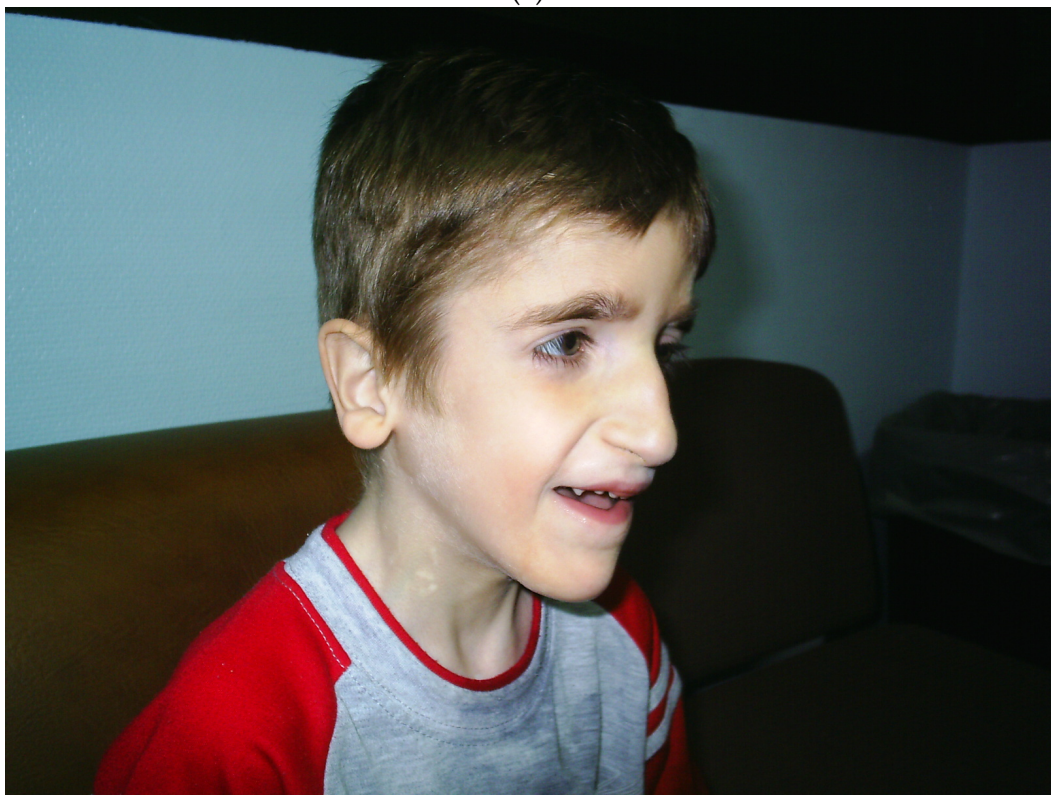

(b)

Fig. 2. Clinical facial features in MWS: 10 years old. Dysmorphic facial features: wide forehead, hypertelorism con antimongoloid palpebral fissure, large dense eyebrows, long eyelashes, low-set ears, prominent uplifted ear lobes, saddle nose, thin upper lip, the face becomes long and thin, with prognathism, and a long, pointed or "chisel-shaped" chin, smiley face. 


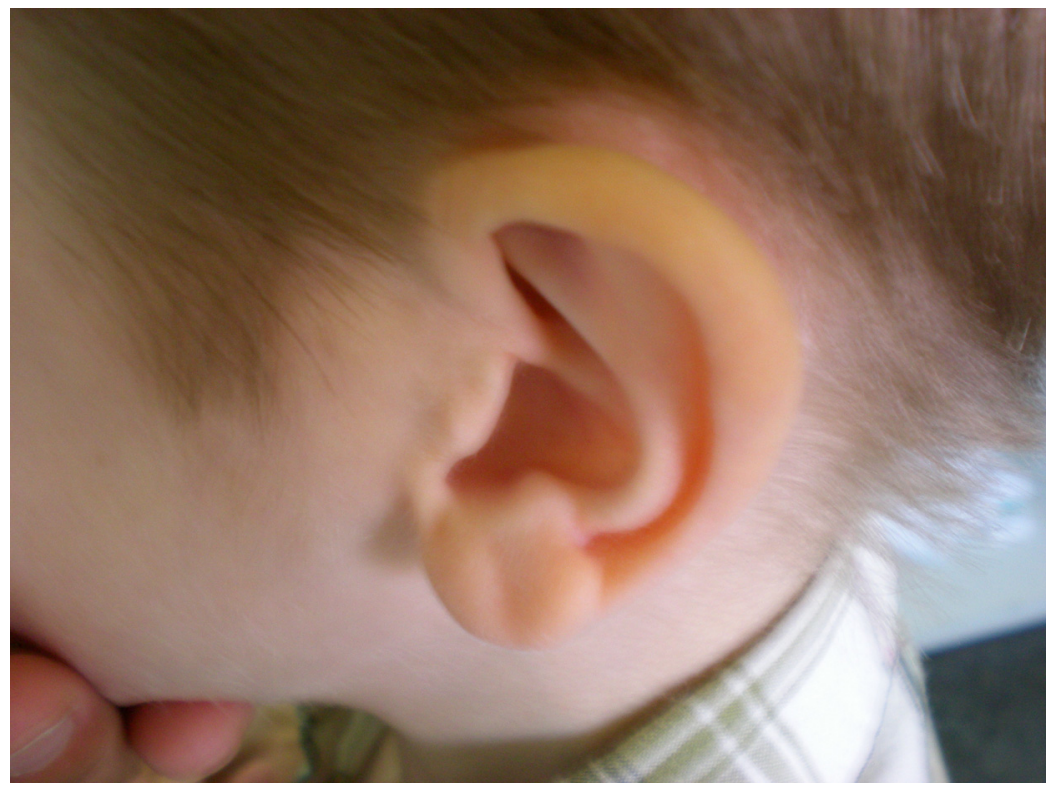

Fig. 3. Clinical facial features in MWS. The ear lobes are very typical. They are large and uplifted with a central depression and have been described as being like "orecchiette pasta" or like "red blood corpuscles" in shape. They do not change significantly with time (with the exception of the central depression, which is less obvious in adults) and are an excellent diagnostic clue

However, the distinct facial features of MWS, in addition to the other typical congenital anomalies, should allow distinguishing these two conditions. Differential diagnosis is also necessary for MWS patients presenting with hypospadias and mental retardation to avoid misdiagnosis as Smith-Lemli-Opitz syndrome, Opitz G/BBB syndrome or X-linked mental retardation-alpha thalassemia syndrome. Again, facial phenotype should be the clue for correct diagnosis.

In this context, we have recently diagnosed a patient with MWS with the help of molecular genetics and neuroimaging (Carrascosa Romero et al., 2009), which we consider an interesting and illustrative case. This patient presented a typical facial phenotype and molecular genetics analysis showed a heterozygous deletion mutation in the ZEB2 gene never described before in the literature. Parents showed no genetic anomalies. Our MWS patient showed some previously described malformations such as patent ductus arteriosus, Tetralogy of Fallot, atrial septal defect (small ostium secundum) and presented with HSCR. Magnetic resonance imaging (MRI) of the brain (Fig. 4, a and b) revealed agenesis of corpus callosum and colpocephaly with an important elevation of the third ventricle, cortical dysgenesis showing pachygyria in the left perisylvian region and decreased mielinization at the biparietal level. The patient also showed severe mental retardation, happy and smiling behavior, developed epilepsy when two years of age and was not able to walk until 4 years old. The finding of CNS demyelinization, detected with neuroimaging, is of special interest. This decrease in oligodendroglia maybe meaningful in the context of neurocristopathies given the role of these cells in nervous system maturation, particularly in the regulation of ventral neuroectodermal progenitor cell fate (Jakovcevski et al., 2009; Pucharcós et al., 1999) 


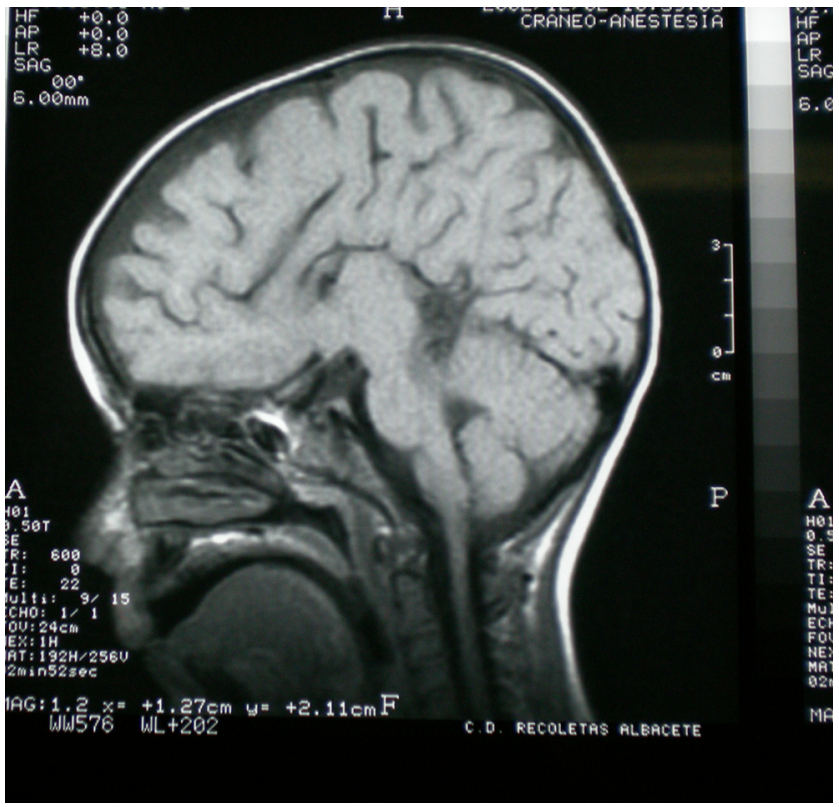

(a)

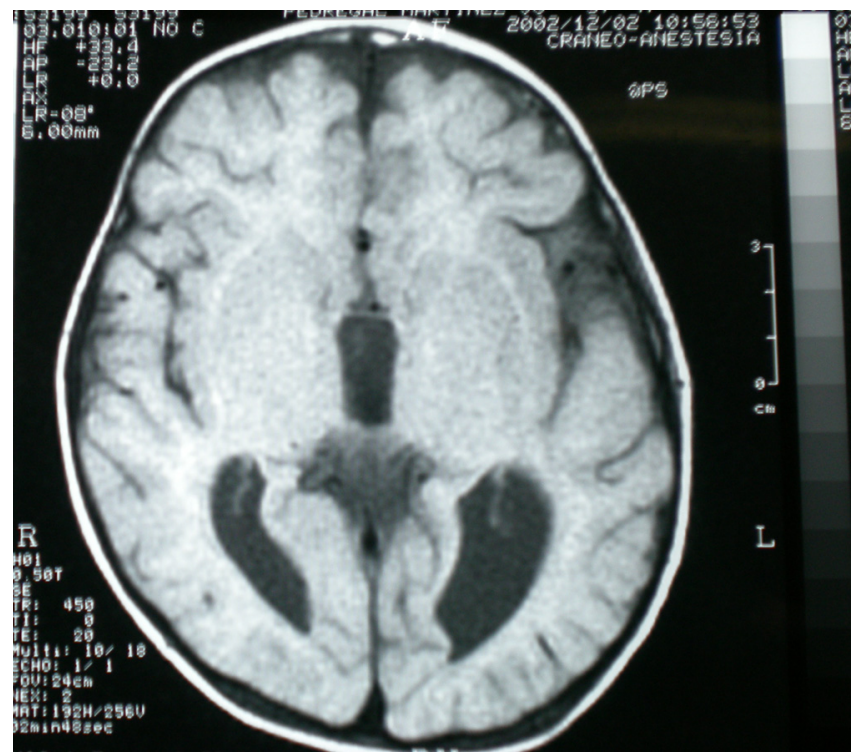

(b)

Fig. 4. a and b. Magnetic resonance imaging (MRI) of our MWS patient brain. Image analysis revealed agenesis of corpus callosum and colpocephaly with an important elevation of the third ventricle, cortical dysgenesis showing pachygyria in the left perisylvian region and decreased mielinization at the biparietal level. 


\subsection{The ZEB2 gene: Expression and role in neurocristopathies}

As mentioned above, at least 100 mutations have been described for the ZEB2 gene (DastotLe Moal et al., 2007) connected with MWS -including the mutation found for our patient (Carrascosa Romero et al., 2009)- which reveals the importance of this gene for the development of this disease.

The ZEB2 gene is $70 \mathrm{~Kb}$ long consists of 10 exons and 9 introns and encodes SIP1 (Smad interacting protein 1). Although its mechanisms of action on morphogenesis and neurogenesis still remain to be clarified, its clinical implications suggest that ZEB2 is involved in the development of the cells from the NC (ENS, craniofacial mesoectoderm), CNS, and cardiac septation, as well as in the development of the median line (agenesis of corpus callosum, urogenital/renal anomalies, pyloric stenosis)(Zweier et al., 2002; Ishihara et al., 2004).

Using mass spectrometry, Verstappen et al. 2008, found that ZEB2 associated with multiple subunits of the NURD complex, which plays a key role in transcriptional repression. Mi2-beta (CHD4; 603277) was identified as a specific cofactor for ZEB2mediated repression of E-cadherin (CDH1; 192090). The N-terminal 289 amino acids of ZEB2 were sufficient for interaction with NURD complex subunits. In vitro studies in Xenopus oocytes showed broad Zeb2 expression at the gastrula stage, with stronger expression in neural tissues and neural crest cells at the neurula stage, suggesting a role in neural development. Endogenous Mi2-beta expression broadly overlapped Zeb2 expression, and antisense morpholino knockdown of Mi2-beta resulted in reduced Zeb2mediated repression of Bmp4 (112262) and decreased induction of neural marker Ncam (116930). Further studies showed that a mutant ZEB2 protein (605802.0014), differing in the first 24 amino acids from the wildtype protein and causing a mild form of MowatWilson syndrome (235730), was unable to interact with the NURD complex and showed decreased transcriptional repression of Bmp4.

To investigate the breadth of clinical variation associated with mutations in ZFHX1B, Yamada et al. 2001, studied DNA samples from 6 patients with clinical features similar to those described for ZFHX1B deficiency, except that they did not have Hirschsprung disease. The results showed the R695X mutation (605802.0002) to be present in 3 cases, with 3 novel mutations being identified in the other 3 patients. All mutations occurred in 1 allele and were de novo events. The results demonstrated that ZFHX1B deficiency is an autosomal dominant complex developmental disorder and that individuals with functional null mutations present with mental retardation, delayed motor development, epilepsy, and a wide spectrum of clinically heterogeneous features suggestive of neurocristopathies at the cephalic, cardiac, and vagal levels.

To clarify the molecular mechanisms underlying the clinical features of Hirschsprung disease-mental retardation syndrome, Van de Putte et al. 2003 generated mice that carried a Zfh $x 1 b$ mutation comparable to those found in several human patients. They showed that Zfhx1b knockout mice did not develop postotic vagal neural crest cells, the precursors of the enteric nervous system that is affected in patients with Hirschsprung disease, and displayed a delamination arrest of cranial neural crest cells, which form the skeletomuscular elements of the vertebral head. This suggests that the gene product is essential for the development of vagal neural crest precursors and the migratory behavior of cranial neural crest in the mouse. Furthermore, they showed that the gene product was involved in the specification of 
neuroepithelium. SIP1-knockout embryos died around embryonic day 9.5, with failed neural tube closure, lack of a sharp boundary between the neural plate and the rest of the ectoderm, and lack of the first branchial arch. It was found that conditional deletion of Zeb2 in mouse neural crest precursors was lethal at embryonic stages. Mutant mice displayed craniofacial and gastrointestinal malformations similar to those of patients with MowatWilson syndrome. In addition, mutant mice had defects in the heart, melanoblasts, and sympathetic and parasympathetic anlagen (Van de Putte et al., 2007).

A single layer of neuroepithelial cells lining the embryonic neural tube gives rise to the entire repertoire of neurons, astrocytes, and oligodendrocytes in the adult central nervous system. Seuntjens et al. 2009, found that conditional SIP1 deletion in young mouse neurons induced premature production of upper layer neurons at the expense of deep layers, precocious and increased generation of glial precursors, and elevated numbers of astrocytes at early postnatal stages. Microarray analysis showed that Ntf3 (162660) and Fgf9 (600921) were over- and prematurely expressed in mutant brains. In the absence of SIP1, there was also a premature peak of MAPK (176948) signalling in neural progenitor cells. It was concluded that SIP1 functions in the postmitotic compartment of the neocortex to control the expression of growth factor genes that feed back to progenitor cells to regulate production of the neurons and glial cells required for corticogenesis.

\section{Conclusion: Relevance of brain imaging and genetics in the diagnosis of neurocristopathies}

The association of HSCR (either isolated or within the context of a specific malformation syndrome) with neuronal migration anomalies is so strong $(23.5 \%)$, that we recommend performing a full evaluation of HSCR patients including: a) specialized molecular genetic studies, b) a complete neurological exploration for all patients diagnosed with HSCR and c) neurologist-monitored brain imaging studies in search for cerebral dysgenesis. Conversely, when confronting cases of children presenting with mental retardation, dysmorphic features and severe constipation, anorectal manometry seems advisable to rule out HSCR.

The development of neuroimaging techniques is now making possible the detailed identification of cerebral dysgenesis compatible with neuronal migration impairments that are at the root of a wide range of brain diseases including epilepsy and mental retardation. The application of neuroimaging techniques in combination with molecular genetics to patients diagnosed with neurocristopathies has an extraordinary potential to connect genotype to phenotype and discover possible brain malformations associated with the mutations causing these syndromes.

\section{References}

Abbracchio MP, Ceruti S. Roles of P2 receptors in glial cells: focus on astrocytes. Purinergic Signal 2006;2:595-604.

Adam MP, Schelley S, Gallagher R, Brady AN, Barr K, Blumberg B, Shieh JTC, Graham J, Slavotinek A, Martin M, Keppler-Noreuil K, Storm AL, Hudgins L: Clinical features and management issues in Mowat-Wilson syndrome. Am J Med Genet 2006; 140 A:2730-2741. 
Al-Humayyd M, White TD. Adrenergic and possible nonadrenergic sources of adenosine $5=$-triphosphate release from nerve varicosities isolated from ileal myenteric plexus. J Pharmacol Exp Ther 1985;233:796-800.

Amiel J, Lyonnet S: Hirschsprung disease, associated syndromes, and genetics: a review. J Med Genet 2001; 38: 729-739.

Antony AC: In utero physiology: role of folic acid in nutrient delivery and fetal development. Am J Clin Nutr. 2007 Feb;85(2):598S-603S.).

Attie T, Pelet A, Edery P et al: Diversity of RET proto-oncogene mutations in familial and sporadic Hirschprung disease. Hum Mol Genet 1995; 4:1381-6.

Barres, B.A: The mystery and magic of glia: a perspective on their roles in health and disease. Neuron. 2008 Nov 6; 60(3):430-40. Review.

Bergles DE, Diamond JS, Jahr CE. Clearance of glutamate inside the synapse and beyond. Curr Opin Neurobiol. 1999 Jun;9(3):293-8. Review.

Boillee S, Yamanaka K, Lobsiger CS, et al. Onset and progression in inherited ALS determined by motor neurons and microglia. Science 2006;312:1389-1392.

Bolande RP: Neurocristopathy: its growth and development in 20 years. Pediatr Pathol Lab Med. 1997 Jan-Feb;17(1):1-25.

Bolande RP: The Neurocristopathy. A unifying concept of disease arising in neural crest maldevelopment . Human Pathol, 1974; 5: 409-429.

Bornstein JC. Purinergic mechanisms in the control of gastrointestinal motility. Purinergic Signal 2008;4:197-212.

Brauer PR: Tierney BJ. Consequences of elevated homocysteine during embryonic development and possible modes of action. Curr Pharm Des. 2004;10(22):2719-32.

Cacheux V, Dastot-Le Moal F, Kaariainen H, Bondurand N, Rintala R, Boissier B, Wilson M, Mowat D, Goossens M. Loss-of-function mutations in SIP1 Smad interacting protein 1 result in a syndromic Hirschsprung disease. Hum Mol Genet. 2001; 10:1503-1510.

Carrascosa Romero MC, Barros Angueira F, Castillo Serrano A, Fernández Córdoba MS, Sorli García M, Quintanilla Mata ML: "Síndrome de Mowat-Wilson con una deleción en el Gen ZEB2 no descrita previamente. Boletín del ECEMC: Revista de Dismorfología y Epidemiología. Serie V. no 8, 2009. ISSN: 0210-3893. pp 9-17.

Carrascosa-Romero MC, Fernandez Cordoba MS, Gonzalvez Piñera jJ, Gutierrez-Junquera C, Pardal-Fernandez JM "Neurocristopathies: a high incidence of cerebral dysgenesis in patients with Hirschsprung's disease." Rev Neurol.2007; 45(12):70712.

Cass K: Aganglionosis: Associated Anomalies. J Pediatr Child Health 1990; 26: 351-354.

Cerruti-Mainardi P, Garavelli L, Pastore G, Virdis R, Pedori S, Godi M, Provera S, Rauch A, Zweier C, Castronovo C, Zollino M, Banchini G, Bernasconi S, Neri G: Mowat-Wilson syndrome and mutation in the Zinc Finger Homeo Box 1B Gene: a new síndrome probably under-diagnosed. Italian J Pediatr 2005, 31:116125.

Cheng $\mathrm{W}$ Au DKK Knowles CH Anand P Tam PKH: Hirschsprung's Disease: A More Generalised Neuropathy? J Pediatr Surg 2001; 36: 296-300. 
Christofi FL. Purinergic receptors and gastrointestinal secretomotor function. Purinergic Signal 2008;4:213-236.

Couly G Aicardi J: Associated morphological anomalies of the face and brain in infants Arch Fr Pediatr. 1988 Feb;45(2):99-104.

Couly G Le Lievre-Ayer C: Laterofacial malformations (maxillomandibular neurocristopathies) associated with anomalies of the brain stem and cranial nerves. Rev Stomatol Chir Maxillofac. 1983;84(5):254-63.

Currie ABM Haddad M Honeyman M Boddy SM: Associated Develpmental Abnormalities of the Anterior End of the Neural Crest: Hirschsprung's Disease, Waardenburg's Syndrome. J Pediatr Surg 1986; 21: 248-250.

Dastot-Le Moal F, Wilson M, Mowat D, Collot N, Niel F, Goossens M: ZFHX1B mutations in patients with Mowat-Wilson syndrome. Hum Mutat 2007, 4:313-321.

De Pontual L Pelet A Trochet D Jaubert F Espinosa-Parrilla Y Munnich A y col: Mutations of the RET gene in isolated and syndromic Hirschsprung's disease in human disclose major and modifier alleles at a single locus. J. Med. Genet. 2006; 43: 419-423.

Fewtrell MS Tam PK Thomson AH Fitchett M Currie J Huson SM Mulligan LM: Hirschsprung's disease associated with a deletion of chromosome 10 (q11.2q21.2): a further link with the neurocristopathies? J Med Genet. 1994 Apr;31(4):325-7.

Freeman MR: Specification and morphogenesis of astrocytes. Science. 2010 Nov 5; 330(6005):774-8.

Garavelli L, Donadio A, Zanacca C, Banchini G, Della Giustina E, Bertani G, Albertini G, Del Rossi C, Zeweier C, Rauch A, Zollino M, Neri G. Hirschsprung disease, mental retardation, characteristic facial features and mutation in the gene ZFHX1B (SIP1): confirmation of the Mowat-Wilson syndrome. Am J Med Genet A.2003; Feb 1; 116A (4):385-8.,

Garavelli L, Mainardi PC: Mowat-Wilson syndrome. Orphanet J Rare Dis. 2007, 2:42 (http://www.OJRD.com/content/2/1/42).

Goldberg RB Shprintzen RJ: Hirschsprung megacolon and cleft palate in two sibs. J Craniofac Genet Dev Biol. 1981; 1: 185-189.

Gulbransen BD, Sharkey KA: Purinergic neuron-to-glia signaling in the enteric nervous system. Gastroenterology 2009;136:1349-1358.

Haydon PG, Carmignoto G.: Astrocyte control of synaptic transmission and neurovascular coupling. Physiol Rev. 2006 Jul;86(3):1009-31. Review

Horn D, Weschke B, Zweier C, Rauch A: Facial phenotype allows diagnosis of MowatWilson syndrome in the absence of Hirschsprung disease. Am J Med Genet 2004, 124 A:102-104.

Hurst JA Markewicz M Kumar K Brett EM: Unknown Syndrome, Hirchsprung's Disease, Microcephaly, and Iris Coloboma: a New Syndrome of Defective Neuronal Migration. J Med Genet 1988; 25: 494-500.

Ishihara N, Yamada K, Yamada Y, Miura K, Kato J, Kuwabara N, Hara Y, Kabayashi Y, Hoshino K, Nomura Y, Mimaki M, Ohya K, Matsushima M, Nitta H, Tanaka K, Segawa M, Ohki T, Ezoe T, Kumagai T, Onuma A, Kurada T, Yoneda M, Yamanaka 
T, Saeki M, Segawa M, Saji T, Nagaya M, Wakamatsu N: Clinical and molecular analysis of Mowat-Wilson syndrome associated with ZFH1B mutations and deletions at 2q22-24.1. J Med Genet 2004, 41:387-393..

Jakovcevski I, Filipovic R, Mo Z, Rakic S, Zecevic N. Oligodendrocyte development and the onset of myelination in the human fetal brain. Front Neuroanat. 2009; 3:5. Epub 2009 Jun 1 (3, article 5):1-15.

Jones MC: The neurocristopathies: reinterpretation based upon the mechanism of abnormal morphogenesis Cleft Palate J. 1990 Apr;27(2):136-40.

Juliá V Albert A Cusi V Gómez A López L Morales L: Hipoganglionismos y displasias neuronales: tienen las mismas malformaciones asociadas que la enfermedad de hirschsprung?. Comunicación al XLII Congreso de la SECP Valencia-2003.

Kusafuka T Puri P: Genetic Aspects of Hirschsprung's Disease. Semin Pediatr Surg 1998; 7: 148-155.

Lister J Irving IM: Neonatal Surgery 3ed ed London, Butterwoths 1990: 408-529.

Luis LA Encinas JL Avila LF: Enfermedad de Hirschsprung: enseñanzas en los últimos 100 casos. Cir Pediatr 2006;19:177-181.

Lurie IW, Supovitz KR, Rosenblum-Vos LS, Wulfsberg EA. Phenotypic variability of del(2) (q22-q23): report of a case with a review of the literature. Genet Couns. 1994; 5:1114.

Martucciello G Pini Prato A Puri P Holschneider AM Meier-Ruge W Jasonni V y col: Controversies concerning diagnostic guidelines for anomalies of the enteric nervous system: a report from the fourth International Symposium on Hirschsprung's disease and related neurocristopathies. J Pediatr Surg. 2005 Oct; 40(10):1527-31.

Mathew A: Anencephaly-Associated Aganglionosis. Am J Med Genet 1998; 80:518-520.

McGaughran J, Sinnott S, Dastot-Le Moal F, Wilson M, Mowat D, Sutton B, Goossens: Recurrence of Mowat-Wilson syndrome in siblings with the same proven mutation. Am J Med Genet 2005; 137A:302-304.

Merkler RC Solish SB Scherzer AL: Meningomyelocele and Hirschsprung Disease: Theoretical and Clinical Significance. Pediatrics 1985; 76: 299-300.

Mograbi B Bocciardi R Bougert I: The Sensitivity of Activated Cys Ret Mutants to Glial Cell Line-Derived Neurotrophic Factor Is Mandatory To Rescue. Neuroectodermic Cells from Apoptosis. Mol Cel Biol.2001; Vol. 21, No. 20:6719-6730.

Mollaaghababa R Pavan WJ: The importance of having your SOX on: role of SOX10 in the development of neural crest-derived melanocytes and glia. Oncogene. 2003 May 19;22(20):3024-34.

Mowat DR, Croaker GDH, Cass DT, Kerr BA, Chaitow J, Adès LC, Chia NL, Wilson MJ: Hirschsprung disease, microcephaly, mental retardation, and characteristic facial features: delineation of a new syndrome and identification of a locus at chromosome 2q22-q23. J Med Genet 1998, 35:617-623.

Mowat DR, Wilson MJ, Goossens M: Mowat-Wilson syndrome. J Med Genet 2003; 40:305310 . 
Nurgali K, Furness JB, Stebbing MJ. Analysis of purinergic and cholinergic fast synaptic transmission to identified myenteric neurons. Neuroscience 2003; 116:335347.

Polly T Coran A: Hirschsprung's Disease in the Newborn. Pediatr Surg Int 1993; 1: 8083.

Pucharcós C, Fuentes JJ, Casas C, de la Luna S, Alcántara S, Arbonés ML, Soriano E, Estivill X, Pritchard M. Alu-splice cloning of human Intersectin (ITSN), a putative multivalent binding protein expressed in proliferating and differentiating neurons and overexpressed in Down syndrome. Eur J Hum Genet. 1999 Sep;7(6):704-12.

Ren J, Bertrand PP. Purinergic receptors and synaptic transmission in enteric neurons. Purinergic Signal 2008;4:255-266.

Romano C Baraitser M Thompson E: A clinical follow-up of British patients with FG syndrome. Clin. Dysmorph. 1994; 3: 104-114.

Sayed M Al-Alaigan S: Agenesis of corpus callosum, hypertrophic Pyloric Stenosis and Hirschsprung Disease: Coincidence or Common Etiology? Neuropediatrics 1996; 27: 204-206.

Scriver CM et al, eds, .2002. "The metabolic and molecular bases of inherited diseases" 8th ed. Chap 251. New York: McGraw-Hill: 6231-55. Volume 2. 8th Edition. McGrawHill, 2002ISBN0079130356, 9780079130358. 6338 pp.

Seuntjens, E., Nityanandam, A., Miquelajauregui, A., Debruyn, J., Stryjewska, A., Goebbels, S., Nave, K.-A., Huylebroeck, D., Tarabykin, V. SIP1 regulates sequential fate decisions by feedback signaling from postmitotic neurons to progenitors. Nature Neurosci. 2009; 12: 1369-1376,

Shahar E Shinawi M: Neurocristopathies presenting with neurologic abnormalities associated with Hirschsprung's disease. Pediatr Neurol. 2003 May;28(5):38591.

Shimotake T, Tanaka S, Fukui R, Makino S, Maruyama R. Neuroglial disorders of central and peripheral nervous systems in a patient with Hirschsprung's disease carrying allelic SOX10 truncating mutation. J Pediatr Surg. 2007Apr;42(4):725-31.

Silengo M, Ferrero GB, Wakamatsu : Pachygyria and cerebellar hypoplasia in a patient with a 2q22-q23 deletion that includes the ZFHX1B gene.Am J Med Genet 2004, 127A:109.

Spritz RA: Piebaldism, Waardenburg syndrome, and related disorders of melanocyte development. Semin Cutan Med Surg. 1997 Mar;16(1):15-23.

Stevens B.: Neuron-astrocyte signaling in the development and plasticity of neural circuits. Neurosignals. 2008;16(4):278-88. Epub 2008 Jul 18.

Tam PKH: An Immunochemical Study With Neuron-Specific-Enolase And Substance P of Human Enteric Innervation. The Normal Developmental Pattern And Abnormal Deviations in Hirschsprung's Disease And Pyloric Stenosis. J Pediatr Surg 1986; 21 : 227-232.

Trainor PA: Specification of neural crest cell formation and migration in mouse embryos. Semin Cell Dev Biol. 2005 Dec;16(6):683-93. 
Turkdogan-Sozuer D Ozek MM Sehiralti V Kurtkaya O Sav A: Hemimegalencephaly and Hirschsprung's Disease: a Unique Association. Pediatr Neurol 1998 ;18: 452-455.

Van de Putte, T., Francis, A., Nelles, L., van Grunsven, L. A., Huylebroeck, D. Neural crestspecific removal of $\mathrm{Zfhx} 1 \mathrm{~b}$ in mouse leads to a wide range of neurocristopathies reminiscent of Mowat-Wilson syndrome. Hum. Molec. Genet. 2007; 16: 14231436.Van de Putte, T., Maruhashi, M., Francis, A., Nelles, L., Kondoh, H., Huylebroeck, D., Higashi, Y. Mice lacking Zfhx1b, the gene that codes for Smadinteracting protein-1, reveal a role for multiple neural crest cell defects in the etiology of Hirschsprung disease-mental retardation syndrome. Am. J. Hum.Genet. 2003; 72: 465-470,

Verstappen, G., van Grunsven, L. A., Michiels, C., Van de Putte, T., Souopgui, J., Van Damme, J., Bellefroid, E., Vandekerckhove, J., Huylebroeck, D. Atypical MowatWilson patient confirms the importance of the novel association between ZFHX1B/SIP1 and NuRD corepressor complex. Hum. Molec. Genet. 2008; 17: 11751183.

Volterra A, Meldolesi J. Astrocytes, from brain glue to communication elements: the revolution continues. Nat Rev Neurosci. 2005 Aug;6(8):626-40. Review

Wakamatsu N, Yamada Y, Yamada K, Ono T, Nomura N, Taniguchi H, Kitoh H, Mutoh N, Yamanaka T, Mushiake K, Kato K, Sonta S, Nagoya M. Mutations in SIP1, encoding Smad interacting protein-1, cause a form of Hirschsprung disease. Nat Genet 2001; 27:369-370.

Williams C, Lossie A, Driscoll D, and the RC Phillips Unit. Angelman syndrome: mimicking conditions and phenotypes. Am J Med Genetics 2001;101:59-64.

Yamada, K., Yamada, Y., Nomura, N., Miura, K., Wakako, R., Hayakawa, C., Matsumoto, A., Kumagai, T., Yoshimura, I., Miyazaki, S., Kato, K., Sonta, S., Ono, H., Yamanaka, T., Nagaya, M., Wakamatsu, N. Nonsense and frameshift mutations in ZFHX1B, encoding Smad-interacting protein 1, cause a complex developmental disorder with a great variety of clinical features. Am. J. Hum. Genet. 2001; 69: 11781185 .

Yamanaka K, Chun SJ, Boillee S, et al. Astrocytes as determinants of disease progression in inherited amyotrophic lateral sclerosis. Nat Neurosci 2008;11:251-253.

Yang Y, Gozen O, Watkins A, Lorenzini I, Lepore A, Gao Y, Vidensky S, Brennan J, Poulsen D, Won Park J, Li Jeon N, Robinson MB, Rothstein JD Presynaptic regulation of astroglial excitatory neurotransmitter transporter GLT1. Neuron. 2009 Mar 26;61(6):880-94.

Zhang W, Segura BJ, Lin TR, et al. Intercellular calcium waves in cultured enteric glia from neonatal guinea pig. Glia 2003;42:252-262.

Zweier C, Albrecht B, Mitulla B, Behrens R, Beese M, Gillessen-Kaesbach G, Rott HD, Rauch A: "Mowat-Wilson" Syndrome with and without Hirschsprung Disease is a distinct, recognizable Multiple Congenital Anomalies-Mental Retardation Syndrome caused by Mutations in the Zinc finger homeobox 1 B gene (ZFHX1B). Am J Med Genet 2002, 108(3):177-181. 
Zweier C, Temple IK, Beemer F, Zackai E, Lerman-Sagie T, Weschke B, Anderson CE, Rauch A: Characterisation of deletions of the ZFHX1B region and genotype-phenotype analysis in Mowat-Wilson syndrome. J Med Genet 2003, 40:601-605. 


\title{
Role of Neuroimaging in Brain Radiosurgery
}

\author{
Tomoyuki Koga and Nobuhito Saito \\ The University of Tokyo \\ Japan
}

\section{Introduction}

Radiosurgery is a therapeutic technique to deliver high dose radiation to the target. It induces radiobiological responses of lesions while minimizing radiation effect to the surrounding area. Gamma knife is one of the representative devices for stereotactic radiosurgery, which is exclusively designed for treatment of cerebral disorders. Gamma knife was developed as the first radiosurgical device in 1967. Professor Lars Leksell at the Karolinska Institute in Sweden developed a stereotactic frame for functional neurosurgery in early 1950s and combined the technique of frame fixation with focused irradiation. The primary role of gamma knife radiosurgery is to control small well-demarcated lesions such as metastatic brain tumors, meningiomas, schwannomas, and pituitary adenomas while preserving function of surrounding brain tissue. It has been used as a primary treatment or in combination with surgery, and some applications have been accepted as standard treatment. Treatment of cerebral arteriovenous malformations has also been drastically changed after emergence of this technology. Arteriovenous malformation in deep locations such as the basal ganglia, thalamus and the brainstem came to be safely treated by radiosurgery. Stereotactic radiosurgery is also employed in the control of functional disorders such as mesial temporal epilepsy and trigeminal neuralgia. As such, gamma knife has been widely accepted in clinical practice in the field of neurosurgery (Koga et al., 2010). On the other hand, the risk of radiation-induced adverse events is not negligible and puts limitations to this technology. Accurate target definition is one of the most important factors to reduce complications because it can reduce unwanted radiation to surrounding normal tissue. The target of radiosurgery is primarily visualized by radiological studies such as angiography, computed tomography and magnetic resonance imaging. Owing to this, the accuracy of treatment largely depends on neuroimaging technology. After introduction of gamma knife, technology of neuroimaging has also been drastically changed as an example of magnetic resonance imaging. Not only neuroimaging itself, but also software to utilize different kinds of imaging modalities for treatment planning of radiosurgery has been developed in the past decades. During almost a half century since treatment of gamma knife radiosurgery was applied for the first patient, technology of radiosurgery has progressed in line with the advances in neuroimaging technology. In this chapter, we discuss technological advances in neuroimaging and consider i) how these have driven more sophisticated radiosurgical treatments and ii) the impact on post radiosurgical outcomes. 

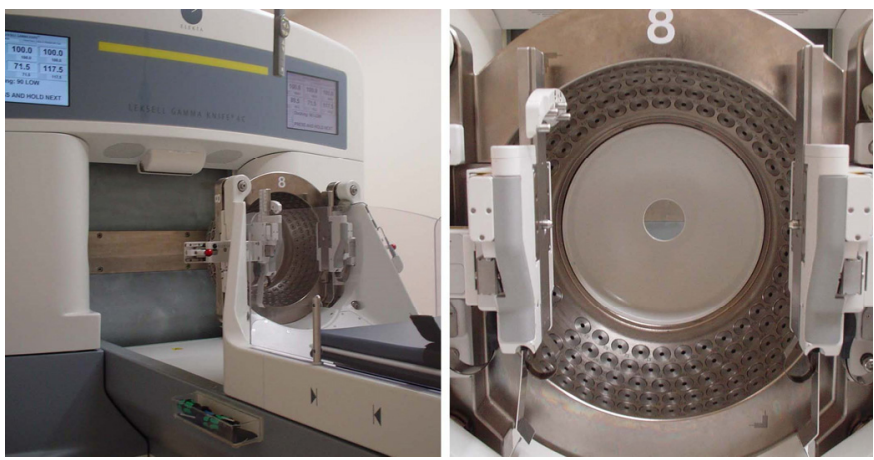

Fig. 1. Structure of gamma knife

Treatment machinery of gamma knife (left). The helmet has approximately 200 portals (collimator, right) through which gamma rays from ${ }^{60} \mathrm{Co}$ are emitted. Those finely narrowed beams of radiation meet at the focal point of gamma knife and give a high target dose.

\begin{tabular}{ll}
\hline Diagnosis & Number of Treatment \\
\hline Metastatic brain tumor & $185,070(36.8 \%)$ \\
Glioma & $26,437(5.3 \%)$ \\
Other malignant tumor & $9,389(1.9 \%)$ \\
\hline Meningioma & $64,115(12.8 \%)$ \\
Vestibular schwannoma & $46,835(9.3 \%)$ \\
Pituitary adenoma & $38,553(7.7 \%)$ \\
Other benign tumor & $26,816(5.3 \%)$ \\
\hline Vascular lesion & $65,084(12.9 \%)$ \\
\hline Trigeminal neuralgia & $32,798(6.5 \%)$ \\
Epilepsy & $2,399(0.5 \%)$ \\
Other functional disorder & $3,264(0.6 \%)$ \\
Ocular lesion & $1,966(0.4 \%)$ \\
\hline Total & 502,726 \\
\hline
\end{tabular}

Table 1. Number of gamma knife treatment in the world until 2008

\section{Integration of three-dimensional images into treatment planning}

Arteriovenous malformation has been treated by gamma knife radiosurgery since it was introduced at first. Emergence of gamma knife has improved treatment outcomes of cerebral arteriovenous malformations, especially deeply located lesions (Sasaki et al., 1998). The goal of radiosurgery for arteriovenous malformations is obliteration of nidus and prevention of devastating hemorrhage. Histopathological study of arteriovenous malformation after radiosurgery showed damage of endothelial cells, followed by thickening of the intimal layer caused by smooth-muscle cell proliferation, and finally obliteration of the lumen by cellular degeneration and hyalinization (Schneider et al., 1997). Minimum dose of 20 Gy to the nidus could completely obliterate nidus in approximately $90 \%$ in the dose response analysis for arteriovenous malformations (Flickinger et al., 2002). In our experience, the rates 
of nidus obliteration confirmed by angiography were $72 \%$ at 3 years and $83 \%$ at 5 years (Shin et al., 2004). In early years of gamma knife treatment, treatment planning for arteriovenous malformation was made based only on angiogram. Because conventional angiogram was two-dimensional projection image, it was difficult to comprehend threedimensional structure of lesions only by angiogram especially when nidus was large or morphologically complicated. Computed tomography or magnetic resonance imaging could depict three-dimensional structure of nidus of arteriovenous malformation, although angiography could show more precise angioarchitecture. Therefore, use of computed tomography or magnetic resonance imaging jointly with angiography for treatment planning facilitated recognizing three-dimensional structure of nidus and reduced the morbidity associated with gamma knife radiosurgery (Shin et al., 2004). More recently, integration of three-dimensional rotational angiography into treatment planning of radiosurgery for arteriovenous malformation has been attempted (Conti et al., 2011). Because three-dimensional rotational angiography can provide both sufficient temporal and spatial resolution, three-dimensional angioarchitecture of nidus can be easily understood by using this technology, and reports of outcomes including obliteration rates and morbidity after treatment with integration of three-dimensional rotational angiography are awaited.

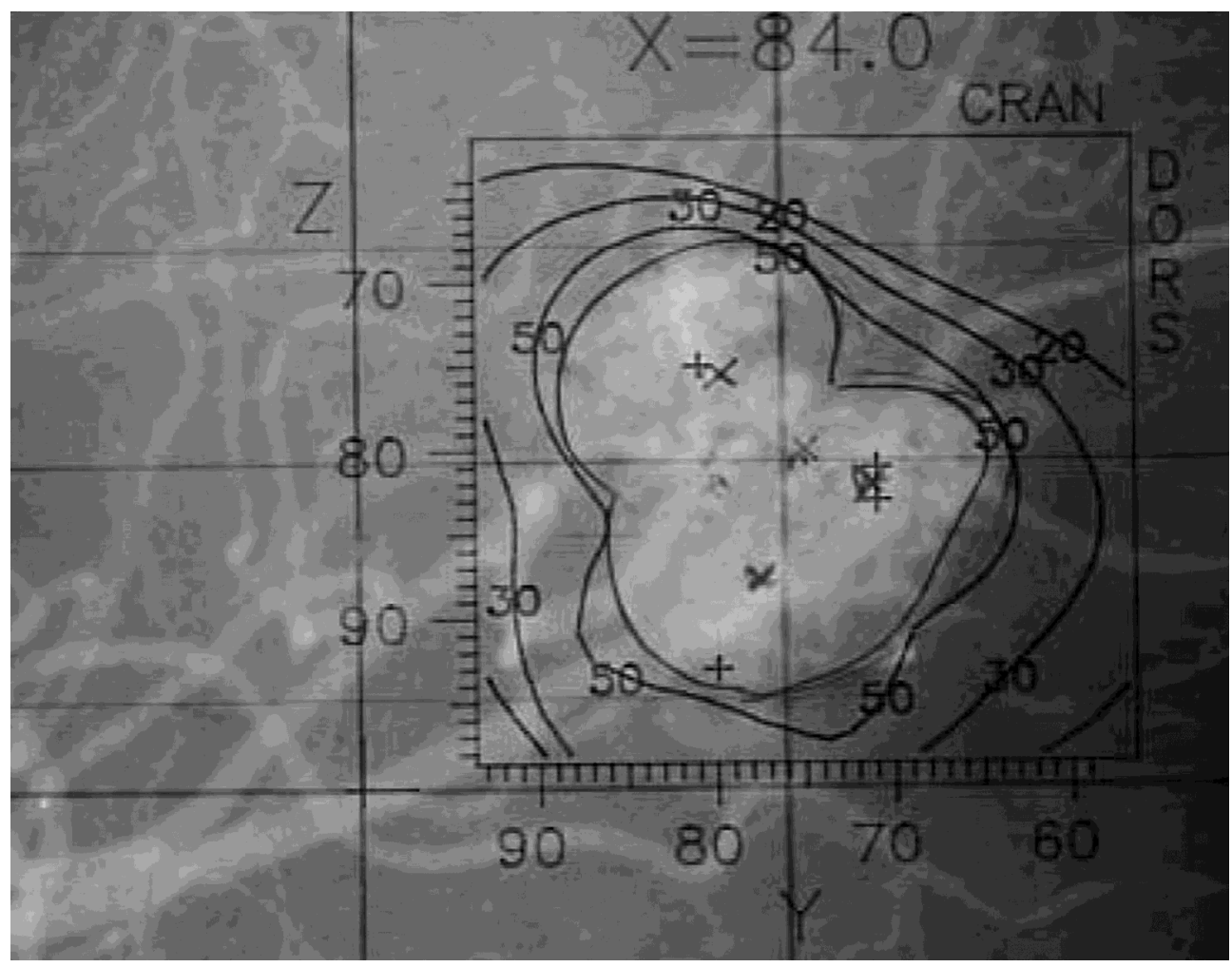

Fig. 2. Treatment planning for arteriovenous malformation based on angiogram. Twodimensional isodose lines (black) were superimposed to the image. It was difficult to recognize three-dimensional angioarchitecture from angiogram only. 

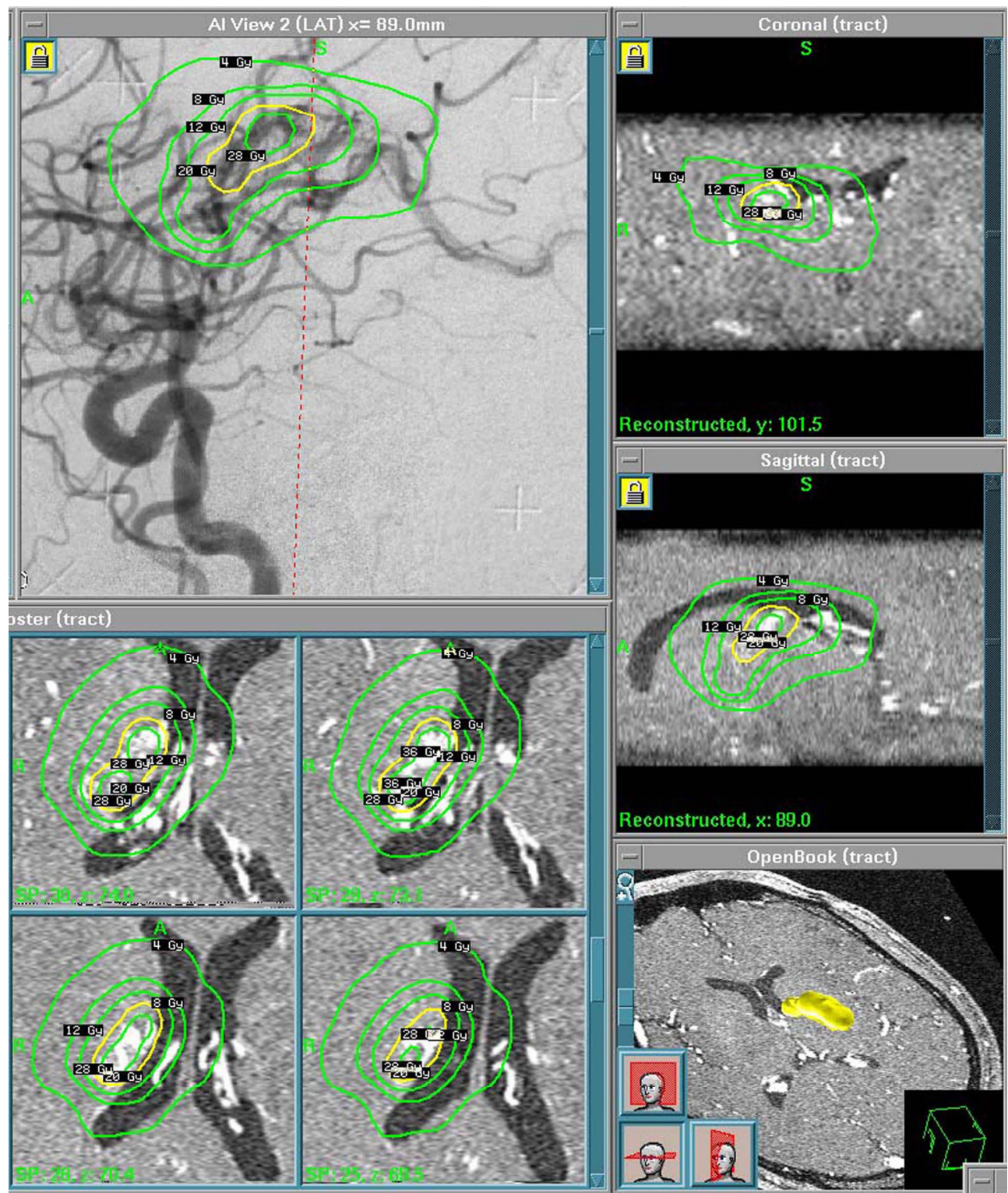

Fig. 3. Treatment planning for a patient with right thalamic arteriovenous malformation. Isodose lines (yellow and green) were simultaneously superimposed to digital subtraction angiogram and magnetic resonance images. Coronal and sagittal images (right upper two images) were simultaneously visible in combination with dose distribution and made recognition of three-dimensional angioarchitecture easier. 


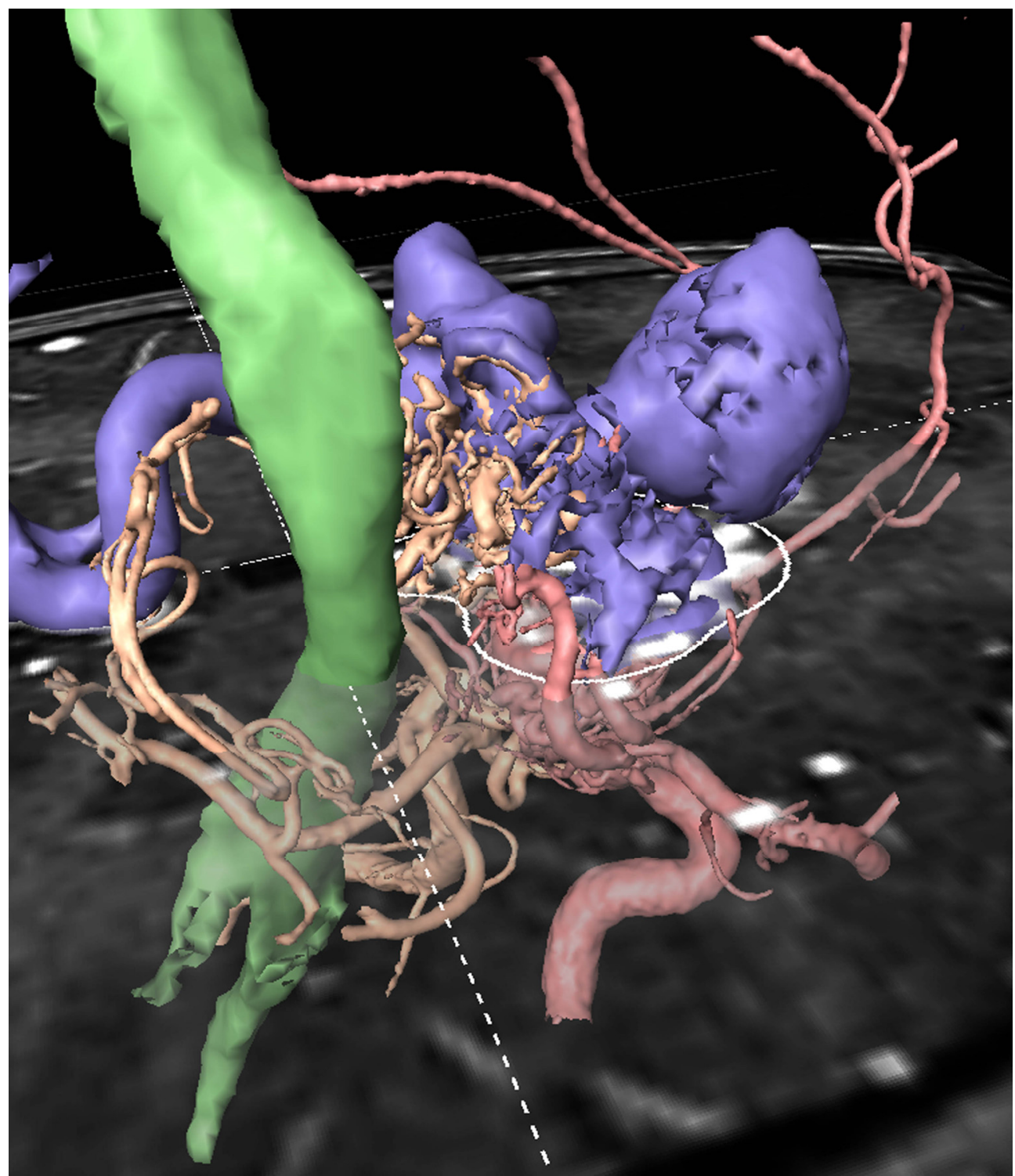

Fig. 4. Three-dimensional rotational angiography in treatment planning for arteriovenous malformation. Nidus and feeder from internal carotid artery (red) and basilar artery (orange) was visible in three-dimensional manner. Drainers (purple) were distinguished from nidus. Isodose line of treatment planning (white line) was superimposed to the image and sufficient coverage by treatment dose was visually confirmed. In this view tractography of the corticospinal tract (green) based on diffusion tensor magnetic resonance imaging was also integrated. 


\section{Integration of multimodal neuroimaging studies into treatment planning}

Since gamma knife was developed, several refinements on its hardware and software have been made. Collimator helmet was modified when the second generation gamma knife was developed and computer workstation was introduced thereafter. The introduction of GammaPlan (Elekta Instrument AB, Stockholm, Sweden), a software for treatment planning workstation, achieved accurate dose planning (Cheung et al., 2000). The third generation of gamma knife was equipped with automatic positioning system, which was machinery that automatically moved the patient's head through a stereotactic frame during irradiation. This model enabled more conformal treatment in shorter time and reduced unwanted radiation exposure to patients compared with prior models (Horstmann \& Van Eck, 2002, Kuo et al., 2004). Thus, the advances in gamma knife radiosurgery greatly depend on the refinement and the development of new devices and software (Kondziolka et al., 2002, Regis et al., 2002). In view of neuroimaging, the significant modification of gamma knife was development of GammaPlan 4C (Elekta Instrument AB, Stockholm, Sweden), which has started to be utilized since 2004. This software enabled coregistration of multimodal neuroimages even taken without frame fixation (Koga et al., 2009). For example, when lesions and bony structures or the orbital fat were indistinguishable on magnetic resonance imaging for planning, coregistered computed tomography helped clearly contour tumor margin along the bony structures.
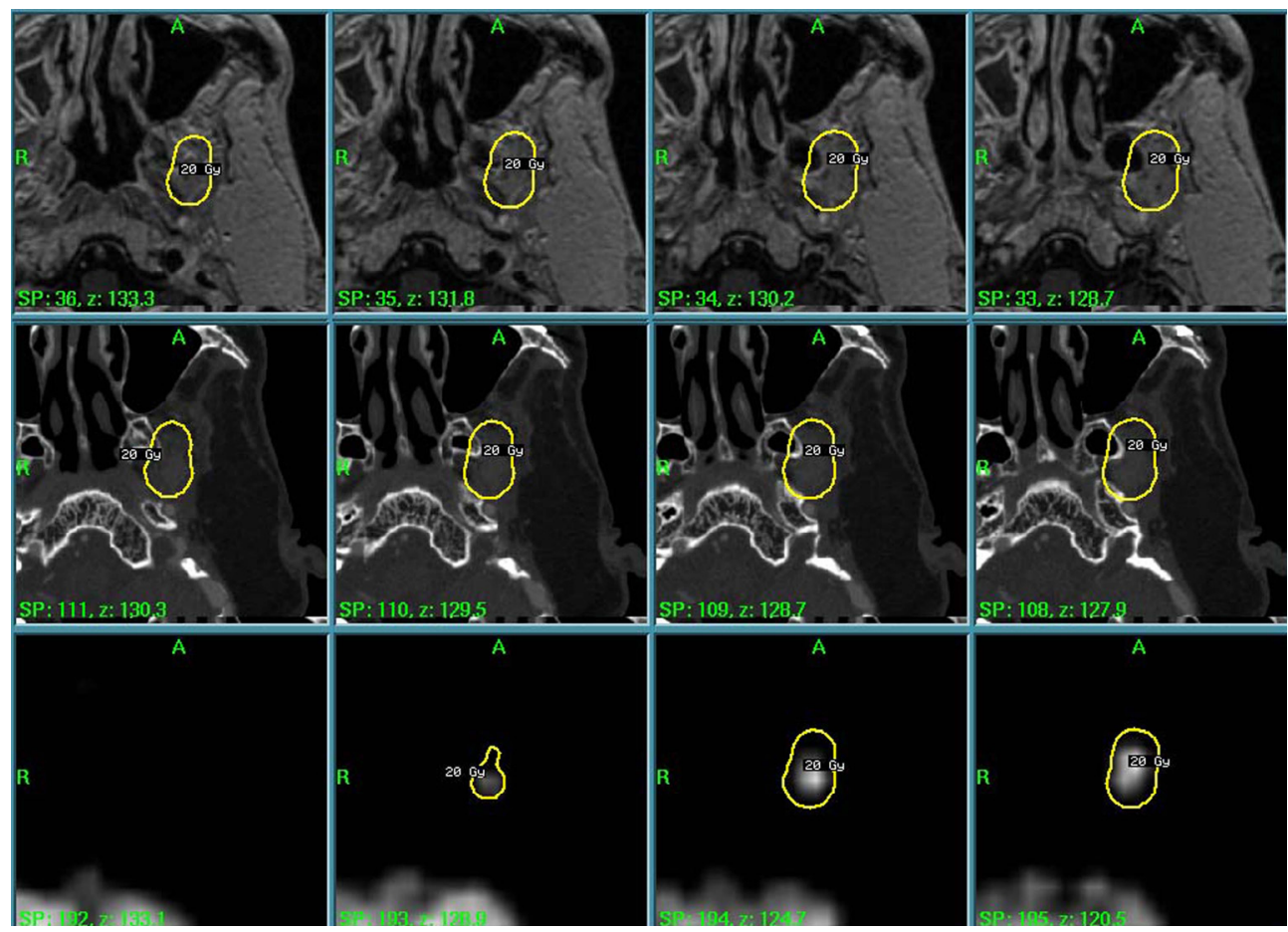

Fig. 5. Treatment planning for a patient with recurrent skull base carcinoma. Computed tomography (middle) and positron emission tomography (lower) was integrated. 
Positron emission tomography (PET) enabled access to metabolic information of lesions at the time of treatment planning for radiosurgery (Levivier et al., 2007, Levivier et al., 2000, Levivier et al., 2002). In our experience, we utilized [ $\left.{ }^{18} \mathrm{~F}\right]$ fluorodeoxyglucose (FDG) PET in patients with malignant tumors to visualize the possibly active component among diffuse enhancement on magnetic resonance images. For example, in a case of recurrent glioblastoma multiforme, ambiguously enhanced lesion on magnetic resonance imaging was suggested to be highly malignant area, because the coregistered FDG-PET showed high uptake at the corresponding area, and this region was irradiated. As another example, 111C-acetate PET was utilized for evaluation of extent of meningioma and response of lesions to radiosurgery (Liu et al., 2010).
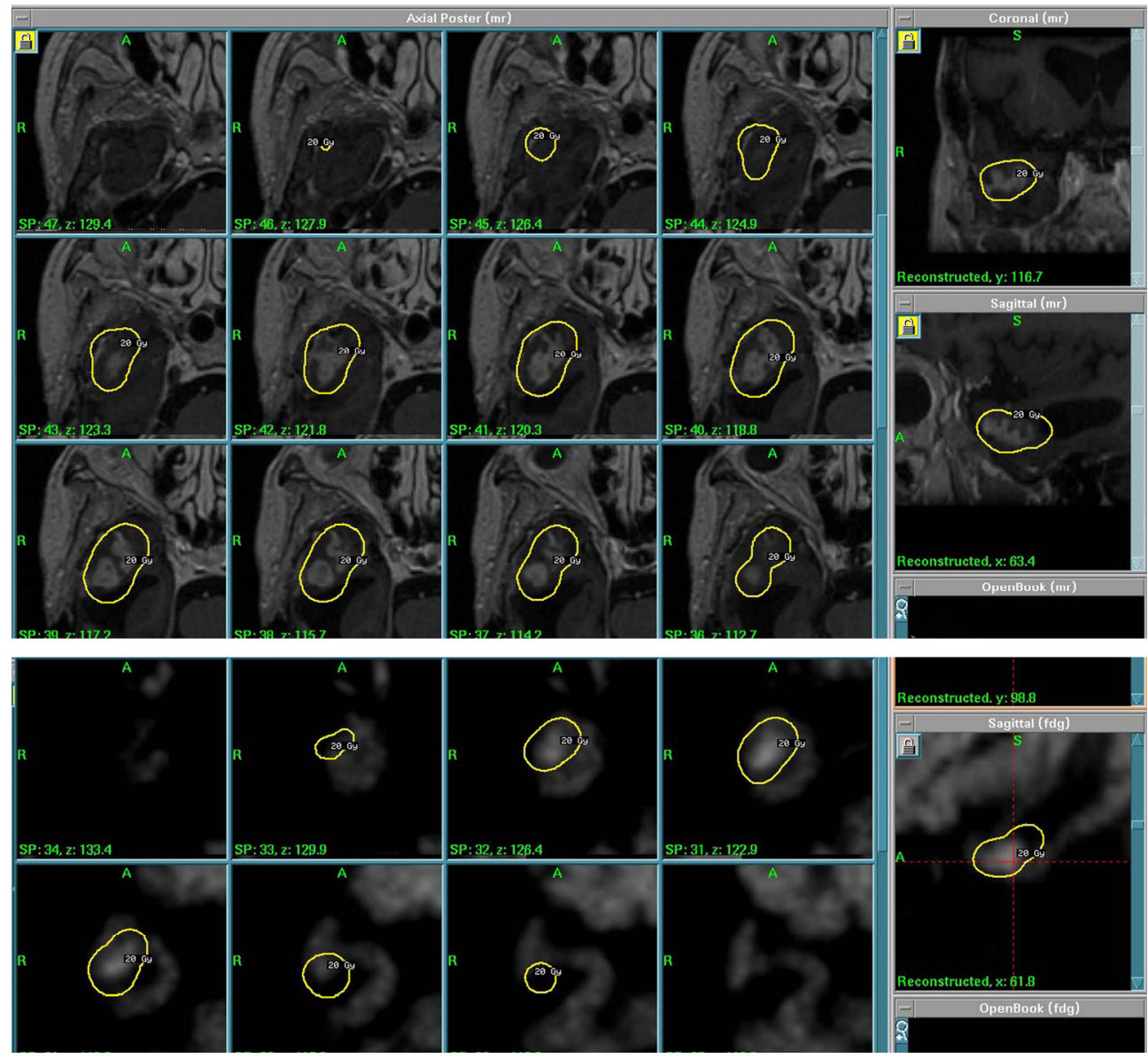

Fig. 6. Treatment planning for a patient with recurrent glioblastoma. An enhanced lesion in the right temporal lobe shown by magnetic resonance imaging (upper). Corresponding area was confirmed as high uptake region by FDG-PET (lower). This lesion was irradiated with margin dose of 20 Gy (yellow line). 


\section{Integration of structural and functional imaging into treatment planning}

Diffusion tensor tractography, one of the major recent advances in neuroimaging, enabled clear visualization of various fibers inside the white matter of the brain, which was not visualized by conventional imaging modalities (Masutani et al., 2003). Clinical application of diffusion tensor tractography were mainly reported as diagnostic tools, and the report on its therapeutic application was quite limited (Maruyama et al., 2005). Although the pointed limitation of tractography is its reliability claiming that there is no guarantee that fibers do not exist where the tracts is not drawn (Holodny et al., 2005, Yamada et al., 2009), intraoperative fiber stimulation analysis proved that tractography reflected functioning pyramidal tracts to some extent (Kamada et al., 2009). Integration of tractography into intraoperative navigation was also developed at our institute (Kamada et al., 2005). However, it contains risks of inevitable brain shift caused by craniotomy or tumor removal, thus leads to poorer accuracy. On the other hand, such a shift does not occur in the setting of integration of tractography into radiosurgery. At our institute, integration of diffusion tensor tractography into radiosurgical treatment planning was started in 2004. While there were a variety of white matter fiber tracts, we considered that the pyramidal tract would be the most important tract in preventing morbidity of radiosurgery because its injury caused motor paresis and led to decline of activity of daily living. (Andrade-Souza et al., 2006, Hadjipanayis et al., 2001) At the same time, the pyramidal tract was practically the easiest one to draw from the technical point of view. (Yamada et al., 2009) The optic radiation and the arcuate fasciculus would be next important and was more difficult to draw. (Catani et al., 2005, Yamamoto et al., 2007) Injury of the optic radiation causes visual disturbance. Verbal function requires participation of a distributed neural system in the dominant hemisphere, and we integrated the arcuate fasciculus tractography in order to preserve this function. For the time being, we are introducing above three tracts, considering them as critical white matter structures to be preserved. By analyzing relationship between occurrence of radiation-induced neuropathy and the maximum dose received by each tract, tolerable doses of each white matter tract were estimated. The estimated tolerable dose for the corticospinal tract was approximately $20 \mathrm{~Gy}$. The dose was $8 \mathrm{~Gy}$ for the optic radiation, 8 Gy and 20 Gy for temporal and frontal fibers of the arcuate fasciculus, respectively (Maruyama et al., 2008, Maruyama et al., 2005, Maruyama et al., 2007). Among 144 patients with arteriovenous malformation who underwent radiosurgical procedure after integration of tractography was started, one patient experienced worsening of pre-existing dysesthesia, one patient exhibited mild transient hemiparesis, and the other two patients who were treated before integration of arcuate fasciculus tractography was started experienced transient speech disturbance during median follow-up of 23 months. Overall, the rate of radiation-induced neuropathy was $2.8 \%$. Since the rates of radiation-induced neuropathy were generally reported as five to $20 \%$ (Andrade-Souza et al., 2005, Flickinger et al., 1999, Maruyama et al., 2005, Pollock et al., 2004, Sasaki et al., 1998), permanent and transient morbidity was suggested to be potentially reduced by integrating diffusion-tensor tractography into treatment planning of radiosurgery (Koga et al., 2011). Two major concerns regarding this new technique were i) whether integration of tractography significantly reduced morbidity and ii) whether alteration of dose planning using tractography compromised obliteration of arteriovenous malformation, which was regarded as cure of this disease. To unveil these issues, further follow-up and accumulation of data of patients who underwent tractography-integrated radiosurgery are awaited. 


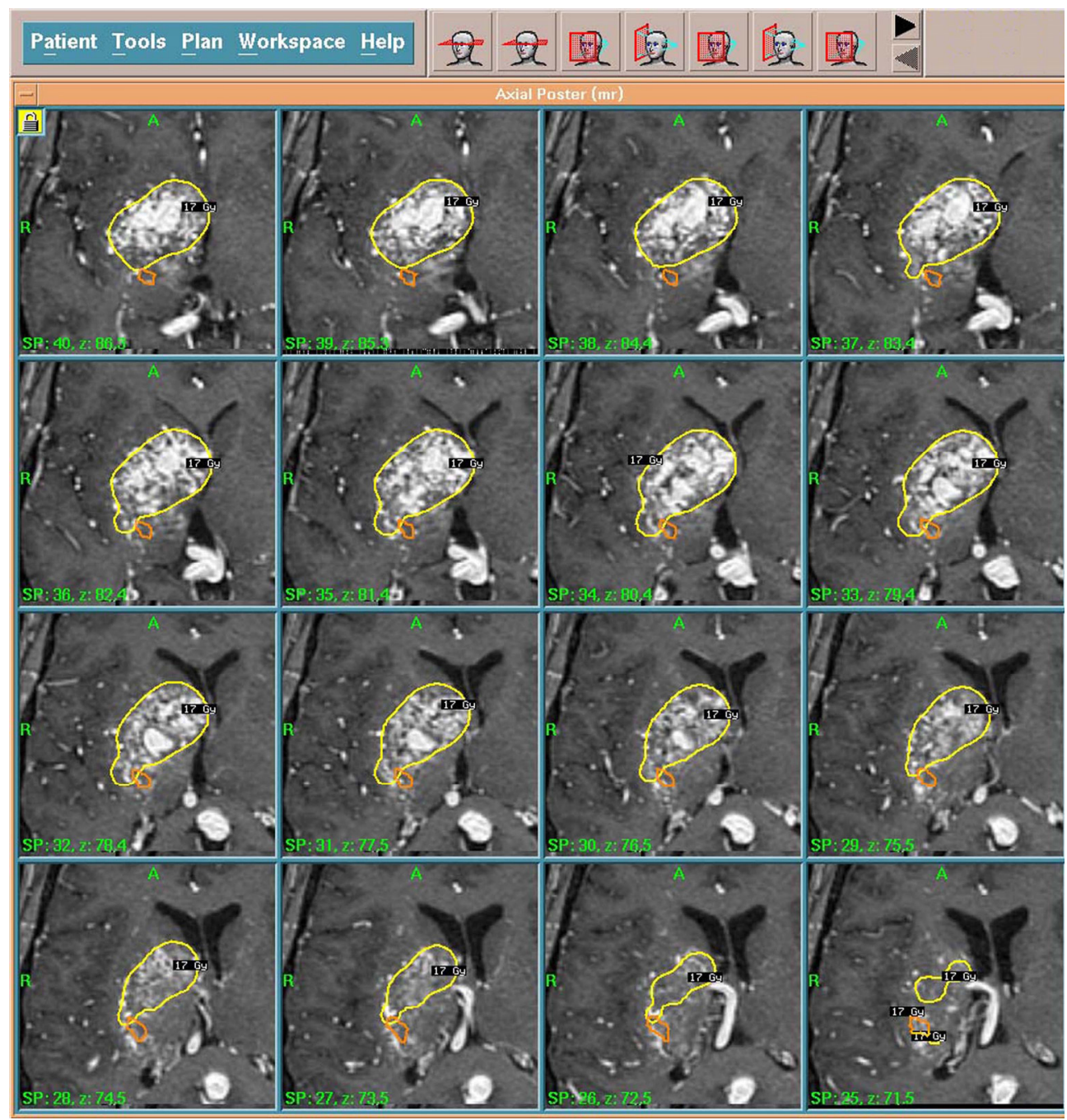

Fig. 7. Treatment planning for a patient with ruptured arteriovenous malformation in the right basal ganglia. Magnetic resonance imaging for treatment planning and integrated diffusion-tensor tractography of the right corticospinal tract showed that the tract (orange) was passing through just behind the nidus of arteriovenous malformation. Therefore, dose distribution at around the posterior portion of the nidus modified so as the dose received by the right corticospinal tract not to exceed $20 \mathrm{~Gy}$, which was considered as tolerable dose of the corticospinal tract. This patient presented mild left hemiparesis at the onset of cerebral hemorrhage. However, no further worsening of hemiparesis was observed until last followup at 23 months after gamma knife treatment. 


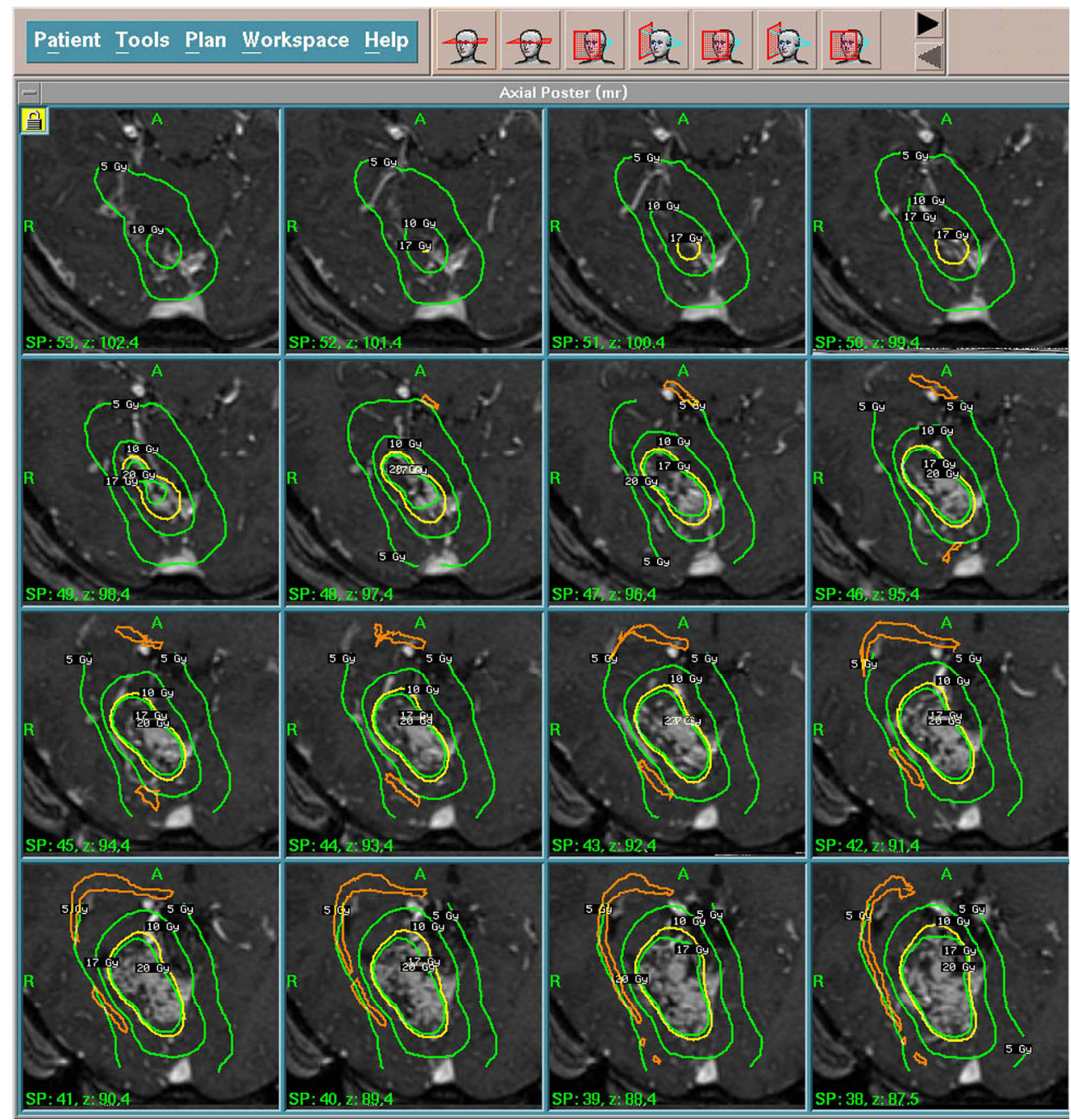

Fig. 8. Treatment planning of gamma knife stereotactic radiosurgery for a patient with unruptured arteriovenous malformation in the right occipital lobe. Magnetic resonance imaging for treatment planning and integrated diffusion-tensor tractography of the right optic radiation showed that the tract (orange) was passing through just lateral to the nidus of arteriovenous malformation. Therefore, dose distribution around the nidus was modified and dose fall-off to the lateral side of nidus was adjusted to be steeper by blocking a part of beams from cobalt sources so as the dose received by the right optic radiation not to exceed $8 \mathrm{~Gy}$, the dose considered as tolerable dose of the optic radiation, while margin of the nidus was irradiated by $20 \mathrm{~Gy}$. This patient presented with headache at the onset. The patient had presented no signs of left visual field defect during the clinical course until the last followup at 16 months after radiosurgery. 
Diffusion tensor tractography was used for CyberKnife stereotactic radiosurgery as well. The technique was utilized in treatment planning for several kinds of cerebral disorders such as arteriovenous malformation, hemangioma and metastatic brain tumor. They successfully reduced the dose received by the optic tract and the corticospinal tract by integrating diffusion tensor tractography of these fibers. They further utilized blood oxygenation level-dependent functional magnetic resonance imaging to visualize the primary motor cortex and reduced the dose delivered to the cortex (Pantelis et al., 2010). Outcomes of this type of treatment are awaited.

As another example of functional imaging, magnetoencephalography was useful to detect firing neurons not only in the cortex, but also in the deeply located structures (Stefan et al., 1990). Since very early stage of gamma knife radiosurgery, magnetoencephalography was used to visualize epileptic foci in treatment planning for focal epilepsy (Hellstrand et al., 1993). Further, magnetoencephalography was as useful to detect functioning cortex as functional magnetic resonance imaging. Use of magnetoencephalography in combination with magnetic resonance imaging and cerebral angiography enabled understanding the relationship between angioarchitecture of arteriovenous malformation nidus and the adjacent sensorimotor cortex (Kamiryo et al., 2002).

\section{Role of neuroimaging in follow-up study after radiosurgery}

Accumulation of radiological knowledge is also important to appropriately follow-up patients after treatment. One of the most difficult issues regarding follow-up of patients with malignant tumors who underwent radiosurgery is differentiation of tumor recurrence and radiation effect including radiation necrosis. Tumor recurrence necessitates additional treatment including surgical resection. On the other hand, radiation necrosis usually responds to administration of steroid. Therefore, these two pathologies should be appropriately differentiated to be appropriately treated. An analysis of correlation between magnetic resonance imaging after radiosurgery and histopathological data revealed that a distinct lesion margin on T2-weigheted images as well as a contrast-enhanced margin on T1weighted images was highly correlated with tumor recurrence, and that the pathology was associated with a higher rate of necrosis when the lesion border on T2-weighted images did not correspond to the contrast-enhanced T1 lesion volume (Kano et al., 2010).

Another example is the findings that predict the risk of hemorrhage from completely obliterated arteriovenous malformations treated by gamma knife radiosurgery. Although complete obliteration of the nidus can be considered as cure of arteriovenous malformation which was treated by radiosurgery, the risk of hemorrhage from obliterated nidus still slightly remains. The annual bleeding rate from completely obliterated arteriovenous malformation was $0.3 \%$ (Shin et al., 2005). A study of contrast-enhanced magnetic resonance imaging findings in angiographically obliterated arteriovenous malformation revealed that increment of the enhanced regions within 1 year of angiographic obliteration compared with the previous measurement was highly associated with hemorrhage after angiographical obliteration. They concluded that patients with this finding should be carefully followed-up by contrast-enhanced magnetic resonance imaging, while contrast-enhanced studies could be reduced for those without the increment of contrast-enhanced area in the first year after obliteration (Kunishima et al., 2010). 


\section{Conclusion}

As described above, radiosurgery using gamma knife was quite dependent on neuroimaging technology because the target of radiosurgery was solely defined by imaging studies. Introduction of basic three-dimensional imaging studies such as computed tomography and magnetic resonance imaging made recognition of threedimensional structure of the target on planning more feasible compared with the planning solely based on two-dimensional projection images such as angiography. In fact, analyses of patient outcomes after radiosurgery proved that the use of threedimensional imaging studies for treatment planning had reduced the morbidity associated with unwanted irradiation. Thus, more accurate target definition enabled by advances in imaging technology truly resulted in safer treatment even in this example of simple progress in the very early stage of brain radiosurgery. Technological advances in computed tomography and magnetic resonance imaging themselves achieved not only images of higher resolution but also imaging studies of completely different quality such as images of vessels and wide variety of sequences of magnetic resonance imaging. Further, metabolic imaging such as positron emission tomography became widely accepted in the field of neuroimaging as well. Technological progress of treatment planning software for gamma knife radiosurgery made it possible to utilize wide variety of neuroimaging studies for process of target definition in planning. By this way, technology of gamma knife radiosurgery could incorporate the fruit of development in neuroimaging and more sophisticated treatment planning based on concise target definition became feasibly available. Furthermore, development such as imaging of neural fibers which was "invisible" on ordinary imaging modalities and visualization of neural function was peculiar to the field of neuroimaging. Protecting neural function of the central nervous system is one of the most important goals of brain radiosurgery. Therefore, as most recent advance in imaging technology, these modalities of neuroimaging were indispensable for development of radiosurgery. Although introduction of neurostructural or functional imaging into treatment planning of brain radiosurgery just became realistic, several recent data suggested that modification of treatment planning to protect white matter visible on diffusion tensor magnetic resonance imaging and functioning cortex visualized in functional magnetic resonance imaging from excessive irradiation during stereotactic radiosurgery contributed to preservation of neural function associated with these structures. Since these kinds of new technology are quite recent progress, it is true that outcomes of patients who underwent radiosurgery using new technology must be carefully followed-up for long period after treatment. However, brain radiosurgery has been developed in this manner in line with recent drastic advances in neuroimaging technology, and technological progress in both radiosurgery and neuroimaging is still in continuation. Therefore, development of safer and more effective brain radiosurgery will be continued in the future as long as progress of neuroimaging technology is on its way.

\section{Acknowledgment}

We are grateful to Dr. Taichi Kin at the University of Tokyo for his help in making a threedimensional computer image of arteriovenous malformation and radiosurgical planning used in this chapter. 


\section{References}

Andrade-Souza, Y. M., Zadeh, G., Scora, D., Tsao, M. N. \& Schwartz, M. L. (year 2005). Radiosurgery for basal ganglia, internal capsule, and thalamus arteriovenous malformation: clinical outcome, Neurosurgery 56(1): 56-64.

Andrade-Souza, Y. M., Ramani, M., Scora, D., Tsao, M. N., TerBrugge, K. \& Schwartz, M. L. (year 2006). Radiosurgical treatment for rolandic arteriovenous malformations, $J$ Neurosurg 105(5): 689-697.

Catani, M., Jones, D. K. \& ffytche, D. H. (year 2005). Perisylvian language networks of the human brain, Ann Neurol 57(1): 8-16.

Cheung, Y. C., Yu, K. N., Ho, R. T. \& Yu, C. P. (year 2000). Stereotactic dose planning system used in Leksell Gamma Knife model-B: EGS4 Monte Carlo versus GafChromic films MD-55, Appl Radiat Isot 53(3): 427-430.

Conti, A., Pontoriero, A., Farago, G., Midili, F., Siragusa, C., Granata, F., Pitrone, A., De Renzis, C., Longo, M. \& Tomasello, F. (year 2011). Integration of Three-Dimensional Rotational Angiography in Radiosurgical Treatment Planning of Cerebral Arteriovenous Malformations, Int J Radiat Oncol Biol Phys.

Flickinger, J. C., Kondziolka, D., Lunsford, L. D., Pollock, B. E., Yamamoto, M., Gorman, D. A., Schomberg, P. J., Sneed, P., Larson, D., Smith, V., McDermott, M. W., Miyawaki, L., Chilton, J., Morantz, R. A., Young, B., Jokura, H. \& Liscak, R. (year 1999). A multi-institutional analysis of complication outcomes after arteriovenous malformation radiosurgery, Int J Radiat Oncol Biol Phys 44(1): 67-74.

Flickinger, J. C., Kondziolka, D., Maitz, A. H. \& Lunsford, L. D. (year 2002). An analysis of the dose-response for arteriovenous malformation radiosurgery and other factors affecting obliteration, Radiother Oncol 63(3): 347-354.

Hadjipanayis, C. G., Levy, E. I., Niranjan, A., Firlik, A. D., Kondziolka, D., Flickinger, J. C. \& Lunsford, L. D. (year 2001). Stereotactic radiosurgery for motor cortex region arteriovenous malformations, Neurosurgery 48(1): 70-76; discussion 76-77.

Hellstrand, E., Abraham-Fuchs, K., Jernberg, B., Kihlstrom, L., Knutsson, E., Lindquist, C., Schneider, S. \& Wirth, A. (year 1993). MEG localization of interictal epileptic focal activity and concomitant stereotactic radiosurgery. A non-invasive approach for patients with focal epilepsy, Physiol Meas 14(2): 131-136.

Holodny, A. I., Watts, R., Korneinko, V. N., Pronin, I. N., Zhukovskiy, M. E., Gor, D. M. \& Ulug, A. (year 2005). Diffusion tensor tractography of the motor white matter tracts in man: Current controversies and future directions, Ann N Y Acad Sci 1064: 88-97.

Horstmann, G. A. \& Van Eck, A. T. (year 2002). Gamma knife model C with the automatic positioning system and its impact on the treatment of vestibular schwannomas, $J$ Neurosurg 97(5 Suppl): 450-455.

Kamada, K., Todo, T., Masutani, Y., Aoki, S., Ino, K., Takano, T., Kirino, T., Kawahara, N. \& Morita, A. (year 2005). Combined use of tractography-integrated functional neuronavigation and direct fiber stimulation, J Neurosurg 102(4): 664-672.

Kamada, K., Todo, T., Ota, T., Ino, K., Masutani, Y., Aoki, S., Takeuchi, F., Kawai, K. \& Saito, N. (year 2009). The motor-evoked potential threshold evaluated by tractography and electrical stimulation, J Neurosurg 111(4): 785-795. 
Kamiryo, T., Cappell, J., Kronberg, E., Woo, H. H., Jafar, J. J., Llinas, R. R. \& Nelson, P. K. (year 2002). Interactive use of cerebral angiography and magnetoencephalography in arteriovenous malformations: technical note, Neurosurgery 50(4): 903-910; discussion 910-901.

Kano, H., Kondziolka, D., Lobato-Polo, J., Zorro, O., Flickinger, J. C. \& Lunsford, L. D. (year 2010). T1/T2 matching to differentiate tumor growth from radiation effects after stereotactic radiosurgery, Neurosurgery 66(3): 486-491; discussion 491-482.

Koga, T., Maruyama, K., Igaki, H., Tago, M. \& Saito, N. (year 2009). The value of image coregistration during stereotactic radiosurgery, Acta Neurochir (Wien) 151(5): 465471; discussion 471.

Koga, T., Shin, M. \& Saito, N. (year 2010). Role of gamma knife radiosurgery in neurosurgery: past and future perspectives, Neurol Med Chir (Tokyo) 50(9): 737-748.

Koga, T., Maruyama, K., Kamada, K., Ota, T., Shin, M., Itoh, D., Kunii, N., Ino, K., Terahara, A., Aoki, S., Masutani, Y. \& Saito, N. (year 2011). Outcomes of Diffusion Tensor Tractography-Integrated Stereotactic Radiosurgery, Int J Radiat Oncol Biol Phys.

Kondziolka, D., Maitz, A. H., Niranjan, A., Flickinger, J. C. \& Lunsford, L. D. (year 2002). An evaluation of the Model C gamma knife with automatic patient positioning, Neurosurgery 50(2): 429-431; discussion 431-422.

Kunishima, K., Shin, M., Kunimatsu, A., Aoki, S., Sasaki, H., Koga, T., Itoh, D., Mori, H., Abe, O., Ohtomo, K. \& Saito, N. (year 2010). Contrast-enhanced magnetic resonance characteristics of arteriovenous malformations after gamma knife radiosurgery: predictors of post-angiographic obliteration hemorrhage, Neurosurgery 67(1): 100109; discussion 109.

Kuo, J. S., Yu, C., Giannotta, S. L., Petrovich, Z. \& Apuzzo, M. L. (year 2004). The Leksell gamma knife Model U versus Model C: a quantitative comparison of radiosurgical treatment parameters, Neurosurgery 55(1): 168-172; discussion 172-163.

Levivier, M., Wikier, D., Goldman, S., David, P., Metens, T., Massager, N., Gerosa, M., Devriendt, D., Desmedt, F., Simon, S., Van Houtte, P. \& Brotchi, J. (year 2000). Integration of the metabolic data of positron emission tomography in the dosimetry planning of radiosurgery with the gamma knife: early experience with brain tumors. Technical note, J Neurosurg 93 Suppl 3: 233-238.

Levivier, M., Wikler, D., Jr., Massager, N., David, P., Devriendt, D., Lorenzoni, J., Pirotte, B., Desmedt, F., Simon, S., Jr., Goldman, S., Van Houtte, P. \& Brotchi, J. (year 2002). The integration of metabolic imaging in stereotactic procedures including radiosurgery: a review, J Neurosurg 97(5 Suppl): 542-550.

Levivier, M., Massager, N., Wikler, D., Devriendt, D. \& Goldman, S. (year 2007). Integration of functional imaging in radiosurgery: the example of PET scan, Prog Neurol Surg 20: 68-81.

Liu, R. S., Chang, C. P., Guo, W. Y., Pan, D. H., Ho, D. M., Chang, C. W., Yang, B. H., Wu, L. C. \& Yeh, S. H. (year 2010). 1-11C-acetate versus 18F-FDG PET in detection of meningioma and monitoring the effect of gamma-knife radiosurgery, J Nucl Med 51(6): 883-891. 
Maruyama, K, Kawahara, N, Shin, M, Tago, M, Kishimoto, J , Kurita, H, Kawamoto, S, Morita, A \& Kirino, T (year 2005). The risk of hemorrhage after radiosurgery for cerebral arteriovenous malformations, N Engl J Med 352(2): 146-153.

Maruyama, K., Kamada, K., Shin, M., Itoh, D., Aoki, S., Masutani, Y., Tago, M. \& Kirino, T. (year 2005). Integration of three-dimensional corticospinal tractography into treatment planning for gamma knife surgery, J Neurosurg 102(4): 673-677.

Maruyama, K., Kamada, K., Shin, M., Itoh, D., Masutani, Y., Ino, K., Tago, M. \& Saito, N. (year 2007). Optic radiation tractography integrated into simulated treatment planning for Gamma Knife surgery, J Neurosurg 107(4): 721-726.

Maruyama, K., Kamada, K., Ota, T., Koga, T., Itoh, D., Ino, K., Aoki, S., Tago, M., Masutani, Y., Shin, M. \& Saito, N. (year 2008). Tolerance of pyramidal tract to gamma knife radiosurgery based on diffusion-tensor tractography, Int J Radiat Oncol Biol Phys 70(5): 1330-1335.

Masutani, Y., Aoki, S., Abe, O., Hayashi, N. \& Otomo, K. (year 2003). MR diffusion tensor imaging: recent advance and new techniques for diffusion tensor visualization, Eur J Radiol 46(1): 53-66.

Pantelis, E., Papadakis, N., Verigos, K., Stathochristopoulou, I., Antypas, C., Lekas, L., Tzouras, A., Georgiou, E. \& Salvaras, N. (year 2010). Integration of functional MRI and white matter tractography in stereotactic radiosurgery clinical practice, Int J Radiat Oncol Biol Phys 78(1): 257-267.

Pollock, B. E., Gorman, D. A. \& Brown, P. D. (year 2004). Radiosurgery for arteriovenous malformations of the basal ganglia, thalamus, and brainstem, J Neurosurg 100(2): 210-214.

Regis, J., Hayashi, M., Porcheron, D., Delsanti, C., Muracciole, X. \& Peragut, J. C. (year 2002). Impact of the model $C$ and Automatic Positioning System on gamma knife radiosurgery: an evaluation in vestibular schwannomas, J Neurosurg 97(5 Suppl): 588-591.

Sasaki, T., Kurita, H., Saito, I., Kawamoto, S., Nemoto, S., Terahara, A., Kirino, T. \& Takakura, K. (year 1998). Arteriovenous malformations in the basal ganglia and thalamus: management and results in 101 cases, J Neurosurg 88(2): 285-292.

Schneider, B. F., Eberhard, D. A. \& Steiner, L. E. (year 1997). Histopathology of arteriovenous malformations after gamma knife radiosurgery, J Neurosurg 87(3): 352-357.

Shin, M., Maruyama, K., Kurita, H., Kawamoto, S., Tago, M., Terahara, A., Morita, A., Ueki, K., Takakura, K. \& Kirino, T. (year 2004). Analysis of nidus obliteration rates after gamma knife surgery for arteriovenous malformations based on long-term followup data: the University of Tokyo experience, J Neurosurg 101(1): 18-24.

Shin, M., Kawahara, N., Maruyama, K., Tago, M., Ueki, K. \& Kirino, T. (year 2005). Risk of hemorrhage from an arteriovenous malformation confirmed to have been obliterated on angiography after stereotactic radiosurgery, J Neurosurg 102(5): 842-846.

Stefan, H., Schneider, S., Abraham-Fuchs, K., Bauer, J., Feistel, H., Pawlik, G., Neubauer, U., Rohrlein, G. \& Huk, W. J. (year 1990). Magnetic source localization in focal epilepsy. Multichannel magnetoencephalography correlated with magnetic resonance brain imaging, Brain 113 ( Pt 5): 1347-1359. 
Yamada, K., Sakai, K., Akazawa, K., Yuen, S. \& Nishimura, T. (year 2009). MR tractography: a review of its clinical applications, Magn Reson Med Sci 8(4): 165-174.

Yamamoto, A., Miki, Y., Urayama, S., Fushimi, Y., Okada, T., Hanakawa, T., Fukuyama, H. \& Togashi, K. (year 2007). Diffusion tensor fiber tractography of the optic radiation: analysis with 6-, 12-, 40-, and 81-directional motion-probing gradients, a preliminary study, AJNR Am J Neuroradiol 28(1): 92-96. 


\title{
The Role of Magnetic Resonance Spectroscopy in the Diagnosis of Ring Enhancing Lesions
}

\author{
Eftychia Kapsalaki ${ }^{1}$, Efstathios D. Gotsis ${ }^{4}$, \\ Ioannis Tsougos ${ }^{2}$ and Konstantinos N. Fountas ${ }^{3}$ \\ ${ }^{1}$ Department of Radiology, \\ ${ }^{2}$ Department of Medical Physics, \\ ${ }^{3}$ Department of Neurosurgery, \\ University Hospital of Larisa, School of Medicine, University of Thessaly, Larissa, \\ ${ }^{4}$ Diagnostic Center Euromedica, Athens, \\ Greece
}

\section{Introduction}

Ring-enhancing intracranial lesions constitute a common and quite puzzling diagnostic dilemma. These lesions may present as solitary or multiple on a routine brain MRI, and are characterized by a contrast enhancing halo and a non enhancing center. The central part may present with low signal intensity on $\mathrm{T} 1$, and high signal intensity on $\mathrm{T} 2$ weighted images. They are usually surrounded by a variable amount of edema. They may be located anywhere in the brain, although the junctional zone of gray-white matter is their most common location [Omuroet al., 2006; Smirniotopoulos et al., 2007]. Their size may vary from a few millimetres to several centimetres.

The differential diagnosis of ring enhancing lesions is quite large. It may include neoplasms, infections, inflammatory processes, or vascular pathologies. The incidence of each pathological entity depends highly on the geographical region and the study population. It is well documented that infections and inflammatory processes are more common in developing countries, while neoplasms and demyelinating lesions are more frequent in developed countries. Clinical history is not always helpful in their differential diagnosis, since more than $50 \%$ of CNS infections may present without fever and no obvious inflicting incident. Moreover, other laboratory tests may not be able to help in their differential diagnosis. In addition, the presenting symptomatology and the clinical examination of these patients are non-specific and frequently overlapping, making thus the establishment of an accurate diagnosis quite difficult.

Routine brain MR imaging is very sensitive in the identification of ring enhancing lesions but it cannot distinguish between neoplastic and non neoplastic lesions, in a large percentage of these cases. Frequently, the differentiation of a tumor from an infection is quite difficult, based solely on conventional MRI. Therefore, advanced MR imaging 
techniques as Diffusion Weighted Imaging (DWI), Perfusion Weighted Imaging (PWI), and proton Magnetic Resonance Spectroscopy (1HMRS) have been employed in the differential diagnosis of these lesions, with variable success rates. These studies are employed in combination with conventional MRI as complimentary imaging tests, and may significantly increase its specificity.

In this chapter, the role of ${ }^{1} \mathrm{HMRS}$ in the differential diagnosis of ring enhancing lesions is going to be discussed. The basic principles of ${ }^{1} \mathrm{HMRS}$, as well as, the typical spectroscopic profiles of the most commonly encountered ring enhancing lesions, are also presented. Furthermore, the implementation of other MR advanced techniques as DWI and PWI along with ${ }^{1} \mathrm{HMRS}$ in the evaluation of patients harbouring ring enhancing lesions, are analyzed.

\section{Basic principles of ${ }^{1} \mathrm{HMRS}$}

Proton MR Spectroscopy ( $\left.{ }^{1} \mathrm{HMRS}\right)$ is a noninvasive imaging technique that may contribute in the preoperative diagnosis of patients with MR ring enhancing lesions. ${ }^{1} \mathrm{HMRS}$ depends on a change in the resonance frequency of the nuclei within the molecules, regarding their chemical bonds, which is based on the chemical shift theory. The resonance frequency difference (chemical shift) is expressed as parts per million or ppm, a value that is independent of the amplitude of the external magnetic field. The value of the chemical shift provides information about the molecular group carrying the hydrogen nuclei, and thus it provides differentiation among several metabolites. Water peak is located at 4,7 ppm, and is much greater than the obtained signal from other hydrogen containing compounds typically identified in the brain parenchyma. Therefore, water signal needs to be suppressed for identifying any other metabolites. The reference frequency used, set at zero ppm, is that of tetra-methyl silane molecule $\mathrm{Si}-\left(\mathrm{CH}_{3}\right)_{4}$, which is symmetrical and has a single proton resonance.

In order to perform in vivo ${ }^{1} \mathrm{HMRS}$, a strong magnetic field of at least $1.5 \mathrm{~T}$ is required. It is generally accepted that, the higher the magnetic field strength, the more metabolites can be identified. Specific sequences for spectroscopic signal acquisition are either Single Voxel Spectroscopy (SVS), which receives the spectrum from a single voxel only, or Chemical Shift Imaging (CSI), which measures spectra in projection, on a slice (2D CSI), or a volume (3D CSI).

\section{Normal brain metabolites}

Proton MRS obtains information about brain tissue metabolism, which cannot be performed by conventional MRI scans [Wilson et al., 2009]. The principal metabolites, which are most commonly identified and evaluated in brain ${ }^{1} \mathrm{HMRS}$, and their characteristic frequencies and concentrations, are summarised in table 1.

At a first glance, a normal brain spectrum contains five to ten resonance peaks (figure 1). $\mathrm{N}$ Acetyl-Aspartate (NAA) is considered to be a unique neuronal marker, reflecting the number of intact neurons in the gray matter, and the density of intact axons in the white matter [Majos et al., 2004]. In general, reduction of NAA is produced by benign and malignant lesions in various proportions, and is indicative of normal neuronal tissue destruction. More specifically, NAA concentration decreases may occur with neuronal dysfunction caused by ischemia, trauma, inflammation, infection, tumor, neurodegenerative processes or reactive gliosis. Therefore, NAA is considered to be a highly sensitive but not specific marker. 
Choline (Cho) constitutes an essential compound of cellular membranes, and is considered to be a sensitive marker of cellular membrane metabolism [Miller et al., 1996]. Hence, variation in choline represents a wide range of abnormalities reflecting cell membrane destruction. Characteristically, tumors, inflammatory processes, demyelinating processes, and hypoxia show increased concentrations of Cho. Contrariwise, the concentration of Cho is decreased in cerebral abscesses.

\begin{tabular}{|c|c|c|}
\hline Metabolite & $\begin{array}{l}\text { Frequency }(\mathrm{ppm}) / \\
\text { Cerebral Concentration } \\
(\mathrm{mmol} / \mathrm{kg})\end{array}$ & Physiological Role \\
\hline N-Acetyl-Aspartate(NAA) & $\begin{array}{l}2.02 \mathrm{ppm} / \\
7.9-16.6 \mathrm{mmol} / \mathrm{kg}\end{array}$ & Neuronal cell marker. \\
\hline Choline (Cho) & $\begin{array}{l}3.2 \mathrm{ppm} / \\
0.9-2.5 \mathrm{mmol} / \mathrm{kg}\end{array}$ & $\begin{array}{l}\text { Marker of cell membrane } \\
\text { metabolism. }\end{array}$ \\
\hline $\begin{array}{l}\text { Creatine / Phosphocreatine } \\
(\mathrm{Cr})\end{array}$ & $\begin{array}{l}3.0 \mathrm{ppm} / \\
5.1-10.6 \mathrm{mmol} / \mathrm{kg}\end{array}$ & $\begin{array}{l}\text { Compounds related to energy } \\
\text { metabolism. }\end{array}$ \\
\hline Alanine(Ala) & $\begin{array}{l}1.5 \mathrm{ppm} / \\
0.2-1.4 \mathrm{mmol} / \mathrm{kg}\end{array}$ & $\begin{array}{l}\text { Is characteristic of meningeal } \\
\text { tumors }\end{array}$ \\
\hline Lipids (Lip) & $\begin{array}{l}0.9,1.4 \mathrm{ppm} / \\
>1.0 \mathrm{mmol} / \mathrm{kg}\end{array}$ & Membrane breakdown product. \\
\hline Lactate (Lac) & $\begin{array}{l}1.33 \mathrm{ppm} / \\
0.4 \mathrm{mmol} / \mathrm{kg}\end{array}$ & $\begin{array}{l}\text { A product of anaerobic } \\
\text { glycolysis. }\end{array}$ \\
\hline $\begin{array}{l}\text { Myo-inositol } \\
(m I)\end{array}$ & $\begin{array}{l}3.6 \mathrm{ppm} / \\
3.8-8.1 \mathrm{mmol} / \mathrm{kg}\end{array}$ & Glial Marker \\
\hline $\begin{array}{l}\text { Glutamate-Glutamine } \\
(\text { "Glx") } \\
\text {-amino-butyric acid } \\
\text { ("GABA") }\end{array}$ & $\begin{array}{l}\text { 2.1-2.4 ppm / } \\
1.3-12.6 \mathrm{mmol} / \mathrm{kg}\end{array}$ & $\begin{array}{l}\text { Intracellular Neurotransmitter } \\
\text { Markers }\end{array}$ \\
\hline Acetate & $1.9 \mathrm{ppm}$ & Characteristic in abscess \\
\hline Succinate / Pyruvate & $2.4 \mathrm{ppm}$ & Characteristic in abscess \\
\hline
\end{tabular}

Table 1. Summary of the principal metabolites that are most commonly evaluated in Magnetic Resonance Spectroscopy (MRS)

Total Creatine $(\mathrm{Cr})$ is a composite peak from the methyl and methylene protons of $\mathrm{Cr}$ and phosphorylated creatine, ( $\mathrm{PCr}$ ). The concentration of total creatine was thought to be relatively constant in the brain. However, with the development of quantitative MRS analysis techniques, it has been demonstrated that the concentration of total $\mathrm{Cr}$ is not constant. It has been shown that $\mathrm{Cr}$ concentrations may vary among different brain regions and among various pathological entities, thus, the usage of $\mathrm{Cr}$ as a reference signal should be applied with extreme caution [Howe et al., 2003]. The concentration of $\mathrm{Cr}$ is reduced in tumors and hypoxia, while $\mathrm{Cr}$ is totally absent in cerebral abscesses.

Lipids are normally absent from a normal brain MR spectrum, and their appearance represents necrosis. Increased concentrations of lipids may be observed in high grade primary and metastatic tumors, but also in abscesses and all necrotic lesions [Gotsis et al., 1996]. 


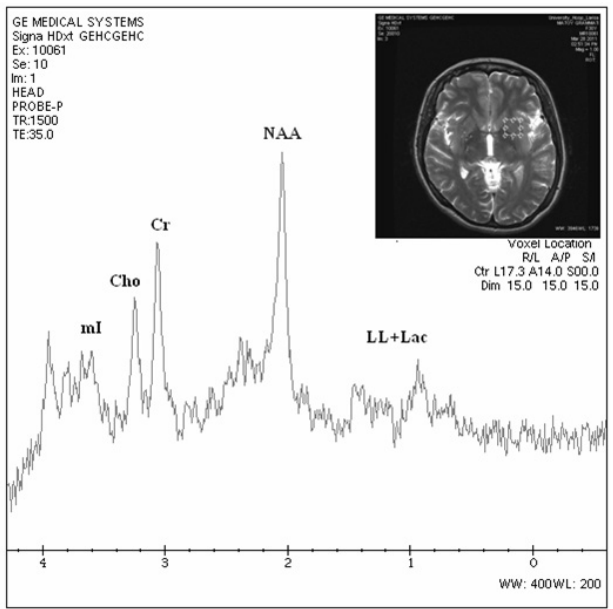

Fig. 1. Typical normal brain MR Spectrum showing the concentrations of Myoinositol (mI), Choline (Cho), Creatines (Cr), N-Acetyl-Aspartate (NAA),lipids (lip)and lactate (Lac)

Lactate signal appears in the same region of proton MR spectrum as lipids, therefore, it may be difficult to be distinguished. Lactate represents a product of anaerobic glycolysis [Barker et al., 1994; Negendank et al., 1996]. Its concentration is increased in ischemia, but also in high grade gliomas, as well as in pyogenic abscesses.

MyoInositol is a rather complex sugar alcohol, which gives rise to four groups of resonances. Its exact function is not completely known, although it has been proposed as a glial marker [Kallenberg et al., 2009]. Its concentration is reduced in high grade gliomas, but is also identified in meningiomas and demyelinating lesions.

Alanine peak appears near, and is often overlapped, by the lipids peak. It represents a discriminating metabolite of tumors of meningeal origin [Shino et al., 1999], but it is also identified in pyogenic anaerobic abscesses and cysticercosis [Kapsalaki et al., 2008]. Aminoacids (acetate and succinate) are not identified in a normal brain spectrum. Their presence is characteristic of brain abscesses.

In a routine clinical spectroscopic study, analysis of the obtained spectrum consists of measurement of the absolute concentrations of the identified metabolites, and calculation of the concentration ratios of the abovementioned metabolites. The most commonly used ratios include NAA/Cr, Cho/Cr, and NAA/Cho. Analysis of the accumulated data provides a characteristic metabolic profile, assigned to specific pathological entities.

\section{Technical limitations of ${ }^{1} \mathrm{HMRS}$}

The clinical use of proton MR spectroscopy presents several technical limitations and pitfalls [Castillo et al., 1996; Fountas et al., 2000; Preul et al., 1996; Rand et al., 1999; Shukla-Dave et al., 2001]. Proton MRS lasts approximately $15 \mathrm{~min}$ and requires the patient's cooperation for avoiding motion artifacts. In single voxel MRS, the obtained region of interest should be appropriately placed at the center of the studied lesion, to avoid signal contamination from the surrounding tissues [Kimura et al., 2001]. It has been reported that even the slightest malpositioning of the voxel may result in up to $50 \%$ signal contamination of the obtained 
spectrum, which may result in misinterpretation of the obtained spectroscopic study and subsequent imaging misdiagnosis. Moreover, calcifications, necrotic areas, and adjacent bony structures should be meticulously excluded from the obtained spectrum. Despite the recent advances in commercially available MRS software packages, the method remains operator dependent, and its accuracy is associated with the experience of the performing spectroscopist and the involved neuroradiologist.

\section{Characteristic MR Spectroscopic profiles of the most common ring enhancing lesions}

\subsection{High grade astrocytomas}

Proton MRS provides information regarding the metabolic profile of a glioma indicating the concentrations of various metabolites in the lesion. The commonly identified spectrum of a high grade glioma in proton MRS presents reduced concentration of NAA, because of destruction of normal neurons, markedly elevated concentrations of Cho due to exponentially increasing cellular populations and increased cell membrane turnover, and decreased $\mathrm{Cr}$ due to a major shift in the utilized cellular metabolic pathways. In regard to the commonly calculated metabolic ratios, the higher the Cho/NAA ratio is, the higher the astrocytoma grade. Therefore, ${ }^{1}$ HMRS may suggest the area of highest malignancy within a non-homogenous glioma and accurately guide an open or stereotactic surgical biopsy. Lac appears infrequently in all grades of astrocytomas. Lipids are detected in high quantity in necrotic gliomas, in the area of the necrosis (figure 2).

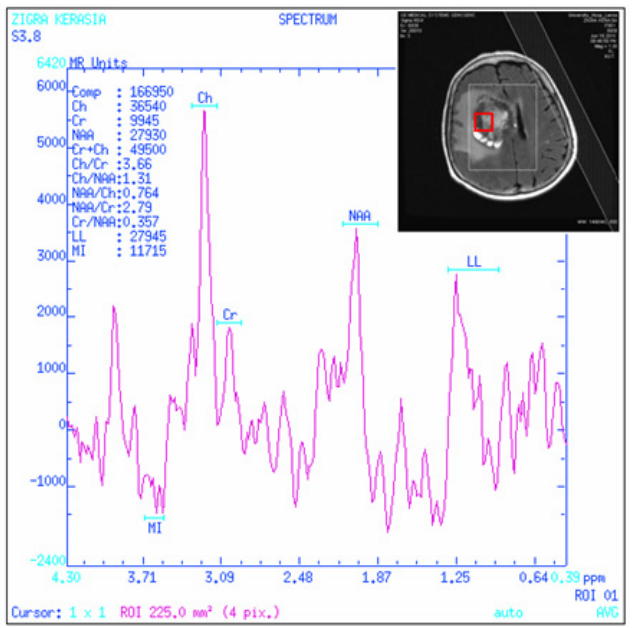

(a)

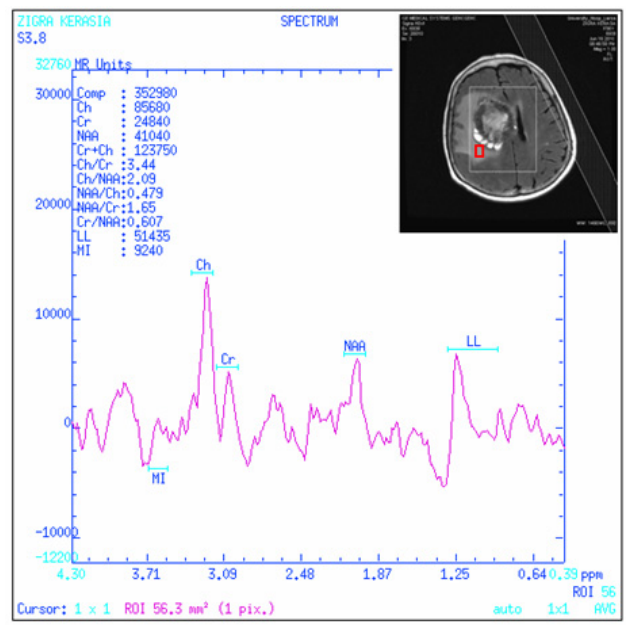

(b)

Fig. 2. a: Proton MR Spectrum of a high grade tumor. DD includes glioblastoma and metastatic lesion. 2b: The presence of increased concentration of Cho in the surrounding brain edema is suggestive of an infiltrative lesion, as a glioma versus a metastatic lesion.

It is well known that gliomas are highly infiltrating brain tumors, and their borders are typically ill-defined and cannot be accurately identified during their surgical removal [Croteau et al., 2001; Fountas et al., 2004; Mikkelsen \& Edvardsen, 1995]. Performing MRS in 
the surrounding edema may facilitate the identification of the glioma borders, and detect the presence and the extent of infiltration of a glioma. The identification of increased Cho and decreased NAA in the surrounding edema is indicative of infiltration, and is not characteristic of other ring enhancing lesions.

\subsection{Metastatic lesions}

The spectra of a ring enhancing metastasic tumor characteristically present very high concentrations of lipids, which are most probably associated with the presence of necrotic areas. Choline levels may also be elevated due to increased cell membrane destruction and turnover. However, the spectra of high grade gliomas and metastatic ring enhancing lesions are not easily distinguished [Burtscher et al., 2000; Opstad et al., 2004]. In such cases, spectra from the surrounding edema may provide additional important information. If normal concentrations of Cho and NAA are measured in a ring-enhancing lesion, this is more likely to be a metastatic lesion. Moreover, in metastatic lesions there is almost no $\mathrm{Cr}$ peak identified, which is not the case in high grade gliomas. (figure 3)

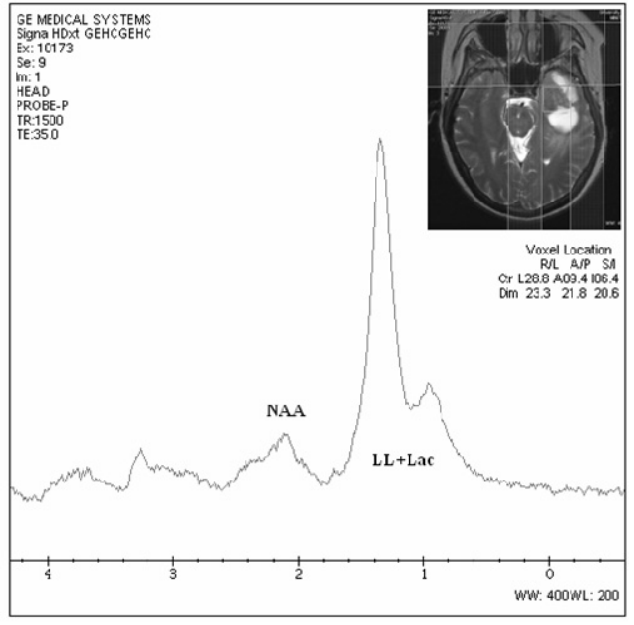

(a)

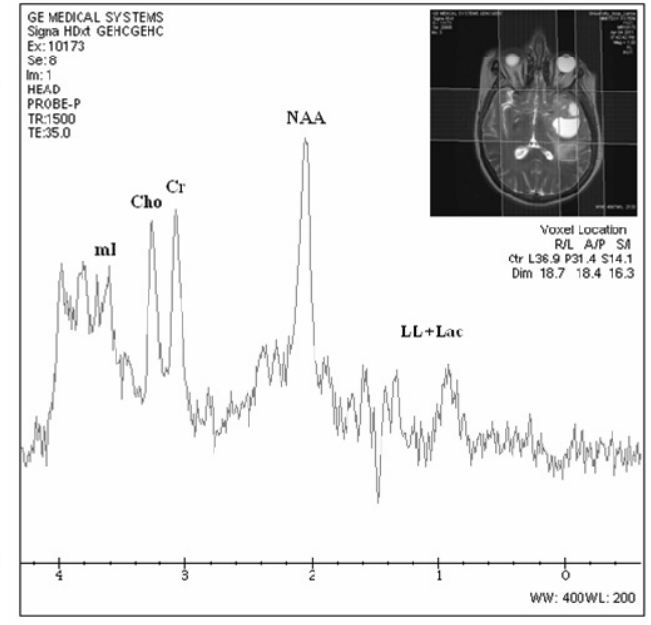

(b)

Fig. 3. a: The obtained spectrum demonstrates a very high peak representing severely increased concentration of lipids, which compresses all other metabolites, and suggests a highly necrotic lesion. Differential diagnosis between metastasis and glioma in this case is not possible. $3 \mathrm{~b}$ : A normal spectrum of the surrounding brain edema suggests that the lesion is more compatible with a metastatic rather than an infiltrative tumor (glioma).

\subsection{Recurrent astrocytomas versus post-radiation necrosis}

Tumor recurrence and radiation-induced necrosis have similar MRI characteristics, and their distinction is usually difficult based solely on conventional MRI. Positron Emission Tomography (PET) has been suggested for differentiating between tumor recurrence and post-radiation necrosis [Kim et al., 2010; Tsuyuguchi et al., 2003] However, PET is a quite expensive imaging modality, with very limited distribution in clinical centers. Nevertheless, 
${ }^{1}$ HMRS has a significant role in their differentiation, considering that in a recurrent glioma the presence of Cho with increased concentration is identified, and the Cho/Cr and Cho/NAA ratios are significantly increased, with absolute values higher than 1.76 [Weybright et al., 2005; Schlemmer et al., 2002]. On the contrary, in cases of post-radiation necrosis, there is a marked reduction in the concentrations of all normally detected metabolites, along with a marked increase of lipids concentration. A study by Tarnawski and coworkers [Tarnawski et al., 2002] indicates that ${ }^{1} \mathrm{H}-\mathrm{MRS}$ has a clear prognostic value for predicting survival in gliomas, and provides a clear diagnosis of recurrence following radiation therapy. In the presence of inflammation, increased levels of Cho may be detected. In such cases, differentiation between radiation necrosis and tumor recurrence with ${ }^{1}$ HMRS alone is almost impossible. Other imaging modalities need to be combined, as perfusion MRI.

\subsection{Abscesses}

Cerebral abscesses contain no normal neurons and no membranous structures in their necrotic lesional center (Fountas et al., 2000; Lai et al., 2005). Therefore, no peaks of NAA, $\mathrm{Cr}$, or Cho should be detected. A typical abscess spectrum shows the presence of cytosolic amino acids (leucine, isoleucine, and valine) [Garg et al, 2004; Kadota et al., 2001; Lai et al., 2002; Remy et al.,1995; Tsui et al., 2002], which are the products of proteolysis caused by enzymes released from neutrophil cells. Because these metabolites have never been detected in neoplasms, their detection is strongly indicative of a cerebral abscess [Kapsalaki et al., 2008]. Lactate is also detected in a large number of cerebral abscesses (figure 4).

Proton MR spectroscopy may also contribute in the identification of the causative organism of an abscess [Lai et al. 2002]. Anaerobic microbial agents are characterized by the presence of lactate, cytosolic amino acids, alanine, acetate, succinate, and lipids. Aerobes and facultative anaerobes are characterized by the presence of lactate, cytosolic amino acids, and the occasional presence of lipids. Streptococcal abscesses are characterized by the presence of lactate, while Staphylococcal infections are associated with the presence of lipids and lactate

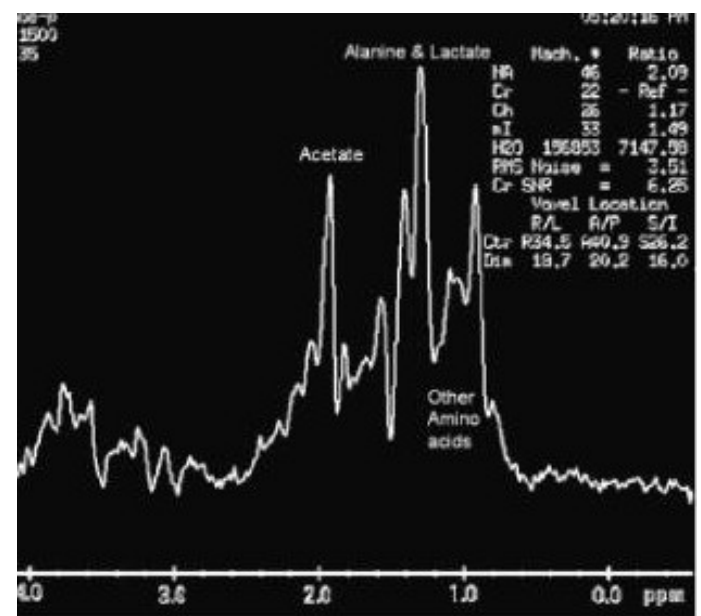

Fig. 4. Characteristic MR spectrum of an abscess. Note the absence of $\mathrm{Cho}, \mathrm{Cr}$, and NAA and also the increased concentrations of various aminoacids (acetate, alanine, lactate). 
[Himmelreich et al., 2005]. It is apparent that identification of the etiologic factor of an abscess contributes significantly in its prompt and timely treatment. Moreover, ${ }^{1}$ HMRS may contribute in the evaluation of the evolution and treatment response of a cerebral abscess. Sequential spectroscopic analysis of a brain abscesses may detect changes in the concentrations of the initially detected metabolites, providing thus a non-invasive methodology for evaluating the evolution of a cerebral abscess, and also its response to the administered antibiotic treatment.

\subsection{Subacute infarct}

Markedly elevated lactate is the key spectroscopic feature of cerebral hypoxia and ischemia, because of a major metabolic shift to anaerobic glycolysis. Choline is also elevated, with variable concentrations, while NAA and $\mathrm{Cr}$ concentrations are reduced. If cerebral infarction ensues, concentration of lipids will also increase. Furthermore, additional MR based advanced imaging modalities, such as Diffusion and Perfusion Weighted Imaging, may significantly contribute in the differential diagnosis in these cases [Parsons et al., 2000]

\subsection{Demyelination}

Proton MRS is a very useful tool in evaluating solitary tumefactive multiple sclerosis (MS) lesions. In acute MS lesions inflammation is the initial pathological change, while in more chronic lesions demyelination occurs. Proton MRS may be a quite sensitive imaging modality for evaluating axonal damage. At the initial presentation of MS, a typical spectrum shows decreased concentration of NAA, increased Choline and myo-inositol (MI) concentrations, and elevated concentration of Lactate, due to inflammation. With progression of an MS plaque, ${ }^{1}$ HMRS shows normalization of MI and Lac levels, while Cho and NAA may remain unchanged. Thus, an MS spectrum may not be always diagnostic of a demyelinating lesion (figure 5). Taking into account the increased concentration of lactate at

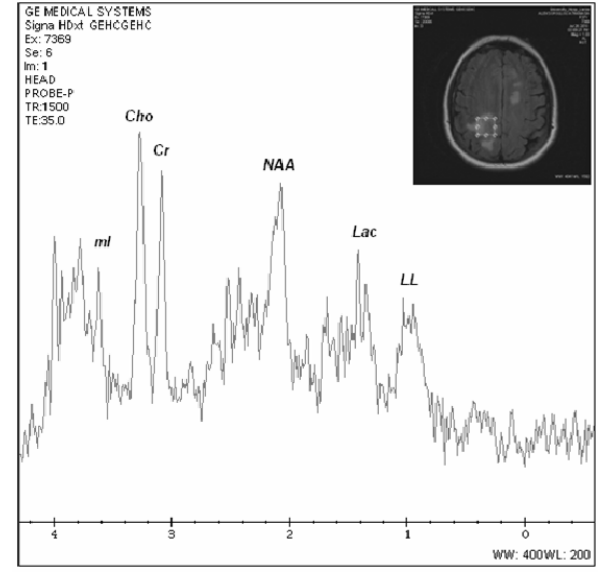

(a)

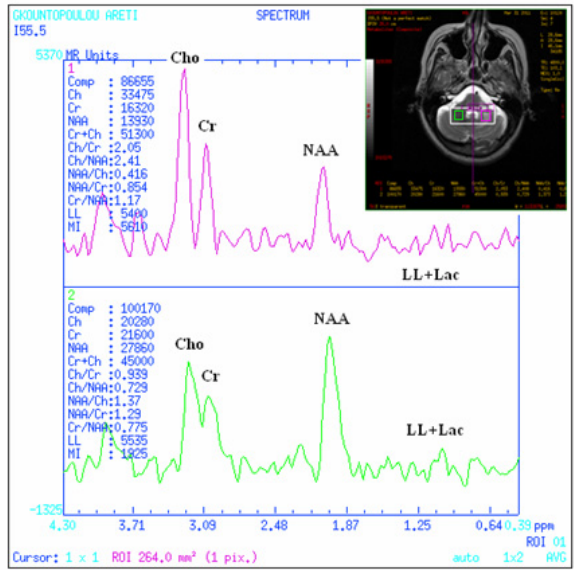

(b)

Fig. 5. (a) Characteristic proton MR spectrum of an acute MS plaque. Note the presence of slightly increased Cho, decreased concentration of NAA, and slightly increased concentration of Lactate (b) Spectrum obtained from a cerebellar demyelinating lesion. This spectrum is not suggestive of demyelination and may be erroneously diagnosed as a spectrum suggestive of a low grade glioma. 
the initial phase, this finding may contribute to the establishment of an accurate diagnosis. However, it has to be emphasized that in cases that a demyelinating process is suspected, completion of the diagnostic investigation with other imaging and laboratory studies is often necessary [De Stefano \& Filippi, 2007; Rovira \& Leon, 2008; Takenaka et al., 2011].

\section{Contribution of Diffusion Weighted Imaging (DWI) and Perfusion Weighted Imaging (PWI) in the distinction of ring enhancing lesions}

\subsection{Diffusion Weighted Imaging}

Diffusion-weighted imaging (DWI) is an MRI technique, which is based on the Brownian motion of molecules. Diffusion-weighted imaging detects the tracing of the microscopic motion of water molecules, thus reflecting the microstructure of local tissue. Free motion of water molecules in all directions is called isotropic diffusion, while motion of water molecules in a specific direction, like a myelinated axon, is called anisotropic diffusion. The diffusion data can be presented as signal intensity, or as an image map of the apparent diffusion coefficient (ADC). Calculation of the ADC requires two or more acquisitions with different diffusion weightings. Increased signal intensity on DWI corresponds to restricted diffusion and low ADC, while low signal intensity on diffusion-weighted images corresponds to normal diffusion and a high ADC.

DWI is a method detecting the diffusion properties of water molecules, and is restricted in subacute ischemia, brain abscesses, and lymhomas. It is usually not restricted in cystic lesions. Application of DWI has been extensively described in the literature [Bükte et al., 2005; Chang et al., 2002]. Cystic lesions and necrotic brain tumors, primary or metastatic, that have a "cystic" central area, show normal diffusion, since the cystic area is caused by liquefaction (necrosis) of the tumor, that permits free motion of the molecules. However, several studies report the presence of restricted diffusion in necrotic brain gliomas, making the use of other imaging modalities mandatory [Chang et al., 2002; Holtas et al., 2000; Lai et al., 2007]. On the contrary, in brain abscesses the cystic part reflects the presence of inflammatory cells, debris and possibly bacteria, that restrict the free motion of the molecules, and thus cause restricted diffusion, which appears with increased signal intensity. However, diffusion may also be restricted in several cases of malignant brain tumors, in acute demyelinating lesions, and in acute encephalitis. In such cases, the employment of ${ }^{1}$ HMRS, along with DWI may contribute in the differentiation of these lesions.

\subsection{Perfusion Weighted Imaging}

Perfusion Weighted Imaging (PWI), is the dynamic contrast imaging of the passage of intravenously injected paramagnetic contrast agent. Perfusion Weighted Imaging requires the acquisition of fast T2* images. After bolus intravenous contrast administration, the T2* images show drop of signal intensity, and as time passes, data are obtained in the form of cerebral blood flow (CBF), cerebral blood volume (CBV), and mean transit time (MTT). A time-intensity curve is generated for each voxel in each MR slice. The time-to-peak (TTP) is the time from the start of the scan until the maximum contrast attenuation occurs. The mean transit time (MTT) is the time it takes the contrast bolus to pass from the arterial to the venous side of the cerebral circulation. The entire area under the curve is a measure of relative cerebral blood volume ( $\mathrm{rCBV})$. Moreover, a measure of relative cerebral blood flow $(\mathrm{rCBF})$ is calculated by dividing the $\mathrm{rCBV}$ by the MTT. 
Perfusion Weighted Imaging is important in the diagnosis of brain tumors and the differentiation of recurrent gliomas from post-radiation necrosis. Malignant high grade gliomas demonstrate neovascularization that is detected by PWI, showing a correlation between microvessel density and histological tumor grade. The higher the tumor grade is, the higher the rCBV will be (Sugahara et al., 1998; Provenzale et al., 2006). Perfusion weighted imaging is of particular importance in the distinction of post-radiation necrosis from glioma recurrence. Especially, when PWI is employed in association with ${ }^{1} \mathrm{HMRS}$, the sensitivity and specificity of both methods increase significantly. When PWI shows a low Cerebral Blood Volume through the area of contrast enhancement, this usually suggests post-radiation necrosis, while high rCBV along with increased levels of Cho, and markedly increased $\mathrm{Cho} / \mathrm{Cr}$, Cho/NAA ratios are more suggestive of glioma recurrence. It has to be pointed out, however, that despite all these recent imaging advances, differentiation of postradiation necrosis from glioma recurrence may not be possible, and the employment of PET scan may be required in these cases.

\section{Conclusions}

The presence of ring enhancing lesions on brain MRI studies constitutes a frequent and quite challenging diagnostic dilemma. The differential diagnosis of lesions presenting as ring enhancing is quite extensive, and varies significantly with patient's age and the geographical region. Unfortunately, clinical history and symptomatology along with conventional MRI cannot accurately differentiate and establish a diagnosis of these lesions. Proton MRS may contribute in their differential diagnosis and may enhance, alone or in combination with other advanced MR Imaging modalities, the specificity and the diagnostic accuracy of conventional MRI. Proton MRS is a non invasive MR based diagnostic modality, which provides a direct spectroscopic signature of the examined brain parenchymal area and its underlying pathology, and an indirect evaluation of the lesion's metabolism. Detection of certain brain metabolites and calculation of their absolute and relative concentrations, are utilized in spectroscopic analysis. $\mathrm{N}$-acetyl-aspartate, Cho, $\mathrm{Cr}$, Lac, Lipids, MI, cytosolic aminoacids, and metabolic ratios of NAA/Cho, NAA/Cr, and Cho/Cr are the most commonly calculated metabolites. Changes in their concentrations may contribute in the differential diagnosis of ring enhancing lesions, since specific spectroscopic profiles exist for most of these lesions. High grade gliomas, metastatic tumors, abscesses, evolving infarcts, and demyelinating lesions demonstrate a specific and characteristic spectrum. In addition, employment of DWI and PWI, may further increase the diagnostic accuracy of ${ }^{1} \mathrm{HMRS}$ and conventional MRI in all these cases. Differentiation of post-radiation necrosis from tumor recurrence remains puzzling, despite all these advanced MR modalities and may require the employment of other imaging methodologies, such as PET. It has to be emphasized that ${ }^{1}$ HMRS carries significant technical limitations, and requires an experienced spectroscopist and neuroradiologist in order to avoid misinterpretation of the obtained data, and subsequently misdiagnosis of the studied lesion.

\section{Acknowledgements}

The authors want to thank Mrs Evdokia Kokoti for her valuable help and support in organizing and submitting this chapter 


\section{References}

Barker, PB., Gillard, JH., van Zijl, PCM., Soher, BJ., Hanley, DF., Agildere, AM., Oppenheimer, SM., \& Bryan, RN. (1994). Acute stroke: evaluation with serial proton MR spectroscopic imaging, Radiology Vol. 192, No. 3 (Setember 1994), pp. 723-732, ISSN 0033-8419

Bükte, Y., Paksoy, Y., Genç, E., \& Uca, AU. (2005) Role of diffusion-weighted MR in differential diagnosis of intracranial cystic lesions, Clin Radiol Vol. 60, No. 3, (March 2005), pp. 375-83, ISSN 0009-9260

Burtscher, IM., \& Holtas, S. (2001). Proton magnetic resonance spectroscopy in brain tumours: clinical applications, Neuroradiology Vol. 43, pp. 345-352, ISSN 0028-3940

Castillo, M., Kwock, L., \& Mukherji, SK. (1996). Clinical applications of proton MR spectroscopy, AJNR Am J Neuroradiol Vol.17, No.1, (January 1996), pp. 1-15, ISSN 0195-6108

Castillo, M., Smith, JK. \& Kwock, L. (2000). Correlation of myo-inositol levels and grading of cerebral astrocytomas, AJNR Am J Neuroradiol Vol. 21, No. 9 (October 2000), pp. 1645-1649, ISSN 0195-6108

Chang, SC., Lai, PH., Chen, WL., Weng, HH., Ho, JT., Wang, JS., Chang, CY., Pan, HB., \& Yang, CF. (2002). Diffusion-weighted MRI features of brain abscess and cystic or necrotic brain tumors: comparison with conventional MRI, Clin Imaging Vol. 26, No. 4 (July-August 2002), pp. 227-36, ISSN 0899-7071

Croteau, D., Scarpace, L., Hearshen, D., Gutiérrez, J., Rock, J., Rosenblum, M., Fisher, J., \& Mikkelsen, T.(2001). Correlation between magnetic resonance spectroscopy imaging and image-guided biopsies: semi-quantitative and qualitative histopathologic analysis of patients with untreated glioma, Neurosurgery Vol. 49, No. 4 (October 2001), pp. 823-829, ISSN 1528-8285

De Stefano, N. \& Filippi, M. (2007). "MR spectroscopy in multiple sclerosis." J Neuroimaging 17 Suppl 1, (April 2007), pp. 31S-35S, ISSN 1051-2284

Fountas, KN., Kapsalaki, EZ., Gotsis, SD., Kapsalakis, JZ., Smisson, HF 3rd., Johnston, KW., Robinson, JS Jr., \& Papadakis, N. (2000). In vivo proton magnetic resonance spectroscopy of brain tumors, Stereotact Funct Neurosurg Vol. 74, No. 2, pp. 83-94, ISSN 1011-6125

Fountas, KN., Kapsalaki, E., Vogel, R., Fezoulidis, I., Robinson, JS., \& Gotsis, ED. (2004). Noninvasive histologic grading of solid astrocytomas using proton magnetic resonance spectroscopy, Stereotact Funct Neurosurg Vol. 82, No. 2-3, pp. 90-97, ISSN1011-6125,

Garg, M., Gupta, RK., Husain, M., Chawla, S., Chawla, J., Kumar, R., Rao, SB., Misr, MK., \& Prasad, KN. (2004). Brain abscesses: etiologic categorization with in vivo proton MR spectroscopy, Radiology Vol. 230, No. 2, (February 2004), pp. 519-527, ISSN 0033-8419

Gotsis, ED., Fountas, K., Kapsalaki, E., Toulas, P., Peristeris, G., \& Papadakis, N. (1996). In vivo proton MR spectroscopy: the diagnostic possibilities of lipid resonances in brain tumors, Anticancer Res Vol. 16, No. 3B, (May- June 1996), pp. 1565-7, ISSN 0250-7005

Himmelreich, U., Accurso, R., Malik, R., Dolenko, B., Somorjai, RL., Gupta, RK., Gomes, L., Mountford, CE., \& Sorrell, TC. (2005). Identification of Staphylococcus aureus brain 
abscesses: rat and human studies with $1 \mathrm{H}$ MR spectroscopy, Radiology Vol. 236, No. 1, (July 2005), pp. 261-270, ISSN 0033-8419

Holtås, S., Geijer, B., Strömblad, LG., Maly-Sundgren, P., Burtscher, IM. (2000). A ringenhancing metastasis with central high signal on diffusion-weighted imaging and low apparent diffusion coefficients, Neuroradiology Vol.24, No. 11, (November 2000), pp. 824-7, ISSN 0195-6108

Howe, FA., Barton, SJ., Cudlip, SA., Stubbs, M., Saunders, DE., Murphy, M., Wilkins, P., Opstad, KS., Doyle, VL., McLean, MA., Bell, BA., \& Griffiths, JR. (2003). Metabolic profiles of human brain tumors using quantitative in vivo $1 \mathrm{H}$ magnetic resonance spectroscopy, Magn Reson Med Vol. 49, No. 2, (February 2003), pp. 223-32, ISSN 1522-2594

Kadota, O., Kohno, K., Ohue, S., Kumon, Y., Sakaki, S., Kikuchi, K., \& Miki, H. (2001). Discrimination of brain abscess and cystic tumor by in vivo proton magnetic resonance spectroscopy, Neuro Med Chir (Tokyo) Vol. 41, No. 3, (March 2001), pp. 121-126, ISSN 0470-8105

Kallenberg, K., Bock, HC., Helms, G., Jung, K., Wrede, A., Buhk, JH., Giese, A., Frahm, J., Strik, H., Dechent, P., \& Knauth, M. (2009). Untreated glioblastoma multiforme: increased myo-inositol and glutamine levels in the contralateral cerebral hemisphere at proton MR spectroscopy, Radiology Vol. 253, No. 3, (December 2009), pp. 805-12, ISSN 0033-8419

Kapsalaki, E., Gotsis, ED., \& Fountas, KN. (2008). The role of proton magnetic resonance spectroscopy in the diagnosis and categorization of cerebral abscesses, Neurosurg Focus Vol. 24, No. 6, pp. E7

Kimura, T., Sako, K., Gotoh, T., Tanaka, K., \& Tanaka, T. (2001). In vivo single-voxel proton MR spectroscopy in brain lesions with ring-like enhancement, NMR Biomed Vol. 14, No. 6 (October 2001), pp. 339-349, ISSN 0952-3480

Kim, YH., Oh, SW., Lim, YJ., Park, CK., Lee, SH., Kang, KW., Jung, HW., \& Chang, KH. (2010). Differentiating radiation necrosis from tumor recurrence in high-grade gliomas: assessing the efficacy of 18F-FDG PET, 11C-methionine PET and perfusion MRI, Clin Neurol Neurosurg Vol. 112, No. 9, (November 2010), pp. 758-65, ISSN 0303-8467

Lai, PH., Ho, JT., Chen, WL., Hsu, SS., Wang, JS., Pan, HB., \& Yang, CF. (2002) Brain abscess and necrotic brain tumor: discrimination with proton MR spectroscopy and diffusion-weighted imaging, AJNR Am J Neuroradiol Vol. 23, No. 8, (September 2002), pp. 1369-1377, ISSN 0195-6108

Lai PH, Li KT, Hsu SS, et al. (2005). Pyogenic brain abscess: findings from in vivo 1.5-T and 11.7-T in vitro proton MR spectroscopy. AJNR Am J Neuroradiol No. 26, pp. 279-288, ISSN 0195-6108

Lai, PH., Hsu, SS., Ding, SW., Ko, CW., Fu, JH., Weng, MJ., Yeh, LR., Wu, MT., Liang, HL., Chen, CK., \& Pan, HB. (2007). Proton magnetic resonance spectroscopy and diffusion-weighted imaging in intracranial cystic mass lesions, Surg Neurol Vol. 68, Suppl 1, pp. S25-36, ISSN 0090-3019

Majos, C., Julia-Sape, M., Alonso, J., Serrallonga, M., Aguilera, C., Acebes, JJ., Arus, C., \& Gili, J. (2004). Brain tumor classification by proton MR spectroscopy: comparison of diagnostic accuracy at short and long TE, Am J Neuroradiol Vol. 25, No. 10, (November-December 2004), pp. 1696-1704, ISSN 0195-6108 
Mikkelsen, T, \& Edvardsen, K. (1995). Invasiveness in nervous system tumors. In: Cancer of the Nervous System (Eds, Black P, Loeffler JS), Blackwell Scientific Publications, ISBN 0781737311, Cambridge, MA

Miller, BL., Chang, L., Booth, R., Ernst, T., Cornford, M., Nikas, D., McBride, D., \& Jenden, DJ. (1996). In vivo 1H MRS choline: correlation with in vitro chemistry/histology, Life Sci Vol. No. 22, pp. 1929-1935, ISSN 0024-3205

Negendank, WG., Sauter, R., Brown, TR., Evelhoch, JL., Falini, A., Gotsis, ED., Heerschap, A., Kamada, K., Lee, BC., Mengeot, MM., Moser, E., Padavic-Shaller, KA., Sanders, JA., Spraggins, TA., Stillman, AE., Terwey, B., Vogl, TJ., Wicklow, K., \& Zimmerman, RA. (1996). Proton magnetic resonance spectroscopy in patients with glial tumors, J Neurosurg Vol. 84, No. 3, (March 1996), pp. 449-58, ISSN 0022-3085

Omuro, AM., Leite, CC., Mokhtari, K., \& Delattre, JY. (2006). Pitfalls in the diagnosis of brain tumours, Lancet Neurol Vol. 5, No. 11, (November 2006), pp. 937-48, ISSN 1474-4422

Opstad, KS.,Murphy, MM., Wilkins, PR., Bell, BA., Griffiths, JR., \& Howe, FA..(2004). Differentiation of metastases from high-grade gliomas using short echo time $1 \mathrm{H}$ spectroscopy, J Magn Reson Imaging Vol. 20, No. 2, (August 2004), pp. 187-192, ISSN 1522-2594

Parsons, MW., Li, T., Barber, PA., Yang, Q., Darby, DG., Desmond, PM., Gerraty, RP., Tress, BM., \& Davis, SM. (2000). Combined (1)H MR spectroscopy and diffusionweighted MRI improves the prediction of stroke outcome, Neurology, Vol. 55, No. 4, (August 22, 2000), pp. 498-505, ISSN 10158618

Preul, MC., Caramanos, Z., Collins, DL., Villemure, JG., Leblanc, R., Olivier, A., Pokrupa, R., \& Arnold, DL. (1996). Accurate, noninvasive diagnosis of human brain tumors by using proton magnetic resonance spectroscopy, Nat Med Vol. 2, No. 3, (March 2003), pp. 323-325, ISSN 1078-8956

Provenzale, JM., Mukundan, S., \& Barboriak, DP. (2006). Diffusion-weighted and perfusion MR imaging for brain tumor characterization and assessment of treatment response , Radiology Vol. 239, No. 3, (June 2006), pp. 632-49, ISSN 0033-8419

Rand, SD., Prost, R., \& Li, SJ.(1999). Proton MR spectroscopy of the brain, Neuroimaging Clin N Am Vol. 9, No. 2, (May 1999), pp. 379-395, ISSN 1052-5149

Remy, M., Grand, S., Lai, ES., Belle, V., Hoffmann, D., Berger, F., Estève, F., Ziegler, A., Le Bas, JF., Benabid, AL., et al. (1995). 1HRMS of human brain abscesses in vivo and in vitro, Magn Reson Med Vol. 34, No. 4, (October 1995), pp. 508-514, ISSN 1522-2594

Rovira, A. \& Leon, A. (2008). MR in the diagnosis and monitoring of multiple sclerosis: an overview, Eur J Radiol Vol. 67, No. 3, (September 2008), pp. 409-14, ISSN 0720-048X

Schlemmer, JP., Bachert, P., Henze, M., Buslei, R., Herfarth, KK., Debus, J., \& van Kaick, G. (2002). Differentiation of radiation necrosis from tumor progression using proton magnetic resonance spectroscopy, Neuroradiology Vol. 44, No. 3, (March 2002), pp. 216-222, ISSN 0028-3940

Shino, A., Nakasu, S., Matsuda, M., Handa, J., Morikawa, S., \& Inubushi, T. (1999). Noninvasive evaluation of the malignant potential of intracranial meningiomas performed using proton magnetic resonance spectroscopy, J Neurosurg Vol. 91, No. 6, (December 1999), pp. 928-934, ISSN 0022-3085

Shukla-Dave, A., Gupta, RK., Roy, R., Husain, N., Paul, L., Venkatesh, SK., Rashid, MR., Chhabra, DK., \& Husain, M. (2001). Prospective evaluation of in vivo proton MR 
spectroscopy in differentiation of similar appearing intracranial cystic lesions, Magn Reson Imaging Vol. 19, No. 1, (January 2001), pp. 103-110, ISSN 0730-725X

Smirniotopoulos, JG., Murphy, FM., Rushing, EJ., Rees, JH., \& Schroeder, JW. (2007). Patterns of contrast enhancement in the brain and meninges, Radiographics Vol. 27, No. 2, (March -April 2007), pp. 525-51, ISSN 0271-5333

Sugahara, T., Korogi, Y., Kochi, M., Ikushima, I., Hirai, T., Okuda, T., Shigematsu, Y., Liang, L., Ge, Y., Ushio, Y., \& Takahashi, M. (1998). Correlation of MR imaging blood volume maps with histologic and angiographic determination of vascularity of gliomas, AJR Am J Roentgenol Vol. 171, No. 6, (December 1998), pp. 1479-1486, ISSN 1546-3141

Takenaka, S., Shinoda, J., Asano, Y., Aki, T., Miwa,K., Ito, T., Yokoyama, K., \& Iwama, T. (2011). Metabolic assessment of monofocal acute inflammatory demyelination using MR spectroscopy and (11) C-methionine-, (11)C-choline-, and (18)Ffluorodeoxyglucose-PET, Brain Tumor Pathol (2011 Mar 26), Epub ahead of print, ISSN 1433-7398

Tarnawski, R., Sokol, M., Pieniazek, P., Maciejewski, B., Walecki, J., Miszczyk, L., \& Krupska,T. (2002). 1H-MRS in vivo predicts the early treatment outcome of postoperative radiotherapy for malignant gliomas, Int J Radiat Oncol Biol Phys Vol. 52, No. 5, (April 1, 2002), pp. 1271-1276, ISSN 0360-3016

Tsui, EYK., Chan, JH., Cheung, YK., Lai, KF., Fong, D., \& Ng, SH.(2002). Evaluation of cerebral abscesses by diffusion-weighted MR imaging and MR spectroscopy, Comput Med Imaging Graph Vol. 26, No. 5, (September- October 2002), pp. 347-351, ISSN 0895-6111

Tsuyuguchi, N., Sunada, I., Iwai, Y., Yamanaka, K., Tanaka, K., Takami, T., Otsuka, Y., Sakamoto, S., Ohata, K., Goto, T., \& Hara, M. (2003). Methionine positron emission tomography of recurrent metastatic brain tumor and radiation necrosis after stereotactic radiosurgery: is a differential diagnosis possible? J Neurosurg, Vol. 98, No. 5, (May 2003), pp., ISSN 0022-3085

Weybright, P., Sundgren, P., Maly, P., Hassan, DG., Nan, B., Rohrer, S., \& Junck, L. (2005). Differentiation between brain tumor recurrence and radiation injury using MR spectroscopy, AJR Am J Roentgenol,Vol. 185, No. 6, (December 2005), pp. 14711476, ISSN 1546-3141

Wilson, M., Davies, NP., Grundy, RG., \& Peet, AC. (2009). A quantitative comparison of metabolite signals as detected by in vivo MRS with ex vivo $1 \mathrm{H}$ HR-MAS for childhood brain tumours, NMR Biomed Vol. 22, No. 2, (Ferbruary 2009), pp. 213-9, ISSN 0952-3480

Xu, V., Chan, H., Lin, AP., Sailasuta, N., Valencerina, S., Tran, T., Hovener, J. \&, Ross, BD. (2008). MR spectroscopy in diagnosis and neurological decision-making, Semin Neurol Vol. 28, No. 4, (September 2008), pp. 407-422, ISSN: 0271-8235 


\title{
The Role of Functional MRI in Intracranial Glioma Resection
}

\author{
Eftychia Z. Kapsalaki', Dimitrios Verganelakis², Ioannis Z. Kapsalakis², \\ Efstathios D. Gotsis ${ }^{2}$ and Kostas N. Fountas ${ }^{1}$ \\ ${ }^{1}$ Departments of Diagnostic Radiology and Neurosurgery University Hospital of Larisa, \\ School of Medicine, University of Thessaly Larissa, \\ ${ }^{2}$ Department of Magnetic Resonance Imaging, Euromedica- Encephalos, Athens,
}

Greece

\section{Introduction}

It is generally accepted that tumor extirpation constitutes the treatment goal in cases of intracranial tumors. It is also well known that intracranial gliomas are infiltrative lesions with ill-defined borders, and their total resection is often quite challenging. Moreover, the presence of a glioma in an eloquent cortical area may make its extirpation even more difficult. It has been demonstrated that extensive glioma resection is associated with prolonged survival and better quality of life, and the overall outcome of patients with intracranial gliomas is associated with the extent of the tumor's surgical resection [Lacroix et al., 2001; McDonald et al., 1999; National Comprehensive Cancer Network, 2007; Sanai \& Berger, 2008; Stafford et al., 1998). Therefore, every effort to achieve maximal tumor resection without jeopardizing vital neuronal functions becomes of paramount importance in cases of intracranial gliomas. Exact knowledge of the cortical topography, accurate identification of all eloquent cortical areas as well as delineation of their relationships with the tumor, constitute key elements in avoiding all functional cortical areas, while aggressive tumor resection is accomplished.

It is well known that conventional imaging studies providing pure structural anatomical information are not sufficient for identifying and localizing functional cortical areas, since there are frequent anatomical variations, and cortical functional center shift due to brain distortion and plasticity, particularly in glioma cases. Various methodologies have been developed for identifying different functional areas of the cerebral cortex and accurately localize them, in regard to the studied tumor on each individual case. Intraoperative electrophysiological studies such as recording of Somato-Sensory Evoked Potentials (SSEPs), Motor Evoked Potentials (MEPs), Direct Cortical Stimulation (DCS), and spontaneous Electro-Myo-Graphy (sEMG) are considered the gold standard for cortical mapping and delineation of functional cortical networks. The major drawback of these methodologies however, is the fact that all are invasive tests and cannot provide all this valuable information preoperatively. Thus, the development of non-invasive tests for cortical mapping seems to be mandatory. Recently, advanced imaging and electrophysiological studies such as Positron Emission Tomography (PET), brain SPECT imaging, functional 
Magnetic Resonance Imaging (fMRI), Magneto-Encephalo-Graphy (MEG) and Magnetic Source Imaging (MSI), as well as high-density Electro-Encephalo-Graphy (hdEEG), have been employed, with various success rates, in cerebral cortical mapping. The wide clinical application of MRI, the high spatial resolution of fMRI compared with all other functional methods, its non-invasive character, and its low cost compared with all other methods, have made fMRI the most popular methodology for cortical functional mapping. Functional MRI can provide the neurosurgeon with critical information for a safe and aggressive surgical planning, thus, allowing maximal tumor resection and minimizing the possibility of a new postoperative neurological deficit.

\section{Functional magnetic resonance imaging: Advantages and drawbacks}

Since its introduction, during the 1990s, functional Magnetic Resonance Imaging has proven to be a powerful technique for the non-invasive imaging of various brain functional centers, such as motor, sensory, language, vision, odor and others. Functional MRI can constructively help towards a safe pre-operative or radiation treatment planning for various pathologies. In neurosurgical practice, fMRI is used in order to assess suitability of patients for resection of tumors and/or resective epilepsy surgery. In neurosurgery, the aim is to maximize the removal of pathological tissue with the minimum post-operative functional deficit. Therefore, the pre-operative knowledge of the proximity of the pathological tissue to the eloquent cortex is of fundamental importance for accurate and safe neurosurgical planning. Functional MRI provides information regarding anatomical variations, as well as tumor related shift of functional cortical centers.

Functional MRI is based on a physiological phenomenon called the Blood-OxygenationLevel-Dependent (BOLD) contrast. BOLD is strongly coupled, to neuronal activity and cerebral hemodynamics [Roy \& Sherrington, 1890]. The BOLD signal expresses small changes in the volume of the oxygenated blood in a specific brain volume during increased neuronal activity. When an examinee is subjected to a stimulus, there is an increased neuronal activity of the corresponding cluster of neurons responsible for this particular function. This is translated into a regionally increased metabolic demand. In order for this demand to be met, the supply of oxygenated blood locally increases, via dilation of the surrounding capillaries. The regional cerebral blood flow (CBF) and volume (CBV) increase with a surplus of oxygen ( $2 \%-6 \%)$. This produces an increased ratio of oxygenated versus de-oxygenated blood in the local system of capillaries and veins, in regard to the adjacent idle neurons. Oxygenated blood contains oxy-hemoglobin, a diamagnetic material, while deoxygenated blood contains de-oxyhemoglobin, a paramagnetic material. Microscopically, this decreased paramagnetism causes less signal loss on $\mathrm{T}_{2}{ }^{*}$ weighted images [Ogawa et al., 1990]. Therefore, a relative signal increase is generated during the active neuron period, with respect to the passive neurons' period. The pulse sequence that is used in order to image the BOLD effect is the Gradient-Echo Echo-Planar-Imaging (GE-EPI), which is $\mathrm{T}_{2}{ }^{*}$ weighted, i.e. sensitive to magnetic susceptibility effects [Stehling et al., 1991]. Thus, the BOLD fMRI response to a neuronal activity is a hemodynamic response function, which is the change in BOLD fMRI signal due to a short period of neural activity. Hemodynamic Response Factor reflects variations in vascular physiology generated by a neuronal activity. The major advantage of fMRI is its non-invasive character, especially when compared to the electrophysiological cortical stimulation studies. In addition, it has a relatively low cost, is reproducible, has a short examination time compared to the electro-cortical stimulation 
studies, and is well tolerated by the vast majority of patients [Giussani et al., $2010 \mathrm{G}$ ]. Moreover, fMRI may be performed in pediatric patients, which is not the case for intraoperative cortical stimulation studies during an awake craniotomy. Functional MRI can test multiple language tasks, and check larger cortical areas for auxiliary language networks [Giussani et al., 2010]. Comparing fMRI with other non-invasive techniques, such as Magneto-Encephalo-Graphy (MEG) and high-density Electro-Encephalo-Graphy (EEG), fMRI has the advantage of studying both cortical and intrasulcal areas, as well as deep brain areas. It has been demonstrated that fMRI has higher spatial resolution than MEG/MSI, PET, SPECT, and hdEEG, and comparable temporal resolution to the MEG/MSI. Furthermore, fMRI does not require intravascular injection of contrast media or radioisotopes.

Several drawbacks of fMRI may however limit its clinical applications. Most importantly, only cooperative patients may undergo an fMRI study, since motion artifacts secondary to head movements, respiratory movements, and cardiac pulsation may influence the quality of the obtained study. Moreover, the patient's ability to understand the functional paradigm and promptly perform the necessary tasks, are essential in obtaining a high quality fMRI. It has to be emphasized that fMRI constitutes an indirect measurement of neuronal activity, and it indicates where this indirect measurement takes place, without however describing the exact underlying mechanism. Besides, fMRI does not exhibit the neuronal networks, which interconnect the various functional clusters of neurons that collaborate for the performance of a particular function. Furthermore, there are other aspects that require attention in interpreting fMRI findings, such as the HRF and the BOLD signal. The HRF is a function: i) of the studied brain area, its regional blood volume and blood flow, ii) of the patient, and iii) of the employed stimulus. Similarly, the BOLD signal may be influenced by: i) hypoxia, ii) hypercapnia, iii) presence of cerebral vascular pathologies, iv) lack of sleep, v) anemia, vi) smoking, vii) various brain degenerative pathologies, viii) proximity of large veins to the activated neuronal clusters (vein effect), and ix) previous brain anatomical injuries.

Since fMRI is based on magnetic susceptibility, hemorrhage within a brain tumor may alter the accuracy of the BOLD effect and misplace the location of the detected signal. Particular attention should be taken in such cases and further preoperative, possibly invasive studies, need to be performed. In high grade gliomas, the presence of neovascularization, the induction of neuro-chemical changes in the cytosol and the subsequent alteration of the regional cerebral blood flow and the oxygen extraction fraction, the presence of arteriovenous shunting, and the presence of tumor-associated edema and mass effect causing mechanical vasoconstriction, as well as the presence of scar tissue secondary to a previous craniotomy may result into significant BOLD signal changes [Atlas et al., 1996; Giussani et al., 2010; Haberg et al., 2004; Krishnan et al., 2004 ]. It has been demonstrated, that in 10-31\% of the performed fMRI studies the obtained data cannot be processed, and this percentage in glioma populations ranges between $0-30 \%$ [Haberg et al., 2004]. Moreover, gliomas are usually surrounded by edema and cause mass effect. During the fMRI study the activation area is located in the brain tissue that may be displaced by the lesion. At the time of the craniectomy, decompression of the brain occurs and alteration of the measured distances between the activation area and the brain tumor may take place. Therefore, we need to refer to the anatomical structures, especially the sulci, rather that the actual distance between the brain tumor and the activation area, when evaluating an fMRI study. 


\subsection{Neurosurgical considerations and clinical experience}

It is widely accepted that surgical resection of intracranial gliomas is the treatment method of choice. Glioma resection reduces the tumor's mass effect on the surrounding brain, reduces the tumor-associated edema, ensures the establishment of an accurate histological diagnosis, and induces residual tumor cells into active mitotic process thus making them more vulnerable to the adjuvant administered radiotherapy and chemotherapy. It has been demonstrated that extensive resection of gliomas is associated with prolonged survival and better quality of life [Lacroix et al., 2001; McDonald et al., 1999; National Comprehensive Cancer Network, 2007; Sanai \& Berger, 2008; Stafford et al., 1998]. Accurate knowledge of the anatomical relationship of a glioma with neighboring eloquent cortical areas is of paramount importance for maximizing tumor resection, minimizing the chance of postoperative neurological deficit, and thus maximizing the patient's safety. Functional MRI has been employed for more than 15 years in the preoperative evaluation of patients harboring intracranial gliomas for identifying, accurately localizing, and intraoperatively avoiding functional cortical centers [Atlas et al., 1996; Berntsen et al., 2010; Bizzi et al., 2008; Fandino et al., 1999; Fitzgerald et al., 1997; Giussani et al., 2010; Haberg et al., 2004; Hirsch et al., 2000; Hoenig et al., 2005; Krasnow et al., 2003; Krishnan et al., 2004; Lehericy et al., 2000; Li et al, 2010; Lurito et al., 2000; Mueller et al., 1996; National Comprehensive Cancer Network, 2007; Petrovich et al., 2005; Pouratian et al., 2002; Puce et al., 1995; Roux et al., Ruge et al., 1999; 2003; Rutten et al., 2002; Sanai \& Berger, 2008; Schulder et al., 1998; Signorelli et al., 2003; Tieleman et al., 2007; Tomczak et al., 2000; Yetkin et al., 1997; Yousry et al., 1995] (Figures 1 \& 2). Several clinical studies have been performed comparing fMRI with intraoperative electrophysiological stimulation studies, with varying results, and frequently contradictory conclusions [Atlas et al., 1996; Berntsen et al., 2010; Bizzi et al., 2008; Fandino et al., 1999; Fitzgerald et al., 1997; Giussani et al., 2010; Haberg et al., 2004; Hirsch et al., 2000; Hoenig et al., 2005; Krasnow et al., 2003; Krishnan et al., 2004; Lehericy et al., 2000; Li et al, 2010; Lurito et al., 2000; Mueller et al., 1996; National Comprehensive Cancer Network, 2007; Petrovich et al., 2005; Pouratian et al., 2002; Puce et al., 1995; Roux et al., 2003; Ruge et al., 1999; Rutten et al., 2002; Sanai \& Berger, 2008; Schulder et al., 1998; Signorelli et al., 2003; Tieleman et al., 2007; Tomczak et al., 2000; Yetkin et al., 1997; Yousry et al., 1995]. Unfortunately, the utilized methodologies vary significantly among these studies, their populations are frequently limited and non-homogenous, and a large number of them are retrospective studies. Therefore, their results are not comparable, in the vast majority of cases. However, a systematic review of the existent clinical studies show that fMRI constitutes a routine clinical practice in many neuro-oncology centers around the world, which provides the opportunity to the performing neurosurgeon for a more realistic and accurate preoperative discussion with the patient, a wiser decision-making process, a safer surgical planning, and a more aggressive tumor resection [Atlas et al., 1996; Berntsen et al., 2010; Bizzi et al., 2008; Fandino et al., 1999; Fitzgerald et al., 1997; Giussani et al., 2010; Haberg et al., 2004; Hirsch et al., 2000; Hoenig et al., 2005; Krasnow et al., 2003; Krishnan et al., 2004; Lehericy et al., 2000; Li et al, 2010; Lurito et al., 2000; Mueller et al., 1996; National Comprehensive Cancer Network, 2007; Petrovich et al., 2005; Pouratian et al., 2002; Puce et al., 1995; Ruge et al., 1999; Roux et al., 2003; Rutten et al., 2002; Sanai \& Berger, 2008; Schulder et al., 1998; Signorelli et al., 2003; Tieleman et al., 2007; Tomczak et al., 2000; Yetkin et al., 1997; Yousry et al., 1995]. Several studies also emphasize that fMRI can localize more accurately motor and sensory cortical areas than language areas, and therefore they indicate the necessity for employing complimentary intraoperative electrophysiological stimulation 
studies, in cases of tumor proximity to language-associated cortical areas [Berntsen et al., 2010; Giussani et al., 2010; Hirsch et al., 2000; Roux et al., 2003; Ruge et al., 1999].

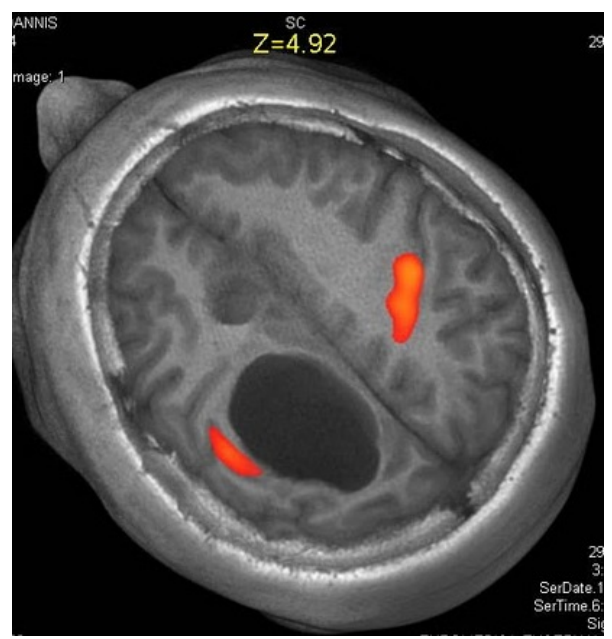

Fig. 1. Preoperative fMRI demonstrating the displacement of the motor cortex and its relationship with a low grade glioma (astrocytoma grade II) during an active right foot plantar flexion.

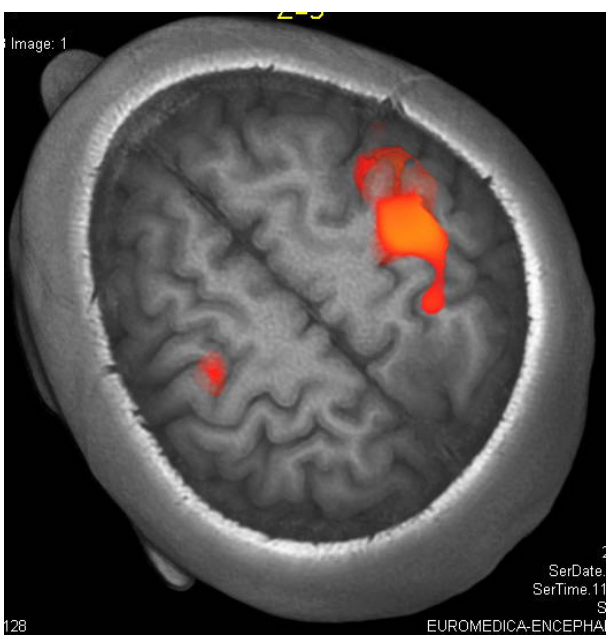

Fig. 2. Preoperative fMRI demonstrating activated hand motor cortex and its relationship to a loe grade glioma during a left fist clentcing paradigm.

In one of the earliest published prospective, clinical studies comparing preoperative fMRI and DCS for identifying cortical motor areas, Yousry et al. [Yousry et al., 1995] reported a series of six patients with gliomas. All participants underwent preoperative fMRI at 1.5T for localizing the motor cortex [Yousry et al., 1995]. A repetitive opening-closing of the hand 
paradigm was used for the fMRI, while DCS was intraoperatively employed for identification and localization of the motor strip [Yousry et al., 1995]. They found that the fMRI accuracy in localizing the motor cortex was $100 \%$ when error margin was confined to 10mm [Yousry et al., 1995]. Likewise, Puce et al. [Puce et al., 1995] in a series of four tumor cases, found in their prospective study good agreement between preoperative fMRI and intraoperative DCS/SSEP, regarding localization of the sensori-motor cortex. Their fMRI protocol included sponge squeezing as a motor task, and electrical stimulation of the median nerve, palm light brushing, and palm air blowing as sensory tasks [Puce et al., 1995]. Mueller et al. [Mueller et al., 1996] reported 10 patients with intracranial tumors and 2 patients with intracranial vascular lesions undergoing preoperative fMRI at $1.5 \mathrm{~T}$ for sensorimotor cortex localization, and intraoperative DCS. Their fMRI protocol included indexthumb tapping as a motor task, and palm light scratching as a sensory task [Mueller et al., 1996]. They found that fMRI accuracy was 100\% in their retrospective study [Mueller et al., 1996]. They also reported that they had no postoperative motor deficits, when their resection distance was more than $5 \mathrm{~mm}$ from the fMRI identified motor cortex [Mueller et al., 1996]. At approximately the same time, Schulder et al. [Schulder et al., 1998] reported a series of 12 patients with various intracranial tumors undergoing preoperative fMRI at 1.5T and intraoperative DCS/SSEP studies, for sensori-motor cortex localization. They reported that fMRI accuracy in their prospective study was $100 \%$, while they concluded that maximal tumor resection and no new postoperative neurological deficits were observed in their series [Schulder et al., 1998]. Contrariwise, Atlas et al. [Atlas et al, 1996] reported a series of seven patients with gliomas, undergoing preoperative fMRI at 1.5T. Their fMRI protocol included single- or multi-stage active finger tapping as motor tasks [Atlas et al, 1996]. They reported that in $28.5 \%$ of their cases fMRI failed to identify the motor cortex, while they postulated that glioblastomas and high-grade gliomas may alter the obtained BOLD signal [Atlas et al, 1996]. Fandino et al. [Fandino et al., 1999] reported similar findings from a series of 11 patients with central area tumors, undergoing preoperative fMRI at $1.5 \mathrm{~T}$ and intraoperative DCS for motor cortex localization. They considered that there was concordance between their fMRI and DCS findings when there was a discrepancy of less than $20 \mathrm{~mm}$ [Fandino et al., 1999]. They found that fMRI accuracy in their series was $82 \%$ [Fandino et al., 1999].

In a more recent study, Lehericy et al. [Lehericy et al., 2000] reported their results from a retrospective study of 26 patients with intracranial tumors, undergoing preoperative fMRI at $1.5 \mathrm{~T}$ and intraoperative DCS for motor cortex localization. Their fMRI protocol included active flexion/extension of fingers/toes, and lip contraction as motor tasks [Lehericy et al., 2000]. They found $92 \%$ agreement between fMRI and DCS results, while fMRI accuracy was $100 \%$ when error margin was $15 \mathrm{~mm}$ [Lehericy et al., 2000]. Similarly, Hirsch et al. [Hirsch et al, 2000] reported their results from a large prospective study of 125 patients with various intracranial pathologies, and of 63 healthy volunteers. All their participants underwent fMRI at 1.5T unit for sensori-motor, language, and visual cortex localization [Hirsch et al, 2000]. Their fMRI protocol included active finger tapping as a motor task, passive tactile hand stimulation as a sensory task, and picture naming by silent speech and object or name listening as language tasks [Hirsch et al, 2000]. They were able to localize with fMRI the central sulcus in $100 \%$ of their healthy volunteers and in $98.4 \%$ of their patients [Hirsch et al, 2000]. They were also able to localize by fMRI the Wernicke's and Broca's areas in $91 \%$ and $77 \%$, respectively, in their patients, while the respective percentages were $100 \%$ and $93 \%$ for their volunteers [Hirsch et al, 2000]. The primary visual cortex was identified by fMRI in 
$100 \%$ of their tested cases [Hirsch et al, 2000]. Haberg et al. [Haberg et al., 2004] reported a series of 21 patients with gliomas, undergoing preoperative fMRI at $1.5 \mathrm{~T}$ for localizing sensori-motor and language associated cortical areas. Their fMRI protocol included active thumb-index opposition, active toe flexion, and active tongue movement as motor tasks, and thinking of a short action-verb and silent construction of a short noun as language paradigms [Haberg et al., 2004]. They reported that $80 \%$ of their patients were able to successfully finish their fMRI studies, while in $20 \%$ of their cases fMRI was unsuccessful due to EPI signal voids, secondary to previous craniotomy, head motion artifacts, and patient's inability to perform the task. They found that $73 \%$ of their patients had no postoperative neurological deficits, while $27 \%$ had a new permanent postoperative deficit [Haberg et al., 2004]. It has to be emphasized however, that $43.3 \%$ of these patients developing new deficits, knew that before the operation, from the obtained fMRI [Haberg et al., 2004]. Similarly, Krishnan et al. [Krishnan et al., 2004] reported their results from a prospective study including 54 patients with various intracranial tumors. All their patients underwent preoperative fMRI at $1.5 \mathrm{~T}$ and intraoperative DCS/SSEP studies for localization of motor cortex [Krishnan et al., 2004]. They examined their total resection rates and the observed morbidity in association with the distance of the resection margin from the fMRI defined motor cortex [Krishnan et al., 2004]. They found that when this distance was $>15 \mathrm{~mm}$ their resection rate was $85.7 \%$, while $53 \%$ of their patients remained neurologically unchanged postoperatively and $47 \%$ were improved [Krishnan et al., 2004]. When this distance was between $10-15 \mathrm{~mm}$, total resection was achieved in $86.6 \%$ of their patients, $13.3 \%$ developed new postoperative neurological deficits, $60 \%$ remained unchanged, while $37.3 \%$ were improved [Krishnan et al., 2004]. When the distance was between 5-10 mm, total resection was observed in $83.3 \%$, while $50 \%$ remained neurologically stable, and the remaining $50 \%$ were postoperatively improved [Krishnan et al., 2004]. Finally, when this distance was between $0-5 \mathrm{~mm}$, total resection was accomplished in $85 \%$, neurological worsening occurred in $35 \%, 50 \%$ remained stable, while $15 \%$ were improved [Krishnan et al., 2004]. They pointed out that the obtained BOLD signal may be varying with the utilized functional paradigm, and they concluded that $\mathrm{fMRI}$ is a valuable tool in surgical planning [Krishnan et al., 2004].

$\mathrm{Li}$ et al. [Li et al, 2010] in a recent study reported their experience from five patients with gliomas, undergoing preoperative fMRI at $3 \mathrm{~T}$ and intraoperative DCS for motor cortex localization. They found that the accuracy of fMRI was 100\% in their series, and postulated that $3 \mathrm{~T}$ field provides an increased BOLD signal [Li et al, 2010]. Berntsen et al. [Berntsen et al., 2010] reported their results from a prospective study including 51 patients with various intracranial lesions (33 patients with gliomas), undergoing preoperative fMRI for sensori-motor cortex localization. Interestingly, 3.9\% of their patients did not undergo surgery because of the preoperative MRI and fMRI findings. They reported that $13 \%$ of their patients could not finish their fMRI study, either due to reduced cooperation or excessive head motion artifacts [Berntsen et al., 2010]. They reported that in $42 \%$ of their cases more than $95 \%$ of the tumor was resected, while their mean residual tumor was $11 \%$ (range: $0-94 \%$ ) [Berntsen et al., 2010]. They also found, that $88 \%$ of their patients had stable postoperative neurological status, while $12 \%$ experienced some worsening [Berntsen et al., 2010]. The lesion to eloquent area distance was related to the amount of tumor residual, and this relationship was statistically significant in their series [Berntsen 
et al., 2010]. They postulated that fMRI accuracy depends highly on the skills of the obtained data analyst [Berntsen et al., 2010].

Several clinical studies with significantly varying results have been published in regard to the language-associated cortical areas identification and localization by employing fMRI [Bizzi et al., 2008; Fandino et al., 1999; Fitzgerald et al., 1997; Giussani et al., 2010; Lurito et al., 2000; Mueller et al., 1996; Pouratian et al., 2002; Roux et al., 2003; Ruge et al., 1999; Rutten et al., 2002; Signorelli et al., 2003; Tomczak et al., 2000; Yetkin et al., 1997]. Mueller et al. [Mueller et al., 1996] reported their results from a series of 12 patients (10 tumor and 2 vascular cases) undergoing fMRI and intraoperative language mapping for languageassociated cortical areas localization. Their fMRI protocol included audible and silent word generation paradigms [Mueller et al., 1996]. They reported that their fMRI findings were concordant to their intraoperative findings in 100\% of their cases [Mueller et al., 1996]. Similarly, Yetkin et al. [Yetkin et al., 1997] reported a series of 28 patients (but only five tumor cases), undergoing preoperative fMRI at 1.5T for language mapping and intraoperative DCS. Their fMRI protocol included silent word generation and number counting tasks, while their DCS protocol included text recitation and number counting [Yetkin et al., 1997]. They found that the fMRI accuracy in localizing language centers was $100 \%$ when the error margin was $20 \mathrm{~mm}$, and $86 \%$ when the error margin was $10 \mathrm{~mm}$ [Yetkin et al., 1997]. Likewise, Ruge et al. [Ruge et al., 1999] reported their results from a series of 21 patients with various intracranial tumors undergoing preoperative fMRI at 1.5T for language mapping and intraoperative DCS. Their fMRI protocol included object naming and word listening, while their DCS paradigms were object naming and number counting [Ruge et al., 1999]. They found concordance between the fMRI and DCS findings in $100 \%$ of their cases [Ruge et al., 1999]. Fitzgerald et al. [Fitzgerald et al., 1997] reported their findings from a series of 11 patients (but only 8 tumor cases), undergoing preoperative fMRI at 1.5T and intraoperative DCS for language mapping. Their fMRI tasks were word reading, verb generation, text listening, and word listening, while their DCS paradigms included object naming and number counting [Fitzgerald et al., 1997]. They reported $81 \%$ sensitivity and $53 \%$ specificity for fMRI when the margin error was $10 \mathrm{~mm}$, while the respective percentages were $92 \%$ and $0 \%$ when the margin error was $20 \mathrm{~mm}$ [Fitzgerald et al., 1997]. Similarly, Signorelli et al. [Signorelli et al., 2003] reported two glioma cases undergoing fMRI at 1.5T and reported excellent fMRI accuracy and high reproducibility.

Contrariwise, Lurito et al. [Lurito et al., 2000] reported three glioma cases undergoing preoperative $\mathrm{fMRI}$ at $1.5 \mathrm{~T}$ and intraoperative DCS for language mapping. They employed object naming and number counting tasks for both fMRI and DCS [Lurito et al., 2000]. They reported good but imperfect correlation between fMRI and DCS findings [Lurito et al., 2000]. Likewise, Tomczak et al. [Tomczak et al., 2000] reported a large series of 41 tumor cases (34 gliomas), undergoing preoperative fMRI at 1.5T and intraoperative DCS for language mapping. Their fMRI paradigm was a semantic test for word relations, while their DCS protocol included speech comprehension, word production, object naming, and text reading [Tomczak et al., 2000]. They reported agreement between their fMRI and DCS findings in only $33.3 \%$ of their cases [Tomczak et al., 2000].

Pouratian et al. [Pouratian et al., 2002] in a more recent study of 10 patients (none of them with glioma) undergoing preoperative fMRI at 3T and intraoperative DCS for language mapping, found that fMRI was more accurate in localizing language-associated areas in the frontal lobe than the temporal and parietal lobes. The observed fMRI sensitivity and 
specificity were $100 \%$ and $66.7 \%$, respectively for the frontal lobe, while the respective percentages for the temporal and parietal lobes were $96.2 \%$ and $69.8 \%$ [Pouratian et al., 2002]. Their fMRI protocol included object naming, word generation, auditory responsiveness, visual responsiveness, and sentence comprehension, while their DCS protocol included only object naming [Pouratian et al., 2002]. Roux et al. [Roux et al., 2003] reported their results from a series of 14 tumor patients (11 gliomas, 3 meningiomas) undergoing preoperative fMRI at 1.5T and intraoperative DCS for language mapping. Their fMRI and DCS protocol included object naming and verb generation paradigms [Pouratian et al., 2002]. They reported 59\% sensitivity and $97 \%$ specificity for fMRI, and they concluded that fMRI cannot be used alone for surgical planning, in critically-located tumors in the language cortical areas [Pouratian et al., 2002].

Rutten et al. [Rutten et al., 2002] reported their findings from a series of 13 epilepsy patients undergoing preoperative fMRI and intraoperative DCS for language mapping. Their fMRI protocol included object naming and verb generation paradigms, while their DCS protocol included only object naming [Rutten et al., 2002]. They reported $100 \%$ sensitivity and $61 \%$ specificity for fMRI in their study [Rutten et al., 2002]. Likewise, Bizzi et al. [Bizzi et al., 2008] reported a large series of tumor cases (28 gliomas, 2 metastatic tumors, and 1 meningioma) undergoing preoperative fMRI at 1.5T and intraoperative DCS for language mapping. Their fMRI and DCS protocol included solely a verb generation paradigm [Bizzi et al., 2008]. They found that fMRI had $80 \%$ sensitivity and $78 \%$ specificity in their series [Bizzi et al., 2008].

\subsection{Future directions of preoperative fMRI in gliomas}

Further development of fMRI techniques may strengthen its role in the preoperative evaluation of patients with gliomas. The wide clinical application of higher magnetic fields may increase fMRI's quality. It has been demonstrated that higher magnetic fields change the relaxation rate $\mathrm{R} 2$ and thus provide better BOLD signal and more accurate fMRI studies [Hoenig et al., 2005; Krasnow et al., 2003; Tieleman et al., 2007] Higher strength magnets allow fMRI studies to be performed in shorter times, and provide the opportunity for almost real time imaging of cortical activation during stimulation [Scarabino et al., 2007]. The development of advanced software packages, allowing further decrease of susceptibility artifacts, may well improve the quality and the accuracy of fMRI [Li et al., 2010].

Moreover, the development of more concrete paradigms and protocols may further improve fMRI's accuracy and reproducibility. It has been shown that loud speech tasks provide more accurate fMRI data than silent speech tasks [Petrovich et al., 2005]. Therefore, the development of multi-stage, loud language tasks examining several aspects of language may further increase fMRI's accuracy. The implementation of other advanced MR imaging techniques such as Diffusion Tensor Imaging and intraoperative fMRI and DTI may allow better identification and localization not only of the cortical languageassociated centers but also their interconnecting networks, and thus may make glioma resection safer [Nimsky, 2011].

The development of paradigms and protocols for testing higher cognitive functions such as memory, emotion, affect, and other high-cognitive functions may further increase the applicability of fMRI in the preoperative evaluation of patients with gliomas. These potential fMRI developments along with the technological evolution of the frameless neuronavigational systems may allow more aggressive and safer resection of gliomas of eloquent cerebral cortex. 


\section{Conclusions}

Functional MRI constitutes a non-invasive imaging modality that lends itself to the identification and localization of eloquent cortical areas and cerebral functional mapping. Its ability to depict neuronal activity is based on the Blood-Oxygenation-Level-Dependent (BOLD) phenomenon, which is correlated with the relative concentrations of oxy- and deoxy-hemoglobin in an activated cerebral region. It represents thus, an indirect evaluating tool of neuronal activity. The acquisition of Gradient Echo-Echo Planar Imaging (GE-EPI) $\mathrm{T}_{2}{ }^{*}$ sequences is required for fMRI studies. Strength of at least $1.5 \mathrm{~T}$ is required for obtaining an adequate resolution fMRI, while several studies postulate that higher magnetic field MR units may provide better quality and more accurate fMRI studies. Functional MRI is characterized by higher spatial resolution than PET, hdEEG, MEG/MSI, or SPECT functional studies, while its temporal resolution is comparable, if not better, to that of all other functional imaging methodologies. Moreover, MR units are easily accessible, and definitely more widely distributed than any of the other methodologies.

Identification and localization of motor, sensory, and language-associated cortical, but also deep-sited areas can be performed with fMRI. Various performance tasks and protocols have been proposed and utilized in clinical practice, with variable success rates. It can be postulated that active thumb-index opposition, active hand squeezing, and active finger tapping are the most commonly employed motor tasks. Palm light brushing, palm air-puff blowing, and median nerve electrical irritation are the most commonly applicable sensory tasks. Object naming, word generation, and number counting are the most popular speech tests, although a wide variability occurs in language tests, and several issues (silent vs. loud language testing) remain still controversial. It has to be emphasized that the selection of the appropriate performance tasks (especially for language) frequently needs to be individualized. Another issue that requires attention is the application of the same language tests during fMRI and intraoperative DCS, when both studies are performed, for standardizing the comparison of these methodologies.

The role of preoperative fMRI in the management of patients with intracranial gliomas is of paramount importance. Functional MRI allows the preoperative localization of sensorimotor and language associated areas, and their relationships with the studied tumor. This information enables the neurosurgeon to discuss with the patient the possibility of total tumor resection, the chance of postoperative neurological deficits, and the calculation of the benefit to risk ratio. Furthermore, it provides the opportunity for a safer surgical planning, and potentially for a more aggressive tumor resection. Functional MRI allows cortical mapping even in cases that DCS during awake-craniotomy is impossible, as in pediatric or psychologically unstable patients. The vast majority of the published clinical studies demonstrate that the accuracy of fMRI in localizing sensori-motor cortex is extremely high, while that of language-associated cortical areas is lower. There are several studies postulating that fMRI language-associated cortical areas may be inaccurate, particularly in cases of temporal or parietal localization of speech areas. Additionally, the presence of scar tissue due to a previous craniotomy, the presence of neovascularization in cases of high grade gliomas, the presence of tumor-induced cellular chemical changes, and the proximity of large veins to the tumor, may confound the accuracy of fMRI.

The development of novel, high-reproducibility, high-resolution software fMRI packages, along with the application of higher magnetic field strength may further increase fMRI's accuracy, and minimize the confounding effect of various glioma-associated parameters. 
The preoperative evaluation with fMRI of higher cognitive functions may allow even safer glioma surgical resection in the near future.

\section{References}

Atlas, S., Howard, RS., Maldjian, J., Alsop, D., Detre, JA., Listerud, J., D'Esposito, M., Judy, KD., Zager, E., \& Stecker, M. (1996). Functional magnetic resonance imaging of regional brain activity in patients with intracerebral gliomas: findings and implications for clinical management, Neurosurgery Vol. 38, No. 2, (February 1996), pp. 329-338, ISSN 0148-396X

Berntsen, EM., Gulati, S., Solheim, O., Kvistad, KA., Torp, SH., Selbekk, T., Unsgård, G., \& Håberg, AK. (2010). Functional magnetic resonance imaging and diffusion tensor tractography incorporated into an intraoperative 3-dimensional ultrasound-based neuronavigation system: impact on therapeutic strategies, extent of resection, and clinical outcome, Neurosurgery Vol. 67, No. 2, (August 2010), pp. 251-264, ISSN 0148-396X

Bizzi, A., Blasi, V., Falini, A., Ferroli, P., Cadioli, M., Danesi, U., Aquino, D., Marras, C., Caldiroli, D., \& Broggi, G. (2008). Presurgical functional MR imaging of language and motor functions: validation with intraoperative electrocortical mapping, Radiology Vol. 248, No. 2, (August 2008), pp. 579-589, ISSN 0033-8419

Fandino, J., Kollias, SS., Wieser, HG., Valavanis, A., \& Yonekawa, Y. (1999). Intraoperative validation of functional magnetic resonance imaging and cortical reorganization patterns in patients with brain tumors involving the primary motor cortex, $J$ Neurosurg Vol. 91, No. 2, (August 1999), pp. 238-250, ISSN 0022-3085

Fitzgerald, DB., Cosgrove, GR., Ronner, S., Jiang, H., Buchbinder, BR., Belliveau, JW., Rosen, BR., \& Benson, RR. (1997). Location of language in the cortex: a comparison between functional MR imaging and electrocortical stimulation, AJNR Am J Neuroradiol Vol. 18, No. 8, (September 1997), pp. 1529-1539, ISSN 0195-6108

Giussani, C., Roux, FE., Ojemann, J., Sganzerla, EP., Pirillo, D., \& Papagno, C. (2010). Is preoperative functional magnetic resonance imaging reliable for language areas mapping in brain tumor surgery? Review of language functional magnetic resonance imaging and direct cortical stimulation correlation studies, Neurosurgery Vol. 66, No. 1, (January 2010), pp. 113-120, ISSN0148-396X

Haberg, A., Kvistad, KA., Unsgard, G., \& Haraldseth, O. (2004). Preoperative blood oxygen level-dependent functional magnetic resonance imaging in patients with primary brain tumors: clinical application and outcome, Neurosurgery Vol. 54, No. 4, (April 2004), pp. 902-915, ISSN 0148-396X

Hirsch, J., Ruge, MI., Kim, KH., Correa, DD., Victor, JD., Relkin, NR., Labar, DR., Krol, G., Bilsky, MH., Souweidane, MM., DeAngelis, LM., \& Gutin, PH. (2000). An integral functional magnetic resonance imaging procedure for preoperative mapping of cortical areas associated with tactile, motor, language, and visual functions, Neurosurgery Vol. 47, No. 3, (September 2000), pp. 711-722, ISSN 0148-396X

Hoenig, K., Kuhl, CK., \& Scheef, L. (2005). Functional 3.0-T MR assessment of higher cognitive function: are there advantages over 1.5-T imaging?, Radiology Vol. 234, No. 3, (March 2005), pp. 860-868, ISSN 0033-8419

Krasnow, B., Tamm, L., Greicius, MD., Yang, TT., Glover, GH., Reiss, AL., \& Menon, V. (2003). Comparison of fMRI activation at 3 and $1.5 \mathrm{~T}$ during perceptual, cognitive, 
and affective processing, Neuroimage Vol. 18, No. 4, (April 2003), pp. 813-826, ISSN 10538119

Krishnan, R., Raabe, A., Hattingen, E., Szelényi, A., Yahya, H., Hermann, E., Zimmermann, M., \& Seifert, V. (2004). Functional magnetic resonance imaging-integrated neuronavigation: correlation between lesion-to-motor cortex distance and outcome, Neurosurgery Vol. 55, No. 4, (October 2004), pp. 904-915, ISSN 0148-396X

Lacroix, M., Abi-Said, D., Fourney, DR., Gokaslan, ZL., Shi, W., DeMonte, F., Lang, FF., McCutcheon, IE., Hassenbusch, SJ., Holland, E., Hess, K., Michael, C., Miller, D., \& Sawaya, R. (2001). A multivariate analysis of 416 patients with glioblastoma multiforme: prognosis, extent of resection, and survival, J Neurosurg Vol. 95, No. 2, (August 2001), pp. 190-198, ISSN 0022-3085

Lehericy, S., Duffau, H., Cornu, P., Capelle, L., Pidoux, B., Carpentier, A., Auliac, S., Clemenceau, S., Sichez, JP., Bitar, A., Valery, CA., Van Effenterre, R., Faillot, T., Srour, A., Fohanno, D., Philippon, J., Le Bihan, D. \& Marsault, C. (2000). Correspondence between functional magnetic resonance imaging somatotopy and individual brain anatomy of the central region: comparison with intraoperative stimulation in patients with brain tumors, J Neurosurg Vol. 92, No. 4, (April 2000), pp. 589-598, ISSN 0022-3085

Li, SW., Wang, JF., Jiang, T., Li, SW., Zhang, WB., Li, ZX., Zhang, Z., Dai, JP., \& Wang, ZC. (2010). Preoperative 3T high field blood oxygen level dependent functional magnetic resonance imaging for glioma involving sensory cortical areas, Chin Med J Vol. 123, No. 8, (April 20), pp. 1006-1010, ISSN

Lurito, JT., Lowe, MJ., Sartorius, C., \& Mathews, VP. (2000). Comparison of fMRI and intraoperative direct cortical stimulation in localization of receptive language areas, J Comput Assist Tomogr Vol. 24, No. 1, (January-February 2000), pp. 99-105, ISSN 0363-8715

McDonald, JD., Chong, BW., Lewine, JD., Jones, G., Burr, RB., McDonald, PR., Koehler, SB., Tsuruda, J., Orrison, WW., \& Heilbrun, MP. (1999). Integration of preoperative and intraoperative functional brain mapping in a frameless stereotactic environment for lesions near eloquent cortex. Technical note, J Neurosurg Vol. 90, No. 3, (March 1999), pp. 591-598, ISSN 0022-3085

Mueller, WM., Yetkin, ZF., Hammeke, TA., Morris, GL 3rd., Swanson, SJ., Reichert, K., Cox, R., \& Haughton, VM. (1996). Functional magnetic resonance imaging mapping of the motor cortex in patients with cerebral tumors, Neurosurgery Vol. 39, No. 3, (September 1996), pp. 515-521, ISSN 0148-396X

National Comprehensive Cancer Network. (2007). Central Nervous System Cancer Guidelines. Jenkintown, PA: NCCN Press

Nimsky, C. (2011). Intraoperative acquisition of fMRI and DTI, Neurosurg Clin N Am Vol. 22, No. 2, (April 2011), pp. 269-277, ISSN 1050-6438

Petrovich, N., Holodny, AI., Tabar, V., Correa, DD., Hirsch, J., Gutin, PH., \& Brennan, CW. (2005). Discordance between functional magnetic resonance imaging during silent speech tasks and intraoperative speech arrest, J Neurosurg Vol. 103, No. 2, (August 2005), pp. 267-274, ISSN 0022-3085

Pouratian, N., Bookheimer, SY., Rex, DE., Martin, NA., \& Toga, AW. (2002). Utility of preoperative functional magnetic resonance imaging for identifying language 
cortices in patients with vascular malformations, Neurosurg Focus Vol. 13, No.4,(October 2002), pp. 21-32, ISSN 1092-0684

Puce, A., Constable, T. Luby, ML., McCarthy, G., Nobre, AC., Spencer, DD., Gore, JC., \& Allison, T. (1995). Functional magnetic resonance imaging of sensory and motor cortex: comparison with electrophysiological localization, J Neurosurg Vol. 83, No. 2, (August 1995), pp. 262-270, ISSN 0022-3085

Roux, FE., Boulanouar, K., Lotterie, JA., Mejdoubi, M., LeSage, JP., \& Berry, I. (2003). Language functional magnetic resonance imaging in preoperative assessment of language areas: correlation with direct cortical stimulation, Neurosurgery Vol. 52, No. 6, (June 2003), pp. 1335-1347, ISSN 0148-396X

Ruge, MI., Victor, J., Hosain, S., Correa, DD., Relkin, NR., Tabar, V., Brennan, C., Gutin, PH., \& Hirsch, J. (1999). Concordance between functional magnetic resonance imaging and intraoperative language mapping, Stereotact Funct Neurosurg Vol. 72, No. 2-4, pp. 95-102, ISSN 0022-3085

Rutten, GJ., Ramsey, NF., van Rijen, PC., Noordmans, HJ., \& van Veelen, CW. (2002). Development of a functional magnetic resonance imaging protocol for intraoperative localization of critical temporoparietal language areas, Ann Neurol Vol. 51, No. 3, (March 2002), pp. 350-360, ISSN 1531-8249

Sanai, N., \& Berger, MS. (2008). Glioma extent of resection and its impact on patient outcome, Neurosurgery Vol. 62, No. 4, (April 2008), pp. 753-764, ISSN 0148-396X

Scarabino, T., Giannatempo, GM., Popolizio, T., Tosetti, M., d'Alesio, V., Esposito, F., Di Salle, F., Di Costanzo, A., Bertolino, A., Maggialetti, A., \& Salvolini, U. (2007). 3.0-T functional brain imaging: a 5-year experience, Radiol Med Vol. 112, No.1, (February 2007), pp. 97-112, ISSN 0033-8362

Schulder, M., Maldjian, JA., Liu, WC., Holodny, AI., Kalnin, AT., Mun, IK., \& Carmel, PW. (1998). Functional image-guided surgery of intracranial tumors located in or near the sensorimotor cortex, J Neurosurg Vol. 89, No. 3, (September 1998), pp. 412-418, ISSN 0022-3085

Signorelli, F., Guyotat, J., Schneider, F., Isnard, J., \& Bret, P. (2003). Technical refinements for validating functional MRI-based neuronavigation data by electrical stimulation during cortical language mapping, Minim Invasive Neurosurg Vol. 46, No. 5, (October 2003), pp. 265-268, ISSN 09467211

Stafford, SL., Perry, A., Suman, VJ., Meyer, FB., Scheithauer, BW., Lohse, CM., \& Shaw, EG. (1998). Primarily resected meningiomas: outcome and prognostic factors in 581 Mayo Clinic patients, 1978 through 1988, Mayo Clin Proc Vol. 73, No. 10, (October 1998), pp. 936-942, ISSN 0025-6196

Tieleman, A., Vandemaele, P., Seurinck, R., Deblaere, K., \& Achten, E. (2007). Comparison between functional magnetic resonance imaging at 1.5 and 3 Tesla: effect of increased field strength on 4 paradigms used during presurgical work-up, Invest Radiol Vol. 42, No. 2, (February 2007), pp. 130-138, ISSN 0020-9996

Tomczak, RJ., Wunderlich, AP., Wang, Y., Braun, V., Antoniadis, G., Görich, J., Richter, HP, \& Brambs, HJ. (2000). fMRI for preoperative neurosurgical mapping of motor cortex and language in a clinical setting, J Comput Assist Tomogr Vol. 24, No. 6, (November-December 2000), pp. 927-934, ISSN 0363-8715

Yetkin, ZF., Mueller, WM., Morris, GL., McAuliffe, TL., Ulmer, JL., Cox, RW., Daniels, DL., \& Haughton, VM. (1997). Functional MR activation correlated with intraoperative 
cortical mapping, AJNR Am J Neuroradiol Vol. 18, No. 7, (August 1997), pp. 1311-5, ISSN 0195-6108

Yousry, TA., Schmid, UD., Jassoy, AG,, Schmidt, D., Eisner, WE., Reulen, HJ., Reiser, MF., \& Lissner, J. (1995). Topography of the cortical motor hand area: prospective study with functional MR imaging and direct motor mapping at surgery, Radiology Vol.195, No.1, (April 1995), pp.23-29, ISSN 0033-8419 


\title{
Neuroimaging in Epileptic Disorders
}

\author{
José Augusto Bragatti \\ Hospital de Clínicas de Porto Alegre \\ Universidade Federal do Rio Grande do Sul \\ Brazil
}

\section{Introduction}

Epilepsy is one of the most common neurologic disorders, and affects about 50 million people around the world. It is a heterogeneous condition characterized by recurrent spontaneous epileptic seizures. There are no age, sex or race discriminations, although incidence of the first epileptic seizure has a bimodal distribution, with peaks in childhood and after 60 years of age (Engel \& Pedley, 2008).

According to their localization, epilepsies and epileptic seizures should be classified as focal (or partial) and generalized. Partial seizures begin in a restricted portion of cerebral cortex. Partial seizures are associated with a restricted area of the of the cerebral cortex and are clasified as either simple or complex, depending on the level of conscious awareness. Simple partial seizures are characterized by brief specific neurologic dysfunction, contingent upon the localization of the epileptogenic focus. By definition, they do not compromise consciousness during the event, and might manifest as motor signs (with a focus involving the primary motor area), sensitive symptoms (focus in the primary somatosensory area), autonomic or psychic signs (mesiobasal areas). Complex partial seizures affect the patient's consciousness from the onset of the event. Both simple and complex partial seizures can propagate to the whole brain and become secondarily generalized seizures. Examples of primary generalized seizures are absence seizures, tonic-clonic generalized seizures, and myoclonic seizures. All those three seizure types are generated by epileptic discharges involving both cerebral hemispheres from their onset. By extension, focal epilepsies are characterized by recurrent spontaneous partial seizures, and generalized epilepsies have generalized seizures as their main seizure type.

Epilepsies can be idiopathic (without an identifiable structural cause), symptomatic (due to a structural lesion), or cryptogenic (with a presumed lesion, not detectable by current available neuroimaging methods). Broadly, idiopathic epilepsies (partial or generalized) begin in the childhood years, have a good prognosis, and go into remission during adolescence. The symptomatic generalized epilepsies encompass a group of epileptic syndromes with very poor prognosis, characterized by extremely refractory seizures and accompanied by neurodevelopmental delay. The symptomatic partial epilepsies depict a large spectrum of epileptic disorders caused by several types of brain lesions. A significant percentage of patients who have seizures not responsive to antiepileptic drugs is affected by this type of epilepsy. Surgical treatment becomes a good therapeutic option for these patients, and the identification of the structural cortical lesion causing their epilepsies is primordial for a good postsurgical prognosis (Panaiyotopoulos, 2010). 
Although the basic phenomena of the epilepsies are basically neurophysiologic in their nature, neuroimaging techniques have provided valuable contributions for a greater understanding on this matter. Magnetic resonance imaging (MRI) has revolutionized assessment and management of patients with refractory epilepsies. Several pathological processes affecting the central nervous system can be identified using MRI and related techniques. Brain lesions classically associated with epilepsy, like hippocampal sclerosis, cortical malformations, gliosis, vascular disorders, and small brain tumors often were not visualized using old techniques, named cranial skull X-ray and brain computed tomography (CT). When it is possible to detect a structural lesion underlying an epileptic condition, the prognosis can be better defined, and the most appropriate treatment (e.g., drug vs. surgery) can be indicated with greater confidence (Mc Donald et al., 2000).

Standard MRI has a fundamental role in epilepsy surgery: to identify the epileptogenic lesion, depicting its topographic relationships with eloquent regions of the brain. Magnetic resonance spectroscopy (MRS) measures metabolites in a defined region of interest, and provides important lateralization information for temporal lobe epilepsies, and localization of neocortical epilepsies. Functional MRI (fMRI) focuses on the identification of eloquent cortex to be spared in planning epilepsy surgery, with the intention to improve outcomes by minimizing postoperative deficits. Diffusion MRI allows visualization of brain tracts, improving the study of epileptic circuitries. Magnetoencephalography (MEG) is a technology used to record spontaneous and evoked magnetic fields generated by cortical neuronal activity, and provides additional data to spike detection primarily performed by electroencephalogram (EEG). Because epilepsy is a functional disorder, several invaluable data can be obtained studying epileptic patients with positron emission tomography (PET) and single photon emission computed tomography (SPECT). PET can map numerous physiochemical brain processes, and ictal SPECT allows measurement of brain perfusion patterns during seizures, which is useful in the preoperative assessment of epileptic patients. Current software development enables integration between a variety of imaging data, aiming to minimize the use of invasive techniques in the investigation of surgical candidates, reducing risk and maximizing results (Duncan, 2009).

In this chapter, the role of different neuroimaging techniques in the study and management of epilepsy will be described and evaluated. A brief description of the technique will be followed by indications and main findings contributing for the comprehension of the epileptic disorders. Because detection of a brain lesion is the first step for a successful surgical treatment of epilepsy, emphasis in the presurgical assessment of epilepsy surgery will be given.

\section{Structural magnetic resonance imaging}

Brain MRI is an important tool to define the presence of a structural abnormality responsible for epilepsy. Acquiring appropriate epilepsy-focused MRI of high quality and diagnosing the important lesions with high sensitivity and specificity are imperative to the definition and understanding of structural brain abnormalities in patients with epilepsy. Moreover, surgical treatment of epileptic patients has a better general outcome when a structural lesion is identified by MRI and further resected. However, brain MRI may be normal in up to one third of patients undergoing anterior temporal lobectomy for refractory temporal lobe epilepsy (Radhakrishnan et al, 1998). 
The technique of MRI is based on the intrinsic properties of atomic nuclei of charge, spin, and magnetism. These properties are enhanced in certain nuclei such as hydrogen, phosphorus, and sodium, which are natural components of the brain tissue. They behave as bar magnets and, when submitted to a magnetic field, will align along the direction of the field. In addition, the nucleus possesses another intrinsic property, which gives it a continuous rotary motion around the nuclear axis. This property is called nuclear spin, or spin angular momentum. Because of the combination of nuclear magnetism and spin, the nucleus rotates around both the direction of an external magnetic field and its own axis. The frequency of the precession around the magnetic field is characteristic of the particular nucleus and the direct linear relationship between frequency and the strength of the magnetic field is fundamental to NMR. This is the ground of the improvement of signal-tonoise ratio (SNR) in MR images acquired in a $3 \mathrm{~T}$ scanner is improved with respect to images acquired in a standard $1.5 \mathrm{~T}$ scanner, as there is a doubling of the fundamental precession frequency.

There are two discrete possibilities for the direction of the precession: one for the nucleus aligned along the direction of the external magnetic field and another one in the opposite direction (anti-aligned). The former state of alignment is preferred and the majority of nuclei will assume this state (low energy). However, a certain proportion of the nuclei will be antialigned (high energy) and it is this population difference that underlies the phenomenon of NMR. In the presence of a pulse of electromagnetic waves whose energy corresponds to the difference between these two states, the energy from these waves will be absorbed by the system and the populations of spins in the two states will be equalized. This perturbed state is temporary and, once the application of the electromagnetic waves is terminated, the nuclei will return to the initial state. The energy previously absorbed by the system will hence be returned in the form of a signal that can be detected by a suitably placed receiver coil. By imposing conditions on the system using magnetic field gradients, this signal will reflect not only the nuclear species from which it originated but also its spatial location. A spatially encoded map of these signals can thereby be formed. As water $(\mathrm{H} 2 \mathrm{O})$ forms more than $80 \%$ of the human body, the nucleus of the hydrogen atom is especially suitable for this technique.

Dedicated protocols for imaging in epilepsy patients have been developed to focus the suspected regions of interest (Jackson and Badawy, 2001). A typical clinical scanning protocol for a patient with refractory epilepsy may include T1-weighted imaging, T2-weighted imaging, fluid-attenuated inversion-recovery (FLAIR) imaging, and threedimensional volume acquisition sequences. This protocol is the basis of the initial screening study used to obtain structural information with good morphology and signal data (Table 1).

T1-weighted imaging (short repetition time [TR] and echo time [TE]) is commonly used to initially define the brain anatomy; thus cerebrospinal fluid (CSF) is black, gray matter is gray, and white matter is bright. These images take longer and have thicker slices and often incomplete brain coverage, but they can be extremely helpful in difficult cases. T2-weighted images (long TR, long TE) usually show the CSF as white and the gray matter of higher signal (brighter) than the white matter. These images typically show pathology as areas of increased signal and are used to assess whether the tissue is abnormal. On FLAIR images, the CSF appears dark, which helps to identify lesions with long T2 relaxation times. Pathology still looks high in signal (bright). In the case of epilepsy, the issue of most concern relates to subtle signal changes in the mesial temporal regions on coronal images, in which these artifacts need to be interpreted with an experienced eye. 


\begin{tabular}{|l|l|l|l|l|l|l|}
\hline & TR & TE & TI & Flip & Thickness & Matrix \\
\hline T2 cor FSE & 5000 & 85 & & 90 & 4 skip 1 & $512 \times 256$ \\
\hline FLAIR cor & 8000 & 129 & 2200 & 90 & 4 skip 1 & $256 \times 224$ \\
\hline T2 cor IR & 5500 & 20 & 200 & 90 & 4 skip 0 & $256 \times 256$ \\
\hline T1 sag & 2000 & 7,5 & 750 & 90 & 5 skip 2 & $256 \times 256$ \\
\hline T2 ax FSE & 5000 & 85 & & 90 & 5 skip 2 & $512 \times 256$ \\
\hline
\end{tabular}

Table 1. Protocol used in MRI exams for epileptic patients at the Service of Radiology of Hospital de Clínicas de Porto Alegre.

A variety of pathologic etiologies are able to be detected using a dedicated epilepsy protocol, with characteristic appearances that may be present, including mesial temporal sclerosis, cortical dysplasia, primary tumors of the brain, vascular malformations, and encephalomalacia. Mesial temporal or hippocampal sclerosis is the most important single cause of intractable MTLE found in surgical series, and it is the most common target for people with epiepsy that requires surgical treatment for refractory complex partial seizures in adulthood. A large body of data confirms the validity of structural abnormalities detected on MRI in pathologically proven HS. HS is histologically characterized by gliosis and neuronal loss in the hippocampus. However, extrahippocampal pathology is often found elsewhere in the temporal lobe, including white matter (Wieser et al., 2004). The key abnormalities in HS are hippocampal atrophy and hyperintense signal on T2-weighted images (Figure 1).

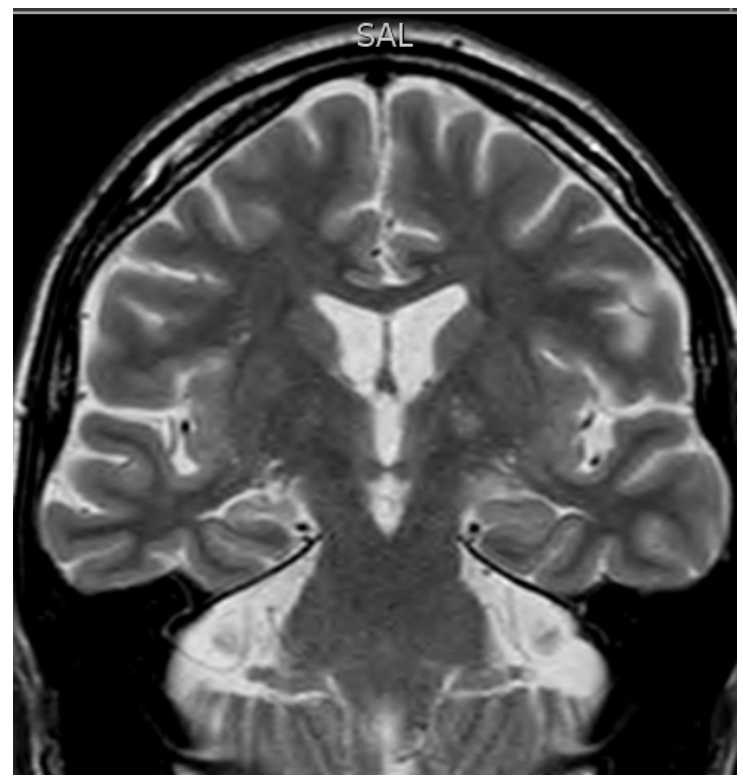

Fig. 1. T2-weighted MRI of right hippocampal sclerosis. Note atrophy and hyperintensity of right hippocampus. 
Other MR findings associated with HS include loss of ipsilateral hippocampal head digitations, widening of the temporal horn (Figure 2), and loss of the white matter adjacent to the collateral sulcus (Table 2). In surgical series of TLE, $43-72 \%$ of patients with the histopathologic features of MTS can be shown (Wieser et al., 2004).

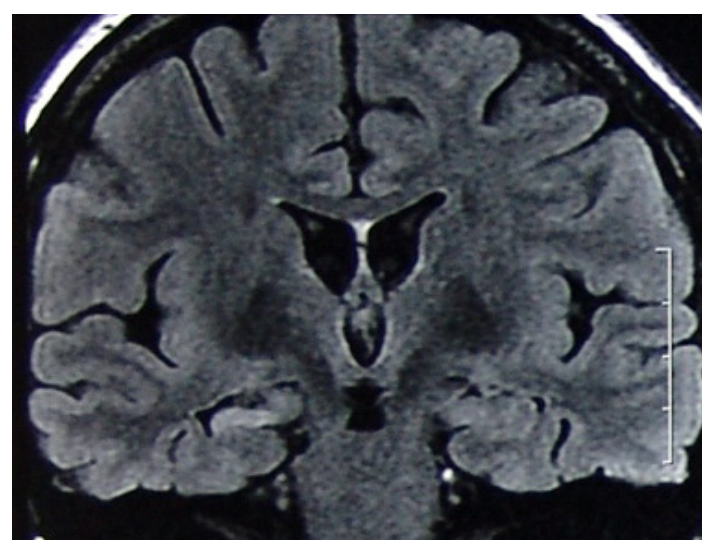

Fig. 2. Right hippocampal sclerosis. Ipsilateral temporal horn dilation and loss of head digitations.

\begin{tabular}{|l|l|}
\hline Hippocampal findings & $\begin{array}{l}\text { Atrophy } \\
\text { Loss of internal architecture } \\
\text { Signal changes }\end{array}$ \\
\hline \multirow{2}{*}{ Temporal lobe (extrahippocampal) findings } & $\begin{array}{l}\text { Dilation of temporal horn } \\
\text { Temporal lobe atrophy } \\
\text { Anterior temporal white matter signal } \\
\text { changes }\end{array}$ \\
\hline \multirow{2}{*}{ Extratemporal lobe findings } & $\begin{array}{l}\text { Fornix atrophy } \\
\text { Thalamic atrophy } \\
\text { Caudate atrophy }\end{array}$ \\
\hline
\end{tabular}

Table 2. MRI findings in hippocampal sclerosis.

Dual pathology is present in at least half of all cases of HS. It is nearly always ipsilateral. This can take one of two forms. In the first case, there is a lesion, clearly unrelated to the HS (Figure 3), and it is usually on the same side as the HS. In the second, the MRI shows changes in other parts of the brain that might be related to a common injury that caused both the HS and changes such as unilateral atrophy and anterior temporal lobe changes.

Malformations of cortical development (MCD) comprise a number of pathological entities that result from derangements of normal cortical development as a result of either abnormal neuronal proliferation, migration, or organization (Barkovich et al., 2005). Developmental disorders of cortex constitute $4 \%$ to $25 \%$ of all epileptogenic lesions in adults and $10 \%$ to $50 \%$ in pediatric epilepsy series (Guerrini, 2005).

Focal cortical dysplasias and diffuse cortical dysplasias are heterogeneous groups of developmental disorders defined by an abnormal cerebral cortical cytoarchitecture. Focal cortical dysplasia (FCD) is the most common form of focal developmental disorder, diagnosed in $20-30 \%$ of patients with medically intractable localization-related epilepsy. 


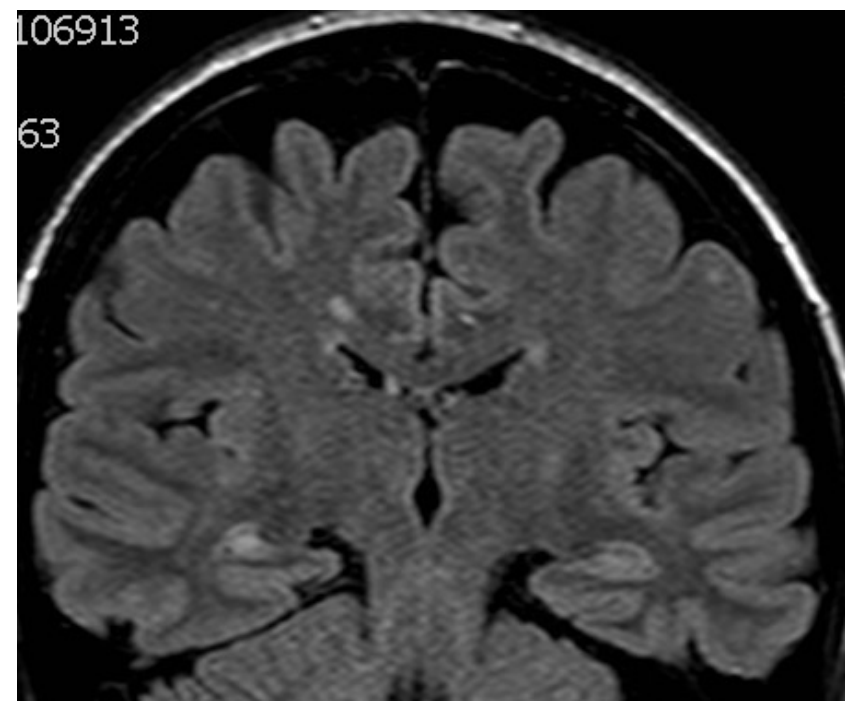

Fig. 3. Right hippocampal sclerosis. In addition, there are several bilateral periventricular hyperintense small lesions.

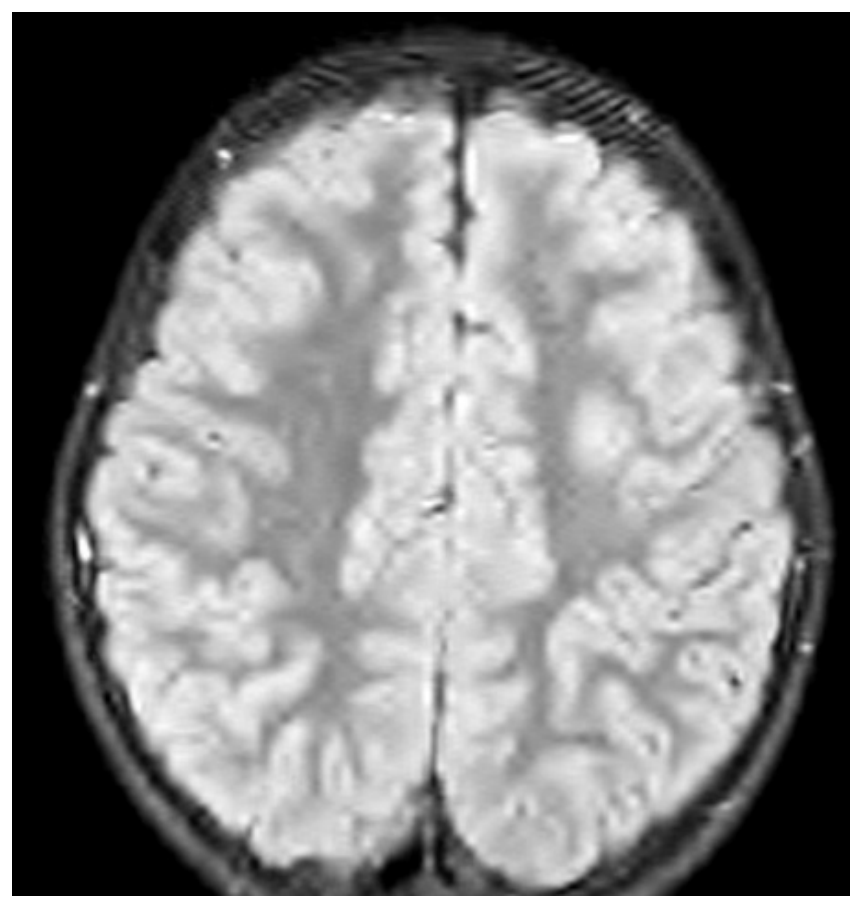

Fig. 4. Axial FLAIR: left frontal hyperintense subcortical lesion, diagnosed as a focal dysplasia by pathological exam. 
MRI has greater resolution than CT imaging and demonstrates abnormal gyral thickening (Figure 4) with underlying T2-weighted white matter changes (Colombo et al., 2003).

Hemimegalencephaly is a disorder of neuronal migration that results in a unilaterally enlarged dysplastic hemisphere. Focal seizures occur in the majority and are typically intractable to AEDs. Hemimegalencephaly may occur as an isolated finding or be associated with other conditions including neurofibromatosis, hypomelanosis of Ito, and the linear nevus syndrome. Brain MRI or CT reveals an enlarged hemisphere with cortical dysplasia with thickening of the mantle or gyri, calcification, other neuronal migrational abnormalities such as schizencephalic clefts (see later), and other anomalies such as agenesis of the corpus callosum. While widespread pathologic changes are seen in the affected hemisphere, microscopic abnormalities have also been noted in the seemingly unaffected hemisphere (Jahan et al., 1997).

Tuberous sclerosis complex (TSC), also known as Bourneville's disease, is one of the common, multisystem, autosomal dominant, neurocutaneous syndromes. TSC presents frequently with infantile spasm or seizures when the congential hamartomas affect the CNS in addition to other organ systems. Imaging techniques such as CT head scan and MRI scans are diagnostic in many cases, with calcified tubers being readily identified by CT brain scans. Tuber calcification and cortical atrophy are common findings, though cortical atrophy and ventricular dilation are more frequently seen with severe epilepsy and with mental retardation. Tuberous sclerosis has imaging features that aresimilar to those of Taylor-type dysplasia. There may not be a clear topographic correlation between tubers and the active epileptogenic zone.

Lissencephaly (including both agyria and pachygyria) is the most severe of the known malformations from abnormal neuronal migration. Less severe defects in the same genes and developmental processes result in subcortical band heterotopia. In this group of malformations, neurons begin migration but are unable to complete it. The imaging findings of lissencephaly vary with the severity of the mutation (Guerrini and Parrini, 2010). When very severe, the cortex is markedly thickened and almost no sulci are formed; when less severe, the cortex is less thickened and a variable number of shallow sulci separate broad gyri. In some cases, a 'cell-sparse zone' is identified between the thin outer cortical layer and the thicker inner cortical layer of neurons whose migration has been arrested.

There are three main groups of heterotopia: periventricular (usually nodular: PNH), subcortical (either nodular or laminar) and leptomeningeal, of which only the first two can be detected by imaging. Nodular heterotopia is one of the more common (15-20\%) forms of cortical malformation, responsible for $2 \%$ of patients with epilepsy. Subcortical band heterotopia or 'double cortex' is a mild form of lissencephaly and classified in that group.

The term "polymicrogyria" defines an excessive number of abnormally small gyri that produce an irregular cortical surface with lumpy aspect. Polymicrogyria can be localized to a single gyrus, involve a portion of one hemisphere, be bilateral and asymmetrical, bilateral and symmetrical or diffuse. Mild forms are difficult to recognize on neuroimaging. The imaging appearance of polymicrogyria varies with the patient's age (Takanashi and Barkovich, 2003). In newborns and young infants, the malformed cortex is very thin with multiple, very small undulations. After myelination, polymicrogyria appears as thickened cortex with irregular cortex white matter junction (Guerrini et al., 2008).

Schizencephaly refers to a full-thickness cleft in the cortex extending to the lateral ventricle and lies near the sylvian fissure. Schizencephaly may be unilateral or bilateral and 
represents a neuronal migrational disorder that affects the telencephalon. If the cortical walls are touching the condition is referred to as "closed lip" and, if separated by CSF, then "open lip schizencephaly". It is thought that schizencephaly and polymicrogyria are similar entities. This is based on the fact that the clefts are lined by polymicrogyria and mechanistically schizencephaly is at the end of the spectrum in polymicrogyria. Whether this is the case in all patients is not known at present.

Pathology plays an important role in determining which patients have seizures. Tumor histology influences seizure frequency, with a seizure incidence of $80-90 \%$ for oligodendrogliomas, $60-75 \%$ for astrocytomas, $55 \%$ for meningiomas, $55 \%$ for metastases, and $35-40 \%$ for malignant gliomas. Astrocytic tumors constitute about $20 \%$ of lesions detected in patients with epilepsy and often involve the parahippocampal gyrus and the amygdala. Although anaplastic tumors or glioblastomas are the most frequent astrocytic tumors; they usually present as a primary tumor problem and are rarely seen in patients with intractable epilepsy. In those patients, fibrillary astrocytomas are most common. MRI typically demonstrates homogeneous masses, with calcifications present in up to $20 \%$ of cases. The location is variable, with cortical lesions extending into the white matter and poorly demarcated. Pilocystic astrocytomas are more frequent in children. On MRI these lesions are sharply demarcated and often lobular. Contrast enhancement is typically present as a result of prominent vascularity. Edema is rare, and calcifications are not seen.

Xanthoastrocytomas usually occur in the first decade of life and have a good prognosis. Cystic components are common in this tumor, which usually occurs in the temporal or parietal region. Oligodendrogliomas are histologically characterized by the presence of compact groups of large, rounded cells with empty cytoplasm, often also with mixed glial components. These tumors are less frequent than astrocytomas and uncommon in patients referred for intractable epilepsy, but, when present, they are located within the temporal lobe and tend to involve the amygdala and the uncus and parahippocampal gyrus, with sparing of the middle and inferior temporal convolutions. The underlying white matter often shows tumor infiltration. Mixed glial lesions are a common tumor type in epilepsy (about $5 \%$ of foreign tissue lesions). Calcifications are often present. Seizures begin typically in childhood. These tumors are seen predominantly in young patients (b14 years). Gangliogliomas tend to spare the lateral temporal neocortex and involve primarily the mesial structures (Figure 5).

Dysembryoplastic neuroepithelial tumors (DNETs) are a common tumor type in patients with intractable epilepsy [22] (Fig. 11). These tumors are usually located in the temporal region or, less commonly, the frontal region. Cysts are uncommon. DNETs involve both gray and white matter. Imaging findings are quite characteristic and, although not pathognomonic of this condition, should strongly suggest the underlying abnormality. Metastatic spread of tumor to the brain is common and constitutes approximately $20 \%$ of brain neoplasms. Seizures are the presenting symptom in about $30 \%$ of patients with cerebral metastasis. Metastases have fairly stereotyped localization (gray-white matter junction) and often have peripheral edema.

eizures are the chief clinical presentation of intracranial vascular malformations, occurring in $24 \%$ to $69 \%$ of arteriovenous malformations (Kraemer and Awad, 1994) and $34 \%$ to $51 \%$ of cavernous hemangiomas (Lee et al., 1998). These abnormalities are less frequent in the temporal lobes than in other regions and can be divided into arteriovenous malformations, cavernous angiomas, capillary telangiectasia, and venous angiomas. The MRI features of cavernous angiomas are fairly characteristic.The lesions have a central core with mixed signals indicating blood by products of different stages. Within the core, methemoglobin 


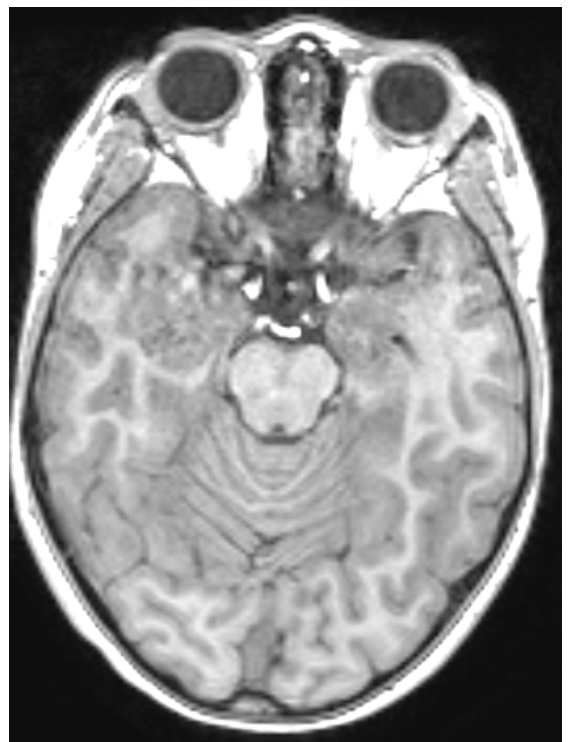

Fig. 5. Heterogenous mass lesion involving the right hippocampus. This ganglioglioma was successfully resected.

$\mathrm{S}$ appears as high signals often intermixed with lower signals. Typically, a complete rim of hypointensity surrounds the central core. This rim is usually hypointense on both long and short echoes, with marked hypointensity in the longer echo. These lesions are void of mass effect or surrounding edema, and they should be easily recognized. Venous angiomas are probably the most common vascular malformation, but they are rarely the cause of chronic epilepsy. For tumors and vascular lesions located in or near eloquent cortex, fMRI nad DTI techniques provide useful information regarding functionally eloquent cortical regions and important white matter tracts, which may be crucial for resecability.

Cortical gliosis can result from a variety of inflammatory, posttraumatic, and cerebrovascular brain insults. Irrespective of the etiology, gliosis usually appears as a region of increased signal change on T2-weighted images and decreased signal on T1-weighted images, often associated with focal volume loss.

Recent advances In neuroimaging techniques have provided additional benefits to the diagnosis of refractory epileptic patients. Modern 3T MRI scanner and sequences can identify focal abnormalities or clarify uncertain findings previously observed (Strandberg et al., 2008). A voxel-based analysis of FLAIR signal intensity detected malformations of cortical development in individual patients, suggesting that this method may be a useful adjunct to the visual reading of MRI scans (Focke et al., 2008). Recent advances in MRI acquisition, such as the PROPELLER sequence, give improved spatial resolution and definition of hippocampal substructures in vivo (Eriksson et al., 2008). Attempts have been made to automate hippocampal volume estimations. A voxel-based approach using estimations of grey matter content has given promising results. It can provide a quantifiable estimative of atrophy, which can aid in the decision about the presence of clinically relevant HA (Bonilha et al., 2009). 


\section{MRI spectroscopy}

MRI spectroscopy and functional MRI are techniques that may complement anatomic MRI. Magnetic resonance spectroscopy (MRS) is a dynamic test of brain neurochemistry. Minuteto-minute changes in phosphorus permit information that reflects changes in cerebral energy metabolism and $\mathrm{pH}$. So, MRS focuses on providing measures of small lowconcentration mobile metabolites. In the presence of magnetic field, molecules and nuclei that have non-zero nuclear spin absorb and emit radiofrequency signals.

MRS has identified alterations that occur in patients with TLE with reduction in the neuronal components (represented by the NAA peak) and increase in the glial elements (creatine and choline) to serve as a noninvasive measure of in vivo function. Areas of interest require large areas of brain to sample, and the resolution of MRS is much lower than PET or SPECT. In nonlseional epilepsy, MRS can potentially detect metabolic changes corresponding to the epileptogenic zone. In cases of TLE with normal MRI, NAA ratios can lateralize the seizure focus in $20 \%$ of patients (Figure 6).

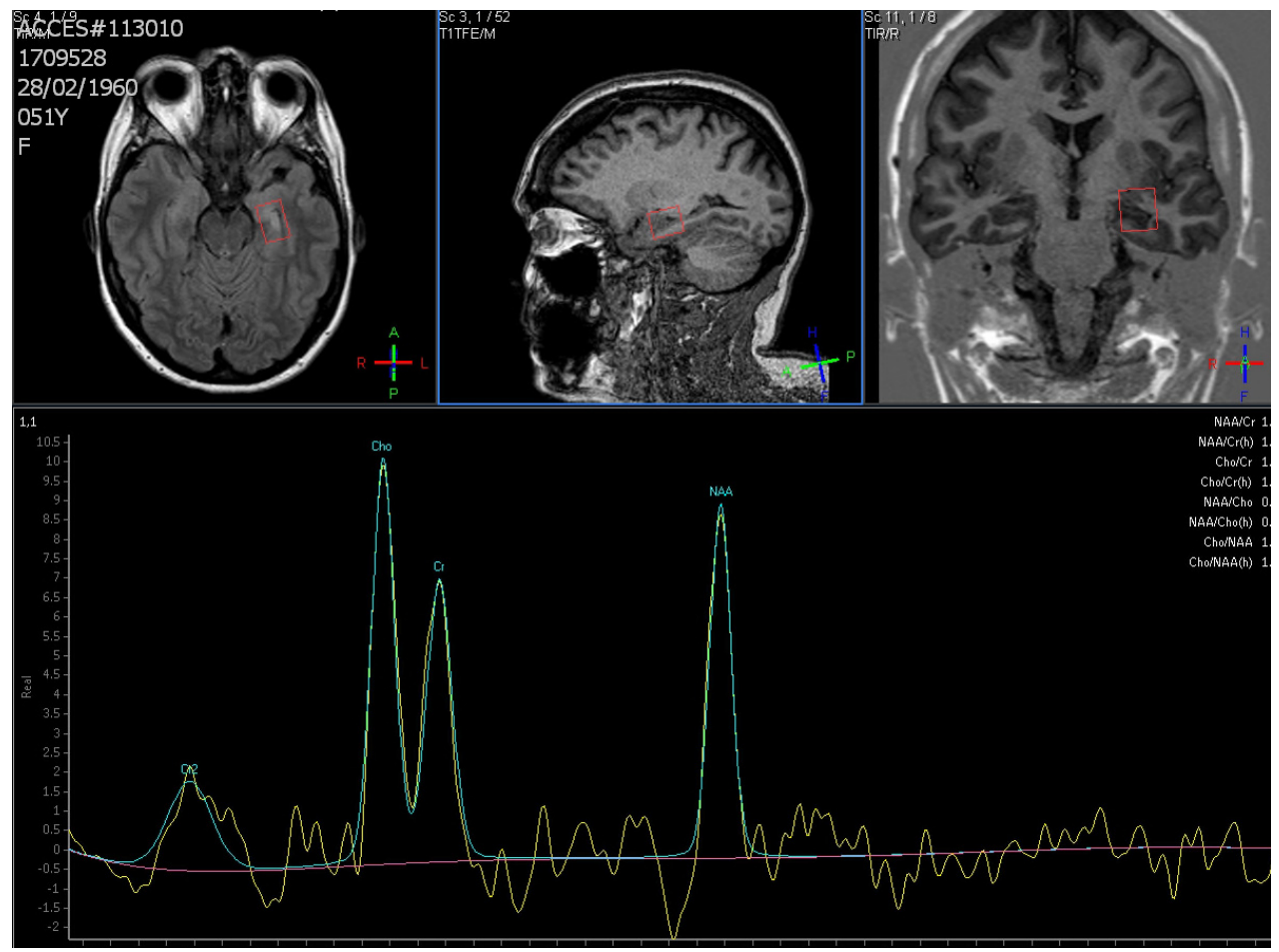

Fig. 6. Left hippocampal sclerosis. Decreased NAA peak.

\section{Diffusion MRI}

Diffusion tensor imaging (DTI) is an imaging technique performed on a standard MRI scanner that is more sensitive to white matter injury than conventional brain MR imaging. DTI reflects the myelin integrity in vivo and the inherent properties of the cell membranes of 
the white matter tracts that restrict the movement of water molecules. Water tends to move preferentially along the horizontal axis of the white matter tracts rather than perpendicular to them (anisotropic diffusion), though the apparent diffusion coefficient (ADC)is the average measure of all directions of water diffusion. The ADC has been considered the surrogate for white matter integrity (Alexander et al., 2007), and DTI may aid in the evaluation of the large fiber white matter tracts. Tractography is user-dependent and very time-consuming. Attempts are being made to automate parts of the process and hence reduce the input needed and the potential bias.

Diffusion-weighted MR techniques are frequently used to assess early signs of cerebral ischemia. Diffusion changes similar to those observed in ischemia may also be present in tumors or infection. Diffusion imaging requires postprocessing, which allows the measurement of summary parameters such as diffusivity and fractional anisotropy

DTI in malformations of cortical development has demonstrated reduced fractional anisotropy and increased mean diffusivity in the white matter adjacent to cortical dysplasias. The disorganization of the underlying white mattertracts can be detected with DTI fiber tractography DTI fiber-tracking has been used to map the major motor and sensory tracts in surgical planning of both lesional and nonlesional epilepsy. In temporal lobe resections, preoperative mapping of Meyer's loopcan help minimize postoperative visual field defects (Taoka et al., 2008).

Asymmetry of connections between temporal and frontal lobes was a predictor of naming difficulties following anterior temporal lobe resection, with greater lateralization of tracts to the dominant hemisphere being associated with greater decline in naming function (Powell et al., 2008).

\section{Functional MRI}

Blood oxygenation level-dependent (BOLD) signals are the basis for generating images created using functional MRI (fMRI). In epilepsy, there is neurovascular coupling to define the relationship between epileptiform neuronal activity that is able to be measured by various techniques including SPECT and PET in addition to fMRI. During an increase in neuronal activity there is a simultaneous increase in the cerebral metabolic rate of glucose and oxygen. Greater regional cerebral metabolic rates result in an increase in regional cerebral blood flow and volume to compensate for the increased demand for oxygen. The robust blood supply delivered to the active region of the seizing brain produces an "uncoupling" between the baseline physiologic cerebral metabolic rate of oxygen and the cerebral blood flow by delivering an increased oxygen load. The difference between the rate of oxygen metabolism and cerebral blood flow forms the basis of the BOLD technique used in generating brain fMRI.

Functional magnetic resonance tomography (fMRI) evaluates cerebral functions, and functional deficits and disturbances. fMRI is pursued in particular for three reasons:

1. To identify the seizure focus for resection

2. To identify eloquent function to be spared during epilepsy surgery

3. To investigate the neurobiology of epilepsy

fMRI provides information about how the brain differs in certain epileptic syndromes or it can be used to assess brain abnormalities in an individual patient. fMRI can also highlight the link between abnormal epileptiform activity, network brain activity, and 
cognitive function. In the clinical situation, fMRI can help to accurately delineate the epileptogenic zone, surrounding eloquent cortex and vital connections between eloquent cortical areas.

Studies of fMRI with simultaneous EEG recordings are revealing more about the spatial distribution of interictal epileptic discharges, and the relation of these sites to the seizure onset zone, as determined with fortuitous ictal EEG-fMRI or intracranial EEG recording and subsequent resective surgery. Paired analyses of cerebral blood-oxygen-level-dependent (BOLD) signal and perfusion during generalized spike-wave activity have shown that the BOLD signal change is on the basis of alterations in cerebral perfusion, indicating preservation of normal neurovascular coupling (Hamandi et al., 2008).

\section{Magnetoencephalography}

MEG is a noninvasive direct measure of the brain's normally inherent electrophysiologic capability of neurons to produce magnetic fields. Transmembrane ion movements in active neurons generate magnetic fields that may be recorded by external devices. Since the magnetic fields produced by the brain are extremely weak, sensitive superconducting quantum interference devices (SQUIDs) and magnetically shielded rooms are used to record the MEG from neuronal sources within the brain. Epileptiform discharges that have been identified on EEG can be measured by averaging techniques and localized to provide information regarding the direction of current flow. The functional information is often combined with structural information from brain MRI to provide three-dimensional information used in the selection process of patients for respective epilepsy surgery. MEG has a high temporal (in milliseconds) and spatial resolution (in millimeters), in contrast to scalp-based EEG. The depth of the generator can be more easily determined, and the sources are recorded extracranially and do not carry the risk that invasive electrodes present. MEG is unaffected by the skull in contrast to scalp-based EEG.

MEG measures current flow tangentially at the surface of the brain, while EEG measures radial flow preferentially, though the two techniques are complementary, permitting information regarding activity along the banks of the sulci or the tops of the gyri.

A number of studies have reported that MEG is superior to EEG with respect to spikedetection sensitivity and localization relative to extratemporal epilepsy (Ossenblok et al., 2007). The inverse problem of source localization is less problematic with MEG than EEG due to the lack of distortion of the magnetic properties with MEG as opposed to the electrical properties with EEG. Therefore, IEDs are in general more abundant in MEG than EEG, the spikes are of briefer duration, and in many patients subpopulations of IEDs are found with different topographic maps, indicating better localizing properties with MEG for interictal IEDs than with EEG. In addition, there has been clinical value noted in patients with recurrent seizures after epilepsy surgery through resection of clusters of MEG source spikes adjacent to the margins of prior resection.

Evoked potentials (EPs) are reproducible electrical currents generated by the synchronous discharge of thousands of similarly oriented and grouped neurons as a response to particular stimuli or actions. MEG measures the magnetic correlates of EPs, referred to as evoked fields (EFs). Somato- sensory evoked fields (SEFs) are most routinely used for presurgical functional mapping for epilepsy surgery around the Rolandic region (Benifla et al., 2009). 


\section{Positron emission tomography}

Positron emission tomography scanning (PET) and single photon emission computed tomography (SPECT) are functional neuroimaging techniques that provide complementary information to anatomic imaging techniques to image the anatomic characteristics of the brain. The most common radioisotope employed for PET is 2-deoxy-2-[18F]fluoro-D-glucose (FDG), or 18F-fluorodeoxyglucose (FDG). FDG is transported into neurons in proportion to glucose consumption of neurons and is trapped rapidly in the cells. PET with FDG, therefore, produces a representation of cerebral energy metabolism. The other energy substrate for brain is oxygen, and $15 \mathrm{O} 2$ PET images can also reflect energy metabolism in brain. This radioactive tag allows heterogeneous rates of regional cerebral glucose utilization to be estimated. In addition to [18F]FDG to image the regional cerebral metabolic rate, and [15O] water to measure regional cerebral blood flow, radiolabeled ligands coupled with dopamine and serotonin permit assessment of neurotransmitter synthesis. Furthermore, benzodiazepine anagonists, NMDA, opiates, tryptophan, and serotonin ligands have been used to evaluate receptors in targeted areas of the brain.

PET scanning has proven useful in the localization of seizure foci and in exploration of the underlying in vivo assessment of physiologic functions of epilepsy in humans (Engel et al., 1990). Interictal regional hypometabolism with PET is seen in $60-90 \%$ of patients with TLE. Interictal hypometabolism with PET can be used in the decision process for selecting patients for surgery, invasive EEG planning, as well as predicting a more favorable postoperative outcome when localized hypometabolism is present, while bilateral temporal hypometabolism portends a poorer postoperative surgical outcome. A recent meta-analysis showed that in TLE, ipsilateral focal hypometabolism was a predictor of good surgical outcome (Willmann et al., 2007)

PET studies with $15 \mathrm{O}$ water are less reliable for identification of the epileptogenic zone and have not been consistent in predicting postoperative seizure outcome. Still, decreased perfusion (similar to SPECT) is able to identify $50 \%$ of patients with epilepsy that reveal an alteration between metabolism and perfusion. PET studies with neurotransmitter receptor markers give clues to neurochemical mechanisms of epilepsy. Opiate receptors, for example, may increase in the region of the epileptogenic zone, possibly as an inhibitory control mechanism, while benzodiazepine receptors decrease.

PET studies suffer from some limitations. They require specialized equipment and personnel and are expensive to obtain. PET studies average metabolic activity over minutes, so rapid events such as seizures are uncommonly analyzed, and briefer events are not able to be detected. Spatial resolution is in theory about 2-3 $\mathrm{mm}$ under ideal conditions (superior to SPECT), but in practical application the resolution may be less. Changes in cerebral blood flow and in the concentration of endogenous compounds that may compete with the radioactive label may greatly affect the results. Finally, PET gives little information on cause and effect.

Depression is a common accompaniment of refractory epilepsy, and understanding the mechanism of this is an important goal. Increased binding of a 5HT-1A receptor antagonist, 18F-MPPF, was found in central serotoninergic networks, particularly the raphe and contralateral insula, ipsilateral hippocampus and bilateral frontal cortex, and there was a correlation with symptoms of depression (Lothe et al., 2008) The most likely explanation was felt to be reduced serotonin concentrations. Longitudinal studies before and after cognitive behaviour therapy, and pharmacological treatment would be of great interest. 


\section{Single photon emission computed tomography}

Single photon emission computed tomography (SPECT) is a cerebral functional neuroimaging technique using a systemically injected or inhaled radiolabeled tracer to measure regional cerebral blood flow. SPECT uses radiotracers labeled with single-photonemitting isotopes to produce images of cerebral function that measure regional cerebral blood flow (rCBF), cerebral blood volume, and blood-brain barrier permeability. A radiotracer that is commonly employed to measure regional cerebral blood flow is hexamethylpropylene amine oxime (HMPAO) coupled with 99mTc (technetium), though other tracers have been utilized. SPECT is similar to PET in that both are functional neuroimaging techniques that measure similar parameters (Figure 7).

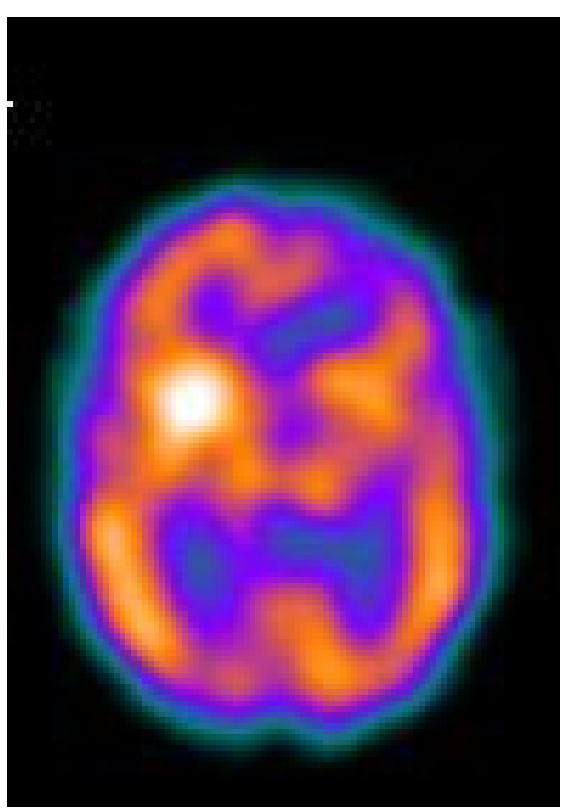

Fig. 7. Ictal SPECT during a right temporal lobe seizure.

Although ictal-interictal subtraction single photon emission computed tomography (SPECT) has been in use for many years as a method of inferring the location of epileptogenic zone, important caveats are still being documented, such as the study of patients with supplementary area seizures in whom ictal SPECT did not localize to the supplementary motor area, but to the anterior cingulate bilaterally (Fukuda eet al., 2006).

The accuracy of ictal SPECT analysis is highest when comparing the ictal with interictal perfusion data. Methodologically, this can be done by traditional side-by-side visual evaluation. Visual comparison of interictal and ictal SPECT is less sensitive than subtraction ictal SPECT co-registered with MRI (SISCOM), but ictal SPECT may be localizing in cases when SISCOM was nonlocalizing due to interictal injection during subclinical seizure activity. SISCOM is sensitive, has a good spatial localization, and is the best method to study ictal propagation patterns in the individual patient (Van Paesschen 
et al. 2007). For SISCOM analysis, interictal and ictal SPECT scans are co-registered using an automatic registration algorithm based on mutual information and the interictal image is then subtracted from the ictal. The difference image is smoothed and can be transformed into a z-score map using the mean and the standard deviation of the differences in all brain voxels. The mean image of the ictal and interictal co-registered images can be used for co-registration to the patient's MRI.

A meta-analysis of SPECT brain imaging in patients with temporal lobe epilepsy (TLE) showed a sensitivity of ictal SPECT localization of 0.97 , relative to diagnostic evaluation without imaging, while this was only 0.44 for inter- ictal SPECT localization (Devous Sr. et al. 1998). Interictal SPECT perfusion imaging on its own, therefore, seems to be inefficient in localizing the seizure onset zone and should only be used as a baseline perfusion measure in the comparison of ictal perfusion images. Ictal SPECT studies of complex focal seizures of extratemporal lobe origin also have an excellent localizing value, but may be more difficult to obtain when the seizures are brief in duration. The sensitivity of ictal SPECT in extratemporal seizures has been reported to be around 90\%. Ictal SPECT, and in particular SISCOM images, are predictive of postsurgical outcome independently of MRI or scalp ictal EEG findings (Cascino et al. 2004).

\section{Conclusion}

To understand the basis of the epileptic disorder, clear definition of the epileptic events (clinical and EEG, functional neuroimaging), the structural abnormalities in the brain, and the clinical context in which seizures occur need to be taken into account. Unless high quality information is obtained in all three of these domains, the basis of the epilepsy in any individual has not been fully assessed. Many levels of information can be acquired using MRI in patients with refractory epilepsy. To make use of all the options available for the investigation of the patient with epilepsy from the perspective of the practicing epileptologist, a well-structured and continuous interaction between the epileptologist and radiologist is necessary. This will lead to acquisition of appropriate epilepsy-focused MR images of high quality and diagnosis of the important lesions with high sensitivity and specificity.

\section{Acknowledgements}

The author is grateful to the Services of Radiology and Nuclear Medicine of the Hospital de Clínicas de Porto Alegre for the images courtesy.

\section{References}

Alexander AL, Lee JE, Lazr M, Field AS.(2007). Diffusion tensor imaging of the brain. Neurotherapeutics, volume 4, issue 3, pp. 316-329, ISSN: 1933-7213.

Barkovich AJ, Kuzniecky RI, Jackson GD, Guerrini R, Dobyns WB (2005). A developmental and genetic classification for malformations of cortical development. Neurology, volume 65, issue 12, pp. 1873-1887, ISSN: 0028-3878.

Benifla M, Sala F, Jane J Jr, et al. (2009). Neurosurgical management of intractable rolandic epilepsy in children: role of resective surgery in eloquent cortex. 
Journal of Neurosurgery: Pediatrics, volume 4, issue 3, pp. 199-216, ISSN: 19330707.

Bonilha L, Halford JJ, Rorden C, et al. (2009) Automated MRI analysis for identification of hippocampal atrophy in temporal lobe epilepsy. Epilepsia, volume 50, issue 2, pp. 228-233, ISSN: 0013-9580.

Cascino GD, So EL, Buchhalter JR, Mullan BP (2004) The current place of single photon emission computed tomography in epilepsy evaluations. Neuroimaging Clinics of North America, volume 14, issue 3, PP. 553-561, ISSN: 1052-5149.

Colombo N, Tassi L, Galli C, et al. (2003) Focal cortical dysplasias: MR imaging, histopathologic, and clinical correlations in surgically treated patiens with epilepsy. American Journal of Neuroradiology, volume 24, issue 4, pp. 724-733, ISSN: 0195-6108.

Devous MD Sr, Thisted RA, Morgan GF, Leroy RF, Rowe CC (1998) SPECT brain imaging in epilepsy: a meta-analysis. Journal of Nuclear Medicine, volume 39, issue 2, pp. 285293, ISSN: 0022-3123.

Duncan J. (2009). The current status of neuroimaging for epilepsy. Current Opinion in Neurology, volume 22, issue 2, pp. 179-184, ISSN: 1350-7540.

Engel J Jr, Henry TR, Risinger MW, et al. (1990). Presurgical evaluation for partial epilepsy: relative contributions of chronic depth-electrode recordings versus FDG-PET and scalp-sphenoidal ictal EEG. Neurology, volume 40, issue 11, pp. 1670-1677, ISSN: 0028-3878.

Engel J., Pedley T. (eds.). (2008). Epilepsy: a comprehensive textbook (2nd Edition). Lippincott Williams \& Wilkins, ISBN 978-0-7817-5777-5, Philadelphia.

Eriksson SH, Thom M, Bartlett PA, et al. (2008). PROPELLER MRI visualizes detailed pathology of hippocampal sclerosis. Epilepsia, volume 49, issue 1, pp. 33-39, ISSN: 0013-9580.

Focke NK, Symms MR, Burdett JL, Duncan JS. (2008). Voxel-based analysis of whole brain FLAIR at 3 T detects focal cortical dysplasia. Epilepsia, volume 49, issue 5, pp. 786793, ISSN: 0013-9580.

Fukuda M, Masuda H, Honma J, et al. (2006) Ictal SPECT in supplementary motor area seizures. Neurological Research, volume 28, issue 8, pp. 845-848, ISSN: 17431328.

Guerrini R (2005). Genetic malformations of the cerebral cortex and epilepsy. Epilepsia, volume 46, issue 1, pp. 32-37., ISSN: 0013-9580.

Guerrini, R., Dobyns, W., Barkovich, A. (2008). Abnormal development of the human cerebral cortex: genetics, functional consequences and treatment options. Trends. in Neurosciences, volume 31, issue 3, pp. 154-162, ISSN: 0166-2236.

Guerrini R, and Parrini E. (2010). Neuronal migration disorders. Neurobiology of Disease, volume 38, issue 2, pp. 154-166, ISSN: 0969-9961.

Hamandi K, Laufs H, Noth U, et al. (2008). BOLD and perfusion changes during epileptic generalised spike wave activity. Neuroimage, volume 39, issue 1, pp. 608-618, ISSN: 1053-8119. 
Jackson GD, Badawy RA. (2011). Selecting patients for epilepsy surgery: identifying a structural lesion. Epilepsy \& Behavior, volume 20, issue 2, pp. 182-189, ISSN: 15255050.

Jahan R, Mischel PS, Curran JG, et al. (1997) Bilateral neuropathologic changes in a child with hemimegalencephaly. Pediatric Neurology, volume 17, issue 4, pp.344-349, ISSN: 0887-8994.

Kraemer DL, Awad IA. (1994). Vascular malformations and epilepsy: clinical considerations and basic mechanisms. Epilepsia, volume 35, supplement 6, pp. S30-S43, ISSN: 00139580 .

Lee HW, Seo DW, Hong SB, et al. (1998) Electroclinicopathologic relationship of epileptogenic foci in cavernous angioma. Epilepsia volume 39, issue 2, pp. 230-231, ISSN: 0013-9580.

Lothe A, Didelot A, Hammers A, et al. (2008) Comorbidity between temporal lobe epilepsy and depression: a [18F]MPPF PET study. Brain, volume 131, issue 10, pp. 2765-2782, ISSN: 0006-8950.

MacDonald BK, Johnson AL, Goodridge DM, et al. (2000). Factors predicting prognosis of epilepsy after presentation with seizures. Annals of Neurology, volume 48, issue 6, pp. 833-841, ISSN: 0364-5134.

Ossenblok P, de Munck JC, Colon A, (2007) Drolsbach W, Boon P. Magnetoencephalography is more successful for screening and localizing frontal lobe epilepsy than electroencephalography. Epilepsia, volume 48, issue 11, pp. 2139-2149, ISSN: 00139580 .

Panayiotopoulos C.P. (ed.). (2010). Atlas of Epilepsies. Springer-Verlag, ISBN 978-1-84882-1279, London.

Powell HW, Parker GJ, Alexander DC, et al.(2008). Imaging language pathways predicts postoperative naming deficits. Journal of Neurology Neurosurgery and Psychiatry, volume 79, issue 3, pp.327-330, ISSN: 0022-3050.

Radhakrishnan K, So EL, Silbert PL, et al (1998). Predictors of outcome of anterior temporal lobectomy for intractable epilepsy: a multivariate study. Neurology, volume 51, issue 2, pp. 465-471, ISSN: 0028-3878.

Strandberg M, Larsson EM, Backman S, Kallen K. (2008). Presurgical epilepsy evaluation using 3T MRI. Do surface coils provide additional information? Epileptic Disorders, volume 10, issue 2, pp. 83-92, ISSN: 1294-9361.

Takanashi J, Barkovich AJ. (2003). The changing MR imaging appearance of polymicrogyria: a consequence of myelination. American Journal of Neuroradiology, volume 24, issue 4, pp. 788-793, ISSN: 0195-6108.

Taoka TM, Sakamoto M, Nakagawa H, et al. (2008). Diffusion tensor tractography of the Meyer loop in cases of temporal lobe resection for temporal lobe epilepsy: correlation between postsurgical visual Field deffect and anterior limito $\mathrm{f}$ Meyer loop on tractography. American Journal of Neuroradiology, volume 29, issue 7, pp. 1329-1334, ISSN: 0195-6108.

Van Paesschen W, Dupont P, Sunaert S, Goffin K, Van Laere K (2007) The use of SPECTand PET in routine clinical practice in epilepsy. Current Opinion in Neurology, volume 20, issue 2, pp. 194-202, ISSN: .1350-7540 
Wieser HG; ILAE Commission on Neurosurgery of Epilepsy (2004). ILAE Commission Report: Mesial temporal lobe epilepsy with hippocampal sclerosis. Epilepsia, volume 45, issue 6, pp. 695-714, ISSN: 0013-9580.

Willmann O, Wennberg R, May $\mathrm{T}$, et al. (2007). The contribution of 18F-FDG PET innpreoperative epilepsy surgery evaluation for patients with temporal lobe epilepsy: a meta-analysis. Seizure, volume 16, issue 6, pp. 509-520, ISSN: 1059-1311. 


\title{
MRI Abnormalities Induced by Seizures
}

\author{
Nuno Canas ${ }^{1}$ and Pedro Soares ${ }^{2}$ \\ ${ }^{1}$ Neurology and Neurophisiology Departments \\ ${ }^{2}$ Neuroradiology Department, \\ Hospital Egas Moniz, \\ Institute of Pharmacology and Neurosciences, Faculty of Medicine, \\ Institute of Molecular Medicine, University of Lisbon, Lisbon, \\ Portugal
}

\section{Introduction}

When computed tomography started to be used routinely in the evaluation of patients presenting with seizures it became evident that some periictal abnormalities disappeared in follow-up studies with no specific treatment except antiepileptics. These findings suggested that those reversible abnormalities were not structural but functional, possibly resulting from the cerebral edema induced by seizure activity (Goulatia et al. 1987; Sammaritano et al., 1985; Sethi et al., 1985).

With the advent of MRI and it's increasingly use in the acute phase of seizures, these seizureinduced abnormalities were also demonstrated, being described as periictal or reversible MRI abnormalities (Cole 2004; Briellmann et al., 2005); since follow-up MRI studies disclosed that irreversible brain damage can occur in the regions previously affected by them, they are now better defined as transient periictal MRI abnormalities (TPMA). In this way, TPMA should only be considered if two conditions are fulfilled: first, the brain MRI signal abnormalities demonstrated periictally must be attributable to seizures and not other causes; and second, that these abnormalities totally or partially reverse on subsequent MRI studies. Besides the detection of TPMA, MRI can provide us with a unique non-invasive diagnostic tool to better understand the physiopathological mechanisms underlying TPMA formation, and to identify and predict the brain damage induced by seizures.

In this chapter, we review the neuroimaging features, differential diagnosis, pathophysiology, electroclinical-imagiological spectrum and outcome of TPMA, highlighting the fundamental role of multi-sequence MRI studies in their investigation.

\section{Clinical and electroencephalographic findings associated with TPMA}

TPMA have been described in several case reports (Amato et al., 2001; Bauer et al., 2006; Chu et al., 2001; Kramer et al., 1987; Senn et al., 2003) and small case series (Canas et al., 2010a; Chan et al., 1996; Huang et al., 2009; Milligan et al., 2009; Raghavendra et al., 2007), the largest not including more than 20 patients (Canas et al., 2010b). This scarcity of reports contrast with the practice of clinicians involved in the evaluation of patients presenting with seizures, where is not so uncommon to find MRI abnormalities induced by them. 
The exact incidence of TPMA is still unknown, with estimated values ranging from $0.007 \%$ (Raghavendra et al., 2007) to $29.4 \%$ (Huang et al., 2009). The best estimation of TPMA incidence that we presently have comes from a prospective study in which MRI was systematically obtained in 34 patients with status epilepticus, with TPMA being disclosed in $20.6 \%$ of them (Goyal et al., 2009). Similar TPMA incidences were reported in the studies that retrospectively reviewed the MRI obtained from patients presenting with status epilepticus, with the periictal signal abnormalities presumably attributable to seizures described in 11.6\% (Milligan et al., 2009) and 29.4\% (Huang et al., 2009) of the patients. Since these results were obtained in patients presenting with status epilepticus, a condition associated with a higher likelihood of inducing TPMA (Cole, 2004), we think that they overestimate the real TPMA incidence, a supposition supported by the much lower values described in the overall population of patients hospitalized for the evaluation of seizure disorders (Raghavendra et al., 2007).

In fact, and although TPMA have been more frequently described in convulsive status epilepticus, complex partial status epilepticus and seizures with a prominent motor component, they could be induced by virtually any type of seizure, a cluster of seizures or other forms of status epilepticus. TPMA can be disclosed in patients with presenting seizures provoked by systemic factors (such as alcohol withdrawal, infections or dialysis disequilibrium) or associated with structural cerebral pathologies, the most common being small-vessel cerebrovascular disease or post-traumatic/ post-stroke encephaloclastic lesions; and can also be demonstrated in patients with a previous history of epilepsy, often difficult to control focal symptomatic or probably symptomatic epilepsies.

The electroencephalographic findings associated with TPMA are also vary variable, with the ictal electroencephalograms generally showing focal continuous or in crescendo epileptiform discharges, rhythmic activity or lateralized periodic epileptiform discharges; in postictal electroencephalograms, the most frequent findings are focal slowing with or without associated epileptiform discharges, lateralized or bilateral periodic epileptiform discharges, frontal intermittent rhythmic delta activity, or no relevant abnormalities.

The reasons why TPMA occur in some but not all patients with seizures is still an unresolved issue. However, some critical variables have been proposed as determinants of TPMA occurrence or outcome, such as seizure type and duration, pre-existing brain lesions, pharmacological interventions (Cole, 2004) and possible hypoperfusion or hypoxia (Milligan et al., 2009). The disclosure of TPMA can also be determined by methodological factors, namely the MRI timing, with ictal or early postictal MRIs increasing the probability of their detection (Briellmann et al., 2005; Goyal et al., 2009).

\section{TPMA neuroimaging features}

TPMA may present with several MRI signal abnormalities, heterogeneous patterns and topographic distribution, contributing to wide neuroimaging features. The knowledge of these features is paramount to recognize TPMA and to make a proper differential diagnosis with other pathologies.

\subsection{Signal abnormalities}

To be visible on an MRI, the seizure-induced MRI abnormalities must be associated with a relatively widespread change in water environment (Briellmann et al., 2005). In this way, the 
intra and/ or extracellular edema induced by seizures are generally hypointense in T1weighted imaging and hyperintense in fluid-attenuated inversion recovery (FLAIR) and T2weighted imaging, with restriction to water on diffusion-weighted imaging (DWI). A pial or cortico-pial contrast enhancement can also be disclosed, particularly in larger TPMAs with a subcortical component. In the cases in which a protonic magnetic resonance spectroscopy (MRS) was obtained periictally, the most consistent findings were an increase in the lactate levels and choline and a decrease in N-acetyl-aspartate (Castillo et al., 2001; Lazeyras et al., 2000; Mueller et al., 2001), with the levels of myoinositol possibly fluctuating during and after seizures (Wellard et al., 2003). Generally, these signal abnormalities are colocalized in the same brain regions.

Although the exact mechanisms underlying these seizure-induced MRI signal changes are still not well known, some have been proposed, mostly based on theoretical models or data from experimental studies (table 1).

\begin{tabular}{|l|l|l|l|}
\hline MRI sequence & Measurement of & $\begin{array}{c}\text { Acute signal } \\
\text { change }\end{array}$ & \multicolumn{1}{|c|}{ Attributed to } \\
\hline T1-weighted & T1-signal & Increase & Cell edema \\
\hline T2-wighted & T2-signal & Increase & Cell edema \\
\hline FLAIR & FLAIR signal & Decrease & Cell edema \\
\hline \multirow{3}{*}{ DWI } & ADC & Decrease & Cytotoxic edema \\
\cline { 2 - 4 } & Lactate & Increase & Vasogenic edema \\
\cline { 2 - 4 } & N-acetyl-aspartate & Increase & Anaerobic metabolism \\
\cline { 2 - 4 } & Choline & Increase & Neuronal loss/ dysfunction \\
\cline { 2 - 4 } & Myoinositol & Decrease & Osmolyte changes \\
\hline \multirow{3}{*}{ With contrast } & $\begin{array}{l}\text { Contrast } \\
\text { enhancement }\end{array}$ & $\begin{array}{l}\text { Cortico-pial } \\
\text { enhancement }\end{array}$ & $\begin{array}{l}\text { Hypervascularization, } \\
\text { vasodilatation, breakdown of the } \\
\text { blood-brain barrier }\end{array}$ \\
\hline
\end{tabular}

Table 1. MRI signal abnormalities induced by seizures.

Several authors highlighted the prominent role of DWI in disclosing TPMA (Kim et al., 2001; Szabo et al., 2005). DWI is the most sensitive MRI-sequence in detecting TPMA (restriction to water diffusion present in more than $90 \%$ of the cases), being frequently the only reliable signal abnormality in small TPMAs restricted to the cerebral cortex or in TPMAs bordering old encephaloclastic lesions (Canas et al., 2010b). In the cases in which attenuation diffusion coefficient (ADC) studies were obtained, some authors reported an increased DWI signal with decreased diffusivity, compatible with cytotoxic edema (Chu et al., 2001; Parmar et al., 2006; Szabo et al., 2005), and others a decreased DWI signal with increased diffusivity, disclosing vasogenic edema (Hong et al., 2004; Wieshmann et al., 1997); other authors, including ourselves, described diverse findings in the TPMA ADC studies, with some 
patients showing a predominantly cytotoxic or vasogenic edema, while others having intermixed areas of both types of edema, mainly cortical cytotoxic edema and subcortical vasogenic edema (Canas et al., 2010a, 2010b; Kim et al., 2001).

The timing of MRI acquisition has been proposed by some authors as one of the factors that could contribute to these apparently non-congruent findings regarding the TPMA ADC signal abnormalities (Briellmann et al., 2005; Hong et al., 2004). Animal experiments disclosed that, early after a seizure, the lactate levels increase and the ADC values decrease, with these changes attributed to the failure of the $\mathrm{Na}^{+} / \mathrm{K}^{+}$-ATPase pump and anaerobic metabolism (Najm et al., 1997; Righini et al., 1994; Wang et al., 1996); whereas the lactate changes are short-lived, the ADC value is maximally decreased at 24 hours, returning to the control level or increasing in the following 48 to 72 hours, when T2-weighted abnormalities emerge (Nakasu et al., 1995; Righini et al., 1994). However, in clinical practice we can observe different MRI signal abnormalities, including increases and decreases in ADC values, in MRIs performed with similar timings regarding the seizure onset, disclosing that other factors must be considered. We believe that one of those factors is the diverse susceptibilities of different brain regions to the pathophysiological mechanisms involved in TPMA formation (see below).

\subsection{Topography and patterns}

The great majority of TPMAs are focal and unilateral, cortically restricted or with a corticosubcortical pattern. Although they can arise anywhere in the cerebral neocortex, they are commonly disclosed in the posterior and perirolandic regions or in the periphery of old encephaloclastic lesions (Silverstein \& Alexander, 1998; Canas et al., 2010b). Less frequently they have a marked subcortical component, with mass effect and gyral effacement, mimicking an intracerebral neoplasm (Canas et al., 2010a; Cohen-Gadol et al., 2004; Hicdonmez et al., 2003); are more diffuse or bilateral; or are associated with remote signal abnormalities, affecting structures anatomically distant from the neocortical seizure focus, including the contralateral cerebellar hemisphere (cerebellar diaschisis), thalamus (more frequently the pulvinar), splenium of the corpus callosum, caudate and globus pallidus (Cole, 2004). In some cases, evanescent T2-weighted and DWI abnormalities are disclosed in cortical regions widely separated from the probable seizure focus, with the signal abnormalities of these migratory lesions and TPMA resolving almost simultaneously (Cole, 2004; Raghavendra et al., 2007). Remote and migratory signal abnormalities are believed to result from seizure propagation through neuronal pathways, and are usually associated with larger neocortical TPMA.

\subsection{Classification}

The first classification for TPMA was proposed by Cole, who classified them into local (colocalized with the epileptic discharge) and remote (affecting distant structures) (Cole, 2004). After that, two comprehensive classification systems to cover the broad spectrum of changes that comprise TPMA were proposed by us (Canas et al., 2010b) and other authors (Raghavendra et al., 2007). These two classifications are based mostly on TPMA patterns and signal abnormalities, facilitating their recognition by Neurologist and Neuroradiologists, and helping in their differential diagnosis.

Raghavendra and colleagues classified TPMA into type I, restricted to the superficial gray matter; type II, affecting the superficial gray and subcortical white matter (type IIa) or with 
the subcortical white matter involvement extending to the adjacent periventricular surface (type IIb); type III, with involvement of the subcortical nuclei associated with any degree of cortical or white matter involvement; type IV, involvement of white matter alone; and type $\mathrm{V}$, with leptomeningeal enhancement and/ or gyriform cortical enhancement. Regarding the differential diagnosis, type I and IIa can mimic encephalitis, type IIb an intracerebral neoplasm and type $\mathrm{V}$, due to the leptomeningeal involvement, meningoencephalitis; type IV can mimic several pathologies and in type $\mathrm{V}$ the ictal onset may have a poor localizing value (Raghavendra et al., 2007).

Our classification has several similarities to the classification proposed by Raghavendra and colleagues (Raghavendra et al., 2007), although we did not find any TPMA restricted to the subcortical white matter (Canas et al., 2010b). We classified TPMA into type 1, restricted to the cerebral cortex, and type 2, cortico-subcortical. Type 2 TPMA can further have a marked subcortical component with mass effect, mimicking a tumor lesion (type 2A-tumor-like); be in the periphery/ bordering an old encephaloclastic lesion (type 2B) or be associated with remote signal abnormalities (type 2C) (table 2).

\begin{tabular}{|c|c|c|c|}
\hline Type & Characteristics & Main MRI features & Major differential diagnosis \\
\hline 1 & Cortically restricted & $\begin{array}{l}\text { Frequently only disclosed } \\
\text { on DWI }\end{array}$ & $\begin{array}{l}\text { Small embolic stroke } \\
\text { Creutzfeldt-Jacob disease }\end{array}$ \\
\hline 2 & Cortico-subcortical & $\begin{array}{l}\text { Any of the TPMA signal } \\
\text { abnormalities }\end{array}$ & $\begin{array}{l}\text { Embolic stroke, Encephalitis, } \\
\text { meningoencephalitis, venous } \\
\text { thrombosis, }\end{array}$ \\
\hline $2 \mathrm{~A}$ & $\begin{array}{l}\text { Prominent } \\
\text { subcortical } \\
\text { component }\end{array}$ & $\begin{array}{l}\text { Mass effect, frequent } \\
\text { contrast enhancement; } \\
\text { marked vasogenic edema }\end{array}$ & $\begin{array}{l}\text { Intracerebral neoplasm, low } \\
\text { grade gliomas }\end{array}$ \\
\hline $2 \mathrm{~B}$ & $\begin{array}{l}\text { Bordering an old } \\
\text { encephaloclastic } \\
\text { lesion }\end{array}$ & $\begin{array}{l}\text { Frequently only disclosed } \\
\text { on DWI }\end{array}$ & Small embolic stroke \\
\hline $2 \mathrm{C}$ & $\begin{array}{l}\text { Associated with } \\
\text { remote lesions }\end{array}$ & $\begin{array}{l}\text { Signal abnormalities in the } \\
\text { contralateral cerebellum, } \\
\text { or the homolateral } \\
\text { pulvinar, caudate, } \\
\text { pallidus }\end{array}$ & $\begin{array}{l}\text { Reversible posterior } \\
\text { leukoencephalopathy } \\
\text { syndrome, stroke, } \\
\text { Creutzfeldt-Jacob disease }\end{array}$ \\
\hline
\end{tabular}

Table 2. Proposed TPMA classification

Topographically, the great majority of type 1, type 2A and 2B TPMA are focal and unilateral, while most type $2 \mathrm{C}$ are larger (hemispheric, lobar, multilobar) and/ or bilateral. Tumor-like TPMA are frequently disclosed in patients with presenting seizures without a known etiology and generally are associated with a good outcome (Canas et al., 2010a); conversely, type $2 \mathrm{C}$ generally occur in patients with a previous history of epilepsy, and are associated with a high percentage of clinical and MRI sequelae (Canas et al., 2010b). The factors predisposing patients with similar types and duration of seizures to develop different types of TPMA remain to be elucidated. 
The wide spectrum of TPMA signal abnormalities, topography and patterns are shown in figures 1 to 7 . To better understand our proposed TPMA classification, a clinical vignette is provided, including the outcome of the disclosed TPMA.
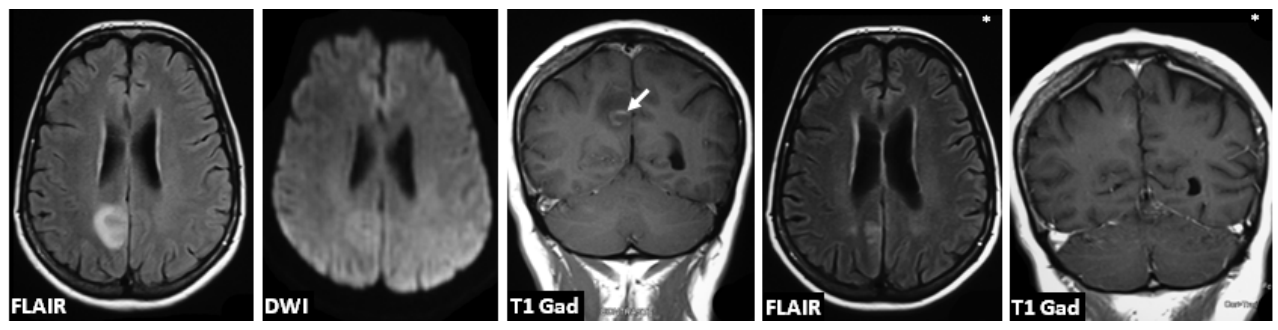

Fig. 1.56 year-old female patient with 3 left focal motor seizures in 1 hour, one with secondarily generalization; there was no history of epilepsy and the seizure etiology was undetermined. The periictal MRI disclosed a pseudotumoral lesion (type 2A TPMA) around the right marginal sulcus, hyperintense on FLAIR, hypointense on T1 and with a cortico-pial pattern of gadolinium (Gad) enhancement (arrow). There was no significant diffusion restriction, thus suggesting vasogenic edema. $\left({ }^{*}\right)$ The follow-up MRI at 2 months showed resolution of the edema and contrast enhancement, with cortical laminar necrosis in the previously affected region; in spite of this, there were no clinical or electroencephalographic sequels.
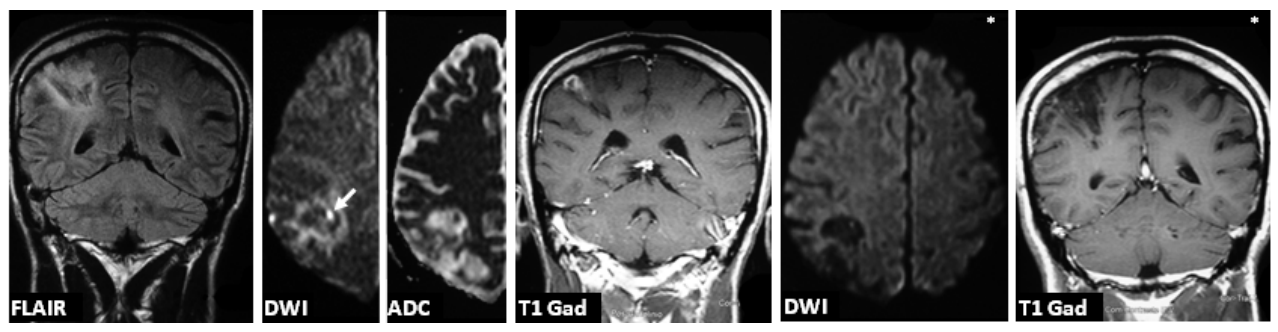

Fig. 2. 45 year-old male patient, with two simple motor seizures affecting the left arm, one with secondarily generalization; he had a left hemiparesis due to previous stroke, but no history of epilepsy; the presenting seizures were induced by alcohol withdrawal. The periictal MRI disclosed an old right parietal ischemic stroke, surrounded by focal diffusion restriction (arrow), gadolinium (Gad) enhancement and FLAIR hyperintensity (type 2B TPMA), attributable to acute seizures and not to a new small embolic stroke. $\left(^{*}\right)$ The followup MRI at 3 months showed resolution of the periictal signal abnormalities, although the patient developed de novo focal epilepsy and aggravated the pre-existing left hemiparesis. 

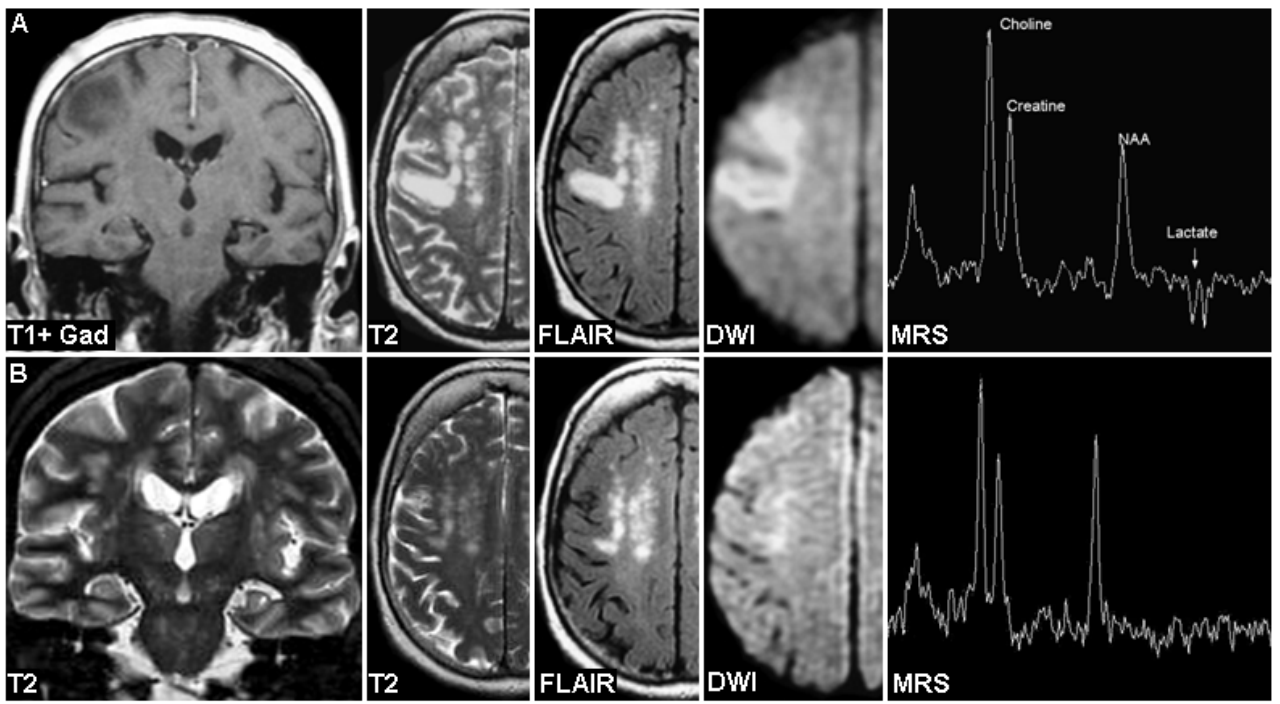

Fig. 3. 73 year-old female patient presenting almost continuous clonic jerks of the left hemiface in the last 5 days; the etiology of the simple motor status epilepticus was attributed to small-vessel cerebrovascular disease. The periictal MRI disclosed a hyperintensity of the right precentral gyrus with subcortical involvement and mass effect on T2 and FLAIR, a T1weighted hypointensity, DWI restriction and no contrast enhancement; lesional MRS revealed a decrease in N-acetyl-aspartate (NAA) and an inverted lactate peak (type 2A TPMA). The Follow-up MRI after 60 months (B) shows resolution of the precentral signal abnormalities, with slight regional brain atrophy, mild recovery of the choline / N-Acetyl A ratio and lactate normalization on MRS. In spite of these residual MRI findings, there were no clinical or electroencephalographic sequels attributable to the TPMA.
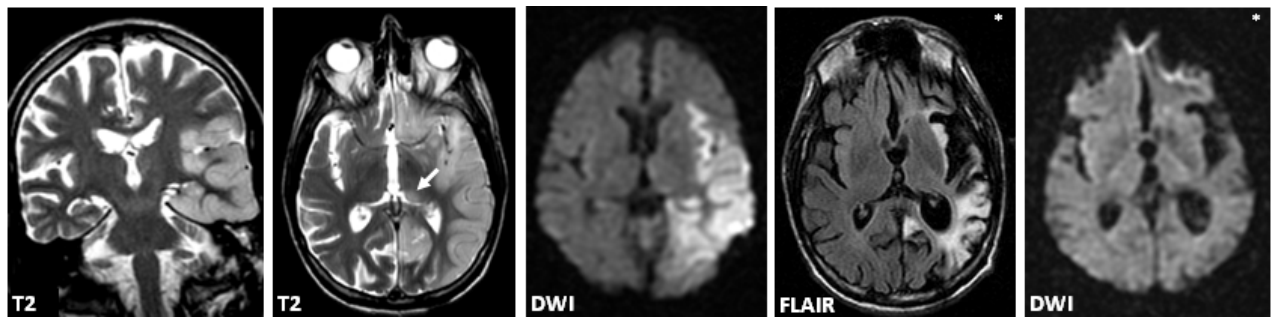

Fig. 4.45 year-old male patient with consciousness impairment in the last 6 hours. The ictal electroencephalogram showed continuous epileptiform discharges over the left temporal region, diagnosing complex partial status epilepticus, attributed to alcohol withdrawal. The periictal MRI disclosed diffuse left hemisphere cortical swelling, T2 hyperintensity and diffusion restriction, associated with remote left pulvinar (arrow) T2 and DWI hyperintensity (type 2C TPMA). (*) The follow-up MRI at 5 months showed resolution of diffusion restriction signal abnormalities and edema, with marked focal atrophy and cortical-subcortical gliosis. The irreversible brain damage that resulted from the TPMA was responsible for Wernicke aphasia and de novo focal symptomatic epilepsy. 

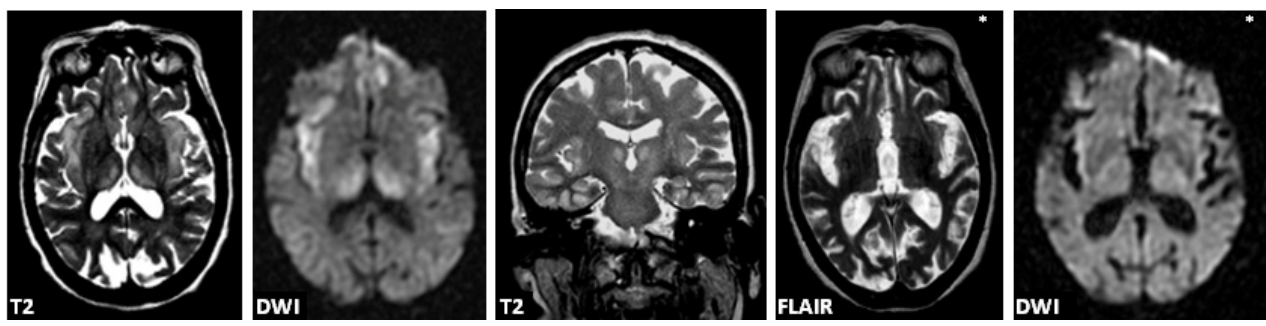

Fig. 5. 59 year-old female patient with a previous history of post-stroke epilepsy. In the last 3 days she presented with stupor, with occasional nystagmoid eye jerks. The ictal EEG allowed the diagnosis of subtle status epilepticus, attributed to a change in the usual antiepileptic medication. The periictal MRI showed bilateral cortico-subcortical T2 and DWI hyperintensities involving the insula, frontal and temporal lobes, the cingulum, the hippocampus and the pulvinar (type 2C TPMA). (*) The follow-up MRI at 3 months showed resolution of diffusion restriction signal abnormalities and aggravation of the diffuse cerebral atrophy. After the status epilepticus control, the patient remained tetraparetic and died after 5 months.
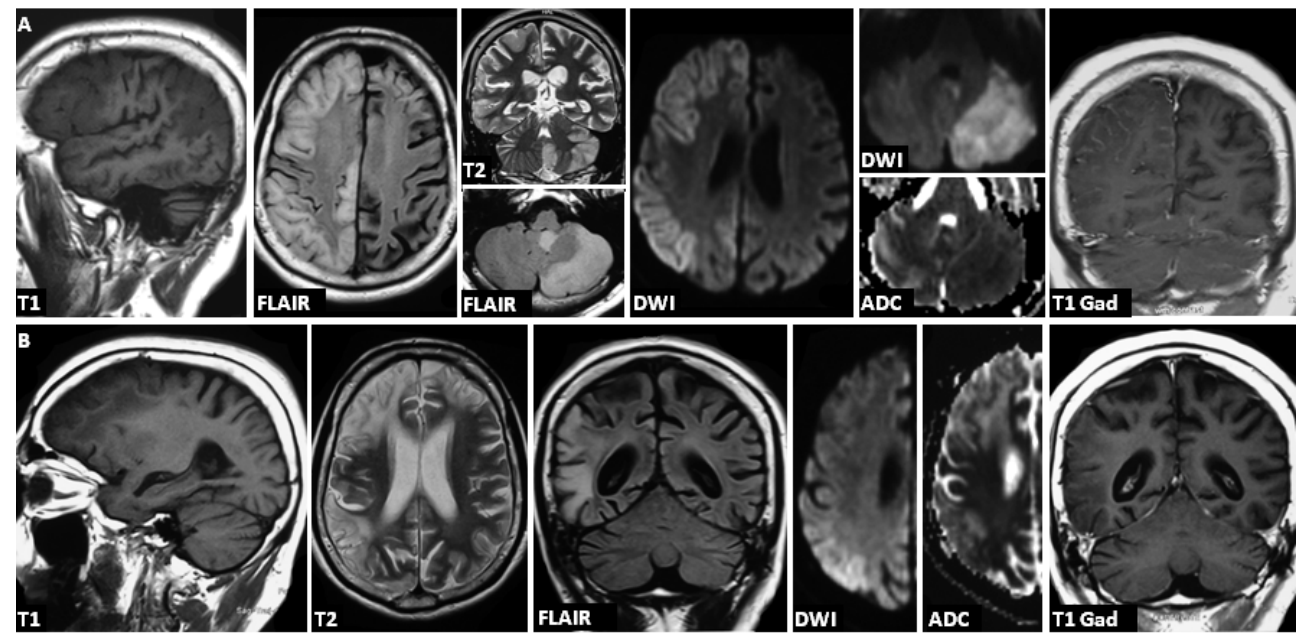

Fig. 6. 35 year-old male patient with cryptogenic refractory temporal lobe epilepsy.

Consciousness impairment with occasional clonic jerks of the left arm in the last 2 days, with the ictal electroencephalogram diagnosing complex partial status epilepticus. The periictal MRI disclosed cortical-subcortical edema and signal changes of the entire right cerebral hemisphere, sparing the rolandic area, with cortical diffusion restriction and cortico-pial contrast enhancement; concomitant contra-lateral cerebellar hemisphere and tonsil signal changes (crossed cerebellar diaschisis) (type 2C TPMA). ( ${ }^{*}$ ) The follow-up MRI at 5 months showed resolution of the edema and contrast enhancement, with cortico-subcortical T2 and FLAIR hyperintensities in the previously affected areas (demyelination and gliosis), and generalized brain atrophy, more marked at the right frontal lobe. After the status epilepticus, the patient developed a left hemiparesis. 

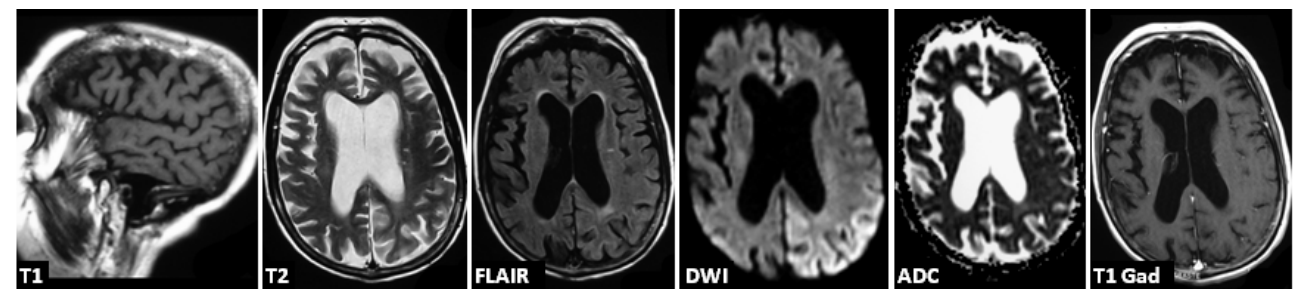

Fig. 7.78 year-old female patient with a previous history of hypertension and diabetes mellitus type II. Admitted with consciousness impairment and occasional jerks of the right arm in the last 3 days; the ictal electroencephalogram disclosed periodic lateralized epileptiform discharges with a higher amplitude over the left posterior regions; the diagnosis of complex partial status epilepticus was admitted, attributable to small-vessel cerebrovascular disease. The periictal MRI disclosed left parieto-occipital cortical diffusion restriction without significant signal changes in the other sequences (type 1 TPMA), which disappeared in follow-up MRIs. After few months, the patient developed de novo partial epilepsy easily controlled with antiepileptics.

\subsection{Differential diagnosis}

The difficult differential diagnosis between the lesions induced by seizures and other pathologies as been described since the earlier computed tomography descriptions (Silverstein \& Alexander, 1998). In the MRI era those difficulties persisted, mostly due to the similar signal abnormalities disclosed on focal TPMA and other pathologies that may present with seizures, namely acute ischemic stroke, intracerebral tumors, reversible posterior leukoencephalopathy syndrome, encephalitis, and venous infarctions resulting from cerebral venous thrombosis.

The differential diagnosis with all these pathologies is particularly relevant in a patient with a first seizure or presenting SE since the attending clinicians may assume that the observed MRI abnormalities are the cause and not the consequence of seizures, a supposition that may possibly lead to unnecessary diagnostic procedures. Although the clinical context in which seizures occur is fundamental to make a correct diagnosis, certain particularities resulting from a detailed MRI evaluation can also contribute for that.

The differential diagnosis of TPMA with embolic acute stroke is mostly relevant, since both situations occur with a prominent cortico-subcortical water restriction on DWI studies. However, TPMA generally do not respect vascular territories, such as in acute stroke (Lansberg et al., 1999), and in uncertain cases a hyperperfusion demonstrated on perfusion computed tomography (Masterson et al., 2009) or MRI (Flacke et al., 2000; Szabo et al., 2005) favors TPMA, while hypoperfusion favors stroke. Other relevant differential diagnosis of TPMA, namely if they have a tumor-like appearance, is with low grade gliomas; in this situation, the results of the DWI studies are very helpful, with TPMA having a marked restriction to water diffusion, generally not present in low grade gliomas (Cha, 2006). Regarding encephalitis and venous infarctions resulting from cerebral venous thrombosis the differential diagnosis with TPMA can only be properly made after the CSF analysis and venous angiography, respectively, excluded these pathologies. The patterns and signal abnormalities of TPMA are so similar to the ones observed in reversible posterior leukoencephalopathy syndrome that solely the clinical context in which they occur can help to make a proper differential diagnosis (Hinchey et al., 1996; Kozak et al., 2007). The same 
applies to TPMA restricted to the cerebral cortex or associated with remote lesions, very similar to the MRI abnormalities observed in Creutzfeldt-Jacob disease (Meissner et al., 2008; Young et al., 2005).

We must highlight that in some cases, and in spite of a detailed clinical history and MRI analysis, the differential diagnosis between TPMA and some of these pathologies can be very difficult. In these situations, the results of subsequent neuroimaging studies obtained some days after seizure control can be very helpful, with the disappearance of the abnormalities strongly favoring the diagnosis of TPMA.

\section{TPMA electroclinical-imagiological spectrum}

Although a high topographic concordance between the clinical, electroencephalographic and MRI findings have been described in most case reports, only recently large case series applied systematically these studies to determine the electroclinical-imagiological spectrum of TPMA. In a recent case series, Huang and colleagues reported an almost complete topographic concordance between the periictal MRI abnormalities and the electroclinical findings in 15 patients presenting with status epilepticus (Huang et al., 2009); similar findings were described in 10 patients with partial status epilepticus, with the periictal DWI abnormalities always colocalized with the ictal electroencephalographic activity disclosed on video-EEG (Di Bonaventura et al., 2009).

In more heterogeneous case series, including patients with different types of seizures and status epilepticus, the degree of this concordance is significantly lower: in 12 patients with a single or a cluster of focal seizures with or without secondarily generalization, Raghavendra and colleagues described a $75 \%$ topographic concordance between reversible periictal MRI abnormalities and the electroclinical findings (Raghavendra et al., 2007); and in our own case series, using more strict topographic criteria, this concordance was only observed in 7 out of 19 patients, with the others presenting several possible combinations between the clinical, MRI and electroencephalographic data: TPMA colocalized with electroencephalographic findings, with patients presenting generalized seizures; partial seizures concordant with focal electroencephalographic findings, with more widespread TPMA; partial seizures concordant with TPMA, with the electroencephalogram showing more diffuse abnormalities; focal TPMA, generalized seizures, and non-focal electroencephalographic abnormalities; colocalized electroencephalographic and clinical findings with "mirror-like" TPMA; and bilateral TPMA associated with diffuse electroencephalographic findings and generalized seizures (Canas et al., 2010b).

The broad electroclinical-imagiological spectrum that we and others describe can be mostly attributed to methodological and clinical factors (table 3). The degree of homogeneity of the clinical, imagiological and electroencephalographic data included for analysis seems to be a determinant methodological factor, with the inclusion of patients of different types of seizures/ status epilepticus and wide timings of MRI and electroencephalographic studies contributing to a lower level of concordance; conversely, a better concordance is associated with a short time-lag between the acquisition of these studies (figure 8) (Cole, 2004; Goyal et al., 2009; Huang et al., 2009). The topographic criteria used for concordance is also very relevant, with more broad criteria, considering concordant electroencephalographic and MRI findings lateralized to the probable seizure focus or admitting generalized seizures associated with focal MRI and electroencephalographic findings as probably generalized, increasing its level (Canas et al., 2010b). Sustained and prolonged focal seizures are also 
highly colocalized with the MRI and electroencephalographic findings (figure 9) (Cole, 2004; Goyal et al., 2009; Huang et al., 2009), in opposition to a systemic seizure etiology, mostly due to its possible associated diffuse electroencephalographic abnormalities (Canas et al., 2010).

\begin{tabular}{|l|l|}
\hline \multicolumn{1}{|c|}{ Methodological } & \multicolumn{1}{c|}{ Clinical } \\
\hline Homogeneity of the analyzed data & Seizure type and duration \\
\hline Time-lag between studies & Seizure etiology \\
\hline Criteria for topographic concordance & Seizure dynamics \\
\hline
\end{tabular}

Table 3. Factors affecting the TPMA electroclinical-imagiological spectrum
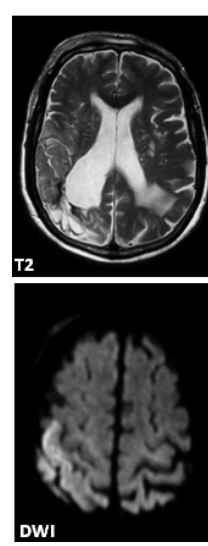
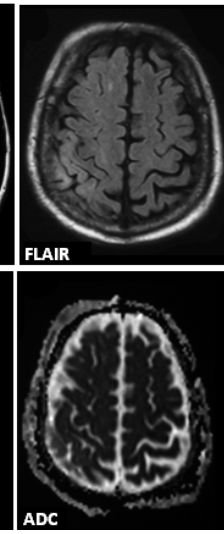

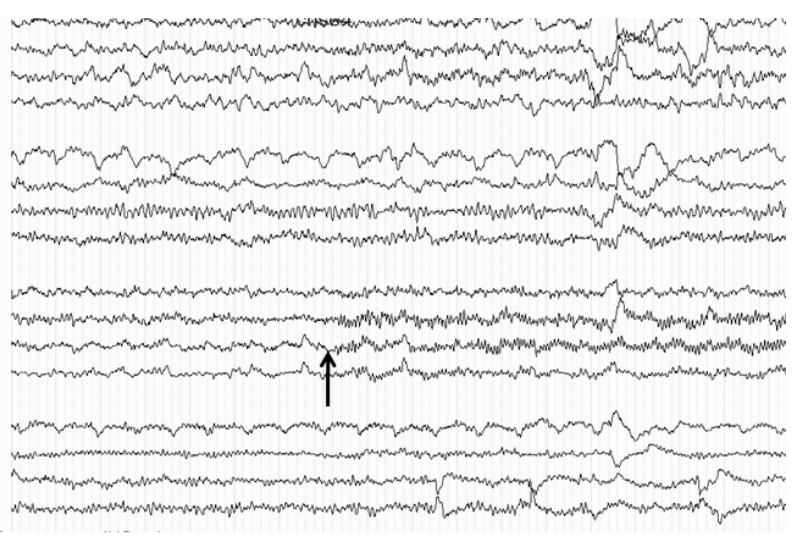

Fig. 8. 67 year-old male patient with post-stroke epilepsy. Hospitalized with recurrent motor seizures of the left-hemibody in the last 7 hours, triggered by low antiepileptic levels. The periictal MRI, obtained within 24 hours after seizure control, disclosed a DWI restriction in the periphery of an old encephaloclastic lesion (type 2B TPMA), which reversed on subsequent studies; the ictal electroencephalogram revealed a seizure-onset in right central region, characterized by rhythmic fast activity (arrow). The short time-lag between studies (ictal electroencephalogram, early postictal MRI) certainly contributed to the excellent electroclinical-imagiological topographic concordance observed in this case.

Another factor that must be considered in the evaluation of the TPMA electroclinicalimagiological spectrum is seizure dynamics. In fact, the rapid seizure propagation from a epileptic focus can contribute for the apparently non-concordant topographic findings that we described (Canas et al., 2010b): in patients presenting generalized seizures with focal colocalized electroencephalographic and MRI findings, the seizures most probably propagated from a epileptic focus defined by the results of those two exams, an hypothesis particularly conceivable in patients presenting structural cerebral abnormalities; when focal concordant clinical and electroencephalographic findings are associated with more widespread MRI abnormalities, the seizure focus is probably defined according to electroclinical criteria, with the more extensive TPMA resulting from the seizure propagation through neuronal pathways; and when the patients present partial seizures concordant with focal TPMA, with electroencephalograms showing more diffuse 
abnormalities (namely periodic lateralized or bilateral epileptiform discharges), the seizure focus is probably defined according to clinical and MRI criteria. The same seizure dynamics probably also justifies all cases of TPMA associated with remote or migratory signal changes and the cases with "mirror-like" TPMA. The determinant role of seizure dynamics to the electroclinical-imagiological spectrum of TPMA was also previously highlighted by other authors (Cole et al., 2004; Goyal et al, 2009).
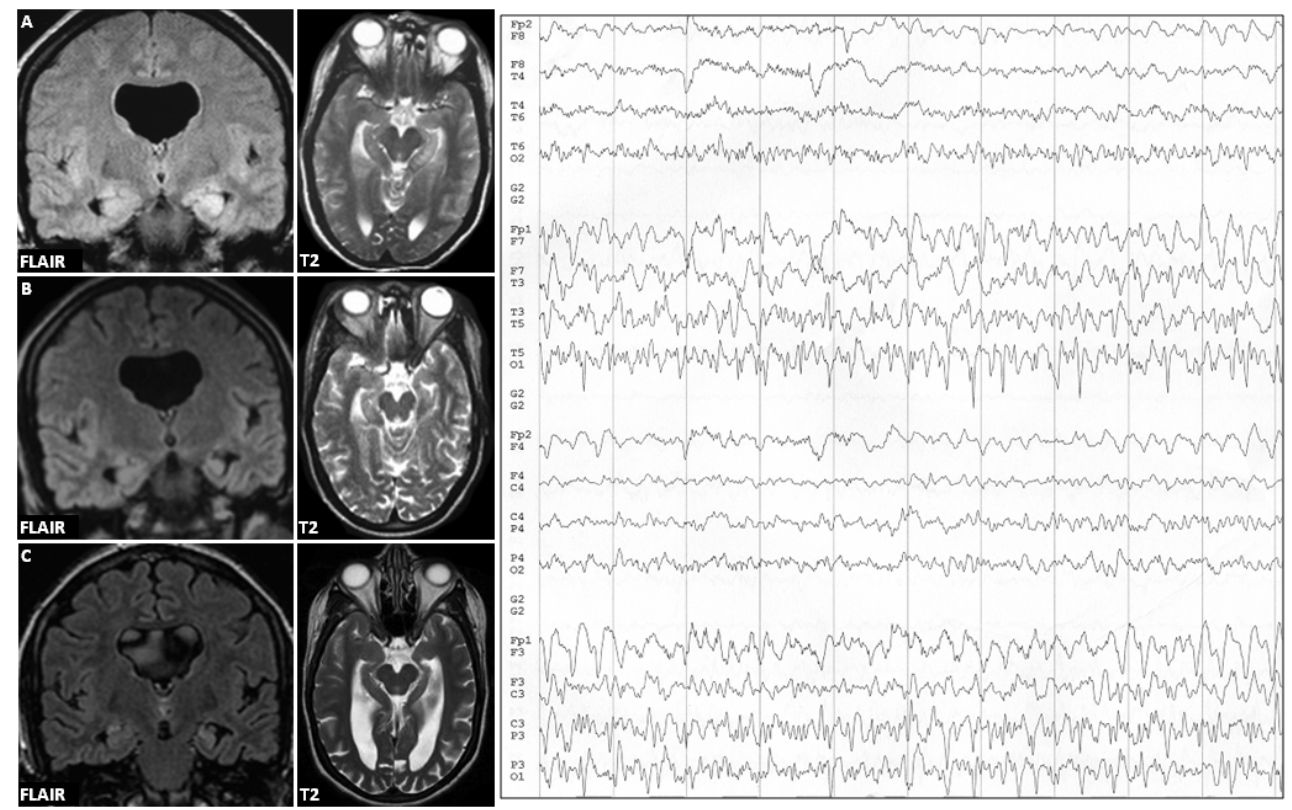

Fig. 9. 23 year-old female patient admitted with behavior abnormalities in the last 3 days. The ictal electroencephalogram showed slowing of the background activity over the left hemisphere and continuous spikes and sharp-waves over the left temporal region, compatible with complex partial status epilepticus. The periictal MRI (A), obtained 4 days after seizure control, disclosed swelling of the left hippocampal formation, with cortical hyperintensity on FLAIR and T2-weighted images (Type 1 TPMA); constitutional alterations of the brain are also seen, being the probable cause of the presenting status epilepticus. After 5 months (B), the follow-up MRI showed reversion of the signal abnormalities; and after 19 months (C) atrophy of the left hippocampus, with mild hyperintensity on T2 and FLAIR, suggestive of hippocampal sclerosis. In this case, in spite of a late periictal MRI, the sustained and prolonged status epilepticus contributed to the topographic concordance between the clinical, electroencephalographic and MRI findings.

\section{TPMA outcome}

In follow-up studies, the periictal MRI abnormalities attributable to seizures may totally reverse or induce irreversible brain damage. In this last situation, focal cortical laminar necrosis, T2 and/or FLAIR hyperintensities, atrophy and a decrease in N-acetyl-aspartate may be observed in the regions previously affected by TPMA, disclosing gliosis and 
neuronal loss (Briellmann et al., 2002, 2005). These focal changes will eventually lead to a new seizure focus and the development of the novo focal epilepsy (Bartolomei et al., 1996; Parmar et al., 2006) or may coexist without any clinical or electroencephalographic evidence of chronic epilepsy (Chu et al., 2001; Senn et al., 2003). In our own series, we found an high incidence $(63.2 \%)$ of clinical, MRI and electroencephalographic sequelae attributable to TPMA, with only small TPMA induced by systemic factors or tumor-like TPMA associated with a better outcome (Canas et al., 2010b); these TPMA sequelae were not only showed in patients with presenting seizures, but also in epileptic patients, with the appearance or aggravation of focal neurological deficits. However, we recognized that some confounding factors could have influenced our results, namely the high incidence of the novo epilepsy in patients with presenting seizure associated with structural brain lesions (small-vessel cerebrovascular disease, old encephaloclastic lesions), possibly the first seizures of a focal symptomatic epilepsy.

At the periictal phase there are some TPMA characteristics that can help to predict their outcome. One of the most relevant is the type of edema disclosed on the DWI/ ADC studies: if TPMA are mainly composed of cytotoxic edema, the probability of inducing irreversible brain damage is higher, with the consequent topographically concordant clinical and electroencephalographic sequels (Canas et al., 2010b; Parmar et al., 2006); on the contrary, the TPMA mainly composed by vasogenic edema partially or totally reverse without any clinical or electroencephalographic consequence (Cohen-Gadol et al., 2004; Raghavendra et al., 2007; Canas et al., 2010a). TPMA affecting the temporal lobe, and particularly the mesial structures (hippocampus and amygdala) may also be associated with a worse outcome, possibly leading to the development of hippocampal sclerosis and the appearance of the novo epilepsy (Bartolomei et al., 1998; Juhazc et al., 1998; see also figure 9). The vulnerability of the temporal structures to seizures was demonstrated in the neuropathological analysis of patients dying from status epilepticus, with a substantial cell loss and gliosis in the hippocampus (Nixon et al., 2001; Pohlmann-Eden et al., 2006); some MRI signal abnormalities have been associated with the development of permanent hippocampal damage, mainly the presence of periictal cytotoxic edema and a lactate peak on protonic

\begin{tabular}{|l|l|l|l|}
\hline \multicolumn{1}{|c|}{ Analyzed data } & \multicolumn{1}{|c|}{ Analysis of } & \multicolumn{1}{c|}{ Worse outcome } & \multicolumn{1}{|c|}{ Better outcome } \\
\hline \multirow{4}{*}{$\begin{array}{l}\text { TPMA } \\
\text { characteristics }\end{array}$} & $\begin{array}{l}\text { Predominant type of } \\
\text { edema }\end{array}$ & Cytotoxic & Vasogenic \\
\cline { 2 - 4 } & Location & Temporal & Extra-temporal \\
\cline { 2 - 4 } & Size & $\begin{array}{l}\text { Large cortico- } \\
\text { subcortical }\end{array}$ & $\begin{array}{l}\text { Small cortically } \\
\text { restricted }\end{array}$ \\
\cline { 2 - 4 } & Type & Type 2B, type 2C & Type 2A (tumor-like) \\
\hline \multirow{4}{*}{$\begin{array}{l}\text { Other MRI } \\
\text { findings }\end{array}$} & $\begin{array}{l}\text { Associated structural } \\
\text { Cerebral disease }\end{array}$ & Yes & No \\
\hline \multirow{2}{*}{ Clinical } & Type of seizures & $\begin{array}{l}\text { Status epilepticus, } \\
\text { convulsive }\end{array}$ & $\begin{array}{l}\text { Single/ short } \\
\text { seizures, non- } \\
\text { convulsive }\end{array}$ \\
\cline { 2 - 4 } & $\begin{array}{l}\text { Etiology of presenting } \\
\text { seizures }\end{array}$ & $\begin{array}{l}\text { Structural cerebral } \\
\text { disease }\end{array}$ & Systemic factors \\
\cline { 2 - 5 } & Preexisting epilepsy & Yes & No \\
\hline
\end{tabular}

Table 4. Proposed factors that may help to predict the TPMA outcome 
MRS (Farina et al., 2004; Parmar et al., 2006), and a T2-weighted hyperintensity in the days following seizures (Provenzale et al., 2008). Finally, we demonstrated that the type of TPMA could help to predict their outcome, with large TPMA associated with remote lesions (type 2C), TPMA in the periphery of cortical lesions (type 2C) and TPMA disclosed in patients with presenting seizures attributed to cerebral brain lesions associated with a higher likelihood of clinical, MRI and electroencephalographic sequels (Canas et al., 2010b).

Based on the available literature and in our own experience, we propose the integrated analysis of some data for the prediction of TPMA outcome. These data, besides including the TPMA neuroimaging features, also consider other clinical and MRI findings (table 4).

\section{Pathophysiological mechanisms underlying TPMA formation and outcome}

Although the exact pathophysiological mechanisms underlying the seizure-induced MRI abnormalities are still not completely elucidated, the results of animal experiments and clinical observations obtained over the last years lead to a conceptual hypothesis, first proposed be Cole (Cole, 2004), and after supported by us and other authors (Canas et al., $2010 \mathrm{~b})$. This hypothesis is based mostly on the capacity of certain brain regions to deal with the perfusion and energy demands triggered by seizure activity, which is determined by their intrinsic threshold; these hypothetical mechanisms, besides explaining some of the periictal MRI changes, could also help to clarify the TPMA electroclinical-imagiological spectrum, outcome, and some apparently contradictory findings described in the literature. The cortical hyperemia associated with provoked or spontaneous seizures is a common finding reported by neurosurgeons. Non-invasive methods, such as single-photon emission computed tomography, positron emission tomography, intracranial doppler and MRangiography, also disclosed that focal seizures are associated with high metabolic demands and a marked blood flow increase to the seizure focus (Juhazc et al., 1998; Theodore, 1999; Doherty et al., 2004). The corresponding hyperperfusion may disrupt the blood-brain barrier and justify the vasogenic edema and contrast enhancement observed in some cases (Wieshmann et al., 1997; Hong et al. 2004); that is supported by the prominent vascular congestion and perivascular hemosiderin deposition that was demonstrated in the rare cases of tumor-like TPMA that were biopsied (McClelland et al., 2005). When this increase in blood flow fails to compensate the focal glucose hypermetabolism, anaerobic metabolism takes place, with the released lactate originating a peak on MRS and contributing to a further damage of the blood-brain barrier. Animal models demonstrated that, if seizures are prolonged and/ or severe, an energy failure of the $\mathrm{Na}^{+} / \mathrm{K}^{+}$-ATPase pump may occur, with water and $\mathrm{Na}^{+}$influx to the cell (Wang et al., 1996); this mechanism, so as an excessive release of glutamate (Sloviter et al., 1985) and an increased membrane ion permeability (McNamara, 1994), may all contribute to cytotoxic edema observed in some cases, with the corresponding neuronal dysfunction and/ or loss explaining the decreased N-acetylaspartate level and the changes in choline and myoinositol observed in protonic MRS. If this events are interrupted when the auto-regulatory vascular mechanisms still can compensate the high metabolic demands of seizure activity, the neuronal damage is minimized, which probably justifies the reversion of TPMA mainly composed of vasogenic edema (Wieshmann et al., 1997; Silverstein \& Alexander, 1998; Cohen-Gadol et al., 2004; Hong et al., 2004); however, if seizure activity continues, irreversible brain damage may occur, justifying the worse clinical outcome of TPMA mainly composed of cytotoxic edema (Chu et al., 2001; Szabo et al., 2005; Parmar et al., 2006) or in which a lactate peak is revealed on MRS (Fazekas et al., 1995; Lazeyras et al., 2005). 
This cascade of events occurring at the seizure focus certainly contributes to the frequently observed electroclinical-imagiological topographic concordance. In spite of this, a high incidence of focal TPMAs are found even when seizures are triggered/ provoked by systemic factors, are primarily generalized or are associated with diffuse EEG findings, contributing to the broad electroclinical-imagiological spectrum that we described (Canas et al., 2010b). We speculate that this can be attributed to the presence of brain regions intrinsically more susceptible to the TPMA pathophysiological mechanisms or to structural brain lesions that determine a lower seizure threshold. We show that in the presence of these lesions, TPMA are generally located in their periphery (type 2B TPMA), with topographically concordant EEG and clinical findings, suggesting that they may define the probable seizure focus. In the absence of these lesions, we and other authors show that TPMA are generally located in the posterior regions (occipital, parieto-occipital and temporo-occipital) (Chu et al., 2001; Kim et al., 2001, Canas et al., 2010b). The preponderance of TPMA in these regions could be attributed to their lower sympathetic innervation, making them more susceptible to the hyperperfusion induced by seizure activity, in a similar way to the cerebral lesions observed in reversible posterior leukoencephalopathy syndrome, with which TPMA share similar pathophysiological mechanisms and MRI signal abnormalities.

\section{Conclusion}

There is overwhelming evidence, obtained from animal experiments and human case reports, that acute seizures per se can induce brain damage and that MRI is a very powerful tool to its detection. In the clinical context where these MRI seizure-induced lesions generally appear the attending clinicians must be familiar not only with their neuroimaging features, but also with their electroclinical spectrum, allowing an accurate diagnosis. Since these seizure-induced MRI lesions are typically obtained fortuitously, several questions still remain without definite answer, namely which factors predispose only some patients to their development, what determine their different patterns, what are the exact physiopathological mechanisms underlying their formation or if the results of the periictal DWI and MRS studies can be reliable markers of epileptogenesis. To effectively answer these questions there is an urgent need for longitudinal multimodal MRI studies applied systematically in patients presenting with seizures.

\section{Acknowledgments}

We are very grateful to our colleagues that referred patients to our studies, namely Dr. Paula Breia, Dr. Sofia Calado, Dr. Ricardo Pestana and Dr. José Vale; and to the Neuroradiologists that helped in the MRI data review, namely Dr. Paulo Saraiva and Dr. Constança Jordão.

\section{References}

Amato C.; Elia M.; Musumeci S.A.; Bisceglie P. \& Moschini M (2001). Transient MRI abnormalities associated with partial status epilepticus: a case report, European Journal of Radiology, Vol.38, No.1, (April 2001), pp. 50-54 
Bauer, G.; Gotwald, T.; Dobesberger, J.; Embacher, N.; Felber, S.; Bauer, R.; Seppi, K.; Walser, G.; Trinka, E. \& Poewe, W. (2006). Transient and permanent magnetic resonance imaging abnormalities after complex partial status epilepticus, Epilepsy $\mathcal{E}$ Behavior, Vol. 8, No.3, (May 2006), pp. 666-671

Bartolomei, F., Regis, J., Donet, A. \& Gastaut, J.L. (1999). Development of focal chronic epilepsy following focal status epilepticus in adult patients, Neurophysiologie Clinique, Vol.29, No3, (June 1999), pp. 271-276

Briellmann, R.S.; Kalnins, R.M.; Berkovic, S.F. \& Jackson, G.D. (2002). Hippocampal pathology in refractory temporal lobe epilepsy: T2-weighted signal change reflects dentate gliosis, Neurology, Vol.58, No2, (January 2002), pp. 265-271

Briellmann, R.S.; Wellard, R.M. \& Jackson, G.D. (2005). Seizure-associated abnormalities in epilepsy: evidence from MR imaging, Epilepsia, Vol.46, No5, (May 2005), pp. 760766

Canas, N.; Soares, P.; Calado, S.; Pestana, R.; Ribeiro, C. \& Vale, J. (2010a). Pathophysiology and long-term outcome of reversible tumor-like lesions induced by presenting status epilepticus, Journal of Neuroimaging, Vol.20, No.2, (April 2010), pp. 169-174

Canas, N.; Breia, P.; Soares, P.; Saraiva, P.; Calado, S.; Jordão, C. \& Vale, J. (2010b). The electroclinical-imagiological spectrum and long-term outcome of transient periictal MRI abnormalities, Epilepsy Research, Vol.91, No.2-3, (October 2010), pp. 240-252

Castillo, M.; Smith, J.K. \& Kwock L. (2001). Proton MR spectroscopy in patients with acute temporal lobe seizures, AJNR American Journal of Neuroradiology, Vol.22, No1, (January 2001), pp. 152-157

Cha, S. (2006). Update on brain tumor imaging: from anatomy to physiology, AJNR American Journal of Neuroradiology, Vol.27, No3, (May 2006), pp. 475-487

Chu, K.; Kang, D.W.; Kim, J.Y.; Chang, K.H. \& Lee, S.K. (2001). Diffusion-weighted magnetic resonance imaging in nonconvulsive status epilepticus, Archives of Neurology, Vol.58, No6, (June 2001), pp. 993-998

Chan, S.; Chin, S.S.M.; Kartha, K.; Nordli, D.R.; Goodman, R.R.; Pedley, T. \& Hilal, S.K. (1996). Reversible signal abnormalities in the hippocampus and neocortex after prolonged seizures, AJNR American Journal of Neuroradiology, Vol.17, No9, (October 1996), pp. 1725-1731

Cohen-Gadol, A.A.; Britton, J.W.; Worrell, G.A. \& Meyer, F.B. (2004). Transient cortical abnormalities on magnetic resonance imaging after status epilepticus: case report, Surgical Neurology, Vol.61, No5, (May 2004), pp. 479-82

Cole A.J. (2004). Status epilepticus and periictal imaging, Epilepsia, Vol.45, (Suppl. 4), pp. 7277

Di Bonaventura, C.; Bonini, F.; Fattouch, J.; Mari, F.; Petrucci, S.; Carnì, M.; Tinelli, E.; Pantano, P.; Bastianello, S.; Maraviglia, B.; Manfredi, M.; Prencipe, M. \& Giallonardo, A.T. (2009). Diffusion-weighted magnetic resonance imaging in patients with partial status epilepticus, Epilepsia, Vol.50, (Suppl. 1), pp. 45-52

Doherty, C.P.; Cole, A.J.; Grant, P.E.; Fischman, A.; Dooling, E.; Hoch, D.B.; White, T.H. \& Cosgrove, G.R. (2004). Multimodal longitudinal imaging of focal status epilepticus, Canadian Journal of Neurological Sciences, Vol.31, No.2, (May 2004), pp. $276-281$ 
Fabene, P.F.; Marzola, P.; Sbarbati, A. \& Bentivoglio, M. (2003). Magnetic resonance imaging of changes elicited by status epilepticus in the rat brain: diffusionweighted and T2-weighted images, regional blood volume maps, and direct correlation with tissue and cell damage, Neuroimage, Vol.18, No.2, (February 2003), pp. 375-89

Farina, L.; Bergqvist, C.; Zimmerman, R.A.; Haselgrove, J.; Hunter, J.V. \& Bilaniuk, L.T. (2004). Acute diffusion abnormalities in the hippocampus of children with newonset seizures: the development of mesial temporal sclerosis, Neuroradiology, Vol.46, No.4, (April 2004), pp. 251-157

Fazekas, F.; Kapeller, P.; Schmidt, R.; Stollberger, R.; Varosanec, S.; Offenbacher, H.; Fazekas, G. \& Lechner, H. (1995). Magnetic resonance imaging and spectroscopy findings after focal status epilepticus, Epilepsia, Vol.36, No.9, (September 1995), pp. 946-949

Flacke, S.; Wullner, U.; Keller, E.; Hamzei, F. \& Urbach, H. (2000). Reversible changes in echo planar perfusion- and diffusion-weighted MRI in status epilepticus, Neuroradiology, Vol.42, No.2, (February 2000), pp. 92-95

Goulatia, R.K.; Verma, A. ; Mishra, N.K. \& Ahuja, G.K. (1987). Disappearing CT lesions in epilepsy, Epilepsia, Vol.28, No.5, (September-October 1987), pp. 523-527

Goyal, M.K.; Sinha, S.; Ravishankar, S. \& Shivshankar, J.J. (2009). Peri-ictal signal changes in seven patients with status epilepticus: interesting MRI observation, Neuroradiology, Vol.51, No3, (March 2009), pp. 151-61

Hicdonmez, T.; Uktu, U.; Turgut, N.; Cobanoglu, S. \& Birgili, B. (2003). Reversible postictal MRI change mimicking structural lesion, Clinical Neurology and Neurosurgery, Vol.105, No.4, (September 2003), pp. 288-290

Hinchey, J.; Chaves, C.; Appignani, B.; Breen, J.; Pao, L.; Wang, A.; Pessin, M.S.; Lamy, C.; Mas, J.L. \& Caplan, L.R. (1996). A reversible posterior leukoencephalopathy syndrome, New England Journal of Medicine, Vol.334, No.8, (February 22, 1996), pp. 494-500

Hong, K.S.; Cho, Y.J.; Lee, S.K.; Jeong, S.W.; Kim, W.K. \& Oh, E.J. (2004). Diffusion changes suggesting predominant vasogenic oedema during partial status epilepticus, Seizure, Vol.13, No.5, (July 2004), pp. 317-321

Huang, Y.C.; Weng, H.H.; Tsai, Y.T.; Huang, Y.C.; Hsiao, M.C.; Wu, C.Y.; Lin, Y.H.; Hsu, H.L. \& Lee, J.D. (2009). Periictal magnetic resonance imaging in status epilecticus, Epilepsy Research, Vol.86, No.1, (September 2009), pp. 72-81

Juhasz, C.; Scheidl, E. \& Szirmai, I. (1998). Reversible focal MRI abnormalities due to status epilepticus. An EEG, single photon emission computed tomography, transcranial Doppler follow-up study, Electroencephalography and Clinical Neurophysiology, Vol.107, No6, (December 1998), pp. 402-407

Kim, J.-A.; Chung, J.I.; Yoon, P.H., Kim; D.I., Chung, T.-S.; Kim, E.-U. \& Jeong, E.-K. (2001). Transient MR signal changes in patients with generalized tonicoclonic seizure or status epilepticus: periictal diffusion-weighted imaging, AJNR Am J Neuroradiology, Vol.22, No6, (June-July 2001), pp. 1149-1160

Kozak, O.S.; Wijdicks, E.F.; Manno, E.M.; Miley, J.T. \& Rabinstein, A.A. (2007). Status epilepticus as initial manifestation of posterior reversible encephalopathy syndrome, Neurology, Vol.69, No9, (August 2007), pp. 894-897 
Kramer, L.E.; Luders, H.; Lesser, R.P.; Weinstein, M.R.; Dinner, D.S.; Morris, H.H. \& Wyllie, E. (1987). Transient focal abnormalities of neuroimaging studies during focal status epilepticus, Epilepsia, Vol.28, No5, (September-October 1987), pp. 528532

Lansberg, M.G.; O’Brien, M.W.; Norbach, A.M.; Moseley, M.E.; Morrell, M. \& Albers, G.W. (1999). MRI abnormalities associated with partial status epilepticus, Neurology, Vol.52, No5, (March 1999), pp. 1021-1027

Lazeyras, F.; Blanke, O.; Zimine, I.; Delavelle, J.; Perrig, S.H. \& Seeck, M. (2000). MRI, (1)HMRS, and functional MRI during and after prolonged nonconvulsive seizure activity, Neurology, Vol.55, No11, (December 2000), pp. 1677-1682

Masterson, K.; Vargas, M.I. \& Delavelle, J. (2009). Postictal deficit mimicking stroke: role of perfusion CT, Journal of Neuroradiology, Vol.36, No.1, (March 2009), pp. 48-51

McClelland, S.; Libien, J.M.; Chin, S.S.; Adams, D.J.; Resor, S.R.; Chan, S. \& Goodman, R.R. (2005). Unusual findings in brain biopsies of two patients with acute magnetic resonance imaging lesions associated with focal seizures, Epilepsia, Vol.46, No.9, (September 2005), pp. 1495-1501

McNamara, J.O. (1994). Cellular and molecular basis of epilepsy, The Journal of Neurosciences, Vol.14, No6, (June 1994), pp. 3413-3425

Meissner, B.; Kallenberg, K.; Sanchez-Juan, P.; Krasnianski, A.; Heinemann, U.; Varges, D.; Knauth, M. \& Zerr, I. (2008). Isolated cortical signal increase on MRI imaging as a frequent lesion pattern in sporadic Creutzfeldt-Jakob disease, AJNR American Journal of Neuroradiology, Vol.29, No8, (September 2008), pp. 1519-1524

Milligan, T.A.; Zamani, A. \& Bromfield, E. (2009). Frequency and patterns of MRI abnormalities due to status epilepticus, Seizure, Vol.18, No2, (March 2009), pp. 104108

Mueller, S.G.; Kollias, S.S.; Trabesinger, A.H.; Buck, A; Boesiger, P. \& Wieser, H.J. (2001). Proton magnetic resonance spectroscopy characteristics of a focal cortical cortical dysgenesis during status epilepticus and in the interictal state, Seizure, Vol.10, No.7, (October 2001), pp. 518-524

Najm, I; Wang, Y; Hong, S.C; Luders, H.O.; Ng, T.C. \& Comair, Y.G. (1997). Temporal changes in proton MRS metabolites after kainic acid-induced seizures in rat brain, Epilepsia, No38, No1, (January 1997), pp. 87-94

Nakasu, Y.; Nakasy, S.; Morikawa, S.; Uemura, S.; Inubushit, T. \& Handa, J. (1995). Diffusion-weighted MRI in experimental sustained seizures elicited with kainic acid, AJNR American Journal of Neuroradiology, Vol.16, No6, (June-July 1995), pp. 1185-1192

Nixon, J.; Bateman, D. \& Moss, T. (2001). An MRI and neuropathological study of a case of fatal status epilepticus, Seizure, Vol.10, No8, (December 2001), pp. 588-91.

Parmar, H.; Lim, S.H.; Tan, N.C. \& Lim, C.C. (2006). Acute symptomatic seizures and hippocampus damage: DWI and MRS findings, Neurology, Vol.66, No11, (June 2006), pp. 1732-1735

Pohlmann-Eden, B.; Gass, A.; Peters, C.N.; Wennberg, R. \& Blumcke, I. (2004). Evolution of MRI changes and development of bilateral hippocampal sclerosis during long 
lasting generalized status epilepticus, Journal of Neurology, Neurosurgery, and Psychiatry, Vol.75, No6, (June 2004), pp. 898-900.

Provenzale, J.M.; Barboriak, D.P.; VanLandingham, K.; MacFall, J.; Delong, D. \& Lewis, D.V. (2008). Hippocampal MRI signal hyperintensity after febril status epilepticus is predictive of subsequent mesial temporal sclerosis, AJR American Journal of Roentegenology, Vol.190, No.4, (April 2008), pp. 976-983

Raghavendra, S.; Ashalatha, R.; Krishnamoorthy, T.; Kesavadas, C.; Thomas, S.V. \& Radhakrishnan, K. (2007). Reversible periictal MRI abnormalities: clinical correlates and long-term outcome in 12 patients, Epilepsy Research, Vol.73, No1, (January 2007), pp. 129-136

Righini, A.; Pierpaoli, C \& Alger, J.R. (1994). Brain parenchyma ADC alterations associated with experimental complex partial status epilepticus, Magnetic resonance imaging, Vol.12, No6, pp. 865-871

Sammaritano, M; Andermann, F.; Melanson, D.; Pappius, H.M,; Camfield, P; Aicardi, J. \& Sherwin, A. (1985). Prolonged focal cerebral edema associated with partial status epilepticus, Epilepsia, Vol.26, No.4, (July-August 1985), pp. 334-339

Senn, P.; Lovblad, K.O.; Zutter, D.; Bassetti, C.; Zeller, O.; Donati, F. \& Schroth, G. (2003). Changes on diffusion-weighted MRI with focal motor status epilepticus: case report, Neuroradiology, Vol.45, No4, (April 2003), pp. 246-249

Sethi P.K.; Kumar B.R.; Madan V.S. \& Mohan V. (1985). Appearing and Disappearing CT scan abnormalities in seizures, Journal of Neurology, Neurosurgery, and Psychiatry, Vol.49, No.9, (September 1985), pp. 866-869

Silverstein, A.M. \& Alexander, J.A. (1998). Acute postictal cerebral imaging. AJNR American Journal of Neuroradiology, Vol.19, No.8, (September 1998), pp. 14851488

Sloviter R.S. \& Dempster D.W. (1985). "Epileptic" brain damage is replicated qualitatively in the rat hippocampus by central injection of glutamate or aspartate but not by GABA or acetylcholine, Brain Research Bulletin, Vol.15, No1, (January 1985), pp. 3960

Szabo, K.; Poepel , K.; Pohlmann-Eden, B.; Hirsch, J.; Back, T.; Sedlaczek, O.; Hennerici, M. \& Gass, A. (2005). Diffusion-weighted and perfusion MRI demonstrates parenchymal changes in complex partial status epilepticus, Brain, Vol.128, Pt 6, (June 2005), pp. 1369-1376

Theodore, W.H. (1999). Cerebral blood flow and glucose metabolism in human epilepsy, Advances in Neurology, Vol.79, pp. 873-881

Wang, Y.; Majors, A. ; Najm, I. ; Xue, M. ; Comair, Y. ; Modic, M. \& Ng, T.C. (1996). Postictal alteration of sodium content and apparent diffusion coefficient in epileptic rat brain induced by kainic acid, Epilepsia, Vol.37, No10, (October 1996), pp. 1000-1006

Wellard, R.M.; Briellmann, R.S.; Prichard, J.W.; Syngeniotis, A \& Jackson, G.D (2003). Myoinositol abnormalities in temporal lobe epilepsy, Epilepsia, Vol.44, No6, (June 2003), pp. 815-821

Wieshmann U.C.; Symms M.R. \& Shorvon S.D. (1997). Diffusion changes in status epilepticus, Lancet, Vol.350, No9076, (August 16, 1997), pp. 493-494 
Young, G.S.; Geschwind, M.D.; Fischbein, N.J.; Martindale, J.L.; Henry, R.H.; Liu, S.; Wong, S.; Liu, H.; Miller, B.L. \& Dillon, W.P. (2005). Diffusion-weighted and fluidattenuation recovery imaging in Creutzfeldt-Jacob disease: high sensitivity and specificity for diagnosis, AJNR American Journal of Neuroradiology, Vol.26, No6, (June-July 2005), pp. 1551-1562 


\title{
Central Nervous System Findings on Magnetic Resonance Imaging in Children with Epilepsy
}

\author{
Teodoro Durá-Travé1, Maria Eugenia Yoldi-Petri' ${ }^{1}$, \\ Joaquin Esparza-Estaún², Fidel Gallinas-Victoriano1, \\ Sergio Aguilera-Albesa ${ }^{1}$ and Amaia Sagastibelza-Zabaleta ${ }^{1}$ \\ ${ }^{1}$ Neurology Pediatric Unit, \\ ${ }^{2}$ Department of Neuroradiology, Navarra Hospital Complex, Pamplona
}

Spain

\section{Introduction}

Epilepsy is a relatively frequent pathology in child populations, with an annual incidence rate ranging from 41 to 67 per 100000 cases $^{1-7}$. In the past decade, many important advances related to epilepsy have arisen. On one hand, new diagnostic methods (video electroencephalography monitoring, structural and/or functional neuroimaging, metabolic and genetic analysis, etc) facilitate the diagnosis and the syndromic classification; on the other hand, the availability of the new antiepileptic drugs is conditioning new therapeutic possibilities ${ }^{8-11}$.

The International League Against Epilepsy classification for epilepsy and epileptic syndromes suggests prognosis consequences, because it allows estimating outcome and therapeutic responses ${ }^{12-15}$. In this way, structural neuroimaging plays an important role in the evaluation, management, and treatment of the child with epilepsy. The role of neuroimaging is to detect an underlying cerebral lesion that may be causally related to the child's seizure disorder. Magnetic resonance imaging (MRI) is the elective of all structural imaging tool. MRI is considerably superior to X-ray CT in terms of its sensitivity and specificity for identifying subtle abnormalities. The principal role of MRI is in the definition of the structural abnormalities that underlie seizure disorders (tumours, malformations of cortical development, hippocampal sclerosis, neurocutaneous diseases, vascular malformations, traumatic lesions, strokes, residual lesions, etc.) and to contribute to the aetiologic diagnosis and classification of the different epilepsies and epileptic syndromes, and thereby provide an accurate prognosis for patients11,16-18. In Spain, there is limited information about its current use in the initial evaluation of pediatric epilepsy and about its performance during the initial diagnosis of epilepsy, which suggests that a descriptive study of MRI findings in epilepsy in children would be necessary.

The aim of this study is to analyze the epidemiological characteristics and proportional distribution of the epilepsy and epileptic syndromes in children, and to describe the central nervous system findings on MRI in these patients. 


\section{Methods}

\subsection{Subjects}

The study is based on data obtained from 457 medical records of a random selection among the patients diagnosed with epilepsy in the Paediatric Neurology Unit of the Navarre Hospital Complex in Pamplona who were examined from January until December 2009. The patients were included on the condition that MRI was performed at diagnosis according to a standardized paediatric seizure protocol.

The Navarra Hospital Complex of Pamplona is the neuropediatric reference center in Navarra and is the place where the neuropediatric and neurophysiology and magnetic resonance imaging units are located. The functional and structural organization of the health service in Navarre provides referral of all patients with suspected seizures or epilepsy from the health care centers or secondary hospitals (located in Tudela and Estella) to the reference hospital. A neuropediatric examination and follow-up are performed, and, finally, a syndromic diagnosis is established in most patients.

Information recorded from every patient includes epidemiologic data (sex, age at onset, and personal and familial history of epilepsy and febrile seizures) and clinical data (seizure types, neurologic findings, and associated pathologic conditions), as well as the results of complementary studies (electroencephalogram and neuroimaging, including genetic, metabolic, and neurophysiologic studies when required) in order to classify seizures according to etiology as idiopathic, symptomatic or cryptogenic.

The International League Against Epilepsy criteria for epileptic seizures and epileptic syndromes and guidelines for epidemiologic studies have been applied for diagnosis and classification ${ }^{12-15,19}$. Therefore, epilepsy was diagnosed at the time of a second unprovoked seizure, and multiple seizures in a 24 -hour period were considered a single episode. Patients with neonatal seizures only, febrile seizure and other acute symptomatic seizures were excluded. The syndromic diagnosis corresponding to each patient was discussed by members of the neuropediatric unit, and a final diagnosis was reached based on concurrence of the members.

\section{Magnetic resonance imaging}

MRI was performed in all patients at the onset of diagnosis according to a standardized paediatric seizure protocol. The standardized pediatric seizure protocol consisted of the following scanning sequences:

- $\quad$ T2-weighted fast spin-echo (FSE) images and proton density-weighted in axial plane with $5 \mathrm{~mm}$ thick slices.

- T2-weighted Fluid attenuated inversion recovery (FLAIR) images in the coronal plane, with $5 \mathrm{~mm}$ thick slices.

- $\quad$ T1-weighted FLAIR images in the sagittal plane, with $5 \mathrm{~mm}$ thick slices.

- $\quad$ T1-weighted FLAIR images in the axial plane, with $3 \mathrm{~mm}$ thick slices.

- $\quad$ Diffusion-weighted (DW) images in the axial plane.

Complementary sequences:

- T2-weighted gradient-echo images in the axial plane, with $5 \mathrm{~mm}$ thick slices, for the detection of calcifications or hemosiderine deposits.

- T2-weighted FSE images in the coronal plane, with $3 \mathrm{~mm}$ thick slices, for the study of temporal lobe seizure foci. 
The scoring system used for MRI examinations in this study classifies various abnormal features by location ${ }^{20}$. Abnormal features include volume loss, leukomalacia/gliosis, encephalomalacia, other white-matter lesions, heterotopia, cortical dysplasia, other graymatter lesions, mass lesions, vascular lesions, ventricular enlargement, prominence of the extra-axial spaces, hippocampal atrophy or signal abnormality, and others structural abnormalities.

Abnormalities on MRI were classified as either significant or nonsignificant based on a system of classification previously developed ${ }^{21-23}$. A significant abnormality was defined as a MRI finding reasonably likely to be related to seizure disorder. These were defined as at least one of the following: leukomalacia/gliosis, encephalomalacia, any gray-matter lesion, mass lesion, hemorrhage, vascular lesion, hippocampal abnormality, ventricular enlargement $>1.5 \mathrm{~cm}$, or prominence of extra-axial fluid spaces $>1.0 \mathrm{~cm}$.

Some potential overlap in scoring encephalomalacia and leukomalacia/gliosis was recognized. Encephalomalacia was generally thought to signify "cystic encephalomalacia" and to involve cortical regions. If there was some signal abnormality consistent with gliosis adjacent to an area of encephalomalacia, this was scored as encephalomalacia. If there were other discrete areas of abnormal signal consistent with gliosis, these were scored as (additional) areas of leukomalacia/gliosis.

All of these studies were read and coded by two neuroradiologists with experience involved in this study. Rater was blind to other rater scoring and to other clinical data (e.g., electroencephalogram findings), except for knowing that the child had presented with a seizure.

The computer program SPSS 17.0 for Windows (Chicago, Illinois, USA) was used to perform the statistical analysis (descriptive study).

\section{Results}

Age distribution was 78 infants (from 1 to 12 months of age), 157 in early childhood (from 1 to 6 years), 134 school-aged children (from 6 to 10 years), and 88 adolescents (from 10 to 15 years).

\subsection{Infants}

The sample consisted of 78 patients (37 males and 41 females). In infants, most of the seizures were symptomatic $(53.8 \%)$, whereas others were idiopathic $(25.6 \%)$ or cryptogenic (20.5\%). Table 1 shows the distribution of the epilepsies and epileptic syndromes in infants. Epilepsies attributed to and organized by structural or metabolic conditions $(34.6 \%)$ and West syndrome $(30.8 \%)$ were the most prevalent syndromes.

Epilepsies attributed to and organized by structural or metabolic conditions $(n=27)$ were secondary to perinatal asphyxia (figure 1), purulent meningitis, congenital malformations of the brain (figure 2), Aicardy syndrome (figure 3), inherited metabolic disorders, phakomatoses (figure 4) and venous thrombosis. Among patients diagnosed with West syndrome, etiology was symptomatic in $62.5 \%$ of the cases $(n=15)$; this means, 6 cases related to perinatal ischaemic or anoxic lesions, 3 cases with Down syndrome, 2 cases with tuberous sclerosis, and 1 case related with pathologies as subcortical band heterotopia (figure 5), semilobar holoprosencephaly, neonatal meningoencephalitis (figure 6) and perinatal intracranial hemorrhage. 


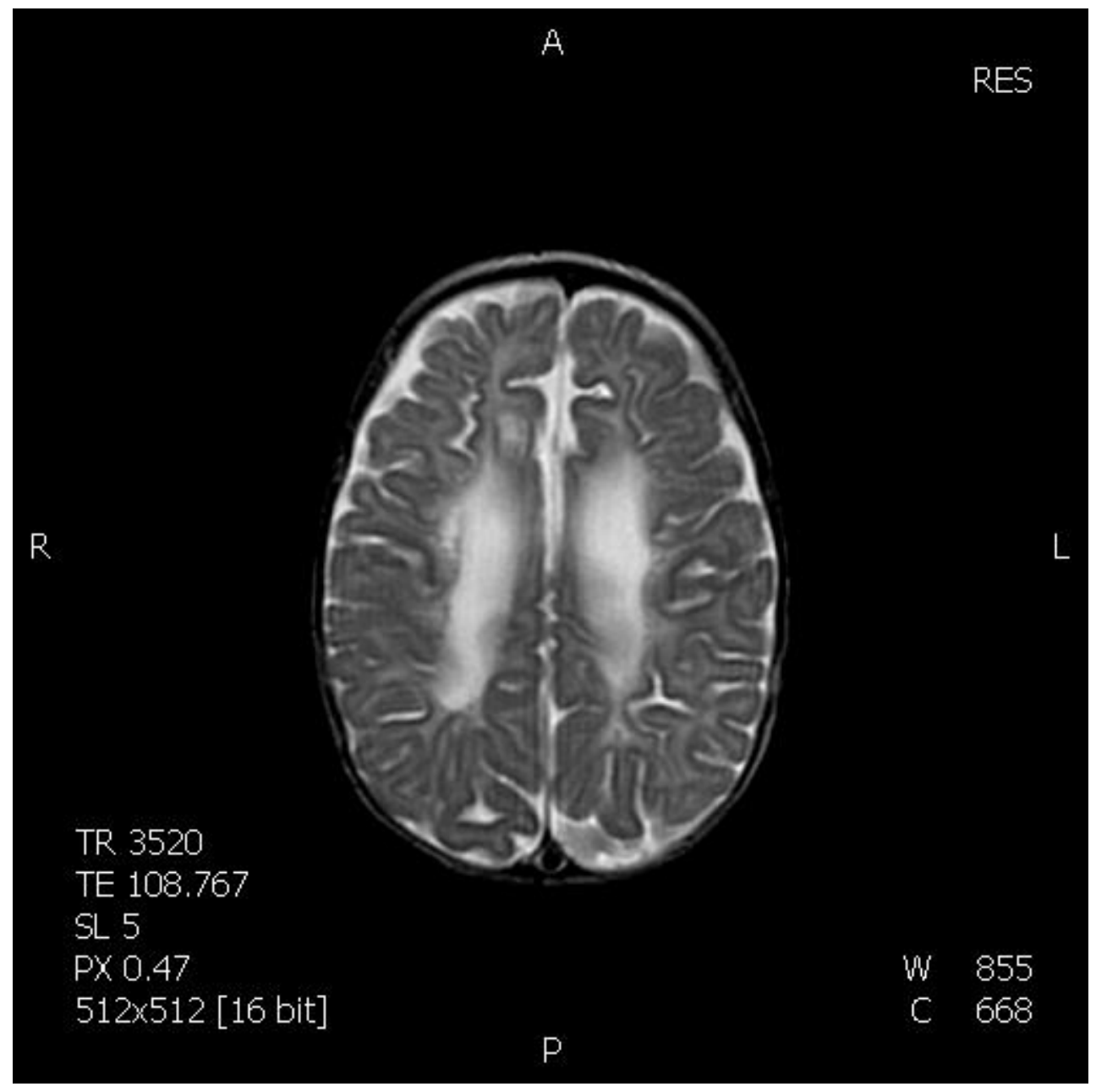

Fig. 1. Chronic-atrophic stage and cystic periventricular leukomalacia: Spin echo (SE) T-2 image showing periventricular white matter atrophy and small cystic lesions adjacent to right ventricle. 


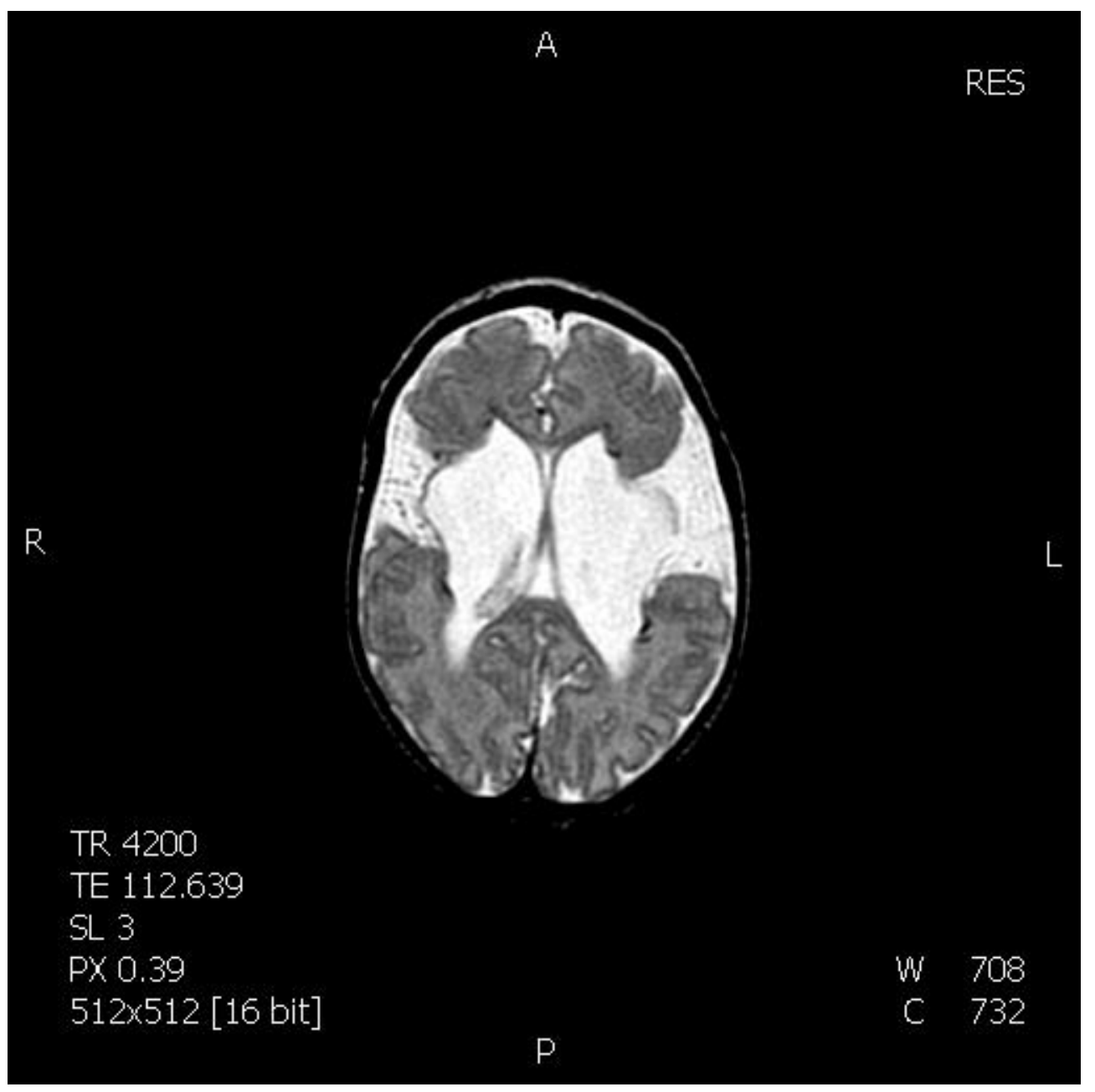

Fig. 2. Open lip schizencephaly: SE T-2 weighted image showing localized parenchymal defects as a split between subarachnoid and ventricular space. Small low-signal periventricular lesions related to calcifications (clinical suspicion of perinatal infection). 


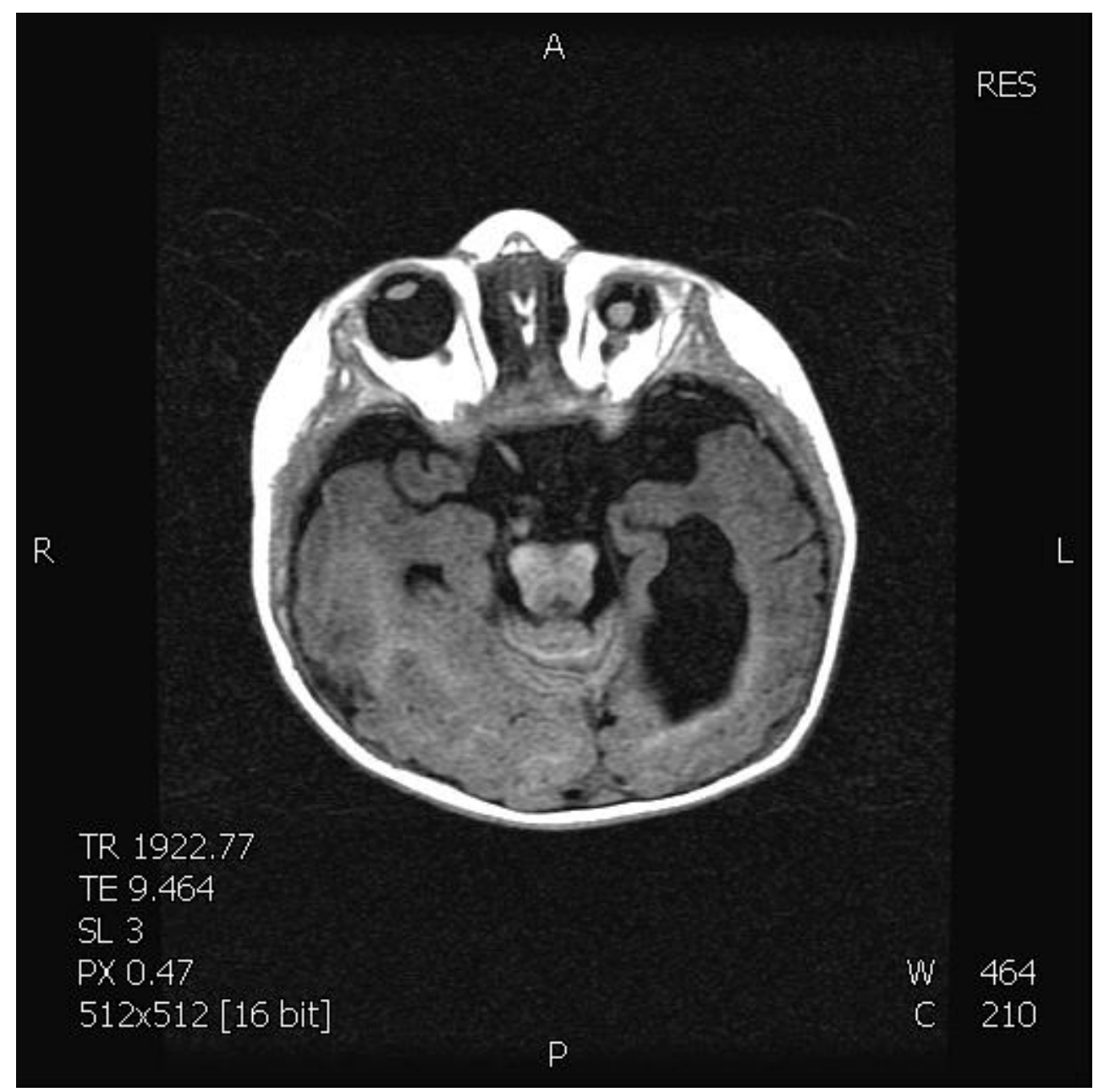

(a) 


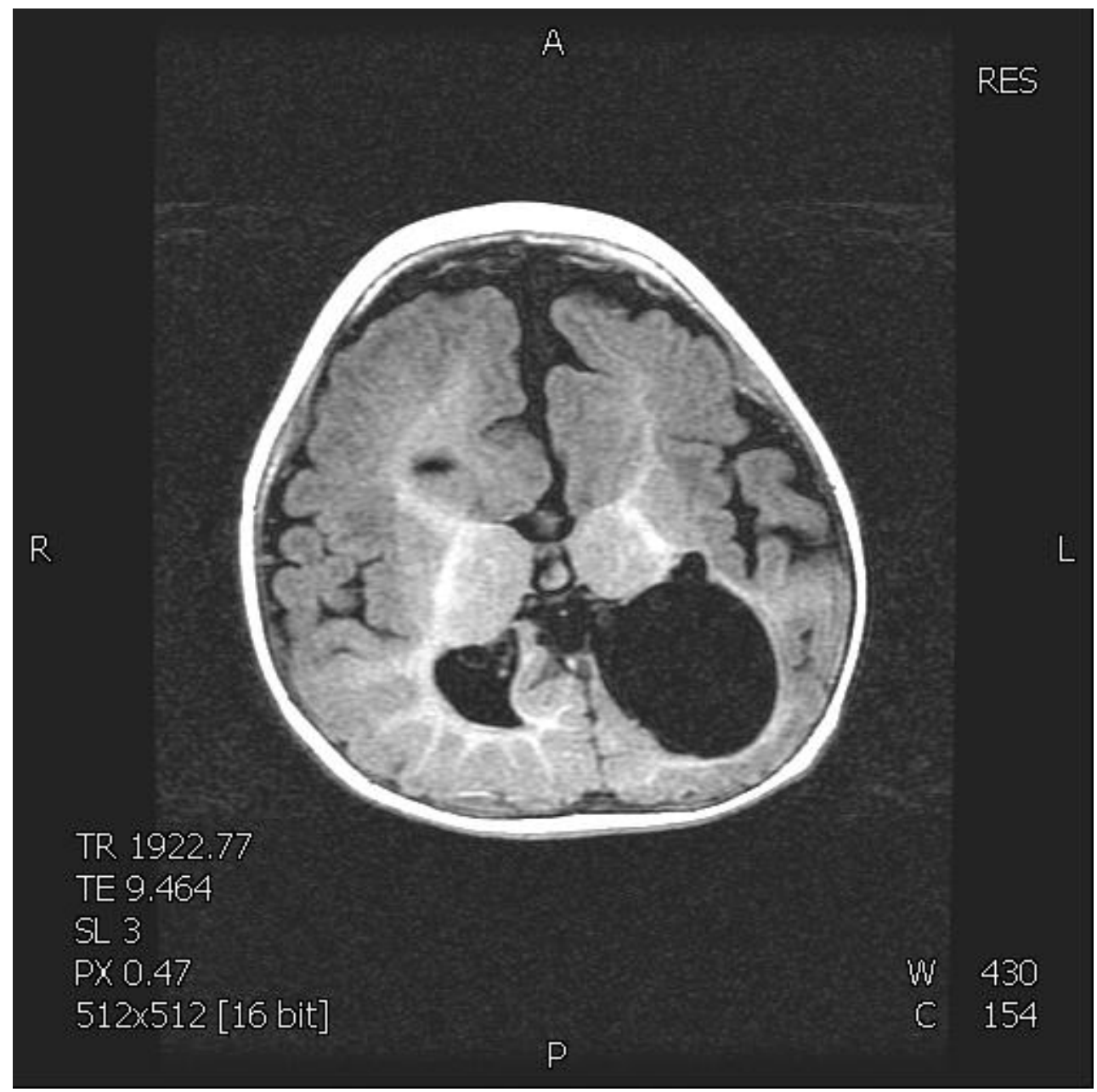

(b) 


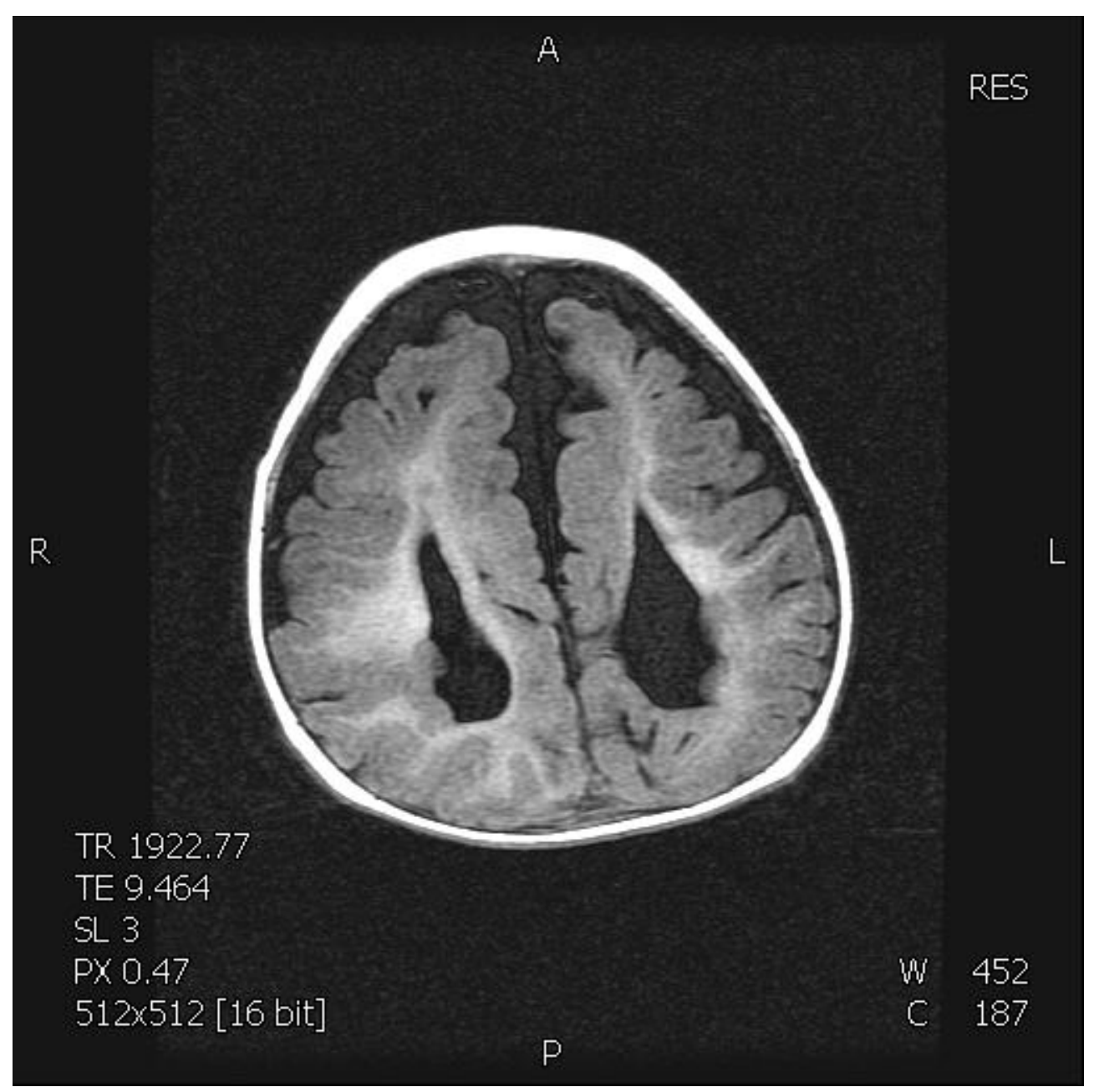

(c)

Fig. 3. (a, b, c). Aicardi syndrome: FLAIR T1 images showing choroid plexus cysts that expand the occipital horn in left ventricle, subependymal heterotopia adjacent to both lateral ventricles, agenesis of the corpus callosum that causes a parallel layout of the lateral ventricles and left microphtalmia with posterior lens luxation. 


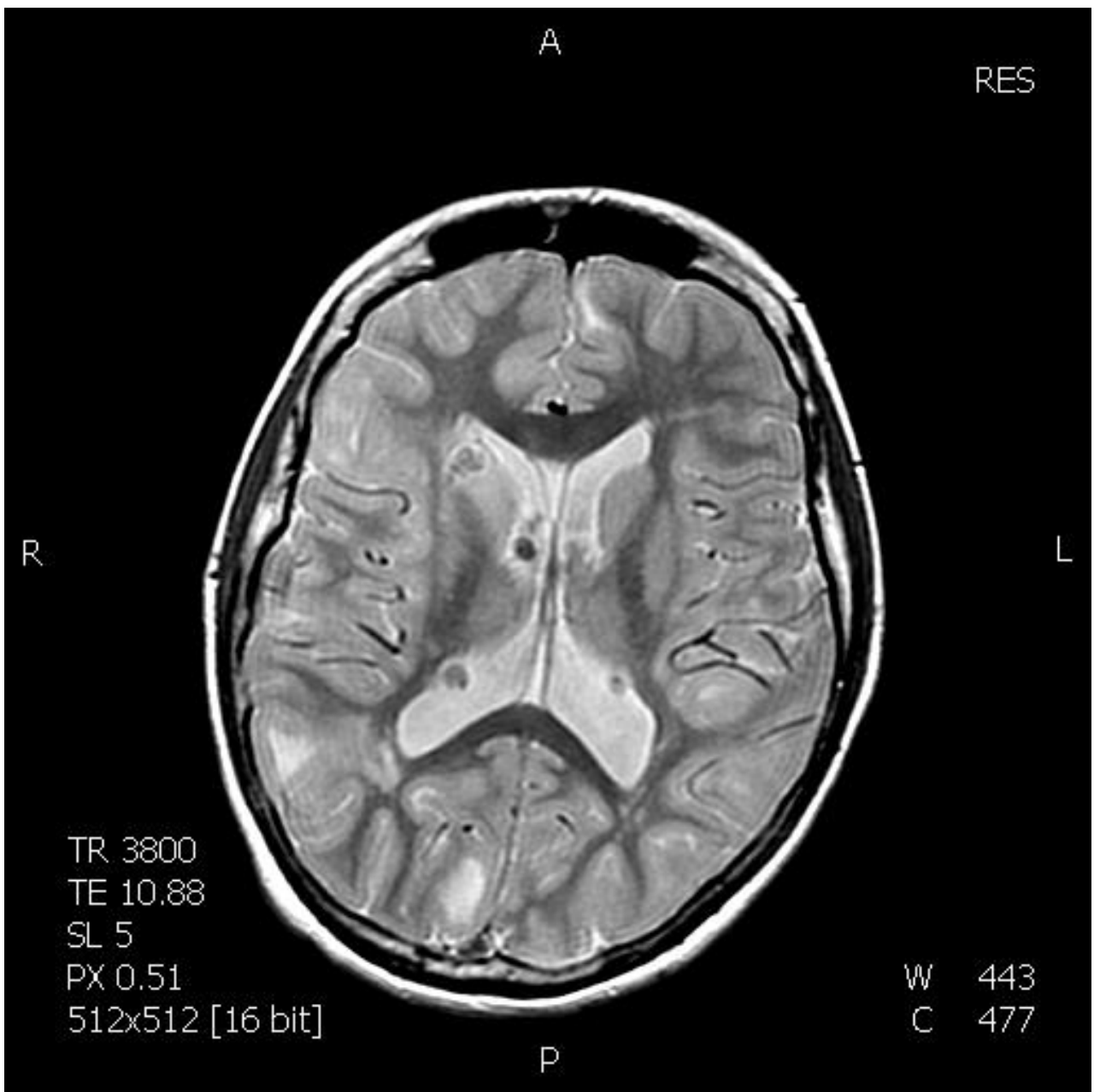

(a) 


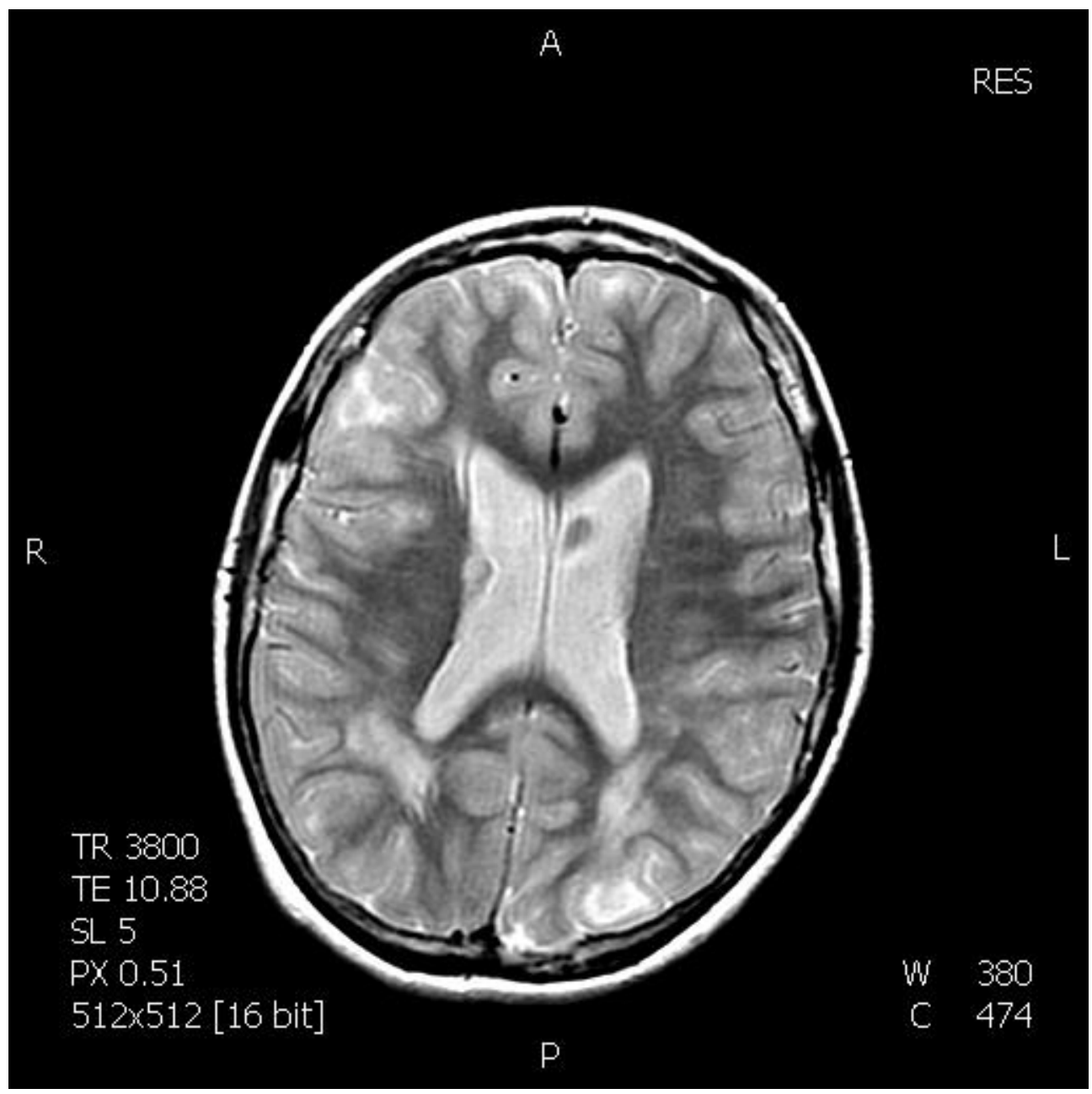

(b)

Fig. 4. (a, b). Tuberous sclerosis: SE 3800/11 images displaying subependymal hamartomas bulging towards the ventricular space, giant cell astrocytomas near the foramen of Monro (the right ventricle astrocytoma showing calcifications), brain hamartomas seen as hyperintense subcortical lesions and portions of dysplastic white matter which follow the pathway of neuronal migration, revealed as linear tracks of radial layout in the white matter. 


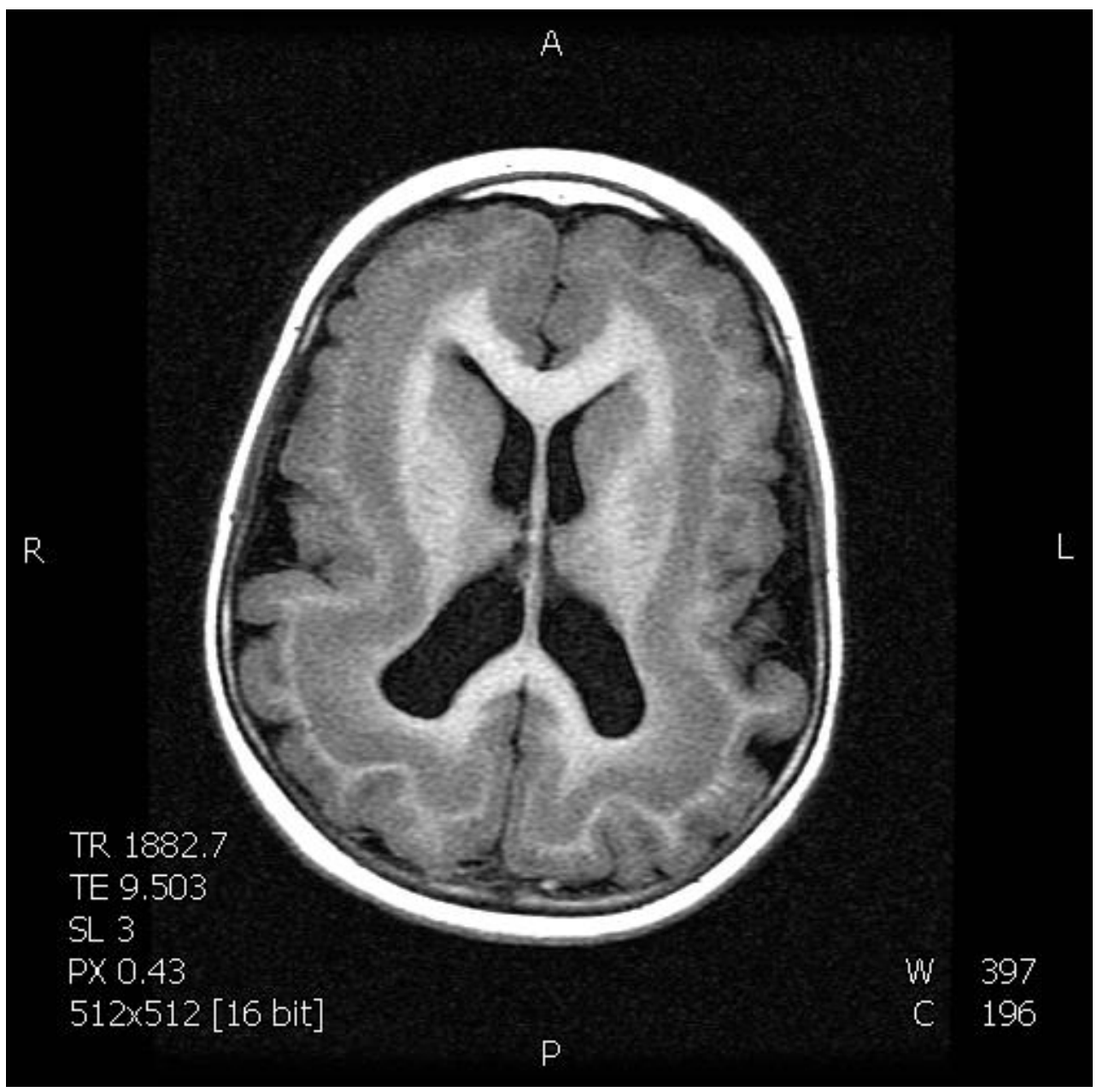

Fig. 5. Band heterotopia: FLAIR T1 images show a thick band of heterotopic grey matter, which is separated from the cortex by a thin layer of myelinated white matter. 


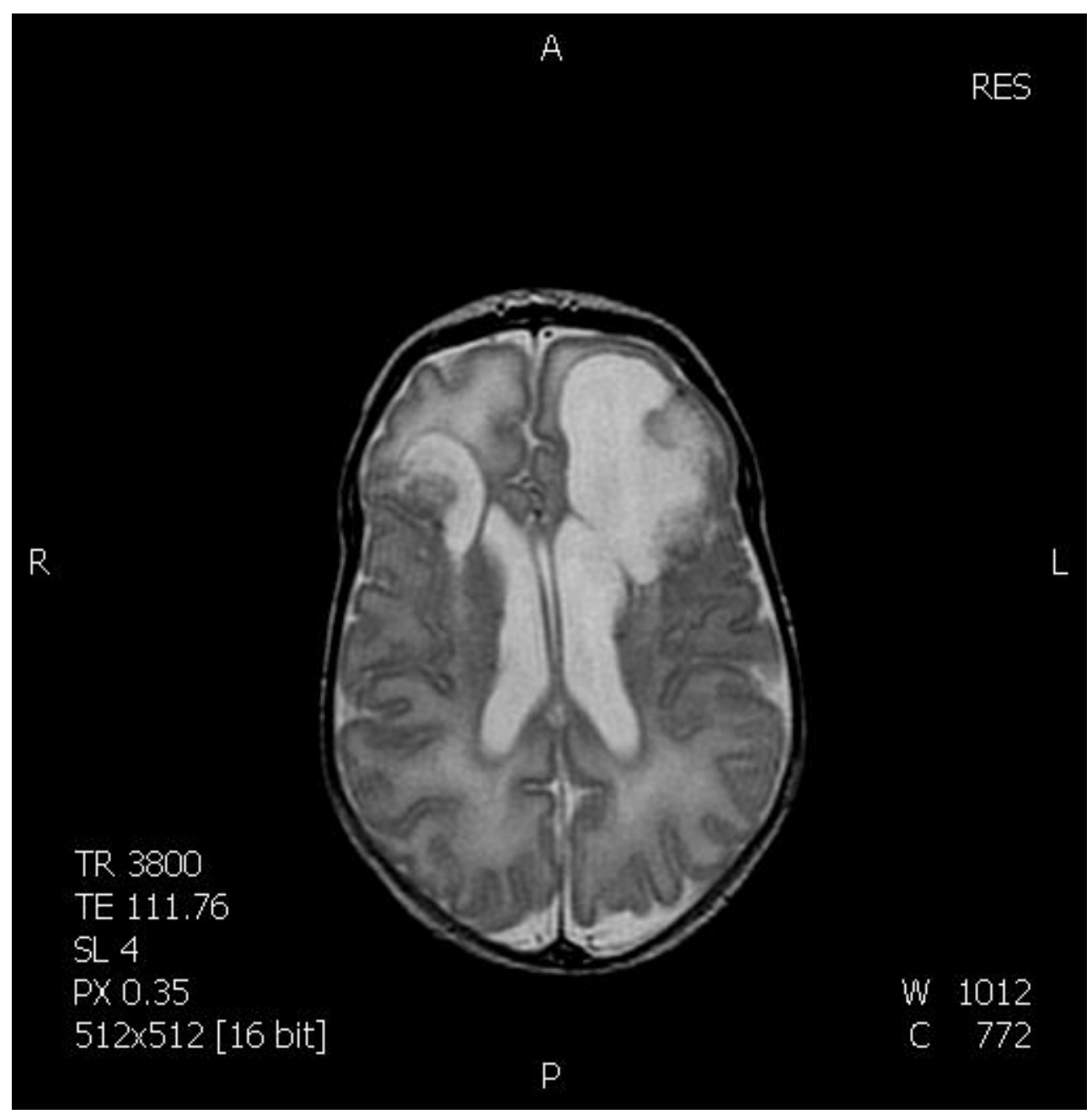

Fig. 6. Frontal bilateral porencephalic lesions as sequelae of meningitis. SE T2 image shows two large frontal porencephalic lesions, being the left one comunicated with the frontal horn. 
Of 78 infants in this study, 42 (53.8\%) demonstrated at least one MRI abnormality. Thirtythree $(42.3 \%)$ manifested MRI abnormalities that were classified as significant because they were considered to be potentially related to the seizure condition.

Table 2 presents the number of infants with MRI abnormalities. Because different abnormalities can affect the same locations for a given child, the sum of abnormalities may exceed the total number of children. Of 42 children with at least one MRI abnormality, the most common abnormalities included white-matter lesions $(28.7 \%)$, volume loss $(22.8 \%)$, gray-matter lesions $(18.8 \%)$ and ventricular enlargement $>1 \mathrm{~cm}$ $(13.9 \%)$.

\begin{tabular}{|c|c|}
\hline West syndrome & 24 \\
\hline Myoclonic epilepsy in infancy & 5 \\
\hline Benign familial infantile epilepsy & 3 \\
\hline Benign non-familial infantile epilepsy & 1 \\
\hline Dravet syndrome & 10 \\
\hline Reflex epilepsy (tactile evoked myoclonic seizures) & 1 \\
\hline Epilepsies of unknown cause (generalised or focal) & 7 \\
\hline Epilepsies associated with structural or metabolic conditions & 27 \\
\hline Perinatal insults & 10 \\
\hline Cerebral infections & \\
\hline Bacterial meningitis (Str. pneumoniae) & 1 \\
\hline Malformations of cortical development & \\
\hline Schizenphalies & 2 \\
\hline Focal cortical dysplasia & 1 \\
\hline Polymicrogiria & 1 \\
\hline Other cerebral malformations & \\
\hline Aicardi syndrome & 1 \\
\hline Holoprosencephaly & 2 \\
\hline Inherited metabolic disorders & \\
\hline Non-ketotic hyperglycinemia & 1 \\
\hline Tay-Sachs disease & 1 \\
\hline Alpers disease & 1 \\
\hline Mucopolysaccharidosis (Hunter syndrome) & 1 \\
\hline Neurocutaneous disorders & \\
\hline Tuberous sclerosis complex & 4 \\
\hline Vascular lesion & \\
\hline Sagittal sinus thrombosis & 1 \\
\hline
\end{tabular}

Table 1. Distribution of the different epilepsies and epileptic syndromes in infants $(n=78)$ 


\begin{tabular}{|l|c|}
\hline MRI abnormality (n=101) & $\mathrm{n}(\%)$ \\
\hline Volume loss & $23(22.8 \%)$ \\
Generalized & 5 \\
Hemisphere & 1 \\
Lobe & 5 \\
Cerebelar & 3 \\
Corpus callosum & 9 \\
\hline White matter lesions & $29(28.7 \%)$ \\
Leukomalacia/gliosis & 15 \\
Encephalomalacia & 5 \\
Other lesions & 9 \\
\hline Gray-matter lesions & $19(18.8 \%)$ \\
Heterotopias & 1 \\
Cortical dysplasias & 7 \\
Other lesions & 11 \\
\hline Vascular lesion & $3(3 \%)$ \\
Hemorrhage & 2 \\
Venous thrombosis & 1 \\
\hline Ventricular enlargement & $14(13.9 \%)$ \\
<1.5cm & 4 \\
$>$ 1.5cm & 10 \\
\hline Prominence of extra-axial fluid space & $1(1 \%)$ \\
$<1.0 c m$ & 1 \\
$>$ 1.0cm & 0 \\
\hline Other structural abnormalities & 2 \\
Agenesis of the corpus callosum & 6 \\
Delayed myelination & $1.9 \%)$ \\
Enlarged perivascular spaces in the corpus callosum & 3 \\
Calcifications & 2 \\
\hline
\end{tabular}

Boldface rows represented "significant abnormalities"

Table 2. MRI abnormalities found in infants diagnosed with epilepsy

\subsection{Early childhood}

The sample was made up of 157 patients (85 males and 72 females). In early childhood, distribution was idiopathic $(44,6 \%)$, cryptogenic $(29.9 \%)$ or symptomatic $(25.5 \%)$. Table 3 shows the distribution of the epilepsies and epileptic syndromes in this period. Epilepsies in which nature of the underlying causes is yet unknown $(29.9 \%)$ and epilepsies attributed to and organized by structural or metabolic conditions $(24.8 \%)$ were the most prevalent syndromes. 
Epilepsies associated to structural or metabolic conditions $(n=39)$ were secondary to perinatal asphyxia (figure 7), cerebral infections (figure 8), congenital malformations of the brain, inherited metabolic disorders, phakomatoses (figure 9), chromosomal abnormalities, arterial infarction (figure 10), arteriovenous malformations and supratentorial tumors.

Of 157 early infants in this study, 45 (28.7\%) had at least one MRI abnormality. Thirty-two (20.4\%) showed MRI abnormalities that were classified as significant because they were considered to be potentially related to the seizure condition.

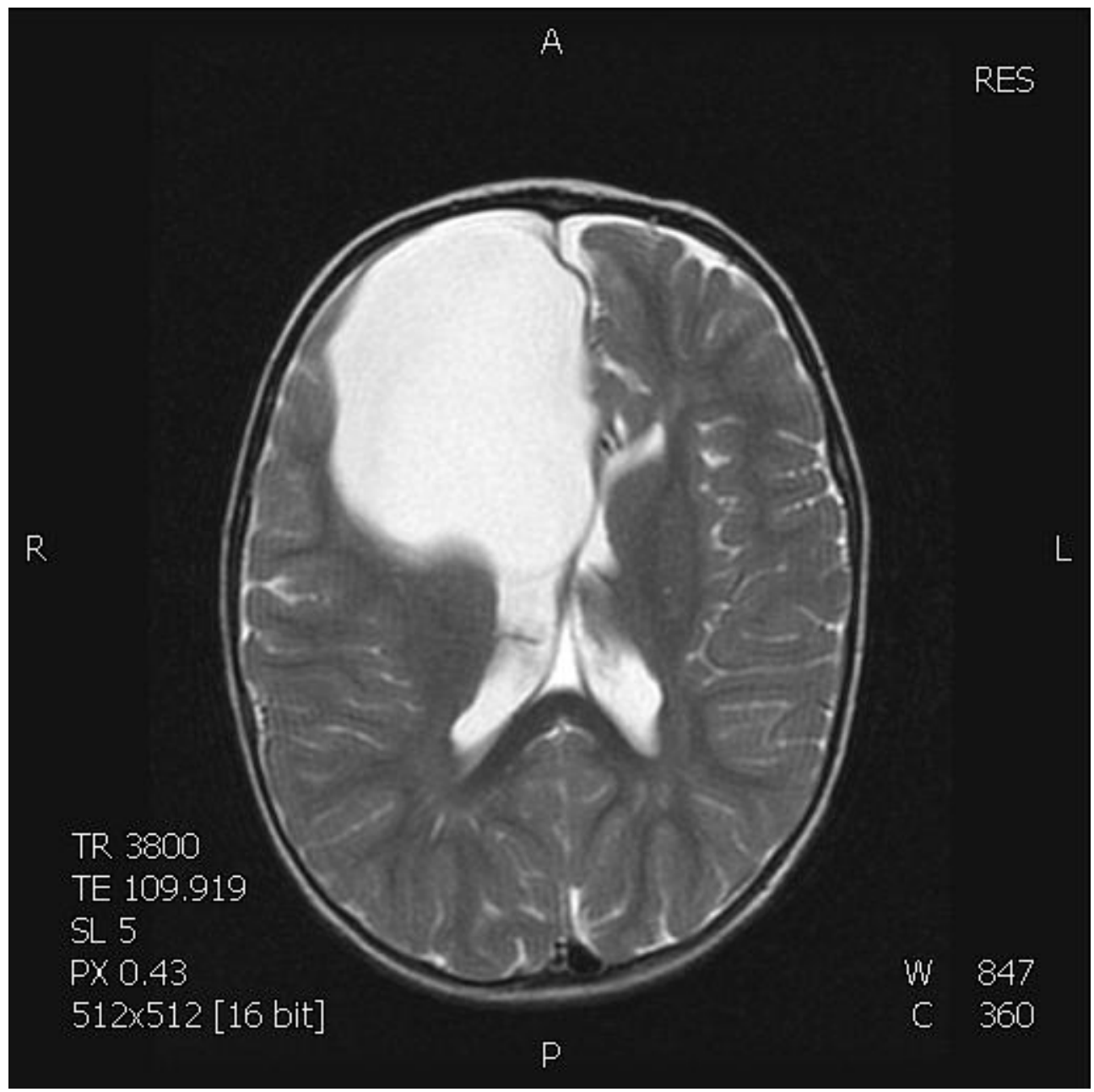

Fig. 7. Porencephalic cyst: T2-weighted SE image displays a wide cyst in the left frontal lobe, which is connected to the lateral ventricle and causes a slight lateral midline displacement of the brain. 


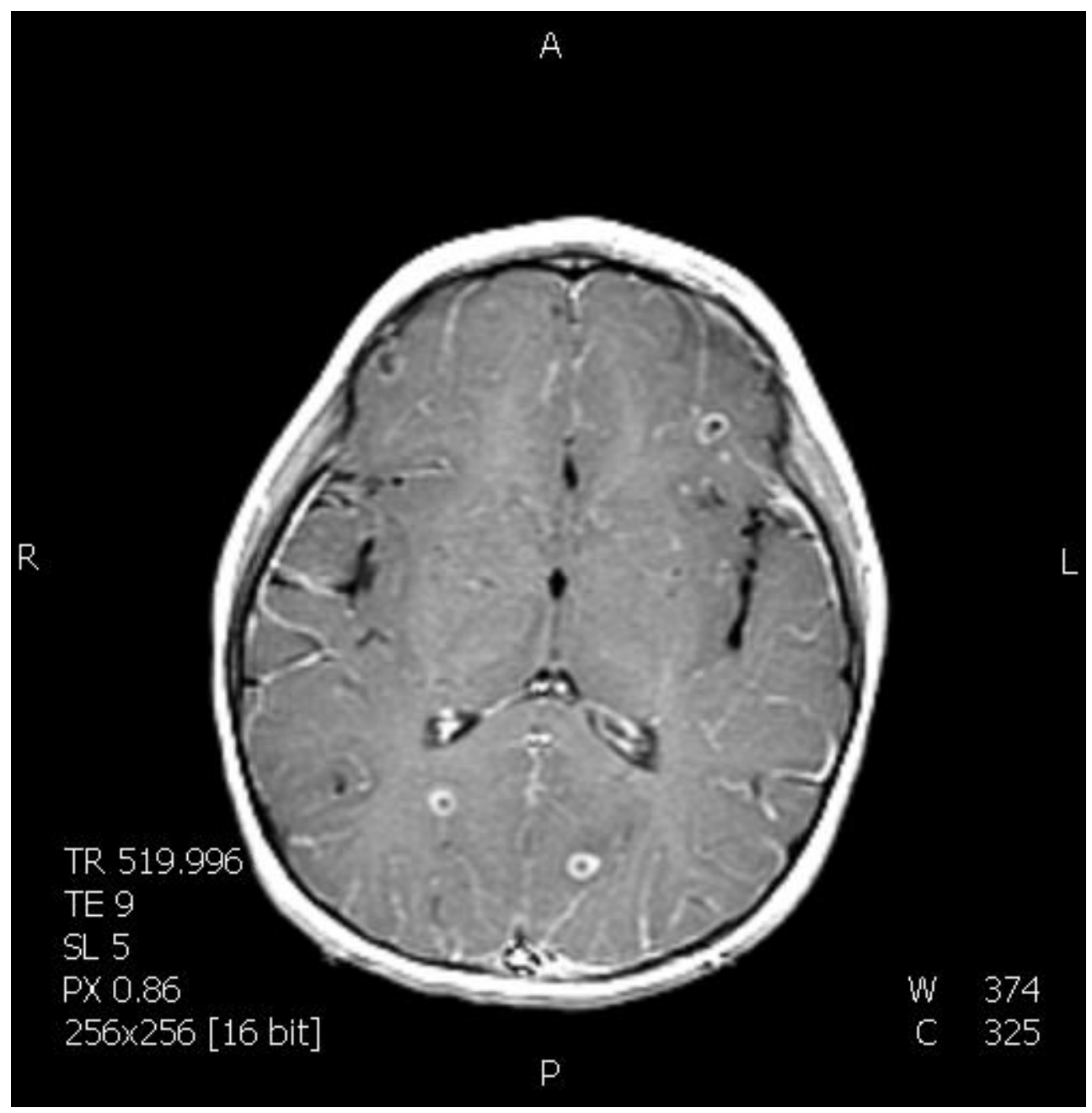

Fig. 8. Neurocysticercosis: T1-weighted SE images (gadolinium-enhanced) shows small cystic intraparenchymal lesions and peripheral enhancement. 


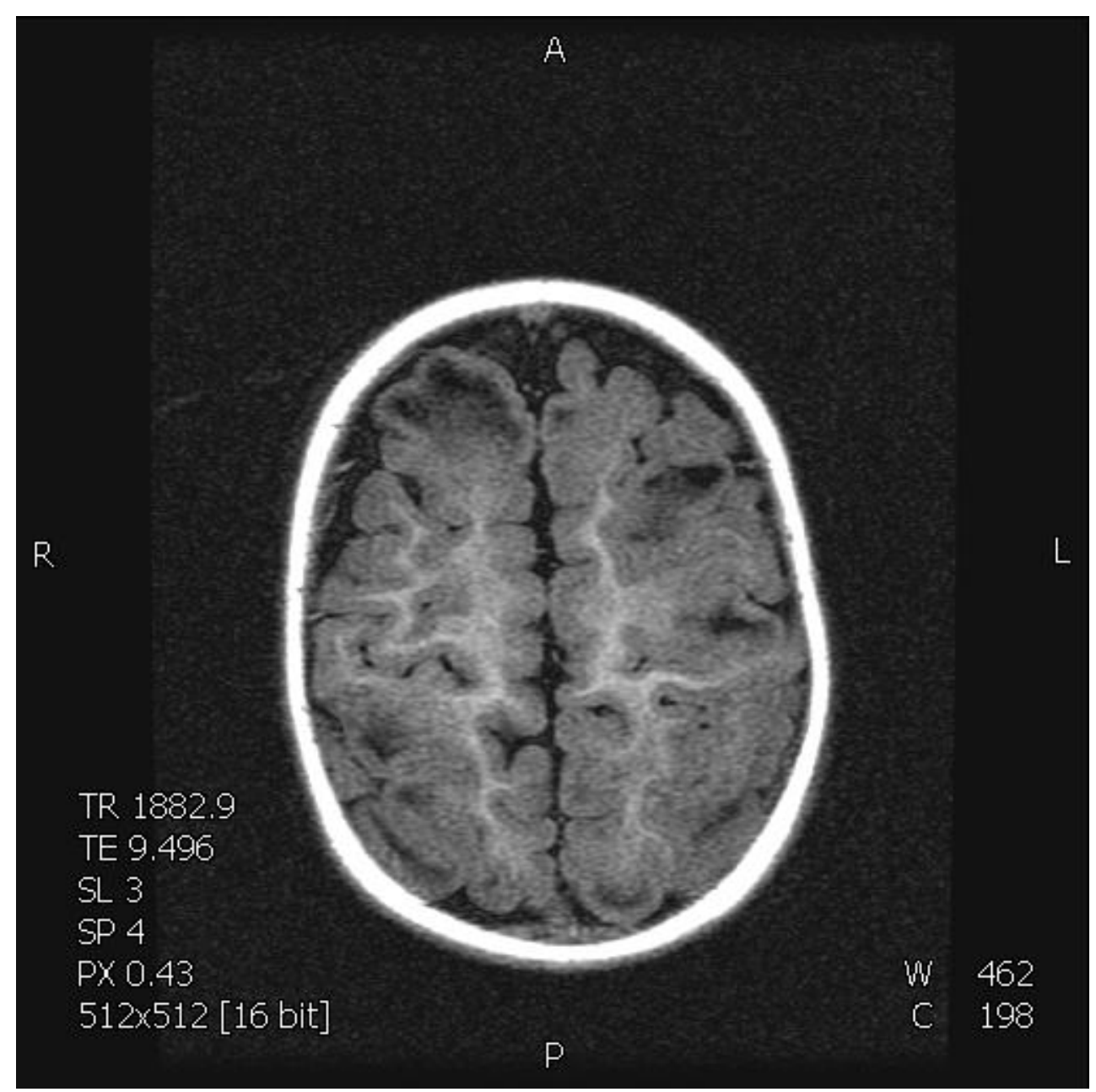

(a) 


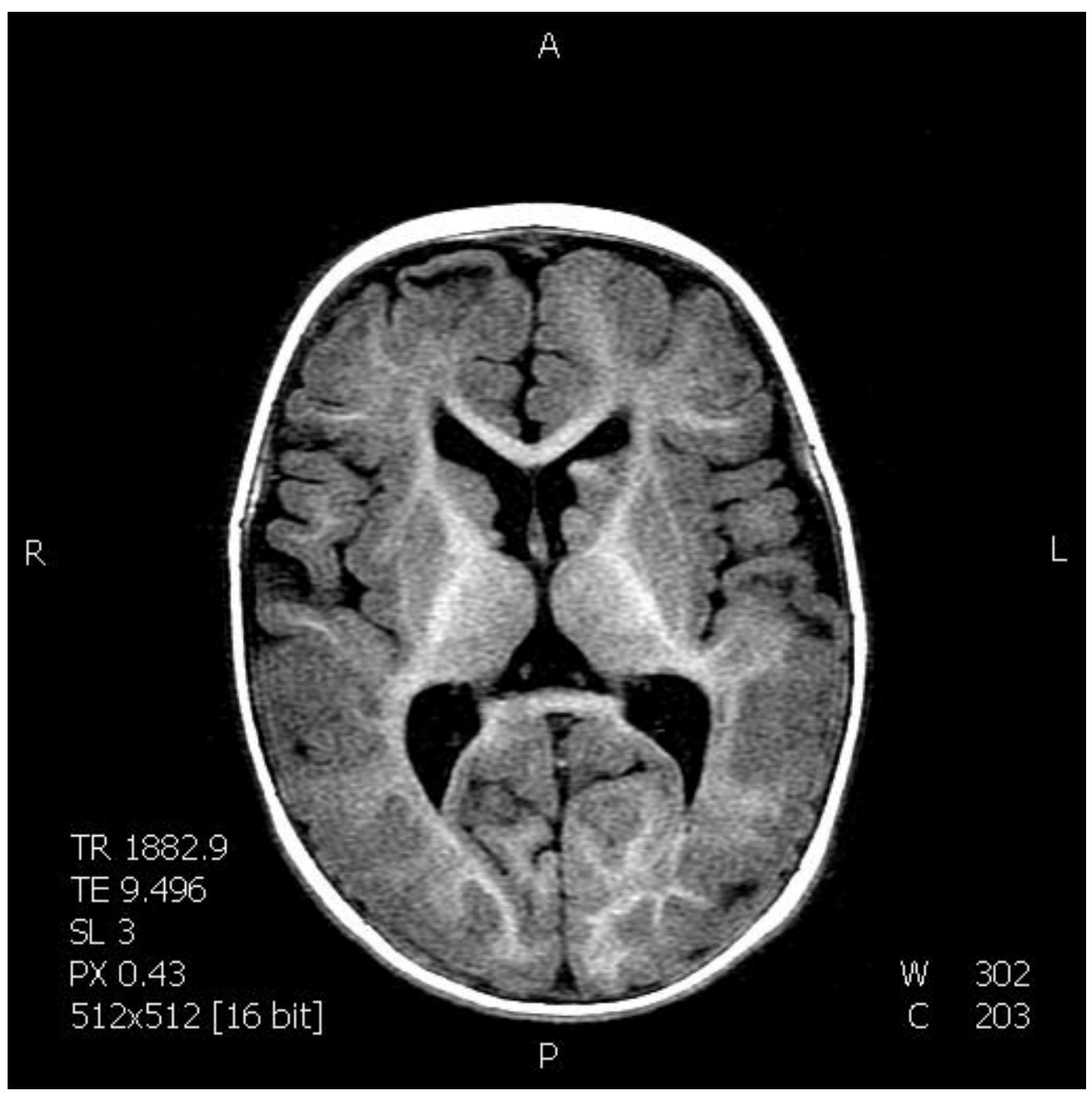

(b) 


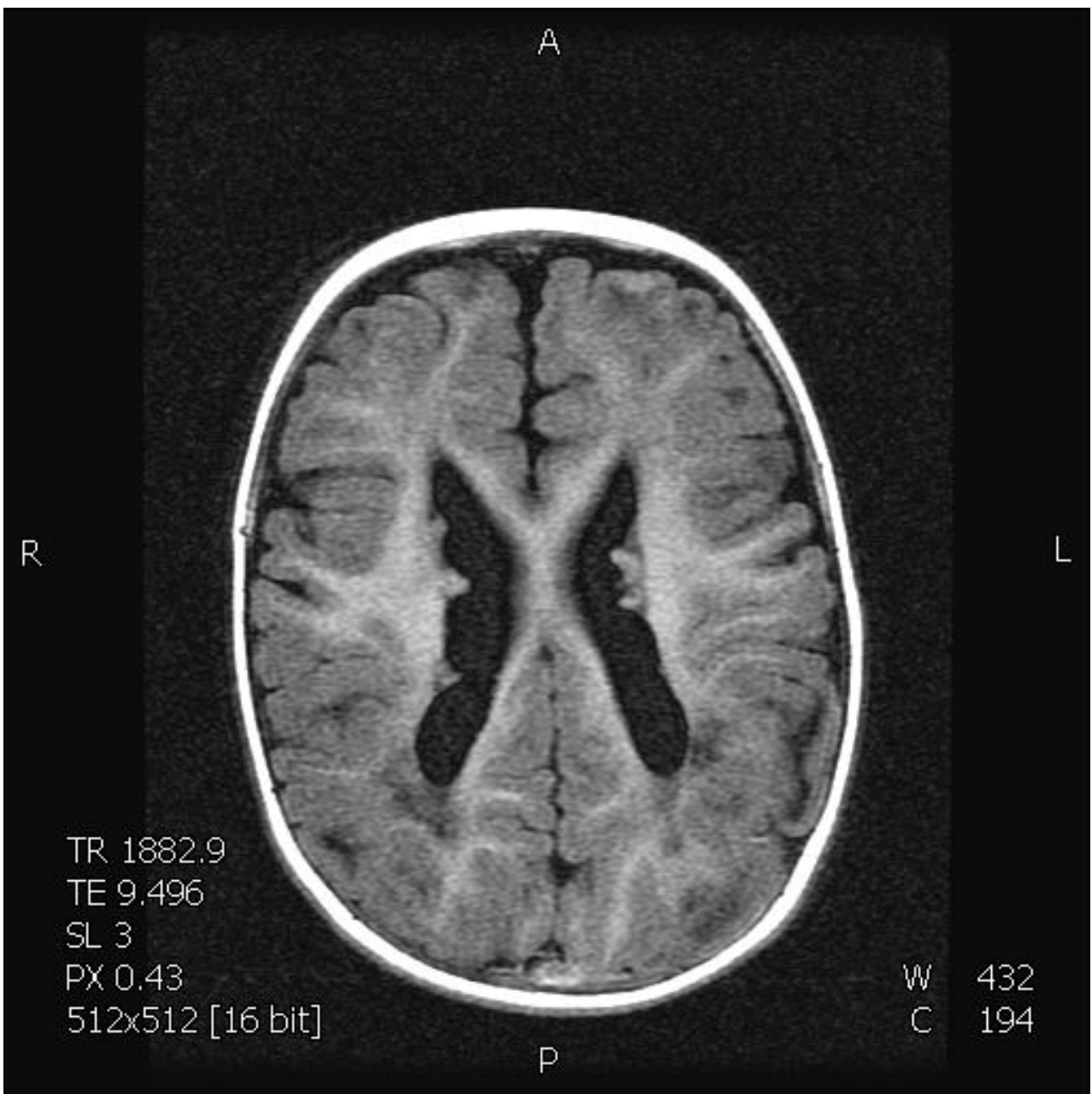

(c)

Fig. 9. $(a, b, c)$. Tuberous sclerosis: T1-weighted FLAIR images reveal cerebral hamartomas as low signal intensity subcortical lesions, subependymal hamartomas casted on the ventricular space and small giant cell astrocytomas adjacent to the foramen of Monro. 


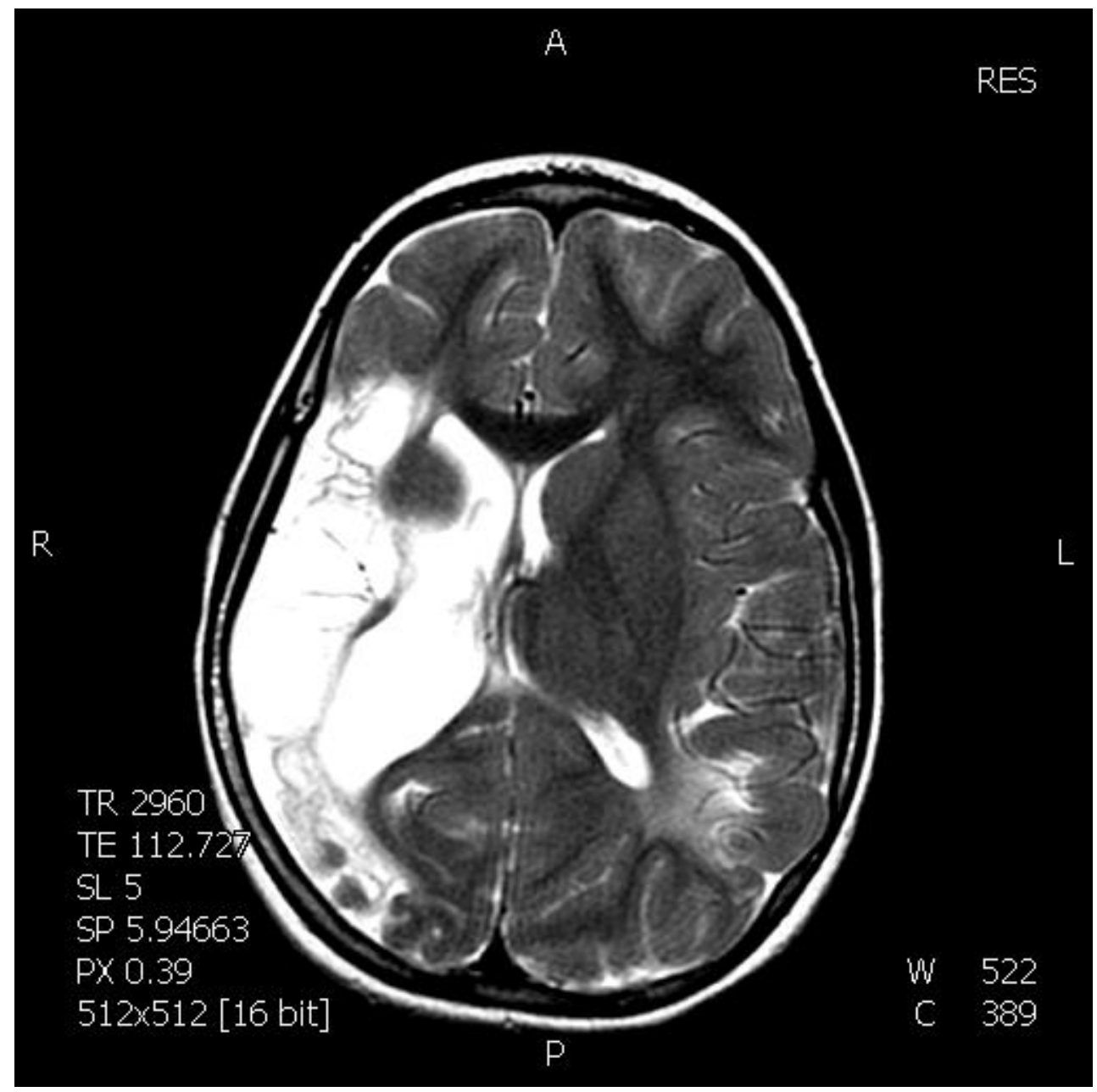

Fig. 10. Post-stroke sequelae in the right middle cerebral artery: T2- weighted SE image showing residual encephalomalacia with volume loss and ex vacuo enlargement of the right ventricle. An area of periventricular leukomalacia in the left occipital lobe can be appreciated.

Table 4 presents the number of early infants with MRI abnormalities (the sum of abnormalities may exceed the total number of children because different abnormalities can affect the same locations for a given patient). In this group of 45 children with at least one MRI abnormality, the most common abnormalities included white-matter lesions $(28.7 \%)$, volume loss $(19.1 \%)$, gray-matter lesions $(21.3 \%)$ and ventricular enlargement (14.9\%). 


\begin{tabular}{|c|c|}
\hline West syndrome & 2 \\
\hline Myoclonic epilepsy & 4 \\
\hline Febrile seizures plus & 1 \\
\hline Panayiotopoulos syndrome & 9 \\
\hline Epilepsy with myoclonic atonic seizures & 10 \\
\hline Benign epilepsy with centrotemporal spikes & 18 \\
\hline Late onset childhood occipital epilepsy (Gastaut type) & 1 \\
\hline Epilepsy with myoclonic absences & 1 \\
\hline Childhood absence epilepsy & 13 \\
\hline Lennox-Gastaut syndrome & 1 \\
\hline Epilepsy with continuous spike-and-wave during sleep & 7 \\
\hline Landau-Kleffner syndrome & 1 \\
\hline Autosomal-dominant nocturnal frontal lobe epilepsy & 1 \\
\hline Epilepsies of unknown cause (generalized or focal) & 46 \\
\hline Epilepsies associated with structural or metabolic conditions & 39 \\
\hline Perinatal insults & 11 \\
\hline Cerebral infections & \\
\hline Cysticercosis & 1 \\
\hline Herpes simplex encephalitis & 2 \\
\hline Malaria & 1 \\
\hline Malformations of cortical development & \\
\hline Focal cortical dysplasia & 2 \\
\hline Heterotopias & 1 \\
\hline Polymicrogiria & 2 \\
\hline Schizenphalies & 2 \\
\hline Others cerebral malformations & \\
\hline Dandy-Walker malformations & 1 \\
\hline Inherited metabolic disorders & \\
\hline Alpers disease & 1 \\
\hline Others & 2 \\
\hline Neurocutaneous disorders & \\
\hline Tuberous sclerosis complex & 2 \\
\hline Chromosomal abnormalities & \\
\hline Down syndrome (trysomy 21 ) & 1 \\
\hline Angelman syndrome & 3 \\
\hline Deletion 8p23 & 1 \\
\hline Deletion $5 q$ syndrome & 1 \\
\hline Duplication $15 q$ syndrome & 1 \\
\hline Stroke & \\
\hline Middle cerebral artery infarction & 2 \\
\hline Vascular malformations & \\
\hline Arteriovenous malformation & 1 \\
\hline Tumors & \\
\hline Supratentorial ependymoma & 1 \\
\hline $\begin{array}{l}\text { Condition with epileptic seizures that do not require a diagnosis of epilepsy } \\
\text { Infantile convulsions with mild gastroenteritis }\end{array}$ & 3 \\
\hline
\end{tabular}

Table 3. Distribution of the different epilepsies and epileptic syndromes in early childhood $(n=157)$ 


\begin{tabular}{|c|c|}
\hline MRI abnormality $(\mathrm{n}=94)$ & $\mathrm{n}(\%)$ \\
\hline Volume loss & $18(19.1 \%)$ \\
\hline Generalized & 6 \\
\hline Hemisphere & 1 \\
\hline Lobe & 2 \\
\hline Cerebelar & 1 \\
\hline Corpus callosum & 8 \\
\hline White matter lesions & $27(28.7 \%)$ \\
\hline Leukomalacia/gliosis & 13 \\
\hline Encephalomalacia & 5 \\
\hline Other lesions & 9 \\
\hline Gray-matter lesions & $20(21.3 \%)$ \\
\hline Heterotopias & 1 \\
\hline Cortical dysplasias & 6 \\
\hline Other lesions & 13 \\
\hline $\begin{array}{c}\text { Vascular lesion } \\
\text { Hemorrhage }\end{array}$ & $\begin{array}{c}2(2.1 \%) \\
2\end{array}$ \\
\hline $\begin{array}{l}\text { Ventricular enlargement } \\
<1.5 \mathrm{~cm} \\
>1.5 \mathrm{~cm}\end{array}$ & $\begin{array}{c}14(14.9 \%) \\
11 \\
3\end{array}$ \\
\hline $\begin{array}{l}\text { Prominence of extra-axial fluid space } \\
<1.0 \mathrm{~cm} \\
>1.0 \mathrm{~cm}\end{array}$ & $\begin{array}{c}1(1.1 \%) \\
1 \\
0\end{array}$ \\
\hline Mass lesion & $1(1.1 \%)$ \\
\hline Other structural abnormalities & $11(11.7 \%)$ \\
\hline Hemisphere asymmetry & 2 \\
\hline Cerebellar tonsils descent & 1 \\
\hline Choroid plexus cyst & 1 \\
\hline Pineal cyst & 1 \\
\hline Mega cisterna magna & 1 \\
\hline Arteriovenous malformations & 1 \\
\hline Delayed myelination & 2 \\
\hline Agenesis of the corpus callosum & 2 \\
\hline
\end{tabular}

Boldface rows represented "significant abnormalities"

Table 4. MRI abnormalities found in early childhood diagnosed with epilepsy

\subsection{School-aged children}

The sample was made up of 134 patients (72 males and 62 females). In school-age children, seizures were idiopathic in $55.2 \%$, cryptogenic in $25.4 \%$ and symptomatic in $19.4 \%$. Table 5 shows the distribution of the epilepsies and epileptic syndromes in school-aged children. Epilepsies in which the nature of the underlying causes is yet unknown (29.1\%), benign epilepsy with centrotemporal spikes $(25.4 \%)$ and childhood absence epilepsy $(18.7 \%)$ were the most prevalent syndromes. 
Epilepsies attributed to structural or metabolic conditions $(n=39)$ were secondary to perinatal asphyxia, cerebral infections, congenital malformations of the brain (figure 11), phakomatoses, arterial infarction, arteriovenous malformations (figure 12), suprasellar arachnoid cyst (figure 13), drug toxicity (figure 14) and desmoplastic neuroepithelial tumors.

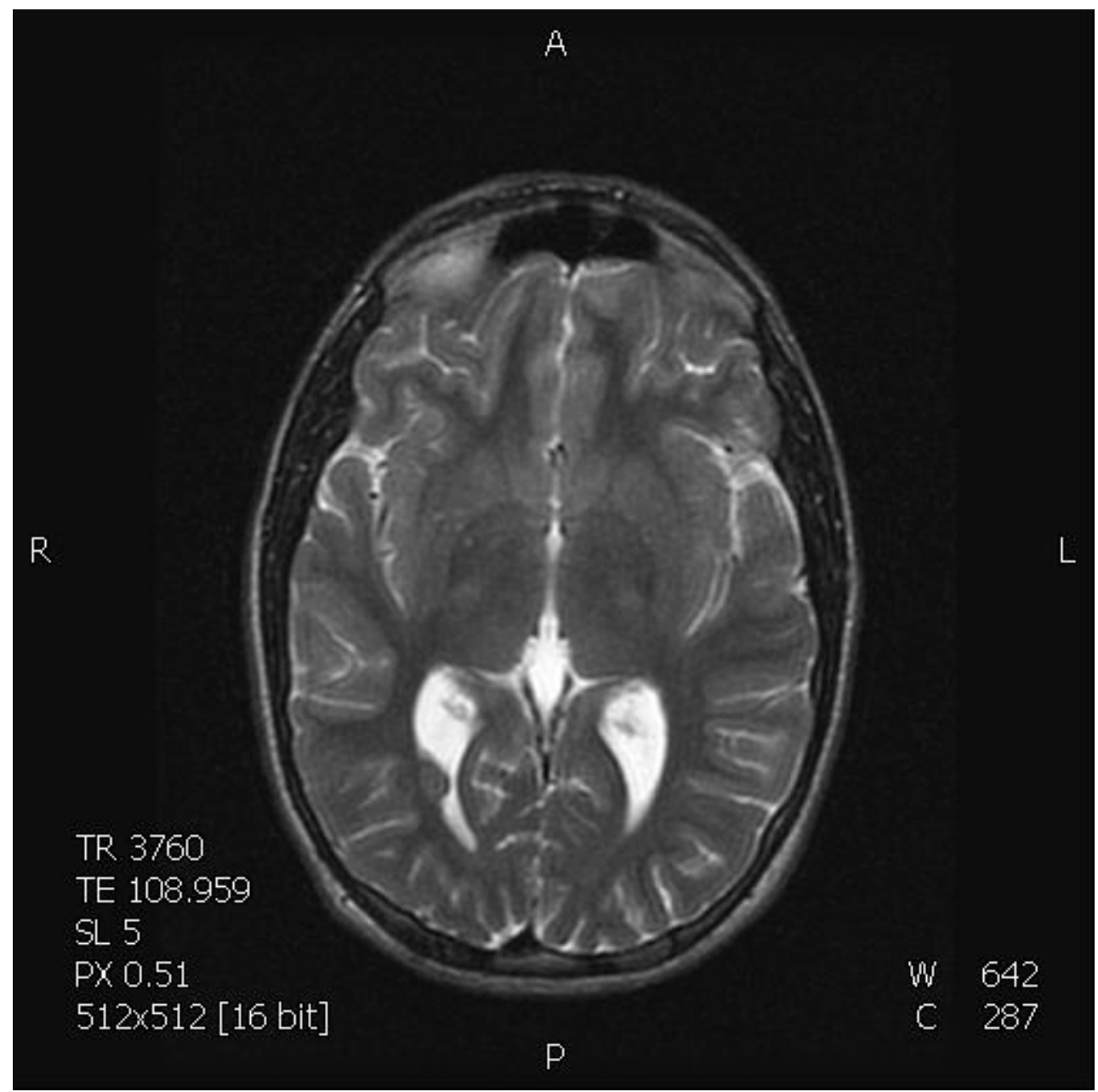

Fig. 11. Subependymal heterotopia: T2-weighted SE image shows a gray matter nodule protruding over the occipital horn of the right ventricle. 


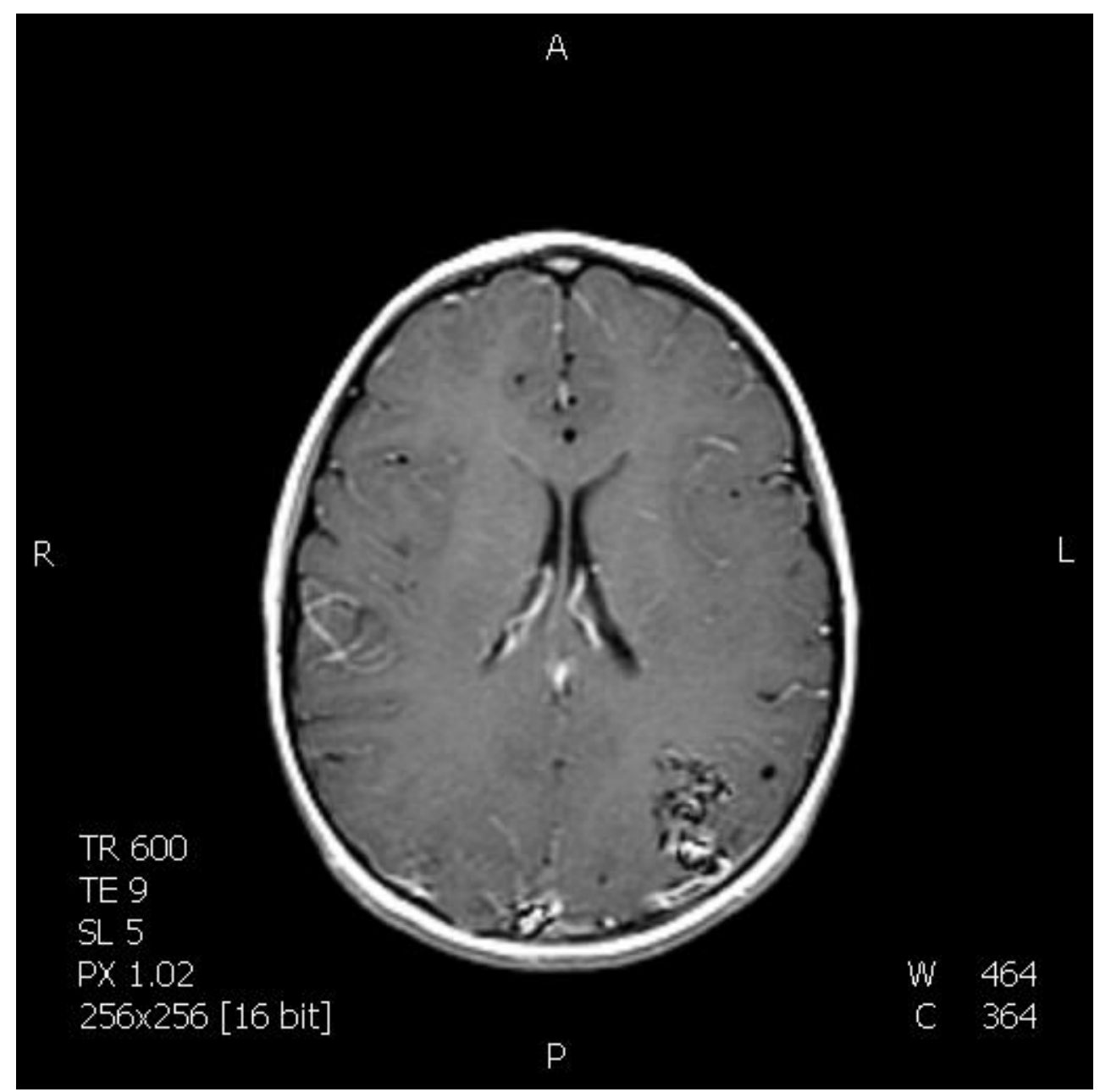

(a) 


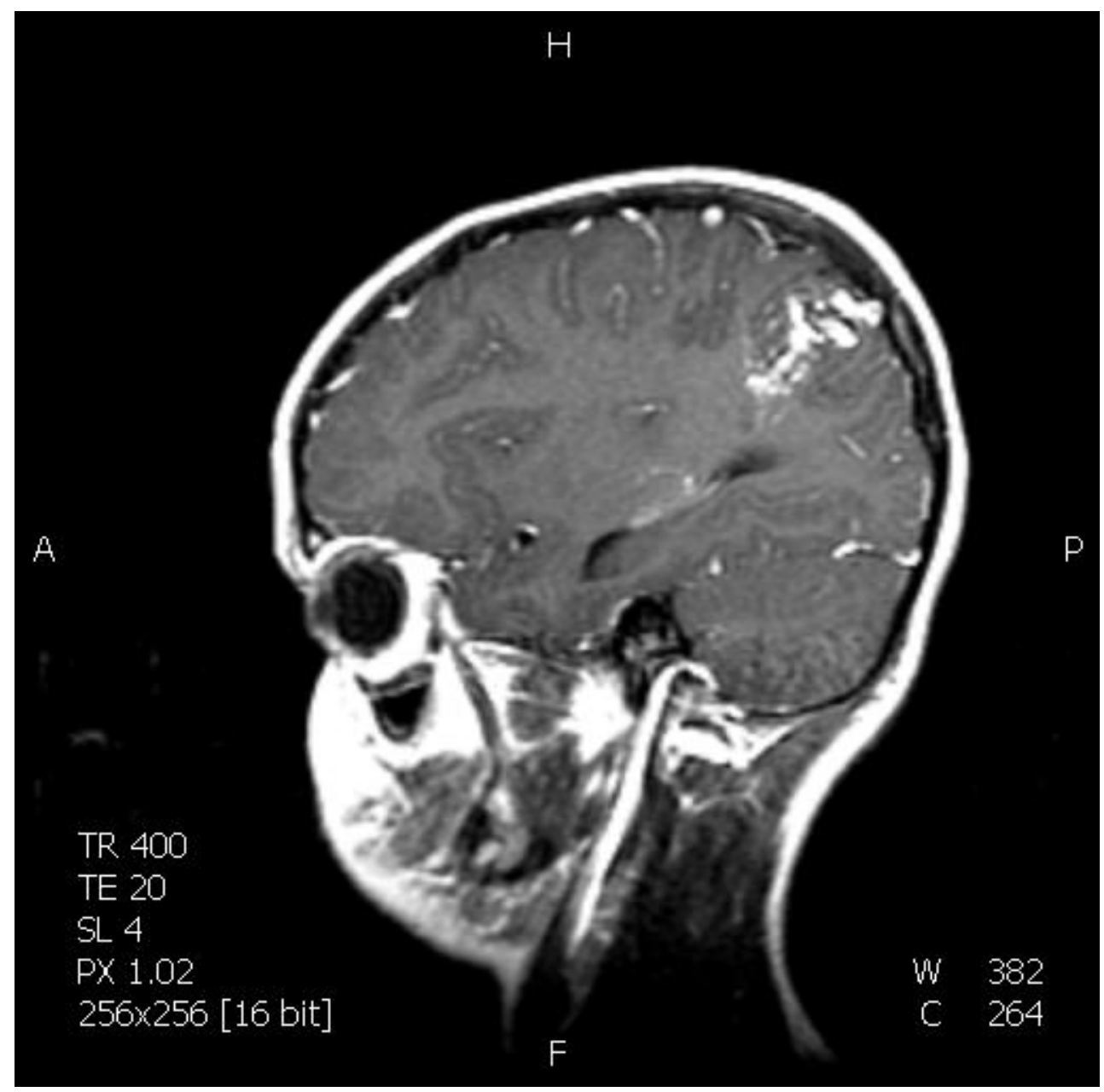

(b)

Fig. 12. $(a, b)$. Arteriovenous malformation (AVM):T1-weighted SE images post contrastenhancement features a tangle of blood vessels in the left parieto-occipital region. Some of them show enhancement and others show absence of blood flow. 


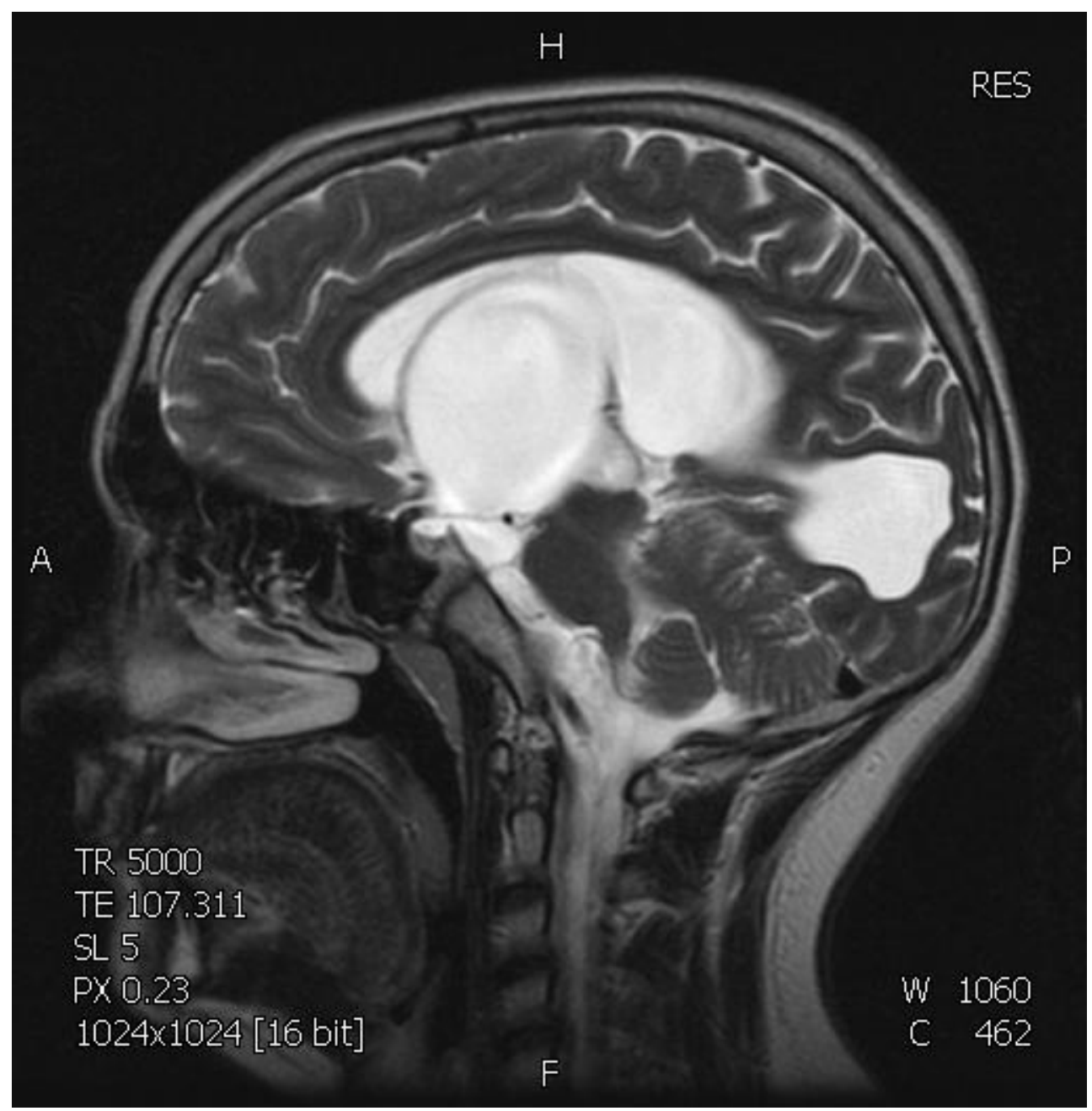

Fig. 13. Suprasellar arachnoid cyst: T2-weighted SE image shows a cystic suprasellar lesion which displaces the III ventricle and causes lateral ventricle hydrocephalus. 


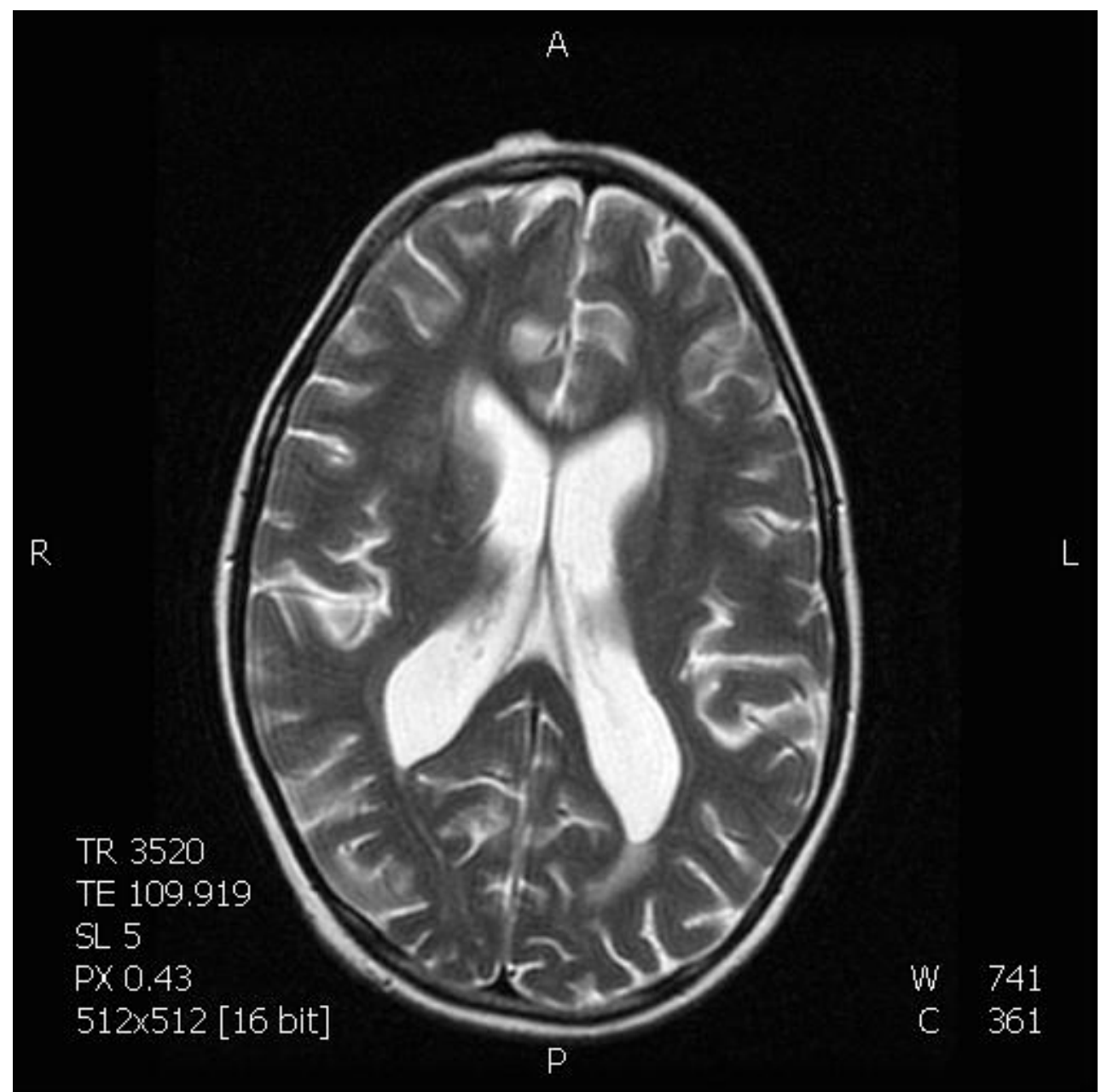

(a) 


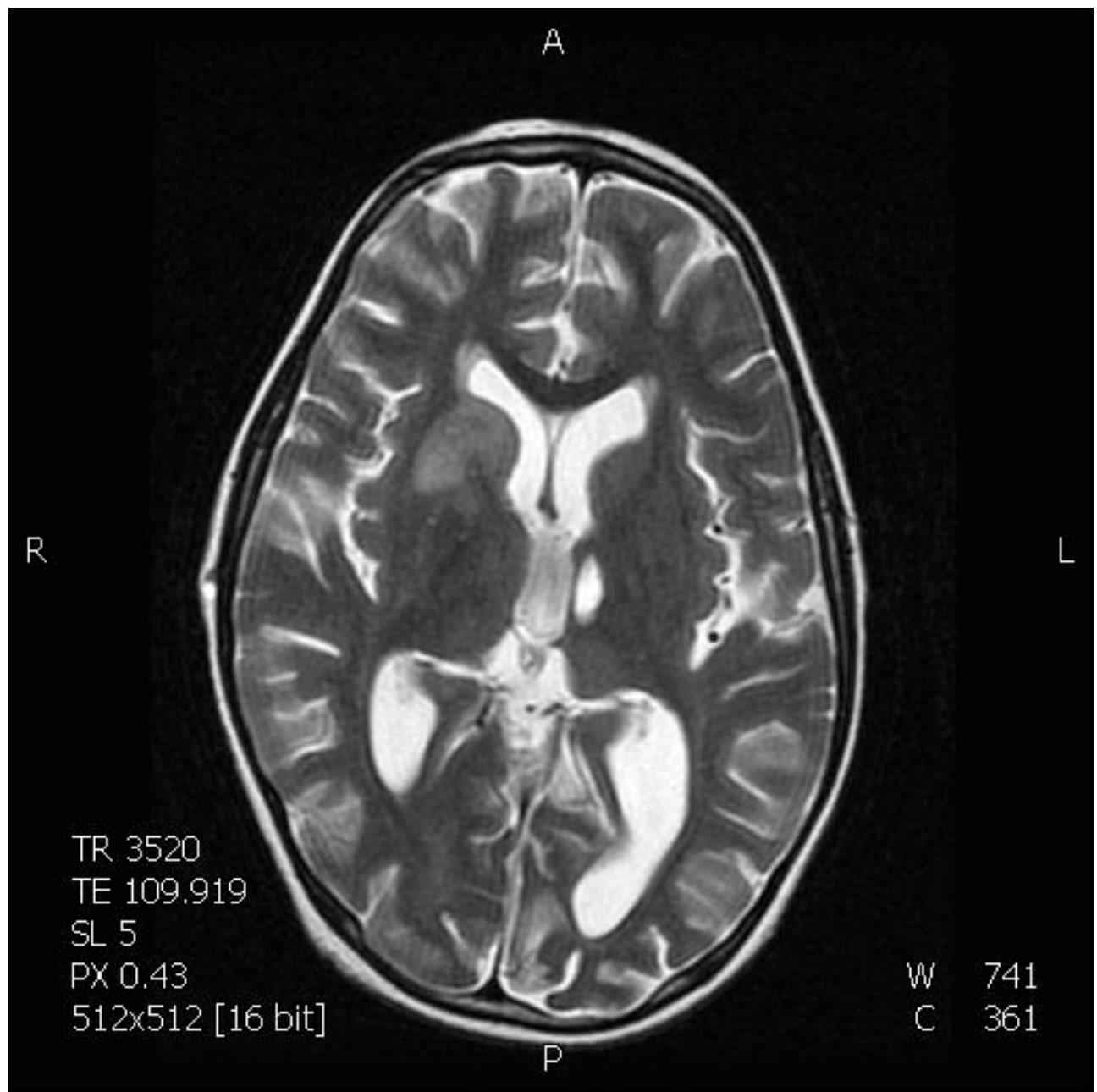

(b)

Fig. 14. $(a, b)$. Chemotherapy-induced toxicity in a boy diagnosed with acute lymphoblastic leukemia. T2-weighted SE images reveal cerebral involvement as patches with hyperintense areas in periventricular white matter, right occipital cortico-subcortical area and the right corpus striatum. In addition, there is a porencephalic lesion in the left thalamic region.

After going through the records of the 134 school-aged children, we found that 28 (20.9\%) had at least one MRI abnormality recorded. Twenty-one $(15.7 \%)$ presented MRI abnormalities that again were considered as significant because they were potentially related to the seizure condition.

Table 6 presents the number of school-age children with MRI abnormalities. From the 28 children with at least one MRI abnormality, the findings included white-matter lesions $(23.4 \%)$, gray-matter lesions $(23.4 \%)$, volume loss $(17 \%)$ and prominence of extra-axial fluid space $(12.8 \%)$ 


\begin{tabular}{|c|c|}
\hline Panayiotopoulos syndrome & 5 \\
\hline Benign epilepsy with centrotemporal spikes & 34 \\
\hline Late onset childhood occipital epilepsy (Gastaut type) & 4 \\
\hline Reflex epilepsy (photosensitive epilepsies) & 1 \\
\hline Childhood absence epilepsy & 25 \\
\hline Lennox-Gastaut syndrome & 1 \\
\hline Epilepsy with continuous spike-and-wave during sleep & 4 \\
\hline Landau-Kleffner syndrome & 1 \\
\hline Epilepsies of unknown cause (focal/generalized) & 39 \\
\hline Epilepsies associated with structural or metabolic conditions & 20 \\
\hline Perinatal insults & 3 \\
\hline Cerebral infections & \\
\hline Subdural empyema & 1 \\
\hline EBV encephalitis & 1 \\
\hline Malformations of cortical development & \\
\hline Subependymal heterotopia & 2 \\
\hline Band heterotopia (double cortex) & 1 \\
\hline Focal cortical dysplasia & 1 \\
\hline Polymicrogyria & 1 \\
\hline Others cerebral malformations & \\
\hline Chiary I malformations & 1 \\
\hline Tumors & \\
\hline Desmoplastic neuroepithelial tumors & 1 \\
\hline Neurocutaneous disorders & \\
\hline Tuberous sclerosis complex & 1 \\
\hline Stroke & \\
\hline Middle cerebral artery infarction & 3 \\
\hline Vascular malformations & \\
\hline Arteriovenous malformations & 1 \\
\hline Cavernous malformations & 1 \\
\hline Prominence of extra-axial fluid space & \\
\hline Suprasellar arachnoid cyst & 1 \\
\hline Drug toxicity & \\
\hline Leukoencephalopathy due to vinca alkaloids & 1 \\
\hline
\end{tabular}

Table 5. Distribution of the different epilepsies and epileptic syndromes in school-aged children $(\mathrm{n}=134)$ 


\begin{tabular}{|l|c|}
\hline MRI abnormality $(\mathrm{n}=47)$ & $\mathrm{n}(\%)$ \\
\hline Volume loss & $8(17 \%)$ \\
Generalized & 3 \\
Hemisphere & 3 \\
Corpus callosum & 2 \\
\hline White matter lesions & $11(23.4 \%)$ \\
Leukomalacia/gliosis & 9 \\
Encephalomalacia & 1 \\
Other lesions & 1 \\
\hline Gray-matter lesions & $11(23.4 \%)$ \\
Heterotopias & 3 \\
Cortical dysplasias & 2 \\
Other lesions & 6 \\
\hline Mass lesion & $1(2.1 \%)$ \\
\hline Vascular lesion & $2(4.3 \%)$ \\
Hemorrhage & 2 \\
\hline Ventricular enlargement & $2(4.3 \%)$ \\
<1.5cm & 1 \\
$>1.5 c m$ & 1 \\
\hline Prominence of extra-axial fluid space & $6(12.8 \%)$ \\
$<1.0 \mathrm{~cm}$ & 5 \\
$>$ 1.0cm & 1 \\
\hline Other structural abnormalities & 2 \\
Calcifications & 1 \\
Lobe asymmetry & 2 \\
Cerebellar tonsils descent & 1 \\
Arteriovenous malformations & $14.9 \%)$ \\
Pineal cyst & 2 \\
\hline
\end{tabular}

Boldface rows represented "significant abnormalities"

Table 6. MRI abnormalities found in school-aged childhood diagnosed with epilepsy

\subsection{Adolescents}

The sample was consisted of 88 patients (39 males and 49 females). In this group of patients, seizures were idiopathic in $69.3 \%$, cryptogenic in $19.3 \%$ and symptomatic in $11.4 \%$ of them. Table 7 shows the distribution of the epilepsies and epileptic syndromes in adolescents. Idiopathic generalized epilepsies with variable phenotypes (46.6\%) and cryptogenic focal epilepsies and/or unknown cause $(19.3 \%)$ were the most prevalent syndromes.

Epilepsies associated to structural or metabolic conditions $(n=9)$ were secondary to perinatal asphyxia, malformations of the brain, strokes (figure 15), vascular malformations, chromosomal abnormalities and ependimal cyst (figure 16). 


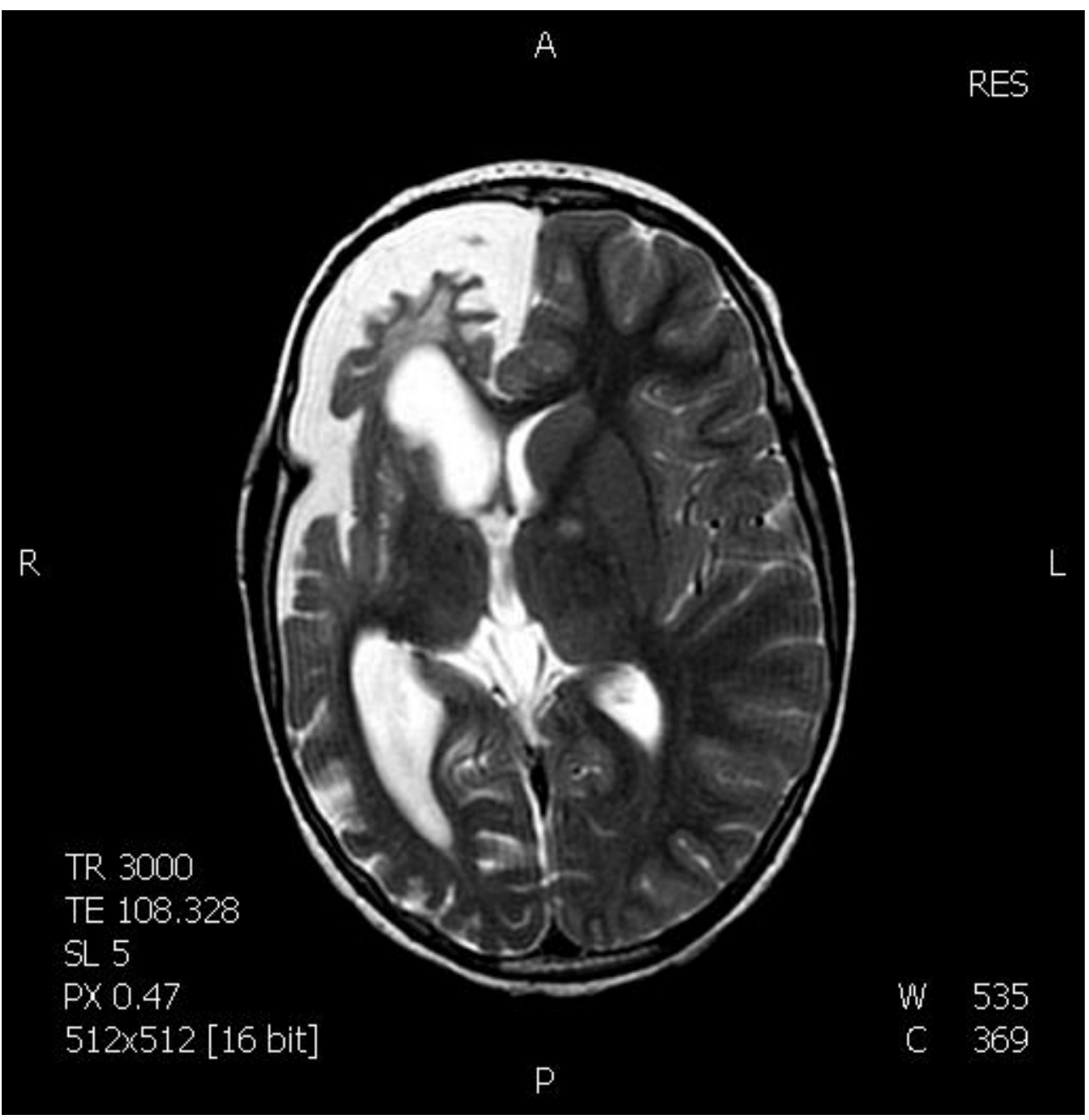

Fig. 15. Right cerebral hemisphere atrophy after ischemic lesion: T2-weighted SE image presents a volume loss in the right hemisphere, atrophy of cortical gyri, leukomalacia and ex vacuo enlargement of lateral ventricle. A hyperintense nodule, compatible with spongiotic intramyelinic change (neurofibromatosis), can be appreciated in the left globus pallidus. 


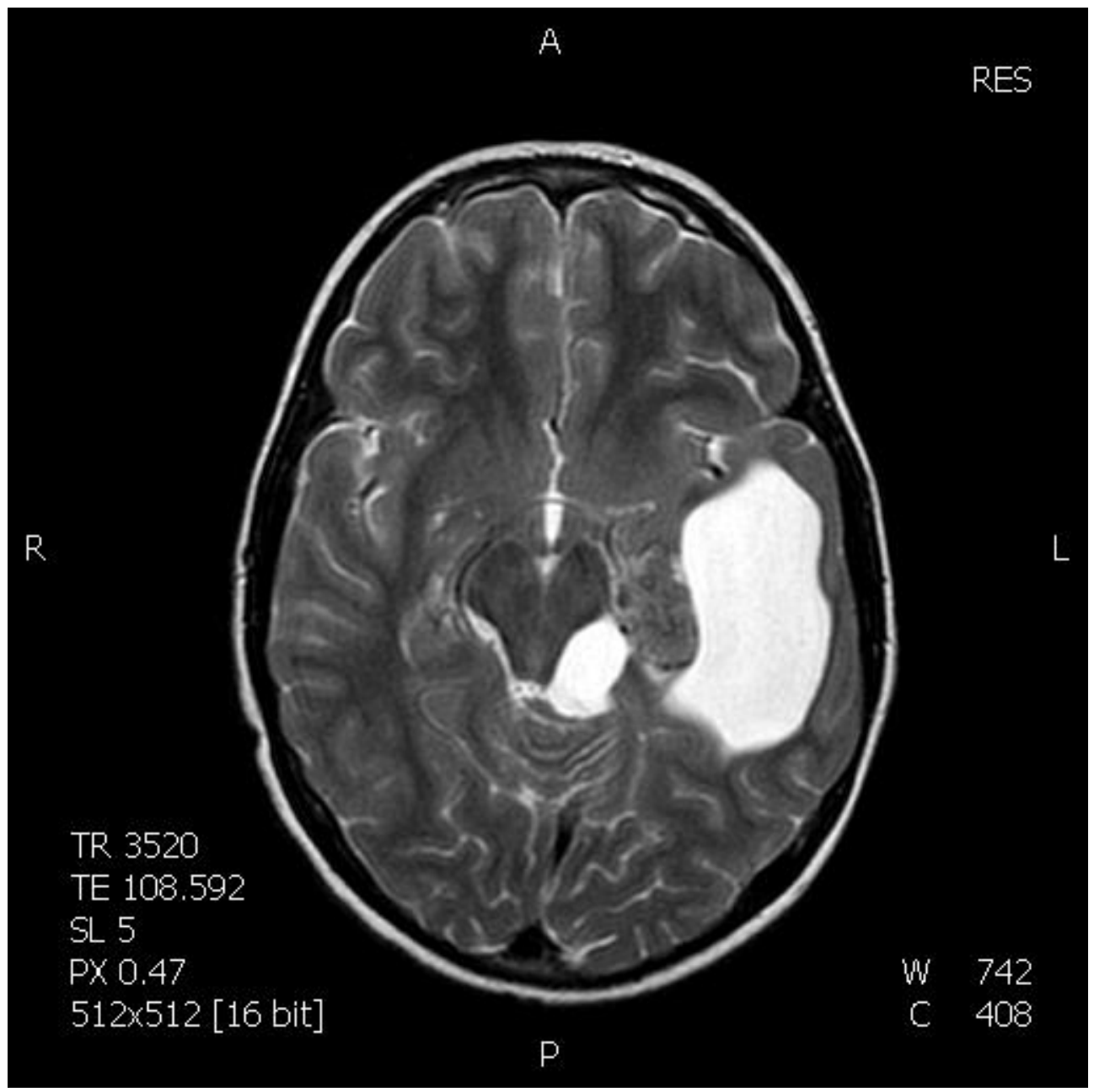

Fig. 16. Ependymal cyst: T2-weighted SE image shows a cystic lesion that expands the left occipital horn and a small brain herniation inside the interpeduncular cistern. 


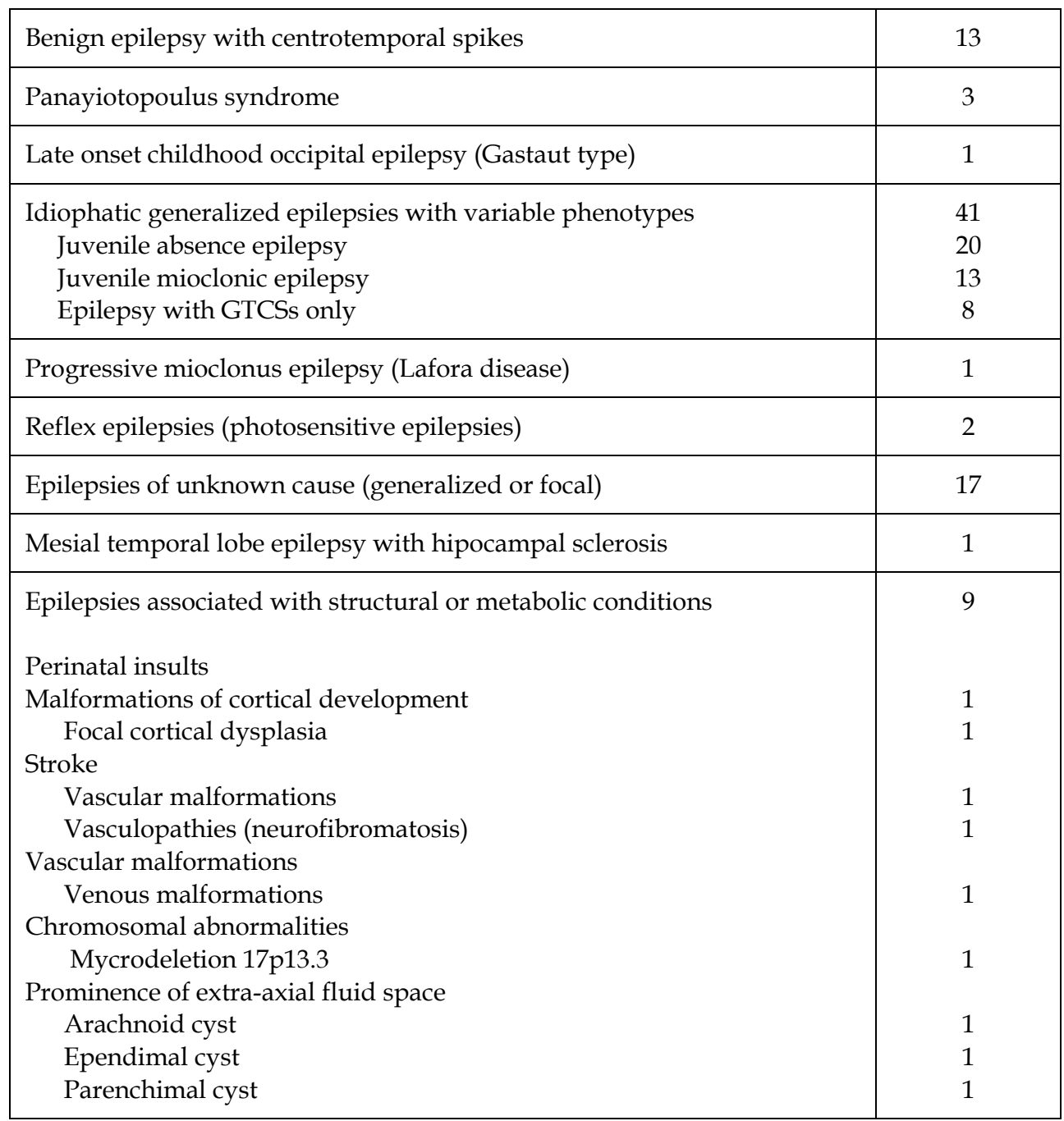

Table 7. Distribution of the different epilepsies and epileptic syndromes in adolescents $(\mathrm{n}=88)$

Of 88 infants in this study, $19(21.6 \%)$ revealed at least one MRI abnormality. Fourteen (15.9\%) manifested significant MRI abnormalities (they might to related to the seizure condition).

Table 8 presents the number of adolescents with MRI abnormalities (the sum of abnormalities may be higher than the total number of children). In 19 studies with at least one MRI abnormality, the most common abnormalities included white-matter lesions $(28.1 \%)$, volume loss $(15.6 \%)$, gray-matter lesions $(12.5 \%)$ and prominence of extra-axial fluid space $(12.5 \%)$. 


\begin{tabular}{|l|c|}
\hline MRI abnormality $(\mathrm{n}=32)$ & $\mathrm{n}(\%)$ \\
\hline Volume loss & $5(15.6 \%)$ \\
Generalized & 1 \\
Hemisphere & 2 \\
Lobe & 1 \\
Cerebelar & 1 \\
\hline White matter lesions & $9(28.1 \%)$ \\
Leukomalacia/gliosis & 7 \\
Encephalomalacia & 2 \\
\hline Gray-matter lesions & $4(12.5 \%)$ \\
Cortical dysplasias & 1 \\
Other lesions & 3 \\
\hline Vascular lesion & $1(3.1 \%)$ \\
Hemorrhage & 1 \\
\hline Ventricular enlargement & $3(9.4 \%)$ \\
$<1.5 \mathrm{~cm}$ & 2 \\
$>1.5 \mathrm{~cm}$ & 1 \\
\hline Prominence of extra-axial fluid space & $4(12.5 \%)$ \\
$<1.0 \mathrm{~cm}$ & 3 \\
$>1.0 \mathrm{~cm}$ & 1 \\
\hline Other structural abnormalities & $6(18.8 \%)$ \\
Lobe asymmetry & 3 \\
Cerebellar tonsils descent & 1 \\
Absence of the posterior pituitary & 1 \\
Arteriovenous malformations & 1 \\
\hline
\end{tabular}

Boldface rows represented "significant abnormalities"

Table 8. MRI abnormalities found in adolescents diagnosed with epilepsy

\subsection{Whole sample}

The whole group was 457 patients (233 males and 224 females). Etiology was considered idiopathic in 225 (49.2\%), cryptogenic in 114 (24.9\%), and symptomatic in 118 (25.8\%). Table 9 shows the distribution of the epilepsies and epileptic syndromes in the overall sample.

There was at least one MRI abnormality in 134 patients $(29.3 \%)$. One hundred $(21.9 \%)$ had significant MRI abnormalities (potentially related to the seizure condition).

Table 10 presents the number of children with MRI imaging abnormalities. As we previously said, the sum of abnormalities may exceed the total number of children, since different abnormalities can affect the same locations in one given patient. Of 134 children with at least one MRI abnormality, the most common abnormalities included white-matter lesions $(27.6 \%)$, volume loss $(19.6 \%)$, gray-matter lesions $(19.6 \%)$ and ventricular enlargement $(12 \%)$. Table 11 shows the distribution of MRI abnormalities found according to age.

Neuroimaging studies achieved in two patients diagnosed with benign epilepsy with centrotemporal spikes, two patients with childhood absence epilepsy, one patient with juvenile myoclonic epilepsy, and other patient with juvenile absence epilepsy revealed MRI abnormalities classified as significant: leukomalacia periventricular (4 cases) or areas of gliosis (2 cases). 


\begin{tabular}{|c|c|}
\hline Infancy & \\
\hline West syndrome & 26 \\
\hline Myoclonic epilepsy in infancy & 9 \\
\hline Benign familial infantile epilepsy & 3 \\
\hline Benign non-familial infantile epilepsy & 1 \\
\hline Dravet syndrome & 10 \\
\hline \multicolumn{2}{|l|}{ Childhood } \\
\hline Febrile seizures plus & 1 \\
\hline Panayiotopoulos syndrome & 17 \\
\hline Epilepsy with myoclonic atonic seizures & 10 \\
\hline Benign epilepsy with centrotemporal spikes & 65 \\
\hline Autosomal-dominant nocturnal frontal lobe epilepsy & 1 \\
\hline Late onset childhood occipital epilepsy (Gastaut type) & 6 \\
\hline Epilepsy with myoclonic absences & 1 \\
\hline Lennox-Gastaut syndrome & 2 \\
\hline Epilepsy with continuous spike-and-wave during sleep & 11 \\
\hline Landau-Kleffner syndrome & 2 \\
\hline Childhood absence epilepsy & 38 \\
\hline \multicolumn{2}{|l|}{ Adolescence } \\
\hline Juvenile absence epilepsy & 20 \\
\hline Juvenile mioclonic epilepsy & 13 \\
\hline Epilepsy with generalized tonic-clonic seizures alone & 8 \\
\hline Progressive mioclonus epilepsy (Lafora disease) & 1 \\
\hline \multicolumn{2}{|l|}{ Less specific age relationship } \\
\hline Reflex epilepsies & 4 \\
\hline \multicolumn{2}{|l|}{ Distinctive constellation } \\
\hline Mesial temporal lobe epilepsy with hipocampal sclerosis & 1 \\
\hline $\begin{array}{l}\text { Epilepsies that the nature of the underlying cause is yet unknown (generalized } \\
\text { or focal) }\end{array}$ & 109 \\
\hline \multicolumn{2}{|l|}{ Epilepsies associated with structural or metabolic conditions } \\
\hline Perinatal insults & 25 \\
\hline Cerebral infections & 7 \\
\hline Cerebral malformations & 22 \\
\hline Inherited metabolic disorders & 7 \\
\hline Neurocutaneous disorders & 7 \\
\hline Tumors & 2 \\
\hline Stroke & 7 \\
\hline Vascular malformations & 4 \\
\hline Vascular lesions & 1 \\
\hline Chromosomal abnormalities & 8 \\
\hline Prominence of extra-axial fluid space & 4 \\
\hline Drug toxicity & 1 \\
\hline \multicolumn{2}{|l|}{ Condition with epileptic seizures that do not require a diagnosis of epilepsy } \\
\hline Infantile convulsions with mild gastroenteritis & 3 \\
\hline
\end{tabular}

Table 9. Distribution of the different epilepsies and epileptic syndromes arranged by age at onset $(n=157)$ 


\begin{tabular}{|c|c|}
\hline MRI abnormality $(\mathrm{n}=275)$ & $\mathrm{n}(\%)$ \\
\hline Volume loss & $54(19.6 \%)$ \\
\hline Generalized & 15 \\
\hline Hemisphere & 7 \\
\hline Lobe & 8 \\
\hline Cerebelar & 5 \\
\hline Corpus callosum & 19 \\
\hline White matter lesions & $76(27.6 \%)$ \\
\hline Leukomalacia/gliosis & 44 \\
\hline Encephalomalacia & 13 \\
\hline Other lesions & 19 \\
\hline Gray-matter lesions & $54(19.6 \%)$ \\
\hline Heterotopias & 5 \\
\hline Cortical dysplasias & 16 \\
\hline Other lesions & 33 \\
\hline Vascular lesion & $8(2.9 \%)$ \\
\hline Hemorrhage & 7 \\
\hline Venous thrombosis & 1 \\
\hline Ventricular enlargement & $33(12 \%)$ \\
\hline$<1.5 \mathrm{~cm}$ & 18 \\
\hline$>1.5 \mathrm{~cm}$ & 15 \\
\hline Prominence of extra-axial fluid space & $12(4.4 \%)$ \\
\hline$<1.0 \mathrm{~cm}$ & 10 \\
\hline$>1.0 \mathrm{~cm}$ & 2 \\
\hline Mass lesion & $2(0.7 \%)$ \\
\hline Other structural abnormalities & $36(13.1 \%)$ \\
\hline Hemisphere asymmetry & 2 \\
\hline Lobe asymmetry & 4 \\
\hline Cerebellar tonsils descent & 3 \\
\hline Choroid plexus cyst & 1 \\
\hline Pineal cyst & 2 \\
\hline Mega cisterna magna & 1 \\
\hline Arteriovenous malformations & 4 \\
\hline Delayed myelination & 8 \\
\hline Agenesis of the corpus callosum & 4 \\
\hline Absence of the posterior pituitary & 1 \\
\hline Calcifications & 5 \\
\hline Enlarged perivascular spaces in the corpus callosum & 1 \\
\hline
\end{tabular}

Boldface rows represented "significant abnormalities"

Table 10. MRI abnormalities found in children diagnosed with epilepsy 


\begin{tabular}{|c||c|c|}
\hline \multicolumn{1}{|c||}{} & \multicolumn{2}{c|}{ Type of abnormalities } \\
\cline { 2 - 3 } Age group & $\begin{array}{c}\text { Significant } \\
\mathrm{n}(\%)\end{array}$ & $\begin{array}{c}\text { Overall } \\
\mathrm{n}(\%)\end{array}$ \\
\hline \hline Infants & $33(42,3 \%)$ & $42(53,8 \%)$ \\
\hline Early infants & $32(20,4 \%)$ & $45(28,7 \%)$ \\
\hline School-aged childhood & $21(15,7 \%)$ & $28(20,9 \%)$ \\
\hline Adolescents & $14(15,9 \%)$ & $19(21,6 \%)$ \\
\hline Total & $100(21,4 \%)$ & $134(29,6 \%)$ \\
\hline
\end{tabular}

Table 11. Distribution of MRI abnormalities found according to age

\section{Discussion}

The community of Navarra has a population of 605876 inhabitants (2008 census, National Institute of Statistics), 88055 of whom are children ( $<15$ years of age; 45105 boys [51.2\%] and 42950 girls [48.5\%]). Although there are no data about the prevalence of infantile epilepsy in Spain, the extrapolation of published data7,24-26 allows estimating that the selected population would account for a high percentage of epilepsy diagnosed children in Navarra. That means, it is a wide enough population to consider as representative for this territory, and, also, the results would contribute to determining the epidemiological characteristics of epilepsy in children. A routine RMI has been requested in all patients with epilepsy as it has been our practice for several years in this Pediatric Neurology Unit in order to aid the formulation of syndromic and etiological classification and, therefore, there is no reason to suspect any statistical bias in the results and conclusions.

According to the data, we confirm that age seems to represent a main factor in the clinical expression of the different childhood epilepsy phenotypes. In fact, relative distribution of the different seizure types and epileptic syndromes was different for each age group, as seen in our series. For example, some epileptic syndromes were predominant in some age groups, such as epilepsies associated to structural or metabolic conditions and West syndrome in infants, epilepsies in which the nature of the underlying causes is yet unknown and epilepsies associated to structural or metabolic conditions in early childhood, or absence and focal benign epilepsies with centrotemporal spikes in school-aged children and idiopathic generalised epilepsies with variable phenotypes in adolescents. Thus, when referring to childhood epilepsy, it would be useful to make reference to the age group of the patients, because characteristics for the epilepsies and epileptic syndromes during childhood seem to be close to the structural and functional changes in the brain since birth to adolescence ${ }^{27-30}$.

Our major finding of this study was that the use of MRI at diagnosis of epilepsy in children demonstrated a high rate of abnormalities findings. There was an inverse relationship between the prevalence of significant MRI abnormalities and age at diagnosis. Thus, the prevalence in the first year of life was $42.3 \%$, in early childhood was $20.4 \%$, in schoolchildren was $15.7 \%$, and in adolescents was $15.9 \%$. White-matter lesions were more 
commonly associated with epilepsies and epileptic syndromes in children at any age, whereas many other children displayed epileptogenic lesions involving the cortex or gray matter. In addition, volume loss, considered a priori to be a non significant abnormality, was also very frequent in this population. Ventricular enlargement, especially mild, was also fairly frequent ( $12 \%$ of total sample).

Structural neuroimaging is recommended for all children with recently diagnosed localization-related or generalized epilepsy who do not have the clinical and electrographic features characteristic of classical idiopathic focal or generalized epilepsy (these include benign epilepsy with centrotemporal spikes, childhood absence epilepsy, juvenile absence epilepsy, and juvenile myoclonic epilepsy) ${ }^{16,31-33}$. However, this series shows how structural abnormalities judged to be etiologically related to the seizure conditions have been found in idiopathic focal or generalized epilepsy, which are consistent with other published data 23,34,35. This possibility suggests that clinicians should consider obtaining structural neuroimaging with an MRI when feasible for all patients with epilepsy, because MRI abnormalities may be present even in children with apparently benign syndromes or cryptogenic seizures, and the discovery of an epileptogenic lesion has potential implications for diagnosis, prognosis and treatment.

Our data cannot be easily compared with those in other studies because the populations are somewhat different23,31-33,36. Our study attempted to acquire MRI scans on all children, whereas previous studies excluded some syndromes or children with seizure onset in infancy and early childhood. Therefore, this series extends earlier work by other investigators in several ways. First, imaging was limited to MRI, the current anatomic "gold standard". Second, imaging was performed at diagnosis. Third, we applied a standardized classification system to MRI findings which permits to present a comprehensive and systematic description of imaging findings. Finally, data were coded directly from magnetic resonance images by two neuroradiologists who read the images firsthand.

In conclusion, the use of MRI and a reliable standardized scoring system in a large sample of children after their diagnosis of epilepsy identified a high rate of abnormalities (134 of 457, $29.3 \%$ ). This may have important implications for practice guidelines in this population. First, some findings that might have been regarded as incidental in the past (e.g., volume loss/ventricular enlargement, and others white-matter abnormalities) appear to be present at the onset of seizures and may therefore be clinically significant. Second, the detection of abnormalities in epileptic syndromes other than localization-related symptomatic/cryptogenic syndromes supports an argument for routine MRI at the onset of any seizure condition.

\section{References}

[1] Hauser WA, Annegers JF, Kurland LT. Incidence of epilepsy and unprovoked seizures in Rochester, Minnesota: 1935-1984. Epilepsia 1993; 34:453-468.

[2] Olafsson E, Hauser WA, Ludvigsson P, Gudmundsson G. Incidence of epilepsy in rural Iceland: a population-based study. Epilepsia 1996; 37:951-955. 
[3] Camfield CS, Camfield PR, Gordon K, Wirrell E, Dooley JM. Incidence of epilepsy in childhood and adolescence: a population-based study in Nova Scotia from 1997 to 1985. Epilepsia 1996; 37:19-23.

[4] Kurtz Z, Tookey P, Ross E. Epilepsy in young people: 23 year follow up of the British National Child Development Study. BMJ 1998; 316:339-342.

[5] Zarrelli MM, Beghi E, Rocca WA, Hauser WA. Incidence of epileptic syndromes in Rochester, Minnesota: 1980-1984. Epilepsia 1999; 40:1708-1714.

[6] Freitag CM, May TW, Pfäfflin M, et al. Incidence of epilepsies and epileptic syndromes in children and adolescents: a populationbased prospective study in Germany. Epilepsia 2001; 42: 979-985.

[7] Durá T, Yoldi ME, Gallinas F. Incidence of Epilepsies and Epileptic Syndromes Among Children in Navarre, Spain: 2002 Through 2005. J Child Neurol 2008; 23: 878-882

[8] Duchowny M, Harvey AS. Pediatric epilepsy syndromes: an udptate and critical review. Epilepsia 1996; 37(suppl 1):26-40.

[9] Berg AT, Testa FM, Levy SR, Shinnar S. Neuroimaging in children with newly diagnosed epilepsy: a community-based study. Pediatrics 2000; 106:527-532.

[10] Camfield P, Camfield C. Epileptic syndromes in childhood: clinical features, outcomes and treatment. Epilepsia 2002; 43(suppl 3):27-32.

[11] Panayiotopoulos CP. EEG and brain imaging. In: Panayiotopoulos CP, ed. A Clinical Guide to Epileptic Syndromes and Their Treatment. 2nd ed. London, UK. SpringerVerlag Publishing. 2007: 129-153.

[12] Commission on Classification and Terminology of the International League Against Epilepsy. Proposal for revised classification of epilepsies and epileptic syndromes. Epilepsia 1989; 30:389-399.

[13] Engel J. A proposed diagnostic scheme for people with epileptic seizures and with epilepsy: report of the ILAE Task Force on Classification and Terminology. Epilepsia 2001; 42:796-803.

[14] Engel J. Report of the ILAE Classification Core Group. Epilepsia 2006; 47:1558-1568.

[15] Berg AT, Berkovic SF, Brodie MJ, Buchhalter J, Cross JH, van Emde Boas W, Engel J, French J, Glauser TA, Mathern GW, Moshé SL, Nordli D, Plouin P, Scheffer IE. Revised terminology and concepts for organization of seizures and epilepsies: Report of the ILAE Commission on Classification and Terminology, 2005-2009. Epilepsia 2010; 51:676-685.

[16] Gaillard WD, Chiron C, Cross JH, Harvey AS, Kuzniecky R, Hertz-Pannier L, Vezina LG. Guidelines for imaging infants and children with recent-onset epilepsy. Epilepsia 2009; 50:2147-2153.

[17] Imaging in paediatric epilepsy. Guissard G, Damry N, Dan B, David P, Sékhara T, Ziereisen F, Christophe C. Arch Pediatr 2005; 12:337-346.

[18] Barkovich AJ. Techniques and methods in pediatric neuroimaging. In: Barkovich AJ, ed. Pediatric Neuroimaging. Fourth ed. Lippincott Williams \& Wilkins. Philadelphia, USA. 2005:1-16.

[19] ILAE Commission Report. The epidemiology of the epilepsies: future directions. Epilepsia 1997; 38:614-618.

[20] Grueneich R, Ris MD, Ball W, Kalinyak KA, Noll R, Vannatta K, Wells R. Relationship of structural magnetic resonance imaging, magnetic resonance perfusion, and other 
disease factors to neuropsychological outcome in sickle cell disease. J Pediatr Psychol 2004; 29:83-92.

[21] Chuang NA, Otsubo H, Chuang SH. Magnetic resonance imaging in pediatric epilepsy. Top Magn Reson Imag 2002; 13:39-60.

[22] Doescher JS, deGrauw TJ, Musick BS, Dunn DW, Kalnin AJ, Egelhoff JC, Byars AW, Mathews VP, Austin JK. (2006) Magnetic resonance imaging (MRI) and electroencephalographic (EEG) findings in a cohort of normal children with newly diagnosed seizures. J Child Neurol 2006; 21:491-495.

[23] Kalnin AJ, Fastenau PS, deGrauw TJ, Musick BS, Perkins SM, Johnson CS, Mathews VP, Egelhoff JC, Dunn DW, Austin JK. Magnetic resonance imaging findings in children with a first recognized seizure. Pediatr Neurol 2008; 39:404-414.

[24] Ochoa C, Palencia R. Study of the prevalence of epilepsy among schoolchildren in Valladolid, Spain. Epilepsia. 1991; 32: 791-797.

[25] Hauser WA. The prevalence and incidence of convulsive disorders in children. Epilepsia. 1994; 35(suppl 2):1-6.

[26] Larsson K, Eeg-Olofsson O. A population based study of epilepsy in children from a Swedish county. Eur J Paediatr Neurol 2006; 10:107-113.

[27] Hauser WA. Seizure disorders: the changes with age. Epilepsia 1992; 33(suppl 4):6-14.

[28] Sillanpää M. Long-term outcome of epilepsy. Epileptic Disord 2000; 2:70-88.

[29] Arzimanoglou A, Guerrini R, Aicardi J. Epilepsy: overview and definitions. In: Aicardi's Epilepsy in Children. 3rd ed. London: Lippincott Williams \& Wilkins; 2004:1-6.

[30] Durá T, Yoldi ME, Gallinas F. Epilepsy in children in Navarre, Spain: epileptic seizure types and epileptic syndromes. J Child Neurol 2007; 22: 823-828.

[31] King MA, Newton MR, Jackson GD, Fitt GJ, Mitchell LA, Silvapulle MJ, Berkovic SF. Epileptology of the first-seizure presentation: a clinical, electroencephalographic, and magnetic resonance imaging study of 300 consecutive patients. Lancet 1998; 52:1007-1011.

[32] Shinnar S, O'Dell C, Mitnick R, Berg AT, Moshe SL. Neuroimaging abnormalities in children with an apparent first unprovoked seizure. Epilepsy Res 2001; 43:261-269.

[33] Berg AT, Testa FM, Levy SR, Shinnar S. Neuroimaging in children with newly diagnosed epilepsy: a community-based study. Pediatrics 2000; 106:527-532.

[34] Gelisse P, Corda D, Raybaud C, Dravet C, Bureau M, Genton P. Abnormal neuroimaging in patients with benign epilepsy with centrotemporal spikes. Epilepsia 2003; 44:372-378.

[35] Labate A, Ventura P, Gambardella A, et al. MRI evidence of mesial temporal sclerosis in sporadic "benign" temporal lobe epilepsy. Neurology 2006; 66:562-565.

[36] Byars AW, deGrauw TJ, Johnson CS, Fastenau PS, Perkins SM, Egelhoff JC, Kalnin A, Dunn DW, Austin JK. The association of MRI findings and neuropsychological functioning after the first recognized seizure. Epilepsia 2007; 48:1067-1074. 


\title{
Robotic Arm and Imaging in Neurosurgical Stereotactic Interventions: Oblique Insular Electrodes Implanted in Patients with Epilepsy
}

\author{
Afif Afif \\ Department of Neurosurgery-A, Neurological Hospital, \\ Hospices Civils de Lyon; Department of Anatomy, \\ Inserm, University Lyon 1, Lyon
}

France

\section{Introduction}

The human insular lobe was initially described by the anatomist Reil JC (Reil, 1809). This lobe is the first in the cerebral cortex that develops and differentiates in the fetus (Streeter, 1912; Kodam, 1926; Afif et al., 2007). A recent study demonstrates that the formation of insular cortex begins by its inferior region that will later become the limen insula (Afif et al., 2007). This cortical development progresses in parallel with that of the middle cerebral artery (MCA) and its branches in the superior and posterosuperior axis (Afif et al., 2007). The insula consists of three short and two long gyri separated by the central insular sulcus, which defines the anterior and posterior insula (Varnavas \& Grand, 1999; Ture et al., 1999; Naidich et al., 2004; Afif \& Mertens, 2010). The opercularization is the result of important development of the surrounding insular cortex (frontal, parietal and temporal lobes). Progressive development over the entire surface of the insular cortex encloses the sylvian fissure.

Due to its deep anatomic position and dense surrounding vascular network, few studies involving direct electrical stimulation (ES) of the insular cortex during surgical procedures have been reported (Penfield \& Jasper, 1954; Penfield \& Faulk, 1955; Oppenheimer et al., 1992; Duffau et al. 2001, 2009).

Immunocytochemistry, functional imaging, direct ES, and lesion studies suggest an important role for the insular cortex. Such roles include language production and grammatical processing (Raichle, 1991; McCarthy et al., 1993; Wise et al., 1999; Price, 2000, 2001; Ackermann \& Riecker, 2004; Isnard et al., 2004; Riecker et al., 2005; Friederici et al., 2006; Kato et al., 2007; Afif et al., 2010a), pain modulation (Tarkka \& Treede, 1993; Frot \& Mauguiere, 1999; Ostrowsky et al., 2000; Peyron et al., 2004; Craig, 2004; Isnard et al., 2005; Brooks et al., 2005 ; Schreckenberger et al., 2005; Mazzola et al., 2006; Henderson et al., 2006, 2007; Afif et al., 2008b), visceral sensory processing (Ostrowsky et al., 2000; Dupont et al., 2003; Isnard et al., 2004), bradycardia (Seeck et al., 2003), dysphagia (Danieles \& Foundas, 1997) and auditory processing (Manes et al., 1999; Lewis et al., 2000; Shergill et al., 2000; Bamiou et al., 2003; Afif et al., 2010b). 
Until recently the insular cortex has not been investigated using depth electrodes because of its anatomical location in the depth of the sylvian fissure and its close anatomical relationship with segments of the middle cerebral artery constituting a "vascular screen" at the surface of the insula (Ture et al., 1999, 2000; Varnavas \& Grand, 1999; Afif \& Mertens, 2010).

Recently, however, Stereoelectroencephalographic (SEEG) recordings on epileptic patients with electrodes stereotactically implanted deep in the brain using either the lateral transopercula approach (Isnard et al., 2000; Isnard et al., 2004; Isnard and Mauguiere, 2005; Ryvlin et al., 2006) or the oblique approach guided by robotic arm (Afif et al., 2008a), have implicated the insular cortex in seizure generation and initial propagation.

These observations emphasize the need to explore this anatomical structure further during invasive pre-surgical evaluation of epileptic patients. Functional studies of the insula should be treated in terms of its gyral and sulcal anatomy. The oblique approach (trans-frontal or trans-parietal) implantation of the electrodes guided by a robotic arm can explore the different insular regions (sulci and gyri) by avoidance of the sylvian vascular network which prevents access to these regions by the lateral approach.

\section{Patient selection criteria}

The non-invasive pre-surgical evaluation included in all cases high resolution magnetic resonance imaging (MRI) (with coronal T1-weighted images perpendicular to the hippocampal axis and T2-weighted images parallel to the hippocampal plane), neuropsychological tests, and prolonged electroencephalography (EEG) video monitoring. MRI scans showed unilateral hippocampal sclerosis in 17 patients $(56.6 \%)$ cortical dysplasia in five patients $(16.7 \%)$, and a cavernoma in two patients $(6.7 \%)$. Six patients $(20 \%)$ were considered cryptogenic (Table 1). Two patients harbored a dysplastic tissue within the insular cortex. The mean duration of epilepsy was $19.4 \pm 11$ years.

Stereoelectroencephalographic SEEG recordings were judged necessary, in all centers using this technique, when non-invasively obtained data were insufficiently concordant, discordant, inconclusive, and/or suggested an early involvement of highly eloquent areas. SEEG exploration within these patients included deep and superficial structures, including the amygdala, hippocampus, cingulate gyrus, SI, SII areas and temporal cortex, with a total of 12 to 16 electrodes per patient by implanting electrodes using the lateral orthogonal approach (mean 14 electrodes / patient).

Electrode implantation into the insular lobe was justified on clinical grounds in each case, either because patients experienced gustatory hallucinations, laryngeal discomfort or throat tightening, paresthesiae or tonic-clonic movements of the face, unpleasant paresthesiae affecting large somatic territories and hyper salivation (Isnard et al., 2000; Isnard et al., 2004), or because scalp video-EEG data suggested the onset of the first ictal electrical change in the perisylvian opercula area or an early spread to the suprasylvian opercular cortex and insula.

The exploration of the insular cortex was elaborated by deferent hospital centers according to the classical lateral trans-opercula (orthogonal) approach (Isnard et al., 2000; Isnard et al., 2004; Isnard \& Mauguiere, 2005). An original oblique approach (trans-frontal or transparietal) was elaborated by the center of university hospital of Grenoble (France) to explore the insula (Afif et al., 2008a, 2008b, 2010b). 


\begin{tabular}{|c|c|c|c|c|c|c|}
\hline P. & $\begin{array}{c}\text { Age } \\
\text { (y) }\end{array}$ & $\begin{array}{l}\text { Lat. } \\
\text { R/L }\end{array}$ & Sexe & Past history & $\begin{array}{l}\text { Age at } 1^{\text {st }} \\
\text { Seizure }\end{array}$ & MRI \\
\hline 1 & 15 & R. & $\mathrm{f}$ & & $6 \mathrm{y}$ & HcS \\
\hline 2 & 28 & R. & $\mathrm{m}$ & H.T. & $20 y$ & Normal \\
\hline 3 & 19 & R. & $\mathrm{f}$ & $\mathrm{FC}(6 \mathrm{mo})$ & $6 \mathrm{mo}$ & HcS \\
\hline 4 & 35 & R. & $\mathrm{f}$ & $\mathrm{FC}(1 \mathrm{y})$ & $12 \mathrm{y}$ & HcS \\
\hline 5 & 37 & R. & $\mathrm{m}$ & H.T. (18 y) & $23 \mathrm{y}$ & T. dysplasia \\
\hline 6 & 11 & L. & $\mathrm{m}$ & & $1 \mathrm{y}$ & TP. dysplasia. \\
\hline 7 & 13 & $\mathrm{~L}$. & $\mathrm{m}$ & & $6 \mathrm{y}$ & Normal \\
\hline 8 & 20 & R. & $\mathrm{f}$ & & $11 \mathrm{mo}$ & F. dysplasia \\
\hline 9 & 22 & R. & $\mathrm{f}$ & H.T. (4 y) & $14 \mathrm{y}$ & FP. dysplasia \\
\hline 10 & 34 & R. & $\mathrm{f}$ & & $3 y$ & Normal \\
\hline 11 & 38 & R. & $\mathrm{m}$ & H.T. $(14 \mathrm{y})$ & $14 \mathrm{y}$ & $\mathrm{HcS}$ \\
\hline 12 & 41 & R. & $\mathrm{f}$ & Meningitis (3 y) & $12 \mathrm{y}$ & HcS \\
\hline 13 & 18 & R. & $\mathrm{m}$ & & $15 \mathrm{y}$ & HcS \\
\hline 14 & 44 & L. & M & $\begin{array}{l}\text { Meningitis (2 y) } \\
+ \text { FC (3 y) }\end{array}$ & $6 y$ & HcS \\
\hline 15 & 42 & $\mathrm{R}$. & $\mathrm{m}$ & & $10 \mathrm{y}$ & HcS \\
\hline 16 & 41 & R. & $\mathrm{f}$ & & $13 \mathrm{y}$ & HcS \\
\hline 17 & 40 & R. & $\mathrm{m}$ & $\mathrm{FC}(10 \mathrm{mo})$ & $2 y$ & HcS \\
\hline 18 & 18 & R. & $\mathrm{f}$ & $\mathrm{FC}(2,5 \mathrm{y})$ & $9 y$ & $\mathrm{HcS}$ \\
\hline 19 & 42 & R. & $\mathrm{m}$ & & $14 \mathrm{y}$ & $\mathrm{HcS}$ \\
\hline 20 & 26 & R. & $\mathrm{f}$ & $\mathrm{FC}(18 \mathrm{mo})$ & $18 \mathrm{y}$ & HcS \\
\hline 21 & 52 & R. & $\mathrm{m}$ & H. T. $(20 y)$ & $20 \mathrm{y}$ & Amygdala Cavernoma \\
\hline 22 & 18 & R. & $\mathrm{m}$ & $\mathrm{FC}(16 \mathrm{mo})$ & $3 y$ & HcS, F. Cavernoma \\
\hline 23 & 27 & R. & $\mathrm{m}$ & H.T. $(9 \mathrm{mo})$ & $16 \mathrm{y}$ & HcS \\
\hline 24 & 26 & L. & $\mathrm{f}$ & FC (20 mo) & $10 \mathrm{y}$ & HcS \\
\hline 25 & 13 & $\mathrm{R}$. & $\mathrm{m}$ & $\begin{array}{c}\text { West syndrom } \\
(10 \mathrm{mo})\end{array}$ & $18 \mathrm{mo}$ & $\begin{array}{l}\text { F. }+ \text { insular cortical } \\
\text { dysplasia }\end{array}$ \\
\hline 26 & 9 & $\mathrm{R}$. & $\mathrm{m}$ & & $1 \mathrm{y}$ & $\begin{array}{l}\text { Orbito-insular cortical } \\
\text { dysplasia }\end{array}$ \\
\hline 27. & 37 & R. & $\mathrm{m}$ & $\begin{array}{c}\text { H.T. (3mo) +FC (9 } \\
\text { mo })\end{array}$ & $3 y$ & HcS \\
\hline 28 & 53 & R. & $\mathrm{m}$ & H.T. (5y) & $9 \mathrm{y}$ & Normal \\
\hline 29 & 14 & R. & $\mathrm{m}$ & & $5 \mathrm{y}$ & Normal \\
\hline 30 & 7 & $\mathrm{R}$. & $\mathrm{f}$ & & $2.5 \mathrm{y}$ & Normal \\
\hline
\end{tabular}

Table 1. Patient details P.: patient; MRI: MR imaging; y: year; mo: month; Lat.: hemispheric dominance for language; L.: left; R.: right; m: male; f: female; H.T.: head trauma; FC: febrile convulsion; HcS: hippocampal sclerosis.

\subsection{Stereotactic implantation of intra-cerebral electrodes}

Targeting of the insular cortex was performed using 3D presurgical T1- MRI images (Imaging parameters included 3D T1 gradient Echo 20/4.6/30 injected sequence, $1.5 \mathrm{~mm}$ thickness, gap: 0) computed with stereotactic software (VoximR, IVS solution, Chemnitz, Germany). The 
avascular characteristics of each trajectory were systematically evaluated by stereotactic and stereoscopic digital cerebral teleangiography (Pixray; Bioscan system, Geneva, Switzerland), as described elsewhere (Bancaud et al., 1967; Talairach et al., 1988; Kahane et al., 1993). Following coagulation of the dural entry point, insular electrodes were implanted through a $2 \mathrm{~mm}$ burr hole using either an anterior (transfrontal) approach (Fig. 1 A,C)

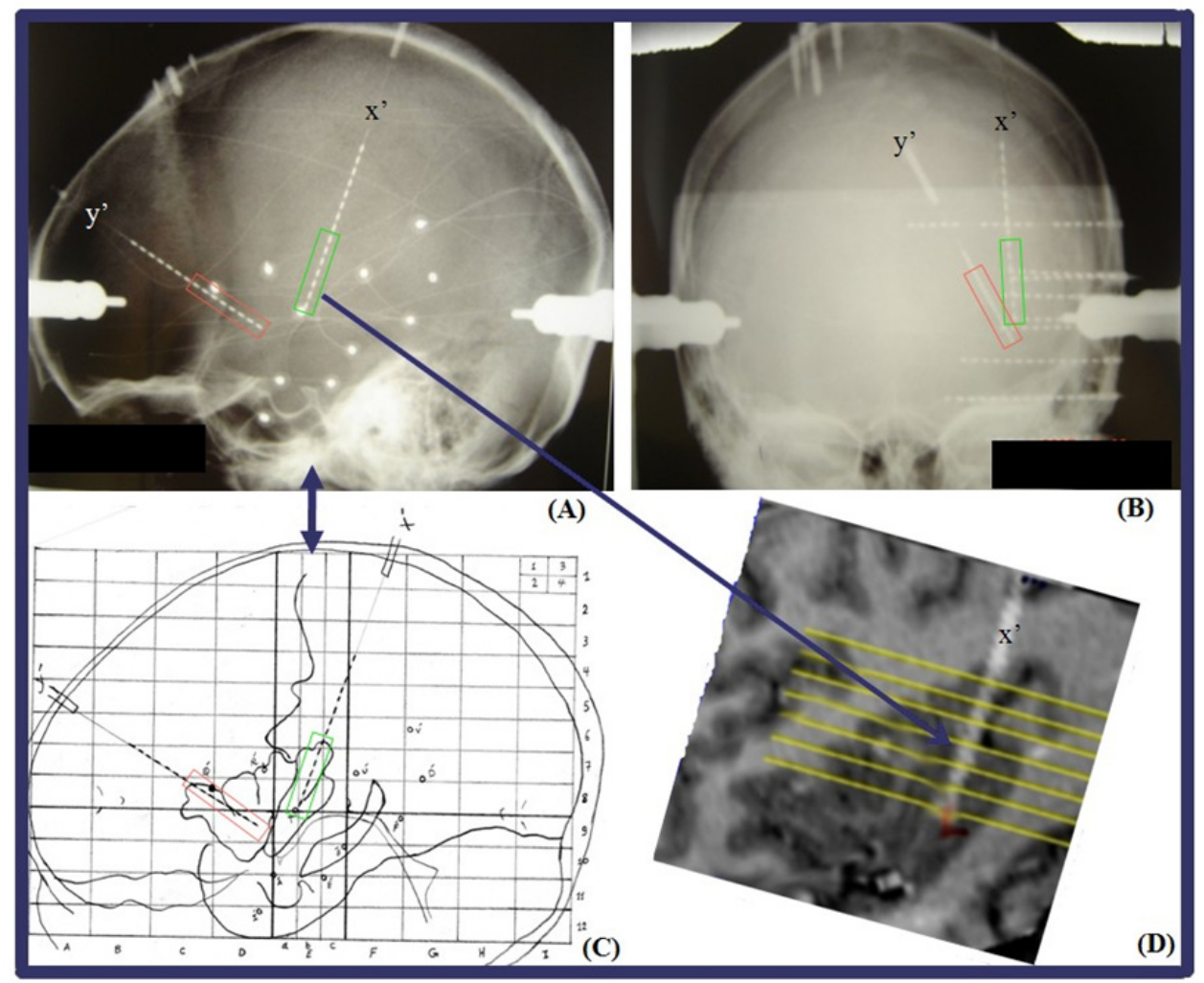

Fig. 1. Locating the insular cortex electrodes: Lateral (A) and frontal (B) X-rays of the patient's head after intracerebral electrode implantation. $\mathrm{y}^{\prime}$ : Anterior oblique electrode ("transfrontal") targeting the anterior and middle short gyrus of the insula; $x^{\prime}$ : Posterior oblique electrode ("transparietal") targeting the post-central gyrus of the insula; boxes indicate the electrode's contacts within the insular cortex. (C): corresponding sagittal patient diagram showing intrinsic anatomical insular structures and implanted electrodes (small letters). We constructed an individual diagram for each patient using different slices from pre-surgical T1-MRI images, in the AC-PC reference system. The box around electrode $y^{\prime}$ represents the contacts within both the anterior and middle short insular gyrus; the box around electrode $x^{\prime}$ represents the contacts within the post-central insular gyrus. (D): image fusion of the pre-operative 3D MRI and the post-operative contrast-enhanced 3D CT scan, using VOXIM stereotactic software. We could then identify the anatomical position of each electrode with reference to the cortical gyri and sulci. The sagittal slice is co-planar to electrode $x^{\prime}$; each perpendicular line on the electrode trajectory pass through one electrode contact. 
with an entry point in the middle frontal gyrus, or a posterior (transparietal) approach (Fig. 1 A, D), entering the brain at the level of the inferior parietal cortex, or both approaches. Similarly to other groups, the lateral orthogonal approach used for all deep brain electrodes (SEEG) stereotactically implanted outside the insular cortex. Electrode insertion was guided by a robotic arm (Neuromate, Renishaw mayfield, Nyon, Switzerland) connected to the stereotactic frame and driven by stereotactic planning software (VoximR, IVS solution, Germany).

In fact, the choice of the oblique approach to explore the insular cortex was decided because the orthogonal approach is less adapted to explore the insula due to several points (Afif et al., 2008b): I) The first one, that the insula lies almost parallel to the sagittal plane in the depth of sylvian fissure behind of the opercula which overlapping irregular folds of the lateral insular surface. II) The second one, that the thickness of the insular cortex is less than the distance covered by two contiguous contacts (up to $5 \mathrm{~mm}$ ), so in SEEG, only one contact of a laterally (trans-opercula) introduced electrode would lie within the insular cortex (Fig. 2). The second contact will be in the mesial part within the extreme capsula or in the lateral part within the opercula. III) The third one, that the dense network of vessels overlying the lateral surface of the insular cortex (Varnavas \& Grand, 1999; Ture et al., 1999; Afif \& Mertens, 2010) prevents the safely access to this region by a lateral orthogonal approach and only the peripherical region near the periinsular sulci can be reached safely.

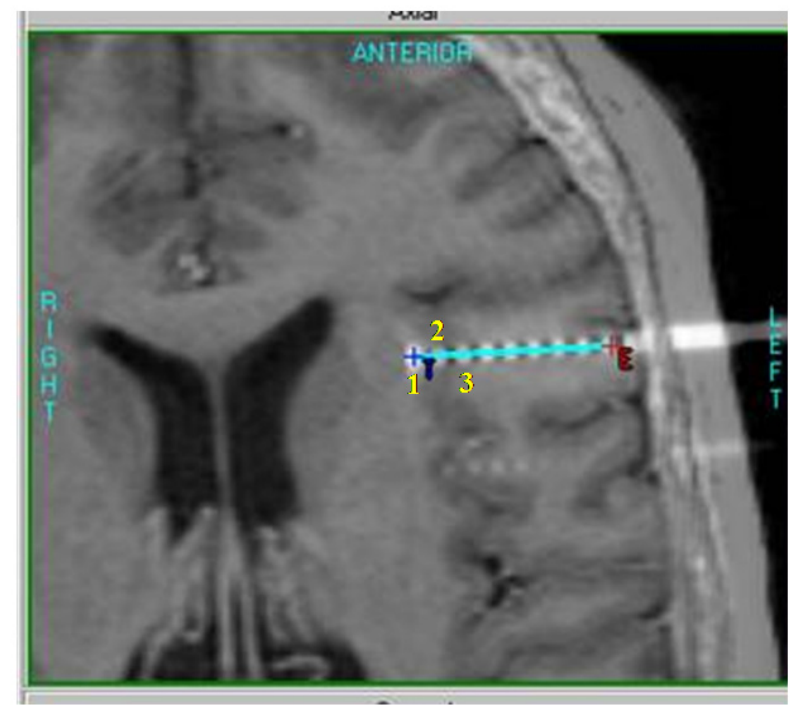

Fig. 2. Example of an orthogonal insular electrode (axial slice): Image fusion of the preoperative 3D MRI and the postoperative contrast-enhanced 3D CT scan of the axial slice using VOXIM stereotactic software. E: Orthogonal trans-opercula insular electrode in SEEG, one contact $\left(n^{\circ}: 1\right)$ in the mesial part within the extreme capsula, one contact $\left(n^{\circ}: 2\right)$ within the insular cortex and one contact $\left(\mathrm{n}^{\circ}: 3\right)$ in the lateral part within the frontal opercula.

The methodology targeted the insular cortex using an oblique approach, which allowed exploration of a larger surface of the insula by one electrode without passing through the 
opercula. Most patients were explored by means of at least one trans-frontal insular electrode, while the choice to use an additional trans-parietal electrode was reserved for patients showing a rapid posterior spread of the ictal electrical discharge on EEG (Afif et al., 2008a). Electrodes (DIXI Besançon, France) were $0.8 \mathrm{~mm}$ in diameter, with 10 to 18 contacts, of $2 \mathrm{~mm}$ in length and $1.5 \mathrm{~mm}$ apart, depending on the length of the electrode (Fig. 3). This allowed to sample different insular regions using only one electrode and to avoid passing through the opercula.
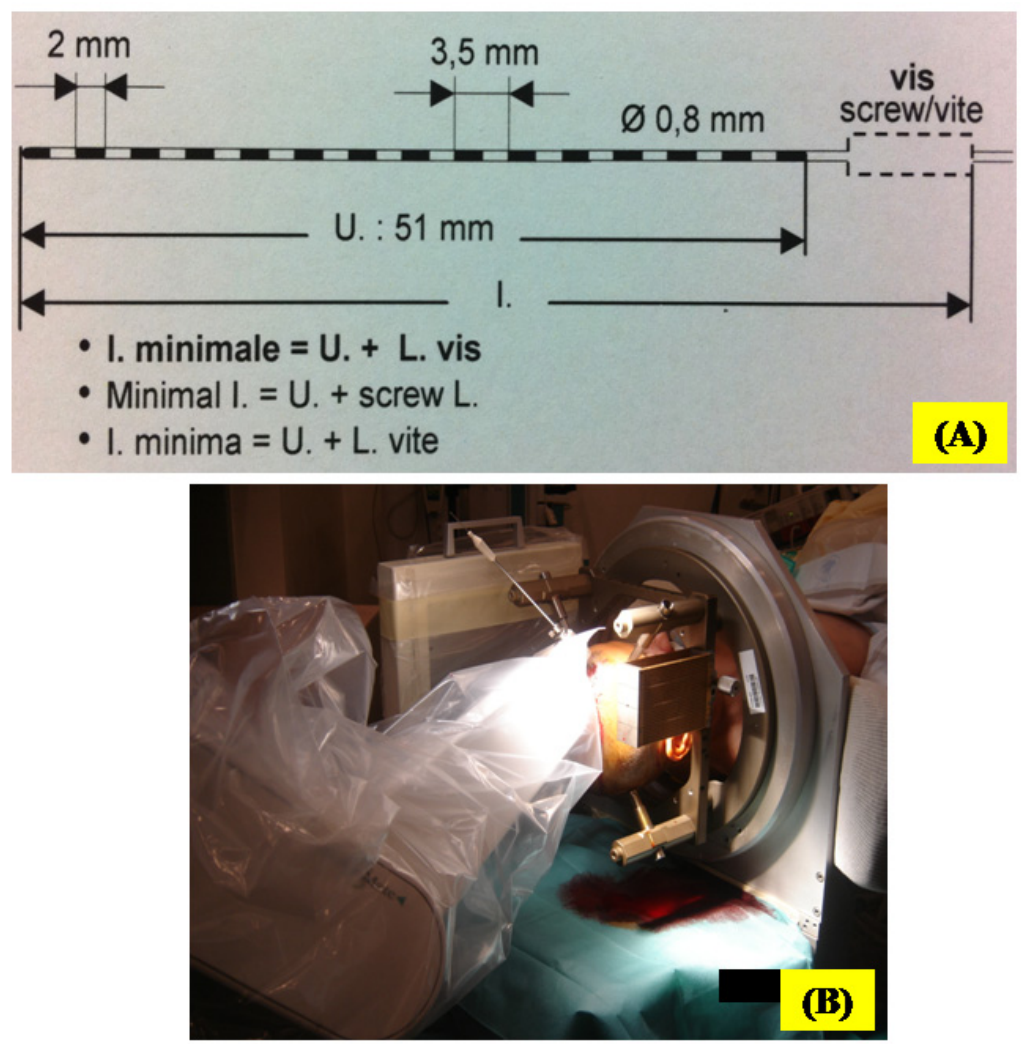

Fig. 3. (A) Drawing of an electrode with 15 contacts: U.: electrode length of 15 contacts $=51$ $\mathrm{mm}, \varnothing$ : diameter of the electrode $=0.8 \mathrm{~mm}$, length of each contact $=2 \mathrm{~mm}$, distance between the two contacts $=1.5 \mathrm{~mm}$. (B) Robotic arm: in the position of an anterior (trans opercular) oblique insular electrode trajectory.

\subsection{Determining the anatomical location of the insular contacts}

Due to the complex anatomy of the insula (its located in the depth of the sylvian fissure and the arteriovenous dense vascular network on the lateral surface of the insula) (Fig. 4) it was necessary to identify with high accuracy the localization of electrical contacts. Two anatomical and one electrophysiological procedure were utilized to accurately establish the location of all contacts clearly located in the insular cortex. 


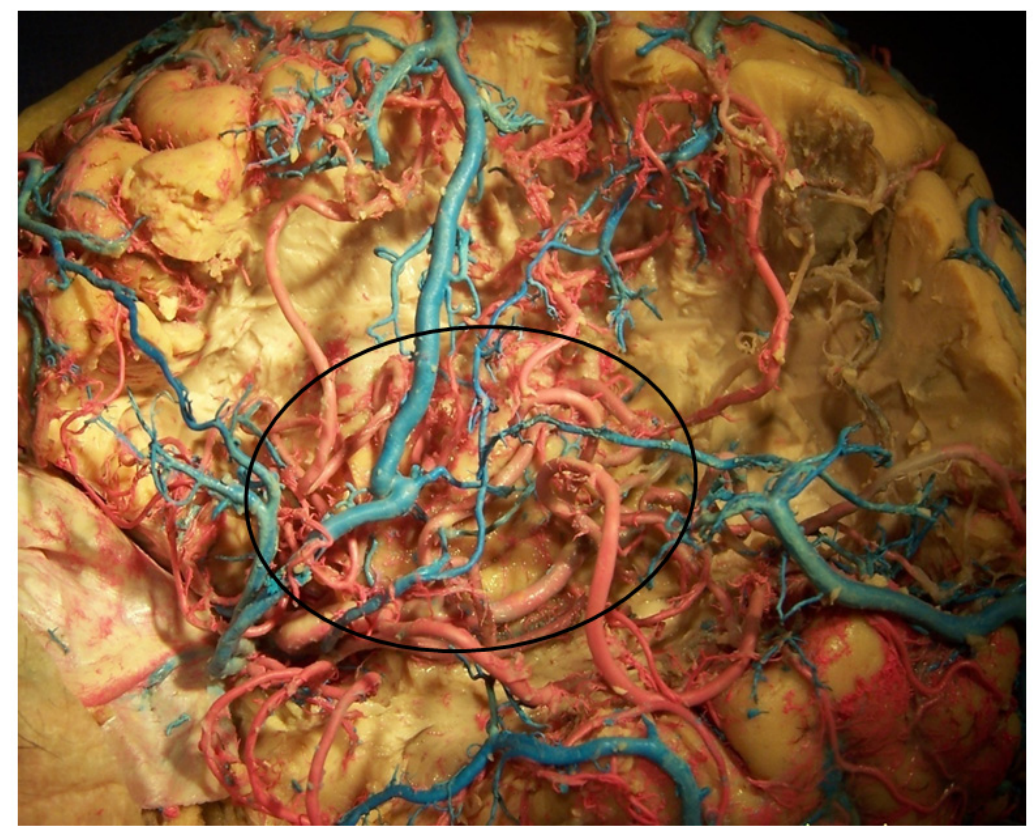

Fig. 4. View of the left insula, showing the MCA collaterals (insular and opercular arteries) and the sylvian venous system on the lateral surface of the insula (in the interior of the cycle) and the supra-sylvian region.

\subsubsection{Anatomy-based procedures}

\section{- Individual insular diagrams:}

This diagram was constructed for each patient using different sagittal slices of presurgical T1 MRI images referenced to the bicommissural plane (AC-PC) reference system (Fig. $1 \mathrm{C}$ ). The electrode contacts localization was identified in reference to insular gyri and sulci in the sagittal diagram (Afif et al., 2008b, 2010b).

- Individual 3D neuro-imaging:

To increase accuracy, a postoperative contrast-enhanced 3D CT scan was performed. All stimulation sites were anatomically located via image fusion between pre-implantation 3D MRI and post-implantation 3D CT scans (Afif et al., 2008b, 2010b). So, the cortical location and anatomical position of each contact were identified in reference to insular gyri in the three dimensions (sagittal, axial and coronal) (Fig. 5). No calculation was needed to reference the anatomical position of the electrical contacts to a standardized system since 3D anatomical localization was obtained by distortion free 3D CT performed immediately after electrode implantation.

In order to represent each stimulation response in a homogeneous manner, a template of the insula, including its sulci and gyri was used. The stimulation sites were located on each patient's individual diagram, before locating it on the insular template with respect to the insular gyri from the individual diagram (Fig. 6). 


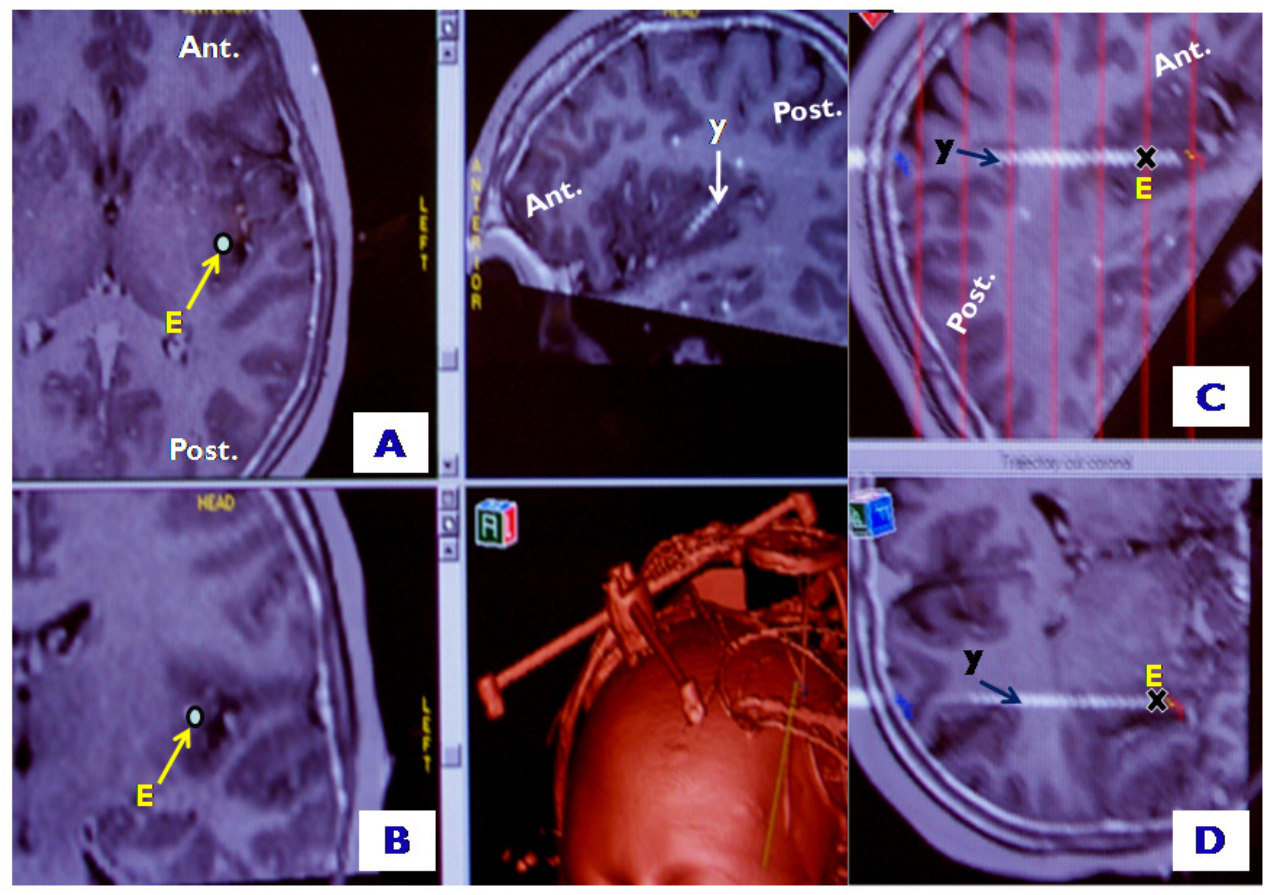

Fig. 5. Example of an oblique insular electrode. Image fusion of preoperative 3D MRI and post-operative contrast-enhanced 3D CT scans using VOXIM stereotactic software. This technique enabled us to identify the contact locations in three dimensions (sagittal, axial and coronal). Axial (A) and frontal (B) section crossing the trajectory of electrode y at the level of the contact $E$ in figures (C) and (D). Lateral (C) and frontal (D) reconstruction of an oblique electrode $(y)$ inserted into the posterior insula using a trans-parietal trajectory. This example clearly demonstrates how oblique implantation enables the exploration of a large part of the posterior insular cortex. E: one contact in the oblique electrode; Ant.: anterior; Post.: posterior. 


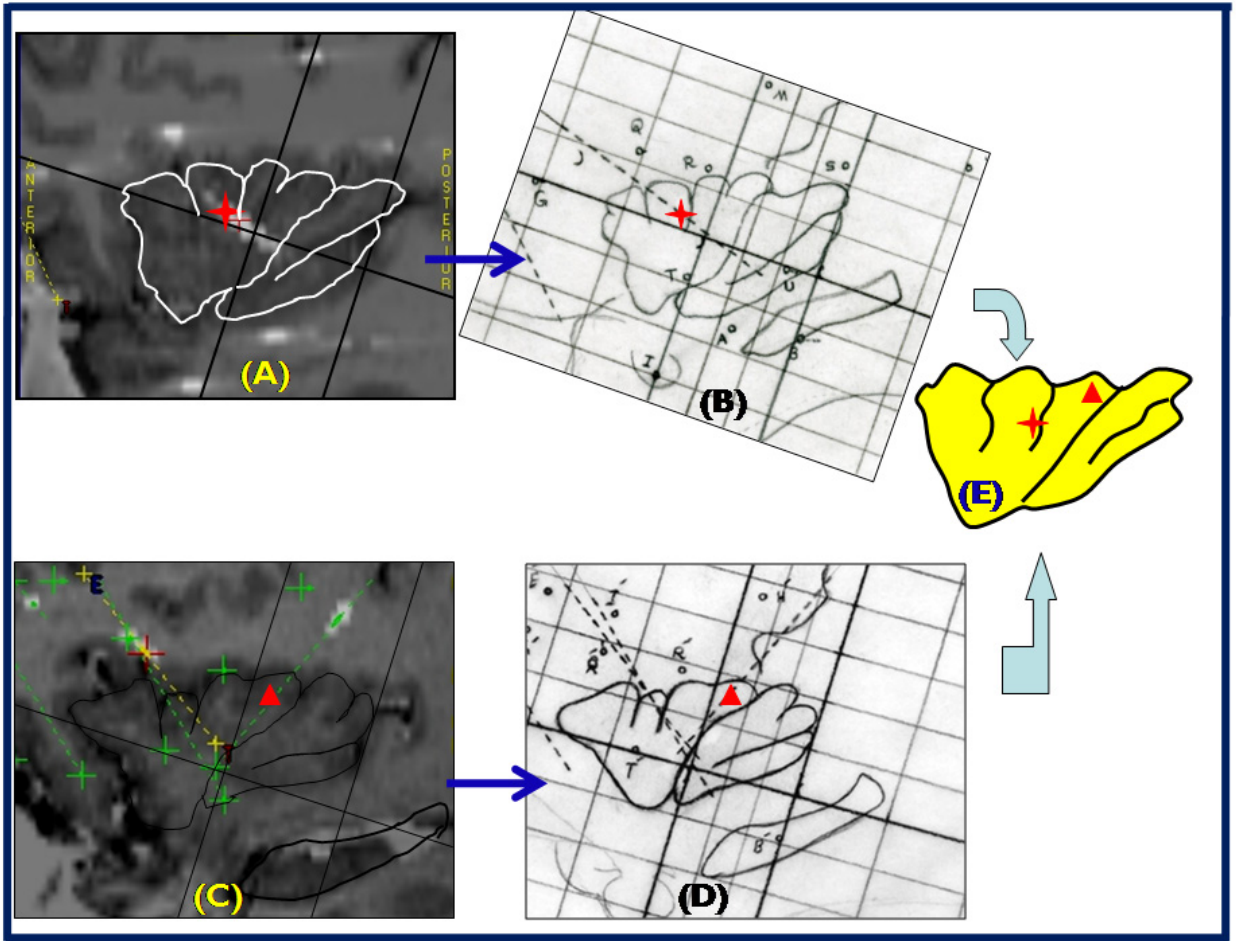

Fig. 6. Our strategy to present the electrical stimulation data. Lateral (A) and (C) individual insula reconstruction in bi-commissural references of 2 patients with the stimulation sites "+ in figure (A) and $\boldsymbol{\Delta}$ in figure (C)". (B) and (D) individual insular diagram (sagittal view) with the stimulation sites between the two contacts stimulated of the 2 patients in figures $\mathrm{A}$ and C. (E) template of the insula, including its sulci and gyri, the stimulation sites were located on the insular template with respect to the insular gyri from the individual diagram.

\subsubsection{Electrophysiological identification}

The localization protocol was based on electrophysiological data enabling us to distinguish between gray and white matter since electrical activity (both voltage and rhythm) decreases significantly in white matter (Fig. 7). All contacts were individually checked by bipolar recording their spontaneous electrical activity. 


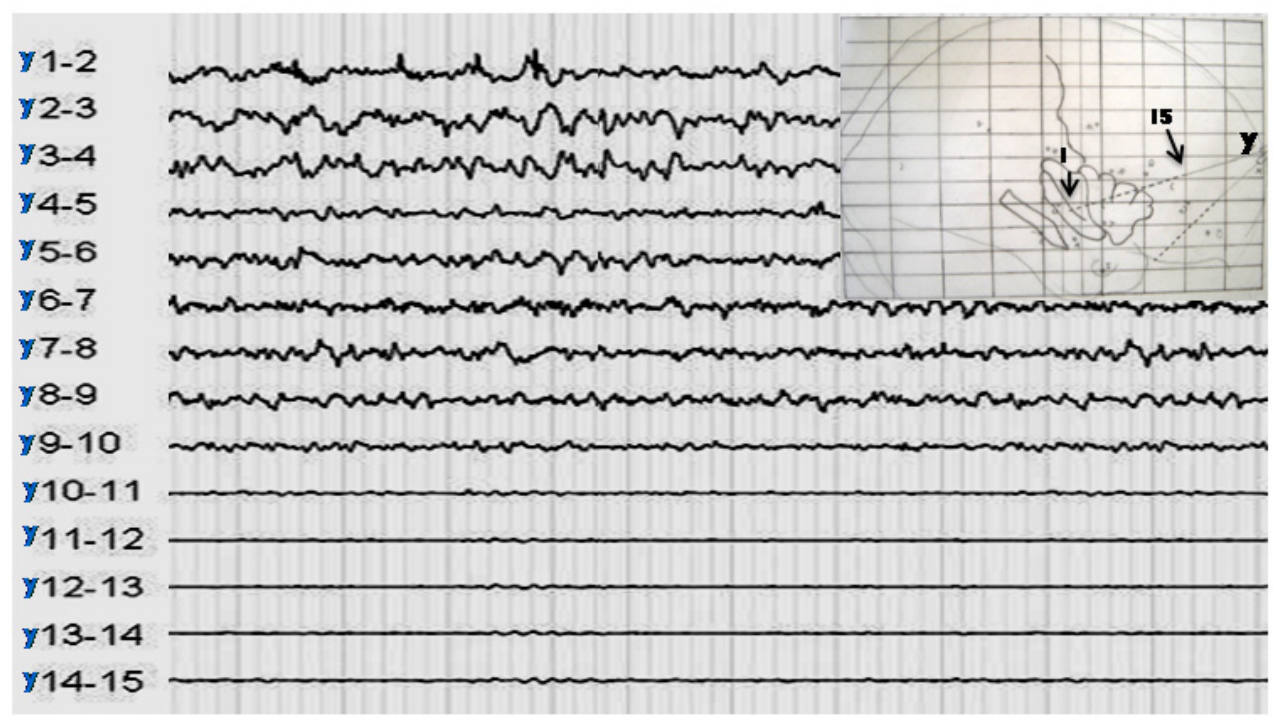

Fig. 7. Bipolar EEG recordings from a right oblique insular electrode. Right oblique insular electrode (y) is shown on a patient's individual diagram. Contact $n^{\circ}: 1$ (the deepest) and contact $n^{\circ}: 15$ (the most superficial). Test recordings were performed on contiguous bipolar electrodes from $y=1-2$ to $y=14-15$. Electrical activity shows typical cortical voltage and rhythms from bipolar electrodes $y=1-2$ to $y=9-10$. Activity drops to typical white substance levels from bipolar electrodes $y=10-11$ to $y=14-15$. Analysis of this EEG activity identifies contact $y=9$ as the last one exploring the insular cortex.

\section{Stimulation procedure}

After implantation, EEG recordings were obtained for several hours per day to monitor interictal and ictal activity. Simultaneous video and EEG recordings were performed enabling a complete online clinical examination of the patient by later analysis.

Following the previously described clinical protocol, ES was applied to every bipole of contiguous contacts selected for EEG recordings (Munari et al., 1993). According to previous studies (Ranck, 1975), the current spread linked to a stimulation intensity of $3 \mathrm{~mA}$ is able to stimulate excitable nervous elements up to a maximum distance of $5 \mathrm{~mm}$. We chose bipolar stimulation known to involve a smaller cortical volume compared to monopolar stimulation, thus leading to more accurate anatomical localization of less than $5 \mathrm{~mm}$ around the stimulated bipole (Nathan et al., 1993). To increase this accuracy by diminishing the current spread, we used only current intensities of bipolar stimulation between 0.2 and 3 mA (Afif et al., 2008b).

The aim of the stimulation protocol was to reproduce part of, or all, the ictal clinical symptomatology and map functionally eloquent areas which needed avoiding during surgery. ES was performed at low frequency (LF) $1 \mathrm{~Hz}$ (pulse width $=3 \mathrm{~ms}$ ) and high 
frequency $(\mathrm{HF}) 50 \mathrm{~Hz}$ (pulse width $=1 \mathrm{~ms}$ ) using a constant current, rectangular pulse generator (Micromed Treviso, Italy). Stimulations were performed after confirming a normal baseline activity of depth EEG recording. During stimulation the electrode containing the stimulated bipole was disconnected from recording amplifiers; electrical activity recordings continued on the other deep brain electrodes. ES was performed at both low frequency $(\mathrm{LF} ; 1 \mathrm{~Hz}$; pulse width $=3 \mathrm{~ms}$ ) and high frequency $(\mathrm{HF} ; 50 \mathrm{~Hz}$; pulse width= 1 $\mathrm{ms})$. Stimulations usually lasted for $40 \mathrm{~s}$ at $1 \mathrm{~Hz}$, and $5 \mathrm{~s}$ at $50 \mathrm{~Hz}$. The patients were asked to report any symptoms they felt as soon as possible and were then immediately examined and questioned. Data on stimulation-evoked phenomena (Kahane et al., 1993; Afif et al., 2008b), only those phenomena not associated with electrical after-discharge and clearly not due to current spread out of insula were considered (Jefferys \& Traub, 1998). Verification of the absence of afterdischarge could be obtained by analyzing the electrical signals obtained during cortical activity recordings on the electrode containing the stimulated bipole as soon as it was reconnected, and on all other deep brain electrodes during and after the stimulation procedure. All stimulation sites were stimulated by both LF and HF protocols starting with LF (Afif et al., 2008b).

\section{SEEG data}

Three groups of patients were identified according to insular involvement during SEEGrecorded seizures (Table 2). Group 1 showed no insula involvement during seizures (Fig. 8a) including patients with temporal lobe epilepsy (TE) and/or with frontal lobe epilepsy (FE). Group 2 showed no insular involvement at seizure onset but involvement after a short delay during seizure evolution (Fig. 8b) including patients suffered from TE and from temporofrontal lobe epilepsy (TFE). Group 3 showed insular involvement at seizure onset (Fig. 8c). Seizures arose either from the insula alone or from both the insula and the frontal operculum.

Compared to the initial hypothesis, SEEG changed the final location of the diagnosed epileptogenic zone in $43.3 \%$ (Afif et al., 2008a). In this study, three patients out of six with an initial hypothesis as suffering from FE were finally considered as having insulo-frontal epilepsy (IFE). Ten out of 14 patients with an initial hypothesis of TFE were finally considered as TE in seven patients, FE with secondary propagation to insula in one patient, and insulo-temporo-frontal epilepsy (ITFE) in the remaining two patients. Thus the SEEG data reduced the proposed surgical resection or disconnection zone in $57.1 \%$ in whom the initial hypothesis was TFE (Table 2).

Another study (Afif et al., 2010a) has shown the importance of SEEG data obtained by recordings and ES of intra-insular oblique electrodes insertions guided by a robotic arm during invasive pre-resection evaluation of epilepsy to identify the insular origin of the evoked clinical responses. This study suggests relationships between insular seizure semiology and evoked clinical responses by ES of insular contacts i.e., the patients with insular involvement at seizure onset or after a short delay during seizure evolution present speech disturbances such as speech arrest, dysarthria, crying and verbal automatism (chic, chic,..) after seizures. These patients reported a speech arrest induced by intra insular HF stimulations during a counting exercise. However, the patients show no insular involvement during seizures. No speech disturbances were described during or after seizures. These patients reported no speech arrest induced by intra insular ES. 


\begin{tabular}{|c|c|c|c|c|c|}
\hline P. & $\begin{array}{c}\text { E.Z. } \\
\text { hypothesis }\end{array}$ & $\begin{array}{c}\text { E.Z. after } \\
\text { SEEG }\end{array}$ & Group & Surgery & $\begin{array}{c}\text { Outco } \\
\text { me }\end{array}$ \\
\hline 1 & R. T.(i) & R. T. & 1 & T. Discon. & Ia \\
\hline 2 & R. T.(i) & R. T. & 1 & T. Discon. & Ia \\
\hline 3 & L. T.(i) & L. T. & 1 & T. Lobect & Ia \\
\hline 4 & L. T.(i) & L. T. & 1 & T. Lobect. & Ib \\
\hline 5 & R. T.(i) & R. T. & 1 & T. Lobect. & Ic \\
\hline 6 & L. T.F.(i) & L. T. & 1 & T..Lobect. & III \\
\hline 7 & L. T.F.(i) & L. F. & 1 & F. Lobect. & Ia \\
\hline 8 & L. F.(i) & L. F. & 1 & F. Lobect. & Ia \\
\hline 9 & R. F.(i) & R. F. & 1 & F. Lobect. & Ia \\
\hline 10 & R. F.(i) & R. F. & 1 & F. Lobect. & IV \\
\hline 11 & R. T.(i) & R. T.(I*) & 2 & T. Discon. & Ia \\
\hline 12 & R. T.(i) & R. T.(I*) & 2 & T. Discon. & Ia \\
\hline 13 & L. T.(i) & L. T.(I*) & 2 & T. Lobect. & Ia \\
\hline 14 & L. T.F.(i) & L. T.(I*) & 2 & T. Discon. & Ia \\
\hline 15 & R. T.F.(i) & R. T.(I*) & 2 & T. Discon. & Ia \\
\hline 16 & R. T.F.(i) & R. T.(I*) & 2 & T. Lobect. & Ia \\
\hline 17 & L. T.F.(i) & L. T.(I*) & 2 & T. Lobect. & Ia \\
\hline 18 & R. T.(i) & R. T.(I*) & 2 & T. Lobect. & Ib \\
\hline 19 & L. T.F.(i) & L. T.(I*) & 2 & T. Lobect. & IIb \\
\hline 20 & L. T.F.(i) & L. T.(I* & 2 & T. Lobect. & IIb \\
\hline 21 & L. T.(i) & L. T.(I* & 2 & T. Lobect. & IId \\
\hline 22 & R. T.F.(i) & R. T.F.(I*) & 2 & T. Lobect. + F. Lesionect. & Ia \\
\hline 23 & R. T.F.(i) & R. T.F.(I* & 2 & F. T. Lobect. & Ia \\
\hline 24 & R. T.F.(i) & R. T.F.(I* & 2 & T. Lobect+ F. Discon. & Ia \\
\hline 25 & R. T.F.(i) & R. T.F.(I* & 2 & T.+F.+ Insula Lobect. & Ia \\
\hline 26 & R. T.F.(i) & R. I.T.F. & 3 & T.+F.+ ant. Insula Lobect. & III \\
\hline 27. & R. T.F.(i) & R. I.T.F. & 3 & T.+F.+ ant. Insula Lobect. & IV \\
\hline 28 & L. F.(i) & L. I.F. & 3 & No surgery & NA \\
\hline 29 & L. F.(i) & L. I.F. & 3 & No surgery & NA \\
\hline 30 & R. F.(i) & R. I.F. & 3 & No surgery & NA \\
\hline
\end{tabular}

Table 2. Summary of the localization of the epileptogenic zone before and after SEEG and case grouping relative to insular involvement and the surgical outcome. TP: temporo-polar; FP: fronto-polar; F: frontal; T: temporal; (i): insular involvement was suspected during seizures prior to SEEG; $\left(\mathrm{I}^{*}\right)$ : secondary insular involvement demonstrated after SEEG evaluation; T.F.: temporo-frontal; I.T.F.: insulo- temporo-frontal; I.F.: insulo-frontal; Discon.: disconnection; Lobect.: lobectomy; Lesionect.: lesionectomy; Outcome according to Engel's classification with at least two year follow-up. 


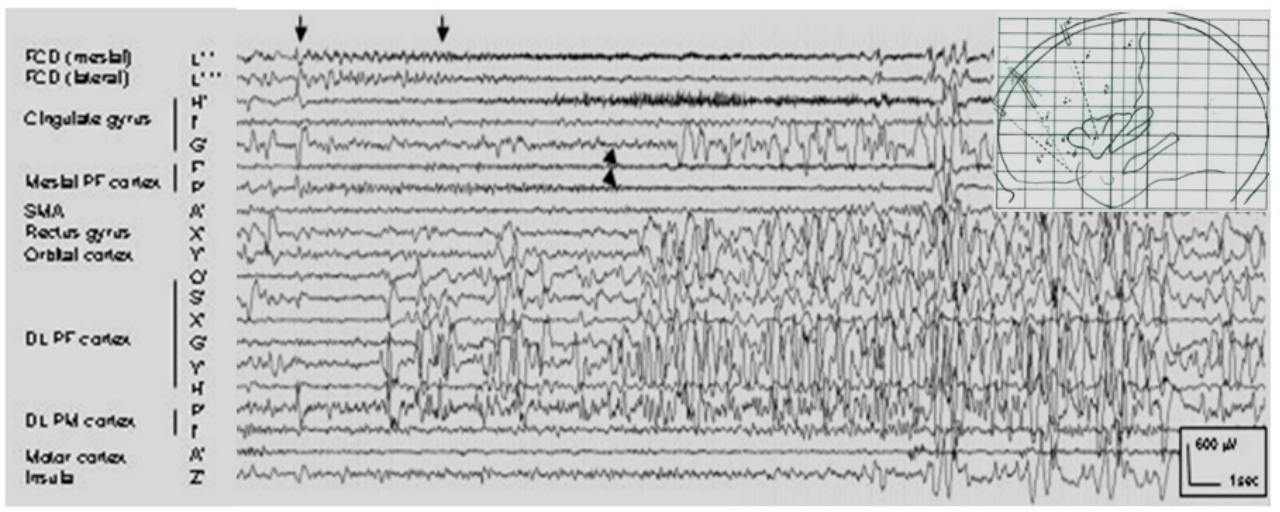

Fig. 8a. No insular involvement during seizure (Group 1) (SEEG trace and individual implantation lateral diagram with an oblique insular electrode). The ictal discharge (fast activity) begins (first arrow) in the lesion FCD (frontal cortical dysplasia) ( $\mathrm{L}^{\prime}$ ) and the anterior cingulate gyrus $\left(\mathrm{H}^{\prime}\right)$ and then (second arrow) becoming more evident later. later (arrowheads) the ictal discharge propagates to the mesial prefrontal cortex $\left(\mathrm{P}^{\prime}\right.$ and $\left.\mathrm{F}^{\prime}\right)$. Other regions are less involved with no involvement of the insular cortex. SMA: supplementary motor area, DLPF: dorsolateral prefrontal, DLPM: dorsolateral premotor.

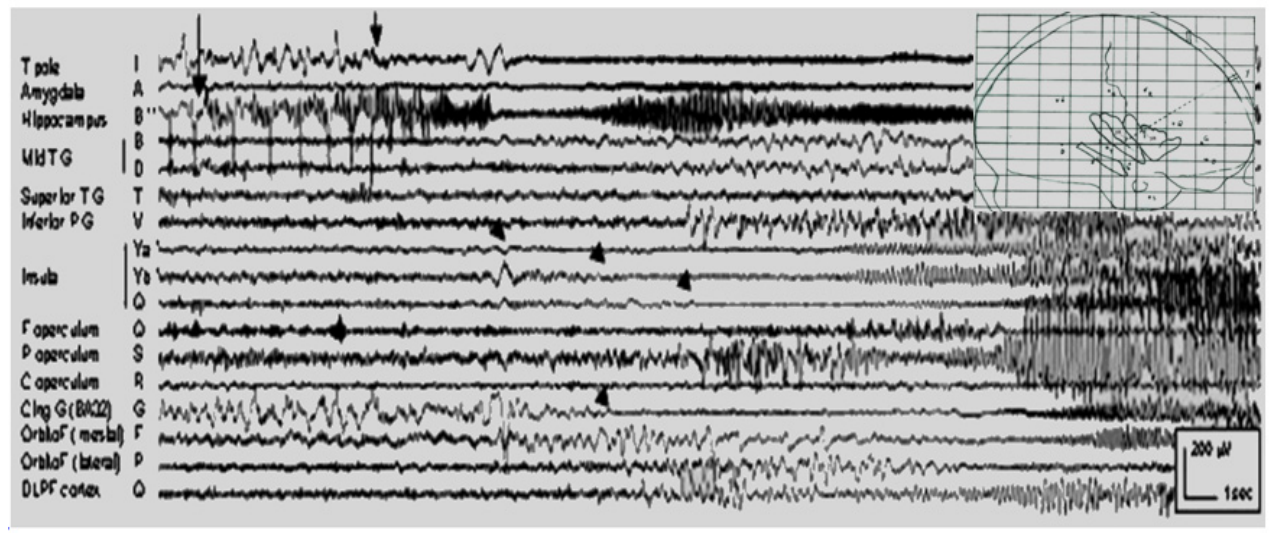

Fig. 8b. Earlier insular involvement during seizure (Group 2) (SEEG trace and individual implantation lateral diagram with an oblique insular electrode). The ictal discharge (fast spikes) begins in the hippocampus (first arrow) then involves the temporal pole and the amygdala (fast activity). The insula becomes involved $3 \mathrm{sec}$ later; the cingulate gyrus $2 \mathrm{sec}$ later (arrowheads). Different parts of the insula are successively involved. Note how the insular discharge is firstly recorded at different levels along the axis of the insular oblique electrode $(\mathrm{Ya}$ and $\mathrm{Yb}$ ) before becoming visible over the recording contacts of the perpendicular insular electrode $(\mathrm{Q})$. T: temporal, P: parietal, F: frontal, C: central, Cing: cingulate, DLPF: dorsolateral prefrontal. Letters refer to the recording electrodes. 


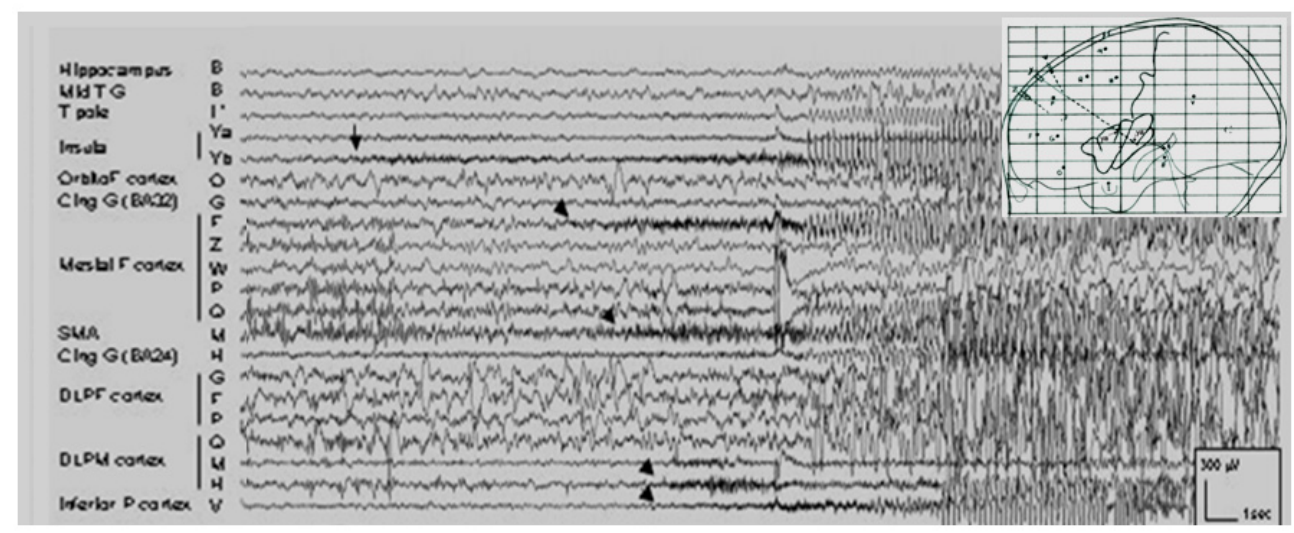

Fig. 8c. Insular involvement at seizure onset (Group 3) (SEEG trace and individual implantation lateral diagram with an oblique insular electrode). The ictal discharge (fast activity) begins in the insula (arrow), involving part of the mesial frontal cortex a few seconds later followed by the dorsolateral frontal cortex and the parietal cortex (arrowheads). Note that only the anterosuperior portion of the insula is involved at seizure onset. T: temporal, F: frontal, P: parietal, Cing: cingulate, SMA: supplementary motor area, DLPF: dorsolateral prefrontal, DLPM: dorsolateral premotor, G: gyrus. Letters refer to the recording electrodes.

\section{Surgical treatment and outcome}

On the basis of SEEG data analysis, 27 patients underwent a tailored surgical resection or disconnection (Afif et al., 2008a). Temporal lobe surgery was performed in 17 cases, frontal lobe surgery in 4 cases, temporo-frontal resection in 3 cases, temporo-frontal resection associated with removal of the anterior part of the insula in 2 cases, and total insulectomy in one patient who was seizure free (Engel's class I) (Engel et al., 1993).

Overall, (Table 2) 20 of the 27 operated patients (74\%) were seizure free (Engel's class I) following surgery, three had rare seizures (Engel's class II), two had a worthwhile improvement (Engel's class III), and two showed no improvement. With respect to insular involvement, eight of the ten operated patients in Group 1 were seizure free (80\%), one was classified in Engel's class III, and one in Engel's class IV. In Group 2, surgery relieved seizures in 12 out of 15 patients $(80 \%)$ while the remaining three patients suffered rare seizures. Surgery was unsuccessful in those patients in Group 3 who underwent surgery (one in Engel's class III, one in Engel's class IV).

With respect to the epileptogenic zone assessed by SEEG, 13 patients out of 17 were seizure free in TE, three were classified as Engel's class II and one as Engel's class III. In FE, three out of four patients were classified as Engel's class I and one as Engel's class IV. In TFE, all four patients were classified as Engel's class I. Neither of the two patients with initial insular involvement became seizure free following operation (Table 2).

The data strongly suggest the usefulness of insular recording to better predict the postoperative outcome and reduced the proposed surgical resection or disconnection zone in the epilepsy. 


\section{Functional data}

Only clinical responses evoked by ES in the absence of after discharge were analyzed (Afif et al., 2008b, 2010a, b). These studies are the first to report clinical responses evoked by ES of the insular cortex in terms of gyral and sulcal anatomy and to propose an anatomofunctional organization scheme of this cortex. The image fusion between the postoperative 3D CT scan with all electrodes still in place and the preoperative 3D MRI enabled us to localize the stimulated sites in three dimensions with respect to the individual gyri and sulci.

All the insular structures present responses by the ES. The relative lack of response to direct ES observed in the anterior short gyrus and pole could be due to under-exploration of these areas (5.4\% and $1.49 \%$ respectively, Table 3) (Afif et al., 2010b).

\begin{tabular}{|l|c|c|c|c|c|c|}
\hline $\begin{array}{l}\text { Anatomical } \\
\text { position }\end{array}$ & $\begin{array}{c}\text { Nmb. of } \\
\text { Stim. } \\
\text { Cont. }\end{array}$ & $\begin{array}{c}\text { Nmb. of } \\
\text { Pats. }\end{array}$ & $\begin{array}{c}\text { Nmb. Of } \\
\text { Clin. } \\
\text { Resp. }\end{array}$ & $\begin{array}{c}\text { Stim. Cont. } \\
\text { versus total }\end{array}$ & $\begin{array}{c}\text { Nmb. of } \\
\text { Pats. with } \\
\text { Resp. }\end{array}$ & $\begin{array}{c}\text { Resp. } \\
\text { versus } \\
\text { total }\end{array}$ \\
\hline Ant. short gyrus & 11 & 8 & 4 & $5.4 \%$ & 1 & $4.8 \%$ \\
\hline Mid. short gyrus & 42 & 17 & 27 & $20.89 \%$ & 10 & $32.5 \%$ \\
\hline $\begin{array}{l}\text { Pre central } \\
\text { gyrus }\end{array}$ & 49 & 19 & 13 & $23.88 \%$ & 10 & $15.6 \%$ \\
\hline $\begin{array}{l}\text { Post central } \\
\text { gyrus }\end{array}$ & 57 & 20 & 36 & $28.3 \%$ & 13 & $43.3 \%$ \\
\hline Post. Long gyrus & 17 & 8 & 3 & $8.4 \%$ & 3 & $3.7 \%$ \\
\hline Insular pole & 3 & 1 & 0 & $1.49 \%$ & 0 & $0 \%$ \\
\hline
\end{tabular}

Table 3. Anatomical positions of electrode contacts and observed responses in insular structures. Ant.: anterior. Mid.: middle. Post.: posterior. Nmb. Of Stim. Cont.: number of stimulated contacts inside the structure. Nmb. of Pats.: number of patients undergoing stimulation to this structure. Nmb. of Clin. Resp.: number of clinical responses in this structure. Stim. Cont. versus total: the number of stimulated contacts in this structure as a percentage of the total number of contacts inside the insular cortex. Nmb. of Pats. with Resp.: number of patients with clinical responses in this structure. Resp. versus total: the number of clinical responses to ES in a structure as a percentage of the total number of responses.

The main responses evoked in this study were classified as sensory (paresthesiae and localized warm sensations), motor, pain, auditory, oropharyngeal, speech disturbances, or neuro-vegetative phenomena. All these responses started immediately after the onset of the electrical stimulus and disappearing with the termination of the stimulus. Table 3 presents the percentage of evoked responses for each insular region.

\subsection{Speech disturbances}

Two different types of speech disturbances were reported (Fig. 9 a): i) speech arrest evoked by HF stimulation of the middle short gyrus (four responses) and one response occurred after stimulating the post-central insular gyrus. ii) episodes of reduced voice intensity in one patient evoked by HF stimulation from a bipolar electrode in the left insula (dominant hemisphere), just superior to the site that induced speech arrest. LF stimulation at this site did not evoke a response (Afif et al., 2010a, 2010b). 


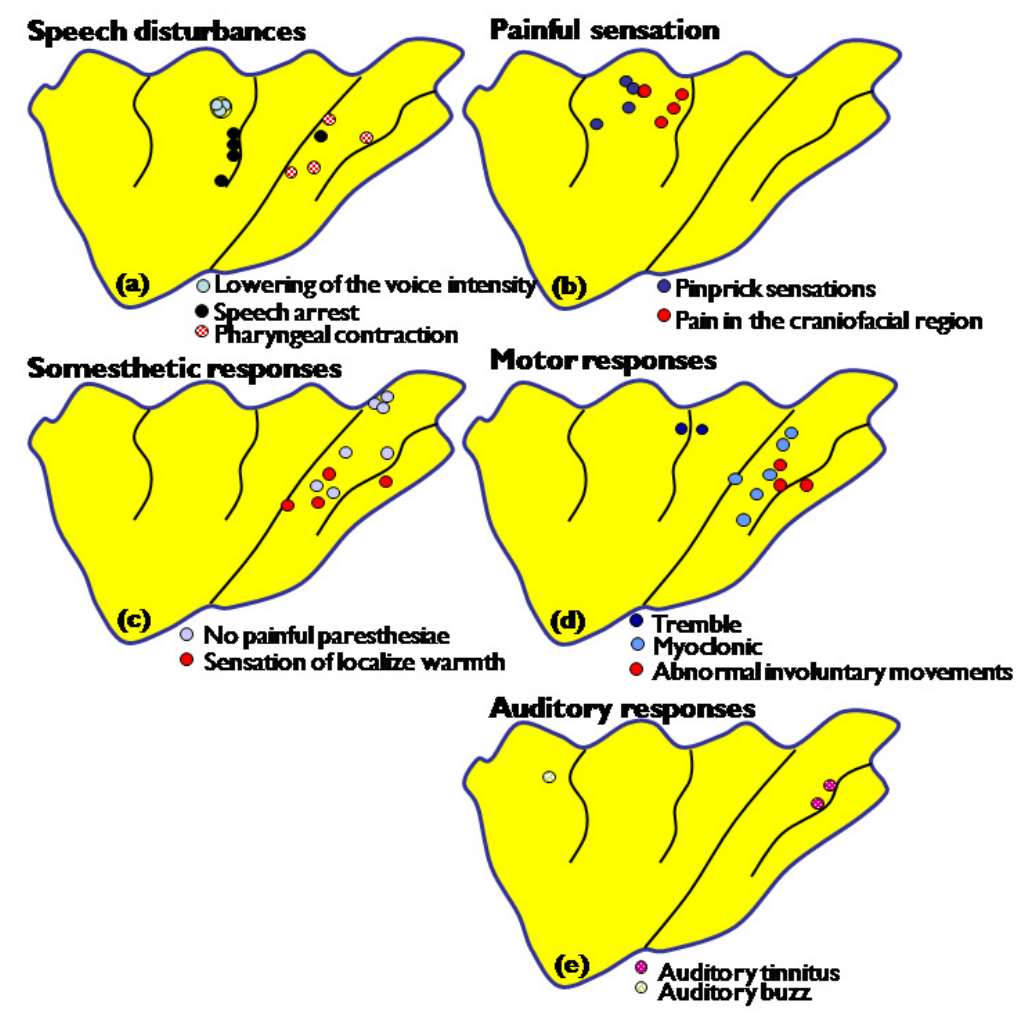

Fig. 9. Insular sites responding to electrical stimulation. The sites corresponding to clinical responses were first located on each patient's individual diagram before being transferred onto the insular template with respect to the insular gyri from the individual diagram. (a): Speech disturbances; (b): Pain; (c): Somesthetic responses; (d): Motor responses; (e): Auditory responses.

The term "speech arrest" used in this study refers to those patients unable to continue counting and speaking during stimulation. The patients later described an inability to continue counting because of an inability to speak (speech arrest) without other associated phenomena, such as pharyngeal constriction, memory deficits, difficulties in performing the movements required for speech, panic or a sensation of asphyxia. $80 \%$ of speech arrest responses involved stimulation of the non-dominant hemispheres, thus suggesting that the dominant cerebral hemisphere may function independently the dominant insula.

One of the patients presented a lowering of the voice intensity responses following stimulation of the bipole situated above and adjacent to the one in which stimulation evoked speech arrest in the middle short gyrus. It is possible that these responses could be the consequence of a disturbance in motor control (pharyngeal construction and breathlessness sensation) or due to current spread to the adjacent area producing speech arrest. $87.5 \%$ of the speech disturbances resulted from stimulating the middle short gyrus of the anterior insula at the anterior edge of the pre-central insular sulcus in both dominant and nondominant hemispheres (Afif et al., 2010a). The one remaining response (12.5\%) was induced 
by stimulating the post-central insular gyrus, in the same region which also precipitated pharyngeal constriction (Fig. 9 a).

The present study suggests the high restriction of the speech activity (articulation, phonation, etc) to the middle short gyrus of the anterior insula. In this study, all patients with insular involvement at seizure onset or after a short delay during seizure evolution present speech disturbances after seizures and after intra insular HF stimulations during a counting exercise (Afif et al., 2010a).

A few studies have been published that used stereotactically implanted deep brain electrodes (SEEG), inserted in a lateral orthogonal way, to stimulate the human insula directly (Ostrowsky et al., 2000; Isnard \& Mauguière, 2005). These studies suggest that the insula is involved in speech disturbances, but localization information for specific gyral or sulcal involvement was not provided. The functional imaging studies have demonstrated the activation of the insula during language-based tasks and grammar exercises (Raichle, 1991; McCarthy et al., 1993; Wise et al., 1999; Riecker et al., 2000, 2005; Friederici et al., 2006). Lesion studies reported speech disturbances relating to the infarctions of the insular cortex (Ferro et al., 1982; Shuren, 1993; Dronkers, 1996; Nagoa et al., 1999)

\subsection{Pain}

Two different types of painful responses were reported (Afif et al., 2008b): I) Pain in the craniofacial region evoked by HF stimulation either as a highly unpleasant headache or a bilateral throat pain that spread to the mouth and was accompanied by a highly unpleasant sensation of suffocation. All sites had been previously stimulated at LF without inducing pain, except for one patient who reported a pinprick sensation, as described further below. II) Pinprick sensations described occurring either bilaterally or ipsilaterally during LF and HF stimulations. These responses were reported in the upper limbs, the neck and abdomen, spreading to the chest and shoulders. All pain-inducing sites were located in the superior part of the middle short gyrus, in both dominant and non-dominant hemispheres (Fig 9 b). No similar responses were reported from other sites within the insular cortex. These findings cannot be due to under-exploration of other insular areas since contacts located in the middle short gyrus only represented $27.2 \%$ of all the insular contacts. Indeed, the post-central gyrus and posterior long gyrus represented $36.7 \%$ of all insular contacts and, furthermore, stimulation here induced $47 \%$ of all observed phenomena (Table 3) (Afif et al., 2008b, 2010b). These present studies are the first to report human painful responses evoked by ES of the insular cortex in terms of gyral and sulcal anatomy and suggest a pain somatotopy within the middle short gyrus of the anterior insula, with a posterior representation of the head and a more anterior representation of the upper limbs and trunk (Fig. $10 \mathrm{~A}$ ).

Few studies have been published using ES of human insular cortex. The present data complement those observed by Penfield and Faulk (1955) which reported a tingling feeling in the upper extremity (arm, hand and fingers) induced by direct stimulation of the anterior insula. No specific location in reference to gyri and sulci was given. Other studies (Ostrowsky et al., 2000; Ostrowsky et al., 2002; Isnard \& Mauguière, 2005), used stereotactically implanted deep brain electrodes (SEEG) inserted orthogonal (trans opercula) to the mid-sagittal plane. These studies suggest that the posterior insula processes both painful and painless somesthetic sensations. Recently, Mazzola et al. (2009) suggested the involvement of the posterior two thirds of the insula in processing pain. Using the insular 

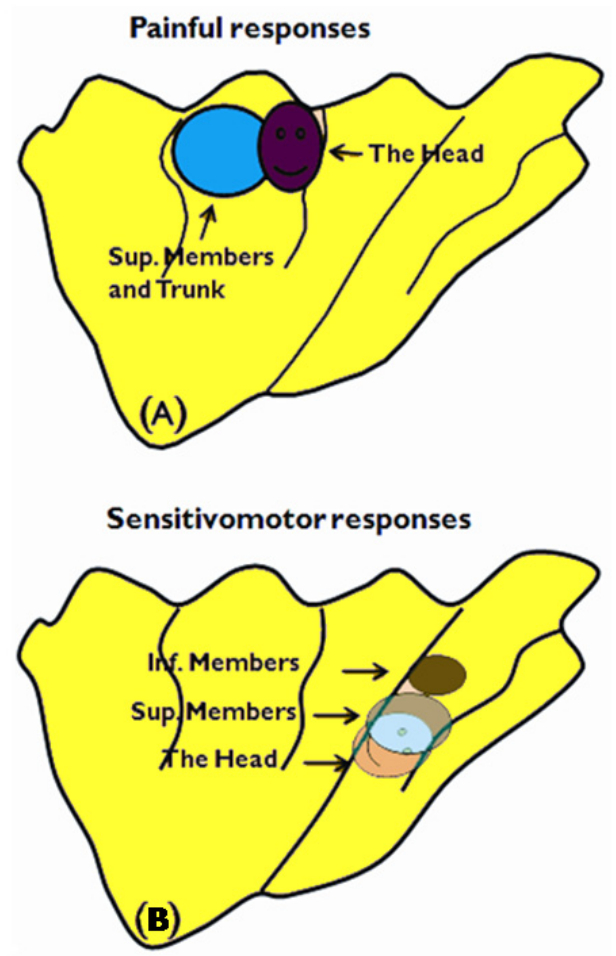

Fig. 10. Somatotopic organization of pain and sensorimotor responses. (A): pain results from stimulating the middle short gyrus of the insula, with a posterior representation of the head and a more anterior representation of the upper limbs and trunk. (B): sensorimotor responses from stimulating the post-central insular gyrus, showing an inferior representation of the head and a more superior representation of the upper and lower limbs.

orthogonal approach exploration, it would be more appropriate to describe the phenomena induced by ES as operculo-insular phenomena (the thickness of the insular cortex is less than the distance covered by two contiguous contacts (Fig. 2), so the bipolar ES will stimulate the overlapping adjacent structures "opercula" of the insula). Moreover, due to the dense network of vessels overlying the insular cortex (Fig. 4), only the peripheral region, near the peri-insular sulci can safely be reached by a lateral orthogonal approach (Afif et al., 2008b, 2010b).

Several functional imaging studies suggest that the posterior operculo-insular complex has a role in pain discrimination and localization (Hua et al., 2005; Baumgärtner et al., 2006). Other studies have identified a region in the anterior insula that becomes activated by noxious stimuli (Brooks et al., 2002, 2005; Henderson et al., 2007). It is interesting to note that the clinical responses evoked by ES within the anterior insular part are usually ipsi- or bilateral to the original peripheral stimulus (Tarkka \& Treede, 1993; Henderson et al., 2006; Afif et al., 2008b). 


\subsection{Somesthetic responses}

Sensory responses $(28.2 \%$ of responses evoked by stimulation sites within the posterior insula) were described as pins and needles or localized warmth contralateral to the ES sites (Fig. 9 c) (Afif et al., 2010b). All these responses were evoked by HF stimulation. Two different types were distinguished: i) Painless paresthesiae or "pins and needles" were reported in the upper limbs, lower limbs and the neck. $71.4 \%$ of these responses were evoked by ES in the non-dominant hemisphere. All responses resulted from electrode contacts stimulated in the post-central insular gyrus. However, paresthesiae in the upper and lower limbs occurred with stimulation in the superior portion of this gyrus. ii) Painless sensations of localized warmth were reported as occurring in the cranial region, contralateral, limbs and in the elbow, evoked by HF stimulation. All these responses were evoked by ES to the middle part of both post-central and posterior insular gyrus. Oppenheimer et al. (1992) noted reports of sensations of warmth when applying ES to the insular cortex, although the precise anatomical site was unknown.

\subsection{Motor responses}

Motor responses (28.2\% of responses evoked by stimulation sites within the posterior insula) were observed in the patients contralateral to the stimulated side $(8$ responses induced by LF and 3 responses by HF) (Afif et al., 2010b). Three different types were distinguished (Fig. $9 \mathrm{~d}$ ): i) Myoclonic phenomena in the upper and lower limbs, evoked by LF stimulation to the post-central insular gyrus. ii) Involuntary movements involved the upper and lower limbs. All of these responses resulted from stimulation to the non-dominant hemisphere, (from the post-central and posterior insular gyrus). iii) Upper limb tremors were evoked by stimulating the anterior and posterior edges of the pre-central insular sulcus in the dominant hemisphere.

In our study the majority of the sensorimotor responses ( $85.3 \%$ of cases) resulted from ES in the peri-central area of the insula, especially the post-central insular gyrus. The sites that induced motor responses also overlapped with those eliciting sensory responses. A new finding which has emerged from our data is that both types (motor and sensory responses) indicated a somatotopic organization in the post-central insular gyrus similar to the periRolandic cortex, with an inferior representation of the head and a more superior representation of the arms and legs (Fig. $10 \mathrm{~B}$ ). It is difficult to determine whether spatial discrimination exists between motor and sensory sites, or whether it results from different motor and sensory neuron densities (neuronal population maps, Roland \& Zilles, 1998), as in the supplementary motor area (Lim et al., 1994). In our study, HF stimulation evoked sensory responses, whereas LF evoked motor responses. Our data complement previous studies that suggest that stimulating the posterior insula can evoke somesthetic sensations (Ostrowsky et al., 2000; Isnard et al., 2004).

\subsection{Oropharyngeal responses}

Eight responses were evoked in 4 patients by HF ( 0.8 to $2.6 \mathrm{~mA})$ stimulation to the pericentral insular cortex and post-central insular gyrus (Fig. 4 E). Four of these responses were described as a pharyngeal constriction and one as a swallowing sensation associated with mastication. The three remaining responses occurred while stimulating the upper part of the middle short gyrus in one patient, who described a feeling of suffocation in the throat. 


\subsection{Auditory responses}

Two types of responses were observed (Fig. 9 e): i) bilateral auditory tinnitus evoked by ES within the upper part of the post-central insular gyrus in the non-dominant side. These responses were different from those known to occur when stimulating the Heschl gyrus, which are contra lateral hallucinations ii) predominantly contra lateral auditory buzz evoked by stimulating the anterior short gyrus. Auditory buzz might be mediated by a distributed network of the anterior insula with the other structures as the anterior cingulate gyrus, hippocampus, parahippocampal gyrus and the thalamus. The data obtained by stimulating the anterior short gyrus complement those from functional imaging studies (Bamiou et al., 2003; Lewis et al., 2000). Another study suggests the activation associated with auditory buzz in the anterior cingulated gyrus, insula, inferior frontal gyrus, middle temporal gyrus, thalamus, left hippocampus and parahippocampal gyrus (Shergill et al., 2000).

\section{Advantages and complications of oblique versus lateral trajectory for placement of insular electrodes}

The insula represents a thin layer of grey matter with a width usually less than $5 \mathrm{~mm}$ and lies almost parallel to the sagittal plane, which consequently allows the placement of a less of two contacts for each electrode implanted orthogonally (Fig. 2) compared to a mean of 7.5 contacts for each electrode implanted with an oblique approach (Afif et al., 2008a).

Firstly, our methodology to target the insula using an oblique approach guided by a robotic arm allows the electrode to cover a larger surface of insula and thus explore the different anatomical parts of the insular cortex without limits in contrast to an orthogonal transopercular approach (Afif et al., 2008a). Secondly, the orthogonal approach may distort any data collected due to anatomical limitations not permitting access to the greatest part of the insula largely covered by the sylvian vascular branches (Fig. 4) (Ture et al., 1999; Varnavas \& Grand, 1999; Afif \& Mertens, 2010). Thirdly, our trajectory enabled the exploration of between two and three distinct insular gyri or even the anterior and posterior insula with one single electrode. This should prove useful when delineating the epileptogenic zone and enable precise tailoring of resection when necessary.

This study provides new arguments in favor of the use of a stereotactic procedure to insert depth recording electrodes in difficult to reach areas such as the insula.

The safety of stereotactic depth electrode implantation has been addressed in numerous studies with severe morbidity with permanent deficit related to electrode implantation ranging from $1 \%$ to $2 \%$ in these series (Binnie et al., 1994; Guenot et al., 2001; Sindou et al., 2006; Tassi et al., 2005). Recently, De Almeida et al. (2006) reported a higher risk of hematoma (2.9\% per hemisphere) in cases where SEEG was performed in frontal epilepsy and when four or more electrodes were implanted. Cossu et al. recently reported the oblique implantation of electrodes in stereotactic conditions in adults (Cossu et al., 2005a) and in children (Cossu et al., 2005b). For these authors, this procedure was mandatory in targeting the frontal or parietal mesial regions, the orbito-basal region, the amygdala and the hippocampus. The oblique approach has also been used to record epileptic activity (Rektor et al., 2002, 2003) from the basal ganglia. In our institution we believe that a safe implantation of electrodes within the insula can be achieved using an oblique stereotactic approach guided by a robotic arm, coupled with preoperative MRI and stereotactic 
angiography. In our study, we observed no morbidity related to the surgical implantation of the oblique insular electrodes.

\section{Conclusion}

Oblique electrodes implanted using a robotic arm in the insular cortex permit the safe exploration of different insular regions and limit potential electrical contamination from adjacent areas, especially the opercular cortex. Insular recordings provide additional presurgical information to allow a tailored surgical approach when necessary and avoid surgery in cases of insular seizure where the insula cannot be removed.

We are not yet able to target one specific part of the insular cortex related to associate clinical phenomena of the epilepsy seizures. The previous results of the insular stimulation studies were not provided in reference to gyral and sulcal anatomy. Consequently, wider sampling of the different sulci and convolutions of insular lobe seems necessary to gather enough information to either exonerate or implicate the insula in a patient's epileptic network. The association of lateral electrodes in the temporal or frontal lobe and oblique electrodes in the insular cortex seems at present to be a good compromise to study multi lobar epilepsy.

The broad sampling of this area by oblique electrodes implanted within the insular cortex has allowed us to construct an original representation that links function to anatomy, according to insular gyri and sulci. The data of this study maybe permeates to target a specific structure of the insular cortex related to the epilepsy seizures manifestations using a functional organization of the insular gyri.

\section{References}

Ackermann, H. \& Riecker, A. (2004) The contribution of the insula to motor aspects of speech production: A review and a hypothesis. Brain Lang 89:320-328.

Afif, A. \& Mertens, P. (2010) Description of sulcal organization of the insular cortex. Surg Radiol Anat 32:491-498.

Afif, A. Bouvier, R. Buenerd, A. Trouillas, J. \& Mertens, P. (2007) Development of the human fetal insular cortex: Study of the gyration from 13 to 28 gestational weeks. Brain Struct Funct 212:335-346.

Afif, A. Chabardes, S. Minotti, L. Kahane, P. \& Hoffmann, D. (2008 a) Safety and usefulness of insular depth electrodes implanted via an oblique approach in patients with epilepsy. Neurosurgery 62:471-479.

Afif, A. Hoffmann, D. Minotti, L. Benabid, AL. \& Kahane, P. (2008 b) Middle short gyrus of the insula implicated in pain processing. Pain 138:546-555.

Afif, A. Minotti, L. Kahane, P. \& Hoffmann, D. (2010 a) Middle short gyrus of the insula implicated in speech production: Intracerebral electric stimulation of patients with epilepsy. Epilepsia 51:206-213.

Afif, A. Minotti, L. Kahane, P. \& Hoffmann, D. (2010 b) Anatomo-Functional Organization of the Insular Cortex: A study using intracerebral electrical stimulation in epileptic patients. Epilepsia 51(11):2305-15. 
Bamiou, DE. Musiek, FE. \& Luxon, LM. (2003) The insula (Island of Reil) and its role in auditory processing Literature review. Brain Res Rev 42(2):143-154.

Bancaud, J. Talairach, J. Bonis, A. Schaub, C. Szikla, G. Morel, P. \& Bordas-Ferrer, M. (1967) La stéréo-électroencéphalographie dans l'épilepsie. Informations neurophysiopathologiques apportées par l'investigation fonctionnelle stéréotaxique. Electroencephalogr Clin Neurophysiol 22:493-495.

Baumgartner, U. Tiede, W. Treede, RD. \& Craig, AD. (2006) Laser-evoked potentials are graded and somatotopically organized anteroposteriorly in the opercul-insular cortex of anesthetized monkeys. J Neurophysiol 96:2802-2808.

Binnie, CD. Elwes, RD. Polkey, CE. \& Volans, A. (1994) Utility of stereoelectroencephalography in preoperative assessment of temporal lobe epilepsy. J Neurol Neurosurg Psychiatry 57:58-65.

Brooks, J. Nurmikko, T. Bimson, W. Singh, K. \& Roberts, N. (2002) fMRI of thermal pain:effects of stimulus laterality and attention. NeuroImage 15:293-301.

Brooks, JCW. Zambreanu, L. Godinez, A. Craig, AD. \& Tracey, I. (2005) Somatotopic organisation of the human insula to painful heat studied with high resolution functional imaging. NeuroImage 27:201-209.

Cossu, M. Cardinale, F. Castana, L. Citterio, A. Francione, S. Tassi, L. Benabid, AL. \& Lo Russo, G. (2005 a) Stereoelectroencephalography in the presurgical evaluation of focal epilepsy: A retrospective analysis of 215 procedures. Neurosurgery 57:706-718.

Cossu, M. Cardinale, F. Colombo, N. Mai, R. Nobili, L. Sartori, I. \& Lo Russo, G. (2005 b) Stereoelectroencephalography in the presurgical evaluation of children with drugresistant focal epilepsy. J Neurosurg 103:333-343.

Craig, AD. (2004) Distribution of trigeminothalamic and spinothalamic lamina I terminations in the macaque monkey. J Comp Neurol 477:119-48.

Daniel, SK. \& Foundas, AL. (1997) The Role of the Insular cortex in Dysphagia. Dysphagia 12:146-156.

De Almeida, AN. Olivier, A. Quesney, F. Dubeau, F. Savard, G. \& Anderman, F.: Efficacy of and morbidity associated with stereoelectroencephalography using computerized tomography- or magnetic resonance imaging-guided electrode implantation. J Neurosurg 104:483-487, 2006.

Dronkers, NF. (1996) A new brain region for coordinating speech articulation. Nature 384:159-61.

Duffau, H. Moritz-Gasser, S. \& Gatignol, P. (2009) Functional outcome after language mapping for insular World Health Organization Grade II gliomas in the dominant hemisphere: experience with 24 patients. Neurosurg Focus 27 (2):E7,

Duffau, H. Bauchet, L. Lehéricy, S. \& Capelle, L.: (2001) Functional compensation of the left dominant insula for language. Neuroreport 12:2159-2163.

Dupont, S. Bouilleret, V. Hasboun, D. Semah, F. \& Baulac, M. (2003) Functional anatomy of the insula: new insights from imaging. Surg Radiol Anat 25:113-119.

Engel, J Jr. Van Ness, PC. \& Rasmussen, TB. Ojemann, LM.: Outcome with respect to epileptic seizures, in Engel J Jr (ed): Surgical Treatment of the Epilepsies. New York, Raven Press, 1993, pp 609-621. 
Ferro, JM. Martins, IP. Pinto, F. \& Castro-Caldas, A. (1982) Aphasia following right striatoinsular infarction in a left-handed child: a clinico-radiological study. Dev Med Child Neurol 24:173-182.

Friederici, AD. Bahlmann, J. Heim, S. Schubotz, RI. \& Anwander, A. (2006) The brain differentiates human and non-human grammars: Functional localization and structural connectivity. Proc Natl Acad Sci U S A 103:2458-2463.

Frot, M. \& Mauguiere, F. (1999) Timing and spatial distribution of somatosensory responses recorded in the upper bank of the sylvian fissure (SII area) in humans. Cereb Cortex 9:854-63.

Guenot, M. Isnard, J. Ryvlin, P. Fischer, C. Ostrowsky, K. Mauguiere, F. \& Sindou, M. (2001) Neurophysiological monitoring for epilepsy surgery: The Talairach SEEG method. StereoElectroEncephaloGraphy. Indications, results, complications and therapeutic applications in a series of 100 consecutive cases. Stereotact Funct Neurosurg 77:29-32.

Henderson, LA. Bandler, R. Gandevia, SC. \& Macefield, VG. (2006) Distinct forebrain activity patterns during deep versus superficial pain. Pain 120:286-96.

Henderson, LA. Gandevia, SC. \& Macefield, VG. (2007) Somatotopic organization of the processing of muscle and cutaneous pain in the left and right insula cortex: A single-trial fMRI study. Pain 128:20-30.

Hua, Le H. Strigo, IA. Baxter, LC. Johnson, SC. \& Craig, AD. (2005) Anteroposterior somatotopy of innocuous cooling activation focus in human dorsal posterior insular cortex. Am J Physiol Regul Integr Comp Physiol 289: R319-R325.

Isnard, J. Guenot, M. Ostrowsky, K. Sindou, M. \& Mauguière, F. (2000) The role of the insular cortex in temporal lobe epilepsy. Ann Neurol 48:614-623.

Isnard, J. Guenot, M. Sindou, M. \& Mauguiere, F. (2004) Clinical manifestations of insular lobe seizures: a stereo-electroencephalographic study. Epilepsia 45:1079-1090.

Isnard, J. \& Mauguiere, F. (2005) [The insula in partial epilepsy]. Rev Neuro (paris)l 161:17-26.

Jefferys, JG. \& Traub, RD. (1998) Electrophysiological substrates for focal epilepsies. Prog Brain Res 116:351-8.

Kahane, P. Tassi, L. Francione, S. Hoffmann, D. Lo Russo, G. \& Munari, C. (1993) [Electroclinical manifestations elicited by intracerebral electric stimulation "shocks" in temporal lobe epilepsy]. Neurophysiol Clin 23:305-326.

Kato, Y. Muramatsu, T. Kato, M. Shintani, M. \& Kashima, H. (2007) Activation of right insular cortex during imaginary speech articulation. Neuroreport 18:505-509.

Kodam, S. (1926) Über die sogenannten Basalganglien, Morphogenetische und pathologischanatomische Untersuchunger. Schweiz Arch Neurol Psychiatr 18:179-246.

Lewis, JM. Beauchamp, MS. \& De Yoe, EA. (2000) A comparison of visual and auditory motion processing in human Cereb Cortex 10:873-888.

Lim, SH. Dinner, DS. Pillay, PK. Luders, H. Morris, HH. Klem, G. Wyllie \& Awad, IA. (1994) Functional anatomy of the human supplementary sensorimotor area : results of extraoperative electrical stimulation. Electroencephalogr Clin Neurophysiol 91:179-93.

Manes, F. Paradiso, S. Springer, JA. Lamberty, G. \& Robinson, RG. (1999) Neglect after right insular cortex infarction. Stroke 30:964-8. 
Mazzola, L. Isnard, J. Peyron, R. Guenot, M. \& Mauguière, F. (2009) Somatotopic organization of pain responses to direct electrical stimulation of the human insular cortex. Pain 146:99-104.

Mazzola, L. Isnard, J. \& Mauguière, F. (2006) Somatosensory and pain responses to stimulation of the second somatosensory area (SII) in humans. A comparison with SI and Insular responses. Cerebral Cortex 16:960-968.

McCarthy, G. Blamier, AM. Rothman, DL. Gruelter, R. \& Shulman, RG. (1993) Echo-planar magnetic resonance imaging studies of frontal cortex activation during word generation in humans. PNAS 90:4952-4956.

Munari, C. Kahane, P. Tassi, L. Francione, S. Hoffmann, D. Lo Russo, G. \& Benabid, AL. (1993) Intracerebral low frequency electrical stimulation: a new tool for the definition of the "epileptogenic area"? Acta Neurochir Suppl (Wien) 58:181-185.

Nagoa, M. Takeda, K. Komori, T. Isozaki, E. \& Hirai, S. (1999) Apraxia of speech associated with an infarct in the pre-central gyrus of the insula. Neuroradiology 41:356-357.

Naidich, TP. Kang, E. Fatterpekar, GM. Delman, BN. Humayun Gultekin, S. Wolfe, D. Ortiz, O. Yousry, I. Weismann, M. \& Yousry, TA. (2004) The Insula : Anatomic Study and MR Imaging Display at 1.5 T. Am J Neuroradiol 25:222-232.

Nathan, SS. Sinha, SR. Gordon, B. Lesser, RP. \& Thakor, NV. (1993) Determination of current density distributions generated by electrical stimulation of the human cerebral cortex. Electroencephalogr Clin Neurophysiol 86:183-192.

Oppenheimer, S. Gelb, A. Girvin, JP. \& Hachinski, V. (1992) Cardiovascular effect of human insular cortex stimulation. Neurology 42:1727-1732.

Ostrowsky, K. Isnard, J. Ryvlin, Ph. Guénot, M. Fischer, C. \& Mauguière, F. (2000) Functional Mapping of the Insular Cortex: Clinical Implication in Temporal Lobe. Epilepsy 41:681-686.

Ostrowsky, K. Magnin, M. \& Ryvlin, Ph. (2002) Representation of pain and somatic sensation in the human insula : a study of responses to direct electrical cortical stimulation. Cereb Cortex 12:376-385.

Penfield, W. \& Jasper, WW. eds. (1954) Epilepsy and the functional anatomy of the human brain. Boston: Little, Brown.

Penfield, W. \& Faulk, ME. Jr. (1955) The insula; further observations on its functions. Brain 78:445-470.

Peyron, R. Schneider, F. Faillenot, I. Convers, P. Barral, F. \& Garcia-larrea, B. (2004) An fMRI study of cortical representation of mechanical allodynia in patients with neuropathic pain. Neurology 23:1838-1846.

Price, CJ. (2000) The anatomy of language: contributions from functional neuroimaging. J Anat 197 pt 3:335-59.

Price, CJ. (2001) Functional-imaging studies of the 19th century neurological model of language. Rev Neurol (Paris) 157:833-6.

Raichle, ME. (1991) Memory mechanisme in the processing of wards and ward-like symbole. In Exploring Brain Functionnal Anatomy With Positron Tomography. Ciba Found Symposiim 163:198-217.

Ranck, JB. (1975) Which elements are excited in electrical stimulation of mammalian central nervous system: a review. Brain Res 98:417-440. 
Reil, JC. (1809) Unterfuchungen uber den Bau des grofsen Gehirns im Menfchen: Vierte Fortsetzung VIII. Arch physiol Halle 9:136-146.

Rektor, I. Kuba, R. \& Brázdil, M. (2002) Interictal and ictal EEG activity in the basal ganglia: An SEEG study in patients with temporal lobe epilepsy. Epilepsia 43:253-262.

Rektor, I. Kaiiovsky, P. Bares, M. Brázdil, M. Streitová, H. Klajblová, H. Kuba, R. \& Daniel, P.: A SEEG study of ERP in motor and premotor cortices and in the basal ganglia. Clin Neurophysiol 114:463-471, 2003.

Riecker, A. Ackermann, H. Wildgruber, D. Dogil, G. \& Grodd, W. (2000) Opposite hemispheric lateralization effects during speaking and singing at motor cortex, insula and cerebellum. Neuroreport 11:1997-2000.

Riecker, A. Mathiak, K. Wildgruber, D. Erb, M. Hertrich, I. Grodd, W. \& Ackermann, H. (2005) fMRI reveals two distinct cerebral networks subserving speech motor control. Neurology 64:700-7006.

Roland, P. \& Zilles, K. (1998) Structral division and functioal fields in the human cerebral cortex, Brain Res Rew 26:87-105.

Ryvlin, P. Minotti, L. Demarquay, G. Hirsch, E. Arzimanoglou, A. Hoffman, D. Guenot, M. Picard, F. Rheims, S. \& Kahane, P. (2006) Nocturnal hypermotor seizures, suggesting frontal lobe epilepsy, can originate in the insula. Epilepsia 47:755-765.

Schreckenberger, M. Siessmeier, T. Viertmann, A. Landvogt, C. Buchholz, HG. Rolke, R. Treede, RD. Bartenstein, P. \& Birklein, F. (2005) The unpleasantness of tonic pain is encoded by the insular cortex. Neurology 64:1175-1183.

Seeck, M. Zaim, S. Chaves-vischer, V. Blanke, O. Maeder-Ingvar, M. Weissert, M. \& Roulet, E. (2003) Ictal bradycardia in a young child with focal cortical dysplasia in the right insular cortex. Eur J Pediatr Neurol 7:177-181.

Shergill, SS. Brammer, MJ. Williams, SC. Murray, RM. \& McGuire, PK. (2000) Mapping auditory hallucinations in schizophrenia using functional magnetic resonance imaging Arch Gen Psychiatry 57:1033-1038.

Shuren, J. (1993) Insula and aphasia. J Neurol 240:216-218.

Sindou, M. Guenot, M. Isnard, J. Ryvlin, P. Fischer, C. \& Mauguière, F.: Temporomesial epilepsy surgery: Outcome and complications in 100 consecutive adult patients. Acta Neurochir (Wien) 148:39-45, 2006.

Streeter, GL. (1912) The development of the nervous system. In: Keibel F, Mall FP (eds) Manual of Human Embryology. Vol. II, Chapter XIV. Lippincott, Philadelphia.

Talairach, J. \& Tournoux, P. (1988) Co-Planar Stereotaxic atlas of the human brain, 3-Dimensional Proportional System: an approach to cerebral imaging. Georg Thieme Verlag, StuttgartNew-York.

Tarkka, IM. \& Treede, RD. (1993) Equivalent electrical source analysis of pain-related somatosensory evoked potentials elicited by a CO2 laser. J Clin Neurophysiol 10:513-9.

Tassi, L. Colombo, N. Cossu, M. Mai, R. Francione, S. Lo Russo, G. Galli, C. Bramerio, M. Battaglia, G. Garbelli, R. Meroni, A. \& Spreafico, R.: Electroclinical, MRI and neuropathological study of 10 patients with nodular heterotopia, with surgical outcomes. Brain 128:321-337, 2005.

Ture, U. Yasargil, DC. Al-Mefty, O. \& Yasargil, MG. (1999) Topographic anatomy of the insular region. J Neurosurg 90:720-733. 
Ture, U. Yaşargil, G. Al-Mefty, O. \& Yaşargil, D. (2000) Arteries of the insula. Neurosurgery 92:676-687.

Varnavas, GG. \& Grand, W. (1999) The insular cortex: morphological and vascular anatomic characteristics. Neurosurgery 44:127-136; discussion 136-128.

Wise, RSJ. Green, J. Buchel, C. \& Scott, SK. (1999) Brain regions involved in articulation. Lancet 353:1057-1061. 


\title{
Multimodal MRI of Cerebral Small Vessel Disease
}

\author{
Bence Gunda ${ }^{1}$, György Várallyay ${ }^{2}$ and Dániel Bereczki ${ }^{1}$ \\ ${ }^{1}$ Department of Neurology \\ ${ }^{2}$ MRI Laboratory, János Szentágothai \\ Knowledge Center Semmelweis University, Budapest \\ Hungary
}

\section{Inroduction}

Cerebral small vessel disease (cSVD) is a spectrum of clinical and imaging abnormalities linked to the pathology of small penetrating arteries and arterioles in the brain irrigating subcortical structures ${ }^{1}$. Accumulating data suggest that cSVD is the most prevalent neurological disorder in the ageing society of the developed world 2,3 . The prevalence of its seemingly asymptomatic manifestations -silent brain infarcts- increases with age from approximately $6-7 \%$ at 60 years to $28 \%$ at 80 years of age according to a recent review ${ }^{4}$. In another study lacunar infarcts were found in $23 \%$ of all subjects over 65 years, and in $43 \%$ of subjects over 80 years of age ${ }^{5}$. Its acute, symptomatic manifestations -lacunar strokesaccount for approximately $20 \%$ of all ischemic strokes $6-8$. Thus improved management of cSVD based on better understanding of the disease is of great importance.

cSVD is characterised by the arteriolosclerosis and/or microatheromatosis of small calibre $(50-500 \mu \mathrm{m})$ cerebral arterial vessels caused by various pathologies ${ }^{1}$. Its most common, sporadic form is related to age and vascular risk factors including hypertension and diabetes in particular. Inherited forms are increasingly recognised with CADASIL (Cerebral Autosomal Dominant Arteriopathy with Subcortical Infarcts and Leukoencephalopathy) being the most prevalent genetic cSVD caused by the mutation of NOTCH 3 gene encoding a transmembrane receptor of vascular smooth muscle cells ${ }^{9,10}$. CADASIL -affecting young to middle aged, otherwise healthy individuals- provides a pure model for cSVD and therefore has been extensively studied ${ }^{11}$. Inflammatory, infective and immunologically mediated forms are usually part of systemic diseases of diverse origin characterised by central nervous system vasculitis ${ }^{12}$. Cerebral amyloid angiopathy (CAA) -a pathological hallmark of Alzheimer's disease- affects small vessels both cortically and subcortically and may also lead to ischemic changes, although it is particularly associated with recurrent lobar haemorrhages ${ }^{12}$. In this chapter we will only focus on the most common and well studied age and vascular risk factor related form of CSVD and CADASIL.

cSVD predominantly affects perforating end-arteries branching usually perpendicularly from a large parent artery. These penetrating arteries irrigate the so called perforator areas including the basal ganglia and internal capsule (lenticulostriate arteries from the anterior cerebral artery (ACA) A1 segment and middle cerebral artery (MCA) M1 segment), the 
thalamus (thalamoperforators from the posterior cerebral artery (PCA) and posterior communicating artery (PCoA)), the pons (pontine perforators from the basilar artery (BA)) and the hemispheric deep white matter -centrum semiovale (perforators from the cortical, leptomeningeal arteries) ${ }^{13-16}$.

The pathological changes in cSVD lead to luminal narrowing, decreased autoregulation and vasoreactivity, and vessel wall damage in the cerebral microvessels resulting in their i. gradual stenosis, ii. sudden occlusion or iii. rupture. As a consequence the subcortical brain tissue suffers from i.: diffuse chronic hypoperfusion and ischemia leading to the progressive disintegration of cerebral white matter17; ii.: acute localised ischemia resulting in lacunar infarcts 18 ; ad iii.: acute major haemorrhages or microbleeds 12,19 . In an advanced state of the disease cerebral atrophy invariably occurs as a remote and/or diffuse consequence of vascular lesion burden ${ }^{20}$. The pathogenesis of cSVD manifestations is summarized in Figure 1. The gradual ischemic tissue damage clinically manifests in progressive vascular cognitive impairment (mainly executive dysfunction) and physical disability (gait disturbances, pseudobulbar palsies, urinary incontinence etc.), whereas acute focal ischemia presents with the so-called lacunar syndromes. Cerebral microbleeds are usually asymptomatic and their clinical significance is yet to be determined.

In this chapter we will summarize recent knowledge about the MRI characteristics of cSVD. Since the cerebral microvasculature cannot be currently visualized in vivo, the consequent parenchymal lesions (lacunar infarcts, white matter lesions, microbleeds and atrophy) have been adopted as markers of $\mathrm{CSVD}^{12}$. We will not discuss the issue of major haemorrhages.

\section{Lacunar infarcts}

\subsection{Definition}

According to the "lacunar hypothesis" first proposed by Fisher small subcortical infarcts of a diameter less than $15 \mathrm{~mm}$-called lacunar infarcts (LI)- result from the sudden occlusion of penetrating arteries due to CSVD in typical locations -the perforator areas (see above) ${ }^{18}$. Infarcts of this type have been linked to particular clinical syndromes with a relatively good prognosis called the lacunar syndromes, most frequent of which are the classical ones: pure motor stroke, pure sensory stroke, ataxic hemiparesis, dysarthria-clumsy hand syndrome and sensorimotor stroke. The concept of lacunar stroke that entered stroke classifications was based on postmortem and CT based studies both with considerable limitations. The pathological studies were limited by the low mortality of lacunar strokes and by the anatomical changes occurring in the chronic stage and/or during fixation. CT has a low sensitivity to detect small infarcts in certain locations (posterior fossa, cortex) and in the acute stage and cannot differentiate between fresh and old lesions. The advent of MRI and especially its newer techniques such as diffusion weighted imaging (DWI), perfusion weighted imaging (PWI) and diffusion tensor imaging (DTI) has slightly modified our understanding of $\mathrm{LIs}^{21}$.

\subsection{Conventional MRI}

Because of their small size visualizing LIs is much more problematic than that of larger territorial infarcts. Compared to CT conventional MRI sequences such as T1 weighted, T2 


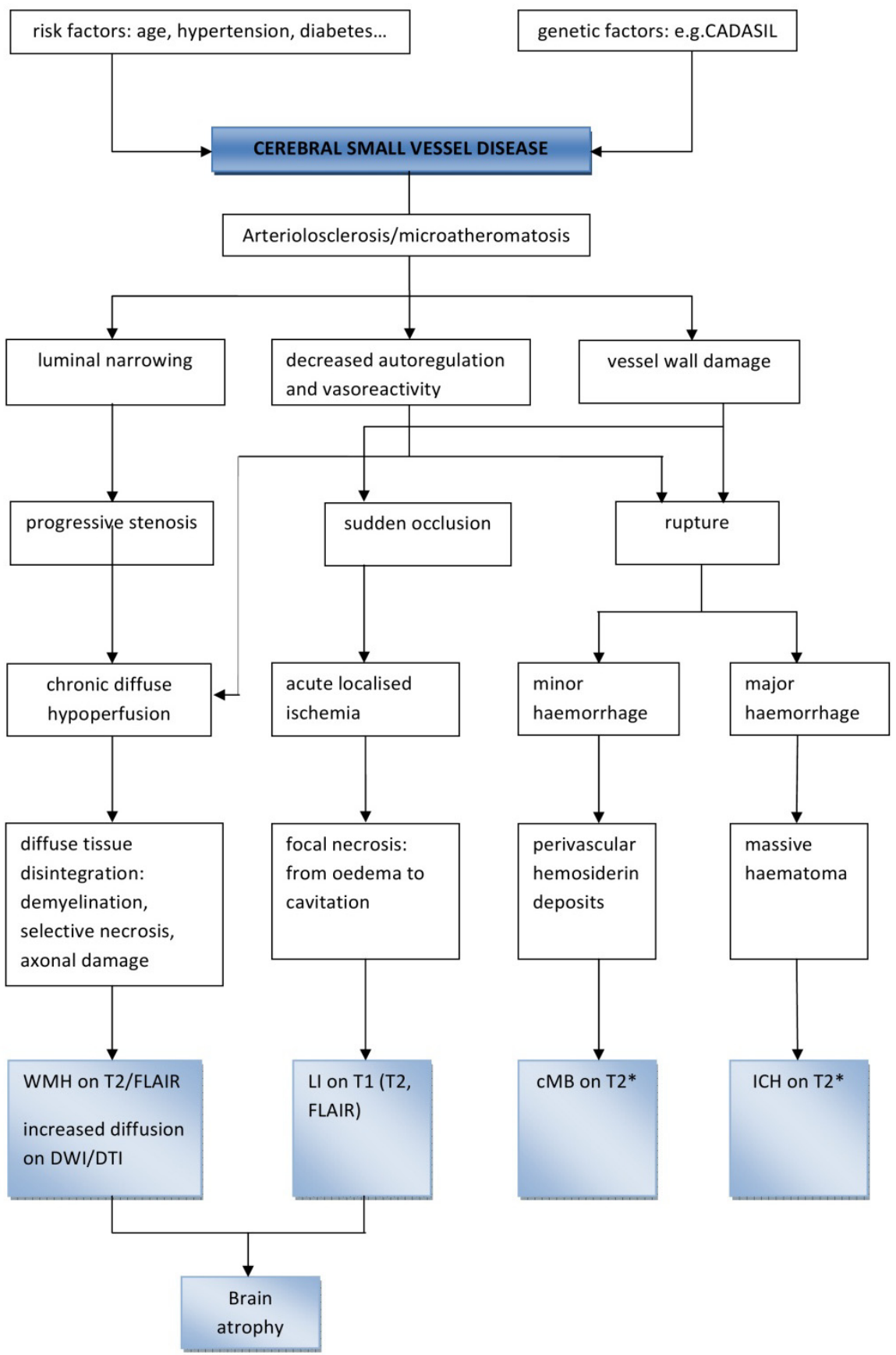

Fig. 1. Pathogenesis of cSVD manifestations. Abbreviations can be found in the text. 
weighted imaging (T1/2WI) have a better spatial resolution, can image the posterior fossa without artifacts and have a better signal/noise ratio. LIs in the chronic stage appear as fluid filled cavities: hypointensities (black holes) on T1WI and hyperintensities on T2WI isointense with CSF. More recent LIs can be seen as hyperintensities on T2WI corresponding to brain tissue with increased water content (oedema). The widely used T2 based fluid attenuation inversion recovery (FLAIR) sequence that nulls the hyperintense signal of free water (mainly CSF) has some advantages over the T2WI. FLAIR is more sensitive in the detection of small, recent infarcts in the proximity of CSF spaces like those in the cortex or next to the ventricles. It can better estimate the age of LIs, because the signal of bulk water in chronic infarcts (cavitations) is nulled as well, whereas the increased bound water content of acute infarcts (solid tissue) is hyperintense ${ }^{22}$. Acute lesions on FLAIR give a relatively stable high signal for several weeks as opposed to the fluctuations in intensity seen on T2W images 23, 24 (Figure 2). However very early ischaemia within the first few hours especially in the lacunar dimension cannot be seen on any of the conventional MR sequences, because the signal abnormality only appears 6-8 hours after symptom onset.

\subsection{DWI}

Acute stage imaging has been revolutionised by the introduction of diffusion weighted imaging (DWI) that shows intracellular cytotoxic oedema resulting from critical cerebral ischaemia within the first few minutes after stroke onset ${ }^{25}$. The energy failure of brain cells results in the accumulation of intracellular bound water leading to a reduced diffusion of free water. This appears as marked hypointensity on the apparent diffusion coefficient (ADC) map which translates into high DWI signal 26-28. In the case of cortical ischemia the reduced ADC returns to normal in 5-10 days 24,29 , while it stays low for a considerably longer period in subcortical disease. Consequently the hyperintensity due to diffusion restriction is also visible for longer ${ }^{30}$. Finally the ADC increases in the chronic stage indicating tissue disintegration/necrosis and vasogenic oedema ${ }^{31}$. At the same time the lesion appearing hyperintense on DWI may remain visible further on as the developing T2 lesion is also seen as high signal (T2 shine through). Therefore DWI and ADC map images have to be interpreted together to judge the age of an ischaemic infarct 32,33 . The sensitivity of this sequence within 6 hours of symptom onset is of $95 \%$ and its specificity is of practically $100 \%$ for territorial infarcts ${ }^{34}$. Although understandably less for small subcortical infarcts, it is still the only reliable tool to visualise hyperacute LIs making it indispensable for acute phase therapy decisions (Figure 2).

\subsection{DTI}

A great proportion of LIs occur along the course of motor pathways whose affection well correlates with the severity of clinical symptoms and mainly determines prognosis. The extent of damage to these pathways can be judged by the diffusion tensor imaging (DTI) that is capable of visualizing white matter tracts ${ }^{35}$. This imaging method is based on the principle that cell membranes constrain the diffusion of water molecules which therefore diffuse longitudinally along axons in the white matter. By measuring diffusion from several directions the net orientation of axons in a voxel of white matter can be determined as a 3 dimensional vector -a "tensor". From these vectors projections of fibres can be generated and displayed as maps of white matter anatomy e.g. in a color-coded way where different colors stand for different directions, and colour brightness for the degree of anisotropy 


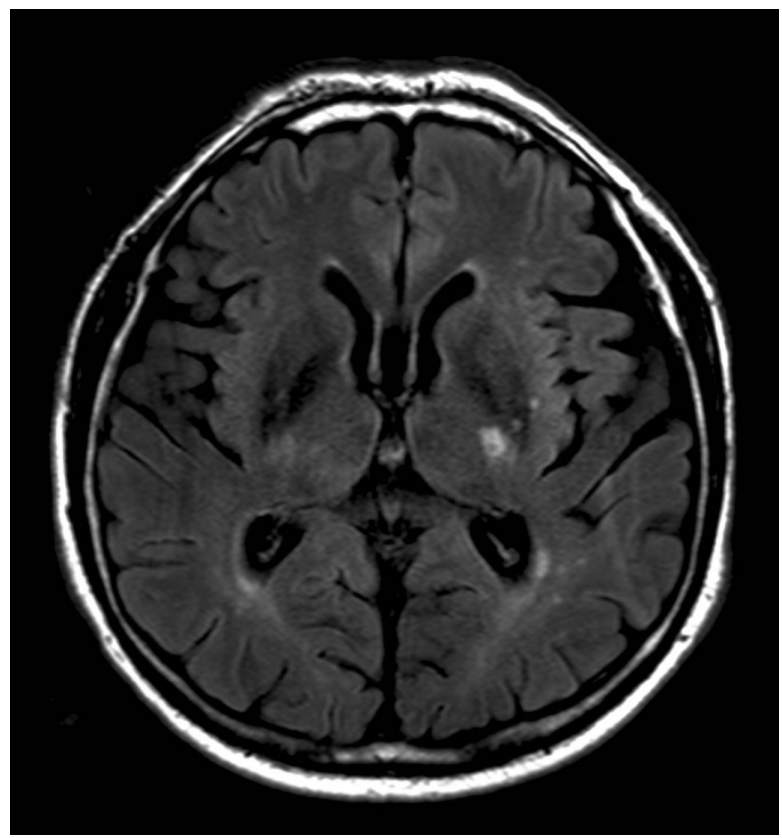

A

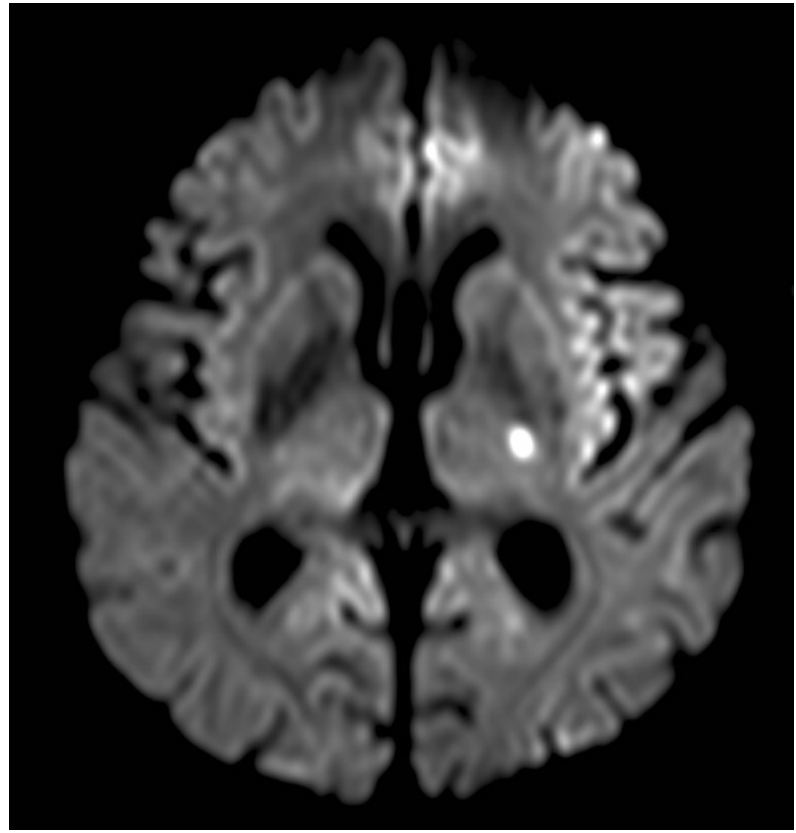

B

Fig. 2. Subacute lacunar infarct in the posterior limb of the left internal capsule in a hypertensive patient on axial FLAIR (A) and DWI trace (B) image. 
(color-coded directional image) ${ }^{36,37}$. Fiber tracking is a further development that enables examiners to visualize fibers passing through a certain region of interest (ROI) or linking two ROIs thus delineating functional systems such as the corticospinal tract (CST). This method has been used to specifically localize small infarcts with regard to the functional pathway of the CST with good topographical accuracy ${ }^{38-40}$. Nevertheless, only a limited number of such tractography studies have been published to date, and few techniques have been assessed for their ability to track through lesions which disrupt tracts.

\subsection{Size criterion}

The size of LIs according to the classical definition is less than $15 \mathrm{~mm}$-a rather arbitrary criterion based on early autopsy studies representing a healed, chronic state ${ }^{18}$. Since then we know that LIs in the acute stage can be significantly larger later undergoing shrinkage by about half their original size ${ }^{41}$. Furthermore surprisingly large infarcts can be caused by single perforator occlusion due to anatomic variations of the branching pattern of the lenticulostriate arteries. More or even all of the penetrating arteries may arise from one common stem 15, 42. Therefore the size criterion for LIs can lead to stroke type misclassification and should be reconsidered ${ }^{43}$.

\subsection{Differentiation of underlying mechanisms}

Apart from cSVD small subcortical infarcts may be caused by emboli of arterial or cardiac origin or critical hypoperfusion in watershed areas due to stenosing large artery disease (LAD). As determining stroke subtype is crucial for further management it is important to make an early etiological diagnosis. Acute lesion patterns on DWI help us to differentiate between the underlying pathomechanisms ${ }^{44}$. The co-existence of a small striatocapsular and one or more distal small cortical lesions points to an embolus originally stuck in the M1 segment obstructing the orifices of the lenticulostriate arteries and later on fragmented and washed further up into one or more small cortical branches of the MCA ${ }^{45,46}$. This scenario is also possible in the posterior circulation -although much less frequently- with the picture of a small brainstem lesion together with a PCA territory thalamic or cortical infarct. Multiple small subcortical lesions in the same vascular territory are associated with LAD (arterioarterial embolism), whereas those in different territories/bilaterally suggest a proximal embolic origin (heart or aortic arch) ${ }^{47}$. In the latter case it is not clear whether they result from repeated embolism or a single embolic shower ${ }^{44}$.

It has also been proposed that multiple small infarcts may also be due to cSVD affecting several vessels contemporaneously ${ }^{48}$. As mentioned earlier subcortical lesions can remain hyperintense on DWI for much longer than cortical ones. Thus several lesions in diffferent vascular territories could arise contemporaneously (i.e. within a few weeks of each other) but not simultaneously and all appear hyperintense on DWI falsely raising the suspicion of an embolic origin. In addition the small perforators arising perpendicularly from large vessels seem hardly accessible for fast moving emboli from an anatomical point of view. Therefore the purely embolic origin of multiple small subcortical DWI lesions in multiple vascular territories remains debated.

Partial borderzone infarcts in the watershed of superficial and deep perforators of the MCA and/or ACA may also appear similar to LIs. They can be seen as a single small lesion or a chain of them (rosary-like pattern) in the centrum semiovale alongside and slightly above the lateral ventricle. The demonstration of ipsilateral carotid artery disease and consequent 
hypoperfusion shown by a perfusion deficit on perfusion weighted imaging (PWI) far exceeding the lesion area leads to diagnosis 49,50 .

\subsection{Differentiation from other small cerebral lesions}

LIs -especially when occurring without overt clinical symptoms, as incidential findingsmay be difficult to distinguish from other hyperintense focal abnormalities on T2 weighted images. Studies correlating these lesions on in vivo and postmortem MR images with brain autopsy findings have identified the following pathologies: silent LIs, dilated VirchowRobin spaces (VRS), foci of demyelination 51 due to incidental multiple sclerosis 52 or insufficient circualtion 53 54, gliosis, minute cysts and ventricular diverticuli 55, 56. Distinction between an infarct, a focal gliosis and a plaque of demyelination is usually impossible on entirely imaging grounds, while the relationship of a diverticulum or cyst to the ventricles and their round shape are differential features 56 .

VRSs are small perivascular spaces surrounding cerebral perforating arteries along their way through the parenchyma serving as drainage pathways for the cerebral interstitial fluid 57. They are small $(<1 \mathrm{~mm})$ CSF isointense foci round shaped in cross section or linear in longitudinal section and run perpendicular to the brain surface ${ }^{58-60}$. Dilated VRSs, that can resemble lacunes, appear as an irregular or ectatic focal expansion of the otherwise regular and smooth VRSs ${ }^{60}$. They are still generally smaller than lacunes usually not exceeding 3 $\mathrm{mm}$ in diameter 61 , whereas lacunes are larger and wedge shaped 59, 60. Dilated VRSs have been associated with ageing 62 , hypertension 63 , widespread white matter lesions 64 , sporadic cSVD 65 61, CADASIL ${ }^{66}$, reduced cognitive function ${ }^{64}$, and vascular dementia ${ }^{65,67}$. However their real clinical significance is a subject of controversy. It is generally accepted that dilated VRSs are related to brain shrinkage around perforating vessels thus representing brain atrophy ${ }^{68}$. In this perspective they can both be regarded as common ageing phenomenon or as a marker of various pathologies. The distinction between normal and pathologically dilated VRSs can be made by judging the appearance of the adjacent brain tissue and the clinical context 60 .

\subsection{Silent cerebral infarcts}

With the increasing use of MRI and the improving image quality an increasing number of patients are found to harbour small cerebral infarcts without any apparent stroke-like symptoms. It has now become clear that LIs only cause clinically evident stroke if they hit main sensorimotor pathways or occur in deep, subcortical nuclei. However the majority of them fall outside of these strategic locations and thus remain silent. Studies have shown that in the general population the prevalence of silent infarcts is fivefold higher than that of stroke, and they can be present in more than one fourth of people over 60 years of age 69-72. They have approximately the same risk factors as symptomatic lacunes with hypertension being the most important 71, 72; and their presence more than doubles the risk of subsequent vascular events, cognitive impairment and dementia 4,73 . The extent of asymptomatic small vessel disease at the time of index stroke has a significant prognostic value for all outcomes 41,73 . These findings have led to a modified understanding of cerebrovascular disease according to which strokes and TIAs -i.e. overt clinical symptoms- are only the tip of the iceberg of cSVD manifestations ${ }^{21}$. Silent infarcts are the underwater majority. It has not yet been evaluated though - and remains doubtful at present- whether the same diagnostic workup and risk factor management would be 
justifiable upon finding a silent infarct as for a clinical stroke. Although by definition silent infarcts lack clinically overt stroke symptoms they progressively lead to less evident cognitive dysfunction, general physical disability and depression. Therefore these infarcts should be referred to as "covert" rather than "silent"4, 74 .

\section{White matter lesions}

\subsection{Definition}

White matter lesions seen in elderly patients and those with arterial hypertension are usually bilateral and more or less symmetrical areas of increased signal on T2 and FLAIR images (hence the name: white matter hyperintensities, $\mathrm{WMH}$ ) located in the hemispheric deep white matter, the basal ganglia and the pons (Figure 3). The term "leukoaraiosis" meaning rarefaction of white matter is a description from the CT era of the same phenomenon ${ }^{75}$. WMHs are generally regarded as a consequence of ischemic brain tissue disintegration due to cSVD. Pathological studies found varying degrees of tissue damage appearing as WMH: from selective loss of myelin, to loss of myelin, axons and oligodendroglia consistent with incomplete infarcts, to near complete infarcts with astrogliosis $76-79$.

\subsection{Differential diagnosis}

Multifocal or diffuse white matter lesions resembling those caused by cSVD can be found in a wide range of central nervous system (CNS) pathologies. These are summarized in Table 1. Their differential diagnosis is based on the complex evaluation of patient history, clinical context, other diagnostic tests and some differences in MRI appearance. Some of these WMHs are also ischemic in origin such as those caused by hypoperfusion 1. in watershed areas due to large artery stenosis, or 2 . in different vascular territories due to various types of CNS vasculitis. These latter can occur either as an isolated CNS affection (primary CNS vasculitis), or as part of a systemic disease (SLE, Sjörgen syndrome, Behcet disease, antiphospholipid syndrome, sarcoidosis etc.) Others are a consequence of multifocal demyelination in multiple sclerosis (MS) and its variants or in central pontine/extrapontine myelinolysis. As opposed to cSVD MS is characterized by ovoidshaped lesions perpendicular to the ventricles (Dawson fingers), frequently found in the corpus callosum, some of which may enhance contrast material. Plaques may also be located in the optic nerves, cerebellum and spinal cord. WMH caused by transient vasogenic edema due to the neurotoxic effect of various complex conditions (preeclampsia/eclampsia, severe hypertension, allogenic bone marrow transplantation, organ transplantation, autoimmune diseases and high dose chemotherapy) has been termed as Posterior reversible encephalopathy syndrome (PRES). The typical pattern of WMH in PRES resembles the watershed zones with a parietal and occipital (posterior) predominance. The subcortical white matter but also the cortex is involved to varying degrees and the lesions always regress ${ }^{80}$. White matter lesions of unclear nature can be seen in some infective diseases / postinfective conditions such as HIV, Lyme-disease or Syphilis related encephalopathies, Progressive multifocal leukoencephalopathy (PML), Subacute sclerosing panencephalitis (SSPE) or Acute disseminated encephalomyelitis (ADEM); and metabolic disorders like leukodystrophies, phenylketonuria and mitochondrial diseases (MELAS) ${ }^{81,82}$. 


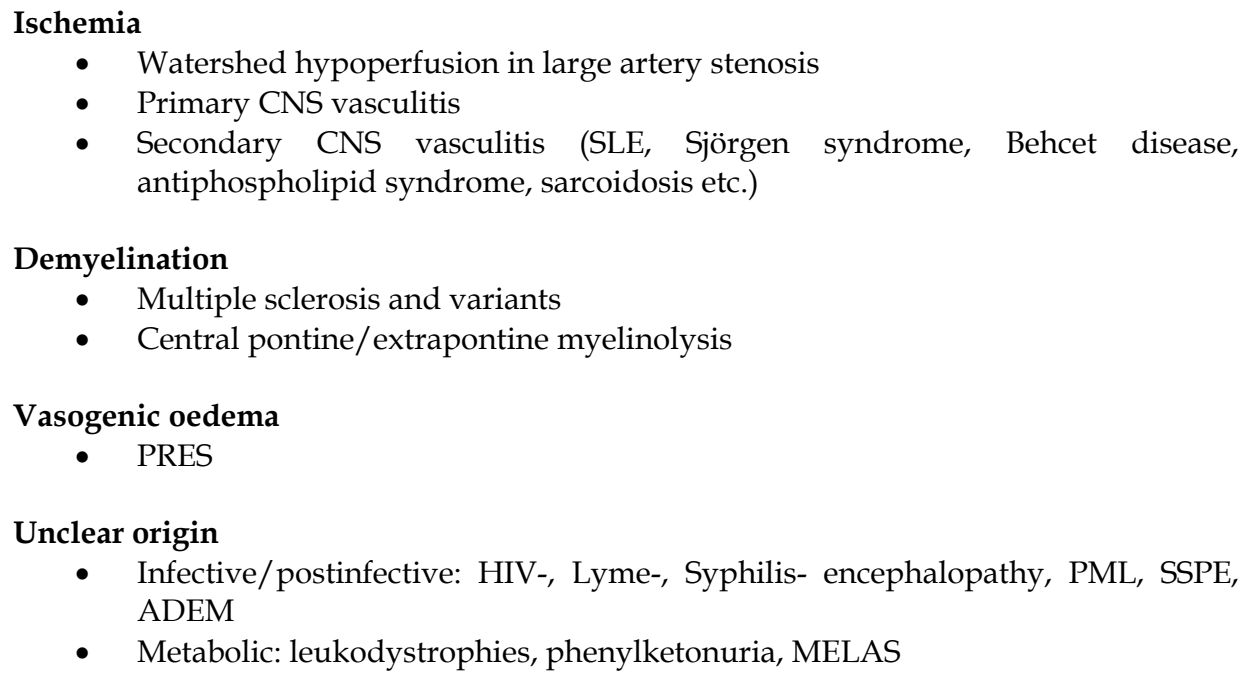

\section{Demyelination}

- Multiple sclerosis and variants

- Central pontine/extrapontine myelinolysis

\section{Vasogenic oedema}

- PRES

\section{Unclear origin}

- Infective/postinfective: HIV-, Lyme-, Syphilis- encephalopathy, PML, SSPE, ADEM

- $\quad$ Metabolic: leukodystrophies, phenylketonuria, MELAS

Table 1. CNS pathologies causing multiple/diffuse white matter lesions. Abbreviations can be found in the text.

\subsection{Evaluation with conventional MRI}

The severity of white matter damage on T2 and FLAIR images can be assessed semiquantitatively by various visual rating scales (proposed by Fazekas ${ }^{83}$, Schmidt ${ }^{84}$, Scheltens ${ }^{85}$, Wahlund ${ }^{86}$ and others) that take into account the location, pattern and extension of $\mathrm{WMH}^{87}$. The mostly used Fazekas-scale which evaluates $\mathrm{WMH}$ in two distinct locations: periventricular and deep subcortical white matter is presented in Table 2 . The mildest forms are seen as smooth periventricular and punctuate deep WMH, whereas irregular periventricular, early confluent and confluent deep WMH represent an increasing severity of tissue damage. Furthermore the three dimensional extension of $\mathrm{WMH}$ can be quantified by volumetric evaluation 88,89 . WMH volumetry is more reproducible and more sensitive for lesion progression than visual scales ${ }^{90}$. However the severity of white matter lesions as assessed by any of the above methods showed only moderate correlations with the clinical status represented by scores of disability and cognitive impairment ${ }^{91-93}$.

\begin{tabular}{|l|l|l|}
\hline & periventricular & deep subcortical \\
\hline $\mathbf{0}$ & absence & no or a single punctate lesion \\
\hline $\mathbf{1}$ & "caps" or pencil-thin lining & multiple punctate lesions \\
\hline $\mathbf{2}$ & smooth „halo" & beginning confluency of lesions \\
\hline $\mathbf{3}$ & irregular PVH extending into deep WM & large confluent lesions \\
\hline
\end{tabular}

Table 2. Fazekas visual rating scale for $\mathrm{WMH}$ (0-6 points $)^{83}$ 


\subsection{Evaluation with non-conventional MRI}

The whole spectrum of microscopic brain tissue changes due to CSVD appears uniformly as $\mathrm{WMH}$ on conventional T2 based MR sequences. In order to obtain information on the degree of underlying tissue damage, non-conventional MRI techniques have been developed such as T1- and T2 relaxation time mapping, magnetisation transfer imaging (MTR) and diffusion tensor imaging (DTI) ${ }^{94}, 95$. This latter technique, that measures the degree and orientation of tissue water diffusivity, has been widely used in various cerebral diseases and conditions including cSVD ${ }^{96}$. As diffusivity partly depends on the density of cells in a given tissue volume (cell membranes and intracellular particles restrict water diffusion), the increase in diffusivity (as measured by a non-oriented derivate of the tensor, the mean diffusivity, MD) is proportional to the degree of ultrastructural tissue disintegration ${ }^{97}$, 98. Region of interest (ROI) based measurements detected increased MD inside but also outside of $\mathrm{WMH}$, in the normal appearing white and subcortical grey matter ${ }^{99}, 100$. DTI can thus show tissue damage "invisible" for conventional MRI. In diffuse cerebral pathologies however -such as cSVD - a global, approach of whole brain diffusion histograms is more informative about the overall disease severity than a ROI analysis. Accordingly, MD histogram parameters have been reported to correlate more with clinical scores than $\mathrm{WMH}$ visual rating scales and volumetric data in cSVD both cross sectionally and longitudinally. Furthermore they were more sensitive than clinical scales in detecting change over time 101-106. There are data indicating that the much simpler, quicker and widely available (DWI) derived ADC can be used similarly to DTI derived MD to quantify brain damage due to cSVD (findings of Gunda et al. to be published)(Figure 3 and 4). Therefore these quantitative MRI techniques seem to be a promising tool in the quantified monitoring of cSVD and could possibly act as surrogate markers in future therapeutic trials.

\section{Cerebral microbleeds}

\subsection{Definition}

Gradient echo (or T2* weighted) imaging is a sequence highly sensitive of blood. With its increasing use the number of visible haemorrhagic brain lesions has grown considerably and even millimetre-sized bleedings in the parenchyma have become detectable. Cerebral microbleeds (cMB) appear as small $(<5 \mathrm{~mm})$, homogenous, rounded foci of low signal intensity on $\mathrm{T}^{*}{ }^{*}$ images ${ }^{107}$ (Figure 5 ). The signal loss is caused by hemosiderin -a paramagnetic blood degradation product that remains in macrophages for several years after haemorrhage indicating previous blood extravasation ${ }^{108}$. Thus the age of cMBs cannot be determined by MRI but the total haemorrhage burden can be assessed. Cerebral microbleeds appear larger on $\mathrm{T} 2 *$ images than the real tissue lesions due to the "blooming effect" of the MR signal ${ }^{109}$. The few studies relating cMBs on MRI to histopathological findings revealed focal hemosiderin deposits from the rupture of small vessels showing evidence of arteriolosclerosis or occasionally amyloid angiopathy clearly indicating an underlying small vessel pathology110,111.

\subsection{Differential diagnosis}

CMBs need to be distinguished from other causes of focal signal loss on $\mathrm{T} 2 *$ images. These include: flow voids of small arteries in cross-section that can be followed on 


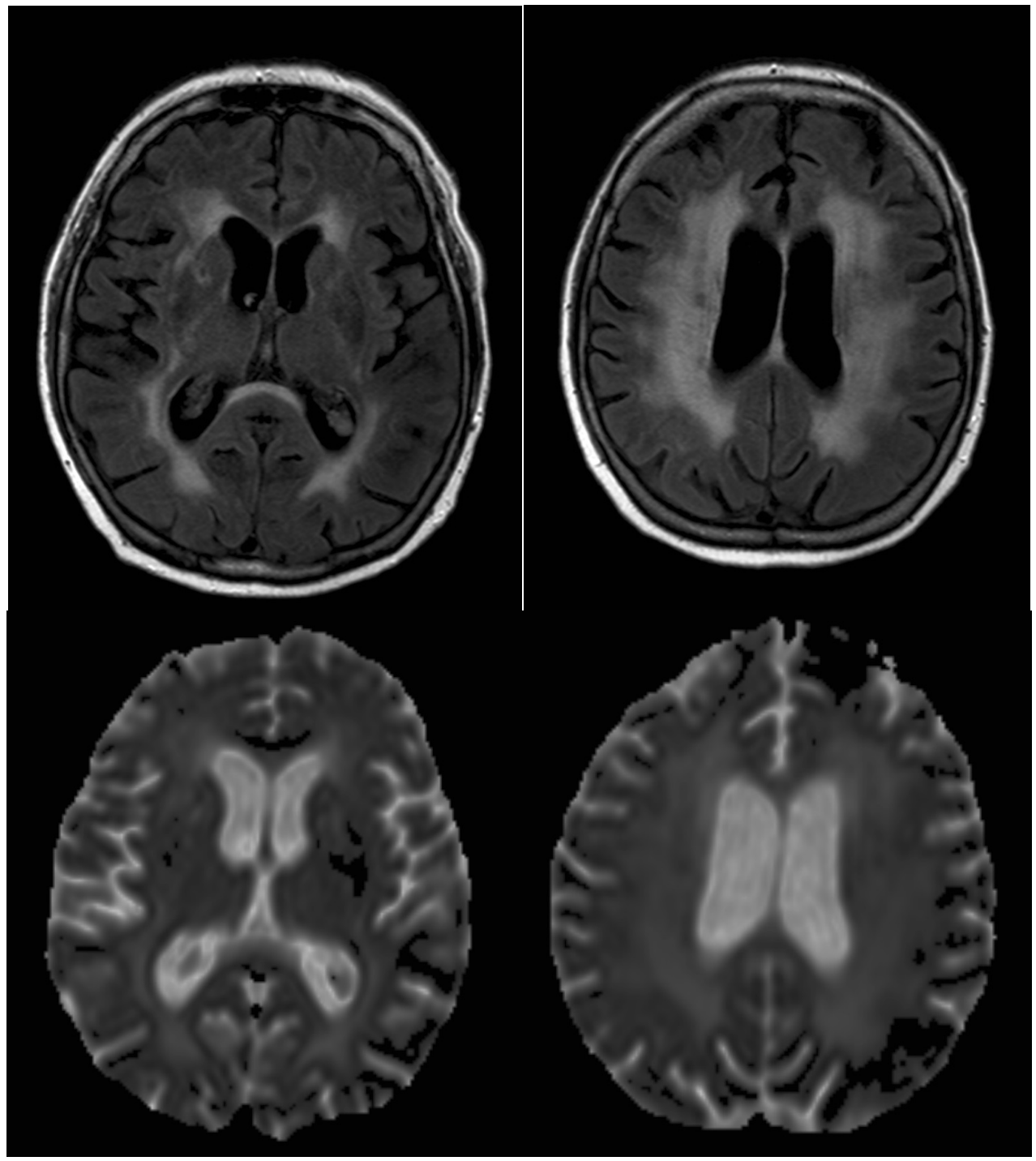

Fig. 3. Diffuse white matter lesions in a 70 year-old hypertensive patient on axial FLAIR image (upper row) and ADC map (lower row). Note the increased diffusion (ADC) corresponding to areas of WMH (FLAIR).

consecutive/neighbouring slices; the usually symmetrical calcifications or iron deposits in the globi pallidi that appear hyperdense on CT; type IV cavernous malformations and capillary teleangiectasias; foci of hypointensity compatible with hemorrhagic shear injury in head trauma, and even artefacts of metallic materials released from mechanical heart valves112, 113. Etiological differentiation of signal loss is based on the location, number and distribution of lesions, associated imaging findings and patient history. 


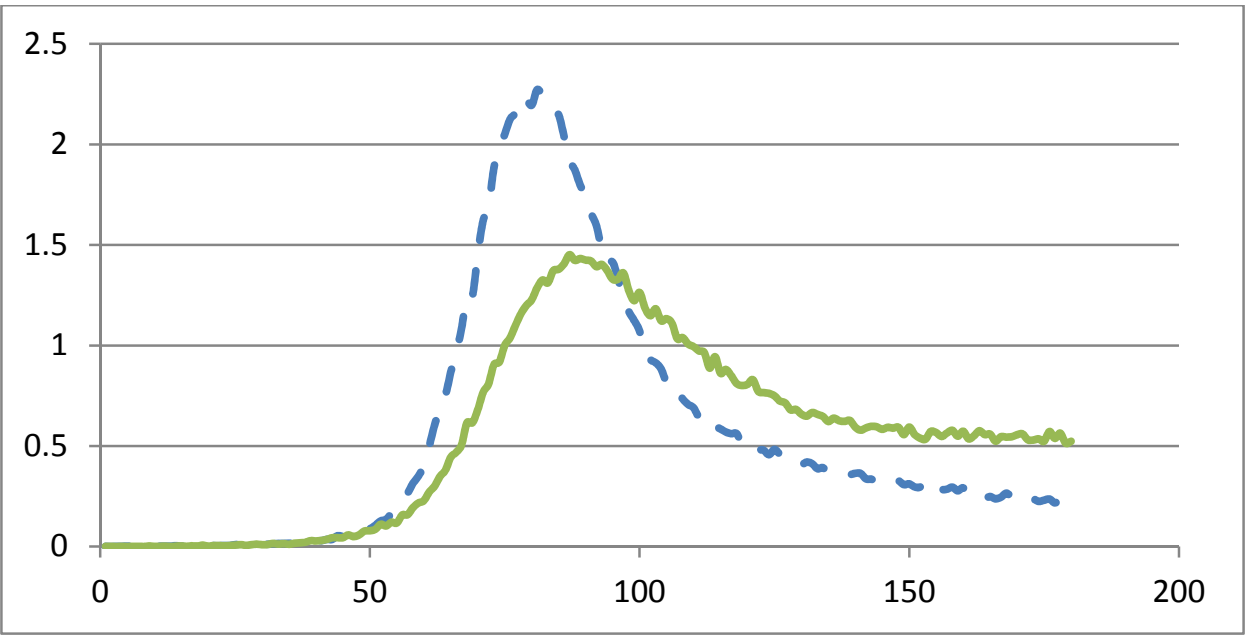

Fig. 4. ADC histogram of the patient on Figure 3 (green line) compared to a normal control (dashed blue line) (X: diffusivity in $10^{-5} \mathrm{~mm}^{2} / \mathrm{s}$, thresholded at $180 ; \mathrm{Y}$ : relative frequency of voxels in \%).

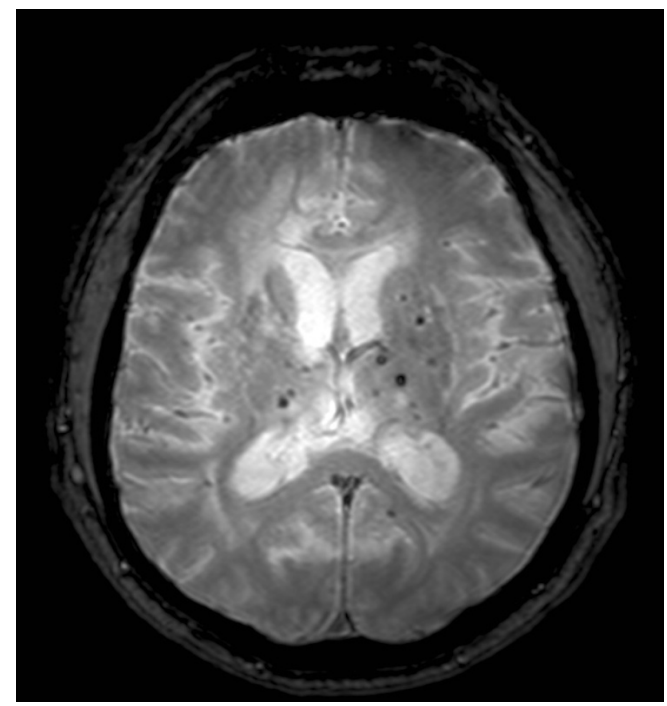

Fig. 5. Multiple cMBs in a 48 year-old hypertensive patient on axial T2* image.

\subsection{Epidemiology}

CMBs have been found in various patient populations as well as healthy elderly. Their occurrence was the most frequent in patients with intracerebral haemorrhage (ICH) and lacunar infarcts (due to hereditary or sporadic cSVD), less so in ischemic stroke patients of other subtypes. The most comprehensive review on cMB published in 2007 pooled data from comparable studies and found the overall prevalence of cMBs to be $5 \%$ among healthy 
adults; $34 \%$ in ischemic stroke patients; and $60 \%$ in patients with $\mathrm{ICH}$. The prevalence according to ischemic stroke subtype was $54 \%$ in lacunar-, $36 \%$ in atherothrombotic - and $19 \%$ in cardioembolic stroke. $38 \%$ of CADASIL patients had cMB. CMBs were more prevalent among patients with recurrent than first-ever stroke ( 44 vs $23 \%$ for ischemic and 83 vs $52 \%$ for haemorrhagic stroke) ${ }^{114}$.

\subsection{Clinical significance}

CMBs were found to be associated with age, hypertension, other manifestations of cSVD (lacunar infarcts and WML), previous ischemic stroke and $\mathrm{ICH}$, and an increased risk of recurrent lacunar infarct or $\mathrm{ICH}$ in those with lacunar infarct or $\mathrm{ICH}^{114}$. These findings further emphasize the common pathophysiological basis for cMB, LI, WML and ICH. Studies have shown that the anatomical distribution of ICHs is similar to that of cMBs in individual patients, but it is not the pre-existing cMBs that evolve into major haemorrhages ${ }^{115}$. Similarly several cases have been reported where patients with cMBs developed major haemorrhage after thrombolysis or antiplatelet therapy remote from the cMBs 116 . Thus cMBs can be considered as markers of a diffuse, bleeding-prone microangiopathy. This raised the important question whether patients with $\mathrm{cMB}$ are at an increased risk of $\mathrm{ICH}$ when treated with antiplatelet, anticoagulant or thrombolytic agents. For the time being there is no sufficient evidence to give a definite answer (some studies reported an increased risk, others not, all of them underpowered to draw firm conclusions) ${ }^{116-120}$. However some stroke centres already incorporate $\mathrm{cMBs}$ in their treatment decisions.

In conclusion cMBs are markers of a haemorrhage-prone cSVD and predictors of recurrent vascular events (be it ischemic or haemorrhagic). At present they cannot be considered as a contraindication to antithrombotic or thrombolytic therapies, but may play a role in the individual stratification of haemorrhagic risk, and may be incorporated in the design of clinical trials of anticoagulation/antiaggregation drugs.

\section{Brain atrophy}

Brain atrophy is best evaluated on T1WI and appears as shrinkage of brain parenchyma with a reduction of cortical thickness and an increase of internal and external CSF spaces. It can be assessed by visually rating the degree of ventricular dilatation and sulcal widening, by measuring the width of sulci or ventricles in a standard location, or by different threedimensional volumetric methods that have now become the methods of choice.

Brain atrophy is a common phenomenon in normal ageing that increases progressively beyond the age of 65 years ${ }^{121}$. This process can be accelerated by numerous cerebral pathologies causing diffuse brain tissue loss such as degenerative diseases (like Alzheimer's disease and other primary dementias) ${ }^{122}$, demyelinating diseases (MS) ${ }^{123}$ and cerebrovascular disorders (Figure 6). In these latter conditions, the importance of brain atrophy has only recently been recognised. A number of imaging studies using quantitative brain volumetry demonstrated atrophy in both focal and diffuse cerebrovascular diseases ${ }^{124-}$ 127. Brain atrophy correlated strongly with the clinical status and cognitive scores, and proved to be a sensitive marker of disease progression in CSVD $104,128,129$. It is now widely accepted that purely subcortical cSVD can lead to cortical volume loss 129,130 . How subcortical ischemic damage leads to cortical atrophy is not fully elucidated, but the diffuse and/or remote effect of lacunar lesions and tissue microstructural changes through Wallerian degeneration, secondary axonal loss due to deinervation and local or remote neuronal apoptosis are possible mechanisms $20,124,131,132$. 

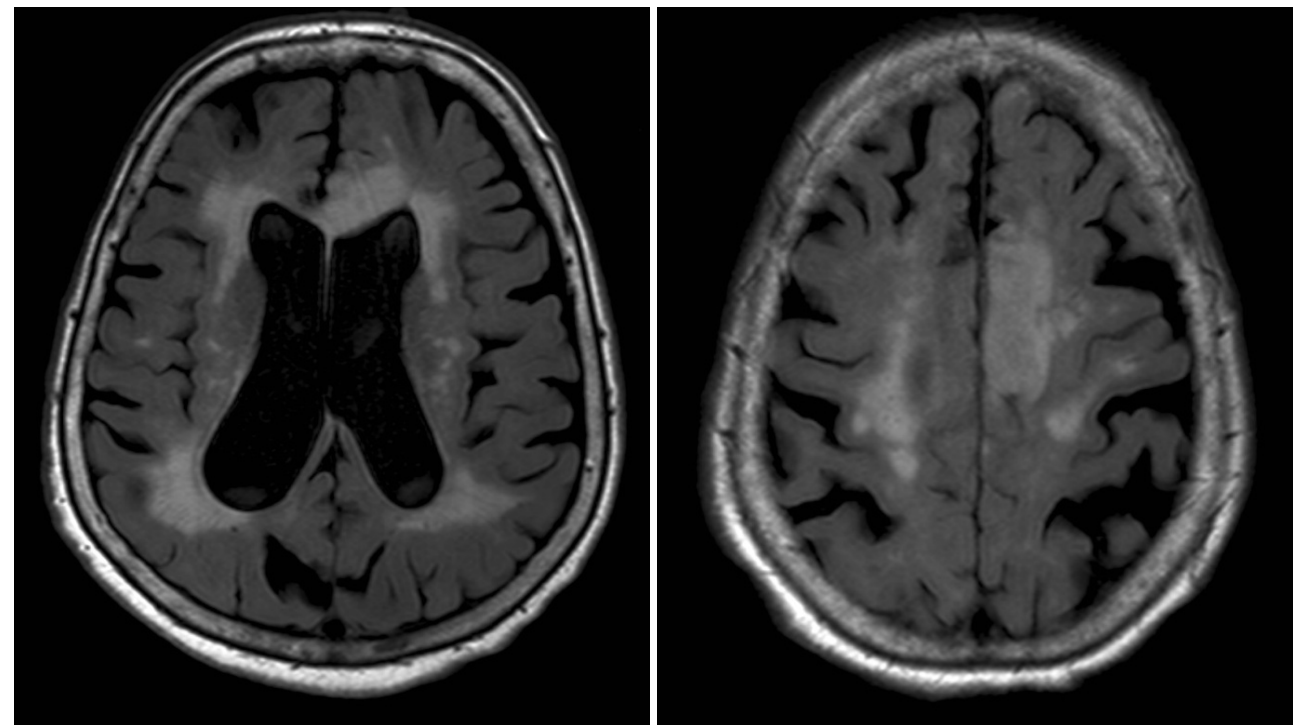

Fig. 6. Widespread WMH and diffuse brain atrophy in a 89 year-old hypertensive patient on axial FLAIR images.
T1
- cavitated, chronic LIs appear hypointens („black hole”)
- good for evaluation of brain atrophy and cortical thickness, volumetry as surrogate marker
T2
- $\quad$ subacute LIs appear hyperintens (fluctuating); good visualisation of VRS
FLAIR
- white matter damage appears as WMH; good for judging deep WMH
- (sub)acute LIs give more stable high signal; good for detection of periventricular LIs, differentiates acute (hyperintens) from chronic (hypointens) LIs
- white matter damage appears as $\mathrm{WMH}$; good for judging both deep and periventricular $\mathrm{WMH}$
T2*
DWI
cMBs appear as small, hypointense foci, marker of bleeding-prone microangiopathy
- the only method to visualize (hyper)acute LIs that give high signal on DWI, low signal on ADC; acute lesion patterns guide differential diagnosis
- chronic LI gives low signal on DWI, ultrastructural tissue damage causes increased diffusivity (high signal on ADC map), whole brain ADC histogram as surrogate marker?
DTI
- localisation of LIs in relation to WM tracts (tractography)
- ultrastructural tissue damage causes increased diffusivity (high signal on MD map), whole brain MD histogram as surrogate marker

Table 3. Utility of different MRI sequences in cSVD. Abbreviations can be found in the text. 
Brain atrophy is an aspecific finding and can be regarded as the final common pathway in the pathophysiology of various cerebral pathologies. As in degenerative diseases, atrophy is now recognized as a strong marker of disease progression in cSVD and thus could serve as a surrogate marker in future clinical trials similarly to whole brain diffusion histogram parameters ${ }^{133}$.

\section{Conclusions, perspectives}

New and continuously developing MRI sequences and postprocessing techniques have greatly helped to explore and better understand cSVD. Diffusion MRI methods have proved to be particularly useful in: i. visualizing hyperacute LIs thus guiding acute phase therapy and etiologic diagnosis (DWI); ii. detecting ultrastructural changes even in otherwise normal appearing WM, and quantifying the global burden of tissue damage in cSVD (whole brain DTI/DWI histogram measures). Brain atrophy -a phenomenon previously considered to be related to cortical disease- is now recognised as a marker of cSVD based on studies using volumetric measures. In the future an increasing use of quantitative MRI techniques (diffusion histograms, volumetry) can be expected as they are more sensitive to the full spectrum of cSVD expressions, and could provide surrogate markers for disease progression in future therapeutic trials for patients with cSVD. The utility of different MRI sequences in cSVD is summarized in Table 3.

\section{Acknowledgements}

The work was partly supported by grants No. ETT 158/2009, and TAMOP-4.2.1.B09/1/KMR.

\section{References}

[1] Lammie GA. Pathology of small vessel stroke. Br Med Bull. 2000;56:296-306

[2] Hachinski V. World stroke day 2008: "Little strokes, big trouble". Stroke. 2008;39:24072420

[3] Thompson CS, Hakim AM. Living beyond our physiological means: Small vessel disease of the brain is an expression of a systemic failure in arteriolar function: A unifying hypothesis. Stroke. 2009;40:e322-330

[4] Vermeer SE, Longstreth WT, Jr., Koudstaal PJ. Silent brain infarcts: A systematic review. Lancet Neurol. 2007;6:611-619

[5] Bryan RN, Wells SW, Miller TJ, Elster AD, Jungreis CA, Poirier VC, Lind BK, Manolio TA. Infarctlike lesions in the brain: Prevalence and anatomic characteristics at $\mathrm{mr}$ imaging of the elderly--data from the cardiovascular health study. Radiology. 1997;202:47-54

[6] Bamford J, Sandercock P, Jones L, Warlow C. The natural history of lacunar infarction: The oxfordshire community stroke project. Stroke. 1987;18:545-551

[7] Petty GW, Brown RD, Jr., Whisnant JP, Sicks JD, O'Fallon WM, Wiebers DO. Ischemic stroke subtypes: A population-based study of incidence and risk factors. Stroke. 1999;30:2513-2516 
[8] Sacco S, Marini C, Totaro R, Russo T, Cerone D, Carolei A. A population-based study of the incidence and prognosis of lacunar stroke. Neurology. 2006;66:1335-1338

[9] Joutel A, Corpechot C, Ducros A, Vahedi K, Chabriat H, Mouton P, Alamowitch S, Domenga V, Cecillion M, Marechal E, Maciazek J, Vayssiere C, Cruaud C, Cabanis EA, Ruchoux MM, Weissenbach J, Bach JF, Bousser MG, Tournier-Lasserve E. Notch3 mutations in cadasil, a hereditary adult-onset condition causing stroke and dementia. Nature. 1996;383:707-710

[10] Artavanis-Tsakonas S, Rand MD, Lake RJ. Notch signaling: Cell fate control and signal integration in development. Science. 1999;284:770-776

[11] Chabriat H, Joutel A, Dichgans M, Tournier-Lasserve E, Bousser MG. Cadasil. Lancet Neurol. 2009;8:643-653

[12] Pantoni L. Cerebral small vessel disease: From pathogenesis and clinical characteristics to therapeutic challenges. Lancet Neurol.9:689-701

[13] Herman LH, Ostrowski AZ, Gurdjian ES. Perforating branches of the middle cerebral artery. An anatomical study. Arch Neurol. 1963;8:32-34

[14] Kaplan HA. The lateral perforating branches of the anterior and middle cerebral arteries. J Neurosurg. 1965;23:305-310

[15] Marinkovic SV, Milisavljevic MM, Kovacevic MS, Stevic ZD. Perforating branches of the middle cerebral artery. Microanatomy and clinical significance of their intracerebral segments. Stroke. 1985;16:1022-1029

[16] Umansky F, Gomes FB, Dujovny M, Diaz FG, Ausman JI, Mirchandani HG, Berman SK. The perforating branches of the middle cerebral artery. A microanatomical study. J Neurosurg. 1985;62:261-268

[17] Pantoni L. Pathophysiology of age-related cerebral white matter changes. Cerebrovasc Dis. 2002;13 Suppl 2:7-10

[18] Fisher CM. Lacunar strokes and infarcts: A review. Neurology. 1982;32:871-876

[19] Greenberg SM, Nandigam RN, Delgado P, Betensky RA, Rosand J, Viswanathan A, Frosch MP, Smith EE. Microbleeds versus macrobleeds: Evidence for distinct entities. Stroke. 2009;40:2382-2386

[20] Jouvent E, Viswanathan A, Mangin JF, O'Sullivan M, Guichard JP, Gschwendtner A, Cumurciuc R, Buffon F, Peters N, Pachai C, Bousser MG, Dichgans M, Chabriat H. Brain atrophy is related to lacunar lesions and tissue microstructural changes in cadasil. Stroke. 2007;38:1786-1790

[21] Gunda B VG, Rudas G, Bereczki D. Challenges in diagnosing cerebral lacunar infarcts. CMIR. 2009;5:75-84

[22] Brant-Zawadzki M, Atkinson D, Detrick M, Bradley WG, Scidmore G. Fluid-attenuated inversion recovery (flair) for assessment of cerebral infarction. Initial clinical experience in 50 patients. Stroke. 1996;27:1187-1191

[23] Ricci PE, Burdette JH, Elster AD, Reboussin DM. A comparison of fast spin-echo, fluidattenuated inversion-recovery, and diffusion-weighted $\mathrm{mr}$ imaging in the first 10 days after cerebral infarction. AJNR Am J Neuroradiol. 1999;20:1535-1542

[24] Lansberg MG, Thijs VN, O'Brien MW, Ali JO, de Crespigny AJ, Tong DC, Moseley ME, Albers GW. Evolution of apparent diffusion coefficient, diffusion-weighted, and $\mathrm{t} 2-$ weighted signal intensity of acute stroke. AJNR Am J Neuroradiol. 2001;22:637-644 
[25] Hjort N, Christensen S, Solling C, Ashkanian M, Wu O, Rohl L, Gyldensted C, Andersen G, Ostergaard L. Ischemic injury detected by diffusion imaging 11 minutes after stroke. Ann Neurol. 2005;58:462-465

[26] Guadagno JV, Jones PS, Fryer TD, Barret O, Aigbirhio FI, Carpenter TA, Price CJ, Gillard JH, Warburton EA, Baron JC. Local relationships between restricted water diffusion and oxygen consumption in the ischemic human brain. Stroke. 2006;37:1741-1748

[27] Hoehn-Berlage M, Norris DG, Kohno K, Mies G, Leibfritz D, Hossmann KA. Evolution of regional changes in apparent diffusion coefficient during focal ischemia of rat brain: The relationship of quantitative diffusion $\mathrm{nmr}$ imaging to reduction in cerebral blood flow and metabolic disturbances. J Cereb Blood Flow Metab. 1995;15:1002-1011

[28] Lin W, Lee JM, Lee YZ, Vo KD, Pilgram T, Hsu CY. Temporal relationship between apparent diffusion coefficient and absolute measurements of cerebral blood flow in acute stroke patients. Stroke. 2003;34:64-70

[29] Schlaug G, Siewert B, Benfield A, Edelman RR, Warach S. Time course of the apparent diffusion coefficient (adc) abnormality in human stroke. Neurology. 1997;49:113-119

[30] Munoz Maniega S, Bastin ME, Armitage PA. A quantitative comparison of two methods to correct eddy current-induced distortions in dt-mri. Magn Reson Imaging. 2007;25:341-349

[31] Knight RA, Dereski MO, Helpern JA, Ordidge RJ, Chopp M. Magnetic resonance imaging assessment of evolving focal cerebral ischemia. Comparison with histopathology in rats. Stroke. 1994;25:1252-1261; discussion 1261-1252

[32] Burdette JH, Elster AD, Ricci PE. Acute cerebral infarction: Quantification of spindensity and $\mathrm{t} 2$ shine-through phenomena on diffusion-weighted $\mathrm{mr}$ images. Radiology. 1999;212:333-339

[33] Geijer B, Sundgren PC, Lindgren A, Brockstedt S, Stahlberg F, Holtas S. The value of b required to avoid $\mathrm{t} 2$ shine-through from old lucunar infarcts in diffusion-weighted imaging. Neuroradiology. 2001;43:511-517

[34] Lovblad KO, Laubach HJ, Baird AE, Curtin F, Schlaug G, Edelman RR, Warach S. Clinical experience with diffusion-weighted $\mathrm{mr}$ in patients with acute stroke. AJNR Am J Neuroradiol. 1998;19:1061-1066

[35] Lie C, Hirsch JG, Rossmanith C, Hennerici MG, Gass A. Clinicotopographical correlation of corticospinal tract stroke: A color-coded diffusion tensor imaging study. Stroke. 2004;35:86-92

[36] Pajevic S, Pierpaoli C. Color schemes to represent the orientation of anisotropic tissues from diffusion tensor data: Application to white matter fiber tract mapping in the human brain. Magn Reson Med. 1999;42:526-540

[37] Wakana S, Jiang H, Nagae-Poetscher LM, van Zijl PC, Mori S. Fiber tract-based atlas of human white matter anatomy. Radiology. 2004;230:77-87

[38] Lai C, Zhang SZ, Liu HM, Zhou YB, Zhang YY, Zhang QW, Han GC. White matter tractography by diffusion tensor imaging plays an important role in prognosis estimation of acute lacunar infarctions. Br J Radiol. 2007;80:782-789 
[39] Lee JS, Han MK, Kim SH, Kwon OK, Kim JH. Fiber tracking by diffusion tensor imaging in corticospinal tract stroke: Topographical correlation with clinical symptoms. Neuroimage. 2005;26:771-776

[40] Yamada K, Mori S, Nakamura H, Ito H, Kizu O, Shiga K, Yoshikawa K, Makino M, Yuen S, Kubota T, Tanaka O, Nishimura T. Fiber-tracking method reveals sensorimotor pathway involvement in stroke patients. Stroke. 2003;34:E159-162

[41] Norrving B. Lacunar infarcts: No black holes in the brain are benign. Pract Neurol. 2008;8:222-228

[42] Cho AH, Kang DW, Kwon SU, Kim JS. Is $15 \mathrm{~mm}$ size criterion for lacunar infarction still valid? A study on strictly subcortical middle cerebral artery territory infarction using diffusion-weighted mri. Cerebrovasc Dis. 2007;23:14-19

[43] Kang DW, Chalela JA, Ezzeddine MA, Warach S. Association of ischemic lesion patterns on early diffusion-weighted imaging with toast stroke subtypes. Arch Neurol. 2003;60:1730-1734

[44] Wessels T, Rottger C, Jauss M, Kaps M, Traupe H, Stolz E. Identification of embolic stroke patterns by diffusion-weighted mri in clinically defined lacunar stroke syndromes. Stroke. 2005;36:757-761

[45] Donnan GA, Bladin PF, Berkovic SF, Longley WA, Saling MM. The stroke syndrome of striatocapsular infarction. Brain. 1991;114 ( Pt 1A):51-70

[46] Gerraty RP, Parsons MW, Barber PA, Darby DG, Desmond PM, Tress BM, Davis SM. Examining the lacunar hypothesis with diffusion and perfusion magnetic resonance imaging. Stroke. 2002;33:2019-2024

[47] Wessels T, Wessels C, Ellsiepen A, Reuter I, Trittmacher S, Stolz E, Jauss M. Contribution of diffusion-weighted imaging in determination of stroke etiology. AJNR Am J Neuroradiol. 2006;27:35-39

[48] Chowdhury D, Wardlaw JM, Dennis MS. Are multiple acute small subcortical infarctions caused by embolic mechanisms? J Neurol Neurosurg Psychiatry. 2004;75:1416-1420

[49] Momjian-Mayor I, Baron JC. The pathophysiology of watershed infarction in internal carotid artery disease: Review of cerebral perfusion studies. Stroke. 2005;36:567-577

[50] Krapf H, Widder B, Skalej M. Small rosarylike infarctions in the centrum ovale suggest hemodynamic failure. AJNR Am J Neuroradiol. 1998;19:1479-1484

[51] Scarpelli M, Salvolini U, Diamanti L, Montironi R, Chiaromoni L, Maricotti M. Mri and pathological examination of post-mortem brains: The problem of white matter high signal areas. Neuroradiology. 1994;36:393-398

[52] Gilbert JJ, Sadler M. Unsuspected multiple sclerosis. Arch Neurol. 1983;40:533-536

[53] Kirkpatrick JB, Hayman LA. White-matter lesions in mr imaging of clinically healthy brains of elderly subjects: Possible pathologic basis. Radiology. 1987;162:509-511

[54] Munoz DG, Hastak SM, Harper B, Lee D, Hachinski VC. Pathologic correlates of increased signals of the centrum ovale on magnetic resonance imaging. Arch Neurol. 1993;50:492-497

[55] Haddad FS, Abla A, Allam C. Ependymal brain cyst. Surg Neurol. 1982;18:246-249

[56] Braffman BH, Zimmerman RA, Trojanowski JQ, Gonatas NK, Hickey WF, Schlaepfer WW. Brain mr: Pathologic correlation with gross and histopathology. 2. 
Hyperintense white-matter foci in the elderly. AJR Am J Roentgenol. 1988;151:559566

[57] Weller RO, Kida S, Zhang ET. Pathways of fluid drainage from the brain-morphological aspects and immunological significance in rat and man. Brain Pathol. 1992;2:277-284

[58] Jungreis CA, Kanal E, Hirsch WL, Martinez AJ, Moossy J. Normal perivascular spaces mimicking lacunar infarction: Mr imaging. Radiology. 1988;169:101-104

[59] Bokura H, Kobayashi S, Yamaguchi S. Distinguishing silent lacunar infarction from enlarged virchow-robin spaces: A magnetic resonance imaging and pathological study. J Neurol. 1998;245:116-122

[60] Groeschel S, Chong WK, Surtees R, Hanefeld F. Virchow-robin spaces on magnetic resonance images: Normative data, their dilatation, and a review of the literature. Neuroradiology. 2006;48:745-754

[61] Rouhl RP, van Oostenbrugge RJ, Knottnerus IL, Staals JE, Lodder J. Virchow-robin spaces relate to cerebral small vessel disease severity. J Neurol. 2008

[62] Heier LA, Bauer CJ, Schwartz L, Zimmerman RD, Morgello S, Deck MD. Large virchow-robin spaces: Mr-clinical correlation. AJNR Am J Neuroradiol. 1989;10:929936

[63] Hiroki M, Miyashita K. Linear hyperintensity objects on magnetic resonance imaging related to hypertension. Cerebrovasc Dis. 2001;11:164-168

[64] Maclullich AM, Wardlaw JM, Ferguson KJ, Starr JM, Seckl JR, Deary IJ. Enlarged perivascular spaces are associated with cognitive function in healthy elderly men. $J$ Neurol Neurosurg Psychiatry. 2004;75:1519-1523

[65] Patankar TF, Mitra D, Varma A, Snowden J, Neary D, Jackson A. Dilatation of the virchow-robin space is a sensitive indicator of cerebral microvascular disease: Study in elderly patients with dementia. AJNR Am J Neuroradiol. 2005;26:1512-1520

[66] Cumurciuc R, Guichard JP, Reizine D, Gray F, Bousser MG, Chabriat H. Dilation of virchow-robin spaces in cadasil. Eur J Neurol. 2006;13:187-190

[67] Erkinjuntti T, Benavente O, Eliasziw M, Munoz DG, Sulkava R, Haltia M, Hachinski V. Diffuse vacuolization (spongiosis) and arteriolosclerosis in the frontal white matter occurs in vascular dementia. Arch Neurol. 1996;53:325-332

[68] Barkhof F. Enlarged virchow-robin spaces: Do they matter? J Neurol Neurosurg Psychiatry. 2004;75:1516-1517

[69] Bots ML, Looman SJ, Koudstaal PJ, Hofman A, Hoes AW, Grobbee DE. Prevalence of stroke in the general population. The rotterdam study. Stroke. 1996;27:1499-1501

[70] Mittelmark MB, Psaty BM, Rautaharju PM, Fried LP, Borhani NO, Tracy RP, Gardin JM, O'Leary DH. Prevalence of cardiovascular diseases among older adults. The cardiovascular health study. Am J Epidemiol. 1993;137:311-317

[71] Vermeer SE, Den Heijer T, Koudstaal PJ, Oudkerk M, Hofman A, Breteler MM. Incidence and risk factors of silent brain infarcts in the population-based rotterdam scan study. Stroke. 2003;34:392-396

[72] Vermeer SE, Koudstaal PJ, Oudkerk M, Hofman A, Breteler MM. Prevalence and risk factors of silent brain infarcts in the population-based rotterdam scan study. Stroke. 2002;33:21-25 
[73] Norrving B. Long-term prognosis after lacunar infarction. Lancet Neurol. 2003;2:238-245

[74] Longstreth WT, Jr., Arnold AM, Beauchamp NJ, Jr., Manolio TA, Lefkowitz D, Jungreis $\mathrm{C}$, Hirsch $\mathrm{CH}$, O'Leary DH, Furberg CD. Incidence, manifestations, and predictors of worsening white matter on serial cranial magnetic resonance imaging in the elderly: The cardiovascular health study. Stroke. 2005;36:56-61

[75] Hachinski VC, Potter P, Merskey H. Leuko-araiosis. Arch Neurol. 1987;44:21-23

[76] Marshall VG, Bradley WG, Jr., Marshall CE, Bhoopat T, Rhodes RH. Deep white matter infarction: Correlation of $\mathrm{mr}$ imaging and histopathologic findings. Radiology. 1988;167:517-522

[77] Revesz T, Hawkins CP, du Boulay EP, Barnard RO, McDonald WI. Pathological findings correlated with magnetic resonance imaging in subcortical arteriosclerotic encephalopathy (binswanger's disease). J Neurol Neurosurg Psychiatry. 1989;52:13371344

[78] Fazekas F, Kleinert R, Offenbacher H, Schmidt R, Kleinert G, Payer F, Radner H, Lechner H. Pathologic correlates of incidental mri white matter signal hyperintensities. Neurology. 1993;43:1683-1689

[79] Fernando MS, O'Brien JT, Perry RH, English P, Forster G, McMeekin W, Slade JY, Golkhar A, Matthews FE, Barber R, Kalaria RN, Ince PG. Comparison of the pathology of cerebral white matter with post-mortem magnetic resonance imaging (mri) in the elderly brain. Neuropathol Appl Neurobiol. 2004;30:385-395

[80] Bartynski WS. Posterior reversible encephalopathy syndrome, part 1: Fundamental imaging and clinical features. AJNR Am J Neuroradiol. 2008;29:1036-1042

[81] Costello DJ, Eichler AF, Eichler FS. Leukodystrophies: Classification, diagnosis, and treatment. Neurologist. 2009;15:319-328

[82] Matthews PM, Tampieri D, Berkovic SF, Andermann F, Silver K, Chityat D, Arnold DL. Magnetic resonance imaging shows specific abnormalities in the melas syndrome. Neurology. 1991;41:1043-1046

[83] Fazekas F CJ, Alavi A, Hurtig HI, Zimmerman RA. Mr signal abnormalities at $1.5 \mathrm{t}$ in alzheimer's dementia and normal aging. AJR Am J Roentgenol. 1987

[84] Schmidt R, Fazekas F, Kapeller P, Schmidt H, Hartung HP. Mri white matter hyperintensities: Three-year follow-up of the austrian stroke prevention study. Neurology. 1999;53:132-139

[85] Scheltens P, Barkhof F, Leys D, Pruvo JP, Nauta JJ, Vermersch P, Steinling M, Valk J. A semiquantative rating scale for the assessment of signal hyperintensities on magnetic resonance imaging. J Neurol Sci. 1993;114:7-12

[86] Wahlund LO, Barkhof F, Fazekas F, Bronge L, Augustin M, Sjogren M, Wallin A, Ader H, Leys D, Pantoni L, Pasquier F, Erkinjuntti T, Scheltens P. A new rating scale for age-related white matter changes applicable to mri and ct. Stroke. 2001;32:1318-1322

[87] Kapeller P, Schmidt R, Enzinger C, Ropele S, Fazekas F. Ct and mri rating of white matter changes. J Neural Transm Suppl. 2002:41-45

[88] Anbeek P, Vincken KL, van Osch MJ, Bisschops RH, van der Grond J. Probabilistic segmentation of white matter lesions in mr imaging. Neuroimage. 2004;21:1037-1044 
[89] Sachdev P, Cathcart S, Shnier R, Wen W, Brodaty H. Reliability and validity of ratings of signal hyperintensities on mri by visual inspection and computerised measurement. Psychiatry Res. 1999;92:103-115

[90] Gouw AA, van der Flier WM, van Straaten EC, Pantoni L, Bastos-Leite AJ, Inzitari D, Erkinjuntti T, Wahlund LO, Ryberg C, Schmidt R, Fazekas F, Scheltens P, Barkhof F. Reliability and sensitivity of visual scales versus volumetry for evaluating white matter hyperintensity progression. Cerebrovasc Dis. 2008;25:247-253

[91] de Groot JC, de Leeuw FE, Oudkerk M, van Gijn J, Hofman A, Jolles J, Breteler MM. Cerebral white matter lesions and cognitive function: The rotterdam scan study. Ann Neurol. 2000;47:145-151

[92] van Straaten EC, Fazekas F, Rostrup E, Scheltens P, Schmidt R, Pantoni L, Inzitari D, Waldemar G, Erkinjuntti T, Mantyla R, Wahlund LO, Barkhof F. Impact of white matter hyperintensities scoring method on correlations with clinical data: The ladis study. Stroke. 2006;37:836-840

[93] Nebes RD, Meltzer CC, Whyte EM, Scanlon JM, Halligan EM, Saxton JA, Houck PR, Boada FE, Dekosky ST. The relation of white matter hyperintensities to cognitive performance in the normal old: Education matters. Neuropsychol Dev Cogn B Aging Neuropsychol Cogn. 2006;13:326-340

[94] Rovaris M, Iannucci G, Cercignani M, Sormani MP, De Stefano N, Gerevini S, Comi G, Filippi M. Age-related changes in conventional, magnetization transfer, and diffusion-tensor $\mathrm{mr}$ imaging findings: Study with whole-brain tissue histogram analysis. Radiology. 2003;227:731-738

[95] Benedetti B, Charil A, Rovaris M, Judica E, Valsasina P, Sormani MP, Filippi M. Influence of aging on brain gray and white matter changes assessed by conventional, mt, and dt mri. Neurology. 2006;66:535-539

[96] Horsfield MA, Jones DK. Applications of diffusion-weighted and diffusion tensor mri to white matter diseases - a review. NMR Biomed. 2002;15:570-577

[97] Mascalchi M, Filippi M, Floris R, Fonda C, Gasparotti R, Villari N. Diffusion-weighted $\mathrm{mr}$ of the brain: Methodology and clinical application. Radiol Med. 2005;109:155-197

[98] Beaulieu C. The basis of anisotropic water diffusion in the nervous system - a technical review. NMR Biomed. 2002;15:435-455

[99] O'Sullivan M, Summers PE, Jones DK, Jarosz JM, Williams SC, Markus HS. Normalappearing white matter in ischemic leukoaraiosis: A diffusion tensor mri study. Neurology. 2001;57:2307-2310

[100] Molko N, Pappata S, Mangin JF, Poupon C, Vahedi K, Jobert A, LeBihan D, Bousser $\mathrm{MG}$, Chabriat H. Diffusion tensor imaging study of subcortical gray matter in cadasil. Stroke. 2001;32:2049-2054

[101] Chabriat H, Pappata S, Poupon C, Clark CA, Vahedi K, Poupon F, Mangin JF, PachotClouard M, Jobert A, Le Bihan D, Bousser MG. Clinical severity in cadasil related to ultrastructural damage in white matter: In vivo study with diffusion tensor mri. Stroke. 1999;30:2637-2643

[102] Holtmannspotter M, Peters N, Opherk C, Martin D, Herzog J, Bruckmann H, Samann $\mathrm{P}$, Gschwendtner A, Dichgans M. Diffusion magnetic resonance histograms as a 
surrogate marker and predictor of disease progression in cadasil: A two-year follow-up study. Stroke. 2005;36:2559-2565

[103] Molko N, Pappata S, Mangin JF, Poupon F, LeBihan D, Bousser MG, Chabriat H. Monitoring disease progression in cadasil with diffusion magnetic resonance imaging: A study with whole brain histogram analysis. Stroke. 2002;33:2902-2908

[104] Nitkunan A, Barrick TR, Charlton RA, Clark CA, Markus HS. Multimodal mri in cerebral small vessel disease: Its relationship with cognition and sensitivity to change over time. Stroke. 2008;39:1999-2005

[105] Charlton RA, Schiavone F, Barrick TR, Morris RG, Markus HS. Diffusion tensor imaging detects age related white matter change over a 2 year follow-up which is associated with working memory decline. J Neurol Neurosurg Psychiatry.81:13-19

[106] Della Nave R, Foresti S, Pratesi A, Ginestroni A, Inzitari M, Salvadori E, Giannelli M, Diciotti S, Inzitari D, Mascalchi M. Whole-brain histogram and voxel-based analyses of diffusion tensor imaging in patients with leukoaraiosis: Correlation with motor and cognitive impairment. AJNR Am J Neuroradiol. 2007;28:1313-1319

[107] Offenbacher H, Fazekas F, Schmidt R, Koch M, Fazekas G, Kapeller P. Mr of cerebral abnormalities concomitant with primary intracerebral hematomas. AJNR Am J Neuroradiol. 1996;17:573-578

[108] Greenberg SM, Finklestein SP, Schaefer PW. Petechial hemorrhages accompanying lobar hemorrhage: Detection by gradient-echo mri. Neurology. 1996;46:1751-1754

[109] Ripoll MA, Siosteen B, Hartman M, Raininko R. Mr detectability and appearance of small experimental intracranial hematomas at $1.5 \mathrm{t}$ and $0.5 \mathrm{t}$. A 6-7-month followup study. Acta Radiol. 2003;44:199-205

[110] Fazekas F, Kleinert R, Roob G, Kleinert G, Kapeller P, Schmidt R, Hartung HP. Histopathologic analysis of foci of signal loss on gradient-echo $\mathrm{t} 2{ }^{*}$-weighted $\mathrm{mr}$ images in patients with spontaneous intracerebral hemorrhage: Evidence of microangiopathy-related microbleeds. AJNR Am J Neuroradiol. 1999;20:637-642

[111] Tanaka A, Ueno Y, Nakayama Y, Takano K, Takebayashi S. Small chronic hemorrhages and ischemic lesions in association with spontaneous intracerebral hematomas. Stroke. 1999;30:1637-1642

[112] Viswanathan A, Chabriat H. Cerebral microhemorrhage. Stroke. 2006;37:550-555

[113] Fiehler J. Cerebral microbleeds: Old leaks and new haemorrhages. Int J Stroke. 2006;1:122-130

[114] Cordonnier C, Al-Shahi Salman R, Wardlaw J. Spontaneous brain microbleeds: Systematic review, subgroup analyses and standards for study design and reporting. Brain. 2007;130:1988-2003

[115] Lee SH, Kwon SJ, Kim KS, Yoon BW, Roh JK. Cerebral microbleeds in patients with hypertensive stroke. Topographical distribution in the supratentorial area. J Neurol. 2004;251:1183-1189

[116] Chalela JA, Kang DW, Warach S. Multiple cerebral microbleeds: Mri marker of a diffuse hemorrhage-prone state. J Neuroimaging. 2004;14:54-57

[117] Derex L, Nighoghossian N, Hermier M, Adeleine P, Philippeau F, Honnorat J, Yilmaz H, Dardel P, Froment JC, Trouillas P. Thrombolysis for ischemic stroke in patients with old microbleeds on pretreatment mri. Cerebrovasc Dis. 2004;17:238-241 
[118] Kakuda W, Thijs VN, Lansberg MG, Bammer R, Wechsler L, Kemp S, Moseley ME, Marks MP, Albers GW. Clinical importance of microbleeds in patients receiving iv thrombolysis. Neurology. 2005;65:1175-1178

[119] Kidwell CS, Saver JL, Villablanca JP, Duckwiler G, Fredieu A, Gough K, Leary MC, Starkman S, Gobin YP, Jahan R, Vespa P, Liebeskind DS, Alger JR, Vinuela F. Magnetic resonance imaging detection of microbleeds before thrombolysis: An emerging application. Stroke. 2002;33:95-98

[120] Nighoghossian N, Hermier M, Adeleine P, Blanc-Lasserre K, Derex L, Honnorat J, Philippeau F, Dugor JF, Froment JC, Trouillas P. Old microbleeds are a potential risk factor for cerebral bleeding after ischemic stroke: A gradient-echo t2*-weighted brain mri study. Stroke. 2002;33:735-742

[121] Enzinger C, Fazekas F, Matthews PM, Ropele S, Schmidt H, Smith S, Schmidt R. Risk factors for progression of brain atrophy in aging: Six-year follow-up of normal subjects. Neurology. 2005;64:1704-1711

[122] Karas GB, Scheltens P, Rombouts SA, Visser PJ, van Schijndel RA, Fox NC, Barkhof F. Global and local gray matter loss in mild cognitive impairment and alzheimer's disease. Neuroimage. 2004;23:708-716

[123] Zivadinov R, Sepcic J, Nasuelli D, De Masi R, Bragadin LM, Tommasi MA, ZambitoMarsala S, Moretti R, Bratina A, Ukmar M, Pozzi-Mucelli RS, Grop A, Cazzato G, Zorzon M. A longitudinal study of brain atrophy and cognitive disturbances in the early phase of relapsing-remitting multiple sclerosis. J Neurol Neurosurg Psychiatry. 2001;70:773-780

[124] Kraemer M, Schormann T, Hagemann G, Qi B, Witte OW, Seitz RJ. Delayed shrinkage of the brain after ischemic stroke: Preliminary observations with voxel-guided morphometry. J Neuroimaging. 2004;14:265-272

[125] Schmidt R, Ropele S, Enzinger C, Petrovic K, Smith S, Schmidt H, Matthews PM, Fazekas F. White matter lesion progression, brain atrophy, and cognitive decline: The austrian stroke prevention study. Ann Neurol. 2005;58:610-616

[126] Preul C, Lohmann G, Hund-Georgiadis M, Guthke T, von Cramon DY. Morphometry demonstrates loss of cortical thickness in cerebral microangiopathy. J Neurol. 2005;252:441-447

[127] Seshadri S, Wolf PA, Beiser A, Elias MF, Au R, Kase CS, D'Agostino RB, DeCarli C. Stroke risk profile, brain volume, and cognitive function: The framingham offspring study. Neurology. 2004;63:1591-1599

[128] Viswanathan A, Godin O, Jouvent E, O'Sullivan M, Gschwendtner A, Peters N, Duering M, Guichard JP, Holtmannspotter M, Dufouil C, Pachai C, Bousser MG, Dichgans M, Chabriat H. Impact of mri markers in subcortical vascular dementia: A multi-modal analysis in cadasil. Neurobiol Aging.31:1629-1636

[129] Fein G, Di Sclafani V, Tanabe J, Cardenas V, Weiner MW, Jagust WJ, Reed BR, Norman D, Schuff N, Kusdra L, Greenfield T, Chui H. Hippocampal and cortical atrophy predict dementia in subcortical ischemic vascular disease. Neurology. 2000;55:16261635 
[130] Jouvent E, Mangin JF, Porcher R, Viswanathan A, O'Sullivan M, Guichard JP, Dichgans M, Bousser MG, Chabriat $\mathrm{H}$. Cortical changes in cerebral small vessel diseases: A 3d mri study of cortical morphology in cadasil. Brain. 2008;131:2201-2208

[131] Viswanathan A, Gray F, Bousser MG, Baudrimont M, Chabriat H. Cortical neuronal apoptosis in cadasil. Stroke. 2006;37:2690-2695

[132] Rao DG, Lyons PR. Wallerian degeneration of the pyramidal tract after a thrombotic stroke. J Neurol Neurosurg Psychiatry. 1998;65:944

[133] Jouvent E, Viswanathan A, Chabriat H. Cerebral atrophy in cerebrovascular disorders. J Neuroimaging.20:213-218 


\title{
Neuroimaging of Intracranial Atherosclerotic Disease
}

\author{
Maria Khan, Imama Naqvi and Ayeesha Kamran Kamal \\ Stroke Program, The Aga Khan University Hospital \\ Pakistan
}

\section{Introduction}

Ischemic stroke is now universally accepted as a heterogeneous disease. The need to properly classify stroke subtypes is increasingly driven by the realization that different mechanisms may require different treatments. Also the risk of recurrent vascular events differs among various ischemic stroke subtypes.

Intracranial atherosclerotic disease (ICAD) is fast emerging as the predominant mechanism of ischemic stroke in the world and particularly in patients of Asian origin (Kim et al, 2006 and Sacco et al, 1995). Also, ICAD is reported to have the highest risk of recurrent ischemic cerebrovascular events being quoted between 25-30\% in two years (Wong et al, 2003 and Mazighi et al, 2006).

\section{Epidemiology}

It is now well recognized that intracranial atherosclerotic disease predominantly affects patients of Asian, African and Hispanic origin. Since these are the regions of the world which are going to harbor the majority of the world's stroke population, it is expected that the disease burden of intracranial atherosclerosis is also going to increase.

The rates of ICAD reported from Asian countries range between 33-56\% depending on what cutoff for stenosis is taken (Huang et al, 1997 and Lee et al, 2003). Caucasians predominantly have extracranial atherosclerosis and rates of intracranial disease vary between 1 and $24 \%$ (e.g., Sacco et al., 1995; Wityk et al., 1996).

Data on the prevalence of asymptomatic stenosis are relatively scarce. Warfarin versus Aspirin in Symptomatic Intracranial disease (WASID) trial reported presence of $27 \%$ asymptomatic disease in patients who also had symptomatic stenosis (Nahab et al, 2008). Studies of healthy Japanese and Korean populations report asymptomatic disease in 3-3.5\% (Park et al, 2006 and Wong et al, 2007). There is a growing need to identify the asymptomatic entity as the process of atherosclerosis can potentially be stalled by timely risk factor management. Several diagnostic modalities are now available and evolving but the focus so far is on the diagnosis of the disease at a stage when it becomes symptomatic.

In this chapter we describe the various radiological presentations of stroke secondary to intracranial atherosclerotic disease followed by a description of the tools available for diagnosing the condition. Non invasive modalities are the main focus as the prevalence of the disease increases and the need to identify asymptomatic disease is also increasingly felt. 
Additionally, with a greater number of patients now being offered endovascular treatment, there is a need to have cost-effective, minimally invasive and reliable methods to follow these patients up.

\section{Infarct patterns}

Literature now exists on infarct patterns associated with intracranial large artery atherosclerosis. The underlying mechanisms leading to ischemia in the diseased arterial territory include artery-to-artery embolism, thrombosis leading to complete occlusion or to local branch occlusion and hemodynamic compromise (Wong et al, 2002). A combination of these factors may also co-exist in some patients. Depending on the mechanism of ischemia, the infarct pattern may differ.

\subsection{Border-zone Infarctions}

Border-zone infarctions have been described with intracranial atherosclerotic disease for decades. Recently the concept of border-zones has been revisited and two types of borderzone infarctions have been described (Yong et al, 2006). Internal border-zone infarctions involve the area between the territory of anterior, middle and posterior cerebral arteries and the territory supplied by the lenticulostriate, anterior choroidal and Heubner arteries. The term cortical border-zone is used for areas located between the cortical supply of the anterior and middle cerebral arteries (anterior cortical border-zone) and between middle and posterior cerebral arteries (posterior cortical border-zone). It is proposed that the two types of infarctions may have different underlying pathophysiology.

\subsubsection{Cortical border-zone infarctions}

For the cortical border-zone infarctions, artery-to-artery embolism has been proposed as causative since the 1980's. Several studies (Torvik et al, 1982, Pollanen et al, 1990, Masuda et al, 1994, Belden et al, 1999) describe border-zone or watershed infarctions resulting from atheromatous large vessel intracranial disease. With Transcranial Doppler monitoring this mechanism has been further validated. Using this modality, impaired clearance of emboli has been documented with middle cerebral artery (MCA) stenosis and patients with this condition were found to have multiple small cerebral infarcts especially in the border-zone territories of MCA with anterior and posterior cerebral arteries (Wong et al, 2002).

\subsubsection{Internal border-zone infarctions}

For internal border-zone infarctions, hemodynamic compromise is believed to be the proximate cause. Infarct patterns described include single lacunes in internal border-zone or a string of pearls or scattered pearl appearance on diffusion weighted imaging (DWI) (Turtzo et al, 2009). The underlying mechanism is believed to be a gradual reduction in the perfusion pressure in the stenosed artery. This leads to development of collateral blood supply from branches of extracranial carotid artery. With progression of disease, these collaterals are unable to maintain the hemodynamics and maximal hypoperfusion occurs in the terminal zones of the distribution of MCA and its perforators. These are the internal border-zone areas and the association of these infarcts with increased oxygen extraction fraction has been documented suggesting hemodynamic compromise as the likely mechanism (Yamauchi et al, 2009). 


\subsection{Cortical territorial infarctions}

Cortically based infarctions in the territory of MCA (anterior territorial infarctions) and PCA (posterior territorial infarctions) have also been described with large vessel atherosclerotic disease. The proposed mechanism is again artery to artery embolism. In case of anterior circulation, as opposed to cardioembolic infarcts, these are usually smaller, and limited to one of the three MCA territories. This can be explained based on the size of cardiac emboli which are larger and likely to give rise to large infarcts. However, with larger emboli occluding proximal MCA or distal internal carotid arteries (ICA), large territorial infarctions may also be seen particularly in the absence of effective collaterals (Rovera et al, 2005). The ischemic cortical lesions may co-exist with smaller cortical or subcortical acute lesions resulting from fragmentation of the embolus.

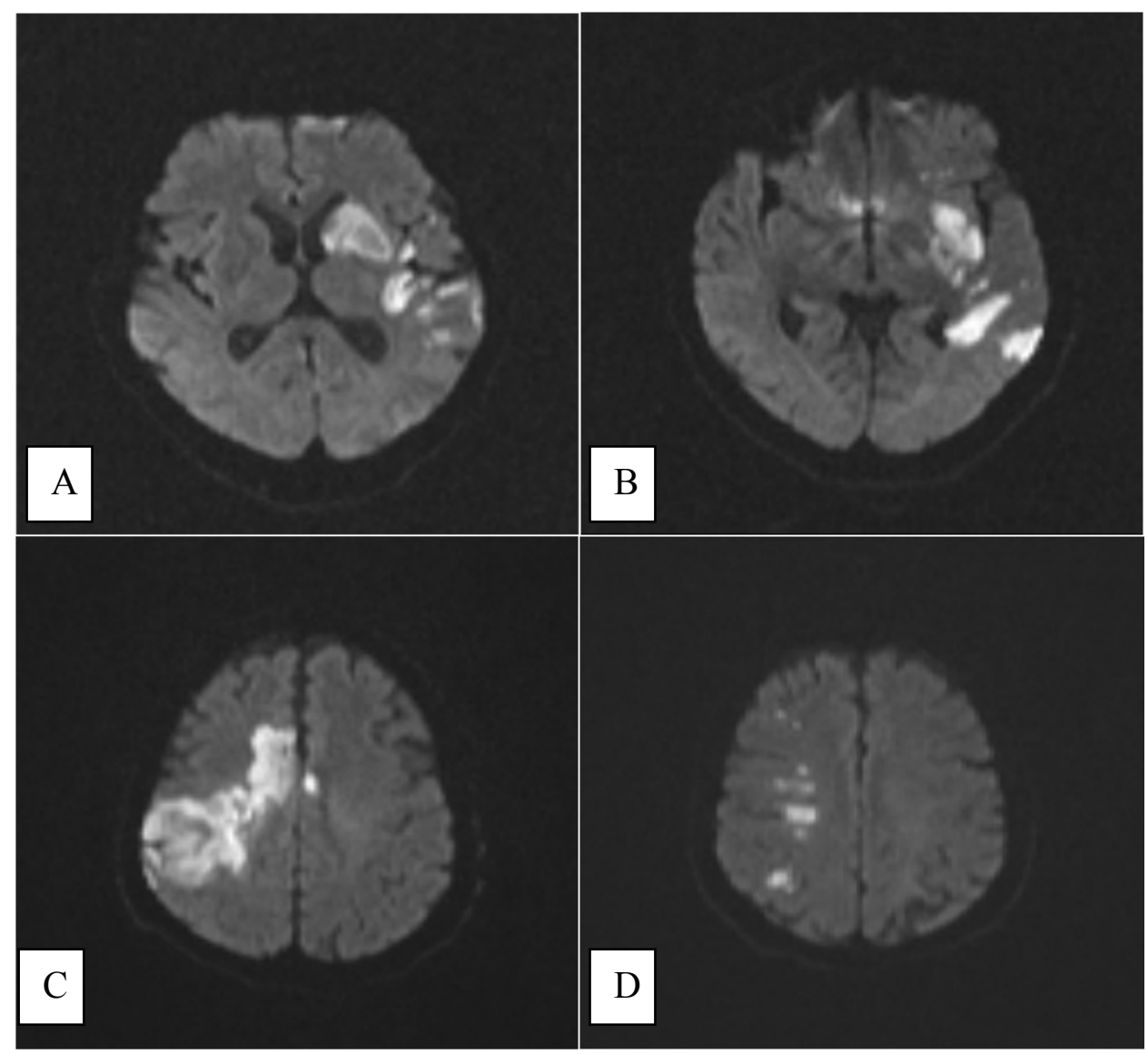

Fig. 1. Diffusion Weighted Images of Infarct Patterns seen with ICAD. A) Multiple infarcts in Right MCA territory, B) Deep MCA perforator infarct along with patchy cortical infarcts, C) cortical infarct in posterior MCA territory, D) String of pearls pattern in Internal borderzone. (Image Courtesy, Stroke Fellowship Program, Aga Khan University Hospital, Karachi, Pakistan) 
In the posterior circulation, large artery atherosclerosis involving the vertebrobasilar arteries leads to infarctions in the territories of posterior inferior cerebellar artery (PICA), superior cerebellar artery (SCA) and PCA. Small territorial and nonterritorial cerebellar infarcts are also described with vertebrobasilar disease (Amarenco et al, 1994).

\subsection{Lacunar infarctions}

Lacunar infarctions in the centrum semiovale have also been described with intracranial atherosclerotic disease. When large $(>1.5 \mathrm{~cm})$ and present in the internal border-zone, these are thought to result from hemodynamic compromise. When small $(<1.5 \mathrm{~cm})$ they may arise secondary to artery to artery embolism. In this case, they may be accompanied by other small acute infarcts in the same large artery territory (Bogousslavsky and Regli, 1992). Posterior circulation lacunes have also been described with large vessel disease, particularly when they occur in isolation.

\subsection{Perforator artery infarctions}

With atherosclerotic involvement of MCA, local branch occlusion has also been described leading to deep perforator artery infarctions. Pial infarctions, those occurring in the vascular territories supplied by the superior and inferior branches of MCA have also been described with MCA disease (Lee et al, 2005). Thrombotic occlusion in the former and distal embolization in the latter is thought to be the underlying mechanism.

\subsection{Multiple acute brain infarctions}

Multiple acute brain infarctions, particularly in the same arterial territory have also been described (Roh et al, 2000 and Kang et al, 2003). The mechanism is believed to be distal embolizations from large vessel unstable plaque. Such infarcts are typically located in a single anterior or posterior arterial territory, but anatomic variations may explain other patterns such as involvement of anterior and posterior circulations simultaneously in case of a fetal-PCA or posterior communicating artery patency, or involvement of bilateral ACA territories in case of a single ACA supplying both hemispheres.

\section{Imaging of vessels}

The diagnosis of intracranial atherosclerotic disease rests on either direct visualization of the vessels or determination of their flow characteristics. Several non-invasive modalities like magnetic resonance angiography (MRA), computerized tomographic angiography (CTA) and transcranial doppler (TCD) are now available for screening and diagnostic purposes. However, the gold standard for diagnosis is still catheter based conventional angiography.

\subsection{Catheter based conventional and Digital Subtraction Angiography (DSA)}

DSA has been the reference standard for evaluation of intracranial stenosis and occlusion for decades. The technique involves injection of a radio-opaque dye via an intra-arterial catheter. Usually the femoral or radial arteries are used. Cerebral angiography is performed after catheterizing the cerebral arteries. The term digital subtraction angiography is used when a computer based software subtracts two images that are obtained before and after administration of contrast media. Modern DSA also incorporates three-dimensional reconstructed images which provide even better details. 
DSA has several advantages over other modalities used for assessing intracranial stenosis. It provides excellent visualization of the anatomy of the vessel, the severity and length of stenosis and the presence of collateral circulation. However, the major disadvantage lies in the high skill required to perform the study. Another disadvantage is the morbidity associated with the procedure including stroke, arterial dissections, puncture site infection, thrombosis of punctured artery leading to limb ischemia and formation of pseudoaneurysms. In addition to this it carries the risk of nephrotoxicity associated with iodinated contrast used in the procedure.
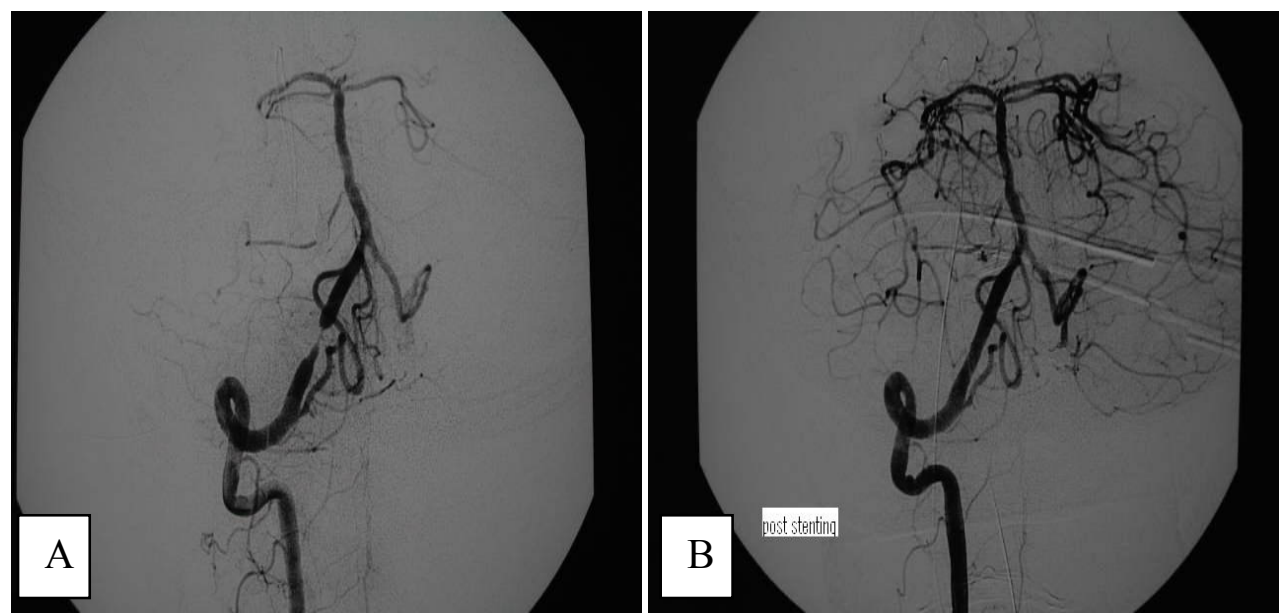

Fig. 2. DSA images showing disease in Right vertebral artery. A) Tight stenosis in Right vertebral artery, B) Post stenting restoration of flow.(Image Courtesy: Dr. Tanveer-ul-Haq, Associate Professor ,Section Head, Vascular and Interventional Radiology, Radiology Department, Aga Khan University Hospital , Karachi, Pakistan)

When conducted for diagnostic purposes, risk associated with the procedure has gone down over the past years. The most recent estimate quotes the risk of stroke at $0.03 \%$ and of arterial dissections at $0.14 \%$. The non-neurologic complications related to the arterial puncture were also not commonly seen (Fifi et al, 2009). In a dedicated unit for cerebral angiographies, the risk of neurologic complications can approach zero (Thiex et al, 2010). Interestingly, the risk of neurological adverse events was higher in patients with known intracranial atherosclerotic disease who underwent cerebral angiography in the WASID trial. $2 \%$ of these patients experienced neurological symptoms although all were transient and $6.1 \%$ had non-neurologic complications (Cloft et al, 2011).

Given the disadvantages of DSA and the increased morbidity and potential mortality associated with it, non-invasive modalities are increasingly being utilized for assessment of intracranial atherosclerotic disease.

\subsection{Magnetic Resonance Angiography (MRA)}

\subsubsection{Phase contrast (PC) and Time of Flight (TOF) MRA}

Phase contrast MRA utilizes the velocity differences and hence the phase shifts in moving spins to provide image contrast in flowing vessels. The phase of magnetization from the 
moving spins is taken as non-zero and that from the stationary spins is zero. Time of Flight MRA depends on the flow and movement of protons in blood through the imaging plane. It derives contrast between flowing blood and stationary tissues by manipulating the magnitude of magnetization. From the moving spins the magnitude is large and from static spins it is small. Both techniques can be used using two or three-dimensional acquisition although 3-D images have a long acquisition time.

The main advantage of the modality lies in its non-invasive nature. There is no radiation exposure and no iodinated contrast exposure. It requires very little user interaction for acquisition and processing of images. When compared to the gold standard DSA, TOF-MRA has sensitivity in the range of $70 \%$ for intracranial stenosis and $81 \%$ for occlusion and a negative predictive value of $98 \%$ for stenosis and $99 \%$ for occlusion (Bash et al, 2005). Another study identified $87 \%$ negative predictive value for detecting a greater than $50 \%$ stenosis (Feldmann et al, 2007). These results may be slightly improved by use of Maximum Intensity Projections (MIP).

There are however, several limitations when using PC and TOF MRA for intracranial stenosis evaluation. Firstly since it depends on changes in blood flow, it is prone to motion artifacts and to changes in the direction of the flow. The spatial resolution is also inferior to DSA and CTA. Another major drawback is its inability to distinguish between high grade stenosis and occlusion. Stenotic lesions are sites where complex, slow or in-phase flow characteristics are seen (Furst et al, 1995). Because of this there are proton spin dephasing artifacts and flow signal intensity loss is seen at severely narrowed sites. In addition, TOF technique requires longer imaging time.
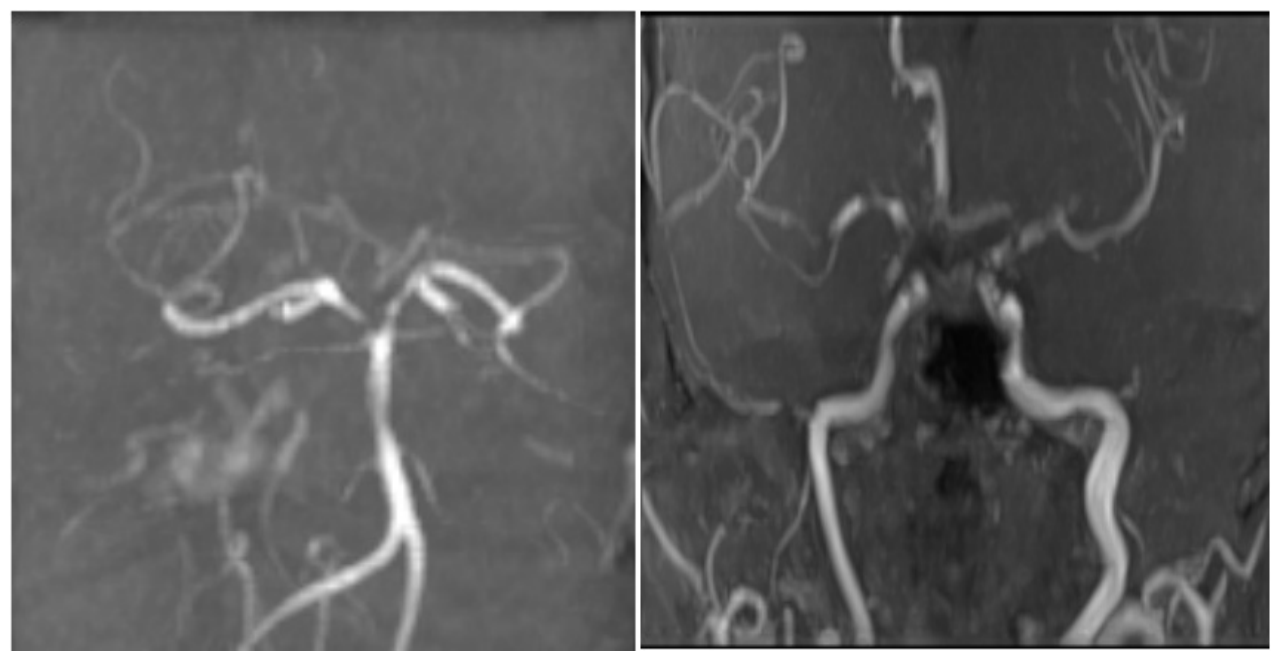

Fig. 3. TOF-MRA images of intracranial stenosis. A) Stenosis seen in bilateral PCAs, B) Severe atherosclerotic disease in supraclinoid ICA, bilateral MCA and ACA. (Image Courtesy, Stroke Fellowship Program, Aga Khan University Hospital, Karachi, Pakistan)

\subsubsection{Contrast Enhanced (CE) MRA}

Some of the disadvantages of TOF and PC MRA can be overcome with the use of gadolinium dye. CE-MRA utilizes the time of flight technique along with use of contrast 
agent to shorten the T1 of the blood so that in the first pass of the contrast agent, the arteries show up in striking contrast to the surrounding stationary tissues and veins. The CE MRA provides better anatomic delineation of vascular structures and can better assess areas of changing flow direction. This is because the technique depends on the intrinsic T1 signal of blood, rather than the flow characteristics and is minimally affected by dephasing seen with complex flow.

A certain degree of expertise is needed to time the image acquisition to minimize venous contamination. If the image is taken too early, arteries will not contrast enhance and if it is taken too late, the image will be contaminated by contrast uptake in the veins and surrounding tissues. For external carotids it is a widely used technique, but for assessment of intracranial vasculature it is still underutilized.

\subsubsection{Quantitative MRA (QMRA)}

Quantitative MRA (QMRA) is a newer technique that utilizes TOF and Phase contrast MRI to determine the vessel anatomy and measure blood flow. This allows for hemodynamic assessment of areas supplied by stenotic vessels. The technique has been shown to be particularly useful in prognosticating future risk of stroke in symptomatic vertebrobasilar disease. Those found to have a low distal flow on QMRA had a 71\% stroke free survival at 2 years as opposed to a $100 \%$ stroke free survival for those with a normal flow (Amin-Hanjani et al, 2005).

QMRA has also found utility in assessment of flow before and after stent placement (Brisman, 2008). More importantly for in-stent restenosis it is now an evolving non-invasive technique. In these patients, CTA and MRA are not of use due to the artifacts produced by the stent and DSA continues to be the mainstay for assessment of stent thrombosis. Recently QMRA has shown promise as a screening tool for in-stent stenosis with $100 \%$ sensitivity and negative predictive value and 92\% specificity (Prabhakaran et al, 2009). QMRA can also be used to determine regional blood flow and indicate the adequacy of collateral flow and overall cerebral hemodynamics in patients of intracranial atherosclerotic disease (Zhao et al, 2007).

\subsubsection{High Resolution MRI (HR-MRI)}

Another emerging technique is HR-MRI which allows for imaging of the atherosclerotic plaque. It creates sufficiently thin slices through the vessel wall to allow for determination of plaque size, composition and biological activity (Yuan et al, 2006). This modality can also help discriminate from other non-atherosclerotic etiologies of vessel narrowing.

Determination of plaque composition in terms of lipids, fibrous tissue and calcium and plaque activity in terms of inflammation can help determine whether a plaque is vulnerable or stable. HR MRI can also detect intraplaque hemorrhage which is another marker of plaque instability and can help predict risk of future vascular events (Turan et al, 2009 and Altaf et al, 2007). Furthermore, imaging the plaque directly can help determine presence of non-stenotic lesions which are generally missed with conventional MRA. Particularly in the evaluation of basilar artery, it was found that HR MRI detected atherosclerotic disease as a cause of pontine infarction in $42 \%$ of cases where TOF MRA and CE MRA had failed to show any basilar disease (Klein et al, 2010). Recently the technique has also found utility in guiding endovascular intervention of basilar artery (Jiang et al, 2011).

In future, HR MRI can potentially provide targets for intervention at an early stage of intracranial atherosclerotic disease, detect non stenotic athterosclerotic lesions, and predict future risk of vascular events based on plaque morphology and characteristics. 


\subsection{Computerized Tomography Angiography (CTA)}

Computed tomography (CT) angiography is a relatively new non-invasive procedure that allows for accurate visualization of vascular structures and differentiates them from adjacent bone and soft tissues. Helical or Spiral CTA is acquired with the X-ray tube continuously rotating in a 360 degree turn while the table transports the patient slowly through the scanner. The slice thickness and the scan time for each helical series determine the extent of anatomic coverage. Axial images are stacked to form a volume of image data that can be processed in various formats like maximum intensity projection, multiplanar reformation and shaded surface display. This allows for 3 dimensional reconstruction of vessels similar to DSA.

An iodinated contrast is injected through a peripheral vein and both arterial and venous phases can be easily obtained. Unlike MRA, CTA is not dependent on flow characteristics of the blood. Instead it relies on relative penetrance of the contrast agent within the blood vessel. New scanners allow smaller slice thickness and hence greater resolution. Additionally, three-dimensional reformatting allows for excellent appreciation of anatomic relationships between bones, soft tissues, and the vascular system. Spiral or helical CTA is now used commonly for the evaluation of intracranial stenosis and provides excellent anatomic visualization.

The sensitivity of CTA for detection of intracranial occlusion is almost $100 \%$ compared to DSA (Nguyen-Huynh et al, 2008 and Bash et al, 2005). For detection of stenotic lesions greater than $50 \%$, the sensitivity is $97.1 \%$ and specificity is $99.5 \%$. With this high sensitivity and specificity it is an excellent tool for screening people with intracranial stenosis. Helical CTA has also been shown to be superior to DSA in detecting vessel patency in posterior circulation where low flow states can produce an impression of occlusion on DSA (Bash et al, 2005).

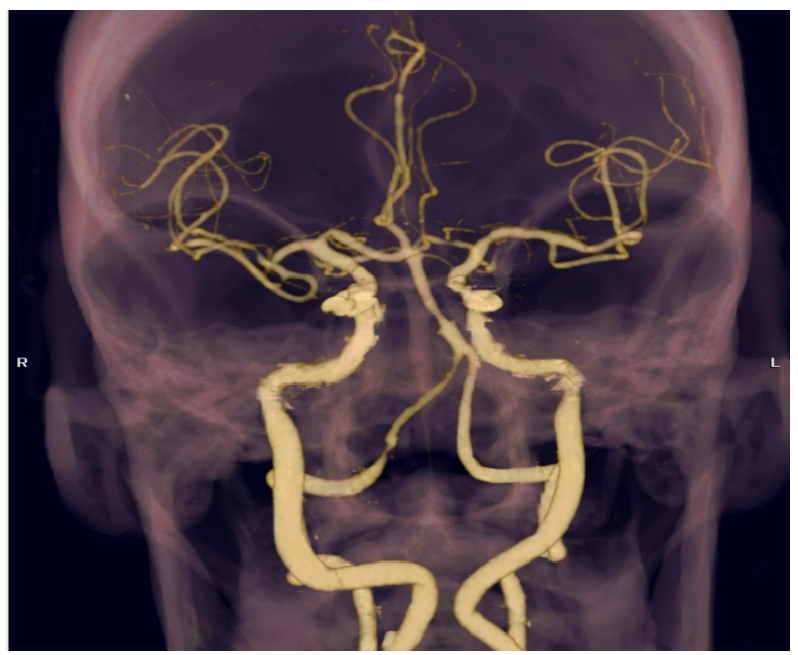

Fig. 4. CTA image demonstrating stenotic lesions in right vertebral, right M2 segment and left M1 segment of MCA. (Image Courtesy: Dr. Tanveer-ul-Haq, Associate Professor ,Section Head, Vascular and Interventional Radiology, Radiology Department, Aga Khan University Hospital , Karachi, Pakistan.) 
The major advantage of CTA lies in its non-invasive nature compared to DSA and better image quality compared to MRA. It takes less time for acquisition of images; it is not affected by motion artifacts and is independent of hemodynamic effects seen with MRA. There is also a high inter-reader reliability in quantitation of stenotic lesions (Bash et al, 2005). Another major advantage over MRA lies in the ability of CTA to accurately depict areas of low flow distal to severely stenotic vessels (Skutta et al, 1999 and Bash et al, 2005). It was previously thought that petrous and cavernous ICA are regions elusive to CTA due to bony or calcium artifacts (Skutta et al, 1999 and Hirai et al 2002). However, newer studies do not report this problem likely because of better post processing and acquisition techniques (Bash et al, 2005).

Limitations of CTA include radiation exposure and risk of nephrotoxicity with the use of intravenous contrast agent. Another limitation to the technique is skill required for optimal contrast gradient-timing to avoid venous contamination and for post processing of the images to avoid contamination with surrounding structures. For accurate visualization of vessels to avoid over or underestimation of stenosis, there is a need for optimally adjusting window and level settings (Bash et al, 2005). CTA may be inferior to MRA in certain respects as it provides no information on flow characteristics across a stenotic lesion.

\subsection{Transcranial Doppler (TCD)}

Transcranial Doppler (TCD) is a useful, non invasive, real time and portable mechanism of investigating 50 to $99 \%$ stenosis of the intracranial arteries. Ultrasound is a travelling wave of energy that has a frequency of more than $20,000 \mathrm{~Hz}$ which is above the audible range for humans. The Doppler effect, first described by Christian Andreas Doppler, is the change or shift in the frequency or wavelength of a wave due to relative movement between the sound scatterer and the receiver. In TCD, the Doppler effect can be used to determine the speed and direction of flow in blood vessels. Many red blood cells move at varying speeds through an insonated vessel and the TCD sample volume is relatively large. The signal received from any sample volume within a vessel is a mixture of the different Doppler frequency shifts forming a spectrum that is displayed visually.

Low frequency ultrasound can penetrate the skull; the temporal bone is the thinnest portion of the skull and allows successful insonation. An inadequate temporal window is a major cause of insufficient evaluation of cerebral vessels during TCD. The prevalence of this finding is $14.5 \%$ and is increased in non white females greater than 60 years of age. (Ratanakorn, 1998). The temporal window allows the ICA, MCA, ACA to be sampled, the occipital allows the posterior circulation to be reviewed, and the ophthalmic allows the collateral information to be gathered.

Spectral waveforms are identified by their direction, depth of insonation, velocity characteristics, and spectral measurements are then performed to report the following parameters for the cerebral circulation: Mean Flow Velocity (MFV) and the Pulsatility Index (PI).

$$
\begin{gathered}
\mathrm{MFV}=\mathrm{PSV}+2 \mathrm{EDV}) / 3 \\
\mathrm{PI}=(\mathrm{PSV}-\mathrm{EDV}) / \mathrm{MFV}
\end{gathered}
$$

where PSV= Peak Systolic Velocity and EDV= End Diastolic Velocity

MFV correlates well with vessel patency and degree of arterial stenosis. The PI is an indirect measure of peripheral resistance. Post stenotic vessels with blunted waveforms have low 
resistance and chronic hypertensives have high PI. In addition TCD can provide information on the presence or absence of microembolic signals and the progression and regression of stenosis.

The advantages of TCD are that the vessels are insonated non-invasively, at the bedside and repeatedly without any harm to the patients. The limitations of TCD include a lack of windows, operator dependency, and the fact that lesser degrees of stenosis are not picked up and that direct evaluation is limited to the basal intracranial vessels.

TCD is a useful adjunct to the diagnosis of ICAD in the following ways

1. Non invasive diagnosis of $50-70 \%$ stenosis and response to therapy

Velocity thresholds that predict a significant MCA stenosis have been reported. As a rule in a vessel with straight walls a 50\% diameter reduction doubles the velocity and a $70 \%$ stenosis can quadruple the velocity at the exit of the stenosis compared with a prestenotic segment or to the contralateral side.

The criteria that are used include MFV, Peak systolic velocity (PSV) and stenotic to prestenotic ratios. The velocity criteria for determining the degree of stenosis in the different vessels are reported from the SONIA study that in addition, reported the Positive and Negative predictive values (PPV and NPV). When using non invasive imaging it is important to correlate the status of the extracranial carotid artery. Collateral flow and decreased volume flow from the proximal carotid stenosis without adequate collateral flow results in false positive and false negative interpretations respectively. To further help avoid false positives a prestenotic to post stenotic MCA velocity ration of $1: \geq 2$ should be used in addition to the MFV threshold.

The relative portability of TCD has led to wide scale applications in population screenings to detect asymptomatic disease and to follow the regression of stenosis and response to therapy in large scale interventional studies. It has therefore been used as a biologic surrogate marker much like carotid Intima media Thickness (IMT). However, it is not clear at this point whether the regressive changes in TCD can predict clinically important amelioration of outcomes.

\section{Characterization of Microembolic Signals (MES)}

When following standardized assessment, the detection of microembolic signals in stenotic arteries in real time can predict the appearance of stroke (Garami and Alexandrov, 2008). Microembolic signals are common in patients with large artery disease and are an independent marker of future stroke risk of intracranial stenosis. In the CLAIR study (Wong et al, 2010) the administration of two antiplatelet agents reduced the appearance of MES in patients with Intracranial stenosis who were given aspirin and clopidogrel within 7 days of their stroke. Whether this reduction in microembolic activity results in reduction of the occurrence of stroke remains to be tested in large scale clinical trials.

\section{Adequacy of Collaterals and Vasomotor Reactivity}

Collaterals have a significant effect on stroke outcomes, and with very severe stenoses they are shown to have a protective effect as opposed to lesser degrees of stenoses in which case the presence of collaterals signifies a greater instability (Liebeskind et al, 2010). TCD can help in evaluation of collaterals at the circle of Willis. Leptomeningeal and other secondary collaterals cannot be evaluated with this technique. Contrast addition to the imaging can help improve its sensitivity. Tests of vasomotor reactivity are also used to assess the adequacy of collaterals. A vasodilatory challenge can be given such as acetazolamide, or 
$\mathrm{CO}_{2}$ inhalation or apnea. Impaired vasomotor reactivity with $\mathrm{CO}_{2}$ has been tested with TCD and shown to correlate with stroke risk in patients with carotid stenosis (Visser et al, 2000).

\subsection{Hemodynamic monitoring}

Direct visualization of the vessels using the above techniques gives a reliable estimate of the anatomic degree of stenosis. Since intracranial atherosclerotic disease is a slowly progressive condition, it leads to chronic hypoperfusion and development of collaterals. The development of collaterals does not guarantee their persistence and they are also prone to hemodynamic fluctuations and occlusion secondary to artery to artery embolization in cases where they originate from cortical vessels. These collaterals can also be visualized using the vessel imaging techniques already described. Whether these collaterals are adequate to maintain perfusion in the territory of stenotic vessel needs evaluation with other modalities of which TCD has already been discussed.

Blood flow through the capillaries is referred to as perfusion and this is responsible for delivering oxygen to the brain tissue. Cerebral blood flow (CBF) and blood volume (CBV) are indicators of brain perfusion. Regional cerebral blood flow is proportional to the regional cerebral metabolism. Initial compensatory mechanism to deal with a decrease in perfusion is vasodilatation. Once maximum vasodialtion has occurred, oxygen extraction fraction increases to maintain the metabolic needs of the cerebral tissue. Regional cerebrovascular reserve (rCVR) is another parameter that can be measured to gauge the vasodilatory capacity of the arterioles.

Several techniques are now available to measure perfusion through the brain. These include positron emission tomography (PET), single-photon emission CT (SPECT), xenon-enhanced $\mathrm{CT}$, perfusion $\mathrm{CT}$ and MRI, and TCD. Addition of acetazolamide challenge to various imaging modalities increases their sensitivity for picking up decreased rCVR.

PET imaging measures cerebral oxygen extraction fraction (OEF) and has been the gold standard so far for identifying hemodynamic failure (Derdeyn et al, 2002). It allows for measurement of CBF, CBV and Mean Transit Time (MTT). It is currently being used to select patients with poor hemodynamic reserve, secondary to ICAD, eligible for external to internal carotid bypass. The benefit of this procedure can then also be gauged by imaging, translating as improved oxygen perfusion in previously occluded areas. Small retrospective studies have shown the outcome of external to internal bypass to be favorable (Nagata et al, 1991 and Mendelowitsch et al, 2004). PET is restricted in its utility because of its high cost and limited availability.

SPECT can be similarly utilized to measure rCVR and when used with acetazolamide challenge it provides additional information regarding hemodynamic status of the brain (Ozgur et al, 2001). This again has been utilized to judge the dependency on external carotid-internal carotid (EC-IC) and leptomeningeal collaterals. Use of acetazolamide challenge allows for assessment of vasomotor reactivity (VMR) as well which when impaired predicts future stroke. Although SPECT is more widely available compared to PET, its disadvantage lies in longer imaging time and inferior spatial resolution (Eskey and Sanelli, 2005).

Two major trials are currently ongoing to recruit patients with poor hemodynamic reserve secondary to atherosclerotic disease and to evaluate the effectiveness of the bypass procedure. These include the Carotid Occlusion Surgery Study in US (COSS) (Grubb et al, 2003) which is using PET and the Japanese EC-IC bypass Trial (JET) (Mizumura et al, 2004) which is utilizing SPECT for hemodynamic monitoring. They have so far validated the imaging modalities to recruit candidates for the procedure. 
Perfusion studies using CT and MRI can also reliably estimate CVR in patients with intracranial stenosis. Perfusion CT provides quantitative values for CBF, CBV and MTT and has recently been shown to have a significant correlation with SPECT parameters. MTT was the most predictive of a decreased CVR in this study even without use of acetazolamide challenge(Kim et al, 2009).

Xenon-enhanced computed tomography (XeCT) has been in use for the past two decades to measure cerebral blood flow and is frequently utilized by revascularization experts to calculate the cerebrovascular reserve capacity (Wintermark et al, 2001). Its major advantage lies in its superior spatial resolution, accuracy, and reproducibility. Xenon gas is inhaled and its concentration is measured in the brain by CT scanner. There is a potential for adverse reaction to the gas besides radiation exposure and motion artifacts can also affect image quality (Wintermark et al, 2005).

To conclude several techniques are available for assessment of cerebral hemodynamics. Each carries its own advantages and disadvantages. PET due to its expense and limited availability is restricted in its utilization, but SPECT and Perfusion CT are reasonable alternatives.

\section{Conclusion}

Intracranial atherosclerosis is the progressive atherosclerotic stenosis of the arteries at the base of the brain. This condition is responsible for $10-50 \%$ of ischemic strokes worldwide. It is more common in Asians, Hispanics, Blacks and South East Asians and thus in absolute numbers is probably the most common cause of stroke in the world. Radiologically, it presents with border-zone ischemia, either in the internal border-zone area or the distal cortical area, with discrete cortical infarction or a lacunar perforator infarction with involvement of the parent vessel. The intracranial stenosis and its associated collaterals can be best quantified by catheter angiography, however this is invasive and not entirely without risk. Non invasively, MRA, CTA and TCD all offer valuable and complementary information. MRA can assess flow and stenosis, however it may overestimate preocclusive stenosis. CTA may be useful in these settings where actual anatomic accuracy is needed to differentiate preocclusive stenosis from complete occlusion since it is not flow dependent. TCD provides real time hemodynamic information to delineate the mechanism of stroke in the individual patient - whether it is a flow related hemodynamic stroke, whether or not regression has occurred or that the patient has microemboli from platelet aggregation. Additionally TCD has good negative predictive value in reporting $50-90 \%$ stenosis. It is postulated that stenosis of less than $50 \%$ may also be responsible for stroke through embolizations from unstable plaque. However, very little is known about this entity except that High resolution MRI can visualize these plaques in vivo. For those with high grade progressive stenosis, the prognosis clearly depends on the extent of collateralization. This can be investigated with PET, SPECT and perfusion CT to best select patients for EC_IC Bypass procedures which are currently investigational.

\section{Acknowledgments}

Dr Maria Khan is a neurovascular fellow whose training is currently funded by Award Number D43TW008660 from the Fogarty International Center and the National Institutes of Neurologic Disorders and Stroke. Dr Ayeesha Kamal is the Principal Investigator for the 
Karachi Intracranial Stenosis Study (KISS) funded by the Higher Education Commission Government of Pakistan. The International Cerebrovascular Translational Clinical Research and Training Program (ICT_CRT) at the Aga Khan University is supported by funds from the Award Number D43TW008660 from the Fogarty International Center and the National Institute of Neurologic Disorders and Stroke. The content is solely the responsibility of the authors and does not necessarily represent the official views of the Fogarty International Center or the National Institutes of Health.

\section{References}

Altaf, N., Beech, A., Goode, S.D., Gladman, J.R., Moody, A.R., Auer, D.P., \& MacSweeney, S.T. (2007) Carotid intraplaque hemorrhage detected by magnetic resonance imaging predicts embolization during carotid endarterectomy. J Vasc Surg, 46, 3136. ISSN 0741-5214

Amarenco, P., Levy, C., Cohen, A., Touboul, P.J., Roullet, E., \& Bousser, M.G. (1994) Causes and mechanisms of territorial and nonterritorial cerebellar infarcts in 115 consecutive patients. Stroke, 25, 105-112. ISSN 0039-2499

Amin-Hanjani, S., Du, X., Zhao, M., Walsh, K., Malisch, T.W., \& Charbel, F.T. (2005) Use of quantitative magnetic resonance angiography to stratify stroke risk in symptomatic vertebrobasilar disease. Stroke, 36, 1140-1145. ISSN 0039-2499

Bash, S., Villablanca, J.P., Jahan, R., Duckwiler, G., Tillis, M., Kidwell, C., Saver, J., \& Sayre, J. (2005) Intracranial vascular stenosis and occlusive disease: evaluation with CT angiography, MR angiography, and digital subtraction angiography. AJNR Am J Neuroradiol, 26, 1012-1021. ISSN 0195-6108

Belden, J.R., Caplan, L.R., Pessin, M.S., \& Kwan, E. (1999) Mechanisms and clinical features of posterior border-zone infarcts. Neurology, 53, 1312-1318. ISSN 0028-3878

Bogousslavsky, J., \& Regli, F. (1992) Centrum ovale infarcts: subcortical infarction in the superficial territory of the middle cerebral artery. Neurology, 42, 1992-1998. ISSN 0028-3878

Brisman, J.L. (2008) Wingspan stenting of symptomatic extracranial vertebral artery stenosis and perioperative evaluation using quantitative magnetic resonance angiography: report of two cases. Neurosurg Focus, 24, E14. ISSN 1092-0684

Cloft, H.J., Lynn, M.J., Feldmann, E., \& Chimowitz, M. Risk of Cerebral Angiography in Patients with Symptomatic Intracranial Atherosclerotic Stenosis. Cerebrovasc Dis, 31, 588-591. ISSN 1015-9770

Derdeyn, C.P., Videen, T.O., Yundt, K.D., Fritsch, S.M., Carpenter, D.A., Grubb, R.L., \& Powers, W.J. (2002) Variability of cerebral blood volume and oxygen extraction: stages of cerebral haemodynamic impairment revisited. Brain, 125, 595-607. ISSN 0006-8950

Eskey, C.J., \& Sanelli, P.C. (2005) Perfusion imaging of cerebrovascular reserve. Neuroimaging Clin N Am, 15, 367-381, xi. ISSN 1052-5149

Felberg, R.A., Christou, I., Demchuk, A.M., Malkoff, M., \& Alexandrov, A.V. (2002) Screening for intracranial stenosis with transcranial Doppler: the accuracy of mean flow velocity thresholds. J Neuroimaging, 12, 9-14. ISSN 1051-2284

Feldmann, E., Wilterdink, J.L., Kosinski, A., Lynn, M., Chimowitz, M.I., Sarafin, J., Smith, H.H., Nichols, F., Rogg, J., Cloft, H.J., Wechsler, L., Saver, J., Levine, S.R., Tegeler, C., Adams, R., \& Sloan, M. (2007) The Stroke Outcomes and Neuroimaging of Intracranial Atherosclerosis (SONIA) trial. Neurology, 68, 2099-2106. ISSN 0028-3878 
Fifi, J.T., Meyers, P.M., Lavine, S.D., Cox, V., Silverberg, L., Mangla, S., \& Pile-Spellman, J. (2009) Complications of modern diagnostic cerebral angiography in an academic medical center. J Vasc Interv Radiol, 20, 442-447. ISSN 1051-0443

Furst, G., Hofer, M., Sitzer, M., Kahn, T., Muller, E., \& Modder, U. (1995) Factors influencing flow-induced signal loss in MR angiography: an in vitro study. J Comput Assist Tomogr, 19, 692-699. ISSN 0363-8715

Garami, Z., \& Alexandrov, A.V. (2009) Neurosonology. Neurol Clin, 27, 89-108, viii. ISSN 0733-8619

Grubb, R.L.Jr., Powers, W.J., Derdeyn, C.P., Adams, H.P.Jr., \& Clarke, W.R. (2003) The Carotid Occlusion Surgery Study. Neurosurg Focus, 14, e9. ISSN 1092-0684

Hirai, T., Korogi, Y., Ono, K., Nagano, M., Maruoka, K., Uemura, S., \& Takahashi, M. (2002) Prospective evaluation of suspected stenoocclusive disease of the intracranial artery: combined MR angiography and CT angiography compared with digital subtraction angiography. AJNR Am J Neuroradiol, 23, 93-101. ISSN 0195-6108

Huang, Y.N., Gao, S., Li, S.W., Huang, Y., Li, J.F., Wong, K.S., \& Kay, R. (1997) Vascular lesions in Chinese patients with transient ischemic attacks. Neurology, 48, 524-525. ISSN 0028-3878

Kang, D.W., Latour, L.L., Chalela, J.A., Dambrosia, J., \& Warach, S. (2003) Early ischemic lesion recurrence within a week after acute ischemic stroke. Ann Neurol, 54, 66-74. ISSN 0364-5134

Kim, E., Sohn, C.H., Na, D.G., Kim, J.E., Chang, K.H., Kim, J.H., \& Jeon, S.J. (2009) Perfusion computed tomography evaluation of cerebral hemodynamic impairment in patients with unilateral chronic steno-occlusive disease: a comparison with the acetazolamide challenge 99mTc-hexamethylpropyleneamine oxime single-photon emission computed tomography. J Comput Assist Tomogr, 33, 546-551. ISSN 0363-8715

Kim, J.T., Yoo, S.H., Kwon, J.H., Kwon, S.U., \& Kim, J.S. (2006) Subtyping of ischemic stroke based on vascular imaging: analysis of 1,167 acute, consecutive patients. J Clin Neurol, 2, 225-230. ISSN 1738-6586

Klein, I.F., Lavallee, P.C., Mazighi, M., Schouman-Claeys, E., Labreuche, J., \& Amarenco, P. Basilar artery atherosclerotic plaques in paramedian and lacunar pontine infarctions: a high-resolution MRI study. Stroke, 41, 1405-1409. ISSN 0039-2499

Lee, D.K., Kim, J.S., Kwon, S.U., Yoo, S.H., \& Kang, D.W. (2005) Lesion patterns and stroke mechanism in atherosclerotic middle cerebral artery disease: early diffusionweighted imaging study. Stroke, 36, 2583-2588. ISSN 0039-2499

Lee, S.J., Cho, S.J., Moon, H.S., Shon, Y.M., Lee, K.H., Kim, D.I., Lee, B.B., Byun, H.S., Han, S.H., \& Chung, C.S. (2003) Combined extracranial and intracranial atherosclerosis in Korean patients. Arch Neurol, 60, 1561-1564. ISSN 0003-9942

Liebeskind, D.S., Cotsonis, G.A., Saver, J.L., Lynn, M.J., Turan, T.N., Cloft, H.J., \& Chimowitz, M.I. Collaterals dramatically alter stroke risk in intracranial atherosclerosis. Ann Neurol. ISSN 0364-5134

Masuda, J., Yutani, C., Ogata, J., Kuriyama, Y., \& Yamaguchi, T. (1994) Atheromatous embolism in the brain: a clinicopathologic analysis of 15 autopsy cases. Neurology, 44, 1231-1237. ISSN 0028-3878

Mazighi, M., Tanasescu, R., Ducrocq, X., Vicaut, E., Bracard, S., Houdart, E., \& Woimant, F. (2006) Prospective study of symptomatic atherothrombotic intracranial stenoses: the GESICA study. Neurology, 66, 1187-1191. ISSN 0028-3878

Mendelowitsch, A., Taussky, P., Rem, J.A., \& Gratzl, O. (2004) Clinical outcome of standard extracranial-intracranial bypass surgery in patients with symptomatic 
atherosclerotic occlusion of the internal carotid artery. Acta Neurochir (Wien), 146, 95-101. ISSN 0001-6268

Mizumura, S., Nakagawara, J., Takahashi, M., Kumita, S., Cho, K., Nakajo, H., Toba, M., \& Kumazaki, T. (2004) Three-dimensional display in staging hemodynamic brain ischemia for JET study: objective evaluation using SEE analysis and 3D-SSP display. Ann Nucl Med, 18, 13-21. ISSN 0914-7187

Nagata, S., Fujii, K., Matsushima, T., Fukui, M., Sadoshima, S., Kuwabara, Y., \& Abe, H. (1991) Evaluation of EC-IC bypass for patients with atherosclerotic occlusive cerebrovascular disease: clinical and positron emission tomographic studies. Neurol Res, 13, 209-216. ISSN 0161-6412

Nahab, F., Cotsonis, G., Lynn, M., Feldmann, E., Chaturvedi, S., Hemphill, J.C., Zweifler, R., Johnston, K., Bonovich, D., Kasner, S., \& Chimowitz, M. (2008) Prevalence and prognosis of coexistent asymptomatic intracranial stenosis. Stroke, 39, 1039-1041. ISSN 0039-2499

Nguyen-Huynh, M.N., Wintermark, M., English, J., Lam, J., Vittinghoff, E., Smith, W.S., \& Johnston, S.C. (2008) How accurate is CT angiography in evaluating intracranial atherosclerotic disease? Stroke, 39, 1184-1188. ISSN 0039-2499

Ozgur, H.T., Kent Walsh, T., Masaryk, A., Seeger, J.F., Williams, W., Krupinski, E., Melgar, M., \& Labadie, E. (2001) Correlation of cerebrovascular reserve as measured by acetazolamide-challenged SPECT with angiographic flow patterns and intra- or extracranial arterial stenosis. AJNR Am J Neuroradiol, 22, 928-936. ISSN 0195-6108

Park, K.Y., Chung, C.S., Lee, K.H., Kim, G.M., Kim, Y.B., \& Oh, K. (2006) Prevalence and risk factors of intracranial atherosclerosis in an asymptomatic korean population. J Clin Neurol, 2, 29-33.ISSN 1738-6586

Pollanen, M.S., \& Deck, J.H. (1990) The mechanism of embolic watershed infarction: experimental studies. Can J Neurol Sci, 17, 395-398. ISSN 0317-1671

Prabhakaran, S., Warrior, L., Wells, K.R., Jhaveri, M.D., Chen, M., \& Lopes, D.K. (2009) The utility of quantitative magnetic resonance angiography in the assessment of intracranial in-stent stenosis. Stroke, 40, 991-993. ISSN 0039-2499

Ratanakorn, D., Kremkau, F.W., Myers, L.G., Meads, D.B., \& Tegeler, C.H. (1998) Mirrorimage artifact can affect transcranial Doppler interpretation. J Neuroimaging, 8, 175177. ISSN 1051-2284

Roh, J.K., Kang, D.W., Lee, S.H., Yoon, B.W., \& Chang, K.H. (2000) Significance of acute multiple brain infarction on diffusion-weighted imaging. Stroke, 31, 688-694. ISSN 0039-2499

Rorick, M.B., Nichols, F.T., \& Adams, R.J. (1994) Transcranial Doppler correlation with angiography in detection of intracranial stenosis. Stroke, 25, 1931-1934. ISSN 0039-2499

Rovira, A., Grive, E., \& Alvarez-Sabin, J. (2005) Distribution territories and causative mechanisms of ischemic stroke. Eur Radiol, 15, 416-426. ISSN 0938-7994

Sacco, R.L., Kargman, D.E., Gu, Q., \& Zamanillo, M.C. (1995) Race-ethnicity and determinants of intracranial atherosclerotic cerebral infarction. The Northern Manhattan Stroke Study. Stroke, 26, 14-20. ISSN 0039-2499

Skutta, B., Furst, G., Eilers, J., Ferbert, A., \& Kuhn, F.P. (1999) Intracranial stenoocclusive disease: double-detector helical CT angiography versus digital subtraction angiography. AJNR Am J Neuroradiol, 20, 791-799. ISSN 0195-6108

Thiex, R., Norbash, A.M., \& Frerichs, K.U. The safety of dedicated-team catheter-based diagnostic cerebral angiography in the era of advanced noninvasive imaging. AJNR Am J Neuroradiol, 31, 230-234. ISSN 0195-6108 
Torvik, A., \& Skullerud, K. (1982) Watershed infarcts in the brain caused by microemboli. Clin Neuropathol, 1, 99-105. ISSN 0722-5091

Turan, T.N., Bonilha, L., Morgan, P.S., Adams, R.J., \& Chimowitz, M.I. Intraplaque hemorrhage in symptomatic intracranial atherosclerotic disease. J Neuroimaging, 21, e159-161. ISSN 1051-2284

Turtzo, L.C., Gottesman, R.F., \& Llinas, R.H. (2009) Diffusion-weighted imaging showing 'pearls' predicts large-vessel disease as stroke etiology. Cerebrovasc Dis, 28, 49-54. ISSN 1015-9770

Wintermark, M., Thiran, J.P., Maeder, P., Schnyder, P., \& Meuli, R. (2001) Simultaneous measurement of regional cerebral blood flow by perfusion CT and stable xenon CT: a validation study. AJNR Am J Neuroradiol, 22, 905-914. ISSN 0195-6108

Wintermark, M., Sesay, M., Barbier, E., Borbely, K., Dillon, W.P., Eastwood, J.D., Glenn, T.C., Grandin, C.B., Pedraza, S., Soustiel, J.F., Nariai, T., Zaharchuk, G., Caille, J.M., Dousset, V., \& Yonas, H. (2005) Comparative overview of brain perfusion imaging techniques. J Neuroradiol, 32, 294-314. ISSN 0150-9861

Wityk, R.J., Lehman, D., Klag, M., Coresh, J., Ahn, H., \& Litt, B. (1996) Race and sex differences in the distribution of cerebral atherosclerosis. Stroke, 27, 1974-1980. ISSN 0039-2499

Wong, K.S., Li, H., Chan, Y.L., Ahuja, A., Lam, W.W., Wong, A., \& Kay, R. (2000) Use of transcranial Doppler ultrasound to predict outcome in patients with intracranial large-artery occlusive disease. Stroke, 31, 2641-2647. ISSN 0039-2499

Wong, K.S., Gao, S., Chan, Y.L., Hansberg, T., Lam, W.W., Droste, D.W., Kay, R., \& Ringelstein, E.B. (2002) Mechanisms of acute cerebral infarctions in patients with middle cerebral artery stenosis: a diffusion-weighted imaging and microemboli monitoring study. Ann Neurol, 52, 74-81. ISSN 0364-5134

Wong, K.S., Ng, P.W., Tang, A., Liu, R., Yeung, V., \& Tomlinson, B. (2007) Prevalence of asymptomatic intracranial atherosclerosis in high-risk patients. Neurology, 68, 20352038. ISSN 0028-3878

Wong, K.S., Chen, C., Fu, J., Chang, H.M., Suwanwela, N.C., Huang, Y.N., Han, Z., Tan, K.S., Ratanakorn, D., Chollate, P., Zhao, Y., Koh, A., Hao, Q., \& Markus, H.S. (2010) Clopidogrel plus aspirin versus aspirin alone for reducing embolisation in patients with acute symptomatic cerebral or carotid artery stenosis (CLAIR study): a randomised, open-label, blinded-endpoint trial. Lancet Neurol, 9, 489-497. ISSN 1474-4422

Yamauchi, H., Nishii, R., Higashi, T., Kagawa, S., \& Fukuyama, H. (2009) Hemodynamic compromise as a cause of internal border-zone infarction and cortical neuronal damage in atherosclerotic middle cerebral artery disease. Stroke, 40, 3730-3735. ISSN 0039-2499

Yong, S.W., Bang, O.Y., Lee, P.H., \& Li, W.Y. (2006) Internal and cortical border-zone infarction: clinical and diffusion-weighted imaging features. Stroke, 37, 841-846. ISSN 0039-2499

Yuan, C., Kerwin, W.S., Yarnykh, V.L., Cai, J., Saam, T., Chu, B., Takaya, N., Ferguson, M.S., Underhill, H., Xu, D., Liu, F., \& Hatsukami, T.S. (2006) MRI of atherosclerosis in clinical trials. NMR Biomed, 19, 636-654. ISSN 0952-3480

Zhao, M., Amin-Hanjani, S., Ruland, S., Curcio, A.P., Ostergren, L., \& Charbel, F.T. (2007) Regional cerebral blood flow using quantitative MR angiography. AJNR Am J Neuroradiol, 28, 1470-1473. ISSN 0195-6108 


\title{
Neuroimaging in Multiple Sclerosis
}

\author{
Elisabeth Andreadou \\ Department of Neurology, Athens National and Kapodistrian University, \\ "Aeginition Hospital", Athens \\ Greece
}

\section{Introduction}

Multiple sclerosis (MS) is a chronic disabling autoimmune disorder of the central nervous system histopathologically characterized by inflammation, multifocal demyelination, axonal damage and neuronal loss. Focal intensive demyelination and white matter infiltration by lymphocytes and mononuclear cells are pathological hallmarks of the disease (Ferguson et al., 1997; Lucchinetti et al., 2000).

Complementary to the clinical evaluation, conventional magnetic resonance imaging (cMRI) plays a prominent role for diagnosis and assessment of patients with multiple sclerosis. It provides reliable detection and quantitative estimation of focal white matter lesions in vivo. Modern criteria involve MRI parameters for the diagnosis of MS and for predicting conversion to clinically definite MS in patients who present with a first clinical episode (eg, unilateral optic neuritis) suggestive of disease onset. A diagnosis of multiple sclerosis is based on showing disease dissemination in space and time and excluding other neurological disorders that can clinically and radiologically mimic multiple sclerosis. However, neurological impairment of patients with MS is poorly associated with the lesion load observed on conventional MRI scans. The discrepancy between clinical and conventional MRI findings in MS is explained, at least partially, by the low sensitivity of conventional MRI in the detection of grey-matter involvement and diffuse damage in white matter.

In order to overcome these limitations new uses of conventional MRI methods have evolved and nonconventional MRI techniques have been developed. These advances are expected to help in understanding the underlying disease processes and the accumulation of irreversible disability and therefore are promising tools in studies of disease evolution and clinical trials.

Optical coherence tomography (OCT) -a new, sensitive and non-invasive transpupillary imaging method- is able to provide accurate and quantitative measurements of axonal loss in retina and therefore holds promise as a biomarker for neurodegeneration in MS.

This chapter reviews the use of cMRI techniques to diagnosis and follow-up of patients with MS. It also summarizes the evolving non-conventional MRI and non-invasive optical techniques, which are sensitive to different aspects of MS pathology and are promising to further increase our understanding of disease pathophysiology and the mechanisms accounting for the accumulation of irreversible disability. 


\section{MRI and the diagnosis of multiple sclerosis}

Since multiple sclerosis is a histopathological diagnosis, clinical criteria have been developed for the diagnosis of MS during life. Initial diagnostic criteria were based on clinical features alone to show disease dissemination in space and in time (Schumacher et al., 1965). The introduction of MRI in clinical practice resulted in improved investigation of multiple sclerosis (Young et al., 1981). Paraclinical evidence to support clinical findings in cases of diagnostic uncertainty was first proposed by Poser and colleagues (Poser et al., 1983). Given that MRI is the most sensitive paraclinical test and that comparable MRI abnormalities may be found in a variety of other diseases and in healthy volunteers, criteria have been developed by which MRIs can be classified as suggestive of multiple sclerosis or not (Fazekas et al., 1988; Paty et al., 1988; Barkhof et al., 1997; Tintore et al., 2000).

With the increased availability of MRI scanners and the high sensitivity and ability of MRI to provide evidence for disease dissemination in both space and time, subsequent consensus criteria relied on MRI findings to complement clinical and other paraclinical data for the diagnosis of MS (McDonald et al., 2001). The 2001 McDonald diagnostic criteria for multiple sclerosis included MRI evidence of dissemination in space (DIS) and dissemination in time (DIT), which allowed a diagnosis of multiple sclerosis to be made in patients with clinically isolated syndrome (CIS) (McDonald et al., 2001). The DIS criteria were those developed for brain MRI by Barkhof and colleagues (1997) and Tintore and colleagues (2000), in addition to allowing one spinal-cord lesion to substitute for a brain lesion. Evidence for DIT requires either a gadolinium enhancing lesion after at least 3 months from CIS onset, or a new T2 lesion developing on a scan subsequent to a reference scan obtained at least 3 months after CIS onset (Table 1).

The incorporation of MRI findings to the diagnostic criteria improved the ability to diagnose MS and exclude alternative diagnoses. Apparently MRI started to play a prominent role in establishing a firm and clear diagnosis of multiple sclerosis and predicting conversion to clinically definite MS in patients who present with a first clinical episode (eg, unilateral optic neuritis) suggestive of disease onset. The necessity of early and accurate diagnosis became particularly important with the advent of disease modifying treatments. Subsequently, the McDonald criteria were revised in 2005 by Polman and colleagues, to simplify their use and increase their sensitivity while maintaining their specificity. These revised criteria strengthen the use of spinal cord lesions in MS diagnosis, allowing a spinal cord lesion to substitute for an infratentorial brain lesion. In 2006, new simpler imaging criteria were proposed with similar high specificity for clinically definite MS (CDMS) and increased sensitivity (Swanton et al., 2006), in which DIS requires at least one T2 lesion in at least two of four locations (juxtacortical, periventricular, infratentorial, and spinal-cord) and DIT requires a new $\mathrm{T} 2$ lesion on a follow-up scan. Thereafter, new data and consensus underlined the need to simplify the revised McDonald Criteria in order to improve their comprehension and utility. Accumulating evidence resulted recently in specific recommendations for revisions to the McDonald Criteria. These proposals concerned the use and interpretation of imaging criteria for DIS and DIT and were based on the simplified criteria of Swanton and colleagues (Polman et al., 2011). The 2010 revisions to the McDonald Criteria simplify the diagnostic process with fewer required MRI examinations, allowing a more rapid diagnosis of MS, with preserved or improved specificity and sensitivity 
compared with past Criteria (Table 2). However, even these latest revisions do not address issues of lesion morphology except to emphasize the importance of Gd enhancement and insufficiently deal with lesion location. Distribution and morphology provide important clues to the suspected nature of the detected lesions. In addition, misapplication of the McDonald criteria can lead to misdiagnosis in subjects without relevant clinical symptoms. Therefore, a diagnosis of MS based on incidental findings on MRI alone, should be avoided (Polman et al., 2011).

\begin{tabular}{|c|c|}
\hline Clinical Presentation & Additional Data Needed \\
\hline $\begin{array}{l}\text { - } 2 \text { or more attacks (relapses) } \\
\text { - } \quad 2 \text { or more objective clinical lesions }\end{array}$ & $\begin{array}{l}\text { None; clinical evidence will suffice } \\
\text { (additional evidence desirable but must be } \\
\text { consistent with MS) }\end{array}$ \\
\hline $\begin{array}{l}\text { - } 2 \text { or more attacks } \\
\text { - } \quad 1 \text { objective clinical lesion }\end{array}$ & $\begin{array}{l}\text { Dissemination in space, demonstrated by: } \\
\text { - } \quad \text { MRI } \\
\text { - } \quad \text { or a positive CSF and } 2 \text { or more MRI lesions } \\
\text { consistent with MS } \\
\text { - } \quad \text { or further clinical attack involving different } \\
\text { site }\end{array}$ \\
\hline $\begin{array}{l}\text { - } 1 \text { attack } \\
\text { - } 2 \text { or more objective clinical lesions }\end{array}$ & $\begin{array}{l}\text { Dissemination in time, demonstrated by: } \\
\text { - } \quad \text { MRI } \\
\text { - } \quad \text { or second clinical attack }\end{array}$ \\
\hline $\begin{array}{l}\text { - } 1 \text { attack } \\
\text { - } 1 \text { objective clinical lesion } \\
\text { (monosymptomatic presentation) }\end{array}$ & $\begin{array}{l}\text { Dissemination in space demonstrated by: } \\
\text { - MRI } \\
\text { - or positive CSF and } 2 \text { or more MRI lesions } \\
\text { and } \\
\text { Dissemination in time demonstrated by: } \\
\text { - MRI } \\
\text { - or second clinical attack }\end{array}$ \\
\hline $\begin{array}{l}\text { Insidious neurological progression } \\
\text { suggestive of MS } \\
\text { (primary progressive MS) }\end{array}$ & 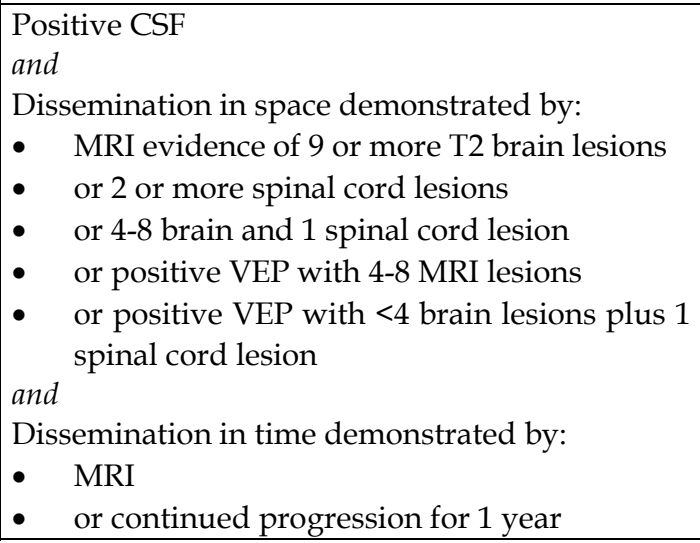 \\
\hline
\end{tabular}

Table 1. 2001 McDonald diagnostic criteria for multiple sclerosis (from McDonald et al., 2001; with permission) 


\begin{tabular}{|c|c|}
\hline Clinical Presentation & Additional Data Needed \\
\hline $\begin{array}{ll}- & 2 \text { or more attacks (relapses; } \\
\text { exacerbations) } & 1 \\
\text { - } & 2 \text { or more objective clinical lesions or } \\
\text { - } & \text { Objective clinical evidence of } 1 \text { lesion } \\
& \text { with reasonable historical evidence of a } \\
\text { prior attack } 2\end{array}$ & None $^{3}$ \\
\hline $\begin{array}{l}\text { - } 2 \text { or more attacks }{ }^{1} \\
\text { - } \quad \text { Objective clinical evidence of } 1 \text { lesion }\end{array}$ & $\begin{array}{l}\text { Dissemination in space, demonstrated by: } \\
\text { - } 1 \text { T2 lesion in at least } 2 \text { of } 4 \text { MS-typical } \\
\text { regions of the CNS (periventricular, } \\
\text { juxtacortical, infratentorial, or spinal } \\
\text { cord) } 4 \\
\text { or } \\
\text { - } \\
\text { Await a further clinical attack } 1 \\
\text { implicating a different CNS site } \\
\end{array}$ \\
\hline $\begin{array}{ll}\text { - } & 1 \text { attack }^{1} \\
\text { - Objective clinical evidence of } 2 \text { or more } \\
\text { lesions }\end{array}$ & $\begin{array}{l}\text { Dissemination in time, demonstrated by: } \\
\text { - Simultaneous presence of } \\
\text { asymptomatic gadolinium-enhancing } \\
\text { and nonenhancing lesions at any time; } \\
\text { or } \\
\text { - A new T2 and/or gadolinium- } \\
\text { enhancing lesion(s) on follow-up MRI, } \\
\text { irrespective of its timing with reference } \\
\text { to a baseline scan; } \\
\text { or } \\
\text { - }\end{array}$ \\
\hline $\begin{array}{l}\text { - } 1 \text { attack }^{1} \\
\text { - } \\
\text { Objective clinical evidence of } 1 \text { lesion } \\
\text { (clinically isolated syndrome) }\end{array}$ & $\begin{array}{l}\text { Dissemination in space and time, } \\
\text { demonstrated by: } \\
\text { For DIS: } \\
\text { 1 or more T2 lesion in at least } 2 \text { of } 4 \text { MS- } \\
\text { typical regions of the CNS (periventricular, } \\
\text { juxtacortical, infratentorial, or spinal cord) }{ }^{4} \\
\text { or } \\
\text { - Await a second clinical attack }{ }^{1} \\
\text { implicating a different CNS site; } \\
\text { and } \\
\text { For DIT: } \\
\text { - Simultaneous presence of } \\
\text { asymptomatic gadolinium-enhancing } \\
\text { and nonenhancing lesions at any time; } \\
\text { or } \\
\text { - A new T2 and/or gadolinium- } \\
\text { enhancing lesion(s) on follow-up MRI, } \\
\text { irrespective of its timing with reference } \\
\text { to a baseline scan; } \\
\text { or } \\
\text { Await a second clinical attack }{ }^{1}\end{array}$ \\
\hline
\end{tabular}




\begin{tabular}{|l|l|}
\hline \multicolumn{1}{|c|}{ Clinical Presentation } & \multicolumn{1}{c|}{ Additional Data Needed } \\
\hline & $\begin{array}{l}1 \text { year of disease progression } \\
\text { (retrospectively or prospectively } \\
\text { determined) plus } 2 \text { of } 3 \text { of the following } \\
\text { criteria: } 4 \\
\text { Insidious neurological progression } \\
\text { suggestive of MS (PPMS) }\end{array}$ \\
$\begin{array}{l}\text { Evidence for DIS in the brain based on } \\
1 \text { or more T2 lesions in the MS- } \\
\text { characteristic (periventricular, } \\
\text { juxtacortical, or infratentorial) regions } \\
\text { Evidence for DIS in the spinal cord } \\
\text { based on } 2 \text { or more T2 lesions in the } \\
\text { cord } \\
\text { Positive CSF (isoelectric focusing } \\
\text { evidence of oligoclonal bands and/or } \\
\text { elevated IgG index) }\end{array}$ \\
\hline
\end{tabular}

${ }^{1}$ An attack is defined as patient-reported or objectively observed events typical of an acute inflammatory demyelinating event in the CNS, current or historical, with duration of at least 24 hours, in the absence of fever or infection.

${ }^{2}$ Clinical diagnosis based on objective clinical findings for 2 attacks is most secure. Historical evidence for 1 past attack can include events with symptoms and evolution characteristic for a prior inflammatory demyelinating episode; at least 1 attack, however, must be supported by objective findings.

${ }^{3}$ No additional tests are required. However, it is desirable that any diagnosis of MS be made with access to imaging based on these Criteria.

${ }^{4}$ Gadolinium-enhancing lesions are not required; symptomatic lesions are excluded from consideration in subjects with brainstem or spinal cord syndromes.

Table 2. The 2010 McDonald Criteria for Diagnosis of MS (from Polman et al., 2011; with permission)

\subsection{Characteristic findings on conventional MRI techniques}

Hydrogen protons of water are highly abundant in our body. Conventional MRI measures alterations in tissue water content and dynamics by proton excitation, thus enabling visualization of edema, inflammation, demyelination and axonal damage in MS lesions. Conventional MRI techniques include T2-weighted, fast fluid-attenuated inversion recovery (FLAIR), and T1-weighted imaging with and without gadolinium (Gd) administration.

In routine evaluation of patients with suspected or known MS, the most useful image acquisition clinical protocols for cMRI are axial dual spin-echo or single late echo T2weighted imaging, axial and sagittal FLAIR, and pre- and post-gadolinium (Gd)-enhanced axial spin-echo T1-weighted imaging (Bakshi et al., 2004). On cMRI scans, detected MS lesions are presented as hyperintensities on T2- weighted images, hypointensities on T1weighted images, and Gd-enhancing foci on postcontrast images.

\subsubsection{T2-weighted imaging}

T2-weighted MRI is sensitive in detecting white matter lesions. In T2-weighted images, MS lesions appear as bright areas against a gray or more neutral background; this hyperintesity reflects an increase in water content and thus is not specific for the underlying pathology. 
The T2 hyperintense lesions represent processes ranging from edema and inflammation to demyelination, remyelination, Wallerian degeneration, axonal loss and gliosis (Fillipi \& Agosta, 2009; Markovic-Plese \& McFarland, 2001). Once detected, a lesion typically persists in the T2 hyperintense stage for many years. The techniques that are commonly used for the identification of T2 hyperintense lesions are conventional spin echo, fast spin echo, and fluid-attenuated inversion recovery (FLAIR) (Filippi et al., 2006). FLAIR imaging is a technique that suppresses the CSF signal, being more sensitive to periventricular and cortical/juxtacortical lesions and less sensitive to infratentorial lesions than T2-weighted MRI (Bakshi et al., 2001).

Given the lack of specificity of hyperintensities on T2-weighted MRI, lesion location and morphology plays a prominent role in distinguishing demyelinating lesions from the common and incidental presence of non-specific white-matter abnormalities, microvascular ischemia, or enlarged perivascular spaces (Fig. 1). MS lesions are primarily found in white matter but also in gray matter and usually affect the periventricular regions, corpus callosum, juxtacortical gray-white matter junction in the white matter, and infratentorial brain regions (brain stem, middle cerebellar peduncles and cerebellar white matter in the posterior fossa) (Bakshi et al., 2004; Barkhof et al., 1997). In some cases, direct involvement of the cerebral cortex also may be apparent (Bakshi et al., 2001). Corpus callosum is commonly affected in MS due to the large amount of myelinated fibres it contains. Callosal demyelinating lesions are usually multiple, small, nodular and characteristically involve callosal-septal interface. Infratentorial lesions are considered specific for multiple sclerosis and are typically located at the surface of the pons, the base of the fourth ventricle, and in the intra-axial trigeminal tract. It is important to note however that they can also be found in small-vessel disorder, with characteristic involvement of the central part of the pons (Kwa et al., 1997). Demyelinating lesions are larger (> 3mm) than punctuate non-specific white matter abnormalities associated with migraine and microvascular ischemia. Characteristically, they have an ovoid shape and appear perpendicular to the long axis of the ventricles in the axial plane. These typical MRI findings reflect the histopathological findings in multiple sclerosis. The periventricular lesions, especially in the corpus callosum, and the ovoid abnormalities with extensions (Dawson' fingers) into the adjacent white matter correlate with the perivenular distribution of multiple sclerosis plaques (GeanMarton et al., 1991).

Between $50 \%$ and $90 \%$ of MS patients show hyperintense T2 lesions in the spinal cord, more frequently located in the cervical and the thoracic area (Lycklama et al., 2003; Agosta \& Filippi, 2007). Cord lesions are usually located peripherally, occupy less than one half of the cord cross-sectional area and rarely involve more than two contiguous vertebral segments in length. Acute lesions may be associated with cord swelling. The presence of characteristic spinal cord abnormalities on MRI increases both sensitivity and specificity to the diagnosis of MS (Fillipi \& Agosta, 2009).

In $50-70 \%$ of patients presenting with CIS clinically silent MRI lesions suggestive of MS are found. The extent of T2 lesions on brain MRI in CIS patients can predict the future risk of MS. The majority of CIS patients with T2 abnormalities (56-88\%), develop clinically definite MS (CDMS) after prolonged (7-14 years) follow-up, whereas a minority of those with normal MRI (approximately 20\%) have a risk for conversion to definite MS (Barkoff et al., 1997; Tintoré et al, 2006). Moreover, brain T2 hyperintense lesion load early in the clinical course of MS is also a predictor of long-term disability (Di Filippo et al., 2010). 


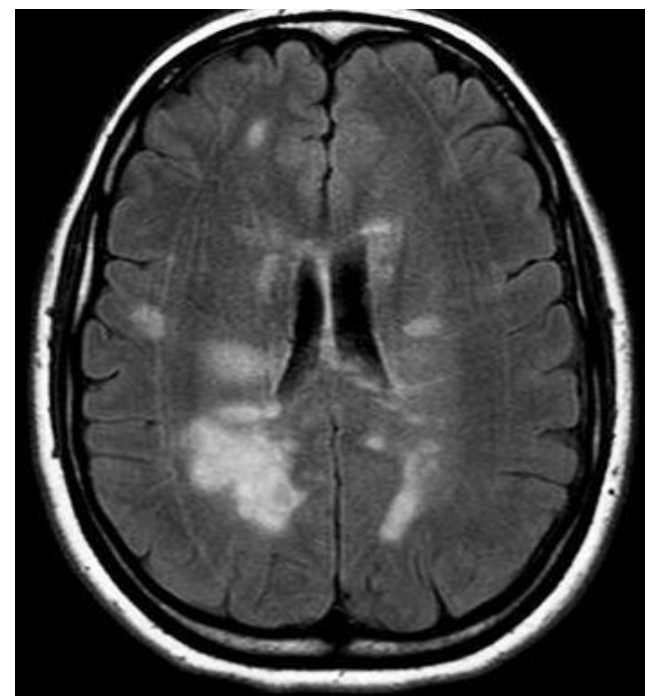

(a) Axial FLAIR image showing typical ovoid periventricular hyperintense MS lesions

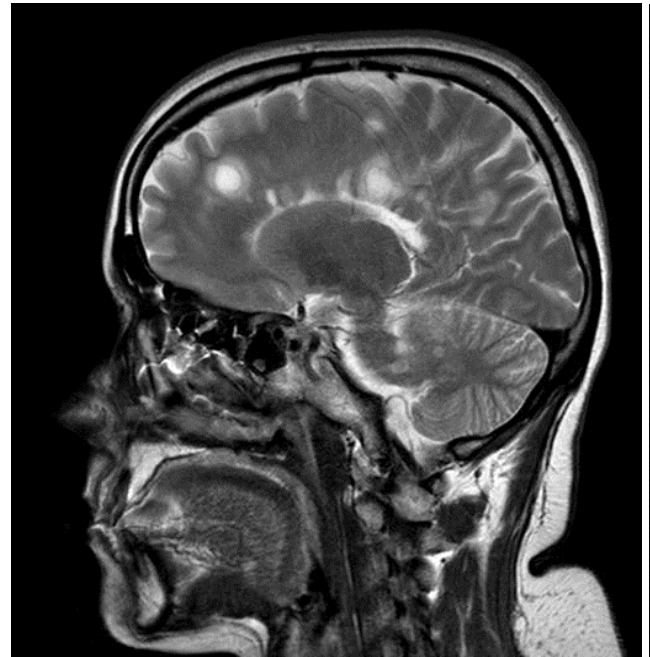

(c) Sagittal T2-weighted image demonstrating lesions in the corpus callosum and periventricular white matter with characteristic extensions (Dawson's fingers)

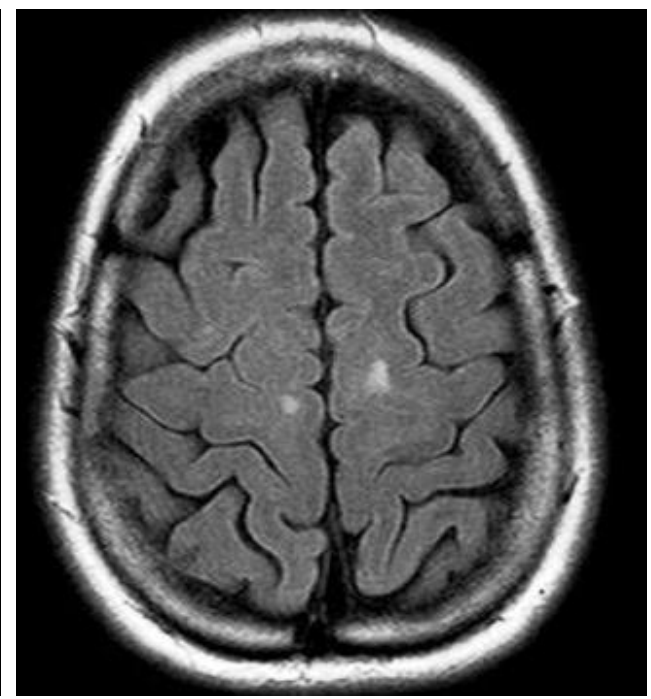

(b) Axial FLAIR scan revealing juxtacortical lesions

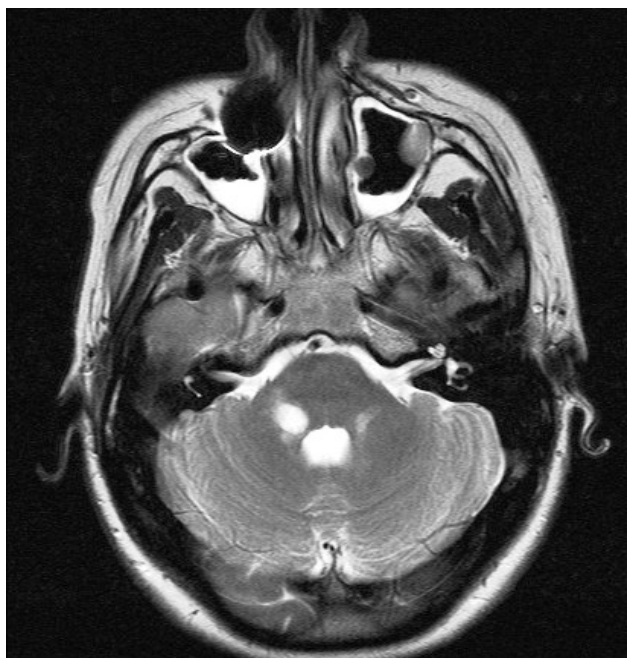

(d) Axial T2 scan showing characteristic infratentorial involvement

Fig. 1. (a-d). Axial and sagittal FLAIR and T2 images showing common sites of MS involvement. 


\subsubsection{T1-weighted imaging}

On noncontrast T1-weighted images (T1-WI), most T2 brain lesions are isointense in white matter; however, some are hypointense (van Walderveen et al., 1998). These hypointense lesions may be acute and reversible within 6 months or chronic (referred to as black holes) (Fig. 2). The acute T1 hypointense lesions are likely to represent oedema and inflammation or demyelination with subsequent remyelination whereas the chronic ones severe demyelination and irreversible axonal loss (Bagnato et al., 2003; Brex et al., 2000; Filippi et al., 2001; Van Walderveen et al., 1998). The percentage of newly formed hypointense lesions that do revert to isointensity varies between 20 and 60\% (van Waesberghe et al., 1998). Larger size of the lesions, longer duration of Gd-enhancement and ring enhancement are associated with the development of persistent T1- hypointense black holes (Mineboo et al., 2005). T1 hypointense lesions correlate better with clinical evolution and disability than T2 lesions and therefore may be a useful biomarker of progressive tissue damage in established MS (Truyen et al., 1996). By contrast to chronic cerebral lesions, chronic spinal cord lesions are isointense on T1-weighted images (Fillipi \& Agosta, 2009).

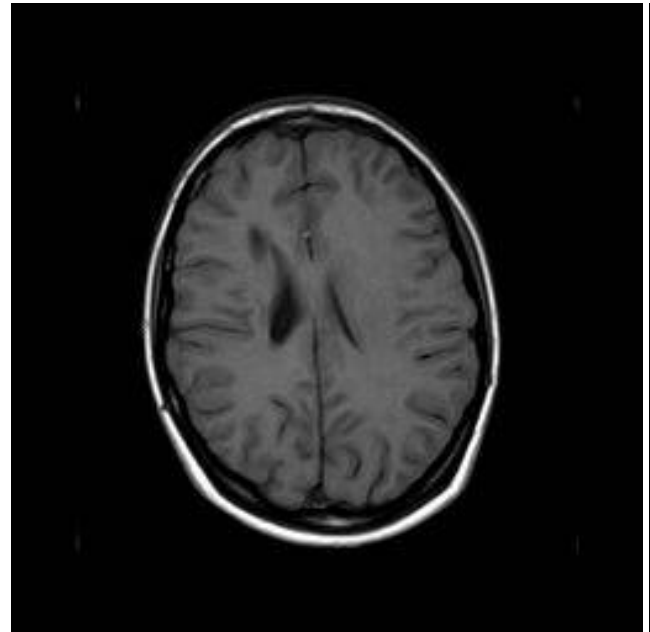

a) Axial T1-weighted noncontrast MRI showing a chronic hypointensity in the right frontal periventricular white matter

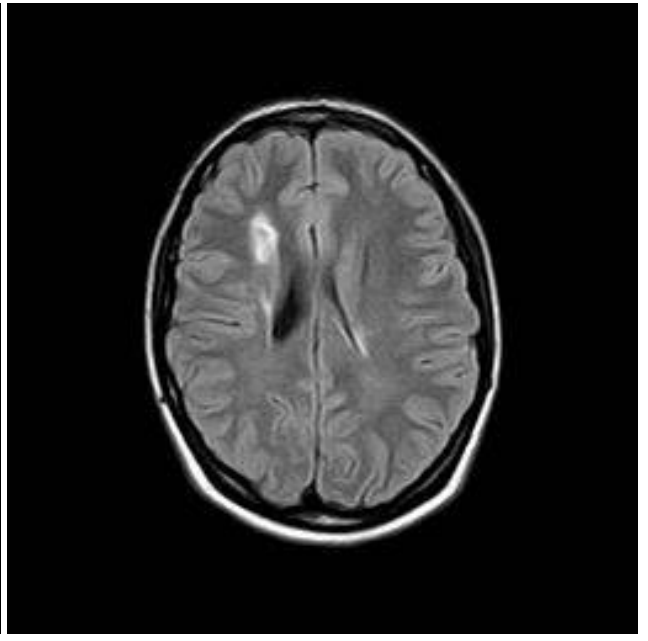

(b) Corresponding hyperintensity on FLAIR scan.

Fig. 2. (a-b) A chronic black hole in a 32-year-old woman with relapsing-remitting MS

\subsubsection{Postcontrast T1-weighted imaging}

On T1-weighted scans, gadolinium injection enables detection of lesions with active inflammation and blood-brain barrier (BBB) disruption, as it is normally excluded from the brain by the BBB. Gd-enhancing lesions on T1-WI typically correspond to areas of high signal intensity on T2-WI and, in some instances, low signal intensity on T1-WI that stem from edema and demyelination (Zivadinov \& Cox, 2007). There are various patterns of gadolinium enhancement in MS lesions that usually persists for 2 - 6 weeks (Cotton et al., 2003): homogeneous, heterogeneous, punctuate, ring, open ring enhancing and tumor-like (Fig. 3). Among them, the incomplete or open-ring morphology (Fig. 3a) is particularly 
characteristic of MS lesions (Masdeu et al., 1996). Compared with homogeneously enhancing plaques, ring-enhancing lesions are larger and have a shorter duration of enhancement (Minneboo et al., 2005). Concentric ring enhancing lesions with central contrast pallor appear in areas of heightened local inflammation (Rovira et al., 1999).

Results of serial MRI studies demonstrate that enhancement usually precedes or accompanies the appearance of most new lesions on T2-WI in all disease subtypes, even in primary-progressive MS (Molyneux et al., 2001). However, Gd- enhancement is more commonly seen in patients with relapsing-remitting (RRMS) than in patients with primary or secondary progressive MS (Thompson et al., 2000) and is considered sensitive to disease activity and predictive of future clinical relapses. Enhancing lesions do not correlate with long-term disability (Kappos et al., 1999), with the exception of ring-enhancing plaques that are usually suggestive of a more aggressive form of MS. Ring-enhancement is associated with severe tissue damage, persisting hypointense T1 lesions and subsequent development of cerebral atrophy (Morgen et al., 2001; Bagnato et al., 2003; Minneboo et al., 2005;). Enhancing lesions are less frequently seen in the cord (Fig. 3b) than in the brain (Filippi \& Agosta, 2009).

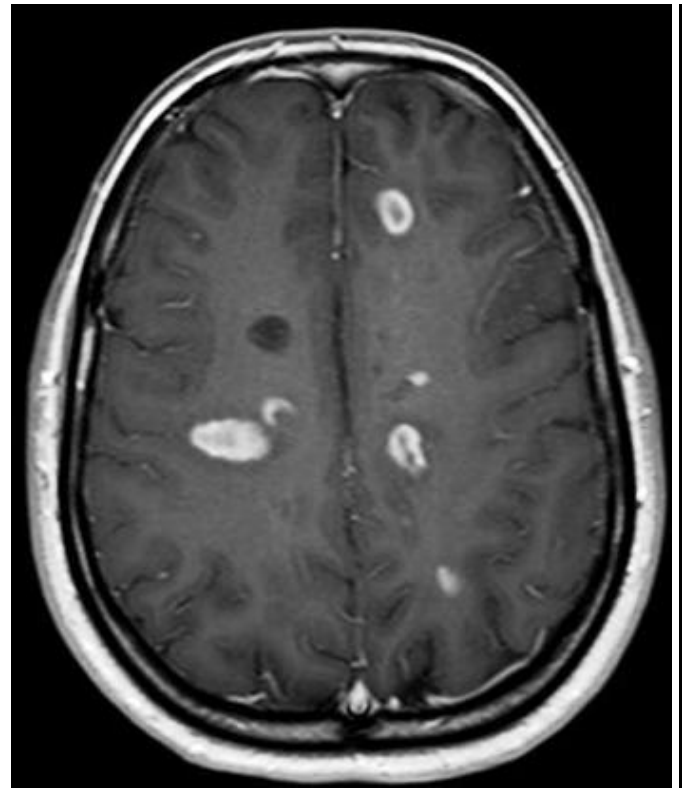

(a) Puctuate, homogeneous, and ring enhancing brain lesions

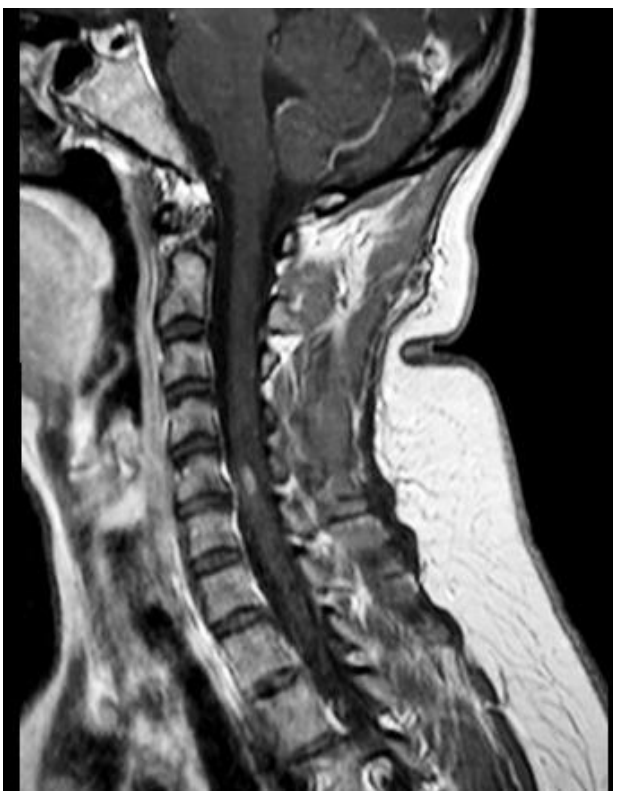

(b) Spinal enhancing lesion

Fig. 3. Characteristic patterns of enhancement on T1-weighted scans

\subsection{Conventional MRI characteristics suggesting alternative diagnoses}

Although existing diagnostic criteria for multiple sclerosis have emphasized that alternative explanation for the clinical presentation must be considered and excluded before a diagnosis of MS can be made, they mainly focus on early diagnosis of patients presenting with a first clinical episode suggestive of MS (e.g., unilateral optic neuritis, internuclear 
ophthalmoplegia, partial myelopathy). Incorporated data from magnetic resonance imaging are used as prognostic for the subsequent development of clinically definite MS, rather than diagnostic (McDonald et al., 2001; Polman et al., 2005, 2011). However, the increased availability of MRI scanners and the sensitivity of this technique in showing asymptomatic lesions of multiple sclerosis, have revealed difficulties in the application of existing MRI criteria, the most common of which is the incidental presence of non-specific white-matter abnormalities on MRI scans of patients who have headache, vertigo or a variety of other common disorders and the inability to differentiate these lesions from those that are typical for MS. Recognition of MRI features that are not suggestive of MS or even suggestive of another disorder is essential, as it underlines the need for extensive evaluation of patients presenting with such abnormalities in order to avoid misdiagnosis.

A set of MRI signs (red flags), which suggest alternative conditions that mimic MS has been defined by the European MAGNIMS group (Charil et al., 2006). It is important to note however that these red flags (Table 3) should be considered in the appropriate clinical context in order to exclude the most common alternatives (Miller et al., 2008).

The distribution of lesions is an important parameter that should be considered in the differential diagnosis of demyelinating lesions. In hypoxic-ischemic diseases lesions follow arterial anatomy, such as borderzone or watershed lesions, lacunes, multifocal basal ganglia lesions and cortical infarcts whereas in multiple sclerosis are perivenular (Enzinger et al., 2006). Moreover, the corpus callosum is not usually involved in hypoxic-ischemic diseases, due to its rich blood supply; on the contrary, this is commonly affected in MS due to the large amount of myelinated fibres it contains, with characteristic involvement of callosalseptal interface (Gean-Marton et al., 1991). Infratentorial lesions are uncommon in smallvessel disorder, but when they occur they are typically located in the central part of the pons (Kwa et al., 1997). The predominance of lesions located at the cortical or subcortical areas, as well as the coexistence of brain infarcts, calcification, or haemorrhages are usually suggestive of systemic immune mediated diseases with CNS involvement, small-vessel vasculitides, or antiphospholipid antibody syndrome (either primary or secondary to systemic immune-mediated diseases). In these disorders, enhancing lesions and T1 black holes are much less common than in multiple sclerosis (Theodoridou \& Settas, 2008). Extensive brainstem and basal ganglia lesions that can be associated with swelling and enhancement in the acute phase and that can shrink or disappear at follow-up may be a manifestation of Behçet's disease (Lee et al., 2001). Bilateral and symmetric white-matter lesions are other important features against the diagnosis of multiple sclerosis. Although adult forms of leucodystrophies, such as adrenoleucodystrophy, metachromatic leucodystrophy, and Krabbe disease may have a MRI pattern resembling that of MS, whitematter lesions tend to be bilateral and symmetric (Barkhof \& Scheltens, 2002). Diffuse and confluent symmetrical T2-weighted hyperintensities in the deep and periventricular white matter with characteristic involvement of the external capsules and temporal poles are considered highly specific for cerebral autosomal dominant arteriopathy with subcortical infarcts and leucoencephalopathy (CADASIL) and may distinguish it from MS (Andreadou et al., 2008; O'Sullivan et al., 2001; Singhal et al., 2005). Moreover, the cortex, corpus callosum and infratentorial regions (except the pons) are rarely affected in CADASIL (Singhal et al., 2005). Furthermore, the presence of multiple microhaemorrhagic foci, which can be seen on gradient-echo images, is another characteristic feature of CADASIL (Dichgans et al., 2002). Another feature pointing against the diagnosis of MS is significant asymmetry of the white-matter abnormalities, which is usually suggestive of ipsilateral carotid disease (Andreadou et al., 2010; Barkhof \& Scheltens, 2002). 


\begin{tabular}{|c|c|}
\hline Brain white matter & Disease \\
\hline Normal & NMO (absent or few lesions), ATM \\
\hline \multicolumn{2}{|l|}{ Lesions } \\
\hline \multicolumn{2}{|l|}{ Morphology } \\
\hline Large lesions & $\begin{array}{l}\text { AMS (sometimes confluent and } \\
\text { perilesional oedema), } \\
\text { BCS (concentric whorls of } \\
\text { alternating rings of enhancement), } \\
\text { PACNS (with mass effect) }\end{array}$ \\
\hline Poorly defined lesion margins & ADEM \\
\hline Absent or rare Dawson fingers & ADEM \\
\hline Absent MRI activity at follow-up & ADEM \\
\hline Isolated lesions with ring enhancement (often complete) & Abscesses \\
\hline Mass effect & Abscesses \\
\hline Large lesions with absent or rare mass effect & PML \\
\hline Multiple bilateral microhaemorrhagic foci & CADASIL, SVD \\
\hline Haemorrhages & PACNS \\
\hline Infarcts & SID, PACNS, SVD \\
\hline \multicolumn{2}{|l|}{ Distribution } \\
\hline Substantially asymmetric distribution of lesions & Ipsilateral carotid disease \\
\hline $\begin{array}{l}\text { Extensive and bilateral periventricular abnormalities in } \\
\text { isolation }\end{array}$ & B12D, ACD \\
\hline Symmetrically distributed lesions & ADEM, AFL \\
\hline Absent or rare corpus callosum and periventricular & \\
\hline lesions & ADEM \\
\hline Frequent sparing of corpus callosum and cerebellum & SVD, CADASIL \\
\hline $\begin{array}{l}\text { Lesions in the centre of corpus callosum, sparing the } \\
\text { periphery }\end{array}$ & Susac's syndrome \\
\hline $\begin{array}{l}\text { T2-hyperintensity of the temporal pole, } \mathrm{U} \text { - fibres at the } \\
\text { vertex, external capsule and insular regions }\end{array}$ & CADASIL \\
\hline $\begin{array}{l}\text { Predominance of lesions at the cortical/subcortical } \\
\text { junction }\end{array}$ & SID \\
\hline Diffuse WM involvement & $\begin{array}{l}\text { NBD, encephalitis (HIVE), SVD, } \\
\text { CADASIL }\end{array}$ \\
\hline Cerebral venous sinus thrombosis & NBD \\
\hline Large and infiltrating brainstem lesions & NBD \\
\hline Pontine lacunar infarcts & CADASIL, SVD \\
\hline $\begin{array}{l}\text { Anterior temporal and inferior frontal lobe involvement, } \\
\text { associated with enhancement or mass effect }\end{array}$ & Encephalitis (HSE) \\
\hline $\begin{array}{l}\text { Multifocal, asymmetrical lesions starting in a } \\
\text { juxtacortical location and progressively enlarging } \\
\text { Pattern of enhancement }\end{array}$ & PML \\
\hline Simultaneous enhancement of all lesions & ADEM, PACNS, sarcoidosis \\
\hline
\end{tabular}




\begin{tabular}{|c|c|}
\hline Punctiform parenchymal enhancement & PACNS, sarcoidosis, NBD \\
\hline $\begin{array}{l}\text { Cortical grey matter } \\
\text { Cortical/subcortical lesions crossing vascular territories } \\
\text { Prevalent involvement versus white matter } \\
\text { Infiltrating lesions that do not remain in grey or white } \\
\text { matter boundaries }\end{array}$ & $\begin{array}{l}\text { MELAS } \\
\text { Encephalitis } \\
\text { Abscesses }\end{array}$ \\
\hline $\begin{array}{l}\text { Deep grey matter } \\
\text { Bilateral lesions }\end{array}$ & $\begin{array}{l}\text { ADEM (at the grey-white-matter } \\
\text { junction), CADASIL }\end{array}$ \\
\hline Lacunar infarcts & CADASIL, SVD \\
\hline T1-hyperintensity of the pulvinar & FD \\
\hline $\begin{array}{l}\text { Multiple discrete lesions in the basal ganglia and } \\
\text { thalamus }\end{array}$ & Susac's syndrome \\
\hline Large and infiltrating basal ganglia lesions & NBD \\
\hline $\begin{array}{l}\text { Infiltrating lesions without respecting grey-matter or } \\
\text { white-matter boundaries }\end{array}$ & Abscesses \\
\hline T2-hyperintense lesions in the dentate nuclei & AFL (CTX) \\
\hline Spinal cord & \\
\hline Large and swelling lesions & $\begin{array}{l}\text { NMO (with corresponding T1 } \\
\text { hypointensity), ADEM, ATM, } \\
\text { Sjogren's syndrome }\end{array}$ \\
\hline $\begin{array}{l}\text { Diffuse abnormalities in the posterior columns } \\
\text { Other }\end{array}$ & $\mathrm{B} 12 \mathrm{D}, \mathrm{ACD}$ \\
\hline Absence of optic-nerve lesions & PML \\
\hline Hydrocephalus & Sarcoidosis \\
\hline Dilation of Virchow-Robin spaces & HHC, PACNS \\
\hline Meningeal enhancement & $\begin{array}{l}\text { Susac's syndrome, PACNS, NBD, } \\
\text { meningitis, Lyme disease, } \\
\text { sarcoidosis }\end{array}$ \\
\hline Regional atrophy & $\begin{array}{l}\text { HHC (hippocampus and } \\
\text { amygdala), NBD (brainstem) }\end{array}$ \\
\hline No "occult" changes in the NAWM & $\begin{array}{l}\text { NMO, Lyme disease, SID (except } \\
\text { in NSLE) }\end{array}$ \\
\hline Diffuse lactate increase on brain MRS & MELAS \\
\hline
\end{tabular}

$\mathrm{ACD}=$ acquired copper deficiency. $\mathrm{ADEM}=$ acute disseminated encephalomyelitis. $\mathrm{AFL}=$ adult forms of leucoencephalopathies. AMS=acute multiple sclerosis (Marburg type). ATM=acute transverse myelitis. $\mathrm{B} 12 \mathrm{D}=$ vitamin $\mathrm{B} 12$ deficiency. $\mathrm{BCS}=$ Balo's concentric sclerosis. $\mathrm{CTX}=$ cerebrotendinous $x$ anthomatosis. $\mathrm{FD}=$ Fabry's disease. $\mathrm{HHC}=$ Hyperhomocysteinaemia. HIVE=HIV encephalitis. HSE=herpes simplex encephalitis. MELAS=mitochondrial encephalopathy with lactic acidosis and stroke-like episodes. MRS=magnetic-resonance spectroscopy. NAWM=normal-appearing white matter. NBD=Behçet's disease with CNS involvement. $\mathrm{NMO}=$ neuromyelitis optica. $\mathrm{NSLE}=$ neuropsychiatric systemic lupus erythematosus. PACNS=primary angiitis of the CNS. PML=progressive multifocal leucoencephalopathy. SID=systemic immune-mediated diseases. SVD=small-vessel disease.

Table 3. MRI characteristics suggestive of alternative conditions that mimic MS (adapted from Charil et al., 2006; with permission). 
By contrast to MS where MRI-detectable spinal cord lesions can be found in the majority of patients with established disease (Lycklama et al., 2003; Agosta and Filippi, 2007), spinal cord abnormalities are not observed in patients with hypoxic-ischemic disease and are rarely reported in other immune-mediated disorders (Bot et al, 2002), and progressive multifocal leucoencephalopathy (Yousry et al., 2006). Another characteristic pointing against the diagnosis of MS is the complete disappearance of spinal cord lesions in systemic immune-mediated diseases after steroid or immunosuppressive treatment (Rovaris et al., 2000). Moreover, incidental spinal cord hyperintense foci do not occur with ageing (Lycklama et al., 2003). Therefore, cord MRI is helpful in patients with normal or equivocal brain MRI and in those more than 50 years old (Agosta \& Filippi, 2007). Longitudinally extensive spinal cord involvement (usually three or more segments), affecting primarily the central part of the spinal cord on axial sections, is typically seen in neuromyelitis optica. Moreover, NMO myelopathy is often associated with swelling and T1 hypointensity in the acute and chronic stages (de Seze et al., 2002; Wingerchuk et al., 2007).

The pattern of enhancement is another useful distinctive characteristic. Simultaneous enhancement of multiple white-matter lesions is usually observed in neurosarcoidosis and primary angiitis of the CNS (PACNS). Moreover, meningeal enhancement should raise the suspicion of PACNS, neurosarcoidosis or neuroBehçet's disease (Campi et al., 2001; Zajicek et al., 1999).

It is important to note that when these MRI features 'not suggestive' of multiple sclerosis are used along with the existing MS diagnostic criteria, they minimize the chance of false positive diagnoses and facilitate the correct diagnosis of other disorders. Therefore, it is suggested to be taken into account in the diagnostic work-up of patients suspected of having MS. In the presence of at least one MRI 'red flag', appropriate additional tests should be performed. However, in cases of equivocal findings, follow-up with repeated imaging will be needed to establish a firm diagnosis (Charil et al., 2006)

\section{MRI and disease progression}

Recognition of the neurodegenerative aspects of MS has rendered the development of techniques to measure disease progression essential. Since conventional MRI is sensitive in detecting disease activity, it has routinely been used to ameliorate not only the accuracy of multiple sclerosis diagnosis but also the prognosis. Conventional MRI assessment of lesions on non-contrast T1-weighted and T2-weighted scans, and on gadolinium-enhanced T1weighted images is now routinely used to monitor the disease course and detect therapeutic effects (Zivadinov \& Leist, 2005). However, measures like the lesion volume and the number of gadolinium-enhancing and T2 lesions, are weakly associated with the clinical status and have insufficient sensitivity and specificity to reveal the extent of histopathological changes occurring in MS. Persistent T1 hypointense lesions can predict development of disability better than the previously mentioned lesion-based MRI measures (Zivadinov \& Leist, 2005) as they are mainly associated with more advanced pathological processes of the disease, i.e. axonal loss, Wallerian degeneration and gliotic changes (Bakshi et al., 2004; Stevenson et al., 2004). Moreover, brain and spinal cord atrophy have been shown to be powerful predictors of clinical impairment and disease progression. Additionally, newer nonconventional MRI techniques that become increasingly available hold significant promise to improve monitoring of MS. Accumulating evidence suggests that metrics derived from non-cMRI techniques correlate better with disability compared to conventional measures. 


\subsection{Detection of subclinical disease activity}

Conventional MRI can be used as a valuable tool for the assessment of disease progression, as it provides objective and sensitive measures of MS activity. In patients with RRMS and SPMS, disease activity is detected 5-10 times more frequently on cMRI scans than with clinical assessment of relapses (Filippi \& Agosta, 2010). Despite the sensitivity of T2weighted images to reveal disease activity and lesions over time (Molyneux et al., 1998), no significant correlation between MRI findings and clinical progression has been demonstrated, except in patients with very early disease (Rovaris et al., 2003). Indeed, both brain T2 hyperintense lesion load early in the clinical course and early progression of T2 burden of MS have been associated with the development of long-term disability (Brex et al., 2002). However, T2 lesion load measurement is not appropriate for the assessment of the advanced stages of the disease (Li et al., 2006). Although differences in T2 lesion load among different MS phenotypes have been consistently reported, being higher in SPMS in comparison to benign MS, RRMS, and PPMS, no significant relationship between T2 lesion load and disability has been demonstrated (Filippi \& Agosta, 2010).

Gd enhancement occurs in almost all new lesions in patients with RRMS or SPMS and can be sometimes detected even before the onset of clinical symptoms (Filippi \& Agosta, 2010). The number of enhancing lesions increases shortly before and during clinical relapses and predicts subsequent MRI activity (Molyneux et al., 1998, Kappos et al., 1999). The burden of MRI activity has been shown to be associated with the subtype of the disease, being higher in RRMS and SPMS in comparison with PPMS and BMS (Kappos et al., 1999). Moreover, patients with SPMS and severe disability show a lower incidence of enhancing lesions when compared to those with mildly disabling RRMS (Filippi et al., 1997). However, a moderate correlation has been demonstrated between the degree of clinical disability and the mean frequency of enhancing lesions in patients with RRMS and SPMS (Filippi \& Agosta, 2010).

T1 black holes and CNS atrophy are useful markers of progressive tissue damage and clinical evolution in established MS. In patients with either RRMS or SPMS, the increase in T1 lesion volume (LV) over time correlates significantly with progressive cerebral atrophy and the change in EDSS score (Sailer et al., 2001; Truyen et al., 1996). Moreover, in patients with CIS, baseline T1 hypointense lesion number and volume are strong predictors of the severity of executive dysfunction (Summers et al., 2008).

\subsection{Detection of brain and spinal cord atrophy}

Since the initial MRI studies in MS, marked atrophy of the brain has been found to be an important feature of the advanced stages of the disease (Filippi \& Agosta, 2010). Subsequently, it was recognised as an early phenomenon that progresses over the disease course. Thus, conventional MRI has been used to measure atrophy of the brain tissue, whole or segmented in white matter (WM) and gray matter (GM). Cerebral volume loss is associated with changes in normal appearing white matter and grey matter (Ge et al., 2000; Horakova et al., 2009). It occurs in all MS subtypes (even in CIS) with a rate of $0.5-1 \%$ per year and is closely related to disability (Anderson et al., 2006; Bermel \& Bakshi, 2006). Moreover, brain atrophy develops in different structures in the different clinical phenotypes of the disease: ventricular enlargement is predominant in RRMS, whereas cortical atrophy is more pronounced in the progressive forms of the disease (Pagani et al., 2005). The most atrophic cortical regions have been consistently found to be the frontal, temporal and parietal lobes (Filippi \& Agosta, 2010). GM atrophy rate has been found to increase with 
disease progression, whereas WM atrophy rate has been reported to remain constant (Fisher et al., 2008). Atrophy of the deep gray matter has been also observed in MS, especially in the posterior basal ganglia and the thalamic regions (Bermel \& Bakshi, 2006; Carone et al., 2006; Filippi \& Agosta, 2010; Henry et al., 2008). Measurement of atrophy in selected pathways, such as the corpus callosum and the corticospinal tract, may also improve the in vivo monitoring of MS progression (Martola et al., 2007). In addition, spinal cord atrophy, more pronounced in the progressive forms of MS, has been reported (Rovaris et al., 2001). It should be mentioned, however, that reduction of the cervical cord size can be observed in the early stages of MS (Brex et al., 2001). CNS atrophy reflects the destructive component of the MS disease process and contributes to irreversible neurological impairment.

Recent advances in volumetric MRI with the use of parallel imaging techniques may improve the use of atrophy metrics as outcome measures in clinical trials of MS therapies. It should be mentioned however, that the anti-inflammatory effect of MS treatments can reduce brain volume (a phenomenon called pseudoatrophy effect), and consequently affect atrophy measurements (Zivadinov et al., 2008). Advances in neuroimaging may help separate true atrophy from pseudoatrophy with the use of methods that can distinguish axonal loss from transient changes in water content.

\subsection{MRI in disease management and evaluation of treatment outcomes}

Since MRI provides information essential in monitoring disease activity and severity of multiple sclerosis, it is a critical component of disease management. In addition to the relapse rate and progression on EDSS, MRI variables play an essential role in determining the short and long-term effects of MS treatments, since the approval of the first therapy for MS by the Food and Drug Administration in 1993 (Rovaris, 2008; Bates, 2011; Freedman, 2011). Short-term and long-term clinical trial results have indicated that currently available disease-modifying therapies (DMTs) are effective in reducing MRI disease activity (Bates, 2011; Freedman, 2011). Moreover, reduction in MRI-detected lesion burden is used as supportive to the clinical findings to show reduction in disease activity. Brain MRI activity is determined as the number of new or enlarging T2-hyperintense lesions and Gd-enhancing lesions, whereas the MRI burden of the disease includes the T2-lesion volume and the T1hypointense LV.

Accumulating evidence suggests that MRI-derived metrics could be used to define treatment options and strategies in individual patients with MS (Beck et al., 2002; Rudick et al., 2004). In RRMS, the accumulation of two or more new T2-hyperintense lesions during a two-year period has been demonstrated to predict poor treatment response more reliably than the persistence of clinical relapses and the presence of enhancing lesions (Rudick et al., 2004). In CIS, the subgroup of patients with enhancing lesions at baseline showed a trend for greater treatment benefit than patients without (Beck et al., 2002). However, only the integration of clinical and MRI data can be considered a reliable approach for the work-up of patients receiving disease-modifying treatments.

Recently, clinical trials have incorporated cerebral volume measurement to determine the efficacy of novel treatments (Bermel \& Bakshi, 2006). However, the current inability to separate true atrophy from pseudoatrophy limits the use of atrophy measures at present. Advances in MRI technology might contribute to a better correlation between clinical and MRI findings, and thus provide relevant information to improve prognosis and predict therapeutic response. 


\section{Nonconventional MRI techniques}

Conventional MRI is unable to disclose the extent of the damage because of its limited sensitivity to structural changes outside the focal lesions and its lack of specificity to the heterogeneous features of the disease. The hyperintense lesions on T2-weighted images represent nonspecific tissue alterations as they reflect changes in water content caused by a variety of processes including edema, inflammation, demyelination, remyelination, Wallerian degeneration, gliosis and axonal loss. Furthermore, neurological impairment of patients with MS is poorly associated with the lesion load observed on conventional MRI scans (Filippi \& Agosta, 2010). During the past decade, other nonconventional MRI techniques-including proton MR spectroscopy, magnetization transfer MRI, diffusion tensor MRI, double inversion recovery sequences, high-field and ultrahigh-field MR imaging, techniques employing cellular MR contrast agents, and functional MRI-have been developed to overcome these limitations, improve the sensitivity and specificity in the detection of MS lesions and characterize and quantify the heterogeneous pathological substrates of the disease. Issues encountered in emerging clinical application of these methods will be addressed.

\subsection{Magnetic resonance spectroscopy}

Conventional MRI describes the physical characteristics of a region of tissue relative to surrounding regions by measuring alterations in tissue water content and dynamics by proton excitation. Proton MR Spectroscopy ( $\left.{ }^{1} \mathrm{H}-\mathrm{MRS}\right)$ is a non invasive method that depicts the chemical properties of a region of brain tissue by investigating other proton-containing cellular metabolites. It provides information on tissue metabolism and function of a selected brain area volume relative to surrounding regions. Therefore it could be used to study biochemical changes occurring in lesions and normal appearing white matter over the course of MS (De Stefano et al., 2007). ${ }^{1} \mathrm{H}-\mathrm{MRS}$ could also be used as a diagnostic tool, although it has not yet moved to clinical practice.

Various compounds can be detected using ${ }^{1} \mathrm{H}-\mathrm{MRS}$. At long echo times four major resonance peaks are revealed from (a) choline-containing phospholipids (Cho), (b) creatine and phospho- creatine (Cr), (c) N-acetyl-aspartate (NAA), and (d) lactate (Lac) methylgroup. NAA, normally present in axons and neurons, reflects neuronal/axonal integrity and therefore appears to be a sensitive biomarker of disease progression (Narayana, 2005). A decline in NAA levels in MS, with correlation with disability, has been shown in several studies. Reduced NAA was observed in lesions, cortical GM and NAWM in early RRMS (Kapeller et al., 2001; Chard et al., 2002). Decrease in NAA levels in chronic WM lesions has been reported in RRMS, SP and PPMS (Davie et al., 1997). Moreover, chronic lesions have higher NAA signal intensities in patients with BMS than those in patients with SPMS, indicating a more efficient tissue integrity recovery in patients with less severe disability (De Stefano et al., 2007). Transient changes in NAA levels in acute MS plaques have been also reported (Narayama et al, 1998). Decreases in NAA concentrations are also known to occur in the NAWM of MS patients (Caramanos et al., 2005). Furthermore, significant reduction in whole-brain NAA levels was found, more pronounced in older than younger patients (Gonen et al., 2000).

Cho and Lac reflect cell membrane metabolism. Increases in these metabolites are considered as chemical correlates of acute inflammatory or demyelinating changes. Indeed, increases in Cho and Lac resonance intensities have been found in acute MS lesions (Davie 
et al., 1994; De Stefano et al., 1995). In large, acute demyelinating lesions, decreases of $\mathrm{Cr}$ have also been seen (De Stefano et al., 1995).

${ }^{1} \mathrm{H}-\mathrm{MRS}$ studies with shorter echo times can detect additional metabolites, such as lipids and myoinositol (mI), which are also regarded as markers of progressing myelin damage. Increase of levels of myoinositol, which is mainly localised in astrocytes, has been shown in early MS (Capeller et al., 2001; De Stefano et al., 1995) and also in chronic lesions, indicating neuronal injury and ongoing astrogliosis (Srinivasan et al., 2005). Amino acids acting as neurotransmitters, such as glutamate, glutamine, and GABA ( $\gamma$-aminobutyric acid), can also be measured. Glutamate levels were found to be increased in acute lesions (Srinivasan et al., 2005). A reduced concentration of glutamate and glutamine in the cortical GM of patients with PPMS has been found (Sastre-Garriga et al., 2005), which was significantly correlated with the EDSS score. Recently, the concentration of glutathione, a marker of oxidative status, was measured by ${ }^{1} \mathrm{H}-\mathrm{MRS}$ at $7.0 \mathrm{~T}$ and was found decreased in the NAWM and gray matter of MS patients compared to healthy controls (Srinivasan et al., 2010).

MRS may provide insight into MS pathogenesis, evaluate the severity of MS and follow disease evolution and therefore is becoming a useful tool in the understanding of the disease. Moreover, MRS measures of brain metabolites seem to be better predictors of clinical disability than is conventional MRI.

\subsection{Magnetization transfer imaging}

Magnetization transfer (MT) is a physical phenomenon in which protons of two or more environments with different magnetic resonance properties exchange magnetization. MT MRI measures quantitatively the continuous exchange of magnetization in the brain tissue between macromolecular bound protons (i.e. protons associated with myelin, cell membranes and proteins) and protons in adjacent free water molecules, thus allowing the calculation of an index, the MT ratio (MTR). Diminution of this index denotes a decreased capacity of the bound protons to exchange magnetization with the surrounding "free" water (Filippi \& Agosta, 2007). The reduction of MTR values in focal lesions is considered as a marker of the extent of tissue damage, primarily associated with demyelination (Schmierer et al., 2004; van Buchem et al., 1996). The relative specificity of MTR to myelin density has been demonstrated by post-mortem imaging and histopathological studies that did not reveal any significant association with axonal density or gliosis (Schmierer et al., 2004).

Voxel-based analysis of MTR changes can provide indirect information about demyelination and remyelination in MS lesions (Chen et al., 2007). Changes of MTR of individual lesion voxels, as well as the mean normalized MTR over all lesion voxels during and after contrast enhancement have been reported (Chen et al., 2008). The mean normalized MTR of Gdenhancing lesions was significantly decreased at the time of lesion enhancement, partially recovered over the subsequent four months, and then appeared to stabilize. Moreover, enhancing lesions were found to have different MTR values, according to their size, pattern, and duration of enhancement. Specifically, MTR was lower in ring-enhancing lesions than in homogeneously enhancing lesions (Silver et al., 1998) and in lesions with longer duration of enhancement (Filippi et al., 1998a), suggesting more severe demyelination. Furthermore, evolution of the mean normalized MTR of individual lesions shows considerable heterogeneity ranging from partial recovery or stability that suggest remyelination, to decline over time that suggests ongoing demyelination (Chen et al., 2008). 
In addition to increased specificity, MTI may also offer increased sensitivity for studies of MS. Focal changes in magnetization transfer were reported to precede the appearance of gadolinium-enhancing lesions (Filippi et al., 1998b). Moreover, microscopic damage in normal-appearing brain tissues was shown on MTI before the development of T2 lesions on cMRI scans (Laule et al., 2003).

Decreased MTR has also been found in normal appearing brain tissue (NABT), in both gray (Ge et al., 2001; Dehmeshki et al., 2003) and white matter (Santos et al., 2002), of patients with established MS. These abnormalities are more pronounced in patients with the progressive forms of MS and tend to worsen over time (Filippi \& Rocca, 2007). Moreover, reduced NABT MTR has been found in patients at presentation with CIS, with significant prognostic value for the subsequent evolution to clinically definite MS (Miller et al., 2005). NAWM and NAGM MTR abnormalities have been found to correlate with clinical disability (Ge et al., 2001; Santos et al., 2002) and cognitive impairment (Ranjeva et al., 2005).

MTI metrics have several limitations that include lack of specificity to the various MS pathological substrates and lack of standard magnetisation transfer data acquisition protocols across different scanners (Horsfield et al., 2003). Improvement of the quantification and specificity of MTI with the use of new analysis methods (Chen et al., 2008) may render this technique a reliable tool not only for studies of disease evolution, but also for diagnostic purposes in every day clinical practice.

\subsection{Diffusion weighted and diffusion tensor imaging}

Diffusion weighted (DWI) and diffusion tensor imaging (DTI) provide information about the tissue fibers by measuring the motion of tissue water molecules in vivo (Rovaris et al., 2005). The mobility of water molecules is diminished in highly organized tissue, like white and gray matter, and consequently, the apparent diffusion coefficient (ADC) is lower in those tissues than in free water. Pathological processes that alter tissue organization can result in abnormal water motion, thus modifying ADC values. Tissue damage in MS, mainly demyelination and axonal degeneration, results in abnormal water motion, and therefore in alteration of the ADC values. Diffusion abnormalities may precede Gd-enhancement in hyperacute MS lesions.

Diffusion tensor imaging (DTI) is a valuable technique based on the fact that diffusion of water molecules within white matter is much greater along the fibres than across them. The diffusion tensor is a mathematical description of the magnitude and predominant direction of water molecules movement (anisotropy) in the three-dimensional space. As the diffusion ellipsoid provides a description of the diffusion tensor, the individual components of the diffusion ellipsoid (called eigenvalues) can also be assessed separately. These components are the longitudinal or axial diffusivity (LD or $\mathrm{AD}$ ), which is the principal eigenvalue of the $\mathrm{DT}$, and the transverse or radial diffusivity (TD or RD) that is the average of the second and third eigenvalues of the DT. Quantitative DTI parameters, such as the fractional anisotropy (FA), which is a normalized measure of the degree of anisotropy, the mean diffusivity (MD), that is the averaged diffusion for detecting WM alterations, and the longitudinal and transverse diffusivities of the diffusion tensor are considered valuable tools in the assessment of focal and widespread white matter tissue damage in patients with MS. FA was found to be decreased (Werring et al., 1999) whereas MD was consistently shown to be increased in MS plaques as well as in normal appearing white matter of MS patients (Lowe 
et al., 2006). However, FA values have been found to be increased in intracortical MS lesions, possibly reflecting intralesional loss of dendrites and activation of microglia (Poonawalla et al., 2008). Transverse diffusivity, which refers to the diffusion across fibers, is believed to be a specific marker for axonal loss and demyelination associated with MS (Oh et al., 2004; Lowe et al., 2006; Henry et al., 2003). Relative increase of TD has been shown in MS, correlating with demyelination and axonal loss (Song et al., 2005). This finding suggests that fragmented or missing myelin permits greater diffusion of water molecules across fibers. Moreover, quantitative variables derived from DTI were found to correlate with clinical disability (Filippi \& Agosta, 2010).

Fiber tractography is a diffusion technique based on the directional movement of water, which allows the generation of non-invasive three-dimensional images of white matter fiber tracts. It is a promising method for in vivo segmentation of the major WM tract fiber bundles in the brain (Mori et al., 2002). In MS patients, DT MRI tractography can be used to segment clinically eloquent WM pathways, such as the corticospinal tracts, the corpus callosum, and the optic radiations and holds promise in enabling visualization and quantification of the degree of axonal loss and demyelination in vivo. However, the application of DTI tractography in MS is limited by the presence of both focal and diffuse alterations of tissue structure, which cause a decrease in anisotropy and consequently an increase in uncertainty of the primary eigenvector of the DT (Pagani et al., 2007). Using DT MRI tractography, a study in patients with optic neuritis showed reduced connectivity values in both left and right optic radiations compared with controls, possibly attributed to trans-synaptic degeneration secondary to optic nerve damage (Ciccarelli et al., 2005). DT MRI tractography has been also used to identify NAWM fibers at risk for degeneration, when they intersect T2-visible lesions (Simon et al., 2006). A recent study found increased interthalamic connectivity in patients with early MS as measured by DTI and tractography when compared with control subjects, suggesting a possible reactive structural reorganization of the fiber tracts within the thalami (Tovar-Moll et al., 2009).

Technical improvements of tensor models may allow better description of water diffusion in more complex architectures such as those with crossing fiber tracts, better segmentation of fiber bundles and better understanding of the relationship between tissue structure and function.

\subsection{Double inversion recovery imaging}

Double inversion recovery (DIR - i.e. two inversion times are used to suppress the signal from both white matter and cerebrospinal fluid) is a novel imaging method that has markedly improved the sensitivity of MRI to detect cortical lesions in vivo (Geurts et al., 2005). Cortical demyelination is an important part of the disease process, especially in more advanced stages (Kutzelnigg et al., 2005), and may contribute to the accumulation of irreversible disability in MS (Calabrese et al., 2009a,b; Calabrese et al., 2010; Roosendaal et al., 2009). Despite improvement, at present only a small number of cortical gray matter lesions can be detected with this technique, compared with pathological studies (Calabrese et al., 2007; Geurts et al., 2008).

Cortical lesions have been detected in all the major MS clinical phenotypes, including patients with CIS suggestive of MS (Calabrese et al., 2009a, b). Remarkably, cortical lesions are more frequently seen in patients with secondary progressive MS than in those with CIS 
or RRMS. Moreover, patients with early RRMS have a greater number of lesions than patients with benign MS (Calabrese et al., 2009a). Longitudinal studies have shown that new cortical lesions continue to form in patients with early RRMS (Calabrese et al., 2009a), and in those with the progressive disease phenotypes over 1 to 2-year periods of follow-up (Calabrese et al., 2009b; Roosendaal et al., 2009). Cortical lesion burden has been associated with progression of disability over the subsequent 2 to 3 years in patients with different disease phenotypes (Calabrese et al., 2009b), as well as with the severity of cognitive impairment in patients with RRMS (Roosendaal et al., 2009).

DIR sequences at $1.5 \mathrm{~T}$ have not been established as yet into the clinical routine probably because of the relatively low signal-to-noise ratio values due to the application of two inversion pulses, longer acquisition times and higher propensity of artefacts. DIR applications at higher magnetic field strengths may overcome these major problems (Wattjes \& Barkhof, 2009).

\subsection{Functional MRI}

Functional MRI (fMRI) is a non-invasive neuroimaging method that enables visualisation not only of the detailed anatomy but also of brain function. It is used to identify changes in brain activation in response to various stimuli and in the resting state. The fMRI signal reflects blood oxygenation level-dependent (BOLD) alterations associated with activation of neural tissue. This mechanism permits the detection of changes in the relationship between blood flow and local oxygen consumption. Upon initiating a task, neural activity in the involved brain region results in increased blood flow and consequently in increased total blood oxygen content (Logothetis et al., 2001; Ogawa et al., 1990). The increased blood oxygen produces an increase in the MRI signal, thus allowing demonstration of abnormal patterns of brain activation caused by tissue injury.

fMRI is not used clinically for the diagnosis of MS; rather it is used to provide insight into disease progression. The most common finding in fMRI studies is increased extent or strength of activation of functional tissue in MS patients compared with controls, implying compensatory processes or reorganization of neuronal activation (Lee et al., 2000; Penner et al., 2003; Reddy et al., 2000; Rocca et al., 2005). At the initial stages of the disease, an increased recruitment of the areas normally involved in the performance of a given task, such as the primary sensorimotor cortex and the supplementary motor area is shown (Rocca et al., 2003). At later stages, bilateral activation of these regions is first observed, followed by a widespread recruitment of additional areas, which are usually recruited by healthy subjects in the performance of more complex or novel tasks (Rocca et al., 2005). Similarly, fMRI studies investigating various cognitive domains in MS patients have shown altered patterns of cortical activation (Audoin et al., 2003; Staffen et al., 2002; Hillary et al., 2003; Li et al., 2004; Cader et al., 2006). Moreover a relationship between cortical activation and fatigue has been shown in several studies suggesting that the underlying mechanism of this common but poorly understood symptom may be the deficiency of neuronal compensation (Filippi et al., 2002; White et al., 2009).

Functional connectivity MRI (resting-state fMRI) is a newer fMRI method that measures low frequency oscillations $(0.01-0.1 \mathrm{~Hz})$ in BOLD signals across the whole brain during rest, thus enabling measurement of functional interactions between brain regions (Cordes et al., 2000). Since it studies the strength of network between anatomically separated brain regions, it is 
considered a promising method to explore disconnectivity effects in MS (van den Heuvel \&, Hulshoff Pol, 2010). Indeed, decreased levels of functional connectivity of regions of the primary motor network have been reported in patients with MS (Lowe et al., 2008).

Since studies with fMRI have demonstrated consistently functional cortical changes in all MS subtypes, this method can be used to assess the role of brain plasticity and cortical adaptive changes in limiting the clinical manifestations of demyelination and tissue loss in MS and therefore explain the discrepancy between clinical and cMRI findings.

\subsection{High and ultrahigh-field MRI}

Magnet field strengths higher than 1.5 Tesla (T) improve image resolution, signal-to-noise ratio and chemical shift. Therefore application of high-field MRI on conventional and quantitative MRI methods offers advantages on brain imaging in MS, as it may give new insights into the microstructural damage and functional reorganization (Wattjes \& Barkhof, 2009). In patients with established MS, high-field MRI has resulted in a higher detection rate of T2 (Wattjes et al., 2009) and gadolinium-enhancing brain (Sicotte et al., 2006) but not spinal cord (Stankiewicz et al., 2009) lesions at 3.0T compared with 1.5T. In CIS patients, however, 3.0T imaging did not substantially affect evidence for disease dissemination in space (Wattjes et al., 2008).

Ultrahigh-field MRI (referred to as imaging at field strength of 7 Tesla or more) provides improved brain lesion load quantification and characterization of the pathological features of MS lesions. It is also likely that detection of GM and spinal cord abnormalities could be improved by ultrahigh-field MRI (Kangarlu et al., 2007).

Both high-field and ultra-high field MRI can improve 1H-MRS by providing better quantification of metabolites in lesions and the normal-appearing brain tissue (white and gray matter) (Srinivasan et al., 2004, 2005, 2009). Magnetisation transfer MRI, diffusion tensor MRI, and fMRI studies in MS may also benefit from higher field strengths (Bakshi et al., 2008; Wattjes \& Barkhof, 2009). Indeed, subtle damage in the NAWM and NAGM Ceccarelli ea al., 2007), as well as diffuse microscopic damage in the absence of macroscopic MS lesions in the thalamus and basal ganglia has been detected by the use of high-field MRI (Tovar-Moll et al., 2009). Finally, higher field MRI might be important for studying iron deposition in the grey matter of patients with MS, which has been postulated to contribute to neurotoxicity (Stankiewicz et al., 2007).

\subsection{New MR contrast agents}

Conventional MRI allows follow-up of lesion development in time and space. Although in clinical practice the number of hyperintense lesions on T2-weighted MRI is considered as a biomarker of disease activity in MS, they represent nonspecific tissue alterations. Commonly, the identification of gadolinium-DTPA (Gd) enhancing lesions on T1weighted scans is considered as evidence for acute inflammation and disruption of the BBB. Gd, a lanthanide chelate, is an extracellular contrast agent that requires relatively high local concentration to achieve sufficient contrast on MRI. Gadofluorine M (Gf), a new Gd-based agent, has been shown in in-vivo experimental studies to have a much higher sensitivity than Gd-DTPA in detecting disruption of the BBB on T1-weighted MRI (Bendszus et al., 2008).

The development and application of novel MR contrast agents allowing cellular and targeted molecular imaging hold promise in exploring inflammation and its regulation by 
cell adhesion molecules in MS. Contrast media composed of iron particles [superparamagnetic particles of iron oxide (SPIO) and ultrasmall particles of iron oxide (USPIO)] are more sensitive compared to Gd-containing agents at similar tissue concentrations (Liu \& Frank, 2009). The size of SPIO particles is $50-150 \mathrm{~nm}$ whereas that of USPIO particles is approximately $10-50 \mathrm{~nm}$. These particles are partly phagocytosed by circulating macrophages upon injection. When circulating macrophages are attracted to inflammatory lesions, they can be detected in vivo by MRI, since in tissue iron particles shorten both the T1 and T2 relaxation time (Vellinga et al., 2008). Several recent studies support the aspect that SPIO/USPIO enhanced MRI allows cellular neuroimaging (Baeten et al., 2008; Chin et al., 2009). Preliminary studies in RRMS patients have shown that some lesions may enhance only with Gd, others only with USPIO, and others with both. Moreover, the same lesions can change their pattern of enhancement over time (Vellinga et al., 2008). Furthermore, USPIO-enhanced MRI may not only disclose a greater number of 'active' MS lesions but may also detect subtle and diffuse inflammatory activity in MS patients not visible on conventional T2-weighted MR sequences and unrelated to Gd-DTPA enhancement (Vellinga et al., 2009).

Additionally, novel cellular MR contrast agents such as polyfluorinated nanoemulsions or activatable paramagnetic sensors and emerging molecular imaging approaches might give new insights into the MS inflammatory process. Myeloperoxidase (MPO) is one of the most abundant enzymes secreted by inflammatory cells and, thus, may serve as a marker of macrophage inflammation. It has been recently shown in an animal model of EAE that myeloperoxidase activity in inflamed tissues can be detected and labelled in vivo by a myeloperoxidase sensitive "smart" molecular imaging probe (JW Chen et al., 2008).

Transferring cellular and targeted molecular imaging approaches from in-vitro to in-vivo MRI and subsequently in clinical use holds promise for exploring MS neuroinflammation in the future.

\section{Optical techniques}

\subsection{Optical coherence tomography (OCT)}

Optical coherence tomography (OCT) is a new, sensitive, non-invasive, transpupilary method that allows direct imaging and quantification of the retinal nerve fibre layer (RNFL), which is principally composed of unmyelinated axons. By using an optical interferometer in conjunction with a low-coherence light source, OCT detects light reflections within a tissue and provides in vivo cross-sectional images at near microscopic resolution (Hrynchak \& Simpson, 2000; Huang et al., 1991). Recent advances in OCT allow differentiation of major retinal layers and analysis of tissue thickness and volume with a resolution of about $3 \mu \mathrm{m}$ (Ko et al., 2004).

Because the retina is the only place where a tissue layer made up of axons can be imaged directly, and the retina and optic nerve are often affected by MS, OCT can be used to assess the impact of MS on the retina by measuring the RNFL thickness and macular volume. The reductions of RNFL thickness and macular volume are associated with axonal loss and secondary retinal ganglion cell loss, respectively. Therefore, OCT shows promise in the detection, assessment and monitoring of neurodegeneration in MS (Barkhof et al., 2009; Petzold et al., 2010).

RNFL thinning has been detected with OCT about 3 months after acute optic neuritis. Moreover, continuing axonal loss in the affected eye for at least 12 months after optic 
neuritis has been found, more pronounced the first 6 months after injury (Costello et al., 2008). In addition, OCT has been shown to be predictive of a clinical outcome (poor visual recovery) (Costello et al., 2006). A recent meta-analysis of time domain OCT showed RNFL thinning in MS patients, both with and without a history of optic neuritis, more pronounced after optic neuritis (Petzold et al., 2010). The estimated RNFL thinning in patients with MS has been found to be greater than the extent expected in normal ageing and has been attributed to retrograde trans-synaptic degeneration and progressive loss of retinal ganglion cells, in addition to the more pronounced thinning observed in case of optic neuritis (Petzold et al., 2010). Furthermore, thinning of the RNFL in MS without optic neuritis was found to correlate with visual and neurological functioning, as well as with paraclinical data (Trip et al., 2005, 2006; Villoslada et al., 2008).

Macular volume loss has also been reported in patients with MS compared with controls, correlating with loss of RNFL (Burkholder et al., 2009; Trip et al., 2006). Recently, a subgroup of MS patients with primary retinal pathology characterized by predominant macular thinning has been described with the use of OCT, in whom there appears to be disproportionate thinning of the inner and outer nuclear retinal layers independent of optic nerve pathology. Moreover, these patients were shown to have accelerated disability progression, implying that this primary process may be associated with a more aggressive form of MS (Saidha et al., 2011).

OCT could be used for analysis of neurodegeneration in MS as well as for monitoring of treatment effects in trials of neuroprotective strategies in MS. Moreover, the integration of OCT into MS research could allow insights into the structure-function relations, thus improving our understanding of the pathophysiology of the disease.

\section{Conclusion}

Advances in neuroimaging have improved our ability to diagnose and monitor MS and have provided insight into the pathophysiology of the disease. Conventional MRI of the central nervous system plays a prominent role in establishing the diagnosis of MS and in differentiating MS-mimics and demyelinating disease subtypes. Moreover, it allows an earlier and accurate diagnosis of the disease, as it can support or even replace some clinical criteria. Early diagnosis is essential to allow earlier therapeutic intervention that appears to be beneficial on delaying the accumulation of irreversible neurologic damage and consequent disability.

In addition cMRI is a useful tool for monitoring disease progression as it is a sensitive imaging biomarker that detects pathological changes prior to the onset of clinical symptoms. Conventional MR scans are effective in detecting clinically silent new white matter inflammatory lesions. In particular, Gd-enhancing lesions are predictive of future clinical relapses. Therefore, cMRI is routinely used for detection of subclinical disease activity and for decisions regarding treatment in individual patients. Moreover, most clinical trials involving new treatments use MRI parameters as outcome measures to show therapeutic effectiveness. Besides Gd-enhancing and T2 lesions, CNS atrophy measurements are being increasingly used as primary MRI end point in clinical trials as they have been shown to be powerful predictors of clinical impairment, disease progression, and the accumulation of long-term physical and neuropsychological disability.

MRI serves also as a research tool in the study of MS. However, cMRI metrics have a limited ability to accurately determine the full extent of inflammatory and neurodegenerative 
pathological processes in MS. The discrepancy between clinical and MRI evolution in MS patients, often referred to as the "clinical-MRI paradox", has led to a growing interest in advanced techniques that can detect the presence of cortical lesions, subtle changes in the normal appearing white and gray matter, as well as adaptive functional changes that limit the clinical consequences of disease-related injury. The development and application of novel MR methods of acquisition and postprocessing aim to provide a more accurate characterization of tissue injury including demyelination, remyelination and axonal damage. These advanced approaches give also insights into the functional and metabolic consequences of MS. Additionally, the combined analysis of different magnetic resonance techniques, sensitive toward different aspects of MS, is likely to improve our understanding of the mechanisms responsible for the accumulation of irreversible neurological deficits in the disease. Emerging applications of these tools may also be useful in measuring the effect of therapies. Beyond MRI, novel imaging approaches such as OCT show promise in detecting and monitoring neurodegeneration in MS. Nevertheless, the new techniques and analysis procedures need to be refined and validated before they can be properly integrated into clinical research and practice. Until that time cMRI metrics will continue to play an important role in clinical practice and in clinical trials. Overuse of MRI in clinical practice, however, should be avoided. It is important to keep in mind that clinical judgment remains essential in the management of the disease and that careful interpretation of the MRI data is needed to avoid misdiagnosis.

\section{References}

Agosta, F. \& Filippi, M. (2007). MRI of spinal cord in multiple sclerosis. Journal of Neuroimaging, Vol.17, Suppl.1, (April 2007), pp. 46S-49S.

Anderson, VM.; Fox, NC. \& Miller DH. (2006). Magnetic resonance imaging measures of brain atrophy in multiple sclerosis. Journal of Magnetic Resonance Imaging, Vol.23, No.5, (May 2006), pp. 605-618.

Andreadou, E.; Papadimas, G. \& Sfagos, C. (2008). A novel heterozygous mutation in the NOTCH 3 gene causing CADASIL. Swiss Medical Weekly, (2008); Vol.138, No.41-42, (October 2008), pp. 614-617

Andreadou, E.; Papadimas, GK.; Sifakis, N. \& Sfagos, C. (2010). Corpus callosum infarct associated with combined variants in circle of Willis. Neurology India, Vol.58, No.5, (September-October 2010), pp. 785-786.

Audoin, B.; Ibarrola, D.; Ranjeva, J. P.; Confort-Gouny, S.; Malikova, I.; Ali-Cherif, A.; Pelletier, J. \& Cozzone, P. (2003). Compensatory cortical activation observed by fMRI during a cognitive task at the earliest stage of MS. Human Brain Mapping, Vol.20, No.2, (October 2003), pp. 51-58.

Baeten, K.; Hendriks, JJ.; Hellings, N.; Theunissen, E.; Vanderlocht, J.; Ryck, LD.; Gelan, J.; Stinissen, P. \& Adriaensens, P. (2008). Visualisation of the kinetics of macrophage infiltration during experimental autoimmune encephalomyelitis by magnetic resonance imaging. Journal of Neuroimmunology, Vol.195, No.1-2, (March 2008), pp. 1-6.

Bagnato, F.; JeVries, N.; Richert, ND. ; Stone, RD.; Ohayon, JM.; McFarland, HF. \& Frank, JA. (2003). Evolution of T1 black holes in patients with multiple sclerosis imaged monthly for 4 years. Brain, Vol.126, No.8, (August 2003), pp. 1782-1789. 
Bakshi, R.; Ariyaratana, S.; Benedict, RHB. \& Jacobs, L. (2001). Fluid-attenuated inversion recovery magnetic resonance imaging detects cortical and juxtacortical multiple sclerosis lesions. Archives of Neurology, Vol.58, No.5, (May 2001), pp. 742-748.

Bakshi, R.; Hutton, GJ.; Miller, JR. \& Radue, EW. (2004). The use of magnetic resonance imaging in the diagnosis and long-term management of multiple sclerosis. Neurology, Vol.63, Suppl. 5, (December 2004), pp. S3- S11.

Bakshi, R.; Thompson, AJ.; Rocca, MA.; Pelletier, D.; Dousset, V.; Barkhof, F.; Inglese, M.; Guttmann, CRG.; Horsfield, MA. \& Filippi, M. (2008). MRI in multiple sclerosis: current status and future prospects. Lancet Neurology, Vol.7, No.7, (July 2008), pp. 615-625

Barkhof, F.; Calabresi, PA.; Miller, DH. \& Reingold, SC. (2009). Imaging outcomes for neuroprotection and repair in multiple sclerosis trials. Nature Reviews, Neurology, Vol.5, No.5, (May 2009), pp. 256-266.

Barkhof, F.; Filippi, M.; Miller, DH.; Scheltens, P.; Campi, A.; Polman, CH.; Comi, G.; Adèr, HJ.; Losseff, N. \& Valk, J. (1997). Comparison of MRI criteria at first presentation to predict conversion to clinically definite multiple sclerosis. Brain, Vol.120, No. 11, (November 1997), pp. 2059-2069.

Barkhof, F. \& Scheltens, P. (2002). Imaging of white matter lesions. Cerebrovascular Diseases, Vol.13, Suppl.2, pp. 21-30.

Bates, DMA. (2011). Treatment effects of immunomodulatory therapies at different stages of multiple sclerosis in short-term trials. Neurology, Vol. 76, No.1 (Suppl 1), (January 2011), pp. S14-S25

Beck, RW.; Chandler, DL.; Cole, SR.; Simon, JH.; Jacobs, LD.; Kinkel, RP.; Selhorst, JB.; Rose, JW.; Cooper, JA.; Rice, G.; Murray, TJ. \& Sandrock, AW. (2002) Interferon beta-1a for early multiple sclerosis: CHAMPS trial subgroup analyses. Annals of Neurology, Vol.51, No.4, (April 2002), pp. 481-490.

Bendszus, M.; Ladewig, G.; Jestaedt, L.; Misselwitz, B.; Solymosi, L.; Toyka, K. \& Stoll, G. (2008). Gadofluorine M enhancement allows more sensitive detection of inflammatory CNS lesions than T2-w imaging: a quantitative MRI study. Brain, Vol.131, No.9, (September 2008), pp. 2341-2352.

Bermel, RA. \& Bakshi, R. (2006). The measurement and clinical relevance of brain atrophy in multiple sclerosis. Lancet Neurology, Vol.5, No.2, (February 2006), pp. 158-170.

Brex, PA.; Ciccarelli, O. ; O’Riordan, JI.; Sailer, M.; Thompson, AJ \& Miller, DH. (2002). A longitudinal study of abnormalities on MRI and disability from multiple sclerosis. The New England Journal of Medicine, Vol.346, No.3, (January 2002), pp. 158-164.

Brex, PA.; Leary, SM. ; O’Riordan, JI.; Miszkiel, KA.; Plant, GT.; Thompson, AJ. \& Miller, DH. (2001). Measurement of spinal cord area in clinically isolated syndromes suggestive of multiple sclerosis. Journal of Neurology, Neurosurgery and Psychiatry, Vol. 70, No.4, (April 2001), pp. 544-547.

Brex, PA.; Parker, GJM.; Leary, SM.; Molyneux, PD.; Barker, GJ.; Davie, CA.; Thompson, AJ. \& Miller, DH. (2000). Lesion heterogeneity in multiple sclerosis: a study of the relations between appearances on $\mathrm{T} 1$ weighted images, $\mathrm{T} 1$ relaxation times, and metabolite concentrations. Journal of Neurology, Neurosurgery and Psychiatry, Vol.68, No.5, (May 2000), pp. 627-632.

Bot, JC.; Barkhof, F.; Lycklama à Nijeholt, G.; van Schaardenburg, D.; Voskuyl, AE.; Ader, HJ.; Pijnenburg, JA.; Polman, CH.; Uitdehaag, BM.; Vermeulen, EG.; Castelijns, JA. (2002). Differentiation of multiple sclerosis from other inflammatory disorders and 
cerebrovascular disease: value of spinal MR imaging. Radiology, Vol.223, No.1, (April 2002), pp. 46-56.

Bruck, W.; Bitsch, A.; Kolenda, H.; Brück, Y.; Stiefel, M. \& Lassmann, H. (1997). Inflammatory central nervous system demyelination: correlation of magnetic resonance imaging findings with lesion pathology. Annals of Neurology, Vol.42, No.5, (November 1997), pp. 783-793.

Burkholder, BM.; Osborne, B.; Loguidice, MJ.; Bisker, E.; Frohman, TC.; Conger, A.; Ratchford, JN.; Warner, C.; Markowitz, CE.; Jacobs, DA.; Galetta, SL.; Cutter, GR.; Maguire, MG.; Calabresi, PA.; Balcer, LJ. \& Frohman, EM. Macular volume determined by optical coherence tomography as a measure of neuronal loss in multiple sclerosis. Archives of Neurology, Vol.66, No.11, (November 2009), pp. 1366-1372.

Cader, S.; Cifelli, A.; Abu-Omar, Y.; Palace, J. \& Matthews, P.M. (2006). Reduced brain functional reserve and altered functional connectivity in patients with multiple sclerosis. Brain, Vol.129, No.2, (February 2006), pp. 527-537.

Calabrese M, Agosta F, Rinaldi F, Mattisi I, Grossi P, Favaretto A, Atzori M, Bernardi V, Barachino L, Rinaldi L, Perini P, Gallo P, Filippi M. (2009). Cortical lesions and atrophy associated with cognitive impairment in relapsing-remitting multiple sclerosis. Archives of Neurology, Vol.66, No.9 (September2006), pp. 1144-1150.

Calabrese, M.; De Stefano, N.; Atzori, M.; Bernardi, V.; Mattisi, I.; Barachino, L.; Morra, A.; Rinaldi, L.; Romualdi, C.; Perini, P.; Battistin, L. \& Gallo, P. (2007). Detection of cortical inflammatory lesions by double inversion recovery magnetic resonance imaging in patients with multiple sclerosis. Archives of Neurology, Vol.64, No.10, (October 2007), pp. 1416-1422.

Calabrese, M.; Filippi, M.; Rovaris, M.; Bernardi, V.; Atzori, M.; Mattisi, I.; Favaretto, A.; Grossi, P.; Barachino, L.; Rinaldi, L; Romualdi, C.; Perini, P. \& Gallo P. (2009a). Evidence for relative cortical sparing in benign multiple sclerosis: a longitudinal magnetic resonance imaging study. Multiple Sclerosis, 2009; Vol.15, No.1, (January 2009), pp. 36-41.

Calabrese, M.; Rocca, MA.; Atzori, M.; Mattisi, I.; Bernardi, V.; Favaretto, A.; Barachino, L.; Romualdi, C.; Rinaldi, L.; Perini, P.; Gallo, P. \& Filippi M. (2009b). Cortical lesions in primary progressive multiple sclerosis: a 2-year longitudinal MR study. Neurology Vol.72, No.15, (April 2009), pp. 1330-1336.

Calabrese, M.; Rocca, M.; Atzori, M.; Mattisi, I.; Favaretto, A.; Perini, P.; Gallo, P. \& Filippi, M. (2010). A three-year MRI study of cortical lesions in relapse-onset multiple sclerosis. Annals of Neurology,; Vol.67, No.3, (March 2010), pp. 376-383.

Campi, A.; Benndorf, G.; Filippi, M.; Reganati, P.; Martinelli, V. \& Terreni, MR. (2001). Primary angiitis of the central nervous system: serial MRI of brain and spinal cord. Neuroradiology, Vol. 43, No.8, (August 2001), pp. 599-607.

Caramanos, Z.; Narayanan, S. \& Arnold, DL. (2005). 1H-MRS quantification of tNA and tCr in patients with multiple sclerosis: a meta-analytic review. Brain, Vol.128, No.11, (November 2005), pp. 2483-2506.

Carone, DA.; Benedict, RH.; Dwyer, MG.; Cookfair, DL.; Srinivasaraghavan, B.; Tjoa, CW. \& Zivadinov, R. (2006b). Semi-automatic brain region extraction (SABRE) reveals superior cortical and deep gray matter atrophy in MS. Neuroimage, Vol.29, No.2, (January 2006), pp. 505-514.

Ceccarelli, A.; Rocca, MA.; Falini, A.; Tortorella P, Pagani E, Rodegher M, Comi G, Scotti G, Filippi M. (2007). Normal-appearing white and grey matter damage in MS: a 
volumetric and diffusion tensor MRI study at 3.0 Tesla. Journal of Neurology, Vol. 254, No.4, (April 2007), pp. 513-518

Chard, DT.; Griffin, CM.; McLean, MA.; Kapeller, P.; Kapoor, R.; Thompson, AJ. \& Miller, DH. (2002). Brain metabolite changes in cortical grey and normal-appearing white matter in clinically early relapsing-remitting multiple sclerosis. Brain, Vol. 125, No.10, (October 2002), pp. 2342-2352.

Charil, A.; Yousry, TA.; Rovaris, M.; Barkhof, F.; De Stefano, N.; Fazekas, F.; Miller, DH.; Montalban, X.; Simon, JH.; Polman, C. \& Filippi, M. (2006). MRI and the diagnosis of multiple sclerosis: expanding the concept of "no better explanation". Lancet Neurology, Vol.5, No.10, (October 2006), pp. 841-852.

Chen, JT.; Kuhlmann, T.; Jansen, GH.; Collins, DL.; Atkins, HL.; Freedman, MS.; O'Connor, PW.; Arnold, DL. \& Canadian MS/BMT Study Group. (2007). Voxel-based analysis of the evolution of magnetization transfer ratio to quantify remyelination and demyelination with histopathological validation in a multiple sclerosis lesion. Neuroimage,Vol.36, No.4, (July 2007), pp. 1152-1158.

Chen, JT., Collins, DL., Atkins, HL.; Freedman, MS.; Arnold, DL. \& Canadian MS/BMT Study Group. (2008). Magnetization transfer ratio evolution with demyelination and remyelination in multiple sclerosis lesions. Annals of Neurology, Vol. 63, No.2, (February 2008), pp. 254-262.

Chen, JW.; Breckwoldt, MO.; Aikawa, E.; Chiang, G. \& Weissleder, R. (2008). Myeloperoxidase-targeted imaging of active inflammatory lesions in murine experimental autoimmune encephalomyelitis. Brain, Vol.131, No.4, (April 2008), pp. 1123-1133.

Chin, CL.; Pai, M.; Bousquet, PF.; Schwartz, AJ.; O'Connor, EM.; Nelson, CM.; Hradil, VP.; Cox, BF.; McRae, BL. \& Fox, GB. (2009). Distinct spatiotemporal pattern of CNS lesions revealed by USPIO-enhanced MRI in MOG-induced EAE rats implicates the involvement of spinoolivocerebellar pathways. Journal of Neuroimmunology, Vol.211, No.1-2, (June 2009), pp. 49-55.

Ciccarelli, O.; Toosy, AT.; Hickman, SJ.; Parker, GJ.; Wheeler-Kingshott, CA.; Miller, DH. \& Thompson, AJ. (2005). Optic radiation changes after optic neuritis detected by tractography-based group mapping. Human Brain Mapping, Vol.25, No.3, (July 2005), 308-316.

Cordes, D.; Haughton, VM.; Arfanakis, K.; Wendt, GJ.; Turski, PA.; Moritz, CH.; Quigley, MA.; Meyerand, ME. (2000). Mapping functionally related regions of brain with functional connectivity MR imaging. American Journal of Neuroradiology, Vol.21, No. 9, (October 2000), pp. 1636-1644.

Costello, F.; Coupland, S.; Hodge, W.; Lorello, GR.; Koroluk, J.; Pan, YI.; Freedman, MS.; Zackon, DH. \& Kardon, RH. (2006). Quantifying axonal loss after optic neuritis with optical coherence tomography. Annals of Neurology, 2006; Vol.59, No.6, (June 2006), pp. 963-69.

Costello, F.; Hodge, W.; Pan, YI.; Eggenberger, E.; Coupland, S. \& Kardon, RH. (2008). Tracking retinal nerve fiber layer loss after optic neuritis: a prospective study using optical coherence tomography. Multiple Sclerosis, Vol.14, No.7, (August 2008), pp. 893-905.

Cotton, F.; Weiner, HL.; Jolesz, FA. \& Guttmann, CR. (2003). MRI contrast uptake in new lesions in relapsing-remitting MS followed at weekly intervals. Neurology, Vol.60, No.4, (February 2003), pp. 640-646. 
Davie, CA.; Barker, GJ.; Thompson, AJ.; Tofts, PS.; McDonald, WI. \& Miller, DH. (1997). 1H magnetic resonance spectroscopy of chronic cerebral white matter lesions and normal appearing white matter in multiple sclerosis. Journal of Neurology, Neurosurgery and Psychiatry, Vol. 63, No.6, (December 1997), pp. 736-742.

Davie, CA.; Hawkins, CP.; Barker, GJ.; Brennan, A.; Tofts, PS.; Miller, DH. \& McDonald WI. (1994). Serial proton magnetic resonance spectroscopy in acute multiple sclerosis lesions. Brain, Vol.117, No.1 (February 1994), pp. 49-58.

Dehmeshki, J.; Chard, DT.; Leary, SM.; Watt, HC.; Silver, NC.; Tofts, PS.; Thompson, AJ. \& Miller, DH. (2003). The normal appearing gray matter in primary progressive multiple sclerosis: a magnetisation transfer imaging study. Journal of Neurology, Vol.250, No1. (January 2003), pp. 67-74.

de Seze, J.; Stojkovic, T.; Ferriby, D.; Gauvrit, JY.; Montagne, C.; Mounier-Vehier, F.; Verier, A.; Pruvo, JP.; Hache, JC. \& Vermersch, P. (2002). Devic's neuromyelitis optica: clinical, laboratory, MRI and outcome profile. Journal of the Neurological Sciences, Vol.197, No.1-2, (May 2002), pp. 57-61.

De Stefano, N.; Filippi, M.; Miller, D.; Pouwels, PJ.; Rovira, A.; Gass, A.; Enzinger, C.; Matthews, PM. \& Arnold DL. (2007). Guidelines for using proton MR spectroscopy in multicenter clinical MS studies. Neurology, Vol.69, No.20, (November 2007), pp. 1942-1952.

De Stefano, N.; Matthews, PM.; Antel, JP.; Preul, M.; Francis, G. \& Arnold, DL. (1995). Chemical pathology of acute demyelinating lesions and its correlation with disability. Annals of Neurology, Vol.38, No.6, (December 1995), pp. 901-909.

Dichgans, M.; Holtmannspotter, M.; Herzog, J.; Peters, N.; Bergmann, M. \& Yousry, TA. (2002). Cerebral microbleeds in CADASIL: a gradient-echo magnetic resonance imaging and autopsy study. Stroke, Vol.33, No.1, (January 2002), pp. 67-71.

Di Filippo, M.; Anderson, VM.; Altmann, DR.; Swanton, JK.; Plant, GT.; Thompson, AJ. \& Miller, DH. (2010). Brain atrophy and lesion load measures over 1 year relate to clinical status after 6 years in patients with clinically isolated syndromes. Journal of Neurology, Neurosurgery and Psychiatry, Vol. 81, No.2, (February 2010), pp. 204-208.

Enzinger, C.; Smith, S.; Fazekas, F.; Drevin, G.; Ropele, S.; Nichols, T.; Behrens, T.; Schmidt, R. \& Matthews, PM. (2006). Lesion probability maps of white matter hyperintensities in elderly individuals: results of the Austrian stroke prevention study. Journal of Neurology, Vol.253, No.8, (August 2006), pp. 1064-1070

Fazekas, F.; Offenbacher, H.; Fuchs, S.; Schmidt, R.; Niederkorn, K.; Horne, S. \& Lechner H. (1988). Criteria for an increased specificity of MRI interpretation in elderly subjects with suspected multiple sclerosis. Neurology, Vol.38, No.12, (December 1988), pp. 1822-1825.

Ferguson, B.; Matyszak, M.K.; Esiri, M.M. \& Perry,VH. (1997). Axonal damage inacute multiple sclerosis lesions. Brain, Vol.120, No.3, (March 1997), pp. 393-399.

Filippi, M. \& Agosta, F. (2007). Magnetization transfer MRI in multiple sclerosis. Journal of Neuroimaging, Vol.17 (Suppl. 1), pp. 22S-26S.

Filippi, M. \& Agosta, F. (2009). Magnetic resonance techniques to quantify tissue damage, tissue repair, and functional cortical reorganization in multiple sclerosis. In: Progress in Brain Research, J. Verhaagen et al. (Eds.), Vol.175, pp. 465-482, ISSN 0079-6123

Filippi, M. \& Agosta, F. (2010). Imaging Biomarkers in Multiple Sclerosis. Journal of Magnetic Resonance Imaging, Vol.31, No.4, (April 2010), pp. 770-788. 
Filippi, M. \& Rocca, MA. (2007). Magnetization transfer magnetic resonance imaging of the brain, spinal cord, and optic nerve. Neurotherapeutics, Vol.4, No.3, (July 2007), pp. 401-13.

Filippi, M.; Rocca, MA.; Arnold, DL.; Bakshi, R.; Barkhof, F.; De Stefano, N.; Fazekas, F.; Frohman, E. \& Wolinsky, JS. (2006). EFNS guidelines on the use of neuroimaging in the management of multiple sclerosis. European Journal of Neurology, Vol.13, No. 4, (April 2006), pp. 313-325.

Filippi, M.; Rocca, MA.; Colombo, B.; Falini, A.; Codella, M.; Scotti, G. \& Comi, G. (2002). Functional magnetic resonance imaging correlates of fatigue in multiple sclerosis. Neuroimage, Vol.15, No.3, (March 2002), pp. 559-567.

Filippi, M.; Rocca, MA. \& Comi, G. (1998a). Magnetization transfer ratios of multiple sclerosis lesions with variable durations of enhancement. Journal of the Neurological Sciences, Vol.159, No.2, (August 1998), pp. 162-165.

Filippi, M., Rocca, M. A., Martino, G., Horsfield, M. A., \& Comi, G. (1998b). Magnetization transfer changes in the normal appearing white matter precede the appearance of enhancing lesions in patients with multiple sclerosis. Annals of Neurology, Vol.43, No.6, (June 1998), pp. 809-814.

Filippi, M.; Rossi, P.; Campi, A.; Colombo, B.; Pereira, C. \& Comi G. (1997). Serial contrastenhanced MR in patients with multiple sclerosis and varying levels of disability. American Journal of Neuroradiology, Vol.18, No.8, (September 1997), pp. 1549-1556.

Fisher, E.; Lee, JC.; Nakamura, K. \& Rudick, RA. (2008). Gray matter atrophy in multiple sclerosis: a longitudinal study. Annals of Neurology, Vol.64, No.3, (September 2008), pp. 255-265.

Freedman, MS. (2011). Long-term follow-up of clinical trials of multiple sclerosis therapies. Neurology, Vol.76, No.1(Suppl. 1), (January 2011), pp. S26-S34.

Ge, Y., Grossman, RI..; Udupa, JK.; Babb, JS.; Kolson, DL. \& McGowan, JC. (2001). Magnetization transfer ratio histogram analysis of gray matter in relapsingremitting multiple sclerosis. American Journal of Neuroradiology, Vol.22, No.3, (March 2001), pp. 470-475.

Ge, Y..; Grossman, RI.; Udupa, JK..; Wei, L.; Mannon, LJ.; Polansky, M. \& Kolson, DL. (2000). Brain atrophy in relapsing-remitting multiple sclerosis and secondary progressive multiple sclerosis: Longitudinal quantitative analysis. Radiology, Vol.214, No.3, (March 2000), pp. 665-670.

Gean-Marton, AD.; Vezina, LG. ; Marton, KI. ; Stimac, GK.; Peyster, RG.; Taveras, JM. \& Davis, KR. (1991). Abnormal corpus callosum: a sensitive and specific indicator of multiple sclerosis. Radiology, Vol.180, No.1, (July 1991), pp. 215-221.

Geurts, JJ.; Blezer, EL.; Vrenken, H.; van der Toorn, A.; Castelijns, JA.; Polman, CH.; Pouwels, PJ.; Bö, L. \& Barkhof, F. (2008). Does high-field MR imaging improve cortical lesion detection in multiple sclerosis? Journal of Neurology, Vol.255, No.2, (February 2008), pp. 183-191.

Geurts, JJ.; Pouwels, PJ.; Uitdehaag, BM.; Polman, CH.; Barkhof, F. \& Castelijns, JA. (2005). Intracortical lesions in multiple sclerosis: improved detection with 3D double inversion-recovery MR imaging. Radiology, Vol.236, No.1, (July 2005), pp. 254-260.

Gonen, O.; Catalaa, I.; Babb, JS.; Ge, Y.; Mannon, LJ.; Kolson, DL. \& Grossman, RI. (2000). Total brain N-acetylaspartate: a new measure of disease load in MS. Neurology, Vol.54, No.1, (January 2000), pp. 15-19.

Henry, RG.; Shieh, M.; Okuda, DT.; Evangelista, A.; Gorno-Tempini, ML. \& Pelletier D. (2008). Regional grey matter atrophy in clinically isolated syndromes at 
presentation. Journal of Neurology, Neurosurgery and Psychiatry, Vol.79, No.11, (November 2008), pp. 1236-1244.

Hillary, FG.; Chiaravalloti, N.D.; Ricker, J.H.; Steffener, J.; Bly, B.M.; Lange, G..; Liu, WC.; Kalnin, AJ. \& DeLuca, J. (2003). An investigation of working memory rehearsal in multiple sclerosis using fMRI. Journal of Clinical and Experimental Neuropsychology, Vol.25, No.7, (October 2003), pp. 965-978.

Horakova, D.; Dwyer, MG.; Havrdova, E.; Cox, JL.; Dolezal, O.; Bergsland, N.; Rimes, B.; Seidl, Z.; Vaneckova, M. \& Zivadinov, R. (2009). Gray matter atrophy and disability progression in patients with early relapsing-remitting multiple sclerosis: a 5-year longitudinal study. Journal of the Neurological Sciences, Vol.282, No.1-2, (July 2009), pp. 112-119.

Horsfield, MA.; Barker, GJ.; Barkhof, F.; Miller, DH.; Thompson, AJ. \& Filippi M. (2003). Guidelines for using quantitative magnetization transfer magnetic resonance imaging for monitoring treatment of multiple sclerosis. Journal of Magnetic Resonance Imaging, Vol.17, No.4, (April 2003), pp. 389-397.

Hrynchak, P. \& Simpson, T. (2000). Optical coherence tomography: An introduction to the technique and its use. Optometry and Vision Science, Vol.77, No.7, (July 2000), pp. 347-56.

Huang, D.; Swanson, EA.; Lin, CP.; Schuman, JS.; Stinson, WG.; Chang, W.; Hee, MR.; Flotte, T.; Gregory, K. \& Puliafito, CA. (1991). Optical coherence tomography. Science, Vol.254, No.5035, (November 1991), pp. 1178-1181.

Kangarlu, A.; Bourekas, EC.; Ray-Chaudhury, A. \& Rammohan, KW. (2007). Cerebral cortical lesions in multiple sclerosis detected by MR imaging at 8 Tesla. American Journal of Neuroradiology, Vol.28, No.2, (February 2007), pp. 262-266.

Kapeller, P.; McLean, MA.; Griffin, CM.; Chard, D.; Parker, GJ.; Barker, GJ.; Thompson, AJ. \& Miller, DH. (2001). Preliminary evidence for neuronal damage in cortical grey matter and normal appearing white matter in short duration relapsing-remitting multiple sclerosis: a quantitative MR spectroscopic imaging study. Journal of Neurology, Vol.248, No.2 (February 2001), pp. 131-138.

Kappos, L.; Moeri, D.; Radue, EW, Schoetzau A, Schweikert K, Barkhof F, Miller D, Guttmann CR, Weiner HL, Gasperini C, Filippi M. (1999). Predictive value of gadolinium-enhanced magnetic resonance imaging for relapse rate and changes in disability or impairment in multiple sclerosis: a meta-analysis. Lancet, Vol.353, No.9157, (March 1999), pp.964-969.

Ko, TH.; Fujimoto, JG.; Duker, JS.; Paunescu, LA.; Drexler, W.; Baumal, CR.; Puliafito, CA.; Reichel, E.; Rogers, AH. \& Schuman, JS. (2004). Comparison of ultrahigh- and standard-resolution optical coherence tomography for imaging macular hole pathology and repair. Ophthalmology, Vol.111, No.11, (November 2004), pp. 20332043.

Kutzelnigg, A.; Lucchinetti, CF.; Stadelmann, C.; Brück, W.; Rauschka, H.; Bergmann, M.; Schmidbauer, M.; Parisi, JE. \& Lassmann H. (2005). Cortical demyelination and diffuse white matter injury in multiple sclerosis. Brain, Vol.128, No.11, (November 2005), pp. 2705-2712.

Kwa, VI.; Stam, J.; Blok, LM. \& Verbeeten, BJr. (1997). T2-weighted hyperintense MRI lesions in the pons in patients with atherosclerosis. Amsterdam Vascular Medicine Group. Stroke Vol.28, No. pp. 1357-1360. 
Laule, C.; Vavasour, IM.; Whittall, KP.; Oger, J.; Paty, DW.; Li, DK. \& Arnold, DL. (2003). Evolution of focal and diffuse magnetisation transfer abnormalities in multiple sclerosis. Journal of Neurology, Vol.250, No.8, (August 2003), pp. 924-931.

Lee, M.; Reddy, H.; Johansen-Berg, H.; Pendlebury, S.; Jenkinson, M.; Smith, S.; Palace, J. \& Matthews, PM. (2000). The motor cortex shows adaptive functional changes to brain injury from multiple sclerosis. Annals of Neurology, Vol.47, No.5, (May 2000), pp. 606-613.

Lee, SH.; Yoon, PH.; Park, SJ. \& Kim, DI. (2001). MRI findings in neuro-Behçet's disease. Clinical Radiology, Vol.56, No.6, (June 2001), pp. 485-494.

Li, DK.; Held, U.; Petkau, J.; Daumer, M.; Barkhof, F.; Fazekas, F.; Frank, JA.; Kappos, L.; Miller, DH.; Simon, JH,.; Wolinsky, JS,; Filippi, M. \& Sylvia Lawry Centre for MS Research. (2006). MRI T2 lesion burden in multiple sclerosis: a plateauing relationship with clinical disability. Neurology, 66, No.9, (May 2006), pp. 1384-1389.

Li, Y.; Chiaravalloti, ND.; Hillary, FG.; Deluca, J.; Liu, WC.; Kalnin, AJ. \& Ricker JH. (2004). Differential cerebellar activation on functional magnetic resonance imaging during working memory performance in persons with multiple sclerosis. Archives of Physical Medicine and Rehabilitation, Vol.85, No.4, (April 2004), pp. 635-639.

Liu, W, \& Frank, JA. (2009). Detection and quantification of magnetically labelled cells by cellular MRI. European Journal of Radiology, Vol.70, No.2, (May 2009), pp. 258-264.

Logothetis, NK.; Pauls, J.; Augath, M.; Trinath, T. \& Oeltermann, A. (2001). Neurophysiological investigation of the basis of the fMRI signal. Nature, Vol. 412, No.6843, (July, 2001), pp. 150-157.

Lowe, MJ.; Beall, EB.; Sakaie, KE.; Koenig, KA.; Stone, L.; Marrie, RA. \& Phillips, MD. (2008). Resting state sensorimotor functional connectivity in multiple sclerosis inversely correlates with transcallosal motor pathway transverse diffusivity. Hum. Brain Mapp. Vol.29, No.7, (July 2008), pp. 818-827.

Lowe, MJ.; Horenstein, C.; Hirsch, JG.; Marrie, RA.; Stone, L.; Bhattacharyya, PK.; Gass, A. \& Phillips, MD. (2006). Functional pathway-defined MRI diffusion measures reveal increased transverse diffusivity of water in multiple sclerosis. NeuroImage, Vol.32, No.3, (September 2006), pp. 1127-1133.

Lucchinetti, C.; Brück, W.; Parisi, J.; Scheithauer, B.; Rodriguez, M. \& Lassmann, H. (2000). Heterogeneity of multiple sclerosis lesions: implications for the pathogenesis of demyelination. Annals of Neurology, Vol.47, No.6, (June 2000), pp. 707-717.

Lycklama, G.; Thompson, A.; Filippi, M.; Miller, D.; Polman, C.; Fazekas, F. \& Barkhof, F. (2003). Spinal-cord MRI in multiple sclerosis. Lancet Neurology, Vol.2, No.9, (September 2003), pp. 555-562.

Markovic-Plese, S. \& McFarland, HF. (2001). Immunopathogenesis of the multiple sclerosis lesion. Current Neurology and Neuroscience Reports, Vol.1, No.3, (May 2001), pp. 257262.

Martola, J.; Stawiarz, L.; Fredrikson, S.; Hillert, J.; Bergström, J.; Flodmark, O. \& Kristoffersen Wiberg M. (2007). Progression of non age-related callosal brain atrophy in multiple sclerosis: a 9-year longitudinal MRI study representing four decades of disease development. Journal of Neurology, Neurosurgery and Psychiatry, Vol.78, No.4, (April 2007), pp. 375-380.

Masdeu, JC.; Moreira, J.; Trasi, S.; Visintainer, P.; Cavaliere, R. \& Grundman, M. (1996). The open ring: a new imaging sign in demyelinating disease. Journal of Neuroimaging, Vol.6, No.2, (April 1996), pp. 104-107. 
McDonald, WI.; Compston, A.; Edan, G.; Goodkin, D.; Hartung, HP.; Lublin, FD.; McFarland, HF.; Paty, DW.; Polman, CH.; Reingold, SC.; Sandberg-Wollheim, M.; Sibley, W.; Thompson, A.; van den Noort, S.; Weinshenker, BY. \& Wolinsky, JS. (2001). Recommended diagnostic criteria for multiple sclerosis: guidelines from the International Panel on the diagnosis of multiple sclerosis. Annals of Neurology, Vol.50, No.1, (July 2001), pp. 121-127.

Miller, D.; Barkhof, F.; Montalban, X.; Thompson, A. \& Filippi, M. (2005). Clinically isolated syndromes suggestive of multiple sclerosis, part 2: non-conventional MRI, recovery processes, and management. Lancet Neurology, Vol.4, No.6, (June 2005), pp. 341-348.

Miller, DH.; Soon, D.; Fernando, KT.; MacManus, DG..;Barker, GJ.; Yousry, TA.; Fisher, E.; O'Connor, P.; Phillips, JT,.; Polman, CH.; Kappos, L.; Hutchinson, M.; Havrdova, E.; Lublin, FD.; Giovannoni, G.; Wajgt, A.; Rudick, R.; Lynn, F.; Panzara, MA.; Sandrock, AW. \& AFFIRM Investigators. (2007). MRI outcomes in a placebo controlled trial of natalizumab in relapsing MS. Neurology, Vol.68, No.17, (April 2007), pp. 1390-1401.

Miller, DH.; Weinshenker, BG.; Filippi, M.; Banwell, BL.; Cohen, JA.; Freedman, MS.; Galetta, SL.; Hutchinson M, Johnson RT, Kappos, L.; Kira, J.; Lublin, FD.; McFarland, HF.; Montalban, X.; Panitch, H.; Richert, JR.; Reingold, SC. \& Polman, $\mathrm{CH}$. (2008). Differential diagnosis of suspected multiple sclerosis: a consensus approach. Multiple Sclerosis, Vol.14, No.9, (November 2008), pp.1157-1174.

Minneboo, A.; Uitdehaag, BM.; Ader, HJ.; Barkhof, F.; Polman, CH. \& Castelijns, JA. (2005). Patterns of enhancing lesion evolution in multiple sclerosis are uniform within patients. Neurology, Vol.65, No.1, (July 2005), pp. 56-61.

Molyneux, PD.; Barker, GJ.; Barkhof, F.; Beckmann, K.; Dahlke, F.; Filippi, M.; Ghazi, M.; Hahn, D,.; MacManus, D.; Polman, C.; Pozzilli, C.; Kappos, L,.;Thompson, AJ.; Wagner, K.; Yousry, T. \& Miller, DH; European Study Group on Interferon Beta-1b in Secondary Progressive MS. (2001). Clinical-MRI correlations in a European trial of interferon beta-1b in secondary progressive MS. Neurology, Vol.57, No.12, (December 2001), pp. 2191-2197.

Molyneux, PD.; Filippi, M.; Barkhof, F.; Gasperini, C.; Yousry, TA.; Truyen, L.; Lai, HM.; Rocca, MA.; Moseley, IF. \& Miller, DH. (1998). Correlations between monthly enhanced MRI lesion rate and changes in T2 lesion volume in multiple sclerosis. Annals of Neurology, Vol.43, No.3, (March 1998), pp. 332-339.

Mori, S.; Kaufmann, WE.; Davatzikos, C.; Stieltjes, B.; Amodei, L.; Fredericksen, K.; Pearlson, GD.; Melhem, ER.; Solaiyappan, M.; Raymond, GV.; Moser HW. \& van Zijl, PC. (2002). Imaging cortical association tracts in the human brain using diffusiontensor- based axonal tracking. Magnetic Resonance in Medicine, Vol.47, No.2, (February 2002), pp. 215-223.

Morgen, K.; Jeffries, NO.; Stone, R.; Martin, R.; Richert, ND.; Frank, JA. \& McFarland, HF. (2001). Ring-enhancement in multiple sclerosis: marker of disease severity. Multiple Sclerosis, Vol.7, No.3, (June 2001), pp. 167-171.

Narayana, PA. (2005). Magnetic resonance spectroscopy in the monitoring of multiple sclerosis. Journal of Neuroimaging, Vol.15, Suppl.4, pp. 46S-57S.

Narayana, PA.; Doyle, T.J. Lai, D., \& Wolinsky, J.S. (1998). Serial proton magnetic resonance spectroscopic imaging, contrast-enhanced magnetic resonance imaging, and quanti- tative lesion volumetry in multiple sclerosis. Annals of Neurology, Vol.43, No.1, (January 1998), pp. 56-71. 
Ogawa, S.; Lee, TE.; Kay, AR. \& Tank, DW. (1990). Brain magnetic resonance imaging with contrast dependent on blood oxygenation. Proceedings of the National Academy of Sciences of the United States of America, Vol.87, No.24, (December 1990), pp. 9868-9872.

Oh, J.; Henry, R. G.; Genain, C.; Nelson, SJ. \& Pelletier, D. (2004). Mechanisms of normal appearing corpus callosum injury related to pericallosal T1 lesions in multiple sclerosis using directional diffusion tensor and H MRS imaging. Journal of Neurology Neurosurgery and Psychiatry, Vol.75, No.9, (September 2004), pp. 1281-1286.

O'Sullivan, M.; Jarosz, JM.; Martin, RJ.; Deasy, N.; Powell, JF. \& Markus, HS. (2001). MRI hyperintensities of the temporal lobe and external capsule in patients with CADASIL. Neurology, Vol.56, No.5, (March 2001), pp. 628-634.

Pagani, E.; Rocca, MA.; Gallo, A.; Rovaris, M.; Martinelli, V.; Comi, G. \& Filippi, M. (2005). Regional brain atrophy evolves differently in patients with multiple sclerosis according to clinical phenotype. American Journal of Neuroradiology, Vol.26, No.2, (February 2005), pp. 341-346.

Pagani, E.; Bammer, R.; Horsfield, MA.; Rovaris, M.; Gass, A.; Ciccarelli, O. \& Filippi, M. (2007). Diffusion MR imaging in multiple sclerosis: technical aspects and challenges. American Journal of Neuroradiology, Vol.28, No.3, (March 2007), pp. 411-420.

Paty, DW.; Oger, JF.; Kastrukoff, LF.; Hashimoto, SA.; Hooge, JP.; Eisen, AA.; Eisen, KA.; Purves, SJ.; Low, MD. \& Brandejs, V. (1988). MRI in the diagnosis of MS: a prospective study with comparison of clinical evaluation, evoked potentials, oligoclonal banding, and CT. Neurology, Vol.38, No.2, (February 1988), pp. 180-185.

Penner, IK.; Rausch, M.; Kappos, L.; Opwis, K. \& Radu, EW. (2003). Analysis of impairment related functional architecture in MS patients during performance of different attention tasks. Journal of Neurology, Vol.250, No.4, (April 2003), pp. 461-472.

Petzold, A.; de Boer, JF.; Schippling, S.; Vermersch, P.; Kardon, R.; Green, A.; Calabresi, PA. \& Polman, C. (2010). Optical coherence tomography in multiple sclerosis: a systematic review and meta-analysis. Lancet Neurology, Vol.9, No.9, (September 2010), pp. 921-932.

Polman, CH.; Reingold, SC.; Edan, G.; Filippi, M.; Hartung, HP.; Kappos, L.; Lublin, FD.; Metz, LM.; McFarland, HF.; O'Connor, PW.; Sandberg-Wollheim, M.; Thompson, AJ.; Weinshenker, BG. \& Wolinsky, JS. (2005). Diagnostic criteria for multiple sclerosis: 2005 revisions to the "McDonald Criteria". Annals of Neurology, Vol.58, No.6, (December 2005), pp. 840-846

Polman, CH.; Reingold, SC.; Banwell, B.; Clanet, M.; Cohen, JA.; Filippi, M.; Fujihara, K.; Havrdova, E.; Hutchinson, M.; Kappos, L.; Lublin, FD.; Montalban, X.; O'Connor, P.; Sandberg-Wollheim, M.; Thompson, AJ.; Waubant, E.; Weinshenker, B. \& Wolinsky, JS. (2011). Diagnostic criteria for multiple sclerosis: 2010 revisions to the McDonald criteria. Annals of Neurology, Vol.69, No.2, (February 2011), pp. 292-302.

Poonawalla, AH.; Hasan, KM.; Gupta, RK.; Ahn, CW.; Nelson, F.; Wolinsky, JS. \& Narayana, PA. (2008). Diffusion-tensor MR imaging of cortical lesions in multiple sclerosis: initial findings. Radiology, Vol.246, No.3, (March 2008), pp. 880-886.

Poser, CM.; Paty, DW.; Scheinberg, L.; McDonald, WI.; Davis, FA.; Ebers, GC.; Johnson, KP.; Sibley, WA.; Silberberg, DH. \& Tourtellotte, WW. (1983). New diagnostic criteria for multiple sclerosis: guidelines for research protocols. Annals of Neurology, Vol.13, No.3, (March 1983), pp. 227-231.

Ranjeva, JP.; Audoin, B.; Au Duong, MV.; Ibarrola, D.; Confort-Gouny, S.; Malikova, I.; Soulier, E.; Viout, P.; Ali-Chérif, A.; Pelletier, J. \& Cozzone, P. (2005). Local tissue 
damage assessed with statistical mapping analysis of brain magnetization transfer ratio: relationship with functional status of patients in the earliest stage of multiple sclerosis. American Journal of Neuroradiology, Vol.26, No.1, (January 2005), pp. 119-127.

Reddy, H.; Narayanan, S,.;Arnoutelis, R.; Jenkinson, M.; Antel, J.; Matthews, PM. \& Arnold, DL. (2000). Evidence for adaptive functional changes in the cerebral cortex with axonal injury from multiple sclerosis. Brain, Vol.123, No.11, (November 2000), pp. 2314-2320.

Rocca, M.; Agosta, F.; Sormani, MP.; Fernando, K.; Tintorè, M.; Korteweg, T.; Tortorella, P.; Miller, DH.; Thompson, A.; Rovira, A,.;Montalban, X.; Polman, C.; Barkhof, F. \& Filippi, M. (2008). A three-year, multi-parametric MRI study in patients at presentation with CIS. Journal of Neurology, Vol. 255, No.5, (May 2008), pp. 683-691.

Rocca, MA.; Colombo, B.; Falini, A.; Ghezzi, A.; Martinelli, V.; Scotti, G.; Comi, G. \& Filippi, M (2005). Cortical adaptation in patients with MS: a cross-sectional functional MRI study of disease phenotypes. Lancet Neurology, Vol.4, No.10, (October 2005), pp. 618-626.

Rocca, MA.; Mezzapesa, DM.; Falini, A.; Ghezzi, A.; Martinelli, V.; Scotti, G.; Comi, G. \& Filippi, M. (2003). Evidence for axonal pathology and adaptive cortical reorganisation in patients at presentation with clinically isolated syndromes suggestive of MS. NeuroImage, Vol.18, No.4, (April 2003), pp. 847-855.

Roosendaal, SD.; Moraal, B.; Pouwels, PJ.; Vrenken, H.; Castelijns, JA.; Barkhof, F. \& Geurts, JJ. (2009). Accumulation of cortical lesions in MS: relation with cognitive impairment. Multiple Sclerosis, Vol.15, No.6, (June 2009), pp. 708-714.

Rovaris, M. (2008). The definition of non-responder to multiple sclerosis treatment: neuroimaging markers. Neurological Scienses, Vol.29, Suppl.2, (September 2008), pp. S222-S224.

Rovaris, M.; Agosta, F.; Sormani, MP.; Inglese, M.; Martinelli, V.; Comi, G. \& Filippi, M. (2003). Conventional and magnetization transfer MRI predictors of clinical multiple sclerosis evolution: a medium-term follow-up study. Brain, Vol.126, No.10, (October 2003), pp. 2323-2332.

Rovaris, M.; Bozzali, M.; Santuccio, G.; Ghezzi, A.; Caputo, D.; Montanari, E.; Bertolotto, A.; Bergamaschi, R.; Capra, R,.; Mancardi, G.; Martinelli, V.; Comi, G. \& Filippi, M. (2001). In vivo assessment of the brain and cervical cord pathology of patients with primary progressive multiple sclerosis. Brain, Vol.124, No.12, (December 2001), pp. 2540-2549.

Rovaris, M.; Gass, A.; Bammer, R.; Hickman, SJ.; Ciccarelli, O.; Miller, DH. \& Filippi, M. (2005). Diffusion MRI in multiple sclerosis. Neurology, Vol.65, No.10, (November 2005), pp. 1526-1532.

Rovaris, M.; Viti, B.; Ciboddo, G.; Capra, R. \& Filippi, M. (2000). Cervical cord magnetic resonance imaging findings in systemic immune-mediated diseases. Journal of the Neurological Scienses, Vol. 176, No.2, (June 2000), pp. 128-130.

Rovira, A.; Alonso, J.; Cucurella, G.; Nos, C.; Tintore, M.; Pedraza, S.; Rio, J. \& Montalban, X. (1999). Evolution of multiple sclerosis lesions on serial contrast-enhanced T1weighted and magnetization-transfer MR images. American Journal of Neuroradiology, Vol.20, No.10, (November-December 1999), pp. 1939-1945.

Rudick, RA. (2004). Impact of disease-modifying therapies on brain and spinal cord atrophy in multiple sclerosis. Journal of Neuroimaging, Vol.14, Suppl.3, (July 2004), pp. 54S-64S. 
Rudick, RA.; Lee, JC.; Simon J.; Ransohoff, RM. \& Fisher, E. (2004). Defining interferon beta response status in multiple sclerosis patients. Annals of Neurology, Vol.56, No.4, (October 2004), pp. 548-555.

Saidha, S.; Syc, SB.; Ibrahim, MA.; Eckstein, C.; Warner, CV.; Farrell, SK.; Oakley, JD.; Durbin, MK.; Meyer, SA.; Balcer, LJ.; Frohman, EM.; Rosenzweig, JM.; Newsome, SD.; Ratchford, JN.; Nguyen, QD. \& Calabresi, PA. (2011). Primary retinal pathology in multiple sclerosis as detected by optical coherence tomography. Brain, Vol. 134, No.2, (February 2011), pp. 518-533.

Sailer, M.; Losseff, NA.; Wang, L.; Gawne-Cain, ML.; Thompson, AJ. \& Miller, DH. (2001). T1 lesion load and cerebral atrophy as a marker for clinical progression in patients with multiple sclerosis: a prospective 18 months follow-up study. European Journal of Neurology, Vol.8, No.1, (January 2001), pp. 37-42.

Santos, AC.; Narayanan, S.,;De Stefano, N.; Tartaglia, MC.; Francis, SJ.; Arnaoutelis, R.; Caramanos, Z.; Antel, JP.; Pike, GB. \& Arnold, DL. (2002). Magnetization transfer can predict clinical evolution in patients with multiple sclerosis. Journal of Neurology, Vol.249, No.6 (June 2002), pp. 662-668.

Sastre-Garriga, J.; Ingle, G.T., Chard, D.T., Ramió-Torrenta', L., McLean, M.A., Miller, D.H. \& Thompson AJ. (2005). Metabolite changes in normal-appearing gray and white matter are linked with disability in early primary progressive multiple sclerosis. Archives of Neurology, Vol.62, No.4, (April 2005), pp. 569-573.

Schmierer, K.; Scaravilli, F.; Altmann, D.R.; Barker, G.J. \& Miller, D.H. (2004). Magnetization transfer ratio and myelin in postmortem multiple sclerosis brain. Annals of Neurology, Vol.56, No.3, (September 2004), pp. 407-415.

Schumacker, GA.; Beebe, G.; Kibler, RF.; Kurland, LT.; Kurtzke, JF.; Mcdowell, F.; Nagler, B.; SIBibley, WA.; Tourtellotte, WW. \& Willmon, TL. (1965). Problems of experimental trials of therapy in multiple sclerosis: report by the panel on the evaluation of experimental trials of therapy in multiple sclerosis. Annals of the New York Academy of Sciences, Vol. 122, (March 1965), pp. 552-568.

Sicotte, NL.; Voskuhl, RR.; Bouvier, S.; Klutch R, Cohen, MS. \& Mazziotta, JC. (2003). Comparison of multiple sclerosis lesions at 1.5 and 3.0 T. Investigative Radiology, Vol.38, No.7, (July 2003), pp. 423-427.

Silver, NC.; Lai, M.; Symms, MR.; Barker, GJ.; McDonald, WI. \& Miller, DH. (1998). Serial magnetization transfer imaging to characterize the early evolution of new MS lesions. Neurology, Vol.51, No.3, (September 1998), pp. 758-764.

Simon, JH.; Zhang, S.; Laidlaw, DH.; Miller, DE.; Brown, M.; Corboy, J. \& Bennett, J. (2006). Identification of fibers at risk for degeneration by diffusion tractography in patients at high risk for MS after a clinically isolated syndrome. Journal of Magnetic Resonance Imaging, Vol.24, No.5, (November 2006), pp. 983-988.

Singhal, S.; Rich, P. \& Markus, HS. (2005). The spatial distribution of MR imaging abnormalities in cerebral autosomal dominant arteriopathy with subcortical infarcts and leukoencephalopathy and their relationship to age and clinical features. AJNR Am J Neuroradiol (2005), Vol.26, No. pp. 2481-87.

Song, SK.; Yoshino, J.; Le, TQ.; Lin, S. J.; Sun, S. W.; Cross, AH. \& Armstrong, RC. (2005). Demyelination increases radial diffusivity in corpus callosum of mouse brain. NeuroImage, Vol.26, No.1, (May 2005), pp. 132-140.

Srinivasan, R.; Ratiney, H.; Hammond-Rosenbluth, KE.; Pelletier, D. \& Nelson, SJ. (2010). MR spectroscopic imaging of glutathione in the white and gray matter at $7 \mathrm{~T}$ with 
an application to multiple sclerosis. Magnetic Resonance Imaging, Vol.28, No.2, (February 2010), pp. 163-170.

Srinivasan, R.; Sailasuta, N.; Hurd, R.; Nelson, S. \& Pelletier, D. (2005). Evidence of elevated glutamate in multiple sclerosis using magnetic resonance spectroscopy at 3 T. Brain, Vol.128, No.5, (May 2005), pp. 1016-1025.

Srinivasan, R.; Vignerion, D.; Sailasuta, N.; Hurd, R. \& Nelson, S. (2004). A comparative study of myo-inositol quantification using LC model at $1.5 \mathrm{~T}$ and $3.0 \mathrm{~T}$ with $3 \mathrm{D} 1 \mathrm{H}$ proton spectroscopic imaging of the human brain. Magnetic Resonance Imaging, Vol.22, No.4, (May 2004), pp. 523-528.

Staffen, W.; Mair, A.; Zauner, H.; Unterrainer, J.; Niederhofer, H.; Kutzelnigg, A.; Ritter, S.; Golaszewski, S.; Iglseder, B. \& Ladurner, G. (2002). Cognitive function and fMRI in patients with multiple sclerosis: evidence for compensatory cortical activation during an attention task. Brain, Vol.125, No.6, (June 2002), pp. 1275-1282.

Stankiewicz, JM.; Neema, M.; Alsop, DC.; Healy, BC.; Arora, A.; Buckle, GJ.; Chitnis, T.; Guttmann, CR.; Hackney, D. \& Bakshi, R. (2009). Spinal cord lesions and clinical status in multiple sclerosis: a $1.5 \mathrm{~T}$ and $3 \mathrm{~T}$ MRI study. Journal of the Neurological Sciences, Vol.279, No.1-2, (April 2009), pp. 99-105.

Stankiewicz, J.; Panter, SS.; Neema, M.; Arora, A.; Batt, CE. \& Bakshi, R. Iron in chronic brain disorders: imaging and neurotherapeutic implications. Neurotherapeutics, Vol.4, No.3, (July 2007), pp. 371-386.

Stevenson, VL.; Ingle, GT.; Miller, DH. \& Thompson, AJ. (2004). Magnetic resonance imaging predictors of disability in primary progressive multiple sclerosis: A 5-year study. Multiple Sclerosis, Vol.10, No.4, (August 2004), pp. 398-401.

Summers, M.; Swanton, J.; Fernando, K.; Dalton C, Miller, DH, Cipolotti, L \& Ron, MA. (2008). Cognitive impairment in multiple sclerosis can be predicted by imaging early in the disease. Journal of Neurology, Neurosurgery and Psychiatry, Vol. 79, No.8, (August 2008), pp. 955-958.

Swanton, JK.; Fernando, KT.; Dalton, CM.; Miszkiel, KA.; Thompson, AJ.; Plant, GT. \& Miller, DH. (2006). Modification of MRI criteria for multiple sclerosis in patients with clinically isolated syndromes. Journal of Neurology, Neurosurgery and Psychiatry, Vol. 77, No.7, (July 2006), pp. 830-833.

Swanton, JK.; Rovira, A.; Tintore, M.; Altmann, DR.; Barkhof, F.; Filippi, M.; Huerga, E.; Miszkiel, KA.; Plant, GT.; Polman, C.; Rovaris, M.; Thompson, AJ.; Montalban, X. \& Miller, DH. (2007). MRI criteria for multiple sclerosis in patients presenting with clinically isolated syndromes: a multicentre retrospective study. Lancet Neurology, Vol.6, No.8, (August 2007), pp. 677-686.

Theodoridou, A. \& Settas, L. (2008). Demyelination in rheumatic diseases. Postgraduate Medical Journal, Vol.84, No.989, (March 2008), pp. 127-132.

Tintore, M.; Rovira, A.; Martinez, M.; Rio, J.; Díaz-Villoslada, P.; Brieva, L.; Borrás, C.; Grivé, E.; Capellades, J. \& Montalban, X. (2000). Isolated demyelinating syndromes: comparison of different MR imaging criteria to predict conversion to clinically definite multiple sclerosis. American Journal of Neuroradiology, Vol.21, No.4, (April 2000), pp. 702-706.

Tintore, M.; Rovira, A.; Rio, J.; Nos, C.; Grivé, E.; Téllez, N.; Pelayo, R.; Comabella, M.; Sastre-Garriga, J. \& Montalban, X. (2006). Baseline MRI predicts future attacks and disability in clinically isolated syndromes. Neurology, Vol. 67, No. 6, (September, 2006), pp. 968-972. 
Thompson, AJ.; Montalban, X.; Barkhof, F.; Brochet, B.; Filippi, M.; Miller, DH.; Polman, CH.; Stevenson, VL. \& McDonald, WI. (2000). Diagnostic criteria for primary progressive multiple sclerosis: a position paper. Annals of Neurology, Vol.47, No. 6, (June 2000), pp. 831-835.

Tovar-Moll, F.; Evangelou, IE.; Chiu, AW.; Richert, ND.; Ostuni, JL.; Ohayon, JM.; Auh, S.; Ehrmantraut, M.; Talagala SL.; McFarland, HF. \& Bagnato F. (2009). Thalamic involvement and its impact on clinical disability in patients with multiple sclerosis: A diffusion tensor imaging study at 3T. American Journal of Neuroradiology, Vol.30, No.7, (August 2009), pp. 1380-1386.

Trip, SA.; Schlottmann, PG.; Jones, SJ.; Altmann, DR.; Garway-Heath, DF.; Thompson, AJ.; Plant, GT. \& Miller, DH. (2005). Retinal nerve fiber layer axonal loss and visual dysfunction in optic neuritis. Annals of Neurology, Vol.58, No.3, (September 2005), pp. 383-391.

Trip, SA.; Schlottmann, PG.; Jones, SJ.; Li, WY.; Garway-Heath, DF.; Thompson, AJ.; Plant, GT. \& Miller, DH. (2006). Optic nerve atrophy and retinal nerve fibre layer thinning following optic neuritis: evidence that axonal loss is a substrate of MRI-detected atrophy. Neuroimage, Vol.31, No.1, (May 2006), pp. 286-293.

Truyen, L.; van Waesberghe, JH.; van Walderveen, MA.; van Oosten, BW.; Polman, CH.; Hommes, OR.; Adèr, HJ. \& Barkhof, F. (1996). Accumulation of hypointense lesions ("black holes") on T1 spin-echoMRI correlates with disease progression in multiple sclerosis. Neurology, Vol.47, No.6, (December 1996), pp. 1469-1476.

van Buchem, MA.; McGowan, JC.; Kolson, DL.; Polansky, M. \& Grossman, RI. (1996). Quantitative volumetric magnetization transfer analysis in multiple sclerosis: Estimation of macroscopic and microscopic disease burden. Magnetic Resonance in Medicine, Vol.36, No.4, (October 1996), pp. 632-636.

van den Heuvel, MP. \& Hulshoff Pol, HE. (2010). Exploring the brain network: A review on resting-state fMRI functional connectivity. European Neuropsychopharmacology, Vol.20, No.8, (August 2010), pp. 519-534

van Waesberghe, JH.; van Walderveen, MA.; Castelijns, JA.; Scheltens, P.; Lycklama à Nijeholt, GJ.; Polman, CH. \& Barkhof, F. (1998). Patterns of lesion development in multiple sclerosis: longitudinal observations with T1-weighted spin-echo and magnetization transfer MR. American Journal of Neuroradiology, Vol.19, No.4, (April 1998), pp. 675-683.

van Walderveen, MA.; Kamphorst, W.; Scheltens, P,.; van Waesberghe, JH.; Ravid, R.; Valk, J.; Polman, CH. \& Barkhof, F. (1998). Histopathologic correlate of hypointense lesions on T1-weighted spin-echo MRI in multiple sclerosis. Neurology, Vol.50, No.5, (May 1998), pp. 1282-1288.

Vellinga, MM.; Oude Engberink, RD.; Seewann, A.; Pouwels, PJ.; Wattjes, MP.; van der Pol, SM.; Pering, C.; Polman, CH.; de Vries, HE.; Geurts, JJ. \& Barkhof, F. (2008). Pluriformity of inflammation in multiple sclerosis shown by ultra-small iron oxide particle enhancement. Brain Vol.131, No.3, (March 2008), pp. 800-807.

Vellinga, MM.; Vrenken, H.; Hulst, HE.; Polman, CH.; Uitdehaag, BM.; Pouwels, PJ.; Barkhof, F. \& Geurts, JJ. (2009). Use of ultrasmall superparamagnetic particles of iron oxide (USPIO)-enhanced MRI to demonstrate diffuse inflammation in the normal-appearing white matter (NAWM) of multiple sclerosis (MS) patients: an exploratory study. Journal of Magnetic Resonance Imaging, Vol.29, No.4, (April 2009), pp.774- 779 . 
Villoslada, P.; Sepulcre, J.; Toledo, J. \& Bejarano, B. (2008). Retinal nerve fiberfibre layer is associated with brain atrophy in multiple sclerosis. Neurology, Vol.71, No.21, (November 2008), pp. 1747.

Wattjes, MP. \& Barkhof, F. (2009). High field MRI in the diagnosis of multiple sclerosis: high field-high yield? Neuroradiology, 2009; Vol.51, No.5, (March 2009), pp. 279-292.

Wattjes, MP.; Harzheim, M.; Lutterbey, GG.; Hojati, F.; Simon, B.; Schmidt, S.; Schild, HH. \& Barkhof, F. (2008). Does high field MRI allow an earlier diagnosis of multiple sclerosis? Journal of Neurology, Vol.255, No.8, (August 2008), pp. 1159-1163.

Werring, DJ.; Clark, CA.; Barker, GJ.; Thompson, AJ. \& Miller, DH. (1999). Diffusion tensor imaging of lesions and normal-appearing white matter in multiple sclerosis. Neurology, Vol.52, No.8, (May 1999), pp. 1626-1632.

White, AT.; Lee, JN.; Light, AR. \& Light, KC. (2009). Brain activation in multiple sclerosis: a BOLD fMRI study of the effects of fatiguing hand exercise. Multiple Sclerosis, Vol.15, No.5, (May 2009), pp. 580-586.

Wingerchuk, DM.; Lennon, VA.; Lucchinetti, CF.; Pittock, SJ. \& Weinshenker, BG. (2007). The spectrum of neuromyelitis optica. Lancet Neurology, Vol.6, No.9, (September 2007), pp. 805-815.

Young, IR.; Hall, AS.; Pallis, CA.; Legg, NJ.; Bydder, GM. \& Steiner, RE. (1981). Nuclear magnetic resonance imaging of the brain in multiple sclerosis. Lancet, 1981; Vol.2, No.8255, (November 1981), pp. 1063-1066.

Yousry, TA.; Major, EO.; Ryschkewitsch, C.; Fahle, G.; Fischer, S.; Hou, J.; Curfman, B.; Miszkiel, K.; Mueller-Lenke,. N.; Sanchez, E.; Barkhof, F.; Radue, EW.; Jäger, HR. \& Clifford, DB. (2006). Evaluation of patients treated with natalizumab for progressive multifocal leukoencephalopathy. New England Journal of Medicine, Vol.354, No.9, (March 2006), pp. 924-933.

Zajicek, JP.; Scolding, NJ.; Foster, O.; Rovaris, M.; Evanson, J.; Moseley, IF.; Scadding, JW.; Thompson, EJ.; Chamoun, V.; Miller, DH.; McDonald, WI. \& Mitchell, D. (1999). Central nervous system sarcoidosis--diagnosis and management. QJM: monthly journal of the Association of Physicians, Vol.92, No.2, (February 1999), pp. 103-117.

Zivadinov, R. \& Cox, JL. (2007). Neuroimaging in multiple sclerosis. International Review of Neurobiology, Vol.79, (May 2007), pp. 449-474

Zivadinov, R. \& Leist, TP. (2005). Clinical-magnetic resonance imaging correlations in multiple sclerosis. Journal of Neuroimaging, Vol.15, Suppl.4, pp. 10S-21S.

Zivadinov, R.; Reder, AT.; Filippi, M.; Minagar, A.; Stüve, O.; Lassmann, H.; Racke, MK.; Dwyer, MG.; Frohman EM. \& Khan, O. Mechanisms of action of disease-modifying agents and brain volume changes in multiple sclerosis. Neurology, Vol.71, No.2, (July 2008), pp. 136-144. 


\title{
Impact of Gray Matter Pathology on Cognitive Function in Multiple Sclerosis
}

\author{
Mike Andrea ${ }^{1,2}$, Guttmann Charles R.G. ${ }^{2}$ and Illes Zsolt ${ }^{1}$ \\ ${ }^{1}$ Division of Clinical and Experimental Neuroimmunology, \\ Department of Neurology, University of Pecs \\ ${ }^{2}$ Center for Neurological Imaging, Brigham and Women's Hospital, \\ Harvard Medical School \\ ${ }^{1}$ Hungary \\ ${ }^{2} U S A$
}

\section{Introduction}

Multiple sclerosis (MS) is a chronic progressive disease associated with both sensory-motor and psychological symptoms including cognitive deficits, affective disturbances, and fatigue. Intellectual disability of MS patients usually manifests itself as a composition of subtle deficits in certain cognitive domains detectable with sensitive neuropsychological test batteries specially developed for the MS population (Benedict et al., 2006b; Rao, 1990). Nevertheless, the effect of cognitive impairment on everyday life activities, employment status, and social relationships is considerable (Amato et al., 1995; Rao et al., 1991b). Cognitive impairment may occur in the earliest stages of the disease (Achiron \& Barak, 2003; Callanan et al., 1989; Deloire et al., 2005; Feinstein et al, 1992; Glanz et al., 2007) even in patients with clinically isolated syndrome (CIS) (Achiron \& Barak, 2003; Feuillet et al., 2007; Glanz et al., 2007; Potagas et al., 2008). Cognitive deficits may develop independently from physical disability particularly in the early stage of the disease and in patients with benign MS (Feuillet et al., 2007; Glanz et al., 2007; Portaccio et al., 2009). However, the presence of cognitive decline has been found to predict a more progressive disease course (Portaccio et al., 2009; Zipoli et al., 2010). Detrimental effects on quality of life and poor disease prognosis underlie the importance of neuropsychological assessment of MS patients in clinical practice.

Magnetic resonance imaging (MRI) is one of the most important paraclinical tools used in the diagnosis of MS. Quantitative measures derived from conventional and advanced MRI methods have been developed to monitor and predict the course of the disease, as well as for in-vivo non-invasive investigation of MS pathophysiology (Filippi \& Rocca, 2010).

Increasing recognition of the functional relevance and prognostic role of MS cognitive impairments has resulted in focused attention on MR correlates of cognitive deficit. Assessment of the global and regional white matter (WM) lesion burden, derived from T2or T1-weighted MRI shows modest association with cognitive status (Arnett et al., 1994; Hohol, et al., 1997; Patti et al., 2009; Rao et al., 1989; Rovaris et al., 2001; Swirsky-Sacchetti et al., 1992). A stronger relationship has been obtained with quantification of the whole and 
regional brain atrophy (Rovaris, et al., 2006; Zivadinov, et al., 2001) considered as a marker of irreversible tissue loss (Miller et al., 2002; Rovaris \& Filippi, 1999). Non-conventional MRI techniques assessing diffuse brain tissue damage outside of the MRI-visible lesions provide potent tools to detect pathological changes underlying MS cognitive deficits than lesionbased and atrophy measures (Filippi et al., 2010).

Recent advances in histopathological and imaging techniques have renewed the appreciation of gray matter (GM) involvement in MS (Pirko et al., 2007). New histopathological methods have found demyelinating lesions in significant portions of the cortex (Albert et al., 2007; Bo et al., 2003; Kidd et al., 1999; Kutzelnigg et al., 2005; Peterson et al., 2001; Wegner et al., 2006). In addition, neuroimaging techniques have detected structural changes in the GM (Pirko et al., 2007). In this review, we summarize the results of recent studies correlating MRI-detectable GM abnormalities with various aspects of MS neuropsychological dysfunction.

\section{Gray matter pathology in multiple sclerosis}

GM pathology is detectable in the earliest disease stages (Chard et al., 2004; De Stefano et al., 2003; Kutzelnigg et al., 2005), and becomes prominent in the progressive phase (Fisniku et al., 2008; Kutzelnigg et al., 2005).

GM damage in MS may result from primary local cortical demyelinating lesions (Bo et al., 2003; Peterson et al., 2001), and from GM atrophy secondary to axonal transections in destructive WM plaques or severe diffuse axonal damage in the normal-appearing WM leading to Wallerian (anterograde or transsynaptic) degeneration, as well as retrograde degeneration (Evangelou et al., 2000; Sepulcre et al., 2009; Trapp et al., 1998). However, the exact relationship between primary and secondary processes leading to GM damage remains poorly understood. Finally, other primary neurodegeneration independent from focal WM or GM lesions has been presumed in the early development of GM atrophy in MS (Sepulcre et al., 2009).

MS is characterized by a significant number of focal cortical lesions (CLs) (Bo et al., 2003; Kidd et al., 1999; Kutzelnigg et al., 2005; Peterson et al., 2001). In addition, demyelination may occur in the deep GM structures, hippocampus, hypothalamus, cerebellum, and spinal cord (Geurts et al., 2009). Pathologically, CLs are characterized by a much milder lymphocyte infiltration, complement deposition, microglial activation, and blood-brain barrier disruption than WM lesions (Bo et al., 2003). Cortical demyelinisation can occur in conjunction with subcortical WM plaques or as small perivascular intracortical lesions. However, the most extensive cortical demyelination is seen as widespread and band-like subpial lesions, which span the cortex over long distances affecting several adjacent gyri and sulci. This type of CL is associated with chronic inflammation of the meninges (Kooi et al., 2009; Magliozzi et al., 2007).

\section{Cognitive impairment in multiple sclerosis}

\subsection{General intellectual abilities}

The estimated prevalence of cognitive impairment in MS ranges between 43 to $70 \%$ (Benedict et al., 2006b; Rao et al., 1991a) both in the earlier and later stages of the disease (Beatty et al., 1990; Piras et al., 2003). Cognitive impairment can be seen irrespective of 
the duration of the disease and is only mildly associated with physical disability (Patti et al., 2009). However, cognitive disturbances seem to be more severe in patients with chronic progressive disease compared to those in the relapsing-remitting (RR) stage (Patti et al., 2009). Both fatigue and depression have been identified as important contributors to cognitive impairment of MS patients (Arnett et al., 2008; Deluca et al., 2004).

MS affects various aspects of general cognitive functioning, including efficiency of information processing, verbal and visuo-spatial memory, executive functioning, attention, and visual perceptual processing. Processing speed, and visual learning and memory seem to be most commonly affected (Benedict et al., 2006b; Rao et al., 1991a).

\subsection{Social cognition, Theory of Mind}

Deficits of general intellectual abilities significantly interfere with everyday life activities and decision-making. In addition, loss of employment status, restriction in social activities, and difficulties in inter-personal relationships frequently occur during the disease. Deficits of social cognition may additionally account for all of these functional limitations. Social cognition is a human mental ability involving the capacity to interpret and predict mental states of other people in terms of thoughts, intentions, desires and beliefs known as Theory of Mind (ToM), also referred as mentalizing and mindreading. ToM ability involves social-perceptual processes that enable mental state decoding from nonverbal cues, such as facial expression, eye gaze, and body postures. Furthermore, ToM involves social cognitive processes that enable complex abstract reasoning about the mental state of others, such as prosody and social content of speech (Stone et al., 1998). Social cognition might be independent, and dissociable from general intelligence (Shamay-Tsoory et al., 2005). Only a few studies investigated social cognition in MS; all demonstrated deficits in facial emotion recognition (Banati et al., 2010; Henry et al., 2009; Jehna et al., 2010; Krause et al., 2009; Ouellet et al., 2010) In addition, decline in complex cognitive inferences relating to the content of the mental state was also described (Banati et al., 2010; Ouellet et al., 2010).

The neural basis of ToM abilities has been widely investigated using advanced neuroimaging methods in healthy subjects and in several clinical conditions showing social cognitive impairment particularly in high-functioning autism and schizophrenia. These studies support the hypothesis that integrated fronto-temporal and temporoparietal circuits are dedicated to mentalizing. Main nodes of these networks were found distributed in the posterior superior temporal sulcus, temporo-parietal junction, temporal pole, medial prefrontal cortex, anterior cingulate cortex, orbitofrontal cortex, and inferior parietal lobule, as well as in subcortical areas, particularly in the amygdala (Adolphs et al., 2002; Frith \& Frith, 2006; Stone, et al., 1998). Another important neural mechanism participating in social cognitive processes is the activation of mirror neurons during observation of movements of others (Williams, 2008). Mirror neuron activation described first in macaque monkeys produces inner simulation of the viewed action thereby enabling the understanding of its intended goal. Electrophysiological studies of monkeys and human functional MRI (fMRI) studies found mirror neurons in the premotor cortex (frontal mirror system), as well as in the inferior parietal cortex and posterior temporal cortex (parietal mirror system) (Williams, 2008). 


\section{Relationship between gray matter findings assessed by MRI and cognitive dysfunction in multiple sclerosis}

\subsection{General intellectual function \\ 4.1.1 Neocortical lesions}

In vivo visualization of CLs is challenging (Geurts et al., 2005a) because CLs are typically small compared to the usual resolution of MRI in clinical practice, have poor contrast with the surrounding normal GM, and have similar signal properties to those of cerebrospinal fluid (CSF) reducing their conspicuity in boundary areas of GM and CSF. Using conventional imaging techniques, CLs are missed in up to $95 \%$ of the cases (Geurts et al., 2005b). In the past few years, large efforts have been devoted to the development of MRI techniques capable of visualizing at least a portion of CLs in vivo (Bagnato et al., 2006; Geurts et al., 2005a; Kangarlu et al., 2007; Mainero et al., 2009; Mike et al., 2011; Nelson Poonawalla et al., 2008; Nelson et al., 2007; Simon et al., 2010). Novel MRI methods have been deployed to address these challenges, including novel pulse sequences, multi-channel and high-resolution imaging, and ultra-high magnetic field strength (Bagnato et al., 2006; Kangarlu et al., 2007; Mainero et al., 2009; Mike et al., 2011; Nelson et al., 2008; Nelson et al., 2007; Simon et al., 2010). Recently, the application of the double inversion recovery (DIR) sequence convincingly demonstrated that CLs are frequent in MS patients (Calabrese et al., 2007; Calabrese et al., 2008), as has been long known from pathological studies (Brownell \& Hughes, 1962, Lumsden, 1970).

We employed a high-resolution 3 Tesla brain MRI protocol that combined multiplanar display of 3 dimensional (3D) fluid attenuated inversion recovery (FLAIR) and T1-weighted 3D inversion recovery spoiled gradient-recalled echo (IR-SPGR) sequences to study the relationship between CLs and cognitive performance of MS patients (Mike et al., 2011). This approach took advantage of the high contrast sensitivity of FLAIR for imaging CLs combined with IR-SPGR to delineate the boundary between cortex and WM (Figure 1.). These sequences are widely available on clinical scanners from multiple vendors and can be integrated in clinical routine with reasonable scan times. Using this clinically applicable MRI method the detected CL load was comparable with the bulk of CLs assessed by specialized MRI methods developed for sensitive CL delineation (Calabrese et al., 2008; Mainero et al., 2009; Nelson et al., 2008; Nelson et al., 2007; Simon et al., 2010).

We explored the relationship between cognitive performance and lesion load of the WM and cortical GM compartments in an MS patient cohort including RR $(n=20)$ and secondary progressive (SP) $(n=6)$ clinical subtypes. Very few studies have examined this previously (Bagnato et al., 2010; Calabrese et al., 2009; Roosendaal et al., 2009). Therefore, a broad range of neuropsychological variables, which could potentially include cortical contributions (e.g. information processing speed, new learning, verbal and non-verbal memory, executive function) had been assessed. After controlling for age, depression and pre-morbid intelligence, CL number, CL volume and WM lesion volume independently predicted the performance of information processing speed and working memory. In addition, CL number also predicted verbal learning and memory.

These findings suggested that both WM and GM lesions influence cognitive performance in MS. However, CLs may have a particularly important contribution to this relationship, as beside information processing speed performance, CL number also significantly correlated with verbal learning abilities. Two recent studies using DIR sequence yielded similar results (Calabrese et al., 2009; Roosendaal et al., 2009). 


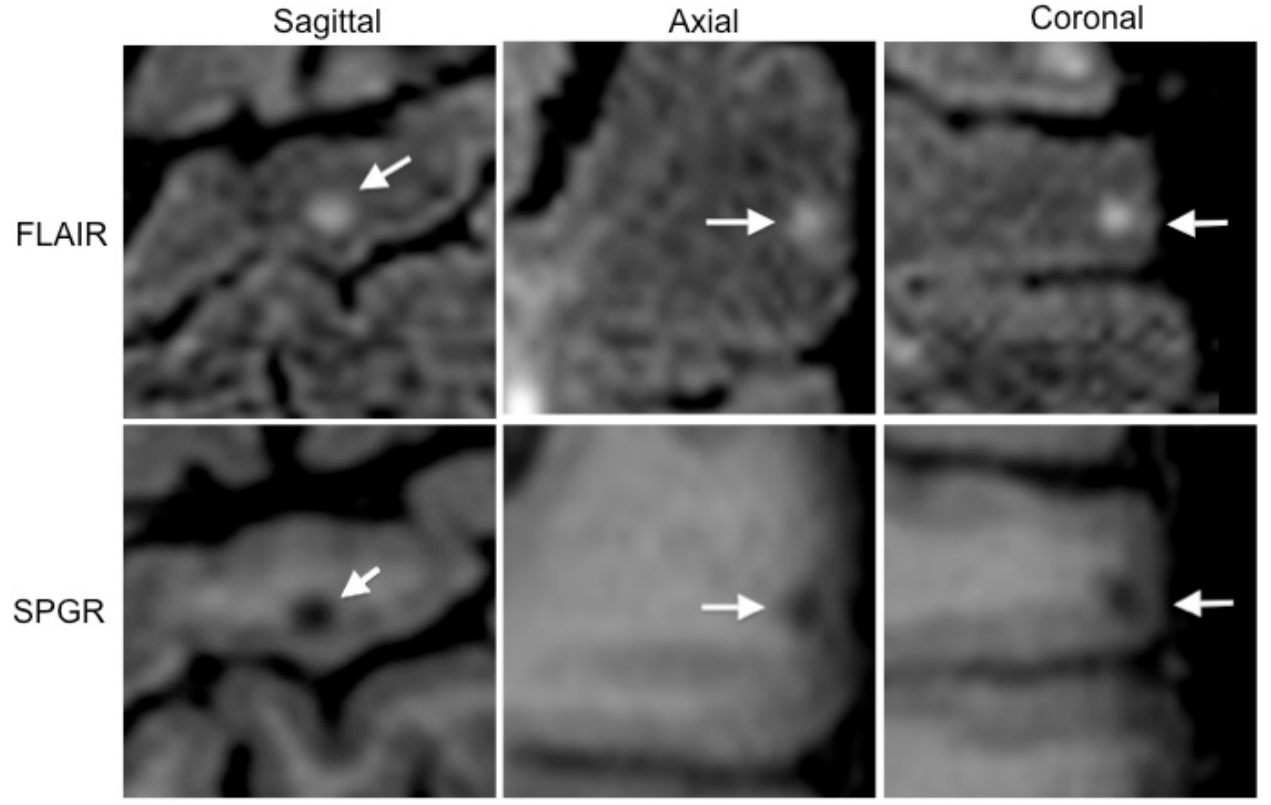

Fig. 1. Cortical lesion shown on multiplanar images

The same cortical lesion involving both the cortex and the adjoining subcortical white matter (Type I lesion) is shown on FLAIR (upper row) and IR-SPGR (lower row) images (arrows), reconstructed in sagittal, axial, and coronal planes. Note that the lesion is conspicuous on FLAIR, while the anatomic location relative to the white matter-gray matter boundary is well defined on the IR-SPGR images.

\subsubsection{T2 hypointensity imaging}

Iron deposition, a possible sign of neurodegeneration, reduces T2 relaxation times, resulting in hypointensity on T2-weighted images. In MS, T2 hypointensity frequently occurs in the thalamus and basal ganglia. T2 hypointense regions in the GM have been shown to correlate with cognitive impairment of MS patients (Brass et al., 2006; Neema et al., 2007).

\subsubsection{Gray matter atrophy}

Several cross-sectional and longitudinal studies have reported GM volume reduction in MS using manual or semi-automated segmentation methods, voxel-based morphometry (VBM) approach, and cortical thickness measurement (Grassiot et al., 2009). GM atrophy has been documented both in the deep gray matter structures (caudate nucleus, thalamus) and in neocortical regions. Recently, the relationship between GM volume decrease and cognitive performance of MS patients has been intensively investigated.

Neocortical volume has been found to be significantly reduced in cognitively impaired compared to cognitively preserved patients (Amato et al., 2004). Similar findings have been reported with cortical thickness analyses of the global brain and parcellated brain areas (Calabrese et al., 2010). Compared to healthy controls, a VBM study found significant 
decrease in GM volume only in MS patients with cognitive impairment in contrast to patients with normal neuropsychological performance (Morgen et al., 2006). Neuropsychological performance of patients with cognitive decline has been documented to correlate with cortical brain volume (Amato et al., 2004; Benedict et al., 2006a; Morgen et al., 2006), as well as cortical thickness (Calabrese et al., 2010). GM volume assessed longitudinally decreased more progressively in MS patients with deteriorating cognitive performance compared to patients with stable or improving cognitive state (Amato et al., 2007).

The relevance of cortical damage in cognitive dysfunction of MS patients has been highlighted by studies of regional cortical atrophy (Calabrese et al., 2010; Morgen et al., 2006; Tekok-Kilic et al., 2007). In a group of cognitively impaired MS patients, GM volume of fronto-temporal brain areas presumed to be involved in neural processing of working memory, information processing, and divided attention correlated with performance on the paced auditory serial addition task (PASAT) test performance (Morgen et al., 2006). Using a semi-automated parcellation analysis of the neocortex, the prefrontal GM volume resulted the strongest predictor of memory and information processing performance in MS patients (Tekok-Kilic et al., 2007).

Impact of subcortical GM atrophy on MS cognitive performance has also been established. Different methods applied for quantification of deep GM structures including the measure of the bicaudate ratio (Bermel et al., 2002), enlargement of the third ventricular width (Benedict et al. 2004a, 2004b, 2006a, Christodoulou et al., 2003; Sanchez et al, 2008; TekokKilic et al., 2007), semi-automated (Houtchens et al., 2007) and automated (Benedict et al. 2009) segmentation of subcortical GM structures provided convergent evidence for a relationship between deep GM damage and neuropsychological impairment. Reduction of thalamic volume was found to be a reliable predictor of cognitive decline in MS even after correction for WM lesion volume, whole brain atrophy and third ventricular with (Benedict et al., 2009; Houtchens et al., 2007). Strong correlations have been reported between caudate atrophy and impaired information processing speed (Bermel et al., 2002), as well as verbal learning and memory (Benedict et al., 2009). Measure of the third ventricle width has been used to infer the atrophy of adjacent GM structures, such as the thalamus (central atrophy). Enlargement of the third ventricle showed a highly significant relationship with both general cognitive impairment and deficits in specific neuropsychological domains even after accounting for the influence of global lesion load measures and whole brain atrophy (Benedict et al., 2004b; Christodoulou et al., 2003; Sanchez et al., 2008).

A recent study investigated the contribution of different GM compartments to cognitive dysfunction in MS patients, and found both the central and cortical atrophy predictive of the presence of cognitive impairment in MS (Tekok-Kilic et al., 2007).

\subsubsection{Non-conventional MRI methods}

Beyond focal lesions and atrophy, MS pathology is characterized by diffuse tissue damage. Conventional morphological MRI methods are insensitive to detect these microscopic structural alterations. Quantitative MRI techniques including magnetization transfer (MT) MRI, diffusion tensor imaging (DTI), and proton MR spectroscopy ( $\left.{ }^{1} \mathrm{H}-\mathrm{MRS}\right)$ detect brain tissue changes on a molecular level, and thereby are able to measure the degree of structural integrity in diffusely affected brain tissue. Abnormalities in the GM assessed with nonconventional MRI methods have been well established in MS (Filippi et al., 2010; Rovaris et 
al., 2006). Studies, addressing the clinical relevance of diffuse GM pathology in the cognitive impairment of MS patients are summarized in the following subsections.

\subsubsection{Magnetization transfer MRI}

The MT ratio (MTR) derived from MT MRI reflects the capacity of macromolecules to exchange magnetization with surrounding water molecules and estimates the relative amounts of free and bound water in the brain (Wolff \& Balaban, 1994). In MS, reduced MTR values may indicate a relative increase in the unbound water fraction due to edema and inflammation, or a reduction in the bound fraction, which may reflect demyelination and axonal loss (Schmierer et al., 2007). MTR values derived from the cortical/subcortical brain tissue were significantly lower in cognitively impaired patients than in those with no impairment (Rovaris, et al., 2000). MTR values of the cortical/subcortical regions showed correlation with cognitive performance of RR MS patients (Rovaris et al., 2000). In CIS patients, using voxel-by-voxel statistical mapping, significant correlations were observed between local MTR values and PASAT scores in the right Brodmann (BA) 4 and the left BA40 (Ranjeva et al., 2006; Ranjeva et al., 2005). A recent study investigated GM MRI correlates of cognitive impairment in different stages of MS. In patients with CIS suggestive for MS, mean cortical MTR has been identified as the only independent predictor of decreased mental processing speed. However, in early RR MS cortical atrophy was found as the strongest correlate of the same cognitive task. These findings suggest that both microscopic tissue disintegrations and atrophy of the cortex are important determinants of MS cognitive performance, however their significance changes with advance of the disease (Khalil et al., 2011). In a study including benign MS patients (BMS), total and regional (frontal, temporal, and occipital) cortical MTR values corrected for WM lesion volume were able to differentiate between cognitively impaired and cognitively preserved MS patients. However, MTR values of cognitively preserved MS patients did not differ from healthy controls. These findings support heterogeneity of the brain structural damage in BMS associating with different clinical prognosis (Amato et al., 2008). In patients with primary progressive (PP) MS, significant correlations have been observed between decrease of MTR value in specific cortical regions (right inferior parietal cortex, right inferior occipital gyrus) and PASAT performance (Khaleeli et al., 2007). In a longitudinal study of PP MS patients, GM MTR independently predicted PASAT score at the 5-year follow-up (Penny et al., 2010).

\subsubsection{Diffusion tensor imaging}

DTI measures the diffusion of water molecules in the brain tissue, which is directionally determined along the fiber tracts. Disturbances in the structural integrity of the brain tissue are reflected by the increase of the mean diffusivity (MD) and decrease of the fractional anisotrophy (FA), parameters derived from DTI technique (Rovaris et al., 2009).

In RRMS, MD of the NAGM moderately correlated with neuropsychological tests measuring semantic fluency, and information processing-working memory (Rovaris et al., 2002). Structural GM damage of BMS patients with cognitive impairment has been demonstrated in a study comparing GM MD between cognitively impaired BMS patients and SP MS patients. MD of the GM showed no difference between BMS patients with cognitive deficit and low physical disability and patients with SP disease course (Rovaris et al., 2008). 


\subsection{Mentalization}

\subsubsection{Focal atrophy of the neocortex impairs mentalization ability of multiple sclerosis patients}

Living with MS frequently associates with disturbed social life including insufficiency in partnerships and family roles, as well as employment status (Halper, 2007). Intact social cognition is fundamental for successful social functioning (Penn et al., 1997). Recently, few studies investigating social cognitive abilities of MS patients have reported deficits in emotion recognition and complex perspective taking abilities (Banati et al., 2010; Henry et al., 2009; Jehna et al., 2010; Krause et al., 2009; Ouellet et al., 2010).

To investigate the impact of cortical pathology on social cognitive dysfunction, we performed an MRI study in a cohort of 49 MS patients. We measured the cortical thickness throughout the whole brain, and analyzed its association with mentalization test performance to indentify cortical areas involved in neural integration processes of mentalization in MS. Performances on neuropsychological tests were corrected for confounding effects of anxiety and depression. Compared to healthy subjects, MS patients performed significantly poorer in verbal (Irony test) and visual (Eyes test) mentalization tasks. These results are in line with previous reports (Banati et al., 2010; Henry et al., 2009; Jehna et al., 2010; Krause et al., 2009; Ouellet et al., 2010).

Cortical thickness was assessed to quantify cortical atrophy as thinning of the cortex. FreeSurfer software, version 4.5 (http://surfer.nmr.mgh.harvard.edu) was applied on 3 Tesla 3D Magnetization Prepared Rapid Gradient Echo (MPRAGE) images for automated reconstruction and measure of cortical thickness characterized by preciosity within a submillimeter range (Dale et al., 1999; Fischl et al., 1999). After correcting for confounding effects of age on cortical thickness, and effects of depression and anxiety on mentalization significant correlations were found between the Eyes test performance and cortical thickness of several focal areas. The Eyes test is an advanced and sensitive tool to detect subtle dysfunctions in facial expression recognition (Baron-Cohen et al., 2001). In the Eyes test, participants are required to judge mental states from photographs portraying only the eye region of the face. The identified frontotemporal cortical areas showing significant correlation with the Eyes test were also reported in previous studies documenting a widely distributed brain network engaged in neural processes of facial emotion recognition (Adolphs et al., 2002; Frith \& Frith, 2006; Sabatinelli et al., 2011). Simulation theory describes a presumed physiologic mechanism participating in the understanding of observed motor actions, motor learning, and mental state decoding as well as empathizing in socialperceptual processes (Bastiaansen et al., 2009; Williams, 2008). Firing of mirror neurons in the brain of the observer simulates the neuronal activation pattern required to execute the observed movement, and thereby enables the understanding of the underlying action goal (Bastiaansen et al., 2009; Williams, 2008). Mirror neurons were identified in prefrontal and premotor areas, and in the parietal and temporal lobe (Bastiaansen et al., 2009; Williams, 2008). In addition, recent studies found evidence on simulation of observed sensations and emotions through mirror neuron activation in the primary somatosensory cortex, anterior insula, and amygdala contributing to empathic understanding (Bastiaansen et al., 2009; Williams, 2008). Previous fMRI studies demonstrated that viewing emotional facial expressions associates with premotor cortex activation presuming a mirror neuron mechanism (Bastiaansen et al., 2009). Inner simulation of the observed facial muscle group contractions is linked to a matching affective state based on personal experiences. 
In conclusion, our study suggests that GM pathology in cortical regions constituting neural networks processing high-level mental information significantly impact the mentalization ability in MS.

\subsubsection{Functional MRI study of mentalization in multiple sclerosis}

Neuroanatomical correlates of emotional face expression recognition deficit in MS were also investigated in an fMRI study. This approach identified decreased insular and ventrolateral prefrontal cortex BOLD activation in MS patients with impaired recognition performance compared to the unimpaired group (Krause et al., 2009).

\section{Conclusion}

Cognitive impairment in MS occurs frequently as mild deficit in different neuropsychological domains. Clinical impact of cognitive dysfunction emerges in every day life situations, employment status, and social environment compromising quality of life of the patients. Diagnostic evaluation of neuropsychological performance in MS has also been proved to be significant from prognostic point of view, as presence of cognitive impairment may predict worse disease course. Recently, involvement of the GM in pathological processes underlying MS has been convergent supported by histopathological and MRI studies. The impact of GM damage on clinical course of the disease and its relationship with WM lesions is under intensive research, in which application of different MRI methods plays a highly important role. Application of conventional structural MRI, and nonconventional MRI techniques has been enabled to quantify GM pathology as atrophy, CL load, and degree of microscopic tissue integration disturbances. Cognitive impairment has been demonstrated to correlate all with these different aspects of GM damage highlighting the relevance of MRI evaluation of the GM in MS. Future studies are needed to further refine the MRI-cognition relationship to improve therapeutic decisions and prognosis of MS, and develop potential treatment strategies targeting neuroprotection.

\section{Acknowledgement}

Dr Mike is a recipient of the 2008 McDonald Fellowship from the Multiple Sclerosis International Federation. The work was supported by the Hungarian National Research Fund (OTKA K77892), and the Hungarian Neuroimaging Foundation.

\section{References}

Achiron, A., \& Barak, Y. (2003). Cognitive impairment in probable multiple sclerosis. J Neurol Neurosurg Psychiatry, Vol.74, No.4, (April 2003), pp. 443-446, ISSN: 0022-3050

Adolphs, R., Baron-Cohen, S., \& Tranel, D. (2002). Impaired recognition of social emotions following amygdala damage. J Cogn Neurosci, Vol.14, No.8, (November 2002) pp. 1264-1274, ISSN: 0898-929X

Albert, M., Antel, J., Bruck, W., \& Stadelmann, C. (2007). Extensive cortical remyelination in patients with chronic multiple sclerosis. Brain Pathol, Vol.17, No.2, (April 2007) 129138, ISSN: 1015-6305

Amato, M. P., Ponziani, G., Pracucci, G., Bracco, L., Siracusa, G., \& Amaducci, L. (1995). Cognitive impairment in early-onset multiple sclerosis. Pattern, predictors, and 
impact on everyday life in a 4-year follow-up. Arch Neurol, Vol.52, No.2, (February 1995) pp. 168-172, ISSN: 0003-9942

Amato, M. P., Bartolozzi, M. L., Zipoli, V., Portaccio, E., Mortilla, M., Guidi, L., et al. (2004). Neocortical volume decrease in relapsing-remitting MS patients with mild cognitive impairment. Neurology, Vol.63, No.1, (July 2004) pp. 89-93, ISSN: 00283878

Amato, M. P., Portaccio, E., Goretti, B., Zipoli, V., Battaglini, M., Bartolozzi, M. L., et al. (2007). Association of neocortical volume changes with cognitive deterioration in relapsing-remitting multiple sclerosis. Arch Neurol, 64(8), 1157-1161, ISSN: 00039942

Amato, M. P., Portaccio, E., Stromillo, M. L., Goretti, B., Zipoli, V., Siracusa, G., et al. (2008). Cognitive assessment and quantitative magnetic resonance metrics can help to identify benign multiple sclerosis. Neurology, Vol.71, No.9, (August 2007) pp. 632638, ISSN: 0028-3878

Arnett, P. A., Rao, S. M., Bernardin, L., Grafman, J., Yetkin, F. Z., \& Lobeck, L. (1994). Relationship between frontal lobe lesions and Wisconsin Card Sorting Test performance in patients with multiple sclerosis. Neurology,Vol.44, No.3 Pt 1, (March 1994) pp. 420-425 ISSN: 0028-3878

Arnett, P. A., Barwick, F. H., \& Beeney, J. E. (2008). Depression in multiple sclerosis: review and theoretical proposal. J Int Neuropsychol Soc, Vol.14, No.5, (September 2008), pp. 691-724, ISSN: 1355-6177

Bagnato, F., Butman, J. A., Gupta, S., Calabrese, M., Pezawas, L., Ohayon, J. M., et al. (2006). In vivo detection of cortical plaques by $M R$ imaging in patients with multiple sclerosis. AJNR Am J Neuroradiol, Vol.27, No.10, (Vovemner-December 2006), pp. 2161-2167, ISSN: 0195-6108

Bagnato, F., Salman, Z., Kane, R., Auh, S., Cantor, F. K., Ehrmantraut, M., et al. (2010). T1 cortical hypointensities and their association with cognitive disability in multiple sclerosis. Mult Scler, Vol.16, No.10, (October 2010), pp. 1203-1212, ISSN: 1352-4585

Banati, M., Sandor, J., Mike, A., Illes, E., Bors, L., Feldmann, A., et al. (2010). Social cognition and Theory of Mind in patients with relapsing-remitting multiple sclerosis. Eur J Neurol, Vol.17, No.3, (March 2010), pp. 426-433, ISSN: 1468-1331

Baron-Cohen, S., Wheelwright, S., Hill, J., Raste, Y., \& Plumb, I. (2001). The "Reading the Mind in the Eyes" Test revised version: a study with normal adults, and adults with Asperger syndrome or high-functioning autism. J Child Psychol Psychiatry, Vol.42, No.2, (February 2001) pp. 241-251, ISSN: 0021-9630

Bastiaansen, J. A., Thioux, M., \& Keysers, C. (2009). Evidence for mirror systems in emotions. Philos Trans R Soc Lond B Biol Sci, Vol.364, No.1528, (August 2009) pp. 2391-2404, ISSN: 00804622

Beatty, W. W., Goodkin, D. E., Hertsgaard, D., \& Monson, N. (1990). Clinical and demographic predictors of cognitive performance in multiple sclerosis. Do diagnostic type, disease duration, and disability matter? Arch Neurol, Vol. 47, No.3, (March 1990), pp.305-308, ISSN: 0003-9942

Benedict, R. H., Carone, D. A., \& Bakshi, R. (2004a). Correlating brain atrophy with cognitive dysfunction, mood disturbances, and personality disorder in multiple sclerosis. $J$ Neuroimaging, Vol.14, No.3 Suppl, (July 2004), pp. 36S-45S, ISSN: 1051-2284 
Benedict, R. H., Weinstock-Guttman, B., Fishman, I., Sharma, J., Tjoa, C. W., \& Bakshi, R. (2004b). Prediction of neuropsychological impairment in multiple sclerosis: comparison of conventional magnetic resonance imaging measures of atrophy and lesion burden. Arch Neurol, Vol.61, No.2, (February 2004), pp. 226-230, ISSN: 00039942

Benedict, R. H., Bruce, J. M., Dwyer, M. G., Abdelrahman, N., Hussein, S., WeinstockGuttman, B., et al. (2006a). Neocortical atrophy, third ventricular width, and cognitive dysfunction in multiple sclerosis. Arch Neurol, Vol.6, No.9, (September 2006), pp. 1301-1306, ISSN: 0003-9942

Benedict, R. H., Cookfair, D., Gavett, R., Gunther, M., Munschauer, F., Garg, N., et al. (2006b). Validity of the minimal assessment of cognitive function in multiple sclerosis (MACFIMS). J Int Neuropsychol Soc, Vol.12, No.4, (July 2006), pp. 549-558, ISSN: 1355-6177

Benedict, R. H., Ramasamy, D., Munschauer, F., Weinstock-Guttman, B., \& Zivadinov, R. (2009). Memory impairment in multiple sclerosis: correlation with deep grey matter and mesial temporal atrophy. J Neurol Neurosurg Psychiatry, Vol.80, No.2, (February 2009), pp. 201-206, ISSN: 0022-3050

Bermel, R. A., Bakshi, R., Tjoa, C., Puli, S. R., \& Jacobs, L. (2002). Bicaudate ratio as a magnetic resonance imaging marker of brain atrophy in multiple sclerosis. Arch Neurol, Vol.59, No.2 (February 2002), pp. 275-280, ISSN: 0003-9942

Bo, L., Vedeler, C. A., Nyland, H. I., Trapp, B. D., \& Mork, S. J. (2003). Subpial demyelination in the cerebral cortex of multiple sclerosis patients. J Neuropathol Exp Neurol, Vol.62, No.7, (July 2003), pp. 723-732, ISSN: 0022-3069

Brass, S. D., Benedict, R. H., Weinstock-Guttman, B., Munschauer, F., \& Bakshi, R. (2006). Cognitive impairment is associated with subcortical magnetic resonance imaging grey matter T2 hypointensity in multiple sclerosis. Mult Scler, Vol.12, No.4, (August 2006), pp. 437-444, ISSN: 1352-4585

Brownell, B., \& Hughes, J. T. (1962). The distribution of plaques in the cerebrum in multiple sclerosis. J Neurol Neurosurg Psychiatry, Vol.25, (November 1962), pp. 315-320, ISSN: 0022-3050

Calabrese, M., De Stefano, N., Atzori, M., Bernardi, V., Mattisi, I., Barachino, L., et al. (2007). Detection of cortical inflammatory lesions by double inversion recovery magnetic resonance imaging in patients with multiple sclerosis. Arch Neurol, Vol.64, No.10, (October 2007), pp. 1416-1422, ISSN: 0003-9942

Calabrese, M., Filippi, M., Rovaris, M., Mattisi, I., Bernardi, V., Atzori, M., et al. (2008). Morphology and evolution of cortical lesions in multiple sclerosis. A longitudinal MRI study. Neuroimage, Vol.42, No.4, (October 2008), pp. 1324-1328, ISSN: 10538119

Calabrese, M., Agosta, F., Rinaldi, F., Mattisi, I., Grossi, P., Favaretto, A., et al. (2009). Cortical lesions and atrophy associated with cognitive impairment in relapsingremitting multiple sclerosis. Arch Neurol,Vol. 66, No.9, (September 2009), pp. 11441150, ISSN: 0003-9942

Calabrese, M., Rinaldi, F., Mattisi, I., Grossi, P., Favaretto, A., Atzori, M., et al. (2010). Widespread cortical thinning characterizes patients with MS with mild cognitive impairment. Neurology, Vol.74, No.4, (January 2010), pp. 321-328, ISSN: 0028-3878 
Callanan, M. M., Logsdail, S. J., Ron, M. A., \& Warrington, E. K. (1989). Cognitive impairment in patients with clinically isolated lesions of the type seen in multiple sclerosis. A psychometric and MRI study. Brain, Vol.112, No.Pt 2, (April 1989), pp. 361-374, ISSN: 0006-8950

Chard, D. T., Griffin, C. M., Rashid, W., Davies, G. R., Altmann, D. R., Kapoor, R., et al. (2004). Progressive grey matter atrophy in clinically early relapsing-remitting multiple sclerosis. Mult Scler, Vol.10, No.4, (August 2004), pp. 387-391, ISSN: 13524585

Christodoulou, C., Krupp, L. B., Liang, Z., Huang, W., Melville, P., Roque, C., et al. (2003). Cognitive performance and MR markers of cerebral injury in cognitively impaired MS patients. Neurology,Vol.60, No.11, (June 2003), pp. 1793-1798, ISSN: 0028-3878

Dale, A. M., Fischl, B., \& Sereno, M. I. (1999). Cortical surface-based analysis. I. Segmentation and surface reconstruction. Neuroimage, Vol.9, No.2, (February 1999), pp. 179-194, ISSN: 1053-8119

De Stefano, N., Matthews, P. M., Filippi, M., Agosta, F., De Luca, M., Bartolozzi, M. L., et al. (2003). Evidence of early cortical atrophy in MS: relevance to white matter changes and disability. Neurology, Vol.60, No.7, April 2003), pp. 1157-1162, ISSN: 0028-3878

Deloire, M. S., Salort, E., Bonnet, M., Arimone, Y., Boudineau, M., Amieva, H., et al. (2005). Cognitive impairment as marker of diffuse brain abnormalities in early relapsing remitting multiple sclerosis. J Neurol Neurosurg Psychiatry, Vol.76, No.4, (April 2005), pp. 519-526, ISSN: 0022-3050

Deluca, J., Christodoulou, C., Diamond, B. J., Rosenstein, E. D., Kramer, N., \& Natelson, B. H. (2004). Working memory deficits in chronic fatigue syndrome: differentiating between speed and accuracy of information processing. I Int Neuropsychol Soc, Vol.10, No.1, (January 2004), pp. 101-109, ISSN: 1355-6177

Evangelou, N., Esiri, M. M., Smith, S., Palace, J., \& Matthews, P. M. (2000). Quantitative pathological evidence for axonal loss in normal appearing white matter in multiple sclerosis. Ann Neurol, Vol.47, No.3, (March 2000), pp. 391-395, ISSN: 0364-5134

Feinstein, A., Youl, B., \& Ron, M. (1992). Acute optic neuritis. A cognitive and magnetic resonance imaging study. Brain,Vol.115, No.Pt 5, (October 1992) pp. 1403-1415, ISSN: 0006-8950

Feuillet, L., Reuter, F., Audoin, B., Malikova, I., Barrau, K., Cherif, A. A., et al. (2007). Early cognitive impairment in patients with clinically isolated syndrome suggestive of multiple sclerosis. Mult Scler, Vol.13, no.1, (January 2007), pp. 124-127, ISSN: 13524585

Filippi, M., \& Rocca, M. A. (2010). Novel MRI approaches to assess patients with multiple sclerosis. Curr Opin Neurol, Vol.23, No.3, (June 2010), pp. 212-217, ISSN: 1350-7540

Filippi, M., Rocca, M. A., Benedict, R. H., DeLuca, J., Geurts, J. J., Rombouts, S. A., et al. (2010). The contribution of MRI in assessing cognitive impairment in multiple sclerosis. Neurology, Vol.75, No.23, (December 2010), pp. 2121-2128, ISSN: 0028-3878

Fischl, B., Sereno, M. I., \& Dale, A. M. (1999). Cortical surface-based analysis. II: Inflation, flattening, and a surface-based coordinate system. Neuroimage, Vol.9, No.2, (February 1999), pp. 195-207, ISSN: 1053-8119

Fisniku, L. K., Chard, D. T., Jackson, J. S., Anderson, V. M., Altmann, D. R., Miszkiel, K. A., et al. (2008). Gray matter atrophy is related to long-term disability in multiple sclerosis. Ann Neurol, Vol.64, No.3, (September 2008), pp. 247-254, ISSN: 0364-5134 
Frith, C. D., \& Frith, U. (2006). The neural basis of mentalizing. Neuron, Vol.50, No.4, (May 2006), pp. 531-534, ISSN: 0896-6273

Geurts, J. J., Bo, L., Pouwels, P. J., Castelijns, J. A., Polman, C. H., \& Barkhof, F. (2005b). Cortical lesions in multiple sclerosis: combined postmortem MR imaging and histopathology. AJNR Am J Neuroradiol, Vol.26, No.3, (March 2005), pp. 572-577, ISSN: 0195-6108

Geurts, J. J., Pouwels, P. J., Uitdehaag, B. M., Polman, C. H., Barkhof, F., \& Castelijns, J. A. (2005a). Intracortical lesions in multiple sclerosis: improved detection with 3D double inversion-recovery MR imaging. Radiology, Vol.236, No.1, (July 2005), pp. 254-260, ISSN: 0033-8419

Geurts, J. J., Stys, P. K., Minagar, A., Amor, S., \& Zivadinov, R. (2009). Gray matter pathology in (chronic) MS: modern views on an early observation. J Neurol Sci, Vol.282, No.1-2, (July 2009), pp. 12-20, ISSN: 0022-510X

Glanz, B. I., Holland, C. M., Gauthier, S. A., Amunwa, E. L., Liptak, Z., Houtchens, M. K., et al. (2007). Cognitive dysfunction in patients with clinically isolated syndromes or newly diagnosed multiple sclerosis. Mult Scler, Vol.13, No.8, (September 2007), pp. 1004-1010, ISSN: 1352-4585

Grassiot, B., Desgranges, B., Eustache, F., \& Defer, G. (2009). Quantification and clinical relevance of brain atrophy in multiple sclerosis: a review. J Neurol, Vol.256, No.9, (September 2009), pp. 1397-1412, ISSN: 0340-5354

Halper, J. (2007). The psychosocial effect of multiple sclerosis: the impact of relapses. J Neurol Sci, Vol.256, Suppl No.1, (May 2007), pp. S34-38, ISSN: 0022-510X

Henry, J. D., Phillips, L. H., Beatty, W. W., McDonald, S., Longley, W. A., Joscelyne, A., et al. (2009). Evidence for deficits in facial affect recognition and theory of mind in multiple sclerosis. J Int Neuropsychol Soc, Vol.15, No.2, (March 2009), pp. 277-285, ISSN: 1355-6177

Hohol, M. J., Guttmann, C. R., Orav, J., Mackin, G. A., Kikinis, R., Khoury, S. J., et al. (1997). Serial neuropsychological assessment and magnetic resonance imaging analysis in multiple sclerosis. Arch Neurol, Vol.54, No.8, (August 1997), pp. 1018-1025, ISSN: 0003-9942

Houtchens, M. K., Benedict, R. H., Killiany, R., Sharma, J., Jaisani, Z., Singh, B., et al. (2007). Thalamic atrophy and cognition in multiple sclerosis. Neurology, Vol.69, No.12, (September 2007), pp. 1213-1223, ISSN: 0028-3878

Jehna, M., Neuper, C., Petrovic, K., Wallner-Blazek, M., Schmidt, R., Fuchs, S., et al. (2010). An exploratory study on emotion recognition in patients with a clinically isolated syndrome and multiple sclerosis. Clin Neurol Neurosurg, Vol.112, No.6, (July 2010), pp. 482-484, ISSN: 0303-8467

Kangarlu, A., Bourekas, E. C., Ray-Chaudhury, A., \& Rammohan, K. W. (2007). Cerebral cortical lesions in multiple sclerosis detected by MR imaging at 8 Tesla. AJNR Am J Neuroradiol, Vol.28, No.2, (February 2007), pp. 262-266, ISSN: 0195-6108

Khaleeli, Z., Cercignani, M., Audoin, B., Ciccarelli, O., Miller, D. H., \& Thompson, A. J. (2007). Localized grey matter damage in early primary progressive multiple sclerosis contributes to disability. Neuroimage,Vol. 37, No.1, (August 2007), pp. 253261, ISSN: 1053-8119

Khalil, M., Enzinger, C., Langkammer, C., Petrovic, K., Loitfelder, M., Tscherner, M., et al. (2011). Cognitive impairment in relation to MRI metrics in patients with clinically 
isolated syndrome. Mult Scler, Vol.17, No.2, (February 2011), pp. 173-180, ISSN: 1352-4585

Kidd, D., Barkhof, F., McConnell, R., Algra, P. R., Allen, I. V., \& Revesz, T. (1999). Cortical lesions in multiple sclerosis. Brain, Vol.12, No. Pt 1, (January 1999), pp. 17-26, ISSN: 0006-8950

Kooi, E. J., Geurts, J. J., van Horssen, J., Bo, L., \& van der Valk, P. (2009). Meningeal inflammation is not associated with cortical demyelination in chronic multiple sclerosis. J Neuropathol Exp Neurol, Vol.68, No.9, (September 2009), pp. 1021-1028, ISSN: 0022-3069

Krause, M., Wendt, J., Dressel, A., Berneiser, J., Kessler, C., Hamm, A. O., et al. (2009). Prefrontal function associated with impaired emotion recognition in patients with multiple sclerosis. Behav Brain Res, Vol.205, No.1, (December 2009), pp. 280-285, ISSN: 0166-4328

Kutzelnigg, A., Lucchinetti, C. F., Stadelmann, C., Bruck, W., Rauschka, H., Bergmann, M., et al. (2005). Cortical demyelination and diffuse white matter injury in multiple sclerosis. Brain, Vol.128, Pt11, (November 2005), pp. 2705-2712, ISSN: 0006-8950

Lumsden C. E. (1970) The neuropathology of multiple sclerosis. In Handbook of clinical neurology. Multiple Sclerosis and other demyelinating diseases. Vinken P.J., Bruyn G. W. (Ed.), pp. 217-309, North Holland Publ., Amsterdam, Netherland

Magliozzi, R., Howell, O., Vora, A., Serafini, B., Nicholas, R., Puopolo, M., et al. (2007). Meningeal B-cell follicles in secondary progressive multiple sclerosis associate with early onset of disease and severe cortical pathology. Brain, Vol.130, Pt4, (April 2007), pp. 1089-1104, ISSN: 0006-8950

Mainero, C., Benner, T., Radding, A., van der Kouwe, A., Jensen, R., Rosen, B. R., et al. (2009). In vivo imaging of cortical pathology in multiple sclerosis using ultra-high field MRI. Neurology, Vol.73, No.12, (September 2009), pp. 941-948, ISSN: 0028-3878

Mike, A., Glanz, B. I., Hildenbrand, P., Meier, D., Bolden, K., Liguori, M., et al. (2011). Identification and Clinical Impact of Multiple Sclerosis Cortical Lesions as Assessed by Routine 3T MR Imaging. AJNR Am J Neuroradiol, Vol.32, No.3, (March 2011), pp. 515-521, ISSN: 0195-6108

Miller, D. H., Barkhof, F., Frank, J. A., Parker, G. J., \& Thompson, A. J. (2002). Measurement of atrophy in multiple sclerosis: pathological basis, methodological aspects and clinical relevance. Brain, Vol.125, Pt 8, (August 2002), pp. 1676-1695, ISSN: 00068950

Morgen, K., Sammer, G., Courtney, S. M., Wolters, T., Melchior, H., Blecker, C. R., et al. (2006). Evidence for a direct association between cortical atrophy and cognitive impairment in relapsing-remitting MS. Neuroimage, Vol.30, No.3, (April 2006), pp. 891-898, ISSN: 1053-8119

Neema, M., Stankiewicz, J., Arora, A., Dandamudi, V. S., Batt, C. E., Guss, Z. D., et al. (2007). T1- and T2-based MRI measures of diffuse gray matter and white matter damage in patients with multiple sclerosis. J Neuroimaging,Vol.17, Suppl.1, (April 2007), pp. 16S-21S, ISSN: 1051-2284

Nelson, F., Poonawalla, A. H., Hou, P., Huang, F., Wolinsky, J. S., \& Narayana, P. A. (2007). Improved identification of intracortical lesions in multiple sclerosis with phasesensitive inversion recovery in combination with fast double inversion recovery 
MR imaging. AJNR Am J Neuroradiol, Vol.28, No.9, (October 2007), pp. 1645-1649, ISSN: 0195-6108

Nelson, F., Poonawalla, A., Hou, P., Wolinsky, J. S., \& Narayana, P. A. (2008). 3D MPRAGE improves classification of cortical lesions in multiple sclerosis. Mult Scler, Vol.14, No.9, (November 2008), pp. 1214-1219, ISSN: 1352-4585

Ouellet, J., Scherzer, P. B., Rouleau, I., Metras, P., Bertrand-Gauvin, C., Djerroud, N., et al. (2010). Assessment of social cognition in patients with multiple sclerosis. J Int Neuropsychol Soc, Vol.16, No.2, (March 2010), pp. 287-296, ISSN: 1355-6177

Patti, F., Amato, M. P., Trojano, M., Bastianello, S., Tola, M. R., Goretti, B., et al. (2009). Cognitive impairment and its relation with disease measures in mildly disabled patients with relapsing-remitting multiple sclerosis: baseline results from the Cognitive Impairment in Multiple Sclerosis (COGIMUS) study. Mult Scler, Vol.15, No.7, (July 2009), pp. 779-788, ISSN: 1352-4585

Penn, D. L., Corrigan, P. W., Bentall, R. P., Racenstein, J. M., \& Newman, L. (1997). Social cognition in schizophrenia. Psychol Bull, Vol.121, No.1, (January 1997), pp. 114-132, ISSN: 0033-2909

Penny, S., Khaleeli, Z., Cipolotti, L., Thompson, A., \& Ron, M. (2010). Early imaging predicts later cognitive impairment in primary progressive multiple sclerosis. Neurology, Vol.74, No.7, (February 2010), pp. 545-552, ISSN: 0028-3878

Peterson, J. W., Bo, L., Mork, S., Chang, A., \& Trapp, B. D. (2001). Transected neurites, apoptotic neurons, and reduced inflammation in cortical multiple sclerosis lesions. Ann Neurol, Vol.50, No.3, (September 2001), pp. 389-400, ISSN: 0364-5134

Piras, M. R., Magnano, I., Canu, E. D., Paulus, K. S., Satta, W. M., Soddu, A., et al. (2003). Longitudinal study of cognitive dysfunction in multiple sclerosis: neuropsychological, neuroradiological, and neurophysiological findings. I Neurol Neurosurg Psychiatry, Vol.74, No.7, (Julay 2003), pp. 878-885, ISSN: 0022-3050

Pirko, I., Lucchinetti, C. F., Sriram, S., \& Bakshi, R. (2007). Gray matter involvement in multiple sclerosis. Neurology, Vol.68, No.9, (February 2001), pp. 634-642, ISSN: 00283878

Portaccio, E., Stromillo, M. L., Goretti, B., Zipoli, V., Siracusa, G., Battaglini, M., et al. (2009). Neuropsychological and MRI measures predict short-term evolution in benign multiple sclerosis. Neurology, Vol.73, No.7, (August 2009), pp. 498-503, ISSN: 00283878

Potagas, C., Giogkaraki, E., Koutsis, G., Mandellos, D., Tsirempolou, E., Sfagos, C., et al. (2008). Cognitive impairment in different MS subtypes and clinically isolated syndromes. J Neurol Sci, Vol.267, No.1-2, (April 2008), pp. 100-106, ISSN: 0022-510X

Ranjeva, J. P., Audoin, B., Au Duong, M. V., Ibarrola, D., Confort-Gouny, S., Malikova, I., et al. (2005). Local tissue damage assessed with statistical mapping analysis of brain magnetization transfer ratio: relationship with functional status of patients in the earliest stage of multiple sclerosis. AJNR Am J Neuroradiol, Vol.26, No.1, (January 2005), pp. 119-127, ISSN: 0195-6108

Ranjeva, J. P., Audoin, B., Au Duong, M. V., Confort-Gouny, S., Malikova, I., Viout, P., et al. (2006). Structural and functional surrogates of cognitive impairment at the very early stage of multiple sclerosis. J Neurol Sci, Vol.245, No.1-2, (June 2006), pp. 161167, ISSN: 0022-510X 
Rao, S. M. (1990). The Cognitive Function Study Group of the National Multiple Sclerosis Society. In: A Manual for the Brief Repetable Battery of Neuropsychological Tests in Multiple Sclerosis. Section of Neuropsychology. Medical College of Wisconsin, Milwaukee, WI

Rao, S. M., Leo, G. J., Haughton, V. M., St Aubin-Faubert, P., \& Bernardin, L. (1989). Correlation of magnetic resonance imaging with neuropsychological testing in multiple sclerosis. Neurology, Vol.39, No.2, Pt1, (February 1989), pp. 161-166, ISSN: 0028-3878

Rao, S. M., Leo, G. J., Bernardin, L., \& Unverzagt, F. (1991a). Cognitive dysfunction in multiple sclerosis. I. Frequency, patterns, and prediction. Neurology, Vol.41, No.5, (May 1991), pp. 685-691, ISSN: 0028-3878

Rao, S. M., Leo, G. J., Ellington, L., Nauertz, T., Bernardin, L., \& Unverzagt, F. (1991b). Cognitive dysfunction in multiple sclerosis. II. Impact on employment and social functioning. Neurology, Vol.41, No.5, (May 1991), pp. 692-696, ISSN: 0028-3878

Roosendaal, S. D., Moraal, B., Pouwels, P. J., Vrenken, H., Castelijns, J. A., Barkhof, F., et al. (2009). Accumulation of cortical lesions in MS: relation with cognitive impairment. Mult Scler, Vol.15, No.6, (June 2009), pp. 708-714, ISSN: 1352-4585

Rovaris, M., \& Filippi, M. (1999). Magnetic resonance techniques to monitor disease evolution and treatment trial outcomes in multiple sclerosis. Curr Opin Neurol, Vol.12, No.3, (June 199), pp. 337-344, ISSN: 1350-7540

Rovaris, M., Filippi, M., Minicucci, L., Iannucci, G., Santuccio, G., Possa, F., et al. (2000). Cortical/subcortical disease burden and cognitive impairment in patients with multiple sclerosis. AJNR Am J Neuroradiol, Vol.21, No.2, (February 2000), pp. 402408, ISSN: 0195-6108

Rovaris, M., Iannucci, G., Falautano, M., Possa, F., Martinelli, V., Comi, G., et al. (2002). Cognitive dysfunction in patients with mildly disabling relapsing-remitting multiple sclerosis: an exploratory study with diffusion tensor MR imaging. J Neurol Sci, Vol.195, No.2, (March 2002), pp. 103-109, ISSN: 0022-510X

Rovaris, M., Comi, G., \& Filippi, M. (2006). MRI markers of destructive pathology in multiple sclerosis-related cognitive dysfunction. J Neurol Sci, Vol.245, No.1-2, (June 2006), pp. 111-116, ISSN: 0022-510X

Rovaris, M., Riccitelli, G., Judica, E., Possa, F., Caputo, D., Ghezzi, A., et al. (2008). Cognitive impairment and structural brain damage in benign multiple sclerosis. Neurology, Vol.71, No.19, (November 2008), pp. 1521-1526, ISSN: 0028-3878

Rovaris, M., Agosta, F., Pagani, E., \& Filippi, M. (2009). Diffusion tensor MR imaging. Neuroimaging Clin N Am, Vol.19, No.1, (February 2009), pp. 37-43, ISSN: 1052-5149

Sabatinelli, D., Fortune, E. E., Li, Q., Siddiqui, A., Krafft, C., Oliver, W. T., et al. (2011). Emotional perception: meta-analyses of face and natural scene processing. Neuroimage, Vol.54, No.3, (February 2011), pp. 2524-2533, ISSN: 1053-8119

Sanchez, M. P., Nieto, A., Barroso, J., Martin, V., \& Hernandez, M. A. (2008). Brain atrophy as a marker of cognitive impairment in mildly disabling relapsing-remitting multiple sclerosis. Eur J Neurol, Vol.15, No.10, (October 2008), pp. 1091-1099, ISSN: 1468-1331

Schmierer, K., Tozer, D. J., Scaravilli, F., Altmann, D. R., Barker, G. J., Tofts, P. S., et al. (2007). Quantitative magnetization transfer imaging in postmortem multiple 
sclerosis brain. J Magn Reson Imaging, Vol.26, No.1, (July 2007), pp. 41-51, ISSN: 1053-1807

Sepulcre, J., Goni, J., Masdeu, J. C., Bejarano, B., Velez de Mendizabal, N., Toledo, J. B., et al. (2009). Contribution of white matter lesions to gray matter atrophy in multiple sclerosis: evidence from voxel-based analysis of T1 lesions in the visual pathway. Arch Neurol, Vol.66, No.2, (February 2009), pp. 173-179, ISSN: 0003-9942

Shamay-Tsoory, S. G., Tomer, R., \& Aharon-Peretz, J. (2005). The neuroanatomical basis of understanding sarcasm and its relationship to social cognition. Neuropsychology, Vol.19, No.3, (May 2005), pp. 288-300, ISSN: 0894-4105

Simon, B., Schmidt, S., Lukas, C., Gieseke, J., Traber, F., Knol, D. L., et al. (2010). Improved in vivo detection of cortical lesions in multiple sclerosis using double inversion recovery MR imaging at 3 Tesla. Eur Radiol, Vol.20, No.7, (July 2010), pp. 1675-1683, ISSN: 0938-7994

Sperling, R. A., Guttmann, C. R., Hohol, M. J., Warfield, S. K., Jakab, M., Parente, M., et al. (2001). Regional magnetic resonance imaging lesion burden and cognitive function in multiple sclerosis: a longitudinal study. Arch Neurol,Vol. 58, No.1, (January 2001), pp. 115-121, ISSN: 0003-9942

Stone, V. E., Baron-Cohen, S., \& Knight, R. T. (1998). Frontal lobe contributions to theory of mind. J Cogn Neurosci, Vol.10, No.5, (September 1998), pp. 640-656, ISSN: 0898-929X

Swirsky-Sacchetti, T., Mitchell, D. R., Seward, J., Gonzales, C., Lublin, F., Knobler, R., et al. (1992). Neuropsychological and structural brain lesions in multiple sclerosis: a regional analysis. Neurology, Vol.42, No.7, (July 1992), pp. 1291-1295, ISSN: 00283878

Tekok-Kilic, A., Benedict, R. H., Weinstock-Guttman, B., Dwyer, M. G., Carone, D., Srinivasaraghavan, B., et al. (2007). Independent contributions of cortical gray matter atrophy and ventricle enlargement for predicting neuropsychological impairment in multiple sclerosis. Neuroimage, Vol.36, No.4, (July 2007), pp. 12941300, ISSN: 1053-8119

Trapp, B. D., Peterson, J., Ransohoff, R. M., Rudick, R., Mork, S., \& Bo, L. (1998). Axonal transection in the lesions of multiple sclerosis. N Engl J Med,Vol.338, No.5, (January 1998), pp. 278-285, ISSN: 0028-4793

Wegner, C., Esiri, M. M., Chance, S. A., Palace, J., \& Matthews, P. M. (2006). Neocortical neuronal, synaptic, and glial loss in multiple sclerosis. Neurology, Vol.67, No.6, (September 2006), pp. 960-967, ISSN: 0028-3878

Williams, J. H. (2008). Self-other relations in social development and autism: multiple roles for mirror neurons and other brain bases. Autism Res, Vol.1, No.2, (April 2008), pp. 73-90, ISSN: 1939-3806

Wolff, S. D., \& Balaban, R. S. (1994). Magnetization transfer imaging: practical aspects and clinical applications. Radiology, Vol.192, No.3, (September 1994), pp. 593-599, ISSN: 0896-6273

Zipoli, V., Goretti, B., Hakiki, B., Siracusa, G., Sorbi, S., Portaccio, E., et al. (2010). Cognitive impairment predicts conversion to multiple sclerosis in clinically isolated syndromes. Mult Scler, Vol.16, No.1, (January 2010), pp. 62-67, ISSN: 1352-4585 
Zivadinov, R., Sepcic, J., Nasuelli, D., De Masi, R., Bragadin, L. M., Tommasi, M. A., et al. (2001). A longitudinal study of brain atrophy and cognitive disturbances in the early phase of relapsing-remitting multiple sclerosis. J Neurol Neurosurg Psychiatry, Vol.70, No.6, (June 2001), pp. 773-780, ISSN: 0022-3050 


\title{
Pseudotumor Cerebri (Idiopathic Intracranial Hypertension) an Update
}

\author{
Eldar Rosenfeld and Anat Kesler \\ Neuro-ophthalmology Unit, Department of Ophthalmology, Tel-Aviv Medical Center, \\ Sackler School of Medicine, Tel Aviv University, Tel Aviv,
}

Israel

\section{Introduction}

\subsection{History}

Historically, several terms have been used to depict pseudotumor cerebri (PTC). In the late 1890 's, Quincke $(1893,1897)$ was the first to describe and name this syndrome - "meningitis serosa" - patients suffering from headache, impaired visual acuity and papilledema. He related the symptoms to a state of elevated intracranial pressure and presumed it was caused by increased secretion of CSF by the autonomic nervous system. In 1904, Nonne (1904) termed this syndrome "pseudotumor cerebri" as the symptoms resembled a suspected intracranial mass. Foley (1955) renamed the condition "benign intracranial hypertension". However, in the late 1980's, Corbett et al (1982) altered the name to idiopathic intracranial hypertension, since the syndrome was not benign as once thought. In some cases, up to $25 \%$ of patients may lose their vision if appropriate treatment measures are not taken. At present, idiopathic intracranial hypertension is the accepted designation.

\subsection{Demographics and epidemiology}

Pseudotumor cerebri (PTC), also known as idiopathic intracranial hypertension (IIH) is a disorder of unknown etiology, predominantly affecting obese women of childbearing age (Ahlskog \& O'Neill, 1982). In the general population, the annual incidence of PTC is estimated between 1-2 per 100,000 (Friedman \& Jacobson, 2002, 2009). The incidence has been reported to have risen to 3.5 per 100,000 in women aged 20 - 44 years and may reach as high as 19 cases per 100,000 in women who are $>20 \%$ over the ideal body weight (Durcan, 1988; Kesler et al 2001; Radhakrishnan et al., 1993, 1994).

PTC is uncommon in men, with female to male ratios reported approximately $4.3: 1$ to $8: 1$ (Binder et al, 2004; Durcan, 1988). The association between obesity and PTC is well established, $>90 \%$ of women and $>60 \%$ of men who suffer from this disorder are obese (Friedman et al., 2002; Radhakrishnan et al, 1994). The syndrome is relatively rare in the pediatric population. In the pre-pubertal population, it seems that obesity is not a risk factor. There is an equal distribution between boys and girls with an estimated incidence of approximately 1 case per 100,000.

\subsection{Presenting signs and symptoms}

Headaches, the most common presenting symptom in all age groups, occur in $>90 \%$ of patients (Binder et al., 2004; Lessell, 1992). A PTC-associated headache has no specific 
characteristics, but is usually more severe and different than previously described headaches. In PTC, the headache is most commonly bifrontal or generalized, usually occurring daily, but may also occur intermittently, worsening in the morning. When cerebral venous pressure is increased by the Valsalva maneuver, the headaches are often exacerbated and accompanied by neck pain.

Transient visual obscurations (TVO), the second most common symptom is more frequently reported in adult patients than in pediatric PTC patients, $72 \%$ vs. $2-53 \%$ respectively (Lessell, 1992). TVO may be unilateral or bilateral, usually lasting less than a minute and often precipitated by a change in posture. TVO indicate the presence of optic disc edema resulting in transient ischemia to the optic nerve head. Over half $(60 \%)$ of PTC patients may experience pulsatile tinnitus as the initial complaint. Pulsatile tinnitus is thought to result from a turbulence created by higher to lower venous pressure around the jugular bulb (Binder et al., 2004).

In patients with PTC, focal neurological deficits are extremely uncommon. An alternative diagnosis should be considered when these deficits occur. Nevertheless, isolated 6th cranial nerve paresis, thought to be attributed to nerve traction from increased intracranial pressure has been observed in approximately $20 \%$ of adult cases (Binder et al., 2004). However, in children, the incidence was found to be as high as 50\% (Cinciripini et al 1999; Lessell, 1992; Rangwala \& Liu, 2007). Paresis of the $3^{\text {rd }}, 4^{\text {th }}, 7^{\text {th }}$ or $12^{\text {th }}$ nerve is extremely rare, occurring mostly in the pediatric population. Young children with PTC may present with irritability rather than headaches (Lessell, 1992), and may consequently develop signs of a posterior

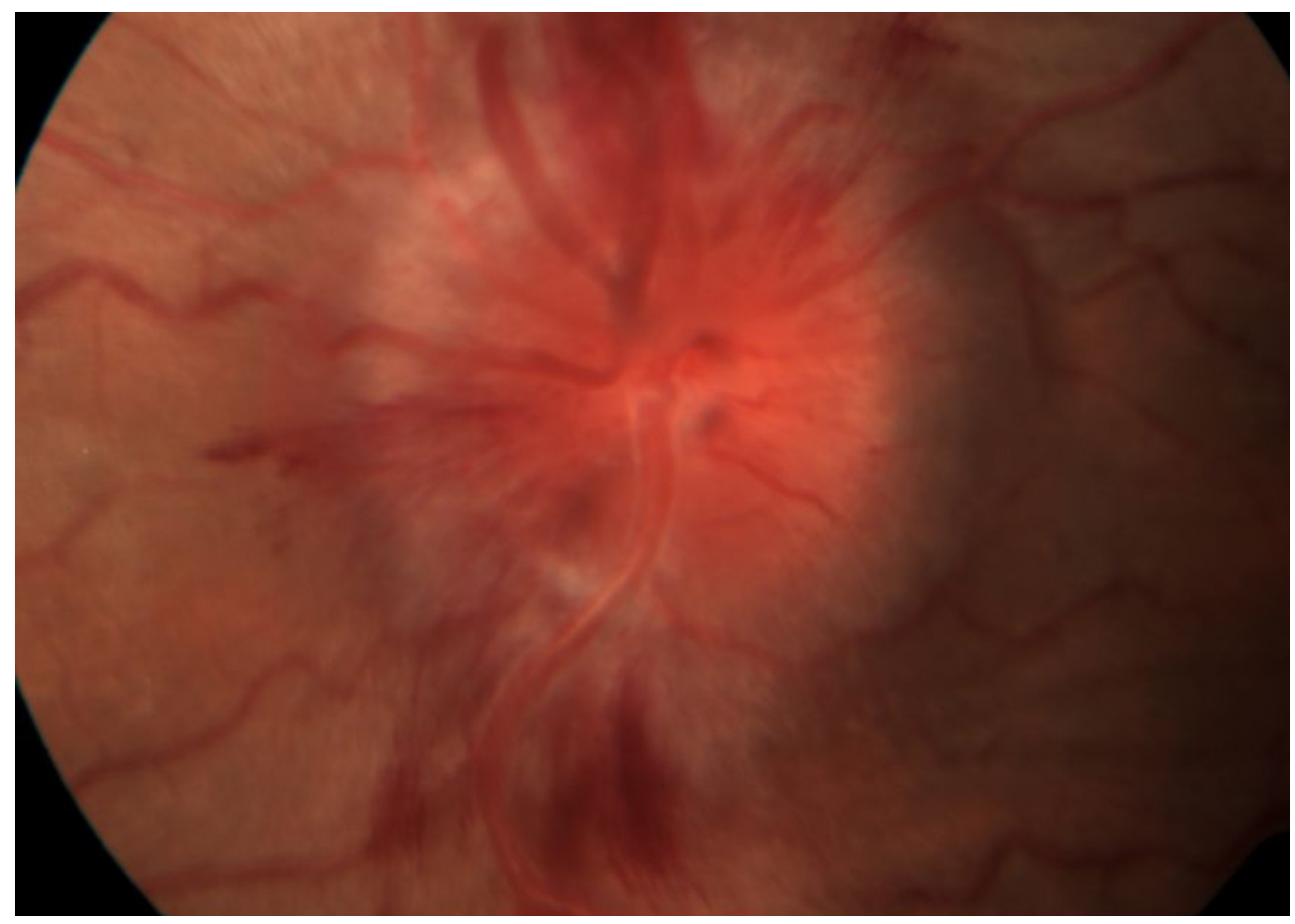

Fig. 1. Papilledema in a 26 year old PTC female patient. 
fossa lesion, including ataxia, facial palsy, nuchal rigidity, malaise, torticollis (Lessell, 1992; Rangwala \& Liu, 2007).

Papilledema is the diagnostic hallmark of PTC and is present in almost all patients (Mathew, 1996) (Figure 1). To note, although, unilateral cases may be encountered in approximately $10 \%$ of cases (Maxner et al., 1997; Wall \& White, 1998) and a small number of patients may not have papilledema at all (Mathew, 1996; Wang et al., 1998; Winner \& Bello, 1996), most cases occur either in very young infants with unfused sutures or in patients with anatomical variants of the endings of the optic nerve sheath (Hayreh, 1977; Killer et al., 1999).

Post-papilledema optic atrophy can occurs in untreated or inadequately treated patients after variable periods of time, usually over several months; in rare cases of fulminant PTC it can appear within weeks of the onset of symptoms. Some patients have persistent chronic papilledema without obvious visual deterioration. Visual field testing is the most sensitive method for detecting visual dysfunction. The most common abnormalities are an enlarged blind spot, generalized constriction and inferior nasal field loss.

\subsection{Diagnosis}

Diagnosis criteria for PTC were reviewed (Binder et al., 2004) (Table 1) and include signs and symptoms attributable to increased ICP, elevated CSF pressure with normal CSF content and normal neuroimaging studies. Other etiologies of intracranial hypertension were excluded. When these criteria are present, neuroimaging is employed to rule out space occupying lesions and sinus vein thrombosis. Lumbar puncture is subsequently performed to measure CSF opening pressure. Generally, an opening pressure of $>250 \mathrm{~mm}$ of water measured in a patient lying in the decubitus position, with outstretched legs and as relaxed as possible, is indicative of increased pressure. A pressure value between 201 and $249 \mathrm{~mm}$ of water is inconclusive (Corbett \& Mehta, 1983); pressure equal to or $<200 \mathrm{~mm}$ of water is considered normal. Often, due to CSF fluctuations, low or inconclusive measurements must be re-evaluated, especially if the clinical picture is indicative of increased ICP.

According to Tibussek et al (2010), a diagnosis of "probable PTC" would be indicated in patients, especially children, with clinical manifestations highly suggestive of PTC, but with a normal CSF opening pressure, presumably due to diurnal fluctuations. Rarely, in these circumstances, is a 24 hour intracranial CSF pressure monitoring, or transducer monitoring for 6 to 24 hours needed to confirm the diagnosis (Spence et al., 1980).

\section{Neuroradiological evaluation}

A detailed section in this chapter is devoted to diverse imaging modalities in patients with PTC. Imaging plays an important role in excluding intracranial tumors and structural or vascular lesions responsible for intracranial hypertension. CT, although adequate in ruling out hydrocephalus and most mass lesions, conditions such as sinus vein thrombosis, meningeal infiltration, and isodense tumors are undetected by a non-enhanced CT.

An MRI will detect almost all changes and by incorporating MRI venography will further enhance the ability to detect sinus vein thrombosis disguised as PTC (Crassard \& Bousser, 2004). In rare cases, imaging of the spinal cord is essential as it excludes rare cases of spinal tumors that cause an increase in intracranial pressure. However, this is typically present with high protein levels in the CSF, therefore making it incompatible with the definition of PTC (Corbett \& Mehta, 1983; Friedman \& Jacobson, 2004; Ridsdale \& Moseley, 1978). Traditionally, slit ventricles were thought to be present in PTC, but a quantitative analysis of 
ventricular volume noted no differences between patients with PTC and age-matched control patients (Jacobson et al., 1990).

Signs of increased intracranial pressure that may be found on imaging studies include empty sella $(70 \%)$, flattening of posterior sclera $(80 \%)$, enhancement of the prelaminar optic nerve $(50 \%)$, distention of the perioptic subarachnoid space $(45 \%)$, vertical tortuosity of the orbital optic nerve $(40 \%)$, and intraocular protrusion of the prelaminar optic nerve $(30 \%)$ (Brodsky \& Vaphiades, 1998).

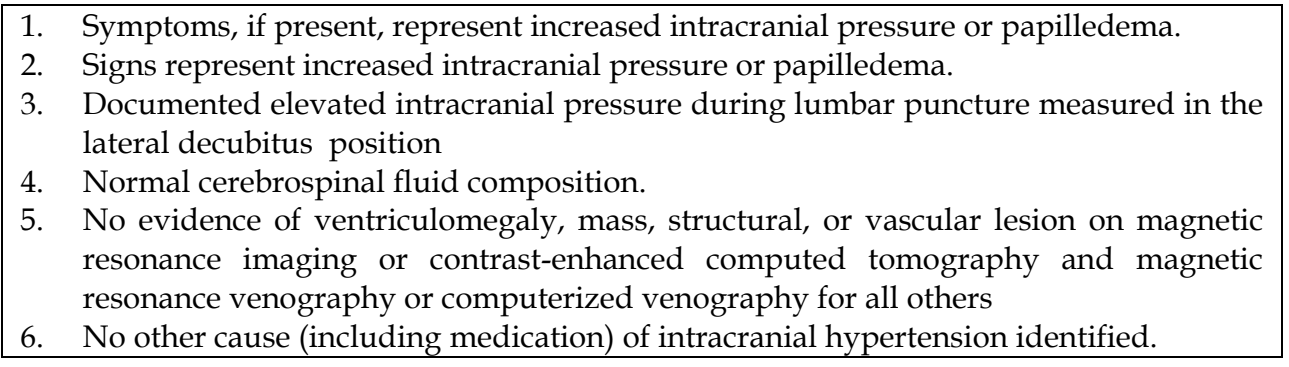

Table 1. Clinical criteria for diagnosing idiopathic intracranial hypertension (adapted from Binder et al 2004)

\section{Pathogenesis}

At present, the pathogenesis of the syndrome is still unknown, however, some explanation must account for elevated intracranial pressure with normal neuroimaging, CSF constituents and neurologic examination (Corbett, 2008). Intracranial pressure is maintained by cerebral arterial pressure which is cerebral autoregulated, resulting in constant intracranial pressure.

CSF is constantly absorbed into the superior sagittal sinus (SSS) at the pacchionian granulation level. This process is carried out by a pressure gradient between the CSF and the venous pressure in the SSS. When there is a rise in the venous pressure, the CSF pressure rises proportionately in order for the CSF to diffuse into the SSS.

According to the Montro-Kellie rule (Greitz et al., 1992; Mokori, 2001), the increase in ICP may be a result of various factors such as an increase in CSF, brain or blood volume. Although many studies have been performed, it is still unclear which factor is responsible for the increase in ICP in cases of PTC.

Different hypotheses have been proposed such as, an increase in cerebral blood volume which was originally proposed by Dandy in 1937. Raichle et al. (1978), using positron emission tomography (PET) found that almost no change occurred in the cerebral blood flow (CBF) in PTC patients, however there were markedly increased cerebral blood or water volumes.

A few years later, Brooks et al (1985) using PET, found no change in cerebral hemodynamics. Recently, Levine demonstrated that vascular compression and dilatation exist in the PTC patient (Levine, 2000).

Bateman et al. (2007) found that the total CBF measured by magnetic resonance (MR) flow quantification and MR venography in the PTC patient, was $46 \%$ more elevated then in the control group, which may be secondary to cerebral vascular autoregulation. On the other hand, Lorberboym et al. (2001) reported a reduction in perfusion, noted on single photon 
emission CT scans in PTC patients a clear correlation between disease severity and CBF reduction. The proposed mechanisms for $\mathrm{CBF}$ changes are an increase of the cerebral vascular resistance, impairment of the $\mathrm{CBF}$ autoregulation, and a decrease of the tissue vascular density as a result of cerebral edema (Bateman, 2004)).

Bicakci et al (2006) recently studied 16 patients with perfusion and diffusion MRI, finding 6 patients with a statistically reduced CBF, and 2 with a marked increase. All other patients' cerebral blood volume did not significantly increase or decrease compared with the control group. Both vasodilatation and compression occurred in the PTC patient depending on the duration of the disease. The authors claimed that a long standing increase in CSF pressure might result in a decrease in CBF. On the other hand, an increase in CBF may be a result of a failure in autoregulation in the first phases of the disease, as suggested by Bateman's study.

\section{Obstruction of venous outflow}

The absence of ventricular dilatation in an elevated ICP condition is most likely explained by the presence of venous hypertension. As the pressure in the SSS rises, so does the CSF pressure due to hindered absorption of CSF. Brain parenchimal turgor increases due to an impending resorption of venous blood.

In 1995, King et al's (1995) series of 9 PTC patients with venous hypertension in the SSS and proximal transverse sinuses, cerebral venography and manometry were performed. The authors were able to observe the appearance of the transverse sinuses, ranging from a smooth tapered narrowing to a discrete intraluminal filling defect resembling mural thrombi.

The authors also found a significant drop in venous pressure at the level of the lateral third of the transverse sinus that was not fully explained by the anatomical finding on venography. Furthermore this gradient was eliminated after performing a cervical puncture which reduced the CSF pressure.

Karahalios et al (1996) also described a dural venous outflow obstruction found on cerebral venography and manometry in 5 out of 10 patients. A high pressure gradient was observed while those without obstruction had elevated right atrial pressure as well as elevated venous sinus pressure. The authors concluded that increased venous pressure was common in PTC, secondary to intracranial venous outlet obstruction and without anatomical obstruction.

In the same study, Karahalios proposed other hypotheses, such as obesity related cardiomyopathy with subsequent congestive heart failure, sleep apnea, carbon dioxide retention and increased intra-abdominal pressure. All these conditions benefited from diuretic therapy which reduced CSF production and also reduced the central plasma volume and hence venous pressure.

A recent study by Nodelmann (2009) observed that jugular vein valve insufficiency in patients with PTC supports the hypothesis that increased ICP may be a result of a more general state of venous hypertension, possibly associated with obesity.

Several studies focusing on the appearance of outflow obstruction on MRI and MRV (Farb et al, 2003; Johnston et al., 2002;) produced inconclusive results due to the wide variations of radiological appearances of posterior fossa dural sinuses, which may be confused with a normal anatomical variant (Lee \& Brazis, 2000).

Higgins et al (2004) published a study comparing 20 patients with PTC who had undergone MRV and a control group of 40 healthy volunteers, strictly selected. Patients with a history of headaches or other signs or symptoms related to cranial venous involvement were 
excluded. All subjects were matched for age and sex. A significant difference was observed in the appearance of the lateral sinus between the 2 groups; bilateral lateral sinus flow gaps were seen in 13 out of 20 (65\%) PTC patients compared to none in the control group.

A new imaging method proposed by Farb et al. (2003) is auto-triggered elliptic-centricordered three-dimensional gadolinium-enhanced MR venography, which may be superior to time of flight MR venography in its flow insensitivity and decreased artifactual signal loss. Using this new technique, bilateral sinovenous stenosis was found in 27 out of 29 (93\%) PTC patients compared to only 4 out of $59(6.5 \%)$ in the control group. The authors concluded that the distal transverse sinus is the area of pressure gradient as described in King et al's study (1995).

Farb et al (2003) described two types of dural narrowing a "long smooth tapered narrowing", indicating an extra luminal compressive stenosis, and the "acutely marginated apparent intraluminal filling defect", indicating an enlarged, partially obstructing, intraluminal arachnoid granulation. They concluded that in PTC patients, increased dural venous pressure is measurable; however, whether this is a primary cause, a contributory factor, or a secondary phenomenon is uncertain.

Two opposing hypotheses can be made. The first proposes that dural sinus stenosis should be considered as the primary cause of PTC (fixed stenosis). Kollar et al. (2001) proposed that the transverse sinus is narrowed or obstructed by venous sinus thrombosis, vasculitis, congenital stenosis, enlarged arachnoid granulations or even heterotropic brain.

A recently published paper by De Lucia et al (2006) supports the suggested speculation that PTC is a long term sequela of previous sinus vein thrombosis or of an unidentified thrombus (Nedelmann et al., 2009; Sussman et al., 1997). In their study, 17 PTC patients without radiographic evidence of thrombosis were compared to healthy controls. The results showed a significant predominance of hypercoagulability markers, including protein $C$ deficiency, increased plasma levels of prothrombin fragment 1 and 2, fibrinopeptide A, gene polymorphism for factor $\mathrm{V}$ leiden mutation, and high titers of cardiolipin antibodies.

The second hypothesis, in contrast to the fixed stenosis theory, suggests a dynamic one, where venous obstruction is the consequence and not the primary cause of intracranial hypertension. An increased intracranial pressure due to some unknown cause will result in a compression of the vascular compartment and dural sinuses which is in agreement with the Monroe-Kellie doctrine (Corbett, 2004). It is presumed that the predisposition to this phenomenon is due to the anatomy of the distal transverse sinus. Studies have shown that after normalization of the CSF pressure, resolution of the dural sinus narrowing occurs.

\section{Endocrinological and metabolic factors}

The association between PTC, female gender and obesity suggests an endocrine basis for this disorder. Reports of PTC occurring in corticosteroid deficient states such as Addison's disease, and following the removal of an ACTH secreting pituitary adenoma (Ross \& Wilson, 1988), implies abnormalities in the adrenal pituitary axis. Furthermore, corticosteroids effectively treat PTC and corticosteroid withdrawal is associated with PTC (Yasargil et al., 1990). However, Soelberg Sørensen et al. (1986) found no consistent abnormality in pituitary, gonadal, thyroid or adrenal function. Multiple studies have documented the clear association between PTC and polycystic ovaries. In one study conducted by Glueck et al. (2003), 15 women out of 38 PTC patients were found to have 
PCOS; 14 were obese, with a body-mass index (BMI) $>30 \mathrm{~kg} / \mathrm{m}^{2}$ and 10 were extremely obese $(\mathrm{BMI}>$ or $=40)$.

\section{Excess CSF production}

Quincke (1893) was the first to describe excess CSF production. The rate of CSF production can be measured through invasive procedures (Walker, 2001). Donaldson found an increased CSF rate, while other studies failed to demonstrate CSF hypersecretion in PTC patients ( Binder et al., 2004; Walker, 2001). A noninvasive technique (MRI) to measure CSF production by recording the flow through the cerebral aqueduct produced highly variable results, which did not support the theory of CSF overproduction in PTC patients (Gideon et al., 1994). In an attempt to rule out this theory, experimental infusion of artificial CSF was injected into the lateral ventricles of dogs which led to ventricular enlargement, not a PTClike syndrome (Greitz et al., 1992; Walker, 2001).

\section{CSF outflow reduction}

This theory, supported by most studies, proposes the pathogenesis of CSF outflow obstruction into the venous system, although existing reports are still controversial. Studies have shown that PTC is associated with CSF outflow impairment (Calabrese et al., 1978; Cameron, 1933; Malm et al., 1992; Martins, 1974) and no histological evidence of arachnoid villi granulation dysfunction. Controversy exists as to whether impairment of CSF outflow at the arachnoid granulation level may be pathophysiological. In infancy, agenesis, deficiency, or dyslasia of the arachnoid villi and granulations result in hydrocephalus, not PTC (Gilles \& Davidson, 1971).

Studies have demonstrated that in cases of elevated intracranial pressure attributed to high protein concentration in the CSF, (spinal tumor, Guillian-Barre syndrome), some patients develop hydrocephalus while others develop a PTC- like syndrome (Feldmann et al., 1986; Raichle et al., 1978; Ridsdale \& Moseley, 1978; Ropper \& Marmarou, 1984). It has been suggested that a high concentration of protein in CSF, may lead directly to impairment of CSF outflow.

\section{Chronic inflammation}

Recent reviews by Binder et al (2004) suggest that increased levels of cytokines and leptins (an adipocyte derivative hormone that circulates in the plasma at levels in proportion to body fat) in the CSF, may contribute to chronic inflammation and pathogenesis of intracranial hypertension in PTC patients.

Hypercoagulable states, devoid of obvious dural sinus thrombosis, have been reported associated with and in some cases used to explain PTC's mechanism. Kesler reported on several individuals found to have antiphospholipid antibodies and hyperfibrinogenemia related to thrombosis (Kesler et al. 2000; Kesler et al., 2010).

\section{Drug associated}

Numerous published reports and studies have described the correlation between certain drugs and vitamins and the development of increased intracranial pressure. These include antibiotics such as tetracycline or minocycline (Giles \& Soble, 1971), fluoroquinolones 
(Winrow \& Supramaniam, 1990), naladixicacids (Cohen, 1973), sulfamethozaxole (Ch'ien, 1976) and hormonal treatments such as oral contraceptives, growth hormones, progesterone (Hamed et al., 1989; Rogers et al., 1999; Walsh et al., 1965), corticosteroid withdrawal (Neville \& Wilson,1970), lithium (Saul et al, 1985) and vitamin A use and its derivatives (Morrice et al., 1960, Spector \& Carlisle, 1984; Visani, 1996) in doses exceeding 50,000 UI in adults and over 20,000 UI in children.

\section{Systemic conditions}

In the literature, various systemic diseases have been found to be associated with PTC, the most common being uremia (Campos \& Olitsky, 1995) Toxic conditions, hypervitaminosis A tetracycline therapy, lithium,prolonged steroid therpy, Steroid withdrawal,. Other diseases include anemia (Capriles, et al. 1963), dysthyroidism (Campos \& Olitsky, 1995; Huseman \& Torkelson, 1984), Addison's disease (Condulis et al., 1997) cerebral sinus thrombosis, and sleep apnea. .

\section{Ancillary tests}

\subsection{Neuroimaging}

The rationale for neuroimaging studies in PTC patients is twofold: prior to obtaining the cerebrospinal fluid, a brain imaging study is required to exclude any condition that would put the patient at risk of herniation, such as a tumor and to assure no secondary cause of increased ICP. The recommended study type has been modified together with the advance in neuroimaging technology. A CT scan is generally adequate as to ensure that the patient is not at risk when undergoing a lumbar puncture. The resolution is insufficient to exclude posterior fossa abnormalities, isodense lesions gliomatosis cerebri or venous sinus thrombosis. As either neuroimaging is acceptable, MRI of the brain with gadolinium is preferred over CT scanning with contrast.

\subsection{MRI}

Brain MRI is typically normal in the PTC patient, with a ventricular size normal for the patient's age. An asymptomatic empty sella is a well known neuroimaging finding in patients with increased intracranial pressure and may be present in over $50 \%$ of cases (Silbergleit et al., 1989). The empty sella (Figure 2) is attributed to longstanding effects of pulsatile CSF under high pressure, leading to downward herniation of an arachnocele through a defect in the diaphragma sella (George, 1989). The incidence of empty sella ranged from $10 \%$ when plain radiographs of the skull were analyzed (Sorenson et al., 1989) to $94 \%$ when third-generation CT scans were analyzed (Gibby et al., 1993).

Over the past few decades, other radiographic evidence of increased ICP in PTC patients has been detected using various neuroimaging techniques. Flattening of the posterior sclera is the most sensitive sign of elevated intracranial pressure, and was observed in $80 \%$ of patients with pseudotumor cerebri in Brodsky's study (Brodsky \& Vaphiades, 1988). The flattening indicated transmission of elevated perioptic CSF pressure to the compressible posterior sclera. Jacobson (1995) found similar findings of bilateral posterior scleral flattening and distension of the perioptic subarachnoid space on MR imaging (Figure 3)in a patient with elevated intracranial pressure and unilateral papilledema. Furthermore, he emphasized that the constellation of acquired hyperopia and choroidal folds may indicate 
the presence of pseudotumor cerebri in rare patients whose distal optic nerves are structurally resistant to developing papilledema.

A study by Jinkins et al (1996) found intraocular protrusion of the swollen optic disc in 10 out of 15 patients with pseudotumor cerebri while examining the prelaminar optic nerves via MRI. The optic disc appeared hypointense to vitreous on T2-weighted images.

Upon administration of intravenous gadolinium, enhancement on T1 and T2 was produced in areas where the blood-brain barrier was absent or disrupted. Intraocular enhancement of the swollen disc was found in 50\% of MR images of patients with PTC resulting from diffuse prelaminar capillary leakage secondary to severe venous congestion. Distension of the perioptic subarachnoid space was present in $45 \%$ of patients with pseudotumor cerebri. A finding of intraocular protrusion of the prelaminar optic nerve can be visualized well on CT scanning (Lam et al., 1997; Jinkins et al., 1996), however, on MRI, no signal differential between the swollen optic disc and the vitreous cavity was observed (Connolly et al., 1992; Foley \& Posner, 1975; 1989; Gass et al., 1996; Gideon et al., 1995; Mashima et al., 1996; Silbergleit et al.).

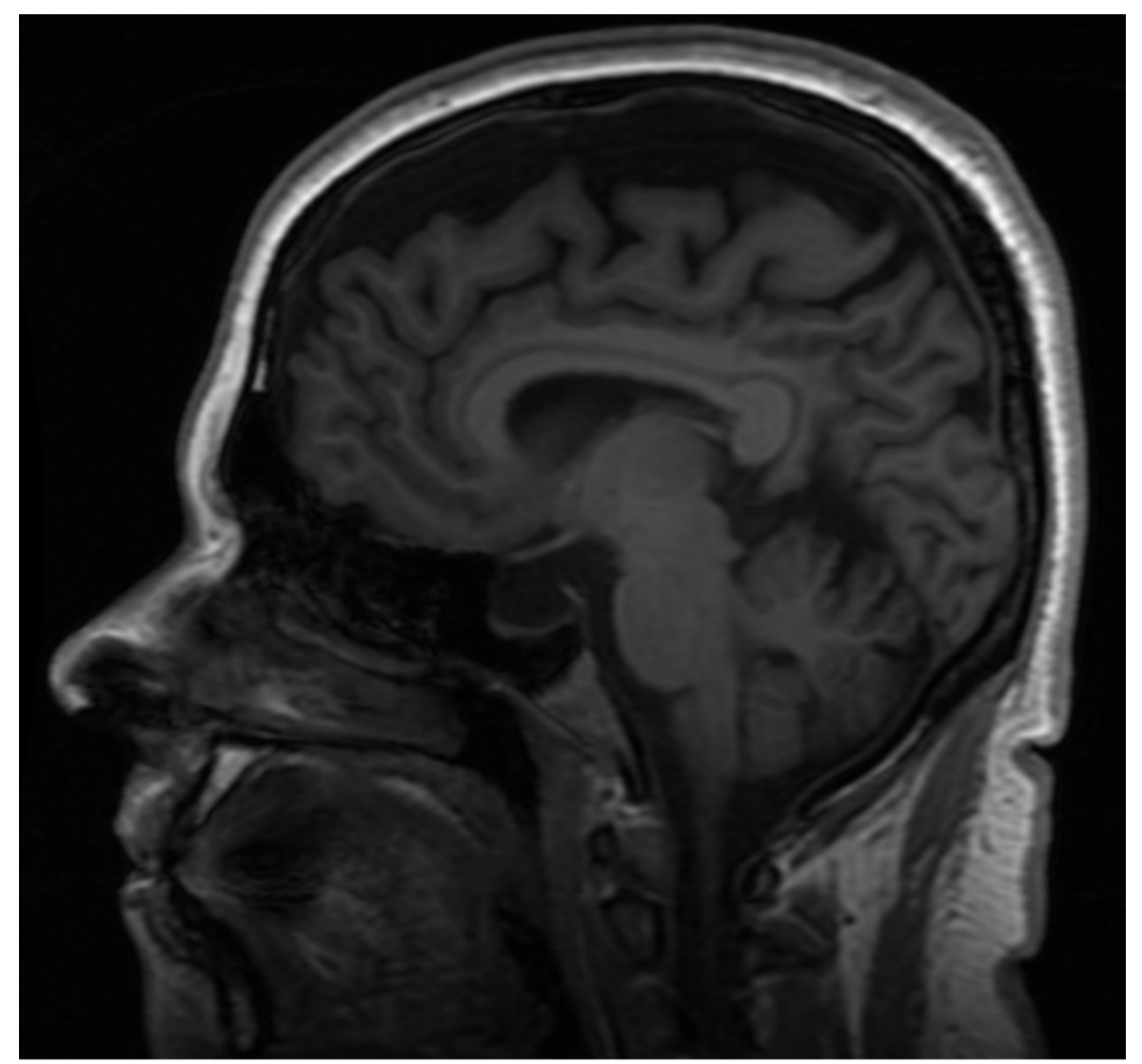

Fig. 2. MRI image demonstrating an empty sella in a 26 year old PTC patient 
Brodsky (1998) observed vertical tortuosity of the optic nerves in 8 out of 20 (40\%) patients with pseudotumor cerebri compared to only one control subject. Since some tortuosity may exist in normal subjects, the ability of axial MR imaging to display relatively minor degrees of horizontal tortuosity, makes it a relatively nonspecific finding. Furthermore, vertical tortuosity of the orbital optic nerve is often accompanied by a "smear sign" on T1-weighted images where the midportion of the optic nerve is displaced from the field of view, causing it to appear obscured by a "smear" of orbital fat. The optic nerve tortuosity or kinking in patients with elevated intracranial pressure is attributable to the distal fixation of the optic nerves by the globes tethered to the orbits by their rectus muscles and check ligaments.

Every patient suspected of PTC must routinely undergo an MRV or CTV examination. Both exams are equally reliable in identifying sinus vein thrombosis and therefore the decision of which examination to perform is entirely up to the expertise of the neurologist at the medical center.

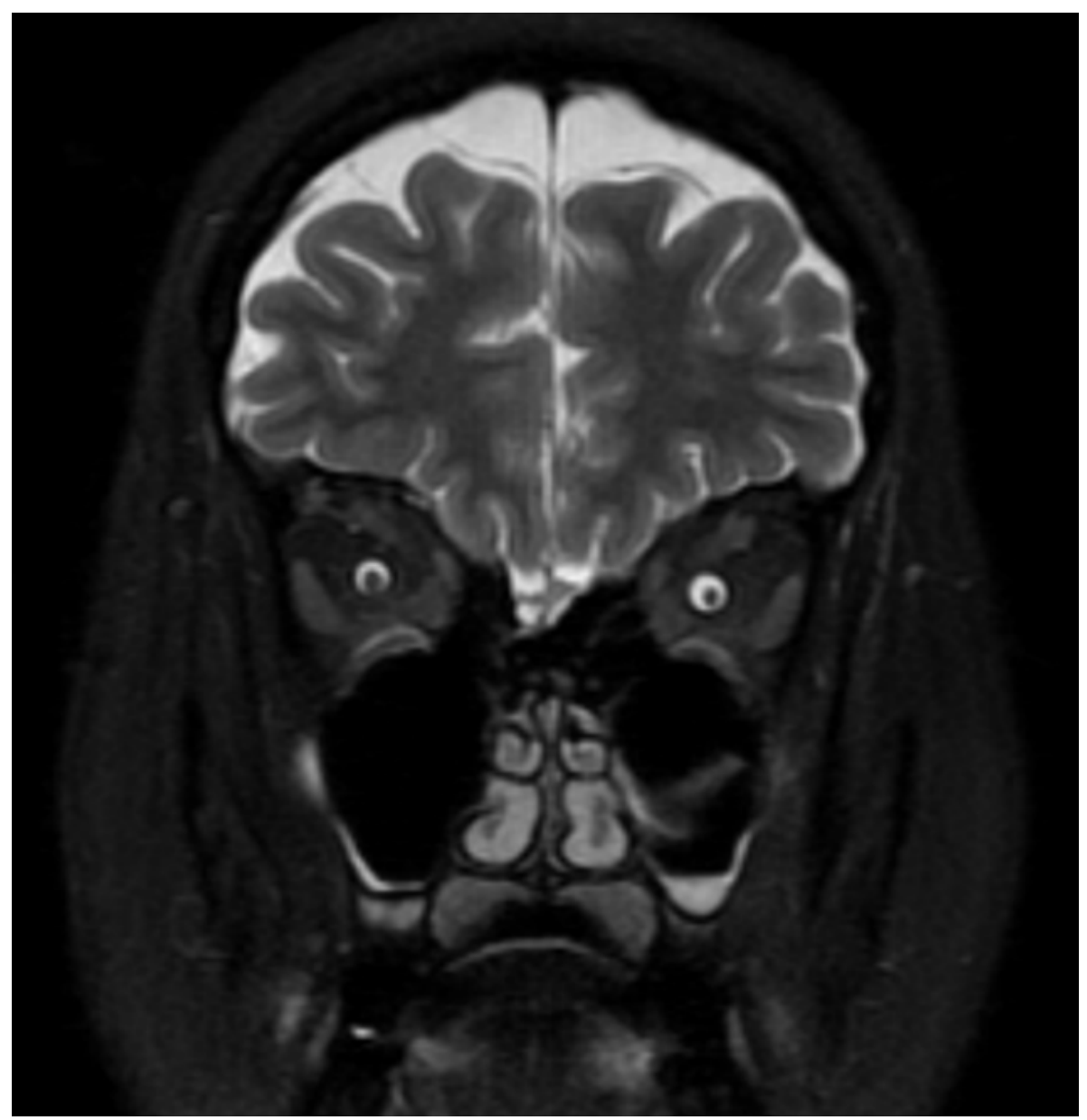

Fig. 3. Coronal T2 MRI image, demonstrating excess of CSF surrounding the optic nerves 


\subsection{MRV}

PTC is consistently associated with venous outflow disturbances. Sinus venous stenosis are found on MR venography in the large majority of PTC patients and may have various conformations, ranging from functional smooth narrowing of sinus segments associated or not associated with definite flow gaps, to segmental hypoplasia or aplasia of one or more central venous collectors. Stenosis is currently believed to be a consequence of primary altered cerebrospinal fluid (CSF) pressure since it may normalize after CSF subtraction with lumbar puncture or shunting procedures (De Simone et al., 2010).

At present, the pathophysiologic mechanism of the elevated intracranial pressure in PTC remains unknown. Evidence suggests that perturbed venous efflux from the head may play a role in the etiology of the disease. Many studies suggest that elevated intracranial venous pressure, an underlying component of PTC, is the result of intracranial venous hypertension due to raised central venous pressure or disturbances of transverse sinuses (TSs) outflow (Brodsky et al., 1998; Friedman \& Jacobson, 2002; Gass et al., 1996; Jacobson et al., 1990).

Occasionally, the clinical picture of PTC is the only clue to the presence of cerebral venous thrombosis, which may be a potentially devastating condition. Thus, every patient diagnosed with PTC must undergo an MRV or CTV to rule out this condition.

MRV is a noninvasive technique effective in visualizing cerebral venous sinuses (Mattle et al., 1991). Different MRV sequences offer the capability of investigating cerebral sinovenous outflow from multiple orientations such as two-dimensional time-offlight (2D-TOF) and three-dimensional phase-contrast (3D-PC) techniques. However, all these techniques have limitations and pitfalls (Ayanzen et al., 2000; Pipe, 2001).

A prospective study by Farb et al. (2003) suggested an auto-triggered elliptic-centric-ordered three-dimensional gadolinium enhanced MR venography (ATECO MRV) to evaluate the cerebral venous outflow of patients with PTC in combination with a novel scoring system. They found substantial bilateral sinovenous stenoses in 27 out of $29(93 \%)$ patients with PTC compared to only 4 out of 59 in the controls and concluded that using ATECO MRV and a novel grading system for quantifying sinovenous stenoses, the authors could identify PTC patients with a sensitivity and specificity of $93 \%$.

Higgins et al. (Higgins et al., 2004) described a distinctive pattern in the signal phase of contrast MRV in PTC patients. They found "signal gaps" in both lateral sinuses of PTC patients, unobserved in their control group. These "signal gaps" may indicate that flow velocities over that specific segment are outside the range prescribed in their study.

This finding does not necessarily mean that a thrombus is present nor does it inevitably indicate that abnormal arachnoid granulations might have caused a local alteration of blood flow. However, it does raise the possibility of stenosis or occlusion.

Higgins et al. (2002) reported the first patient with refractory PTC and transverse sinus stenosis treated with a stent. Direct cerebral venography and manometry confirmed the presence of stenoses with raised pressure, proximal to the obstructions. Dilation of one of the sinuses with a stent reduced the pressure gradient with dramatic symptomatic improvement.

Current evidence (Higgins et al., 2003; Metellus et al.; Ogungbo et al., 2003; 2005; Rajpal et al., 2005) suggests that selected patients with PTC may benefit from a transverse sinus (TS) stent. Restoring the patency of stenotic TSs with a stent in patients with refractory IIH resulted in a resolution or significant improvement in headache and papilledema. 


\subsection{CT and CTV}

Neuroimaging utilizing CT offers rapid image acquisition, wide availability and excellent spatial resolution. The speed and ability of a CT to detect acute blood or bone abnormality makes this technique very valuable in traumatic cases.

Due to the rise in availability of MRI and MRV during the past few decades, and their known advantages over CT scans, the use of a CT as a neuroimaging tool in diagnosing PTC patients has lessened over the years. Current studies in the literature are focusing mainly on MRI and MRV findings in neuroimaging rather the CT.

Almost 25 years ago, Jinkins (1987) first reported on the reversal of the optic nerve head in a variety of conditions associated with increased intracranial pressure, including pseudotumor cerebri. Enlarged optic nerve sheaths have also been observed in patients with pseudotumor cerebri. Weisberg (1985) found that 2/28 patients had enlarged optic nerves, although no measurements were provided and no control values were available. Six out of 28 patients were described as having a "small-sized ventricular system." These examinations were performed on a second-generation CT scanner, without controls and in a non-blinded manner.

Almost two decades ago, Gibby et al. (1993) published a paper on CT findings in their PTC patients. The purpose of their study was to evaluate and compare orbital and cerebral CT findings in PTC patients with those of age and sex matched controls. Ventricular size in both groups was evaluated. Their findings matched Huckman et al's (1976) who found no differences between ventricular sizes of patients with pseudotumor cerebri and those of a control population.

Empty sellae have been associated with many conditions causing increased intracranial pressure. Gibby et al. in 1993 observed empty sellae in 5\% of 788 autopsies (Kesler et al., 2001). In the PTC group, 16 (94\%) out of 17 patients had at least partial empty sella. The degree of empty sellae was significantly greater than in the controls. The high prevalence noted in patients with pseudotumor cerebri is compatible with their chronic elevated intracranial pressure, which averaged $370 \mathrm{~mm} \mathrm{H2O}$. CT angiography uses a high speed spiral scanner, providing excellent vessel resolution with a 3-dimensional capability, comparable to an MRA. The technique requires iodinated dye and ionizing radiation and takes approximately 15 minutes. Sensitivities in detecting aneurysms $>3 \mathrm{~mm}$ or stenosis $>70 \%$ are approximately $95 \%$. Some centers prefer the use of a CTA over an MRA in identifying cerebral aneurysms, including those causing ocular motor cranial nerve palsies.

\subsection{Visual field perimetry}

Visual field testing by either automated Humphrey static or manual Goldmann perimetry reveals enlargement of the physiological blind spot in virtually all patients with PTC. Other common visual field defects include inferonasal loss and generalized constriction of the fields. Central defects, arcuate and altitudinal defects may occur but are highly unlikely. However, if found, a search is warranted for another cause, unless a large serous retinal detachment from a high-grade optic edema, spreading to the macula area, is found.

Almost all PTC patients suffer from some type of visual field loss over time. In prospective (Wall \& George, 1987; Wall \& George, 1991) studies of patients with PTC, visual field loss in at least 1 eye (other than an enlargement of the blind spot due to the edematous optic nerve) was found in $96 \%$ of patients using a Goldmann perimetry, a disease specific strategy and in $92 \%$ with automated perimetry. Approximately one third of this visual field loss was mild and was usually not noticed by the patient (Wall \& George, 1991). 
Wall et al's study (1991) showed that with treatment, about 50\% of patients experience a significant visual field improvement. They further demonstrated that the only subgroup of patients who had a worsening of their visual fields were those with a recent weight gain. This was the only factor significantly associated with a decline in vision.

\subsection{Ultrasound of optic nerve}

Ultrasound has been used to identify intracranial hypertension by measuring the optic nerve sheath diameter. The modality is extremely safe and does not have any contraindications for use, except for ocular globe injuries (Munk et al., 1991). In most modern medical facilities, multipurpose ultrasound units with high-frequency transducers (>7.5 MHz) provide high lateral and axial precision (Berges et al., 2006).

Hayreh (1964) in an experimental study of monkeys and humans showed that the subarachnoid spaces surrounding the optic nerve is in continuity with the intracranial cavity, and therefore changes in cerebrospinal fluid pressure may be transmitted along the optic nerve sheath (Hayreh, 1964). The retrobulbar enlarged optic nerve sheath can therefore inflate as a consequence of raised pressure in the cerebrospinal fluid.

It has been confirmed that the optic nerve sheath diameter (ONSD) increases in patients with intracranial hypertension (Blaivas et al., 2005; Geeraerts et al., 2007; Girisgin et al., 2007; Karakitsos et al., 2006; Malayeri et al., 2005; Munk et al., 1991; Salgarello et al., 1996; Stone, 2009). ONSD alterations are correlated with head CT scan results in brain injured adults (Karakitsos et al., 2006) as well as with the invasive and noninvasive measurements of the ICP (Soldatos et al., 2008).

During US evaluation, it is important to perform a $30^{\circ}$ test to evaluate excess of fluids within the arachnoid sheaths of the optic nerve. The patient is asked to turn approximately $30^{\circ}$ toward the probe placed temporally on the globe. The measurements in abduction are compared with the measurements at the primary position. If fluid excess is present, the diameter at $30^{\circ}$ will be smaller than that found in the primary position.

\subsection{Optical coherence tomography (OCT)}

OCT is a non-invasive imaging technique yielding high-resolution, cross-sectional images of the retina. More importantly, it is a valuable modality used to identify the status of the retinal nerve fiber layer as well as to measure macular thickness. The technique is principally based on measuring the time required for light to reflect from the tissue to an external detector (i.e. "echo time delay"). Duration of time and intensity of the backscattered light corresponds directly to the depth and density of the tissue being imaged. OCT produces in vivo cross-sections which can be serially acquired (in thin sections) in order to obtain tomographic images (Schuman et al., 2004).

The principles underlying OCT are similar to those of ultrasound imaging, except that light is utilized instead of sound. The axial resolution of OCT images is at least 1-2 orders of magnitude greater than those of ultrasound. OCT images of the retina provide a spatial resolution as low as 3-5 microns (Huang et al., 1991; Schuman et al., 2004).

Currently, two main types of OCT are utilized in practice: the time-domain, employing low-coherence interferometry for imaging tissue structures, and the spectral-domain OCT. Spectral-domain imaging provides faster and more spatially detailed images than the time-domain method. In addition, spectral domain OCT allows for imaging at a speed of 18,000 to approximately 40,000 A-scans per second, fast enough to eliminate artifacts from 
eye movements. It also provides a greater resolution of the retina with subsequent better visualization of the laminar structure and clarification of details at the cellular level within the nuclear layers (Choi et al., 2008). Several reports have also described particularly good reproducibility of RNFL and optic disc measurements with the newer spectral-domain technique (Gonzalez-Garcia, et al., 2009; Leung et al., 2009; Menke et al., 2008).

Computer-aided image processing algorithms have been developed to estimate NFL thickness from circumpapillary OCT images acquired in cylindrical sections surrounding the optic disc. A circumpapillary scan pattern of a typically $3 / 4 \mathrm{~mm}$ diameter is used, because it effectively intercepts all nerve fibers originating from the optic disc, thus enabling a quantitative measurement of the circumferential variations in NFL thickness around the optic disc as well as visualization of the nerve fiber bundles.

Studies have demonstrated the effectiveness of time-domain OCT in providing a quantitative measure of the severity of disease, response to treatment and follow up monitoring of patients with PTC (Ophir et al., 2005; Rebolleda \& Munoz-Negrete, 2009). RNFL thickness decreased correspondingly, with the improvement recorded at the patients' clinical evaluation. The study outcomes provided a basis for using OCT to predict which patients would ultimately fare better in terms of visual functional status (Rebolleda \& Munoz-Negrete, 2009).

Precise measurements obtained with OCT allow the physician to more accurately evaluate the treatment effect than the subjective interpretation of the funduscopic appearance of the optic nerve heads. Recently, studies have emphasized the importance of viewing the macula in these patients. Hoye et al. (2001) evaluated the detection and monitoring of sub-retinal fluids extending from the optic nerve head to the macula in patients with papilledema. The authors found that the fluids accounted for the reduced visual acuity in 7 out of 55 patients, and when the volume of sub-retinal fluids decreased, an improvement in the visual acuity function was noted.

Future OCT assessment of papilledema will be evaluating the total optic nerve head disc volume and maximal optic nerve head height, as these measures potentially correlate with the response to medication and ultimate visual outcome. At present, clinical evaluation of the optic nerve head volume, better reflects the overall degree of optic nerve head edema rather than the thickness of the RNFL alone, since volume measurement incorporates the volume of both the total optic nerve height and the sub-retinal hypo reflective space. (Johnson et al., 2009).

\subsection{Fluorescein angiography}

Fluorescein angiography was initially developed as a tool for studying retinal vascular flow characteristics. Extensive use of fluorescein angiography and technique refinement has allowed the clinician and researcher to better understand the pathophysiologic and histopathologic changes of fundus disease in vivo. Fluorescein angiography may aid the clinician in accurately diagnosing papilledema by the appreciation of leakage or late hyperfluorescence surrounding the optic disc. Blocked hypofluorescence may be noted at the optic nerve head during papilledema as a result of bleeding, which is present at the level of the neurosensory retina, blocking the fluorescence from the underlying choroid (Rabb et al., 1978). 


\section{Management}

There are nonsurgical and surgical approaches to PTC, decisions based on symptomatology and visual function status. If a headache is controlled by common analgesics and no optic nerve dysfunction is observed, therapy may not be required. Asymptomatic patients with preserved vision and minimal papilledema warrant only frequent follow-ups as well as monitoring disc swelling and visual function, including visual fields (Nonne, 1904).

\subsection{Medical (nonsurgical) approach}

Acetazolamide, a carbonic anhydrase inhibitor that decreases CSF production by the choroid plexus, is generally accepted as first-line medication, although its efficacy has not been proven in prospective trials. Based on our experience, a starting regimen of acetazolamide $500 \mathrm{mg}$, orally two or three times per day, is preferred. The dose can be increased to a total of 3 grams per day, if necessary. Major side effects include diuresis, loss of appetite, abnormal taste (metallic taste with carbonated beverages) paresthesias of the lips, fingers and toes, malaise, renal colic and metabolic acidosis (Corbett et al., 1982). Most of these side effects resolve with potassium and magnesium-rich dietary supplements such as bananas and oranges. Severe adverse effects experienced with acetazolamide treatment include acute tubular necrosis, hepatic dysfunction, and aplastic anemia.

Teratogenic effects in animals such as limb malformations and cortical dysgenesis have been reported (Quincke, 1893). Although sacrococcygeal teratoma in neonates (Tibussek et al., 2010) have been documented in the past, there is little clinical or experimental evidence to support any adverse effect of the drug on pregnancy outcome in humans (Spence et al., 1980). If acetazolamide fails, topiramate, an anti-epileptic drug, may be used. Its therapeutic effects are due to its carbonic anhydrase inhibitory properties. The drug is particularly useful as a prophylaxis for headaches, appetite suppression and weight loss (Nonne, 1904). The dose should be built up slowly over weeks ( $25 \mathrm{mg} /$ week) in order to reduce the risk of cognitive side effects. It is of utmost importance to regularly check the intraocular pressure (IOP) of patients undergoing this treatment, as elevation of IOP is a known side effect of topamax.

Short-term oral corticosteroids may be considered as a treatment option in patients presenting with severe headaches, marked papilledema, and very high intracranial pressure. High doses of intravenous corticosteroid treatment may occasionally be administered when there is rapidly progressive vision loss or while the patient awaits surgery (Mathews et al., 2003).

Treatment medication is usually given over a long period of time. ICP-lowering agents may be tapered and eventually discontinued when the patient's visual status and optic nerve appearance have improved and stabilized. Patients should be periodically monitored post treatment, since recurrences are common. If symptoms reoccur, reinstitution of medications is usually indicated (Friedman \& Jacobson, 2004).

Weight loss is a crucial part of the treatment program, as even moderately obese patients may significantly benefit from a sensible diet and exercise program (Mathews et al., 2003). Medications known as associated risk factors for pseudotumor syndromes, such as vitamin A, vitamin A derivatives, and tetracycline should be discontinued if possible.

\subsection{Lumbar puncture}

In the past, repeated lumbar punctures were an acceptable treatment modality due to improvement of symptoms after the procedure. Nowadays, this type of treatment approach 
is less acceptable. Currently, lumbar puncture may be indicated in pregnant women or when the clinical picture points to rapidly declining vision, thus temporarily lowering the CSF pressure while planning a more aggressive treatment (Friedman \& Jacobson, 2004). In the past serial lumbar punctures (e.g., twice weekly) have been proposed as an alternative to surgery for patients with papilledema, when the disease cannot be controlled medically. Complications of the procedure such as infection, tonsillar herniation, radiculopathy, and arachnoiditis are rare.

\subsection{Surgical approach}

The most frequent and accepted indication for surgery is a progressive loss of vision despite maximal medical therapy. Surgery should be carried out as soon as a visual field defect worsens or remains unimproved despite maximal treatment. Medical treatment fails in approximately $18 \%$ to $22 \%$ of patients with PTC (Burgett et al., 1997; Corbett \& Thompson, 1989; Lorberboym et al., 2001; Lueck \& Mcllwaine, 2002; Pearson et al., 1991). According to Friedman et al (2004), ophthalmological indications for surgical intervention are severe or rapid visual loss at onset ("malignant $\mathrm{IIH}^{\prime}$ ), severe papilledema causing macular edema or exudates without improvement while undergoing medical treatment. Even if there is no consensus among the specialists (Friedman \& Jacobson, 2004), surgery should be considered in the management of intractable headaches (Binder et al., 2004; Mathews et al., 2003). Surgical treatment options include optic nerve sheath fenestration (ONSF) and CSF diversion procedures. A CSF shunt reduces intracranial hypertension, whereas ONSF focuses on protecting the vulnerable optic nerve head.

\subsection{Optic nerve sheath decompression (ONSD)}

The mechanism by which ONSD works has not been clarified, but several theories have been suggested. Keltner (1988) suggests that it may provide a filtering effect, with a subsequent decrease in the local CSF pressure, improvement of the peripapillary circulation (Keltner, 1988), or produce a generalized decrease in ICP. According to another hypothesis, the scarring of the arachnoid by the procedure itself may protect the nerve head from elevated CSF pressure (Friedman \& Jacobson, 2004). Technically, ONSD is performed by uncovering the optic nerve sheath through a lateral orbitotomy or through a medial approach via a transconjunctival incision. Multiple linear incisions are made or a window is cut into the anterior dural that covers the optic nerve sheath, creating a CSF drainage outlet (Mathews et al., 2003).

The overall complication rate of ONSD ranges from $4.8 \%$ to $45 \%(151,165,166,167,168)$, the most common being extraocular motility problems due to lateral rectus palsy which is usually transient, and papillary abnormalities. Other complications may include long-term and transient blindness due to ischemic injury to the optic nerve, orbital hemorrhage, visual field defects and globe perforation (Friedman \& Jacobson, 2004). In the majority of patients, post-ONSD vision stabilizes or improves in the long-term, but as many as $32 \%$ of operated eyes may experience deterioration following initially successful surgery (McHenry \& Spoor, 1993). A reoperation can be performed, but based on clinical experience, a shunting procedure is recommended in these cases.

\subsection{Lumbar peritoneal shunt (L-P shunt)}

Although an L-P shunt is considered an effective procedure, failure and low pressurerelated headaches are common. Shunts may be efficiently placed in the lumbar cistern, 
cisterna magna, or the ventricles. The lumboperitoneal (L-P) technique has traditionally been the method of choice in PTC.

A review of the literature Binder et al (2004) found that the efficacy of L-P shunting is maintained as long as the shunt remains patent. The failure rate for LP shunts range from $38 \%$ to $64 \%$ (Burgett et al., 1997; Gupta et al., 2007; Johnston et al., 1988). Major causes include catheter obstruction, over-shunting (low pressure headaches), catheter migration, and lumbar radiculopathy.

\subsection{Ventriculoperitoneal (V-P) shunts}

A V-P shunt is difficult to perform due to relatively small or normal ventricle size. However, this technique is becoming increasingly popular in treating PTC (Binder et al., 2004; Maher et al., 2001; Tulipan et al., 1998).

In the long-term, V-P shunts offer advantages compared to the L-P shunt method, especially with regard to shunt revision (Kang, 2000; Maher et al., 2001). The procedure does not present the risk of inducing a Chiari I malformation, and may be less likely to over-drain.

A wider range of shunts are available for the V-P route. Usually, PTC patients benefit from relatively high-pressure valves (possibly with an antisiphon system to limit over-drainage), which are able to retain sufficient intracranial CSF to compensate the changing intracranial volume conditions and limit the collapse of the ventricular system around the shunt catheter. Flow-regulated valves have also been proposed (Garton, 2004).

\subsection{Subtemporal decompression}

The first neurosurgical technique to treat PTC patients, by a subtemporal decompression, was performed in 1937 (Dandy, 1937). Dandy performed a unilateral subtemporal craniectomy with excellent initial results in alleviating headaches and preventing visual loss. The long-term efficacy of the procedure was uncertain, since a high rate of morbidity and complications were reported, including seizures, infections, focal brain damage, cosmetic disfigurement, intracranial hematomas, and further visual deterioration (Binder et al., 2004). After introducing stenting procedures for treating $\mathrm{IIH}$, this procedure became obsolete; nonetheless, subtemporal decompression is still an option, when other surgical methods have failed.

\subsection{Endovascular stenting}

It is highly controversial whether venous sinus narrowing is the cause or the result of elevated intracranial pressure. Based on the frequent findings in MRI venography of narrowed transverse sinuses, endovascular stenting of the venous sinuses has been recently advocated by some authors (Higgins et al., 2002; Higgins et al., 2003; Metellus et al., 2005; Metellus et al., 2007; Donnet et al., 2008; Paquet et al., 2008).

Higgins et al (2002) was the first to report on a 30 year old patient with refractory PTC, papilledema and bilateral TS stenosis found on an MR venogram, that was successfully treated with dilation of 1 of the sinuses with a stent, thus reducing the pressure gradient with dramatic symptomatic improvement.

As of today, only about 40 patients with PTC, treated with sinovenous stent placement, have been reported in the literature. Most were women aged 15-65, symptomatic with PTC for 2 weeks to 15 years. After stent placement, 33 out of the $40(82.5 \%)$, reported a significant improvement in their headaches. Papilledema improved or resolved in 30 out of $33(91 \%)$ 
patients who presented with active papilledema. Although the promising initial results of long term efficiency of the procedure still needs to be proven, further investigation is still warranted to prove the procedure as a useful treatment technique.

\subsection{Pregnancy and PTC}

Current reports suggest that pregnant patients with PTC can be safely managed similarly to nonpregnant patients with PTC. No increase in the rate of spontaneous abortion or fetal wastage has been reported. A therapeutic abortion to limit the progression of the disease is not indicated. (Tang et al., 2004).

Acetazolamide had previously been considered as the preferred therapy after 20 weeks of gestation, since sacrococcygeal teratoma was reported with earlier use (Digre et al., 1984). A recent report of 12 women treated with acetazolamide for PTC during pregnancy, showed no adverse pregnancy outcomes in terms of fetal loss or congenital malformation. Acetazolamide at high doses may produce birth defects in animals, but there is little clinical or experimental evidence to support any adverse effect on pregnancy outcome in humans. If the clinical situation mandates acetazolamide use in PTC, the drug can be offered after appropriate informed consent (Lee et al., 20050). Management of labor and indications for cesarean delivery for a parturient with PTC are controversial. Regular labor may be allowed with a cesarean delivery reserved for obstetric indications.

\section{Natural history and visual prognosis}

The natural history of PTC is unknown. In some cases, it is a self-limited condition, while in others ICP may remain elevated for many years even if systemic and visual symptoms resolve. In some patients, the process may last from months to years. Individuals with mild to moderate visual loss tend to recover vision following medical therapy. Papilledema usually resolves after a few weeks or months, but many patients are left with some residual disc elevation, especially nasally. Severe visual impairment may be a serious and permanent complication of PTC. PTC produces significant visual impairment in approximately $25 \%$ of patients. The risk of visual loss in the pediatric PTC population is similar to that of adults (Corbett \& Thompson, 1989). Recurrent symptoms have been reported in 8 to $37 \%$ of patients, years after being diagnosed (Corbett \& Thompson, 1989). Visual deterioration in PTC patients is usually gradual, but in cases of fulminant papilledema, blindness may appear rather quickly. In Corbett et al's (1989) follow up study of 5 - 41 years after the initial diagnosis of 57 patients, revealed severe visual impairment in 14 patients (24.6\%). In Kesler et al's experience (2004), recurrence was frequently associated with weight gain. The longterm prognosis and visual outcome of 54 patients with IIH was observed over a period of 6.2 years. The results showed that recurrences occurred in almost $40 \%$ of the cases. None of these exacerbations occurred during the first 10 months, and none occurred while the patients continued treatment.

\section{References}

Ahlskog, J.E., \& O'Neill, B.P. (1982). Pseudotumor cerebri. Annals of Internal Medicine, Vol. 97, No. 2 (August 1982), pp. 249-256 
Ayanzen, R.H., Bird, C.R., Keller, P.J., McCully, F.J., Theobald, M.R., \& Heiserman, J.E. (2000). Cerebral MR venography: normal anatomy and potential diagnostic pitfalls. American Journal of Neuroradiology Vol. 21, No. 1, (January 2000), pp. 74-78

Balcer, L.J., Liu, G.T., Forman, S., Pun, K., Volpe, N.J., Galetta, S.L., \& Maguire, M.G. (1999). Idiopathic intracranial hypertension: relation of age and obesity in children. Neurology Vol. 52, No. 4, (March 1999), pp. 870-872

Bateman, G.A., Smith, R.L., \& Siddique, S.H. (2007). Idiopathic hydrocephalus in children and idiopathic intracranial hypertension in adults: two manifestations of the same pathophysiological process? Journal of Neurosurgery, Vol. 107, No. 6 Suppl, pp. 439444.

Bateman, G.A. (2004). Idiopathic intracranial hypertension: priapism of the brain? Medical Hypotheses, Vol. 63, No. 3, (March 2004), pp, 549-552.

Berges, O., Koskas, P., Lafitte, F., \& Piekarski, J.D. (2006). Sonography of the eye and orbit with a multipurpose ultrasound unit. Journal of Radiology, Vol. 87, No. 4 Pt 1, pp. 345-353.

Bicakci, K., Bicakci, S., \& Aksungur, E. (2006). Perfusion and diffusion magnetic resonance imaging in idiopathic intracranial hypertension. Acta Neurologica Scandinavica, Vol. 114, No. 3, (September 2006), pp. 193-197.

Binder, D.K., Horton, H.C., Lawton, M.T., \& McDermott, M.W. (2004). Idiopathic intracranial hypertension. Neurosurgery Vol. 54, No. 3, (March 2004), pp. 538-552.

Blaivas, M., Theodoro, D., \& Sierzenski, PR. (2003). Elevated intracranial pressure detected by bedside emergency ultrasonography of optic nerve sheath. Academic Emergency Medicine, Vol. 10, No. 4, (April 2003), pp. 376-381.

Brodsky, M.C., \& Vaphiades, M. (1998). Magnetic resonance imaging in pseudotumor cerebri. Ophthalmology Vol. 105, No. 5, (September 1998), pp. 1686-1693.

Brooks, D.J., Beaney, R.P., Leenders, K.L., Marshall, J., Thomas, D.J., \& Jones, T. (1985). Regional cerebral oxygen utilization, blood flow, and blood volume in benign intracranial hypertension studied by positron emission tomography. Neurology Vol. 35, No. 7, (July 1985), pp. 1030-1034.

Burgett, R.A., Purvin, V.A., \& Kawasaki, A. (1997). Lumboperitoneal shunting for pseudotumor cerebri. Neurology Vol. 49, No. 3, (September 1997), pp. 734-739.

Busch, W. (1951). Die Monphologie den Sella Turcica und ihre Heziehungen zur Hypophyse. Virchows Archiv A: Pathological Anatomy Histopathology, Vol. 320, No. 5, (September 1951), pp. 437-458.

Campos, S.P., \& Olitsky, S. (1995). Idiopathic intracranial hypertension after l-thyroxine therapy for acquired primary hypothyroidism. Clinical Pediatrics, Vol. 34, No. 6, (June 1995), pp. 334-337.

Calabrese, V.P., Selhorst, J.B., \& Harbison, J.W. (1978). CSF infusion test in pseudotumor cerebri. Transactions of the American Neurological Association, Vol. 103, pp. 146-150.

Cameron, A.J. (1933). Marked papilloedema in pulmonary emphysema. British Journal of Ophthalmology, Vol. 17, No. 3, (March 1933), pp. 167-169.

Capriles, L.F. (1963). Intracranial hypertension and iron-deficiency anemia. Archives of Neurology, Vol. 9, pp. 147-153.

Chang, D., Nagamoto, G., \& Smith, W.E. (1992). Benign intracranial hypertension and chronic renal failure. Cleveland Clinic Journal of Medicine,Vol. 59, No. 4, (July-August 1992), pp. 419-422. 
Ch'ien, L.T. (1976). Intracranial hypertension and sulfamethoxazole. New England Journal of Medicine, Vol. 283, No. 1, (July 1970), pp. 47.

Choi, S.S., Zawadzki, R.J., Keltner, J.L., \& Werner, J.S. (2008). Changes in cellular structures revealed by ultra-high resolution retinal imaging in optic neuropathies. Investigative Ophthalmology E Visual Science, Vol. 49, No. 5, (May 2008), pp. 2103-2119.

Cinciripini, G.S., Donahue, S., \& Borchert, M.S. (1999). Idiopathic intracranial hypertension in prepubertal pediatric patients: characteristics, treatment, and outcome. American Journal of Ophthalmology, Vol. 127, No. 2, (February 1999), pp. 178-182.

Corbett, J.J. (2008). The first Jacobson Lecture. Familial idiopathic intracranial hypertension. Journal of Neuroophthalmology, Vol. 28, No. 4, (December 2008), pp. 337-347.

Corbett, J.J., \& Mehta, M.P. (1983). Cerebrospinal fluid pressure in normal obese subjects and patients with pseudotumor cerebri. Neurology Vol. 33, No. 10, (October 1983), pp. 1386-1388.

Cohen, D.N. (1973). Intracranial hypertension and papilledema associated with nalidixic acid. American Journal of Ophthalmology, Vol. 76, No. 5, (November 1973), pp. 680682.

Condulis, N., Germain, G., Charest, N., Levy, S., \& Carpenter, T.O. (1997). Pseudotumor cerebri: a presenting manifestation of Addison's disease. Clinical Pediatrics, Vol. 36, No. 12, (December 1997), pp. 711-713.

Connolly, M.B., Farrell, K., Hill, A., \& Flodmark, O. (1992). Magnetic resonance imaging in pseudotumor cerebri. Development Medicine and Child Neurology, Vol. 34, No. 12, (December 1992), pp. 1091-1094.

Corbett, J.J. (2004) Increased intracranial pressure: idiopathic and otherwise. Journal of Neuro-Ophthalmology, Vol. 24, No. 2, (June 2004), pp. 103-105.

Corbett, J.J., Savino, P.J., Thompson, H.S., et al. (1982).Visual loss in pseudotumor cerebri. Follow-up of 57 patients from five to 41 years and a profile of 14 patients with permanent severe visual loss. Archives of Neurology, Vol. 39, No. 8, (August 1982), pp. 461-474.

Crassard, I., \& Bousser, M.G. (2004). Cerebral venous thrombosis. State of the art. Journal of Neuro-Ophthalmology, Vol. 24, No. 10, (October 1989), pp. 156-163.

Dandy, W.E. (1937). Intracranial pressure without brain tumor: diagnosis and treatment. Annals of Surgery, Vol. 106, No. 4, (October 1937), pp. 492-513.

De Lucia, D., Napolitano, M., Di Micco, P., et al. (2006). Benign intracranial hypertension associated to blood coagulation derangements. Thrombosis Journal Vol. 24, No. 4, (December 2006), pp. 21.

De Simone, R., Ranieri, A., \& Bonavita, V. (2010). Advancement in idiopathic intracranial hypertension pathogenesis: focus on sinus venous stenosis. Neurological Sciences, Vol. 31, Supplement 1, (June 2010), pp. S33-S39.

Digre, K.B., Varner. M.W., \& Corbett, J.J. (1984). Pseudotumor cerebri and pregnancy. Neurology, Vol. 34, No. 6, (June 1984), pp. 721-729.

Donnet, A., Metellus, P., Levrier, O., et al. (2008). Endovascular treatment of idiopathic intracranial hypertension: clinical and radiologic outcome of 10 consecutive patients. Neurology, Vol. 70, No.8, (February 2008), pp. 641-647.

Durcan, F.J., Corbett, J.J., \& Wall, M. (1988). The incidence of pseudotumor cerebri: population studies in Iowa and Louisiana. Archives of Neurology, Vol. 45, No. 8, (August 1988), pp. 875-877. 
Farb, R.I., Vanek, I., Scott, J.N., Procopis, P. \& Antony, J. (2003). Idiopathic intracranial hypertension: the presence and morphology of sinovenous stenosis. Neurology, Vol. 60, No. 9, (May 2003), pp. 1418-1424.

Farb, R.I.,Vanek, I., Scott, J.N. et al. (2003). Idiopathic intracranial hypertension: The prevalence and morphology of sinovenous stenosis. Neurology Vol. 60, No. 9, (May 2003), pp. 1418-1424.

Feldmann, E., Bromfield, E., Navia, B., Pasternak, G.W., \& Posner, J.B. (1986). Hydrocephalic dementia and spinal cord tumor. Archives of Neurology, Vol. 43, No. 7, (July 2006), pp. 714-718.

Foley, K.M. \& Posner, J.B. (1975). Does pseudotumor cerebri cause the empty sella syndrome? Neurology, Vol. 25, No. 6, (June 1975), pp. 565-569.

Foley, J. (1955). Benign forms of intracranial hypertension. Toxic and otitic hydrocephalus. Brain, Vol. 78, No. 1, pp. 1-41.

Friedman, D.I., \& Jacobson, D.M. (2002). Diagnostic criteria for idiopathic intracranial hypertension. Neurology, Vol. 59, No. 10, (November 2002), pp.1492-1495.

Friedman, D.I. \& Jacobson, D.M. (2004). Idiopathic intracranial hypertension. State of art. Journal of Neuro-Ophthalmology, Vol. 24, No. 2, (June 2004), pp. 138-145.

Garton, H.J.L. (2004). Cerebrospinal fluid diversion procedures. Journal of NeuroOphthalmology, Vol. 24, No. 2, (June 2004), pp. 146-155.

Gass, A., Barker, G.J., Riordan-Eva, P., et al. (1996). MRI of the optic nerve in benign intracranial hypertension. Neuroradiology Vol. 38, No. 8, (November 1996), pp. 769 773.

Geeraerts, T., Launey, Y., Martin, L., et al. (2007). Ultrasonography of the optic nerve sheath may be useful for detecting raised intracranial pressure after severe brain injury. Intensive Care Medicine, Vol. 33, No. 10, (October 2007), pp. 1704-1711.

George, A.E. (1989). Idiopathic intracranial hypertension: pathogenesis and the role of MR imaging. Radiology, Vol. 170 (1 Pt 1), pp. 21-22.

Gideon, P., Sfrensen, P.S., Thomsen, C. et al. (1995). Increased brain water self- diffusion in patients with idiopathic intracranial hypertension. American Journal of Neuroradiology, Vol. 16, No. 2, (February 1995), pp. 381-387.

Gideon, P., Sorensen, P.S., Thomsen, C., et al (1994) Assessment of CSF dynamics and venous flow in the superior sagittal sinus by MRI in idiopathic intracranial hypertension: a preliminary study. Neuroradiology, Vol. 36, No. 5, (July 1994), pp. 350-354.

Gibby, W.A., Cohen, M.S., Goldberg, H.I., \& Sergott, R.C. (1993). Pseudotumor cerebri: CT findings and correlation with vision loss. American Journal of Roentgenology, Vol. 160, No. 1, (January 1993), pp. 143-146.

Giles, C., \& Soble, A. (1971). Intracranial hypertension and tetracycline therapy. American Journal of Ophthalmology, Vol. 72, No. 2, (November 1971), pp. 981-982.

Gilles, F.H., \& Davidson, R.I. (1971). Communicating hydrocephalus associated with deficient dysplastic parasagittal arachnoidal granulations. Journal of Neurosurgery, Vol. 35, No. 4, (October 1971), pp. 421-426.

Girisgin, A.S., Kalkan, E., Kocak, S., et al. (2007). The role of optic nerve ultrasonography in the diagnosis of elevated intracranial pressure. Emergency Medicine Journal, Vol. 24, No. 4, (April 2007), pp. 251-254. 
Glueck, C.J., Iyengar, S., Goldenberg, N., Smith, L.S., \& Wang, P. (2003). Idiopathic intracranial hypertension: associations with coagulation disorders and polycysticovary syndrome. Journal of Laboratory and Clinical Medicine, Vol. 142, No. 1, (July 2003), pp. 35-45.

Gonzalez-Garcia, A.O., Vizzeri, G., Bowd, C., et al. (2009). Reproducibility of RTVue retinal nerve fiber layer thickness and optic disc measurements and agreement with stratus optical coherence tomography measurements. American Journal of Ophthalmology, Vol. 147, No. 6, (June 2009), pp. 1067-1074.

Greitz, D., Wirestam, R., Franck, A., et al. (1992). Pulsatile brain movement and associated hydrodynamics studied by magnetic resonance phase imaging. The Monro-Kellie doctrine revisited. Neuroradiology, Vol. 34, No. 5, pp. 370-380.

Gupta, A.K., Gupta, A., Kumar, S., \& Lal, V. (2007). Endoscopic endonasal management of pseudotumor cerebri: is it effective? Laryngoscope, Vol. 117, No. 7, (July 2007), pp. 1138-1142.

Hamed, L.M., Glaser, J.S., Schatz, N.J., \& Perez, T.H. (1989). Pseudotumor cerebri induced by danazol. American Journal of Ophthalmology, Vol. 107, No. 2, (February 1989), pp. 105-110.

Hayreh, S.S. (1964). Pathogenesis of oedema of the optic disk (papilloedema), a preliminary report. British Journal of Ophthalmology. Vol. 48, (October 1964), pp. 522-543.

Hayreh, S.S. (1977). Optic disc edema in raised intracranial pressure. VI. Associated visual disturbances and their pathogenesis. Archives of Ophthalmology, Vol. 95, No. 9, (September 1977), pp. 1566-1579.

Higgins, J.N., Owler, B.K., Cousins, C., et al. (2002). Venous sinus stenting for refractory benign intracranial hypertension. Lancet, Vol. 359, No. 9302, (January 2002), pp. 228230.

Higgins, J.N.P., Cousins, C., Owler, B.K., et al. (2003). Idiopathic intracranial hypertension: 12 cases treated by venous sinus stenting. Journal of Neurology, Neurosurgery $\mathcal{E}$ Psychiatry, Vol. 74, No. 12, (December 2003), pp. 1662-1666.

Higgins, J.N.P., Gillard, J.H., Owler, B.K., Harkness, K., \& Pickard, J.D. (2004). MR venography in idiopathic intracranial hypertension: unappreciated and misunderstood. Journal of Neurology, Neurosurgery \& Psychiatry, Vol. 75, No. 4, (April 2004), pp. 621-625.

Holt, G.R., \& Holt, J.E. (1983). Incidence of eye injuries in facial fractures: an analysis of 727 cases. Otolaryngology- Head and Neck Surgery. Vol. 91, No. 3, (June 1983), pp. 276-279.

Hoye, V.J., III, Berrocal, A.M., Hedges, T.R., III, \& Maro-Quireza, M.L. (2001). Optical coherence tomography demonstrates subretinal macular edema from papilledema. Archives of Ophthalmology, Vol. 119, No. 9, (September 2001), pp. 1287-1290.

Huang, D., Swanson, E.A., Lin, C.P., et al. (1991). Optical coherence tomography. Science, Vol. 254, No. 5035, (November 1991), pp. 1178-1181.

Huckman, M.S., Fox, J.S., Ramsey, R.G., et al. (1976). Computed tomography in the diagnosis of pseudotumor cerebri. Radiology, Vol. 119, No. 3, (June 1976), pp. 593-597.

Huseman, C.A., \& Torkelson, R.D. (1984). Pseudotumor cerebri following treatment of hypothalamic and primary hypothyroidism. American Journal of Diseases of Children, Vol. 138, No. 10, (October 1984), pp. 927-931. 
Jacobson, D.M. (1995). Intracranial hypertension and the syndrome of acquired hyperopia with choroidal folds. Journal of Neuroophthalmology, Vol. 15, No. 3, (September 1995), pp. 178-185.

Jacobson, D.M., Karanjia, P.N., Olson, K.A., \& Warner, J.J. (1990). Computed tomography ventricular size has no predictive value in diagnosing pseudotumor cerebri. Neurology, Vol. 40, No. 9, (September 1990), pp. 1454-1455.

Jinkins, J.R. (1987). "Papilledema": neuroradiologic evaluation of optic disk protrusion with dynamic orbital CT. American Journal of Radiology, Vol. 149, No. 4, (October 1987), pp. 793-802.

Jinkins, J.R., Athale, S., Xiong, L., et al. (1996). MR of optic papilla protrusion in patients with high intracranial pressure. American Journal of Neuroradiology, Vol. 17, No. 4, (April 1996), pp. 665-668.

Johnson, L.N., Diehl, M.L., Hamm, C.W., Sommerville, D.N., \& Petroski, G.F. (2009). Differentiating optic disc edema from optic nerve head drusen on optical coherence tomography. Archives of Ophthalmology, Vol. 127, No. 1, (January 2009), pp. 45-49.

Johnston, I., Besser, M., \& Morgan, M. (1988). Cerebrospinal fluid diversion in the treatment of benign intracranial hypertension. Journal of Neurosurgery, Vol. 69, No. 2, (August 1988), pp. 195-202.

Johnston, I., Kollar, C., Dunkley, S., Assaad, N., \& Parker, G. (2002). Cranial venous outflow obstruction in the pseudotumour syndrome: incidence, nature and relevance. Journal of Clinical Neuroscience, Vol. 9, No. 3, (May 2002), pp. 273-278.

Kang, S. (2000). Efficacy of lumbo-peritoneal versus ventriculoperitoneal shunting for management of chronic hydrocephalus following aneurismal subarachnoid hemorrhage. Acta Neurochirurgia (Wien), Vol. 142, No. 1, pp. 45-49.

Karahalios, D.G., Rekate, H.L., Khayata, M.H., \& Apostolides, P.J. (1996). Elevated intracranial venous pressure as a universal mechanism in pseudotumor cerebri of varying etiologies. Neurology, Vol. 46, No. 1, (January 1996), pp. 198-202.

Karakitsos, D., Soldatos, T., Gouliamos, A., Armaganidis, A., et al. (2006). Transorbital sonographic monitoring of optic nerve diameter in patients with severe brain injury. Transplantation Proceedings, Vol. 38, No. 10, (December 2006), pp. 3700-3706.

Kelman, S.E., Heaps, R., Wolf, A., \& Elman, M.J. (1992). Optic nerve decompression surgery improves visual function in patients with pseudotumor cerebri. Neurosurgery, Vol. 30, No. 3, (March 1992), pp. 391-395.

Keltner, J.L. (1988). Optic nerve sheath decompression. How does it work? Has its time come? Archives of Ophthalmology, Vol. 106, No. 10, (October 1988), pp. 1365-1369.

Kesler, A., Ellis, M.H., Reshef, T., Kott, E., \& Gadoth, N. (2000). Idiopathic intracranial hypertension and anticardiolipin antibodies. Journal of Neurology Neurosurgery $\mathcal{E}$ Psychiatry, Vol. 68, No. 3 (March 2000), pp. 379-380.

Kesler, A., Goldhammer, Y., \& Gadoth, N. (2001). Do men with pseudomotor cerebri share the same characteristics as women? A retrospective review of 141 cases. Journal of Neuroophthalmology, Vol. 21, No.1, (March 2001), pp. 15-7.

Kesler, A., Hadayer, A., Goldhammer, Y., Almog, Y. \& Korczyn, A.D. (2004). Idiopathic intracranial hypertension: risk of recurrences. Neurology, Vol. 63, No. 9, (November 2004), pp. 1737-1739. 
Kesler, A., Kliper, E., Assayag, E.B., et al. (2010). Thrombophilic factors in idiopathic intracranial hypertension: a report of 51 patients and a meta-analysis. Blood Coagulation \& Fibrinolysis, Vol. 21. No. 4, (June 2010), pp. 328-33.

Killer, H.E., Laeng, H.R., \& Groscurth, P. (1999). Lymphatic capillaries in the meninges of the human optic nerve. Journal of Neuro-Ophthalmology, Vol. 19, No. 4, (December 1999), pp. 222-228.

King, J.O., Mitchell, P.J,, Thomson, K.R., \& Tress, B.M. (1995). Cerebral venography and manometry in idiopathic intracranial hypertension. Neurology, Vol. 45, No. 12, (December 1995), pp. 2224-2228.

Kollar, C., Parker, G., \& Johnston, I. (2001). Endovascular treatment of cranial venous sinus obstruction resulting in pseudotumor syndrome. Report of three cases. Journal of Neurosurgery, Vol. 94, No. 4, (April 2001), pp. 646-651.

Lam, B.L., Glasier, C.M., \& Feuer, W.J. (1997). Subarachnoid fluid of the optic nerve in normal adults. Ophthalmology, Vol. 104, No. 10, (October 1997), pp. 1629-1633.

Lee, A.G., \& Brazis, P.W. (2000), Magnetic resonance venography in idiopathic pseudotumor cerebri. Journal of Neuroophthalmology, Vol. 20, No. 1, (March 2000), pp. 12-13.

Lee, A.G., Pless, M., Falardeau, J., et al. (2005). The use of acetazolamide in idiopathic intracranial hypertension during pregnancy. American Journal of Ophthalmology, Vol. 139, No. 5 (May 2005), pp. 855-915.

Lessell, S. (1992). Pediatric pseudotumor cerebri (idiopathic intracranial hypertension). Survey of Ophthalmology, Vol. 37, No. 3, (November 1992), pp. 155-166.

Leung, C.K., Cheung, C.Y., Weinreb, R.N., et al. (2009). Retinal nerve fiber layer imaging with spectral-domain optical coherence tomography: a variability and diagnostic performance study. Ophthalmology, Vol. 116, No. 7, (July 2009), pp. 1257-1263.

Levine, D.N. (2000). Ventricular size in pseudotumor cerebri and the theory of impaired CSF absorption. Journal of the Neurological Sciences, Vol. 177, No. 2, (August 2000), pp. 8594.

Lorberboym,M., Lampl, Y., Kesler, A., Sadeh, M., \& Gadot, N. (2001). Benign intracranial hypertension: correlation of cerebral blood flow with disease severity. Clinical Neurology and Neurosurgery, Vol. 103, No.1, (April 2001), pp. 33-36.

Lueck, C., \& McIlwaine, G. (2002). Interventions for idiopathic intracranial hypertension. Cochrane Database System Review, Vol 3, pp. CD003434.

Maher, C.O., Garrity, J.A., \& Meyer, F.B. (2001). Refractory idiopathic intracranial hypertension treated with stereotactically planned ventriculoperitoneal shunt placement. Neurosurgery Focus, Vol. 10, No. 2, (February 2001), pp. 1-4.

Malayeri, A.A., Bavarian, S., \& Mehdizadeh, M. (2005). Sonographic evaluation of optic nerve diameter in children with raised intracranial pressure. Journal of Ultrasound Medicine, Vol. 24, No. 2, (February 2005), pp. 143-147.

Malm, J., Kristensen, B., Markgren, P., \& Ekstedt, J. (1992). CSF hydrodynamics in idiopathic intracranial hypertension: a longterm study. Neurology, Vol. 42, No. 4, (April 1992), pp. 851-858.

Mashima Y, Oshitari K, Imamura Y, et al.(1996) High-resolution magnetic resonance imaging of the intraorbital optic nerve and subarachnoid space in patients with papilledema and optic atrophy. Arch Ophthalmol Vol. 114, No. 10, (October 1996), pp. 1197-203. 
Mathew, N.T., Ravishankar, K., \& Sanin, L.C. (1996). Coexistence of migraine and idiopathic intracranial hypertension without papilledema. Neurology, Vol. 46, No. 5, (May 1996), pp. 1226-1230.

Mathews, M.K., Sergott, R.C. \& Savino, P.J. (2003). Pseudotumor cerebri. Current Opinion in Ophthalmology, Vol. 14, No. 6, (December 2003), pp. 364-370.

Mattle, H.P.,Wentz ,K.U., Edelman, R., et al. (1991). Cerebral venography with MR. Radiology, Vol. 178, No. 2, (February 1991), pp. 453-458.

Martins, A.N. (1973). Resistance to drainage of cerebrospinal fluid: clinical measurement and significance. Journal of Neurology Neurosurgery \& Psychiatry, Vol.36, No. 2, (April 1973), pp. 313-318.

Maxner, C.E., Freeman, M.I. \& Corbett, J.J. (1987). Asymmetric papilledema and visual loss in pseudotumor cerebri. Canadian Journal Neurological Sciences, Vol. 4, No. 4, (November 1987), pp. 593-596.

McHenry, J.G., \& Spoor, T.C. (1993). Optic nerve sheath fenestration for treatment of progressive ischemic optic neuropathy. Archives of Ophthalmology, Vol. 111, No. 12, (December 1993), pp. 1601-1602.

Menke, M.N., Knecht, P., Sturm,V., Dabov, S., \& Funk, J. (2008). Reproducibility of nerve fiber layer thickness measurements using 3D fourier-domain OCT. Investigative Ophthalmology \& Visual Science, Vol. 49, No. 12, (December 2008), pp. 5386-5391.

Metellus, P., Levrier, O., Fuentes, S., et al. (2005). Endovascular treatment of benign intracranial hypertension by stent placement in

the transverse sinus: therapeutic and pathophysiological considerations illustrated by a case report [in French]. Neurochirurgie, Vol. 51, No. 2, (May 2005), pp. 113-120.

Mokri, B. (2001). The Monro-Kellie hypothesis: applications in CSF volume depletion. Neurology, Vol. 56, No. 12, (June 2001), pp. 1746-1748.

Morrice, G., Havener, W.H., \& Kapetanxky, F. (1960). Vitamin A intoxication as a cause of pseudotumor cerebri. Journal of the American Medical Association, Vol. 173, (August 1960), pp. 1802-1805.

Munk, P.L., Vellet, A.D., Levin, M., Lin, D.T., \& Collyer, R.T. (1991). Sonography of the eye. American Journal of Roentgenology, Vol. 157, No. 5, (November 1991), pp. 1079-1086.

Nedelmann, M., Kaps, M., \& Mueller-Forell, W. (2009). Venous obstruction and jugular valve insufficiency in idiopathic intracranial hypertension. Journal of Neurology, Vol. 256, No. 6, (June 2009), pp. 964-969.

Neville, B.G.R., \& Wilson, J. (1970). Benign intracranial hypertension following corticosteroid withdrawal in childhood. British Medical Journal, Vol. 3, No. 5722, (September 1970), pp. 554-556.

Nonne, M. (1904). Ueber Falle vom Symptomkomplex "tumor cerebri" mit Ausgang in Heilung (pseudotumor cerebri). Dtsch Z Nervenheil, Vol. 27, pp. 169-216.

Ogungbo, B., Roy, D., Gholkar, A., et al. (2003). Endovascular stenting of the transverse sinus in a patient presenting with benign intracranial hypertension. British Journal of Neurosurgery, Vol. 17, No. 6, (December 2003), pp. 565-568.

Ophir, A., Karatas, M., Ramirez, J.A., \& Inzelberg, R. (2005). OCT and chronic papilledema. Ophthalmology, Vol. 112, No. 12, (December 2005), pp. 2238.

Paquet, C., Poupardin, M., Boissonnot, M., et al.(2008). Efficacy of unilateral stenting in idiopathic intracranial hypertension with stenosis: a case report. European Neurology, Vol. 60, No. 1, (May 2008), pp. 47-48. 
Pearson, P.A., Baker, R.S., Khorram, D., \& Smith, T.J. (1991). Evaluation of optic nerve sheath fenestration in pseudotumor cerebri using automated perimetry. Ophthalmology, Vol. 98, No. 1, (January 1991), pp. 99-105.

Pipe, J.G. (2001). Limits of time-of-flight magnetic resonance angiography. Topics in Magnetic Resonance, Vol. 12, No. 13, (June 2001), pp. 163-174.

Plotnik, J.L., \& Kosmorsky, G.S. (1993). Operative complications of optic nerve sheath decompression. Ophthalmology, Vol. 100, No. 5, (May 1993), pp. 683-690.

Quincke, H. (1893). Meningitis serosa. Samml Klin Vortr, Leipzig, Vol. 67: Inn Med 23:655.

Quincke H (1897) Ueber meningitis serosa und verwande Zustande. Dtsch Z Nervenheil, Vol. 9, pp. 140-168.

Rabb, F., Burton, T.C., Schatz, H., \& Yannuzzi, L.A. (1978) Fluorescein angiography of the fundus: A schematic approach to interpretation. Survey of Ophthalmology, Vol. 22, No. 6, (May 1978), pp. 387-403.

Radhakrishnan, K., Ahlskog, J.E., Cross, S.A., et al. (1993). Idiopathic intracranial hypertension (pseudotumor cerebri). Descriptive epidemiology in Rochester, Minn, 1976 to 1990. Archives of Neurology, Vol. 50, No. 1, (January 1993), pp. 78-80.

Radhakrishnan, K., Ahlskog, J.E., Garrity, J.A., \& Kurland, L.T. (1994). Idiopathic intracranial hypertension. Mayo Clinic Proceedings, Vol. 69, No. 2, (February 1994), pp. 169-180.

Raichle, M.E., Grubb, R.L., Jr, Phelps, M.E., Gado, M.H. \& Caronna, J.J. (1978). Cerebral hemodynamics and metabolism in pseudotumor cerebri. Annals of Neurology, Vol. 4, No. 2, (August 1978), pp. 104-111.

Rajpal, S., Niemann, D.B., \& Turk, A.S. (2005). Transverse venous sinus stent placement as treatment for benign intracranial hypertension in a young male: case report and review of the literature. Journal of Neurosurgery, Vol. 102, No. 3(suppl), (April 2005), pp. 342-346.

Rangwala, L.M., \& Liu, G.T. (2007). Pediatric idiopathic intracranial hypertension. Survey of Ophthalmology, Vol. 52, No. 6, (November 2007), pp. 597-617.

Rebolleda, G., \& Munoz-Negrete, F.J. (2009). Follow-up of mild papilledema in idiopathic intracranial hypertension with optical coherence tomography. Investigative Ophthalmology \& Visual Science, Vol. 50, No. 11, (November 2009), pp. 5197-5200.

Reid, A.C., Teasdale, G.M., Matheson, M.S., \& Teasdale, E.M. (1981). Serial ventricular volume measurements: further insights into the aetiology and pathogenesis of benign intracranial hypertension. Journal of Neurology Neurosurgery \& Psychiatry, Vol. 44, No. 7, (July 1981), pp. 636-40.

Ridsdale, L., \& Moseley, I. (1978). Thoracolumbar intraspinal tumours presenting features of raised intracranial pressure. Journal of Neurology Neurosurgery $\mathcal{E}$ Psychiatry, Vol. 41, No. 8, (August 1978), pp. 737-745.

Rogers, A.H., Rogers, G.L., Bremer, D.L., \& McGregor, M.L. (1999). Pseudotumor cerebri in children receiving recombinant human growth hormone. Ophthalmology, Vol. 106, No. 6, (June 1999), pp. 1186-1190.

Ropper, A.H., \& Marmarou, A. (1984). Mechanism of pseudotumor in Guillain-Barré syndrome. Archives of Neurology, Vol. 41, No. 3, (March 1984), pp. 259-261,

Ross, D.A \& Wilson, C.B. (1988). Results of transsphenoidal microsurgery for growth Hormone secreting pituitary adenoma in a series of 214 patients. Journal of Neurosurgery, Vol. 68, No. 6, (June 1988), pp. 854-867. 
Rothman, M.I., \& Zoarski, G.H. (2003). The orbit. In: Textbook of Radiology and Imaging Vol. 2. 7th ed, Sutton, D. pp. 1573-1595, Churchill Livingstone, London.

Salgarello T., Tamburrelli, C., Falsini, B., Giudiceandrea, A., \& Colotto, A. (1996). Optic nerve diameters and perimetric thresholds in idiopathic intracranial hypertension. British Journal of Ophthalmology, Vol. 80, No 6, (June 1996), pp. 509-514.

Saul, R.F., Hamburger, H.A., \& Selhorst, J.B. (1985). Pseudotumor cerebri secondary to lithium carbonate. Journal of the American Medical Association, Vol. 253, No. 19, (May 1985), pp. 2869-2870.

Schuman, J.S., Puliafito, C.A., \& Fujimoto, J.G. (2004). Optical coherence tomography of ocular diseases. Slack Incorporated, Thorofare, NJ.

Silbergleit, R., Junck, L., Gebarski, S.S., \& Hatfield, M.K. (1989). Idiopathic intracranial hypertension (pseudotumor cerebri): MR imaging. Radiology, Vol. 170, No. 1, (January 1989), pp. 207-209.

Soelberg Sørensen, P., Gjerris, F., \& Svenstrup, B. (1986). Endocrine studies in patients with pseudotumor cerebri. Estrogen levels in blood and cerebrospinal fluid. Archives of Neurology, Vol. 43, No. 9, (September 1986), pp. 902-906.

Soldatos, T., Karakitsos, D., Chatzimichail, K., et al. (2008). Optic nerve sonography in the diagnosis evaluation of adult brain injury. Critical Care, Vol. 12, No. 3, (May 2008), pp. R67

Sorensen, P.S., Thomsen, C., \& Gjerris, F. et al. (1989). Increased brain water content in pseudotumor cerebri measured by magnetic resonance imaging of brain water self diffusion. Neurology Research, Vol. 11, No. 3, (September 1989), pp. 160-4.

Spector, R.H., \& Carlisle, J. (1984). Pseudotumor cerebri caused by a synthetic vitamin A preparation. Neurology, Vol. 34, No. 11, (November 1984), pp. 1509-1511.

Spence, J.D., Amacher, A.L \& Willis, N.R. (1980). Benign intracranial hypertension without papilledema: role of 24-hour cerebrospinal fluid pressure monitoring in diagnosis and management. Neurosurgery Vol.7, No. 4, (October 1980), pp. 326-336.

Spoor, T.C., \& McHenry, J.G. (1993). Long-term effectiveness of optic nerve sheath decompression for pseudotumor cerebri. Archives of Ophthalmology, Vol. 111, No. 5, (May 1993), pp. 632-635.

Stone, M.B. (2009). Ultrasound diagnosis of papilledema and increased intracranial pressure in pseudotumor cerebri. American Journal of Emergency Medicine, Vol. 27, No. 3, (March 2009), pp. e1-376

Sussman, J., Leach, M., Greaves, M., Malia, R., \& Davies-Jones, GA. (1997). Potentially prothrombotic abnormalities of coagulation in benign intracranial hypertension. Journal of Neurology Neurosurgery \& Psychiatry, Vol. 62, No. 3, (March 197), pp. 229233.

Tang, R.A., Dorotheo, E.U., Schiffman, J.S., \& Bahrani, H.M. (2004). Medical and surgical management of idiopathic intracranial hypertension in pregnancy. Current Neurology and Neuroscience Reports, Vol. 4, No. 5, (September 2004), pp. 398-409.

Tibussek, D., Schneider, D.T., Vandemeulebroecke, N., et al. (2010). Clinical spectrum of the pseudotumor cerebri complex in children. Child's Nervous System, Vol. 26, No. 3, (March 2010), pp. 313-321.

Tulipan, N., Lavin, P.J., \& Copeland M.(1998). Stereotactic ventriculoperitoneal shunt for idiopathic intracranial hypertension: technical note. Neurosurgery Vol. 43, No. 1, (July 1998), pp. 175-176. 
Visani, G., Manfroi, S., Tosi, P., \& Martinelli, G. (1996). All-trans-retinoic acid and pseudotumor cerebri. Leukemia \& Lymphoma. Vol. 23, No. 5-6, (November 1996), pp. 437-442.

Walker, R.W.H. (2001). Idiopathic intracranial hypertension: any light on the mechanism of the raised pressure? Journal of Neurology Neurosurgery \& Psychiatry, Vol. 71, No. 1, (July 2001), pp. 1-5.

Wall, M., \& George, D. (1987). Visual loss in pseudotumor cerebri. Incidence and defects related to visual field strategy. Archives of Neurology, Vol. 44, No. 2, (February 1987), pp. 170-175.

Wall, M., \& George, D. (1991). Idiopathic intracranial hypertension. A prospective study of 50 patients. Brain. Vol. 114, No. 1A, (January 1991), pp. 155-180.

Wall, M., \& White, W.N. II. (1998). Asymmetric papilledema in idiopathic intracranial hypertension: prospective interocular comparison of sensory visual function. Investigative Ophthalmology \& Visual Science, Vol. 39, No. 1, (January 1998), pp. 134142.

Walsh, F.B., Clark, D.B., Thompson, R.S., \& Nicholson, D.H. (1965). Oral contraceptives and neuro-ophthalmologic interest. Archives of Ophthalmology, Vol. 74, No. 5, (November 1965), pp. 628-640.

Wang, S.J., Silberstein, S.D., Patterson, S., \& Young, W.B. (1998). Idiopathic intracranial hypertension without papilledema: a case-control study in a headache center. Neurology Vol. 51, No. 1, (July 1998), pp.245-249.

Weisberg, L.A. (1985). Computed tomography in benign intracranial hypertension. Neurology, Vol. 35, No. 7, (July 1985), pp.1075-8.

Wessel, K., Thron, A., Linden, D., et al. (1987). Pseudotumor cerebri: clinical and neuroradiological findings. European Archives of Psychiatry $\&$ Neurological Sciences, Vol. 237, No. 1, pp. 54-60.

Winner, P., \& Bello, L. (1996). Idiopathic intracranial hypertension in a young child without visual symptoms or signs. Headache, Vol. 36, No. 9, (October 1996), pp. 574-576.

Winrow, A.P., \& Supramaniam, G. (1990). Benign intracranial hypertension after ciprofloxacin administration. Archives Disease of Childhood, Vol. 65, No. 10, (October 1990), pp.1165-1166.

Worsham, F. Jr, Beckman, E.N., \& Mitchell, E.H. (1978). Sacrococcygeal teratoma in a neonate. Association with maternal use of acetazolamide. Journal of the American Medical Association, Vol. 240, No. 3, (July 1978), pp. 251-2.

Yaşargil, M.G., Curcic, M., Kis, M., et al. (1990). Total removal of craniopharyngiomas. Approaches and long-term results in 144 patients. Journal of Neurosurgery, Vol. 73, No. 1, (July 1990), pp. 3-11. 


\title{
Dopamine Transporter Imaging for Distinguishing Between Idiopathic Parkinson's Disease and Secondary Parkinsonism
}

\author{
Chin-Chang Huang1, Tzu-Chen Yen² and Chin-Song Lu ${ }^{3}$ \\ ${ }^{1}$ Department of Neurology, Chang Gung Memorial Hospital \\ and Chang Gung University College of Medicine, Taipei \\ ${ }^{2}$ Department of Nuclear Medicine, Chang Gung Memorial \\ Hospital and Chang Gung University \\ ${ }^{3}$ Department of Neurology, Chang Gung Memorial Hospital \\ and Chang Gung University
}

Taiwan

\section{Introduction}

Idiopathic Parkinson's disease (IPD), first described by James Parkinson in 1817, is a sporadic neurodegenerative disorder. The main clinical features include masked face, resting tremor, bradykinesia, rigidity, festinating gait, and loss of postural reflexes. The clinical features are most insidious and usually asymmetric at onset. The asymmetry may persist even in a late stage and progress slowly. The pathological findings are characterized by loss of pigmented dopamine neurons in the substantia nigra, particularly the pars compacta and locus ceruleus, and the presence of Lewy bodies. The cause of IPD remains unknown.

Parkinsonism (PM) is not a single disease but a common clinical presentation. The clinical syndrome is characterized by tremors, bradykinesia, rigidity, and postural instability. Exposure to toxins such as 1-methyl-4-phenyl-1,2,3,6-tetrahydropyridine (MPTP), which was sold as "synthetic heroin," manganese $(\mathrm{Mn})$, carbon disulfide $\left(\mathrm{CS}_{2}\right)$, carbon monoxide (CO), methanol, cyanide, and other organic solvents may cause brain damage, leading to features similar to PM. Many neurodegenerative disorders may present with PM, including progressive supranuclear palsy (PSP), multiple system atrophy (MSA), spinocerebellar atrophy (SCA), and corticobasal sundrome (CBS). Several genetic diseases, including doparesponsive dystonia (DRD), Wilson's disease (WD), and Huntington's disease (HD), may cause degeneration in the basal ganglia or affect the dopaminergic pathway. Furthermore, some dementia syndromes may be associated with PM, including vascular parkinsonism (multiple infarct parkinsonism), dementia with Lewy bodies (DLB), and frontotemporal dementia, and parkinsonism linked to chromosome 17 (FTD-17).

The main treatment of IPD includes the use of dopamine, dopamine agonists, monoamine oxidase inhibitors, and catechol-o-methyltransferase inhibitors. The above medications are 
usually effective in IPD patients, whereas their effects are usually limited in patients with secondary parkinsonism. Although definite diagnosis of IPD is based on typical pathological findings, early diagnosis is very important as it leads to early treatment.

IPD and PM are distinguished on the basis of the onset of symptoms, symmetry of clinical features, characteristics of tremors, rigidity, bradykinesia, and other associated symptoms, such as cognitive impairment, limitation of eye ball movement, ataxia, and autonomic dysfunction. In addition, information concerning family history, smoking and alcohol exposure, diabetes with hypertension, and exposure to toxic substances are also essential for diagnosis. Despite differences in the clinical features of IPD and PM, definite diagnosis may be difficult; therefore, reliable imaging is helpful for early and accurate diagnosis.

\section{Dopamine transporter (DAT) scan}

Dopamine transport is one of the primary mechanisms that can modulate the dopaminergic tone via an active transport system that involves the re-uptake of dopamine. Cocaine analogues including (1r) $2 \beta$-carbomethoxy-3 $\beta$-(4-iodophenyl) tropane ( $\beta$-CIT), and 123I-FPCIT have been developed as single photon emission computed tomography (SPECT) imaging agents. Both agents can bind at the DAT site of dopamine neuron terminals in normal human subjects and IPD patients. In addition, $99 \mathrm{mTc}$-TRODAT-1 is a promising 99mTc-labelled radiotracer for imaging DAT in the human brain. Since a cyclotron and welltrained radiochemists are required for clinical usage of ${ }^{123}$ I- $\beta$-CIT and 123I-FP-CIT SPECT, they are more difficult to use in clinical settings. ${ }^{99 \mathrm{mTc}-T R O D A T-1}$ is much easier to prepare and can be made in many nuclear medicine departments. Previous studies have shown that 99mTc-TRODAT-1 is very reliable in detecting dopamine neurons in the striatum; therefore, it is an important tool for understanding the role of DAT in various neurological diseases.

\section{DAT scan in IPD}

Similar to ${ }^{123}$ I- $\beta$-CIT and ${ }^{123}$ I-FP-CIT, $99 \mathrm{mTc}$-TRODAT-1 activity in the basal ganglia can demonstrate a stable target/non-target ratio, and at a reduced level in IPD patients than in healthy volunteers. Serial 99mTc-TRODAT-1 SPECT images taken 2, 3, and $4 \mathrm{~h}$ after injection of $925 \mathrm{MBq}$ 99mTc-TRODAT into healthy volunteers show a consistent increase of the uptake with time. Furthermore, the relative concentration of 99mTc-TRODAT- 1 in the basal ganglia regions decreases significantly with age in healthy volunteers. The rate of decline is significantly faster in young individuals than in the elderly. The effect seems to occur during young adulthood, particularly in individuals younger than 40 years. The putamen/occipital and caudate/occipital ratios show a statistically significant difference between IPD patients and healthy volunteers.

\section{Secondary parkinsonism}

\subsection{Toxin-induced PM}

\subsubsection{1-Methyl-4-phenyl-1,2,3,6-tetrahydropyridine (MPTP)}

MPTP is a byproduct of a meperidine analogue, 1-methyl-4-proprion-oxypeperidine (MPPP), which is a synthetic heroin. Injection of the contaminated synthetic drug may cause the victims to develop acute severe parkinsonian features such as bradykinesia and severe rigidity in about 7 days. Since its discovery, MPTP has been used in animal models of 
parkinsonism, which is responsive to dopamine and dopamine agonist treatment. Although dementia and autonomic dysfunction, typical dyskinesia, prominent wearing off phenomena, and psychiatric impairments in MPTP victims occur more rapidly than in subjects with IPD, the clinical features of these individuals are indistinguishable from those of IPD patients. The MPTP toxin may damage the dopamine neurons in the substantia nigra via 1-methyl-4-phenyl-pyridinium (MPP+), a metabolite of MPTP, that may inhibit the production of ATP and stimulate the formation of superoxide radicals. The neurotoxic effect of MPTP is permanent, even though the patients have an excellent response to levodopa treatment. 6F-Dopa positron emission tomography (PET) of the brain showed that a subclinical exposure to MPTP might result in a reduction of fluorodopa uptake in the striatum. In some experimental studies that used brain SPECT, 99m Tc-TRODAT-1 binding was significantly lower in the MPTP-treated monkeys than in the control monkeys.

\subsubsection{Manganese (Mn) intoxication}

Chronic exposure to manganese may induce parkinsonism similar to IPD. However, the clinical features of manganism, including lower body parkinsonism, frequent gait disturbance (particularly cock gait), increased dystonia, and reduced action tremor, also differ from IPD. In addition, in Mn-induced PM, a reduced response to anti-parkinsonian drugs, gait-freezing during turns, and difficulty in walking backwards were also noted. Although relative symmetry was noted, clinical asymmetry was also reported. Unlike patients with multiple system atrophy, patients with manganism did not show postural hypotension, sexual dysfunction, and sphincter disturbance.

Brain magnetic resonance imaging (MRI) is a promising technique to demonstrate the presence of manganese in the brain. T1-weighted MR images showed an increased intensity in the globus pallidus area of welders, smelters, patients undergoing parenteral nutrition, and in patients with hepatic failure. However, the increase in signal intensity in T1weighted MR images only indicates an exposure to manganese in recent months but does not indicate manganism.

Previous PET scans with 6-FD had shown a normal nigrostriatal dopaminergic uptake in the caudate or putamen in manganism patients. In addition, brain PET scans with raclopride showed a mild decrease (less than 20\%) of caudate dopamine D2 receptors. However, the minimal decrease of D2 receptor density could not account for the prominent clinical features in manganese intoxication patients.

Both 6-FD PET and DAT are sensitive detectors for dopamine neurons. In a previous study, DAT density with 123I- $\beta$-CIT SPECT was decreased in PM patients with manganese exposure. However, these findings seemed to be more consistent with IPD than with Mninduced parkinsonism. The brain 99mTc-TRODAT-1 SPECT showed no significant changes in the putamen and the putamen/caudate ratio of manganism patients and normal controls. However, a statistically significant decrease was noted in the uptake of 99mTc-TRODAT-1 in the putamen area of IPD patients than in the manganism patients. Figure 1 shows the DAT findings in a manganism patient, an IPD patient, and a normal control. The data indicate that presynaptic dopaminergic terminals are not the main targets of chronic manganese intoxication. Pathologic changes in monkeys after manganese chloride injection included prominent gliosis in the globus pallidus and in the substantia nigra pars reticularis that differs from the target lesion-substantia nigra pars compacta in IPD. 


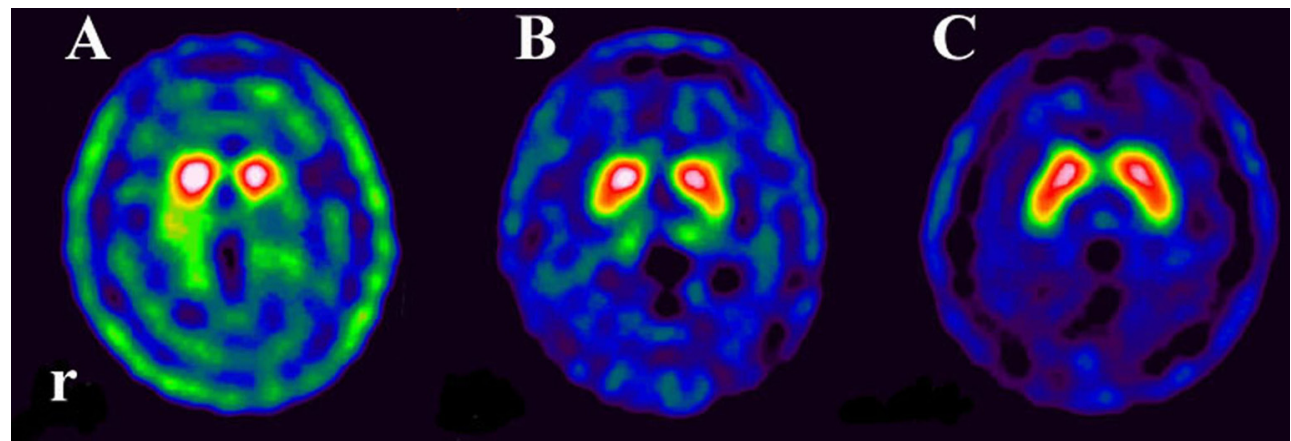

Fig. 1. The uptakes of 99mTc -TRODAT-1 brain SPECT were decreased in the corpus striatum particularly in the left side in a PD patient (A), and nearly normal in a patient with chronic manganism (B) and a normal control (C). $r=$ right.

\subsubsection{Carbon disulfide $\left(\mathrm{CS}_{2}\right)$ intoxication}

$\mathrm{CS}_{2}$ is a colorless liquid organic solvent frequently used in the production of viscose rayon fibers and cellophane films. Acute exposure to $\mathrm{CS}_{2}$ may cause psychosis, delirium, seizures,

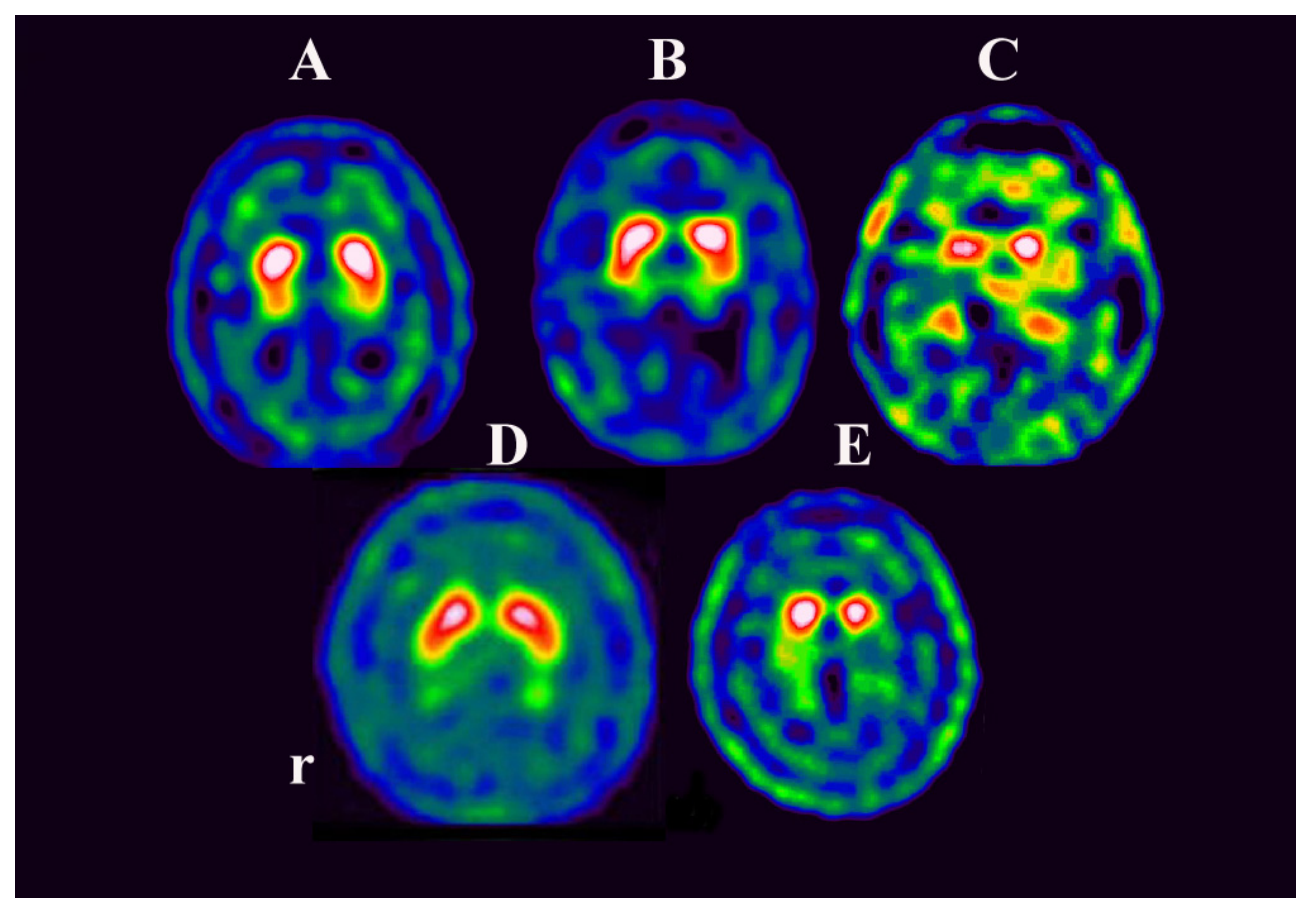

Fig. 2. Normal DAT bindings with 99mTc -TRODAT-1 brain SPECT were noted in 2 patients with $\mathrm{CS}_{2}$ intoxication (A), and (B) but a decreased DAT binding in another patient who had IPD with $\mathrm{CS}_{2}$ exposure $(\mathrm{C})$, as compared with those of a normal control (D) and a PD patient (E). r=right. 
and even death. Chronic exposure to $\mathrm{CS}_{2}$ manifests as a diffuse encephalopathy including parkinsonism, intention tremor, emotional lability, and neurobehavioral disorders as well as polyneuropathy. Brain MRI may reveal diffuse hyperintense lesions in T2-weighted images in the subcortical white matter, basal ganglia, and brainstem. A brain CT perfusion study showed a decrease of regional cerebral flow and prolonged regional mean transit time in the subcortical white matter and the basal ganglia. The diffuse white matter lesions are better explained by vascular insufficiency than demyelination. In $\mathrm{CS}_{2}$ intoxicated patients with parkinsonism, brain 99mTc-TRODAT-1 SPECT showed a normal uptake of the dopamine transporter, indicating a normal presynaptic dopaminergic pathway (Figure 2). Therefore, $\mathrm{CS}_{2}$ intoxication-induced parkinsonism is probably due to post-synaptic lesions in the basal ganglia rather than the presynaptic dopaminergic pathway.

\subsubsection{Carbon monoxide (CO) intoxication}

Acute CO intoxication may induce hypoxic changes in the brain with variable degree of consciousness disturbance from confusion, delirium, and stupor to deep coma. Most patients recover after appropriate oxygen therapy; however, sequelae such as dystonia and cognitive impairment may persist. Approximately $0.2-40 \%$ of survivors developed delayed encephalopathy within 2 months. The common manifestations include cognitive changes, sphincter disturbance, akinetic mutism, and parkinsonian features. Brain MRI studies showed hyperintense lesions in the basal ganglia, particularly in the globus pallidus and subcortical white matter. A steady improvement was found after 1-2 years of supportive therapy; however, residual parkinsonism may develop in some patients. Moreover, a poor response to levodopa is noted. Brain 99mTc-TRODAT-1 may show a normal uptake in the basal ganglia, indicating that the presynaptic pathway of the nigrostriatral system is normal.

\subsubsection{Others: Methanol and cyanide}

Acute intoxication with methanol may cause metabolic acidosis and severe anionic gaps, leading to blindness and parkinsonism including masked face, rigidity, bradykinesia, gait disturbance, and dystonia. Brain MRI may show damage in the bilateral putaminal areas. Acute cyanide intoxication may also cause parkinsonism such as hypomimia, rigidity, and gait disturbance within a few days, and subsequent dystonia and dementia. The response to levodopa therapy is usually disappointing. Table 1 summarizes the clinical features and DAT findings in toxin-induced PM.

\subsection{Other neurodegenerative parkinsonian syndromes \\ 4.2.1 Progressive supranuclear palsy (PSP)}

PSP, first described in the early 1900s, is a devastating neurodegenerative disease. In 1963, Steele, Richardson, and Olszewski reported a series of patients with pathologically confirmed heterogeneous system degeneration. The syndrome is characterized by parkinsonism, axial rigidity, frequent falls, vertical gaze palsy, pseudobulbar palsy, and dementia. In addition, atypical features include asymmetrical parkinsonism, dystonia, tremor, apraxia, and pure akinesia. The pathological changes include neuronal loss, neurofibrillary tangles, and gliosis in the basal ganglia, brainstem, and cerebral cortex. The response to levodopa treatment for parkinsonian symptoms is usually poor. The most 
common subtypes of PSP syndrome include Richardson's syndrome (RS) and progressive supranuclear palsy-parkinsonism (PSP-P). The clinical features of RS are similar to the classic type of PSP, whereas PSP-P has features similar to IPD, such as asymmetric onset of symptoms, tremor, and initial response to levodopa.

\begin{tabular}{|c|c|c|c|c|c|}
\hline & MPTP & $\mathrm{Mn}$ & $\mathrm{CS}_{2}$ & $\mathrm{CO}$ & Methanol \\
\hline \multicolumn{6}{|l|}{ Clinical features } \\
\hline Parkinsonism & + & + & + & + & + \\
\hline Rigidity & + & + & + & + & + \\
\hline Tremor & + & Less & - & - & - \\
\hline Bradykinesia & + & + & + & + & + \\
\hline $\begin{array}{l}\text { Loss of postural } \\
\text { reflex }\end{array}$ & + & + & + & + & + \\
\hline Mental disorders & + & + & + & + & + \\
\hline Cerebellar sign & - & Less & + & - & - \\
\hline Polyneuropathy & - & - & + & - & - \\
\hline $\begin{array}{l}\text { Autonomic } \\
\text { dysfunction }\end{array}$ & + & + & + & - & - \\
\hline \multicolumn{6}{|l|}{ Neuroimaging } \\
\hline Brain CT/MRI & $\mathrm{N} / \mathrm{N}$ & $\begin{array}{c}\mathrm{N} /+ \\
\text { (T1 high) }\end{array}$ & $\begin{array}{c}+/+ \\
\text { (Vascular } \\
\text { changes) }\end{array}$ & $\begin{array}{l}+/+ \\
(\mathrm{GP})\end{array}$ & $\begin{array}{c}+/+ \\
\text { (Putamen } \\
\text { lesion) }\end{array}$ \\
\hline $\begin{array}{l}\text { DAT uptake in } \\
\text { striatum }(99 \mathrm{mTc}- \\
\text { TRODAT-1 SPECT) }\end{array}$ & $\begin{array}{l}\text { Decrease } \\
\quad \text { (in } \\
\text { monkey) }\end{array}$ & $\mathrm{N}$ & $\mathrm{N}$ & $\mathrm{N}$ & NA \\
\hline Prognosis & Permanent & Deterioration & Poor & $\begin{array}{l}\text { Partial } \\
\text { recovery }\end{array}$ & Blindness \\
\hline $\begin{array}{l}\text { Response to } \\
\text { Levodopa }\end{array}$ & Good & No & No & No & Poor \\
\hline Source of exposure & $\begin{array}{l}\text { Synthetic } \\
\text { heroin }\end{array}$ & $\begin{array}{l}\text { Smelter, } \\
\text { miner, welder }\end{array}$ & $\begin{array}{l}\text { Viscose } \\
\text { rayon } \\
\text { worker }\end{array}$ & $\begin{array}{l}\text { Accidental, } \\
\text { suicidal } \\
\text { attempts }\end{array}$ & Accidental \\
\hline
\end{tabular}

+: presence; -: absence; GP: globus pallidus; N: normal; NA: not available

MPTP: 1-methyl-4-phenyl-1, 2, 3, 6-tetrahydropyridine; Mn: manganese; $\mathrm{CS}_{2}$ : carbon disulfide; $\mathrm{CO}$ : carbon monoxide.

Table 1. Toxins induced secondary parkinsonism

Dopamine transporter (DAT) scans with ${ }^{123}$ I- $\beta$-CIT showed a reduction of DAT activities in the caudate and putamen areas, particularly the caudate areas in PSP patients. However, the dopamine D2 receptor images with IBZM were variable. The inconsistent findings are probably because of the grouping of both RS and PSP-P types. In our previous studies with 
99mTc-TRODAT-1 scans, the mean striatal uptake was reduced in the RS group than in the PSP-P group, even though uptake did not reach statistical significance. The putamen/caudate ratios were significantly different between IPD and PSP patients. However, there was no difference between RS and PSP-P patients. In the IBZM scan, the uptake was significantly reduced in the RS group, but mildly increased in the PSP-P group. The data indicate that DAT imaging is helpful to distinguish PSP-P from IPD patients in the early stages. DAT activities showed a greater decrease in the RS group than in the PSP-P group. In addition, activities of the D2 receptor were reduced in the RS group but not in the PSP-P group.

\subsubsection{Multiple system atrophy (MSA)}

Multiple system atrophy (MSA) was originally described as 3 distinct disorders: olivopontocerebellar atrophy (OPCA), Shy-Drager syndrome (SDS), and striatonigral degeneration (SND). MSA is a sporadic progressive neurodegenerative disease characterized by variable degrees of parkinsonism, cerebellar ataxia, and autonomic dysfunction. According to the motor dysfunction, MSA can be divided into 2 subtypes: parkinsonian type (MSA-P) and cerebellar type (MSA-C). The pathologic changes reveal a variable involvement of neuronal loss in the corpus striatum, globus pallidus, substantia nigra, locus ceruleus, Edinger-Westphal nucleus, olivary nuclei, cerebellar peduncles, cerebellar Purkinje cells, intermediolateral column, and Onuf's nucleus of the spinal cord. The diagnosis of MSA is still based on clinical criteria. The clinical distinction between MSA$\mathrm{P}$ and IPD is sometimes difficult, particularly in the early stages, because both have a good response to levodopa.

In IPD patients, a severe reduction of DAT uptake in the putamen and relative sparing of the caudate nucleus is noted. However, a variable uptake of $6-18 \mathrm{~F}-$ fluorodopa was noted in MSA patients. $99 \mathrm{mTc}$-TRODAT-1-brain SPECT revealed a more symmetrical reduction of the striatal binding in MSA-P and MSA-C patients; this was in contrast with the greater asymmetric reduction seen in IPD patients. In addition, the reduction of $\mathrm{P} / \mathrm{O}$ and $\mathrm{S} / \mathrm{O}$ ratios is greater for the MSA-P patients than for the MSA-C patients. $\mathrm{P} / \mathrm{C}$ ratios showed that MSA$\mathrm{P}$ and IPD patients have a similar pattern of nigral involvement but that MSA-C patients had a different pattern.

\subsubsection{Spinocerebellar degeneration (SCA)}

Hereditary ataxias are a clinically and genetically heterogeneous group of disorders transmitted most frequently as autosomal dominant or autosomal recessive traits. Three common phenotypes including SCA1, SCA2 and SCA3 (Machado-Joseph disease, MJD) are characterized by variable degrees of cerebellar signs, pyramidal dysfunction, anterior horn cell involvement, and/or peripheral neuropathy but some patients may develop parkinsonian symptoms, which may also respond to levodopa treatment.

\subsubsection{SCA1}

The early pictures include cerebellar syndrome and upper motor neuron signs. Later, ophthalmoplegia, slow saccades, and a sensory predominant polyneuropathy, amyotrophy, chorea, and dystonia may develop. Dysarthria, dysphagia, and cognitive impairment are also noted. The gene mutation is an unstable CAG expansion in the ataxin 1 gene on chromosome 6p. Brain 99mTc-TRODAT-1 SPECT imaging revealed a decrease of dopamine transport in the striatum. 


\subsubsection{SCA2}

SCA2 has a wider phenotypical spectrum than SCA1. The presence of slow saccades and peripheral neuropathy early in the disease may lead to the diagnosis of SCA2. In addition, dystonia, levodopa-responsive parkinsonism, and cognitive decline are also noted. The mutation is a CAG expansion in the ataxin 2 gene on chromosome 12 with alleles ranging from 32-64 (normal, 15-31). 99mTc-TRODAT-1 SPECT of the brain showed a significantly asymmetric reduction of the striatal dopamine transporter in these patients; this was similar to the finding in IPD patients. The presynaptic impairment of nigrostriatal function is probably the reason for levodopa responsiveness.

\subsubsection{SCA3}

This is the most prevalent type of spinocerebellar ataxia. The clinical manifestations include cerebellar and brainstem signs such as facial and tongue fasciculations or myokymia, with facial atrophy, and dysphonia. Non-cerebellar eye signs such as slow saccades, impairment in conjugate eyeball movement, ophthalmoparesis, ptosis, eyelid retraction, and blepharospasm have also been reported. Dystonia is commonly seen. In addition, the parkinsonian features may respond to dopamine therapy. The mutation is an unstable CAG expansion in the ataxia 3 gene on chromosome 14 with 53-86 CAG repeats (normal limit < 47). ${ }^{99 \mathrm{mTc}-T R O D A T-1}$ scan of the brain revealed a significant decrease in the uptake of tracers in MJD patients than in healthy controls. The decreased uptakes of 99mTc-TRODAT-1 indicated a defect in the nigrostriatal dopaminergic pathway in symptomatic MJD patients with and without extrapyramidal signs. However, the severity of the DAT abnormality did not correlate well with the length of the CAG repeat, age at disease onset, or disease duration.

\subsubsection{Corticobasal syndrome (CBS)}

Corticobasal syndrome was first described in 1967 in 3 patients who had asymmetric motor symptoms with an involvement of frontoparietal atrophy and neuronal loss at autopsy. CBS is an adult onset and slowly progressive degeneration with asymmetric akinetic-rigid syndrome. A limited response to levodopa treatment is noted in such patients. Some other extrapyramidal symptoms include tremor, dystonia, cortical dysfunction, cortical sensory impairment, apraxia, and alien hand phenomenon. Brain MRI may show focal cortical atrophy, particularly in the parietal lobe. Brain ${ }^{18}$ F-FDG PET reveals a frequently asymmetric hypometabolism in both the cerebral hemispheres. Brain 99mTc-TRODAT SPECT reveals an asymmetric involvement in the corpus striatum with equal involvement in both caudate and putamen regions.

The clinical and DAT findings in the above-described neurodegenerative diseases are shown in Table 2.

\subsection{Gene-related parkinsonism/dystonia degenerative diseases \\ 4.3.1 Dopa-responsive dystonia (DRD)}

Dopa-responsive dystonia, also known as Segawa's disease, is characterized by foot dystonia since childhood, diurnal fluctuation, and a dramatic and sustained response to low-dosage levodopa. Some patients with DRD may also show adult-onset parkinsonism similar to IPD. Pathologic degeneration of dopaminergic nigral cells is found in IPD, whereas synthesis defects in dopamine neurons without cell loss are noted in DRD. 


\begin{tabular}{|c|c|c|c|c|c|c|c|c|}
\hline & \multicolumn{2}{|l|}{ PSP } & \multicolumn{2}{|l|}{ MSA } & \multicolumn{3}{|l|}{ SCA } & \multirow[t]{2}{*}{ CBS } \\
\hline & $\overline{\mathrm{RS}}$ & PSP-P & MSA-P & MSA-C & SCA1 & SCA2 & SCA3 & \\
\hline Clinical features & & & & & & & & \\
\hline Parkinsonism & +(sym) & $+($ asym) & + & + & + & + & + & $+($ asym $)$ \\
\hline Rigidity & $+($ axial $)$ & $+($ axial $)$ & + & + & + & + & + & $+($ asym $)$ \\
\hline Tremor & - & + & + & + & $\begin{array}{c}+ \\
\text { (action) }\end{array}$ & $\begin{array}{c}+ \\
\text { (action) }\end{array}$ & $\begin{array}{c}+ \\
\text { (action) }\end{array}$ & + \\
\hline Bradykinesia & + & + & + & + & + & + & + & - \\
\hline $\begin{array}{l}\text { Loss of } \\
\text { postural reflex }\end{array}$ & + & + & + & + & + & + & + & - \\
\hline $\begin{array}{l}\text { Akinetic- rigid } \\
\text { svndrome }\end{array}$ & + & - & + & - & - & - & - & + \\
\hline Dystonia & $+($ facial $)$ & $+($ facial $)$ & - & - & + & + & + & + \\
\hline Retrocollis & + & + & (anticollis) & - & - & - & - & - \\
\hline $\begin{array}{l}\text { Cognitive } \\
\text { dysfunction }\end{array}$ & + & + & + & - & + & - & - & + \\
\hline $\begin{array}{l}\text { Alien hand } \\
\text { Cortical }\end{array}$ & - & - & - & - & - & - & - & + \\
\hline $\begin{array}{l}\text { sensory } \\
\text { impairment }\end{array}$ & - & - & - & - & - & - & - & + \\
\hline Cerebellar sign & - & - & + & + & + & + & + & - \\
\hline Ataxia & - & - & - & + & + & + & + & - \\
\hline Slow saccade & - & - & - & & + & + & + & - \\
\hline $\begin{array}{l}\text { Peripheral } \\
\text { neuropathy }\end{array}$ & - & - & - & - & + & + & - & - \\
\hline $\begin{array}{l}\text { Autonomic } \\
\text { dysfunction }\end{array}$ & + & + & + & + & - & $?$ & $?$ & $?$ \\
\hline EOM limitation & $+(\mathrm{VGP})$ & $+(\mathrm{VGP})$ & - & - & + & - & + & - \\
\hline Ptosis & - & - & - & - & - & - & + & - \\
\hline Fasciculation & - & - & - & - & - & - & + & - \\
\hline Face & - & - & - & - & - & - & + & - \\
\hline Tongue & - & - & - & - & - & - & + & - \\
\hline Dysarthria & + & + & + & + & + & + & + & - \\
\hline Dysphagia & + & + & + & + & + & + & + & - \\
\hline Apraxia & - & - & - & - & - & - & - & + \\
\hline $\begin{array}{l}\text { Response to } \\
\text { Levodopa } \\
\text { Neuroimaging }\end{array}$ & Poor & $\begin{array}{l}\text { Initial } \\
\text { good }\end{array}$ & $\begin{array}{l}\text { Initial } \\
\text { good }\end{array}$ & Partial & Partial & $\begin{array}{l}\text { Initial } \\
\text { good }\end{array}$ & Partial & $\begin{array}{c}+ \\
\text { (limited) }\end{array}$ \\
\hline $\mathrm{CT} / \mathrm{MRI}$ & $+/+$ & $+/+$ & $+/+$ & $+/+$ & $+/+$ & $+/+$ & $+/+$ & $+/+$ \\
\hline DAT uptake & $\mathrm{D}$ & $\mathrm{D}$ & D (sym) & D (sym) & $\mathrm{D}$ & $\begin{array}{c}\mathrm{D} \\
\text { (asym) }\end{array}$ & $\mathrm{D}$ & $\mathrm{D}$ (asym) \\
\hline IBZM uptake & $\mathrm{D}$ & $\begin{array}{l}\text { Increase } \\
\text { (mild) }\end{array}$ & $\begin{array}{l}\text { Possible } \\
\text { D }\end{array}$ & $\begin{array}{l}\text { Possible } \\
\text { D }\end{array}$ & NA & NA & NA & $\mathrm{D}$ \\
\hline 6 FD-PET uptake & NA & NA & $\mathrm{D}$ & D & NA & NA & NA & $\mathrm{D}$ (asym) \\
\hline
\end{tabular}

+: presence; -: absence; D: decrease; I: increase; sym: symmetrical; asym: asymmetrical; VGP: vertical gaze palsy; NA: not available

Table 2. Clinical features and DAT data in neurodegenerative diseases 
Molecular genetic studies revealed a mutation in GTP cyclohydrolase 1 (GCH1) in autosomal-dominant inherited DRD and mutations in tyrosine hydroxylase $(T H)$ in autosomal-recessive inherited DRD. In DRD patients, ${ }^{18}$ F-Dopa PET reveals normal uptakes in the corpus striatum; these findings may distinguish DRD from IPD, which reveals a decreased uptake even in the early stage of IPD patients. Dopamine transporter images with 99mTc-TRODAT-1 SPECT also show a normal uptake in DRD, indicating that presynaptic nigrostriatal dopaminergic terminals are normal (Figure 3 ).

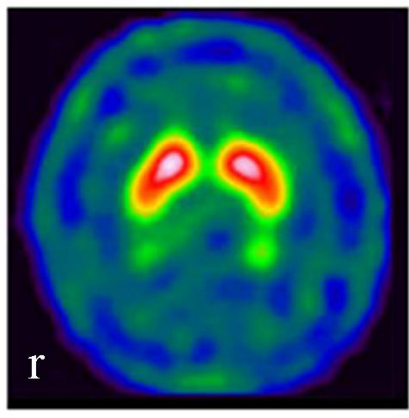

A

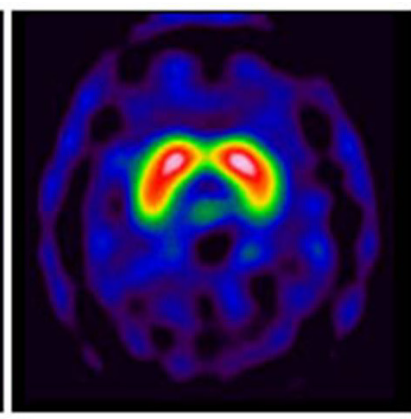

B

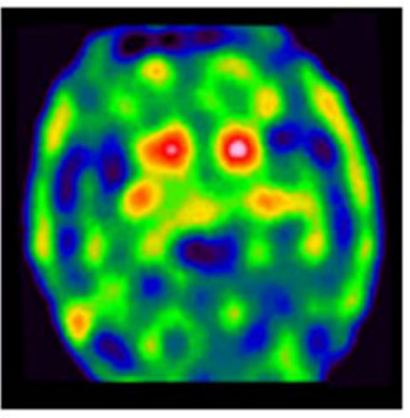

C

Fig. 3. The 99mTc -TRODAT-1 brain SPECT shows a normal uptake in the putamen and caudate in a patient with DRD (B) compared with those in a normal control (A) and a PD patient $(C)$. A reduction of the uptake in the corpus striatum, particularly in the right side was observed in the PD patient. $r=$ right.

\subsubsection{Wilson's disease (WD)}

Wilson's disease, hepatolenticular degeneration, is an autosomal recessive disorder characterized by a decreased serum concentration of ceruloplasmin, low serum copper concentration, and excessive deposition of copper in the liver, brain, and other organs.

The most common neurological manifestations include akinetic-rigid syndrome, dystonia, and cerebellar ataxia with action tremor. Pathologically, the most severely affected lesions include the basal ganglia involving the putamen, caudate, and globus pallidus. A brain CT scan may show low-density lesions with cystic degeneration in the basal ganglia, particularly the putamen and globus pallidus, as well as cortical atrophy and ventricular enlargement. Brain MRI reveals increased signal intensities in T2-weighted images of the lenticular nuclei, thalamus, and brainstem including the pons, midbrain, and even the substantia nigra. Occasionally, double panda signs were found in the brainstem. A poor therapeutic response to levodopa is noted in WD patients. However, brain 6F-DOPA PET studies have shown an involvement of the nigrostriatal presynaptic dopaminergic pathway. In addition, SPECT with 123 I-iodobenzamide (123I-IBZM) and PET images with ${ }^{18} \mathrm{~F}-$ methylspiperone have showed a reduction of postsynaptic striatal D2 receptor, reflecting striatal neuronal damage. Some DAT studies with ${ }^{123}$ I- $\beta$-CIT SPECT disclosed a severe or differential loss of the DAT in the striatum of WD patients, indicating a presynaptic defect in the terminals of the nigrostriatal dopaminergic neurons. However, in some WD patients with akinetic-rigid syndrome, a normal presynaptic dopaminergic pathway may occur; brain MRI also reveals the involvement of substantia nigra in these patients. (Figure 4). 

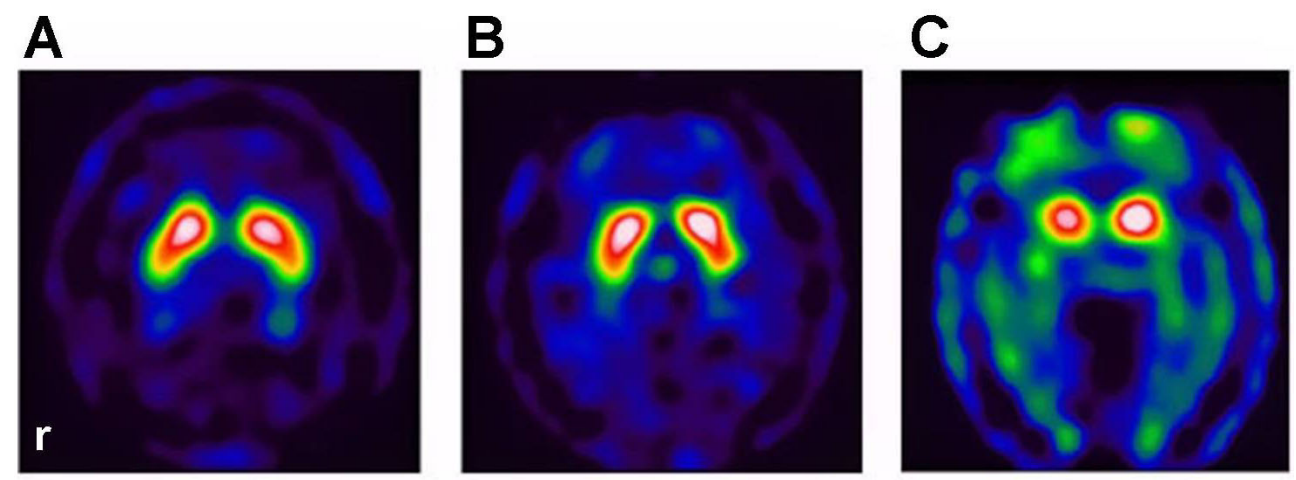

Fig. 4. Demonstration of 99mTc-TRODAT-1 uptake in an age-matched normal control (A), a WD patient (B) and a PD patient (C). Normal uptake of 99mTc-TRODAT-1 in the putamen and caudate nucleus was noted in a normal control and a WD patient (A and B). In PD patient, there was an asymmetrically decreased 99mTc-TRODAT-1 uptake, predominantly in the putamen $(\mathrm{C}) . \mathrm{r}=$ right.

\subsubsection{Huntington's disease (HD, Westphal type)}

Huntington's disease, the most common cause of hereditary chorea, is an autosomal dominant disorder caused by an expansion of an unstable trinucleotide repeat in chromosome 4 . The most striking feature is the appearance of chorea movements that seem purposeless and abrupt. However, some patients may present with the so-called akineticrigid variant form (Westphal variant). This form of the disease is rapidly progressive with a fatal outcome in less than 10 years after the onset of symptoms. Brain CT/MRI show enlarged ventricles with atrophy of the caudate nucleus. MRI of patients with the a kineticrigid form of the disease may reveal T2 hyperintense lesions in the striatum. FDG-PET may show hypometabolism in the caudate and putamen regions.

The clinical features and neuroimages of DRD, WD, and HD with Westphal variant are summarized in Table 3.

\subsection{Dementia syndromes with parkinsonism}

\subsubsection{Vascular parkinsonism (VP) or multiple infarct parkinsonism}

Vascular parkinsonism is characterized by clinical symptoms of gait disturbance with freezing, lower body parkinsonism, and loss of postural reflexes. Tremor is rarely seen. The onset is usually insidious and the course is progressive. Brain MRI usually reveals hyperintense T2-weighted signals in the basal ganglia and/or white matter; these findings are compatible with those of multiple infarctions. Hypertension is a common risk factor for the disorder. A poor or insufficient response to anti-parkinsonian drugs is also noted in these patients. Early diagnosis of VP is important because the prognosis and response to treatment in these patients are different from those of patients with IPD. However, VP may have a wide spectrum of clinical features, which make the differential diagnosis of these diseases difficult. A study using DAT with ${ }^{99 m T c}$-TRODAT-1 showed that specific binding in the putamen and caudate areas was slightly lower in VP patients than in healthy individuals; however, a significant decrease in the uptake of 99mTc-TRODAT-1 in the 
striatum was noted in IPD patients. A significant striatal asymmetry was observed in IPD patients but not in VP patients.

\begin{tabular}{lccc}
\hline & DRD & WD & HD (Westphal) \\
\hline Clinical features & & & - \\
Diurnal fluctuation & + & - & + \\
Chorea & - & - & + \\
Dystonia & + & + & + \\
Parkinsonism & + & + & - \\
Cerebellar sign & - & + & + \\
Cognitive impairment & - & + & - \\
Liver dysfunction & - & + & - \\
Autonomic dysfunction & - & Partial & No \\
Response to levodopa & Excellent & & Abn/ Abn (caudate \\
Neuroimaging & & Abn/Abn & atrophy) \\
CT/MRI & N/N & Abn & NA \\
& & Abn & Abn \\
DAT-SPECT & $\mathrm{N}$ & Abn & NA \\
FDG-PET & $\mathrm{N}$ & $\mathrm{N}$ & \\
6 FD-PET & & & \\
\hline
\end{tabular}

+: presence; -: absence; N: normal; Abn: abnormal; NA: not available

Table 3. Clinical features and DAT findings in gene- related PM/dystonia degenerative diseases

\subsubsection{Dementia with Lewy bodies (DLB)}

DLB is the second most common cause of neurodegenerative dementia after Alzheimer's disease (AD). The diagnostic criteria of DLB were established by the consensus conference for DLB in 2005. In the early stage of DLB, deficits in attention, executive function, and visuospatial ability are very prominent. The core clinical features include fluctuation of cognition, visual hallucination, and spontaneous parkinsonism. Recent suggestive features include REM sleep behavioral disorder, severe neuroleptic sensitivity, and low dopamine transporter uptake in the basal ganglia on SPECT or PET imaging. Supportive features of DLB diagnosis include repeated falls, syncope, transient loss of consciousness, autonomic dysfunction, depression, systematized delusions, or hallucinations. In brain MRI, atrophy of the cortical or hippocampus is lower in DLB patients than in AD patients. In ${ }^{18} \mathrm{~F}-\mathrm{FDG}$ PET or SPECT, maximal hypometabolism was noted in the parieto-occipital area in DLB patients; however, maximal hypoperfusion was noted in the tempo-parietal cortex in AD patients.

Serial DAT with I-123 $\beta$-CIT brain SPECT also demonstrated progressive striatal dopaminergic loss in DLB and Parkinson's disease with dementia, but not in AD. These findings have a high specificity $(94 \%)$ in distinguishing between DLB and AD.

A brain DAT with TRODAT-1 SPECT also demonstrated a decreased uptake in the striatum, including the putamen and caudate regions, but the DLB patients had relatively symmetric lesions and IPD patients had asymmetric lesions. 


\subsubsection{Frontotemporal dementia with parkinsonism-17 (FTDP-17)}

Frontotemporal dementia (FTD) can be divided into 3 major subtypes, including frontotemporal lobe dementia (FTLD), semantic dementia (SD), and progressive nonfluent aphasia (PNFA). The characteristic behavior changes include disinhibition, social withdrawal, diminished insight, loss of empathy, perseverance, and stereotypic behaviors. Semantic dementia may present with progressive loss of semantic knowledge, and although speech remain fluent, it becomes empty. Semantic dementia usually manifests as a fluent

\begin{tabular}{|c|c|c|c|}
\hline & $\mathrm{VaD}$ & DLB & FTDP-17 \\
\hline \multicolumn{4}{|l|}{ Clinical features } \\
\hline Parkinsonism & + & + & + \\
\hline Rigidity & + & + & + \\
\hline Tremor & - & - & - \\
\hline Bradykinesia & + & + & + \\
\hline $\begin{array}{l}\text { Lose of postural } \\
\text { reflex }\end{array}$ & + & + & + \\
\hline Language problem & + & - & + \\
\hline Focal sign & + & - & + \\
\hline Dysarthria & + & - & - \\
\hline Dysphagia & + & - & - \\
\hline Dementia & + & + & + \\
\hline Hallucination & + & + & - \\
\hline Cognitive fluctuation & - & + & - \\
\hline Personality changes & + & + & + \\
\hline Syncope & - & + & - \\
\hline $\begin{array}{l}\text { Autonomic } \\
\text { dysfunction }\end{array}$ & - & + & - \\
\hline $\begin{array}{l}\text { Response to } \\
\text { levodopa }\end{array}$ & Poor & Poor & Poor \\
\hline \multicolumn{4}{|l|}{ Neuroimages } \\
\hline $\mathrm{CT} / \mathrm{MRI}$ & Abn/Abn & Abn/Abn & Abn/Abn \\
\hline DAT-TRODAT & Normal & Abn & NA \\
\hline FDG-PET & Abn & Abn & Abn \\
\hline 6FD-PET & NA & Abn & NA \\
\hline
\end{tabular}

+: Presence; -: absence; Abn: abnormal; NA: not available

Table 4. Clinical features and DAT findings in dementia syndromes 
dysphasia with impairment in semantic verbal memory and an associative agnosia in individuals with more left temporal lobe involvement. Prosopagnosia may occur with right temporal damage. Progressive non-fluent aphasia is characterized by aphasia with stuttering and agrammatism. The executive function and working memory are usually impaired. The typical neuroimaging findings are asymmetrical atrophy of the anterior temporal lobe in SD and atrophy of the left inferior frontal lobe and anterior insular cortex in PNFA. In addition, there is overlap of clinical manifestations between AD and FTD. These 3 subtypes of FTD often overlap motor syndromes such as amyotrophic lateral sclerosis (ALS) and parkinsonism.

FTDP-17 is a distinct disease characterized by personality changes, executive dysfunction, memory deterioration, and parkinsonism. Motor disturbances include bradykinesia, axial and limb rigidity, and postural instability. Early manifestations include behavioral changes such as disinhibition, impaired social function, judgment and planning, and global dementia. Parkinsonism in FTDP-17 is unresponsive to levodopa. Table 4 summarizes the clinical manifestations in vascular parkinsonism, DLB, and FTDP-17.

\section{Conclusion}

The clinical features of IPD and PM are very similar, but some manifestations differ. The treatment and prognosis also differ. The response to treatment with levodopa is variable; therefore, definite diagnosis is very important. Early and accurate differentiation between IPD and PM has been markedly improved by recent developments in neuroimaging, particularly the 99mTc-TRODAT-1 SPECT, which is not only easy and economical to prepare and use in a wide variety of applications but also reliable in understanding the role of DAT in various neurological diseases. Most importantly, early and correct diagnosis leads to earlier and, therefore, more effective treatment with levodopa, when appropriate.

\section{Abbreviations:}

CBS: corticobasal syndrome

$\beta$-CIT: (1r) $2 \beta$-carbomethoxy-3 $\beta$-(4-iodophenyl) tropane

CO: carbon monoxide

$\mathrm{CS}_{2}$ : carbon disulfide

DAT: dopamine transporter

DRD: dopa-responsive dystonia

DLB: dementia with Lewy bodies

6-FD: 6-fluorodopa

FDG: fluorodeoxyglucose

FTD: frontotemporal dementia

FTDP-17: frontotemporal dementia with parkinsonism linked to chromosome 17

HD: Hungtington's disease

123I-IBZM scan: I-123-iodobenzamide $\mathrm{D}_{2}$ receptor scan

IPD: idiopathic Parkinson's disease

MJD: Machado-Joseph disease

Mn: manganese

MPTP: 1-methyl-4-phenyl-1,2,3,6-tetrahydropyridine 
MSA: multiple system atrophy

MSA-C: multiple system atrophy-cerebellar subtype

MSA-P: multiple system atrophy-parkinsonism subtype

PET: positron emission tomography

PM: Parkinsonism

PSP: progressive supranuclear palsy

PSP-P: progressive supranuclear palsy-parkinsonism

RS: Richardson syndrome

SCA: spinocerebellar atrophy

SPECT: single photon emission computed tomography

99mTc-TRODAT-1: Tc-99m labeled radiotracer for imaging DAT

VP: vascular parkinsonism

WD: Wilson's disease

\section{References}

\section{Idiopathic Parkinson's disease}

[1] Parkinson J (Sir James). "An essay on the shaking palsy" Whittingham and Rowland for Sherwood, Neely and Jones. London: 1817.

[2] Lee CS, Schulzer M, Mak EK, Hammerstad JP, Calne S, Calne DB. Patterns of asymmetry do not change over the course of idiopathic parkinsonism: implication for pathogenesis. Neurology 1995; 45: 435-439.

[3] Braak H, Del Tredici K, Rub U, de Vos RA, Jansen Stear EN, Braak E. Staging of brain pathology related to sporadic Parkinson's disease. Neurobiol Aging 2003; 24: 197211.

[4] Robottom BJ, Weiner WJ, Shulman LM. Parkinsonism In: Lisak RP, Truong DD, Carroll WM, Bhidayasiri R. eds. International Neurology: A Clinical Approach. London: Wiley-Blackwell; 2009: 152-158.

[5] Fahn S, Przedborski S. Parkinson disease. In Rowland LP, Pedley TA, eds. Merritt's Neurology. 12th ed. Philalelphia, Lippincott Williams \& Wilkins 2010: 751-769.

[6] Schapira AHV. Parkinson's disease. BMJ 1999;318:311-314.

[7] Innis RB, Seiby JB, Scanley BE, et al. Single photon emission computed tomographic imaging demonstrates loss of striatal dopamine transporters in Parkinson disease. Proc Natl Acad Sci USA 1993;90:11965-11969.

[8] Booji J, Tissingh G, Boer GJ, et al. [123I]FP-CIT SPECT shows a pronounced decline of striatal dopamine transporter labeling in early and advanced Parkinson's disease. J Neurol Neurosurg Psychiatry 1997;62:133-140.

[9] Kao PF, Tzen KY, Yen TC, Lu CS, Weng YH, Wey SP, Ting G. The optimal imaging time for $\left[{ }^{99 \mathrm{mTC}}\right.$ ]TRODAT-1/SPECT in normal subjects and patients with Parkinson's disease. Nucl Med Comm 2001; 22: 151-154.

[10] Seibyl JP, Marek KL, Quinlan D, et al. Decreased single-photon emission computed tomographic [123I]-CIT striatal uptake correlates with symptom severity in Parkinson's disease. Ann Neurol 1995;38:589-598. 
[11] Marek KL, Seibyl JP, Zoghbi S, et al. [123I]-CIT/SPECT imaging demonstrates bilateral loss of dopamine transporters in hemi-Parkinson's disease. Neurology 1996;46:231237.

[12] Polymeropoulos MH, Lavedan C, Leroy E, et al. Mutation in the alpha-synuclein gene identified in families with Parkinson's disease. Science 1997;276:20452047.

[13] Parkes JD, Marsden CD, Rees JE, et al. Parkinson's disease, cerebral arteriosclerosis, and senile dementia. Q J Med 1974;43:49-61.

[14] Tissingh G, Booij J, Winogrodzka A, van Royen EA, Wolters EC. IBZM- and CIT-SPECT of the dopaminergic system in parkinsonism. J Neural Transm 1997;50(suppl):3137.

[15] Morrish PK, Rakshi JS, Bailey DL, Sawle GV, Brooks DJ. Measuring the rate of progression and estimating the preclinical period of Parkinson's disease with [18F]dopa PET. J Neurol Neurosurg Psychiatry 1998;64:314-319.

[16] Brooks DJ. The early diagnosis of Parkinson's disease. Ann Neurol 1998;44(suppl):S10S18.

\section{Manganism}

[17] Barbeau A. Manganese and extrapyramidal disorders (a critical review and tribute to Dr. George C. Cotzias). Neurotoxicology 1984;5:13-36.

[18] Huang CC, Chu NS, Lu CS, et al. Chronic manganese intoxication. Arch Neurol 1989;46:1104-6.

[19] Mena I, Court J, Fuenzalida S, Papavasiliou PS, Cotzias GC. Modification of chronic manganese poisoning treatment with L-dopa or 5-OH tryptophane. N Engl J Med 1970;282:5-10.

[20] Calne DB, Chu NS, Huang CC, Lu CS, Olanow W. Manganism and idiopathic parkinsonism: similarities and differences. Neurology 1994;44:1583-1585.

[21] Wolters EC, Huang CC, Clark C, et al. Positron emission tomography in manganese intoxication. Ann Neurol 1989;26:647-651.

[22] Shinotoh H, Snow BJ, Chu NS, et al. Presynaptic and postsynaptic striatal dopaminergic function in patients with manganese intoxication: a positron emission tomography study. Neurology 1997;48:1053-1056.

[23] Huang CC, Lu CS, Chu NS, et al. Progression after chronic manganese exposure. Neurology 1993;43:1479-483.

[24] Huang CC, Chu NS, Lu CS, Chen RS, Calne DB. Long term progression in chronic manganism: ten years follow up. Neurology 1998;50:698-700.

[25] Huang CC, Weng YH, Lu CS, Chu NS, Yen TC. Dopamine transporter binding in chronic manganese intoxication. J Neurol 2003; 250: 1335-1339.

\section{Carbon disulfide}

[26] Aaserud O, Hommeren OJ, Tvedt B, Nakstad P, Mowe G, Efskind J, Russel D, Jörgensen EB, Nyber-Hansen R, Rootwelt K, Gjerstad L. Carbon disulfide exposure and neurotoxic sequelae among viscose rayon workers. Am J Ind Med 1990;18:25-37. 
[27] Hageman G, van der Hoek J, van Hout M, van der Laan G, Steur EJ, de Bruin W, Herholz K. Parkinsonism, pyramidal signs, polyneuropathy, and cognitive decline after long-term occupational solvent exposure. J Neurol 1999;246:198206.

[28] Huang CC, Chu CC, Chen RS, Lin SK, Shih TS. Chronic carbon disulfide encephalopathy. Eur Neurol 1996;36:364-368.

[29] Huang CC, Chu CC, Chu NS, Wu TN. Carbon disulfide vasculopathy: a small vessel disease. Cerebrovasc Dis 2001;11:240-250.

[30] Huang CC, Yen TC, Shih TS, Chang HY, Chu NS. Dopamine transporter binding study in differentiating carbon disulfide-induced parkinsonism from idiopathic parkinsonism. Neurotoxicology 2004;25:341-347.

[31] Chuang WL, Huang CC, Chen CJ, Hsieh YC, Kuo HC, Shih TS. Carbon disulfide encephalopathy: cerebral microangiopathy. NeuroToxicology 2007; 28: 387-393.

\section{Carbon monoxide intoxication}

[32] Kim JH, Chang KH, Song IC, et al. Delayed encephalopathy of acute carbon monoxide intoxication: diffusivity of cerebral white matter lesions. AJNR 2003; 24: 1592-1597.

[33] Hampson NB, Hauff NM. Risk factors for short-term mortality from carbon monoxide poisoning treated with hyperbaric oxygen. Crt Care Med 2008; 36: 2523-2527.

[34] Chu K, Jung KH, Kin HJ, et al. Diffusion-weighted MRI and 99mTc-HMPAO SPECT in delayed relapsing type of carbon monoxide poisoning: evidence of delayed cytotoxic edema. Eur Neurol 2004; 51: 98-103.

[35] Hsiao CL, Kuo HC, Huang CC. Delayed encephalopathy after carbon monoxide intoxication - long-term prognosis and correlation of clinical manifestations and neuroimages. Acta Neurol Taiwan 2004; 13: 64-70.

\section{Dopa responsive dystonia}

[36] Segawa M, Nomura Y, Tanaka S, Hakamada S, Nagata E, Soda M, Kase M. Hereditary progressive dystonia with marked diurnal fluctuation on its pathophysiology based on the characteristics of clinical and polysomnographical findings. In: Fahn S, Marsden CD, Calne DB (eds) Advances in Neurology, vol. 50, Dystonia 2, Raven Press, New York; 1988:367-376.

[37] Nygaard TG, Marsden CD, Fahn S. Dopa-responsive dystonia: long-term treatment response and prognosis. Neurology 1991;41:174-181.

[38] Nygaard TG, Takahashi H, Heiman GA, Snow BJ, Fahn S, Calne DB. Long-term treatment response and fluorodopa positron emission tomographic scanning of parkinsonism in a family with dopa-responsive dystonia. Ann Neurol 1992;32:603608.

[39] Ichinose H, Ohye T, Takahashi E, et al. Hereditary progressive dystonia with marked diurnal fluctuation caused by mutations in the GTP cyclohydrolase gene. Nat Genet 1994;8:236-242.

[40] Furukawa Y, Shimadzu M, Rajput AH, et al. GTP cyclohydrolase I gene mutations in hereditary progressive and dopa-responsive dystonia. Ann Neurol 1996;39:609-617. 
[41] Ludecke B, Knappskog PM, Clayton PT, et al. Recessively inherited L-DOPAresponsive parkinsonism in infancy caused by a point mutation (L205P) in the tyrosine hydroxylase gene. Hum Mol Genet 1996;5:1023-1028.

[42] Snow BJ, Nygaard TG, Takahashi H, Calne DB. Positron emission tomographic studies of dopa-responsive dystonia and early-onset idiopathic parkinsonism. Ann Neurol 1993;34:733-738.

[43] Huang CC, Yen TC, Weng YH, Lu CS. Normal dopamine transporter binding in dopa responsive dystonia. J Neurol 2002; 249: 1016-1020.

\section{Progressive supranuclear palsy}

[44] Burn DJ, Lees AJ. Progressive supranuclear palsy: where are we now? Lancet Neurol 2002;1:359-369.

[45] Williams DR, De Silva R, Paviour DC, Pittman A, Watt HC, Kilford L, et al. Characteristics of two distinct clinical phenotypes in pathologically proven progressive supranuclear palsy: Richardson's syndrome and PSP-parkinsonism. Brain 2005;128:1247-1258.

[46] Williams DR, Holton JL, Strand C, Pittman A, De Silva R, Lees AJ, et al. Pathological tau burden and distribution distinguishes progressive supranuclear palsyparkinsonism from Richardson's syndrome. Brain 2007;130:1566-1576.

[47] Kim YJ, Ichise M, Ballinger JR, Vines D, Erami SS, Tatschida T, et al. Combination of dopamine transporter and D2 receptor SPECT in the diagnostic evaluation of PD, MSA, and PSP. Mov Disord 2002;17:303-312.

[48] Antonini A, Benti R, De Notaris R, Tesei S, Zecchinelli A, Sacilotto G, et al. 123IIoflupane/SPECT binding to striatal dopamine transporter (DAT) uptake in patients with Parkinson's disease, multiple system atrophy, and progressive supranuclear palsy. Neurol Sci 2003;24:149-150.

[49] Pirker W, Asenbaum S, Bencsits G, Prayer D, Gerschlager W, Deecke L, et al. [123I]betaCIT SPECT in multiple system atrophy, progressive supranuclear palsy, and corticobasal degeneration. Mov Disord 2000;15:1158-1167.

[50] Burn DJ, Sawle GV, Brooks DJ. Differential diagnosis of Parkinson's disease, multiple system atrophy, and Steele-Richardson-Olszewski syndrome: discriminant analysis of striatal 18F-dopa PET data. J Neurol Neurosurg Psychiatry 1994;57:278284.

[51] Oyanagi C, Katsumi Y, Hanakawa T, Hayashi T, Thuy DD, Hashikawa K, et al. Comparison of striatal dopamine D2 receptors in Parkinson's disease and progressive supranuclear palsy patients using [123I] iodobenzofuran single-photon emission computed tomography. J Neuroimaging 2002;12:316-324.

\section{Multiple system atrophy}

[52] Hierholzer J, Cordes M, Venz S, Schelosky L, Harisch C, Richter W, et al. Loss of dopamine-D2 receptor binding sites in Parkinsonian plus syndromes. J Nucl Med 1998;39:954-960.

[53] Arnold G, Schwarz J, Tatsch K, Kraft E, Wachter T, Bandmann O, et al. SteeleRichardson-Olszewski-syndrome: the relation of dopamine D2 receptor binding and subcortical lesions in MRI. J Neural Transm 2002;109:503-512. 
[54] Schwarz J, Tatsch K, Arnold G, Ott M, Trenkwalder C, Kirsch CM, et al. 123Iiodobenzamide-SPECT in 83 patients with de novo parkinsonism. Neurology 1993;43:S17-S20.

[55] Lin WY, Lin KJ, Weng YH, Yen TZ, Shen LH, Liao MH, Lu CS. Preliminary studies of differential impairments of the dopaminergic system in subjects of progressive supranuclear pslay. Nucl Med Commun 2010;31:974-980.

[56] Wenning GK, Tison F, Ben-Shlomo Y, et al. Multiple system atrophy: a review of 203 pathologically proven cases. Mov Disord 1997;12:133-147.

[57] Wenning GK, Ben-Shlomo Y, Hughes A, Deniel SE, Lees A, Quinn NP. What clinical features are most useful to distinguish definite multiple system atrophy from Parkinson's disease? J Neurol Neurosurg Psychiatry 2000;68:434-440.

[58] Gilman S, Low PA, Quinn N, et al. Consensus statement on the diagnosis of multiple system atrophy. J Neurol Sci 1999;163:94-98.

[59] Wenning GK, Ben-Shlomo Y, Magalhaes M. Clinicopathological study of 35 cases of multiple system atrophy. J Neurol Neurosurg Psychiatry 1995;58:160-166.

[60] Brooks DJ, Ibanez V, Sawle GV, et al. Differing patterns of striatal 18F-dopa uptake in Parkinson's disease, multiple system atrophy, and progressive supranuclear palsy. Ann Neurol 1990;28:547-555.

[61] Gilman S, Koeppe RA, Junck L, et al. Decreased striatal monoaminergic terminals in multiple system atrophy detected with positron emission tomography. Ann Neurol 1999;45:769-777.

[62] Lu CS, Weng YH, Chen MC, Chen RS, Tzen KY, Wey SP, Ting G, Chang HC, Yen TC. [99mTc]-TRODAT-1 imaging of multiple system atrophy. J Nucl Med 2004;1:49-55.

\section{Spinocerebellar degeneration}

[63] Gispert S, Twell R, Orozco G, et al. Chromosomal assignment of the second locus for autosomal dominant cerebellar ataxia (SCA2) to chromosome 12q23-24.1. Nat Genet 1993;4:295-299.

[64] Pulst SM, Nechiporuk A, Nechiporuk T, et al. Moderate expansion of a normally biallelic trinucleotide repeat in spinocerebellar ataxia type 2. Nat Genet 1996;14:269-276.

[65] Cancel G, Durr A, Didierjean O, et al. Molecular and clinical correlation in spinocerebellar ataxia 2: a study of 32 families. Hum Mol Genet 1997;6:709-715.

[66] Sasaki H, Wakisaka A, Sanpei K, et al. Phenotype variation correlates with CAG repeat length in SCA2 - a study of 28 Japanese patients. J Neurol Sci 1998;159:202-208.

[67] Lu CS, Wu Chou YH, Yen TC, Tsai CH, Chen RS, Chang HC. Dopa-responsive Parkinsonism phenotype of spinocerebellar atokia type 2. Mov Disord 2002; 17: 1046-1051.

[68] Onoders O, Idezuka J, Igarashi S, et al. Progressive atrophy of cerebellum and brainstem as a function of age and the size of expanded CAG repeats in the MJD1 gene in Machado-Joseph disease. Ann Neurol 1998;43:288-296.

[69] Kawaguchi Y, Okamoto T, Taniwaki M, et al. CAG expansions in a novel gene for Machado-Joseph disease at chromosome 14q32.1. Nat Genet 1994;8:221-227.

[70] Murata Y, Tamaguchi S, Kawakami H, et al. Characteristic magnetic resonance imaging findings in Machado-Joseph disease. Arch Neurol 1998;55:33-37. 
[71] Ishibashi M, Sakai T, Matsuishi T, et al. Decreased benzodiazepine receptor binding in Machado-Joseph disease. J Nucl Med 1998;39:1518-1520.

[72] Shinotoh H, Thiessen B, Snow BJ. Fluorodopa and raclopride PET analysis of patients with Machado-Joseph disease. Neurology 1997;49:1133-1136.

[73] Soong BW, Liu RS. Positron emission tomography in asymptomatic gene carriers of Machado-Joseph disease. J Neurol Neurosurg Psychiatry 1998;64:499-504.

[74] Yen TC, Tzen KY, Wey SP, Ting G. Decreased dopamine transporter binding in Machado-Joseph disease. J Nucl Med 2000;41:994-998.

[75] Yen TC, Tzen KY, Chen MC, et al. Dopamine transporter concentration is reduced in asymptomatic Machado-Joseph disease gene carriers. J Nucl Med 2002;43:153-159.

[76] Matilla T, McCall A, Subramony SH, Zoghbi HY. Molecular and clinical correlations in spinocerebellar ataxia type 3 and Machado-Joseph disease. Ann Neurol 1995;38:6872.

[77] Lu CS, Chang HC, Kuo PC. The parkinsonian phenotype of spinocerebellar ataxia type 3 in a Taiwanese family. Parkinsonism Rel Disord 2004; 10: 369-373.

\section{Corticobasal syndrome}

[78] Gibb WR, Luthert PJ, Marsden CD. Corticobasal degeneration. Brain 1989;112:11711192.

[79] Lang AE, Riley DE, Bergeron C. Cortical-basal ganglionic degeneration. In: Calne DB, ed. Neurodegenerative Diseases. Philadelphia: W.B. Saunders; 1994:877-894.

[80] Eidelberg D, Dhawan V, Moeller JR, et al. The metabolic landscape of cortico-basal ganglionic degeneration: regional asymmetries studied with positron emission tomography. J Neurol Neurosurg Psychiatry 1991;54:856-862.

[81] Grimes DA, Lang AE, Bergeron CB. Dementia as the most common presentation of cortical-basal ganglionic degeneration. Neurology 1999;53:1969-1974.

[82] Grisoli M, Fetoni V, Savoiardo M, Girotti F, Bruzzone MG. MRI in corticobasal degeneration. Eur J Neurol 1995;2:547-552.

[83] Lutte I, Laterre C, Bodart JM, De Volder A. Contribution of PET studies in diagnosis of corticobasal degeneration. Eur Neurol 2000;44:12-21.

[84] Pirker W, Asenbaum S, Bencsits G, et al. [123I]- $\beta$-CIT SPECT in multiple system atrophy, progressive supranuclear palsy, and corticobasal degeneration. Mov Disord 2000;15:1158-1167.

[85] Lai SC, Tsai CC, Yen TC, Lu CS. 18F-FDG PET and 99mTc-TRODAT-1 SPECT observations in a case of corticobasal degeneration. Ann Nucl Med Sci 2002; 15: 9396.

\section{Wilson's disease}

[86] Walshe JM. Wilson's disease In: Vinken PJ, Bruyn GW, Klawans HL, eds. Handbook of Clinical Neurology. Vol 49. Extrapyramidal Disorders. Amsterdam: NorthHolland; 1986:223-238.

[87] Marsden CD. Wilson's disease. Q J Med 1987;248:959-966.

[88] Williams JB, Walshe JM. Wilson's disease: an analysis of the cranial computerized tomographic appearances found in 60 patients and the changes in response to treatment with chelating agents. Brain 1981;104:735-752. 
[89] Roh JK, Lee TG, Wie BA, et al. Initial and follow-up brain MRI findings and correlation with the clinical course in Wilson's disease. Neurology 1994;44:1064-1068.

[90] Huang CC, Chu NS. Acute dystonia with thalamic and brainstem lesions after initial penicillamine treatment in Wilson's disease. Eur Neurol 1998;39:32-37.

[91] Morgan JP, Preziosi TJ, Bianchine JR. Ineffectiveness of L-DOPA as a supplement to penicillamine in a case of Wilson's disease. Lancet 1970;ii:659.

[92] Snow BJ, Bhatt M, Martin WRW, Li D, Calne DB. The nigrostriatal dopaminergic pathway in Wilson's disease studied with positron emission tomography. J Neurol Neurosurg Psychiatry 1991;54:12-17.

[93] Oertel WH, Tatsch K, Schwavz J, et al. Decrease of D2 receptors indicated by 123IIodobenzamide single-photon emission computed tomography relates to neurological deficit in treated Wilson's disease. Ann Neurol 1992;32:743-748.

[94] Oder W, Brucke T, Kollegger H, Spatt J, Asenbaum S, Deecke L. Dopamine D2 receptor binding is reduced in Wilson's disease: correlation of neurological deficits with striatal 123I-iodobenzamide binding. J Neural Trans 1996;103:1093-1103.

[95] Jeon B, Kim JM, Jeong JM, Kim KM, Chang YS, Lee DS, Lee MC. Dopamine transporter imaging with [123I]- $\beta$-CIT demonstrates presynaptic nigrostriatal dopaminergic damage in Wilson's disease. J Neurol Neurosurg Psychiatry 1998;65:60-64.

[96] Barthel H, Sorger D, Kuhn HJ, Wagner A, Kluge R, Hermann W. Differential alteration of the nigrostriatal dopaminergic system in Wilson's disease investigated with [123I]- $\beta$-CIT and high-resolution SPET. Eur J Nucl Med 2001;28:1656-1663.

[97] Huang CC, Chu NS, Yen TC, Wai YY, Lu CS. Dopamine transporter binding in Wilson's disease. Can J Neurol Sci 2003; 30: 163-167.

\section{Vascular parkinsonism}

[98] Tzen KY, Lu CS, Yen TC, et al. Differential diagnosis of Parkinson's disease and vascular parkinsonism by 99mTc-TRODAT-1. J Nucl Med 2001;42:408-413.

\section{Dementia with Lewy body}

[99] Mckeith IG, Dickson DW, Lowe J, et al. Diagnosis and management of dementia with Lewy bodies: third report of DLB consortium. Neurology 2005; 65: 1863-1872.

[100] Burn DJ. Cortical Lewy body disease and Parkinson's disease dementia. Curr Opin Neurol 2006; 19: 572-579.

\section{Frontotemporal dementia}

[101] Mesulam MM. Primary progressive aphasia. Ann Neurol 2001; 49: 425-432.

[102] Cairns NJ, Bigio EH, Mackenzie IR, et al. Neuropathologic diagnostic and nosolgic criteria for frontotemporal lobar degeneration: consensus of the consortium for frontotemporal lobar degeneration. Acta Neuropathol 2007; 114: 5-22.

[103] Scarmeas N, Honig LS. Frontotemporal degenerative dementias. Clin Neurosci Res 2004; 3: 449-460.

[104] Foster NL, Heidebrink JL, Clark CM, et al. FDG-PET improves accuracy in distinguishing frontotemporal dementia and Alzheimer's disease. Brain 2007; 130: 2616-2635. 
[105] Rabinovici GD, Frust AJ, O'Neil JP, et al. ${ }^{11}$ C-PIB PET imaging in Alzheimer's disease and frontotemporal lobar degeneration. Neurology 2007; 68: 1205-1212.

[106] Hutton M, Lendon CL, Rizzu P. Association of missense and 5'-splice-site mutations in tau with the inherited dementia FTDP-17. Nature 1998; 393: 702-705. 


\title{
Neuroimaging in Fragile X-Associated Tremor/Ataxia Syndrome (FXTAS)
}

\author{
Laia Rodriguez-Revenga ${ }^{1,2}$, Beatriz Gómez-Ansón ${ }^{3,4}$, \\ Esther Granell Moreno ${ }^{3}$, Javier Pagonabarraga ${ }^{4,5}$ and Montserrat Mila1,2 \\ ${ }^{1}$ Biochemistry and Molecular Genetics Department, Hospital Clinic, IDIBAPS Barcelona \\ ${ }^{2}$ CIBER de Enfermedades Raras (CIBERER), Barcelona \\ ${ }^{3}$ Neuroradiology Unit, Radiology Department, Hospital Sant Pau, Barcelona \\ ${ }^{4}$ CIBER de Enfermedades Neurodegenerativas (CIBERNED), Barcelona \\ ${ }^{5}$ Neurology Service, Hospital Sant Pau, Barcelona
} Spain

\section{Introduction}

Fragile X syndrome (FXS, OMIM \#300624) is the most common form of inherited mental retardation. The real incidence of the syndrome is not known, but epidemiological studies indicate that it is responsible for mental retardation in 1 in 4,000-6,000 males and in 1 in 7,000-10,000 females of European descendent (for review Hagerman, 2002). In a study performed in Catalonia it gave an incidence of 1:2,466 male and 1:8,333 females (Rife et al., 2003). It is also important to highlight the high incidence of premutation carriers, 1 in 1,233 males and 1 in 411 females (Rife et al., 2003). FXS is inherited as X-linked dominant trait, with a reduced penetrance ( $80 \%$ for males and $30 \%$ for females).

In 1991 the responsible gene was identified by positional cloning and named the fragile $X$ mental retardation-1 gene (FMR1) (Oberle et al., 1991; Verkerk et al., 1991; Yu et al., 1991). The FMR1 gene is located in the long arm of the $\mathrm{X}$ chromosome at $\mathrm{Xq} 27.3$, it expands 17 exons, and $40 \mathrm{~kb}$ of genomic DNA. It transcribes an mRNA of $3.9 \mathrm{~kb}$, and the translated protein is called fragile $\mathrm{X}$ mental retardation protein (FMRP). The lack of this protein, which plays an important role in synaptogenesis and synaptic plasticity (Basell \& Warren, 2008), is the cause of the FXS.

FXS is almost exclusively caused by a dynamic mutation, a CGG repeat expansion in the $5^{\prime}$ untranslated region of the FMR1 gene (Oberle et al., 1991; Verkerk et al., 1991; Yu et al., 1991). In a normal situation the number of CGG repeats is polymorphic with alleles between 6 to about 54, with the most common allele presenting 30 CGG repeats. In this situation the CpG island, which is located in the promoter of the gene acting as a switch depending on its methylation status, is unmethylated. Thus the gene is active, FMR1 is transcribed and translated. Repeats at this size remain stable upon transmission. A second class of alleles that overlaps with the upper range of the wild-type is those with $\sim 40-55$ CGGs. This range, known as the gray zone, no expands to full mutation but it is transmitted slightly unstable to subsequent generations with the possibility of creating a premutated allele. In the premutation, alleles range from 55 to about 200 CGG repeats. At this situation, the FMR1 
gene is also transcribed as the CpG island is unmethylated. Therefore, premutated carriers have normal or lightly reduced synthesis of FMRP and they are asymptomatic for FXS. However, they have risk of having affected descendence since the number of CGG is unstable, and in each cellular division it can increase, and can be transmitted with a higher number of repeats to the next generation. Finally, when the CGG number is beyond 200 repeats (known as full mutation), the CpG island is methylated, and as consequence, the gene is transcriptionally silenced, and no protein is translated. These individuals are always affected if they are males and in about $30 \%$ of females.

In this chapter we review the clinical, molecular and neuroradiological aspects of FXTAS syndrome, a late-onset neuropsychiatric degenerative disorder that occurs predominantly in male carriers of the FMR1 premutation. Based on our experience we describe in detail the different aspects that characterize the syndrome as well as the new findings.

\section{Fragile $X$ syndrome premutation and FXTAS identification}

The possibility of clinical involvement in carriers of premutation expansions (55 to 200 CGG repeats) of FMR1 gene was initially discounted, since carrier mothers of FXS children have a normal cognitive functioning (Bennetto et al., 2001; Reiss et al., 1993). Even though, and contrary to expectation, there are reported several subgroups of male and female premutation carriers displaying features consistent with the typical clinical spectrum of FXS. For instance, Riddle and co-workers (1998) and Hagerman (2002) described a group of premutation carriers with prominent ears and joint laxity; or with learning disabilities, attention deficit/hyperactivity disorder (ADHD), or difficulty with math (Riddle et al., 1998, reviewed in Hagerman \& Hagerman 2004). In addition, some carriers have emotional problems, including anxiety, obsessional thinking, schizotypy, and/or depression (Hagerman et al., 2002; Rodriguez-Revenga et al., 2008a). Such findings were observed to be more likely to occur in carriers with a lowered FMRP levels and particularly in those carrier females with larger CGG expansions (>100 repeats) (Johnston et al., 2001).

There are, however, two forms of clinical involvement among carriers of premutation alleles that are not consistent with the clinical spectrum of the FXS. These two disorders are the primary ovarian insuffiency (FXPOI) and the fragile X-associated tremor/ataxia syndrome (FXTAS). FXPOI, which refers to the cessation of menses before age 40, is seen in $\sim 20 \%$ of female who carry permutation alleles. Therefore, the genetic counseling for premutated women has to include a fertility advice since delaying the reproduction may not be a good option.

FXTAS, the second form of clinical involvement described among permutation carriers, was identified in 2001 by Hagerman and co-workers as a late-onset neurodegenerative disorder (Hagerman et al., 2001). It took more than 10 years after the FMR1 gene identification to recognize FXTAS as a FMR1 permutation associated phenotype. One explanation for this is that the movement disorder experienced by older carriers, who were thought to be clinically normal, was not associated with the FXS (a childhood disorder) affecting children. Mothers of FXS children, being seen in clinics, were often expressing concerns about their fathers (FMR1 premutation carriers) who were experiencing problems with hand tremor and unsteady gait. When Hagerman and co-workers evaluated these male carriers they found that they all have a common neurological profile, consisting of intention tremor and gait ataxia (reviewed in Hagerman \& Hagerman 2004). Therefore, FXTAS was firstly identified among older male carriers of premutation alleles, including progressive action tremor and 
ataxia with associated radiological findings (Hagerman \& Hagerman 2004). However, to date FXTAS has also been described among premutated women although it has been suggested that it occurs less frequently and that the phenotype is milder with older age at onset (Hagerman et al., 2001; Jacquemont et al., 2004a). An explanation for this difference is the presence of a second normal allele and a random X-inactivation of the premutated one; however, there may be additional sex-specific effects that reduce penetrance among females (Hagerman \& Hagerman, 2004).

Not all FMR1 premutation carriers develop FXTAS and it remains unknown which carriers will do so and when. It has been estimated that at least one-third of all male carriers will develop a FXTAS syndrome, although the penetrance increases with age, exceeding $50 \%$ for men aged $70-90$ years. Moreover, there is significant variability in the progression of neurological dysfunction (Hagerman \& Hagerman 2004; Jacquemont et al., 2004a, 2004b). In an attempt to provide an estimation of FXTAS penetrance among premutation carriers in Spanish FXS families, we evaluated 398 families among which 151 were composed of at least three generations. Our results showed that signs of FXTAS were detected in $16.5 \%$ of female premutation carriers and in $45.5 \%$ of premutated males older than 50 years. Overall, the mean age and the mean of CGG repeat number for the FXTAS men group was of $72.05 \pm 6.85$ and $85 \pm 21.5$ (mean \pm SD), respectively. Similarly it was of 75.8 years old \pm 10.2 and 82 CGG repeats \pm 18 (mean \pm SD) for the FXTAS women group (Rodriguez-Revenga et al., 2009).

The description and characterization of FXTAS syndrome is of great interest to the population, because the prevalence of FMR1 premutation in the general population is relatively high. Several studies have been performed in order to determine the real role of FXTAS in undiagnosed adult patients with movement disorders. The results obtained in European populations ranges from 0\% to 4\% (Brussino et al.,2005; Macpherson et al., 2003; Van Esch et al., 2005; Zuhlke et al., 2004). Our studies show an estimated FXTAS prevalence of $2 \%$ among patients presenting with ataxia or movement disorders of unknown etiology (Rodriguez-Revenga et al., 2007, 2008b). Although large studies are necessary to better define FXTAS prevalence in this kind of population, on the basis of premutation male frequency in general population, the prevalence of FXTAS has been estimated in $\sim 1 / 3,000$ males aged over 50 years of age $(\sim 1 / 10,000$ males of all ages) (Hagerman \& Hagerman, 2004).

\section{Clinical and cognitive overview}

FXTAS syndrome is a neurodegenerative disease that eventually appears in adult subjects who carry a CGG repeat length between 55 and 200 trinucleotides in the FMR1 gene of chromosome $X$ (Jacquemont et al., 2007). These subjects are categorized as FMR1 premutation carriers. As stated above, it was originally described in grandparents of children with FXS. Different specialized centres in diagnosing and attending children with FXS observed that their grandparents and uncles were more likely to develop, from 50 years onwards, a stereotyped clinical picture characterized by unsteadiness while walking and action tremor in both hands (Hagerman et al., 2001).

Neurological examination of children's relatives disclosed the presence of predominant intention tremor in hands and wide-base cerebellar ataxia. Both symptoms used to follow a progressive course, and were often accompanied by progressive cognitive and behavioral disturbances (Hagerman et al., 2001). 
Clinical descriptions published in the past 10 years have demonstrated that FMR1 premutation carriers are very prone to develop cerebellar dysfunction from the age of 50 years. Seveirty of symptoms usually impairs gait and associates mild to moderate dysmetria, repeated falls, intention tremor, and speech difficulties. The combination of cerebellar dysfunction, cognitive impairment and the appearance of characteristic radiologic features in the brain MRI has been claimed to constitute a nosological entity that has been named Fragile X-associated Tremor/ Ataxia syndrome (FXTAS).

In the only study about the natural history of patients with FXTAS, this syndrome has been observed to represent a disabling condition impacting on motor daily activities, thinking, and social skills after 15 years of evolution (Jacquemont et al., 2003).

Mean age of onset of FXTAS is 60 years. Mild intention tremor, affecting both hands, symmetrically, is usually the first symptom. Within the first 5 years, tremor becomes more apparent and some balance and speech problems develop. At this stage, balance problems are mild and are only noticed by patients as a feeling of unsteadiness while walking for long periods of time or when turning. Between 5 and 15 years from disease onset balance problems progress in severity and are usually the main complain, provoking repeated falling and limiting the ability to drive, walking autonomously in the streets, or using public transportation. At this stage, moreover, is when the first cognitive and behavioural disturbances appear. After 15 years, autonomous walk is greatly impaired, and patients need the help of a walker or the supervision of another person. Finally, dysphagia and severe ataxia are associated with immobility and recurrent urinary and respiratory infections (Leehey et al., 2007).

The penetrance of FXTAS is not well established. Penetrance increases with age, and it has been reported to range from $15 \%$ at 50 years of age to $75 \%$ at age 80 (Jacquemont et al., 2004b).

Being a relatively newly described condition, the clinical spectrum of the disease has expanded in the past years. As in other diseases, such as Parkinson's disease, FXTAS was initially conceived as a predominant motor disease. New data have evidenced that many clinically relevant cognitive and behavioural disturbances may also develop in FXTAS patients. Also, while FXTAS was originally described in males, the number of women developing FXTAS symptoms has increased exponentially in the past five years. Women have been reported to develop the same motor, cognitive and behavioural problems than males, although women are more likely to develop a milder form of the disease (Coffey et al., 2008). Nevertheless, cognitive dysfunction severe enough to accomplish criteria for dementia has been reported in FXTAS women with inactivation of the healthy Xchromosome (Rodriguez-Revenga et al., 2010).

Cognitive impairment in FXTAS is characterized by recent episodic memory problems, difficulties in sustained attention, and other executive problems such as organizing new material and inhibiting automatic responses. In males, initially mild cognitive problems can accomplish criteria for dementia after 5 to 10 years of evolution (Grisby et al., 2007). Extension of the neurodegenerative process to prefrontal and temporal structures seems to account for the progression of cognitive defects in FXTAS patients (Seritan et al., 2008). While balance problems and tremor seem to be the consequence of the progressive degeneration of middle cerebellar peduncles and associated cerebellar structures, both cognitive and behavioural problems in FXTAS are associated with diffuse cortical and white-matter subcortical degeneration (Hashimoto et al., 2011). 
The dysexecutive problems of FXTAS patients affect different prefrontal dominions. Cognitive tasks dependent on the dorsolateral prefrontal cortex are affected since the early stages of the disease. Problems in working memory, set-shifting and mental flexibility have been described in patients with mild ataxia and tremor (Grisby et al., 2007). Later in the disease, cognitive alterations more dependent on the medial prefrontal cortex, such as difficulties in inhibiting automatic responses, arise. Response inhibition in go-no go tasks, inability to perform Luria's promotor series, and signs of environmental dependency were seen not only to limit the ability of FXTAS' patients to cope with cognitive strategies in the daily life, but also accounted for the behavioral disorders of the disease (Moore et al., 2004a). Memory problems develop due to both prefrontal and medial temporal dysfunction. Numerous eosinophylic inclusions have been observed bilaterally in the hippocampus and the entorrhinal cortex. Progressive deterioration and atrophy of medial temporal lobe structures explains the progressive nature of temporal disorientation and both verbal and visual recent episodic memory. (Grisby et al., 2008). In comparison with Alzheimer's disease, FXTAS patients show a higher impairment in dysexecutive tasks and less memory impairment, and in comparison with Parkinson's disease, visuospatial skills seem to be more preserved. In addition, compared to both Parkinson's disease and dementia with Lewy bodies, FXTAS patients are less likely to manifest visual hallucinations and psychosis (Grisby et al., 2008).

Neuropsychiatric disturbances in FXTAS are characterized by a change in premorbid personality. Patients with FXTAS, as in frontotemporal dementia, may develop irritability, psychomotor agitation, a tendency to selfishness, blunted emotions, apathy, and sometimes even social and personal disinhibition. Similarities with frontotemporal dementia correlate with the evidence of predominant medial prefrontal and anteromedial temporal atrophy in neuroimaging studies (Bacalman et al., 2006).

In summary, fronto-temporal cognitive and behavioral disturbances seem to characterize FXTAS patients. Frontal cognitive symptoms are present in early stages and progress through disease evolution, medial prefrontal behavioral disturbances appear in the middle stages and progress also in severity, and finally, from medium to late FXTAS stages, temporal cognitive symptoms impair even more global cognitive function and lead to dementia. A better delineation of each one of these components would help to refine the progression of cognitive and behavioural symptoms in different FXTAS subgroups (age, gender, etc...).

Other neurological disturbances described in FXTAS patients are the development of rigidakinetic parkinsonism with predominant axial involvement (35\%), unilateral resting tremor $(10 \%)$, peripheral neuropathy $(30 \%)$, dysautonomia $(40-50 \%$; urinary urgency, erectile dysfunction, orthostatic hypotension), nistagmus (10\%), and hyporreflexia (10\%).

In some patients, postural and intention tremor may present in isolation, making the diagnosis of essential tremor very likely. Reported cases stress the importance of considering the diagnosis of FXTAS in patients diagnosed of essential tremor who develop ataxia or with a family history of mental retardation (Leehey et al., 2003). Other patients present with isolated and progressive adult-onset ataxia. Currently, genetic testing for FXTAS must be considered in any patient with ataxia developing after the age of 50 . The screening of FXTAS in a prospective series of patients with multiple system atrophy showed that $4 \%$ of patients with an initial diagnosis of multiple system atrophy of the cerebellar type were actually FMR1 premutation carriers (Kamm et al., 2005). Taking into account the 
consequences of a diagnosis of FXTAS on genetic counselling, FXTAS should be categorized as a new kind of spinocerebellar ataxia (Milà et al., 2009).

\section{Molecular genetics overview}

FXTAS is an allelic disorder to the FXS, and therefore should be considered as a distinct neurodegenerative disorder. In fact, the molecular mechanism leading to FXTAS is distinct from the FMR1 silencing mechanism and/or a deficit in FMRP operating in FXS. In premutated patients the FMR1 gene is rarely silenced and FMRP levels are generally normal or only slightly lowered (Hagerman \& Hagerman, 2004) (Fig.1a). The only known molecular abnormality among premutation carriers is the presence of markedly elevated levels $(\sim 2-8$ fold) of FMR1 mRNA (Fig.1b).

a

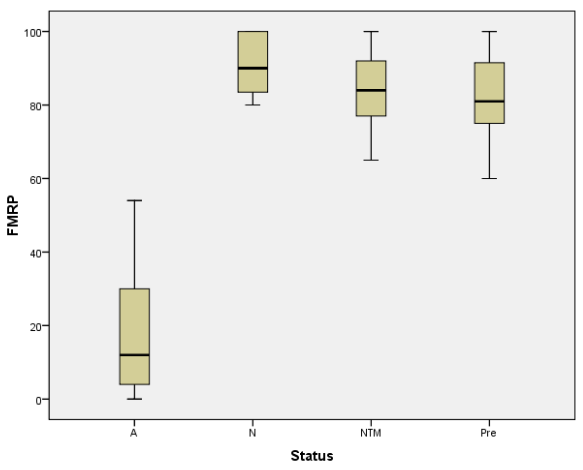

b

FMR1 mRNA levels

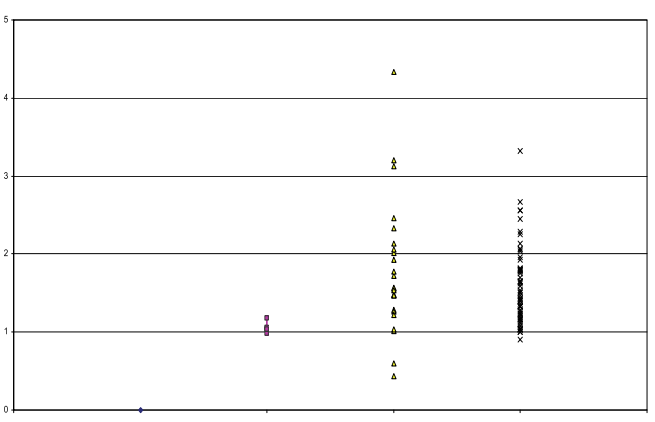

A N NTM Pre

Fig. 1. a) Schematic representation of FMRP levels detected in A: FXS affected individuals, $\mathrm{N}$ : individuals with a normal CGG repeat number; NTM: FMR1 premutation male carriers and Pre: FMR1 premutation female carriers. B) Schematic representation of FMR1 mRNA levels detected in A: FXS affected individuals, $\mathrm{N}$ : individuals with a normal CGG repeat number; NTM: FMR1 premutation male carriers and Pre: FMR1 premutation female carriers.

The increased transcriptional activity of the FMR1 gene seems to be positively correlated with the size of the CGG repeat. That is, CGG repeats in the upper range (100-200 CGG) result in average 5-8 fold elevation, whereas CGGs in the lower range (50-100 CGG) result in an average 2-fold elevation (Kenson et al., 2001; Oostra \& Willemsen, 2003; Tassone et al., 2000a, 2000b). Although the precise mechanism for this overexpression is unknown, several possible mechanisms have been postulated. A feedback mechanism suggests that the cell attempts to compensate for reduced levels of FMRP by increasing the amount of available FMR1 transcript (reviewed in Galloway \& Nelson, 2009; Tassone \& Hagerman, 2003). Alternatively, it is likely that the increasing length of the CGG repeat near the FMR1 promoter proportionally opens the chromatin, allowing more ready access to transcription factors (Tan el al., 2009). The presence of these elevated levels of abnormal (expanded CGG repeat) FMR1 mRNA led to propose an RNA "toxic gain-of-function" model for FXTAS, in which the mRNA itself, with the abnormal CGG repeat tract, is causative of the neurological 
disorder (Greco et al., 2002: Hagerman \& Hagerman, 2004; Hagerman et al., 2001: Jacquemont et al., 2003). Although several evidences support the RNA-based mechanism, the precise form of how the CGG-repeat RNA is responisble for FXTAS pathogenesis is not yet resolved (Garcia-Arocena \& Hagerman, 2010) The same RNA “toxic gain-of-function" mechanism has been proposed for myotonic dystrophy (DM1 and DM2), in which either the expanded repeat tract of CUG in DM1 or CCUG in DM2 sequestered CUG-binding proteins that disrupts mRNA processing of other genes or transport of other mRNAs (Mankodi \& Thornton, 2002). In fact, this model has been demonstrated for DM1 by placing an expanded CTG tract in 3' UTR region of the DMPK mRNA in a transgenic mouse (Mankodi et al., 2000). A part from this finding, FXTAS and myotonic dystrophy have another important similarity that supports the RNA gain-of-function mechanism. Both disorders show nuclear inclusions produced as a result of the binding proteins sequestered by the respectively mRNA, with a cytotoxic effect that lead to cell death. In a study performed by Greco and coworker (2002) eosinophilic intranucelear inclusions in neurons and astrocytes throughout the cortex and in deep cerebellar nuclei of FXTAS post-mortem samples were reported. Furthermore, in a subsequent study, there is described a highly significant association between CGG length and both the number of inclusions and the age of death, which correlates with the progressive character of the disease (Greco et al., 2006). The intranuclear inclusions associated with FXTAS have characteristic features different than those found in tauopathies (e.g., Pick disease), synucleinopathies (e.g., Lewy body dementias and Parkinson disease) or polyglutamine disorders (SCAs). It is important to note that, unlike the polyglutamine disorders, there is no known structurally abnormal protein with FXTAS (reviewed in Galloway \& Nelson, 2009; Iwahashi et al., 2006). Taken together, these facts define FXTAS as a new class of inclusion disorder.

In order to test the RNA gain-of-function hypothesis for FXTAS, a "knock-in" mouse model has been generated in which the endogenous mouse CGG repeat was replaced by a human CGG tract carrying 98 CGGs (Bontekoe et al., 2001; reviewed in Oostra \& Willemsen, 2009). Further studies of the brain of these expanded-repeat mice (at 20-72 weeks) evidenced elevated Fmr1 mRNA levels and ubiquitin-positive intranuclear inclusions (Willemsen et al., 2003). An increase was also observed in both the number and the size of the inclusions in specific brain region during the course of life (Oostra \& Willemsen, 2003). The presence of inclusions in this mouse, that has normal levels of FMRP, provides evidences against a protein-deficiency model for FXTAS, and supports a direct role of the Fmr1 gene, by either CGG expansion per se or by elevated Fmr1 mRNA levels, in the pathology.

There are several other animal and cell-based studies that provide evidence of direct RNA toxicity (Galloway \& Nelson, 2010; reviewed in Garcia-Arocena \& Hagerman, 2010). Most of these studies have demostrated a sequestartion of several candidates CGG-repeat binding proteins from their normal function. Remarkably, they have also demonstrated at least partial rescue of the wild-type phenotype by overexpressing the sequestered protein. The number of candidate proteins for sequestration has been lately increased and surprisingly, it has also been shown that inclusions are dynamic structures that expanded over time, resulting in giant inclusions (Sellier et al., 2010). Continuous enlargements of CGG RNA aggregates suggest that these repeats may constantly recruit proteins, implying a founding RNA-protein interaction event that would subsequently trap other proteins through indirect RNA-protein or protein-protein interactions (Sellier et al., 2010). The protein components of FXTAS inclusions fell into eight major functional categories, including: histone family; intermediate filament; microtubule; myelin-associated proteins; RNA-binding proteins; 
stress-related proteins; chaperones and ubiquitin-proteasome-related proteins (reviewed Galloway \& Nelson, 2010).

Interestingly, an antisense transcript, $A S F M R 1$, has recently been identified to overlap the CGG repeat region of the FMR1 gene (Ladd et al., 2007). Similar to FMR1, the ASFMR1 transcript is silenced in full mutation individuals and overexpressed in permutation carriers. However, whether ASFMR1 contributes to the pathogenesis of either FXTAS or FXS remains to be determined (Tan et al., 2009).

\section{Neuroimaging findings - Conventional MRI strenghts and limitations}

FXTAS was originally described in men, older than 50 years of age, having a typical clinical picture of progressive intention tremor, and cerebellar ataxia (Hagerman et al 2001). Magnetic resonance imaging (MRI) of the brain was obtained in these patients searching for specific features, and the finding of hyperintensities in the cerebellar white matter and middle cerebellar peduncles on T2-weighted images was reported as a characteristic feature and called the "MCP sign" (Fig. 2a), as it was seen in nearly all FXTAS-premutated carriers, and not in controls (Brunberg et al., 2002). Other typical MR features described originally in patients with FXTAS included cerebellar, pontine, and cerebral atrophy, as well as white matter hyperintensities (Fig. 2 b-d) (Brunberg et al., 2002).

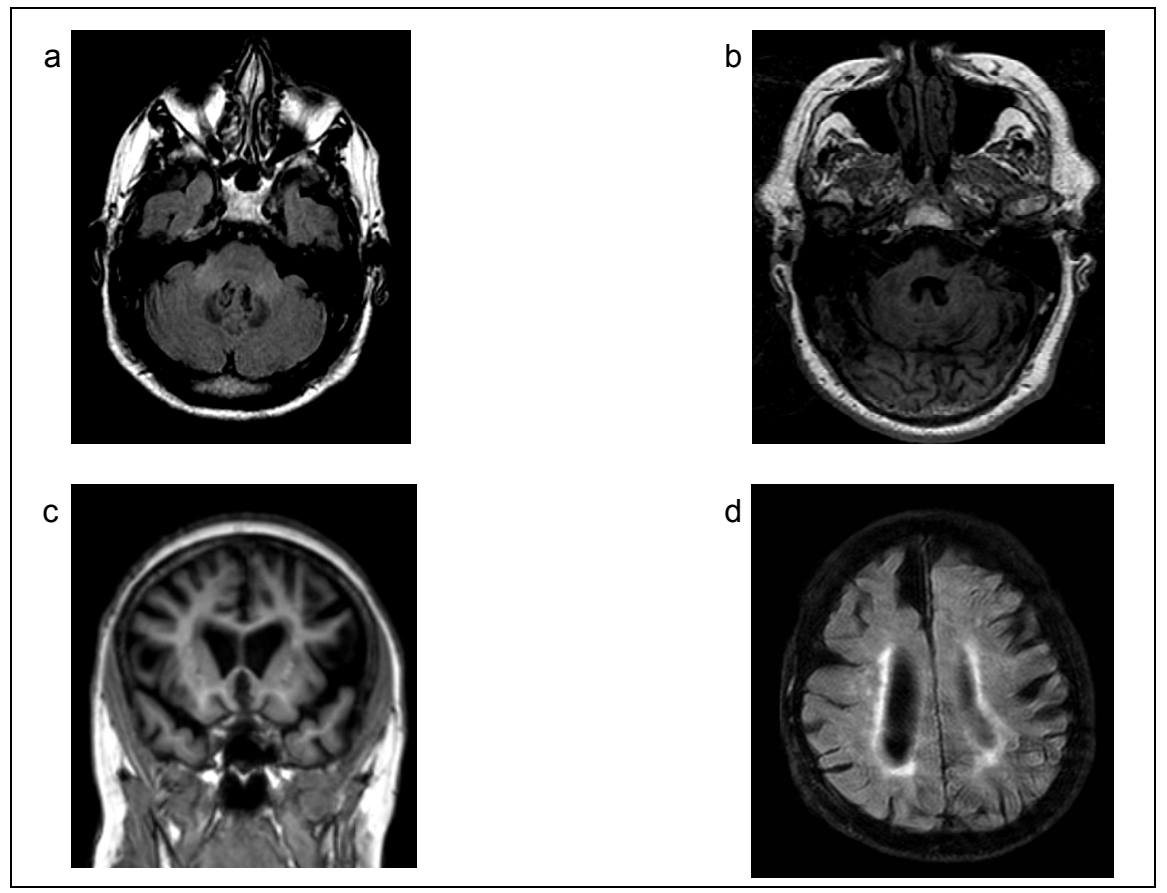

Fig. 2. MR images showing characteristic findings in FXTAS. 1a. Axial FLAIR- MCP sign (middle cerebellar peduncle hyperintensities); 1b. Axial T1- Cerebellar and pontine atrophy; 1c. Coronal T1- Cerebral atrophy; 1d. Axial FLAIR- Hyperintensities in the cerebral white matter. 
Thus, characteristic findings described on conventional MRI in patients with FXTAS, were classified into two categories -major and minor criteria-, and proposed, together with clinical findings, as diagnostic criteria for FXTAS (Jacquemont et al., 2003). Major criteria included the MCP sign, as well as white matter and brainstem hyperintensities. Cerebellar and brain atrophy were proposed as minor criteria (Jacquemont et al., 2003) (Table 1).

\begin{tabular}{|l|l|}
\hline Examination and Degree & Observation \\
\hline Radiological: & \\
\hline Major & MRI white matter lesions in MCPs and or brain stem \\
\hline Minor & MRI white matter lesions in cerebral white matter \\
\hline Minor & Moderate-to-severe generalized atrophy \\
\hline Clinical: & \\
\hline Major & Intention tremor \\
\hline Major & Gait ataxia \\
\hline Minor & Parkinsonism \\
\hline Minor & Moderate-to-severe short-term memory deficiency \\
\hline Minor & Executive function deficit \\
\hline
\end{tabular}

Inclusion criterion: CGG repeat number between 55 and 200.

Note. Data described by Jacquemont et al. 2003.

Table 1. Clinical Criteria for FXTAS. The diagnostic categories described by Jaquemont et al. 2003 are as follows:

Definite (1 major radiological sign plus 1 major clinical symptom)

Probable (1 major radiological sign plus 1 minor clinical symptom or two major clinical symptoms)

Possible (1 minor radiological sign plus 1 major clinical symptom)

Early neuropathological, postmortem studies of the brain of patients with FXTAS revealed intranuclear inclusions in neurons and astrocytes throughout the cortex and in deep cerebellar nuclei, but not in Purkinje cells of the cerebellum (Greco et al., 2002). Further evidence has shown that there is significant cerebral and cerebellar white matter disease, associated astrocytic pathology in the cerebral white matter, and intranuclear inclusions in both brain and spinal cord (Greco et al., 2006). Additionally, there seems to be an association between the number of CGG repeats and the number of intranuclear inclusions in neurons and astrocytes, so that CGG repeat has been suggested as a predictor for clinical and neuropathological involvement (Greco et al., 2006). Unfortunately, there is no histopathological evidence from brains of premutated, non-FXTAS subjects, who died because of an unrelated condition, being either asymptomatic neurologically, or little symptomatic. This evidence would certainly add in the understanding of the pathogenetic processes underlying glial and neuronal damage, and eventual neurological dysfunction. Brain banks and collaborative actions may represent a good opportunity in this regard.

Penetrance of FXTAS among premutation carriers has been studied, and reported to be relevant, mainly in men (Jacquemont et al., 2004). As premutation carriers are relatively common in the general population, it has been proposed that older men with ataxia and intention tremor should be screened for the FMR1 mutation (Jacquemont 2004). Using the previously described criteria for FXTAS (Jacquemont et al., 2003), a study among adult Spanish patients with ataxia, revealed an estimated FXTAS prevalence varying between 
$1.15 \%$ for males, and 3\% for females (Rodriguez-Revenga et al., 2007). Similarly, a frequency of $1.6 \%$ of patients with FXTAS has been reported among adult patients with movement disorders who tested negative for the Huntington gene (Rodriguez-Revenga et al., 2008b). The importance of neuroradiological findings that could be used as an additional screening tool for FXTAS is demonstrated by these studies.

In this regard, the MCP sign, which was originally proposed to be a characteristic and specific feature for FXTAS, that could be used for screening purposes, has been also reported in patients with other forms of adult-onset cerebellar ataxia, thus lacking specificity for FXTAS (Okamoto et al., 2003). Patients with atypical parkinsonism, and particularly those having a clinical picture including dysautonomia, and ataxia -a condition currently known as the cerebellar form of Multiple System Atrophy (MSA)-, may show the MCP sign on MRI, so that middle cerebellar peduncles hyperintensities in a patient with parkinsonism should be regarded as a non-specific finding, which can be seen in FXTAS, but also in MSA (Kamm et al., 2005). Additionally, the sensitivity of the MCP sign may be less than previously thought, as the frequency of the MCP sign among women with FXTAS seems to be less (Hagerman et al., 2004), and its presence among premutated men with subtle neurological, psychiatric, or cognitive dysfunction remains unknown.

As more evidence among FMR1 premutation carriers developing neurological features, and particularly parkinsonism, is being gained, the spectrum and variability of MRI features becomes broader. Also, the severity of disease, which may relate to CGG repeat number, or other unknown factors, may influence the presence and magnitude of MRI findings. In this context, a correlation between CGG repeat length and reductions in IQ and cerebellar volume, and increased ventricular volume and whole-brain white matter hyperintensities, have been reported in FMR1 premutation carriers (Cohen et al., 2006). It may well then be the case, that patients with a longer duration of neurological disturbances, or a greater severity, are those showing the so-known "typical MRI findings" of FXTAS. In this regard, more evidence is needed among younger premutated patients. Additionally, premutated women and women with FXTAS seem to have a different phenotype (Berry et al., 2004; Hagerman et al., 2004; Hessl et al., 2005), so that the frequency and relevance of MRI findings in women may be different from that in men. In this regard, less pronounced reductions in cerebellar volume and a lower incidence of the MCP sign has been reported in women with FXTAS compared to men (Adams et al., 2007). Also, an absence of significant associations between reduced cerebellar volumes and increased FXTAS severity, and increased length of the CGG repeat expansion was reported in women, differently from men having FXTAS (Adams et al., 2007).

Conventional MRI, as including T1- and T2- weighted MR images, suffers from several limitations as a tool to investigate patients with a neurodevelopmental disorder that develop a neurodegenerative process later in life, as happens in the FMR1 premutation/FXTAS condition. Today, with the advent of high field strength MRI, and stronger gradients, there are more specific sequences such as susceptibility- or gradient-echo-weighted MR-images, which can provide more specific assessment of mechanisms underlying neuronal degeneration, such as iron deposition. Iron deposition is seen with normal aging in specific brain structures. Up to date, increased and/or iron deposition in certain brain structures has not been demonstrated among FXTAS patients, to the best of our knowledge, but this may only be a question of time or of the cohorts being studied.

Also, recently FLAIR imaging has somehow substituted T2-weighted imaging for the assessment of white matter and brainstem T2- signal changes. Findings on FLAIR images 
may not be exactly the same as seen on T2-weighted images, and thus, more evidence is needed with these more recent techniques. Finally, Arterial Spin Labeling, a non-invasive MRI method that allows detection of specific perfusion patterns of involvement linked to brain metabolism, is currently being applied to several neurodegenerative brain conditions, and may also be useful in the context of FMR1 premutation/FXTAS.

\section{Advances in neuroimaging in FXTAS and future needs}

The advancing field of Neuroradiology, and particularly of MRI, has provided insight in neurodegenerative conditions. Typical MRI findings have been described, that allow prompt and more precise characterization of many conditions, such as FXTAS. Additionally, MRI has provided non-invasive markers of disease, which may be potentially useful in early and differential diagnosis, in prognosis, and eventually in therapeutic response. This is the case for triplet expansion, genetic conditions, such as Huntington's disease (HD), which offers some similarities to FXTAS. Originally, conventional MRI was used and typical MRI findings were described in HD, but recently, more sophisticated imaging methods, such as MRSpectroscopy, have been applied not only to patients with HD, but also to asymptomatic carriers, searching for markers for early diagnosis and conversion to disease. In this regard, metabolic (Gomez-Anson et al., 2007) and structural (Gomez-Anson et al., 2009) MR-alterations in the prefrontal regions of asymptomatic HD carriers have been recently described, linked to neuropsychological dysfunction, and proposed as early markers for disease, related to underlying pathology. Knowledge in field of FMR1 premutation carriers, who will eventually develop FXTAS, may follow a similar course. Although until now, mainly conventional MRI features have been described, there is growing evidence from more sophisticated MR techniques, which has and will continue adding knowledge in the field.

Advances in Neuroimaging in FMR1 premutation/FXTAS may, perhaps, come from two distinct contributions. Firstly, more recent developments in MR are being applied to these patients. This is particularly the case of functional MR techniques, which not only provide information about structural brain changes in these patients, but also of brain functioning. As an example, MR-Spectroscopy (MRS) is a technique which allows studying non-invasively brain metabolism in vivo. Metabolic information can be linked to cellular pools, thus to histopathological changes, and to neuropsychological and clinical features. There is very little evidence about MRS in FXTAS. However, MRS changes indicating neuronal loss/dysfunction have been described in the pons of patients with FXTAS having the typical MCP sign on conventional imaging (Ginestroni et al., 2007). More recent evidence has shown altered metabolism on MRS in the middle cerebellar peduncles of patients with FMR1 premutation, which may be more marked in FXTAS (Gomez-Anson et al., abstract 2007). These findings indicate a potential usefulness of MRS, adding in differential diagnosis of patients with ataxias, and being able, perhaps, to identify those patients that will develop FXTAS. However, more longitudinal evidence and larger cohorts of carriers and patients from multicentric studies are needed in this regard. Functional MRI may also prove to be useful in the field of, as it has been recently demonstrated that there is altered prefrontal cortex activity underlying executive and memory deficits in permutated carriers and patients (Hashimoto et al., 2011).

Secondly, contributions from more sophisticated postprocessing tools will certainly add during the near future in the field. In this context, assessment and quantification of volume changes in the brain is now feasible non-invasively using MRI and volumetric techniques. One of these techniques, the Voxel Based Morphometry method (VBM) allows 
determination of focal changes of grey and white matter density in the brain on MRI (Ashburner et al., 2000). However, the evidence of volumetric studies in FXTAS and premutation carriers is scarce (Gomez-Anson et al., 2007; Hashimoto et al., 2011; Moore et al., 2004b). Automated postprocessing tools are also being currently applied to study regional changes of volume in the brain, which may be specific to a condition, and related to certain cognitive tasks. This is the case of the hippocampus and memory impairment, which are relevant in Alzheimer's disease, for example (Sanchez-Benavides et al., 2010). Evidence of the application of these tools to research in the FXTAS condition is still lacking.

The potential usefulness of neuroimaging in providing insight in FXTAS becomes more evident as cognitive decline in FXTAS is resulting in an important field of research. As the phenotypic spectrum has expanded among FMR1 premutated carriers, and FXTAS patients, psychiatric and cognitive disturbances are increasingly being recognized as relevant features

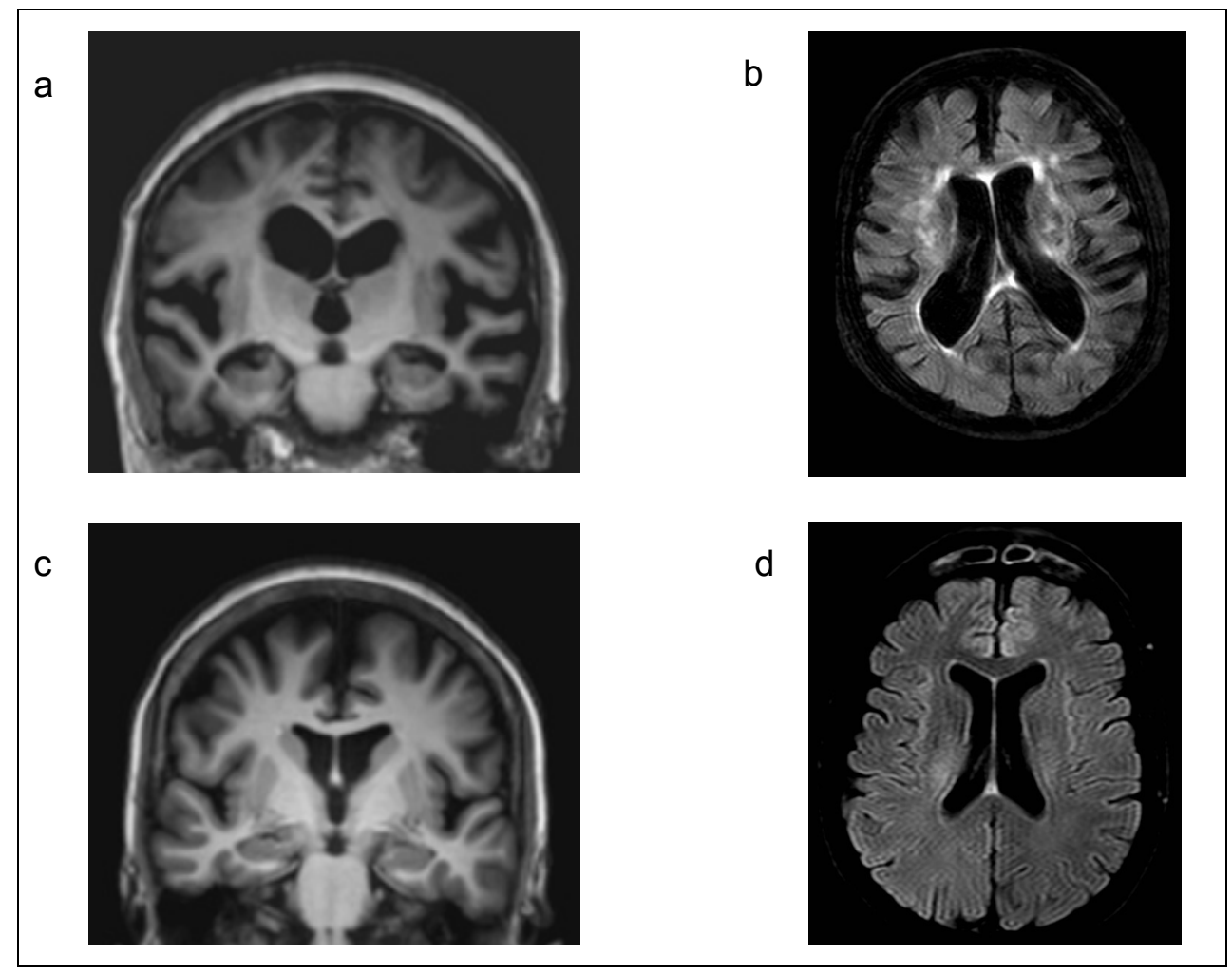

Fig. 3. Magnetic resonance imaging findings of two female patients with dementia and FXTAS. A) Coronal T1-weighted, 3D MPRAGE MR image of patient 1 shows asymmetrical frontal, and bilateral, symmetrical, medial temporal atrophy. B) Axial 3D FLAIR of patient 1 shows marked hyperintensities in the cerebral white matter. C) Coronal T1-weighted, 3D MPRAGE MR image of patient 2 shows moderate cerebral atrophy involving the frontal lobes, while the medial temporal lobes appeared normal.; K) Axial 3D FLAIR of patient 2 shows no marked $\mathrm{HI}$ in the white matter. 
(Sevin et al., 2009). Cognitive decline in FXTAS usually occurs in men, after a long duration of extrapyramidal disturbances, but it may also occur in women. Typically in men, a clinical picture similar distinct from Alzheimer's disease (Seritan et al., 2008), and more similar to patients with fronto-temporal lobar degeneration occurs (FTLD) (Burgeois et al., 2009).

Although there is little evidence from MRI in these patients, particularly in women (Al Hinti et al., 2007; Karmon et al., 2008), more recently, two cases of mother-to-daughter transmission were reported (Rodriguez-Revenga et al., 2010). In these two female patients with FXTAS and dementia, a radiological pattern of brain atrophy resembling that of patients with FTLD, was described. However, radiological heterogeneity may be large in this condition, as shown by the fact that prominent white matter hyperintensities were only seen in one case, as had been previously described (Al Hinti et al., 2007; Karmon et al., 2008), the second case lacking these, as well as hippocampal atrophy (Fig. 3).

There is very recent evidence about the use of VBM for studying the brain changes of FMR1 premutated carriers, with and without FXTAS, and their correlation to neuropsychology (Hashimoto et al., 2011). In this study, patients with FXTAS demonstrated a distinct pattern of grey matter volume loss, involving multiple cortical and subcortical regions. This included different parts of the cerebellum, as well as of the medial surface of the brain, including the dorsomedial prefrontal cortex, anterior cingulate and precuneus. Additional volume loss was seen in the lateral prefrontal cortex, orbitofrontal cortex, amygdala, and insula. More interestingly, there were significant correlations between grey matter loss in different brain regions, behavioral scales, and CGG repeats (Hashimoto et al., 2011).

\section{Conclusion}

FXTAS is a relatively new disorders that is currently regarded as a late-onset neurodegenerative disorder; however, the underlying pathogenic process may begin very early in life. It is essential to fathom the molecular mechanism of FXTAS and to increase our knowledge of disease pathology in order to understand of FXTAS disease progression. Although the RNA gain-of-function hypothesis for FXTAS is well accepted, further analysis of the pathogenic effect of the expanded CGG-repeat FMR1 mRNA are still required. A better understanding of the molecular basis of FXTAS should shed light on therapeutic approaches that will combat neurodegeneration and improve cognitive and motor performance. Furthermore, it can also help unraveling common mechanisms in other neurodegenerative diseaser which will bring hope to treatments more effective and specific to the underlying dysfunction.

MRI findings in FXTAS patients classically include middle cerebellar and white matter hyperintensities, as well as cerebellar, pontine and brain atrophy. Neuroimaging, and particularly MR techniques, offer an excellent opportunity to gain insight into the FXTAS condition. In vivo biomarkers may be identified non-invasively, which may be potentially useful in improving recognition, and early characterization of patients with FXTAS, so that treatment strategies can be developed and applied.

As the prevalence of premutated alleles is relatively high in general population, FXTAS may represent one of the more common monogenic causes of tremor, ataxia, and dementia. For this reason, it is probably that many carriers with FXTAS are being seen by a clinical 
specialist without awareness of the underlying genetic basis for the symptoms. The early diagnosis of those patients not only benefits themselves but also the rest of the family that should be advised for the FXS.

\section{Acknowledgments}

This work has received financial support from grant PI09/0413 and FISS PI/770 both financed by "Instituto Carlos III". We would like to acknowledge GIRMOGEN and the Catalan Association of Fragile $X$ syndrome as well as all FXS families that collaborate with us. The CIBER de Enfermedades Raras is an initiative of the ISCIII

\section{References}

Adams, JS., Adams, PE., Nguyen, D., Brunberg, JA., Tassone, F., Zhang, W., Koldewyn, K., Rivera, SM., Grigsby, J., Zhang, L., DeCarli, C., Hagerman, PJ., \& Hagerman, RJ. (2007). Neurology 28,69,851-859.

Al-Hinti, JT., Nagan, N., \& Harik, SI. (2007). Alzheimer Disease \& Associated Disorders 21,262264.

Ashburner, J., \& Friston, KJ. (2000). Neuroimage 11,805-821.

Bacalman, S., Farzin, F., Bourgeois, JA., Cogswell, J., Goodlin-Jones, BL., Gane, LW., Grigsby, J., Leehey, MA., Tassone, F., \& Hagerman, RJ. (2006). Journal of Clinical Psychiatry 67,87-94.

Bassell, GJ., \& Warren, ST. (2008). Neuron 60,201-214.

Bennetto, L; Pennington, BF; Porter, D; Taylor, AK; Hagerman, RJ. (2001). Neuropsychology 15,290-299.

Berry-Kravis, E., Potanos, K., Weinberg, D., Zhou, L., \& Goetz, CG. (2004). Annals of Neurology 57,144-147.

Bontekoe, CJ; Bakker, CE; Nieuwenhuizen, IM; van der Linde, H; Lans, H; de Lange, D; Hirst, MC; \& Oostra, BA. (2001). Human Molecular Genetics 10,1693-1639.

Bourgeois, JA., Coffey, SM., Rivera, SM., Hessl, D., Gane, LW., Tassone, F., Greco, C., Finucane, B., Nelson, L., Berry-Kravis, E., Grigsby, J., Hagerman, PJ., \& Hagerman, RJ. (2009). Journal of Clinical Psychiatry 70,852-862.

Brunberg J., Jacquemont S., Hagerman RJ., Berry-Kravis, E., Grigsby, J., Leehey, M., Tassone, F., Brown, T., Greco, C., \& Hagerman. PJ. (2002). American Journal of Neuroradiology.23,1757-1766.

Brussino, A., Gellera, C., Saluto, A., Mariotti, C., Arduino, C., Castellotti, B., Camerlingo, M., de Angelis, V., Orsi, L., Tosca, P., Migone, N., Taroni, F., \& Brusco, A. (2005) Neurology 64,145-147.

Coffey, SM., Cook, K., Tartaglia, N., Tassone, F., Nguyen, DV., Pan, R., Bronsky, HE., Yuhas, J., Borodyanskaya, M,, Grigsby, J., Doerflinger, M., Hagerman, PJ., \& Hagerman, RJ. (2008) American Journal of Medical Genetics Part A 146,1009-1016.

Cohen, S., Masyn, K., Adams, J., Hessl, D., Rivera, S., Tassone, F., Brunberg, J., DeCarli, C., Zhang, L., Cogswell, J., Loesch, D., Leehey, M., Grigsby, J., Hagerman, PJ., \& Hagerman, R. (2006). Neurology 67,1426-1431.

Galloway, JN., \& Nelson, DL. (2009) Future Neurology 4,785. 
Garcia-Arocena, D., \& Hagerman PJ. (2010). Human Molecular Genetics 19,R83-R89.

Ginestroni, A., Guerrini, L., Della Nave, R., Tessa, C., Cellini, E., Dotti, MT., Brunori, P., De Stefano, N., Piacentini, S., \& Mascalchi, M. (2007). AJNR - American Journal of Neuroradiology. 28,486-488.

Gómez-Ansón, B., Alegret, M., Muñoz, E., Sainz, A., Monte, GC., \& Tolosa, E. (2007). Neurology 68,906-910.

Gómez Ansón, B., Monte, GC., Rotger, R., Rodriguez-Revenga, L., Mila, M., \& Capurro, S. (2007). MR characterization of premutated carriers of the fragile $X$ syndrome: a VBM and 1H-MRS study. Proceedings of Congress of European Society of Neuroradiology (ESNR), Genova, September 2007.

Gómez-Ansón, B., Alegret, M., Munoz, E., Monte, GC., Alayrach, E., Sanchez, A., Boada, M., \& Tolosa, E. (2009). Parkinsonism \& Related Disorders 15,213-219.

Greco, CM., Hagerman, RJ., Tassone, F., Chudley, AE., Del Bigio, MR., Jacquemont, S., Leehey, M., \& Hagerman, PJ. (2002). Brain 125,1760-1771.

Greco, CM., Berman, RF., Martin, RM., Tassone, F., Schwartz, PH., Chang, A., Trapp, BD., Iwahashi, C., Brunberg, J., Grigsby, J., Hessl, D., Becker, EJ., Papazian, J., Leehey, MA., Hagerman, RJ., \& Hagerman, PJ. (2006). Brain 129:243-255.

Grigsby, J., Brega, AG., Leehey, MA., Goodrich, GK., Jacquemont, S., Loesch, DZ., Cogswell, JB., Epstein, J., Wilson, R., Jardini, T., Gould, E., Bennett, RE., Hessl, D., Cohen, S., Cook, K., Tassone, F., Hagerman, PJ., \& Hagerman, RJ. (2007). Movement Disorders 22,645-650.

Grigsby, J., Brega, AG., Engle, K., Leehey, MA., Hagerman, RJ., Tassone, F., Hessl, D., Hagerman, PJ., Cogswell, JB., Bennett, RE., Cook, K., Hall, DA., Bounds, LS., Paulich, MJ., \& Reynolds, A. (2008). Neuropsychology 22,48-60.

Hagerman, RJ., Leehey, M., Heinrichs, W., Tassone, F., Wilson, R., Hills, J., Grigsby, J., Gage, B., \& Hagerman, PJ. (2001). Neurology 57,127-130.

Hagerman, RJ. (2002). Physical and behavioral phenotype, In: Fragile X Syndrome: Diagnosis, treatment and research Hagerman RJ \& Hagerman PJ (3rd edition). The Johns Hopkins University Press, Baltimore, MD.

Hagerman, PJ., \& Hagerman, RJ. (2004). The American Journal of Human Genetics 74,805816.

Hashimoto, RI., Backer, KC., Tassone, F., Hagerman, RJ., \& Rivera, SM. (2011). Movement Disorder 11.

Hessl, D., Tassone, F., Loesch, DZ., Berry-Kravis, E., Leehey, MA., Gane, LW., Barbato, I., Rice, C., Gould, E., Hall, DA., Grigsby, J., Wegelin, JA., Harris, S., Lewin, F., Weinberg, D., Hagerman, PJ., \& Hagerman, RJ. (2005). American Journal of Medical Genetics Part A 139,115-121.

Iwahashi, CK., Yasui, DH., An, HJ., Greco, CM., Tassone, F., Nannen, K., Babineau, B., Lebrilla, CB., Hagerman, RJ., \& Hagerman, PJ. (2006). Brain 129,256-271.

Jacquemont, S., Hagerman, RJ., Leehey, M., Grigsby, J., Zhang, L., Brunberg, JA., Greco, C., Des Portes, V., Jardini, T., Levine, R., Berry-Kravis, E., Brown, WT., Schaeffer, S., Kissel J., Tassone, F., \& Hagerman, PJ. (2003). The American Journal of Human Genetics 72,869-878. 
Jacquemont, S., Hagerman, RJ., Leehey, MA., Hall, DA., Levine, RA., Brunberg, JA., Zhang, L., Jardini, T., Gane, LW., Harris, SW., Herman, K., Grigsby, J., Greco, CM., BerryKravis, E., Tassone, F., \& Hagerman, PJ. (2004a). JAMA, the Journal of the American Medical Association 291,460-469.

Jacquemont, S., Farzin, F., Hall, D., Leehey, M., Tassone, F., Gane, L., Zhang, L., Grigsby, J., Jardini, T., Lewin, F., Berry-Kravis, E., Hagerman, PJ., \& Hagerman, RJ. (2004b). American Journal Of Mental Retardation 109,154-164.

Jacquemont, S., Hagerman, R., Hagerman, PJ., Leehey, MA. (2007). The Lancet Neurology $6,45-55$.

Johnston, C., Eliez, S., Dyer-Friedman, J., Hessl, D., Glaser, B., Blasey, C., Taylor, A., \& Reiss, A. (2001). American Journal of Medical Genetics 103,314-319.

Kamm, C., Healy, DG., Quinn, NP., Wüllner, U., Moller, JC., Schols, L., Geser, F., Burk, K., Børglum, AD., Pellecchia, MT., Tolosa, E., del Sorbo, F., Nilsson, C., Bandmann, O., Sharma, M., Mayer, P., Gasteiger, M., Haworth, A., Ozawa, T., Lees, AJ., Short, J., Giunti, P., Holinski-Feder, E., Illig, T., Wichmann, HE., Wenning, GK., Wood, NW., Gasser, T., \& European Multiple System Atrophy Study Group. (2005). Brain 128,1855-1860.

Karmon, Y., \& Gadoth, N. (2008). Journal of Neurology, Neurosurgery \& Psychiatry 79,738739.

Kenneson, A., Zhang, F., Hagedorn, CH., \& Warren, ST. (2001). Human Molecular Genetics 10,1449-1454.

Ladd, PD., Smith, LE., Rabaia, NA., Moore, JM., Georges, SA., Hansen, RS., Hagerman, RJ., Tassone, F., Tapscott, SJ., \& Filippova, GN. (2007). Human Molecular Genetics 16,3174-3187.

Leehey, MA., Munhoz, RP., Lang, AE., Brunberg, JA., Grigsby, J., Greco, C., Jacquemont, S., Tassone, F., Lozano, AM., Hagerman, PJ., \& Hagerman, RJ. (2003). Archives of Neurology 60,117-121.

Leehey, MA., Berry-Kravis, E., Min, SJ., Hall, DA., Rice, CD., Zhang, L., Grigsby, J., Greco, CM., Reynolds, A., Lara, R., Cogswell, J., Jacquemont, S., Hessl, DR., Tassone, F., Hagerman, R., \& Hagerman PJ. (2007). Movement Disorders 22,203-206.

Macpherson, J., Waghorn, A., Hammans, S., \& Jacobs, P. (2003). Human Genetics 112,619620.

Mankodi, A., Logigian, E., Callahan, L., McClain, C., White, R., Henderson, D., Krym, M., \& Thornton, CA. (2000). Science 289,1769-1773.

Mankodi, A., \& Thornton, CA. (2002). Current Opinion in Neurology 15,545-552.

Milà, M., Madrigal, I., Kulisevsky, J., Pagonabarraga, J., Gómez, B., Sánchez, A., \& Rodríguez-Revenga, L. (2009). Medicina Clínica 133, 252-4.

Moore, CJ., Daly, EM., Schmitz, N., Tassone, F., Tysoe, C., Hagerman, RJ., Hagerman, PJ., Morris, RG., Murphy, KC., \& Murphy, DG. (2004a). Neuropsychologia 42,1934-1947.

Moore, CJ., Daly, EM., Tassone, F., Tysoe, C,. Schmitz, N., Ng, V., Chitnis, X., McGuire, P., Suckling, J., Davies, KE., Hagerman, RJ., Hagerman, PJ., Murphy, KC., \& Murphy, DG. (2004b). Brain 127,2672-2681. 
Oberle, I., Rousseau, F., Heitz, D., Kretz, C., Devys, D., Hanauer, A., Boue, J., Bertheas, MF., \& Mandel, JL. (1991). Science 252,1097-1102.

Okamoto, J., Tokiguchi, S., Furusawa, T., Ishikawa, K., Quardery, AF., Shinbo, S., \& Sasai S. (2003). AJNR Am American Journal of Neuroradiology 24,1946-1954.

Oostra, BA., \& Willemsen, R. (2003). Human Molecular Genetics 12,R249-R257.

Oostra BA., \& Willemsen R. (2009). Biochimica et Biophysica Acta 1790,467-477.

Reiss, AL., Freund, L., Abrams, MT., Boehm, C., \& Kazazian, H. (1993). The American Journal of Human Genetics 52,884-894.

Riddle, JE., Cheema, A., Sobesky, WE., Gardner, SC., Taylor, AK., Pennington, BF., \& Hagerman, RJ. (1998). American Journal Of Mental Retardation 102,590-601.

Rife, M., Badenas, C., Mallolas, J., Jimenez, L., Cervera, R., Maya, A., Glover, G., Rivera, F., \& Mila, M. (2003). Genetic Testing 7,339-343.

Rodriguez-Revenga, L., Gómez-Anson, B., Muñoz, E., Jiménez, D., Santos, M., Tintoré, M., Martín, G., Brieva, L., \& Milà, M. (2007). Molecular Neurobiology 35,324-328.

Rodriguez-Revenga, L., Madrigal, I., Alegret, M., Santos, M., \& Milà, M. (2008a). Psychiatric Genetics 18,153-155.

Rodriguez-Revenga, L., Santos, MM., Sánchez, A., Pujol, M., Gómez-Anson, B., Badenas, C., Jiménez, D., Madrigal, I., \& Milà, M. (2008b). Genetic Testing 12,135-138.

Rodriguez-Revenga, L., Madrigal, I., Pagonabarraga, J., Xunclà, M., Badenas, C., Kulisevsky, J., Gomez, B., \& Milà, M. (2009). European Journal of Human Genetics $17,1359-1362$.

Rodriguez-Revenga, L., Pagonabarraga, J., Gómez-Anson, B., López-Mourelo, O., Xunclà. M., \& Milà, M. (2010). Neurology 75,1370-1376.

Sánchez-Benavides, G., Gómez-Ansón, B., Sainz, A., Vives, Y., Delfino, M., \& PeñaCasanova, J. (2010). Psychiatry Research 181,219-225.

Sellier, C., Rau, F., Liu, Y., Tassone, F., Hukema, RK., Gattoni, R., Schneider, A., Richard, S., Willemsen, R., Elliott, DJ., Hagerman, PJ., \& Charlet-Berguerand, N. (2010). The EMBO Journal 29,1248-1261.

Seritan, AL., Nguyen, DV., Farias, ST., Hinton, L., Grigsby, J., Bourgeois, JA., \& Hagerman, RJ. ( 2008). American Journal of Medical Genetics Part B: Neuropsychiatric Genetics 147,1138-1144.

Sevin, M., Kutalik, Z., Bergman, S, Vercelletto, M., Renou, P., Lamy, E., Vingerhoets,FJ., Di Virgilio, G., Boisseau, P., Bezieau, S., Pasquier, L., Rival, JM., Beckmann, JS., Damier, P., \& Jacquemont. S. (2009). Journal of Medical Genetics 46,818-824.

Tan, H., Li, H., \& Jin, P. (2009). Neuroscience Letters 466,103-108.

Tassone, F., Hagerman, RJ., Taylor, AK., Gane, LW., Godfrey, TE., \& Hagerman, PJ. (2000a). The American Journal of Human Genetics 66,6-15.

Tassone, F., Hagerman, RJ., Taylor, AK., Mills, JB., Harris, SW., Gane, LW., \& Hagerman, PJ. (2000b). American Journal of Medical Genetics 91,144-1152.

Tassone, F., \& Hagerman, PJ. (2003). Cytogenetic and Genome Research 100,124-128.

Van Esch, H., Matthijs, G., \& Fryns, JP. (2005). Annals of Neurology 57,932-933.

Verkerk, AJ., Pieretti, M., Sutcliffe, JS., Fu, YH., Kuhl, DP., Pizzuti, A., Reiner, O., Richards, S., Victoria, MF., Zhang, FP., et al. (1991). Cell 65,905-914. 
Willemsen, R., Hoogeveen-Westerveld, M., Reis, S., Holstege, J., Severijnen, LA., Nieuwenhuizen, IM., Schrier, M., van Unen, L., Tassone, F., Hoogeveen, AT., Hagerman, PJ., Mientjes, EJ., \& Oostra, BA. (2003). Human Molecular Genetics 12,949959.

Yu, S., Pritchard, M., Kremer, E., Lynch, M., Nancarrow, J., Baker, E., Holman, K., Mulley, JC., Warren, ST., Schlessinger, D; et al. (1991). Science 252,1179-1181.

Zuhlke, Ch., Budnik, A., Gehlken, U., Dalski, A., Purmann, S., Naumann, M., Schmidt, M., Burk, K., \& Schwinger, E. (2004). Journal of Neurology 251:1418-1419. 


\title{
Non-Conventional MRI Techniques in Neurophychiatric Systemic Lupus Erythematosus (NPSLE): Emerging Tools to Elucidate the Pathophysiology and Aid the Diagnosis and Management
}

\author{
Efrosini Z. Papadaki ${ }^{1}$ and Dimitrios T. Boumpas ${ }^{2}$ \\ ${ }^{1}$ Department of Radiology \\ 2Internal Medicine and Rheumatology, \\ University of Crete School of Medicine, Heraklion
}

Greece

\section{Introduction}

Systemic Lupus Erythematosus (SLE) is an autoimmune inflammatory disorder affecting multiple organ systems. $30-40 \%$ of the patients manifest variable neuropsychiatric symptoms leading to significant morbidity and mortality. CNS involvement could be primary if directly related to SLE activity in the CNS or secondary to treatment, infections, metabolic abnormalities or other systemic manifestations such as hypertension (Futrell et al,1992). Primary NPSLE is divided into focal and diffuse disease. Focal NPSLE is characterized by focal neurologic deficits and is strongly associated with the occurrence of thromboembolic events. Diffuse primary NPSLE is a group of neurologic, psychiatric, and cognitive syndromes that vary from overt neurologic and psychiatric symptoms (eg. seizures, psychosis) to more subtle signs such as headache, mood disturbances, anxiety disorders or mild cognitive dysfunction. Neuropsychiatric lupus (NPSLE) manifestations can occur in the absence of either serologic activity or other systemic disease manifestations (Sibbit et al., 1999). Thus, in clinical practice the diagnosis of primary NPSLE is rather presumptive, after the exclusion of alternative causes of the neuropsychiatric symptoms.

There is no single diagnostic test that is sensitive and specific for SLE-related neuropsychiatric manifestations. The assessment of individual patients is based on clinical, neurologic and rheumatologic evaluation, immunoserologic testing, brain imaging, and psychiatric and neuropsychological assessment. These examinations are used to support or refute the clinical diagnostic impression, rule out alternative explanations, and form the basis for prospective monitoring of clinical evolution and response to treatment interventions. The lack of a diagnostic gold standard makes the correct diagnosis of primary NPSLE a challenge. This realization has led us in developing under the auspices of the European League Against Rheumatology (EULAR) evidence and expert based recommendations for the management of NPSLE (Bertsias et al, 2010). 
Magnetic resonance imaging (MRI) is the current modality of choice in the imaging assessment of NPSLE patients, due to its high sensitivity in detecting even small alterations in tissue water content (Sibbitt et al., 1999). However, the variable pathologic substrate of the NPLSE lesions result in low MRI specificity. Conventional MRI reveals lesions in about $50-75 \%$ of NPSLE patients, depending on the disease activity and the severity and kind of the neurological manifestations (focal or diffuse). In patients with focal symptoms conventional MRI commonly detects small, discrete, frontal-parietal subcortical or periventricular white matter lesions, hyperintense on T2 sequences, that usually represent small acute or chronic infarcts, or even microhemorrhages. Unfortunately, these lesions are not specific for NPSLE and exhibit no clinical correlation. Additional intravenous Gadolinium administration is helpful in differentiating acute from chronic lesions. Mild-to-moderate cerebral atrophy could also be detected by T1 sequences, associated with both generalized and focal brain injury.

In NPSLE patients with diffuse neurological manifestations conventional neuroimaging usually fails to demonstrate abnormalities that explain these symptoms. Additionally, histopathological studies confirm the presence of extensive diffuse parenchymal and cerebrovascular injury that could not be identified by the conventional MRI techniques. On the contrary, brain tissue microscopic structural, hemodynamic or metabolic changes could be assessed by advanced, non-conventional quantitative neuroimaging techniques, such as Diffusion Weighted Imaging (DWI), Diffusion Tensor Imaging (DTI), Perfusion Weighted Imaging (PWI), Magnetization Transfer Imaging (MTI) and Magnetic Resonance Spectroscopy (MRS). Although not available in the current daily practice, these techniques are promising for the better understanding of the NSPLE, and improvement of the diagnostic work-up and treatment.

We critically review the literature on brain imaging in NPSLE, with special emphasis on non-conventional neuroimaging techniques in order to access their contribution to diagnosis, understanding of the pathophysiology and clinical management.

\section{Histopathological changes in NPSLE}

The underlying pathologic basis of NP-SLE is still under investigation (Bertsias GK, Boumpas DT, 2010). Pathological studies revealed multiple microinfarcts, noninflammatory thickening of small vessels with intimal proliferation, small-vessel occlusion, and intracranial embolism or hemorrhage (Van Dam et al., 1991). True vasculitis, with inflammatory infiltrate and fibrinoid necrosis is relatively rare, occurring in 6-9\% of cases (Van Dam et al., 1991). Vasculopathy is most common. SLE vasculopathy affects predominantly arterioles and capillaries, resulting in vessel tortuosity, vascular hyalinization, endothelial proliferation, and perivascular inflammation or gliosis. This vasculopathy could be related to both acute inflammation and ischemia (Hanly et al., 1992; Van Dam et al., 1991).

According to a recent histopathological study (Sibbit et al., 2010) the basic underlying pathologic process of NPSLE is cerebrovascular injury associated with disease activity and thromboembolism, resulting in focal and diffuse brain ischemia, small and large brain infarcts, focal and diffuse brain edema, brain hemorrhage, and focal and diffuse parenchymal injury. Multiple coexisting pathogenic mechanisms including, thromboembolism by cardiac valvular lesions, hypercoagulability, diffuse endothelial injury, and excitotoxicity could not be excluded (Roldan et al., 2006, 2008). 
Four patterns of cerebrovascular disease NPSLE have been suggested: (1) an antiphospholipid antibody cerebrovasculopathy characterized by bland thromboses, thrombotic microangiopathy, and arterial intimal fibrous hyperplasia; (2) a diffuse cerebrovasculopathy characterized by endothelial injury associated with increased SLE disease activity, glomerulonephritis, hypertension, and perhaps neuroexcitotoxic antibodies; (3) thromboembolic NPSLE directly caused by cardiac valvular lesions; and (4) mixed cerebrovascular NPSLE with simultaneous aspects of antiphospholipid- associated thrombosis, increased disease activity, and thromboembolic valvular lesions (Sibbit et al.,2010).

\section{Conventional MRI}

Conventional pre- and postcontrast-enhanced brain MRI appears normal in approximately one-third of both symptomatic and asymptomatic NPSLE patients (Chinn et al., 1997; Jennings et al., 2004). Hyperintense white matter lesions are revealed in up to $70 \%$ of SLE patients. In the majority of MRI studies in which white matter lesions were quantified, patient groups with NPSLE (active and inactive) showed a significantly higher number and total volume of white matter hyperintensities compared to non-NPSLE ( Appenzeller et al., 2008; Castellino et al., 2008).

According to a recent study (Luyendijk et al, 2011), that reviewed retrospectively the MRI exams of the first episode of active NPSLE in 74 patients, 4 types of findings were observed : 1) focal hyperintensities in white matter $(49 \%$ of all patients and $84 \%$ of patients with abnormalities on MRI) or both white matter and gray matter (5\%), suggestive of vasculopathy or vasculitis (Figure 1), 2) more widespread, confluent hyperintensities in the WM, suggestive of chronic hypoperfusion due to the same mechanisms, 3) diffuse cortical GM lesions (12\%), compatible with an immune response to neuronal components or postseizure vasogenic edema, and 4) absence of MRI abnormalities, despite active signs and symptoms $(42 \%)$. Small punctate focal white matter lesions, the most common imaging finding, are followed in prevalence by cortical atrophy, ventricular dilation and diffuse white matter changes.

The small focal lesions- hyperintense on T2-weighted or FLAIR imaging- typically occur in periventricular and subcortical white matter, especially in the frontoparietal regions. They usually represent small resolved infarcts or focal areas of reduced neuronal density, but in some cases they may be the result of acute infarcts, focal edema, or even acute microhemorrhages (Sibbitt et al., 2010).The corresponding T1-weighted images often appearing normal (Ainiala et al, 2005), while FLAIR images have been shown to be more sensitive for detecting these lesions than T2 images. Periventricular lesions have been particularly associated with the antiphospholipid syndrome (APS) and can be impossible to differentiate on MRI from multiple sclerosis (Peterson et al., 2005).

Although the focal hyperintense white matter lesions are often considered nonspecificsince they are indistinguishable from age-related small vessel disease -they may occur much earlier and in greater numbers in SLE subjects. These findings also seem to be more common in patients with NP-SLE in the presence of antiphospholipid antibodies, although no clinical correlation was observed in most studies (Ainiala et al., 2005). In a large prospective population-based study involving healthy individuals, the presence of focal hyperintense white matter lesions was associated with cognitive impairment (Vermeer et al., 2003). In addition, correlations have been observed between white matter 
hyperintensities and both cumulative SLE related injury scores, including neuropsychiatric damage (SLICC/ACRDI), and separate neuropsychiatric component scores of SLE-injury indices (neuro-SLEDAI and neuro-SLICC) (Appenzeller et al., 2008; Ainiala et al., 2005).

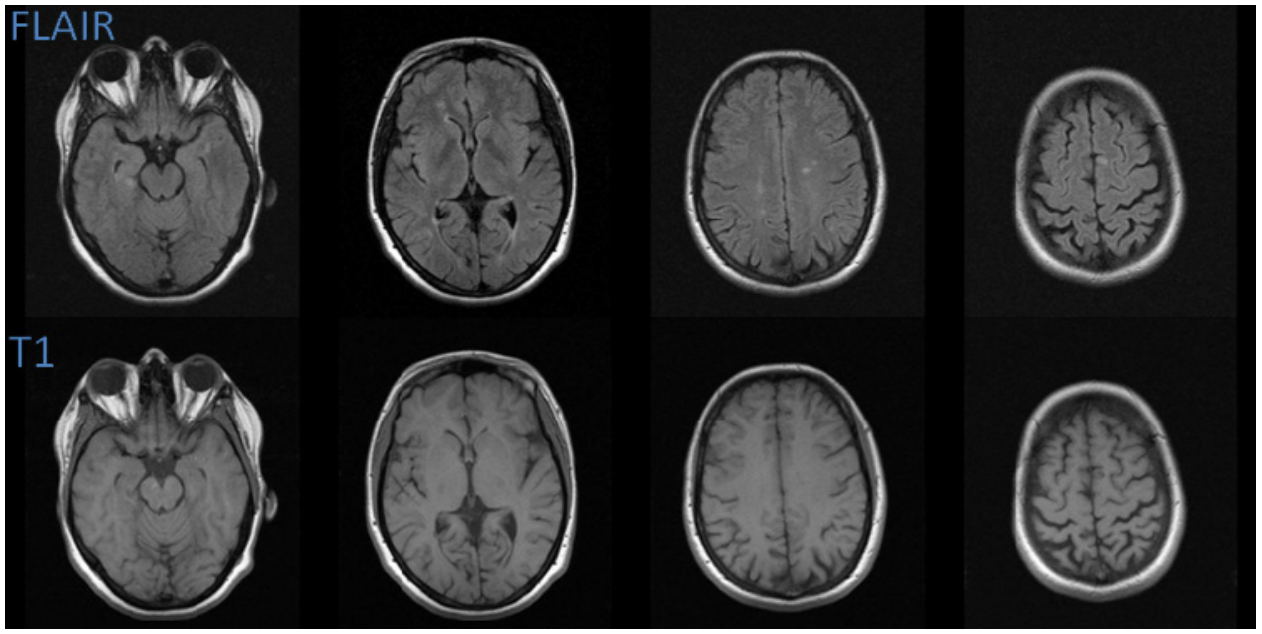

Fig. 1. FLAIR and T1 MR images of a 44 years female with NPSLE reveal multiple focal punctuate lesions-hyperintense on FLAIR and hypointense or isointense on T1- at the periventricular and subcortical white matter, semioval center and the cortex of the left frontal lobe.

Besides aging, white mater hyperintensities are also associated with hypertension, valvular heart disease, and migraine, conditions that commonly occur secondary or concomitantly to NPSLE. Consequently, it is not possible to differentiate NPSLE from other vasculopathies using conventional MRI. The differentiation of acute active disease from old chronic lesions is also difficult. It has been noted that the presence of indiscrete lesion borders, intermediate intensity of T2 lesions and grey matter lesions are all indicative of active disease (Sibbitt et al., 1999). The use of gadolinium has also been shown to be helpful in delineating active inflammatory lesions that usually enhance (Miller et al., 1992). Quantitation of T2 values has also been shown to be helpful in distinguishing active from chronic lesions (Sibbitt et al., 1995). T2 values appear to be increased in the normal appearing frontal grey matter of patients with active diffuse neurological syndromes.

MRI studies may show extensive bilateral, potentially reversible, white matter abnormalities in the cerebral hemispheres, the brainstem, or the cerebellum usually associated with active NPSLE - the so-called "acute posterior leukoencephalopathy,"( Sibbitt et al., 1999). The reversible lesions of acute leukoencephalopathy of NPSLE have been attributed to focal cerebral edema associated with blood vessel injury and microhemorrhages (Sibbitt et al, 2010).

Atrophy was described in 6-12\% of SLE patients (Appenzeller et al., 2005,2008) and is associated with multiple factors such as disease duration (Cauli et al., 1994), corticosteroid use (Appenzeller et al., 2005; Ainiala et al, 2005), older age (Appenzeller et al, 2005), 
antiphospholipid antibodies (Appenzeller et al., 2008; Provenzale et al., 1996) and the presence of hyperintense white matter lesions (Appenzeller et al., 2005; Chinn et al., 1997). The loss of tissue within the brain is thought to result from a combination of both myelin damage and axonal loss, followed by Wallerian degeneration, and the loss of extracellular space and vascular compartments. The histopathological findings associated with MRIvisible cerebral atrophy were highly variable and included multiple infarcts and reduced neuronal density, suggesting that atrophy in NPSLE may be associated with both generalized and focal brain injury. However, normal histological appearance was also noted in some atrophic brains (Sibbitt et al., 2010).

Cerebral atrophy is usually measured from T1-weighted MRI scans, where good contrast between the cerebral spinal fluid (CSF) and the brain parenchyma is observed (Appenzeller et al., 2008). Because high spatial resolution images allow brain volume measurement with the greatest accuracy and precision, three-dimensional gradient methods are generally preferred. As cerebral atrophy can be measured serially on MRI scans of the brain by linear or volumetric measurements, it has been proposed as a means of monitoring the progression of SLE (Appenzeller et al., 2005, 2008).

Regional atrophy in SLE patients has also been described involving cortical and subcortical regions, with particular clinical significance. Selective involvement of the amygdala in patients with SLE and anti-NMDAR antibodies has been found (Emmer et al., 2006). Cognitive impairment may be present more frequently in both corpus callosum and hippocampal atrophy (Appenzeller et al., 2006, 2008). Mild or clinically insignificant spinal cord pathology has also been described in SLE, which might be secondary to Wallerian degeneration of long tract fibers passing through damaged areas of the brain (Benedetti et al., 2007).

MR Angiography detects medium-to-large vessels involvement. Since a small vessel vasculopathy represents the major histopathological background of brain involvement in NPSLE angiographic techniques are usually negative, although angiographic arterial stenoses or occlusions are rarely reported (Weiner et al., 1991). Multiple mechanisms for angiographic arterial stenosis or occlusion have been considered, including coagulopathy, cardiogenic embolism, atherosclerosis from long-term steroid use and anticoagulant or antiplatelet therapy, vasculitis due to SLE or infection, or a combination of these processes (Devinsky et al., 1998).

Conventional MRI techniques are particularly useful in NPSLE patients with acute focal neurologic deficits, although they cannot always differentiate lesions indicating active acute NPSLE from chronic lesions that represent past NPSLE. The differential diagnosis usually includes thromboembolic events due to vasculopathy, lupus-related CNS vasculitis, antiphospholipid antibodies (APL-Ab)- mediated thrombosis, microangiopathy (including thrombotic thrombocytopenic purpura), Libman-Sacks endocarditis, and accelerated atherosclerosis. The pathogenesis in many patients is probably multifactorial. Accurate assessment is crucial, as treatment for these alternative diagnoses differs. Immunosuppressive agents are typically used for suspected vasculitis, while lifelong anticoagulation is the mainstay of therapy for APL-Ab-mediated thromboembolic events.

In diffuse NPSLE presentation conventional MRI could be unremarkable since it doesn't give information about damage in normal-appearing tissue (Sibbitt et al., 1999). Nonconventional MRI techniques, sensitive to microstructural, hemodynamic and biochemical 
characteristics of the tissues have been developed, that could detect gray and white matter abnormalities in NPSLE patients, otherwise occult by conventional imaging.

\section{Advanced MRI methods}

\subsection{Diffusion-weighted imaging}

Diffusion-weighted imaging (DWI) is a magnetic resonance technique that is based on the random, incoherent (brownian) motion of protons on the molecular scale. In free water, proton-containing molecules move unrestricted in all directions, a situation that is referred to as isotropy. In highly structured tissue, such as the corticospinal tract, molecules encounter fewer barriers when moving in a craniocaudal direction than in directions perpendicular to it. This situation gives rise to preferential molecular movement in a certain direction, which is known as anisotropy. DWI can be used to measure diffusivity in the brain, providing signal proportional to the molecular diffusion of water molecules (Schaefer et al., 2000).

Diffusion Tensor Imaging (DTI) is a DWI technique that permits assessment of the preferential direction of proton diffusivity (Ulug et al., 1999). DTI offers increased resolution compared to conventional MRI regarding white matter microstructure by measurement of water diffusion through cellular compartments in vivo (Pierpaoli et al., 1996). Compared to more isotropic movement of water in gray matter, water diffusion in white matter moves anisotropically, meaning that water diffuses preferentially along the length of the axon compared to perpendicular to the axon. This anisotropic diffusion of water appears to be due to the highly structured axonal membranes and their associated myelin sheaths (Cascio et al., 2007). By tracking the diffusion of water in the brain, the measure fractional anisotropy (FA) and mean diffusivity (MD) can be derived. Higher FA (and lower MD) suggests greater axonal coherence and myelination. Measures of FA are usually considered to be overall measures of axonal integrity, reflecting either increased axonal caliber, increased myelin thickness, increased fiber coherence in a given direction, or some combination of these factors. In contrast, MD, is a measure of the average molecular motion independent of the constraints of tissue boundaries, and is affected by cellular size and degradations in tissue integrity (Pierpaoli et al., 1996). One way to assess the magnitude of diffusion is by calculating the apparent diffusion coefficient (ADC), an index of mean diffusivity, for individual pixels on average apparent diffusion coefficient (ADC) maps. Average diffusion coefficient (ADC) maps provide information on the microstructure of tissue and can be very useful in the detection of disease ADC values that can be assessed locally in regions of interest (ROIs). Another way is the generation of ADC histograms for the whole brain (Nusbaum et al., 2000). Such measures consisted of mean ADC values of the whole brain volume and descriptive parameters of ADC histograms of the whole brain, such as peak height. Relatively few studies have emerged showing water diffusivity changes in NPSLE. DWI was first used by Moritani and co-workers (Moritani et al., 2001) who detected acute or subacute lesions in 9 of 20 patients with SLE (45\%). Two main patterns of acute or subacute brain parenchymal lesions were described. The first include hyperintense lesions with decreased ADC indicating acute or subacute infarction due to primary or secondary arterial stenosis or occlusion (Figure 2) and the second isointense or slightly hyperintense lesions on diffusion-weighted images with increased ADC representing vasogenic edema, with or without microinfarcts, due to small-vessel vasculopathy or hypertensive encephalopathy (Figure 3). 


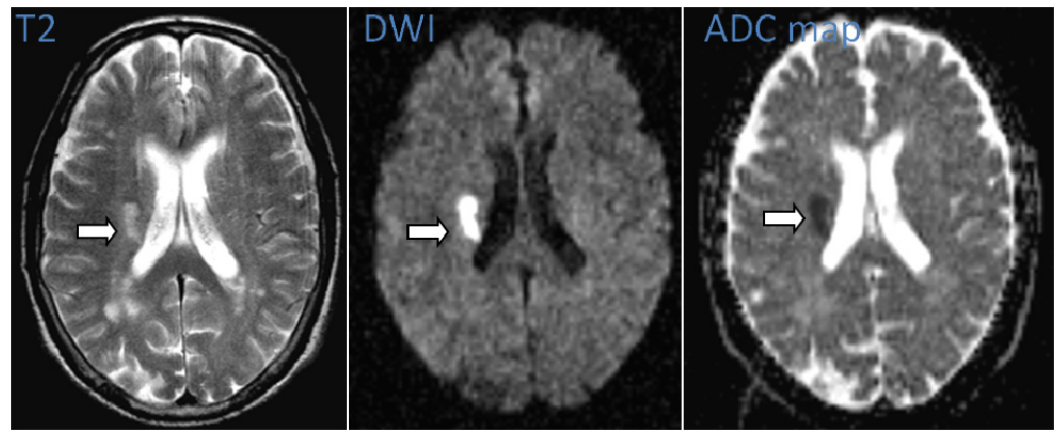

Fig. 2. Hyperintense lesion at the right corona radiata with increased signal intensity on DWI and decreased signal intensity on ADC map due to acute infarction.
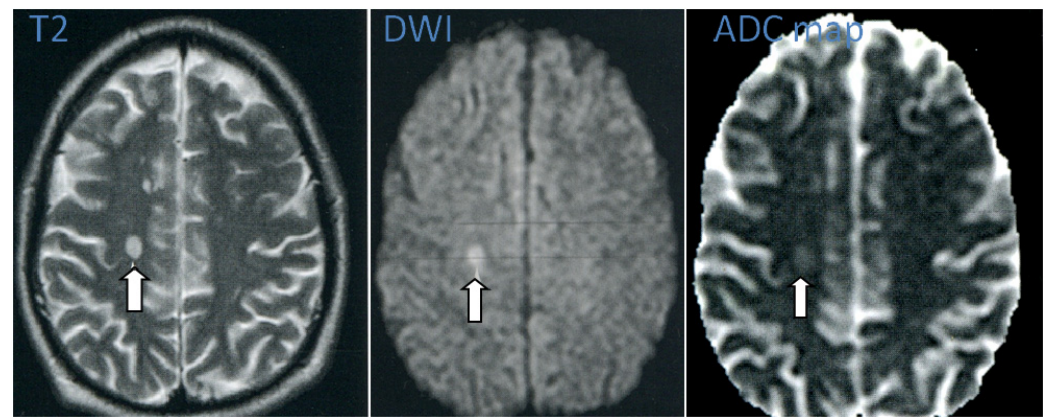

Fig. 3. Hyperintense lesion at the right semioval center with increased signal intensity on DWI and increased signal intensity on ADC map, due to vasogenic edema.

DWI then was used in NPSLE patients to provide quantitative measures of the integrity of the entire brain (Bosma et al., 2003). Using ADC histograms of the whole brain, in a group of 11 patients with a history of non focal NPSLE and 10 healthy volunteers they found changes in NPSLE patients who had no relevant changes on conventional MRI, that correlated with their clinical symptoms. The ADC histograms of the NPSLE group were, on average, significantly lower and broader, with a significant decrease of the peak height and a significant increase in the number of pixels with higher ADC values compared to the healthy subjects. The flatter and broader histograms in the NPSLE group indicate that, in these patients, widespread increased motility of free-water protons occurs. The subtle white matter hyperintensities that were visible on conventional MR images seemed unlikely to be responsible for the significantly different diffusion pattern in NPSLE because their number and sizes were small. These findings suggest that widespread damage exists in the brain parenchyma of NPSLE patients, invisible with conventional MRI. However, the method used in that study did not permit assessment of which parts of the brain were responsible for the observed changes in the ADC histograms of the whole brain.

Welsch and co-workers investigated 21 acute NPSLE patients and 21 healthy volunteers using also ADC histograms (Welsch et al., 2007).Whole-brain histograms, gray matter only 
histograms, and white matter only histograms were calculated for each subject. They found increased mean ADC values in acute NPSLE, not only in the whole-brain histograms, but also in the gray matter only and white matter only, indicating that the cerebral alterations is not limited to one tissue compartment.

Zhang and co-workers used a region of interest (ROI) approach for ADC and FA measurements to assess a cohort of 34 patients diagnosed with SLE, and 29 healthy volunteers (Zhang et al., 2007). They found early diffusion changes (higher diffusion values and lower FA values) in the frontal lobe, the genu of the corpus callosum, and the anterior internal capsule in patients with SLE, although routine MRI findings were negative. Another group (Hughes et al., 2007) compared 8 female NPSLE patients, with new onset of symptoms, to 20 healthy controls using diffusion tensor imaging (DTI) and an ROI approach. They found that these patients differed from controls in a wide range of normal appearing gray and white matter regions including the insular cortex, thalamus, parietal and frontal white matter, and corpus callosum. These findings are suggestive of the presence of subtle and widespread damage in the brain parenchyma in NPSLE patients.

Recently Jung and co-workers (Jung et al., 2010) using DTI and the tract-based spatial statistics (TBSS) analysis technique assessed white matter abnormalities in 17 NPSLE patients, 16 SLE patients without NPSLE, and 20 age- and gender-matched controls. According to their findings there were no significant FA or MD differences observed between the SLE patients without NPSLE and the matched controls, although many of the SLE patients had subcortical white matter and periventricular lesions. In contrast, when comparing the acute NPSLE patients to controls, or the acute NPSLE patients to patients with SLE without NPSLE they found decreased FA and increased MD values especially at the corpus callosum and the left anterior corona radiata reflecting diffuse white matter abnormalities. They suggest that either the acute effects of the NPSLE disease, or its treatment, results in white matter changes discernable with conventional MRI techniques. This study provides new evidence that FA and MD may have diagnostic use in NPSLE by demonstrating, for the first time, regional brain specificity, and by distinguishing NPSLE from SLE patients, further indicating the potential diagnostic specificity of this technique for patients in the acute stage of this difficult disease.

Using the same analysis technique Emmer and co-workers (Emmer et al., 2010) investigated 12 patients with SLE (7 with NPSLE) and 28 healthy controls with DTI and found reduction in the FA values of patients with SLE as compared with normal subjects, indicating reduced integrity at particular white matter tracts. The integrity of the subcortical white matter tracts of the occipital, parietal and posterior frontal lobe was relatively preserved, whereas the frontobasal and temporal regions including the inferior fronto-occipital fasciculus, the fasciculus uncinatus, as well as the fornix, the posterior limb of the internal capsule (corticospinal tract), and the anterior limb of the internal capsule (anterior thalamic radiation) seem to be predominantly involved.

The increased ADC values, as well as the decreased FA values, could be explained by reduced structural integrity of the brain parenchyma with permanent loss of neurons and demyelination in patients with NPSLE. This will allow the interstitial water molecules to move freely in a less restricted environment. Normally, the ADC values decrease and the FA values increase by the restriction of motion in a particular direction, for example, due to the boundaries of myelin sheets. Alternatively, loss of structural brain integrity would allow interstitial water molecules to move in a more unrestricted environment, thus resulting in an 
increase in ADC and decrease in FA. A breakdown of the myelin sheets, as in demyelination, would result in less restricted movement of the water molecules transverse to the fiber tracts. The observed increase in gray matter ADC can be interpreted as consistent with inflammation and/or vasculitis of the gray matter.

It has been suggested that the abnormal diffusion findings seen in the brain of NPSLE patients are not mainly related to hypoxic/anaerobic events, but, rather, indicate a host response to injury, such as an inflammatory reaction, membrane activation, or demyelination (Sibbitt et al., 1997). Such a theory can be supported by histopathology studies in which gliosis and demyelination has been described in the brain parenchyma of patients with NPSLE (Hanly et al., 1992). Immune-mediated vascular or neuronal injury was postulated and subsequent neuronal and metabolic dysfunction resulting in edematous processes that increase water content in WM regions of the brain.

The white matter damage in NPSLE could be the indirect result of subtle noxious influences in NPSLE, such as repeated episodes of acute inflammation in small vessels. This could cause priming or activation of the wall of these small vessels by complement and/or antiendothelial antibodies. Priming or activation of the vessel wall could subsequently lead to vasculopathy and microinfarcts or subtle hypoperfusion in the small vessels of the brain (Zvaifler et al., 1982). Altered cerebral blood flow was previously demonstrated with PET and SPECT in NPSLE patients (Kuschner et al., 1990; Kao et al., 1999). If hypoperfusion occurs, it might be responsible for the metabolic abnormalities reported in NPSLE patients, as suggested by decreased high energy phosphate levels in phosporus-31 MR spectroscopic studies and decreased brain oxygen consumption in PET studies. The end stage of this process might be axonal loss and associated demyelination. It is also possible that antibodies aimed directly against myelin, leading to direct white matter damage through a pervasive attack on axonal myelin sheaths or the oligodendrocytes from which they are derived. The involvement of the white matter could be, finally, the reflection of axonal damage through Wallerian degeneration caused by damage to the gray matter (Emmer et al., 2010).

Diffusion differences affecting acute NPSLE patients could also be related to therapy, whereby the introduction of corticosteroids, or other immunosuppression drugs (i.e. cyclophosphamide), and/or disease modifying antirheumatic drugs, can affect water content in the brain parenchyma. Another possible mechanism may be related to platelet or fibrin macro- or microembolism from Libman-Sacks endocarditis or anticardiolipin antibodies causing multiple areas of macroscopic or microscopic ischemia, infarctions, and microhemorrhages with surrounding edema (Roldan et al., 2006).

Undoubtedly, one of the more useful applications of DWI in clinical practice is the assessment of cerebro-vascular accidents; in these conditions DWI allows early detection (within $1 \mathrm{~h}$ ) of acute ischemic insult showing a reduction of ADC due to cytotoxic edema which occurs rapidly after the onset of ischemia (Figure 2). DWI also permits to discriminate between recent (with restricted diffusivity) and old (with normal diffusivity) hyperintense ischemic lesions which can be otherwise undistinguishable with conventional MRI. Furthermore, for its ability to differentiate between vasogenic and cytotoxic edema, DWI could be also useful in SLE patients to discriminate between inflammatory and ischemic lesions (Iguchi et al., 2007). Increased diffusion occurs although conventional MRI findings are negative in some cases, which suggests that DTI is more sensitive and that quantitative diffusion measurements can be used in detecting early signs of SLE or, furthermore, monitoring disease evaluations. The findings of diffusion-weighted imaging may help guide 
the choice of treatment and predict patient outcome in patients with SLE and CNS involvement.

\subsection{Perfusion weighted imaging}

A variety of imaging techniques have been used to assess cerebral perfusion, beginning with positron emission tomography (PET). PET is a nuclear medicine technique which explores both brain glucose metabolism and cerebral blood flow (CBF). In patients with NPSLE multiple areas of hypometabolism were detected in both MRI normal-appearing white and grey matter regions (Otte et al., 1997). Although PET has high sensitivity (abnormal in 100\% of patients with active NPSLE), it lacks specificity and due to its high radiation dose, high cost and limited availability, is rarely applied in the daily clinical practice.

Over the past two decades, two more perfusion imaging techniques, single photon emission computed tomography (SPECT), and perfusion-weighted MRI (PWI), were introduced. These techniques have been used to evaluate a variety of disease states, most commonly acute and chronic ischemia. SPECT is based on the radio-tracer uptake by viable neuronal cells and explores brain perfusion that is the result of both CBF and neuronal integrity. The most commonly observed abnormalities detected by SPECT in patients with NPSLE are diffuse, focal or multifocal areas of decreased uptake corresponding to hypoperfusion, which -according to some authors- correlates with NP disease severity and activity (Colamussi et al., 1995). Other SPECT studies reported CBF abnormalities in NPSLE without correlation to disease activity or serological parameters. There is also no significant associations between perfusion parameters and NP symptoms (Waterloo et al., 2001). A recent voxel-based SPECT study found no difference in perfusion parameters between healthy controls and SLE patients with inactive NP involvement. However the authors found a global hypoperfusion in active NPSLE patients compared to healthy controls, which was mainly located in the cortical gray matter (Appenzeller et al, 2008). Although SPECT has high sensitivity (abnormal in 86$100 \%$ of patients with major NPSLE) it lacks specificity (abnormal in 10-50\% of SLE patients without NPSLE) and has limited anatomic resolution.

The most commonly used MR perfusion technique is dynamic susceptibility contrast (DSC) imaging. This is a non-invasive dynamic process based on the MR signal changes during the first pass of the intravenously injected contrast agent (a gadolinium chelate) through the vasculature. The change in signal intensity is then measured and perfusion maps are generated, most commonly encountering cerebral blood volume (CBV), cerebral blood flow (CBF), time to peak (TTP), or mean transit time (MTT)values. Nowadays PWI is widely used in the acute stroke, for evaluation of the salvageable tissue and the brain neoplasms to detect neoangiogenesis. This technique could be useful in NPSLE and antiphospholipid syndrome (APS) patients (Figure 4) at risk of cerebro-vascular ischemic events, but reports on the use of PWI in NPSLE are still very few and limited.

The first multimodality approach in patients with SLE was performed by Borelli and coworkers (Borelli et al., 2003) using simultaneously MRI, DWI, PWI and SPECT in 20 SLE patients. They found that SPECT was more sensitive than PWI in detecting brain hypoperfused areas, probably due to the different aspects of brain perfusion explored by these two techniques. PWI is a dynamic process related to the blood supply to the anatomical districts of the brain, while SPECT images reflect the distribution of a flow tracer 
whose uptake at the neuronal level may, however, be in part influenced by the metabolic status of the nervous tissue. Therefore the combination of these techniques might yield more information about the underlying pathogenetic mechanism of brain hypoperfusion.

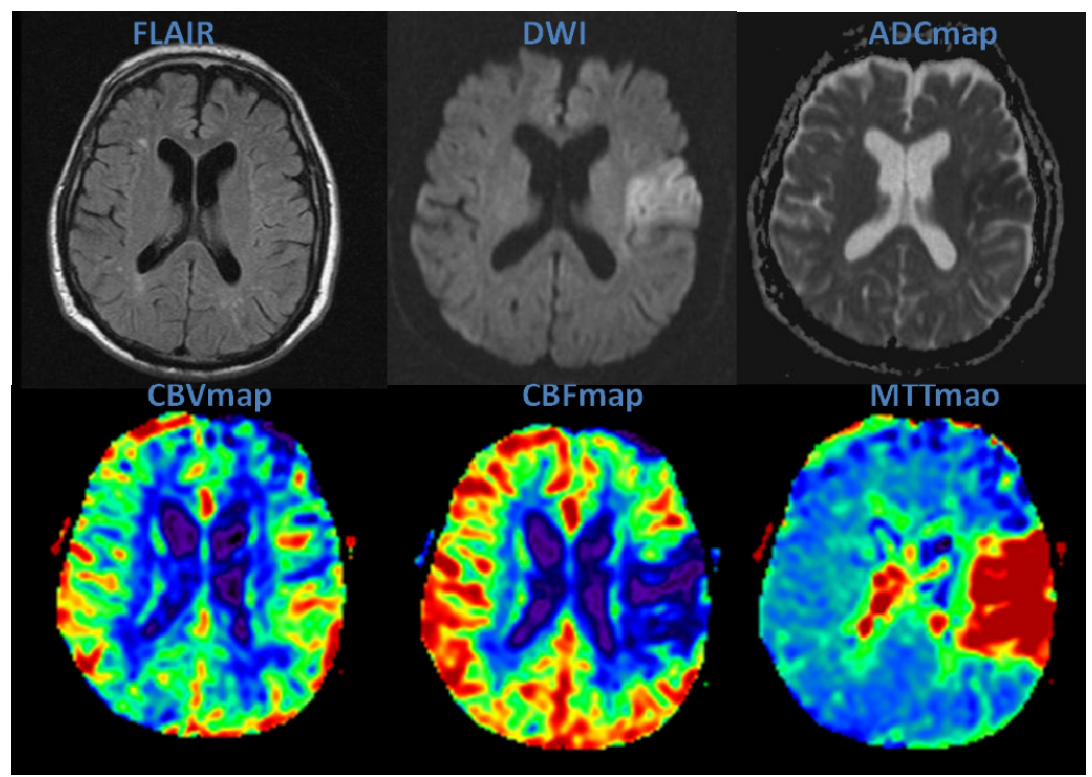

Fig. 4. Imaging of an acute ischemic lesion in a 45 yrs old female with NPSLE and antiphospholipid syndrome (APS), 4 hours after the onset of the symptoms. FLAIR is unremarkable, while the lesion is revealed on DWI and ADC map as hyperintense and hypointense area, respectively, due to cytotoxic edema. Dynamic Susceptibility Contrast (DSC) MR Perfusion technique shows mild increased cerebral blood volume (red on CBV map), decreased cerebral blood flow (dark blue on CBF map) and increased mean transit time (red on MTT map)

Two recent studies with application of dynamic susceptibility contrast perfusion MRI in SLE patients have opposite results. In the first study 15 active NPSLE, 26 inactive NPSLE and 11 control subjects were investigated and no signs of focal or global abnormalities in the perfusion parameters of the patients were found (Emmer et al., 2010). Furthermore, no significant differences were found when comparing patients with a specific SLE criterion (lupus anti-coagulant LAC, anti-cardiolipin antibodies or APS).The main limitation of this study is the fact that the CBF and CBV values have been calibrated to normal white matter, assuming that perfusion in the contralateral white matter is normal. This assumption could be erroneous in SLE patients with diffuse NP symptoms, like cognitive dysfunction or headache.

In the second study (Gasparovic et al., 2010)the DSCMRI technique was performed in 42 SLE patients and 19 healthy controls. They demonstrated higher CBV and CBF throughout cortical and white matter regions in the SLE patients (with or without lesions) relative to the control group. According to the authors the higher global CBF and CBV values may be due 
to reactive physiologic or pathogenic factors underlying SLE, such as vasomotor instability, compensatory mechanisms for resolving injury, low-grade excitotoxicity due to antibody impairment of N-methyl D-aspartate (NMDA) receptors, or inflammatory factors. This global hyperperfusion in SEL patients is in contrast with most SPECT studies that reveal patchy hypoperfused areas in SLE patients. More studies should be performed to SLE and NPSLE patients with application of both SPECT and MRI perfusion techniques to further delineate this issue.

\subsection{Magnetization Transfer Imaging (MTI)}

MTI is a quantitative MRI technique that is sensitive to macroscopic and microscopic brain tissue changes (Grossman et al., 1994; Wolff et al., 1994) and more sensitive to the presence of disease than conventional MRI. In MRI, the magnetic characteristics of free-water protons determine the contrasts of the images. MTI is based on the magnetization transfer that occurs between the bound pool of macromolecule-related protons in biologic tissues and the pool of free water protons. In MTI, a saturating RF pulse selectively reduces the magnetization of the bound pool. Due to the interactions between the two proton pools, the magnetization of the free-water pool is also reduced, which is called the MT effect. Tissue factors that affect the amount of this magnetization transfer are the concentration of macromolecules and the surface chemistry and biophysical dynamics of the macromolecules (Wollf et al., 1994). Macromolecules that contribute to the MT effect in the brain are the cholesterol component of myelin, cerebrosides, and phospholipids (Koenig et al., 2001).

The amount of MT is expressed by the magnetization transfer ratio (MTR) value. MTR values can be easily calculated in regions of interest (ROIs) to assess local tissue composition. In most diseases affecting the brain, MTR values are reduced, presumably due to dilution or destruction of macromolecules (Finelli et al., 1998). Using this approach it has been proved that MTI could detect abnormalities in brain tissue that are not visible in conventional MRI in cases of multiple sclerosis (Rovaris et al., 2000).Whole brain assessment can be also performed using MTR histograms for quantification of the disease burden of the whole brain (van Buchem et al., 1997). According to this volumetric method, the intracranial volume (ICV) is segmented, and the MTR values of the segmented brain are displayed as a histogram. Gray and white matter have different mean MTRs, which is probably mainly due to different concentrations of myelin. Still, the histograms for gray matter and white matter overlap substantially. The summation of these 2 histograms results in a histogram that is characteristic of the whole brain. The shape of this whole-brain histogram reflects conditions that affect the gray and white matter. MTR histograms of normal brains are characterized by the presence of a single, sharp peak, indicating that most normal brain voxels have approximately the same MTR values due to relative homogeneous tissue composition and microstructure. Demyelination, edema, atrophy and gliosis could impair MTR and the MTR histograms shape become flattened with decreased peak height reflecting dyshomogeneity of the brain tissue. In this way MTI allows the evaluation of brain tissue integridy, while simultaneously permits quantification of structural damage.

Studies that compared MS patients with normal controls using MTI revealed that the MTR histogram peak height was the volumetric MTI parameter that differed most significantly (van Buchem et al., 1998). This peak height was found to be decreased in MS patients. It was suggested that the peak height was a measure of the amount of residual normal brain tissue, and therefore inversely reflected the disease burden of the brain (van Buchem et al., 1998). In 
several MS studies, this measure was found also to correlate with measures of cognitive and neurologic functioning (van Buchem et al., 1998).

MTI has been applied in NPSLE patients without visible abnormalities on conventional MRI. All these studies used MTR histograms for the quantitative assessment of the whole brain abnormalities. Bosma and co-workers (Bosma et al., 2000) applied the MTI technique to 11 patients with NPSLE, 11 patients with SLE without history of NPSLE (non-NPSLE) and 10 healthy volunteers. In this study, for every patient, an MTR histogram was created after correction for the ICV and another was created after correction for brain volume. In MTR histograms that are adjusted for the ICV, the peak height reflects both the integrity of brain parenchyma and the extent of atrophy. A different distribution of the brain voxels, in terms of changes in MTR values due to disorders of brain parenchyma or a reduction in the total number of brain voxels due to atrophy, can cause a decrease in the peak height. The peak height of the MTR histograms corrected for brain volume is affected only by the aspect of brain parenchyma, and not by the volume of the brain and atrophy (van Buchem et al., 1998). In the group of NPSLE patients, the average peak height of the histograms corrected for intracranial volume and the peak height normalized for brain volume were significantly lower than in either the non-NPSLE patients or the healthy controls at the same position of the peaks. This suggests the presence, in NPSLE, not only of atrophy, but also of an abnormality of the remaining brain parenchyma. The latter disorder in NPSLE is probably primarily present in the normal appearing parenchyma, since the low number and small size of hyperintense lesions observed in these patients cannot solely account for the decreased peak height of the histograms. Since myelin is a major contributing factor to the MT effect in the brain, the decreased peak heights of the MTR histogram corrected for brain volume might have originated from demyelination along with axonal loss. Edema and gliosis are also considered to give rise to abnormal MTRs (Dousset et al., 1992).

In another study (Bosma et al., 2000) MTI was performed to 9 patients with active nonthromboembolic NPSLE, 10 patients with chronic NPSLE, 10 patients with SLE and no history of NPSLE (non-NPSLE), 10 patients with inactive MS, and 10 healthy control subjects, to investigate whether volumetric MTI analysis with MTR histograms can demonstrate abnormalities in patients in the acute stage of diffuse NPSLE and compare these findings with those from chronic SLE, non-SLE and MS. The MTR histograms of both the non-NPSLE group and the healthy controls were similar. There was flattening of the histograms in the active NPSLE group, but with a shift toward higher MTRs. These changes indicate that the uniformity of the brain in patients with active NPSLE is lower than that in SLE patients with no history of NPSLE as well as that in healthy controls, suggesting that patients with active NPSLE can be distinguished from SLE patients with no history of NPSLE by use of MTR histogram analysis. The shift of the MTR histogram peak to the right that was observed in active NPSLE suggest an all-over improvement in the exchange of saturation from the pool of bound protons to that of the free water protons. Increased MTRs were also found in an experimental model of acute inflammation in MS, experimental allergic encephalomyelitis, during the early phase of inflammation in that disease (Dousset et al., 1992). Similar processes may occur during the active phase of NPSLE. In this study, all MTR histogram parameters in chronic NPSLE and MS were identical. Since NPSLE and MS are diseases with a different biologic behavior, it is unlikely that these similarities suggest a 
similar pathogenesis. Instead, the similarities indicate that different diseases may give rise to a common final pathway (i.e., gliosis and demyelination) that results in similar changes in the MTR. These results also demonstrate that in the chronic stage of diffuse brain diseases, MTR histograms may not be able to differentiate between different diseases.

The same group (Bosma et al., 2002) further investigated the relationship between quantitative estimates of global brain damage based on magnetization transfer imaging (MTI) and the cerebral functioning, as measured by neurologic, psychiatric, and cognitive assessments, as well as the disease duration in patients with NPSLE. They performed MTI to 24 patients with NPSLE and found significant correlations between the descriptive measures of the MTR histograms and: a) the neurologic functioning quantified by the Kurtzke's Expanded Disability Status Scale (EDSS) b) the cognitive functions with the Wechsler Adult Intelligence Scale Revised (WAIS-R), a standardized psychometric test for assessing intelligence and c) the psychiatric functioning by the Hospital Anxiety and Depression Scale (HADS) questionnaire. Since the EDSS score mainly assesses motor skills, these findings suggest that atrophy and diffuse microscopic damage of the remaining brain parenchyma in NPSLE affect, among other areas, brain regions that are responsible for motor skills. Atrophy and diffuse microscopic cerebral damage also contribute the development of psychiatric symptoms, like anxiety and depression and cognitive impairment. There is no correlation between the quantitative estimates of global brain damage and age, SLE duration, or time elapsed since the first occurrence of neuropsychiatric symptoms suggesting that the accumulation of brain damage over time has a nonlinear aspect.

Dehmeshki and co-workers (Dehmeshki et al., 2002) developed an alternative way of analyzing and globally characterizing MTR histograms by using multivariate discriminant analysis (MDA) and correctly assigned patients with MS to clinical subgroups. The same group proceed to a study in SLE patients in order to explore the diagnostic potential of MDA for assigning patients with SLE to different subgroups of patients with on the basis of MTR histograms (Dehmeshki et al., 2002). MTI was performed to 9 patients with active NPSLE, 10 patients with chronic NPSLE, 10 patients with SLE and no history of NPSLE (non-NPSLE), 10 patients with inactive MS, and 10 healthy control subjects. Three binary comparisons were made: First, comparison of active NPSLE versus past NPSLE groups which is important because in patients with NPSLE, a new episode of neuropsychiatric symptoms could be due to an active intrinsic SLE-related brain process - that is, a recurrent active phase of NPSLE - or to extrinsic processes such as the side effects of drug use. Secondly, comparison of active NPSLE from non-NPSLE which is also important because an acute episode of neuropsychiatric symptoms could be due to active NPSLE or to extrinsic processes in patients with SLE. Finally, comparison of patients with active and/or past NPSLE with those who have MS which important because in patients who present with neuropsychiatric symptoms, the differential diagnosis includes SLE and MS, and these diseases are notoriously difficult to differentiate. In this study, MDA proved to be effective in assigning the majority of individual patients to disease categories in the given binary comparisons. In addition, MDA was shown to be considerably more effective for categorizing patient groups on the basis of MTR histograms than the conventional method of analyzing MTR histograms and might be of help in clinical practice.

Steens and co-workers (Steens et al., 2004) performed MTI in 24 SLE patients with a history of diffuse neuropsychiatric symptoms and 24 healthy controls to assess the distribution of 
MTI abnormalities over gray matter (GM) and white matter (WM) in SLE patients without explanatory MRI evidence of focal disease. MTR maps were calculated for GM and WM separately, and GM and WM MTR histograms were generated. Significantly lower peak height and mean MTR of the gray matter were found in NPSLE patients as compared with healthy controls indicative of parenchymal brain damage specifically in the GM in SLE patients with a history of NP symptoms and without explanatory focal abnormalities seen on MRI. This observation supports the model of neuronal damage in diffuse NPSLE, and can be explained by the greater susceptibility of GM to the sequelae of small-vessel disease and hypoperfusion. Small-vessel disease itself may also increase blood-brain barrier permeability, which facilitates the entrance of antineuronal antibodies. In either case, because of the higher concentration of neurons in GM, the GM will be particularly affected.

The same group (Steens et al., 2006) examined the correlation between gray and white matter magnetization transfer ratio (MTR) parameters and the presence of IgM and IgG anticardiolipins antibodies (aCLs) and lupus anticoagulant in 18 patients with NPSLE, but without cerebral infarcts on conventional magnetic resonance imaging. Lower gray and white matter mean MTR and peak location were observed in IgM aCL-positive patients than in IgM aCL-negative patients. No significant differences were found in MTR histogram parameters with respect to IgG aCL and lupus anticoagulant status, nor with respect to antidsDNA or anti- ENA (extractable nuclear antigen) status.

Various autoantibodies have been implicated in the pathogenesis of NPSLE, including anticardiolipin antibodies (aCLs) . Because of their prothrombotic tendency, aCLs may cause cerebral infarctions and as such they are correlated with focal neurological syndromes (Denburg, Denburg, 2003). In a study of Steens and co-workers (Steens et al., 2006) MTI parameters demonstrated brain damage in aCL-positive SLE patients in the absence of cerebral infarcts on conventional MRI. These findings suggest that, apart from giving rise to macroscopic cerebral infarctions, aCLs may play a role in the pathogenesis of diffuse microscopic brain damage in NPSLE. According to the authors there are at least three possible explanations for how aCLs could be involved to diffuse microscopic brain damage in NPSLE. First, the thrombotic tendency of antiphospholipid antibodies, including aCLs, may cause aggregation of thrombocytes and an increase in blood viscosity (Scolding, Joseph 2002; Connor, Hunt 2003). This may affect blood flow in small cerebral blood vessels in particular and cause widespread hypoperfusion, which subsequently causes ischaemic damage to brain tissue. Second, aCLs may activate endothelial cells and cause a diffuse small-vessel vasculopathy - a neuropathological finding that was reported as long ago as 1968 (Connor, Hunt 2003; Scolding, Joseph 2002). The resulting increase in blood-brain barrier permeability permits entrance to the brain parenchyma of substances such as circulating antibodies. Third, it has been shown in vitro that IgG aCLs themselves may interfere with glutamatergic pathways by a mechanism involving over-activation of the $\mathrm{N}$ methyl-d-aspartate receptor (Andreasi et al., 2001).

Emmer and co-workers (Emmer et al., 2006) proved that changes in the clinical status of individual NPSLE patients correspond to changes in the MTR peak height and MTI is a valuable objective measure for following a clinical course in NPSLE. 19 active or inactive NPSLE patients underwent MTI on at least two separate occasions. Their neuropsychiatric status between the first and the second MRI sessions was classified as deteriorated, stable, or improved. In all clinically deteriorated patients the MTR peak height decreased between the first and second scans. In all clinically improved patients the MTR peak height increased 
between the first and second scans, indicating that brain involvement in NPSLE patients, as detected by MTI, is at least partly reversible. The nature of the pathophysiological substrate of the reversible MTR changes in NPSLE patients is unclear. According to the authors reversible changes in the integrity of the parenchyma are probably due to edema, since neuronal loss would not show such a degree of reversibility, and neuronal apoptosis is not a common finding in postmortem studies of NPSLE. Inflammation influences the vessel walls and permits extravasation of fluid and inflammatory mediators into the brain tissue that leads to inflammatory brain edema

\subsection{Magnetic Resonance Spectroscopy}

Proton Magnetic Resonance Spectroscopy (MRS) is an MRI technique that permits study of the metabolites in tissue and provides qualitative and quantitative information about some brain metabolites displayed as spectra. The position of metabolites peaks in the spectrum is determined by its molecular characteristics. Studies in humans have used hydrogen -MRS (H-MRS) and rarely phosphorous-MRS (P-MRS). In normal water-suppressed, localized HMRS of human brain at echo times (TEs) between $135 \mathrm{msec}$ and $270 \mathrm{msec} 3$ major neurometabolites are revealed: 1. N-acetylaspartate (NAA) with peak at 2.0 parts per million, which can be used as a neuronal marker, because is found exclusively in neurons. NAA is reduced in conditions associated with neuronal loss, such as stroke or neuronal degenerative disorders. However, several studies have shown reversible decreases in NAA in a number of conditions have also been recognized, emphasizing that neuronal dysfunction can also lead to a decrease in NAA (Rudkin, Arnold, 1999). 2. Choline (Cho). Choline peak consists of choline, phosphocholine and glycerocholine. Cho is increased during increased cell -membrane turn over or during active myelin breakdown when these choline-containing membrane phospholipids are released. Increase Cho is detected in demyelination, remyelination, inflammation or gliosis (Rudkin, Arnold, 1999). 3. Creatine and phosphocreatine $(\mathrm{Cr})$. Total creatine concentration is relatively constant throughout the brain, and is used as an internal reference to normalize NAA and choline. Nevertheless there is loss of creatine in tissue necrosis (Rudkin, Arnold, 1999).

When performing MRS with short TE $(30-35 \mathrm{msec})$ some other metabolites could also be revealed, such as myo-inositol (mI), which is an osmolyte and astrocyte marker and is usually increased in demyelination, inflammation and gliosis. Lactate is elevated in anaerobic glycolysis and is not seen in normal brain spectra. The lactate peak is above the baseline when the TE is low $(20-35 \mathrm{msec})$ or high $(270-288 \mathrm{msec})$. At an intermediate TE (135-144 msec), the lactate peak inverts to project below the baseline, a feature that enables its distinction from lipids and some macromolecules seen at a similar location on the spectrum (1.35 parts per million).

Commonly used spectroscopic techniques include the single- voxel spectroscopy which allows evaluation of only small volumes of tissue, and the multivoxel technique that allows examination of different areas of the brain at the same time and also permits the derivation of metabolite maps. The selection of appropriate MRS techniques, including measurement parameters such as repetition time (TR) and TE, depends on the clinical question. Short TE (20-35 msec) evaluations are required when there is a need for detection of metabolites with short relaxation times, such as glutamine, glutamate or $\mathrm{mI}$, whereas studies with long TE (135-270 msec) are sufficient for the detection of the major metabolites such as NAA, Cho, Cr, and lactate/lipids (Rudkin, Arnold, 1999) 
Several studies have been performed using MRS in patients with SLE with or without neuropsychiatric manifestations. Most of them used single voxel MRS and proved reduction of the NAA/Cr and NAA/Cho ratios and elevation of Cho/Cr ratio in patients with SLE, not only in lesions, but also in normal-appearing white matter, when compared to controls. Nevertheless no dinstiction between acute and chronic disease has been demonstrated (Sibbit et al., 1997; Davie et al., 1995; Friedman et al., 1998). The NAA/Cr ratio was negatively correlated to the degree of atrophy in SLE patients, suggesting that cerebral atrophy in SLE in associated with neuronal damage (Sibbitt et al., 1994; Chinn et al., 1997).

Decreased NAA/Cr ratio was found in white mater lesions of patients with NPSLE with no correlation with neurololgic or psyschiatric involvement (Davie et al., 1995). Patients with white matter lesions also had a more pronounced reduction in NAA/Cr ratio, when compared with patients without lesions, suggesting that neurophychiatric manifestations are associated with a complex multifocal and diffuse neurotoxic process (Brooks et al., 1999). Cerebrovascular abnormalities with small vessel injury, that underlie diffuse cerebral injury in SLE, (small focal lesions), are primarily associated with a decrease NAA/Cr ratio, while medium vessel injury is primarily associated with an increased Cho/Cr ratio (Friedman et al., 1998). It is well known that small focal lesions are also observed in healthy adults, often associated with older age. However, if neurometabolic changes are observed within these lesions, it could be inferred that these white matter lesions represent a serious pathologic process resulting in focal neuronal death or injury (Brooks et al., 1997).

The amount of reduction in NAA/Cr ratio is associated with disease activity and the severity of clinical manifestations (Sibbitt et al., 1997). The same group failed to demonstrate lactate even in severely ill patients with major NPSLE, suggesting that extensive, anaerobic metabolism is not a fundamental characteristic of NPSLE, although this was contraindicated by others (Sundgren et al., 2005).They also found that increased lipid-macromolecules peaks at $1,2 \mathrm{ppm}$ is an indicator of disease activity representing inflammatory cell infiltration, membrane activation, degradation or demyelination. Appenzeller and co-workers (Appenzeller et al., 2005) also demonstrated that the reduction in NAA / Cr ratio correlated with disease activity, independently of CNS manifestations, and that NAA/ $\mathrm{Cr}$ ratio in normal-appearing white matter returned to normal range after remission.

Sabet and co-workers (Sabet et al., 1998) proved that NAA/Cr ratio was lower and Cho/Cr was higher in SLE patients with antiphospholipid antibody syndrome (aPLS) compared to those without aPLS. However thrombotic phenomena are most closely associated with the $\mathrm{Cho} / \mathrm{Cr}$ ratio elevation in patients with SLE. Elevated Cho/ $\mathrm{Cr}$ ratio was observed in focal lesions and normal-appearing tissues of patients with SLE-aPLS consistent with infarct, activation of cellular membranes, catabolism of myelin, or inflammation. Cho/ $\mathrm{Cr}$ ratio was increased in normal-appearing tissues, suggesting exaggerated injury to normal-appearing tissue in patients with SLE-aPLS consistent with widespread microinfarction.

Reduction of NAA/Cr ratio and elevation of $\mathrm{Cho} / \mathrm{Cr}$ ratio seem to reflect the cerebral metabolic disturbance related to the severity of neuropsychiatric symptoms regardless of the presence of abnormal MRI findings (Lim et al., 2000). In that study metabolic changes at the basal ganglia, besides the normal appearing white matter, was also found, indicating small vessel injury. Reduction of $\mathrm{NAA} / \mathrm{Cr}$ ratio and elevation of $\mathrm{Cho} / \mathrm{Cr}$ ratio in normal appearing white matter, related to the severity of neuropsychiatric symptoms was also proved by others (Axford et al., 2001; Handa et al., 2003; Castellino et al., 2005). Axford and co-workers also performed quantitative absolute assessment of the metabolites and found 
increased absolute concentration of $\mathrm{mI}$ in NPSLE patients symptoms, that could be the result of inflammation, with a trend to be reversible in patients with minor. The authors suggest that raised $\mathrm{mI}$, but normal NAA, that characterized SLE minor, may be due to result of vasculitis and inflammatory sequelae, which may be reversible if treated early enough. In contrast with the patients with SLE minor, the patients with SLE major had both increased $\mathrm{mI}$ and decreased NAA that may reflect gliosis and irreversible neuronal loss.

Castellino and co-workers (Castellino et al., 2005) assessed metabolites at hypoperfused and normoperfused brain areas according to SPECT finding and found that NAA/Cr ratio was reduced in hypoperfused areas, and Cho/Cr ratio was elevated in normoperfused areas, while new white matter lesions were developed on previous areas of hypoperfusion and $\mathrm{NAA} / \mathrm{Cr}$ ratio reduction indicating that increased $\mathrm{Cho} / \mathrm{Cr}$ ratio in normal-appearing white matter may predict the appearance of white matter lesions.

The NAA reduction has been also correlated with cognitive dysfunction and extent of brain damage (Sibbit et al., 1997; Lim et al., 2000). Increased choline was also associated with the presence of cognitive dysfunction in patients with SLE (Kozora et al., 2005) a finding that was further proved by other studies (Lapteva et al., 2006; Filley et al., 2009). Significant correlation was found between cognitive scores and higher $\mathrm{Cho} / \mathrm{Cr}$ ratio of the dorsolateral prefrontal cortex and white matter (Lapteva et al., 2006) or the left frontal white matter (Filley et al., 2009).

A recent study (Brooks et al, 2010) investigated most-mortem histopathological changes at autopsy in NPSLE patients and matched them voxel-by-voxel with the neurometabolites. They found that neurometabolite abnormalities were closely associated with underlying histopathological changes in the brain. Elevated choline levels were independently associated with gliosis, vasculopathy, and edema, while reduced creatine levels were associated with reduced neuronal-axonal density and gliosis. Reduced NAA levels were associated with reduced neuronal-axonal density and the presence of lactate was associated with necrosis, microhemorrhages, and edema. They suggest that altered neurometabolites in NPSLE patients, as determined by MRS, are a grave prognostic sign, indicating serious underlying histologic brain injury.

Few studies incorporated a multisequence MRI approach in patients with NPSLE to investigate the relationship between the different non-conventional MRI techniques. Emmer and co-workers (Emmer et al., 2008) applied MTI and MRS in SLE and NPSLE patients and healthy controls and found that there was significant association between the MTR histogram peak height of the whole brain parenchyma and the white and gray matter and the NAA/Cr ratio, indicating that demyelination and neuronal/axonal damage often occur together in patients with a history of NPSLE. The Cho/Cr ratio showed no significant association with any MTR parameters. According to another study that investigated the relationship between magnetization transfer imaging (MTI), diffusion weighted imaging (DWI), proton magnetic resonance spectroscopy (H-MRS), and T2 relaxometry findings in patients with primary neuropsychiatric systemic lupus erythematosus (NPSLE) (Bosma et al., 2004), significant correlations were found between the different metrics and cerebral atrophy. The correlations between MTI and DWI parameters indicate that demyelination in NPSLE patients is associated with increased diffusivity, due to either to a breakdown of myelin or a discrete increase of CSF spaces-including perivascular changes-associated with cerebral atrophy. The association between prolonged T2 relaxation time and increased diffusivity could be based not only on atrophy, but also on the presence of gliosis. 


\section{Conclusion}

NPSLE is characterized by variable, focal or diffuse, neuropsychiatric symptoms, leading to significant morbidity and mortality. NPSLE manifestations can occur in the absence of either serologic activity or other systemic lupus manifestations and there is no single sensitive and specific diagnostic test. Thus, in clinical practice the diagnosis of primary NPSLE is rather presumptive, after the exclusion of alternative causes of the neuropsychiatric symptoms. In patients with focal symptoms conventional MRI commonly detects small, discrete, hyperintense, frontal-parietal subcortical or periventricular white matter lesions, or even microhemorrhages, which are not specific for NPSLE and exhibit no clinical correlation. In diffuse NPSLE presentation conventional MRI could be totally unremarkable.

Non- conventional MRI techniques, namely diffusion weighted imaging (DWI), diffusion tensor imaging (DTI), perfusion weighted imaging (PWI), Magnetization transfer imaging (MTI) and magnetic resonance spectroscopy (MRS) have been developed. These techniques are sensitive to microstructural (DWI, DTI,MTI ), hemodynamic (PWI) and biochemical (MRS) characteristics of the tissues, and could detect gray and white matter abnormalities in NPSLE patients, otherwise occult by conventional imaging. Vasculopathy is the most common pathologic finding in NSPLE, while true vasculitis is rather uncommon. SLE vasculopathy affects predominantly arterioles and capillaries and could be related to both acute inflammation and hypoperfusion resulting in ischemia, demyelination, axonal loss and gliosis. These pathologic findings lead to particular changes of the non-conventional MRI metrics, such as reduced increased diffusivity, decreased fractional anisotropy, decreased MTR values, decreased NAA levels, and elevated choline levels, not only in focal lesions but mostly in normal appearing white and gray matter in patients with NPSLE. These quantitative changes are related to disease activity and severity, cognitive performance, and also the presence of antiphospholipid antibody syndrome and/or anticardiolipin antibodies, while could affect with the prognosis of new lesions and clinical deterioration. Although there are some inconsistencies in the various reports published thus far reflecting heterogeneity in patient selection, sample size and diagnostic criteria used, , we believe that there is enough data to support the use in daily clinical practice at tertiary care centers of non- conventional MRI techniques for the diagnostic work up of SLE patients with neuropsychiatric manifestations. For places whereby these modalities are not available the EULAR guidelines recommend at a minimum an MRI protocol (brain and spinal cord) that includes conventional MRI sequences (T1/T2, FLAIR), diffusion-weighted imaging (DWI), and gadolinium-enhanced T1 sequences (Bertsias et al., 2010).

\section{References}

Ainiala H, Dastidar P, Loukkola J, Lehtimaki T, Korpela M \&Peltola J (2005) Cerebral MRI abnormalities and their association with neuropsychiatric manifestations in SLE: apopulation-based study. Scand J Rheumatol ; 34:376-382

Andreassi C, Zoli A, Riccio A, Scuderi F, Lombardi L, Altomonte L \&Eboli ML(2001). Anticardiolipin antibodies in patients with primary antiphospholipid syndrome: a correlation between IgG titre and antibody-induced cell dysfunctions in neuronal cell cultures. Clin Rheumatol; 20:314-318.

Appenzeller S, Rondina JM, Li LM, Costallat LT \&Cendes F (2005) Cerebral and corpus callosum atrophy in systemic lupus erythematosus. Arthritis Rheum; 52:2783-2789 
Appenzeller S, Carnevalle AD, Li LM, Costallat LT \& Cendes F (2006) Hippocampal atrophy in systemic lupus erythematosus. Ann Rheum Dis; 65:1585-1589

Appenzeller S, Amorim BJ, Ramos CD, Rio PA, de C Etchebehere EC, Camargo EE, Cendes F \& Costallat LT (2007) Voxel-based morphometry of brain SPECT can detect the presence of active central nervous system involvement in systemic lupus erythematosus. Rheumatology (Oxford); 46:467-472.

Appenzeller S, Bonilha L, Rio PA, Min Li L, Costallat LT \& Cendes F (2007) Longitudinal analysis of gray and white matter loss in patients with systemic lupus erythematosus. Neuroimage 34:694-701

Appenzeller S, Pike BG , Clarke AE, (2008) Magnetic Resonance Imaging in the Evaluation of Central Nervous System Manifestations in Systemic Lupus Erythematosus Clinic Rev Allerg Immunol; 34:361-366

Axford JS, Howe FA, Heron C \& Griffiths JR. (2001) Sensitivity of quantitative (1)H magnetic resonance spectroscopy of the brain in detecting early neuronal damage in systemic lupus erythematosus. Ann Rheum Dis; 60:106-11.

Benedetti B, Rovaris M, Judica E, Donadoni G, Ciboddo G \& Filippi M (2007) Assessing "occult" cervical cord damage in patients with neuropsychiatric systemic lupus erythematosus using diffusion tensor MRI) J Neurol Neurosurg Psychiatry. J Neurol Neurosurg Psychiatry 78:893-895

Bertsias GK, Ioannidis JP, Aringer M, Bollen E, Bombardieri S, Bruce IN, Cervera R, Dalakas M, Doria A, Hanly JG, Huizinga TW, Isenberg D, Kallenberg C, Piette JC, Schneider M, Scolding N, Smolen J, Stara A, Tassiulas I, Tektonidou M, Tincani A, van Buchem MA, van Vollenhoven R, Ward M, Gordon C.\& Boumpas DT.(2010) EULAR recommendations for the management of systemic lupus erythematosus with neuropsychiatric manifestations: report of a task force of the EULAR standing committee for clinical affairs. Ann Rheum Dis.;69(12):2074-82.

Bertsias GK,Boumpas DT. (2010) Pathogenesis, diagnosis and management of neuropsychiatric SLE manifestations. Nat Rev Rheumatol. Jun;6(6):358-67.

Bosma GP, van Buchem MA \& Rood MJ, (2000),: Comparison of ADC histograms of patients with neuropsychiatric systemic lupus erythematosus and healthy volunteers. Proc Intl Soc Mag Res Med 8:1244.

Bosma GP, Rood MJ, Zwinderman AH, Huizinga TW \& van Buchem MA.(2000) Evidence of central nervous system damage in patients with neuropsychiatric systemic lupus erythematosus, demonstrated by magnetization transfer imaging. Arthritis Rheum;43:48-54.

Bosma GPTh, Rood MJ, Huizinga TWJ, De Jong BA, Bollen ELEM \& van Buchem MA. (2000) Detection of cerebral involvement in patients with active neuropsychiatric systemic lupus erythematosus using magnetization transfer imaging. Arthritis Rheum; 43: 2428-36.

Bosma GP, Middelkoop HA, Rood MJ, Bollen EL, Huizinga TW \& van Buchem MA. (2002) Association of global brain damage and clinical functioning in neuropsychiatric systemic lupus erythematosus. Arthritis Rheum;46:2665-72

Bosma GP, Huizinga TW, Mooijaart SP \& van Buchem MA. (2003) Abnormal brain diffusivity in patients with neuropsychiatric systemic lupus erythematosus. AJNR;24: 850-4. 
Bosma GP, Steens SC, Petropoulos H, Admiraal-Behloul F, van den Haak A, Doornbos J,Huizinga TW, Brooks WM, Harville A, Sibbitt WL Jr \& van Buchem MA. (2004) Multisequence magnetic resonance imaging study of neuropsychiatric systemic lupus erythematosus. Arthritis Rheum;50:3195-202.

Borrelli M, Tamarozzi R, Colamussi P, Govoni M, Trotta F,\& Lappi S. (2003) Evaluation with MR, perfusion MR and cerebral flow SPECT in NPSLE patients. Radiol Med;105:482-9.

Brooks WM, Sabet A, Sibbitt WL Jr, Barker PB, van Zijl PC, Duyn JH \& Moonen CT. (1997) Neurochemistry of brain lesions determined by spectroscopic imaging in systemic lupus erythematosus. J Rheumatol;24:2323-9.

Brooks WM, Jung RE, Ford CC, Greinel EJ \& Sibbitt WL Jr.(1999) Relationship between neurometabolite derangement and neurocognitive dysfunction in systemic lupus erythematosus. J Rheumatol;26:81-5.

Brooks WM, Sibbitt WL, Mario Kornfeld M,Jung RE,Bankhurst AD \& Roldan CA (2010) The Histopathologic Associates of Neurometabolite Abnormalities in Fatal Neuropsychiatric Systemic Lupus Erythematosus Arthritis and Rheumatism; 62: 2055-2063

Cascio CJ, Gerig G \& Piven J (2007) Diffusion tensor imaging: Application to the study of the developing brain. J Am Acad Child Adolesc Psychiatry;46(2):213-223.

Castellino G, Govoni M, Padovan M, Colamussi P, Borrelli M \& Trotta F. (2005)Proton magnetic resonance spectroscopy may predict future brain lesions in SLE patients: a functional multiimaging approach and follow up. Ann Rheum Dis;64:1022-7.

Castellino G, Padovan M, Bortoluzzi A, Borrelli M, Feggi L, Caniatti ML Trotta F \& Govoni M. (2008) Single photon emission computed tomography and magnetic resonance imaging evaluation in SLE patients with and without neuropsychiatric involvement. Rheumatology (Oxford); 47(3):319-23.

Cauli A, Montaldo C, Peltz MT, Nurchis P, Sanna G, Garau P Pala R, Passiu G \& Mathieu A. (1994) Abnormalities of magnetic resonance imaging of the central nervous system in patients with systemic lupus erythematosus correlate with disease severity. Clin Rheumatol 13:615-618

Chinn RJ, Wilkinson ID, Hall-Craggs MA, Paley MN, Shortall E, Carter S Kendall BE, Isenberg DA, Newman SP,\& Harrison MJ. (1997) Magnetic resonance imaging of the brain and cerebral proton spectroscopy in patients with systemic lupus erythematosus. Arthritis Rheum 40:36-46

Colamussi P, Giganti M, Cittanti C, Dovigo L, Trotta F, Tola MR, Tamarozzi R, Lucignani G \& Piffanelli A. (1995) Brain single-photonemission tomography with 99mTcHMPAO in neuropsychiatric systemic lupus erythematosus: relations with EEG and MRI findings and clinical manifestations. Eur J Nucl Med;22:17-24.

Connor P, Hunt BJ. (2003): Cerebral haemostasis and antiphospholipid antibodies. Lupus, 12:929-934.

Davie CA, Feinstein A, Kartsounis LD, Barker GJ, McHugh NJ, Walport MJ, Ron MA, Moseley IF, McDonald WI \& Miller DH. (1995).Proton magnetic resonance spectroscopy of systemic lupus erythematosus involving the central nervous system. J Neurol;242:522-8. 
Dehmeshki J , Van Buchem MA, Bosma GPT, Huizinga TWJ \& Tofts PS, (2002) Systemic Lupus Erythematosus: Diagnostic Application of Magnetization Transfer Ratio Histograms in Patients with Neuropsychiatric Symptoms-Initial Results Radiology; 222:722-728

Devinsky O, Petito CK \& Alonso DR. (1998) Clinical and neuropathological findings in systemic lupus erythematosus: the role of vasculitis, heart emboli and thrombotic thrombocytopenic purpura. Ann Neurol; 23:380-384.

Denburg SD, Denburg JA: (2003) Cognitive dysfunction and antiphospholipid

antibodies in systemic lupus erythematosus. Lupus; 12:883-890.

Emmer BJ, van der Grond J, Steup-Beekman GM, Huizinga TWJ \& van Buchem MA(2006) Selective Involvement of the Amygdala in Systemic Lupus Erythematosus PLoS Med;3(12): e499.

Emmer BJ, Steens SC, Steup-Beekman GM, van der Grond J, Admiraal-Behloul F, Olofsen H, Bosma GP, Ouwendijk WJ, Huizinga TW \& van Buchem MA.(2006)Detection of change in CNS involvement in neuropsychiatric SLE: a magnetization transfer study. J Magn Reson Imaging;24:812-6.

Emmer BJ. Steup-Beekman GM, Steens SCA, Huizinga TWJ,van Buchem MA. \& van der Grond J(2008) Correlation of Magnetization Transfer Ratio Histogram Parameters With Neuropsychiatric Systemic Lupus Erythematosus Criteria and Proton Magnetic Resonance Spectroscopy Association of Magnetization Transfer Ratio Peak Height With Neuronal and Cognitive Dysfunction Arthritis and Rheumatism;58(5): 1451-1457

Emmer BJ, Veer IM, Steup-Beekman GM, Huizinga TWJ, van der Grond J \& van Buchem MA (2010)Tract-Based Spatial Statistics on Diffusion Tensor Imaging in Systemic Lupus Erythematosus Reveals Localized Involvement of White Matter Tracts Arthritis Rheum; 62 (12): 3716-3721

Emmer BJ, Osch MJ, Wu O, Steup-Beekman GM, Steens SC, Huizinga TW, van Buchem MA. \& van der Grond J. (2010) Perfusion MRI in Neuro-Psychiatric Systemic Lupus Erthemathosus J Magn Reson Imaging;32:283-288

Filley CM, Kozora E, Brown MS, Miller DE ,West SG, David B. Arciniegas DB, Grimm A. \& Zhang L. (2009) White Matter Microstructure and Cognition in Nonneuropsychiatric Systemic Lupus Erythematosus Cog Behav Neurol;22:38-44

Finelli DA.(1998) Magnetization transfer in neuroimaging. Magn Reson Imaging Clin North Am;6:31-52.

Friedman SD, Stidley CA, Brooks WM, Hart BL. \& Sibbitt WL Jr (1998). Brain injury and neurometabolic abnormalities in systemic lupus erythematosus. Radiology;209:7984 .

Futrell N, Schultz LR. \& Millikan C.(1992) Central nervous system disease in patients with systemic lupus erythematosus. Neurology.;42:1649-57.

Gasparovic CM, Roldan CA, Sibbitt WI Jr, Qualls CR, Mullins PG, Sharrar JM, Yamamoto JJ, \& Bockholt JH(2010)Elevated Cerebral Blood Flow and Volume in Systemic Lupus Measured by Dynamic Susceptibility Contrast Magnetic Resonance Imaging. J Rheumatol;37;1834-1843

Grossman RI, Gomori JM, Ramer KN, Lexa FJ \& Schnall MD.(1994) Magnetization transfer: theory and clinical applications in neuroradiology.Radiographics;14:279-90. 
Grunwald F, Schomburg A, Badali A, Ruhlmann J, Pavics L. \& Biersack HJ. (1995) 18FDG PET and acetazolamide-enhanced 99mTc- HMPAO SPET in systemic lupus erythematosus. Eur J Nucl Med;22:1073-7.

Handa R, Sahota P, Kumar M, Jagannathan NR, Bal CS, Gulati M, Tripathi BM \& Wali JP. (2003);In vivo proton magnetic resonance spectroscopy (MRS) and single photon emission computerized tomography (SPECT) in systemic lupus erythematosus (SLE). Magn Reson Imaging;21:1033-7.

Hanly JG, Walsh NM \& Sangalang V.(1992) Brain pathology in systemic lupus erythematosus. J Rheumatol;19:732-741.

Hoeffner EG, (2005) Cerebral Perfusion Imaging(J Neuro-Ophthalmol;25: 313-320)

Hughes M, Sundgren PC, Fan X, Foerster B, Nan B, Welsh RC, Williamson JA,Attwood J, Maly PV, Chenevert TL, McCune W \& Gebarski S. (2007). Diffusion tensor imaging in patients with acute onset of neuropsychiatric systemic lupus erythematosus: a prospective study of apparent diffusion coefficient, fractional anisotropy values, and eigenvalues in different regions of the brain. Acta Radiol; 48(2):213-222.

Jennings JE, Sundgren PC, Attwood J, McCune J \& Maly P (2004) Value of MRI of the brain in patients with systemic lupus erythematosus and neurologic disturbance Neuroradiology; 46: 15-21

Jung RE, Caprihan A, Chavez RS, Flores RA, Sharrar J,Qualls CR, Sibbitt W, Roldan CA (2010) Diffusion tensor imaging in neuropsychiatric systemic lupus erythematosus BMC Neurology, 10:65

Kao CH, Lan JL, ChangLai SP, Liao KK, Yen RF, \& Chieng PU.(1999).The role of FDG-PET, HMPAO-SPECT and MRI in the detection of brain involvement in patients with systemic lupus erythematosus. Eur J Nucl Med 26:129-134.

Kelly MC, Denburg JA. (1987) Cerebrospinal fluid immunoglobulins and neuronal antibodies in neuropsychiatric systemic lupus erythematosus and related conditions. J Rheumatol;14:740-4.

Koenig SH. (1991) Cholesterol of myelin is the determinant of gray-white contrast in MRI of brain. Magn Reson Med;20:285-91.

Kodama K, Okada S, Hino T, Takabayashi K, Nawata Y, Uchida Y, Yamanouchi N, Komatsu N, Ikeda T. \& Shinoda N (1995).Single photon emission computed tomography in systemic lupus erythematosus with psychiatric symptoms. J Neurol Neurosurg Psychiatry;58:307-11.

Kozora E, Arciniegas DB, Filley CM, Ellison MC, West SG, Brown MS. \& Simon JH. (2005). Cognition, MRS neurometabolites, and MRI volumetrics in non-neuropsychiatric systemic lupus erythematosus: preliminary data. Cogn Behav Neurol; 18:159-62.

Kushner MJ, Tobin M, Fazekas F, Chawluk J, Jamieson D,Freundlich B, Grenell S, Freemen L. \& Reivich M (1990). Cerebral blood flow variations in CNS lupus. Neurology;40:99-102.

Lapteva L, Nowak M, Yarboro CH, Takada K, Roebuck-Spencer T, Weickert T, Bleiberg J, Rosenstein D, Pao M, Patronas N, Steele S, Manzano M, van der Veen JW, Lipsky PE, Marenco S, Wesley R, Volpe B, Diamond B. \& Illei GG.(2006) Anti-N-methyl-Daspartate receptor antibodies, cognitive dysfunction, and depression in systemic lupus erythematosus. Arthritis Rheum.;54(8):2505-14. 
Lim MK, Suh CH, Kim HJ, Cho YK, Choi SH, Kang JH, Park W \& Lee JH. (2000).Systemic lupus erythematosus: brain MR imaging and single voxel hydrogen $1 \mathrm{MR}$ spectroscopy. Radiology;217: 43-9

Luyendijk J, Steens SC, Ouwendijk WJ, Steup-Beekman GM, Bollen EL, van der Grond J, Huizinga TW, Emmer BJ. \& van Buchem MA(2011) Neuropsychiatric systemic lupus erythematosus: lessons learned from magnetic resonance imaging. Arthritis Rheum. ;63(3):722-32.

Miller DH, Buchanan N, Barker G Morrissey SP, Kendall BE, Rudge P, Khamashta M, Hughes GR,. \& McDonald WI. (1992). Gadolinium enhanced magnetic resonance imaging in the central nervous system in systemic lupus erythematosus. J Neurol; 239: 460-464.

Nusbaum AO, Tang CY, Wei T, Buchsbaum MS \& Atlas SW.(2000): Whole-brain diffusion MR histograms differ between MS subtypes. Neurology, 54:1421-1427.

Otte A, Weiner SM, Peter HH, Mueller-Brand J, Goetze M, Moser E, Gutfleisch J, Hoegerle S, Juengling FD \& Nitzsche EU. (1997). Brain glucose utilization in systemic lupus erythematosus with neuropsychiatric symptoms: a controlled positron emission tomography study. Eur J Nucl Med;24:787-91.

Peterson PL, Axford JS. \& Isenberg D (2005) Imaging in CNS lupus Best Practice \& Research Clinical Rheumatology; 19(5): 727-739.

Pierpaoli C, Jezzard P, Basser PJ, Barnett A. \& Di Chiro G (1996): Diffusion tensor MR imaging of the human brain. Radiology; 201(3):637-648.

Provenzale JM, Barboriak DP, Allen NB. \& Ortel TL (1996) Patients with antiphospholipid antibodies: CT and MR findings of the brain. Am J Roentgenol ;167: 1573-1578

Roldan CA, Gelgand EA, Qualls CR. \& Sibbitt WL Jr. (2006) Valvular heart disease is associated with nonfocal neuropsychiatric systemic lupus erythematosus. J Clin Rheumatol; 12(1):3-10.

Roldan CA, Qualls CR, Sopko KS. \& Sibbitt WL Jr.(2008) Transthoracic versus transesophageal echocardiography for detection of Libman- Sacks endocarditis: a randomized controlled study. J Rheumatol;35:224-9.

Rovaris M, Viti B, Ciboddo G Gerevini S, Capra R, Iannucci G, Comi G. \& Filippi M. (2000) Brain involvement in systemic immune mediated diseases: magnetic resonance and magnetization transfer imaging study. J Neurol Neurosurg Psychiatry; 68:170-177

Rudkin TM, Arnold DL. (1999) Proton magnetic resonance spectroscopy for the diagnosis and management of cerebral disorders. Arch Neurol;56:919-26.

Sabet A, Sibbitt WL Jr, Stidley CA, Danska J. \& Brooks WM.(1998)Neurometabolite markers of cerebral injury in the antiphospholipid antibody syndrome of systemic lupus erythematosus. Stroke;29:2254-60

Scolding NJ, Joseph FG (2002) The neuropathology and pathogenesis of systemic lupus erythematosus. Neuropathol Appl Neurobiol; 28:173-189.

Schaefer PW, Grant PE. \& Gonzalez RG (2000) Diffusion-weighted MRimaging of the brain. Radiology; 217:331-345.

Sibbitt Jr.WL, BrooksWM, Haseler LJ \& Griffey RH. (1995) Spin-spin relaxation of brain tissues in systemic lupus erythematosus. A method for increasing the sensitivity of magnetic resonance imaging for neuropsychiatric lupus. Arthritis Rheum; 38(6): 810-818. 
Sibbitt Jr WL, Haseler LJ, Griffey RR, Friedman SD\& Brooks WM. (1997)Neurometabolism of active neuropsychiatric lupus determined with proton MR spectroscopy. AJNR Am J Neuroradiol;18: 1271-1277.

Sibbitt WL Jr, Sibbitt RR. \&Brooks WM.(1999)Neuroimaging in neuropsychiatric SLE. Arthritis Rheum;42:2026-38.

Sibbitt WL Jr, Brooks WM, Kornfeld M, Hart BL, Bankhurst AD,\& Roldan CA (2010) Magnetic Resonance Imaging and Brain Histopathology in Neuropsychiatric Systemic Lupus Erythematosus. Semin Arthritis Rheum; 40:32-52

Steens SC, Admiraal-Behloul F, Bosma GP, Steup-Beekman GM, Olofsen H, le Cessie S, Huizinga TWJ \& van Buchem MA.(2004) Selective gray matter damage in neuropsychiatric lupus: a magnetization transfer imaging study. Arthritis Rheum ; 50:2877-81.

Steens SC, Bosma GP, Steup-Beekman GM , le Cessie S, Huizinga TWJ. \& van Buchem MA (2006)Association between microscopic brain damage as indicated by magnetization transfer imaging and anticardiolipin antibodies in neuropsychiatric lupus Arthritis Research \& Therapy; $8:$ 1186-1892.

Stojanovich L, Zandman-Goddard G, Pavlovich S \& Sikanich N (2007) Psychiatric manifestations in systemic lupus erythematosus. Autoimmun Rev 6:421-426

Sundgren PC, Jennings J, Attwood JT, Nan B, Gebarski S, McCune WJ, Pang Y. \& Maly P. (2005) MRI and 2D-CSI MR spectroscopy of the brain in the evaluation of patients with acute onset of neuropsychiatric systemic lupus erythematosus. Neuroradiology;47:576-85.

Ulug AM, Moore DF, Bojko AS.\& Zimmerman RD.(1999) Clinical use of diffusion-tensor imaging for diseases causing neuronal and axonal damage. AJNR Am J Neuroradiol ;20:1044-8.

Van Buchem MA, Grossman RI, Armstrong C, Polansky M, Miki Y, Heyning FH BoncoeurMartel MP, Wei L, Udupa JK, Grossman M, Kolson DL \& McGowan JC.(1998) Correlation of volumetric magnetization transfer imaging with clinical data in MS. Neurology;50:1609-17.

Van Dam AP (1991) Diagnosis and pathogenesis of CNS lupus. Rheumatol Intl 11: 1-11

Vermeer SE, Priens ND, den Heijer T, Hofman A, Koudstaal PJ. \& Breteler MB (2003) Silent brain infarcts and the risk of dementia and cognitive decline. $\mathrm{N}$ Engl J Med 348:1215-1222

Waterloo K, Omdal R, Sjoholm H, Koldingsnes W, Jacobsen EA, Sundsfjord JA, Husby G. \& Mellgren SI. (2001). Neuropsychological dysfunction in systemic lupus erythematosus is not associated with changes in cerebral blood flow. J Neurol;248: 595-602.

Weiner DK, Allen NB. (1991)Large vessel vasculitis of the central nervous system in systemic lupus erythematosus: report and review of the literature.J Rheumatol; 18:748-751

Welsh RC, Rahba H, Foerster B, Thurnher M \& Sundgren PC. (2007)Brain Diffusivity in Patients with Neuropsychiatric Systemic Lupus Erythematosus with New Acute Neurological Symptoms J of Magnetic Resonance Imaging ;26:541-551

Wolff SD, Balaban RS.(1994) Magnetization transfer imaging: practical aspects and clinical applications. Radiology;192:593-9. 
Yuh WTC, Ueda T. \& Male YJE.(1999) Diagnosis of microvasculopathy in CNS vasculitis: value of perfusion and diffusion imaging. J Magn Reson Imaging;10:310-3.

Zhang L, Harrison M, Heier LA, Zimmerman RD, Ravdin L, Lockshin M. \& Ulug AM: (2007) Diffusion changes in patients with systemic lupus erythematosus. Magn Reson Imaging; 25(3):399-405.

Zvaifler NJ, Bluestein HG.(1982) The pathogenesis of central nervous system manifestations of systemic lupus erythematosus. Arthritis Rheum;25:862-6. 


\title{
Central Nervous System Tuberculosis
}

\author{
Shahina Bano, Vikas Chaudhary ${ }^{2}$ and Sachchidanand Yadav 3 \\ ${ }^{1}$ Department of Radiodiagnosis, G.B. Pant \\ Hospital \& Maulana Azad Medical College, New Delhi \\ 'Department of Radiodiagnosis, Employees' State Insurance \\ Corporation (ESIC) Model Hospital, Gurgaon, Haryana \\ ${ }^{3}$ Department of Radiodiagnosis, Dr. Ram Manohar \\ Lohia Hospital \& PGIMER, New Delhi, \\ India
}

\section{Introduction}

Tuberculosis is a formidable disease worldwide because of its highly infectious nature and propensity for latency. The increasing prevalence of tuberculosis in both immunocompetent and immunocompromised individuals in recent years makes this disease a topic of universal concern. The disease has insidious onset and can affect virtually any organ system in the body, including the central nervous system (CNS). The CNS tuberculosis can mimic a number of other disease entities, and therefore it is important to be familiar with the various radiologic features of CNS tuberculosis to ensure early, accurate diagnosis. In this chapter we discuss various possible presentation of central nervous system tuberculosis involving the brain and spine.

\section{Pathophysiology}

Most tuberculous infections of the central nervous system are caused by Mycobacterium tuberculosis, as a result of hematogenous spread from a primary location, either the lung or gastrointestinal tract. Initially, small tuberculous lesions (Rich's foci) develop in the CNS, either during the stage of bacteraemia of the primary tuberculous infection or shortly afterwards. These initial tuberculous lesions may be inoculated in the meninges; the subpial and subependymal surface of the brain or the spinal cord, and may remain dormant for years. Later, rupture or growth of one or more of these small tuberculous lesions produces various types of CNS tuberculosis. The type and extent of lesion depend upon the number and virulence of bacilli and the immune response of the host. ${ }^{1} \mathrm{~A}$ tubercular rupture into the subarachnoid space results in TB meningitis; where as deep lesions cause tuberculoma or abscesses. TB meningitis may cause inflammatory changes in cranial/spinal nerves and the blood vessels. The inflammation of blood vessels (vasculitis) subsequently results in thrombosis and infarction. Hydrocephalous can occur secondary to impedance of CSF circulation and absorption. The inflammatory exudates may also surround the spinal cord producing tuberculous arachnoiditis. Infrequently, infection spreads to the CNS from a site of discal TB, tuberculous otitis, or osteogenic tubercular foci in the spine or cranial vault. ${ }^{2}$

Pathologically, a tuberculoma is composed of central core of caseous necrosis surrounded by a capsule of collagenous tissues and an outer layer of mononuclear inflammatory cells (including plasma cells \& lymphocytes), epitheloid cells and multinucleated Langerhans' 
giant cells. A tuberculoma harbours few tubercular bacilli within the necrotic center and the capsule. Outside the capsule, there is parenchymal edema and astrocyte proliferation. Unlike caseous tuberculoma, a tubercular abscess has purulent center rich in tubercular bacilli, and lacks epithelioid giant cell granulomatous reaction in its wall. ${ }^{3}$

\section{Clinical features}

CNS TB usually has signs and symptoms of increased intracranial pressure or spaceoccupying lesions in the brain or spine. Signs of meningitis includes constitutional symptoms (such as low grade fever, headache, nausea, vomiting, lethargy), meningismus, confusion, seizure, papilloedema, cranial nerve palsies (commonly second, third, fourth, sixth and seventh nerves), focal neurological deficits, stupor and coma. Patients with brain and spinal cord tuberculomas will have physical signs and symptoms consistent with the location of the lesion, which may include altered mental status, visual changes, hemiparesis/hemiplegia or seizure as seen with brain lesions; while weakness of extremities or bowel and bladder symptoms as seen with spinal cord lesions. 4,5

\section{Classification of ens tuberculosis}

The manifestations of CNS tuberculosis are highly variable; however we have tried to include various possible presentations of CNS tuberculosis in this chapter as summarized in the box 1 given below:

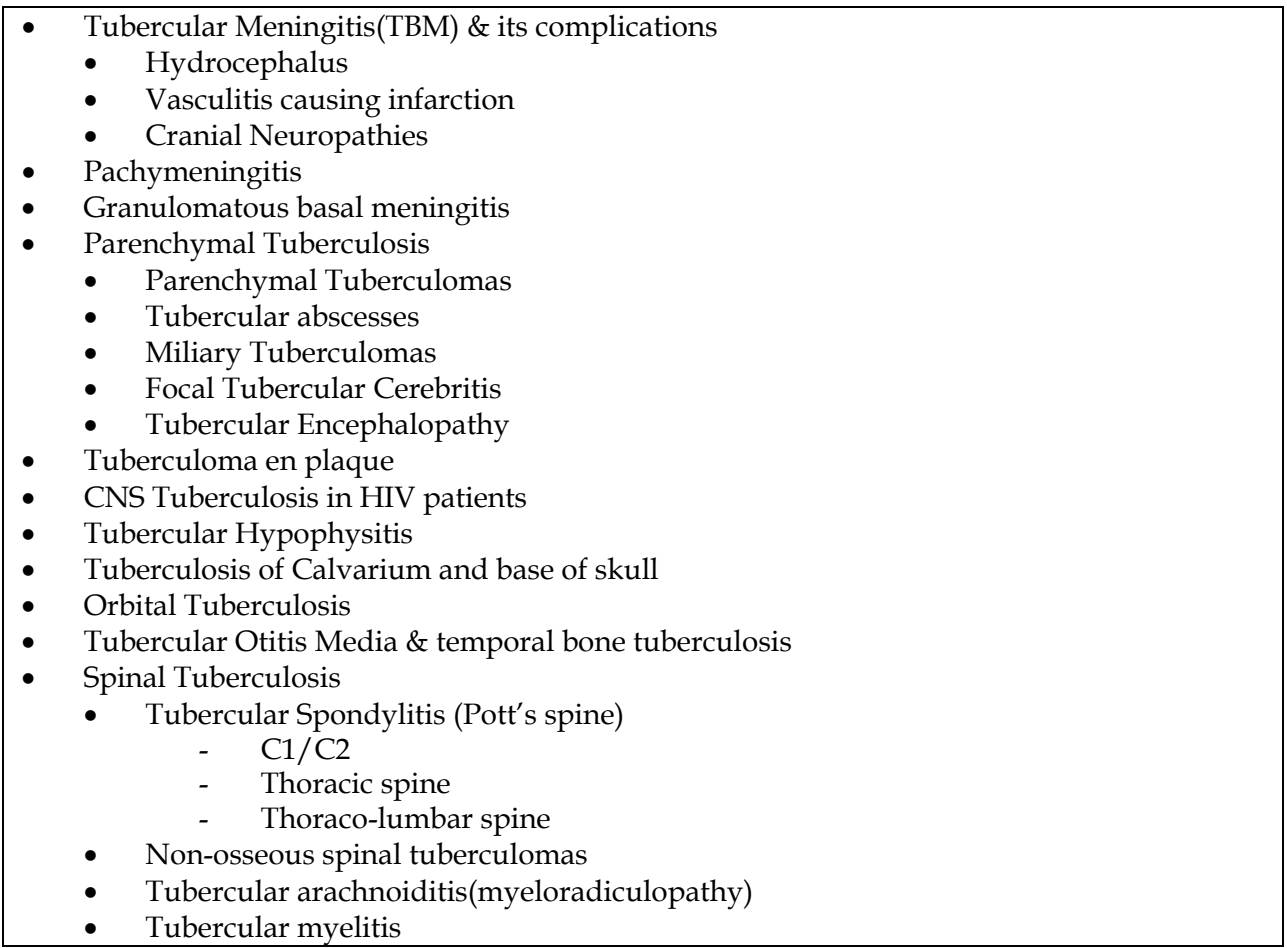

Box 1. Variable presentation of CNS tuberculosis 


\section{Imaging features}

\subsection{Tuberculous meningitis}

Tuberculous meningitis (TBM) is the most common presentation of CNS TB and is seen most frequently in children and adolescents. TBM develops when a meningeal, subpial or subependymal tuberculous focus (Rich focus) ruptures into the subarachnoid space or into the ventricular system. Important features of TBM are: 6

1. Enhancing basal exudates

2. Progressive hydrocephalus

3. Vasculitis \& Infarction, and

4. Cranial neuropathies

\subsubsection{Basal exudates}

In tuberculous meningitis, there is formation of thick, gelatinous exudate as a result of cellmediated immunity. Initially the exudate is largely confined to basal subarachnoid areas, but rapidly extends to involve the basal cisterns, particularly the interpeduncular and the suprasellar cisterns. From these sites the exudate spreads to the ambient cistern, prepontine cistern, sylvian fissures, cerebral convexities, to the ependymal surfaces of the ventricles and over choroid plexus. 7,8 On noncontrast $\mathrm{CT}$, the most common finding of cranial tuberculous meningitis is obliteration of basal cistern by isodense or slightly hyperdense exudates. Contrast enhanced CT demonstrates thickening and intense homogenous enhancement of the basal meninges, giving characteristic spider leg appearance [figure1]. MRI is more sensitive than CT in depicting these abnormalities. The basal exudates are best appreciated on FLAIR sequences, while the cisternal enhancement is better demonstrated on postgadolinium MRI images [Fgure2]. ${ }^{9}$ The brain parenchyma immediately beneath the exudate shows various degrees of edema. The meningeal enhancement can extend over the surface of the brain convexities, along tentorium and the sylvian fissures. 6,7 Isolated sylvian fissure involvement by tuberculous meningitis is also known. ${ }^{10}$ Extension into the ventricular system may cause ependymitis (abnormal enhancement of ventricular linings), or choroid plexitis (enlarged, enhancing choroid plexus).., 8

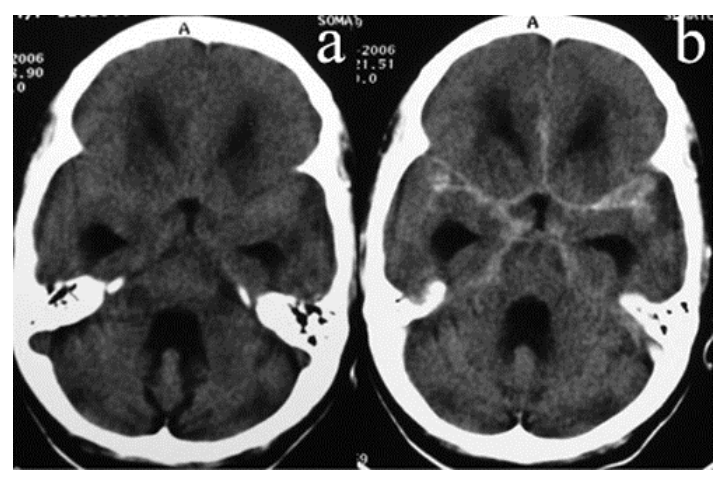

Fig. 1. Tuberculous meningitis. NCCT scan of the brain(a) shows effacement of basal cisterns by isodense exudates. CECT scan(b) shows dense enhancement of thickened and inflamed basal meninges along the basal cisterns (giving characteristic spider leg appearance), tentorium and the sylvian fissure with evidence of hydrocephalus and periventricular ooze. 

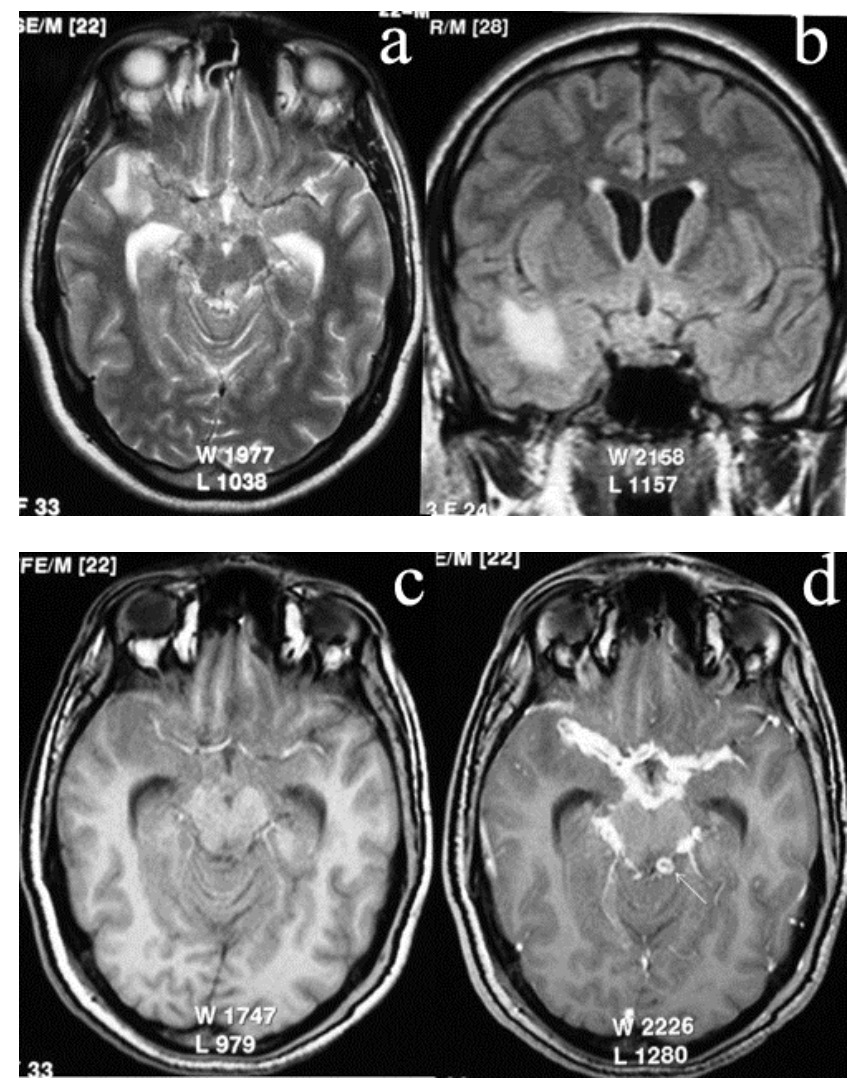

Fig. 2. Tuberculous meningitis. Axial T2W(a), and Fluid attenuated inversion recovery(b) MR images shows abnormal complex hyperintensity in the suprasellar, interpeduncular and perimesencephalic cisterns. Axial T1W(c) and corresponding post contrast image demonstrates diffuse enhancement of the basal cisterns. Few ring-enhancing tuberculomas (arrow) are also noted within the cistern.

\subsubsection{Hydrocephalus}

Hydrocephalus is the most common sequel of tuberculous meningitis. Communicating hydrocephalus [Figure1,2] is the most frequently observed form, usually caused by obstruction to CSF flow by thick gelatinous inflammatory exudates within the basal cisterns and over the brain convexities.11,12 Less commonly, the hydrocephalus may be obstructive, due to obstruction of cerebral aqueduct or fourth ventricular foramen by focal parenchymal lesion with mass effect or due to entrapment of the ventricle by granulomatous ependymitis. ${ }^{13,14}$ The presence of periventricular ooze suggests high pressure hydrocephalus. The incidence of hydrocephalus increases with the duration of disease and is associated with poor prognosis especially in children. The progress of hydrocephalus may be followed up by sequential CT or MRI scan. ${ }^{15}$ 


\subsubsection{Vasculitis \& infarction}

In TB meningitis, the basal exudates are maximally localized to the circle of Willis, and produce a vasculitis like syndrome. The vasculitis is initiated by either direct invasion of vessel wall by mycobacteria or may result from secondary extension of adjacent arachnoiditis. Thus a consequent inflammatory change in the arteries and veins may lead to spasm or thrombosis of the vessels with resulting infarction. ${ }^{6}$ The vessels at the base of the brain are most severely affected, including the terminal segment of common carotid artery and proximal segment anterior, middle and posterior cerebral arteries.10,16,17 The middle cerebral and its branches are most often affected, especially the medial striate and thalamoperforating arteries supplying the basal ganglia and thalami. ${ }^{14,16,17}$ Cortical infarctions resulting from the involvement of cortical vessels are less common. The infarcts are commonly bilateral, often hemorrhagic, and relatively more common in infants and children than in adults. Although both CT and MRI can demonstrate the infarction, MRI is more sensitive than CT for demonstration of these infarcts. Infarcts appear as low density regions on CT scan, while as areas of prolonged T1 and T2 relaxation on MR images. Diffusion weighted images (DWI) are gold standard for the diagnosis of acute infarction, which appears bright on DWI, and shows decrease signal on corresponding apparent diffusion coefficient(ADC) map[Figure3]. MR angiography is useful in follow-up of patients with vasculitis secondary to TB meningitis. The angiogram demonstrates a triad of narrowing of arteries at the base of the brain, narrowed or occluded small or medium sized arteries with early draining veins, and wide sweep of pericallosal arteries secondary to hydrocephalus [Figure4]. ${ }^{16,17}$

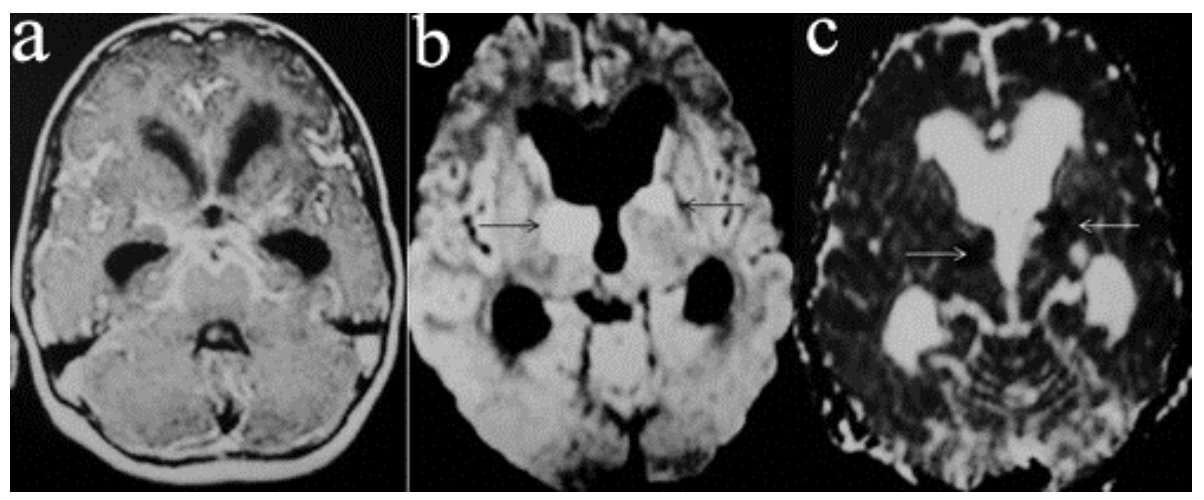

Fig. 3. Tuberculous meningitis with acute infarct. Post contrast axial MR image (a) shows enhancing basal exudates with associated hydrocephalus. Diffusion weighted image(b) and corresponding apparent diffusion coefficient(c) mapping reveals acute infarct in bilateral basal ganglia (due to vasculitis) showing high signal intensity on b1000 and low signal intensity on ADC map. 


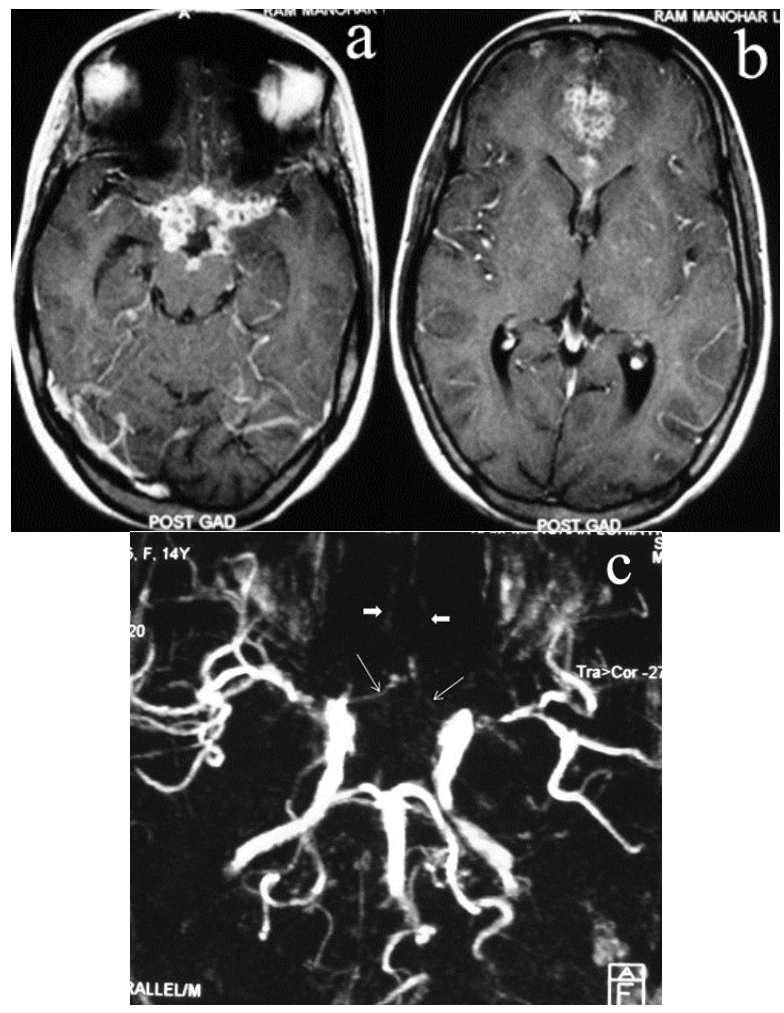

Fig. 4. Basal granulomatous meningitis with vascular changes. Axial T1W post contrast MR images of the brain at two different levels $(a, b)$ shows basal meningitis with multiple conglomerate tuberculomas in suprasellar cistern and along anterior interhemispheric fissure. TOF MR angiogram(c) demonstrates severe narrowing of bilateral anterior cerebral arteries(thin arrows), and splaying of pericallosal arteries (thick arrows) by these conglomerate masses.

\subsubsection{Cranial neuropathies}

Cranial nerve palsies occurs in $20-40 \%$ of patients and may be the presenting feature of TB meningitis. Cranial nerve involvement is partly due to vascular compromise resulting in ischemia of the nerve or may be due to entrapment of the nerves by the exudates. Most commonly affected cranial nerves are II, III, IV, VI, VII. The affected cranial nerves are best evaluated by MRI. They appear thickened and hyperintense on T2-weighted sequences, and show marked enhancement on postgadolinium images. Constructive Interference at Steady State (CISS), which is a T2-weighted, 3D-Gradient echo sequence is particularly used for evaluating cranial nerves around the brain stem. Optochiasmatic arachnoiditis, compression of optic chiasma by third ventricular dilatation (in case of hydrocephalus) and optic nerve granuloma are common factors associated with vision loss in these patients. On MRI optochiasmatic arachnoiditis is characterized by perichiasmal enhancement (of basal exudates), hypertrophy and enhancement of chiasma and cisternal segment of both optic nerves. Associated dilatation of third and lateral ventricles is also evident.7,8,19,20 


\subsection{Pachymeningitis}

Chronic tubercular infection of dura mater results in pachymeningitis, a rare manifestation, seen as focal or diffuse thickening and enhancement of the dura. Common sites include cavernous sinus, floor of middle cranial fossa, tentorium and the cerebral convexity. On noncontrast $\mathrm{CT}$, the affected dura has plaque like appearance with or without calcification. On MRI, thickened dura appears hypointense to gray matter both on T1 and T2-weighted images. Post contrast study shows intense homogenous enhancement of thickened pachymeninges [Figure5]. Important differential diagnosis includes neurosarcoidosis, meningioma and lymphoma. ${ }^{21}$

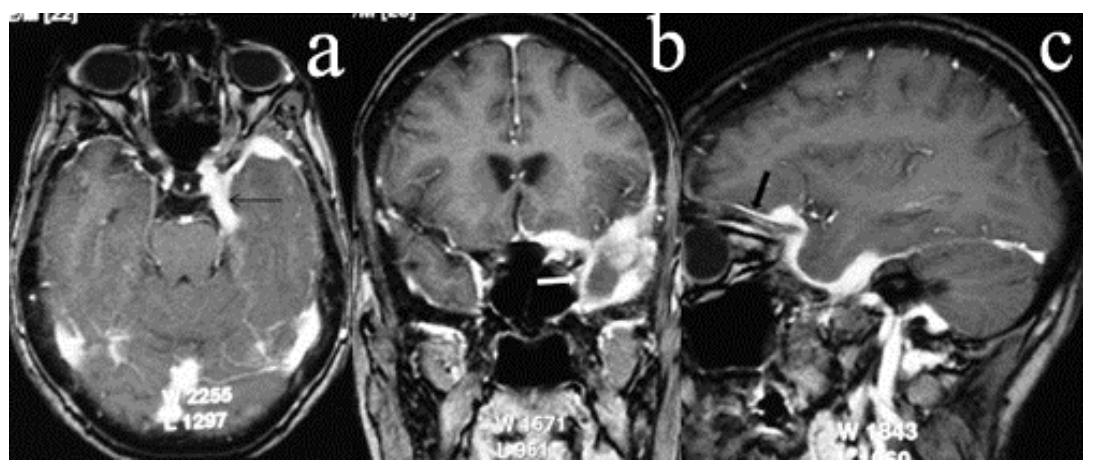

Fig. 5. Pachymeningitis. Post contrast T1W axial(a), coronal(b) and sagittal(c) images of brain show intensely enhancing, thickened pachymeninges(dura mater) encircling the left temporal lobe(thick white arrow), floor of ipsilateral anterior cranial fossa (thick black arrow) and the left cavernous sinus (thin black arrow). The process seems to be progressive with further extension to involve ipsilateral tentorium and cerebral convexity, where dura appears mild thickened.

\subsection{Granulomatous basal meningitis}

Granulomatous basal meningitis is another, relatively uncommon presentation of intracranial tuberculosis. It is characterised by diffuse or circumscribed granulomatous involvement of meninges at the skull base. It commonly causes compression of optic nerves and the optic chiasma producing visual disturbances. On CT scan, it is seen as an irregular lumpy enhancing mass, superimposed on dense basal enhancement. On MRI, the granulomatous basal mass is hypointense on T2-weighted images and shows intense but heterogeneous postgadolinium enhancement. 9,22

\subsection{Parenchymal tuberculosis}

\subsubsection{Parenchymal tuberculomas}

Tuberculous granuloma (tuberculoma) is the most common form of parenchymal lesion. ${ }^{6}$ Tuberculoma can occur at all age group; however, its incidence is higher in pediatric population. ${ }^{11}$ These are usually located at corticomedullary junction and periventricular region, as expected for hematogenous dissemination. They are mostly infratentorial in children, and supratentorial in adults.23,24 Common locations where tubercuomas can be found includes cerebrum, cerebellum, brainstem, basal ganglia, subarachnoid space, cisterns and fissures Rarely, they can be found within the ventricle (lateral ventricle being the most 
common site), cavernous sinus, sella turcica, hypophysis, hypothalamus, sphenoid sinus and mastoid air cells.25,26 Parenchymal tuberculomas can be single or multiple, with or without coexisting meningitis. ${ }^{27}$ Both the parenchymal and intraventricular tuberculoma may be associated with hydrocephalus. ${ }^{28}$

On CT, the noncaseating granulomas are solid, isodense or hyperdense in attenuation and show homogenous contrast enhancement, while the caseating granulomas enhance peripherally. Moderate to marked perilesional edema is frequently present. The 'target sign' seen on CECT is characterized by a central calcific nidus surrounded by rim of enhancement. This sign is highly suggestive of, but not pathognomonic of tuberculosis.29,30 Solitary ring enhancing lesion on $\mathrm{CT}$ in patients presenting with seizures present a diagnostic dilemma since the granuloma of tuberculosis and cysticerus both may have similar morphological appearances. Some authors have tried to differentiate these two entities on basis of clinical signs and radiological (CT) findings. In patients with tuberculoma there is evidence of raised intracranial tension with progressive neurological deficit. The ring enhancing lesion is usually greater than $20 \mathrm{~mm}$ in size, show irregular margin and cause midline shift. On contrary, neurocysticercus granuloma is less than $20 \mathrm{~mm}$ in size, rounded disc like with no significant mass effect. Enlarging lesions on repeat CT after 8-12 weeks of anticonvulsant therapy could be due to different etiologies and should be biopsied. 31

The MR features of individual tuberculoma will depend on whether the lesion is noncaseating, caseating with a solid center or caseating with a liquid center. ${ }^{32,33}$ The Noncaseating granuloma is usually hypointense on T1-weighted images(T1WI), hyperintense on T2-weighted images(T2WI) and shows homogenous nodular enhancement on post gadolinium images. The caseating granuloma(s) with solid center [Figure6]. appears hypointense to isointense on T1WI (may have a slight hyperintense rim) and strikingly hypointense on $\mathrm{T} 2 \mathrm{~W}$ images. On contrast administration the lesion shows peripheral rim enhancement. The relative hypointensity on T2WI is attributed to high cellular density of central core of tuberculoma. The caseating granuloma with central liquefaction of caseous material appears hypointense on T1WI and hyperintense on T2WI with peripheral hypointense rim which represents the capsule of tuberculoma. The rim enhancement occurs after gadolinium administration. These lesions are indistinguishable from pyogenic abscess on imaging. At this stage, diffusion weighted images(DWI) may reveal diffusion restriction within the tuberculoma [Figure6]. ${ }^{34}$ A variable degree of vasogenic edema surrounds the lesion, and is relatively more prominent in the early stages of granuloma formation.

MR spectroscopy has been used to differentiate tuberculoma from pyogenic abscesses and neoplasms (both primary and secondary). On proton MRS [Figure6], these lesions show a large lipid peak at $0.9,1.3,2.0$ and 2.8 ppm, highly specific for tuberculomas, more choline and less NAA and creatine. The choline/creatine ratio is greater than 1 in all tuberculomas. Caseous material typical of tuberculomas has high lipid content. ${ }^{35}$ Pyogenic brain abscesses show lipid and lactate peak at $1.3 \mathrm{ppm}$ and amino acid peak (e.g. valine, leucine and isoleucine) at $0.9 \mathrm{ppm} .{ }^{36}$ The lesions such as metastases and high grade gliomas may show lipid peak in addition to significantly elevated Choline/NAA ratio. ${ }^{35}$

Magnetization transfer imaging (MTI) improves the detectability of these lesions with more number of tuberculomas detected on precontrast T1-weighted MT-SE images, compared to routine spin echo (SE) sequences and postcontrast T1-weighted MT-SE images. 
Conventional SE invisible lesions (isointense on T1-and T2-weighted images) can be easily picked up on MT images because of lower transfer of magnetization in tuberculomas as compared to surrounding brain parenchyma. The presence of lipid within tuberculoma is probably responsible for lowering magnetization transfer. Detection of more lesions on precontrast T1-weighted MT-SE image as compared to postcontrast T1-weighted MT-SE image suggests lack of breach of the blood-brain barrier in some of the lesions. Thus the improved estimate of disease load on precontrast T1-weighted MT-SE images helps to better assess to response to specific therapy. The Quantitative MT (i.e. MT ratio) further helps to differentiate $\mathrm{T} 2$ hypointense tubercular granulomas from similar appearing lesions of neurocysticercosis. The MT ratio is significantly lower in tuberculomas as compared to cysticercus granulomas. ${ }^{37}$
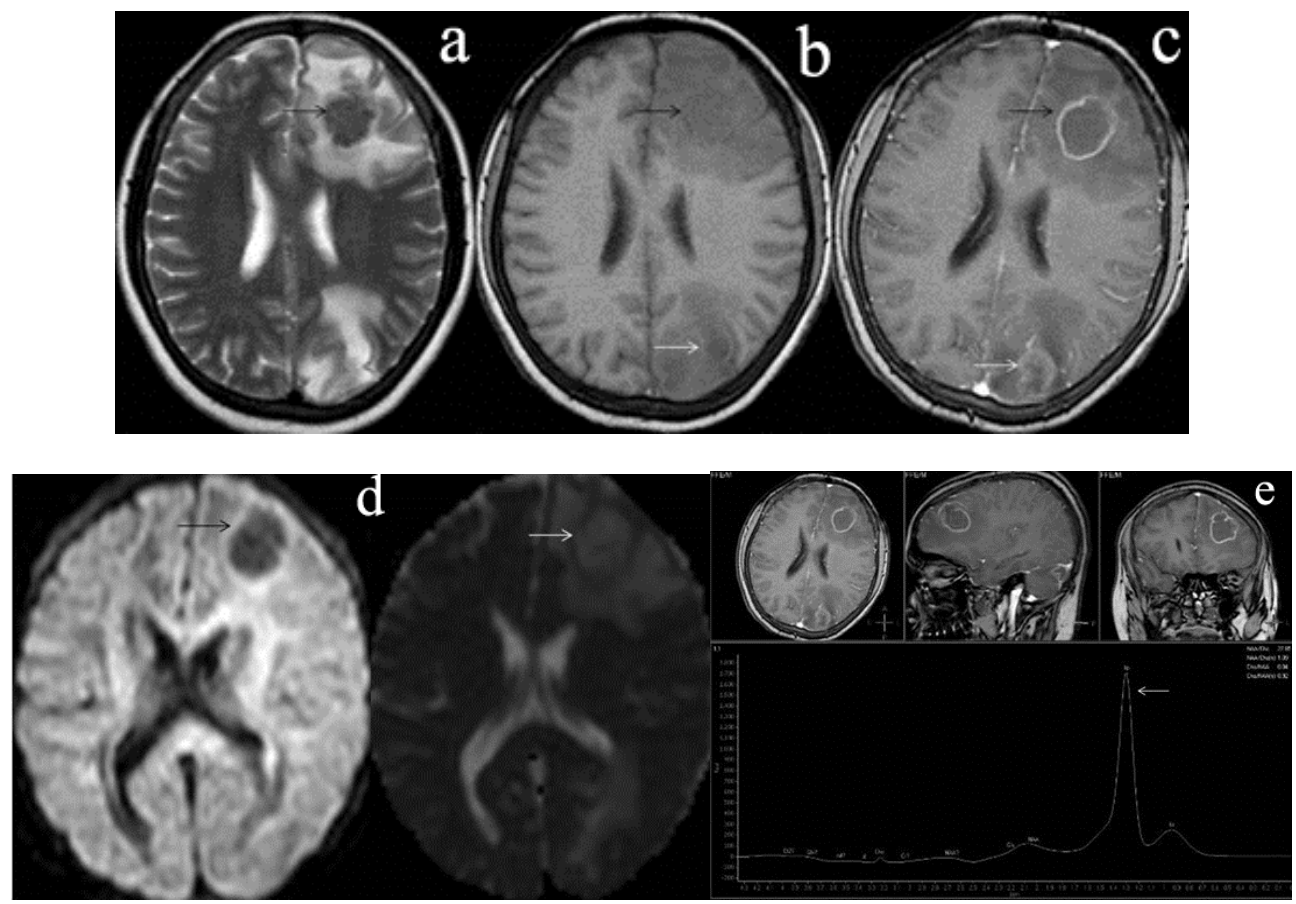

Fig. 6. Caseating tuberculoma. Axial T2W MR image(a) of brain shows profoundly hypointense lesion in left frontal lobe(black arrow) with marked perilesional oedema. The lesion demonstrates isointense core with slight hyperintense rim on T1W image(b), and thin peripheral ring enhancement on gadolinium-enhanced image(c). Multiple similar lesions were present involving both supra and infratentorial compartments, note similar lesion in left occipito-parietal region(white arrow). The solid portion of the lesion shows no diffusion restriction on DWI and corresponding ADC mapping(d). Single voxel MR spectroscopy(e) done at $\mathrm{TE}=30$ shows large lipid peak at $1.33 \mathrm{ppm}$ (arrow), with marked reduction in other metabolites. Large lipid peak corresponds to high lipid content within the caseous material. 
Healed tuberculomas and the inflammatory exudates may calcify (in up to $23 \%$ cases) and these are more evident on CT [Figure7]. On MRI, the calcifications are better appreciated on gradient recalled echo (GRE- T2*WI) and Susceptibility weighted images (SWI). ${ }^{30}$ Multiple conglomerate tuberculomas generally impose no difficulty in making a correct diagnosis; however, a solitary parenchymal tuberculoma needs to be differentiated from neurocysticercosis, pyogenic abcesses, primary or metastatic neoplasm.

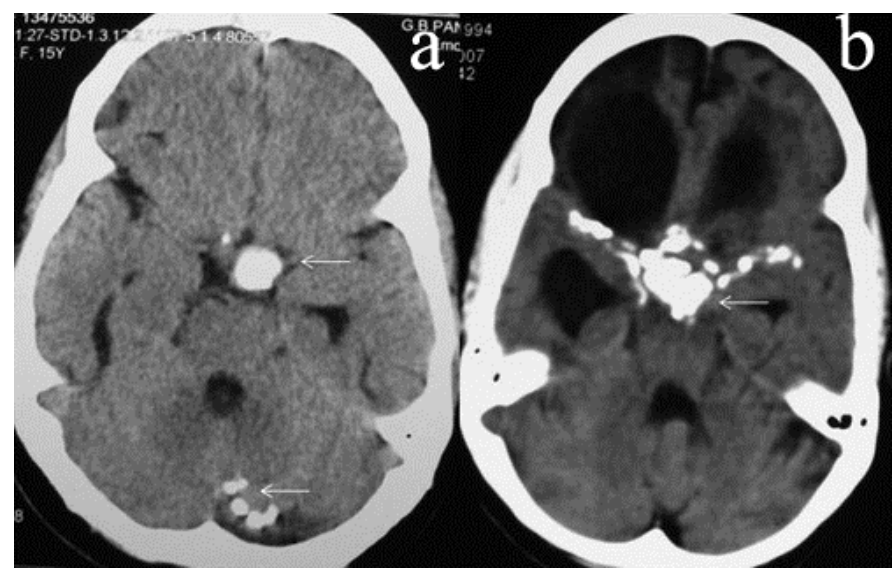

Fig. 7. Healed tuberculomas and basal exudates. Axial NCCT brain of two different patients demonstrate discrete calcified granulomas (arrow) in suprasellar cistern and left cerebellar hemisphere(a), and densely calcified basal exudates(arrow) in suprasellar region and along the M1 segment of bilateral middle cerebral arteries(b).

\subsubsection{Tubercular abscesses}

Tubercular abscess is a rare manifestation of CNS tuberculosis, occurring in less than $10 \%$ cases. They are found more frequently in elderly and immunocompromised patients. The patient is acutely ill with focal neurological deficit. TB abscesses have a more accelerated clinical course. ${ }^{24}$

On imaging, a TB abscess may be indistinguishable from a caseous tuberculoma with central liquefaction or a pyogenic abscess. However, a TB abscess is usually solitary and larger than tuberculoma. Perilesional edema and mass effect is more as compared to tuberculoma. On CT and MRI [Figure 8], it is often multinucleated and shows thin, smooth peripheral wall enhancement on post contrast images. ${ }^{38}$ Proton MRS and MTI help to differentiate tuberculous from pyogenic brain abscesses. On MRS, a pyogenic abscess demonstrates amino acid peak at $0.9 \mathrm{ppm}$, which is characteristically absent in tubercular abscess. On MTI, MT ratio of a tubercular abscess is lower than that found in pyogenic abscess. ${ }^{36}$ The role of DWI is conflicting in making correct diagnosis of tuberculoma as well as tubercular abscess. The reports in the literature vary regarding findings on diffusion weighted images with regard to both the tuberculoma and tubercular abscess. Some papers report slightly increased diffusivity in tuberculoma, and significantly higher in tubercular abscesses, 36 others report decreased diffusivity, ${ }^{39}$ while still others report diffusion characteristic similar to the normal brain. 40 


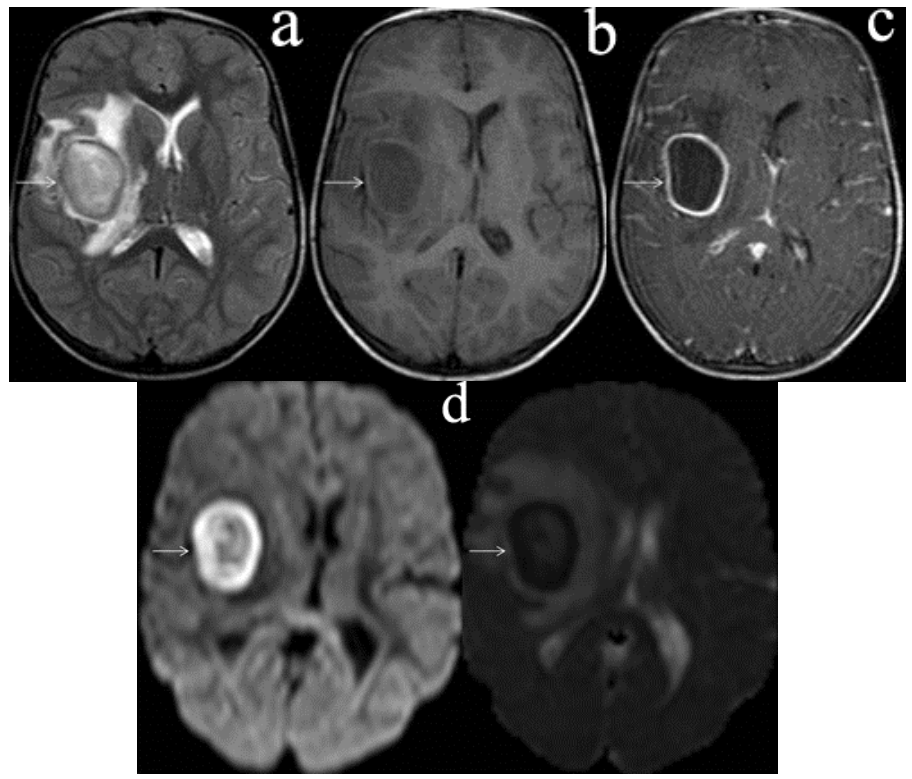

Fig. 8. Tubercular abscess. Axial T2W(a), T1W (b), and CEMR(c) images of another patient shows solitary ring enhancing lesions with liquified center, involving right basal ganglia region(arrow). There is associated marked perilesional edema with mass effect. The lesion demonstrates restriction on DWI and corresponding ADC mapping (d). MRS(not shown) revealed findings consistent with tubercular abscess.

\subsubsection{Miliary tuberculomas}

Miliary tuberculosis of the brain may be a part of generalized pathological process, with primary focus situated in the lung or elsewhere. The condition is subtle with no clinical evidence of brain involvement. On imaging, these lesions are small, less than $5 \mathrm{~mm}$ in size;

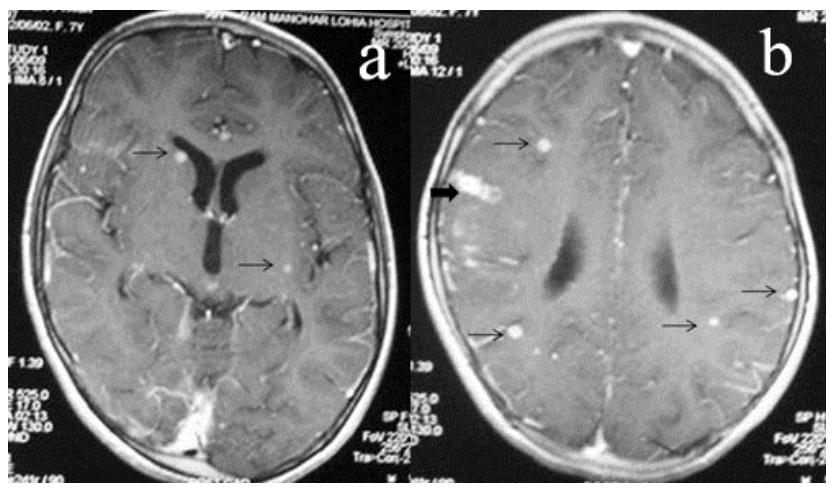

Fig. 9. Miliary tuberculosis. Post contrast T1W axial MR images of brain at two different levels reveal intense nodular enhancing small granulomas (thin arrow) randomly distributed throughout the brain parenchyma. Few larger lesions (thick arrow) were also seen along with these miliary nodules. 
located at cortico-medullary junction and in the distribution of perforating vessels. They appear as high signal intensity foci throughout the brain parenchyma on T2WI and show intense nodular enhancement on post gadolinium images [Figure 9]. Contrast enhanced MRI is more sensitive than CECT for detecting these lesions. ${ }^{41,42}$

\subsubsection{Focal tubercular cerebritis}

This entity was described by Jinskin based on retrospective analysis of five patients. CT imaging shows intense focal gyral enhancement. On MRI, focal tuberculous cerebritis appears hypointense on T1, hyperintense on T2 and shows small areas of patchy contrast enhancement on post gadolinium images. 30

\subsubsection{Tubercular encephalopathy}

Tubercular encephalopathy is a diffuse cerebral disorder characterized by convulsion, stupor and coma, without signs of meningeal irritation or focal neurological deficit. It is exclusively seen in infants and children receiving antitubercular treatment. Imaging shows unilateral or bilaterally symmetrical cerebral white matter edema, occasionally with perivascular demyelination or hemorrhagic leukoencephalopathy. A picture resembling post-infectious demyelinating encephalomyelitis may be observed. The pathological basis suggested for TB encephalopathy is an allergic delayed type IV hypersensitivity reaction due to cell mediated immunity to tubercular protein. 43,44

\subsection{Tuberculoma en plaque}

An en plaque meningeal tuberculoma is a rare manifestation, seen as dural based, massforming localized meningeal process which morphologically resembles en plaque meningioma or meningeal metastases. They are commonly seen along the frontal and parietal convexities, tentorium, interhemispheric fissures and in the posterior fossa. On noncontrast CT, these lesions are hyperdense in appearance. On MRI, they appear isointense on T1WI, hypo to hyperintense on T2WI, and show homogenous or peripheral contrast enhancement, depending on the presence or absence of central caseation [Figure 10]. Prominent feeding meningeal vessels may also be evident. 45,46

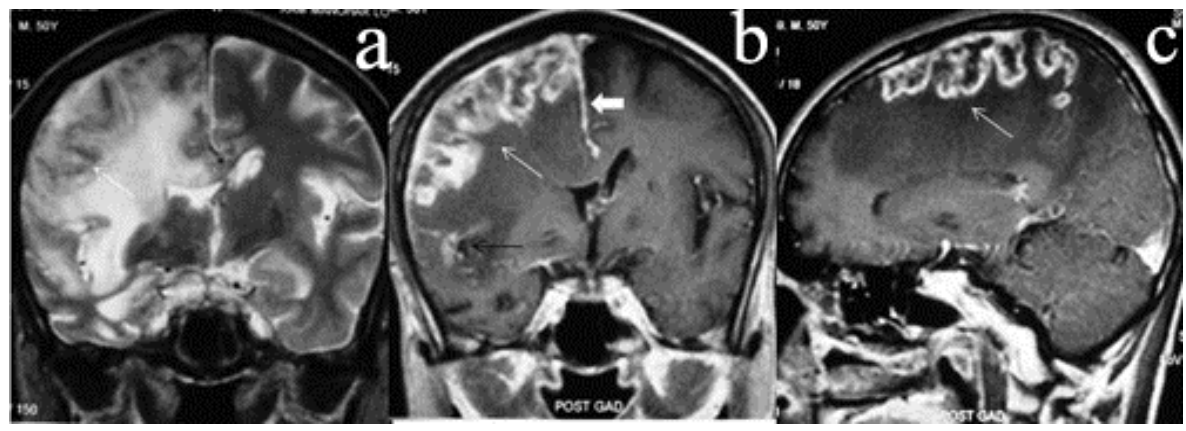

Fig. 10. En-plaque tuberculoma. T2W coronal image(a) shows predominantly hypointense dura based lesion widely spread along the right cerebral convexity, with associated marked vasogenic white matter edema and mass effect. Post contrast T1W coronal(b) and sagittal(c) image shows irregular peripheral rim enhancement of the lesion(thin white arrow). Also note, thickened enhancing dura along the falx (thick white arrow) and right cerebral convexity; and right sylvian fissure meningitis(thin black arrow). 


\subsection{CNS tuberculosis in AIDS}

Although TB infection of CNS in AIDS patients follows a rapidly progressive course, the spectrums of imaging findings are similar to those of the non-immunocompromised patients. Meningitis, cerebral abscesses and tuberculomas are often observed in combination with one another [Figure11]. The differential diagnosis includes other opportunistic infections and primary or secondary CNS lymphoma. ${ }^{47}$

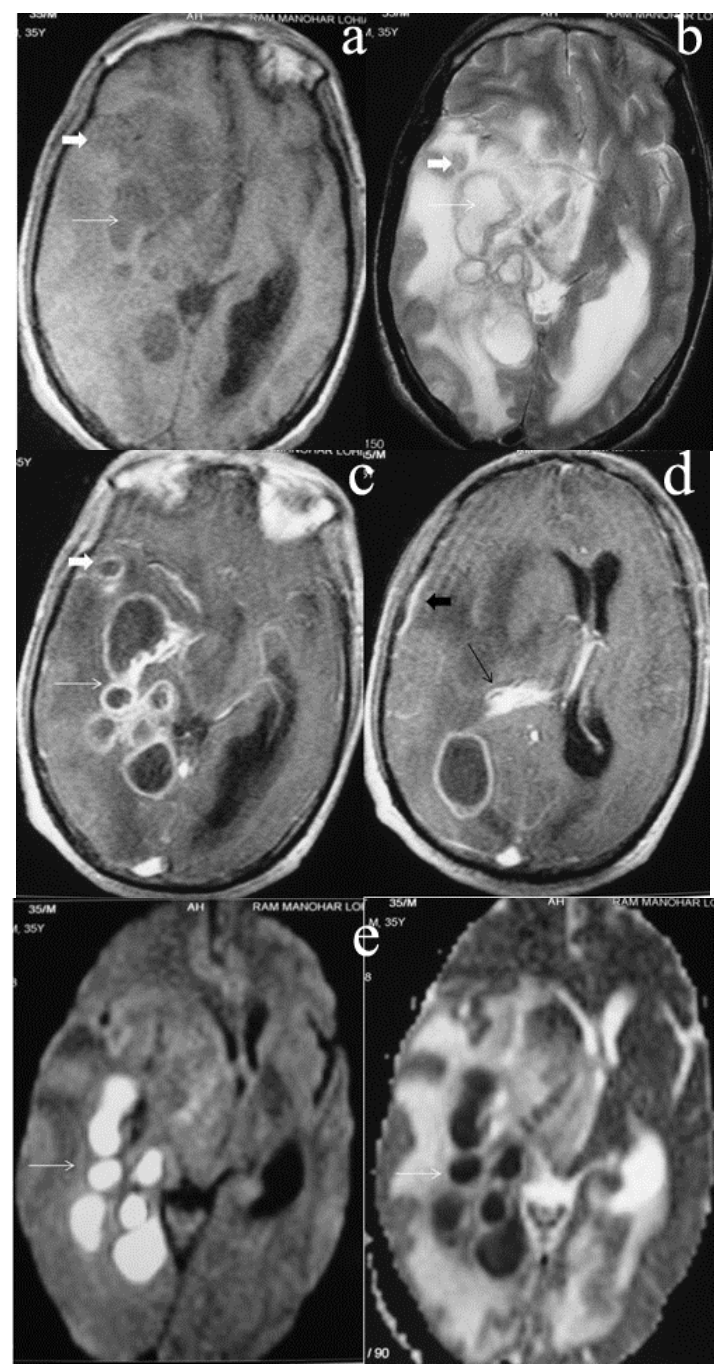

Fig. 11. CNS tuberculosis in AIDS. Axial T1W(a), T2W(b), post contrast(c) and diffusion weighted(d) MR images of brain demonstrate multiple cerebral abscesses (thin white arrows), tuberculomas(thick white arrow), choroid plexitis (thin black arrow) and dural thickening(thick black arrow). Note associated marked perilesional vasogenic edema with significant mass effect. 


\subsection{Tuberculous hypophysitis}

Tubercular hypophysitis is an extremely rare entity that commonly presents with enlargement of pituitary gland, mimicking a pituitary adenoma. On MR imaging, the gland is diffusely enlarged with a thickened stalk, seen infrequently. The thickening and enhancement of stalk and surrounding dura differentiates these lesions from pituitary adenoma[Figure12]. However, these are non-specific findings and are also seen with tuberculous meningitis, sarcoidosis, syphilis and eosinophilic granuloma. ${ }^{48}$

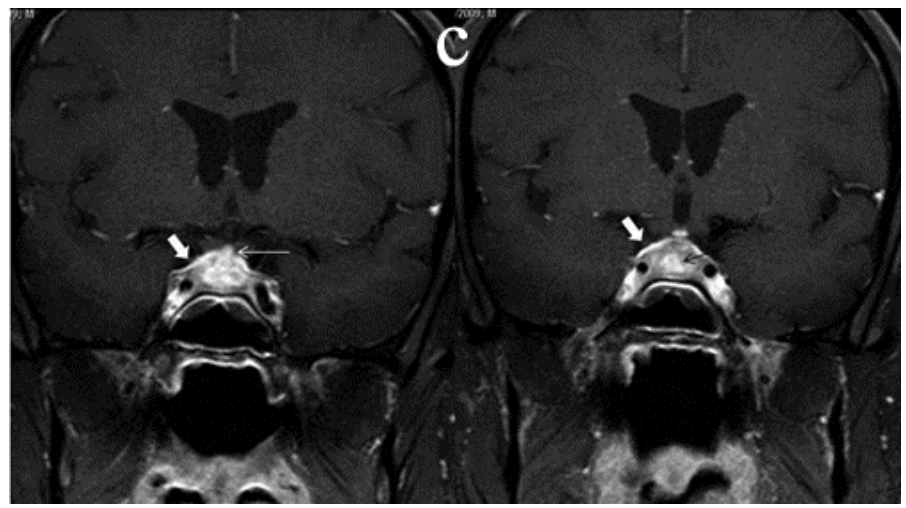

Fig. 12. Tuberculous hypophysitis. Post contrast T1W coronal MR images of a young adult male showing enlarged pituitary gland with intra-glandular ring enhancing tuberculoma (thin black arrow), and thick enhancing pituitary stalk(thin white arrow). Thickening and enhancement of diaphragma sellae (thick white arrow) is also noted. The patient had associated pulmonary tuberculosis and showed complete resolution of the pituitary lesion after a course of antitubercular therapy.

\subsection{Tuberculosis of calvarium and base of skull}

Isolated calvarial tuberculosis is a rare condition; and commonly occurs secondary to hematogenous spread from primary focus elsewhere. Frontal and parietal bones are most commonly involved followed by occipital and sphenoid bone. Calvarial tuberculosis may present as a subgaleal swelling (Pott's puffy tumor) with a discharging sinus when the outer table is involved. Inner table involvement which is relatively more common is associated with formation of underlying extradural granulation tissue. Both tables involvement is not uncommon. The bony lesions are usually osteolytic, and appear as a well defined punched out defect with central sequestrum. Rarely sclerosis may be seen. Cranial sutures do not prevent the spread of granulation tissue, and hence extensive destruction can occur before a sinus or swelling becomes apparent. Despite the dura mate, which is an effective barrier to the spread of infection, subdural empyema, meningitis, and parenchymal granulomas may be encountered. CT scan of the brain [Figure13] helps in assessing the extent of bone destruction, scalp swelling and extent of intracranial involvement. 49,50

Tuberculous ostitis of skull base (spheno-clival) is very rare in occurrence. The usual clinical picture is of jugular foramina syndrome. The involvement of skull base may be either by contiguous spread of infection from the adjacent site, or via hematogenous route from primary focus elsewhere. On imaging [Figure14], there is destruction of the skull base with enhancing soft tissue mass that may cause compression of adjacent structures. Associated 


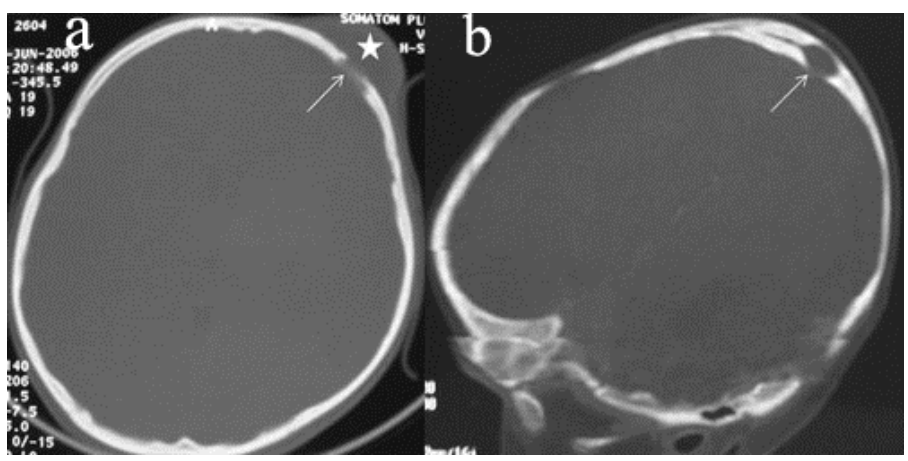

Fig. 13. Calvarial tuberculosis. NCCT head (bone window) of two different patients. First case(a) shows a large subgaleal soft tissue swelling (Pott's puffy tumor)(asterix) with destruction of both outer and inner table(arrow). Second case(b) shows a well defined lytic lesion involving right parietal bone with destruction of inner table but intact outer table(arrow). Both the cases showed no intracranial extension of the lesion.

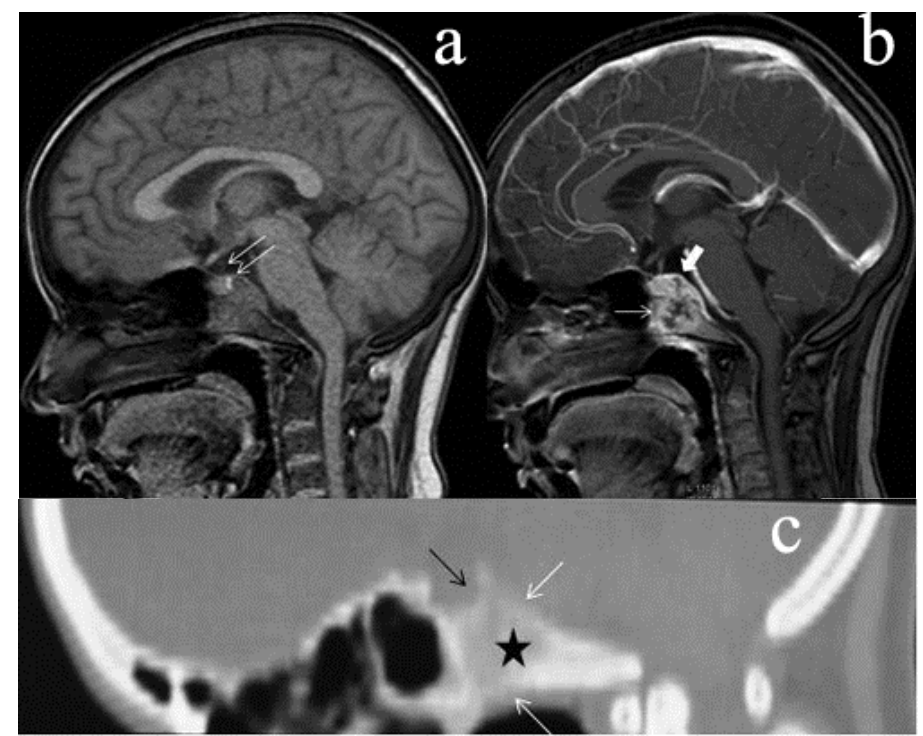

Fig. 14. Spheno-clival tuberculosis. Sagittal plain (a) and post contrast(b) T1W MR image of brain demonstrates an intraosseous soft tissue lesion in anterior part of body of clivus, showing heterogeneous contrast enhancement(thin white arrow). The dura along the superior aspect of the clivus shows thickening and enhancement(thick white arrow). Soft tissue along the inferior margin of the clivus also shows inflammatory changes. Note, the pituitary gland and the stalk(double white arrow) which appears normal in morphology and signal intensity. Sagittal NCCT skull(c) demonstrates destruction of anterior half of the body of clivus(asterix) and erosion of both antero-superior and antero-inferior cortical margin of the clivus(thin white arrow). The floor of sella tursica and the posterior clinoid process also shows rarefaction (thin black arrow). 
meningitis may further complicate the disease producing multiple cranial neuropathies. The imaging characteristic mimics a malignant tumor (e.g. Cordoma), making the diagnosis of tuberculosis difficult, hence a high degree of clinical suspicion is mandatory. ${ }^{51,52}$

\subsection{Orbital tuberculosis}

Tuberculosis of orbit is rare, usually occurring in pediatric age group. Hematogenous spread from a primary tubercular focus or contiguous spread from paranasal sinuses may affect the orbit. The disease is usually unilateral and has slow progressive course. The imaging findings include involvement of bony orbital wall (producing cortical irregularity, destruction, thickening or sclerosis) and lacrimal gland, with extraconal inflammatory mass or frank abscess formation. The patient may present with isolated preseptal thickening. Infratemporal and intracranial extension is not uncommon [Figure15]. MRI is the imaging modality of choice in these cases. Post contrast, fat suppression techniques significantly

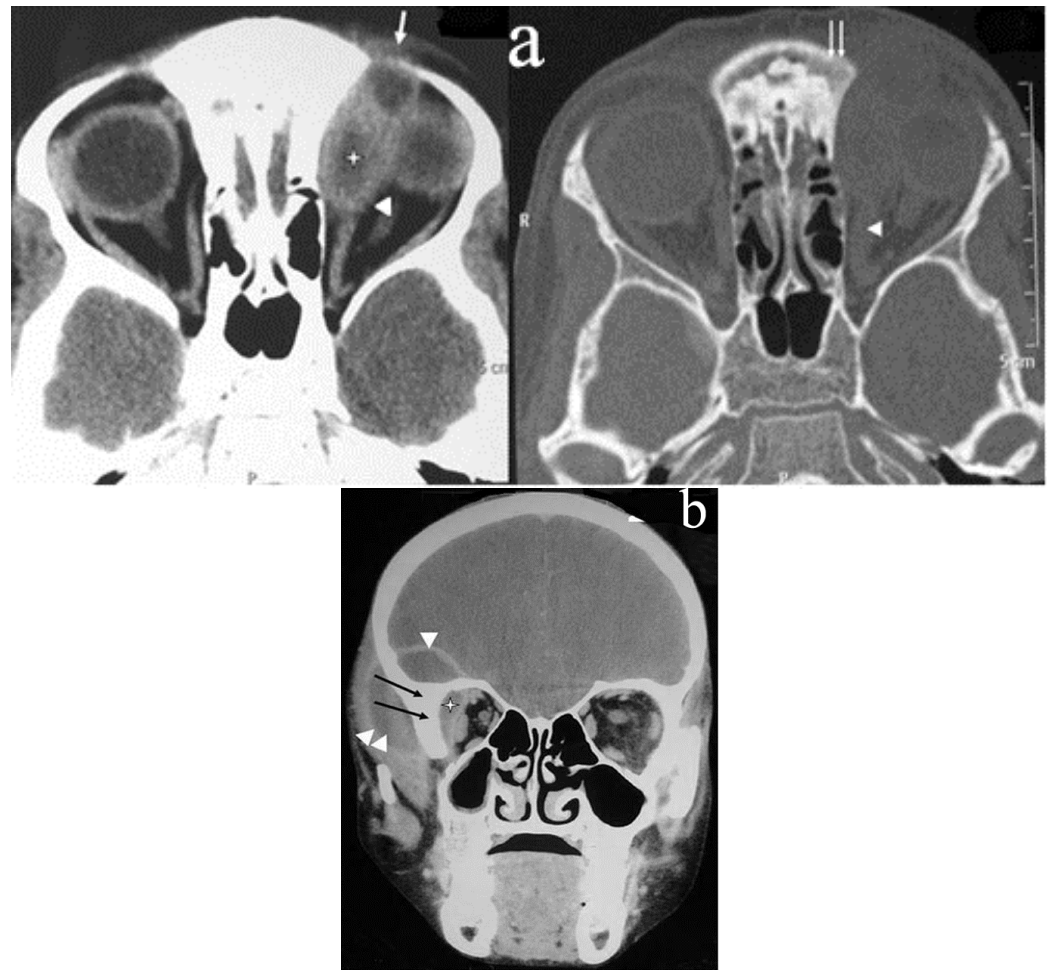

Fig. 15. Orbital tuberculosis. Axial CECT orbit(a) shows a medially situated extraconal orbital abscess(asterix) with extension into preseptal tissue and root of nasion(single thin arrow). Adjacent medial rectal muscle is displaced medially by the extraconal mass(arrow head). Irregularity and destruction of nasion(double thin arrows) is also present. Coronal CECT orbit (b) of another patient shows a laterally situated extraconal lacrimal gland abscess(asterix) which extends intracranially as epidural abscess(single arrow head) and into the infratemporal fossa (double arrow heads). Ipsilateral zygomatic bone appears thickened (double black arrows). (From IJRI 2010; 20:6-10. 
improve the visualization of subtle masses. Non-tuberculous infection, vascular malformations and various neoplasms should always be considered in differential diagnosis of orbital masses. 53

\subsection{Tubercular otitis media and tuberculosis of temporal bone}

Tubercular otitis media (TOM), a relatively uncommon condition, may be a part of widespread central nervous system disease or hematogenous spread from a primary tubercular focus elsewhere. The disease is more common in infants and children. It may be unilateral or bilateral. The patient usually presents with profuse painless otorrhea. High resolution $\mathrm{CT}$ is the modality of choice for imaging the temporal bone. Imaging shows middle ear soft tissue mass with destruction of bony walls. In addition, mucosal thickening of bony external auditory canal (EAC), extension of soft tissue mass into EAC and destruction of osscicles may also be documented. However, the scutum is always preserved in a case of TOM. T1-weighted post gadolinium MR images demonstrate the extent of inflammatory changes, evident as enhancing granulation tissues. Important complications include conductive deafness, facial palsy, cochlear involvement with labyrinthitis, sensory neural hearing loss and intracranial dissemination of infection. Mastoiditis and sinus formation may occur. Important differential includes other bacterial infections, cholesteatoma, fungal granulomas, wegener's granulomatosis, Langerhans cell histiocytosis. ${ }^{4}$

\subsection{Spinal tuberculosis}

\subsubsection{Tuberculous Spondylitis (pott's spine)}

Tuberculous Spondylitis is a leading cause of paraplegia. In developing countries, spinal tuberculosis affects younger age groups, including infants and children. In developed countries, it mostly affects the elderly. However, due to HIV epidemic, its incidence has increased among younger age groups. The disease has insidious onset and indolent course. The lower dorsal and lumbar spines are most commonly affected, followed by cervical spine. The atlanto-axial region involvement is relatively uncommon. The disease process results from hematogenous spread of infection to the vertebral body via paravertebral venous plexus of Batson. Infection usually begins in anterior part of vertebral body within the cancellous bone adjacent to the end plate or anteriorly under the periosteum of the vertebral body. Destruction of end plate allows the spread of infection to the adjacent intervertebral disc, and subsequently to the additional spinal segment. Subsequent spread of infection to other vertebral bodies may also occur via subligamentous route, with sparing of intervertebral disc [Figure16]. Thus the classic pattern of involvement of more than one vertebral body together with the intervening disc is seen in TB spine [Figure17]. Skip lesions are not uncommon. Occasionally, tuberculous spondylitis affects only one vertebral body, sparing the adjacent disc. The pedicle and posterior element involvement is rare. The spread of infection into the paraspinal tissues results in the formation of paravertebral soft tissue inflammatory mass (phlegmon) and/or frank abscess [Figure16,17]. Intraspinal extension is also frequent. 55,56,57,58 Neurological deficits are commonly associated with spinal tuberculosis of cervical region, particularly when cranio-vertebral junction or C1-C2 spine is involved [Figure18]. The neurological deficit is usually caused by significant thecal or cord compression by displaced bony fragment, epidural inflammatory mass and/or abscess. Death may occur due to atlanto-axial instability or cervico medullary compression.59,60 


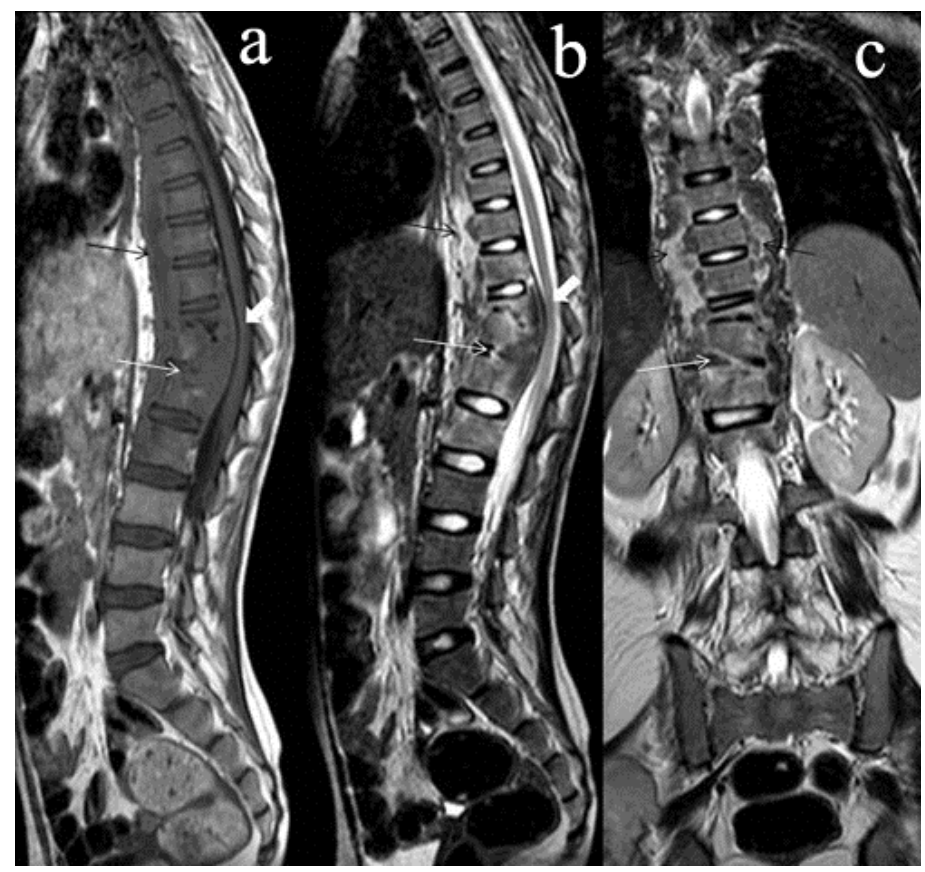

Fig. 16. Tuberculous spondylitis (Pott's spine) with subligamentous spread of the disease. Sagittal T1W(a), T2W(b), and Coronal T2W(c) image of dorso-lumbar spine demonstrates primary involvement of D12-L1 vertebral bodies and the intervening discs (thin white arrow) by the disease process with contiguous spread if the infection cranially (to involve all dorsal vertebrae) and caudally(to involve L2 vertebra) along the subligamentous route(thin black arrow) with sparing of intervening discs(except at D12-L1 level). Note, marrow signal intensity changes in all the involved vertebral bodies, and an epidural phlegmon at D12-L1 level causing localized cord compression (thick white arrow).

Conventional radiograph of spine demonstrates end plate irregularity, destruction of vertebral body and involvement of intervertebral disc. Reactive sclerosis is not a feature on initial presentation. CT scans [Figure19] characteristically demonstrate extensive bone destruction and large paraspinal abscesses. Large paravertebral abscesses and subligamentous spread of infection may produce anterior scalloping of the vertebral bodies. Calcification within the abscess is virtually diagnostic for tuberculosis. If left untreated, the infection eventually results in vertebral collapse and anterior wedging, leading to kyphosis and gibbus formation with healing, fusion of vertebral bodies (bony ankylosis) occurs in most cases. In patients with neurological deficit CT can define the extent of epidural compression, detection of bony fragment within the spinal canal and atlanto-axial instability. ${ }^{61,62} \mathrm{MRI}$ is the modality of choice for evaluating intraspinal and subligamentous spread of the infection, cervico medullary junction and nerve root compression, intervertebral disc changes and vertebral skip lesions. Contrast enhanced MRI is particularly useful for demonstrating intraosseous, epidural and paraspinal soft tissue involvement. $56,57,58$ 

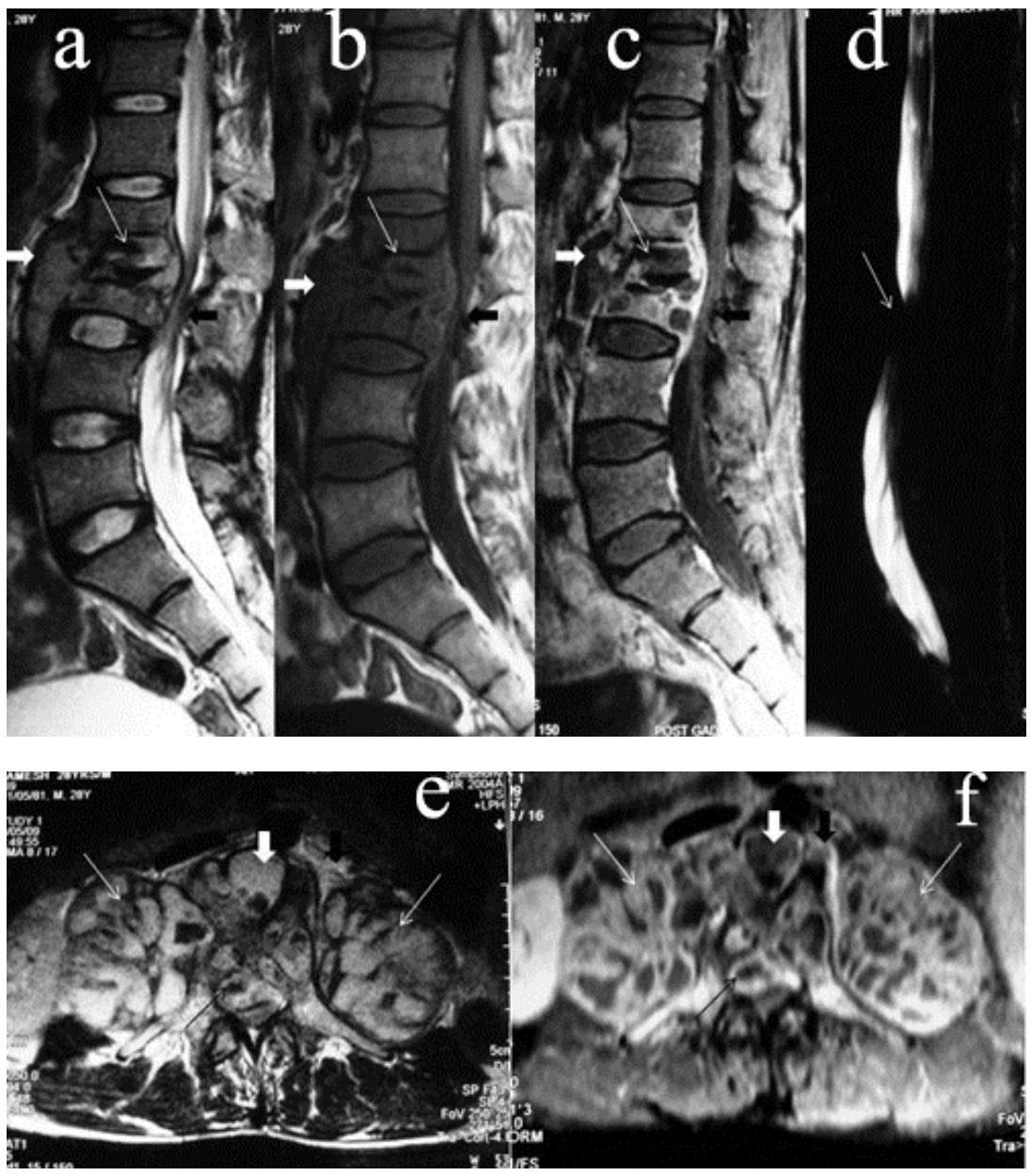

Fig. 17. Tuberculous spondylitis with involvement of intervening disc. Sagittal T2W(a), $\mathrm{T} 1 \mathrm{~W}(\mathrm{~b})$ and post contrast(c) MR of lumbar spine demonstrates involvement of L2-L3 vertebral bodies and the intervening discs by the disease process, showing osseous destruction and heterogeneous contrast enhancement(thin white arrow). Associated heterogeneous enhancing prevertebral soft tissue(thick white arrow) and an epidural phlegmon/abscess(thick black arrow) is also present at the same level. The epidural phlegmon/abscess causes severe lumbar canal stenosis and compression of conus and cauda equina nerve roots, producing a CSF cut off sign on MR myelogram (d) (thin white arrow). Multiple intraosseous lesions (thick white arrow), subligamentous inflammatory mass(thick black arrow), bilateral psoas abscesses (thin white arrow) and epidural phlegmon causing cord compression (thin black arrow) are well appreciated on axial T2W(e) and T1W post contrast image(f). 


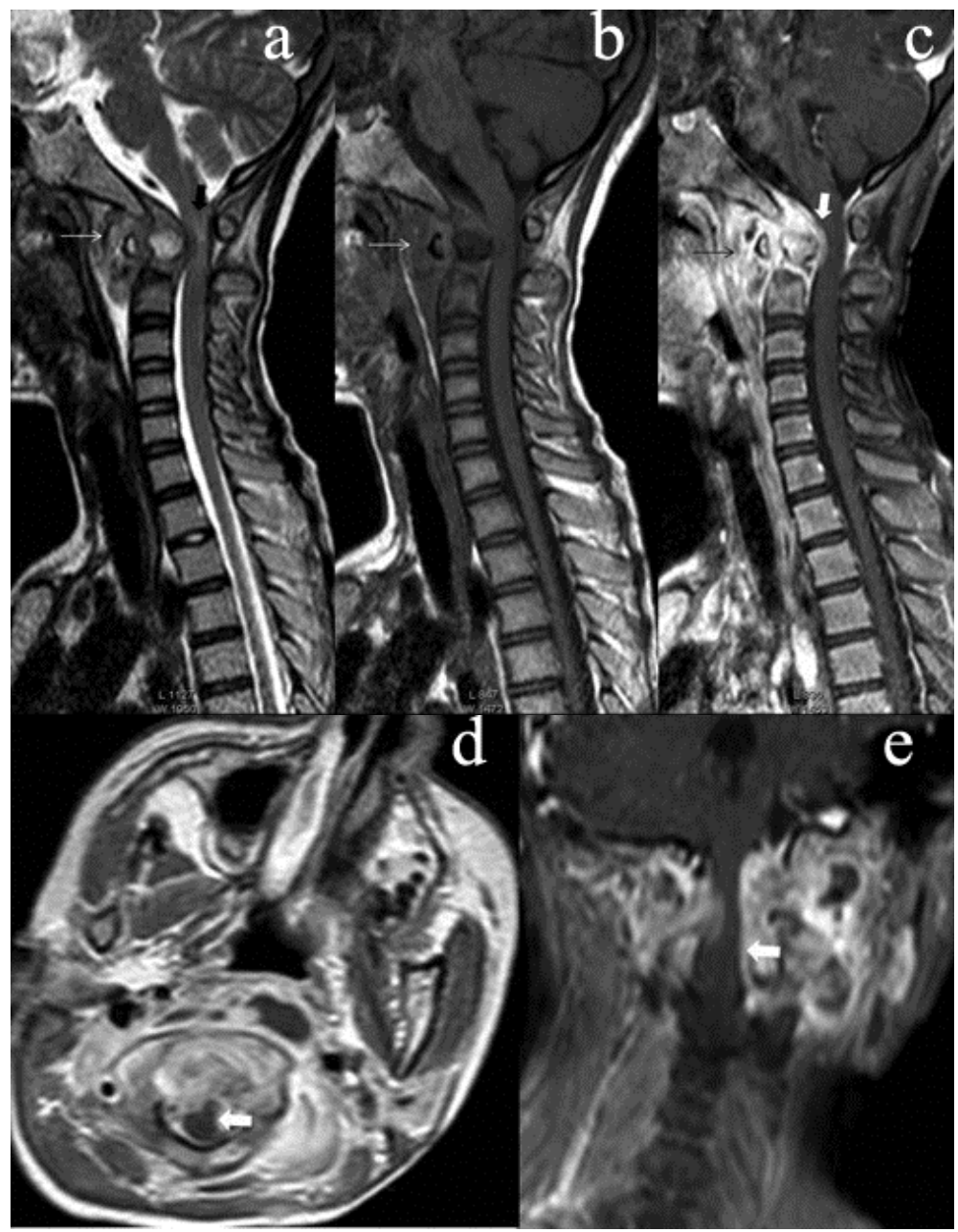

Fig. 18. Tuberculous spondylitis (C1-C2 vertebrae). Sagittal T2W(a), T1W(b) and post contrast(c) image of cervical spine shows enhancing inflammatory soft tissue mass (thin arrows) surrounding the anterior arch of $\mathrm{C} 1$ vertebra, tip of odontoid process (os odontoideum) and posterior part of the clivus, appearing isointense to the cord both on T1W and $\mathrm{T} 2 \mathrm{~W}$ image. Note subligamentous spread of infection along anterior aspect of $\mathrm{C} 2-\mathrm{C} 3$ vertebral body. There is associated atlanto-axial dislocation and cervicomedullary junction compression both by the posteriorly displaced odontoid tip and epidural-intraspinal phlegmonatous inflammatory tissue (thick white arrow), best appreciated on post contrast sagittal(c), axial(d) and coronal(e) images. The compressed cervical cord shows focal T2 hyperintense(thick black) signal suggestive of compressive myelopathy. Intraosseous marrow signal changes involving anterior arch of atlas, odontoid tip and posterior part of clivus is also present. There is severe narrowing of nasopharynx and the oropharynx by the prevertebral inflammatory mass. 


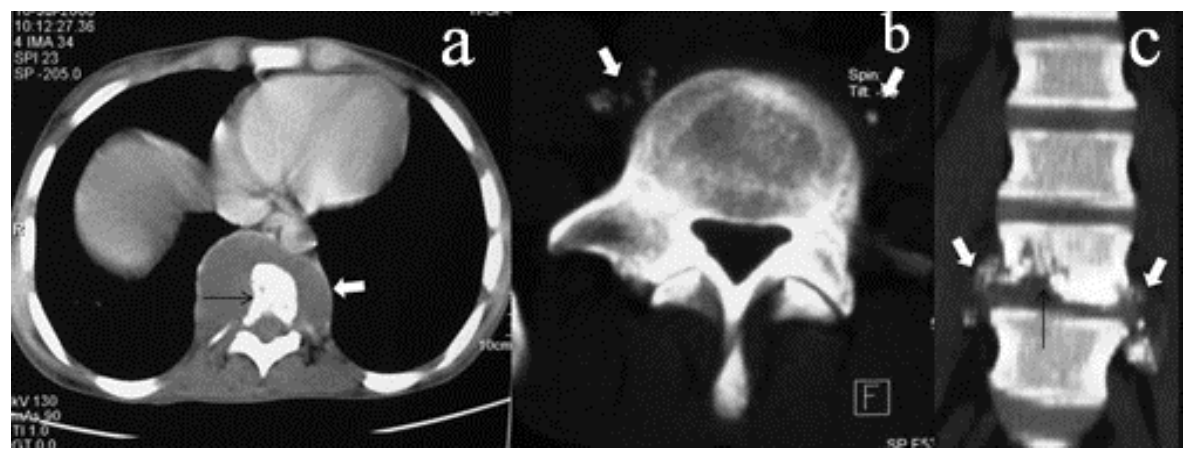

Fig. 19. Tuberculous spondylitis (Pott's spine). Axial (a,b) and coronal (c) noncontrast CT spine demonstrates marked osseous and intervertebral disc destruction(thin black arrow), and calcified/ non-calcified paravertebral abscesses(thick white arrows).

The major differential diagnosis is low grade pyogenic osteomyelitis (e.g. brucellosis), metastatic disease and fungal infections. Tuberculosis is characteristically associated with little or no reactive sclerosis, a feature that helps to distinguish it from pyogenic infections of spine. ${ }^{58}$ Tuberculosis rarely affects the posterior vertebral elements, including pedicles, in contrast to metastatic disease. ${ }^{62}$ Anterior scalloping of vertebral bodies can also be seen with paravertebral lymphadenopthy, metastatic or lymphomatous deposits. ${ }^{58,62} \mathrm{CT}$ guided needle biopsy is very useful in establishing the diagnosis in case of uncertainty.

\subsubsection{Non-osseous spinal tuberculomas}

Spinal tuberculoma are very rare presentation of non-osseous spinal tuberculosis, characterized as extradural $(64 \%)$, intramedullary $(8 \%)$, or intradural extramedullary (IDEM) (1\%) according to their location.63 Most of the subdural (IDEM) tuberculomas are detected as a result of paradoxical response to antitubercular therapy for meningitis, ranging from three months to one year. ${ }^{64}$ Intramedullary tuberculomas are mostly induced by hematogenous dissemination from primary focus in the lung, or via CSF seeding, and rarely local spread of spinal tuberculosis. ${ }^{65}$ MRI is imaging modality of choice for these lesions. IDEM tuberculoma may present as a single, dural based ring enhancing lesion or as a long segment enhancing soft tissue mass. En plaque IDEM tuberculoma may mimic meningioma. IDEM tuberculomas commonly cause spinal cord and nerve root compression; however, may or may not be associated with arachnoiditis [figure20].64,66 Concurrent IDEM tuberculoma and syringomyelia has also been reported. 67 Intramedullary tuberculomas have specific findings on MRI, and hence can be diagnosed accurately on imaging. T2WI shows a typical "target sign" demonstrating low signal center (caseous material) surrounded by high signal rim (peripheral infective granulation tissue). This "target sign" is a valuable indicator and differentiates tuberculoma from other intramedullary lesions. Intravenous contrast administration shows sharp margin with peripheral rim enhancement. Associated syrinx and/or arachnoiditis may or may not be present with these lesions [Figure21].65 


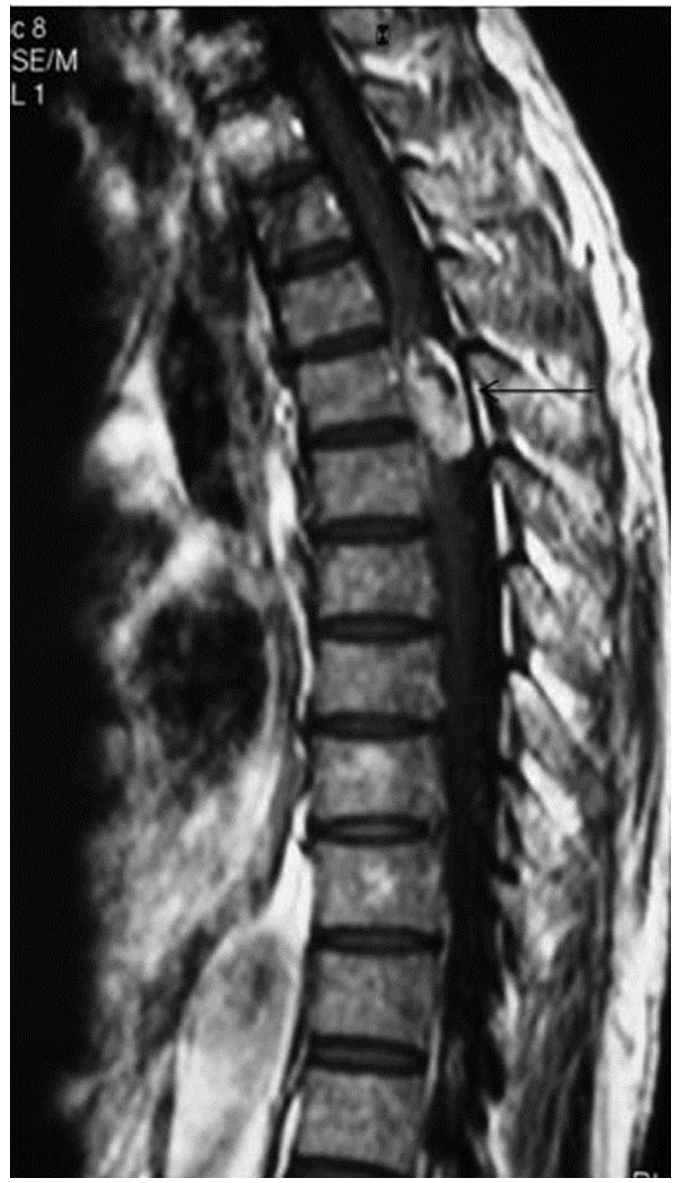

Fig. 20. Intradural extramedullary spinal tuberculoma. Contrast enhanced T1-weighted Sagittal image demonstrates an intradural extramedullary (arrow) lesion in posterior subarachnoid space, at mid-dorsal spine level, showing dense nodular enhancement with a linear non-enhancing area. There is localized spinal cord compression and widening of dorsal subarachnoid space. No associated syrinx or arachnoiditis is present. 

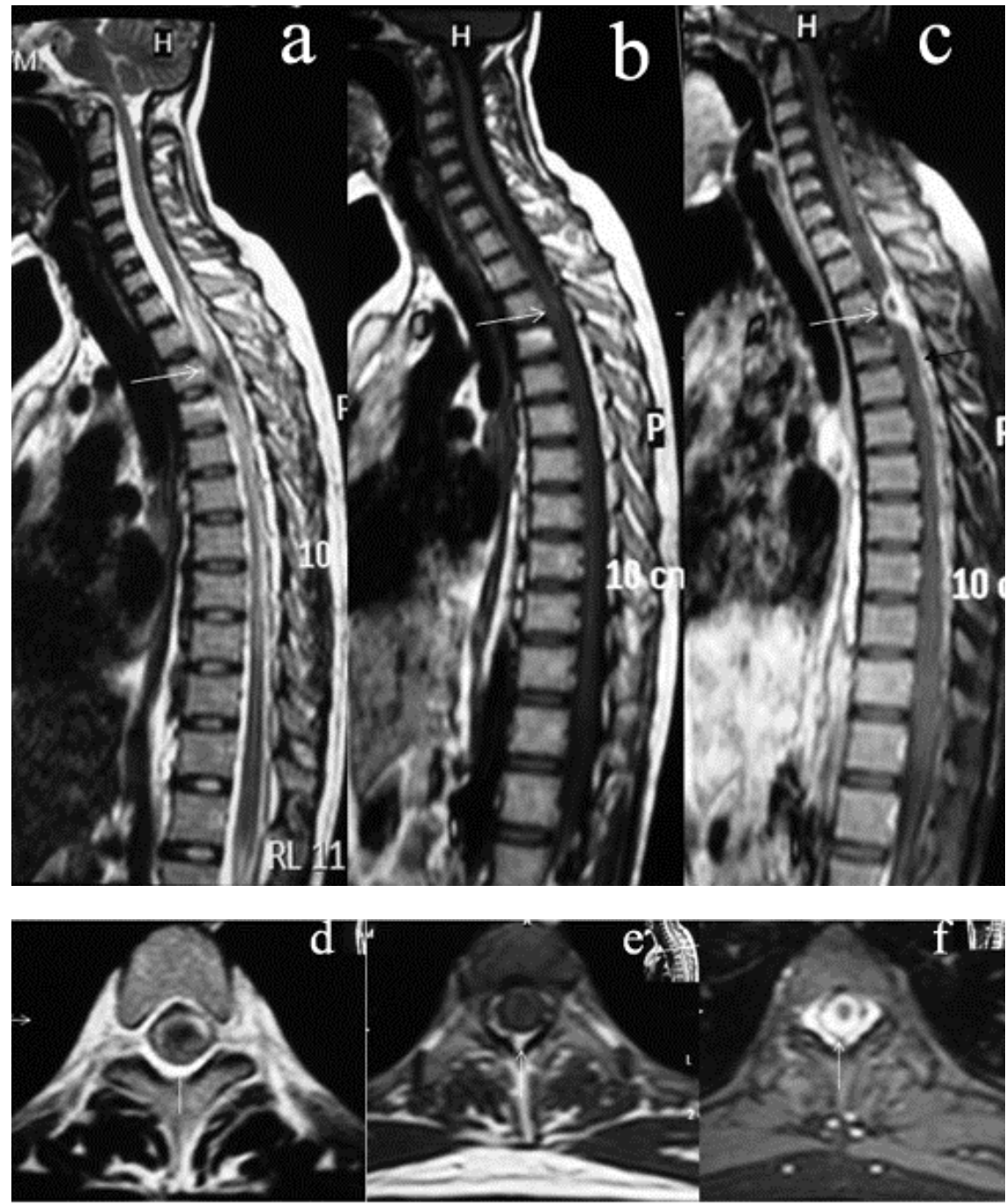

Fig. 21. Intramedullary tuberculoma with arachnoiditis. Sagittal T2W(a), T1W(b) and post contrast(c) image of thoracic spine shows an intramedullary space occupying lesion (thin white arrow) at D3 level with loss of normal cord-CSF interface posteriorly from D1-D11 level and few thin septations within posterior subarachnoid space, best appreciated on $\mathrm{T} 2 \mathrm{~W}$ image. The intramedullary SOL appears iso-hypointense on T1W image, shows typical "target sign" on T2W image and peripheral rim enhancement on contrast administration. There is obliteration and intense enhancement of posterior subarachnoid space from D1-D11 level with scattered thin CSF loculations consistent with arachnoiditis (thin black arrow). Note, a short segment syrinx situated cranial to the intramedullary lesion and T2 hyperintense signal within the cord caudal to this lesion. Intramedullary tuberculoma is well appreciated on axial T2W(d), T1W(e) and post contrast(f) images. 


\subsubsection{Spinal tubercular arachnoiditis (myeloradiculopathy)}

Spinal tuberculous arachnoiditis is an inflammatory condition that involves the leptomeninges along the spinal tract and often manifests as myeloradiculopathy. Previously, it was known as adhesive spinal arachnoiditis or chronic adhesive arachnoiditis. Clinically patient presents with progressive spinal cord and/or nerve root dysfunction, usually accompanied by constitutional symptoms. This condition may result from downward extension of intracranial tuberculous meningitis (most common) or a tuberculous lesion primarily arising in the spinal meninges, or extension from tuberculous spondylitis. The thoracic region is most commonly affected followed by the lumber and cervical region. The involvement may be focal, multifocal or diffuse. The inflammatory exudate surrounds the spinal cord and the nerve roots, causing obliteration of spinal subarachnoid space (SAS). On imaging [figure22], there is increased CSF signal intensity within the SAS on T1WI due to elevated protein content of CSF with resultant loss of spinal cord outline in cervico-thoracic region. Thickening and clumping of nerve roots in the lumber region is frequently seen with arachnoiditis. Meningeal involvement has been described in patients with arachnoiditis; it represents ongoing meningeal inflammation and may constitute an early sign of arachnoiditis. Contrast enhanced MR images show linear enhancement of surface of spinal cord and nerve roots, or plaque like enhancement of dura-arachnoid mater complex which can obliterate the subarachnoid space. ${ }^{68}$ Enhancing subarachnoid space nodular lesions may represents IDEM tuberculoma or vascularized fibrous tissue. In chronic adhesive

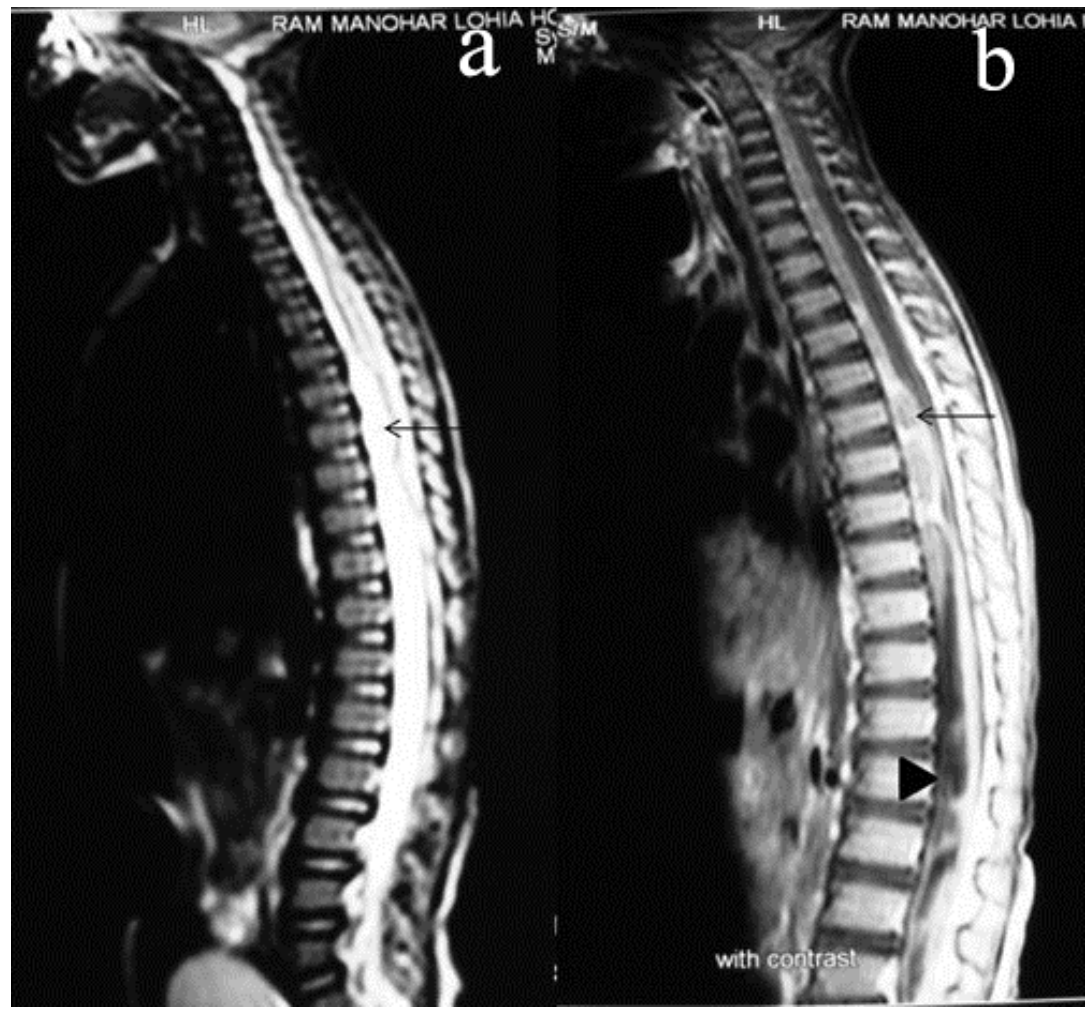




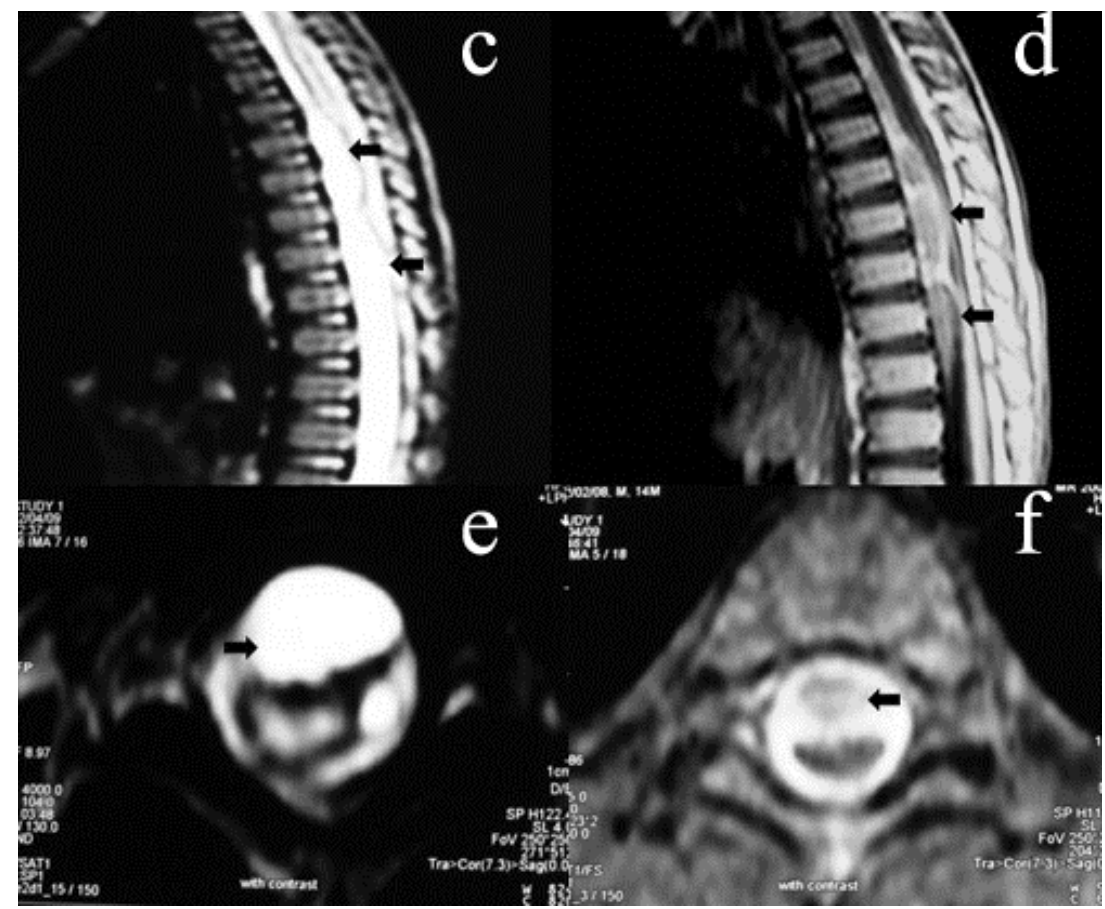

Fig. 22. Tuberculous spinal arachnoiditis with ascending infection to the brain. Sagittal T2W (a) and T1W post contrast(b) MR image of whole spine demonstrates loss of normal cord-CSF interface with obliteration of anterior subarachnoid space(SAS) by thick inflammatory exudates appearing hyperintense on T2W image, and showing intense enhancement on post contrast image (thin black arrow). Few scattered intervening septae are also present within the anterior SAS (at upper cervical and mid thoracic level) forming loculations. These loculations containing the inflammatory tissue have pseudomass appearance (on sagittal and axial T2W/post contrast image-c,d,e,f), which causes severe cord compression (thick black (arrow). Thickening and clumping of cauda equine nerve roots is evident in lumbar region (arrow head). The obliteration and intense enhancement of anterior subarachnoid space along the entire spinal canal, scattered pseudomasses in upper cervical (please see figure5b-sagittal post gad. image) and mid thoracic level, and clumping of cauda equine nerve roots in lumbar region is consistent with spinal arachnoiditis.

arachnoiditis, the spinal SAS is irregularly obstructed with formation of CSF loculations. ${ }^{32}$ The CSF loculations usually show normal CSF signal intensity on T1-and T2WI and do not enhance on post contrast images. The recognition of CSF loculation is important as they may cause cord compression, necessitating surgical intervention. The spinal cord changes are frequently seen in these patients and may include myelitis, cord edema, syringomyelia, infarction, myelomalacia, cavitation or atrophy. ${ }^{32}$

Spinal tuberculous arachnoiditis must be distinguished from other possible causes of arachnoiditis, for example subarachnoid hemorrhage or iatrogenic cause. 


\subsubsection{Tubercular myelitis}

Tuberculous myelitis, an important cause of paraparesis, and usually occurs as a secondary event in the course of common forms of tuberculous meningitis. The cervico-thoracic segment of the spinal cord is most commonly involved. MR Imaging features of TB myelitis are similar to those of cerebritis. There is diffuse cord swelling with signal abnormality. Most spinal cord lesions appear as hyperintense on T2, isointense to hypointense on T1weighted images, and show segmental enhancement on post contrast images [Figure23].

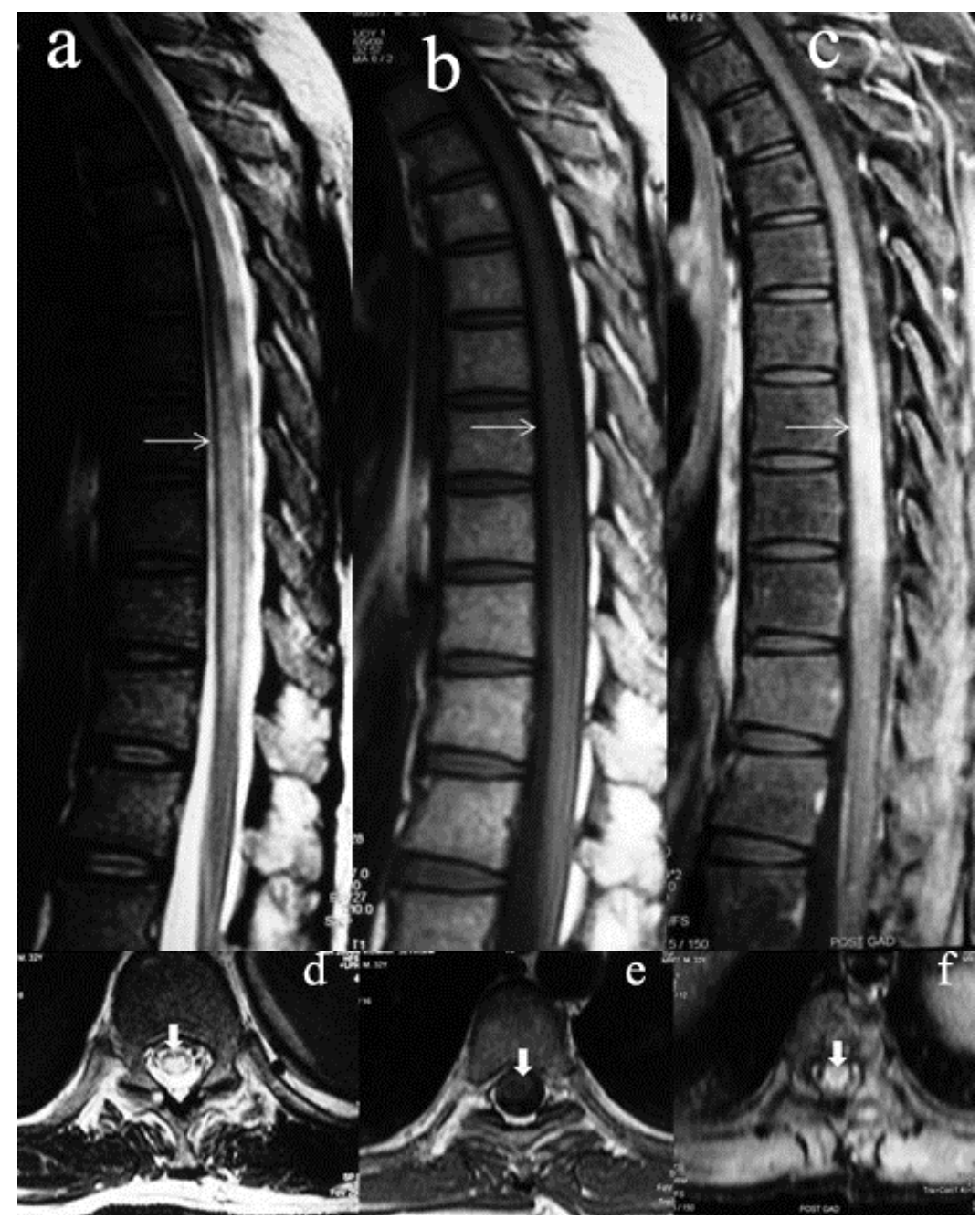

Fig. 23. Tuberculous Myelitis. Sagittal T2W(a), T1W(b) and post contrast(c) MRI of dorsolumbar spine shows diffuse cord swelling and edema appearing hypointense on T1W and hyperintense on $\mathrm{T} 2 \mathrm{~W}$ image and showing intense central contrast enhancement on post gadolinium images(thin white arrow). These changes are better appreciated (thick white arrow) on axial T2W(d), T1W(e) and post contrast image(f). 
Intramedullary abscess demonstrates central necrotic area with clearly defined marginal enhancement. Cord atrophy, cavitation and syringomyelia may be associated with poor outcome. Differential diagnosis includes cord contusion, cord infarction due to vasculitis, acute transverse myelitis, and demyelinating diseases. 69,70

\section{Differential diagnosis}

The important differential diagnosis of CNS tuberculosis has been already discussed with individual manifestations. Broadly, the conditions which may mimic cranial and spinal tuberculosis on imaging include other infectious and non-infectious inflammatory disease such as fungal infection, sarcoidosis, primary and metastatic neoplastic disease.

\section{Role of imaging in the evaluation of cns tuberculosis}

Role of imaging has been dealt in detail with individual manifestation of CNS tuberculosis (vide supra). In nut shell contrast-enhanced MRI is superior to CT scanning or unenhanced MRI for the demonstration of CNS TB, and is currently the best modality for demonstration of meningeal disease, parenchymal abnormalities, for assessment of complication and monitoring the disease. The multiplanar capability of MRI offers additional advantage for localization of lesions. CT is particularly used for evaluation of osseous changes, calcification, and intracranial shunt in cases of hydrocephalus.

\section{Management of cns tuberculosis}

Inspite of rapid advances in the management of pulmonary tuberculosis, currently no general agreement about the form of chemotherapy or optimal duration of treatment has been reached. The World Health Organization ${ }^{71}$ has put CNS tuberculosis under TB treatment category1, and recommended an initial phase therapy with streptomycin, isoniazid, rifampicin and pyrazinamide for 2-months, followed by a seven month continuation phase with isoniazid and rifampicin. The duration of therapy should be at least 6-months, and in some cases up to 12-months treatment is required. A similar drug regimen has been recommended for all forms of CNS tuberculosis. A four-drug regimen is needed to treat atypical mycobacteria ( $\mathrm{M}$ avium intracellulare) in persons with HIV infection. Current recommended therapy for HIV patients include azithromycin and clarithromycin in combination with ethambutol or clofazimine. ${ }^{72,73,74}$

Role of corticosteroids in the treatment of CNS tuberculosis is controversial. It is believed that corticosteroids improve both the survival rate and neurological outcome in patients with TB meningitis. However they should be used with caution in pediatric population. The response to corticosteroids may be dramatic with rapid resolution of basal exudates and tuberculomas on serial imaging. The main argument against using corticosteroids is that they decrease meningeal inflammation, and in turn can affect CSF penetration of antituberculous drugs. ${ }^{74,75}$

Surgical procedures in patients with tuberculous meningitis are primarily directed to the treatment of hydrocephalus. Serial lumbar puncture along with diuretics is used as temporary measures to relieve raised intracranial pressure. Ventriculo-peritoneal or ventriculo-atrial shunts are permanent measures, which relieve the signs and symptoms of hydrocephalus and significantly improve the sensorium and neurological deficit. However, 
these shunts may require replacement due to blockage by high protein content of CSF. Early shunting in combination with drug therapy offers best therapeutic outcome.

Intracranial tuberculoma, which may behave as single space occupying lesion causing midline shift and increased intracranial pressure, and that fail to respond to chemotherapy should be removed surgically. ${ }^{72,76}$ In chronic adhesive spinal arachnoiditis, the CSF loculations in SAS may cause cord compression, necessitating surgical intervention. ${ }^{32}$

\section{Paradoxical response of tuberculomas to the treatment}

Paradoxical enlargement of pre-existing tuberculoma or appearance of new intracranial and spinal tuberculoma in patients receiving effective antituberculous therapy has been noted in the past. This paradoxical phenomenon is thought to be due to result of an immunological reaction. These lesions are usually discovered accidently when follow-up scan is performed routinely or when new neurological signs develop during the course of antitubercular therapy. Concomitant steroid therapy probably has a preventive role against these focal lesions. However, with continuation of antituberculous therapy, eventual resolution of these tuberculoma usually occurs. In case of unresponsiveness to medical therapy, surgery is recommended. 77,78

\section{Prognosis}

The single most important factor determining the prognosis in cases of CNS tuberculosis is the stage of tuberculous meningitis at which the treatment has been started. If treatment is started at stage I (prodromal phase with no definite neurological symptoms) the mortality and morbidity is very low, whereas in stage III (loss of sensorium, convulsions, focal neurological deficit, and involuntary movements) almost $50 \%$ patient die, and those who recover may have some form of neurological deficit. Stage II patients (signs of meningeal irritation, slight or no clouding of sensorium, minor cranial nerve palsies, and no neurological deficit) have intermediate prognosis. ${ }^{74,79}$

\section{Summary}

CNS tuberculosis is rare but serious complication and its early recognition and treatment is imperative. Early diagnosis can prevent further deterioration and result in better prognosis. Imaging plays a very important role in establishing the diagnosis of CNS tuberculosis. A radiologist should maintain a high degree of suspicion when patients with tuberculosis risk factors present with neurologic complains. Various imaging modalities, CSF studies, and brain biopsy if necessary, can aid in establishing the diagnosis of CNS tuberculosis. Pharmacological regimen is the mainstay of treatment, although various other options such as addition of corticosteroid and surgical intervention are also recommended as per requirement.

\section{References}

[1] Rich AR, Mccordock HA. Pathogenesis of tubercular meningitis. Bull John Hopkins Hosp 1933; 52:5-13.

[2] Leonard MK. Tuberculosis: forms of tuberculosis. 2002 Oct 1. Available at: www.medscape.com/viewarticle/534783?rssm Accessed May 25, 2006. 
[3] Dastur DK. Neurotuberculosis. In: Minckler J, ed. Pathology of the nervous system, Vol. 3. New York: McGraw-Hill 1972;2412-2422.

[4] Nicolls DJ, King M, Holland D, Bala J, del Rio C. Intracranial tuberculomas developing while on therapy for pulmonary tuberculosis. Lancet Infect Dis. 2005; 5(12):795-801.

[5] Bayindir C, Mete O, Bilgic B. Retrospective study of 23 pathologically proven cases of central nervous system tuberculomas. Clin Neurol Neurosurg. 2006;108 (4):353-357.

[6] Wallace RC, Brutons EM, Beret FF, et al. Intracranial tuberculosis in children. CT appearance and clinical outcome. Pediatric Radiol. 1991;21:241-246.

[7] Barkovich AJ. Infections of the nervous system. In: Barkovich AJ (Ed). Pediatric Neuroimaging (4th edn). Lippincott Williams and Wilkins 2005;801-805.

[8] Synder RD, Bacterial infections of the nervous system. In: Berg BO, ed. Neurologic aspects of pediatrics. Boston: Butterworth-Heinemann, 1992;195-226.

[9] Shah, GV. Central nervous system tuberculosis. Neuroimaging Clin North Am 2000;10(2),355-374.

[10] Klingensmith WC, Datu J, Tuberculous meningitis of the sylvian fissure. Clin Nucl Med. 1978; 3:315-317.

[11] Jamieson DH. Imaging intracranial tuberculosis in childhood. Paediatr Radiol.1995;25:165-170.

[12] Kioumehr F, Dadsetan MR, Rooholamini SA, et al. Central nervos system tuberculosis: MRI. Neuroradiology. 1994;36:93-96.

[13] Rovira M, Romero F, Torrent $\mathrm{O}$, et al. Intracranial tuberculoma. MR Imaging. Neuroradiology. 1989;31:299-302.

[14] Sheller JR, DesPrez RM. CNS tuberculosis. Neurol Clin 1986;4:143-158.

[15] Fischbein N, Dillon W, Barkovich A (Eds). Tuberculosis. In: Teaching atlas of brain imaging. Thieme 2000;165-168.

[16] Hsuh EY, Chi a LG, Shen We. Location of cerebral infarctions in tuberculous meningitis. Neuroradiol 1992;34:197.

[17] Reid H, Fallon RJ. Bacterial infections. In Adams JH Duchen L (Eds). Greenfields Neuropathology (5th edn). New York: Oxford University Press 1992;317-342.

[18] Gupta RK, Gupta S, Singh D, Sharma B, Kohli A, Gujral RB. MR imaging and angiography in tuberculous meningitis. Neuroradiol 1994;36:87-92.

[19] Garg RK, Tuberculosis of central nervous system. Postgrad Med J. 1999;75:133-140.

[20] Silverman IE, Liu GT, Bilaniuk LT, Volpe NJ, Galetta SL. Tuberculous meningitis with blindness and perichiasmal involvement on MRI. Pediatr Neurol. 1995;12(1):65-67.

[21] S. Prabhakar, R. Bhatia, V. Lal, Paramjeet Singh. Hypertrophic Pachymeningitis : Varied Manifestations of a Single Disease Entity. Neurol India. 2002;50: 45-52.

[22] Beşkonakli E, Çayli S, Turgut M, Yalçinlar Y. Primary giant granulomatous basal meningitis: An unusual presentation of tuberculosis. Child Nerv Syst 1998;14:79-81.

[23] Welchman JM. CT of intracranial tuberculomata. Clin Radiol 1979;30:567-579.

[24] Bhargava, Tandon PN. Intracranial tuberculomas: A CT study. Br J Radiol 1980;53:935945.

[25] Altenbesak S, Baytok V, Alhan E, et al. Suprasellar tuberculoma causing endocrinological disorders and initiating craniopharyngioma. Paeditr Neurosurg 1995;23:328-331.

[26] Esposito V, Fraioli B, Ferrante L, et al. Intrasellar tuberculoma: Case report. Neurosurgery 1987;21:721-723. 
[27] Sze G. Infections and inflammatory diseases. In:Stark DD, Bradley WG Jr. Magnetic resonance imaging. St. Louis, MO: CV Mosby, 1988:316-343.

[28] Desai K, Nadkarni T, Bhatjiwale M, Goel A. Intraventricular tuberculoma. Neurol Med Chir (Tokyo) 2002; 42:501-3.

[29] Van Dyk A. CT of intracranial tuberculosis with specific reference to the 'target sign.' Neuroradiology. 1988;30:329-336.

[30] Jinkins JR. Computed tomography of intracranial tuberculosis. Neuroradiology. 1991;33:126-135.

[31] Rajshekhar V, Haran RPO, Prakash GS, et al. Differentiating solitary small cysticercus granulomas and tuberculomas in patients with epilepsy. J Neurosurg 1993;78:402407.

[32] Jinkins JR. Gupta R, Chang KH, Rodriguez-Carbajal J. MR imaging of central nervous system tuberculosis. Radiol clin North Am 1995;33(4):771-789.

[33] Gupta RK, Jena A, Sharma DK, et al. MR imaging of intracranial tuberculomas. J Comput Assist Tomogr. 1988;12:280-285.

[34] Gupta RK, Prakash M, Mishra AM, et al. Role of diffusion weighted imaging in differentiation of intracranial tuberculoma and tuberculous abscess from cysticercus granulomas - a report of more than 100 lesions. Eur J Radiol. 2005;55(3):384-392.

[35] Gupta RK, Poptani M, Kohli A, et al. In vivo localized proton magnetic resonance spectroscopy of intracranial tubercolomas. Ind J Med Res. 1995;101:19-24.

[36] Gupta RK, Vatsal DK, Husain N, Chawla S, Prasad KN, Roy R, Kumar R, Jha D, Husain M. Differentiation of tuberculous from pyogenic brain abscesses with in vivo proton MR spectroscopy and magnetization transfer MR imaging. Am J Neuroradiol 2001;22:1503-1509.

[37] Gupta RK, Kathuria MK, Pradhan S. Magnetization transfer MR imaging in central nervous system tuberculosis. Am J Neuroradiol 1990;20:867-875.

[38] Whitener DR. Tuberculous brain abscess. Report of a case and review of the literature. Arch Neurol 1978;35:148-155.

[39] Bulakbasi N, Kocaoglu M, Ors F, Ucoz T. Combination of single-voxel proton MR spectroscopy and apparent diffusion coefficient calculation in the evaluation of common brain tumors. AJNR Am J Neuroradiol 2003;24:225-233.

[40] Kaminogo M, Ishimaru H, Morikawa M, Suzuki Y, Shibata S. Proton MR spectroscopy and diffusion weighted MR imagingfor the diagnosis of intracranial tuberculomas. Report of two cases. Neurol Res 2002;24:537-543.

[41] Withman RR, Johnson RH, Roberts DL. Diagnosis of military tuberculosis by cerebral computed tomography. Arch Intern Med 1979;139:479-480.

[42] Gee GT, Bazan C III, Jinkins JR. Miliary tuberculosis involving the brain: MR findings. AJR 1992;159:1075-1076.

[43] Dastur DK, Manghani DK, Udani PM. Pathology and pathogenetic mechanisms in neurotuberculosis. Radiol Clin North Am 1995;33:733-52.

[44] Udani PM, Dastur DK. Tuberculous encephalopathy with and without meningitis: clinical features and pathological correlations. J Neurol Sci 1970;10:541-61.

[45] Ng SH, Tang LM, Lui TN, et al. Tuberculoma en plaque: CT. Neuroradiology 1996; 38:453-5. 
[46] Dubey S, Devi BI, Jawalkar VK, Bhat DI. Tuberculoma en plaque: a case report. Neurol India 2002; 50:497-9.

[47] Villoria MF, Fortea F, Moreno S, Munoz L, Manero M, Benito C. MR imaging and CT of central nervous system tuberculosis in the patient with AIDS. Radiol Clin North Am. 1995 Jul:33(4):805-820.

[48] Bhaya A. Granulomatous hypophysitis - A rare entity mimicking pituitary adenoma. Indian J Radiol Imaging 1999;9:203-4.

[49] Diyora B, Kumar R, Modgi R, Sharma A. Calvarial tuberculosis: A report of eleven patients. Neurology India. 2009;57(5):607-612.

[50] Abhijit AR, Arpit MN, Datta M, Ashish JC, Ranjeet SN, Sudhir F and Veena LB. Imaging Features of Calvarial Tuberculosis: A Study of 42 Cases. American Journal of Neuroradiology 25:409-414, March 2004.

[51] Shenoy SN, Raja A. Tuberculous granuloma of the spheno-clival region. Neurology India. 2004; 52(1):129-130.

[52] Indira DB, Tyagi AK, Bhat DI, Santosh V. Tuberculous osteitis of clivus. Neurol India 2003;51:69-70.

[53] Narula MK, Chaudhary V, Baruah D, Kathuria M, Anand R. Pictorial essay: Orbital tuberculosis. Indian J Radiol Imaging. 2010;20:6-10.

[54] M.H. Rho, D.W. Kim, S.S. Kim, Y.S. Sung, J.S. Kwon, S.W. Lee. Tuberculous Otomastoiditis on High-Resolution Temporal Bone CT: Comparison with Nontuberculous Otomastoiditis with and without Cholesteatoma. AJNR Am J Neuroradiol.2007;28:493-496.

[55] McGuinness F. Tuberculous spondylitis. In McGuinness F (ed). Clinical imaging of nonpulmonary tuberculosis. Springer, Berline Heidelberg New York, pp 43-80.

[56] Sharif HS. Role of MR imaging in the management of spinal infections. AJR Am J Roentgenol.1992;158:1333-1345.

[57] Sharif HS, Morgan JL, Al-Shahed MS, et al: Role of CT and MR imaging in the management of tuberculous spondylitis. Radiol Clin North Am. 1995;33:787-804.

[58] Smith AS, Weinstein MA, Mizushima A et al: MR imaging of characteristics of tuberculous spondylitis vs vertebral osteomyelitis. AJNR. 1989;10:619-625.

[59] Akhaddar A, Gourinda H, Gazzaz M, Elmadhi T, Elalami Z, Miri A. Craniocervical junction tuberculosis in children. Rev Rhum Engl Ed. 1999;66(12):739-742.

[60] Allali F, Benomar A, EL Yahyaoui M, Chkili T, Hajjaj-Hassouni N . Atlantoaxial tuberculosis: three cases. Joint bone spine.2000;67(5):481-484.

[61] Zamiati W, Jiddane M, El Hassani MR, Chakir N, Boukkrissi N. Contribution of spiral CT scan and MRI in spinal tuberculosis (Spanish). J of Neuroradiology. J de Neuroradiologe. 1999;26:27-34.

[62] Resnick D. Tuberculous infection. In: Resnick D, ed. Diagnosis of bone and joint disorders. 3rd ed. London, United Kingdom: Saunders, 2002; 2524-2545.

[63] Dastur HM. Diagnosis and neurosusgical treatment of tuberculosis disease of the CNS. Neurosurg Rev 1983; 6: 111-17.

[64] Roca B. Intradural extramedullary tuberculoma of the spinal cord: a review of reported cases. J Infect. 2005;50(5):425-31.

[65] Ming Lu. Imaging Diagnosis of Spinal Intramedullary Tuberculoma:Case Reports and Literature Review. J Spinal Cord Med. 2010;33(2):159-162. 
[66] Shim DM, Kyum S, Kim TK, Chae SU. Intradural Extramedullary Tuberculoma Mimicking En Plaque Meningioma Clinics in Orthopedic Surgery 2010; 2:260-263.

[67] Sanser G, Guven C, Murat K, Bektas A. Syringomyelia and Intradural Extramedullary Tuberculoma of the Spinal Cord as a Late Complication of Tuberculous Meningitis. Turkish Neurosurgery 2010; 20: 561-565.

[68] SharmaA, Goyal M, Mishra NK, Gupta V, Gaikwad SB. MR imaging of tubercular spinal arachnoiditis. AJR Am J Roentgenol1997;168(3):807-812.

[69] Mohammad W, Hiba A , Bhojo K, Humera A. Neuroimaging of Tuberculous Myelitis: Analysis of Ten Cases and Review of Literature. Journal of Neuroimaging. 2006;16(3):197-205.

[70] Trivedi R, Saksena S, Gupta RK. Magnetic resonance imaging in central nervous system tuberculosis. Indian J Radiol Imaging. 2009 November; 19(4): 256-265.

[71] Harries A, Maher D. TB: a clinical manual for South East Asia. Geneva:World Health Organisation, 1997.

[72] Berger JR. Tuberculous meningitis. Curr Opin Neurol 1994;7:191-200.

[73] Small PM. Schecter GF, Goodman PC, Sande MA, Chaisson RE, Hopewell PC. Treatment of tuberculosis in patients with advanced human immunodeficiency virus infection. N Engl J Med 1991;324:289-94.

[74] Holdiness MR. Management of tuberculous meninglitis. Drugs 1990;39:224-33.

[75] Schoeman JF, Vanzyl LF, Laubscher JA, Donald PR. Effect of cortico- steroids on intracranial pressure, computed tomographic findings, and clinical outcome in young children with tuberculous meningitis. Pediatrics 1997;99:226-31.

[76] Leonard JM, Des Prez RM. Tuberculous meningitis. Infect Dis Clin North Am 1990;4:769-87.

[77] Afghani B, Lieberman JM: Paradoxical enlargement or development of intracranial tuberculomas during therapy : case report and review. Clin Infect Dis 1994;19:1092-1099.

[78] Nomura S, Akimura T, Kitahara T, Nagomi K, Suzuki M: Surgery for expansion of spinal tuberculoma during antituberculous chemotherapy: a case report. Pediatr Neurosurg 2001;35:153-157.

[79] Medical Research Council. Streptomycin in tuberculosis trials committee. Streptomycin treatment of tuberculous meningitis. Lancet 1948;i:582-96. 


\title{
Imaging of Metabotropic Glutamate Receptors (mGluRs)
}

\author{
Zhaoda Zhang and Anna-Liisa Brownell \\ Athinoula A. Martinos Biomedical Imaging Center \\ Massachusetts General Hospital \\ Harvard Medical School, Charlestown, \\ Massachusetts \\ USA
}

\section{Introduction}

The ubiquitous amino acid L-glutamate is thought to act as a neurotransmitter at the majority of synapses in the brain. It mediates the major excitatory pathways in the brain, and is referred to as an excitatory amino acid (EAA). The EAA plays a role in a variety of physiological processes, such as long-term potentiation (learning and memory), the development of synaptic plasticity, motor control, respiration, cardiovascular regulation, emotional states and sensory perception (Bliss \& Collingridge, 1993).

The excessive or inappropriate stimulation of EAA receptors leads to neural cell damage or loss by a mechanism known as excitotoxicity (Lucas \& Newhouse, 1957; Oney, 1978). EAA receptors are classified in two general types (Kornhuber \& Weller, 1997). Receptors that are directly coupled to the opening of cation channels in the cell membranes of the neuron are termed 'ionotropic', which include NMDA, AMPA, and kainate receptors. The second type of receptors are the G-protein or second messenger-linked 'metabotropic' EAA receptors. This second type is coupled to multiple second messenger systems that lead to enhanced phosphoinositide hydrolysis, activation of phospholipase D, increase or decrease in cAMP formation, and changes in ion channel function (Kozikowski et al., 1998).

Metabotropic glutamate receptors belong to Class C of a superfamily of G-protein coupled receptors (GPCRs). Class C GPCRs possess a large extracellular domain that is responsible for endogenous ligand recognition (Pin et al., 2003), in addition to the seven strand transmembrane domain, which is characteristic of all GPCRs. The mGluRs possess a large bi-lobed extracellular N-terminus of $\sim 560$ amino acids which has been shown by mutagenesis studies to confer glutamate binding, agonist activation of the receptor, and subtype specificity for group selective agonists (Schoepp et al., 1999).

Since mGluRs have neuromodulatory role in the control of both glutamatergic and GABAergic neurotransmission, there has been much interest to develop novel mGluR ligands for therapeutic purposes of a variety of neurological and psychiatric conditions. The mGluRs have been proposed to be involved in physiological and pathophysiological processes of a number of CNS disorders, including anxiety, pain, depression, neurodegenerative disorders, schizophrenia, epilepsy, and drug abuse. In order to 
characterize the role of mGluRs in different physiological processes there is a need to identify novel compounds, which are highly potent and specific for an mGluR group or a subtype. Such compounds are needed to further investigate mGluR function, and as potential therapeutic agents for a variety of neurological diseases, which are associated with the abnormal activation of mGluRs. A large amount of pharmacological agents acting at metabotropic glutamate receptors have been described in the literature (Guitart \& Khurdayan, 2005; Kew, 2004; Layton, 2005; Marino et al., 2005; Rudd \& McCauley, 2005; Schoepp et al., 1999; Slassi et al., 2005; Williams \& Lindsley, 2005; Yang, 2005). According to the mode of binding, these mGluR pharmacological agents can be classified into competitive and non-competitive agents. Based on the mode of action, they can be classified into agonists, antagonists, and positive/negative/neutral modulators (Layton, 2005). Competitive agonists and antagonists bind to the same orthosteric binding site as endogenous glutamate (Niswender et al., 2005; Ritzen et al., 2005; Rudd \& McCauley, 2005), which is a cleft between the two lobes in the extracellular N-terminus. Their binding ability depends on how much they can stabilize the closed conformation (Kew, 2004). These ligands received earliest research interest and have been well developed (Schoepp et al., 1999). They are all glutamate analogs or substituted glycines, which imply that they have poor selectivity within their group. In addition, competitive agonists and antagonists have structural carboxyl and amino groups, which make them too polar to penetrate the blood brain barrier (BBB) (Kew, 2004).

Starting from 1996 (Annoura et al., 1996), a number of different types of non-competitive negative, positive and neutral allosteric modulators have been developed as mGluR ligands (Niswender et al., 2005; Ritzen et al., 2005). These ligands modulate mGlu receptor activity by binding to allosteric binding sites that are located in the seven strand transmembrane domain. The allosteric binding sites are structurally distinct from the classical agonist orthosteric binding site (Williams \& Lindsley, 2005). Positive and negative modulators thus offer a potential for improved selectivity for individual mGluR family members compared to competitive agonists and antagonists at the glutamate site (Kew, 2004). Positive allosteric modulators (PAM)s have little or no effect on the receptor but can significantly enhance the effect of endogenous ligand. Correspondingly negative allosteric modulators inhibit the activity of orthosteric agonists in a noncompetitive manner. These ligands are structurally diverse and not amino acid derivatives. They are lipophilic and have much better CNS penetrating ability. Thus, positive and negative modulators with high subtype selectivity, and appropriate lipophilicity are good candidates for mGluR radiotracer development. There will be no competitive binding of this kind of tracers with endogenous glutamate, which might otherwise decrease the availability in vivo, and thus decrease the sensitivity of potential ligands.

During the last fifteen years the subtype selective modulators have been identified for mGluR1, mGluR2, mGluR3, mGluR4, mGluR5, mGluR7 and mGluR8. Based on these modulators, several positron emission tomography radiotracers have been developed for in vivo imaging of specific mGluRs. Presently, three mGluR ligands have been used for human studies. They have been developed as negative allosteric modulators for mGluR5. In this review we intend to summarize the radiotracers which have characteristics to be developed as tracers for in vivo PET imaging to investigate modulation of mGluRs in normal and pathological conditions. Emphasis will also be given to the highly potent and subtype selective allosteric modulators which are candidates for radiolabeling with ${ }^{18} \mathrm{~F}$ or ${ }^{11} \mathrm{C}$. 


\section{Metabotropic glutamate receptors and their physiological function}

Recent molecular cloning studies have revealed the existence of eight different subtypes of mGluRs. The mGluR subtypes can be divided into three different groups according to their sequence similarities, signal transduction mechanism, and pharmacological profiles to agonists (Pin \& Duvoisin, 1995). The first group comprising mGluR1 and mGluR5 is coupled to stimulating of phosphoinositide hydrolysis/Ca2+ signal transduction (Schoepp et al., 1994). The second group, consisting of mGluR2 and mGluR3, is negatively coupled through adenylate cyclase to cAMP formation (Tanabe et al., 1997). The third group, containing mGluR4, mGluR6, mGluR7 and mGluR8, is also negatively linked to adenylate cyclase activity but shows a different agonist preference (Conn \& Pin, 1997; Tanabe et al., 1997).

The neuroanatomical localization of Group I and Group II mGluRs in the rodent brain, as assessed by immunohistochemical or in situ hybridization techniques, has revealed overlapping, yet distinct patterns of expression of these receptors. In order to better characterize the roles of mGluRs in physiological processes, there is a need to identify novel compounds that are highly potent and specific for an mGluR group or a subtype. Such compounds are needed as pharmacological tools for further investigation of mGluR function, and as potential therapeutic agents for the treatment of diseases or conditions including epilepsy, cerebral ischemia, pain, spinal cord injury, 'neurotoxicity' and chronic neurodegenerative diseases (e.g. Parkinson's and Huntington's disease), which are associated with abnormal activation of mGluRs (Aguirre et al., 2001; Blakely, 2001; Calabresi et al., 1999; Keyvani et al., 2001; Marino et al., 2001; O'Neill, 2001; Popoli et al., 2001; Rao et al., 2000; Rouse et al., 2000).

It is known that glutamate can act as a neurotoxin when energy supplies are compromised. This has stimulated a hypothesis that injury to neurons in some neurological conditions may be caused, partly, by over stimulation of glutamate receptors and/or glutamate transporters. These neurological conditions may be acute insult like stroke or chronic neurodegenerative states like Parkinson's or Huntington's disease or dementia. To better explore the roles of mGluRs in physiological and pathological processes, there is a need to learn more about functional behavior of these receptors in vivo.

\section{PET radiotracer development}

Positron emission tomography (PET) has become an important clinical diagnostic and research modality, and also a valuable technology in drug discovery and development (Cai et al., 2008). PET tracers have been used for the imaging and quantification of biochemical processes. PET tracers play a critical role for assessing in vivo distribution of specific receptors in normal and disease conditions to understand underlying mechanisms of physiology and pathology. Moreover, PET tracers serve as invaluable biomarkers during the clinical development of potential therapeutic mGluR modulators, in which the receptor occupancy of potential drug candidates in the brain is measured (Passchier et al., 2002; Sharma \& Lindsley, 2007). In vivo receptor occupancy can help to answer many vital questions in the drug discovery and development process such as whether potential drugs reach their molecular targets, the relationship between therapeutic dose and receptor occupancy, the correlation between receptor occupancy and plasma drug levels, and the duration of time a drug remains at its target (Passchier et al., 2002). In PET imaging a small amount of tracer is injected into a living object. The tracer is labeled with a short-lived 
radioisotope, which emits positrons as it decays. The positrons collide with electrons resulting in high-energy photons that escape from the object and are detected by the PET scanner. Carbon-11 $\left(\mathrm{t}_{1 / 2}=20.4 \mathrm{~min}\right)$ and fluorine-18 $\left(\mathrm{t}_{1 / 2}=109.7 \mathrm{~min}\right)$ are the most commonly used radionuclides in PET imaging (Miller et al., 2008). The characteristics of successful PET tracers include high affinity, high selectivity over other mGluR subtypes as well as other receptors, suitable pharmacological properties including lipophilicity, metabolic stability, no radiolabeled metabolites that can penetrate into the brain, and the chemical structure of the precursor to allow fast labeling.

\subsection{Allosteric modulators and radiotracers for Group I mGluRs}

The group I receptors mGluR1 and mGluR5 exhibit different patterns of expression in the CNS. The distribution of mGluR1 is found throughout the human brain with high levels in the olfactory bulb, thalamus, hippocampus, lateral septum, superior colliculus and cerebellum (Olive, 2009). Inhibition of mGluR1 has been suggested as potential treatment for various psychiatric disorders including schizophrenia, anxiety, and neuropathic pain.

The mGluR5 is usually found in postsynaptic neurons with moderate to high density in the frontal cortex, caudate, putamen, nucleus accumbens, olfactory tubercle, and hippocampus, whereas in contrast to expression patterns of mGluR1, the density in the cerebellum is low (Olive, 2009). Dysfunction of mGluR5 is implicated in a variety of diseases in the CNS, including anxiety, depression, schizophrenia, Parkinson's disease, and drug addiction or withdrawal.

\subsubsection{Allosteric modulators and radiotracers for mGluR1}

A variety of mGluR1 modulators have been reported in the literature. Competitive mGluR1 agonists and antagonists historically have been amino acid derivatives, which display poor potency, lack of selectivity and unsatisfactory BBB penetration (Layton, 2005). Although a number of selective competitive mGluR1 ligands appear in literature, they are not good candidates for potential PET tracers. None of the existing orthosteric ligands has a binding affinity (or potency) of $\mathrm{IC}_{50} / \mathrm{K}_{\mathrm{i}} / \mathrm{K}_{\mathrm{d}}$ less than $20 \mathrm{nM}$ with an acceptable selectivity over other members in the same group. There is a consensus that identification of highly potent and subtype selective competitive mGluR ligands has been difficult due to a high degree of sequence similarity at the orthosteric binding site to which the endogenous agonist binds (Layton, 2005; Williams \& Lindsley, 2005). Alternatively, several structural types of mGluR1 allosteric modulators have been reported in literature, including negative and positive allosteric modulators which show high binding affinity, high selectivity and good lipophilicity (Layton, 2005).

CPCCOEt (1) was the first reported mGluR1 negative allosteric modulator (Fig.1). Before 2008, only compound 4 (3,5-dimethyl PPP) (Micheli et al., 2003b) and a quinoline derivative 5 (JNJ16259685) (Lavreysen et al., 2004b; Mabire et al., 2005) had reported binding affinity (or potency) less than $20 \mathrm{nM}$ (Table 1). 2,4-Dicarboxy-pyrrole ester 4 (3,5-dimethyl PPP), as a racemic mixture, is a highly potent and subtype-selective noncompetitive antagonist of mGluR1, having $\mathrm{IC}_{50}$ of $16 \mathrm{nM}$ at rat mGluR1 and > 1000-fold selectivity over mGluR 2, 4, and 5 (Micheli et al., 2003b). Pharmacological studies of its two enantiomers showed that the S-enantiomer had the same activity as the racemic mixture, while the R-enantiomer was less potent $(40 \mathrm{nM})$. Although compound 4 had a poor stability to rat plasma esterase $\left(\mathrm{t}_{1 / 2}=12\right.$ min versus $2.8 \mathrm{~h}$ in mice), a good CNS accumulation was observed $5 \mathrm{~min}$ after intravenous administration with a brain/plasma ratio of 20 (Micheli et al., 2003b). Compound 5 (JNJ- 
16259685) demonstrated high specificity over other mGlu receptor subtypes and a fast brain penetration with high receptor occupancy after subcutaneous administration (Lavreysen et al., 2004b). In addition to 5, a series of quinoline derivatives have been synthesized. The in vitro pharmacological data showed that they are highly potent noncompetitive mGluR1 antagonists (Mabire et al., 2005) with high binding affinity. However, the quinoline derivatives have issues of poor aqueous solubility and poor stability to human liver microsomes (Layton, 2005; Mabire et al., 2005).

Since 2008, many new compounds (Fig. 1 and Table 1) have been reported having binding affinity (or potency) less than $20 \mathrm{nM}$ and high selectivity over other mGluRs. These compounds are diverse heterocyclic compounds including mono-, di- and tri-cyclic structures. Some of these compounds or their derivatives are amenable to radiolabeling with fluorine-18 or carbon-11. For example, a series of potent 2-fluoro-3-pyridyl-triazol derivatives such as FTIDC (10) and FPTQ (11) have been developed.. These derivatives are relatively easy to label with fluorine-18 at 2-pyridine position. Other compounds such as $\mathbf{1 2}$ are amenable to radiolabeling with carbon-11.

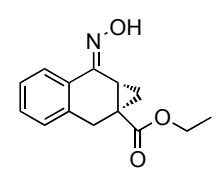

1, CPCCOEt<smiles>CO[C@H]1CC[C@H](C(=O)c2ccc3nc4c(cc3c2)CCCO4)CC1</smiles>

5, JNJ-16259685

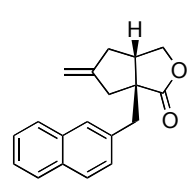

2, BAY36-7620<smiles>CCn1c(C)nc(N2C=Cc3ccccc3C=C2)c(C#N)c1=O</smiles>

3, EM-TBPC<smiles>CCCOC(=O)c1[nH]c(C)c(C(=O)OC(C)C(C)(C)C)c1C</smiles>

4, 3,5-dimethyl PPP<smiles>CCc1ccc(-n2cnc3c(sc4nccc(N(C)C)c43)c2=O)cc1</smiles>

6<smiles></smiles><smiles>C[C@H]1CC[C@H](NC(=O)c2cnc(N3CCC(CO)CC3)cn2)CC1</smiles><smiles>CC=C(CC)S(N)(=O)=O</smiles><smiles>C/C(=C(/N)C1=CCN(C(=O)N(C)C(C)C)CC1)N(N)c1cccnc1F</smiles>

10, FTIDC<smiles>COCCN(C)Cc1ccc2nc3sc(C(=O)N(C)C4CCCCC4)cn3c2c1</smiles>

11, FPTQ

12, YM-202074<smiles>c1nc(NC2CCCCCC2)c2cc(OCCN3CCOCC3)ncc2n1</smiles>

13<smiles>CC(C)Nc1cc(-c2csc(N(C)C(=O)c3ccc(F)cc3)n2)ncn1</smiles>

14<smiles>Cc1ccc(-n2cnc3c(sc4nc(N)nc(N)c43)c2=O)cc1</smiles>

15<smiles>C/C(=C(\N)c1ccc2c(c1)CN(C(C)C)C2=O)N(N)c1ccc(F)cc1F</smiles>

16, MK-5435

Fig. 1. Chemical structures of mGluR1 negative modulators.

MGluR1 expression is localized throughout the nervous system (Layton, 2005; Spooren et al., 2003). The distribution of mGluR1 in the peripheral nervous system (Bhave et al., 2001; Lesage, 2004; Skerry \& Genever, 2001) and in the CNS has been studied using various methods including radioligand autoradiography and immunohistochemical techniques (Lavreysen et al., 2003; Lavreysen et al., 2004a; Shigemoto \& Mizuno., 2000; Simonyi et al., 2005). MGlu1 receptors have been observed in the cerebellum, thalamus, hippocampus and 


\begin{tabular}{|c|c|c|c|c|c|}
\hline Compound & $\begin{array}{c}\text { Rat } \\
\text { mGluR1 } \\
\text { IC }_{50} \\
\text { (nM) }\end{array}$ & $\begin{array}{c}\text { Human } \\
\text { mGluR1 } \\
\text { IC }_{50} \\
\text { (nM) }\end{array}$ & Selectivity & In vivo properties & References \\
\hline $\begin{array}{c}\mathbf{1} \\
(\mathrm{CPCCOEt})\end{array}$ & & $\begin{array}{c}1500- \\
6500\end{array}$ & $\begin{array}{l}>15 \text { over } \\
\text { mGluR2, 4, 5, } \\
7,8\end{array}$ & & $\begin{array}{l}\text { (Litschig et } \\
\text { al., 1999; Ott } \\
\text { et al., 2000) }\end{array}$ \\
\hline $\begin{array}{c}2 \\
(\text { Bay36- } \\
7620)\end{array}$ & 160 & & $\begin{array}{l}>100 \text { over } \\
\text { mGluR 2, 3, } \\
4,5,7,8\end{array}$ & $\begin{array}{l}30 \% \text { receptor } \\
\text { occupancy in } \\
\text { cerebellum and } \\
\text { thalamus (s.i.) }\end{array}$ & $\begin{array}{l}\text { (Carroll et } \\
\text { al., 2001) }\end{array}$ \\
\hline $\begin{array}{c}3 \\
(\mathrm{EM}-\mathrm{TBPC})\end{array}$ & 130 & & $\begin{array}{l}\text { No binding } \\
\text { for rat } \\
\text { mGluR5 }\end{array}$ & & $\begin{array}{c}\text { (Malherbe et } \\
\text { al., 2003) }\end{array}$ \\
\hline $\begin{array}{c}4 \\
(3,5- \\
\text { dimethyl } \\
\text { PPP })\end{array}$ & 16 & & $\begin{array}{l}>1000 \text { over } \\
\text { mGluR2, 4, } 5\end{array}$ & $\begin{array}{l}\text { Good CNS } \\
\text { exposure with } \\
\text { brain/plasma ratio } \\
\text { of } 20\end{array}$ & $\begin{array}{l}\text { (Micheli et } \\
\text { al., 2003a; } \\
\text { Micheli et } \\
\text { al., 2003b) }\end{array}$ \\
\hline $\begin{array}{c}5 \\
(\mathrm{JNJ}- \\
16259685)\end{array}$ & 3 & 0.55 & $\begin{array}{l}>400 \text { over rat } \\
\text { mGluR5; } \\
>20,000 \text { over } \\
\text { human } \\
\text { mGluR5 }\end{array}$ & $\begin{array}{l}\text { Fast brain } \\
\text { penetration and } \\
\text { high receptor } \\
\text { occupancy (s.i.) }\end{array}$ & $\begin{array}{l}\text { (Lavreysen } \\
\text { et al., 2004b; } \\
\text { Mabire et } \\
\text { al., 2005) }\end{array}$ \\
\hline 6 & $\mathrm{~K}_{\mathrm{i}}=5$ & 3 & $\begin{array}{l}\mathrm{IC}_{50}=442 \mathrm{nM} \\
\text { for human } \\
\text { mGluR5; } \\
\mathrm{K}_{\mathrm{i}}=194 \mathrm{nM} \\
\text { for rat } \\
\text { mGluR5 }\end{array}$ & $\begin{array}{l}\text { Demonstrated } \\
\text { efficacy in various } \\
\text { in vivo animal } \\
\text { models }\end{array}$ & $\begin{array}{l}\text { (Zheng et } \\
\text { al., 2005) }\end{array}$ \\
\hline 7 & $\mathrm{~K}_{\mathrm{i}}=0.4$ & 2.9 & $\begin{array}{l}>1,000 \mathrm{nM} \\
\text { for human } \\
\text { mGluR5 }\end{array}$ & $\begin{array}{l}\text { Demonstrated } \\
\text { activity in the rat } \\
\text { spinal nerve } \\
\text { ligation } \\
\text { neuropathic pain } \\
\text { model (SNL } \\
\text { model) with ED } 50 \\
\text { of } 5.1 \mathrm{mg} / \mathrm{kg} \text {. }\end{array}$ & $\begin{array}{c}\text { (Wu et al., } \\
\text { 2007) }\end{array}$ \\
\hline 8 & $\mathrm{~K}_{\mathrm{i}}=9$ & & & $\begin{array}{l}\text { LogD=3.3; human } \\
\text { liver microsomal } \\
\text { metabolic stability: } \\
\mathrm{Cl}_{\text {int }}<7 \mu \mathrm{l} / \mathrm{min} / \mathrm{mg}\end{array}$ & $\begin{array}{c}\text { (Owen et al., } \\
\text { 2007) }\end{array}$ \\
\hline 9 & & 127 & $\begin{array}{l}>100,000 \mathrm{nM} \\
\text { for human } \\
\text { mGluR5 }\end{array}$ & $\begin{array}{l}\text { Solubility: } 42 \mu \mathrm{M} \text {; } \\
\text { microsomal } \\
\text { clearance: }<2.5 \\
\text { L/h/kg; } \\
\text { quantitative } \\
\text { bioavailability }\end{array}$ & $\begin{array}{c}\text { (Wang et al., } \\
\text { 2007b) }\end{array}$ \\
\hline
\end{tabular}




\begin{tabular}{|c|c|c|c|c|c|}
\hline Compound & $\begin{array}{c}\text { Rat } \\
\text { mGluR1 } \\
\text { IC }_{50} \\
\text { (nM) }\end{array}$ & $\begin{array}{c}\text { Human } \\
\text { mGluR1 } \\
\text { IC }_{50} \\
\text { (nM) }\end{array}$ & Selectivity & In vivo properties & References \\
\hline $\begin{array}{c}10 \\
(\text { FTIDC) }\end{array}$ & 5.8 & 5.8 & $\begin{array}{l}6200 \mathrm{nM} \text { for } \\
\text { human } \\
\text { mGluR5; } \\
>1720 \text { over } \\
\text { mGluR2, 4, 6, } \\
7,8\end{array}$ & $\begin{array}{l}\text { LogD=2.1; } \\
\text { demonstrated } \\
\text { efficacy in (S)-3,5- } \\
\text { DHPG-induced } \\
\text { face-washing } \\
\text { behavior in mice }\end{array}$ & $\begin{array}{c}\text { (Suzuki et } \\
\text { al., 2009; } \\
\text { Suzuki et al., } \\
\text { 2007a) }\end{array}$ \\
\hline $\begin{array}{c}11 \\
\text { (FPTQ) }\end{array}$ & 14 & 3.6 & & & $\begin{array}{l}\text { (Suzuki et } \\
\text { al., 2009) }\end{array}$ \\
\hline $\begin{array}{c}12 \\
(\mathrm{YM}- \\
202074)\end{array}$ & $\begin{array}{c}8.6 \\
\mathrm{~K}_{\mathrm{i}}=4.8\end{array}$ & & $\begin{array}{l}>1000 \text { for rat } \\
\text { mGluR2, 3, 4, } \\
6,7 ;>100 \text { for } \\
\text { rat mGluR5; }\end{array}$ & $\begin{array}{l}\text { Showed efficacy } \\
\text { for } \\
\text { neuroprotection in } \\
\text { rats suffering from } \\
\text { transient focal } \\
\text { cerebral ischemia; }\end{array}$ & $\begin{array}{l}\text { (Kohara et } \\
\text { al., 2008) }\end{array}$ \\
\hline 13 & $\mathrm{~K}_{\mathrm{i}}=6$ & & & $\begin{array}{l}\text { CSF: } \mathrm{C}_{\mathrm{u}}=0.5 ; \mathrm{HLM}: \\
\mathrm{Cl}_{\text {int }}=24 \\
\mu \mathrm{l} / \mathrm{min} / \mathrm{mg}\end{array}$ & $\begin{array}{l}\text { (Mantell et } \\
\text { al., 2009) }\end{array}$ \\
\hline 14 & & 5.1 & $\begin{array}{l}7000 \mathrm{nM} \text { for } \\
\text { human } \\
\text { mGluR5; } \\
>10,000 \mathrm{nM} \\
\text { for human } \\
\text { mGluR2, } 8\end{array}$ & $\begin{array}{l}\text { Mouse } \\
\text { brain/plasma } \\
\text { concn } 0.17 \\
\text { nmol/g/0.19 } \mu \mathrm{M} ; \\
\text { Rat F: } 53 \%, \mathrm{~T}_{1 / 2}: 2.3 \\
\mathrm{~h}, \mathrm{CLp:} 28 \\
\mathrm{~mL} / \mathrm{min} / \mathrm{kg} \text {; Rat } \\
\text { PPI disruption } \\
\text { model MED } 1.0 \\
\text { mg/kg, PO; Mouse } \\
\text { hyperlocomotion } \\
\text { model MED } 0.3 \\
\text { mg/kg, PO }\end{array}$ & $\begin{array}{c}\text { (Satoh et al., } \\
\text { 2009) }\end{array}$ \\
\hline 15 & $\mathrm{~K}_{\mathrm{i}}=9.3$ & 2.1 & $\begin{array}{l}>3000 \mathrm{nM} \text { for } \\
\text { human } \\
\text { mGluR5 }\end{array}$ & $\begin{array}{l}\text { Rat PK, (10 } \\
\text { mg/kg), AUC (ng } \\
\text { h/mL): 965; Brain } \\
\text { concn @ } 6 \mathrm{~h} \\
\text { (ng/g): 100; } \\
\text { Brain/ plasma: } 0.9\end{array}$ & $\begin{array}{l}\text { (Sasikumar } \\
\text { et al., 2010) }\end{array}$ \\
\hline $\begin{array}{c}16 \\
(\mathrm{MK}-5435)\end{array}$ & & 4.3 & $\begin{array}{l}1500 \mathrm{nM} \text { for } \\
\text { human } \\
\text { mGluR5 }\end{array}$ & & $\begin{array}{c}\text { (Hostetler et } \\
\text { al., 2011) }\end{array}$ \\
\hline
\end{tabular}

Table 1. In vitro and in vivo pharmacological profiles for mGluR1 negative allosteric modulators. 
<smiles>CCCN1Cc2cc(-c3nnn(-c4cccnc4F)c3C)ccc2C1=O</smiles>

$\left[{ }^{18} \mathrm{~F}\right] \mathrm{MK}-1312$<smiles>Cc1c(C2=CCN(C(=O)N(C)C(C)C)CC2)nnn1-c1cccnc1F</smiles>

$\left[{ }^{18} \mathrm{~F}\right] \mathrm{FTIDC}$<smiles>COCCN(C)Cc1ccc2nc3sc(C(=O)N(C)C4CCCCC4)cn3c2c1</smiles><smiles>Cc1c(-c2ccc3ncccc3c2)nnn1-c1cccnc1F</smiles>

Fig. 2. PET ligands for mGluR1

spinal cord (Karakossian \& Otis, 2004; Lavreysen et al., 2003; Shigemoto \& Mizuno, 2000; Spooren et al., 2003). Tritium-labeled highly potent and subtype-selective radioligands were used earlier in mapping mGluR1 ex vivo (Yang, 2005). Presently, demands on PET radioligands are increasing due to the advantage of in vivo noninvasive imaging techniques to investigate pathophysiological processes.

In 2002, a carbon-11 labeled CPCCO-Me analog was described in the literature (Yu \& Brownell, 2002), but no animal studies were conducted. In the series of quinoline derivatives (represented by 5), several compounds are amenable to radiolabeling with either fluorine-18 or carbon-11. Carbon-11 labeling would not be preferred in the methyl ether positions, in spite of methyl ether position is very popular in ${ }^{11} \mathrm{C}$-methylation, since O-demethylation of the methoxy groups on the quinoline moiety and the cyclohexyl ring are the major metabolic pathways (Mabire et al., 2005). Therefore, practical methods must be developed to label the methyl groups elsewhere in the molecule. Accordingly, Huang et al. successfully labeled a quinoline derivative, providing the first PET tracer, $\left[{ }^{11} \mathrm{C}\right] \mathrm{JNJ}-16567083$, suitable for in vivo imaging of mGluR1 (Huang et al., 2005). [11C]JNJ-16567083 (17) is an analog of JNJ16567083 (5). In vitro binding experiments showed that JNJ16567083 (cold compound) possesses high affinity for rat mGluR1 $\left(K_{i}=0.87 \mathrm{nM}\right)$ and low affinity for mGluR5 $\left(K_{i}=2366\right.$ $\mathrm{nM}) . E x$ vivo biodistribution studies in rats showed that $\left[{ }^{11} \mathrm{C}\right] \mathrm{JNJ}-16567083$ has high brain uptake and its binding in brain is specific to mGluR1. MicroPET imaging experiments in rats indicated that radioactivity entered the brain rapidly and was localized over time in brain regions with high densities of mGluR1, such as the cerebellum and striatum. Activity in cerebellum peaked at $\sim 10 \mathrm{~min}$ after intravenous injection. Radioactivity uptake was highest in the cerebellum, followed by striatum and hippocampus. However, evaluation of this PET tracer in higher species has not been reported.

Yanamoto et al. have labeled an mGluR1 antagonist YM-202074 (12, $\left.\mathrm{K}_{\mathrm{i}}=4.8 \mathrm{nM}\right)$ with ${ }^{11} \mathrm{C}$ and evaluated its potential as a PET ligand for mGluR1 (Yanamoto et al., 2010). In vitro autoradiographic study demonstrated that [11C]YM-202074 (21, Fig.2) had high specific binding with mGluR1 in the rat cerebellum and its regional distribution was consistent with the distribution pattern of mGluR1 in the brain. However, the total accumulation of 
[11C]YM-202074 in the brain was very low including lipophilic radiometabolites hampering its usefulness for in vivo imaging.

Prahakaran et al. have reported the synthesis for in vitro and in vivo evaluation of [11C]MMTP (20) as a potential PET ligand for mGluR1 (Prabhakaran et al., 2010). Synthesis of the corresponding desmethyl precursor was achieved by demethylation of the methoxyphenyl compound MMTP in 90\% yield. Methylation using [ $\left.{ }^{11} \mathrm{C}\right] \mathrm{MeOTf}$ in presence of $\mathrm{NaOH}$ afforded [ ${ }^{11} \mathrm{C}$ ]MMTP in 30\% yield (EOS) with $>99 \%$ chemical and radiochemical purities and with a specific activity of $3-5 \mathrm{Ci} / \mu \mathrm{mol}(\mathrm{n}=6)$. The total synthesis time was 30 min from EOB. In vitro autoradiography using phosphor imaging demonstrated that the radiotracer bound selectively mGlu1 receptors in slide-mounted sections of postmortem human brain containing cerebellum, hippocampus, prefrontal cortex and striatum. PET studies in anesthetized baboon showed that [11C]MMTP penetrates the BBB and accumulates in cerebellum, a region of high expression of mGluR1.

Recently, a $\left.{ }^{18 F-l a b e l e d ~ t r i a z o l e ~ a n a l o g ~[18 F] F T I D C ~(19, ~ K i ~=~} 3.9 \mathrm{nM}\right)$ (Ohgami et al., 2009) was presented for imaging of mGluR1 showing high uptake in the rat brain. In addition, Fujinaga et al. have labeled a triazole analog, FPTQ (11, $\mathrm{IC}_{50}=3.6 \mathrm{nM}$ and $1.4 \mathrm{nM}$ for human and mouse mGluR1, respectively) (Fujinaga et al., 2011). [18F]FPTQ (22) was synthesized by $\left[{ }^{18} \mathrm{~F}\right]$ fluorination of the corresponding 2-bromo-3-pyridyl precursor with potassium $\left[{ }^{18} \mathrm{~F}\right]$ fluoride. At the end of synthesis, $35-50 \mathrm{mCi}(\mathrm{n}=8)$ of $\left[{ }^{18} \mathrm{~F}\right] \mathrm{FPTQ}$ was obtained with $>98 \%$ radiochemical purity and 3.2-6.4 $\mathrm{Ci} / \mu \mathrm{mol}$ specific activity using $89-108 \mathrm{mCi}$ of $\left[{ }^{18} \mathrm{~F}\right]$ fluoride. In vitro autoradiography showed that $\left[{ }^{18} \mathrm{~F}\right] \mathrm{FPTQ}$ had high specific binding with mGluR1 in the rat brain. Biodistribution study using a dissection method and small-animal PET showed that $\left[{ }^{18} \mathrm{~F}\right] \mathrm{FPTQ}$ had high uptake in the rat brain. The uptake of radioactivity in the cerebellum was reduced by unlabeled FPTQ and mGluR1-selective ligand JNJ-16259685 (Fujinaga et al., 2011), indicating that [18F]FPTQ had in vivo specific binding to mGluR1. However, because of a low amount of radiolabeled metabolite present in the brain, this compound may have limiting use for in vivo imaging of mGluR1 by PET.

Hostetler et al. have reported a PET radioligand, [18F]MK-1312 (18), which was radiolabeled with fluorine-18 via nucleophilic displacement of the corresponding 2-chloropyridine precursor with [18F]potassium fluoride (Hostetler et al., 2011). [18F]MK-1312 was synthesized $(\mathrm{n}=25)$ in good yield $(46 \pm 15 \%)$ with $>98 \%$ radiochemical purity and high specific activity $(2.5 \pm 1.4 \mathrm{Ci} / \mu \mathrm{mol})$. In vitro autoradiographic studies with [18 $\mathrm{F}] \mathrm{MK}-1312$ in rhesus monkey and human brain tissue slices revealed an uptake distribution consistent with the known distribution of mGluR1, with the highest uptake in the cerebellum, moderate uptake in the hippocampus, thalamus, and cortical regions, and the lowest uptake in the caudate and putamen. In vitro saturation binding studies in rhesus monkey and human cerebellum homogenates confirmed that $\left.{ }^{18} \mathrm{~F}\right] \mathrm{MK}-1312$ binds to a single binding site with a Bmax/Kd ratio of 132 and 98, respectively. PET studies in rhesus monkey with [18F]MK-1312 showed high brain uptake and a regional distribution consistent with in vitro autoradiography results. Blockade of $\left[{ }^{18} \mathrm{~F}\right] \mathrm{MK}-1312$ uptake with mGluR1 allosteric antagonist MK-5435 dosedependently reduced tracer uptake in all regions of gray matter. These results show that $[18 \mathrm{~F}] \mathrm{MK}-1312$ is a promising PET tracer for clinical studies to determine mGluR1 occupancy of MK-5435.

In summary, several PET radioligands have been developed using highly potent and subtype-selective mGluR1 negative allosteric modulators. Although they showed efficacy in studying the distribution of mGluR1, some compounds may have limited applications 
because of low brain uptake and/or brain penetrating radiometabolites. [18F]MK-1312 is the most advanced mGluR1 PET tracer, which has demonstrated efficacy in rhesus monkey.

Although all the published mGluR1 PET tracers are radiolabeled mGluR1 negative allosteric modulators, mGluR1 positive allosteric modulators can also be used for developing mGluR1 PET tracers. Several papers have been published about the functional differences between antagonist and agonist tracers in imaging G-protein coupled receptors, including dopamines D2 receptor (Hwang et al., 2004; Wilson et al., 2005), serotonin receptors (Kumar et al., 2006; Prabhakaran et al., 2006) and mGlu receptors. GPCRs have been postulated to exist in interconvertible high-affinity and low-affinity states. The high-affinity sites are G-protein coupled, whereas the low-affinity sites are those uncoupled with G-protein. Antagonist radiotracers bind with equal affinity to both the high- and low-affinity forms of the receptor, and they do not provide information about in vivo affinity of the receptor for antagonist. On the contrary, agonist radioligands bind only to high-affinity form of the receptor, thus giving valuable information about in vivo affinity of the receptor for agonists in normal and abnormal states. Concerning the binding sites of allosteric modulators in the seven strand transmembrane domain, there is no evidence for difference between negative and positive modulators in terms of their binding to high-affinity or low-affinity states of mGlu receptors (Kew \& Kemp, 2005). Fig. 3 illustrates structures of some representative positive allosteric mGluR1 modulators reported (Knoflach et al., 2001; Layton, 2005; Wichmann et al., 2002).

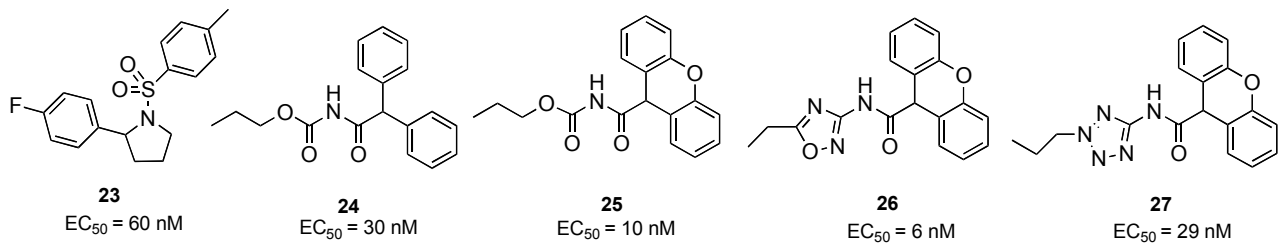

Fig. 3. Chemical structures of mGluR1 positive allosteric modulators.

\subsubsection{Allosteric modulators and radiotracers for mGluR5}

Since the first selective mGluR5 antagonist was identified in 1999 (Varney et al., 1999), a large number of potent, subtype selective and structurally diverse allosteric modulators have been described. SIB1757 (28) and SB1893 (29) were discovered through random screening. Subsequent optimization by replacement of the trans-olefinic tether in SIB1893 (29) with a C $\equiv$ C triple bond led to MPEP (30), which demonstrated a dramatically improved mGluR5 antagonist activity (Gasparini et al., 1999). Various structure-activity relationship (SAR) studies have been done on MPEP, in which chemical modifications were done to each of the three regions of the lead molecule, identifying a series of highly potent and selective diaryl (heteroaryl) acetylenes as mGluR5 noncompetitive antagonists. By assumption that the (2-methyl-1,3-thiazo-4-yl)ethynyl group is one of the best structural parts to achieve mGluR5 antagonist activity further SAR studies on MTEP (31) identified more high-profile ligands containing thiazole moiety as mGluR5 noncompetitive antagonists such as (33) (Iso et al., 2006). Many PET tracers have been synthesized by radiolabeling on the derivatives of MPEP and MTEP.

A major concern with acetylenes in potential drugs is the possibility of chemical or metabolic reactivity (Milbank et al., 2007). Terminal acetylenes are well known to be 
mechanism-based CYP-inactivators (Testa \& Jenner, 1981) and there is an increasing body of information suggesting that internal acetylenes can be activated by CYPs (Fontana et al., 2005; Foroozesh et al., 1997; Shimada et al., 2007) or even undergo uncatalyzed addition of glutathione (Chen et al., 2002; Mutlib et al., 1999). Mutlib et al. reported that incubation of MPEP with triple-labeled glutathione gave compounds with molecular weights and fragmentations consistent with both activated and unactivated addition of GSH to the alkyne (Mutlib et al., 2005). These events are potential sources for hepatic or idiosyncratic toxicity. To avoid a potential metabolic liability, many research groups have designed and synthesized mGluR5 negative allosteric modulators without the acetylene structure. Some structures such as $\mathbf{3 7}$ to $\mathbf{4 8}$ are given in Fig. 4, which may be useful for development of a new PET tracer.

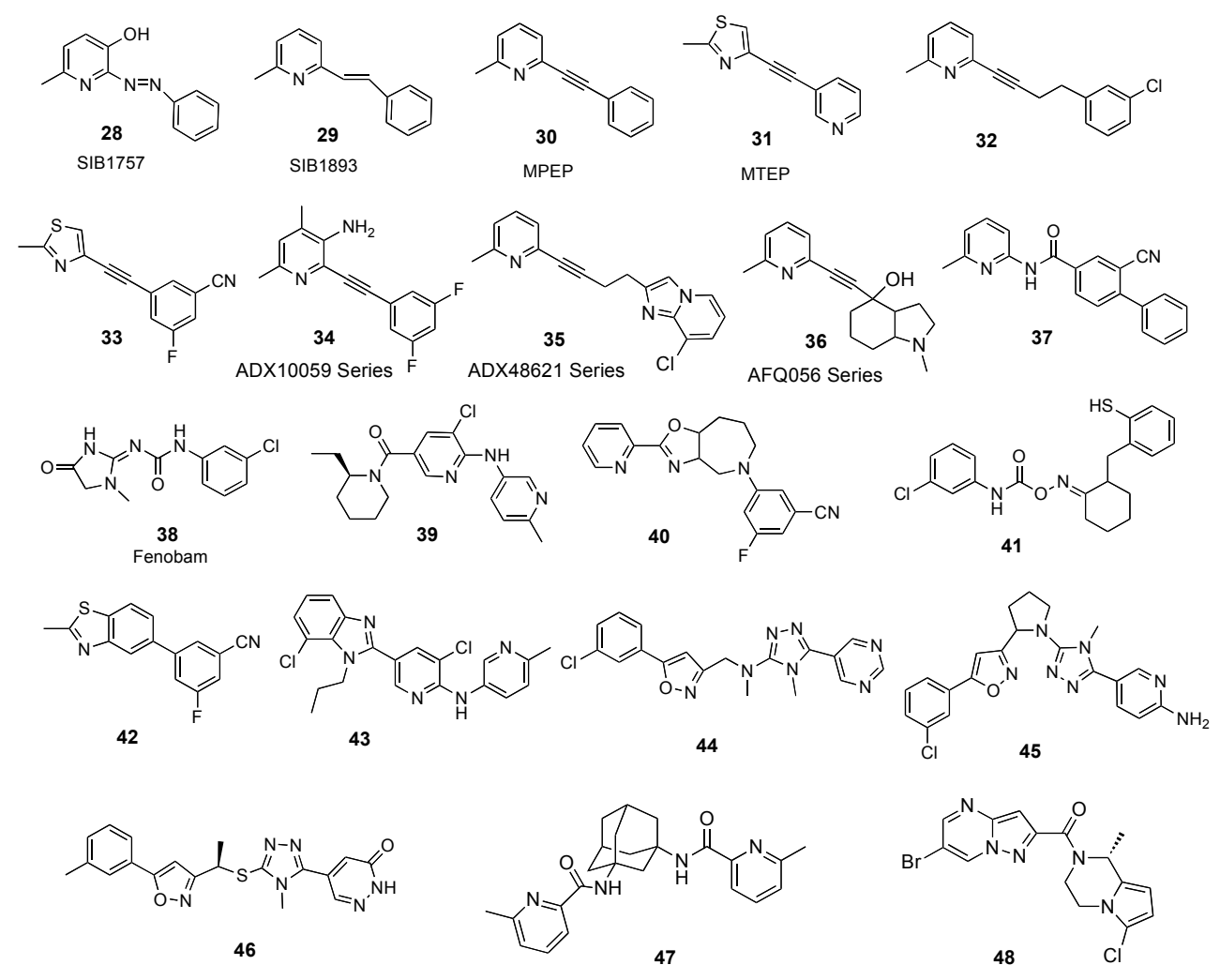

Fig. 4. Chemical structures of mGluR5 negative allosteric modulators

Since the discovery of the first mGluR5 positive modulator, DFB (49, Fig. 5) (O'Brien et al., 2003), Merck has reported three series of positive allosteric modulators for mGluR5, which are benzaldazine, benzamide and pyrazole series, exemplified by DFB, CPPHA (50) (O'Brien et al., 2004) and CDPPB (51) (Kinney et al., 2005; Lindsley et al., 2004), respectively. Subsequent structure-activity relationship study on CDPPB identified several nanomolar potent pyrazole ligands (De Paulis et al., 2006). Although these compounds are potent with an $\mathrm{EC}_{50}$ value of less than $20 \mathrm{nM}$, their poor binding affinity $\left(\mathrm{K}_{\mathrm{i}}\right)$ and high lipophilicity 


\begin{tabular}{|c|c|c|c|c|}
\hline Compound & $\begin{array}{c}\text { Rat } \\
\text { mGluR5 } \\
\text { IC }_{50} \\
\text { (nM) }\end{array}$ & $\begin{array}{l}\text { Human } \\
\text { mGluR5 } \\
\text { IC }_{50} \\
(\mathrm{nM})\end{array}$ & In vivo properties & References \\
\hline $\begin{array}{c}\mathbf{2 8} \\
(\mathrm{SIB} 1757)\end{array}$ & & & & $\begin{array}{c}\text { (Varney et al., } \\
\text { 1999) }\end{array}$ \\
\hline $\begin{array}{c}29 \\
(\mathrm{SIB} 1893)\end{array}$ & & & & $\begin{array}{c}\text { (Varney et al., } \\
1999)\end{array}$ \\
\hline $\begin{array}{c}30 \\
\text { (MPEP) }\end{array}$ & $K_{i}=12$ & 2 & & $\begin{array}{l}\text { (Cosford et al., } \\
\text { 2003) }\end{array}$ \\
\hline $\begin{array}{c}31 \\
(\mathrm{MTEP})\end{array}$ & $K_{i}=16$ & 5 & $\begin{array}{l}\text { MTEP is more potent than } \\
\text { MPEP in vivo (rats) in both a } \\
\text { receptor occupancy assay and } \\
\text { in the fear-potentiated startle } \\
\text { model of anxiety. }\end{array}$ & $\begin{array}{l}\text { (Cosford et al., } \\
\text { 2003) }\end{array}$ \\
\hline 32 & 5 & & & $\begin{array}{l}\text { (Bach et al., } \\
\text { 2006) }\end{array}$ \\
\hline 33 & $\begin{array}{c}0.8 \\
\mathrm{~K}_{\mathrm{i}}=0.9\end{array}$ & & & $\begin{array}{c}\text { (Kulkarni et al., } \\
\text { 2009) }\end{array}$ \\
\hline $\begin{array}{c}34 \\
\text { ADX10059 } \\
\text { Series } \\
\end{array}$ & & & $\begin{array}{l}\text { Positive data from phase II } \\
\text { clinical studies in both GERD } \\
\text { and acute migraine. }\end{array}$ & $\begin{array}{l}\text { (Keywood et al., } \\
\text { 2009; Marin \& } \\
\text { Goadsby, 2010) }\end{array}$ \\
\hline $\begin{array}{c}35 \\
\text { ADX48621 } \\
\text { Series }\end{array}$ & & & $\begin{array}{l}\text { Showed efficacy in } \\
\text { nonhuman primate model of } \\
\text { PD-LID. }\end{array}$ & (Emmitte, 2011) \\
\hline $\begin{array}{c}36 \\
\text { AFQ056 } \\
\text { Series } \\
\end{array}$ & & & $\begin{array}{l}\text { Reported improvements in } \\
\text { certain aberrant behaviors in } \\
\text { clinical trial for treating FXS. }\end{array}$ & (Emmitte, 2011) \\
\hline 37 & $\begin{array}{c}0.8 \\
\mathrm{~K}_{\mathrm{i}}=22\end{array}$ & & $\begin{array}{l}\text { Showed efficacy for } \\
\text { anxiolytic activity in the } \\
\text { Vogel assay. }\end{array}$ & $\begin{array}{l}\text { (Milbank et al., } \\
\text { 2007) }\end{array}$ \\
\hline $\begin{array}{c}38 \\
\text { (Fenobam) }\end{array}$ & & & $\begin{array}{l}\text { Using prepulse inhibition as } \\
\text { an outcome measure for } \\
\text { treating FXS, } 50 \% \text { of patients } \\
\text { responded according to the } \\
\text { predefined criteria of efficacy. }\end{array}$ & $\begin{array}{l}\text { (Berry-Kravis et } \\
\text { al., 2009; Porter } \\
\text { et al., 2005) }\end{array}$ \\
\hline 39 & 32 & & & $\begin{array}{l}\text { (Spanka et al., } \\
2010)\end{array}$ \\
\hline 40 & & 16 & $\begin{array}{l}\text { Showed good brain } \\
\text { penetration, robust receptor } \\
\text { occupancy and short half-life } \\
\text { in rodent. }\end{array}$ & $\begin{array}{l}\text { (Burdi et al., } \\
\text { 2010) }\end{array}$ \\
\hline 41 & $\begin{array}{c}109 \\
K_{i}=9.1\end{array}$ & & & $\begin{array}{c}\text { (Galambos et } \\
\text { al., 2010) }\end{array}$ \\
\hline 42 & 61 & & $\begin{array}{l}\text { Showed efficacy in the OSS } \\
\text { model. }\end{array}$ & $\begin{array}{l}\text { (Lindsley et al., } \\
\text { 2011) }\end{array}$ \\
\hline 43 & 24 & & $\begin{array}{l}\text { Showed a robust anxiolytic- } \\
\text { like effect. }\end{array}$ & $\begin{array}{l}\text { (Carcache et al., } \\
\text { 2011) }\end{array}$ \\
\hline
\end{tabular}




\begin{tabular}{|c|c|c|c|c|}
\hline Compound & $\begin{array}{c}\text { Rat } \\
\text { mGluR5 } \\
\text { IC }_{50} \\
\text { (nM) }\end{array}$ & $\begin{array}{c}\text { Human } \\
\text { mGluR5 } \\
\text { IC }_{50} \\
\text { (nM) }\end{array}$ & In vivo properties & References \\
\hline 44 & 20 & & Rat $\mathrm{B} / \mathrm{P}$ ratio $=0.16$. & $\begin{array}{c}\text { (Isaac \& } \\
\text { Waallberg, } \\
\text { 2009) }\end{array}$ \\
\hline 45 & $<3$ & & Rat $\mathrm{B} / \mathrm{P}$ ratio $=0.085$. & $\begin{array}{l}\text { (Granberg \& } \\
\text { Holm, 2009) }\end{array}$ \\
\hline 46 & 19 & & Rat $\mathrm{B} / \mathrm{P}$ ratio $=0.26$. & $\begin{array}{l}\text { (Granberg \& } \\
\text { Holm, 2010) }\end{array}$ \\
\hline 47 & $\mathrm{~K}_{\mathrm{i}}=6.7$ & & & $\begin{array}{c}\text { (Jimenez et al., } \\
\text { 2010) }\end{array}$ \\
\hline 48 & 7.8 & 25 & & $\begin{array}{c}\text { (Henrich et al., } \\
\text { 2009) }\end{array}$ \\
\hline
\end{tabular}

Table 2. In vitro and in vivo pharmacological profiles for mGluR5 negative allosteric modulators.

$(\log \mathrm{P})$ prevent them from being good candidates for radiotracer because high lipophilicity decreases brain penetration. Bessis et al. reported a fourth structural series represented by ADX47273 (52) (Bessis et al., 2005). Recently, many mGluR5 positive allosteric modulators, 5360, have been reported to have an $\mathrm{EC}_{50}$ value below $20 \mathrm{nM}$ (Fig. 5) (Varnes et al., 2011; Williams et al., 2011). However, no PET tracers have been developed from this class of compounds.
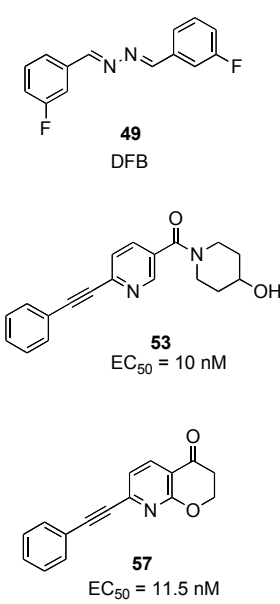
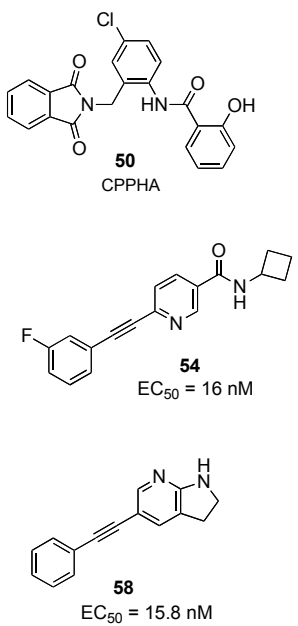
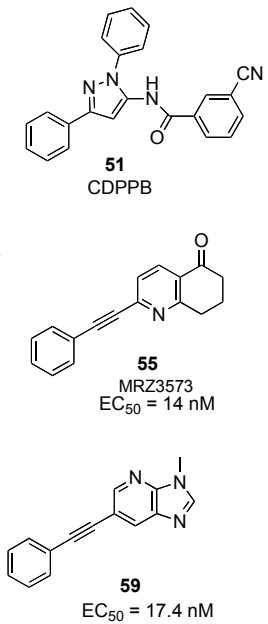
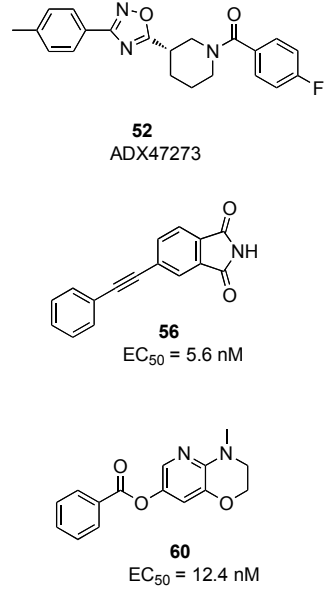

Fig. 5. Chemical structures of mGluR5 positive allosteric modulators

The discoveries of noncompetitive allosteric modulators with high binding affinity and subtype-selectivity entitle the exploration of the physiological functions of mGluR5 in normal and pathological states. Although in vitro and ex vivo studies using selective mGluR5 allosteric antagonists labeled with tritium (Cosford, 2003; Gasparini et al., 2002) have played important roles in elucidating the distribution and functions of mGluR5, PET tracers are needed for the in vivo quantitative visualization of mGluR5 in a living body and to conduct longitudinal studies of modulation of mGluR5 expression. 


\subsubsection{PET imaging studies of mGluR5 function}

MPEP and MTEP have provided leads to some radioligand candidates for imaging human mGlu5 receptors with PET in vivo. Great effort was done to identify suitable positronemitting radiotracers for noninvasive imaging of mGluRs. To date, more than 15 mGluR5selective PET ligands labeled with ${ }^{18} \mathrm{~F}$ or ${ }^{11} \mathrm{C}$ have been reported (Fig. 6) (Ametamey et al., 2006; De Paulis et al., 2006; Hamill et al., 2005; Honer et al., 2007; Krause et al., 2003; Musachio et al., 2003; Patel et al., 2005; Sanchez-Pernaute et al., 2008; Sime'on et al., 2007; Wang et al., 2007a; Yu, 2005; Zhu et al., 2007).

In 2005, Hamill and colleagues from Merck demonstrated the first successful PET imaging of mGluR5 in rhesus monkeys using [18F]F-MTEB (61) (Hamill et al., 2005; Patel et al., 2005). This compound was highly selective and bound with high affinity $\left(\mathrm{IC}_{50}=80 \mathrm{pM}\right)$ to the receptor. However, the synthesis of this tracer in the cyclotron gave low yields $(2-5 \%)$, which limited its potential utility as a ligand for clinical trials in humans.

Brownell et al. have synthesized and radiolabeled five noncompetitive antagonists for mGluR5: [11C]M-MPEP (62) (Yu et al., 2005), [11C]M-PEPy (63) (Sanchez-Pernaute et al., 2008), [ $\left.{ }^{11} \mathrm{C}\right] \mathrm{MPEP}$ (64) (Yu et al., 2005), [18F]FMTEP (65) (Zhu et al., 2007) and ${ }^{18}$ F]FPEB (66) (Wang et al., 2007a) and conducted in vivo PET imaging studies in different disease models to investigate modulation of mGluR5 function. It was found in these studies that accumulation of pyridine derivatives $\left[{ }^{11} \mathrm{C}\right] \mathrm{M}-\mathrm{MPEP}(62),\left[{ }^{11} \mathrm{C}\right] \mathrm{M}-\mathrm{PEPy}(63),\left[{ }^{11} \mathrm{C}\right] \mathrm{MPEP}(\mathbf{6 4})$ and [18F]FMTEP (65) into the brain was fast and the highest accumulation was reached in 1-5 min followed by fast washout, suggesting little retention by high affinity receptor binding. This creates limitation to obtain statistically meaningful imaging data without overdosing the object with radiation or saturating the receptor binding sites with accompanying cold compound. These ligands have limitation, due to high lipophilicity, unfavorable brain uptake kinetics, or a high rate of metabolism, though they possess favorable in vitro pharmacological profiles. For PET ligands to be used in the central nervous system, a postulated lipophilicity coefficient $(\log \mathrm{D}$ or $\log \mathrm{P})$ value should be between 2 and 3 for good brain accumulation. The compounds [ $\left.{ }^{11} \mathrm{C}\right] \mathrm{ABP} 688$ (67) (Ametamey et al., 2006) and [18F]FPEB (66) (Patel et al., 2007; Wang et al., 2007a) have better binding profile for imaging studies of mGluR5. The $\log \mathrm{D}$ value of 2.3 for $\left[{ }^{11} \mathrm{C}\right] \mathrm{ABP} 688$ and the $\log P$ value of 2.8 for [18F]FPEB suggest that the two compounds are sufficiently lipophilic for the BBB penetrating. Both compounds have good binding properties with a $K_{i}$ Value of $0.2 \mathrm{nM}$ for [18F]FPEB and a $\mathrm{K}_{\mathrm{d}}$ value of $1.7 \mathrm{nM}$ for [11C]ABP688. The brain uptake of both compounds is highly selective, with high accumulation in mGluR5-rich brain regions such as the hippocampus, striatum and cortex. Blocking studies by coinjection of [ $\left.{ }^{11} \mathrm{C}\right] \mathrm{ABP} 688$ and corresponding unlabeled compound revealed up to $80 \%$ specific binding in these regions, whereas in cerebellum, a region with negligible mGluR5 density, no significant changes in radioactivity uptake were observed (Ametamey et al., 2006). Specific binding of compounds [11C]ABP688 and $\left.{ }^{18}{ }^{18} \mathrm{~F}\right] \mathrm{FPEB}$ were also demonstrated with mGluR5-knockout mice which exhibited a homogeneous background level accumulation throughout the brain (Black et al., 2010). The metabolism studies of $\left[{ }^{11} \mathrm{C}\right] \mathrm{ABP} 688$ and $\left[{ }^{18} \mathrm{~F}\right] \mathrm{FPEB}$ indicated that more than $95 \%$ of the radioactivity found in the brain was parent compound $30 \mathrm{~min}$ after injection for [11C]ABP688 and $78 \%$ for [18F]FPEB. Both compounds have been translated to human studies to investigate mGluR5 function.

Siméon and colleagues of the NIH reported a new high affinity radioligand, [18F]-SP203 (68), for mGluR5 (Sime'on et al., 2007). [18F]-SP203 has high affinity $\left(\mathrm{IC}_{50}=36 \mathrm{pM}\right)$ and potency in a phosphoinositol hydrolysis assay $\left(\mathrm{IC}_{50}=0.71 \mathrm{pM}\right)$ for mGluR5. It demonstrates a high 
uptake in mGlu5 receptor rich regions of the rat and rhesus brain. The major advantage of this tracer over $\left[{ }^{18} \mathrm{~F}\right] \mathrm{F}-\mathrm{MTEB}$ is its high radiochemical yield $(87 \%)$ and easy radiosynthesis. This ligand is presently in NIH administrated clinical trial.

[11C]M-FPEP (69, $\mathrm{K}_{\mathrm{D}} 1.2 \mathrm{nM}$ and $\mathrm{B}_{\max } 84.5 \mathrm{f}_{\mathrm{mol}} / \mathrm{mg}$ ) has an even biodistribution in all brain regions demonstrating that this tracer lacks specific binding (Ametamey et al., 2003). Compound 70 showed little retention by the receptor (Krause et al., 2003). Compound 71 (rat $K_{\mathrm{i}} 0.23 \mathrm{nM}$ ) had a good brain uptake and slow washout, with high concentration in striatum, frontal cortex and cerebellum of monkey (Hamill et al., 2005). However, the
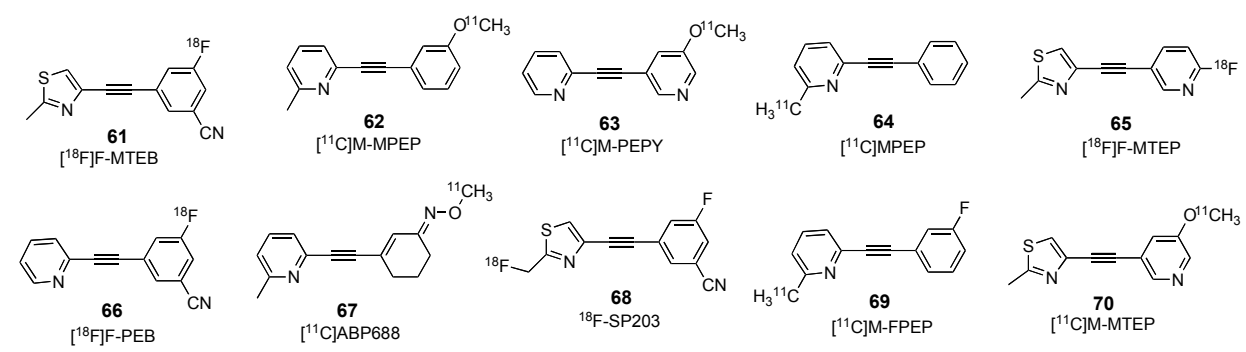

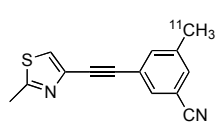

71
$\left.{ }^{11} \mathrm{C}\right] \mathrm{M}-\mathrm{MTEB}$

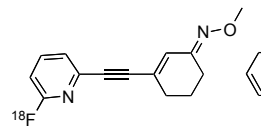

72

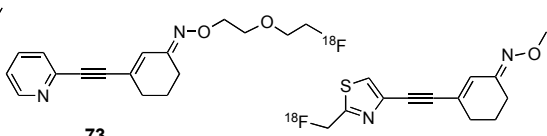

73

$\left[{ }^{18}\right.$ F $]$ FTECMO

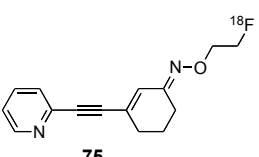

75

$\left[{ }^{18} \mathrm{~F}\right] \mathrm{FE}-\mathrm{DABP} 688$

Fig. 6. Chemical structures of mGluR5 PET tracers.

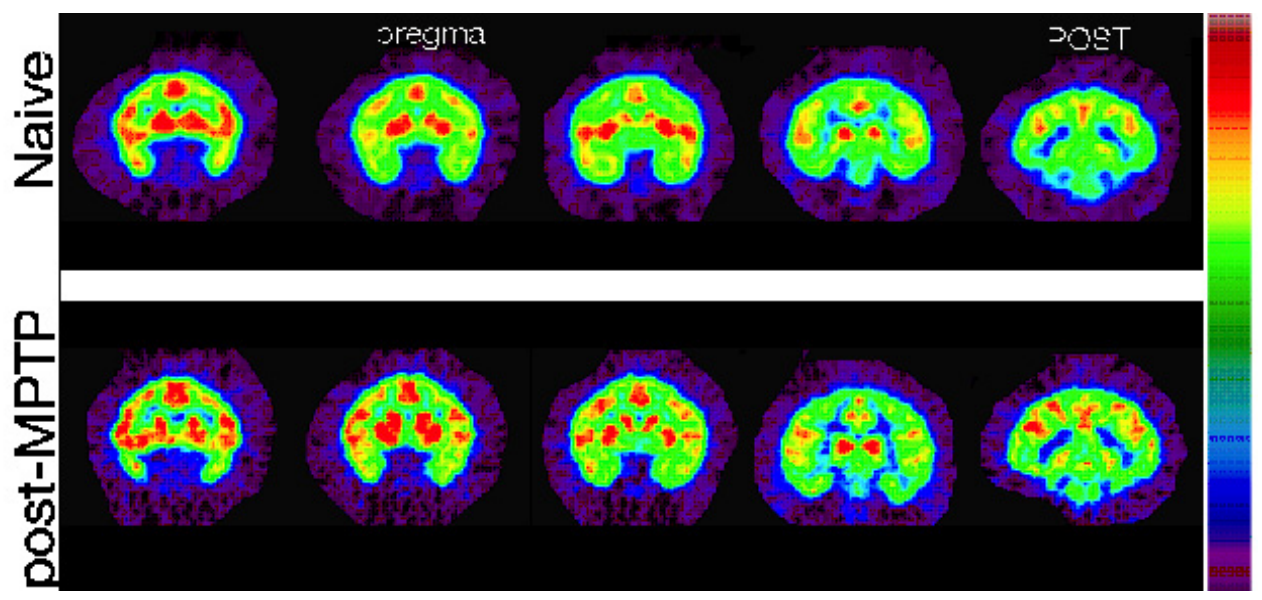

Fig. 7. Expression of mGluR5 in the brain of a naïve (top) and a symptomatic parkinsonian primate, using the highly selective tracer [18F]FPEB (3-[18F]fluoro-5-(2-

pyridinylethynyl)benzonitrile). Primate Parkinson's disease (PD) was introduced by low dose long-term systemic administration of MPTP. In PD monkey accumulation of [18F]FPEB was enhanced compared to naïve monkey in several brain areas including caudate, putamen, accumbens and SN/VTA. Distribution of [ $\left.{ }^{18} \mathrm{~F}\right] \mathrm{FPEB}$ accumulation is illustrated at $60-70 \mathrm{~min}$ after administration of radioligand (1.2-1.5 mCi iv., specific activity $1.9 \mathrm{Ci} / \mu \mathrm{mol})$. 
cerebellum is an area with fairly low mGluR5 expression indicating that $\mathbf{7 1}$ may have nonspecific binding.

Four derivatives, 72-75, were developed of ABP688. PET imaging with 72 (Lucatelli et al., 2009) did not allow visualization of mGluR5-rich brain regions in the rat brain due to fast washout and rapid defluorination. Compound 73 (Baumann et al., 2010a) was reported to have the high binding affinity to mGluR5. Further in vitro evaluation and in vivo imaging are needed for characterization of this ligand. Baumann et al. (Baumann et al., 2010b) reported that although [18F]-FTECMO (74) displayed optimal lipophilicity $\left(\log \mathrm{D}_{\mathrm{pH} 7.4}=1.6 \pm 0.2\right)$ and high stability in rat and human plasma as well as sufficient stability in rat liver microsomes, PET imaging with [18F]-FTECMO in Wistar rats showed low brain uptake. Uptake of radioactivity into the skull was observed suggesting in vivo defluorination. Honer et al. reported that $\left[{ }^{18} \mathrm{~F}\right]-\mathrm{FE}-\mathrm{DABP} 688$ (75) have optimal lipophilicity $(\log \mathrm{D} 2.1 \pm 0.1)$ and high plasma stability (Honer et al., 2007). Saturation assays of [18F]-FE-DABP688 revealed a single high affinity binding site with a dissociation constant $\left(\mathrm{K}_{\mathrm{d}}\right)$ of $1.6 \pm 0.4 \mathrm{nM}$ and a $\mathrm{B}_{\max }$ value of $119 \pm 24 \mathrm{fmol} / \mathrm{mg}$ protein. PET scanning indicated radioactivity uptake in mGluR5-rich regions such as the hippocampus, striatum and cortex, and radioactivity accumulation in the cerebellum, a region with negligible mGluR5 density, was significantly lower. Biodistribution studies showed a similar distribution pattern of [18F]-FE-DABP688 binding in the brain. The hippocampus-to-cerebellum and striatum-to-cerebellum ratios were $1.81 \pm 0.16$ and $1.93 \pm 0.36$, respectively. Blocking studies using coinjection of [18F]-FE-.

DABP688 and unlabeled M-MPEP $(1 \mathrm{mg} / \mathrm{kg})$ revealed more than $45 \%$ replacement in the hippocampus and striatum, thus demonstrating the in vivo specificity of tracer binding. This result shows that $\left[{ }^{18} \mathrm{~F}\right]-\mathrm{FE}-\mathrm{DABP} 688$ may be a useful PET tracer for imaging mGluR5

\subsection{Allosteric modulators and radiotracers for group II mGluRs}

Group II mGluRs have been shown to be expressed in several brain areas. The expression patterns of Group II receptors in the rodent brain parallel those of mGluR5, although the overall abundance of mGluR2/3 receptors appears slightly reduced as compared with that of mGluR5 (Olive, 2009). Expression levels of mGluR2/3 receptors are high in the olfactory bulb and hippocampus, and moderate in the dorsal striatum, nucleus accumbens, amygdala, anterior thalamic nuclei, cerebral cortex and cerebellum. Low levels of mGluR2/3 are found in the pallidum, colliculi, ventral midbrain and hypothalamus.

Group II mGluRs act in the hippocampus to decrease synaptic transmission and glutamate release when activated. These receptors have been targeted extensively by potential neuroprotective agents to develop treatments for anxiety, schizophrenia, Alzheimer's disease, Parkinson's disease, pain, drug withdrawal, and epilepsy (Rudd \& McCauley, 2005).

\subsubsection{Allosteric modulators for mGluR2}

Over the past decade, a number of highly potent $\left(\mathrm{EC}_{50}\right.$ in subnanomolar) mGluR2 agonists and antagonists with high binding affinity $\left(\mathrm{K}_{\mathrm{i}}<2 \mathrm{nM}\right)$ have been identified (Rudd \& McCauley, 2005; Yasuhara et al., 2006). However, their mGluR2-selectivity over mGluR3 in the same group is fairly low with the highest potency ratio being 6.5 (Dominguez et al., 2005). A high potency ratio does not necessarily imply a high binding affinity ratio, whereas the specific binding of a radiotracer depends much on the binding affinity ratio. Considering a low subtype-selectivity and unfavorable brain penetration of classical mGluR2 agonists and antagonists, the focus has presently been to develop noncompetitive allosteric modulators. When the allosteric binding sites on glutamate receptors within a group are sufficiently different it is possible to develop subtype selectivity modulators. 


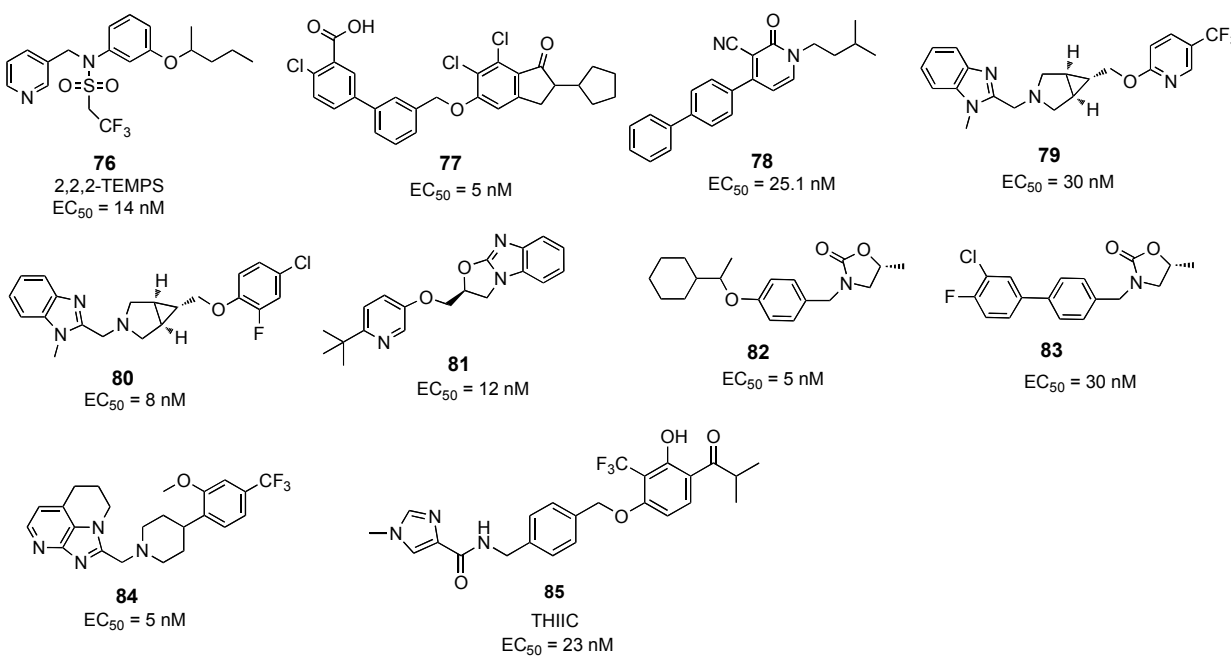

Fig. 8. Chemical structures of mGluR2 positive allosteric modulators

Many series of selective mGluR2 positive allosteric modulators have been reported to date. Figure 8 shows the compounds that were reported to have an $\mathrm{EC}_{50}$ value of less than $30 \mathrm{nM}$. They are N-aryl-N-(pyridylmethyl)ethanesulfonamides (76) (Barda et al., 2004; Johnson et al., 2003), biphenyl-indanones (77) (Bonnefous et al., 2005), 1,4-disubstituted 3-cyanopyridone derivatives (78) (Imogai et al., 2007), 3-(Imidazolyl methyl)-3-azabicyclo[3.1.0]hexan-6-yl)methyl ethers (79 and 80) (Zhang et al., 2008), oxazolobenzimidazoles (81) (Garbaccio et al., 2010), 3-Benzyl-1,3-oxazolidin-2-ones (82 and 83) (Duplantier et al., 2009), 2-((4-(2-methoxy-4-(trifluoromethyl)phenyl)piperidin-1yl)methyl)-5,6-dihydro-4H-imidazo[4,5,1-ij][1,7]naphthyridine (84) (Efremov et al., 2008) and THIIC (85) (Fell et al., 2011).
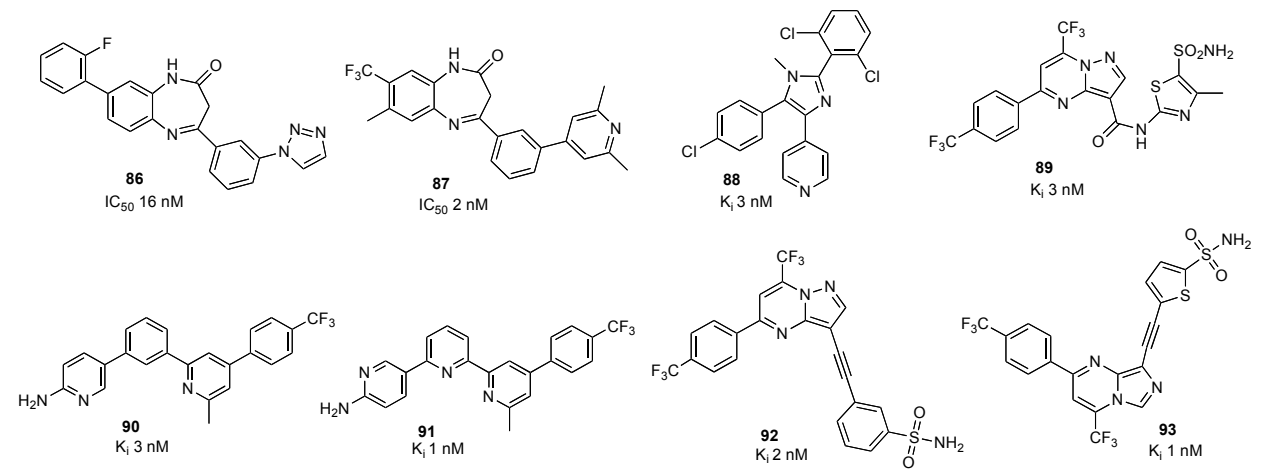

Fig. 9. Chemical structures of mGluR2/3 positive allosteric modulators

Several series of compounds have been developed as mGluR2 or mGluR2/3 allosteric antagonists, which include 8-ethynyl-1,3-dihydrobenzo[b][1,4]diazepin-2-one derivatives (86 and 87) (Woltering et al., 2007; Woltering et al., 2008a; Woltering et al., 2008b; Woltering et al., 2010), imidazole derivatives (88) (Gatti McArthur et al., 2006b), pyrazolopyrimidines 
(89) (Gatti McArthur et al., 2006c), Pyridine and pyrimidine derivatives (90 and 91) (Gatti Mcarthur et al., 2007), acetylenyl-pyrazolo-pyrimidine derivatives (92 and 93) (Gatti McArthur et al., 2006a). Representative compounds listed in Fig. 9 exhibit high binding affinity towards mGluR2, however, their binding selectivity over mGluR3 is either very low or is not disclosed.

Currently, no positron emitting radioligand has been developed for imaging mGluR2.

\subsubsection{PET imaging studies of mGluR2/3 expression}

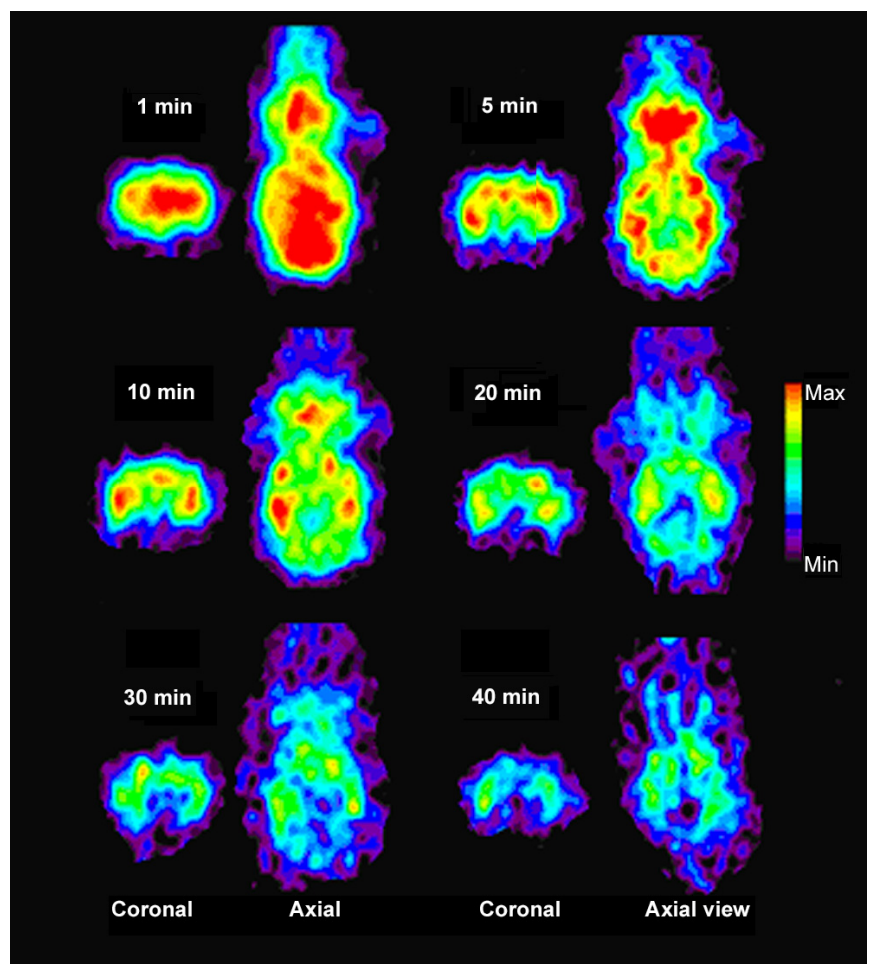

Fig. 10. To investigate preliminary imaging characteristics of (S,S,S)-2-(2-

carboxycyclopropyl)-2-(3-[11C]methoxyphenethyl) glycine dimethyl ester ([11C]CMG) 0.4-0.5 $\mathrm{mCi}$ of $\left[{ }^{11} \mathrm{C}\right] \mathrm{CMG}$ was administered iv. into the anesthetized (isoflurane $1.5 \%$ with $\mathrm{O} 2$ flow of $1 \mathrm{~L} / \mathrm{min}$ ) rats (male Spraque Dawley) in a microPET scanner (P4, Concord Microsystems). Dynamic volumetric data were acquired in 6 rats for $60 \mathrm{~min}$. Fast reversible binding was observed in several cortical areas, hippocampus, striatum and olfactory bulb, the sites which are known to express group II mGluRs. The maximum binding (1.1-1.6\% of the injected dose per $\mathrm{cm} 3$ ) was observed $2 \mathrm{~min}$ after administration. These data provide a foundation for future development of specific PET imaging ligands for group II mGluRs. Coronal and axial slices of $\left.{ }^{11} \mathrm{C}\right] \mathrm{CMG}$ distribution in the rat brain from $1 \mathrm{~min}$ till $40 \mathrm{~min}$ after administration of the radioligand are illustrated. Color coded images are normalized to each other and correspond the acquisition time of $1 \mathrm{~min}$ at the same midbrain level (coronal slice at bregma $-1.6 \mathrm{~mm}$; axial slice at bregma $-5.4 \mathrm{~mm}$ ). 


\subsubsection{Allosteric modulators for mGluR3}

Eli Lilly and Company reported the first series of compounds, 1-(heteroaryl)-3-(2,4dichlorobenzyl)amino-pyrolidine, acting as mGluR3 negative allosteric modulators (Britton et al., 2006). Figure 11 shows the chemical structures of two most potent ligands reported in the patent. Compounds, 94 and 95, have an $\mathrm{IC}_{50}$ value of $77 \mathrm{nM}$, which is insufficient for in vivo detection of the receptors. Further SAR studies are needed to find more potent ligands. No PET radioligands have been identified for mGluR3 so far.
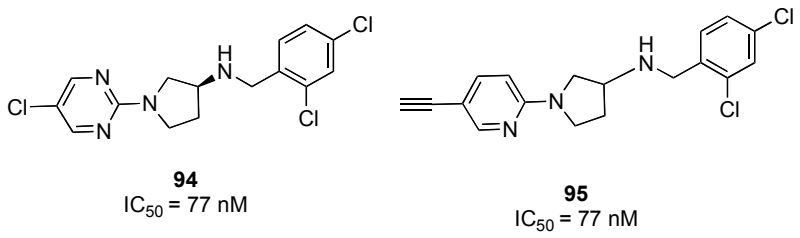

Fig. 11. Chemical structures of mGluR3 negative allosteric modulators

\subsection{Allosteric modulators and radiotracers for Group III mGluRs}

Group III metabotropic glutamate receptors are mGluR4, mGluR6, mGluR7 and mGluR8. There is no publication reporting mGluR6 allosteric ligands.

\subsubsection{Allosteric modulators for mGluR4}<smiles>O=C(Nc1ccccc1)C1CC2Oc3ccccc3/C(=N/O)C21</smiles>

96

(-)-PHCCC

$\mathrm{EC}_{50}: 4.7 \mathrm{mM}$<smiles></smiles>

97

VU0359516 $\mathrm{EC}_{50}: 380 \mathrm{nM}$<smiles>O=C(O)[C@H]1CCCC[C@H]1C(=O)Nc1cc(Cl)cc(Cl)c1</smiles>

8 VU0155041
$\mathrm{EC}_{50}: 0.75 \mathrm{mM}$

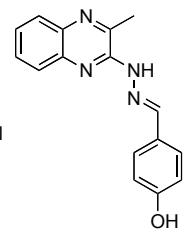

99 VU0001171 $\mathrm{EC}_{50}: 0.65 \mathrm{mM}$

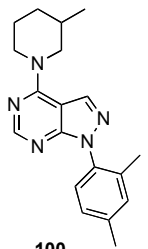

100

Vu0080241

$\mathrm{EC}_{50}: 5.0 \mathrm{mM}$

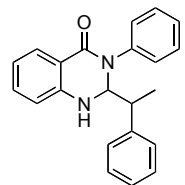

101

VU0092145 $\mathrm{EC}_{50}: 3.0 \mathrm{mM}$

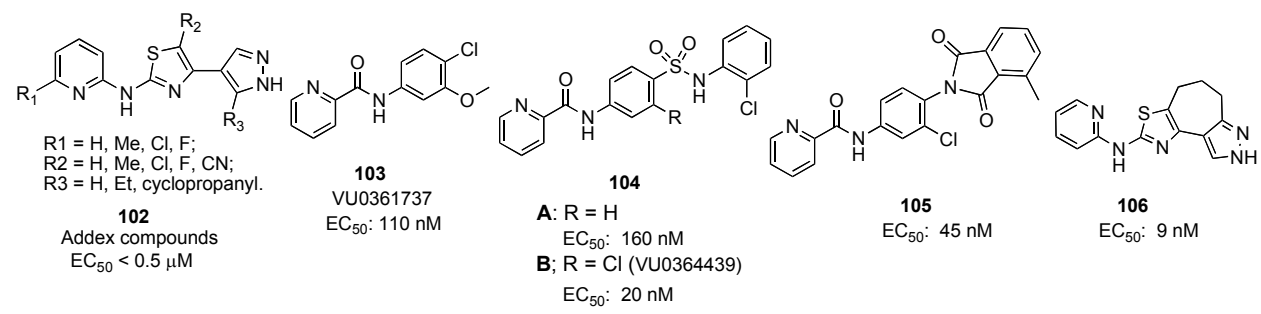

Fig. 12. Chemical structures of mGluR4 positive allosteric modulators

MGluR4 has received much attention lately due to its implication in several diseases, such as PD, epilepsy, and anxiety. There has been substantial progress in identifying positive allosteric modulators for mGluR4. The compound PHCCC (96, Fig. 12), a partial selective mGluR4 potentiator, has been studied for many years. Unfortunately PHCCC and other early disclosed mGluR4 PAMs such as 98-101 (Fig.12) are deficient in their BBB penetration (Engers et al., 2009). The potencies of these compounds are also relatively low (EC50: 0.65 - 
$5.0 \mu \mathrm{M})$ and SAR studies around these structures have given 'flat' results. Addex Pharma disclosed a series of heteroaromatic compounds (102 in Fig. 12) as positive allosteric modulators for mGluR4, with many compounds having $\mathrm{EC}_{50}<0.5 \mu \mathrm{M}$ (Bolea \& Celanire, 2009). However, no other information was reported about these compounds.

Two research groups; Addex Pharma (Bolea, 2009) and Vanderbilt University (Engers et al., 2009), have independently disclosed a series of small arylamide compounds as a new class of mGluR4 PAMs. Engers et al (Vanderbilt University) found from a high-throughput screening that there were a number of small arylamide compounds having mGluR4 PAM activity (Engers et al., 2009). They reported studies on SAR and in vitro and in vivo pharmacokinetic parameters in rat. The most potent compound in this series was $\mathbf{1 0 3}$ shown in Fig. 12. Researchers at Merck presented two new compounds, 104A and 105, with improved activity (Reynolds, 2008). Engers et al. further studied SAR of 4(phenylsulfamoyl)phenylacetamide derivatives and found that 104B was the most potent $(19.8 \mathrm{nM})$ mGluR4 positive allosteric modulator reported to date (Engers et al., 2010). Doller and co-workers (Lundbeck Research USA) have recently reported on a series of tricyclic thiazolopyrazole derivatives including compound 106, which was identified as a very potent and orally available compound with excellent brain penetration and good physicochemical properties (Hong et al., 2011).

\subsubsection{PET imaging studies of mGluR4 expression}

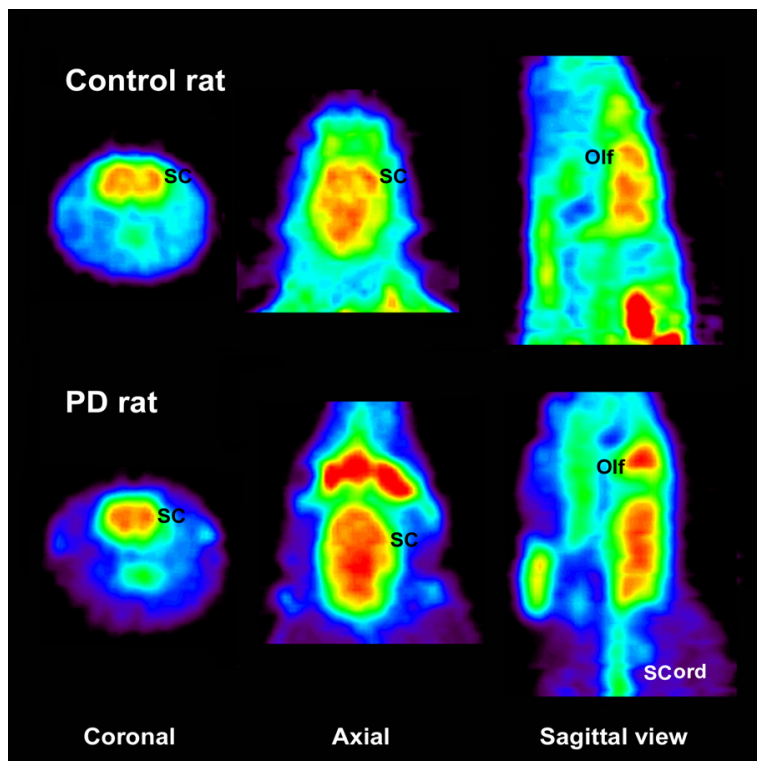

Fig. 13. Distribution of [11C]methyl-PHCCC between 10-20 min after administration of radioligand in a control $(1.2 \mathrm{mCi}$ iv. $)$ and $\mathrm{PD}(1.1 \mathrm{mCi}$ iv.) rat brain. Coronal and axial views localize cortex at the level of S1 and S2 areas. It is noticeable that the accumulation of [11C]methyl-PHCCC is enhanced in PD rat in the areas of subthalamic nucleus and spinal cord. The motor neurons in the ventral horn in the spinal cord express mGluR4 and the observed enhanced accumulation of mGluR4 ligand, $\left.{ }^{11} \mathrm{C}\right]$ methyl-PHCCC is an indication of excess glutamate. This is the first time, when this aspect has been demonstrated in vivo in a PD model. 


\subsubsection{Allosteric modulators for mGluR7}

It is reported that mGluR7 is widely expressed in the central nervous system and is primarily located on presynaptic terminals in brain regions such as the hippocampus, amygdala, and locus coeruleus. Mitsukawa et al. developed the first selective allosteric agonist of mGluR7, AMN082 (107), which has an $\mathrm{EC}_{50}$ value of $64-290 \mathrm{nM}$ and it is brain penetrating (Mitsukawa, 2005). However, converting it to a PET tracer is not straightforward. Researchers of Banyu Pharmaceutical Co reported a series of isoxazolopyridone derivatives as allosteric mGluR7 antagonists (Suzuki et al., 2007b). Compound MDIP (108) that was identified by random screening displayed mGluR7 antagonistic activity $\left(\mathrm{IC}_{50}=20 \mathrm{nM}\right)$ and had no detectable activity on other mGluRs at 1000 nM. However, MDIP showed poor metabolic stability (predicted $\mathrm{F}_{\mathrm{H}}: 34 \%$ ) on rat hepatocyte assay and low aqueous solubility $(0.17 \mu \mathrm{g} / \mathrm{mL}, \mathrm{pH} 7.4)$. It is assumed that poor metabolic stability and low aqueous solubility may be due to its high lipophilicity ( $\left.\log \mathrm{D}_{7.4}: 3.5\right)$. Recently, Nakamura et al. have identified some isoxazolopyridone derivatives with potent mGluR7 antagonistic activity and metabolic stability, in which MMPIP (109) with improved physicochemical properties and metabolic stability showed good oral bioavailability and brain penetrability in rats (Nakamura et al., 2010).
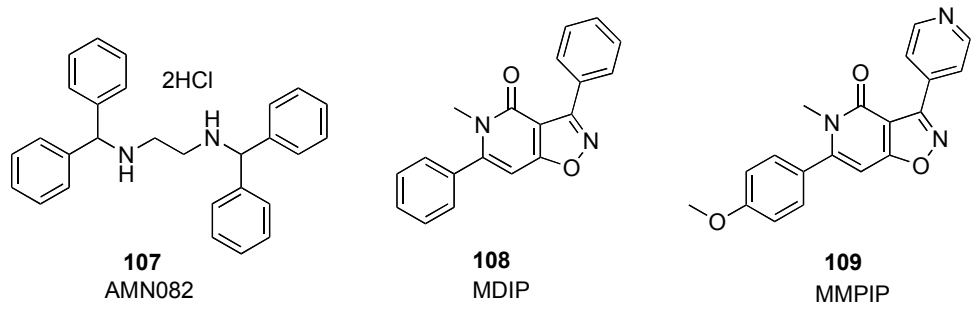

Fig. 14. Chemical structures of mGluR7 modulators

\subsubsection{Allosteric modulators for mGluR8}

Recently, AstraZeneca developed a positive allosteric modulator for mGluR8 (Duvoisin et al., 2010; Duvoisin et al., 2011). The compound AZ12216052 as injected into the amygdale, reduced measures of anxiety. There is no PET ligand available and AZ12216052 does not cross blood brain barrier.

\section{Conclusion}

Glutamate is an interesting transmitter since it can participate also on glutamate metabolism to be converted to glutamine and its function as a neurotransmitter can be investigated based on its receptor functions. To understand the diverse physiological effects of glutamate it is important to know molecular identity of mGluRs expressed in distinct subpopulations of neurons. For instance, group I mGluRs are coupled to phospholipase C and subsequent production of inositol triphosphates and induces intracellular calcium release in Purkinje cells and hippocampal CA1 neurons, but the same receptor types are also coupled to inhibition of voltage-dependent calcium channel in hippocampal neurons without intracellular diffusible messengers (Choi \& Lovinger, 1996). Group II mGluRs can be coupled to inhibition of cyclic AMP cascade in neural and glial cells while they are also linked to rapid-onset regulation of various channels including calcium channels and G- 
protein. The group III mGluRs-mediated effect is inhibition of neurotransmission through suppression of presynaptic voltage-dependent calcium channels (Pekhletski et al., 1996). This basic functional information of mGluRs has been obtained with in situ hybridization, immunohistochemistry and ex vivo studies with tritium labeled antibodies. While ex vivo studies can provide accurate endpoint information in steady state, they cannot provide information of the active inhibitory or stimulating effects in the system or interplay with other systems. To obtain functional information in real time, the investigation has to be done by using in vivo imaging methods. However, a lack of specific agonists and antagonists has limited the precise characterization of the role of individual metabotropic glutamate receptors in glutamatergic neurotransmission and hampered progress in identifying the physiological and pathological roles of mGluRs in vivo.

Recently, the modern computational chemistry has opened a wide range of technical approaches to design and construct molecules for imaging and to simulate their molecular targets. This technology has been used to design molecules for tracking different mGluRs. Especially, approach of allosteric compounds relies on sophisticated design of threedimensional arrangement of the tracer molecules responsible for the biological activity. Pharmacophore models can be constructed based on known biological activity. Design of novel allosteric modulators is an iterative process where structure-activity relationship information generated in the biological assays guides how to make structural alternations towards the optimal compound. Recently several non-competitive structurally diverse mGluR ligands have been published. These ligands, positive, negative and neutral modulators, bind to the allosteric binding sites located in the seven strand transmembrane domain. Based on these modulators, a number of radiotracers useful for imaging specific metabotropic glutamate receptors have been developed and their in vivo biological properties have been characterized.

Development of metabotropic glutamate receptor ligands will open a new perspective for molecular imaging. Modulation of receptor functions might be used as diagnostic tools as well as to follow progression/regression of neural diseases. Presently, three mGluR ligands have been used in human studies. They are developed as negative allosteric modulators for mGluR5. For example, concerning PD, the death of dopamine neurons in the substantia nigra pars compacta causes a loss of dopamine in the basal ganglia. Dopamine modulation of neurotransmission in the striatum and other basal ganglia structures is crucial to gate cortical and thalamic excitatory input through the direct and indirect pathways. By using in vivo PET imaging studies and $\left[{ }^{18} \mathrm{~F}\right] \mathrm{FPEB}$ we have found an upregulation of mGluR5 expression following dopamine denervation in animal models of PD (Figures 7 \& 13), which probably represents a local compensatory mechanism, directed to dampen an excessive excitability of striatopallidal neurons. Drugs targeting the mGluR5 might provide new approaches by selectively reducing glutamate transmission in the areas where it is abnormally enhanced. In addition, we and others have found enhanced mGluR5 expression in several brain areas related to the indirect pathway in models of L-DOPA induced dyskinesias and some studies have shown promising therapeutic results after using mGluR5 antagonists. In gut glutamate is the main energy source and its neurotransmission is conducted by vagal afferents. The gut expresses also mGlu5 receptors and we have localized them with [18F]FPEB. This phenomenon has raised a hypothesis that gut-brain axis as well as interplay with dopamine transmission might contribute to obesity.

Even mGlu2 receptors had the earliest interest as targets for drug development and Eli Lilly developed several potent ligands targeted to mGluR2 there is not yet any specific allosteric 
modulators available for imaging purposes of mGluR2 function. The earlier compounds were missing receptor selectivity and sensitivity for imaging purposes since sequence similarity at the orthosteric binding site to which endogenous agonists bind.

Present application of glutamate transmission has evoked an active drug development especially to develop allosteric modulators for neurodegenerative disorders, pain and schizophrenia. It should be noted that these disorders are affected also by modulation of dopaminergic system supporting hypothesis of interplay of these powerful transmitter systems. Future pharmacological and imaging studies will show which specific ligands acting at individual receptor subtypes could be used as sensitive indicators for diagnostic imaging. Therefore, there is an urgent need for development of allosteric modulators as imaging ligands for different of mGluRs for human use.

\section{Acknowledgement}

This work was supported by the NIH grant NIBIB-EB12864 to A-LB.

\section{References}

Aguirre, J. A., Andbjer, B., Gonzalez-Baron, S., Hansson, A., Stromberg, I., et al. (2001). Group I mGluR antagonist AIDA protects nigral DA cells from MPTP-induced injury. NeuroReport, Vol. 12, pp. 2615-7

Ametamey, S. M., Kessler, L., Honer, M., Auberson, Y., Gasparini, F., Schubiger, P. A. (2003). Synthesis and evaluation of [11C]MFPEP as a PET ligand for imaging the metabotropic glutamate receptor subtype 5 (mGluR5). J. Label. Compd. Radiopharm., Vol. 46, pp. S188

Ametamey, S. M., Kessler, L. J., Honer, M., Wyss, M. T., Buck, A., et al. (2006). Radiosynthesis and preclinical evaluation of ${ }^{11} \mathrm{C}-\mathrm{ABP} 688$ as a probe for imaging the metabotropic glutamate receptor subtype 5. J. Nucl. Med., Vol. 47, pp. 698-705

Annoura, H., Fukunaga, A., Uesugi, M., Tatsuoka, T., Horikawa, Y. (1996). A novel class of antagonists for metabotropic glutamate receptors, 7-(hydroxyimino) cyclopropa[b]chromen-1a-carboxylates. Bioorg Med chem Lett, Vol. 6, pp.763-6

Bach, P., Nilsson, K., Svensson, T., Bauer, U., Hammerland, L., et al. (2006). Structureactivity relationships for the linker in a series of pyridinyl-alkynes that are antagonists of the metabotropic glutamate receptor 5 (mGluR5). Bioorg. Med. Chem. Lett., Vol. 16, pp. 4788-91

Barda, D A., Wang, Z-Q., Britton, T. C., Henry, S. S., Jagdmann, G. E., et al. (2004). SAR study of a subtype selective allosteric potentiator of metabotropic glutamate 2 receptor, N-(4-phenoxyphenyl)-N-(3-pyridinylmethyl)ethanesulfonamide. Bioorg. Med. Chem. Lett., Vol. 14, pp. 3099-102

Baumann, C., Mu, L., Johannsen, S., Honer, M., Schubiger, P., Ametamey, S. (2010a). Structure-activity relationships of fluorinated (E)-3-((6-methylpyridin-2-yl)ethynyl) cyclohex-2-enone-O-methyloxime (ABP688) derivatives and the discovery of a high affinity analogue as a potential candidate for imaging metabotropic glutamate recepors subtype 5 (mGluR5) with positron emission tomography (PET). J. Med. Chem., Vol. 53, pp. 4009-17 
Baumann, C., Mu, L., Wertli, N., Krämer, S., Honer, M, et al. (2010b). Syntheses and pharmacological characterization of novel thiazole derivatives as potential mGluR5 PET ligands. Bioorg. Med. Chem., Vol. 18, pp. 6044-54

Berry-Kravis, E., Hessl, D., Coffey, S., Hervey, C., Schneider, A., et al. (2009). A pilot open label, single dose trial of fenobam in adults with fragile $\mathrm{X}$ syndrome. J. Med. Genet., Vol 46, pp. 266-71

Bessis, A-S., Bonnet, B., Le Poul, E., Rocher, J-P., Epping-Jordan, M. (2005). Application: WO Patent No. 2004-IB3822 2005044797

Bhave, G., Karim, F., Carlton, S., Gereau, R. (2001). Peripheral group I metabotropic glutamate receptors modulate nociception in mice. Nat. Neurosci., Vol. 4, pp. 417-23

Black, Y., Xiao, D., Pellegrino, D., Kachroo, A., Brownell, A., Schwarzschild, M. (2010). Protective effect of metabotroic glutamate mGluR5 receptor elimination in a 6hydroxydopamine model of Parkinson's disease. Neurosci. Lett., Vol. 486, pp. 161-5

Blakely, R. (2001). Neurobiology. Dopamine's reversal of fortune. Science, Vol 293, pp. 2407-9

Bliss, T., Collingridge, G. (1993). A synaptic model of memory: long-term potentiation in the hippocampus. Nature, Vol 361, pp. 31-9

Bolea, C. (2009). Application: WO Patent No. 2008-EP59043 2009010454

Bolea, C., Celanire, S. (2009). WO Patent No. 2009/010455

Bonnefous, C., Vernier, J-M., Hutchinson, J. H., Gardner, M. F., Cramer, M., et al. (2005). Biphenyl-indanones: Allosteric potentiators of the metabotropic glutamate subtype 2 receptor. Bioorg. Med. Chem. Lett., Vol. 15, pp. 4354-8

Britton, T., Dehlinger, V., Dell, C., Dressman, B., Myers, J., Nisenbaum, S. (2006). PCT Int Appl 2006; WO 2006/044454.

Burdi, D., Hunt, R., Fan, L., Hu, T., Wang, J., et al. (2010). Design, synthesis, and structureactivity relationships of novel bicyclic azole-amines as negative allosteric modulators of metabotropic glutamate receptor 5. J. Med. Chem., Vol. 53, pp. 710718

Cai, L., Lu, S., Pike, V. (2008). Chemistry with [18F]fluoride ion. Eur. J. Org. Chem., Vol. 73, pp. 2853-73

Calabresi, P., Centonze, D., Pisani, A., Bernardi, G. (1999). Metabotropic glutamate receptors and cell-type-specific vulnerability in the striatum: implication for ischemia and Huntington's disease. Exp Neurol, Vol. 158, pp. 97-108

Carcache, D., Vranesic, I., Blanz, J., Desrayaud, S., Fendt, M., Glatthar, R. (2011). Benzimidazoles as potent and orally active mGlu5 receptor antagonists with an improved PK profile. ACS Med. Chem. Lett., Vol. 2, pp. 58-62

Carroll, F. Y., Stolle, A., Beart, P. M., Voerste, A., Brabet, I., et al. (2001). BAY 36-7620: a potent non-competitive mGlu1 receptor antagonist with inverse agonist activity. Mol. Pharmacol., Vol. 59, pp. 965-73

Chen, H., Shockcor, J., Chen, W., Espina, R., Gan, L-S., Mutlib, A. E. (2002). Delineating novel metabolic pathways of DPC 963, a non-nucleoside reverse transcriptase inhibitor, in rats. Characterization of glutathione conjugates of postulated oxirene and benzoquinone imine intermediates by LC/MS and LC/NMR. Chem. Res. Toxicol, Vol. 15, pp. 388-99

Choi, S., Lovinger, D. M. (1996). Metabotropic glutamate receptor modulation of voltagegated $\mathrm{Ca}^{2+}$ channels involves multiple receptor subtypes in cortical neurons. $J$ Neurosci, Vol. 16, pp. 36-45 
Conn, J., Pin, J. (1997). Pharmacology and functions of metabotropic glutamate receptors. Annu Rev Pharmacol Toxicol, Vol. 37, pp. 205-37

Cosford, N., Roppe, J., Tehrani, L., Schweiger, E. J., Seiders, T. J., Chaudary, A., Rao, S., Varney, M. A. (2003). [ $\left.{ }^{3} \mathrm{H}\right]-M e t h o x y m e t h y l-M T E P$ and $[3 \mathrm{H}]-$ methoxy-PEPy: potent and selective radioligands for the metabotropic glutamate subtype 5 (mGlu5) receptor. Bioorg. Med. Chem. Lett., Vol. 13, pp. 351-4

Cosford, N., Tehrani, L., Roppe, J., Schweiger, E., Smith, N., et al. (2003). 3-[(2-Methyl-1,3thiazol-4-yl)ethynyl]-pyridine: A potent and highly selective metabotropic glutamate subtype 5 receptor antagonist with anxiolytic activity. J. Med. Chem., Vol. 46, pp. 204-6

De Paulis, T., Hemstapat, K., Chen, Y., Zhang, Y., Saleh, S., et al. (2006). Substituent effects of $\mathrm{N}-(1,3-$ diphenyl-1H-pyrazol-5-yl)benzamides on positive allosteric modulation of the metabotropic glutamate-5 receptor in rat cortical astrocytes. J. Med. Chem., Vol. 49, pp. 3332-44

Dominguez, C., Prieto, L., Valli, M. J., Massey, S. M., Bures, M., et al. (2005). Methyl Substitution of 2-Aminobicyclo[3.1.0]hexane 2,6-Dicarboxylate (LY354740) Determines Functional Activity at Metabotropic Glutamate Receptors: Identification of a Subtype Selective mGlu2 Receptor Agonist. J. Med. Chem., Vol. 48 , pp. $3605-12$

Duplantier, A. J., Efremov, I., Candler, J., Doran, A. C., Ganong, A. H., et al. (2009). 3-Benzyl1,3-oxazolidin-2-ones as mGluR2 positive allosteric modulators: Hit-to lead and lead optimization. Bioorg. Med. Chem. Lett., Vol. 19, pp. 2524-9

Duvoisin, R. M., Pfankuch, T., Wilson, J. M., Grabell, J., Chhajlani, V., et al. (2010). Acute pharmacological modulation of mGluR8 reduces measures of anxiety. Behav. Brain Res., Vol. 212, pp. 168-73

Duvoisin, R. M., Villasana, L., Davis, M. J., Winder, D. G., Raber, J. (2011). Opposing roles of mGluR8 in measures of anxiety involving non-social and social challenges. Behav. Brain Res., Vol. 221, pp. 50-4

Efremov, I., Rogers, B., Duplantier, A., Zhang, L., Maklad, N. (2008). WO Patent No. 2008/012622

Emmitte, K. A. (2011). Recent advances in the design and development of novel negative allosteric modulators of mGlu5. ACS Chem. Neurosci., ACS ASAP

Engers, D., Gentry, P., Williams, R., Bolinger, J., Weaver, D., et al. (2010). Synthesis and SAR of novel, 4-(phenylsulfamoyl)phenylacetamide mGlu4 positive allosteric modulators (PAMs) identified by functional high-throughput screening (HTS). Bioorg. Med. Chem. Lett., Vol. 20, pp. 5175-8

Engers, D., Niswender, C., Weaver, C., Jadhav, S., Menon, U., et al. (2009). Synthesis and evaluation of a series of heterobiarylamides that are centrally penetrant metabotropic glutamate receptor 4 (mGluR4) positive allosteric modulators (PAMs). J. Med. Chem., Vol. 52, pp. 4115-8

Fell, M. J., Witkin, J. M., Falcone, J. F., Katner, J. S., Perry, K. W., et al. (2011). N-(4-((2(trifluoromethyl)-3-hydroxy-4-(isobutyryl)phenoxy)methyl)benzyl)-1-methyl-1Himidazole-4-carboxamide (THIIC), a novel metabotropic glutamate 2 potentiator with potential anxiolytic/antidepressant properties: in vivo profiling suggests a link between behavioral and central nervous system neurochemical changes. $J$. Pharmacol. Exp. Ther., Vol. 336, pp. 165-77 
Fontana, E., Dansette, P., Poli, S. (2005). Cytochrome P450 Enzymes Mechanism Based Inhibitors: Common Sub-Structures and Reactivity. Curr. Drug. Metab., Vol. 6, pp. 413-54

Foroozesh, M., Primrose, G., Guo, Z., Bell, L., Alworth, W., Guengerich, F. (1997). Aryl acetylenes as mechanism-based inhibitors of cytochrome P450-dependent monooxygenase enzymes. Chem. Res. Toxicol., Vol. 10, pp. 91-102

Fujinaga, M., Yamasaki, T., Kawamura, K., Kumata, K., Hatori, A., et al. (2011). Synthesis and evaluation of 6-[1-(2-[18F]fluoro-3-pyridyl)-5-methyl-1H-1,2,3-triazol-4yl]quinoline for positron emission tomography imaging of the metabotropic glutamate receptor type 1 in brain. Bioorg. Med. Chem., Vol. 19, pp. 102-10

Galambos, J., Wágner, G., Nógrádi, K., Bielik, A., Molnár, L., et al. (2010). Carbamoyloximes as novel non-competitive mGlu5 receptor antagonists. Bioorg. Med. Chem. Lett., Vol. 20, pp. $4371-5$

Garbaccio, R. M., Brnardic, E. J., Fraley, M. E., Hartman, G. D., Hutson, P. H., et al. (2010). Discovery of oxazolobenzimidazoles as positive allosteric modulators for the mGluR2 receptor. ACS Med. Chem. Lett., Vol. 1, pp. 406-10

Gasparini, F., Andres, H., Flor, P. J., Heinrich, M., Inderbitzin, W., et al. (2002). [ ${ }^{3 \mathrm{H}]-\mathrm{M}-}$ MPEP, a potent, subtype-selective radioligand for the metabotropic glutamate receptor subtype 5. Bioorg. Med. Chem. Lett., Vol. 12, pp. 407-9

Gasparini, F., Lingenhohl, K., Stoehr, N., Flor, P. J., Heinrich, M., et al. (1999). 2-Methyl-6(phenylethynyl)-pyridine (MPEP), a potent, selective and systemically active mGlu5 receptor antagonist. Neuropharmacology, Vol. 38, pp. 1493-503

Gatti McArthur, S., Goetschi, E. Palmer, W. S., Wichmann, J., Woltering, T. J. (2006a). Application: WO Patent No. 2006-EP2334 2006099972

Gatti McArthur, S., Goetschi, E., Wichmann, J. (2006b). WO Patent No. 2006/082002

Gatti McArthur, S., Goetschi ,E., Wichmann, J., Woltering, T. J. (2006c). Application: WO Patent No. 2006-EP940 2006084634

Gatti Mcarthur, S., Goetschi, E., Wichmann, J., Woltering, T. J. (2007). Application: WO Patent No. 2007-EP52560 2007110337

Granberg, K., Holm, B. (2009). Application: WO Patent No. 2008-SE51195 2009054792

Granberg, K., Holm, B. (2010). Application: WO Patent No. 2010-SE50440 2010123451

Guitart, X., Khurdayan, V. (2005). Metabotropic glutamate receptors as therapeutic targets. Drug News Perspect, Vol. 18, pp. 587-93

Hamill, T. G., Krause, S., Ryan, C., Bonnefous, C., Govek, S., et al. (2005). Synthesis, characterization, and first successful monkey imaging studies of metabotropic glutamate receptor subtype 5 (mGluR5) PET radiotracers. Synapse (Hoboken, NJ, U. S.), Vol. 56, pp. 205-16

Henrich, M., Weil, T., Mueller, S., Nagel, J., Gravius, A., et al. (2009). Application: WO Patent No. 2009-EP616 2009095254

Honer, M., Stoffel, A., Kessler, L., Schubiger, P., Ametamey, S. (2007). Radiolabeling and in vitro and in vivo evaluation of [(18)F]-FE-DABP688 as a PET radioligand for the metabotropic glutamate receptor subtype 5. Nucl. Med. Biol., Vol. 34, pp. 973-80

Hong, S.-P., Liu, K. G., Ma, G., Sabio, M., Uberti, M. A., Bacolod, M. D., Peterson, , J., Zou, Z. Z., Robichaud, A. J., and Doller, D. (2011) Tricyclic thiazolopyrazole derivatives as novel, potent, selective, and orally available metabotropic glutamate receptor 4 positive allosteric modulators. J. Med. Chem. Vol 54, pp. 5070-81 
Hostetler, E. D., Eng, W., Joshi, A. D., Sanabria-Bohorquez, S., Kawamoto, H., et al. (2011). Synthesis, characterization, and monkey PET studies of [18F]MK-1312, a PET tracer for quantification of mGluR1 receptor occupancy by MK-5435. Synapse (Hoboken, NJ, U. S.), Vol. 65, pp. 125-35

Huang, Y., Narendran, R., Bischoff, F., Guo, N., Zhu, Z., et al. (2005). A positron emission tomography radioligand for the in vivo labeling of metabotropic glutamate 1 receptor: (3-ethyl-2-[11C]methyl-6-quinolinyl)(cis- 4methoxycyclohexyl)methanone. J. Med. Chem., Vol. 48, pp. 5096-9

Hwang, D-R., Narendran, R., Huang, Y., Slifstein, M., Talbot, P. S., et al. (2004). Quantitative analysis of (-)-N-11C-propyl-norapomorphine in vivo binding in nonhuman primates. J. Nucl. Med., Vol. 45, pp. 338-46

Imogai, H. J., Cid-Nunez, J. M., Andres-Gil, J. I., Trabanco-Suarez, A. A., OyarzabalSantamarina J., et al. (2007). Application: WO Patent No. 2007-EP52442 2007104783

Isaac, M., Waallberg, A. (2009). Application: WO Patent No. 2008-SE51197 2009054794

Iso, Y., Grajkowska, E., Wroblewski, J. T., Davis, J., Goeders, N. E., et al. (2006). Synthesis and structure-activity relationships of 3-[(2-Methyl-1,3-thiazol-4yl)ethynyl]pyridine analogues as potent, noncompetitive metabotropic glutamate receptor subtype 5 antagonists; Search for cocaine medications. J. Med. Chem., Vol. 49, pp. 1080-100

Jimenez, H. N., Li, G., Doller, D., Grenon, M., White, A. D., et al. (2010). Application: WO Patent No. 2009-US50934 2010011570

Johnson, M. P., Baez, M., Jagdmann, G. E., Jr., Britton, T. C., Large, T. H., et al. (2003). Discovery of allosteric potentiators for the metabotropic glutamate 2 receptor: synthesis and subtype selectivity of N-(4-(2-Methoxyphenoxy)phenyl)-N-(2,2,2trifluoroethylsulfonyl)pyrid-3-ylmethylamine. J. Med. Chem., Vol. 46, pp. 3189-92

Karakossian, M., Otis, T. (2004). Excitation oof cerebellar interneurons by group I metabotropic glutamate receptors. J. Neurophysiol., Vol. 92, pp. 1558-65

Kew, J. (2004). Positive and negative allosteric modulation of metabotropic glutamate receptors: emerging therapeutic potential. Pharmacol Ther, Vol. 104, pp. 233-44

Kew, J., Kemp, J. (2005). Ionotropic and metabotropic glutamate receptor structure and pharmacology. Psychopharmacology, Vol. 179, pp. 4-29

Keyvani, K., Bosse, F., Reinecke, S., Paulus, W., Witte, O. (2001). Postlesional transcriptional regulation of metabotropic glutamate receptors: implications for plasticity and excitotoxicity. Acta Neuropathol, Vol. 101, pp. 79-84

Keywood, C., Wakefield, M., Tack, J. (2009). A proof-of-concept study evaluating the effect of ADX 10059, a metabotropic glutamate receptor-5 negative allosteric modulator, on acid exposure and symptoms in gastro-oesophageal reflux disease. Gut, Vol. 58, pp. 1192-9

Kinney, G. G., O'Brien, J. A., Lemaire, W., Burno, M., Bickel, D. J., et al. (2005). A novel selective positive allosteric modulator of metabotropic glutamate receptor subtype 5 has in vivo activity and antipsychotic-like effects in rat behavioral models. $J$. Pharmacol. Exp. Ther., Vol. 313, pp. 199-206

Knoflach, F., Mutel, V., Jolidon, S., Kew, J. N. C., Malherbe, P., et al. (2001). Positive allosteric modulators of metabotropic glutamate 1 receptor: characterization, mechanism of action, and binding site. Proc. Natl. Acad. Sci. U. S. A., Vol. 98, pp. 13402-7 
Kohara, A., Takahashi, M., Yatsugi, S-i., Tamura, S., Shitaka, Y., et al. (2008). Neuroprotective effects of the selective type 1 metabotropic glutamate receptor antagonist YM-202074 in rat stroke models. Brain Res., Vol. 1191, pp. 168-79

Kornhuber, J., Weller, M. (1997). Psychotogenicity and N-methyl-D-aspartate receptor antagonism: implications for neuroprotective pharmacotherapy. Biol Psychiatry, Vol. 41, pp. 135-44

Kozikowski, A. P., Steensma, D., Araldi, G. L., Tueckmantel, W., Wang, S., et al. (1998). Synthesis and biology of the conformationally restricted ACPD analog, 2aminobicyclo[2.1.1] hexane-2,5-dicarboxylic acid-I, a potent mGluR agonist. J. Med. Chem., Vol. 41, pp. 1641-50

Krause, S., Hamill, T., Seiders, T., et al. (2003). In vivo characterizations of PET ligands for the mGluR5 receptor in rhesus monkey. Mol. Imaging Biol., Vol. 5, pp. 166

Kulkarni, S., Zou, M-F., Cao, J., Deschamps, J., Rodriguez, A., et al. (2009). Structure-activity relationships comparing $\mathrm{N}$-(6-Methylpyridin-yl)-substituted aryl amides to 2methyl-6-(substituted-arylethynyl)pyridines or 2-methyl-4-(substituted-arylethynyl thiazoles as novel metabotropic glutamate receptor subtype 5 antagonists. J. Med. Chem., Vol. 52, pp. 3563-75

Kumar, J. S. D., Majo, V. J., Hsiung, S-C., Millak, M. S., Liu, K-P., et al. (2006). Synthesis and in vivo validation of [O-methyl-11C]-2-[4-[4-(7-methoxy-1-naphthalenyl)-1piperazinyl]butyl]-4-methyl-2H-[1,2,4]triazine-3,5-dione: A novel 5-HT1A receptor agonist positron emission tomography ligand. J. Med. Chem., Vol. 49, pp. 125-34

Lavreysen, H., Janssen, C., Bischoff, F., Langlois, X., Leysen, J., Lesage, A. (2003). [3H]R214127: a novel high-affinity radioligand for the mGlu1 receptor reveals a common binding site shared by multiple allosteric antagonists. Mol. Pharmacol., Vol. 63, pp. 1082-93

Lavreysen, H., Pereira, S., Leysen, J., Langlois, X., Lesage, A. (2004a). Metabotropic glutamate 1 receptor distribution and occupancy in the rat brain: a quantitative autoradiographic study using [ $\left.{ }^{3} \mathrm{H}\right] \mathrm{R} 214127$. Neuropharmacology, Vol. 46, pp. 609-19

Lavreysen, H., Wouters, R., Bischoff, F., Nobrega Pereira, S., Langlois, X., et al. (2004b). JNJ16259685, a highly potent, selective and systemically active mGlu1 receptor antagonist. Neuropharmacology, Vol. 47, pp. 961-72

Layton, M. (2005). Subtype-selective noncompetitive modulators of metabotropic glutamate receptor subtype 1 (mGluR1). . Curr Top Med Chem, Vol. 5, pp. 859-67

Lesage, A. (2004). Role of group I metabotropic glutamate receptors mGlu1 and mGlu5 in Nociceptive signalling. Curr. Neuropharmacology, Vol. 2, pp. 363-93

Lindsley, C., Bates, B., Menon, U., Jadhav, S., Kane, A., et al. (2011). (3-Cyano-5fluorophenyl)biaryl negative allosteric modulators of mGlu5: Discovery of a new tool compound with activity in the OSS mouse model of addiction. ACS Chem. Neurosci., Vol. 2, ASAP

Lindsley, C. W., Wisnoski, D. D., Leister, W. H., O'Brien, J. A., Lemaire, W., et al. (2004). Discovery of positive allosteric modulators for the metabotropic glutamate receptor subtype 5 from a series of N-(1,3-Diphenyl-1H- pyrazol-5-yl)benzamides that potentiate receptor function in vivo. J. Med. Chem., Vol. 47, pp. 5825-8

Litschig, S., Gasparini, F., Rueegg, D., Stoehr, N., Flor, P. J., et al. (1999). CPCCOEt, a noncompetitive metabotropic glutamate receptor 1 antagonist, inhibits receptor signaling without affecting glutamate binding. Mol. Pharmacol., Vol. 55, pp. 453-61 
Lucas, D., Newhouse, J. (1957). The toxic effect of sodium L-glutamate on the inner layers of the retina. AMA Arch Opthalmol, Vol. 58, pp. 193-201

Lucatelli, C., Honer, M., Salazar, J-F., Ross, T. L., Schubiger, P., Ametamey, S. (2009). Synthesis, radiolabeling, in vitro and in vivo evaluation of $\left[{ }^{18} \mathrm{~F}\right]-\mathrm{FPECMO}$ as a positron emission tomography radioligand for imaging the metabotropic glutamate receptor subtype 5. Nuclear Medicine and Biology, Vol. 36, pp. 613-22

Mabire, D., Coupa, S., Adelinet, C., Poncelet, A., Simonnet, Y., et al. (2005). Synthesis, structure-activity relationship, and receptor pharmacology of a new series of quinoline derivatives acting as selective, noncompetitive mGlu1 antagonists. J. Med. Chem., Vol. 48, pp. 2134-53

Malherbe, P., Kratochwil, N., Knoflach, F., Zenner, M-T., Kew, J. N. C., et al. (2003). Mutational analysis and molecular modeling of the allosteric binding site of a novel, selective, noncompetitive antagonist of the metabotropic glutamate 1 receptor. J. Biol. Chem., Vol. 278, pp. 8340-7

Mantell, S. J., Gibson, K. R., Osborne, S. A., Maw, G. N., Rees, H., et al. (2009). In vitro and in vivo SAR of pyrido[3,4-d]pyramid-4-ylamine based mGluR1 antagonists. Bioorg. Med. Chem. Lett., Vol. 19, pp. 2190-4

Marin, JC. A., Goadsby, P. J. (2010). Glutamatergic fine tuning with ADX-10059: a novel therapeutic approach for migraine? Expert Opin. Invest. Drugs, Vol. 19, pp. 555-61

Marino, M., Hess, J., Liverton, N. (2005). Targeting the metabotropic glutamate receptor mGluR4 for the treatment of diseases of the central nervous system. . Curr Top Med Chem, Vol. 5, pp. 885-95

Marino, M., Wittmann, M., Bradley, S., Hubert, G., Smith, Y., Conn, P. (2001). Activation of group I metabotropic glutamate receptors produces a direct excitation and disinhibition of GABAergic projection neurons in the substantia nigra pars reticulata. J Neurosci, Vol. 21, pp. 7001-12

Micheli, F., Di Fabio, R., Bordi, F., Cavallini, P., Cavanni, P., et al. (2003a). 2,4Dicarboxypyrroles as selective non-competitive mGLUR1 antagonists: further characterization of 3,5-dimethylpyrrole-2,4-dicarboxylic acid 2-propyl ester 4-(1,2,2trimethylpropyl) ester and structure-activity relationships. Bioorg. Med. Chem. Lett., Vol. 13, pp. 2113-8

Micheli, F., Di Fabio, R., Cavanni, P., Rimland, J.M., Capelli, A. M., et al. (2003b). Synthesis and pharmacological characterization of 2,4-dicarboxy-pyrroles as selective noncompetitive mGluR1 antagonists. Bioorg. Med. Chem., Vol. 11, pp. 171-83

Milbank, J., Knauer, C., Augelli-Szafran, C., Sakkab-Tan, A., Lin, K., et al. (2007). Rational design of 7-arylquinolines as non-competitive metabotropic glutamate receptor subtype 5 antagonists. Bioorg. Med. Chem. Lett., Vol. 17, pp. 4415-8

Miller, P., Long, N., Vilar, R., Gee, A. (2008). Synthesis of 11C, 18F, 15O, and 13N radiolabels for positron emission tomography. Angew. Chem. int. ed, Vol. 47, pp. 8998-9033

Mitsukawa, K,. Yamamoto, R., Ofner, S., Nozulak, J., Pescott, O., et al. (2005). A selective metabotropic glutamate receptor 7 agonist: activation of receptor signaling via an allosteric site modulates stress parameters in vivo. Proc Natl Acad Sci U S A, Vol. 102, pp. $18712-7$

Musachio, J., Ghose, S., Toyama, H., et al. (2003). Two potential mGluR5 PET radioligands, [11C]M-MPEP and [11C]Methoxy-PEPy - synthesis and initial PET evaluation in rats and monkeys in vivo. Mol. Imaging Biol., Vol. 5, pp. 168 
Mutlib, A., Lam, W., Atherton, J., Chen, H., Galatsis, P., Stolle, W. (2005). Application of stable isotope labeled glutathione and rapid scanning mass spectrometers in detecting and characterizing reactive metabolites. Rapid Commun. Mass Spectrom., Vol. 19, pp. 3482-92

Mutlib, A. E., Chen, H., Nemeth, G. A., Markwalder, J. A., Seitz, S. P., et al. (1999). Identification and characterization of efavirenz metabolites by liquid chromatography/mass spectrometry and high field NMR: species differences in the metabolism of efavirenz. Drug Metab. Dispos., Vol. 27, pp. 1319-33

Nakamura, M., Kurihara, H., Suzuki, G., Mitsuya, M., Ohkubo, M., Ohta, H. (2010). Isoxazolopyridone derivatives as allosteric metabotropic glutamate receptor 7 antagonists. Bioorganic \& Medicinal Chemistry Letters, Vol. 20, pp. 726-9

Niswender, C., Jones, C., Conn, P. (2005). New therapeutic frontiers for metabotropic glutamate receptors. Curr Top Med Chem, Vol. 5, pp. 847-57

O'Brien, J. A., Lemaire, W., Chen, T-B., Chang, R. S. L., Jacobson, M. A., et al. (2003). A family of highly selective allosteric modulators of the metabotropic glutamate receptor subtype 5. Mol. Pharmacol., Vol. 64, pp. 731-40

O'Brien, J., Lemaire, W., Wittmann, M. (2004). A novel selective allosteric modulator potentiates the activity of native metabotropic glutamate receptor subtype 5 in rat forebrain. J Pharmacol Exp Ther, Vol. 309, pp. 568-77

O'Neill, M. (2001). Pharmacology and neuroprotective actions of mGlu receptor ligands. . Dev Med Child Neurol Suppl, Vol. 86, pp. 13-5

Ohgami, M., Haradahira, T., Takai, N., Zhang, M-R. (2009). Eur. J. Nucl. Med. Mol. Imag., Vol. 36, pp. $\mathrm{S} 310$

Olive, M. F. (2009). Metabotropic glutamate receptor ligands as potential therapeutics for addiction. Curr. Drug Abuse Rev., Vol. 2, pp. 83-98

Oney, J. (1978). Neurotoxicity of excitatory amino acids. New York: Raven Press. 27 pp.

Ott, D., Floersheim, P., Inderbitzin, W., Stoehr, N., Francotte, E., et al. (2000). Chiral resolution, pharmacological characterization, and receptor docking of the noncompetitive mGlu1 receptor antagonist (+-)-2-hydroxyimino- 1a,2-dihydro-1H7-oxacyclopropa[b]naphthalene-7a-carboxylic acid ethyl ester. J. Med. Chem., Vol. 43, pp. 4428-36

Owen, D. R., Dodd, P. G., Gayton, S., Greener, B. S., Harbottle, G. W., et al. (2007). Structureactivity relationships of novel non-competitive mGluR1 antagonists: A potential treatment for chronic pain. Bioorg. Med. Chem. Lett., Vol. 17, pp. 486-90

Passchier, J., Gee, A., Willemsen, A., Vaalburg, W., Waarde, Av. (2002). Measuring drug related-receptor occupancy with positron emission tomography. Methods, Vol. 27, pp. 278-86

Patel, S., Hamill, T., Connolly, B., Jagoda, E., Li, W., Gibson, R. (2007). Species differences in mGluR5 binding sites in mammalian central nervous system determined using in vitro binding with [18F]F-PEB. Nuclear Medicine and Biology, Vol. 34, pp. 1009-17

Patel, S., Ndubizu, O., Hamill, T., Chaudhary, A., Burns, H. D., et al. (2005). Screening cascade and development of potential positron emission tomography radiotracers for mGluR5: in vitro and in vivo characterization. Mol Imaging Biol, Vol. 7, pp. 31423 
Pekhletski, R., Gerlai, R., Overstreet, L. S., et al. (1996). Impaired cerebellar synaptic plasticity and motor performance in mice lacking the mGluR4 subtype of metabotropic glutamate receptor. J Neurosci, Vol. 16, pp. 6364-73.

Pin, J., Duvoisin, R. (1995). Review: neurotransmitter receptors I. The metabotropic glutamate receptors: structure and functions. Neuropharmacology, Vol. 34, pp. 1-26

Pin, J-P., Galvez, T., Prezeau, L. (2003). Evolution, structure, and activation mechanism of family 3/C G-protein-coupled receptors. . Pharmacol Ther, Vol. 98, pp. 325-54

Popoli, P., Pezzola, A., Torvinen, M., et al. 2001. The selective mGlu(5) receptor agonist CHPG inhibits quinpirole-induced turning in 6-hydroxydopamine-lesioned rats and modulates the binding characteristics of dopamine $\mathrm{D}(2)$ receptors in the rat striatum: interactions with adenosine A(2a) receptors. Neuropsychopharmacology. Vol. 25, pp. 505-13

Porter, R. H. P., Jaeschke, G., Spooren, W., Ballard, T. M., Buttelmann, B., et al. (2005). Fenobam: A clinically validated nonbenzodiazepine anxiolytic is a potent, selective, and noncompetitive mGlu5 receptor antagonist with inverse agonist activity. $J$. Pharmacol. Exp. Ther., Vol. 315, pp. 711-21

Prabhakaran, J., Majo, V. J., Milak, M. S., Kassir, S. A., Palner, M., et al. (2010). Synthesis, in vitro and in vivo evaluation of [11C]MMTP: A potential PET ligand for mGluR1 receptors. Bioorg. Med. Chem. Lett., Vol. 20, pp. 3499-501

Prabhakaran, J., Parsey, R. V., Majo, V. J., Hsiung, S-C., Milak, M. S., et al. (2006). Synthesis, in vitro and in vivo evaluation of [O-methyl-11C] 2-\{4-[4-(3methoxyphenyl)piperazin-1-yl]-butyl\}-4-methyl-2H-[1,2,4]-triazine-3,5-dione: A novel agonist 5-HT1A receptor PET ligand. Bioorg. Med. Chem. Lett., Vol. 16, pp. 2101-4

Rao, A., Hatcher, J., Dempsey, R. (2000). Neuroprotection by group I metabotropic glutamate receptor antagonist in forebrain ischemia of gerbil. Neurosci Lett, Vol. 293, pp. 1-4

Reynolds, I. J. (2008). Metabotropic glutamate receptors as therapeutic targets in Parkinson's disease. 6th international meeting on metabotropic glutamate receptors. Taoromino, Sicily, Italy

Ritzen, A., Mathiesen, J., Thomsen, C. (2005). Molecular pharmacology and therapeutic prospects of metabotropic glutamate receptor allosteric modulators. Basic Clin Pharmacol Toxicol, Vol. 97, pp. 202-13

Rouse, S., Marino, M., Bradley, S., Award, H., Wittmann, M., Conn, P. (2000). Distribution and roles of metabotropic glutamate receptors in the basal ganglia motor circuit: implications for treatment of Parkinson's disease and related disorders. Pharmacol Ther, Vol. 88, pp. 427-35

Rudd, M., McCauley, J. (2005). Positive allosteric modulators of the metabotropic glutamate receptor subtype 2 (mGluR2). . Curr Top Med Chem, Vol. 5, pp. 869-84

Sanchez-Pernaute, R., Wang, J. Q., Kuruppu, D., Cao, L., Tueckmantel, W., et al. (2008). Enhanced binding of metabotropic glutamate receptor type 5 (mGluR5) PET tracers in the brain of parkinsonian primates. Neuroimage, Vol. 42, pp. 248-51

Sasikumar, T. K., Qiang, L., Burnett, D. A., Greenlee, W. J., Li, C., et al. (2010). A-ring modifications on the triazafluorenone core structure and their mGluR1 antagonist properties. Bioorg. Med. Chem. Lett., Vol. 20, pp. 2474-7 
Satoh, A., Nagatomi, Y., Hirata, Y., Ito, S., Suzuki, G., et al. (2009). Discovery and in vitro and in vivo profiles of 4-fluoro-N-[4-[6-(isopropylamino)pyrimidin-4-yl]-1,3thiazol-2-yl]-N-methylbenzamide as novel class of an orally active metabotropic glutamate receptor 1 (mGluR1) antagonist. Bioorg. Med. Chem. Lett., Vol. 19, pp. 5464-8

Schoepp, D., Goldsworthy, J., Johnson, B., Salhoff, C., Baker, S. (1994). 3,5dihydroxyphenylglycine is a highly selective agonist for phosphorinositide-linked metabotropic glutamate receptors in the rat hippocampus. J Neurochem, Vol. 1994, pp. 769-72

Schoepp, D. D., Jane, D. E., Monn, J. A. (1999). Pharmacological agents acting at subtypes of metabotropic glutamate receptors. Neuropharmacology, Vol. 38, pp. 1431-76

Sharma, S., Lindsley, C. (2007). A new high affinity PET tracer for the metabotropic glutamate receptor subtype 5 (mGluR5). Curr. Top. Med. Chem., Vol. 7, pp. 1541-2

Shigemoto, R., Mizuno, N. (2000). Metabotropic glutamate receptors - immunocytochemical and in situ hybridization analyses. Handbook Chemical Neuroanat., Vol. 18, pp. 63-98

Shimada, T., Murayama, N., Okada, K., Funae, Y., Yamazaki, H., Guengerich, F. P. (2007). Different mechanisms for inhibition of human cytochromes P450 1A1, 1A2, and 1B1 by polycyclic aromatic inhibitors. Chem. Res. Toxicol., Vol. 20, pp. 489-96

Sime'on, F., Brown, A., Zoghbi, S., Patterson, V., Innis, R., Pike, V. (2007). Synthesis and simple ${ }^{18}$ F-labeling of 3-fluoro-5-(2-(2-(fluoromethyl)thiazol-4-yl)ethynyl benzonitrile as a high affinity radioligand for imaging monkey brain metabotropic glutamate subtype-5 receptors with positron emission tomography. J. Med. Chem., Vol. 50, pp. 3256-66

Simonyi, A., Ngomba, R. T., Storto, M., Catania, M. V., Miller, L. A., et al. (2005). Expression of groups I and II metabotropic glutamate receptors in the rat brain during aging. Brain Res., Vol. 1043, pp. 95-106

Skerry, T., Genever, P. (2001). Glutamate signaling in non-neuronal tissues. Trends Pharmacol. Sci., Vol. 22, pp. 174-81

Slassi, A., Isaac, M., Edwards, L., Minidis, A., Wensbo, D., et al. (2005). Recent advances in non-competitive mGlu5 receptor antagonists and their potential therapeutic applications. Curr. Top. Med. Chem. (Sharjah, United Arab Emirates), Vol. 5, pp. 897911

Spanka, C., Glatthar, R., Desrayaud, S., Fendt, M., Orain, D., et al. (2010). Piperidyl amides as novel, potent and orally active mGlu5 receptor antagonists with anxiolytic-like activity. Bioorganic \& Medicinal Chemistry Letters, Vol. 20, pp. 184-8

Spooren, W., Ballard, T., Gasparini, F., Amalric, M., Mutel, V., Schreiber, R. (2003). Insight into the function of group I and group II metabotropic glutamate (mGlu) receptors: behavioural characterization and implications for the treatment of CNS disorders. Behav. Pharmacol., Vol. 14, pp. 257-77

Suzuki, G., Kawagoe-Takaki, H., Inoue, T., Kimura, T., Hikichi, H., et al. (2009). Correlation of receptor occupancy of metabotropic glutamate receptor subtype 1 (mGluR1) in mouse brain with in vivo activity of allosteric mGluR1 antagonists. J. Pharmacol. Sci. (Tokyo, Jpn.), Vol. 110, pp. 315-25

Suzuki, G., Kimura, T., Satow, A., Kaneko, N., Fukuda, J., et al. (2007a). Pharmacological characterization of a new, orally active and potent allosteric metabotropic glutamate receptor 1 antagonist, 4-[1-(2-fluoropyridin-3-yl)-5-methyl-1H-1,2,3- 
triazol-4-yl]-N-isopropyl-N-methyl-3,6-dihydropyridine-1(2H)-carboxamide (FTIDC). J. Pharmacol. Exp. Ther., Vol. 321, pp. 1144-53

Suzuki, G., Tsukamoto, N., Fushiki, H., Kawagishi, A., Nakamura, M., et al. (2007b). In vitro pharmacological characterization of novel isoxazolopyridone derivatives as allosteric metabotropic glutamate receptor 7 antagonists. J. Pharmacol. Exp. Ther., Vol. 323, pp. 147-56

Tanabe, Y., Nomura, A., Masu, M., Shigemoto, R., Mizuno, N., Nakanishi, S. (1997). Signal transduction, pharmacological properties, and expression patterns of two metabotropic glutamate receptors, mGluR3 and mGluR4. J Neurosci, Vol. 13, pp. 1372-8

Testa, B., Jenner, p. (1981). Inhibitors of Cytochrome P-450s and Their Mechanism of Action. Drug Metab. Rev., Vol. 12, pp. 1-117

Varnes, J., Marcus, A., Mauger, R., Throner, S., Hoesch, V., et al. (2011). Discovery of novel positive allosteric modulators of the metabotropic glutamate receptor 5 (mGlu5). Bioorganic \& Medicinal Chemistry Letters, Vol. 21, pp. 1402-6

Varney, M. A., Cosford, N. D., Jachec, C., Rao, S. P., Sacaan, A., et al. (1999). SIB-1757 and SIB-1893: selective, noncompetitive antagonists of metabotropic glutamate receptor type 5. J Pharmacol Exp Ther, Vol. 290, pp. 170-81

Wang, J-Q., Tueckmantel, W., Zhu, A., Pellegrino, D., Brownell, A-L. (2007a). Synthesis and preliminary biological evaluation of 3-[18F]fluoro-5-(2pyridinylethynyl)benzonitrile as a PET radiotracer for imaging metabotropic glutamate receptor subtype 5. Synapse (Hoboken, NJ, U. S.), Vol. 61, pp. 951-61

Wang, X., Kolasa, T., El Kouhen, O. F., Chovan, L. E., Black-Shaefer, C. L., et al. (2007b). Rapid hit to lead evaluation of pyrazolo[3,4-d]pyrimidin-4-one as selective and orally bioavailable mGluR1 antagonists. Bioorg. Med. Chem. Lett., Vol. 17, pp. 4303-7

Wichmann, J., Bleicher, K., Vieira, E., Woltering, T., Knoflach, F., Mutel, V. (2002). Alkyl diphenylacetyl, 9H-xanthene- and 9H-thioxanthene-carbonyl carbamates as positive allosteric modulators of mGlu1 receptors. Farmaco, Vol. 57, pp. 989-92

Williams, D. J., Lindsley, C. (2005). Discovery of positive allosteric modulators of metabotropic glutamate receptor subtype 5 (mGluR5). Curr Top Med Chem, Vol. 5, pp. 825-46

Williams, R., Manka, J., Rodriguez, A., Vinson, P., Niswender, C., et al. (2011). Synthesis and SAR of centrally active mGlu5 positive allosteric modulators based on an aryl acetylenic bicyclic lactam scaffold. Bioorg. Med. Chem. Lett., Vol. 21, pp. 1350-3

Wilson. A. A., McCormick, P., Kapur, S., Willeit, M., Garcia, A., et al. (2005). Radiosynthesis and evaluation of [11C]-(+)-4-propyl-3,4,4a,5,6,10b-hexahydro-2H-naphtho[1,2b] 11,4 ]oxazin-9-ol as a potential radiotracer for in vivo imaging of the dopamine D2 high-affinity state with positron emission tomography. J. Med. Chem., Vol. 48, pp. 4153-60

Woltering, T. J., Adam, G., Alanine, A., Wichmann, J., Knoflach, F., et al. 2007. Synthesis and characterization of 8-ethynyl-1,3-dihydro-benzo[b][1,4]diazepin-2-one derivatives: New potent non-competitive metabotropic glutamate receptor $2 / 3$ antagonists. Part 1. Bioorg. Med. Chem. Lett., Vol. 17, pp. 6811-5

Woltering, T. J., Adam, G., Wichmann, J., Goetschi, E, Kew, J. N. C., et al. (2008a). Synthesis and characterization of 8-ethynyl-1,3-dihydro-benzo[b][1,4]diazepin-2-one 
derivatives: Part 2. New potent non-competitive metabotropic glutamate receptor 2/3 antagonists. Bioorg. Med. Chem. Lett., Vol. 18, pp. 1091-5

Woltering, T. J., Wichmann, J., Goetschi, E., Adam, G., Kew, J. N. C., et al. (2008b). Synthesis and characterization of 1,3-dihydro-benzo[b][1,4]diazepin-2-one derivatives: Part 3. New potent non-competitive metabotropic glutamate receptor 2/3 antagonists. Bioorg. Med. Chem. Lett., Vol. 18, pp. 2725-9

Woltering, T. J., Wichmann, J., Goetschi, E., Knoflach, F., Ballard, T. M., et al. (2010). Synthesis and characterization of 1,3-dihydro-benzo[b][1,4]diazepin-2-one derivatives: Part 4. In vivo active potent and selective non-competitive metabotropic glutamate receptor 2/3 antagonists. Bioorg. Med. Chem. Lett., Vol. 20, pp. 6969-74

Wu, W-L., Burnett, D. A., Domalski, M., Greenlee, W. J., Li, C., et al. (2007). Discovery of orally efficacious tetracyclic metabotropic glutamate receptor 1 (mGluR1) antagonists for the treatment of chronic pain. J. Med. Chem., Vol. 50, pp. 5550-3

Yanamoto, K., Konno, F., Odawara, C., Yamasaki, T., Kawamura, K., et al. (2010). Radiosynthesis and evaluation of [11C]YM-202074 as a PET ligand for imaging the metabotropic glutamate receptor type 1. Nuclear Medicine and Biology, Vol. 37, pp. $615-24$

Yang, Z-Q. (2005). Agonists and antagonists for group III metabotropic glutamate receptors 6, 7, and 8. Curr Top Med Chem, Vol. 5, pp. 913-8

Yasuhara, A., Sakagami, K., Yoshikawa, R., Chaki, S., Nakamura, M., Nakazato, A. (2006). Synthesis, in vitro pharmacology, and structure-activity relationships of 2aminobicyclo[3.1.0] hexane-2,6-dicarboxylic acid derivatives as mGluR2 antagonists. Bioorg. Med. Chem., 14, pp. 3405-20

Yu, M., Brownell, A-L. (2002). Synthesis of C-11 CPCCOMe, a potent PET ligand for imaging mGluR1 in vivo. Molecular Imaging, Vol. 1, pp. 230

Yu, M., Tueckmantel, W., Wang, X., Zhu, A., Kozikowski, A., Brownell, A-L. (2005). Methoxyphenylethynyl, methoxypyridylethynyl and phenylethynylderivatives of pyridine: synthesis, radiolabeling and evaluation of new PET ligands for metabotropic glutamate subtype 5 receptors. . Nucl. Med. Biol., Vol. 32, pp. 631-40

Yu, M., Tueckmantel, W., Wang, X., Zhu, A., Kozikowski, A. P., Brownell, A-L. (2005). Methoxyphenylethynyl, methoxypyridylethynyl and phenylethynyl derivatives of pyridine: synthesis, radiolabeling and evaluation of new PET ligands for metabotropic glutamate subtype 5 receptors. Nucl Med Biol, Vol. 32, pp. 631-40

Zhang, L., Rogers, B. N., Duplantier, A. J., McHardy, S. F., Efremov, I., et al. (2008). 3(Imidazolylmethyl)-3-aza-bicyclo[3.1.0]hexan-6-yl)methyl ethers: A novel series of mGluR2 positive allosteric modulators. Bioorg. Med. Chem. Lett., Vol. 18, pp. 5493-6

Zheng, G. Z., Bhatia, P., Daanen, J., Kolasa, T., Patel, M., et al. (2005). Structure-activity relationship of triazafluorenone derivatives as potent and selective mGluR1 antagonists. J. Med. Chem., Vol. 48, pp. 7374-88

Zhu, A., Wang, X., Yu, M., Wang, J-Q., Brownell, A-L. (2007). Evaluation of four pyridine analogs to characterize 6-OHDA induced modulation of mGluR5 function in rat brain using microPET studies. J. Cereb. Blood. Flow. Metab., Vol. 27, pp. 1623-31 


\title{
Molecular Imaging of $\alpha 7$ Nicotinic Acetylcholine Receptors In Vivo: Current Status and Perspectives
}

\author{
Peter Brust and Winnie Deuther-Conrad \\ Helmholtz-Zentrum Dresden - Rossendorf \\ Research Site Leipzig \\ Germany
}

\section{Introduction}

Nicotine, named after the French diplomat Jean Nicot who brought the tobacco plant (Nicotiana tabacum) to France, isolated in 1828 as the major pharmacologically active compound in this plant (Posselt \& Reimann, 1828), structurally identified between 1890 and 1893 (Pinner, 1893; Pinner \& Wolffenstein, 1891), and first synthesized chemically in 1903 (Pictet, 1903), acts on various subtypes of nicotinic acetylcholine receptors (nAChRs) in the brain and in the periphery (Changeux, 2010; Langley, 1906).

Besides tobacco, nicotine is found in plants of the nightshade family (solanaceae) such as tomato, potato, peppers and aubergine (eggplant) but also in tea plants (Schep et al., 2009). Accordingly, it is regularly taken up by the great majority of the human population with a mean daily dietary intake of approximately $1.4 \mu \mathrm{g}$ per day (Siegmund et al., 1999). The alkaloid is readily absorbed by the lung or intestinal tissue, distributed by the blood and transported across the blood-brain barrier (Allen \& Lockman, 2003; Oldendorf et al., 1979). When inhaled it takes about seven seconds for nicotine to reach the brain (Rose et al., 2010), where it binds with high affinity to the heteromeric a $4 \beta 2$ and the homomeric a7 nAChRs, the two most abundant nAChR populations (Changeux, 2010). In the brain, nAChRs are involved in attention and cognition, locomotion, vigilance control, and rewarding mechanisms (Changeux, 2010; Graef et al., 2011), and they are suggested to play a major role in brain development (Hruska et al., 2009; Ross et al., 2010).

Notably, nicotinic receptors, and in particular $\mathrm{a} 7 \mathrm{nAChR}$, are not only expressed on neurons but virtually on all cell types present in the brain including astrocytes (Sharma \& Vijayaraghavan, 2001), microglia (De Simone et al., 2005; Suzuki et al., 2006), oligodendrocyte precursor cells (Sharma \& Vijayaraghavan, 2002), and endothelial cells (Hawkins et al., 2005). Accordingly, neuronal and non-neuronal expression of a7 nAChR has also been found in peripheral organs (Albuquerque et al., 2009; Sharma \& Vijayaraghavan, 2002).

Molecular imaging in vivo as considered in this review relates exclusively to the use of radiolabelled receptor ligands, although occasionally optical imaging has been used to investigate the cholinergic system (Prakash \& Frostig, 2005). Molecular imaging of a4 $\beta 2$ nAChR in vivo has recently been reviewed (Horti et al., 2010; Sabri et al., 2008). Therefore, the current review is focussed on neuroimaging of a7 nAChRs. 


\section{Role of $\alpha 7$ nicotinic receptors in normal brain function}

a7 nAChRs, discovered in 1990 (Couturier et al., 1990), belong to the superfamily of multisubunit ligand-gated ion channels and mediate the effects of the endogenous neurotransmitter acetylcholine. Homomeric $a 7 \mathrm{nAChR}$ is functionally distinct from the heteromeric nAChRs due to lower affinity to the agonists acetylcholine and nicotine, and higher affinity to the antagonistic snake venom a-bungarotoxin (a-BGT). Agonist binding induces a change in conformation of all five subunits of the a7 nAChR and leads to opening of the cation-conducting channel across the plasma membrane, probably by cis-trans prolyl isomerisation (Lummis et al., 2005). Regarding ion selectivity, a7 nAChR is known to have the highest permeability to $\mathrm{Ca}^{2+}$ ions within all $\mathrm{nAChR}$ subtypes (Dajas-Bailador et al., 2002; Gilbert et al., 2009; Sharma \& Vijayaraghavan, 2001). Therefore, the activation of a7 nAChR changes the intracellular $\mathrm{Ca}^{2+}$ homoestasis both directly as well as indirectly, the latter via voltage-dependent membrane-spanning $\mathrm{Ca}^{2+}$ channels as well as $\mathrm{Ca}^{2+}$ release channels and pumps in the endoplasmatic reticulum. Downstream events of this $\mathrm{Ca}^{2+}$ signalling result in (i) immediate effects, such as neurotransmitter release, (ii) short-term effects, such as receptor desensitisation and recovery, and (iii) long-lasting adaptive effects, such as neuroprotection or changes in the plasticity of the brain via gene expression (Leonard, 2003; Radcliffe \& Dani, 1998; Shen \& Yakel, 2009). Dependent on the cell-specific pattern of intracellular signalling in neurons with a7 nAChRs located post-, pre- and extrasynaptically (Berg \& Conroy, 2002; Frazier et al., 1998; Schilström et al., 2000), these complex functional properties explain the involvement of the $a 7 \mathrm{nAChR}$ in physiological processes of neurotransmission as well as its role in both acute and chronic neuropathologies (Fig. 1).

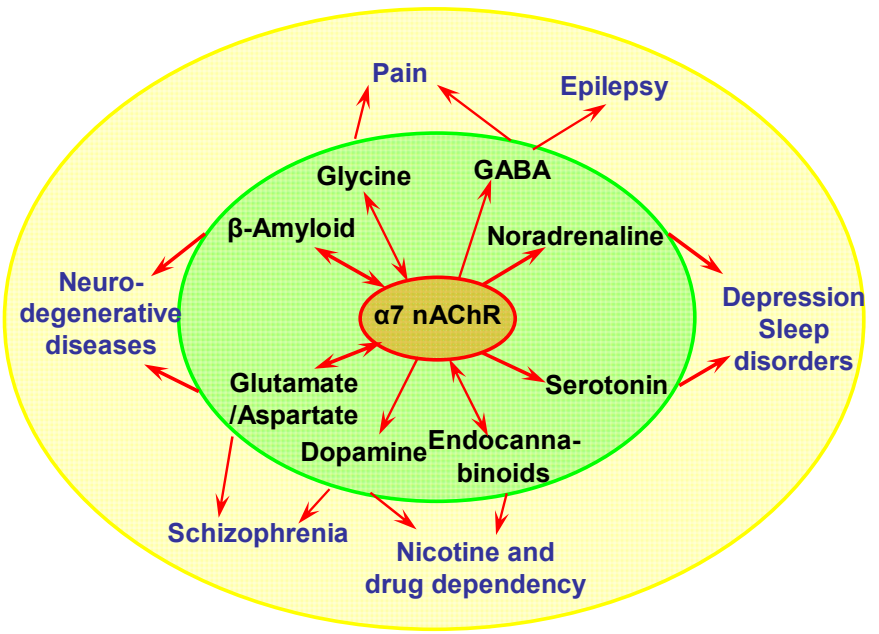

Fig. 1. Involvement of a7 nAChRs in physiological and pathophysiological processes of neurotransmission.

For example, presynaptic a7 nAChRs regulate, either directly or via modulation of glutamate release, the activity profiles of neurotransmitters such as GABA (Albuquerque et al., 1997; Liu et al., 2001), noradrenaline (Fu et al., 1999; Li et al., 1998), or dopamine (Kulak 
et al., 1997; Northrop et al., 2010) and thereby mediate neuronal adaptation related to development, learning, memory, attention, pain perception, and reward. Furthermore, a7 $\mathrm{nAChRs}$ mediate postsynaptic responses in serotonergic neurons involved in maintaining the waking state (Galindo-Charles et al., 2008). An assumed contribution of a7 nAChRs to the formation of endocannabinoids (Stella \& Piomelli, 2001) and a proposed regulation of $a 7$ nAChR activity by anandamide (van der Stelt \& Di Marzo, 2005) is consistent with a functional interaction of neuromodulating systems involved in drug dependency (McPartland et al., 2008).

\begin{tabular}{|c|c|c|c|c|}
\hline Species & Brain region & $\begin{array}{l}\text { Radioligand, } \\
\text { concentration }\end{array}$ & Receptor binding* & Referenz \\
\hline \multirow[t]{7}{*}{ Human } & $\begin{array}{l}\text { Nucleus } \\
\text { reticularis }\end{array}$ & [125I]a-BGT, $1 \mathrm{nM}$ & 5-12 nM & (Spurden et al., 1997) \\
\hline & $\begin{array}{l}\text { Nucleus } \\
\text { geniculatis lat. }\end{array}$ & [125I]a-BGT, $1 \mathrm{nM}$ & $2 \mathrm{nM}$ & (Spurden et al., 1997) \\
\hline & $\begin{array}{l}\text { Dorsolateral } \\
\text { prefrontal cortex }\end{array}$ & {$\left[{ }^{125 I}\right] \mathrm{a}-\mathrm{BGT}, 5 \mathrm{nM}$} & $9-12 \mathrm{nM}$ & (Mathew et al., 2007) \\
\hline & Cingulate cortex & [125I]a-BGT, $2.6 \mathrm{nM}$ & $\sim 16 \mathrm{nM}$ & (Marutle et al., 2001) \\
\hline & Temporal cortex & [125I]a-BGT, $2.6 \mathrm{nM}$ & $\sim 8 \mathrm{nM}$ & (Marutle et al., 2001) \\
\hline & Hippocampus & [125I]a-BGT, $1.2 \mathrm{nM}$ & $2-8 \mathrm{nM}$ & $\begin{array}{l}\text { (Hellström-Lindahl \& } \\
\text { Court, 2000) }\end{array}$ \\
\hline & Cerebellum & [25I] a-BGT, $1 \mathrm{nM}$ & $1-3 \mathrm{nM}$ & (Lee et al., 2002) \\
\hline \multirow[t]{2}{*}{ Monkey } & Cortex & [125]]iodo-MLA & $6 \mathrm{nM}\left(\mathrm{B}_{\max }\right)$ & (Kulak et al., 2006) \\
\hline & Striatum & $\begin{array}{l}\text { [125]]iodo-MLA } \\
{[125[] \mathrm{a}-\mathrm{BGT}, 3 \mathrm{nM}}\end{array}$ & $\begin{array}{l}3-4 \mathrm{nM}\left(\mathrm{B}_{\max }\right) \\
7.5 \mathrm{nM}\end{array}$ & $\begin{array}{l}\text { (Kulak et al., 2006) } \\
\text { (Quik et al., 2005) }\end{array}$ \\
\hline \multirow[t]{11}{*}{ Rat } & Cortex & {$\left[{ }^{3} \mathrm{H}\right] \mathrm{MLA}, 5 \mathrm{nM}$} & $6-16 \mathrm{nM}$ & (Mugnaini et al., 2002) \\
\hline & & [ $\left.{ }^{3} \mathrm{H}\right] \mathrm{MLA}, 20 \mathrm{nM}$ & $\sim 30 \mathrm{fmol} / \mathrm{mg}$ protein & (Davies et al., 1999) \\
\hline & & $\begin{array}{l}{\left[{ }^{125} \mathrm{I}\right] \mathrm{a}-\mathrm{BGT}, 10 \mathrm{nM}} \\
{\left[{ }^{125} \mathrm{I}\right] \mathrm{a}-\mathrm{BGT}}\end{array}$ & $\begin{array}{l}\sim 40 \mathrm{fmol} / \mathrm{mg} \text { protein } \\
\sim 1.1 \mathrm{nM}\left(\mathrm{B}_{\max }\right)\end{array}$ & $\begin{array}{l}\text { (Davies et al., 1999) } \\
\text { (Christensen et al., 2010) }\end{array}$ \\
\hline & Thalamus & {$\left[{ }^{3} \mathrm{H}\right] \mathrm{MLA}, 5 \mathrm{nM}$} & $0.9-21 \mathrm{nM}$ & (Mugnaini et al., 2002) \\
\hline & Hippocampus & {$\left[{ }^{3} \mathrm{H}\right] \mathrm{MLA}, 5 \mathrm{nM}$} & 6-182 nM & (Mugnaini et al., 2002) \\
\hline & & {$\left[{ }^{3} \mathrm{H}\right] \mathrm{MLA}, 20 \mathrm{nM}$} & $\sim 70 \mathrm{fmol} / \mathrm{mg}$ protein & (Davies et al., 1999) \\
\hline & & [125I]a-BGT, $10 \mathrm{nM}$ & $\sim 70 \mathrm{fmol} / \mathrm{mg}$ protein & (Davies et al., 1999) \\
\hline & & {$\left[{ }^{125} \mathrm{I}\right] \mathrm{a}-\mathrm{BGT}$} & $\sim 1.2 \mathrm{nM}\left(\mathrm{B}_{\max }\right)$ & (Christensen et al., 2010) \\
\hline & Hypothalamus & {$\left[{ }^{3} \mathrm{H}\right] \mathrm{MLA}, 5 \mathrm{nM}$} & $14-34 \mathrm{nM}$ & (Mugnaini et al., 2002) \\
\hline & & [3H]MLA, $20 \mathrm{nM}$ & $\sim 55 \mathrm{fmol} / \mathrm{mg}$ protein & (Davies et al., 1999) \\
\hline & & [125I]a-BGT, $10 \mathrm{nM}$ & $\sim 50 \mathrm{fmol} / \mathrm{mg}$ protein & (Davies et al., 1999) \\
\hline \multirow[t]{11}{*}{ Mouse } & Cortex & {$\left[{ }^{3} \mathrm{H}\right] \mathrm{MLA}, 2 \mathrm{nM}$} & $1-5 \mathrm{fmol} / \mathrm{mg}$ protein & (Whiteaker et al., 1999) \\
\hline & & [125I]a-BGT, $1.2 \mathrm{nM}$ & $\sim 8 \mathrm{nM}$ & (Svedberg et al., 2002) \\
\hline & & [125I]a-BGT, 2 nM & $0-3 \mathrm{fmol} / \mathrm{mg}$ protein & (Whiteaker et al., 1999) \\
\hline & Thalamus & {$\left[{ }^{3} \mathrm{H}\right] \mathrm{MLA}, 2 \mathrm{nM}$} & $1-20 \mathrm{fmol} / \mathrm{mg}$ protein & (Whiteaker et al., 1999) \\
\hline & & [125I]a-BGT, 2 nM & $0-12 \mathrm{fmol} / \mathrm{mg}$ protein & (Whiteaker et al., 1999) \\
\hline & & [125I]a-BGT, $1.2 \mathrm{nM}$ & $\sim 3 \mathrm{nM}$ & (Svedberg et al., 2002) \\
\hline & Hippocampus & {$\left[{ }^{3} \mathrm{H}\right] \mathrm{MLA}, 2 \mathrm{nM}$} & 0-9 fmol/mg protein & (Whiteaker et al., 1999) \\
\hline & & [125I]a-BGT, 2 nM & $0-4 \mathrm{fmol} / \mathrm{mg}$ protein & (Whiteaker et al., 1999) \\
\hline & & [125I]a-BGT, $1.2 \mathrm{nM}$ & $\sim 12 \mathrm{nM}$ & (Svedberg et al., 2002) \\
\hline & Hypothalamus & {$\left[{ }^{3} \mathrm{H}\right] \mathrm{MLA}, 2 \mathrm{nM}$} & $1-12 \mathrm{fmol} / \mathrm{mg}$ protein & (Whiteaker et al., 1999) \\
\hline & & [125I]a-BGT, 2 nM & $1-6 \mathrm{fmol} / \mathrm{mg}$ protein & (Whiteaker et al., 1999) \\
\hline
\end{tabular}

Table 1. Quantitative in vitro autoradiographic studies on $\mathrm{a} 7 \mathrm{nAChR}$ binding of various radioligands in the brains of different species, ${ }^{*} \mathrm{nM}=\mathrm{fmol} / \mathrm{mg}$ wet weight 
Qualitatively, the expression pattern of $a 7 \mathrm{nAChR}$ is similar in rodent and primate brain (Han et al., 2000), although a comprehensive and parallel quantitative analysis of a7 nAChR protein expression in the brain of different species, expected to facilitate the translation of experimental data on the imaging of a7 $\mathrm{nAChR}$ from in vitro and in vivo animal models into clinical application, is still warranted. In general, regions with high- to moderate-density of a7 nAChR gene expression and [125I]a-BGT binding are related to learning and memory such as thalamic and hippocampal structures, the horizontal limb of the diagonal band of Broca, and the nucleus basalis of Meynert (Alkondon et al., 2007; Breese et al., 1997; FabianFine et al., 2001; Hellström-Lindahl et al., 1999; Schulz et al., 1991; Spurden et al., 1997). However, species differences exist regarding the total number of binding sites of a7 nAChR specific radioligands (Han et al., 2003) with for example a lower amount of [125I]a-BGT binding in the monkey hippocampus or the human thalamus and cortex compared with the same regions of rat brain (Breese et al., 1997) (Tab. 1).

\section{Alterations of $\alpha 7 \mathrm{nAChR}$ in diseased brain}

The World Health Organization has classified dependence on the use of drugs including tobacco as a disease in 1965. During the following decades convincing evidence was obtained that nicotine is the key factor in tobacco addiction and that nicotinic acetylcholine receptors are of importance (Stolerman, 1990). It has been suggested that a7 nAChRs in the ventral tegmental area mediate nicotine's stimulatory effect on mesolimbocortical dopaminergic function and consequently its reinforcing and dependence-producing properties (Nomikos et al., 2000). As shown in rats, exposure to tobacco smoke not only induced nicotine dependence but increased the a7 nAChR density in the CA2/3 area (+ $25 \%$ ) and the stratum oriens (+18\%) of the hippocampus (Small et al., 2010).

With respect to clinical considerations, a close association between nicotine addiction and schizophrenia has been found (Lohr \& Flynn, 1992). Consistent with the hypothesis, that a gene-mediated dysfunction of a7 nAChR (Dome et al., 2010; Freedman et al., 1997; Stephens et al., 2009) underlies impairments seen in schizophrenia (Nomikos et al., 2000), the density of hippocampal [125I]a-BGT binding sites was decreased in schizophrenic patients (Freedman et al., 1995) but was at control levels in schizophrenic smokers (Mexal et al., 2010).

Evidence for an involvement of a7 nAChR in Alzheimer's disease (AD) was obtained at about 30 years ago from data showing a significantly reduced number of [125I]a-BGT binding sites in the mid-temporal gyrus from demented patients (Davies \& Feisullin, 1981). During the last decade, comparable results were obtained by analysing other neurodegenerative diseases. Lewy body dementia (DLB) and Parkinson's disease have also been associated with alterations in the transcription or translation of the a7 subunit (Burghaus et al., 2003; Court et al., 2000; Nordberg, 2001; Wevers \& Schröder, 1999), indicating a hypocholinergic tone due to for example reduced levels of a7 mRNA and protein in the hippocampus and reticular nucleus in AD and DLB (Court et al., 1999; Guan et al., 2000; Hellström-Lindahl et al., 1999). Functional interactions of $\beta$-amyloid with $\alpha 7$ $\mathrm{nAChR}$, revealed in vitro (Wang et al., 2000), and the colocalization of both in AD support the hypothesis that neuronal degeneration in $\mathrm{AD}$ might also be triggered by $\beta$-amyloidinitiated and $\alpha 7 \mathrm{nAChR}$-mediated inflammatory processes (Bencherif \& Lippiello, 2010). 
Interestingly, also in traumatic brain injury (TBI), regarded as risk factor for AD (Fleminger et al., 2003), significantly lowered a7 nAChR densities were found in rats and pigs (Hoffmeister et al., 2010). The resulting cholinergic hypofunction may attenuate the antiinflammatory effect of acetylcholine (Rosas-Ballina \& Tracey, 2009) and thus contribute to the process of neurodegeneration (Conejero-Goldberg et al., 2008).

Other diseases with potential involvement of a7 nAChR include epilepsy and attention deficit hyperactivity disorder (ADHD). While some forms of epilepsy have recently been associated with alterations of a 4 subtype expression (Raggenbass \& Bertrand, 2002), there is experimental evidence that $\mathrm{a} 7 \mathrm{nAChR}$ may play a role in epileptogenesis (Dobelis et al., 2003). Based on similarities between schizophrenia and ADHD with regard to a number of disturbances in attention it has been hypothesized that the a7 subunit gene may be of significance in ADHD although experimental data are still missing (Kent et al., 2001). Previous attempts to treat ADHD patients with nicotine (Levin et al., 1996; Potter \& Newhouse, 2004) are currently repeated in a Phase II study with the selective a7 nAChR ligand TC-5619 by Targacept Inc.

\section{4. $\alpha 7 \mathrm{nAChR}$ as target for drug development}

Because the activation of $a 7 \mathrm{nAChR}$ persistently affects synaptic transmission, multiple neurotransmitter and neuropeptide systems, and eventually brain plasticity (Leonard, 2003; Radcliffe \& Dani, 1998; Shen \& Yakel, 2009), a7 nAChR has been assessed as a potential target for the rational design of drugs for neuroprotective and neuropsychiatric indications. The large number of studies on receptor structure and pharmacology makes a7 nAChR an extensively investigated receptor protein and the continued development of orthosteric ligands and allosteric modulators by the pharmaceutical industry testifies the importance of efforts to assess $\alpha 7 \mathrm{nAChR}$ expression and functionality in the living human brain (Bunnelle et al., 2004; Mazurov et al., 2006).

Evidence of a correlation between $a 7 \mathrm{nAChR}$ properties and brain performance has been provided by studies on the attentional and cognitive enhancement obtained by a7 nAChR agonists (Feuerbach et al., 2009; Levin et al., 1999; Roncarati et al., 2009) and positive allosteric modulators (Faghih et al., 2008; Timmermann et al., 2007) as well as on a7 nAChR related pharmacotherapeutic approaches for schizophrenia (Freedman et al., 2008; Olincy et al., 2006; Tregellas et al., 2011), and dementia (Bacher et al., 2010; Kitagawa et al., 2003; Thomsen et al., 2010). Furthermore, electrophysiological (Hurst et al., 2005; Ng et al., 2007) and behavioural data (Bitner et al., 2010; Pacini et al., 2010; Tietje et al., 2008) highlight the potential of $\mathrm{a} 7 \mathrm{nAChR}$ as therapeutic target for neurodegenerative diseases. The close connection between $\alpha 7 \mathrm{nAChR}$ signalling, inflammation, and neurodegeneration makes $\alpha 7$ $\mathrm{nAChR}$ auspicious also for medicinal control of inflammation as an epiphenomenon of many brain disorders (Conejero-Goldberg et al., 2008; de Jonge \& Ulloa, 2007; Rosas-Ballina \& Tracey, 2009).

\section{Noninvasive imaging of $\alpha 7$ receptors in normal and diseased brain}

Far beyond what can be analysed postmortem, the non-invasive and real-time investigation of $a 7 \mathrm{nAChR}$ by means of molecular imaging techniques provides the assessment of temporal and spatial changes in receptor distribution and density during disease progression and drug treatments. 


\subsection{Technical requirements}

The most advanced system for non-invasive diagnostic and therapeutic neuroreceptor imaging is positron emission tomography (PET) (Antoni \& Langström, 2008; Hagooly et al., 2008; Heiss \& Herholz, 2006). In PET, the quantitative detection of the distribution of radiolabeled molecules in vivo with high resolution and sensitivity leads to functional images of brain biochemistry and physiology (Spanoudaki \& Ziegler, 2008). PET has now become an advanced nuclear medicine imaging technique integrated into routine clinical use (Galban et al., 2010) and a highly sophisticated tool for experimental animal research (Lancelot \& Zimmer, 2010; Xi et al., 2011).

Receptor ligands used for PET are radiolabelled with short-lived positron-emitting isotopes such as $15 \mathrm{O},{ }^{13} \mathrm{~N},{ }^{11} \mathrm{C}$, and ${ }^{18} \mathrm{~F}$ with half-lives of $2,10,20.4$, and $109.6 \mathrm{~min}$, respectively. The spatial resolution of recently developed clinical PET systems with about 2-3 $\mathrm{mm}$ allows tracing of radioligand distribution even within small cerebral nuclei in human brain (Heiss et al., 2004; Lecomte, 2009; Wienhard et al., 2002), and a detailed regional analysis also in rodents (Lancelot \& Zimmer, 2010; Lecomte, 2009; Xi et al., 2011) can be achieved with dedicated small-animal PET scanners. To overcome the problem of the anatomic classification of areas with increased or diminished radioligand accumulation, coregistration of brain anatomy with MRI or CT is needed. Software-based approaches used for computerized anatomical alignment have been very successful in brain imaging because of the relatively fixed and uniform structure of the head, and both manual and automated systems have been developed in the last years (Slomka \& Baum, 2009). Through the use of multimodal approaches delineation of small-sized but receptor-rich brain areas is considerably improved (Heiss, 2009). During the last decade hybrid PET-CT scanners have been developed, where two gantries for PET and CT are placed back-to-back (Mawlawi \& Townsend, 2009). Technically even more challenging is the development of hybrid PET-MRI scanners because of the sensitivity of the photomultiplier tubes of the standard PET detectors to even low magnetic fields. This problem has been solved only recently (Pichler et al., 2008; Pichler et al., 2006) and was first successfully accomplished for small-animal designs (Judenhofer et al., 2008). Very recently, fully integrated PET-MRI systems which allow simultaneous data acquisition have been developed as clinical research instruments, and four prototypes of integrated hybrid PET-MRI scanners were installed at two PET centres in Europe (Germany) and the United States so far. However, several technological and methodical issues have to be addressed before PET-MRI can establish itself as a routine clinical tool (von Schulthess \& Schlemmer, 2009).

\subsection{Radiotracer development}

PET technology, using radionuclides with high specific radioactivity and the opportunity to specifically label a chemical compound by substituting a stable atom with its radioactive counterpart, combined with quantitative measurements of radioactivity, is the preferred modality for molecular imaging (Antoni \& Langström, 2008). Despite of some of the limitations in instrumentation discussed above, the bottleneck for broad clinical applications in neuroimaging is the limited availability of suitable radioligands. Among positronemitting isotopes only ${ }^{11} \mathrm{C}$ and ${ }^{18} \mathrm{~F}$ are applicable for imaging of neurotransmitter-related components in the brain. Their short half-live $\left({ }^{11} \mathrm{C}: \mathrm{t}_{1 / 2}=20.4 \mathrm{~min},{ }^{18} \mathrm{~F}: \mathrm{t}_{1 / 2}=109.6 \mathrm{~min}\right.$ ) allows repeated investigations in the same patient or the same animal with short time intervals. Accordingly, the patient or the animal can be considered as its own reference 
following a pharmacological intervention. For use in a satellite concept (i.e. with no on-site cyclotron available at the PET center), there is a special demand for PET radioisotopes with longer half-life such as ${ }^{18} \mathrm{~F}$.

Even though the basic mechanisms of radioligand-target interactions in vitro and in vivo are identical, in vivo imaging requires some additional factors that have to be taken into account. In addition to high-affinity binding and supreme selectivity towards the biological target, key requirements for all types of radioligands, suitable physicochemical properties gain special importance for brain imaging with PET. For example not only the transfer of the radioligand across the blood-brain barrier (BBB) is determined by its lipophilicity (Davson \& Segal, 1996; Liu et al., 2010) but also the non-specific binding (Waterhouse, 2003). High accumulation and prolonged retention in the target region with target-to-background ratios of desirably more than 5 are closely related to both the affinity of the radioligand and the density of its potential binding sites, which are small compared to the concentration of nontarget proteins. Because saturation of binding sites may be obtained at comparably low radioligand concentrations, the concentration of the radioligand applied has to be about 1000 -fold lower than the pharmacological threshold. In other words, high specific activity in the range of $50-500 \mathrm{GBq} / \mu \mathrm{mol}$ has to be achieved, feasible nowadays with both ${ }^{11} \mathrm{C}$ - and ${ }^{18} \mathrm{~F}-$ labeled radioligands (Antoni \& Langström, 2008).

In summary, high target affinity, specificity, sensitivity, metabolic stability and appropriate pharmacokinetics are among the most important features for a good in vivo neuroreceptorimaging agent. Despite the fact that over the past decade a great variety of a7 $\mathrm{nAChR}$ selective agents have been developed, so far there are only a few radioligands which fulfil at least some of these criteria and will be discussed below.

\subsection{Imaging of $\alpha 7 \mathrm{nAChR}$ in animal and human brain}

Although a radiopharmaceutical for PET imaging of a7 nAChR that fulfills all the abovementioned pre-conditions is still missing, there is general agreement to develop ligands, which bind to the orthosteric site of the a7 $\mathrm{nAChR}$. The steric and electronic requirements of this site are met by structurally diverse classes of compounds as reviewed recently (Toyohara et al., 2010a), and potential ligands originate from for example benzylidene anabasein compounds such as GTS-21 (Meyer et al., 1998), or the quinuclidine framework such as AR-R-17779, both shown in Fig. 2 (Bodnar et al., 2005; Mazurov et al., 2005; Mullen et al., 2000; Tatsumi et al., 2005). A recently developed highly selective fluorescent a7 nicotinic receptor ligand is restricted to in vitro studies because of its chemical structure (Hone et al., 2010).

Despite this basic knowledge and promising experimental data obtained in vitro, the imaging of a7 $\mathrm{nAChR}$ in vivo is still in its infancy. This is not only due to the inadequate in vivo performance caused by an insufficient target specificity of radioligands such as the nonnegligible $5 \mathrm{HT}_{3} \mathrm{R}$ binding of otherwise promising quinuclidine-based tracers (Pomper et al., 2005) (Table 2).

Compared to the heteromeric a $4 \beta 2 \mathrm{nAChRs,} \mathrm{imaging} \mathrm{of} \alpha 7 \mathrm{nAChR}$ is challenged by the much lower expression of this target, which is illustrated by the up to 100 -fold lower density of binding sites of a7-specific [125I]a-BGT in comparison to a4 $\beta 2$-specific [ $\left.{ }^{3} \mathrm{H}\right]$ nicotine in different nuclei of human thalamus (Spurden et al., 1997). Furthermore, the outcome of preclinical studies in primates can hardly be predicted from biodistribution studies in rodents. While in the monkey brain high target-to-nontarget ratios were obtained for the 
diazabicyclooctane derivatives [11C]A-582941 and [11C]A-844606, both failed with regard to regional distribution and selectivity in the mouse brain (Toyohara et al., 2010b) (Tab. 2).<smiles>COc1ccc(C=C2CCCN=C2c2cccnc2)c(OC)c1</smiles>

GTS-21

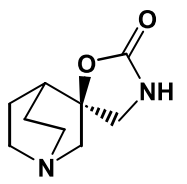

AR-R-17779

Fig. 2. Lead structures for development of radioligands for neuroimaging of a7 nAChR

Recently, the 1,4-diazabicyclo-[3.2.2]nonane skeleton (Bunnelle et al., 2004) has been identified as new structure to improve the receptor-ligand interaction, and both ${ }^{18} \mathrm{~F}$ substituted compounds such as [18F]NS10743 (Peters et al., 2007) and those for labelling with ${ }^{11} \mathrm{C}$ such as [11C]CHIBA-1001 (Hashimoto et al., 2008; Toyohara et al., 2009) and [11C]NS12857 (Lehel et al., 2009) have been designed (Table 2). As illustrated by the data in Tab. 2, the general suitability of these derivatives for imaging of a7 $\mathrm{nAChR}$ is supported by preclinical PET studies in pigs (Deuther-Conrad et al., 2011; Lehel et al., 2009) and nonhuman primates (Hashimoto et al., 2008) as well as a first clinical study (Toyohara et al., 2009). However, substantial enhancement in the affinity of the a7 nAChR PET ligands is required to improve image analysis, modelling, and eventually quantification of a7 nAChR in brain diseases. Considering the low density of $\mathrm{a} 7 \mathrm{nAChR}$ in brain, the target affinity of the currently most promising tracers [11C]CHIBA-1001 ( $K_{\mathrm{i}} \sim 35 \mathrm{nM}$; (Hashimoto et al., 2008; Toyohara et al., 2009) and [18F]NS10743 ( $K_{\mathrm{i}} \sim 10 \mathrm{nM}$; (Deuther-Conrad et al., 2009) has proved insufficient, because dissociation constants of $\geq 10 \mathrm{nM}$ result in baseline binding potential values considerably lower than the threshold value of 2 (Koeppe, 2001). NS14490, a novel diazabicyclononane derivative which has been developed by NeuroSearch and radiolabelled in collaboration with the authors, possesses a $K_{\mathrm{i}}$ value of $\sim 3 \mathrm{nM}$ in vitro (Deuther-Conrad and colleagues, unpublished), and the ligand distribution pattern of [18F]NS14490 has been assessed in a first proof-of-principle experiment (Fig. 3).
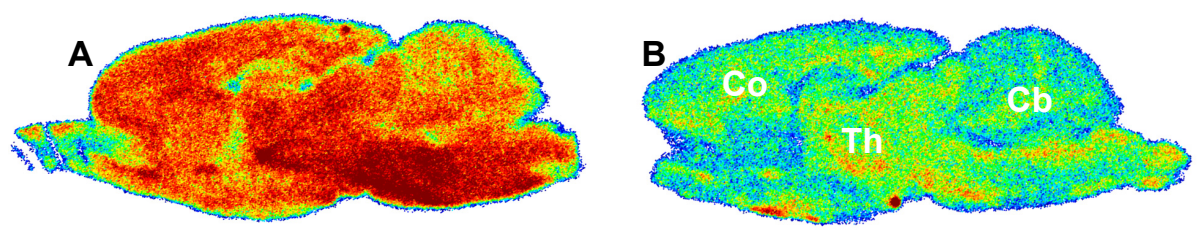

Fig. 3. In vitro autoradiography on the distribution of [18F]NS14490 in rat brain (sagittal slices, $12 \mu \mathrm{m}) . A=\left[{ }^{18} \mathrm{~F}\right] \mathrm{NS} 14490$, total binding; $\mathrm{B}=\mathrm{Co}$-incubation of [18F]NS14490 with 20 $\mu \mathrm{M}$ methyllycaconitine; Abbreviations: $\mathrm{Co}=$ cortex; $\mathrm{Cb}=$ cerebellum; Th=thalamus. 


\begin{tabular}{|c|c|c|c|}
\hline PET radioligand & $\begin{array}{l}\text { Species; } \\
\text { Study type }\end{array}$ & Main findings & Reference \\
\hline$\left[{ }^{125} \mathrm{I}\right] 4$ & $\begin{array}{l}\text { CD1 mice } \\
\text { Biodistribution }\end{array}$ & $\begin{array}{l}\text { Very limited uptake of radioactivity } \\
\text { in the brain; } \\
\text { No evidence of receptor blockade }\end{array}$ & $\begin{array}{l}\text { (Pomper et al., } \\
2005)\end{array}$ \\
\hline$\left[{ }^{11} \mathrm{C}\right] 1$ & $\begin{array}{l}\text { SPRD rats } \\
\text { Biodistribution }\end{array}$ & $\begin{array}{l}\text { No regionally selective or specific } \\
\text { binding }\end{array}$ & (Dolle et al., 2001) \\
\hline$\left[{ }^{[11} \mathrm{C}\right] \mathrm{N}$ & $\begin{array}{l}\text { Sus scrofa } \\
\text { domestica } \\
\text { Dynamic PET } \\
\text { scan }\end{array}$ & $\begin{array}{l}\text { High uptake in the pig brain; } \\
\text { Distribution as reported in primates; } \\
\text { Lack of in vivo displacement }\end{array}$ & (Lehel et al., 2009) \\
\hline$\left[{ }^{18} \mathrm{~F}\right] \mathrm{NS} 1$ & $\begin{array}{l}\text { Sus scrofa } \\
\text { domestica } \\
\text { Dynamic PET } \\
\text { scan }\end{array}$ & $\begin{array}{l}\text { High uptake in the pig brain; } \\
\text { Blocking significantly reduced } \\
\text { binding potential in regions with } \\
\text { high radioactivity uptake }\end{array}$ & $\begin{array}{l}\text { (Deuther-Conrad } \\
\text { et al., 2011) }\end{array}$ \\
\hline [2/4-methoxy & $\begin{array}{l}\text { Papio anubis } \\
\text { Dynamic PET } \\
\text { scan }\end{array}$ & $\begin{array}{l}\text { Very high initial uptake followed by } \\
\text { rapid clearance; Radiometa-bolites } \\
\text { penetrate the BBB; } \\
\text { High nonspecific binding consistent } \\
\text { with the low affinity for a7 nAChR }\end{array}$ & (Kim et al., 2007) \\
\hline$\left.{ }^{11} \mathrm{C}\right] \mathrm{Me}$ & $\begin{array}{l}\text { Macaca mulatta } \\
\text { Dynamic PET } \\
\text { scan }\end{array}$ & $\begin{array}{l}R \text {-enantiomer with high uptake of } \\
\text { radioactivity in the brain and a7 } \\
\text { nAChR-related distribution }\end{array}$ & $\begin{array}{l}\text { (Ogawa et al., } \\
2010)\end{array}$ \\
\hline$\left[{ }^{76} \mathrm{Br}\right] \mathrm{SSR} 180711$ & $\begin{array}{l}\text { Macaca mulatta } \\
\text { Dynamic PET } \\
\text { scan }\end{array}$ & $\begin{array}{l}\text { Substantial and heterogenous brain } \\
\text { accumulation; } \\
\text { Uptake reduced to background level } \\
\text { of the cerebellum by pretreatment } \\
\text { with the a7 nAChR agonist } \\
\text { SSR180711 }\end{array}$ & $\begin{array}{l}\text { (Hashimoto et al., } \\
\text { 2008) }\end{array}$ \\
\hline$\left.{ }^{11} \mathrm{C}\right] \mathrm{A}-844606$ & $\begin{array}{l}\text { Macaca mulatta } \\
\text { Dynamic PET } \\
\text { scan }\end{array}$ & $\begin{array}{l}\text { Regional distribution consistent with } \\
\text { a7 } \mathrm{nAChR} \text { expression }\end{array}$ & $\begin{array}{l}\text { (Toyohara et al., } \\
\text { 2010b) }\end{array}$ \\
\hline$\left[{ }^{11} \mathrm{C}\right] \mathrm{CHIBA}-100$ & $\begin{array}{l}\text { Clinical PET } \\
\text { study } \\
\text { (one healthy } \\
\text { male subject) }\end{array}$ & $\begin{array}{l}\text { Selective uptake in the regions of the } \\
\text { hippocampus, cortex and basal } \\
\text { ganglia; gradual washouts; } \\
\text { cerebellum with lowest binding }\end{array}$ & $\begin{array}{l}\text { (Toyohara et al., } \\
\text { 2009) }\end{array}$ \\
\hline
\end{tabular}

Table 2. Findings on in vivo biodistribution and PET imaging studies on the binding of a7 $\mathrm{nAChR}$ specific radioligands in brain of different species. 


\section{Noninvasive imaging of $\alpha 7 \mathrm{nAChR}$ in other diseases - Reality and vision}

For another derivative, NS14492, an $\mathrm{IC}_{50}$ value of $4.5 \mathrm{nM}$ was reported. It was radiolabelled with ${ }^{11} \mathrm{C}$ and investigated in pigs, where the radioligand showed the capability of measuring in vivo occupancy at $\alpha 7 \mathrm{nAChR}$ (Ettrup et al., 2010).

With regard to molecular imaging, the development of quantitative approaches to visualise a7 nAChR outside the brain is another big challenge because comparably low receptor densities have to be expected also in peripheral organs. Experimental radiotracer studies discussed above provided evidence of specific a7 receptor binding not only in the adrenals with reported receptor densities of less than $10 \mathrm{fmol} / \mathrm{mg}$ in human tissue (Mousavi et al., 2001) but also in heart, muscle, gut, kidney, thymus, pancreas and liver (Deuther-Conrad et al., 2009).

In general, imaging the concentration, distribution and occupancy of neuroreceptors involved in respiratory and cardiovascular disorders is a very attractive research area as it can provide new insights in the aetiology of these diseases as well as means to diagnose them (Hagooly et al., 2008). Regarding a7 nAChR, the presence of these receptors in microvascular endothelial cells has been shown and their involvement in the regulation of microvascular permeability and angiogenesis has been suggested (Egleton et al., 2009; Li \& Wang, 2006; Moccia et al., 2004).

Furthermore, nicotinic a7 receptors are part of a neural circuit where acetylcholine transmitted via the vagus nerve is thought to control cytokine release as part of the cholinergic anti-inflammatory pathway (Tracey, 2002). This pathway may protect organs such as heart or kidney from ischemic injury (Li et al., 2010; Sadis et al., 2007; Yeboah et al., 2008) and could be of importance in patients with autoimmune diseases known to be characterized by suppressed vagus nerve activity (Bruchfeld et al., 2010). Accordingly, neuroimmunomodulation mediated by a7 $\mathrm{nAChR}$ agonists is regarded as a future therapeutic approach (Bencherif et al., 2011; Kumar \& Sharma, 2010).

Nicotinic a7 receptors are also regarded as a powerful regulator of responses that stimulate cancer cells (Egleton et al., 2008; Schuller, 2009). In particular, evidence of the involvement of nicotinic a7 receptors in the control of basal cell proliferation and differentiation pathways in lung and the participation of these receptors in airway remodelling during brochopulmonary diseases led to the assumption that $\mathrm{a} 7 \mathrm{nAChRs}$ are of relevance for lung development, injury, repair, and carcinogenesis (Maouche et al., 2009). Because the a7 $\mathrm{nAChR}$ is the most predominantly expressed nAChR subtype in bronchial epithelial cells (Paleari et al., 2009) and mRNA for $a 7 \mathrm{nAChR}$ has been detected not only in normal lung cells but in most human lung cancer cell lines (Egleton et al., 2008; Plummer et al., 2005), it has been hypothesized that a desensitization of a7 nAChR in heavy smokers with a prolonged exposure to nicotine could lead to squamous metaplasia (Tournier \& Birembaut, 2011). While in an early investigation of small cell carcinomas of the lung no specific [125I]-abungarotoxin binding could be demonstrated, probably due to a sub-threshold density of the a7 receptor protein related to this particular type of cancer (Cunningham et al., 1985), not only all of 50 investigated non-small cell lung cancer (NSCLC) cell lines expressed the a7 subtype (Paleari et al., 2009) but also all out of 52 investigated NSCLC patients expressed a7 receptor mRNA and protein and the values were higher in smoking patients with squamous carcinomas than those with adenocarcinomas (Paleari et al., 2008).

Besides lung cancer, a7 nAChR-mediated signalling has been implicated in the growth and metastasis of colon cancer (Wei et al., 2009; Wei et al., 2011; Ye et al., 2004), probably due to 
the activity of the endogenous allosteric a7 nAChR modulator SLURP-1 and the upregulation of the downstream signalling molecule NF-kB (Chernyavsky et al., 2010; Pettersson et al., 2008; Ye et al., 2004). Also the development of keratinocyte carcinoma, the most prevalent skin cancer and the most common cancer in United States (Albert \& Weinstock, 2003), may depend on $\mathrm{a} 7 \mathrm{nAChR}$ expression and regulation. Interestingly, antagonisation of nAChR activity by SLURP-1 and -2 prevented the tobacco nitrosamineinduced malignant transformation of oral keratinocytes (Arredondo et al., 2007), cells known to express a7 nAChR (Chernyavsky et al., 2010). Further cancers with known a7 nAChR expression include breast, pancreas, and prostate carcinomas (Al-Wadei et al., 2009; Dasgupta et al., 2009; Hirata et al., 2010; Hruska et al., 2009).

Based on this evidence, $\mathrm{a} 7 \mathrm{nAChR}$ is considered a primary target in ongoing research on pathogenesis of a variety of cancers. Furthermore, the quantitative imaging of diseaserelated changes in the expression of peripheral $a 7 \mathrm{nAChR}$ by PET is highly desirable for the validation of novel approaches in diagnostics and development of cancer-specific therapy.

\section{Conclusion}

Generally, the development of imaging approaches to non-invasively quantify a7 nAChR receptors in and outside the brain is expected to help in the generation and testing of novel hypotheses supporting the understanding of pathogenetic processes and promoting novel diagnostic and therapeutic concepts. The clinical significance of a malfunction of a7 nAChR, involved in particular cell-type and pathology specific modulating and signalling cascades, can be assessed on molecular level with an imaging-supported spatiotemporal quantification of a7 $\mathrm{nAChR}$ protein. In this context, the imaging technique must be sensitive enough not only to identify but also to assess the dynamics and quantity of even subtle changes in the amount of functional a7 nAChR, which is despite its physiological importance expressed at comparably low levels in the brain and periphery. PET techniques offer the highest achievable resolution of functional processes in the body in four dimensions by imaging of a7 $\mathrm{nAChR}$ with further optimised PET radiotracers, which might be based for instance on the currently most promising diazabicyclononane derivatives.

\section{References}

Al-Wadei, H. A., Al-Wadei, M. H. \& Schuller, H. M. (2009). Prevention of pancreatic cancer by the beta-blocker propranolol. Anti-Cancer Drugs, Vol.20, No.6, (July 2009), pp 477-482, ISSN 0959-4973

Albert, M. R. \& Weinstock, M. A. (2003). Keratinocyte carcinoma. CA: A Cancer Journal for Clinicians, Vol.53, No.5, (September 2003), pp 292-302, ISSN 0007-9235

Albuquerque, E. X., Pereira, E. F., Alkondon, M. \& Rogers, S. W. (2009). Mammalian nicotinic acetylcholine receptors: from structure to function. Physiological Reviews, Vol.89, No.1, (Jan 2009), pp 73-120, ISSN 0031-9333

Albuquerque, E. X., Alkondon, M., Pereira, E. F., Castro, N. G., Schrattenholz, A., Barbosa, C. T., Bonfante-Cabarcas, R., Aracava, Y., Eisenberg, H. M. \& Maelicke, A. (1997). Properties of neuronal nicotinic acetylcholine receptors: pharmacological characterization and modulation of synaptic function. Journal of Pharmacology and Experimental Therapeutics, Vol.280, No.3, (March 1997), pp 1117-1136, ISSN 00223565 
Alkondon, M., Pereira, E. F. \& Albuquerque, E. X. (2007). Age-dependent changes in the functional expression of two nicotinic receptor subtypes in CA1 stratum radiatum interneurons in the rat hippocampus. Biochemical Pharmacology, Vol.74, No.8, (October 2007), pp 1134-1144, ISSN 0006-2952

Allen, D. D. \& Lockman, P. R. (2003). The blood-brain barrier choline transporter as a brain drug delivery vector. Life Sciences, Vol.73, No.13, (August 2003), pp 1609-1615, ISSN 0024-3205

Antoni, G. \& Langström, B. (2008). Radiopharmaceuticals: molecular imaging using positron emission tomography. Handb Exp Pharmacol, No.185 Pt 1, 2008), pp 177-201, ISSN 0171-2004

Arredondo, J., Chernyavsky, A. I. \& Grando, S. A. (2007). SLURP-1 and -2 in normal, immortalized and malignant oral keratinocytes. Life Sciences, Vol.80, No.24-25, (May 2007), pp 2243-2247, ISSN 0024-3205

Bacher, I., Rabin, R., Woznica, A., Sacco, K. A. \& George, T. P. (2010). Nicotinic receptor mechanisms in neuropsychiatric disorders: Therapeutic Implications. Primary Psychiatry, Vol.17, No.1, (January 2010), pp 35-41, ISSN 1082-6319

Bencherif, M. \& Lippiello, P. M. (2010). Alpha7 neuronal nicotinic receptors: the missing link to understanding Alzheimer's etiopathology? Medical Hypotheses, Vol.74, No.2, (February 2010), pp 281-285, ISSN 0306-9877

Bencherif, M., Lippiello, P. M., Lucas, R. \& Marrero, M. B. (2011). Alpha7 nicotinic receptors as novel therapeutic targets for inflammation-based diseases. Cellular and Molecular Life Sciences, Vol.68, No.6, (March 2011), pp 931-949, ISSN 1420-682X

Berg, D. K. \& Conroy, W. G. (2002). Nicotinic $\alpha 7$ receptors: synaptic options and downstream signaling in neurons. Journal of Neurobiology, Vol.53, No.4, (December 2002), pp 512-523, ISSN 0022-3034

Bitner, R. S., Bunnelle, W. H., Decker, M. W., Drescher, K. U., Kohlhaas, K. L., Markosyan, S., Marsh, K. C., Nikkel, A. L., Browman, K., Radek, R., Anderson, D. J., Buccafusco, J. \& Gopalakrishnan, M. (2010). In vivo pharmacological characterization of a novel selective $\alpha 7$ neuronal nicotinic acetylcholine receptor agonist ABT-107: preclinical considerations in Alzheimer's disease. Journal of Pharmacology and Experimental Therapeutics, Vol.334, No.3, (September 2010), pp 875-886, ISSN 0022-3565

Bodnar, A. L., Cortes-Burgos, L. A., Cook, K. K., Dinh, D. M., Groppi, V. E., Hajos, M., Higdon, N. R., Hoffmann, W. E., Hurst, R. S., Myers, J. K., Rogers, B. N., Wall, T. M., Wolfe, M. L. \& Wong, E. (2005). Discovery and structure-activity relationship of quinuclidine benzamides as agonists of $\alpha 7$ nicotinic acetylcholine receptors. Journal of Medicinal Chemistry, Vol.48, No.4, (February 2005), pp 905-908, ISSN 0022-2623

Breese, C. R., Adams, C., Logel, J., Drebing, C., Rollins, Y., Barnhart, M., Sullivan, B., Demasters, B. K., Freedman, R. \& Leonard, S. (1997). Comparison of the regional expression of nicotinic acetylcholine receptor alpha7 mRNA and [125I]- $\alpha-$ bungarotoxin binding in human postmortem brain. Journal of Comparative Neurology, Vol.387, No.3, (October 1997), pp 385-398, ISSN 0021-9967

Bruchfeld, A., Goldstein, R. S., Chavan, S., Patel, N. B., Rosas-Ballina, M., Kohn, N., Qureshi, A. R. \& Tracey, K. J. (2010). Whole blood cytokine attenuation by cholinergic agonists ex vivo and relationship to vagus nerve activity in rheumatoid arthritis. Journal of Internal Medicine, Vol.268, No.1, (July 2010), pp 94-101, ISSN 0955-7873 
Bunnelle, W. H., Dart, M. J. \& Schrimpf, M. R. (2004). Design of ligands for the nicotinic acetylcholine receptors: the quest for selectivity. Curr Top Med Chem, Vol.4, No.3, (February 2004), pp 299-334, ISSN 1568-0266

Burghaus, L., Schütz, U., Krempel, U., Lindstrom, J. \& Schröder, H. (2003). Loss of nicotinic acetylcholine receptor subunits $\alpha 4$ and $\alpha 7$ in the cerebral cortex of Parkinson patients. Parkinsonism Relat Disord, Vol.9, No.5, (June 2003), pp 243-246, ISSN 13538020

Changeux, J. P. (2010). Nicotine addiction and nicotinic receptors: lessons from genetically modified mice. Nature Reviews Neuroscience, Vol.11, No.6, (June 2010), pp 389-401, ISSN 1471-003X

Chernyavsky, A. I., Arredondo, J., Galitovskiy, V., Qian, J. \& Grando, S. A. (2010). Upregulation of nuclear factor- $\kappa \mathrm{B}$ expression by SLURP-1 is mediated by $\alpha 7-$ nicotinic acetylcholine receptor and involves both ionic events and activation of protein kinases. Am J Physiol Cell Physiol, Vol.299, No.5, (November 2010), pp C903911, ISSN 0363-6143

Christensen, D. Z., Mikkelsen, J. D., Hansen, H. H. \& Thomsen, M. S. (2010). Repeated administration of $\alpha 7$ nicotinic acetylcholine receptor (nAChR) agonists, but not positive allosteric modulators, increases $\alpha 7 \mathrm{nAChR}$ levels in the brain. Journal of Neurochemistry, Vol.114, No.4, (August 2010), pp 1205-1216, ISSN 0022-3042

Conejero-Goldberg, C., Davies, P. \& Ulloa, L. (2008). Alpha7 nicotinic acetylcholine receptor: A link between inflammation and neurodegeneration. Neuroscience and Biobehavioral Reviews, Vol.32, No.4, (April 2008), pp 693-706, ISSN 0149-7634

Court, J., Spurden, D., Lloyd, S., McKeith, I., Ballard, C., Cairns, N., Kerwin, R., Perry, R. \& Perry, E. (1999). Neuronal nicotinic receptors in dementia with Lewy bodies and schizophrenia: $\alpha$-bungarotoxin and nicotine binding in the thalamus. Journal of Neurochemistry, Vol.73, No.4, (October 1999), pp 1590-1597, ISSN 0022-3042

Court, J. A., Martin-Ruiz, C., Graham, A. \& Perry, E. (2000). Nicotinic receptors in human brain: topography and pathology. Journal of Chemical Neuroanatomy, Vol.20, No.3-4, (December 2000), pp 281-298, ISSN 0891-0618

Couturier, S., Bertrand, D., Matter, J. M., Hernandez, M. C., Bertrand, S., Millar, N., Valera, S., Barkas, T. \& Ballivet, M. (1990). A neuronal nicotinic acetylcholine receptor subunit $(\alpha 7)$ is developmentally regulated and forms a homo-oligomeric channel blocked by alpha-BTX. Neuron, Vol.5, No.6, (December 1990), pp 847-856, ISSN 0896-6273

Cunningham, J. M., Lennon, V. A., Lambert, E. H. \& Scheithauer, B. (1985). Acetylcholine receptors in small cell carcinomas. Journal of Neurochemistry, Vol.45, No.1, (July 1985), pp 159-167, ISSN 0022-3042

Dajas-Bailador, F. A., Mogg, A. J. \& Wonnacott, S. (2002). Intracellular Ca ${ }^{2+}$ signals evoked by stimulation of nicotinic acetylcholine receptors in SH-SY5Y cells: contribution of voltage-operated $\mathrm{Ca}^{2+}$ channels and $\mathrm{Ca}^{2+}$ stores. Journal of Neurochemistry, Vol.81, No.3, (May 2002), pp 606-614, ISSN 0022-3042

Dasgupta, P., Rizwani, W., Pillai, S., Kinkade, R., Kovacs, M., Rastogi, S., Banerjee, S., Carless, M., Kim, E., Coppola, D., Haura, E. \& Chellappan, S. (2009). Nicotine induces cell proliferation, invasion and epithelial-mesenchymal transition in a variety of human cancer cell lines. International Journal of Cancer, Vol.124, No.1, (January 2009), pp 36-45, ISSN 0020-7136 
Davies, A. R., Hardick, D. J., Blagbrough, I. S., Potter, B. V., Wolstenholme, A. J. \& Wonnacott, S. (1999). Characterisation of the binding of $[3 \mathrm{H}]$ methyllycaconitine: a new radioligand for labelling $\alpha 7$-type neuronal nicotinic acetylcholine receptors. Neuropharmacology, Vol.38, No.5, (May 1999), pp 679-690, ISSN 0028-3908

Davies, P. \& Feisullin, S. (1981). Postmortem stability of $\alpha$-bungarotoxin binding sites in mouse and human brain. Brain Research, Vol.216, No.2, (July 1981), pp 449-454, ISSN 0006-8993

Davson, H. \& Segal, M. B. (1996). Physiology of the CSF and blood-brain barriers, CRC Press, ISBN 0849344727, Boca Raton, USA

de Jonge, W. J. \& Ulloa, L. (2007). The $\alpha 7$ nicotinic acetylcholine receptor as a pharmacological target for inflammation. British Journal of Pharmacology, Vol.151, No.7, (August 2007), pp 915-929, ISSN 0007-1188

De Simone, R., Ajmone-Cat, M. A., Carnevale, D. \& Minghetti, L. (2005). Activation of alpha7 nicotinic acetylcholine receptor by nicotine selectively up-regulates cyclooxygenase-2 and prostaglandin E2 in rat microglial cultures. Journal of Neuroinflammation, Vol.2, No.4, (January 2005), pp 1-10, ISSN 1742-2094

Deuther-Conrad, W., Fischer, S., Hiller, A., Nielsen, E. O., Timmermann, D. B., Steinbach, J., Sabri, O., Peters, D. \& Brust, P. (2009). Molecular imaging of $\alpha 7$ nicotinic acetylcholine receptors: design and evaluation of the potent radioligand [18F]NS10743. Eur J Nucl Med Mol Imaging, Vol.36, No.5, (May 2009), pp 791-800, ISSN 1619-7070

Deuther-Conrad, W., Fischer, S., Hiller, A., Becker, G., Cumming, P., Xiong, G., Funke, U., Sabri, O., Peters, D. \& Brust, P. (2011). Assessment of a7 nicotinic acetylcholine receptor availability in porcine brain with [18F]NS10743. . Eur J Nucl Med Mol Imaging, (March 2011), $\mathrm{p}$ in press, ISSN 1619-7070

Dobelis, P., Hutton, S., Lu, Y. \& Collins, A. C. (2003). GABAergic systems modulate nicotinic receptor-mediated seizures in mice. Journal of Pharmacology and Experimental Therapeutics, Vol.306, No.3, (September 2003), pp 1159-1166, ISSN 0022-3565

Dolle, F., Valette, H., Hinnen, F., Vaufrey, F., Demphel, S., Coulon, C., Ottaviani, M., Bottlaender, M. \& Crouzel, C. (2001). Synthesis and preliminary evaluation of a carbon-11-labelled agonist of the $\alpha 7$ nicotinic acetylcholine receptor. Journal of Labelled Compounds \& Radiopharmaceuticals, Vol.44, No.11, (October 2001), pp 785795, ISSN 0362-4803

Dome, P., Lazary, J., Kalapos, M. P. \& Rihmer, Z. (2010). Smoking, nicotine and neuropsychiatric disorders. Neuroscience and Biobehavioral Reviews, Vol.34, No.3, (March 2010), pp 295-342, ISSN 0149-7634

Egleton, R. D., Brown, K. C. \& Dasgupta, P. (2008). Nicotinic acetylcholine receptors in cancer: multiple roles in proliferation and inhibition of apoptosis. Trends in Pharmacological Sciences, Vol.29, No.3, (March 2008), pp 151-158, ISSN 0165-6147

Egleton, R. D., Brown, K. C. \& Dasgupta, P. (2009). Angiogenic activity of nicotinic acetylcholine receptors: implications in tobacco-related vascular diseases. Pharmacology and Therapeutics, Vol.121, No.2, (February 2009), pp 205-223, ISSN 0163-7258

Ettrup, A., Mikkelsen, J. D., Palner, M., Lehel, S., Madsen, J., Timmermann, D. B., Peters, D. \& Knudsen, G. M. (2010) [11C]NS14492 as a novel PET ligand for imaging cerebral 
$\alpha 7$ nicotinic receptors: in vivo evaluation and drug occupancy measurements (abstract). Society of Neuroscience San Diego:November 13-17, 2010, F39.

Fabian-Fine, R., Skehel, P., Errington, M. L., Davies, H. A., Sher, E., Stewart, M. G. \& Fine, A. (2001). Ultrastructural distribution of the $\alpha 7$ nicotinic acetylcholine receptor subunit in rat hippocampus. Journal of Neuroscience, Vol.21, No.20, (October 2001), pp 7993-8003, ISSN 0270-6474

Faghih, R., Gopalakrishnan, M. \& Briggs, C. A. (2008). Allosteric modulators of the $\alpha 7$ nicotinic acetylcholine receptor. Journal of Medicinal Chemistry, Vol.51, No.4, (February 2008), pp 701-712, ISSN 0022-2623

Feuerbach, D., Lingenhoehl, K., Olpe, H. R., Vassout, A., Gentsch, C., Chaperon, F., Nozulak, J., Enz, A., Bilbe, G., McAllister, K. \& Hoyer, D. (2009). The selective nicotinic acetylcholine receptor $\alpha 7$ agonist JN403 is active in animal models of cognition, sensory gating, epilepsy and pain. Neuropharmacology, Vol.56, No.1, (January 2009), pp 254-263, ISSN 0028-3908

Fleminger, S., Oliver, D. L., Lovestone, S., Rabe-Hesketh, S. \& Giora, A. (2003). Head injury as a risk factor for Alzheimer's disease: the evidence 10 years on; a partial replication. Journal of Neurology, Neurosurgery and Psychiatry, Vol.74, No.7, (July 2003), pp 857-862, ISSN 0022-3050

Frazier, C. J., Rollins, Y. D., Breese, C. R., Leonard, S., Freedman, R. \& Dunwiddie, T. V. (1998). Acetylcholine activates an $\alpha$-bungarotoxin-sensitive nicotinic current in rat hippocampal interneurons, but not pyramidal cells. Journal of Neuroscience, Vol.18, No.4, (February 1998), pp 1187-1195, ISSN 0270-6474

Freedman, R., Hall, M., Adler, L. E. \& Leonard, S. (1995). Evidence in postmortem brain tissue for decreased numbers of hippocampal nicotinic receptors in schizophrenia. Biological Psychiatry, Vol.38, No.1, (July 1995), pp 22-33, ISSN 0006-3223

Freedman, R., Olincy, A., Buchanan, R. W., Harris, J. G., Gold, J. M., Johnson, L., Allensworth, D., Guzman-Bonilla, A., Clement, B., Ball, M. P., Kutnick, J., Pender, V., Martin, L. F., Stevens, K. E., Wagner, B. D., Zerbe, G. O., Soti, F. \& Kem, W. R. (2008). Initial phase 2 trial of a nicotinic agonist in schizophrenia. American Journal of Psychiatry, Vol.165, No.8, (August 2008), pp 1040-1047, ISSN 0002-953X

Freedman, R., Coon, H., Myles-Worsley, M., Orr-Urtreger, A., Olincy, A., Davis, A., Polymeropoulos, M., Holik, J., Hopkins, J., Hoff, M., Rosenthal, J., Waldo, M. C., Reimherr, F., Wender, P., Yaw, J., Young, D. A., Breese, C. R., Adams, C., Patterson, D., Adler, L. E., Kruglyak, L., Leonard, S. \& Byerley, W. (1997). Linkage of a neurophysiological deficit in schizophrenia to a chromosome 15 locus. Proceedings of the National Academy of Sciences of the United States of America, Vol.94, No.2, (January 1997), pp 587-592, ISSN 0027-8424

Fu, Y., Matta, S. G. \& Sharp, B. M. (1999). Local $\alpha$-bungarotoxin-sensitive nicotinic receptors modulate hippocampal norepinephrine release by systemic nicotine. Journal of Pharmacology and Experimental Therapeutics, Vol.289, No.1, (April 1999), pp 133-139, ISSN 0022-3565

Galban, C. J., Galban, S., Van Dort, M. E., Luker, G. D., Bhojani, M. S., Rehemtulla, A. \& Ross, B. D. (2010). Applications of molecular imaging. Prog Mol Biol Transl Sci, Vol.95, (September 2010), pp 237-298, ISSN 1877-1173

Galindo-Charles, L., Hernandez-Lopez, S., Galarraga, E., Tapia, D., Bargas, J., Garduno, J., Frias-Dominguez, C., Drucker-Colin, R. \& Mihailescu, S. (2008). Serotoninergic 
dorsal raphe neurons possess functional postsynaptic nicotinic acetylcholine receptors. Synapse, Vol.62, No.8, (August 2008), pp 601-615, ISSN 0887-4476

Gilbert, D., Lecchi, M., Arnaudeau, S., Bertrand, D. \& Demaurex, N. (2009). Local and global calcium signals associated with the opening of neuronal $\alpha 7$ nicotinic acetylcholine receptors. Cell Calcium, Vol.45, No.2, (February 2009), pp 198-207, ISSN 0143-4160

Graef, S., Schönknecht, P., Sabri, O. \& Hegerl, U. (2011). Cholinergic receptor subtypes and their role in cognition, emotion, and vigilance control: An overview of preclinical and clinical findings. Psychopharmacology, Vol.DOI: 10.1007/s00213-010-2153-8, (January 2011)ISSN 0033-3158

Guan, Z. Z., Zhang, X., Ravid, R. \& Nordberg, A. (2000). Decreased protein levels of nicotinic receptor subunits in the hippocampus and temporal cortex of patients with Alzheimer's disease. Journal of Neurochemistry, Vol.74, No.1, (January 2000), pp 237-243, ISSN 0022-3042

Hagooly, A., Rossin, R. \& Welch, M. J. (2008). Small molecule receptors as imaging targets. Handb Exp Pharmacol, No.185 Pt 2, 2008), pp 93-129, ISSN 0171-2004

Han, Z. Y., Le Novere, N., Zoli, M., Hill, J. A., Jr., Champtiaux, N. \& Changeux, J. P. (2000). Localization of $\mathrm{nAChR}$ subunit mRNAs in the brain of Macaca mulatta. European Journal of Neuroscience, Vol.12, No.10, (October 2000), pp 3664-3674, ISSN 0953-816x

Han, Z. Y., Zoli, M., Cardona, A., Bourgeois, J. P., Changeux, J. P. \& Le Novere, N. (2003). Localization of $\left[{ }^{3} \mathrm{H}\right]$ nicotine, $\left[{ }^{3} \mathrm{H}\right]$ cytisine, $\left[{ }^{3} \mathrm{H}\right]$ epibatidine, and $\left[{ }^{[25 I}\right]$ alphabungarotoxin binding sites in the brain of Macaca mulatta. Journal of Comparative Neurology, Vol.461, No.1, (June 2003), pp 49-60, ISSN 0021-9967

Hashimoto, K., Nishiyama, S., Ohba, H., Matsuo, M., Kobashi, T., Takahagi, M., Iyo, M., Kitashoji, T. \& Tsukada, H. (2008). [11C]CHIBA-1001 as a novel PET ligand for $\alpha 7$ nicotinic receptors in the brain: a PET study in conscious monkeys. PLoS ONE, Vol.3, No.9, 2008), p e3231, ISSN 1932-6203

Hawkins, B. T., Egleton, R. D. \& Davis, T. P. (2005). Modulation of cerebral microvascular permeability by endothelial nicotinic acetylcholine receptors. American Journal of Physiology - Heart and Circulatory Physiology, Vol.289, No.1, (July 2005), pp H212-219, ISSN 0363-6135

Heiss, W. D. (2009). The potential of PET/MR for brain imaging. European Journal of Nuclear Medicine and Molecular Imaging, Vol.36 Suppl 1, (March 2009), pp S105-112, ISSN 1619-7070

Heiss, W. D. \& Herholz, K. (2006). Brain receptor imaging. Journal of Nuclear Medicine, Vol.47, No.2, (February 2006), pp 302-312, ISSN 0161-5505

Heiss, W. D., Habedank, B., Klein, J. C., Herholz, K., Wienhard, K., Lenox, M. \& Nutt, R. (2004). Metabolic rates in small brain nuclei determined by high-resolution PET. Journal of Nuclear Medicine, Vol.45, No.11, (November 2004), pp 1811-1815, ISSN 0161-5505

Hellström-Lindahl, E. \& Court, J. A. (2000). Nicotinic acetylcholine receptors during prenatal development and brain pathology in human aging. Behavioural Brain Research, Vol.113, No.1-2, (August 2000), pp 159-168, ISSN 0166-4328

Hellström-Lindahl, E., Mousavi, M., Zhang, X., Ravid, R. \& Nordberg, A. (1999). Regional distribution of nicotinic receptor subunit mRNAs in human brain: comparison between Alzheimer and normal brain. Brain Research. Molecular Brain Research, Vol.66, No.1-2, (March 1999), pp 94-103, ISSN 0169-328x 
Hirata, N., Sekino, Y. \& Kanda, Y. (2010). Nicotine increases cancer stem cell population in MCF-7 cells. Biochemical and Biophysical Research Communications, Vol.403, No.1, (December 2010), pp 138-143, ISSN 0006-291X

Hoffmeister, P. G., Donat, C. K., Schuhmann, M. U., Voigt, C., Walter, B., Nieber, K., Meixensberger, J., Bauer, R. \& Brust, P. (2010). Traumatic Brain Injury Elicits Similar Alterations in alpha7 Nicotinic Receptor Density in Two Different Experimental Models. NeuroMolecular Medicine, Vol.DOI: 10.1007/s12017-010-81364, (September 2010)ISSN 1535-1084

Hone, A. J., Whiteaker, P., Mohn, J. L., Jacob, M. H. \& McIntosh, J. M. (2010). Alexa Fluor 546-ArIB[V11L;V16A] is a potent ligand for selectively labeling alpha 7 nicotinic acetylcholine receptors. Journal of Neurochemistry, Vol.114, No.4, (August 2010), pp 994-1006, ISSN 0022-3042

Horti, A. G., Gao, Y., Kuwabara, H. \& Dannals, R. F. (2010). Development of radioligands with optimized imaging properties for quantification of nicotinic acetylcholine receptors by positron emission tomography. Life Sciences, Vol.86, No.15-16, (April 2010), pp 575-584, ISSN 0024-3205

Hruska, M., Keefe, J., Wert, D., Tekinay, A. B., Hulce, J. J., Ibanez-Tallon, I. \& Nishi, R. (2009). Prostate stem cell antigen is an endogenous lynx1-like prototoxin that antagonizes $\alpha 7$-containing nicotinic receptors and prevents programmed cell death of parasympathetic neurons. Journal of Neuroscience, Vol.29, No.47, (November 2009), pp 14847-14854, ISSN 0270-6474

Hurst, R. S., Hajos, M., Raggenbass, M., Wall, T. M., Higdon, N. R., Lawson, J. A., Rutherford-Root, K. L., Berkenpas, M. B., Hoffmann, W. E., Piotrowski, D. W., Groppi, V. E., Allaman, G., Ogier, R., Bertrand, S., Bertrand, D. \& Arneric, S. P. (2005). A novel positive allosteric modulator of the $\alpha 7$ neuronal nicotinic acetylcholine receptor: in vitro and in vivo characterization. Journal of Neuroscience, Vol.25, No.17, (April 2005), pp 4396-4405, ISSN 0270-6474

Judenhofer, M. S., Wehrl, H. F., Newport, D. F., Catana, C., Siegel, S. B., Becker, M., Thielscher, A., Kneilling, M., Lichy, M. P., Eichner, M., Klingel, K., Reischl, G., Widmaier, S., Rocken, M., Nutt, R. E., Machulla, H. J., Uludag, K., Cherry, S. R., Claussen, C. D. \& Pichler, B. J. (2008). Simultaneous PET-MRI: a new approach for functional and morphological imaging. Nature Medicine, Vol.14, No.4, (April 2008), pp 459-465, ISSN 1078-8956

Kent, L., Green, E., Holmes, J., Thapar, A., Gill, M., Hawi, Z., Fitzgerald, M., Asherson, P., Curran, S., Mills, J., Payton, A. \& Craddock, N. (2001). No association between CHRNA7 microsatellite markers and attention-deficit hyperactivity disorder. American Journal of Medical Genetics, Vol.105, No.8, (December 2001), pp 686-689, ISSN 0148-7299

Kim, S. W., Ding, Y. S., Alexoff, D., Patel, V., Logan, J., Lin, K. S., Shea, C., Muench, L., Xu, Y., Carter, P., King, P., Constanzo, J. R., Ciaccio, J. A. \& Fowler, J. S. (2007). Synthesis and positron emission tomography studies of C-11-labeled isotopomers and metabolites of GTS-21, a partial alpha7 nicotinic cholinergic agonist drug. Nuclear Medicine and Biology, Vol.34, No.5, (July 2007), pp 541-551, ISSN 0969-8051

Kitagawa, H., Takenouchi, T., Azuma, R., Wesnes, K. A., Kramer, W. G., Clody, D. E. \& Burnett, A. L. (2003). Safety, pharmacokinetics, and effects on cognitive function of 
multiple doses of GTS-21 in healthy, male volunteers. Neuropsychopharmacology, Vol.28, No.3, (March 2003), pp 542-551, ISSN 0893-133X

Koeppe, R. A. (2001). A panel discussion on the future of pharmacology and experimental tomography, In: Physiological imaging of the brain with PET, A. Gjedde, S. B. Hansen, G. M. Knudsen \& O. B. Paulson, (Eds.), 402, Academic Press, ISBN 0-12-285751-8, New York, USA

Kulak, J. M., Carroll, F. I. \& Schneider, J. S. (2006). [125I]Iodomethyllycaconitine binds to $\alpha 7$ nicotinic acetylcholine receptors in monkey brain. European Journal of Neuroscience, Vol.23, No.10, (May 2006), pp 2604-2610, ISSN 0953-816X

Kulak, J. M., Nguyen, T. A., Olivera, B. M. \& McIntosh, J. M. (1997). Alpha-conotoxin MII blocks nicotine-stimulated dopamine release in rat striatal synaptosomes. Journal of Neuroscience, Vol.17, No.14, (July 1997), pp 5263-5270, ISSN 0270-6474

Kumar, V. \& Sharma, A. (2010). Is neuroimmunomodulation a future therapeutic approach for sepsis? International Immunopharmacology, Vol.10, No.1, (January 2010), pp 9-17, ISSN 1567-5769

Lancelot, S. \& Zimmer, L. (2010). Small-animal positron emission tomography as a tool for neuropharmacology. Trends in Pharmacological Sciences, Vol.31, No.9, (September 2010), pp 411-417, ISSN 0165-6147

Langley, J. N. (1906). Croonian Lecture, 1906: On Nerve Endings and on Special Excitable Substances in Cells. Proceedings of the Royal Society of London. Series B: Biological Sciences, Vol.78, No.524, (September 1906), pp 170-194 Online ISSN 1471-2954

Lecomte, R. (2009). Novel detector technology for clinical PET. European Journal of Nuclear Medicine and Molecular Imaging, Vol.36 Suppl 1, (March 2009), pp S69-85, ISSN 16197070

Lee, M., Martin-Ruiz, C., Graham, A., Court, J., Jaros, E., Perry, R., Iversen, P., Bauman, M. \& Perry, E. (2002). Nicotinic receptor abnormalities in the cerebellar cortex in autism. Brain, Vol.125, No.Pt 7, (July 2002), pp 1483-1495, ISSN 0006-8950

Lehel, S., Madsen, J., Ettrup, A., Mikkelsen, J. D., Timmermann, D. B., Peters, D. \& Knudsen, G. M. (2009). [11C]NS-12857: A novel PET ligand for $\alpha 7$-nicotinergic receptors. Journal of Labelled Compounds \& Radiopharmaceuticals, Vol.52, ( 2009), pp S379-S379, ISSN 0362-4803

Leonard, S. (2003). Consequences of low levels of nicotinic acetylcholine receptors in schizophrenia for drug development. Drug Development Research, Vol.60, No.2, (October 2003), pp 127-136, ISSN (electronic) 1098-2299

Levin, E. D., Bettegowda, C., Blosser, J. \& Gordon, J. (1999). AR-R17779, and $\alpha 7$ nicotinic agonist, improves learning and memory in rats. Behavioural Pharmacology, Vol.10, No.6-7, (November 1999), pp 675-680, ISSN 0955-8810

Levin, E. D., Conners, C. K., Sparrow, E., Hinton, S. C., Erhardt, D., Meck, W. H., Rose, J. E. \& March, J. (1996). Nicotine effects on adults with attention-deficit/hyperactivity disorder. Psychopharmacology, Vol.123, No.1, (January 1996), pp 55-63, ISSN 00333158

Li, D. L., Liu, B. H., Sun, L., Zhao, M., He, X., Yu, X. J. \& Zang, W. J. (2010). Alterations of muscarinic acetylcholine receptors-2, 4 and $\alpha 7$-nicotinic acetylcholine receptor expression after ischaemia / reperfusion in the rat isolated heart. Clinical and Experimental Pharmacology and Physiology, Vol.37, No.12, (December 2010), pp 11141119, ISSN 0143-9294 
Li, X., Rainnie, D. G., McCarley, R. W. \& Greene, R. W. (1998). Presynaptic nicotinic receptors facilitate monoaminergic transmission. Journal of Neuroscience, Vol.18, No.5, (March 1998), pp 1904-1912, ISSN 0270-6474

Li, X. W. \& Wang, H. (2006). Non-neuronal nicotinic $\alpha 7$ receptor, a new endothelial target for revascularization. Life Sciences, Vol.78, No.16, (March 2006), pp 1863-1870, ISSN 0024-3205

Liu, X., Testa, B. \& Fahr, A. (2010). Lipophilicity and its relationship with passive drug permeation. Pharmaceutical Research, Vol.DOI: 10.1007/s11095-010-0303-7, (October 2010)ISSN 0724-8741

Liu, Y., Ford, B., Mann, M. A. \& Fischbach, G. D. (2001). Neuregulins increase $\alpha 7$ nicotinic acetylcholine receptors and enhance excitatory synaptic transmission in GABAergic interneurons of the hippocampus. Journal of Neuroscience, Vol.21, No.15, (August 2001), pp 5660-5669, ISSN 0270-6474

Lohr, J. B. \& Flynn, K. (1992). Smoking and schizophrenia. Schizophrenia Research, Vol.8, No.2, (December 1992), pp 93-102, ISSN 0920-9964

Lummis, S. C., Beene, D. L., Lee, L. W., Lester, H. A., Broadhurst, R. W. \& Dougherty, D. A. (2005). Cis-trans isomerization at a proline opens the pore of a neurotransmittergated ion channel. Nature, Vol.438, No.7065, (November 2005), pp 248-252, ISSN 1476-4687

Maouche, K., Polette, M., Jolly, T., Medjber, K., Cloez-Tayarani, I., Changeux, J. P., Burlet, H., Terryn, C., Coraux, C., Zahm, J. M., Birembaut, P. \& Tournier, J. M. (2009). $\alpha 7$ nicotinic acetylcholine receptor regulates airway epithelium differentiation by controlling basal cell proliferation. American Journal of Pathology, Vol.175, No.5, (November 2009), pp 1868-1882, ISSN 1525-2191

Marutle, A., Zhang, X., Court, J., Piggott, M., Johnson, M., Perry, R., Perry, E. \& Nordberg, A. (2001). Laminar distribution of nicotinic receptor subtypes in cortical regions in schizophrenia. Journal of Chemical Neuroanatomy, Vol.22, No.1-2, (July 2001), pp 115126, ISSN 0891-0618

Mathew, S. V., Law, A. J., Lipska, B. K., Davila-Garcia, M. I., Zamora, E. D., Mitkus, S. N., Vakkalanka, R., Straub, R. E., Weinberger, D. R., Kleinman, J. E. \& Hyde, T. M. (2007). Alpha7 nicotinic acetylcholine receptor mRNA expression and binding in postmortem human brain are associated with genetic variation in neuregulin 1. Human Molecular Genetics, Vol.16, No.23, (December 2007), pp 2921-2932, ISSN 0964-6906

Mawlawi, O. \& Townsend, D. W. (2009). Multimodality imaging: an update on PET/CT technology. European Journal of Nuclear Medicine and Molecular Imaging, Vol.36 Suppl 1, (March 2009), pp S15-29, ISSN 1619-7070

Mazurov, A., Hauser, T. \& Miller, C. H. (2006). Selective $\alpha 7$ nicotinic acetylcholine receptor ligands. Current Medicinal Chemistry, Vol.13, No.13, (October 2006), pp 1567-1584, ISSN 0929-8673

Mazurov, A., Klucik, J., Miao, L., Phillips, T. Y., Seamans, A., Schmitt, J. D., Hauser, T. A., Johnson, R. T., Jr. \& Miller, C. (2005). 2-(Arylmethyl)-3-substituted quinuclidines as selective $\alpha 7$ nicotinic receptor ligands. Bioorganic and Medicinal Chemistry Letters, Vol.15, No.8, (April 2005), pp 2073-2077, ISSN 0960-894X 
McPartland, J. M., Blanchon, D. J. \& Musty, R. E. (2008). Cannabimimetic effects modulated by cholinergic compounds. Addiction Biology, Vol.13, No.3-4, (September 2008), pp 411-415, ISSN 1355-6215

Mexal, S., Berger, R., Logel, J., Ross, R. G., Freedman, R. \& Leonard, S. (2010). Differential regulation of $\alpha 7$ nicotinic receptor gene (CHRNA7) expression in schizophrenic smokers. Journal of Molecular Neuroscience, Vol.40, No.1-2, (January 2010), pp 185195, ISSN 0895-8696

Meyer, E. M., Kuryatov, A., Gerzanich, V., Lindstrom, J. \& Papke, R. L. (1998). Analysis of 3(4-hydroxy, 2-methoxybenzylidene)anabaseine selectivity and activity at human and rat $\alpha 7$ nicotinic receptors. Journal of Pharmacology and Experimental Therapeutics, Vol.287, No.3, (December 1998), pp 918-925, ISSN 0022-3565

Moccia, F., Frost, C., Berra-Romani, R., Tanzi, F. \& Adams, D. J. (2004). Expression and function of neuronal nicotinic ACh receptors in rat microvascular endothelial cells. Am J Physiol Heart Circ Physiol, Vol.286, No.2, (February 2004), pp H486-491, ISSN 0363-6135

Mousavi, M., Hellström-Lindahl, E., Guan, Z. Z., Bednar, I. \& Nordberg, A. (2001). Expression of nicotinic acetylcholine receptors in human and rat adrenal medulla. Life Sciences, Vol.70, No.5, (December 2001), pp 577-590, ISSN 0024-3205

Mugnaini, M., Tessari, M., Tarter, G., Merlo Pich, E., Chiamulera, C. \& Bunnemann, B. (2002). Upregulation of $\left[{ }^{3} \mathrm{H}\right]$ methyllycaconitine binding sites following continuous infusion of nicotine, without changes of $\alpha 7$ or $\alpha 6$ subunit mRNA: an autoradiography and in situ hybridization study in rat brain. European Journal of Neuroscience, Vol.16, No.9, (November 2002), pp 1633-1646, ISSN 0953-816X

Mullen, G., Napier, J., Balestra, M., DeCory, T., Hale, G., Macor, J., Mack, R., Loch, J., 3rd, Wu, E., Kover, A., Verhoest, P., Sampognaro, A., Phillips, E., Zhu, Y., Murray, R., Griffith, R., Blosser, J., Gurley, D., Machulskis, A., Zongrone, J., Rosen, A. \& Gordon, J. (2000). (-)-Spiro[1-azabicyclo[2.2.2]octane-3,5'-oxazolidin-2'-one], a conformationally restricted analogue of acetylcholine, is a highly selective full agonist at the $\alpha 7$ nicotinic acetylcholine receptor. Journal of Medicinal Chemistry, Vol.43, No.22, (November 2000), pp 4045-4050, ISSN 0022-2623

Ng, H. J., Whittemore, E. R., Tran, M. B., Hogenkamp, D. J., Broide, R. S., Johnstone, T. B., Zheng, L., Stevens, K. E. \& Gee, K. W. (2007). Nootropic $\alpha 7$ nicotinic receptor allosteric modulator derived from $\mathrm{GABA}_{\mathrm{A}}$ receptor modulators. Proceedings of the National Academy of Sciences of the United States of America, Vol.104, No.19, (May 2007), pp 8059-8064, ISSN 0027-8424

Nomikos, G. G., Schilström, B., Hildebrand, B. E., Panagis, G., Grenhoff, J. \& Svensson, T. H. (2000). Role of $\alpha 7$ nicotinic receptors in nicotine dependence and implications for psychiatric illness. Behavioural Brain Research, Vol.113, No.1-2, (August 2000), pp 97103, ISSN 0166-4328

Nordberg, A. (2001). Nicotinic receptor abnormalities of Alzheimer's disease: therapeutic implications. Biological Psychiatry, Vol.49, No.3, (February 2001), pp 200-210, ISSN 0006-3223

Northrop, N. A., Smith, L. P., Yamamoto, B. K. \& Eyerman, D. J. (2010). Regulation of glutamate release by $\alpha 7$ nicotinic receptors: differential role in methamphetamineinduced damage to dopaminergic and serotonergic terminals. Journal of 
Pharmacology and Experimental Therapeutics, Vol.DOI:10.1124/jpet.110.177287, (December 2010)ISSN 0022-3565

Ogawa, M., Nishiyama, S., Tsukada, H., Hatano, K., Fuchigami, T., Yamaguchi, H., Matsushima, Y., Ito, K. \& Magata, Y. (2010). Synthesis and evaluation of new imaging agent for central nicotinic acetylcholine receptor $\alpha 7$ subtype. Nuclear Medicine and Biology, Vol.37, No.3, (April 2010), pp 347-355, ISSN 0969-8051

Oldendorf, W., Braun, L. \& Cornford, E. (1979). pH dependence of blood-brain barrier permeability to lactate and nicotine. Stroke, Vol.10, No.5, (September 1979), pp 577581, ISSN 0039-2499

Olincy, A., Harris, J. G., Johnson, L. L., Pender, V., Kongs, S., Allensworth, D., Ellis, J., Zerbe, G. O., Leonard, S., Stevens, K. E., Stevens, J. O., Martin, L., Adler, L. E., Soti, F., Kem, W. R. \& Freedman, R. (2006). Proof-of-concept trial of an $\alpha 7$ nicotinic agonist in schizophrenia. Archives of General Psychiatry, Vol.63, No.6, (Jun 2006), pp 630-638, ISSN 0003-990X

Pacini, A., Mannelli, L. D., Bonaccini, L., Ronzoni, S., Bartolini, A. \& Ghelardini, C. (2010). Protective effect of alpha7 nAChR: Behavioural and morphological features on neuropathy. Pain, Vol.150, No.3, (September 2010), pp 542-549, ISSN 0304-3959

Paleari, L., Cesario, A., Fini, M. \& Russo, P. (2009). $\alpha 7-$ Nicotinic receptor antagonists at the beginning of a clinical era for NSCLC and Mesothelioma? Drug Discov Today, Vol.14, No.17-18, (September 2009), pp 822-836, ISSN 1359-6446

Paleari, L., Catassi, A., Ciarlo, M., Cavalieri, Z., Bruzzo, C., Servent, D., Cesario, A., Chessa, L., Cilli, M., Piccardi, F., Granone, P. \& Russo, P. (2008). Role of $\alpha 7$-nicotinic acetylcholine receptor in human non-small cell lung cancer proliferation. Cell Proliferation, Vol.41, No.6, (December 2008), pp 936-959, ISSN 0960-7722

Peters, D., Olsen, G. M., Nielsen, E. O., Timmermann, D. B., Loechel, S. C., Mikkelsen, J. D., Hansen, H. B., Redrobe, J. P., Christensen, J. K. \& Dyhring, T. (2007). Novel 1,4diaza-bicyclo[3.2.2]nonyl oxadiazolyl derviatives and their medical use, WO/2007/138037

Pettersson, A., Nordlander, S., Nylund, G., Khorram-Manesh, A., Nordgren, S. \& Delbro, D. S. (2008). Expression of the endogenous, nicotinic acetylcholine receptor ligand, SLURP-1, in human colon cancer. Autonomic and Autacoid Pharmacology, Vol.28, No.4, (October 2008), pp 109-116, ISSN1474-8665

Pichler, B. J., Judenhofer, M. S. \& Pfannenberg, C. (2008). Multimodal imaging approaches: PET/CT and PET/MRI. Handb Exp Pharmacol, No.185 Pt 1, 2008), pp 109-132, ISSN 0171-2004

Pichler, B. J., Judenhofer, M. S., Catana, C., Walton, J. H., Kneilling, M., Nutt, R. E., Siegel, S. B., Claussen, C. D. \& Cherry, S. R. (2006). Performance test of an LSO-APD detector in a 7-T MRI scanner for simultaneous PET/MRI. Journal of Nuclear Medicine, Vol.47, No.4, (April 2006), pp 639-647, ISSN 0161-5505

Pictet, A. (1903). Synthese de la nicotine. Comptes Rendus de l Academie des Sciences, Vol.137, (November 1903), pp 860-862, ISSN 0764-4469

Pinner, A. (1893). Ueber Nicotin. Die Constitution des Alkaloids. V. Mittheilung. Berichte der deutschen chemischen Gesellschaft, Vol.26, No.1, (January 1893), pp 292-305, ISSN 1099-0682

Pinner, A. \& Wolffenstein, R. (1891). Ueber Nicotin. Berichte der deutschen chemischen Gesellschaft, Vol.24, No.1, (January 1891), pp 61-67, ISSN 1099-0682 
Plummer, H. K., 3rd, Dhar, M. \& Schuller, H. M. (2005). Expression of the $\alpha 7$ nicotinic acetylcholine receptor in human lung cells. Respiration Research, Vol.6, (April 2005), p 29, ISSN 1465-993X

Pomper, M. G., Phillips, E., Fan, H., McCarthy, D. J., Keith, R. A., Gordon, J. C., Scheffel, U., Dannals, R. F. \& Musachio, J. L. (2005). Synthesis and biodistribution of radiolabeled $\alpha 7$ nicotinic acetylcholine receptor ligands. Journal of Nuclear Medicine, Vol.46, No.2, (February 2005), pp 326-334, ISSN 0161-5505

Posselt, W. \& Reimann, L. (1828). Chemische Untersuchungen des Tabaks und Darstellung des eigenthümlichen wirksamen Princips dieser Pflanze. Geiger's Magazin für Pharmacie und die dahin einschlagenden Wissenschaften, Vol.24, 1828), pp 138-161,

Potter, A. S. \& Newhouse, P. A. (2004). Effects of acute nicotine administration on behavioral inhibition in adolescents with attention-deficit/hyperactivity disorder. Psychopharmacology, Vol.176, No.2, (November 2004), pp 182-194, ISSN 0033-3158

Prakash, N. \& Frostig, R. D. (2005). What has intrinsic signal optical imaging taught us about NGF-induced rapid plasticity in adult cortex and its relationship to the cholinergic system? Molecular Imaging and Biology, Vol.7, No.1, (January 2005), pp 14-21, ISSN 1536-1632

Quik, M., Vailati, S., Bordia, T., Kulak, J. M., Fan, H., McIntosh, J. M., Clementi, F. \& Gotti, C. (2005). Subunit composition of nicotinic receptors in monkey striatum: effect of treatments with 1-methyl-4-phenyl-1,2,3,6-tetrahydropyridine or L-DOPA. Molecular Pharmacology, Vol.67, No.1, (January 2005), pp 32-41, ISSN 0026-895X

Radcliffe, K. A. \& Dani, J. A. (1998). Nicotinic stimulation produces multiple forms of increased glutamatergic synaptic transmission. Journal of Neuroscience, Vol.18, No.18, (September 1998), pp 7075-7083, ISSN 0270-6474

Raggenbass, M. \& Bertrand, D. (2002). Nicotinic receptors in circuit excitability and epilepsy. Journal of Neurobiology, Vol.53, No.4, (December 2002), pp 580-589, ISSN 0022-3034

Roncarati, R., Scali, C., Comery, T. A., Grauer, S. M., Aschmi, S., Bothmann, H., Jow, B., Kowal, D., Gianfriddo, M., Kelley, C., Zanelli, U., Ghiron, C., Haydar, S., Dunlop, J. \& Terstappen, G. C. (2009). Procognitive and neuroprotective activity of a novel $\alpha 7$ nicotinic acetylcholine receptor agonist for treatment of neurodegenerative and cognitive disorders. Journal of Pharmacology and Experimental Therapeutics, Vol.329, No.2, (May 2009), pp 459-468, ISSN 0022-3565

Rosas-Ballina, M. \& Tracey, K. J. (2009). The neurology of the immune system: neural reflexes regulate immunity. Neuron, Vol.64, No.1, (October 2009), pp 28-32, ISSN 0896-6273

Rose, J. E., Mukhin, A. G., Lokitz, S. J., Turkington, T. G., Herskovic, J., Behm, F. M., Garg, S. \& Garg, P. K. (2010). Kinetics of brain nicotine accumulation in dependent and nondependent smokers assessed with PET and cigarettes containing ${ }^{11} \mathrm{C}$-nicotine. Proceedings of the National Academy of Sciences of the United States of America, Vol.107, No.11, (Mar 16 2010), pp 5190-5195, ISSN 0027-8424

Ross, R. G., Stevens, K. E., Proctor, W. R., Leonard, S., Kisley, M. A., Hunter, S. K., Freedman, R. \& Adams, C. E. (2010). Research review: Cholinergic mechanisms, early brain development, and risk for schizophrenia. Journal of Child Psychology and Psychiatry and Allied Disciplines, Vol.51, No.5, (May 2010), pp 535-549, ISSN 00219630 
Sabri, O., Kendziorra, K., Wolf, H., Gertz, H. J. \& Brust, P. (2008). Acetylcholine receptors in dementia and mild cognitive impairment. Eur J Nucl Med Mol Imaging, Vol.35 Suppl 1, (March 2008), pp S30-45, ISSN 1619-7070

Sadis, C., Teske, G., Stokman, G., Kubjak, C., Claessen, N., Moore, F., Loi, P., Diallo, B., Barvais, L., Goldman, M., Florquin, S. \& Le Moine, A. (2007). Nicotine protects kidney from renal ischemia/reperfusion injury through the cholinergic antiinflammatory pathway. PLoS ONE, Vol.2, No.5, (May 2007), pp e469 (461-468), ISSN 1932-6203

Schep, L. J., Slaughter, R. J. \& Beasley, D. M. (2009). Nicotinic plant poisoning. Clinical Toxicology (Philadelphia), Vol.47, No.8, (September 2009), pp 771-781, ISSN 1556-3650

Schilström, B., Fagerquist, M. V., Zhang, X., Hertel, P., Panagis, G., Nomikos, G. G. \& Svensson, T. H. (2000). Putative role of presynaptic $\alpha 7^{*}$ nicotinic receptors in nicotine stimulated increases of extracellular levels of glutamate and aspartate in the ventral tegmental area. Synapse, Vol.38, No.4, (December 2000), pp 375-383, ISSN 0887-4476

Schuller, H. M. (2009). Is cancer triggered by altered signalling of nicotinic acetylcholine receptors? Nature Reviews Cancer, Vol.9, No.3, (March 2009), pp 195-205, ISSN 1474$175 X$

Schulz, D. W., Loring, R. H., Aizenman, E. \& Zigmond, R. E. (1991). Autoradiographic localization of putative nicotinic receptors in the rat brain using ${ }^{125}$ I-neuronal bungarotoxin. Journal of Neuroscience, Vol.11, No.1, (January 1991), pp 287-297, ISSN 0270-6474

Sharma, G. \& Vijayaraghavan, S. (2001). Nicotinic cholinergic signaling in hippocampal astrocytes involves calcium-induced calcium release from intracellular stores. Proceedings of the National Academy of Sciences of the United States of America, Vol.98, No.7, (March 2001), pp 4148-4153, ISSN 0027-8424

Sharma, G. \& Vijayaraghavan, S. (2002). Nicotinic receptor signaling in nonexcitable cells. Journal of Neurobiology, Vol.53, No.4, (December 2002), pp 524-534, ISSN 0022-3034

Shen, J. X. \& Yakel, J. L. (2009). Nicotinic acetylcholine receptor-mediated calcium signaling in the nervous system. Acta Pharmacol Sin, Vol.30, No.6, (June 2009), pp 673-680, ISSN 1671-4083

Siegmund, B., Leitner, E. \& Pfannhauser, W. (1999). Determination of the nicotine content of various edible nightshades (Solanaceae) and their products and estimation of the associated dietary nicotine intake. Journal of Agricultural and Food Chemistry, Vol.47, No.8, (August 1999), pp 3113-3120, ISSN 0021-8561

Slomka, P. J. \& Baum, R. P. (2009). Multimodality image registration with software: state-ofthe-art. European Journal of Nuclear Medicine and Molecular Imaging, Vol.36 Suppl 1, (March 2009), pp S44-55, ISSN 1619-7070

Small, E., Shah, H. P., Davenport, J. J., Geier, J. E., Yavarovich, K. R., Yamada, H., Sabarinath, S. N., Derendorf, H., Pauly, J. R., Gold, M. S. \& Bruijnzeel, A. W. (2010). Tobacco smoke exposure induces nicotine dependence in rats. Psychopharmacology, Vol.208, No.1, (January 2010), pp 143-158, ISSN 0033-3158

Spanoudaki, V. C. \& Ziegler, S. I. (2008). PET \& SPECT instrumentation. Handb Exp Pharmacol, No.185 Pt 1, 2008), pp 53-74, ISSN 0171-2004

Spurden, D. P., Court, J. A., Lloyd, S., Oakley, A., Perry, R., Pearson, C., Pullen, R. G. \& Perry, E. K. (1997). Nicotinic receptor distribution in the human thalamus: 
autoradiographical localization of $\left[{ }^{3} \mathrm{H}\right]$ nicotine and $\left[{ }^{125} \mathrm{I}\right]$ alpha-bungarotoxin binding. Journal of Chemical Neuroanatomy, Vol.13, No.2, (July 1997), pp 105-113, ISSN 0891-0618

Stella, N. \& Piomelli, D. (2001). Receptor-dependent formation of endogenous cannabinoids in cortical neurons. European Journal of Pharmacology, Vol.425, No.3, (August 2001), pp 189-196, ISSN 0014-2999

Stephens, S. H., Logel, J., Barton, A., Franks, A., Schultz, J., Short, M., Dickenson, J., James, B., Fingerlin, T. E., Wagner, B., Hodgkinson, C., Graw, S., Ross, R. G., Freedman, R. \& Leonard, S. (2009). Association of the 5'-upstream regulatory region of the $\alpha 7$ nicotinic acetylcholine receptor subunit gene (CHRNA7) with schizophrenia. Schizophrenia Research, Vol.109, No.1-3, (April 2009), pp 102-112, ISSN 0920-9964

Stolerman, I. P. (1990). Behavioural pharmacology of nicotine: implications for multiple brain nicotinic receptors. Ciba Foundation Symposium, Vol.152, (June 1990), pp 3-16; discussion 16-22, ISSN 0300-5208

Suzuki, T., Hide, I., Matsubara, A., Hama, C., Harada, K., Miyano, K., Andra, M., Matsubayashi, H., Sakai, N., Kohsaka, S., Inoue, K. \& Nakata, Y. (2006). Microglial $\alpha 7$ nicotinic acetylcholine receptors drive a phospholipase C/IP3 pathway and modulate the cell activation toward a neuroprotective role. Journal of Neuroscience Research, Vol.83, No.8, (June 2006), pp 1461-1470, ISSN 0360-4012

Svedberg, M. M., Svensson, A. L., Johnson, M., Lee, M., Cohen, O., Court, J., Soreq, H., Perry, E. \& Nordberg, A. (2002). Upregulation of neuronal nicotinic receptor subunits $\alpha 4, \beta 2$, and $\alpha 7$ in transgenic mice overexpressing human acetylcholinesterase. Journal of Molecular Neuroscience, Vol.18, No.3, (June 2002), pp 211-222, ISSN 0895-8696

Tatsumi, R., Fujio, M., Satoh, H., Katayama, J., Takanashi, S., Hashimoto, K. \& Tanaka, H. (2005). Discovery of the $\alpha 7$ nicotinic acetylcholine receptor agonists. (R)-3'-(5Chlorothiophen-2-yl)spiro-1-azabicyclo[2.2.2]octane-3,5'-[1',3'] oxazolidin-2'-one as a novel, potent, selective, and orally bioavailable ligand. Journal of Medicinal Chemistry, Vol.48, No.7, (April 2005), pp 2678-2686, ISSN 0022-2623

Thomsen, M. S., Hansen, H. H., Timmerman, D. B. \& Mikkelsen, J. D. (2010). Cognitive improvement by activation of $\alpha 7$ nicotinic acetylcholine receptors: from animal models to human pathophysiology. Current Pharmaceutical Design, Vol.16, No.3, (January 2010), pp 323-343, ISSN 1381-6128

Tietje, K. R., Anderson, D. J., Bitner, R. S., Blomme, E. A., Brackemeyer, P. J., Briggs, C. A., Browman, K. E., Bury, D., Curzon, P., Drescher, K. U., Frost, J. M., Fryer, R. M., Fox, G. B., Gronlien, J. H., Hakerud, M., Gubbins, E. J., Halm, S., Harris, R., Helfrich, R. J., Kohlhaas, K. L., Law, D., Malysz, J., Marsh, K. C., Martin, R. L., Meyer, M. D., Molesky, A. L., Nikkel, A. L., Otte, S., Pan, L., Puttfarcken, P. S., Radek, R. J., Robb, H. M., Spies, E., Thorin-Hagene, K., Waring, J. F., Ween, H., Xu, H., Gopalakrishnan, M. \& Bunnelle, W. H. (2008). Preclinical characterization of A582941: a novel $\alpha 7$ neuronal nicotinic receptor agonist with broad spectrum cognition-enhancing properties. CNS Neuroscience $\mathcal{E}$ Therapeutics, Vol.14, No.1, (Spring 2008), pp 65-82, ISSN 1755-5930

Timmermann, D. B., Gronlien, J. H., Kohlhaas, K. L., Nielsen, E. O., Dam, E., Jorgensen, T. D., Ahring, P. K., Peters, D., Holst, D., Chrsitensen, J. K., Malysz, J., Briggs, C. A., Gopalakrishnan, M. \& Olsen, G. M. (2007). An allosteric modulator of the $\alpha 7$ 
nicotinic acetylcholine receptor possessing cognition-enhancing properties in vivo. Journal of Pharmacology and Experimental Therapeutics, Vol.323, No.1, (October 2007), pp 294-307, ISSN 0022-3565

Tournier, J. M. \& Birembaut, P. (2011). Nicotinic acetylcholine receptors and predisposition to lung cancer. Current Opinion in Oncology, Vol.23, No.1, (January 2011), pp 83-87, ISSN 1040-8746

Toyohara, J., Wu, J. \& Hashimoto, K. (2010a). Recent development of radioligands for imaging $\alpha 7$ nicotinic acetylcholine receptors in the brain. Curr Top Med Chem, Vol.10, No.15, (October 2010a), pp 1544-1557, ISSN 1568-0266

Toyohara, J., Ishiwata, K., Sakata, M., Wu, J., Nishiyama, S., Tsukada, H. \& Hashimoto, K. (2010b). In vivo evaluation of $\alpha 7$ nicotinic acetylcholine receptor agonists [11C]A582941 and [11C]A-844606 in mice and conscious monkeys. PLoS ONE, Vol.5, No.2, (February 2010b), p e8961, ISSN 1932-6203

Toyohara, J., Sakata, M., Wu, J., Ishikawa, M., Oda, K., Ishii, K., Iyo, M., Hashimoto, K. \& Ishiwata, K. (2009). Preclinical and the first clinical studies on [11C]CHIBA-1001 for mapping $\alpha 7$ nicotinic receptors by positron emission tomography. Annals of Nuclear Medicine, Vol.23, No.3, (May 2009), pp 301-309, ISSN 0914-7187

Tracey, K. J. (2002). The inflammatory reflex. Nature, Vol.420, No.6917, (December 2002), pp 853-859, ISSN 0028-0836

Tregellas, J. R., Tanabe, J., Rojas, D. C., Shatti, S., Olincy, A., Johnson, L., Martin, L. F., Soti, F., Kem, W. R., Leonard, S. \& Freedman, R. (2011). Effects of an alpha 7-nicotinic agonist on default network activity in schizophrenia. Biological Psychiatry, Vol.69, No.1, (January 2011), pp 7-11, ISSN 0006-3223

van der Stelt, M. \& Di Marzo, V. (2005). Anandamide as an intracellular messenger regulating ion channel activity. Prostaglandins and Other Lipid Mediators, Vol.77, No.1-4, (September 2005), pp 111-122, ISSN 1098-8823

von Schulthess, G. K. \& Schlemmer, H. P. (2009). A look ahead: PET/MR versus PET/CT. European Journal of Nuclear Medicine and Molecular Imaging, Vol.36 Suppl 1, (March 2009), pp S3-9, ISSN 1619-7070

Wang, H. Y., Lee, D. H., D'Andrea, M. R., Peterson, P. A., Shank, R. P. \& Reitz, A. B. (2000). $\beta$-Amyloid(1-42) binds to $\alpha 7$ nicotinic acetylcholine receptor with high affinity. Implications for Alzheimer's disease pathology. Journal of Biological Chemistry, Vol.275, No.8, (Feb 25 2000), pp 5626-5632,

Waterhouse, R. N. (2003). Determination of lipophilicity and its use as a predictor of bloodbrain barrier penetration of molecular imaging agents. Mol Imaging Biol, Vol.5, No.6, (November 2003), pp 376-389, ISSN 1536-1632

Wei, P. L., Chang, Y. J., Ho, Y. S., Lee, C. H., Yang, Y. Y., An, J. \& Lin, S. Y. (2009). Tobaccospecific carcinogen enhances colon cancer cell migration through $\alpha 7$-nicotinic acetylcholine receptor. Annals of Surgery, Vol.249, No.6, (June 2009), pp 978-985, ISSN 0003-4932

Wei, P. L., Kuo, L. J., Huang, M. T., Ting, W. C., Ho, Y. S., Wang, W., An, J. \& Chang, Y. J. (2011). Nicotine enhances colon cancer cell migration by induction of fibronectin. Annals of Surgical Oncology, Vol.DOI: 10.1245/s10434-010-1504-3, (January 2011)ISSN 1068-9265

Wevers, A. \& Schröder, H. (1999). Nicotinic acetylcholine receptors in Alzheimer's disease. J Alzheimers Dis, Vol.1, No.4-5, (November 1999), pp 207-219, ISSN 1387-2877 
Whiteaker, P., Davies, A. R., Marks, M. J., Blagbrough, I. S., Potter, B. V., Wolstenholme, A. J., Collins, A. C. \& Wonnacott, S. (1999). An autoradiographic study of the distribution of binding sites for the novel $\alpha 7$-selective nicotinic radioligand $\left[{ }^{3} \mathrm{H}\right]-$ methyllycaconitine in the mouse brain. European Journal of Neuroscience, Vol.11, No.8, (August 1999), pp 2689-2696, ISSN 0953-816X

Wienhard, K., Schmand, M., Casey, M. E., Baker, K., Bao, J., Eriksson, L., Jones, W. F., Knoess, C., Lenox, M., Lercher, M., Luk, P., Michel, C., Reed, J. H., Richerzhagen, N., Treffert, J., Vollmar, S., Young, J. W., Heiss, W. D. \& Nutt, R. (2002). The ECAT HRRT: Performance and first clinical application of the new high resolution research tomograph. Ieee Transactions on Nuclear Science, Vol.49, No.1, (February 2002), pp 104-110, ISSN 0018-9499

Xi, W., Tian, M. \& Zhang, H. (2011). Molecular imaging in neuroscience research with smallanimal PET in rodents. Neuroscience Research, Vol.doi:10.1016/j.neures.2010.12.017, (January 2011)ISSN 0168-0102

Ye, Y. N., Liu, E. S., Shin, V. Y., Wu, W. K. \& Cho, C. H. (2004). The modulating role of nuclear factor- $\kappa \mathrm{B}$ in the action of $\alpha 7$-nicotinic acetylcholine receptor and cross-talk between 5-lipoxygenase and cyclooxygenase-2 in colon cancer growth induced by 4-(N-methyl-N-nitrosamino)-1-(3-pyridyl)-1-butanone. Journal of Pharmacology and Experimental Therapeutics, Vol.311, No.1, (October 2004), pp 123-130, ISSN 0022-3565

Yeboah, M. M., Xue, X. Y., Javdan, M., Susin, M. \& Metz, C. N. (2008). Nicotinic acetylcholine receptor expression and regulation in the rat kidney after ischemiareperfusion injury. American Journal of Physiology-Renal Physiology, Vol.295, No.3, (September 2008), pp F654-F661, ISSN 0363-6127 


\title{
Advances in MR Imaging of Leukodystrophies
}

\author{
Eva-Maria Ratai ${ }^{1}$, Paul Caruso ${ }^{1}$ and Florian Eichler ${ }^{2}$ \\ ${ }^{1}$ Department of Radiology, \\ ${ }^{2}$ Department of Neurology, \\ Massachusetts General Hospital, \\ Harvard Medical School, Boston, MA, \\ USA
}

\section{Introduction}

Leukodystrophies are hereditary disorders of white matter that impair brain that is initially normally formed and developed $(1,2)$. They can affect brain myelin throughout life. The disorders are commonly progressive in nature and ultimately fatal. First manifestations are often cognitive deterioration and neuropsychological problems. Motor and balance difficulties occur, as do visual abnormalities. Classic leukodystrophies lead to a vegetative state or death within months to years. In general, the earlier the onset of symptoms, the more progressive the disease course.

Most leukodystrophies are monogenetic disorders. The mutant gene often encodes an enzyme or protein that maintains neuronal and/or glial health and is responsible for regulation of brain metabolism. Many enzymes involved play a role in lipid metabolism. The inability to degrade or synthesize substrate leads to an upstream excess or downstream lack of vital lipids. This can cause a wide range of pathology, from inflammatory demyelination to axonal degeneration and microglial activation. Hence, the often cited prominent demyelination is only one manifestation in leukodystrophies, and myelinforming oligodendrocytes are not the only cells affected in these disorders.

Yet, there are some common characteristics that distinguish the pathology in leukodystrophies from that of other disorders. MRI has played a seminal role in visualization of the lesion pattern. (3). The demyelinating lesions are usually confluent and symmetric. Many of the classic disorders, such as X-linked adrenoleukodystrophy (X-ALD), metachromatic leukodystrophy (MLD) and Krabbe, show relative sparing of subcortical fibers. Yet again, other disorders have early involvement of the $U$ fibers and other unique characteristics, such as cystic rarefaction and degeneration.

Hereditary disorders that do not show demyelination but rather hypomyelination have come to increasing attention (4). Common neurological features in hypomyelinating disorders are developmental delay, nystagmus, cerebellar ataxia and spasticity. One of the better characterized hypomyelinating disorders is Pelizaeus Merzbacher disease (PMD). Beyond this classic disorder, a multitude of other hypomyelinating disorders exist. Only about half of the patients with evidence of hypomyelination on MRI come to a definitive diagnosis. Yet, specific clinical and MRI features can be pathognomonic and lead to diagnosis. 
The current review aims to present the diagnostic MRI lesion patterns of leukodystrophies, as well as the utility of advanced MR techniques. While some of these techniques are already established in clinical practice, others are still experimental in nature and require future validation.

\section{Conventional brain MRI lesion patterns in LD}

Overall, MR imaging has made an enormous contribution to the field of leukodystrophies due to the precise lesion pattern evident on MRI (5). Many leukodystophies classically show imaging features that, in some cases, are pathognomonic and, in some cases, highly suggestive of the diagnosis. Both demyelinating and hypomyelinating disorders carry distinct features and are listed in Tables 1 and 2. While the patterns of maturation of white matter are similar on T1 and T2 weighted images, white matter appears to mature at a later time on T2 weighted images. This is crucial, as it indicates that T1 weighted imaging may be more sensitive to immature myelin than $\mathrm{T} 2$ weighted imaging.

In its most severe form, X-ALD is a lethal neurodegenerative disorder with inflammatory demyelination. Defective peroxisomal beta-oxidation causes accumulation of very long-chain fatty acids (VLCFA) in tissues and plasma, particularly in the nervous system and adrenal glands. At least four clinical phenotypes have been delineated: childhood cerebral (CCALD), adult cerebral, adrenomyeloneuropathy (AMN), and female heterozygotes for X-ALD.

In CCALD, the posterior regions of the brain are involved in $80-90 \%$ and the frontal regions are involved in $5-10 \%(6,7)$. The lesion evolves in a symmetric confluent fashion starting in the splenium or genu of the corpus callosum and spreading into the periventricular white matter (Figure 1). The arcuate fibers are most often spared. In the acute phase, a garland of contrast enhancement is present. In the final stages, white matter atrophy is seen. The systematic progression has given rise to a scoring system of 34 points $(8,9)$. This pattern is markedly different than that seen in the adult form of the disease, AMN, a noninflammatory chronic axonopathy.

In Krabbe disease, the parieto-occipital lesions are also present, although a garland of contrast enhancement is never seen $(10,11)$. MLD shows more diffuse involvement of both frontal and posterior regions of the brain (12). Involvement of the corpus callosum is seen early, although not as striking as that seen in ALD or GLD. A tigroid pattern is often apparent in the centrum semiovale. In contrast to ALD, the outer subarachnoid spaces are not enlarged in MLD, even in the most advanced stages of disease.

Other disorders have characteristic early involvement of the subcortical fibers. Children with Canavan disease present with an enlarged head ("megalencephaly"), but show less behavioral changes than other leukodystrophies of infancy. $(13,14)$. Their MRI shows diffuse involvement of white matter including the subcortical $U$ fibers (15). There is also involvement of basal ganglia and other gray matter structures. Alexander disease is another leukodystrophy that often manifests with megalencephaly (16). Imaging studies of the brain typically show cerebral white matter abnormalities, preferentially affecting the frontal region, although unusual variants are coming to increasing attention $(17,18)$.

The MRI of vanishing white matter disease (VWMD) also has a characteristic pattern. It shows progressive loss of white matter over time on proton density and FLAIR images (19-21). The findings on autopsy confirm the white matter rarefaction and cystic 
degeneration suggested by the MRI. Regions of relative sparing include the U-fibers, corpus callosum, internal capsule, and the anterior commissure. The cerebellar white matter and brainstem show variable degrees of involvement but do not undergo cystic degeneration.

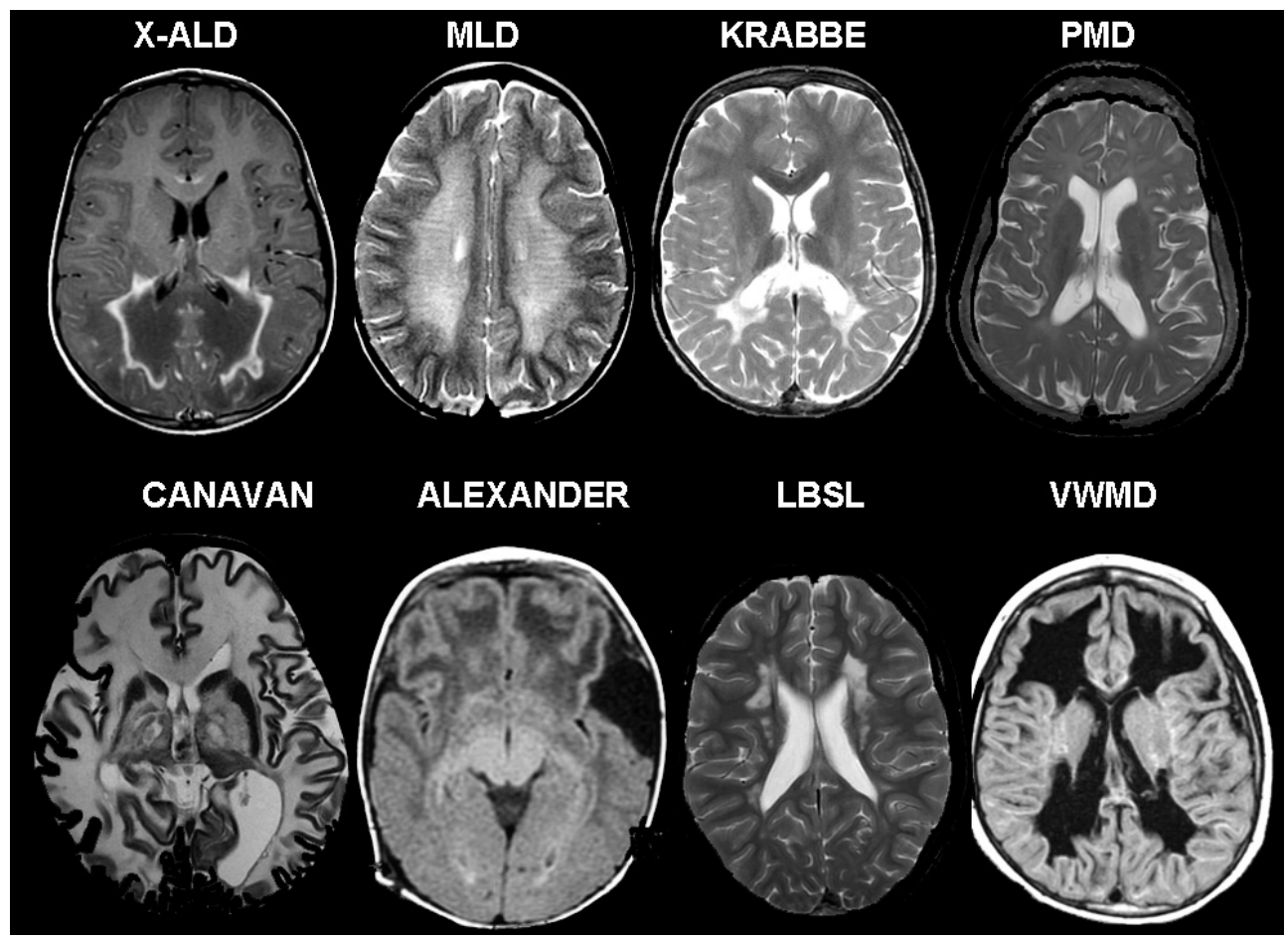

Fig. 1. Lesion Patterns on Conventional MRI in Leukodystrophies and Hypomyelinating Disorders

Brainstem lesion patterns can also often provide critical clues to the diagnosis. Leukoencephalopathy with Brainstem and Spinal Cord Involvement and Elevated White Matter Lactate (LBSL) has recently been described and shows distinct involvement of the pyramidal tracts, medial lemniscus, mesencephalic trigeminal tracts, corticobulbar tracts, and superior cerebellar peduncles. (22). Recognition of the distinct lesion pattern led to the identification of the responsible gene DARS2, which encodes mitochondrial aspartyl-tRNA synthetase.

In hypomyelinating disorders, the boundaries between gray and white matter often appear "blurred" (4). The T2 hypointensity of the white matter is milder in hypomyelination than in demyelination and other white matter pathology. Overall, the brain MRI in hypomyelination looks like that of a young child, with less well distinguished gray and white matter. As opposed to "delayed myelination," the pattern on brain MRI is unchanged, as in myelination is "stuck" on two MRIs 6-12 months apart in a child older than one year of age. While at first glance, hypomyelinating disorders may all have a similar MRI 
appearance, there are a group of disorders for which the MRI scan can provide clues to the diagnosis (see Table 2). In these individual disorders attention to the deep gray matter structures will often reveal a characteristic "signature" (4).

\begin{tabular}{|c|c|c|}
\hline Leukodystrophy & $\begin{array}{l}\text { Mutated } \\
\text { genes }\end{array}$ & Characteristics on Brain MRI \\
\hline $\begin{array}{l}\text { Aicardi-Goutières } \\
\text { syndrome }(78,79)\end{array}$ & TREX1 & $\begin{array}{l}\text { Often with microcephaly and intracranial } \\
\text { calcifications (CT) }\end{array}$ \\
\hline Alexander Disease & GFAP & $\begin{array}{l}\text { Macrocephaly with frequent frontal lesion } \\
\text { predominance in childhood, variants with } \\
\text { abnormalities in medulla and spinal cord, } \\
\text { ventricular garlands }\end{array}$ \\
\hline Canavan Disease & ASPA & $\begin{array}{l}\text { Macrocephaly, subcortical U fibers and } \\
\text { basal ganglia involved }\end{array}$ \\
\hline $\begin{array}{l}\text { Cerebral autosomal } \\
\text { dominant arteriopathy } \\
\text { with subcortical infarcts } \\
\text { and leukoencephalopathy } \\
\text { (CADASIL) }\end{array}$ & NOTCH3 & $\begin{array}{l}\text { Multiple small hemorrhages. Widened } \\
\text { perivascular spaces in the centrum } \\
\text { semiovale and basal ganglia }\end{array}$ \\
\hline $\begin{array}{l}\text { Cerebrotendineous } \\
\text { Xanthomatosis }\end{array}$ & CYP27A1 & $\begin{array}{l}\text { Cerebellar lesions, calcifications visible on } \\
\mathrm{CT}\end{array}$ \\
\hline $\begin{array}{l}\text { Globoid Leukodystrophy } \\
\text { (Krabbe Disease) }\end{array}$ & GALC & $\begin{array}{l}\text { Posterior predominance, no contrast } \\
\text { enhancement }\end{array}$ \\
\hline $\begin{array}{l}\text { Leukoencephalopathy } \\
\text { with Brain Stem and } \\
\text { Spinal Cord Involvement } \\
\text { and Elevated Lactate }\end{array}$ & DARS2 & $\begin{array}{l}\text { Characteristic brainstem pattern: pyramidal } \\
\text { tracts, cerebellar connections and } \\
\text { intraparenchymal trajectories of trigeminal } \\
\text { nerve }\end{array}$ \\
\hline $\begin{array}{l}\text { Megalencephalic } \\
\text { Leukodystrophy with } \\
\text { Cysts }(80,81)\end{array}$ & $\begin{array}{l}\text { MLC1, } \\
\text { GLIALCAM }\end{array}$ & $\begin{array}{l}\text { Macrocephaly with swelling of cerebral } \\
\text { white matter and cystic lesions (bilateral } \\
\text { anterior temporal lobes) }\end{array}$ \\
\hline $\begin{array}{l}\text { Metachromatic } \\
\text { leukodystrophy }\end{array}$ & ARSA & $\begin{array}{l}\text { Diffuse with initial subcortical sparing, } \\
\text { "tigroid pattern" in centrum semiovale }\end{array}$ \\
\hline $\begin{array}{l}\text { Vanishing White Matter } \\
\text { Disease }\end{array}$ & EIF2B1-5 & $\begin{array}{l}\text { Confluent cystic degeneration, white } \\
\text { matter signal appears CSF-like }\end{array}$ \\
\hline $\begin{array}{l}\text { X-Linked } \\
\text { Adrenoleukodystrophy }\end{array}$ & ABCD1 & $\begin{array}{l}\text { CCALD: posterior predominance with } \\
\text { contrast enhancement in acute phase } \\
\text { AMN: corticospinal tracts and dorsal } \\
\text { columns, no contrast enhancement }\end{array}$ \\
\hline
\end{tabular}

Table 1. Characteristics on Brain MRI in Leukodystrophies 


\begin{tabular}{|c|c|c|}
\hline Hypomyelinating Disorder & Mutated genes & Characteristics on Brain MRI \\
\hline Fucosidosis & FUCA1 & T2 hypointensity globus pallidus \\
\hline GM2 gangliosidoses & HEXA, HEXB & $\mathrm{T} 2$ hyperintensity in the basal ganglia \\
\hline $\begin{array}{l}\text { Hypomyelination with } \\
\text { atrophy of the basal } \\
\text { ganglia and cerebellum }\end{array}$ & unknown & $\begin{array}{l}\text { Atrophy of the basal ganglia and } \\
\text { cerebellum }\end{array}$ \\
\hline $\begin{array}{l}\text { Hypomyelination, } \\
\text { Hypodontia, } \\
\text { Hypogonadotropic } \\
\text { Hypogonadism }\end{array}$ & unknown & $\begin{array}{l}\text { Early cerebellar atrophy with absence of } \\
\text { putamen }\end{array}$ \\
\hline $\begin{array}{l}\text { Pelizaeus-Merzbacher } \\
\text { Disease }\end{array}$ & PLP1 & $\begin{array}{l}\text { Homogeneous } \mathrm{T} 2 \text { hyperintensity of } \\
\text { cerebral white matter }\end{array}$ \\
\hline $\begin{array}{l}\text { Pelizaeus-Merzbacher- } \\
\text { like Disease }\end{array}$ & $\begin{array}{l}\text { GJC2, } \\
\text { SLC16A2 }\end{array}$ & Pontine T2 hyperintensity \\
\hline
\end{tabular}

Table 2. Characteristics on Brain MRI in Hypomyelinating Disorders

Advanced magnetic resonance (MR) imaging techniques, such as proton MR spectroscopic and diffusion tensor (DT) MR imaging, permit the investigation of changes in metabolite levels and water diffusion parameters in leukodystrophy patients. Both metabolite measures and water diffusion parameters offer an opportunity to assess the degree of axonal loss and demyelination in the leukodystrophies.

\section{Proton MR spectroscopy}

Magnetic resonance spectroscopy (MRS) offers the unique ability to measure metabolite levels in vivo in a non-invasive manner $(23,24,25)$. These metabolite quantifications can be used to identify disease, measure the severity of an injury, or monitor a patient's response to treatment. Table 3 shows the most well characterized metabolite abnormalities detected by MRS in leukodystrophies.

The resonances seen in the brain by MRS are typically low weight molecules (see Figure 2). In the normal brain, the most prominent peak arises from N-acetylaspartate (NAA) at 2.0 ppm. The other major peaks include creatine $(\mathrm{Cr})$ and phosphocreatine (phospho- $\mathrm{Cr}$ ), which are observed at 3.0 and 3.3 ppm, respectively, as well as choline containing compounds. $1 \mathrm{H}$ MR spectra acquired with short echo times are characterized by additional resonances from myo-inositol (MI) at $3.5 \mathrm{ppm}$, and glutamate and glutamine, which overlap with each other so that they are often referred to as Glx, at $\sim 2.5 \mathrm{ppm}$. Under normal conditions, the lactate concentration is very low in the adult brain. This resonance (observed as a doublet) occurs at $1.32 \mathrm{ppm}$.

NAA within the adult brain is found exclusively in neurons, serving as a marker of neuronal density and viability and reported to be decreased in a number of neurological disorders. 
Leukodystrophy or Hypomyelinating Disorder

Systemic metabolites

Unknown

Urine N-

Canavan Disease

Cerebrotendineous

Xanthomatosis

Globoid Leukodystrophy

(Krabbe Disease)

GM2 gangliosidoses

Hypomyelination with atrophy of the basal ganglia and cerebellum

Leukoencephalopathy with Brain

Stem and Spinal Cord

Involvement and Elevated Lactate

Leukoencephalopathy associated Arabinitol and ribitol with a disturbance in the metabolism of polyols $(83,84)$

Megalencephalic

Leukodystrophy with Cysts

Pelizaeus-Merzbacher Disease Unknown

Vanishing White Matter Disease

X-Linked

Adrenoleukodystrophy
Unknown

Unknown in urine, plasma and CSF

Unknown

Decreased ratio of asialotransferrin to transferrin in CSF

Plasma very long chain fatty acids
Brain metabolites abnormalities on proton MR spectroscopy

Infantile form: myoinositol elevations in white and gray matter, decreased Nacetylaspartate

Highly elevated Nacetylaspartate

Lipid peaks seen in cerebellum

Choline and myoinositol elevations, decreased $\mathrm{N}$-acetylaspartate Infantile: variable choline, myoinositol and Nacetylaspartate.

Late onset: decreased Nacetylaspartate

Increased myoinositol and creatine

Decreased N-acetylaspartate and increased myo-inositol, choline and lactate

Elevated levels of arabinitol and ribitol (coupled resonances between 3.5 and 4ppm)

Decreased ratio of $\mathrm{N}$ acetylaspartate to Creatine

Variable reports on changes in $\mathrm{N}$-acetylaspartate and choline

Within cystic white matter complete absence of all metabolites

CCALD: Choline elevations within normal appearing white matter, elevations of lactate within the lesion.

AMN: decreased Nacetylaspartate in adrenomyeloneuropathy

Table 3. Brain Metabolites Abnormalities on Proton MR Spectroscopy 
NAA is the source of acetyl in myelin membrane biosynthesis (26) and is coupled to lipid metabolism and energy generation (27). Creatine serves as a marker for energy-dependent systems in cells and it tends to be low in processes that have low metabolism, such as necrosis and infarction. As $\mathrm{Cr}$ and phospho-Cr are in equilibrium, the $\mathrm{Cr}$ peak is thought to remain stable in size, despite bioenergetic abnormalities that occur with multiple pathologies. Consequently, the $\mathrm{Cr}$ resonance is often used as an internal standard.

The choline (Cho) resonance arises from signals of several soluble components that resonate at $3.2 \mathrm{ppm}$. This resonance contains contributions primarily from glycerophosphocholine (GPC), phosphocholine (PCho), and Cho. Changes in this resonance are commonly seen with diseases that have alterations in membrane turnover and in inflammatory and gliotic processes $(28,29)$. The function of MI is not fully understood, although it is believed to be an essential requirement for cell growth, an osmolyte, and a storage form for glucose (30). MI is primarily located in glia, and an increase in MI is commonly thought to be a marker of gliosis (31). Lactate is produced by anaerobic metabolism, and increased lactate has been found during hypoxia (32), mitochondrial diseases (33,34), seizures (35), and in the first hours after birth $(36,37)$.

In the developing brain, Cho and MI are the dominant peaks in the MR spectrum. Their levels are high compared to $\mathrm{Cr}$. In contrast, NAA levels are low in newborns and increase with age, while Cho and MI decrease with age. During the first 6 months of life, these metabolic changes are most rapid, leveling off at about 30 months of age (50). These changes are crucial, as both hypomyelination and delayed myelination affect changes of these metabolites.

In X-ALD, proton MRS sometimes shows metabolite abnormalities beyond the margins of disease depicted by conventional MR imaging (38). The white matter lesion in children with X-ALD shows reduced NAA/Cr and increased $\mathrm{Cho} / \mathrm{Cr}, \mathrm{MI} / \mathrm{Cr}$ and $\mathrm{Glx} / \mathrm{Cr}$ (30). Spectroscopic changes in normal appearing white matter (NAWM) that precede disease progression in patients with X-ALD have been described (39). The changes are an increase in choline and a decrease in NAA. They occur in areas where subsequent lesion progression is observed, but not in the remainder of the brain. These areas may represent a zone of impending or beginning demyelination.

Adrenomyeloneuropathy (AMN) is the adult variant of X-ALD. The disease pathology is usually limited to spinal cord and peripheral nerves ("'pure $\mathrm{AMN}^{\prime \prime}$ ) but shows cerebral involvement on histopathology. MRS studies showed reduced global NAA/Cho and NAA/Cr compared to controls. These changes are most prominent in internal capsule and parieto-occipital white matter. Decreased ratios of NAA in the absence of Cho/Cr elevation suggest prominent axonal involvement (40). Furthermore, Dubey et al demonstrated that the Expanded Disability Status Scale (EDSS) score inversely correlated with global NAA/Cr, suggesting a potential role of axonal injury in clinical disability in pure AMN. (40). Brain involvement demonstrable by MRI is rare in female subjects heterozygous for X-ALD, including those who have clinical evidence of spinal cord involvement. Nevertheless, NAA levels are reduced in the corticospinal projection fibers in female subjects with normal results on MRI, suggesting axonal dysfunction (41).

Canavan disease is caused by a deficiency in aspartoacylase (ASPA), an enzyme involved in the process of degrading NAA to aspartate and acetate. Deficiency leads to the accumulation of NAA, which impairs normal myelination and results in spongiform degeneration of the brain $(42,43)$. The elevations in NAA can be detected by MR spectroscopy in vivo (Figure 2), a diagnostic clue that can then be confirmed by urine measurement of NAA. The distinctly higher NAA peak can even be detected in the newborn 


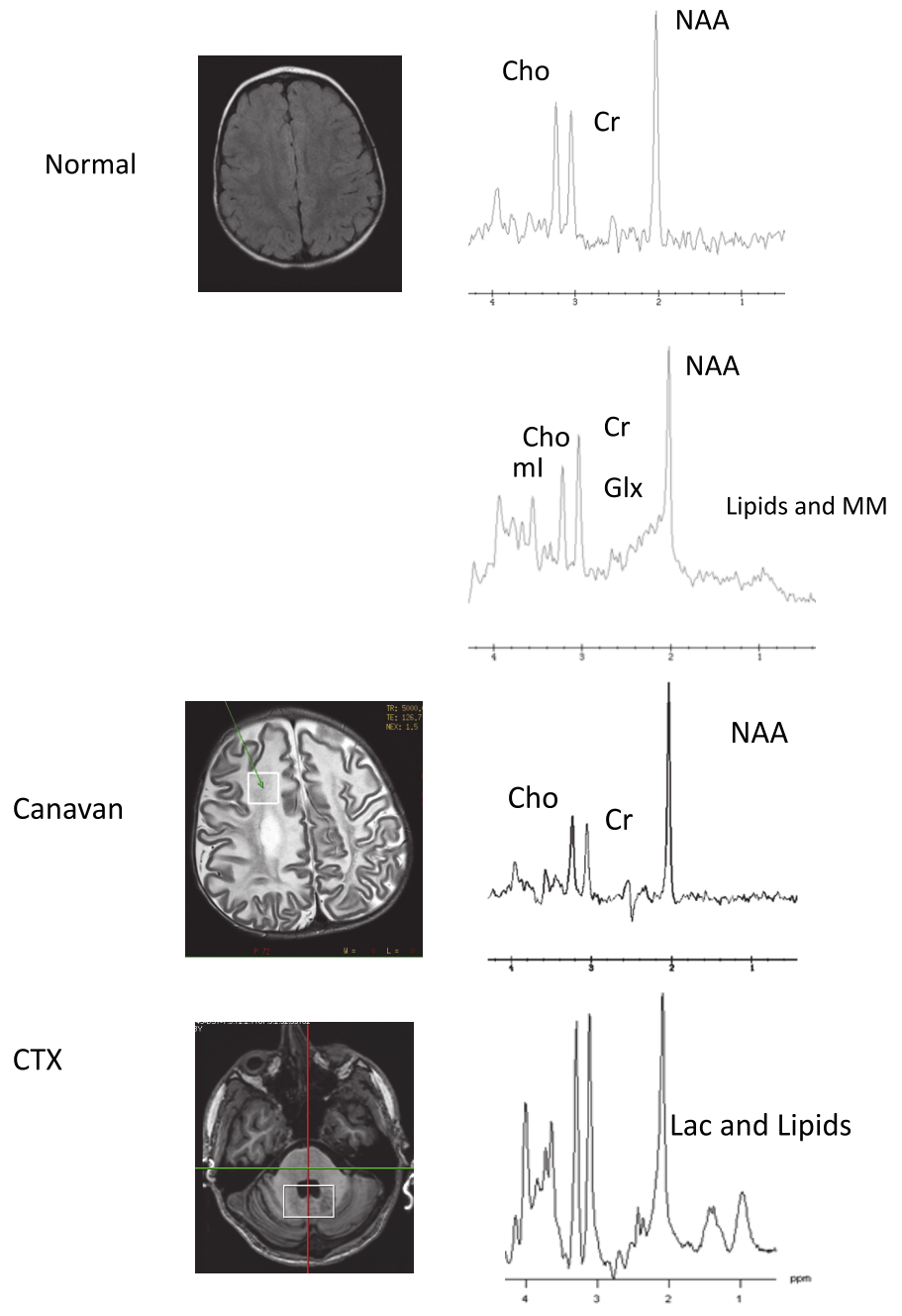

ALD
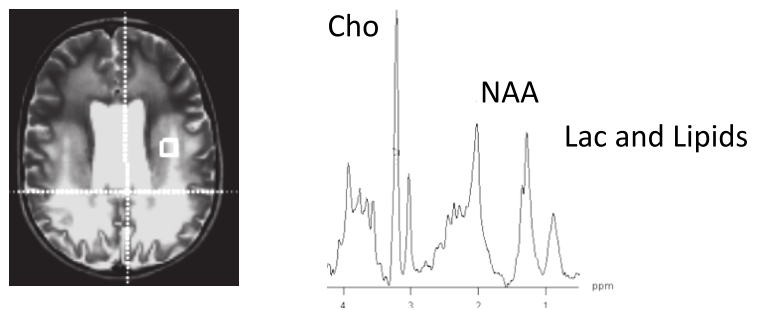

Fig. 2. The spectra of a normal brain are shown at both long echo time (top), TE $=250-280$ $\mathrm{ms}$, and short echo time (bottom), $\mathrm{TE}=20-50 \mathrm{~ms}$. The spectra for the disease states are as follows: the Canavan spectra are acquired at long echo time, and the ALD and CTX spectra are acquired at short echo time. 
although a radiologist only familiar with MR spectra in adults may not recognize the elevation in the newborn as pathologic.

LBSL is a disorder clinically characterized by slowly progressive signs of pyramidal, cerebellar and dorsal column dysfunction. LBSL shows a very distinct MRI pattern, with selective involvement of cerebral and cerebellar white matter and brainstem and spinal tracks, while U-fibers are spared (44). In LBSL, MR spectroscopy characteristically reveals decreased NAA and increased lactate, Cho and MI in the white matter, suggesting axonal damage and gliosis (45). Lately, mutations in the DARS2 gene, which encodes mitochondrial aspartyl-tRNA synthetase, have been identified as the underlying defect.

Cerebrotendinous xanthomatosis (CTX) is a rare but treatable disorder characterized by a defect in the metabolic pathway of cholesterol (46). Symptoms in infancy include diarrhea, cataracts and psychomotor retardation. In adulthood, the spectrum of neurologic dysfunction includes mental retardation leading to dementia, psychiatric symptoms, premature retinal aging, and epileptic seizures. The most distinctive MR imaging abnormalities are bilateral T2 hyperintensities in the dentate nuclei and adjacent cerebellar white matter (47). An MRS study showed a reduction in NAA levels and the presence of a lactate peak (see Figure 2). NAA decreases are attributed to neuroaxonal damage due to neurotoxic deposition of cholesterol (48). A recent case report described the presence of abnormal lipid peaks at 0.9 and $1.3 \mathrm{ppm}$ in the cerebellar hemisphere (49). These peaks can either be attributed to membrane breakdown or they may serve as surrogate markers of major lipid storage, with a potential role in monitoring therapeutic response. In addition, one patient had an increase in MI concentration, pointing to gliosis and astrocytic proliferation (50).

The MR spectra of patients with 4H-Syndrome, a rare form of hypomyelinating leukodystrophy, reveals low $\mathrm{Cho} / \mathrm{Cr}$ and NAA/Cr, while a prominent MI peak can be observed $(51,52)$. Low Cho levels are indicative of hypomyelination due to decreased membrane synthesis and turnover. In a new syndrome characterized by Hypomyelination with Atrophy of the Basal Ganglia and Cerebellum (H-ABC), MI and $\mathrm{Cr}$ levels are found to be elevated in the cerebral white matter, while NAA and choline levels are normal (53). These findings suggest that neither axonal loss nor active demyelination occurs in the setting of gliosis. In Pelizaeus Merzbacher disease, another hypomyelinating disorder, there have been discrepant reports on metabolite abnormalities detected by MRS. In part, these findings may be explained by the concurrent pathophysiologic processes of hypomyelination, gliosis, and neuronal loss over time (54).

\section{Diffusion tensor imaging}

While conventional MRI and MR spectroscopy show greatest utility in the diagnoses of leukodystrophies, other techniques, such as diffusion tensor imaging, are starting to be applied to leukodystrophies as well. Diffusion tensor (DT) MRI measures diffusivity of free water molecules in the brain. From the diffusion tensor, one can calculate indices that describe features of the orientationally averaged water diffusivity (isotropic part) and the water molecule displacements affected by the orientation of a regularly ordered structure in the tissue (anisotropic diffusion). Compared to gray matter, in which diffusion shows less directional dependence, white matter apparent diffusion is very anisotropic. (55). This 
property, termed fractional anisotropy (FA), depends on the orientation of the white matter tracks and on the degree and integrity of myelination (56-59).

In X-ALD, DT imaging may reflect the different abnormal white matter (AWM) zones in patients with X-ALD (60). We and others have observed that FA decreases and the isotropic apparent diffusion coefficient (iADC) increases over the zones toward the center of the lesion. The decrease in mean FA indicates the loss of an ordered structure governing the directionality of water molecule displacement. The increase in mean iADC suggests an increase in free water and a decrease in structures that restrict water diffusion.

In X-ALD, a strong correlation exists between NAA levels and FA, reinforcing the concept that both reflect axonal integrity. However, proton MR spectroscopic imaging reveals a low NAA level in regions with normal MR and DT imaging findings (61). In contrast, DT imaging showed no abnormalities outside the lesion on T2-weighted images. Further, the membrane turnover and cell accumulation associated with beginning demyelination, recognized in the enhanced choline and creatine signal intensity on proton MR spectroscopic imaging, did not have an effect on diffusion parameters. This suggests that proton MR spectroscopic imaging may have a higher sensitivity than both conventional MR and DT imaging in the early detection of abnormalities related to demyelination or axonal loss in X-ALD patients.

However, in other instances, DTI has proven utility over proton MRSI. In pure AMN patients, DTI-based three-dimensional fiber tracking has shown occult tract-specific cerebral microstructural abnormalities in patients who had a normal conventional brain magnetic resonance image (62). This advance in MR imaging demonstrates that the corticospinal tract abnormalities in AMN reflect a centripetal extension of the spinal cord long-tract distal axonopathy.

DTI anisotropy has also proved useful in Krabbe disease (63, 64). In particular, measurements of white matter metabolites may help assess disease progression and determine optimal candidates for treatment options. Patients with Krabbe disease who underwent stem cell transplantation within the 1st month of life showed substantially smaller decreases in anisotropy ratios than those who were treated later. These findings correlate well with global assessments of disease progression as recognized by neurodevelopmental evaluations and conventional MR imaging.

DT MR imaging studies of cerebral white matter development in human premature and term infants demonstrated that, in general, the apparent diffusion coefficient decreases while relative anisotropy increases with brain maturation. The most prominent regional difference at term is the increased relative anisotropy in the internal capsule, indicative of high directionality of diffusion, which could in part be related to myelination. Axonal diameter increases before and during myelination. This diameter change could also contribute in an important way to the diminished water diffusion perpendicular to the orientation of the fiber and thereby contribute to the increase in relative anisotropy. As anisotropy precedes myelination changes seen on conventional imaging, it is expected that quantitative analysis of diffusion parameters would add to the field of hypomyelinating disorders. Yet, little or no data is currently available in this group of disorders. Clearly, more systematic research is needed in regards to hypomyelination and DTI. 


\section{Recent applications of advanced MR technology}

One major technological advance has been imaging at higher field strength. Magnetic resonance imaging at 4 and 7T allows for better visualization of lesion architecture, white matter tracts, and gray-white matter distinction compared with 1.5T. The field of proton MR spectroscopy has also benefited from higher field strength $(65,66)$. Better spectral resolution results from improved signal-to-noise ratio and chemical-shift dispersion, and this in turn leads to more reliable detection of metabolites such as myoinositol and glutamine.

Using 7T MRSI, decreases in NAA in the cortex of X-ALD patients were detected, which appears greater in male hemizygotes than in female heterozygotes and most pronounced with the occurrence of white matter lesions in males. (66). Although the cytoarchitecture of the cerebral cortex generally appears normal in X-ALD, scattered neuronal loss can be seen in gray matter during a pathologic examination. Both ratios of myoinositol and choline to creatine were found to be higher in normal appearing white matter of adult ALD patients with brain lesions compared to those without lesions. Yet, the interpretation of 7T MRSI data also poses challenges. Voxels close to the scalp show poor water and lipid suppression. Also, the quantification of spectral data in the presence of substantial radiofrequency excitation field (B1) variations is difficult. Therefore, focus has shifted on using adiabatic pulses to compensate for radiofrequency inhomogeneity and reduce the chemical shift displacement error $(67,68)$.

Overcoming some of the shortcomings of DTI, novel methods now exist to map complex fiber architectures of white matter and other brain tissues. Diffusion spectrum imaging (DSI) allows resolution of regions of 3 way fiber crossings (69-71). On DTI this was not possible as fiber crossing led to decreases in FA, making it difficult to distinguish pathological changes from normal fiber crossings. The years ahead will likely bring more studies employing DSI in leukodystrophy patients.

Advances have also been in the development of ex vivo MRI and novel magnetic resonance contrast agents for imaging of autopsy specimen. In metachromatic leukodystrophy, postmortem studies have demonstrated the pathological correlate of the "tigroid stripes" characteristically seen on conventional imaging (72). Through direct correlation of postmortem MR imaging on $1 \mathrm{~cm}$ thick blocks with neuropathology staining, the authors were able to show that perivascular clusters of glial cells containing lipid material corresponded to the stripes on MRI.

Using contrast agents ex vivo, the corresponding substrate of imaging can be further elucidated. One such example is luxol fast blue that displays a binding affinity for myelinated constituents of the brain (73). The specificity of luxol fast blue for lipid constituents results in an increase in longitudinal and transverse relaxation rates of tissue dependent on myelination status. The relaxation rates of white matter increase sufficiently to permit T1-weighted images of ex vivo samples that are similar in contrast behavior to T1-weighted in vivo imaging. The contrast increases in MR images of LFB-stained ex vivo brain tissues enhancing delineation between upper lamina and the more myelinated lower lamina.

Other advances in MR technology have brought great practical benefits. Sedation and anesthesia represent risks in advanced brain disease of LD patients. Using new techniques, such as propeller MRI, it has become possible to oversample $\mathrm{k}$ space and, thereby, compensate for motion and allow follow-up MR imaging without sedation $(74,75)$. As an 
alternative to these retrospective motion-correction techniques, it is also possible to prospectively correct motion in structural imaging and single-voxel spectroscopy using image-based navigators (76-78). In patients with more advanced leukodystrophies, these advances may allow for imaging without sedation and thereby give insight into the more advanced stages of disease.

Overall, not one advanced imaging technology is expected to bring about a breakthrough in the leukodystrophies. Rather, the multimodal approach with coregistration of high resolution imaging with advanced spectroscopic and diffusion imaging will lead to new pathophysiological insights in the years to come. Different diseases, varying phenotypes, and stages within the disease will require varying imaging modalities.

\section{Conclusions}

MRI has allowed for much progress in the field of leukodystrophies. Prior to arrival of MRI, the specific vulnerability of brain white matter was not well understood. Today, MRI has helped define disorders through the recognition of specific lesion patterns and their evolution over time. This has also led to identification of novel leukodystrophies and the genes underlying these disorders. Even in previously well characterized disorders, MRI patterns have shed light on disease mechanisms.

The understanding of the pathology and molecular basis of leukodystrophies has in turn allowed for new insight into the significance of MRI changes and elucidated the capabilities of MR techniques. Brain MRI today is a valuable tool in monitoring disease progression and the success of therapeutic interventions in leukodystrophies. Advances in new techniques encourage a multimodal approach employing a variety of sequences sensitive to different brain tissue characteristics. Together, these techniques will be able to provide clues to the early stages of disease - insight not gained by pathology in the past.

\section{References}

[1] Costello DJ, Eichler AF, Eichler FS. Leukodystrophies: classification, diagnosis, and treatment. Neurologist 2009;15(6):319-28.

[2] Kohlschutter A, Bley A, Brockmann K, Gartner J, Krageloh-Mann I, Rolfs A, et al. Leukodystrophies and other genetic metabolic leukoencephalopathies in children and adults. Brain Dev;32(2):82-9.

[3] van Der Knaap MS. Magnetic Resonance of Myelination and Myelin Disorders, 3rd edition. 2005.

[4] Steenweg ME, Vanderver A, Blaser S, Bizzi A, de Koning TJ, Mancini GM, et al. Magnetic resonance imaging pattern recognition in hypomyelinating disorders. Brain;133(10):2971-82.

[5] Schiffmann R, van der Knaap MS. Invited article: an MRI-based approach to the diagnosis of white matter disorders. Neurology 2009;72(8):750-9.

[6] Moser H SK, Watkins P, Powers J, Moser A. X-linked adrenoleukodystrophy. 8th ed. New York: McGraw Hill; 2000. 
[7] Melhem ER, Barker PB, Raymond GV, Moser HW. X-linked adrenoleukodystrophy in children: review of genetic, clinical, and MR imaging characteristics. AJR Am J Roentgenol 1999;173(6):1575-81.

[8] Loes DJ, Hite S, Moser H, Stillman AE, Shapiro E, Lockman L, et al. Adrenoleukodystrophy: a scoring method for brain MR observations. AJNR Am J Neuroradiol 1994;15(9):1761-6.

[9] Loes DJ, Fatemi A, Melhem ER, Gupte N, Bezman L, Moser HW, et al. Analysis of MRI patterns aids prediction of progression in X-linked adrenoleukodystrophy. Neurology 2003;61(3):369-74.

[10] Wenger DA SK, Suzuki Y, Suzuki K. Galactosylceramide lipidosis: globoid cell leukodystrophy (Krabbe disease). 2001:3669-94.

[11] Loes DJ, Peters C, Krivit W. Globoid cell leukodystrophy: distinguishing early-onset from late-onset disease using a brain MR imaging scoring method. AJNR Am J Neuroradiol 1999;20(2):316-23.

[12] Eichler F, Grodd W, Grant E, Sessa M, Biffi A, Bley A, et al. Metachromatic leukodystrophy: a scoring system for brain MR imaging observations. AJNR Am J Neuroradiol 2009;30(10):1893-7.

[13] Kumar S, Mattan NS, de Vellis J. Canavan disease: a white matter disorder. Ment Retard Dev Disabil Res Rev 2006;12(2):157-65.

[14] Surendran S, Matalon KM, Tyring SK, Matalon R. Molecular basis of Canavan's disease: from human to mouse. J Child Neurol 2003;18(9):604-10.

[15] Janson CG, McPhee SW, Francis J, Shera D, Assadi M, Freese A, et al. Natural history of Canavan disease revealed by proton magnetic resonance spectroscopy (1H-MRS) and diffusion-weighted MRI. Neuropediatrics 2006;37(4):209-21.

[16] van der Knaap MS, Naidu S, Breiter SN, Blaser S, Stroink H, Springer S, et al. Alexander disease: diagnosis with MR imaging. AJNR Am J Neuroradiol 2001;22(3):541-52.

[17] van der Knaap MS, Salomons GS, Li R, Franzoni E, Gutierrez-Solana LG, Smit LM, et al. Unusual variants of Alexander's disease. Ann Neurol 2005;57(3):327-38.

[18] van der Knaap MS, Ramesh V, Schiffmann R, Blaser S, Kyllerman M, Gholkar A, et al. Alexander disease: ventricular garlands and abnormalities of the medulla and spinal cord. Neurology 2006;66(4):494-8.

[19] van der Knaap MS, Barth PG, Gabreels FJ, Franzoni E, Begeer JH, Stroink H, et al. A new leukoencephalopathy with vanishing white matter. Neurology 1997;48(4):84555.

[20] van der Knaap MS, Leegwater PA, Konst AA, Visser A, Naidu S, Oudejans CB, et al. Mutations in each of the five subunits of translation initiation factor eIF2B can cause leukoencephalopathy with vanishing white matter. Ann Neurol 2002;51(2):264-70.

[21] Leegwater PA, Vermeulen G, Konst AA, Naidu S, Mulders J, Visser A, et al. Subunits of the translation initiation factor eIF2B are mutant in leukoencephalopathy with vanishing white matter. Nat Genet 2001;29(4):383-8.

[22] Scheper GC, van der Klok T, van Andel RJ, van Berkel CG, Sissler M, Smet J, et al. Mitochondrial aspartyl-tRNA synthetase deficiency causes eukoencephalopathy 
with brain stem and spinal cord involvement and lactate elevation. Nat Genet 2007;39(4):534-9.

[23] Barker PB, Horska A. Neuroimaging in leukodystrophies. J Child Neurol 2004;19(8):559-70.

[24] Barker PB BB, De Stefano N, Gullapalli R, Lin DDM Clinical MR Spectroscopy: Techniques and Applications. 2010.

[25] Lin A, Ross BD, Harris K, Wong W. Efficacy of proton magnetic resonance spectroscopy in neurological diagnosis and neurotherapeutic decision making. NeuroRx, 2(2), 197-214, review (2005).

[26] Chakraborty G, Mekala P, Yahya D, Wu G, Ledeen RW. Intraneuronal Nacetylaspartate supplies acetyl groups for myelin lipid synthesis: evidence for myelin-associated aspartoacylase. J Neurochem 2001;78(4):736-45.

[27] Moffett JR, Ross B, Arun P, Madhavarao CN, Namboodiri AM. N-Acetylaspartate in the CNS: from neurodiagnostics to neurobiology. Prog Neurobiol 2007;81(2): 89-131.

[28] Pouwels PJ, Kruse B, Korenke GC, Mao X, Hanefeld FA, Frahm J. Quantitative proton magnetic resonance spectroscopy of childhood adrenoleukodystrophy. Neuropediatrics 1998;29(5):254-64.

[29] Tzika AA, Ball WS, Jr., Vigneron DB, Dunn RS, Nelson SJ, Kirks DR. Childhood adrenoleukodystrophy: assessment with proton MR pectroscopy. Radiology 1993;189(2):467-80.

[30] Ross BD. Biochemical considerations in $1 \mathrm{H}$ spectroscopy. Glutamate and glutamine; myo-inositol and related metabolites. NMR Biomed 1991;4(2):59-63.

[31] Brand A, Richter-Landsberg C, Leibfritz D. Multinuclear NMR studies on the energy metabolism of glial and neuronal cells. Dev Neurosci 1993;15(3-5):289-98.

[32] Kreis R, Arcinue E, Ernst T, Shonk TK, Flores R, Ross BD. Hypoxic encephalopathy after near-drowning studied by quantitative $1 \mathrm{H}$-magnetic resonance spectroscopy. J Clin Invest 1996;97(5):1142-54.

[33] Castillo M, Kwock L, Green C. MELAS syndrome: imaging and proton MR spectroscopic findings. AJNR Am J Neuroradiol 1995;16(2):233-9.

[34] Mathews PM, Andermann F, Silver K, Karpati G, Arnold DL. Proton MR spectroscopic characterization of differences in regional brain metabolic abnormalities in mitochondrial encephalomyopathies. Neurology 1993;43(12):2484-90.

[35] Breiter SN, Arroyo S, Mathews VP, Lesser RP, Bryan RN, Barker PB. Proton MR spectroscopy in patients with seizure disorders. AJNR Am J Neuroradiol 1994;15(2):373-84.

[36] Barkovich AJ, Miller SP, Bartha A, Newton N, Hamrick SE, Mukherjee P, et al. MR imaging, MR spectroscopy, and diffusion tensor imaging of sequential studies in neonates with encephalopathy. AJNR Am J Neuroradiol 2006;27(3):533-47.

[37] Barkovich AJ, Westmark KD, Bedi HS, Partridge JC, Ferriero DM, Vigneron DB. Proton spectroscopy and diffusion imaging on the first day of life after perinatal asphyxia: preliminary report. AJNR Am J Neuroradiol 2001;22(9):1786-94. 
[38] Holshouser BA, Ashwal S, Luh GY, Shu S, Kahlon S, Auld KL, et al. Proton MR spectroscopy after acute central nervous system injury: outcome prediction in neonates, infants, and children. Radiology 1997;202(2):487-96.

[39] Kruse B, Barker PB, van Zijl PC, Duyn JH, Moonen CT, Moser HW. Multislice proton magnetic resonance spectroscopic imaging in X-linked adrenoleukodystrophy. Ann Neurol 1994;36(4):595-608.

[40] Eichler FS, Barker PB, Cox C, Edwin D, Ulug AM, Moser HW, et al. Proton MR spectroscopic imaging predicts lesion progression on MRI in X-linked adrenoleukodystrophy. Neurology 2002;58(6):901-7.

[41] Dubey P, Fatemi A, Barker PB, Degaonkar M, Troeger M, Zackowski K, et al. Spectroscopic evidence of cerebral axonopathy in patients with "pure" adrenomyeloneuropathy. Neurology 2005;64(2):304-10.

[42] Fatemi A, Barker PB, Ulug AM, Nagae-Poetscher LM, Beauchamp NJ, Moser AB, et al. MRI and proton MRSI in women heterozygous for X-linked adrenoleukodystrophy. Neurology 2003;60(8):1301-7.

[43] Tsai G, Coyle JT. N-acetylaspartate in neuropsychiatric disorders. Prog Neurobiol 1995;46(5):531-40.

[44] Grodd W, Krageloh-Mann I, Klose U, Sauter R. Metabolic and destructive brain disorders in children: findings with localized proton MR spectroscopy. Radiology 1991;181(1):173-81.

[45] van der Knaap MS, Scheper GC. Leukoencephalopathy with Brain Stem and Spinal Cord Involvement and Lactate Elevation. 1993.

[46] Uluc K, Baskan O, Yildirim KA, Ozsahin S, Koseoglu M, Isak B, et al. Leukoencephalopathy with brain stem and spinal cord involvement and high lactate: a genetically proven case with distinct MRI findings. J Neurol Sci 2008;273(1-2):118-22.

[47] Salen G, Berginer V, Shore V, Horak I, Horak E, Tint GS, et al. Increased concentrations of cholestanol and apolipoprotein B in the cerebrospinal fluid of patients with cerebrotendinous xanthomatosis. Effect of chenodeoxycholic acid. N Engl J Med 1987;316(20):1233-8.

[48] De Stefano N, Dotti MT, Mortilla M, Federico A. Magnetic resonance imaging and spectroscopic changes in brains of patients with cerebrotendinous xanthomatosis. Brain 2001;124(Pt 1):121-31.

[49] Pilo de la Fuente B, Ruiz I, Lopez de Munain A, Jimenez-Escrig A. Cerebrotendinous xanthomatosis: neuropathological findings. J Neurol 2008;255(6):839-42.

[50] Embirucu EK, Otaduy MC, Taneja AK, Leite CC, Kok F, Lucato LT. MR spectroscopy detects lipid peaks in cerebrotendinous xanthomatosis. AJNR Am J Neuroradiol;31(7):1347-9.

[51] Outteryck O, Devos D, Jissendi P, Boespflug-Tanguy O, Hopes L, Renard D, et al. 4H syndrome: a rare cause of leukodystrophy. J Neurol;257(10):1759-61.

[52] Wolf NI, Harting I, Boltshauser E, Wiegand G, Koch MJ, Schmitt-Mechelke T, et al. Leukoencephalopathy with ataxia, hypodontia, and hypomyelination. Neurology 2005;64(8):1461-4. 
[53] van der Knaap MS, Linnankivi T, Paetau A, Feigenbaum A, Wakusawa K, Haginoya K, et al. Hypomyelination with atrophy of the basal ganglia and cerebellum: followup and pathology. Neurology 2007;69(2):166-71.

[54] Cecil KM. MR spectroscopy of metabolic disorders. Neuroimaging Clin N Am 2006;16(1):87-116, viii.

[55] Basser PJ, Mattiello J, LeBihan D. MR diffusion tensor spectroscopy and imaging. Biophys J 1994;66(1):259-67.

[56] Mori S, Crain BJ, Chacko VP, van Zijl PC. Three-dimensional tracking of axonal projections in the brain by magnetic resonance imaging. Ann Neurol 1999;45(2):265-9.

[57] Wakana S, Jiang H, Nagae-Poetscher LM, van Zijl PC, Mori S. Fiber tract-based atlas of human white matter anatomy. Radiology 2004;230(1):77-87.

[58] Mori S, Oishi K, Faria AV. White matter atlases based on diffusion tensor imaging. Curr Opin Neurol 2009;22(4):362-9.

[59] Wakana S, Caprihan A, Panzenboeck MM, Fallon JH, Perry M, Gollub RL, et al. Reproducibility of quantitative tractography methods applied to cerebral white matter. Neuroimage 2007;36(3):630-44.

[60] Ito R, Melhem ER, Mori S, Eichler FS, Raymond GV, Moser HW. Diffusion tensor brain MR imaging in X-linked cerebral adrenoleukodystrophy. Neurology 2001;56(4):544-7.

[61] Eichler FS, Itoh R, Barker PB, Mori S, Garrett ES, van Zijl PC, et al. Proton MR spectroscopic and diffusion tensor brain $M R$ imaging in X-linked adrenoleukodystrophy: initial experience. Radiology 2002;225(1):245-52.

[62] Dubey P, Fatemi A, Huang H, Nagae-Poetscher L, Wakana S, Barker PB, et al. Diffusion tensor-based imaging reveals occult abnormalities in adrenomyeloneuropathy. Ann Neurol 2005;58(5):758-66.

[63] Escolar ML, Poe MD, Smith JK, Gilmore JH, Kurtzberg J, Lin W, et al. Diffusion tensor imaging detects abnormalities in the corticospinal tracts of neonates with infantile Krabbe disease. AJNR Am J Neuroradiol 2009;30(5):1017-21.

[64] Provenzale JM, Escolar M, Kurtzberg J. Quantitative analysis of diffusion tensor imaging data in serial assessment of Krabbe disease. Ann N Y Acad Sci 2005;1064:220-9.

[65] Oz G, Tkac I, Charnas LR, Choi IY, Bjoraker KJ, Shapiro EG, et al. Assessment of adrenoleukodystrophy lesions by high field MRS in non-sedated pediatric patients. Neurology 2005;64(3):434-41.

[66] Ratai E, Kok T, Wiggins C, Wiggins G, Grant E, Gagoski B, et al. Seven-Tesla proton magnetic resonance spectroscopic imaging in adult X-linked adrenoleukodystrophy. Arch Neurol 2008;65(11):1488-94.

[67] Tannus A, Garwood M. Adiabatic pulses. NMR Biomed 1997;10(8):423-34.

[68] Andronesi OC, Ramadan S, Ratai EM, Jennings D, Mountford CE, Sorensen AG. Spectroscopic imaging with improved gradient modulated constant adiabaticity pulses on high-field clinical scanners. J Magn Reson;203(2):283-93. 
[69] Wedeen VJ, Wang RP, Schmahmann JD, Benner T, Tseng WY, Dai G, et al. Diffusion spectrum magnetic resonance imaging (DSI) tractography of crossing fibers. Neuroimage 2008;41(4):1267-77.

[70] Schmahmann JD, Pandya DN, Wang R, Dai G, D'Arceuil HE, de Crespigny AJ, et al. Association fibre pathways of the brain: parallel observations from diffusion spectrum imaging and autoradiography. Brain 2007;130(Pt 3):630- 53.

[71] Hagmann P, Sporns O, Madan N, Cammoun L, Pienaar R, Wedeen VJ, et al. White matter maturation reshapes structural connectivity in the late developing human brain. Proc Natl Acad Sci U S A;107(44):19067-72.

[72] van der Voorn JP, Pouwels PJ, Kamphorst W, Powers JM, Lammens M, Barkhof F, et al. Histopathologic correlates of radial stripes on MR images in lysosomal storage disorders. AJNR Am J Neuroradiol 2005;26(3):442-6.

[73] Blackwell ML, Farrar CT, Fischl B, Rosen BR. Target-specific contrast agents for magnetic resonance microscopy. Neuroimage 2009;46(2):382-93.

[74] Tamhane AA, Arfanakis K. Motion correction in periodically-rotated overlapping parallel lines with enhanced reconstruction (PROPELLER) and turboprop MRI. Magn Reson Med 2009;62(1):174-82.

[75] Forbes KP, Pipe JG, Karis JP, Farthing V, Heiserman JE. Brain imaging in the unsedated pediatric patient: comparison of periodically rotated overlapping parallel lines with enhanced reconstruction and single-shot fast spin-echo sequences. AJNR Am J Neuroradiol 2003;24(5):794-8.

[76] Hess AT TM, Andronesi OC, Meintjes EM, van der Kouwe AJW. Real-time Motion and B0 corrected single voxel spectroscopy using volumetric navigators. Magnetic Resonance in Medicine (in press) 2011.

[77] Tisdall MD HA, van der Kouwe AJW. Selective k-space Reacquisition in Anatomical Brain Sequences using EPI Navigators. 2010.

[78] White N, Roddey C, Shankaranarayanan A, Han E, Rettmann D, Santos J, et al. PROMO: Real-time prospective motion correction in MRI using image-based tracking. Magn Reson Med;63(1):91-105.

[79] Orcesi S, La Piana R, Fazzi E. Aicardi-Goutieres syndrome. Br Med Bull 2009;89:183-201.

[80] Uggetti C, La Piana R, Orcesi S, Egitto MG, Crow YJ, Fazzi E. Aicardi-Goutieres syndrome: neuroradiologic findings and follow-up. AJNR Am J Neuroradiol 2009;30(10):1971-6.

[81] Lopez-Hernandez T, Ridder MC, Montolio M, Capdevila-Nortes X, Polder E, Sirisi S, et al. Mutant GlialCAM causes megalencephalic leukoencephalopathy with subcortical cysts, benign familial macrocephaly, and macrocephaly with retardation and autism. Am J Hum Genet;88(4):422-32.

[82] Lopez-Hernandez T, Sirisi S, Capdevila-Nortes X, Montolio M, Fernandez-Duenas V, Scheper GC, et al. Molecular mechanisms of MLC1 and GLIALCAM mutations in megalencephalic leukoencephalopathy with subcortical cysts. Hum Mol Genet. 
[83] van der Knaap MS, Wevers RA, Struys EA, Verhoeven NM, Pouwels PJ, Engelke UF, et al. Leukoencephalopathy associated with a disturbance in the metabolism of polyols. Ann Neurol 1999;46(6):925-8.

[84] Moolenaar SH, van der Knaap MS, Engelke UF, Pouwels PJ, Janssen-Zijlstra FS, Verhoeven NM, et al. In vivo and in vitro NMR spectroscopy reveal a putative novel inborn error involving polyol metabolism. NMR Biomed 2001;14(3):167-76. 